\title{
High-Throughput Calculations of Magnetic Topological Materials
}

\author{
Yuanfeng Xu, ${ }^{1}$ Luis Elcoro, ${ }^{2}$ Zhida Song, ${ }^{3}$ Benjamin J. Wieder, ${ }^{4,5,3}$ M. G. Vergniory,,${ }^{6,7}$ \\ Nicolas Regnault, ${ }^{8,3}$ Yulin Chen, ${ }^{9,10,11,12}$ Claudia Felser, ${ }^{13,14}$ and B. Andrei Bernevig ${ }^{3,1,15, \text { * }}$ \\ ${ }^{1}$ Max Planck Institute of Microstructure Physics, 06120 Halle, Germany \\ ${ }^{2}$ Department of Condensed Matter Physics, University of the Basque Country UPV/EHU, Apartado 644, 48080 Bilbao, Spain \\ ${ }^{3}$ Department of Physics, Princeton University, Princeton, New Jersey 08544, USA \\ ${ }^{4}$ Department of Physics, Massachusetts Institute of Technology, Cambridge, MA 02139, USA \\ ${ }^{5}$ Department of Physics, Northeastern University, Boston, MA 02115, USA \\ ${ }^{6}$ Donostia International Physics Center, P. Manuel de Lardizabal 4, 20018 Donostia-San Sebastian, Spain \\ ${ }^{7}$ IKERBASQUE, Basque Foundation for Science, Bilbao, Spain \\ ${ }^{8}$ Laboratoire de Physique de l'Ecole normale supérieure, \\ ENS, Université PSL, CNRS, Sorbonne Université, \\ Université Paris-Diderot, Sorbonne Paris Cité, Paris, France \\ ${ }^{9}$ School of Physical Science and Technology, ShanghaiTech University, Shanghai 201210, China \\ ${ }^{10}$ ShanghaiTech Laboratory for Topological Physics, Shanghai 200031, China \\ ${ }^{11}$ Clarendon Laboratory, Department of Physics, University of Oxford, Oxford OX1 3PU, UK \\ ${ }^{12}$ State Key Laboratory of Low Dimensional Quantum Physics, \\ Department of Physics and Collaborative Innovation Center of Quantum Matter, Tsinghua University, Beijing 100084, China \\ ${ }^{13}$ Max Planck Institute for Chemical Physics of Solids, Dresden D-01187, Germany \\ ${ }^{14}$ Center for Nanoscale Systems, Faculty of Arts and Science, \\ Harvard University, 11 Oxford Street, LISE 308Cambridge, MA 021138, USA \\ ${ }^{15}$ Physics Department, Freie Universitat Berlin, Arnimallee 14, 14195 Berlin, Germany
}

(Dated: February 11, 2023)

\begin{abstract}
The discoveries of intrinsically magnetic topological materials, including semimetals with a large anomalous Hall effect and axion insulators [1 3], have directed fundamental research in solid-state materials. Topological Quantum Chemistry [4 has enabled the understanding of and the search for paramagnetic topological materials [5, 6]. Using magnetic topological indices obtained from magnetic topological quantum chemistry (MTQC) [7, here we perform the first high-throughput search for magnetic topological materials. here we perform a high-throughput search for magnetic topological materials based on first-principles calculations. We use as our starting point the Magnetic Materials Database on the Bilbao Crystallographic Server, which contains more than 549 magnetic compounds with magnetic structures deduced from neutron-scattering experiments, and identify 130 enforced semimetals (for which the band crossings are implied by symmetry eigenvalues), and topological insulators. For each compound, we perform complete electronic structure calculations, which include complete topological phase diagrams using different values of the Hubbard potential. Using a custom code to find the magnetic co-representations of all bands in all magnetic space groups, we generate data to be fed into the algorithm of MTQC to determine the topology of each magnetic material. Several of these materials display previously unknown topological phases, including symmetry-indicated magnetic semimetals, three-dimensional anomalous Hall insulators and higher-order magnetic semimetals. We analyse topological trends in the materials under varying interactions: 60 per cent of the 130 topological materials have topologies sensitive to interactions, and the others have stable topologies under varying interactions. We provide a materials database for future experimental studies and open-source code for diagnosing topologies of magnetic materials.
\end{abstract}

\section{CONTENTS}

I. Introduction

II. Workflow

III. Topological phase diagrams

3

IV. High-quality topological materials

V. Consistency with previous works

6
VI. Chemical Categories

VII. Discussion

VIII. Conclusion

IX. Methods

A. A brief introduction to Magnetic Topological Quantum Chemistry (MTQC)

B. Material statistics in the BCSMD

14

C. Computational methods

1. Convention setting of the magnetic unit cell 
2. Parameters setting in $a b$ initio calculations

17

3. Magnetic VASP2trace package

4. Construction of Wannier tight-binding

Hamiltonian and surface states calculation

D. Comparison of the ground state energy between different magnetic configurations of several compounds

E. Comparisons between different exchange-correlation potentials

1. Band structure calculations with GGA functional

2. Band structure calculations with meta-GGA functional

F. Comparisons between $\mathrm{LDA}+\mathrm{U}$ and LDA + Gutzwiller methods

G. Topological phase diagrams of the topological materials that predicted by MTQC

H. Physical interpretations for the TI classified by MTQC

1. Definitions for the stable indices of MSG 2.4

2. Definitions for the stable indices of MSG 47.249

3. Definitions for the stable indices of MSG 81.33

4. Definitions for the stable indices of MSG 83.43

5. Definitions for the stable indices of MSG 143.1

6. Stable indices of the magnetic TIs

I. Compatibility-relations along high-symmetry paths of the symmetry enforced semimetals

J. Detailed discussion of the ideal magnetic TI and SMs

1. Higher-order topology of the ideal Axion insulator $\mathrm{NpBi}$

2. Topological phase diagram of the ideal antiferromagnetic nodal-line semimetal $\mathrm{CeCo}_{2} \mathrm{P}_{2}$

3. Topological phase diagram of the antiferromagnetic Dirac semimetal $\mathrm{MnGeO}_{3}$

4. Weyl nodes, Nodal-lines and Anomalous Hall effect in $\mathrm{Mn}_{3} \mathrm{ZnC}$

K. Fragile bands in the magnetic materials

L. Magnetic moments for each materials with different Coulomb interactions

1. Ferro(Ferri)magnetic materials
18

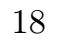

19

19
M. Band structures and detailed information

123

References

225

\section{INTRODUCTION}

Non-magnetic topological materials have dominated the landscape of topological physics for the past two decades. Research in this field has led to a rapid succession of theoretical and experimental discoveries; notable examples include the theoretical prediction of the first topological insulators (TIs) in two [8, 9] and three spatial dimensions [10, topological crystalline insulators [11, Dirac and Weyl semimetals [1218, and non-symmorphic topological insulators and semimetals [19 23]. Though topological materials were once believed to be rare and esoteric, recent advances in nonmagnetic topological materials have found that TIs and enforced semimetals (ESs) are much more prevalent than initially thought. In 2017 Topological Quantum Chemistry (TQC) and the equivalent method of symmetry-based indicators provided a description of the universal global properties of all possible atomic limit band structures in all non-magnetic symmetry groups, in real and momentum space [4, 24, 27]. This allowed for a classification of the non-magnetic, non-trivial (topological) band structures through high-throughput methods that have changed our understanding of the number of topological materials existent in nature. About $40 \%-50 \%$ of all non-magnetic materials can be classified as topological at the Fermi level [5, 6, 28, leading to a "periodic table" of topological materials.

These breakthroughs in non-magnetic materials have not yet been matched by similar advances in magnetic compounds, due to a multitude of challenges. First, although a method for classifying band topology in the 1651 magnetic and nonmagnetic space groups (MSGs and SGs, respectively) was recently introduced [29], there still does not exist a theory similar to TQC or equivalent methods [4, 24,27] by which the indicator groups in Ref. 29] can be linked to topological (anomalous) surface (and hinge) states. Second, a full classification of the magnetic co-representations and compatibility relations has not yet been tabulated. Third, code to compute the magnetic co-representations from $a b$ initio band structures does not exist. Fourth, and finally, even if all the above were available, the $a b$ initio calculation of magnetic compounds is notoriously inaccurate for complicated magnetic structures beyond ferromagnets. Specifically, unless the magnetic structure of a material is known a priori, then the $a b$ initio calculation will likely converge to a misleading ground state. This has rendered the number of accurately predicted magnetic topological materials to be less than 10 [1, 3, 30,39].

In the present work and in ref. [7], we present substantial advances towards solving all of the above challenges - which we have made freely 
available to the public on internet repositories (https://www.cryst.ehu.es/cryst/checktopologicalmagmat) -covering four years of our work on the subject, and over 70 years [40] of research on the group theory, symmetry, and topology of magnetic materials. We present a full theory of magnetic indices, co-representations, compatibility relations, code with which to compute the magnetic co-representations directly from ab initio calculations, and we perform full local density approximation (LDA) + Hubbard U calculations on 549 magnetic structures, which have been accurately tabulated through the careful analysis of neutron-scattering data. We predict several novel magnetic topological phases in real materials, including higher-order magnetic Dirac semimetals with hinge arcs [41, magnetic chiral crystals with long Fermi arcs, Dirac semimetals with nodes not related by time-reversal symmetry, Weyl points and nodal lines in non-collinear antiferrimagnets, and ideal axion insulators with gapped surface states and chiral hinge modes [42, 43.

\section{WORKFLOW}

Starting from the material database MAGNDATA MAGNDATA on the Bilbao Crystallographic Server (BCS) (the BCSMD), which contains portable magnetic structure files determined by neutron scattering experiments of more than 707 magnetic structures, we select 549 high-quality magnetic structures for the ab initio calculations. We take the magnetic configurations provided by BCSMD as the initial inputs and then perform ab initio calculations incorporating spin-orbit coupling. LDA $+\mathrm{U}$ are applied for each material with different Hubbard $U$ parameters to obtain a full phase diagram. Then, we calculate the character tables of the valence bands of each material using the MagVasp2trace package. By feeding the character tables into the machinery of MTQC, that is, the Check Topological Magnetic Mat. (https://www.cryst.ehu.es/cryst/checktopologicalmagmat) function on BCS [7, we identify the corresponding magnetic co-representations (irreps) and classify the materials into different topological categories. Here we define six topological categories:

1. Band representation. Insulating phase consistent with atomic insulators.

2. Enforced semimetal with Fermi degeneracy (ESFD). Semimetal phase with a partially filled degenerate level at a high symmetry point in the Brillouin zone.

3. Enforced semimetal. Semimetal phase with un-avoidable level crossings along high symmetry lines or in high-symmetry planes in the Brillouin zone.
4. Smith-index semimetal (SISM). Semimetal phase with un-avoidable level crossings at generic points (away from high symmetry points/lines/planes) in the Brillouin zone.

5. Stable topological insulator. Insulating phase inconsistent with atomic insulators. The topology (inconsistency with atomic insulators) is stable against being coupled to atomic insulators.

6. Fragile topological insulator. Insulating phase inconsistent with atomic insulators. The topology is unstable against being coupled to certain atomic insulators.

Further details about BCSMD, the calculation methods, the MagVasp2trace package, the identification of magnetic irreps, and definitions of the topological categories are given in the Methods.

\section{TOPOLOGICAL PHASE DIAGRAMS}

With the irreps successfully identified, we classify 403 magnetic structures with convergent ground states into the six topological categories. We find that there are 130 materials (about $32 \%$ of the total) that exhibit nontrivial topology for at least one of the $U$ values in the phase diagram. We sort these materials into four groups based on their U-dependence: (1) 50 materials belong to the same topological categories for all values of U. These are the most robust topological materials. (2) 49 materials, on the other hand, are nontrivial at $\mathrm{U}=0$ but become trivial when $\mathrm{U}$ is larger than a critical value. (3) 20 materials have non-monotonous dependence on $\mathrm{U}$ : they belong to one topologically nontrivial categories at $\mathrm{U}=0$ and change to a different topologically nontrivial category at a larger value of U. (4) Six materials are trivial at $\mathrm{U}=0$ but become nontrivial after a critical value of $\mathrm{U}$. The topology of these six interesting materials is thus driven by electron-electron interactions. The materials in this category are: $\mathrm{CaCo}_{2} \mathrm{P}_{2}, \quad \mathrm{YbCo}_{2} \mathrm{Si}_{2}, \quad \mathrm{Ba}_{5} \mathrm{Co}_{5} \mathrm{ClO}_{13}$, $\mathrm{U}_{2} \mathrm{Ni}_{2} \mathrm{Sn}, \mathrm{CeCoGe}_{3}$, and CeMnAsO. The self-consistent calculations of the remaining 5 materials do not converge for at least one value of $U$, and hence the phase diagrams are not complete. Complete classifications of the converged materials are tabulated in Appendix G: the corresponding band structures are given in Appendix M. In Table Table [1] we summarize the total number of topological materials in each magnetic space group (MSG) at different values of U. We have also provided full topological classifications and band structures of each material on the Topological Magnetic Materials Database (https://www.topologicalquantumchemistry.fr/magnetic).

In the scheme of MTQC, the stable magnetic topological insulators and SISMs are characterized by non-zero stable indices. These indices can be understood as generalizations of the $\mathrm{Fu}$-Kane parity criterion for 
a three-dimensional topological insulator 44. A complete table of the stable indices and the index-implied topological invariants, which include (weak) Chern numbers, the axion $\theta$ angle, and magnetic higher-order topological insulator (HOTI) indices, is given in ref. [7. In Appendix $\mathrm{H}$, we present examples of stable indices relevant to the present work, as well as their physical interpretations. Alhough there are many $(1,651)$ magnetic and nonmagnetic space groups, we find in ref. [7] that the stable indices of all of the MSGs are dependent on minimal indices in the set of the so-called minimal groups. Thus, to determine the stable indices of a material, we first subduce the representation of the MSG formed by the material to a representation of the corresponding minimal group - a subgroup of the MSG on which the indices are dependent. We then calculate the indices in the minimal group. Using this method, we find a tremendous variety of topological phases among the magnetic materials studied in this work, including axion insulators 45 48, mirror topological crystalline insulators 11, three-dimensional quantum anomalous Hall insulators, and SISMs. A complete table of the topology of all of the magnetic materials studied in this work is provided in Appendix $\mathrm{G}$.

We additionally discover many ESFD and enforced semimetal materials, in which unavoidable electronic band crossings respectively occur at high-symmetry $\mathbf{k}$ points or on high-symmetry lines or planes in the Brillouin zone. For each of the ESFD and enforced semimetal magnetic materials, we tabulate the $\mathbf{k}$ points where unavoidable crossings occur (see Appendix I).

We did not discover any examples of magnetic materials for which the entire valence manifold is fragile topological. However, as will be discussed below, we discovered many magnetic materials with well isolated fragile bands in their valence manifolds, thereby providing examples of magnetic fragile bands in real materials.

\section{HIGH-QUALITY TOPOLOGICAL MATERIALS}

We here select several representative "high-quality" topological materials with clean band structures at the Fermi level: NpBi in MSG $224.113\left(P n \overline{3} m^{\prime}\right)$ (antiferromagnetic stable topological insulator), $\mathrm{CaFe}_{2} \mathrm{As}_{2}$ in MSG $64.480\left(C_{A} m c a\right.$ ) (antiferromagnetic stable topological insulator), NpSe in MSG 228.139 $\left(F_{S} d \overline{3} c\right) \quad$ (antiferromagnetic ESFD), $\mathrm{CeCo}_{2} \mathrm{P}_{2}$ in MSG $126.386 \quad\left(P_{I} 4 / n n c\right)$ (antiferromagnetic enforced semimetal), $\mathrm{MnGeO}_{3}$ in $\mathrm{MSG} 148.19\left(R \overline{3}^{\prime}\right)$ (antiferromagnetic enforced semimetal), $\mathrm{Mn}_{3} \mathrm{ZnC}$ in MSG $139.537\left(14 / \mathrm{mm}^{\prime} \mathrm{m}^{\prime}\right)$ (non-collinear ferrimagnetic enforced semimetal), as shown in Fig. 1.

To identify the stable topologies of the antiferrimagnets $\mathrm{NpBi}$ and $\mathrm{CaFe}_{2} \mathrm{As}_{2}$, we calculate

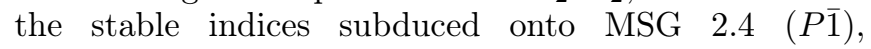

a subgroup of MSG $224.113\left(\operatorname{Pn} \overline{3} m^{\prime}\right)$ and 64.480 $\left(C_{A} m c a\right)$.

The stable indices in MSG $2.4(P \overline{1})$ are defined using only parity (inversion) eigenvalues [7, 29, 42, 49, 51]:

$$
\begin{array}{r}
\eta_{4 I}=\sum_{K} n_{K}^{-} \bmod 4 \\
z_{2 I, i}=\sum_{K, K_{i}=\pi} n_{K}^{-} \bmod 2
\end{array}
$$

where $K$ sums over the eight inversion-invariant momenta, and $n_{K}^{-}$is the number of occupied states with odd parity eigenvalues at momentum $K . z_{2 I, i}$ is the parity of the Chern number of the Bloch states in the plane $k_{i}=\pi$. As explained in Appendix $\mathrm{H}_{1}$, $\eta_{4 I}=1,3$ correspond to Weyl semimetal (WSM) phases with odd-numbered Weyl points in each half of the BZ; $\eta_{4 I}=2$ indicates an axion insulator phase provided that the band structure is fully gapped and the weak Chern numbers in all directions are zero. The inversion eigenvalues of $\mathrm{NpBi}$ with $U=2 \mathrm{eV}$ and $\mathrm{CaFe}_{2} \mathrm{As}_{2}$ with $U=2 \mathrm{eV}$ are tabulated in Table II. The corresponding band structures are shown in Fig. 2 a and Fig. 2 b, respectively. Both $\mathrm{NpBi}$ and $\mathrm{CaFe}_{2} \mathrm{As}_{2}$ have the indices $\left(\eta_{4 I}, z_{2 I, 1}, z_{2 I, 2}, z_{2 I, 3}\right)=(2,0,0,0)$. As shown in Appendix $\mathrm{H} 6$, the $\eta_{4 I}$ index of $\mathrm{NpBi}$ is the same for $U=0,2,4$, $6 \mathrm{eV}$; whereas the $\eta_{4 I}$ index of $\mathrm{CaFe}_{2} \mathrm{As}_{2}$ is 2 for $U=0$, $1,2 \mathrm{eV}$ and 0 for $U=3,4 \mathrm{eV}$. We have confirmed that both $\mathrm{NpBi}$ and $\mathrm{CaFe}_{2} \mathrm{As}_{2}$ have vanishing weak Chern numbers, implying that the $\eta_{4 I}=2$ phases must be axion insulators.

An axion insulator is defined by a nontrivial $\theta$ angle, which necessitates a quantized magneto-electric response in the bulk and chiral hinge modes on the boundary 43 , 45, 48. We have calculated the surface states of $\mathrm{NpBi}$ and find that, as expected, the (001) surface is fully gapped (Fig. 2a). Owing to the $C_{3}$-rotation symmetry of the MSG 224.113, the (100) and (010) surfaces are also gapped. Therefore, a cubic sample with terminating surfaces in the (100), (010) and (001) directions, as shown in Fig. 2a, exhibits completely gapped surfaces. However, as an axion insulator, it must exhibit chiral hinge modes when terminated in an inversion-symmetric geometry [42, 43]. We predict that the chiral hinge modes exist on the edges shown in Fig. 2a. More details about $\mathrm{NpBi}$ are provided in Appendix $\mathrm{J} 1$.

Next, we discuss representative examples of magnetic topological semimetals. The antiferrimagnet NpSe with $U=2 \mathrm{eV}, 4 \mathrm{eV}$ and $6 \mathrm{eV}$ is an ESFD with a partially-filled degenerate band at the $\Gamma$ point, where the lowest conduction bands and the highest valence bands meet in a fourfold degeneracy (Fig. 11. The antiferrimagnet $\mathrm{CeCo}_{2} \mathrm{P}_{2}$ is an enforced semimetal at all the $U$ values used in our calculations. For $U=$ $0 \mathrm{eV}$ and $2 \mathrm{eV}$, we predict $\mathrm{CeCo}_{2} \mathrm{P}_{2}$ to be a Dirac semimetal protected by $C_{4}$ rotation symmetry. Because the Dirac points in $\mathrm{CeCo}_{2} \mathrm{P}_{2}$ lie along a high-symmetry line $(\Gamma \mathrm{Z})$ whose little group contains $4 \mathrm{~mm}$, we see that 
TABLE I. Topological categories vary with $U$. Shown are the number of magnetic topological insulators/SISMs and enforced semimetals/ESFDs in each MSG for different values of the Hubbard interaction $U=0 \mathrm{eV}, 2 \mathrm{eV}$ and $4 \mathrm{eV}$. For $U=0$, there are 38 topological insulators/SISMs and 73 enforced semimetals/ESFDs in total. For $U=2$ eV, the numbers of topological insulators/SISMs and enforced semimetals/ESFDs decrease to 27 and 58, respectively. For $U=4$ eV, the numbers of topological insulators/SISMs and enforced semimetals/ESFDs decrease to 24 and 57, respectively. Choosing the value of $U$ for each material for which the magnetic moments calculated ab initio lie closest to their experimentally measured values, there are 29 topological insulators/SISMs and 62 enforced semimetals/ESFDs.

\begin{tabular}{|c|c|c|c|c|c|c|c|c|c|c|c|c|c|c|c|c|c|c|c|c|}
\hline MSG & $\begin{array}{r}\mathrm{TI} \\
\mathrm{U}=0\end{array}$ & $\begin{array}{l}\mathrm{s} / \mathrm{SISI} \\
\mathrm{U}=2\end{array}$ & $\begin{array}{l}\overline{M s} \\
\mathrm{U}=4\end{array}$ & $\begin{array}{r}\mathrm{ES} \\
\mathrm{U}=0\end{array}$ & $\begin{array}{l}\mathrm{s} / \mathrm{ESH} \\
\mathrm{U}=2\end{array}$ & $\begin{array}{l}\mathrm{Ds} \\
\mathrm{U}=4\end{array}$ & MSG & $\begin{array}{c}\mathrm{TI} \\
\mathrm{U}=0\end{array}$ & $\begin{array}{r}\mathrm{s} / \mathrm{SIS} \\
\mathrm{U}=2\end{array}$ & $\begin{array}{l}\overline{M s} \\
\mathrm{U}=4\end{array}$ & $\begin{array}{r}\mathrm{ES} \\
\mathrm{U}=0\end{array}$ & $\begin{array}{c}\mathrm{s} / \mathrm{ESH} \\
\mathrm{U}=2\end{array}$ & $\begin{array}{l}\mathrm{Ds} \\
\mathrm{U}=4\end{array}$ & MSG & $\begin{array}{c}\mathrm{TI} \\
\mathrm{U}=0\end{array}$ & $\begin{array}{l}\mathrm{S} / \mathrm{SIS} \\
\mathrm{U}=2\end{array}$ & $\begin{array}{l}\overline{M s} \\
\mathrm{U}=4\end{array}$ & $\begin{array}{r}E S \\
U=0\end{array}$ & $\begin{array}{c}\mathrm{s} / \mathrm{ESF} \\
\mathrm{U}=2\end{array}$ & $\begin{array}{l}\mathrm{FDs} \\
\mathrm{U}=4\end{array}$ \\
\hline 2.7 & 1 & 0 & 1 & 0 & 1 & 0 & 62.447 & 0 & 1 & 0 & 1 & 0 & 0 & \begin{tabular}{|l|}
129.416 \\
\end{tabular} & 0 & 0 & 1 & 0 & 1 & 0 \\
\hline 4.7 & 0 & 1 & 0 & 0 & 0 & 0 & 62.450 & 2 & 2 & 1 & 2 & 1 & 2 & 130.432 & 0 & 1 & 0 & 1 & 0 & 1 \\
\hline 11.54 & 1 & 0 & 1 & 0 & 0 & 0 & 63.462 & 0 & 2 & 0 & 2 & 0 & 2 & 132.456 & 0 & 1 & 0 & 1 & 0 & 1 \\
\hline 11.57 & 1 & 0 & 0 & 0 & 0 & 0 & 63.463 & 0 & 1 & 0 & 1 & 0 & 1 & 134.481 & 3 & 0 & 1 & 1 & 2 & 0 \\
\hline 12.62 & 2 & 0 & 1 & 0 & 1 & 0 & 63.464 & 1 & 1 & 1 & 1 & 0 & 1 & 135.492 & 0 & 2 & 0 & 2 & 0 & 2 \\
\hline 12.63 & 0 & 0 & 0 & 0 & 0 & 0 & 63.466 & 0 & 0 & 2 & 0 & 1 & 1 & 138.528 & 1 & 0 & 1 & 0 & 1 & 0 \\
\hline 13.73 & 2 & 0 & 2 & 0 & 1 & 0 & 63.467 & 0 & 0 & 0 & 0 & 1 & 0 & 139.536 & 0 & 1 & 0 & 1 & 0 & 1 \\
\hline 14.75 & 0 & 1 & 0 & 0 & 0 & 0 & 64.480 & 3 & 0 & 2 & 0 & 0 & 1 & 139.537 & 0 & 1 & 0 & 1 & 0 & 1 \\
\hline 14.80 & 1 & 0 & 0 & 0 & 0 & 0 & 65.486 & 0 & 0 & 0 & 1 & 0 & 1 & 140.550 & 0 & 2 & 1 & 2 & 1 & 2 \\
\hline 15.89 & 2 & 1 & 3 & 0 & 3 & 0 & 65.489 & 1 & 0 & 0 & 1 & 0 & 1 & 141.556 & 0 & 1 & 0 & 0 & 1 & 0 \\
\hline 15.90 & 4 & 0 & 0 & 0 & 0 & 0 & 67.510 & 0 & 0 & 1 & 0 & 0 & 0 & 141.557 & 1 & 4 & 0 & 1 & 0 & 1 \\
\hline 18.22 & 0 & 1 & 0 & 0 & 0 & 0 & 70.530 & 0 & 1 & 0 & 0 & 0 & 0 & 148.19 & 0 & 1 & 0 & 1 & 0 & 1 \\
\hline 33.154 & 0 & 1 & 0 & 0 & 0 & 0 & 71.536 & 0 & 1 & 0 & 1 & 0 & 1 & 155.48 & 0 & 0 & 0 & 0 & 0 & 1 \\
\hline 36.178 & 0 & 0 & 0 & 0 & 0 & 1 & 73.553 & 1 & 0 & 1 & 0 & 1 & 0 & 161.69 & 0 & 1 & 0 & 1 & 0 & 0 \\
\hline 38.191 & 0 & 1 & 0 & 0 & 0 & 0 & 74.559 & 0 & 1 & 0 & 1 & 0 & 1 & 161.71 & 0 & 2 & 0 & 2 & 0 & 0 \\
\hline 49.270 & 0 & 1 & 0 & 1 & 0 & 1 & 85.59 & 0 & 1 & 0 & 1 & 0 & 1 & 165.95 & 0 & 1 & 0 & 0 & 0 & 0 \\
\hline 49.273 & 0 & 1 & 1 & 0 & 1 & 0 & 88.81 & 0 & 1 & 0 & 0 & 0 & 0 & 166.101 & 1 & 3 & 0 & 3 & 1 & 2 \\
\hline 51.295 & 0 & 1 & 0 & 1 & 0 & 1 & 92.114 & 0 & 1 & 0 & 1 & 0 & 1 & 166.97 & 0 & 1 & 0 & 1 & 0 & 1 \\
\hline 51.298 & 0 & 1 & 0 & 1 & 0 & 1 & 107.231 & 0 & 0 & 0 & 1 & 0 & 1 & 167.108 & 0 & 1 & 0 & 0 & 0 & 0 \\
\hline 53.334 & 0 & 0 & 0 & 0 & 1 & 0 & 114.282 & 0 & 1 & 0 & 0 & 0 & 0 & 185.201 & 1 & 0 & 0 & 0 & 0 & 0 \\
\hline 57.391 & 1 & 0 & 1 & 0 & 1 & 0 & 123.345 & 0 & 1 & 0 & 1 & 0 & 1 & 192.252 & 0 & 2 & 0 & 2 & 0 & 2 \\
\hline 58.398 & 0 & 1 & 0 & 0 & 0 & 0 & 124.360 & 1 & 3 & 0 & 4 & 0 & 4 & 194.268 & 0 & 0 & 0 & 0 & 0 & 1 \\
\hline 58.399 & 0 & 2 & 0 & 2 & 0 & 2 & 125.373 & 0 & 1 & 0 & 1 & 0 & 1 & 205.33 & 1 & 0 & 0 & 0 & 0 & 0 \\
\hline 59.407 & 0 & 1 & 0 & 0 & 0 & 0 & 126.386 & 0 & 1 & 0 & 1 & 0 & 1 & 222.103 & 0 & 1 & 0 & 1 & 0 & 1 \\
\hline 59.416 & 0 & 0 & 1 & 0 & 1 & 0 & 127.394 & 1 & 1 & 1 & 1 & 1 & 1 & 224.113 & 2 & 1 & 2 & 0 & 2 & 0 \\
\hline 60.431 & 1 & 0 & 0 & 0 & 0 & 0 & 127.397 & 0 & 1 & 0 & 1 & 0 & 1 & 227.131 & 0 & 1 & 0 & 0 & 0 & 0 \\
\hline 61.439 & 0 & 0 & 1 & 0 & 0 & 0 & 128.408 & 0 & 1 & 0 & 1 & 0 & 1 & 228.139 & 2 & 1 & 0 & 3 & 0 & 3 \\
\hline 62.441 & 0 & 3 & 0 & 0 & 0 & 0 & 128.410 & 0 & 4 & 0 & 4 & 0 & 4 & Total & 38 & 27 & 24 & 73 & 58 & 57 \\
\hline
\end{tabular}

TABLE II. Parities and topological indices of two magnetic topological insulators. Shown are the numbers of occupied bands with odd/even parity eigenvalues at the eight inversion-invariant points $\left(\eta_{\alpha}\right)$ for the magnetic topological insulators NpX (where $\mathrm{X}=\mathrm{Sb}, \mathrm{Bi}$ ) and $\mathrm{XFe}_{2} \mathrm{As}_{2}$ (where $\mathrm{X}=\mathrm{Ca}, \mathrm{Ba}$ ) with $U=2 \mathrm{eV}$. The $\eta_{4 I}=2$ phase corresponds to an axion insulator or Weyl semimetal phase with even pairs of Weyl points at generic locations in the Brillouin zone interior (given vanishing weak Chern numbers), and $\eta_{4 I}=1,3$ corresponds to a Weyl semimetal phase with odd number of Weyl points at generic locations within

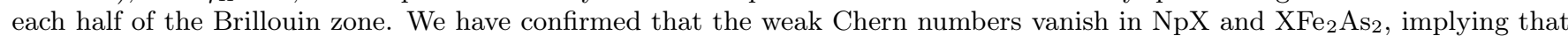
both materials are axion insulators.

\begin{tabular}{c|llllllll|c}
\hline \hline$\Lambda_{\alpha}$ & $(0,0,0)$ & $(\pi, 0,0)$ & $(0, \pi, 0)$ & $(\pi, \pi, 0)$ & $(0,0, \pi)$ & $(\pi, 0, \pi)$ & $(0, \pi, \pi)$ & $(\pi, \pi, \pi)$ & $\left(\eta_{4 I}, z_{2 I, 1}, z_{2 I, 2}, z_{2 I, 3}\right)$ \\
\hline $\mathrm{NpX}$ & $58 / 22$ & $40 / 40$ & $40 / 40$ & $40 / 40$ & $40 / 40$ & $40 / 40$ & $40 / 40$ & $48 / 32$ & $(2,0,0,0)$ \\
\hline $\mathrm{XFe}_{2} \mathrm{As}_{2}$ & $50 / 46$ & $48 / 48$ & $48 / 48$ & $52 / 44$ & $48 / 48$ & $52 / 44$ & $52 / 44$ & $48 / 48$ & $(2,0,0,0)$ \\
\hline \hline
\end{tabular}

$\mathrm{CeCo}_{2} \mathrm{P}_{2}$ is a higher-order topological semimetal that exhibits flat-band-like higher-order Fermi-arc states on mirror-invariant hinges, analogous to the hinge states recently predicted 41] and experimentally observed [52] in the non-magnetic Dirac semimetal $\mathrm{Cd}_{3} \mathrm{As}_{2}$. For $U=4$ $\mathrm{eV}$ and $6 \mathrm{eV}, \mathrm{CeCo}_{2} \mathrm{P}_{2}$ becomes a nodal ring semimetal protected by the mirror symmetry $M_{z}$. As detailed in Appendix J2, the transition between the two enforced semimetal phases is completed by two successively band inversions at $\Gamma$ and $\mathrm{Z}$, which removes the Dirac node and creates the nodal ring, respectively. The band structure of the nodal ring semimetal phase at $U=6 \mathrm{eV}$ is plotted in Fig. 1 $1 \mathrm{~d}$. The antiferromagnet $\mathrm{MnGeO}_{3}$ is a $C_{3}$-rotation-protected Dirac semimetal, in which the number of Dirac nodes changes with the value of $U$. For $U=0 \mathrm{eV}, 1 \mathrm{eV}, 3 \mathrm{eV}$ and $4 \mathrm{eV}$, we predict $\mathrm{MnGeO}_{3}$ to have two Dirac nodes along the high-symmetry line $\Gamma F$; for $U=2 \mathrm{eV}$, we observe four Dirac nodes along the same high-symmetry line. In Fig. 11, we plot the band structure of $\mathrm{MnGeO}_{3}$ using $U=4 \mathrm{eV}$. We next 

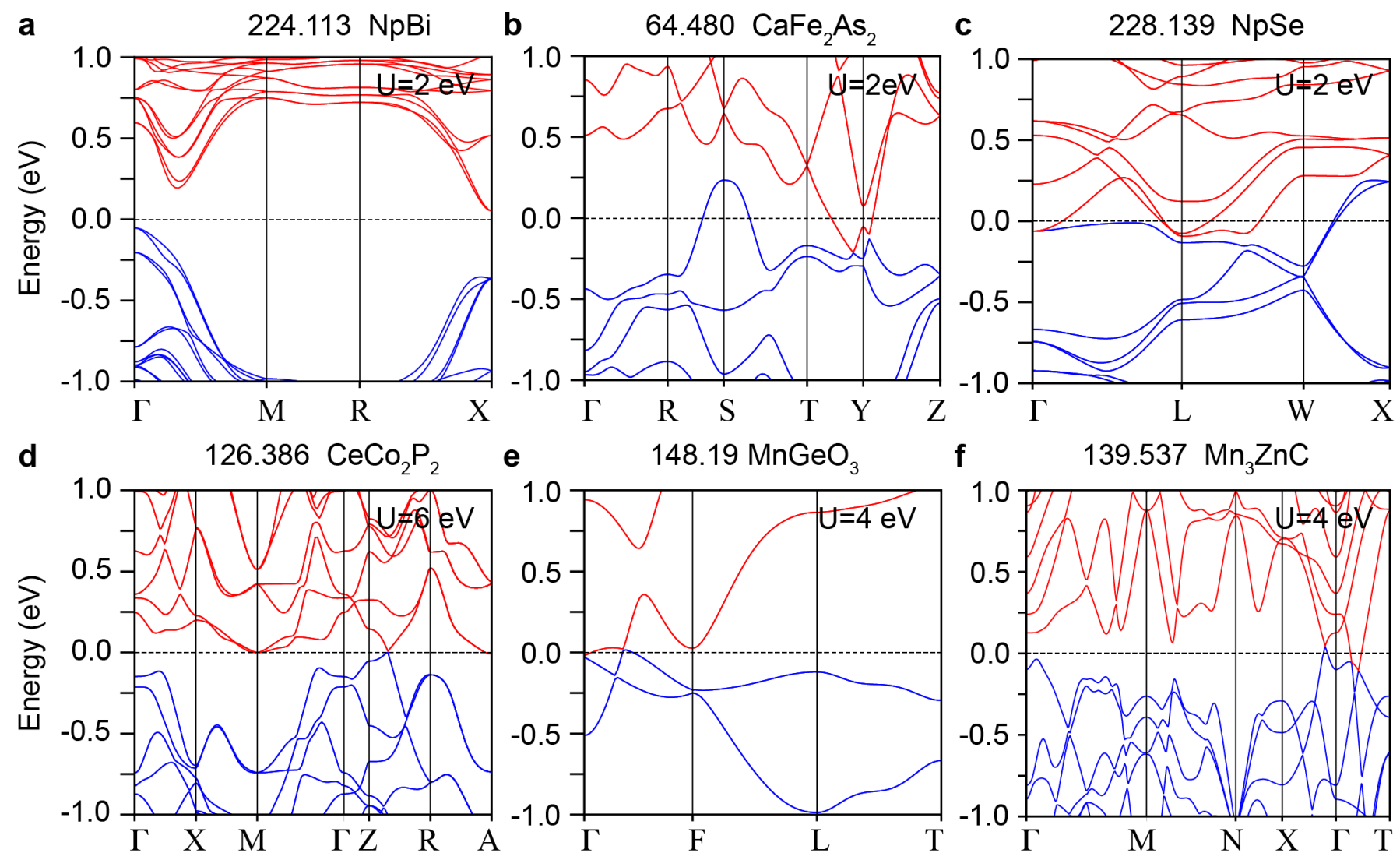

FIG. 1. Band structures of the 'high-quality' magnetic topological materials predicted by MTQC. (a, b) The antiferromagnetic axion topological insulators, $\mathrm{NpBi}$ and $\mathrm{CaFe}_{2} \mathrm{As}_{2}$. Although there are Fermi pockets around $S$ and $Y$ in $\mathrm{CaFe}_{2} \mathrm{As}_{2}$, the insulating compatibility relations are fully satisfied. We note that there is a small gap (about $5 \mathrm{meV}$ ) along the path $\mathrm{T}-\mathrm{Y}$; this indicates that the valence bands are well separated from the conduction bands, and thus have a well defined topology. (c) The antiferromagnetic ESFD NpSe, which has a partially filled fourfold degeneracy at $\Gamma$. (d) The antiferromagnetic nodal-line semimetal $\mathrm{CeCo}_{2} \mathrm{P}_{2}$. A gapless nodal ring protected by mirror symmetry lies in the Z-R-A plane. (e) The antiferromagnetic Dirac semimetal $\mathrm{MnGeO}_{3}$. One of the two Dirac nodes protected by the $C_{3}$-rotation symmetry lies along the high-symmetry line $\Gamma-\mathrm{F}$. Note that there is a small bandgap at the $\Gamma$ point. (f) The non-collinear ferrimagnetic Weyl semimetal $\mathrm{Mn}_{3} \mathrm{ZnC}_{\text {. }}$

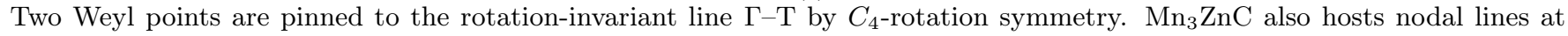
the Fermi level $E_{F}$; we specifically observe five nodal rings protected by the mirror symmetry $\left(M_{z}\right)$ in the plane $k_{z}=0$. The sequential number of each MSG in the BNS setting and the chemical formula of each material are provided on the top of each panel.

predict the non-collinear ferrimagnet $\mathrm{Mn}_{3} \mathrm{ZnC}$ to be an ES with symmetry-enforced Weyl points coexisting with the Weyl nodal rings (Fig. 1f). Two of the Weyl points in $\mathrm{Mn}_{3} \mathrm{ZnC}$ are pinned by the $C_{4}$-rotation symmetry to the high-symmetry line $\Gamma \mathrm{T}$, and we observe five nodal rings protected by the mirror symmetry $M_{z}$ in the $k_{z}=$ 0 plane. In time-reversal-breaking Weyl semimetals, divergent Berry curvature near Weyl points can give rise to a large intrinsic anomalous Hall conductivity [1, 2, 33, 34, 53. We thus expect there to be a large anomalous Hall effect in $\mathrm{Mn}_{3} \mathrm{ZnC}$. As detailed in Appendix J4, we have specifically calculated the the anomalous Hall conductivity of $\mathrm{Mn}_{3} \mathrm{ZnC}$ to be about 123 $\Omega^{-1} \cdot \mathrm{cm}^{-1}$.

The surface states of the enforced semimetals $\mathrm{CeCo}_{2} \mathrm{P}_{2}$ and $\mathrm{MnGeO}_{3}$ are shown in Fig. 2p,c, respectively. Because the bulk states of $\mathrm{CeCo}_{2} \mathrm{P}_{2}$ and $\mathrm{MnGeO}_{3}$ have clean Fermi surfaces, the surface states are well separated from the bulk states, and should be observable in experiment. For the Dirac semimetal $\mathrm{MnGeO}_{3}$, we observe a discontinuous Fermi surface (Fermi-arc) on the surface (Fig. 2d). In Appendix J, we provide further details of our surface-state calculations.

\section{CONSISTENCY WITH PREVIOUS WORKS}

Our magnetic materials database (https://www.topologicalquantumchemistry.fr/magnetic) includes several topological materials that have previously been reported but whose topology was not known to be protected by symmetry eigenvalues. For example, the non-collinear magnet $\mathrm{Mn}_{3} \mathrm{Sn}$ in MSG $63.463\left(\mathrm{Cm}^{\prime} \mathrm{cm}^{\prime}\right)$ has been reported as a magnetic 

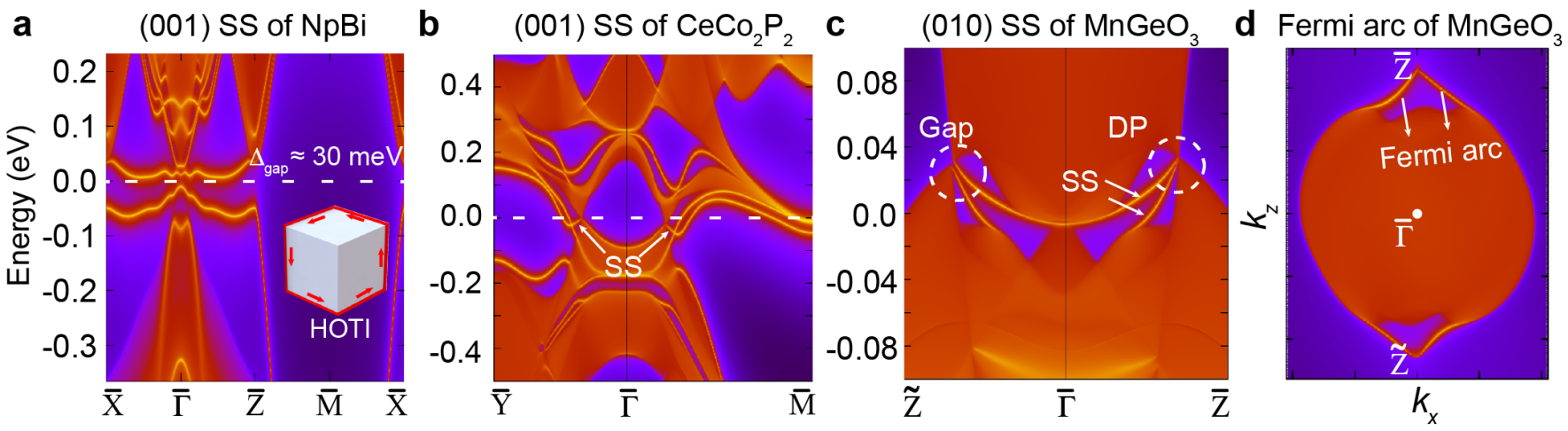

FIG. 2. Topological surface states of representative magnetic topological insulator and enforced semimetal phases. (a) The (001) surface state of the axion insulator $\mathrm{NpBi}$, which has an energy gap of $30 \mathrm{meV}$. The inset shows a schematic of the chiral hinge states on a cubic sample. (b) The (001) surface state of the enforced semimetal $\mathrm{CeCo}_{2} \mathrm{P}_{2}$. The drumhead-like topological surface states connect the projections of the bulk nodal rings. (c) The (010) surface state of the enforced semimetal $\mathrm{MnGeO}_{3}$. The bulk Dirac point along the $\bar{\Gamma}-\bar{Z}$ line is protected by $C_{3}$ symmetry. However, because time-reversal symmetry is broken, the projected band crossing on $\bar{\Gamma}-\tilde{Z}$ (along $-k_{z}$ ) is no longer protected, and is instead weakly gapped. The coordinates of $\bar{Z}$ and $\tilde{Z}$ on the (010) surface are $\left(0, k_{z}=\pi / c\right)$ and $\left(0, k_{z}=-\pi / c\right)$, respectively. (d) The surface Fermi arcs connecting the Dirac points on the (010) surface of $\mathrm{MnGeO}_{3}$.

Weyl semimetal candidate with six pairs of Weyl points [31, 54. In our $\mathrm{LDA}+\mathrm{U}$ calculation, for $U=0 \mathrm{eV}, 1$ $\mathrm{eV}$ and $2 \mathrm{eV}$, we find $\mathrm{Mn}_{3} \mathrm{Sn}$ to be classified instead as a magnetic topological insulator category with the index $\eta_{4 I}=2 . \quad \eta_{4 I}=2$ can correspond to several different topological phases (which we emphasize are not all topological insulators): (1) an axion insulator, (2) a three-dimensional quantum anomalous Hall state with even weak Chern number (not determinable from symmetry eigenvalues) [55, or (3) a Weyl semimetal phase with an even number of Weyl points in half of the BZ (not determinable from symmetry eigenvalues). Thus our calculations on $\mathrm{Mn}_{3} \mathrm{Sn}$ for $U=0,1,2 \mathrm{eV}$ are consistent with the results in refs. 31, 54. We emphasize that if the six Weyl points in half of the Brillouin zone were pairwise annihilated without closing a gap at the inversion-invariant momenta, then the gapped phase would either be an axion insulator or a three-dimensional quantum anomalous Hall state. When $\mathrm{U}$ is further increased to $3 \mathrm{eV}$ and $4 \mathrm{eV}$, a topological phase transition occurs, driving the $\eta_{4 I}=2$ phase into a gapless enforced semimetal phase.

\section{CHEMICAL CATEGORIES}

In Table III we classify the topological magnetic materials predicted by MTQC into three main chemical categories, and 11 sub-categories, through a consideration of their magnetic ions and chemical bonding. Detailed descriptions of each category are given in the Methods. Of the materials listed in Table III most antiferromagnetic insulators, which are well studied experimentally in the case of the so-called Mott insulators, appear to be trivial. We observe that most of the materials in Table III are identified as topological enforced semimetals or ESFDs, which are defined by small densities of states at the Fermi level, and hence lie chemically at the border between insulators and metals.

\section{DISCUSSION}

A large number of the topological materials predicted in this work (see Appendix $\mathrm{G}$ for a complete tabulation) can readily be synthesized into single crystals for the exploration of their unusual physical properties and the confirmation of their topological electronic structures in different phase categories. These include materials with non-trivial topology over the full range of $\mathrm{U}$ values used in our calculations (for example, $\mathrm{Mn}_{3} \mathrm{Ge}$, $\mathrm{Mn}_{3} \mathrm{Sn}, \mathrm{Mn}_{3} \mathrm{Ir}, \mathrm{LuFe}_{4} \mathrm{Ge}_{2}$, and $\mathrm{YFe}_{4} \mathrm{Ge}_{2}$ ), materials sensitive to $U$ (for example, $\mathrm{NdCo}_{2}$ and $\mathrm{NdCo}_{2} \mathrm{P}_{2}$ ), and interaction-driven topological materials (for example, $\mathrm{U}_{2} \mathrm{Ni}_{2} \mathrm{Sn}$ and $\mathrm{CeCuGe}_{3}$ ).

We did not find any examples of materials whose entire valence manifolds are fragile topological. However, it is still possible for well isolated bands within the valence manifold to be fragile topological if they can be expressed as a difference of band representations. We find many examples of energetically well isolated fragile branches among the occupied bands. We tabulate all the fragile branches close to the Fermi level in Appendix $\mathrm{K}$.

We emphasize that there also exist topological insulators and topological semimetals (for example, Weyl semimetals) that cannot be diagnosed through symmetry eigenvalues, which in this work are classified as trivial band representations [4. It is worth mentioning that even the topologically trivial bands may also be 


\begin{tabular}{|c|c|c|}
\hline Categories & Properties & Materials \\
\hline $\mathrm{I}-\mathrm{A}$ & Non-collinear Manganese compounds & $\mathrm{Mn}_{3} \mathrm{GaC}, \mathrm{Mn}_{3} \mathrm{ZnC}, \mathrm{Mn}_{3} \mathrm{CuN}, \mathrm{Mn}_{3} \mathrm{Sn}, \mathrm{Mn}_{3} \mathrm{Ge}, \mathrm{Mn}_{3} \mathrm{Ir}, \mathrm{Mn}_{3} \mathrm{Pt}, \mathrm{Mn}_{5} \mathrm{Si}_{3}$ \\
\hline I-B & Actinide Intermetallic & $\mathrm{UNiGa}_{5}, \mathrm{UPtGa}_{5}, \mathrm{NpRhGa}_{5}, \mathrm{NpNiGa}_{5}$ \\
\hline $\mathrm{I}-\mathrm{C}$ & Rare earth intermetallic & $\begin{array}{l}\mathrm{NdCo}_{2}, \quad \mathrm{TbCo}_{2}, \quad \mathrm{NpCo}_{2}, \quad \mathrm{PrAg} \quad \mathrm{DyCu}, \quad \mathrm{NdZn}, \quad \mathrm{TbMg}, \quad \mathrm{NdMg}, \\
\mathrm{Nd}_{5} \mathrm{Si}_{4}, \quad \mathrm{Nd}_{5} \mathrm{Ge}_{4}, \quad \mathrm{Ho}_{2} \mathrm{RhIn}_{8}, \quad \mathrm{Er}_{2} \mathrm{CoGa}_{8}, \quad \mathrm{Nd}_{2} \mathrm{RhIn}_{8}, \quad \mathrm{Tm}_{2} \mathrm{CoGa}_{8}, \\
\mathrm{Ho}_{2} \mathrm{RhIn}_{8}, \mathrm{DyCo}_{2} \mathrm{Ga}_{8}, \mathrm{TbCo}_{2} \mathrm{Ga}_{8}, \mathrm{Er}_{2} \mathrm{Ni}_{2} \mathrm{In}, \mathrm{CeRu}_{2} \mathrm{Al}_{10}, \mathrm{Nd}_{3} \mathrm{Ru}_{4} \mathrm{Al}_{12}, \\
\mathrm{Pr}_{3} \mathrm{Ru}_{4} \mathrm{Al}_{12}, \mathrm{ScMn}_{6} \mathrm{Ge}_{6}, \mathrm{YFe}_{4} \mathrm{Ge}_{4}, \mathrm{LuFe}_{4} \mathrm{Ge}_{4}, \mathrm{CeCoGe}_{3}\end{array}$ \\
\hline II-A & Metallic Iron pnictides & $\begin{array}{lllll}\mathrm{LaFeAsO}, \quad \mathrm{CaFe}_{2} \mathrm{As}_{2}, \quad \mathrm{EuFe}_{2} \mathrm{As}_{2}, & \mathrm{BaFe}_{2} \mathrm{As}_{2}, & \mathrm{Fe}_{2} \mathrm{As}, & \mathrm{CaFe}_{4} \mathrm{As}_{3}, \\
\mathrm{LaCrAsO}, \mathrm{Cr}_{2} \mathrm{As}, \mathrm{CrAs}, \mathrm{CrN} & & & \end{array}$ \\
\hline II-B & Semiconducting manganese & $\begin{array}{l}\mathrm{BaMn}_{2} \mathrm{As}_{2} \mathrm{BaMn}_{2} \mathrm{Bi}_{2}, \mathrm{CaMnBi}_{2}, \mathrm{SrMnBi}_{2}, \mathrm{CaMn}_{2} \mathrm{Sb}_{2}, \mathrm{CuMnAs} \text {, } \\
\mathrm{CuMnSb}, \mathrm{Mn}_{2} \mathrm{As}\end{array}$ \\
\hline II-C & $\begin{array}{l}\text { Rare earth intermetallic compounds with } \\
\text { the composition } 1: 2: 2\end{array}$ & $\begin{array}{llllll}\mathrm{PrNi}_{2} \mathrm{Si}_{2}, & \mathrm{YbCo}_{2} \mathrm{Si}_{2}, & \mathrm{DyCo}_{2} \mathrm{Si}_{2}, & \mathrm{PrCo}_{2} \mathrm{P}_{2}, & \mathrm{CeCo}_{2} \mathrm{P}_{2}, & \mathrm{NdCo}_{2} \mathrm{P}_{2}, \\
\mathrm{DyCu}_{2} \mathrm{Si}_{2}, & \mathrm{CeRh}_{2} \mathrm{Si}_{2}, & \mathrm{UAu}_{2} \mathrm{Si}_{2}, & \mathrm{U}_{2} \mathrm{Pd}_{2} \mathrm{Sn}, & \mathrm{U}_{2} \mathrm{Pd}_{2} \mathrm{In}, & \mathrm{U}_{2} \mathrm{Ni}_{2} \mathrm{Sn}, \\
\mathrm{U}_{2} \mathrm{Ni}_{2} \mathrm{In}, \mathrm{U}_{2} \mathrm{Rh}_{2} \mathrm{Sn} & & & & \\
\end{array}$ \\
\hline II-D & \begin{tabular}{|l} 
Rare earth ternary compounds of the \\
composition $1: 1: 1$
\end{tabular} & $\mathrm{CeMgPb}, \mathrm{PrMgPb}, \mathrm{NdMgPb}, \mathrm{TmMgPb}$ \\
\hline III-A & \begin{tabular}{lll|} 
Semiconducting & Actinides/Rare earth \\
Pnictides & & \\
\end{tabular} & $\mathrm{HoP}, \mathrm{UP}, \mathrm{UP}_{2}, \mathrm{UAs}, \mathrm{NpS}, \mathrm{NpSe}, \mathrm{NpTe}, \mathrm{NpSb}, \mathrm{NpBi}, \mathrm{U}_{3} \mathrm{As}_{4}, \mathrm{U}_{3} \mathrm{P}_{4}$ \\
\hline III-B & Metallic oxides & $\mathrm{Ag}_{2} \mathrm{NiO}_{2}, \mathrm{AgNiO}_{2}, \mathrm{Ca}_{3} \mathrm{Ru}_{2} \mathrm{O}_{7}$, Double perovskite $\mathrm{Sr}_{3} \mathrm{CoIrO}_{6}$ \\
\hline III-C & Metal to insulator transition compounds & $\mathrm{NiS}_{2}, \mathrm{Sr}_{2} \mathrm{Mn}_{3} \mathrm{As}_{2} \mathrm{O}_{2}$ \\
\hline III-D & $\begin{array}{l}\text { Semiconducting and insulating oxides, } \\
\text { borates, hydroxides, silicates, phosphate }\end{array}$ & $\begin{array}{l}\mathrm{LuFeO}_{3}, \mathrm{PdNiO}_{3}, \mathrm{ErVO}_{3}, \mathrm{DyVO}_{3}, \mathrm{MnGeO}_{3}, \mathrm{Tm}_{2} \mathrm{Mn}_{2} \mathrm{O}_{7}, \mathrm{Yb}_{2} \mathrm{Sn}_{2} \mathrm{O}_{7}, \\
\mathrm{~Tb}_{2} \mathrm{Sn}_{2} \mathrm{O}_{7}, \mathrm{Ho}_{2} \mathrm{Ru}_{2} \mathrm{O}_{7}, \mathrm{Er}_{2} \mathrm{Ti}_{2} \mathrm{O}_{7}, \mathrm{~Tb}_{2} \mathrm{Ti}_{2} \mathrm{O}_{7}, \mathrm{Cd}_{2} \mathrm{Os}_{2} \mathrm{O}_{7}, \mathrm{Ho}_{2} \mathrm{Ru}_{2} \mathrm{O}_{7}, \\
\mathrm{Cr}_{2} \mathrm{ReO} 6, \mathrm{NiCr}_{2} \mathrm{O}_{4}, \mathrm{MnV}_{2} \mathrm{O}_{4}, \mathrm{Co}_{2} \mathrm{SiO}_{4}, \mathrm{Fe}_{2} \mathrm{SiO}_{4}, \mathrm{PrFe}_{3}\left(\mathrm{BO}_{3}\right)_{4}, \\
\mathrm{KCo}_{4}\left(\mathrm{PO}_{4}\right)_{3}, \mathrm{CoPS}_{3}, \mathrm{SrMn}\left(\mathrm{VO}_{4}\right)(\mathrm{OH}), \mathrm{Ba}_{5} \mathrm{Co}_{5} \mathrm{ClO}_{13}, \mathrm{FeI}_{2}\end{array}$ \\
\hline
\end{tabular}

TABLE III. The magnetic topological materials identified in this work.

interesting if the occupied bands form Wannier functions centred at positions away from the atoms, because a Wannier centre shift in three-dimensional insulators leads to the appearance of topological corner states, like those of quantized 'quadrupole' insulators 41. Topological phases characterized by displaced Wannier functions are known as obstructed atomic limits; we leave their high-throughput calculation for future studies.

\section{CONCLUSION}

We have performed LDA $+\mathrm{U}$ calculations on 549 existent magnetic structures and have successfully classified 403 using the machinery of MTQC 7]. We find that 130 materials (about $32 \%$ of the total) have topological phases as we scan the U parameter. Our results suggest that a large number of previously synthesized magnetic materials are topologically nontrivial. We highlight several 'high-quality' magnetic topological materials that should be experimentally examined for topological response effects and surface (and hinge) states.

Acknowledgements We thank U. Schmidt, I. Weidl, W. Shi and Y. Zhang. We acknowledge the computational resources Cobra in the Max Planck Computing and Data Facility (MPCDF), the HPC Platform of ShanghaiTech University and Atlas in the Donostia International Physics Center (DIPC). Y.X. is grateful to D. Liu for help in plotting some diagrammatic sketches. B.A.B., N.R., B.J.W. and Z.S. were primarily supported by a Department of Energy grant (DE-SC0016239), and partially supported by the National Science Foundation (EAGER grant DMR 1643312), a Simons Investigator grant (404513), the Office of Naval Research (ONR; grant N00014-14-1-0330), the NSF-MRSEC (grant DMR-142051), the Packard Foundation, the Schmidt Fund for Innovative Research, the BSF Israel US foundation (grant 2018226), the ONR (grant N00014-20-1-2303) and a Guggenheim Fellowship (to B.A.B.). Additional support was provided by the Gordon and Betty Moore Foundation through grant GBMF8685 towards the Princeton theory programme. L.E. was supported by the Government of the Basque Country (Project IT1301-19) and the Spanish Ministry of Science and Innovation (PID2019-106644GB-I00). M.G.V. acknowledges support from the Diputacion Foral de Gipuzkoa (DFG; grant INCIEN2019-000356) from Gipuzkoako Foru Aldundia and the Spanish Ministerio de Ciencia e Innovación (grant PID2019-109905GB-C21). Y.C. was supported by the Shanghai Municipal Science and Technology Major Project (grant 2018SHZDZX02) and a Engineering and Physical Sciences Research Council (UK) Platform Grant (grant EP/M020517/1). C.F. acknowledges financial support by the DFG under Germany's Excellence Strategy through the Würzburg-Dresden Cluster of Excellence on Complexity and Topology in Quantum Matter (ct.qmat EXC 2147, project-id 390858490), an ERC Advanced Grant (742068 'TOPMAT'). Y.X. and B.A.B. were also supported by the Max Planck Society.

Author contributions B.A.B. conceived this work; Y.X. and M.G.V. performed the first-principles 
calculations. L.E. wrote the code for calculating the irreducible representations and checking the topologies of materials. Y.X., Z.S., B.J.W. and B.A.B. analysed the calculated results, B.J.W. determined the physical meaning of the topological indices with help from L.E., Z.S. and Y.X. C.F. performed chemical analysis of the magnetic topological materials. N.R. built the topological material database. All authors wrote the main text and Y.X. and Z.S. wrote the Methods and the Supplementary Information.

\section{Competing interests}

The authors declare no competing interests.

Correspondence and requests for materials should be addressed to B.A.B.

Corresponding authors

Correspondence to B. Andrei Bernevig,

\section{METHODS}

Concepts Here we give a brief introduction to MTQC [4, 7, 56, 58, and the definitions of six topological classes. A magnetic band structure below the Fermi level is partially described by the irreducible co-representations (irreps) formed by the occupied electronic states at the high-symmetry $\mathbf{k}$ points, which are defined as the momenta whose little groups - the groups that leave the momenta unchanged - are maximal subgroups of the space group. If the highest occupied (valence) band and the lowest unoccupied (conduction) band are degenerate at a high-symmetry $\mathbf{k}$ point, then we refer to the material as an enforced semimetal with Fermi degeneracy (ESFD) [4]. Depending on whether the irreps at high-symmetry points satisfy the so-called compatibility relations [4, 24, 25, 58] - which determine whether the occupied bands must cross with unoccupied bands along high-symmetry lines or planes (whose little groups are non-maximal) - band structures can then be further classified as insulating (along high-symmetry lines and planes) or enforced semimetals (ES). ES-classified materials generically feature band crossings along high-symmetry lines or planes. If a band structure satisfies the compatibility relations, it can be a trivial insulator, whose occupied bands form a BR [4, a topological semimetal with crossing nodes at generic momenta [Smith-index semimetal (SISM) or non-symmetry-indicated topological semimetal - a system which satisfies all compatibility relations but exhibits Weyl-type nodes], or a TI. Some of the topological semimetals and insulators can be diagnosed through their irreps: If the irreps do not match a BR, then the band structure must be a topological insulator or a SISM. There are two types of topological insulators: Stable TIs [26, 27, 59, 60, which include crystalline and higher-order TIs (TCIs and HOTIs, respectively) 61 65], and fragile TIs [60, 66 70]. Stable TIs remain topological when coupled to trivial or fragile bands, whereas fragile
TIs, on the other hand, can be trivialized by being coupled to certain trivial bands, or even other fragile bands [41, 43]. In the accompanying paper [7], we explicitly identify all of the symmetry-indicated stable electronic (fermionic) TIs and topological semimetals, specifically detailing the bulk, surface, and hinge states of all symmetry-indicated stable TIs, TCIs, and HOTIs in all 1651 spinful (double) SGs and MSGs.

To summarize, using MTQC, we divide an electronic band structure into one of six topological classes: BRs, ESFD, ES, SISM, stable TI, and fragile TI, among which only BRs are considered to be topologically trivial. If a band structure satisfies the compatibility relations along high-symmetry lines and planes, and has a nontrivial value of a stable index, then, unlike in the nonmagnetic SGs, it is possible for the bulk to be a topological (semi)metal [7]. We label these cases as Smith-index semimetals (SISMs). See Appendix A for a more detailed description of the six topological classes.

Magnetic Materials Database We perform high-throughput calculations of the magnetic structures listed on BCSMD [71. BCSMD contains portable structure files, including magnetic structure data and symmetry information, for 707 magnetic structures. The magnetic structures of all the materials are determined by neutron scattering experiments. We thus consider it reasonable and experimentally motivated to use the crystal and magnetic structures provided on the BCSMD as the initial inputs for $a b$ initio calculations, instead of letting our theoretical ab-initio codes predict the magnetic ground-state. We emphasize that predictions of topological magnetic materials based on theoretically calculated magnetic structures, rather than experimentally measured structures, are more likely to predict unphysical (and possibly incorrect) magnetic ground states. From the 707 magnetic structures on the BCSMD, we omit 63 structures with lattice-incommensurate magnetism and 95 alloys, as they do not have translation symmetry and hence are not invariant under any MSG. We apply ab initio calculations for the remaining 549 structures. These magnetic structures belong to 261 different MSGs, including 29 chiral MSGs and 232 achiral MSGs (chiral MSGs are defined as MSGs without improper rotations or combinations of improper rotations and time reversal; all other MSGs are achiral). In Appendix B, we list the number of materials with experimentally obtained magnetic structures in each MSG.

Calculation Methods We performed ab initio calculations incorporating spin-orbital coupling (SOC) using VASP [72. Because all of the magnetic materials on BCSMD with translation symmetry contain at least one correlated atom with $3 d, 4 d, 4 f$, or $5 f$ electrons, we apply a series of $\mathrm{LDA}+U$ calculations for each material with different Hubbard- $U$ parameters to obtain a full phase diagram. For all of the $3 d$ valence orbitals and the atom $\mathrm{Ru}$ with $4 d$ valence orbitals, we take $U$ as $0 \mathrm{eV}, 1$ $\mathrm{eV}, 2 \mathrm{eV}, 3 \mathrm{eV}$ and $4 \mathrm{eV}$. The other atoms with $4 d$ valence 
electrons usually do not exhibit magnetism or have weak correlation effects, and hence are not considered to be correlated in our calculations. Conversely, atoms with $4 f$ and $5 f$ valence electrons have stronger correlation effects, so we take $U$ for atoms with $4 f$ or $5 f$ valence electrons to be $0 \mathrm{eV}, 2 \mathrm{eV}, 4 \mathrm{eV}$ and $6 \mathrm{eV}$. If a material has both $d$ and $f$ electrons near the Fermi level, we fix the $U$ parameter of the $d$ electrons as 2 $\mathrm{eV}$, and take $U$ of the $f$ electrons to be $0 \mathrm{eV}, 2 \mathrm{eV}$, $4 \mathrm{eV}$ and $6 \mathrm{eV}$ sequentially. We also adopt four other exchange-correlation functionals in the LDA $+U$ scheme to check the consistency between different functionals. Further details of our first-principles calculations are provided in Appendix CD, Eand F.

Of the 549 magnetic structures that we examined, 403 converged self-consistently to a magnetic ground state within an energy threshold of $10^{-5} \mathrm{eV}$ per cell. For 324 of the 403 converged materials, magnetic moments matching the experimental values (up to an average error of $50 \%$ ) were obtained for at least one of the values of $\mathrm{U}$ used to obtain the material phase diagram. We stress that these are good agreements for calculations on these strongly correlated states. However, for the other 79 materials, the calculated magnetic momenta always notably diverged from the experimental values (see Appendix L for a complete comparison of the experimental and ab initio magnetic moments). The differences can be explained as follows. First, we consider only the spin components, but not the orbital components, of the magnetic moments in our current ab initio calculations. This can result in a large average error for compounds with large spin-orbital coupling. Second, because the average error is defined relative to the experimental moments, the 'error' (measured as a percentage) is likely to be larger when the experimental moments are small. In this case, the random, slight changes in the numerically calculated moments have an outsized effect on the reported error percentage. Last but not least, mean-field theory applied in the $\mathrm{LDA}+U$ calculations is not a good approximation for some strongly correlated materials, which should be checked further with more advanced methods. Although the prediction of magnetic structure with mean-field theory is sometimes not reliable for strongly correlated materials, it is worth comparing the energy difference between the magnetic structures from neutron scattering and the other possible magnetic structures. In Appendix D, we selected several topological materials and compared their energies with some possible magnetic configurations and different $U$. We find that their experimental magnetic configurations have the lowest energies, and hence are theoretically favoured. Finally, we have additionally performed self-consistent calculations of the charge density at different values of $U$, which we used as input for our band structure calculations. In Appendix M. we provide a complete summary of results.

Considering the possible underestimation of the band gap by generalized gradient approximations (GGA), electronic structures of 23 topological mateirals are further confirmed by the calculations using the modified Becke-Johnson potential 73. As shown in Appendix E2 both the features of bands near Fermi level and the topological classes obtained from modified Becke-Johnson potential are consistent with $\mathrm{LDA}+U$ calculations. Because of the limitations of the $\mathrm{LDA}+U$ method, we have also performed the more costly LDA + Gutzwiller 74 calculations in two of the topological materials identified in this work- $\mathrm{CeCo}_{2} \mathrm{P}_{2}$ and $\mathrm{MnGeO}_{3}$, both classified as enforced semimetal-to confirm the bulk topology. As shown in Appendix F the strong correlations renormalize the quasiparticle spectrum by a factor of quasiparticle weight, but do not change the band topology. The surface-state calculations have been performed using the WannierTools package ??.

Identification of the magnetic irreps Using the self-consistent charge density and potentials, we calculate the Bloch wavefunctions at the high-symmetry momenta in the Brillouin zone and then identify the corresponding magnetic irreps using the MagVasp2trace package, which is the magnetic version of Vasp2trace package [75. (See Appendix C for details about MagVasp2trace). The little group $G_{\mathbf{k}}$ of a high symmetry point $\mathbf{k}$ is in general isomorphic to an MSG. For little groups without anti-unitary operations, we calculate the traces of the symmetry representations formed by the wavefunctions, and then decompose the traces into the characters of the small irreps of the little group $G_{\mathbf{k}}$. For little groups with anti-unitary operations, we calculate only the traces of the unitary operations and decompose the representations into the irreps of the maximal unitary subgroup $G_{\mathbf{k}}^{U}$ of $G_{\mathbf{k}}$. Since anti-unitary operations in general lead to additional degeneracies, specifically enforcing two irreps of $G_{\mathbf{k}}^{U}$ to become degenerate and form a co-representation, we check whether the additional degeneracies hold in the irreps obtained. Because VASP does not impose anti-unitary (magnetic) symmetries, degeneracies labelled by magnetic co-representations may exhibit very small splittings in band structures generated by VASP. In these cases, we reduce the convergence threshold and re-run the self-consistent calculation until the splitting is specifically small $(\leq 10 \%)$ compared to the smallest energy gap across all of the high-symmetry momenta.

The algorithm and methods designed in this work are also applicable to future high-throughput searches for magnetic topological materials 76 .

Details of the chemical catagories Considering the magnetic ions and chemical bonding of the magnetic materials, we classify the topological magnetic materials predicted in this work into the following 11 chemical categories.

(I-A) Non-collinear manganese compounds, which have received considerable recent attention owing to their unusual combination of a large anomalous Hall effect and net-zero magnetic moments. The symmetry of the non-collinear antiferromagnet spin structure allows 
for a non-vanishing Berry curvature, the origin of the unusual anomalous Hall effect. Examples of non-collinear manganese compounds include the hexagonal Weyl semimetals $\mathrm{Mn}_{3} \mathrm{Sn}, \mathrm{Mn}_{3} \mathrm{Ge}$ and the well-studied cubic antiferromagnetic spintronic-material $\mathrm{Mn}_{3} \mathrm{Ir}$, as well as the inverse perovskite compounds $\mathrm{Mn}_{3} \mathrm{Y}$, which represent 'stuffed' versions of the cubic $\mathrm{Mn}_{3} \mathrm{Y}$ compounds.

(I-B,C) Intermetallic materials, containing rare-earth atoms or actinide atoms, which are typically antiferromagnets. The variation of the Hubbard $U$ changes the band structures slightly in these materials, but not the topological character.

(II-A) The $\mathrm{ThCr}_{2} \mathrm{Si}_{2}$ structure and related structures, which have received attention because of the high-temperature iron pnictide superconductors in this group. In these materials, the transition-metal layers and the pnictide layers form square lattices. The square nets of the pnictides act as a driving force for a topological band structure [77]. Several of the antiferromagnetic undoped prototypes, such as $\mathrm{CaFe}_{2} \mathrm{As}_{2}$, are topological antiferromagnets. This suggests the possibility of topological superconductivity in these materials, like that recently found in $\mathrm{FeTe}_{0.55} \mathrm{Se}_{0.45}$ [8].

(II-B) Semiconducting manganese pnictines, which occur when iron is substituted with manganese, leading to materials that are trivial when insulating and gapped, but which become topological antiferromagnets when their gap is closed. By increasing the Hubbard $U$, the antiferromagnetic phases of these compounds can be converted into trivial insulators. The antiferromagnetic insulators and semimetals in this class can also be converted into ferromagnetic metals by doping.

(II-C) Transition metals in combination with rare earth or actinide atoms form compounds of the $\mathrm{ThCr}_{2} \mathrm{Si}_{2}$ structure type. Here the antiferromagnetic ordering comes from the Thorium-position in the $\mathrm{ThCr}_{2} \mathrm{Si}_{2}$ structure type.

(II-D) Rare earth ternary compounds of the composition 1:1:1.

(III-A,B,C,D) The third class of magnetic materials are ionic compounds, of which most have been experimentally determined to be insulating. Within the density functional approximation, several of the compounds have been identified as topological nontrivial metals, such as oxides, borates, hydroxides, silicates, phosphates and $\mathrm{FeI}_{2}$. By increasing the Hubbard $U$, a topologically trivial gap can be opened in these materials.

Additional data and discussion can be found online in the Supplementary information.

Data availability All data are available in the Supplementary Information and at https://www.topologicalquantumchemistry.fr/magnetic. The codes required to calculate the character table of magnetic materials are available at https://www.cryst.ehu.es/cryst/checktopologicalmagmat. 


\section{Appendix A: A brief introduction to Magnetic Topological Quantum Chemistry (MTQC)}

The symmetry group property of a band structure is fully described by the multiplicities of the irreducible co-representations (irreps) formed by the occupied bands at all the maximal $K$-points. In the present paper, we define the 1 st band to the $N_{e}$ th band as the "occupied" bands, where $N_{e}$ is the number of electrons. Maximal $k$-points are defined as the high symmetry momenta whose little groups are maximal subgroups of the magnetic space group. The maximal $K$-points of each magnetic space group are supplied in the magnetic vasp2trace package. We denote the little group at the momentum $K$ as $G_{K}$, the $i$ th irrep of $G_{K}$ as $\rho_{K}^{i}$, and its multiplicity of $\rho_{K}^{i}$ formed by the occupied bands as $m\left(\rho_{K}^{i}\right)$. For example, the Brillouin zone (BZ) of the 2D space group generated from inversion $(I)$ and translations has four maximal $k$-points: $\Gamma(0,0), \mathrm{X}(\pi, 0), \mathrm{Y}(0, \pi), \mathrm{M}(\pi, \pi)$, all of which have the same little group: $C_{i}=\{E, I\}$. Here $E$ is the identity. $C_{i}$ has two types of irreps: the even $(+)$ and the odd $(-)$. Thus a band structure is characterized by the eight integers $m\left(\rho_{\Gamma, \mathrm{X}, \mathrm{Y}, \mathrm{M}}^{+,-}\right)$. For convenience, we introduce the symmetry-data-vector [4]

$$
B=\left(m\left(\rho_{\Gamma}^{+}\right), m\left(\rho_{\Gamma}^{-}\right), m\left(\rho_{\mathrm{X}}^{+}\right), m\left(\rho_{\mathrm{X}}^{-}\right), m\left(\rho_{\mathrm{Y}}^{+}\right), m\left(\rho_{\mathrm{Y}}^{-}\right), m\left(\rho_{\mathrm{M}}^{+}\right), m\left(\rho_{\mathrm{M}}^{-}\right)\right)^{T} .
$$

The symmetry property of a band structure is fully described by the symmetry-data-vector.

Enforced semimetal with Fermi degeneracy. In some materials, the highest occupied band and the lowest empty band are degenerate at some maximal $K$-points, and the degeneracy is protected by the MSG. We call such states enforced semimetals with Fermi degeneracy (ESFD) 4, 79]. ESFD does not have a well defined symmetry-data-vector $B$. See FIG. 1c of the main text for examples of ESFD.

For band structures where the occupied bands are gapped from the empty bands along all the high symmetry lines, the multiplicities $m$ necessarily satisfy the compatibility relations [4, 25, 29, 56, 58, 80. We consider two maximal $k$-points $K_{1,2}$ and a path $k$ between them. On the one hand, since $G_{k}$ is a subgroup of $G_{K_{1}}$, the irreps of $G_{k}$ formed by the occupied bands in $k$ near to $K_{1}$ can be obtained by subduction of the irreps of $G_{K_{1}}$ formed by occupied bands at $K_{1}$. On the other hand, the irreps of $G_{k}$ formed by the occupied bands in $k$ near to $K_{2}$ can also be obtained by subduction of the irreps at $K_{2}$. If the irreps of $G_{k}$ obtained at the two ends $K_{1}$ and $K_{2}$ are not the same, then there must be a symmetry protected level crossing along the path $k$. In other words, in order to guarantee the path $k$ is gapped, the irreps at $K_{1}$ and $K_{2}$ must reduce to the same set of irreps of $G_{k}$. This requirement establishes the compatibility relations along $k$. The full compatibility relations can be obtained by applying this analysis to all the inequivalent paths in the BZ. [56]

In the example of $2 \mathrm{D}$ space group with inversion $P \overline{1}$, all the momenta except $\Gamma, \mathrm{X}, \mathrm{Y}, \mathrm{M}$ have the same little group: the identity group. Thus for any two maximal $k$-points, there is only one inequivalent path connecting them, and both even and odd irreps reduce to the identity irrep of the identity group. The compatibility-relation is nothing but the restriction that the two maximal $k$-points have the same number of occupied bands. We can write the compatibility relations as

$$
m\left(\rho_{\Gamma}^{+}\right)+m\left(\rho_{\Gamma}^{-}\right)=m\left(\rho_{\mathrm{X}}^{+}\right)+m\left(\rho_{\mathrm{X}}^{-}\right)=m\left(\rho_{\mathrm{Y}}^{+}\right)+m\left(\rho_{\mathrm{Y}}^{-}\right)=m\left(\rho_{\mathrm{M}}^{+}\right)+m\left(\rho_{\mathrm{M}}^{-}\right) .
$$

Since we define the "occupied" bands as the 1st band to the $N_{e}$ th band, there are always $N_{e}$ occupied levels at any momentum and hence Eq.A2 is automatically satisfied. However, most other magnetic space groups have more compatibility relations than the band number restriction; these compatibility relations can be broken in materials.

Enforced Semimetals. Band structures breaking the compatibility relations are referred to as enforced semimetals (ESs). The inversion case is not a good example for ES because the compatibility-relation is satisfied by definition. Please see Jfor example of ES.

We now classify the possible band structures allowed by compatibility relations. The strategy is that we first enumerate all the atomic insulators and then, for any given band structure from DFT, compare its irreps with those of the atomic insulators. A band structure must be topologically nontrivial if its irreps are not consistent with any atomic insulator; otherwise can be either trivial/nontrivial. Following the terminology of Zak [81 83], we refer to atomic insulators as band representations (BRs) and to the generators of the BRs as elementary BRs (EBRs).

We take the $2 \mathrm{D}$ space group with inversion $P \overline{1}$ as an example to illustrate the concept of EBRs. There are four maximal Wyckoff positions in each unit cell, $a(0,0), b\left(\frac{1}{2}, 0\right), c\left(0, \frac{1}{2}\right), d\left(\frac{1}{2}, \frac{1}{2}\right)$. Maximal Wyckoff positions are defined as positions with site-symmetry-groups which are maximal subgroups of the space group. In this example, the site-symmetry-groups of $a, b, c, d$ are all isomorphic to $C_{i}=\{E, I\}$. Since $C_{i}$ only has two types of irreps (even and odd), we can add either $s$ orbital (even) $/ p$ orbital (odd) at each position. We can then obtain eight different EBRs. To see that they are EBRs, we consider an atomic insulator formed by two orbitals at two general positions, $(x, y)$, $(1-x, 1-y)$, which transform into each other under the inversion operation at $d$ position. We can recombine the two orbitals to form a bonding state and an anti-bonding state at the $d$ position. Thus this atomic insulator can be generated from two EBRs at the $d$ position. The symmetry-data-vectors of the eight EBRs can be calculated by acting 
the symmetry operators on the corresponding Bloch wave functions. The wave functions are Fourier transformations of the local orbitals

$$
\left|\phi_{\xi, \alpha, \mathbf{k}}\right\rangle=\frac{1}{\sqrt{N}} \sum_{\mathbf{R}} e^{i \mathbf{k} \cdot\left(\mathbf{R}+\mathbf{t}_{\alpha}\right)}\left|\xi, \mathbf{R}+\mathbf{t}_{\alpha}\right\rangle
$$

Here $\xi= \pm$ is the parity of the local orbital, $\alpha=a, b, c, d$ is the Wyckoff position, $\mathbf{t}_{\alpha}$ is the position vector of the Wyckoff position, $\mathbf{R}$ sums over all lattice vectors, and $N$ is the system size. Since $I\left|\xi, \mathbf{R}+\mathbf{t}_{\alpha}\right\rangle=\xi\left|\xi,-\mathbf{R}-\mathbf{t}_{\alpha}\right\rangle$, we obtain

$$
I\left|\phi_{\xi, \alpha, \mathbf{k}}\right\rangle=\xi \frac{1}{\sqrt{N}} \sum_{\mathbf{R}} e^{i \mathbf{k} \cdot\left(\mathbf{R}+\mathbf{t}_{\alpha}\right)}\left|\xi,-\mathbf{R}-\mathbf{t}_{\alpha}\right\rangle=\xi \frac{1}{\sqrt{N}} \sum_{\mathbf{R}^{\prime}} e^{-i \mathbf{k} \cdot\left(\mathbf{R}^{\prime}+\mathbf{t}_{\alpha}\right)}\left|\xi, \mathbf{R}^{\prime}+\mathbf{t}_{\alpha}\right\rangle,
$$

where the lattice vector $\mathbf{R}^{\prime}$ is $-\mathbf{R}-2 \mathbf{t}_{\alpha}$. If $\mathbf{k}$ is one of the maximal $k$-points $(\Gamma, \mathrm{X}, \mathrm{Y}, \mathrm{M})$, we can calculate the parity of the Bloch wave function as

$$
\left\langle\phi_{\xi, \alpha, \mathbf{k}}|I| \phi_{\xi, \alpha, \mathbf{k}}\right\rangle=\xi \frac{1}{N} \sum_{\mathbf{R}^{\prime}} e^{-i 2 \mathbf{k} \cdot\left(\mathbf{R}^{\prime}+\mathbf{t}_{\alpha}\right)}=\xi e^{-i 2 \mathbf{k} \cdot \mathbf{t}_{\alpha}} .
$$

We have made use of the fact that $2 \mathbf{k}$ is a reciprocal lattice vector and hence $2 \mathbf{k} \cdot \mathbf{R}=0 \bmod 2 \pi$. For $\alpha=a$, the Bloch wave function has the same parity at all the four maximal $k$-points because $e^{-i 2 \mathbf{k} \cdot \mathbf{t}_{\alpha}}=1$. Thus the EBR induced from the orbital with parity \pm at the $a$ position form the irreps $\rho_{\Gamma}^{ \pm}, \rho_{\mathrm{X}}^{ \pm}, \rho_{\mathrm{Y}}^{ \pm}, \rho_{\mathrm{M}}^{ \pm}$. The symmetry-data-vectors $\mathrm{A} 1$ of these two EBRs are

$$
E B R_{+, a}=(1,0,1,0,1,0,1,0)^{T}, \quad E B R_{-, a}=(0,1,0,1,0,1,0,1)^{T} .
$$

For $\alpha=b, c, d$, the Bloch wave function has different parities at the four maximal $k$-points because $e^{-i 2 \mathbf{k} \cdot \mathbf{t}_{\alpha}}$ can be either $1 /-1$. For example, for $\alpha=b, e^{-i 2 \mathbf{k} \cdot \mathbf{t}_{\alpha}}$ equals to 1 and -1 at $\Gamma, \mathrm{Y}$ and $\mathrm{X}$, M, respectively. Thus the EBR induced from the orbital with parity \pm at the $b$ position form the irreps $\rho_{\Gamma}^{ \pm}, \rho_{\mathrm{X}}^{\mp}, \rho_{\mathrm{Y}}^{ \pm}, \rho_{\mathrm{M}}^{\mp}$. The corresponding symmetry-data-vectors are

$$
E B R_{+, b}=(1,0,0,1,1,0,0,1)^{T}, \quad E B R_{-, b}=(0,1,1,0,1,0,1,0)^{T} .
$$

Similarly, one can derive the symmetry-data-vectors of EBRs induced from $c, d$ positions as

$$
\begin{aligned}
& E B R_{+, c}=(1,0,1,0,0,1,0,1)^{T}, \quad E B R_{-, c}=(0,1,0,1,1,0,1,0)^{T}, \\
& E B R_{+, d}=(1,0,0,1,0,1,1,0)^{T}, \quad E B R_{-, d}=(0,1,1,0,1,0,0,1)^{T} .
\end{aligned}
$$

Stable TI. We consider an example where the occupied band form a single odd irrep at $\Gamma$ and three even irreps at X, Y, M respectively. The corresponding symmetry-data-vector can be written as

$$
B_{1}=(0,1,1,0,1,0,1,0)^{T} .
$$

$B_{1}$ is not one of the EBRs; It is also not a sum of EBRs, because all EBRs have even number of odd irreps in Eqs. A6 to A9. It is also not a sum of EBRs because $B_{1}$ has only one band but any sum of EBRs has at least two bands. Thus $B_{1}$ must be topological. According to the Fu-Kane-like formula for Chern insulators 84]

$$
(-1)^{C}=\prod_{K=\Gamma, \mathrm{X}, \mathrm{Y}, \mathrm{M}} \prod_{n} \lambda_{n}(K),
$$

where $C$ is the Chern number, $n$ is the index of occupied bands, and $\lambda_{n}(K)$ is the parity of $n$th band at the momentum $K$, the band structure has an odd Chern number.

One notices that $B_{1}$ can be written as a linear combination of EBRs with fractional coefficients

$$
B_{1}=-\frac{1}{2} E B R_{-, a}+\frac{1}{2} E B R_{-, d}+\frac{1}{2} E B R_{-, c}+\frac{1}{2} E B R_{-, d},
$$

but cannot be written as an integer combination of EBRs. It is a general principle that if a band structure cannot be written as a linear combination of EBRs unless the coefficients are fractional numbers, the band structure must have stable topology. Such stable topology implied by symmetry eigenvalues is characterized by the stable index (SI) (also 
referred to as symmetry-based indicator [60, 80, $)$. Eq. (A11) can be thought as an example of SI. Readers can refer to supplementary information of Ref. [60, 70, for technical details.

Smith-index semimetal. In magnetic space groups, some symmetry-data-vectors are not compatible with gapped state and implies topological Weyl semimetal (WSM), even when all of the compatibility relations are satisfied. In this work, the WSM phase implied by symmetry eigenvalue is named as Smith-index semimetal (SISM).

From the MTQC theory, we have found several MSGs with SI corresponding to WSM phase. All of these MSGs have a minimal subgroup MSG $2.4(P \overline{1}) /$ MSG $81.33(P \overline{4})$. For the MSGs with minimal subgroup $P \overline{1}$ (with only inversion symmetry), the topologies are described by the stable indices group $\mathbb{Z}_{4} \times \mathbb{Z}_{2}^{3}$. We found the stable index $\eta_{4 I} m o d 2$ is the parity of the Chern number difference between $k z=0$ and $k z=\pi$ planes. Thus $\eta_{4 I}=1,3$ correspond to the WSM phase with odd number of Weyl points in one half Brillouin zone [7. For the MSGs with minimal subgroup $P \overline{4}$, they have the SI group $\mathbb{Z}_{4} \times \mathbb{Z}_{2}^{2}$. We find one of the two $z_{2}$ indices can be interpreted as [7] $\delta_{2 S}=\frac{c_{\pi}-c_{0}}{2} m o d 2$, where $c_{0, \pi}$ are the Chern numbers in the $k_{z}=0, \pi$ planes. Thus, when this $\delta_{2 S}$ index is nonzero, $k_{z}=0, \pi$ planes must have different Chern numbers and hence Weyl nodes must appear in between the two planes.

Fragile TI. If the $B$ vector of a state cannot be written as a sum of EBRs, but can be written as a difference of EBRs, then the state is at least a fragile TI. 60, 66, 70, 85, 87. We say "at least" because the state can also have a stable topology which cannot be diagnosed through symmetry eigenvalues but through Berry phases. Now we give an example in the inversion case. We consider that the occupied bands form two odd irreps at $\Gamma$ and three pairs of even irreps at X, Y, M respectively. The corresponding symmetry-data-vector is double of $B_{1}$, i.e.,

$$
B_{2}=(0,2,2,0,2,0,2,0)^{T} .
$$

Since $B_{2}=2 B_{1}$, we can write the $B_{2}$ as a linear combination of EBRs with integer coefficients, and one of the coefficients is negative

$$
B_{2}=-E B R_{-, a}+E B R_{-, d}+E B R_{-, c}+E B R_{-, d}
$$

This decomposition implies that, after being coupled to a trivial band forming the $E B R_{-, a}, B_{2}$ becomes trivial because it can be written as a sum of EBRs as $E B R_{-, d}+E B R_{-, c}+E B R_{-, d}$. Therefore, $B_{2}$ is at least a fragile TI. Readers can refer to Ref. [70] for more examples and complete classifications of eigenvalue implied fragile TIs.

\section{Appendix B: Material statistics in the BCSMD}

Ignoring the magnetic materials with incommensurate structures, there are 644 materials (including 95 alloys) with 261 different MSGs in the Bilbao Crystallographic Server Magnetic database(BCSMD) [71, 88]. We provide the number of materials in each MSG in Table IV] Detailed information about each of the magnetic materials can be obtained on the BCSMD website (http://webbdcrista1.ehu.es/magndata). Based on the stable topological classifications of MSGs [7, 29], we classify the MSGs into four types.

Type A The MSGs that have stable topological indices, which are indicated by red color. There are 435 materials in BCSMD with Type A MSGs.

Type B In this type of MSGs, given the electron number, one can immediately identify whether a material is ESFD. This type of MSGs are indicated by blue color. There are 34 materials with Type B MSGs in BCSMD.

Type C Among Type B MSGs, some also have stable topological indices, which are indicated by green color. There are 19 materials in BCSMD with Type C MSG.

Type D The other MSGs that do not belong to Type A/Type B are indicated by black color. There are 183 materials with Type D MSG.

We also emphasize that for an ES/ESFD, if the crossing point occurs at a k-point whose little co-group is chiral, the crossing point must necessarily carry a nonzero chiral charge [89 91. The chiral MSGs have been tagged in Table IV.

\begin{tabular}{|c|c|c|c|c|c|c|c|}
\hline MSG & Count & MSG & Count & MSG & Count & MSG & Count \\
\hline$\overline{1.3 P_{S} 1^{*}}$ & 4 & 33.144 Pna $2_{1}$ & 3 & $63.462 C m^{\prime} c^{\prime} m$ & 2 & $138.528 P_{c} 4_{2} / \mathrm{ncm}$ & 1 \\
\hline $2.4 P \overline{1}$ & 4 & $33.147 P n a^{\prime} 2_{1}^{\prime}$ & 2 & $63.463 C m c^{\prime} m^{\prime}$ & 1 & $138.529 \mathrm{P}_{\mathrm{C}} 4_{2} / \mathrm{ncm}$ & 1 \\
\hline $2.6 P \overline{1}^{\prime}$ & 3 & $33.148 P^{\prime} a^{\prime} 2_{1}$ & 3 & $63.464 \mathrm{Cm}^{\prime} \mathrm{cm}^{\prime}$ & 4 & $139.535 \mathrm{I}^{\prime} / \mathrm{mmm}^{\prime}$ & 1 \\
\hline $2.7 P_{S} \overline{1}$ & 34 & $33.149 P_{a} n a 2_{1}$ & 1 & $63.466 C_{c} \mathrm{mcm}$ & 2 & $139.536 \mathrm{I}^{\prime} / \mathrm{m}^{\prime} \mathrm{m}^{\prime} \mathrm{m}$ & 4 \\
\hline $4.10 P_{a} 2_{1}{ }^{*}$ & 7 & $33.150 P_{b} n a 2_{1}$ & 1 & $63.467 \mathrm{C}_{a} \mathrm{mcm}$ & 1 & $139.537 \mathrm{I} 4 / \mathrm{mm}^{\prime} \mathrm{m}^{\prime}$ & 2 \\
\hline $4.12 P_{C} 2_{1}{ }^{*}$ & 1 & $33.154 P_{C} n a 2_{1}$ & 3 & $63.468 C_{A} m \mathrm{~cm}$ & 1 & $140.550 I_{c} 4 / \mathrm{mcm}$ & 6 \\
\hline
\end{tabular}

TABLE IV: The number of magnetic materials per magnetic space group in BCSMD 


\begin{tabular}{|c|c|c|}
\hline $4.7 P 2_{1}{ }^{*}$ & 3 & $\mid 35.167 \mathrm{Cm}^{\prime} m 2^{\prime}$ \\
\hline $4.9 P 2_{1}^{\prime *}$ & 3 & $36.174 \mathrm{Cm}^{\prime} c 2_{1}^{\prime}$ \\
\hline $5.13 C 2^{*}$ & 1 & $36.176 \mathrm{Cm}^{\prime} \mathrm{c}^{\prime} 2_{1}$ \\
\hline $5.15 C 2^{\prime *}$ & 1 & $36.178 C_{a} m c 2_{1}$ \\
\hline $5.16 C_{c} 2^{*}$ & 3 & $38.191 \mathrm{Am}^{\prime} \mathrm{m}^{\prime} 2$ \\
\hline $6.20 \mathrm{Pm}^{\prime}$ & 1 & $38.192 A_{a} m m 2$ \\
\hline $7.27 P_{a} c$ & 1 & $39.201 \quad A_{b} b m 2$ \\
\hline $7.29 P_{b} c$ & 1 & $41.217 A_{b} b a 2$ \\
\hline $8.35 C_{c} m$ & 1 & $42.223 F_{S} m m 2$ \\
\hline $8.36 C_{a} m$ & 4 & $43.227 F d^{\prime} d^{\prime} 2$ \\
\hline $9.39 C c^{\prime}$ & 2 & $45.237 I b^{\prime} a 2^{\prime}$ \\
\hline $9.40 C_{c} c$ & 3 & $46.243 \operatorname{Im}^{\prime} a 2^{\prime}$ \\
\hline $9.41 C_{a} c$ & 3 & $49.270 \mathrm{Pc}^{\prime} \mathrm{cm}^{\prime}$ \\
\hline $10.49 P_{C} 2 / m$ & 1 & $49.273 P_{c} c \mathrm{~cm}$ \\
\hline $11.54 P 2_{1}^{\prime} / m^{\prime}$ & 2 & $50.282 \mathrm{~Pb}^{\prime} a n^{\prime}$ \\
\hline $11.55 P_{a} 2_{1} / m$ & 2 & $51.295 \mathrm{Pmm}^{\prime} \mathrm{a}^{\prime}$ \\
\hline $11.57 P_{C} 2_{1} / m$ & 3 & $51.298 P_{a} m m a$ \\
\hline $12.58 \mathrm{C} 2 / \mathrm{m}$ & 1 & $52.310 P n^{\prime} n^{\prime} a$ \\
\hline $12.60 \mathrm{C} 2^{\prime} / \mathrm{m}$ & 4 & 52.312Pn $P n^{\prime} a^{\prime}$ \\
\hline $12.62 C 2^{\prime} / m^{\prime}$ & 9 & $52.315 P_{b} n n a$ \\
\hline $12.63 C_{c} 2 / m$ & 8 & $53.334 P_{B} m n a$ \\
\hline $12.64 C_{a} 2 / m$ & 5 & $53.335 P_{C} m n a$ \\
\hline $13.67 P 2^{\prime} / c$ & 1 & $54.350 P_{B} c c a$ \\
\hline $13.69 P 2^{\prime} / c^{\prime}$ & 1 & $54.352 P_{I} c c a$ \\
\hline $13.70 P_{a} 2 / c$ & 1 & $55.355 \mathrm{~Pb}^{\prime} \mathrm{am}$ \\
\hline $13.73 P_{A} 2 / c$ & 3 & $55.356 \mathrm{Pbam}^{\prime}$ \\
\hline $13.74 P_{C} 2 / c$ & 4 & $55.361 P_{c}$ bam \\
\hline $14.75 P 2_{1} / c$ & 9 & $56.369 P c^{\prime} c^{\prime} n$ \\
\hline $14.77 P 2_{1}^{\prime} / c$ & 3 & $56.372 P_{b} \mathrm{ccn}$ \\
\hline $14.78 P 2_{1} / c^{\prime}$ & 5 & $56.373 P_{c} c c n$ \\
\hline $14.79 P 2_{1}^{\prime} / c^{\prime}$ & 8 & $56.374^{\prime} P_{A} c c n^{\prime}$ \\
\hline $14.80 P_{a} 2_{1} / c$ & 20 & $57.389 P_{A} b \mathrm{~cm}$ \\
\hline $14.81 P_{b} 2_{1} / c$ & 1 & $57.391 P_{C} b c m$ \\
\hline $14.82 P_{c} 2_{1} / c$ & 6 & $58.395 P^{\prime} n^{\prime} m$ \\
\hline $14.83 P_{A} 2_{1} / c$ & 1 & $58.398 P^{P n n^{\prime}} \mathrm{m}^{\prime}$ \\
\hline $14.84 P_{C} 2_{1} / c$ & 10 & $58.399 P n^{\prime} n^{\prime} m^{\prime}$ \\
\hline $15.85 C 2 / c$ & 6 & $58.404 P_{I} n n m$ \\
\hline $15.87 C 2^{\prime} / c$ & 4 & $59.407 P m^{\prime} m n$ \\
\hline $15.88 C 2 / c^{\prime}$ & 2 & $59.409 \mathrm{Pm}^{\prime} \mathrm{m}^{\prime} n$ \\
\hline $15.89 C 2^{\prime} / c^{\prime}$ & 11 & 59.410Pmm $\mathrm{P}^{\prime}$ \\
\hline $15.90 C_{c} 2 / c$ & 28 & $59.416 P_{I} m m n$ \\
\hline $15.91 C_{a} 2 / c$ & 3 & $60.419 \mathrm{~Pb}^{\prime} \mathrm{cn}$ \\
\hline $18.19 P 2_{1} 2_{1}^{\prime} 2^{\prime *}$ & 1 & $60.422 P b^{\prime} c^{\prime} n$ \\
\hline $18.22 P_{B} 2_{1} 2_{1} 2^{*}$ & 1 & $60.431 P_{C} b c n$ \\
\hline $19.25 P 2_{1} 2_{1} 2_{1}^{*}$ & 2 & 61.433 Pbca \\
\hline $19.27 P 2_{1}^{\prime} 2_{1}^{\prime} 2_{1}^{*}$ & 1 & $61.437 P b^{\prime} c^{\prime} a^{\prime}$ \\
\hline $19.28 P_{c} 2_{1} 2_{1} 2_{1}{ }^{*}$ & 1 & $61.439 P_{C} b c a$ \\
\hline $19.29 P_{C} 2_{1} 2_{1} 2_{1}^{*}$ & 1 & 62.441 Pnma \\
\hline $20.34 C 22^{\prime} 2_{1}^{\prime *}$ & 1 & $62.443 P n^{\prime} m a$ \\
\hline $20.37 C_{A} 222_{1}^{*}$ & 1 & $62.444 P m^{\prime} a$ \\
\hline 26.66 Pmc2 $2_{1}$ & 2 & 62.445 Pnma' \\
\hline $26.68 P m^{\prime} c 21^{\prime}$ & 2 & 62.446Pn'm $\mathrm{Pn}^{\prime} a$ \\
\hline $26.72 P_{b} m c 2_{1}$ & 3 & 62.447 Pnm ${ }^{\prime} a^{\prime}$ \\
\hline
\end{tabular}

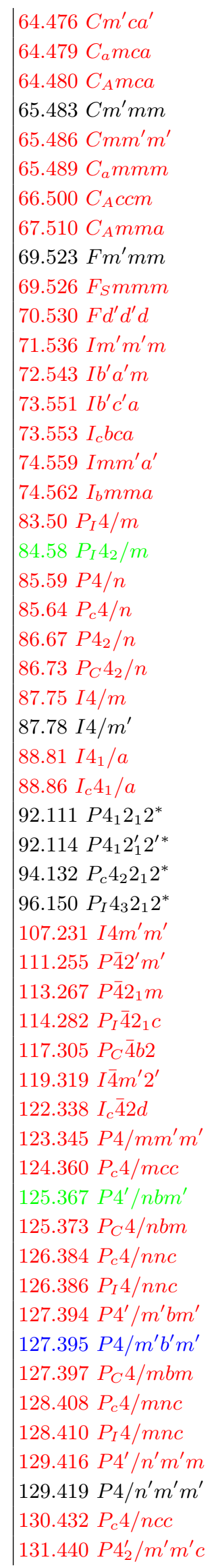

\begin{tabular}{|c|c|}
\hline 1 & $141.554 \mathrm{I}_{1}^{\prime} / \mathrm{am}^{\prime} \mathrm{d}$ \\
\hline 1 & $141.555 \mathrm{I}_{1}^{\prime} / a m d^{\prime}$ \\
\hline 13 & $141.556 I 4_{1}^{\prime} / a^{\prime} m^{\prime} d$ \\
\hline 1 & $141.557 \mathrm{I}_{1} / \mathrm{am}^{\prime} \mathrm{d}^{\prime}$ \\
\hline 2 & $142.568 I 4_{1}^{\prime} / a^{\prime} c d^{\prime}$ \\
\hline 2 & $146.10 R 3^{*}$ \\
\hline 5 & $146.12 R_{I} 3^{*}$ \\
\hline 1 & $148.17 R \overline{3}$ \\
\hline 1 & $148.19 R \overline{3}^{\prime}$ \\
\hline 3 & $148.20 R_{I} \overline{3}$ \\
\hline 2 & $152.35 P 3_{1} 2^{\prime} 1^{*}$ \\
\hline 2 & $154.41 P 3_{2} 21^{*}$ \\
\hline 1 & $154.44 P_{c} 3_{2} 21^{*}$ \\
\hline 1 & $155.48 R_{I} 32^{*}$ \\
\hline 2 & $157.53 \mathrm{P} 31 \mathrm{~m}$ \\
\hline 1 & $157.55 \mathrm{P}^{2} \mathrm{~m}^{\prime}$ \\
\hline 1 & $159.64 P_{c} 31 c$ \\
\hline 2 & $161.69 R 3 c$ \\
\hline 1 & $161.71 R 3 c^{\prime}$ \\
\hline 1 & $161.72 R_{I} 3 c$ \\
\hline 1 & $162.78 P_{c} \overline{3} 1 \mathrm{~m}$ \\
\hline 1 & $164.89 P \overline{3} m^{\prime} 1$ \\
\hline 3 & $165.94 P \overline{3}^{\prime} c^{\prime} 1$ \\
\hline 1 & $165.95 P \overline{3} c^{\prime} 1$ \\
\hline 3 & $165.96 P_{c} \overline{3} c 1$ \\
\hline 1 & $166.101 R \overline{3} m^{\prime}$ \\
\hline 1 & $166.102 R_{I} \overline{3} m$ \\
\hline 1 & $166.97 R \overline{3} m$ \\
\hline 1 & $167.103 R \overline{3} c$ \\
\hline 1 & $167.106 R \overline{3}^{\prime} c^{\prime}$ \\
\hline 1 & $167.107 R \overline{3} c^{\prime}$ \\
\hline 1 & $167.108 R_{I} \overline{3} c$ \\
\hline 1 & $173.129 P 6_{3}$ \\
\hline 1 & $173.131 P 6_{3}^{\prime}$ \\
\hline 1 & $174.136 P_{c} \overline{6}$ \\
\hline 1 & $176.145 P 6_{3}^{\prime} / m$ \\
\hline 1 & $185.197 \mathrm{P}_{3} \mathrm{~cm}$ \\
\hline 1 & $185.199 P 6_{3}^{\prime} c^{\prime} m$ \\
\hline 1 & $185.200 P 6_{3}^{\prime} \mathrm{cm}^{\prime}$ \\
\hline 4 & $185.201 P 6_{3} c^{\prime} m^{\prime}$ \\
\hline 1 & 186.207 $P 6_{3} m^{\prime} c^{\prime}$ \\
\hline 1 & $188.220 P_{c} \overline{6} c 2$ \\
\hline 1 & 189.223P $\overline{6}^{\prime} 2^{\prime} m$ \\
\hline 1 & $189.224 P \overline{6}^{\prime} 2 m^{\prime}$ \\
\hline 2 & $192.252 P_{c} 6 / m c c$ \\
\hline 1 & $193.259 P 6_{3}^{\prime} / \mathrm{m}^{\prime} \mathrm{cm}^{\prime}$ \\
\hline 1 & $193.260 \mathrm{P}_{3} / \mathrm{mc}^{\prime} \mathrm{m}^{\prime}$ \\
\hline 1 & $194.268 P 6_{3}^{\prime} / \mathrm{m}^{\prime} \mathrm{m}^{\prime} \mathrm{c}$ \\
\hline 5 & $203.26 F d \overline{3}$ \\
\hline 3 & 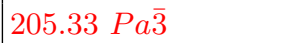 \\
\hline 1 & $216.77 F_{S} \overline{4} 3 m$ \\
\hline 2 & $222.103 P_{I} n \overline{3} n$ \\
\hline 1 & 224.113 Pn $\overline{3} m^{\prime}$ \\
\hline
\end{tabular}




\begin{tabular}{|c|c|c|c|c|c|c|c|}
\hline $27.82 P_{c} c c 2$ & 1 & 62.448Pn'ma' & 5 & $132.456 P_{c} 4_{2} / \mathrm{mcm}$ & 1 & 227.131 Fd $\overline{3} m^{\prime}$ & 1 \\
\hline $29.101 P c^{\prime} a 2_{1}^{\prime}$ & 4 & $62.449 P n^{\prime} m^{\prime} a^{\prime}$ & 4 & $134.481 \mathrm{P}_{C} 4_{2} / \mathrm{nnm}$ & 3 & $228.139 F_{S} d \overline{3} c$ & 3 \\
\hline $29.104 P_{a} c a 2_{1}$ & 5 & $62.450 P_{a} n m a$ & 5 & $135.492 P_{c} 4_{2} / m b c$ & 2 & 229.143 Im $\overline{3} m^{\prime}$ & 1 \\
\hline $29.105 P_{b} c a 2_{1}$ & 1 & $62.452 P_{c} n m a$ & 1 & $136.499 \mathrm{P}_{2}^{\prime} / \mathrm{mnm}^{\prime}$ & 2 & 230.148 Ia $\overline{3} d^{\prime}$ & \\
\hline $29.110 P_{I} c a 2_{1}$ & 1 & $62.453 P_{A} n m a$ & 1 & $136.503 P 4_{2} / m^{\prime} n^{\prime} m^{\prime}$ & 1 & & \\
\hline $31.129 P_{b} m n 2_{1}$ & 3 & $63.459 \mathrm{Cm}^{\prime} \mathrm{cm}$ & 1 & $136.506 \mathrm{P}_{I} 4_{2} / \mathrm{mnm}$ & 1 & & \\
\hline $32.137 \mathrm{~Pb}^{\prime} a 2^{\prime}$ & 1 & $63.461 \mathrm{Cmcm}^{\prime}$ & 1 & $138.525 \mathrm{P}_{2} / n c^{\prime} \mathrm{m}^{\prime}$ & 1 & & \\
\hline
\end{tabular}

* Chiral MSG

In the magnetic material database, all of the materials have distinct chemical formulae/different MSGs except for the 15 materials tabulated in Table $\mathrm{V}$. The 15 compounds are reported having the same chemical formulae and MSGs but different magnetic moments in two independent neutron experiments. The differences between them have been described in Table V. These differences consist in the experimental temperature/lattice parameters. In this work, we have performed the abinitio calculations for all of them.

TABLE V: The 15 compounds that have the same chemical formulae and MSG but with different magnetic moments are tabulated together.

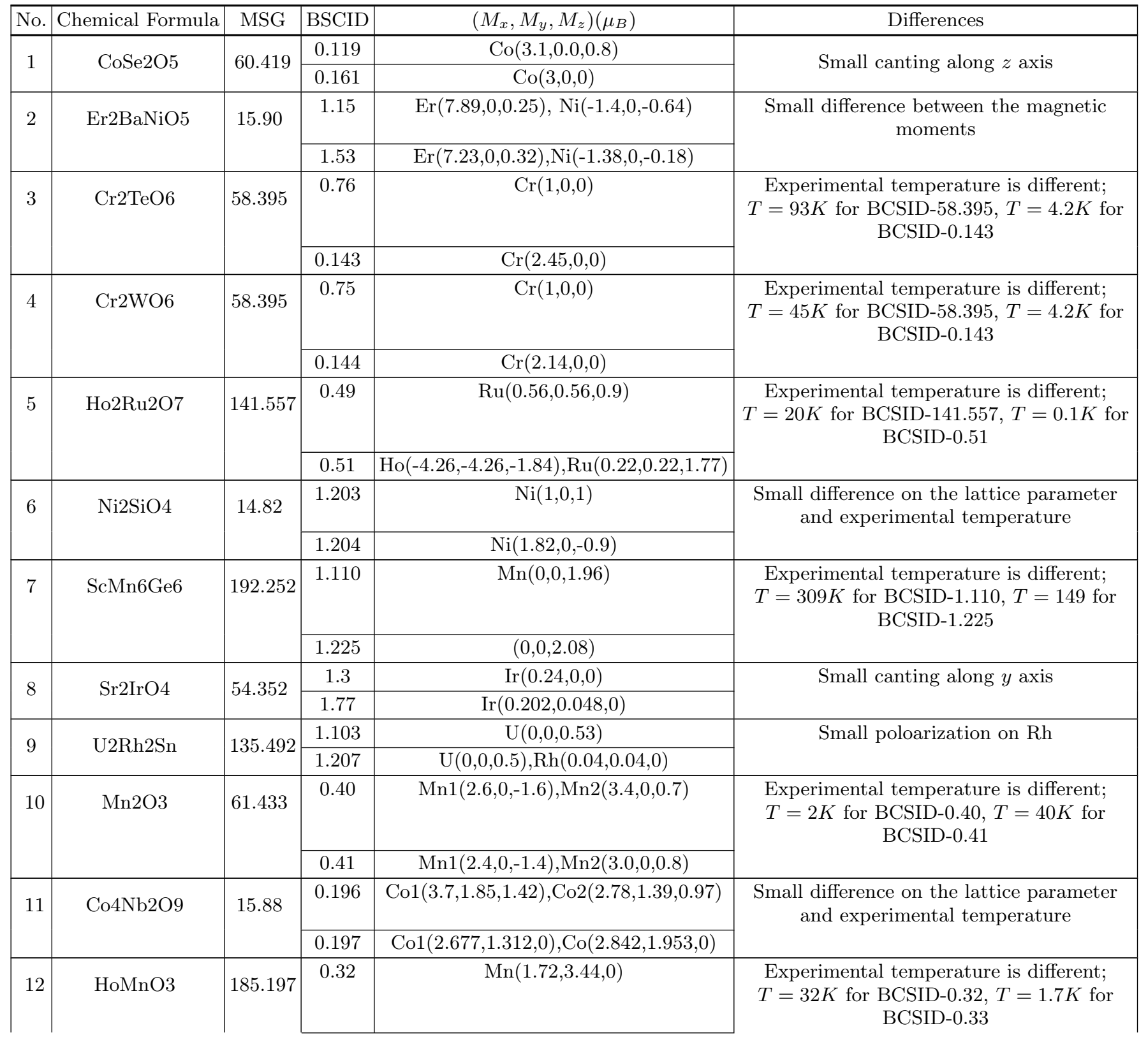




\begin{tabular}{|c|c|c|c|c|c|}
\hline & & & 0.33 & $\operatorname{Mn}(1.76,3.52,0), \operatorname{Ho}(0,0,2.87)$ & \\
\hline \multirow{2}{*}{13} & \multirow{2}{*}{ FeI2 } & \multirow{2}{*}{12.62} & 3.14 & $\mathrm{Fe}(0,0,1)$ & \multirow[t]{2}{*}{ Different lattice parameters } \\
\hline & & & 1.0 .13 & $\mathrm{Fe}(0,0,1)$ & \\
\hline \multirow[t]{2}{*}{14} & \multirow[t]{2}{*}{$\mathrm{Co} 2 \mathrm{SiO} 4$} & \multirow[t]{2}{*}{62.441} & 0.218 & Co1 $(0.94,3.14,0.47), \operatorname{Co} 2(0,3.64,0)$ & \multirow{2}{*}{$\begin{array}{l}\text { Small difference on the experimental } \\
\text { temperature }\end{array}$} \\
\hline & & & 0.219 & Co1 $(1.2,3.64,0.57), \operatorname{Co} 2(0,3.35,0)$ & \\
\hline \multirow[t]{2}{*}{15} & \multirow[t]{2}{*}{$\mathrm{CuMnSb}$} & \multirow[t]{2}{*}{16.72} & 1.233 & $\operatorname{Mn}(2.53,1.39,2.53)$ & \multirow{2}{*}{$\begin{array}{l}\text { Small difference on the lattice parameter } \\
\text { and experimental temperature }\end{array}$} \\
\hline & & & 1.265 & $\mathrm{Mn}(2.25,2.25,2.25)$ & \\
\hline
\end{tabular}

\section{Appendix C: Computational methods}

\section{Convention setting of the magnetic unit cell}

We read the crystalline parameters and magnetic moments from the magnetic structure files, whose datatype are 'mcif', provided by BCSMD In the BCSMD website, lattice parameters of the magnetic unit cell are in the convention called working setting $(\vec{a}, \vec{b}, \vec{c})$ and it can be transformed to the standard convention $\left(\vec{a}_{s}, \vec{b}_{s}, \vec{c}_{s}\right)$ by the transformation matrix $T_{s}=\{T \mid \vec{\tau}\}$ as,

$$
\left(\vec{a}_{s}, \vec{b}_{s}, \vec{c}_{s}\right)=T \cdot(\vec{a}, \vec{b}, \vec{c})+\vec{\tau}
$$

where the transformation matrix $T_{s}=\{T \mid \vec{\tau}\}$ of each material has been supplied in the BCSMD website.

While, in the ab initio calculations, we adopt the primitive magnetic unit cell. The primitive lattice vectors $\left(\vec{p}_{1}, \vec{p}_{2}\right.$, $\left.\vec{p}_{3}\right)$ can be obtained by transforming the lattice vectors in standard convention $\left(\vec{a}_{s}, \vec{b}_{s}, \vec{c}_{s}\right)$ with the transformation matrix $M_{X}$,

$$
\left(\vec{p}_{1}, \vec{p}_{2}, \vec{p}_{3}\right)=\left(\vec{a}_{s}, \vec{b}_{s}, \vec{c}_{s}\right) \cdot M_{X}
$$

where $M_{X}$ has been supplied in the VASP2trace package (www.cryst.ehu.es/cryst/checktopologicalmat) and $X$ is the lattice type of the magnetic unit cell.

\section{Parameters setting in ab initio calculations}

We perform all of the first-principle calculations using the Vienna ab initio simulation package(VASP); the generalized gradient approximation (GGA) with the Perdew-Burke-Ernzerhof (PBE) type exchange-correlation potential was adopted. For each material, we set the cutoff energy for plane wave basis as 1.2 times larger than the suggested value in the pseudo-potential files. In the $a b$ initio calculations, the initial magnetic moments are set to the experimental values provided by BCSMD website. The convergence accuracy of self-consistent calculations is $10^{-5} \mathrm{eV}$ and spin-orbital coupling (SOC) has been included. For magnetic cells containing less than 50 atoms, the Brillouin zone (BZ) sampling is performed by using $\mathrm{k}$ grids with a $9 \times 9 \times 9$ mesh in self-consistent calculations. We reduce the grids to $5 \times 5 \times 5$ if there are more than 50 atoms in the magnetic primitive cell to save calculational costs. We implement the ab initio calculations on the MPG supercomputer Cobra and Draco with $960 \mathrm{CPU}$ cores in total and the supercomputer in ShanghaiTech University with $560 \mathrm{CPU}$ cores. For benchmarking, we calculate the simple compound $\mathrm{CeCo}_{2} \mathrm{P}_{2}$ (with 10 atoms per magnetic primitive cell) on the Cobra supercomputer with 80 Skylake cores at $2.4 \mathrm{GHz}$. The time used is $15 \mathrm{~min} 34 \mathrm{~s}$ for the self consistent calculations and $15 \mathrm{~min} 45 \mathrm{~s}$ for the band structure calculations with $240 \mathrm{k}$ points. For the complex compound $\mathrm{Sr}_{3} \mathrm{CoIrO}_{6}$ with 66 atoms per magnetic primitive cell, it costs $7 \mathrm{~h} 50 \mathrm{~min}$ for the self consistent calculations and $6 \mathrm{~h} 42 \mathrm{~min} 53 \mathrm{~s}$ for the band structure calculation with $200 \mathrm{k}$ points.

Since all of the magnetic materials contain at least one correlated element, we also perform the $\mathrm{L}(\mathrm{S}) \mathrm{DA}+\mathrm{U}$ calculations for all of the magnetic materials using the VASP. For the $\mathrm{L}(\mathrm{S}) \mathrm{DA}+\mathrm{U}$ calculations, we adopt the simplified (rotationally invariant) approach and set the Hubbard $\mathrm{U}$ as 1, 2,3,4 eV for the $d$ electrons and 2, 4, $6 \mathrm{eV}$ for the $f$ electrons. For the materials which have both $d$ and $f$ electron, we set $\mathrm{U}$ of $d$ electron as $2 \mathrm{eV}$ and the $\mathrm{U}$ of $f$ electron as $2,4,6 \mathrm{eV}$. 
Similar with the TQC for paramagnetic materials, we also provide the maximal $\mathbf{k}$ vectors for each magnetic space group in the BCS website. Based on the self-consistent charge density files, we calculate the wave functions at the magnetic maximal $\mathbf{k}$ vectors and obtain the characters using the MagVASP2trace package.

\section{Magnetic VASP2trace package}

In TQC, the in house VASP2trace package [75] is used to calculate the character tables of paramagnetic materials. It read the unitary symmetry operators from the output files of VASP and can identify the space group. While the anti-unitary symmetries are absent and VASP2trace cannot identify the magnetic space groups (MSGs).

In the MTQC, we revise the VASP2trace package to calculate the character tables of magnetic materials and supply the symmetry file for each MSG. The magnetic VASP2trace (MagVASP2trace) [? ] reads the magnetic symmetries from the symmetry files that we supply, instead of reading them from the output files of VASP. The symmetry file contains both unitary operations and anti-unitary operations. Both $\mathrm{SO}(3)$ and $\mathrm{SU}(2)$ matrix in the symmetry files are written in the basis of primitive lattice vectors.

MagVASP2trace adopts both $\mathrm{SO}(3)$ and $\mathrm{SU}(2)$ matrix in the convention used in the BCS website (https://www.cryst.ehu.es/) and generate the trace.txt file that contains all of the magnetic symmetry operators and the character tables of the occupied bands at the magnetic maximal $\mathbf{k}$ vectors.

\section{Construction of Wannier tight-binding Hamiltonian and surface states calculation}

We construct the tight-binding Hamiltonians of $\mathrm{NpBi}, \mathrm{CeCo}_{2} \mathrm{P}_{2}, \mathrm{MnGeO}_{3}$ and $\mathrm{Mn}_{3} \mathrm{ZnC}$ using the Wannier90 package 92. We generate the maximally localized Wannier functions (MLWFs) for $5 p$ orbitals on Bi, $5 f$ and $6 d$ orbitals on $\mathrm{Np}$ for the magnetic TI NpBi. For the magnetic NLSM $\mathrm{CeCo}_{2} \mathrm{P}_{2}$, the MLWFs for $3 p$ orbitals on $\mathrm{P}, 3 d$ orbitals on Co, $4 f$ and $5 d$ orbitals on Ce are constructed. For the magnetic DSM MnGeO 3 , we generate the MLWFs for $4 s$ orbitals on Ge, $2 p$ orbitals on $\mathrm{O}$, and $3 d$ orbitals on $\mathrm{Mn}$. For the ferrimagnetic $\mathrm{ES}_{3} \mathrm{Mn}_{3} \mathrm{ZnC}$, we generate the MLWFs for $4 s, 4 p$ and $3 d$ orbitals on Zn, $2 p$ orbitals on C and $3 d$ orbitals on Mn.

The surface states are calculated with the Green's function method using the WannierTools package [93, 94, and the results are shown in FIG. 2 of main text and FIG. 32,33

\section{Appendix D: Comparison of the ground state energy between different magnetic configurations of several compounds}

We select the three magnetic topological materials NpBi with BCSID-3.7, $\mathrm{CeCo}_{2} \mathrm{P}_{2}$ with BCSID-1.253 and $\mathrm{MnGeO}_{3}$ with BCSID-0.125 to compare the energy difference between different magnetic structures, respectively. As shown in Figure 3 , there are three possible magnetic structures for each material, where AFM-I and AFM-II are the assumed configurations and the AFM-III phase is the one obtained from neutron experiments. The relative ground state energies at each $\mathrm{U}$ for the three materials are tabulated in Table VI. For $\mathrm{NpBi}$ and $\mathrm{CeCo}_{2} \mathrm{P}_{2}$, AFM-III phase always has the lowest ground state energy at different U. For $\mathrm{MnGeO}_{3}$, there is only one exception, i.e. the AFM-I phase of $\mathrm{MnGeO}_{3}$ with $\mathrm{U}=0$, that has lower energy than the AFM-III phase. With increasing $\mathrm{U}$, the experimental AFM-III phase lowers its energy to become the lowest.

The comparisons in Table VI indicate that the magnetic configurations obtained from neutron experiments are favorable with the lowest ground state energy.

TABLE VI: The relative ground state energy of $\mathrm{NpBi}, \mathrm{CeCo}_{2} \mathrm{P}_{2}$ and $\mathrm{MnGeO}_{3}$ in three possible magnetic structures with different $U$ added. The magnetic structures are shown in Figure 3, where the magnetic configurations AFM-III are obtained from neutron scattering experiments.

\begin{tabular}{c|c|c|c|c}
\hline Materials (BCSID) & $\mathrm{U}(\mathrm{eV})$ & $\mathrm{E}(\mathrm{AFM}-\mathrm{I})(\mathrm{eV})$ & $\mathrm{E}(\mathrm{AFM}-\mathrm{II})(\mathrm{eV})$ & $\mathrm{E}(\mathrm{AFM}-\mathrm{III})(\mathrm{eV})$ \\
\hline \multirow{4}{*}{ NpBi (BCSID: 3.7) } & 0 & 0.079 & 0.091 & 0 \\
\cline { 2 - 5 } & 2 & 2.108 & 2.125 & 1.995 \\
\cline { 2 - 5 } & 4 & 3.049 & 3.05 & 2.999 \\
\cline { 2 - 5 } & 6 & 3.538 & 3.543 & 3.525 \\
\hline & 0 & 0.388 & 0.388 & 0 \\
\cline { 2 - 5 }
\end{tabular}

$\mathrm{CeCo}_{2} \mathrm{P}_{2}$ (BCSID: 1.253$)$ 

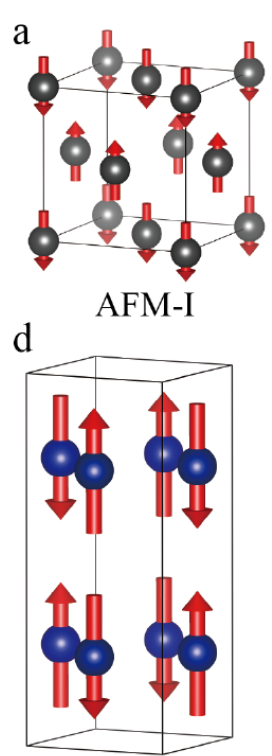

AFM-I

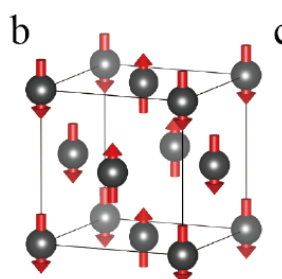

AFM-II

e

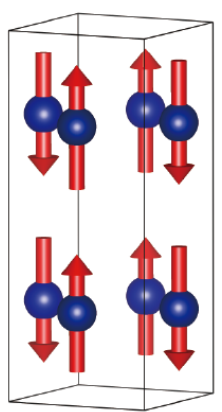

AFM-II

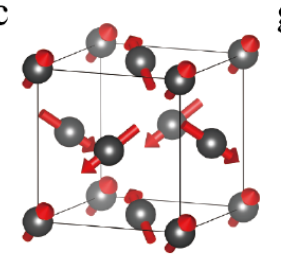

AFM-III

f

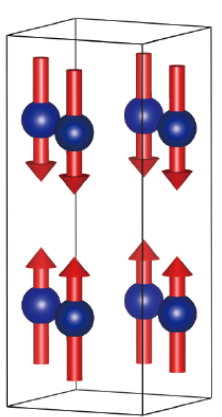

AFM-III

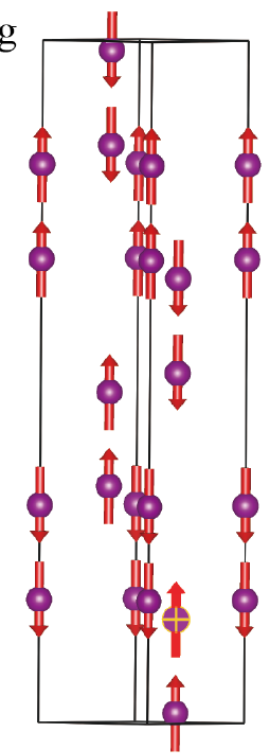

AFM-I $\mathrm{h}$

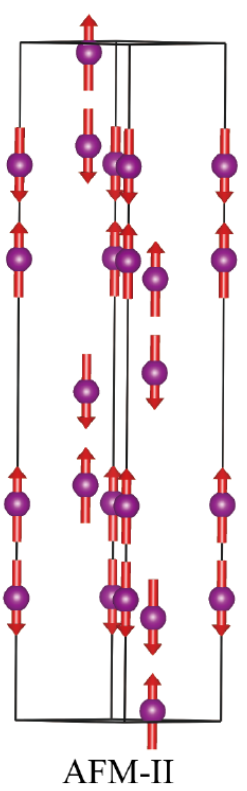

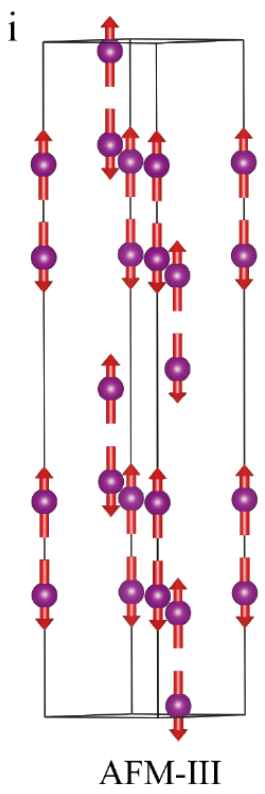

FIG. 3. Three possible magnetic structures for (a-c) NpBi, (d-f) $\mathrm{CeCo}_{2} \mathrm{P}_{2}$ and (g-i) $\mathrm{MnGeO}_{3}$, where the AFM-III phase in (c)(f)(i) are the ones from neutron scattering experiments.

\begin{tabular}{c|c|c|c|c} 
& 2 & 9.159 & 9.243 & 8.468 \\
& 4 & 10.666 & 10.729 & 9.973 \\
& 6 & 11.955 & 12.098 & 11.263 \\
\hline \multirow{3}{*}{$\mathrm{MnGeO}_{3}$ (BCSID: 0.125) } & 0 & -0.4 & 0.164 & 0 \\
\cline { 2 - 5 } & 1 & 6.845 & 6.532 & 6.437 \\
\cline { 2 - 5 } & 2 & 12.713 & 12.151 & 12.068 \\
\cline { 2 - 5 } & 3 & 17.703 & 17.132 & 17.068 \\
\cline { 2 - 5 } & 4 & 22.095 & 21.589 & 21.529 \\
\hline
\end{tabular}

Appendix E: Comparisons between different exchange-correlation potentials

\section{Band structure calculations with GGA functional}

We adopt five different exchange-correlation functional methods, including Perdew-Wang 91 (91), AM05 (AM), revised PBE (RE), revised PBE with Pade Approximation (RP) and Ceperley-Alder functional (CA), to check the topologies and the band structures that obtained by the PBE (PE) method. The topology and band structure comparisons of the topological materials $\mathrm{BaFe}_{2} \mathrm{As}_{2}, \mathrm{CeCo}_{2} \mathrm{P}_{2}, \mathrm{NpBi}$, and $\mathrm{MnGeO}_{3}$ are shown in the FIG. 4.7. The comparisons indicate that different exchange-correlation functional methods have minor effect on the band structures but do not change the topologies of these materials.

\section{Band structure calculations with meta-GGA functional}

To further check the band structures and topologies obtained from LDA $+\mathrm{U}$ calculations, we have also performed $\mathrm{ab}$ initio calculations with the modified Becke-Johnson $(\mathrm{mBJ})$ [3] potential for 23 topological compounds. They are $\mathrm{Mn}_{3} \mathrm{Ir}$ (BCSID-0.108), $\mathrm{Mn}_{3} \mathrm{Sn}$ (BCSID-0.200), $\mathrm{Mn}_{3} \mathrm{Pt}$ (BCSID-1.143), $\mathrm{MnGeO}_{3}$ (BCSID-0.125), $\mathrm{Mn}_{2} \mathrm{As}$ (BCSID-1.132), $\mathrm{CaFe}_{2} \mathrm{As}_{2}$ (BCSID-1.52), $\mathrm{Cd}_{2} \mathrm{Os}_{2} \mathrm{O}_{7}$ (BCSID-0.2), $\mathrm{NiCr}_{2} \mathrm{O}_{4}$ (BCSID-0.4), $\mathrm{PbNiO}_{3}$ (BCSID-0.21), $\mathrm{LuFeO}_{3}$ (BCSID-0.117), $\mathrm{LuFe}_{4} \mathrm{Ge}_{2}$ (BCSID-0.140), $\mathrm{NiS}_{2}$ (BCSID-0.150), $\mathrm{Mn}_{3} \mathrm{Ge}$ (BCSID-0.203), $\mathrm{Co}_{2} \mathrm{SiO}_{4}$ (BCSID-0.218), $\mathrm{CrN}$ (BCSID-1.28), $\mathrm{ScMn}_{6} \mathrm{Ge}_{6}$ (BCSID-1.110), $\mathrm{CaCo}_{2} \mathrm{P}_{2}$ (BCSID-1.252), $\mathrm{CeCo}_{2} \mathrm{P}_{2}$ (BCSID-1.253), $\mathrm{GdIn}_{3}$ (BCSID-1.81), $\mathrm{Mn}_{3} \mathrm{ZnC}$ (BCSID-2.19), NpBi (BCSID-3.7), NpSe (BCSID-3.10) and NpSb (BCSID-3.12). 


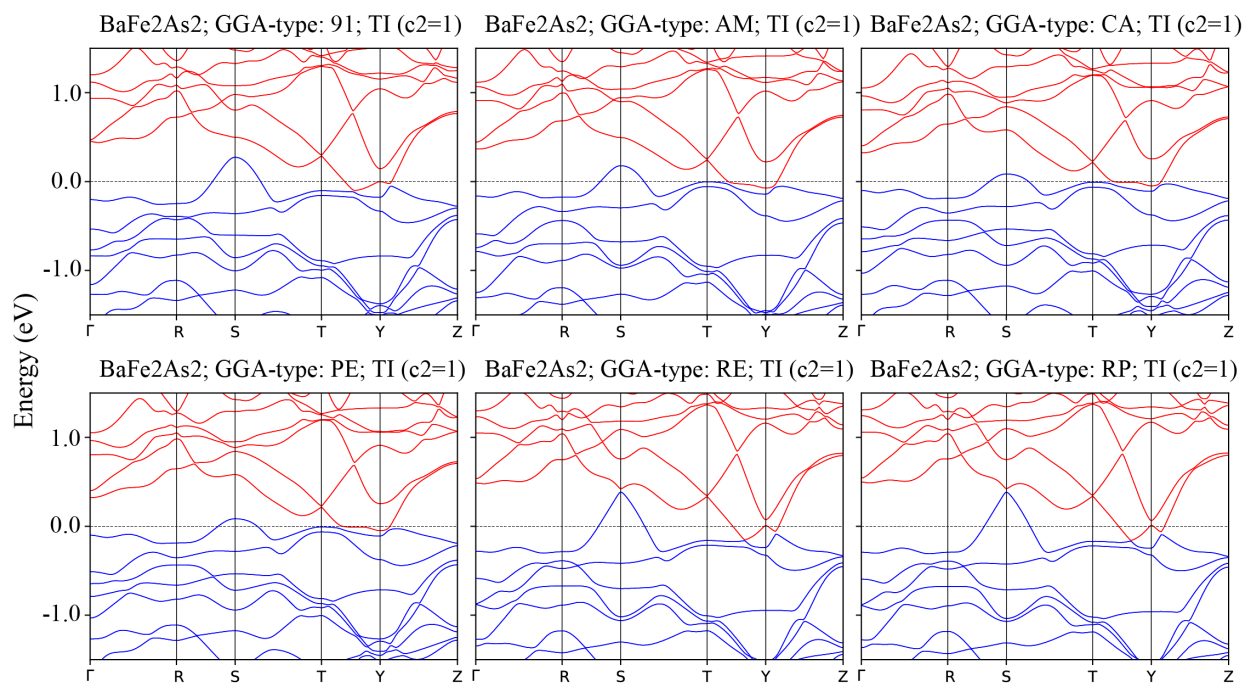

FIG. 4. The band structures and topology of $\mathrm{BaFe}_{2} \mathrm{As}_{2}$ obtained by different exchange-correlation functional methods. The topology is maintained for different methods, indicating a TI with topological index $c_{2}=1$. The Hubbard $\mathrm{U}$ of $3 d$ electron is set to $1 \mathrm{eV}$.

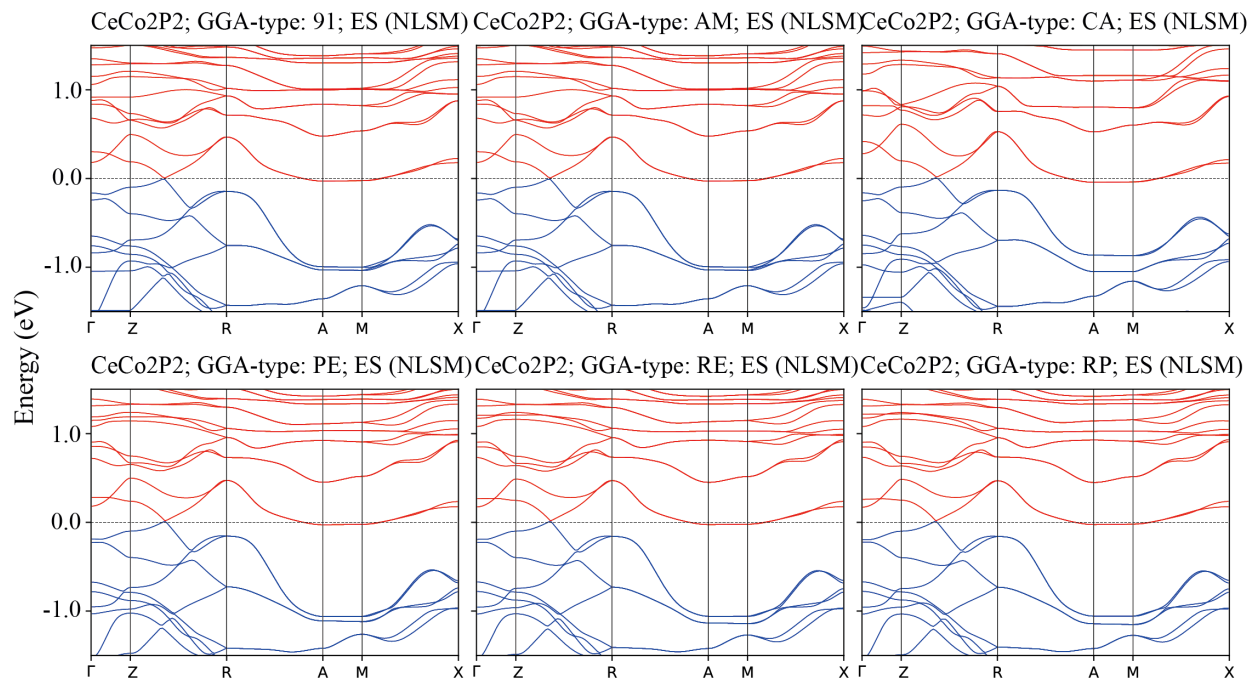

FIG. 5. The band structures and topology of $\mathrm{CeCo}_{2} \mathrm{P}_{2}$ obtained by different exchange-correlation functional methods. The topology is maintained ES (also NLSM) for the different methods. The Hubbard U of $3 d$ and $4 f$ electron are set to 2 and 6 $\mathrm{eV}$, respectively.

Apart from NpSe (BCSID-3.10), we find slightly different band structures with MBJ and LDA+U methods. However, comparing these 2 band structures and its topology, we can always find a value of $U$ that reproduces the MBJ calculations. As shown in FIG. $8-29$, we have found the correct value of U for each compound. Using the correct value of $\mathrm{U}$, we can reproduce the the band structures and topology at the Fermi level consistent with the results obtained from $\mathrm{mBJ}$. 


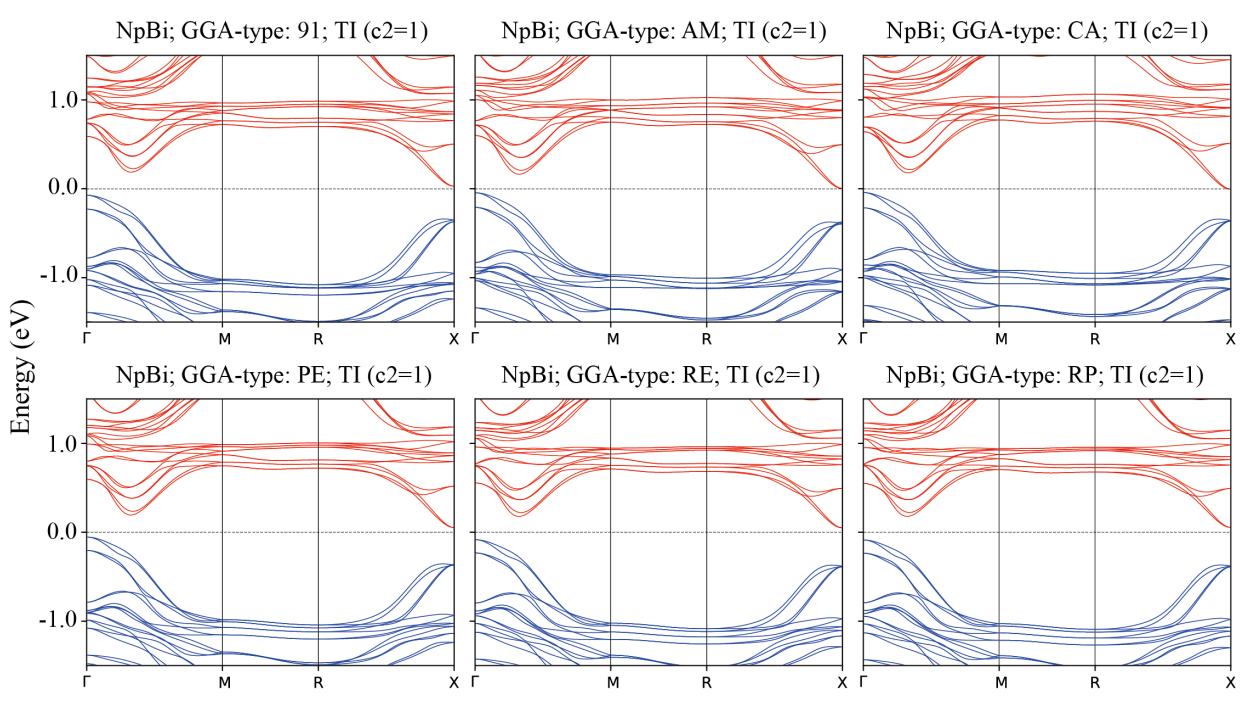

FIG. 6. The band structures and topology of NpBi obtained by different exchange-correlation functional methods. The topology is maintained TI with topological index $c_{2}=1$ for the different methods. The Hubbard $\mathrm{U}$ of $5 f$ electron is set to $2 \mathrm{eV}$.

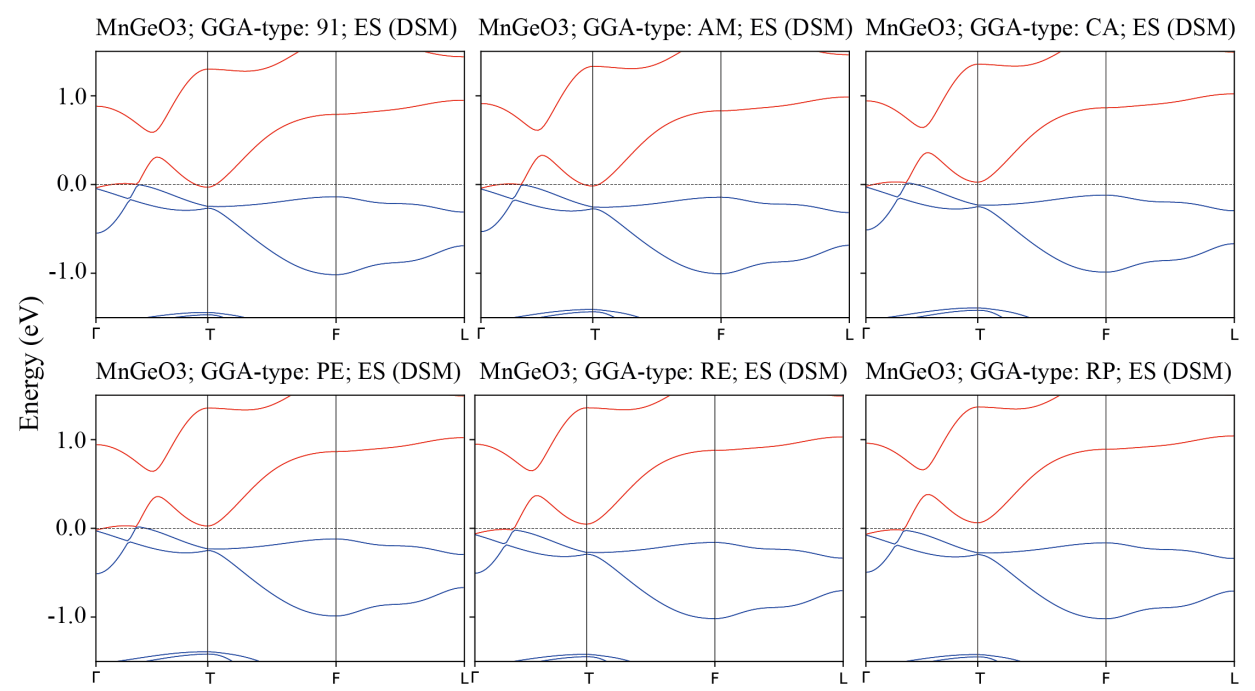

FIG. 7. The band structures and topology of $\mathrm{MnGeO}_{3}$ obtained by different exchange-correlation functional methods. The topology is maintained ES (also DSM) for the different methods. The Hubbard U of $3 d$ electron is set to $4 \mathrm{eV}$.
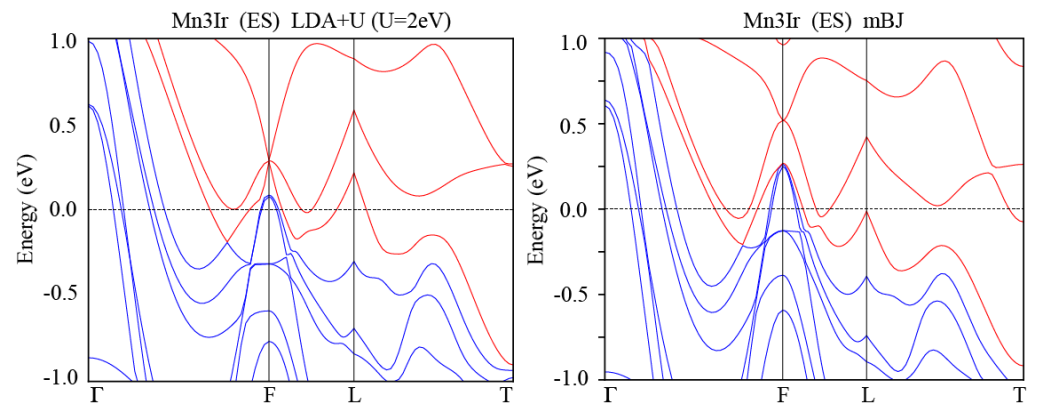

FIG. 8. Band structures of the ES $\mathrm{Mn}_{3} \mathrm{Ir}$ obtained from $\mathrm{LDA}+\mathrm{U}(U=2 e V)$ and mBJ methods. 

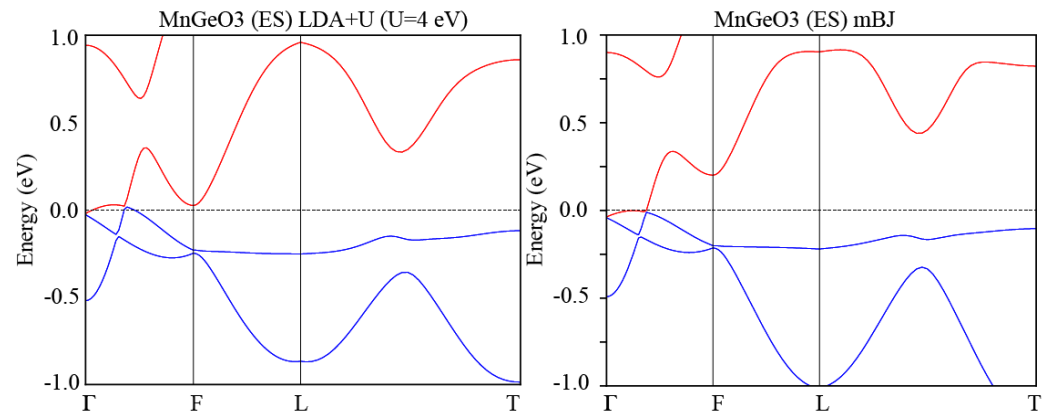

FIG. 9. Band structures of the ES $\mathrm{MnGeO}_{3}$ obtained from $\mathrm{LDA}+\mathrm{U}(\mathrm{U}=4 \mathrm{eV})$ and mBJ methods.
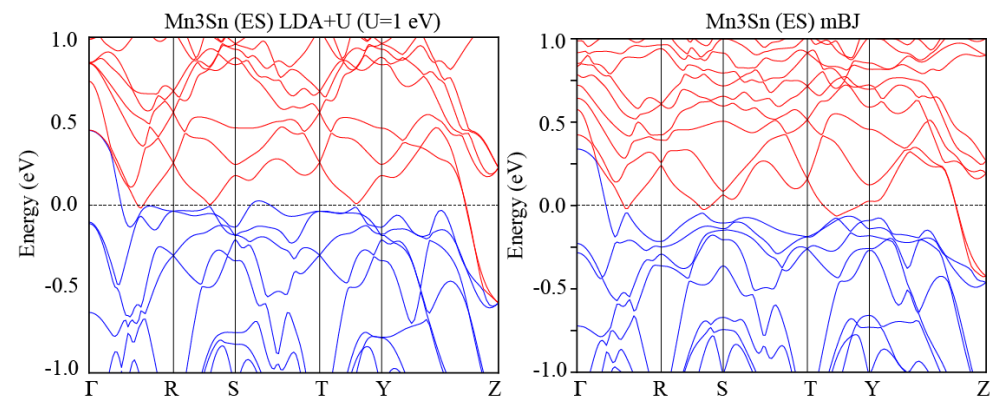

FIG. 10. Band structures of the $\mathrm{ES} \mathrm{Mn}_{3} \mathrm{Sn}$ obtained from $\mathrm{LDA}+\mathrm{U}(\mathrm{U}=1 \mathrm{eV})$ and mBJ methods.
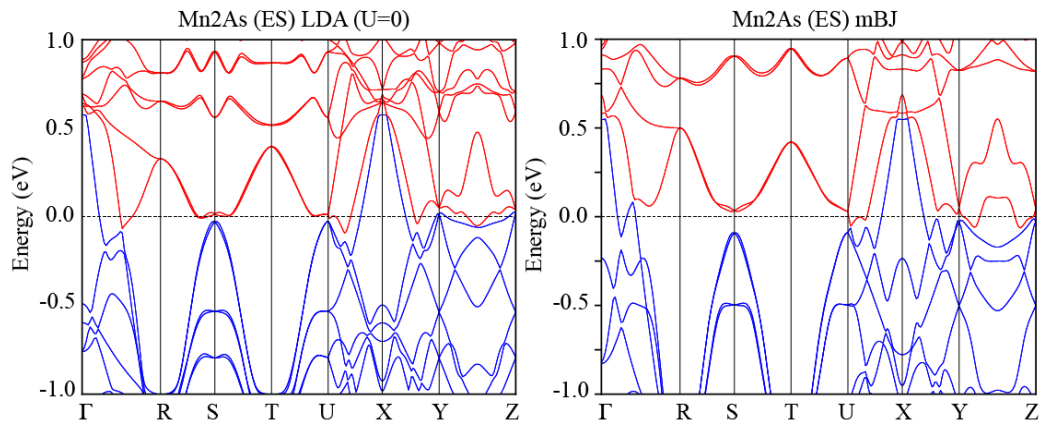

FIG. 11. Band structures of the $\mathrm{ES} \mathrm{Mn}_{2} \mathrm{As}$ obtained from $\mathrm{LDA}+\mathrm{U}(\mathrm{U}=0 \mathrm{eV})$ and mBJ methods.
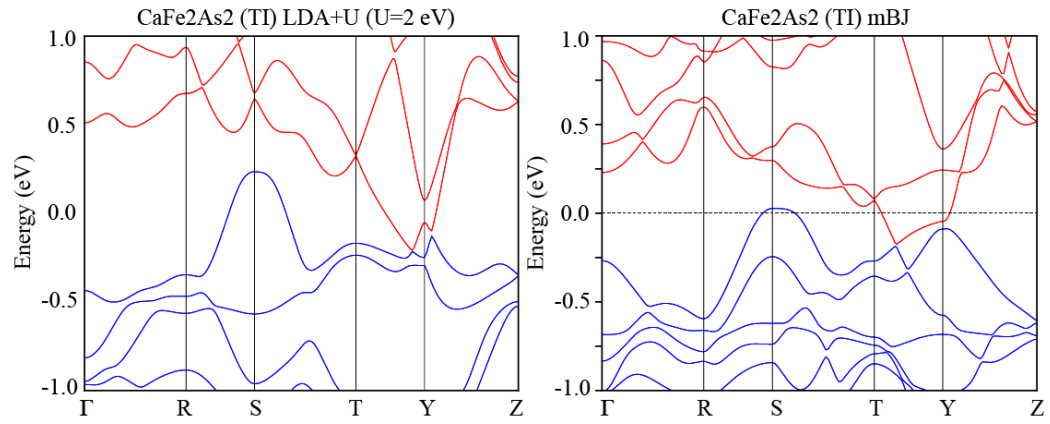

FIG. 12. Band structures of the TI $\mathrm{CaFe}_{2} \mathrm{As}_{2}$ obtained from $\mathrm{LDA}+\mathrm{U}(U=2 \mathrm{eV})$ and mBJ methods. 

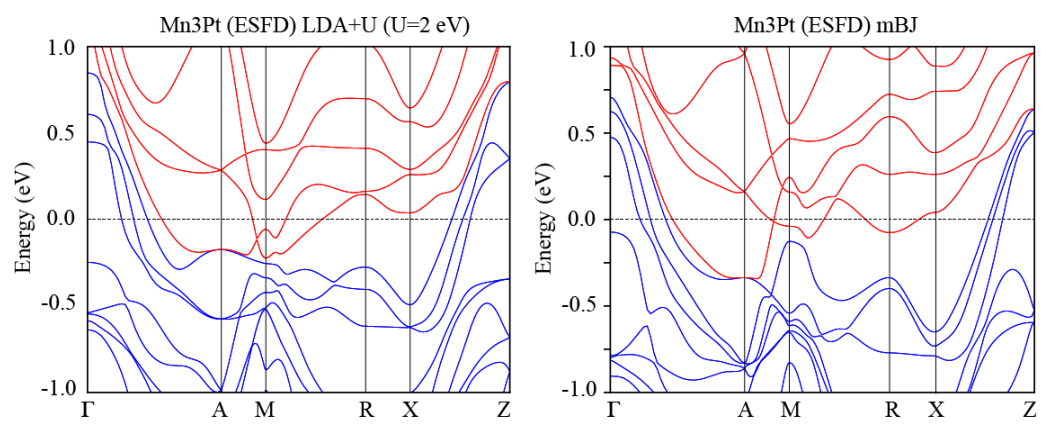

FIG. 13. Band structures of the ESFD $\mathrm{Mn}_{3} \mathrm{Pt}$ obtained from $\mathrm{LDA}+\mathrm{U}(U=2 \mathrm{eV})$ and mBJ methods.
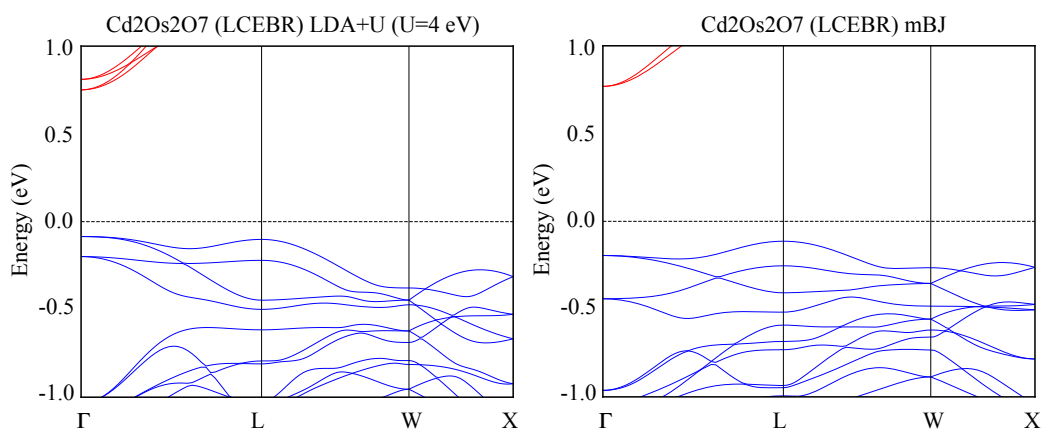

FIG. 14. Band structures of $\mathrm{Cd}_{2} \mathrm{Os}_{2} \mathrm{O}_{7}$ obtained from LDA $+\mathrm{U}(U=2 e V)$ with LCEBR phase and mBJ method with LCEBR phase.
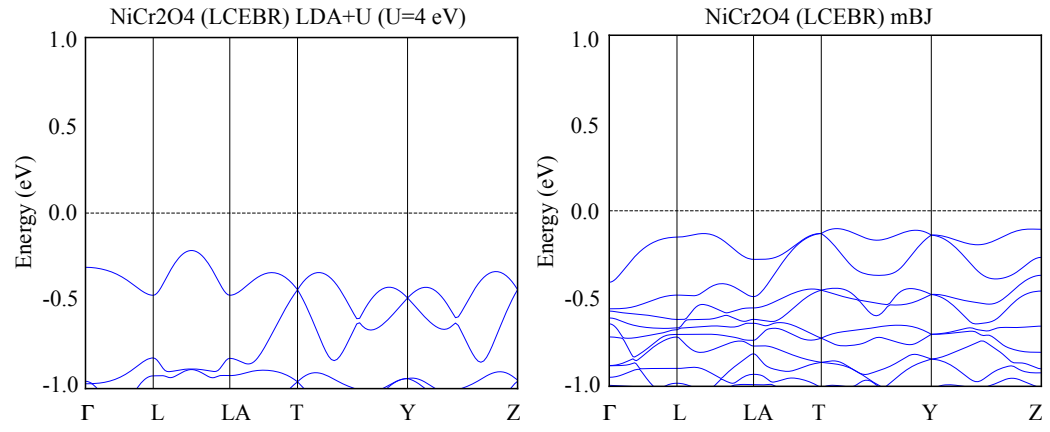

FIG. 15. Band structures of the $\mathrm{NiCr}_{2} \mathrm{O}_{4}$ obtained from LDA $+\mathrm{U}(U=4 \mathrm{eV})$ with LCEBR phase and mBJ method with LCEBR phase.
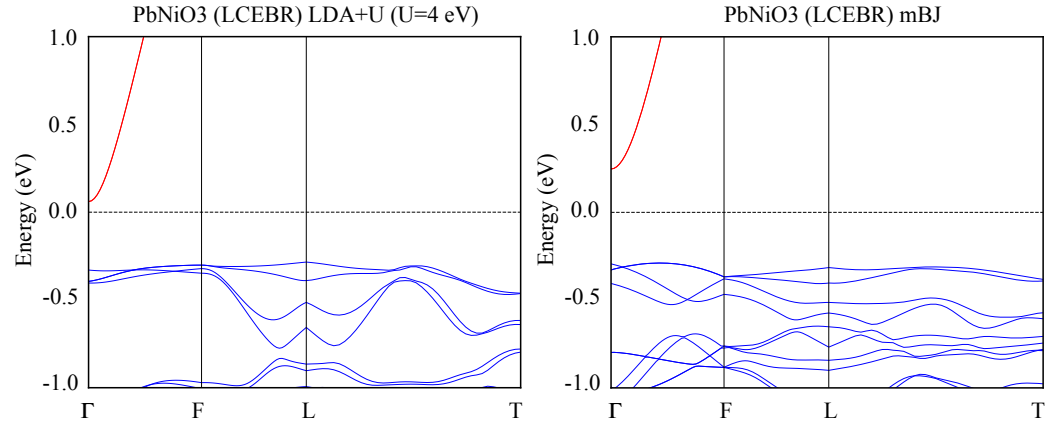

FIG. 16. Band structures of $\mathrm{PbNiO}_{3}$ obtained from LDA $+\mathrm{U}(U=4 \mathrm{eV})$ with LCEBR phase and mBJ methods with LCEBR phase. 

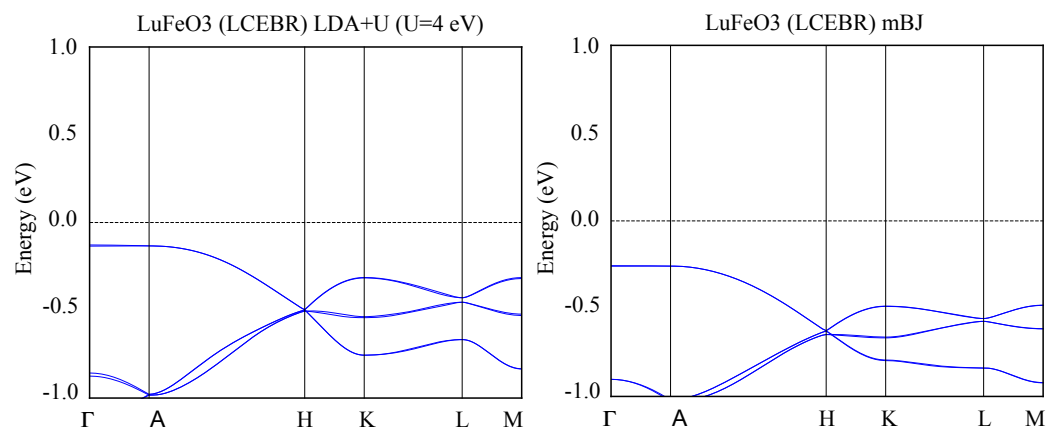

FIG. 17. Band structures of the $\mathrm{LuFeO}_{3}$ obtained from LDA $+\mathrm{U}(U=4 \mathrm{eV})$ with LCEBR phase and mBJ methods with LCEBR phase.
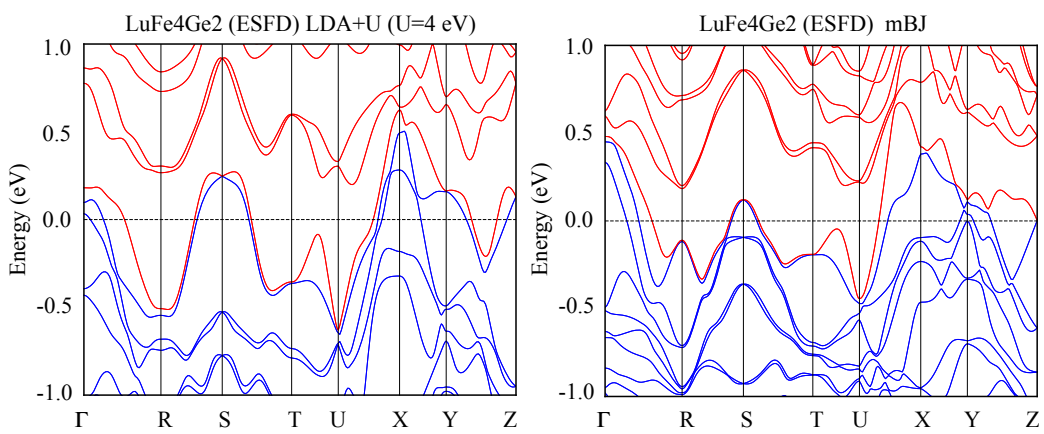

FIG. 18. Band structures of the ESFD $\mathrm{LuFe}_{4} \mathrm{Ge}_{2}$ obtained from $\mathrm{LDA}+\mathrm{U}(U=4 e V)$ and mBJ methods.
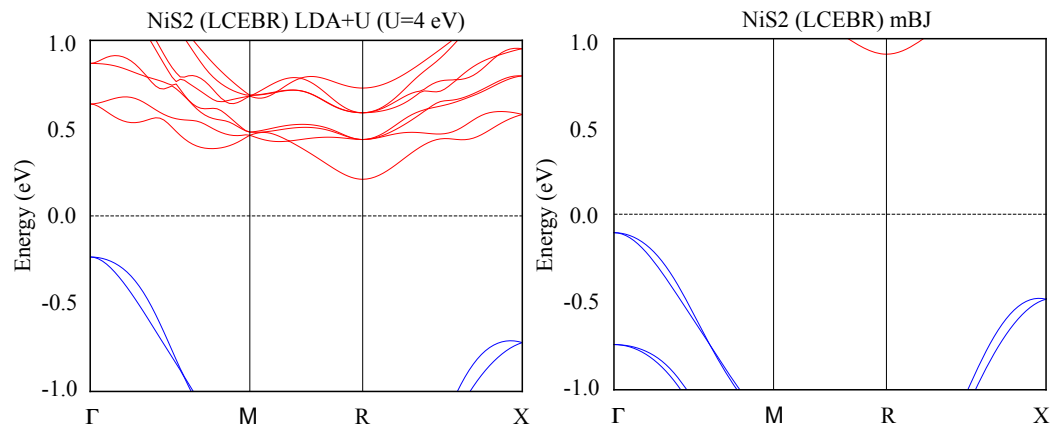

FIG. 19. Band structures of $\mathrm{NiS}_{2}$ obtained from LDA $+\mathrm{U}(U=2 e V)$ with LCEBR phase and mBJ methods with LCEBR phase.
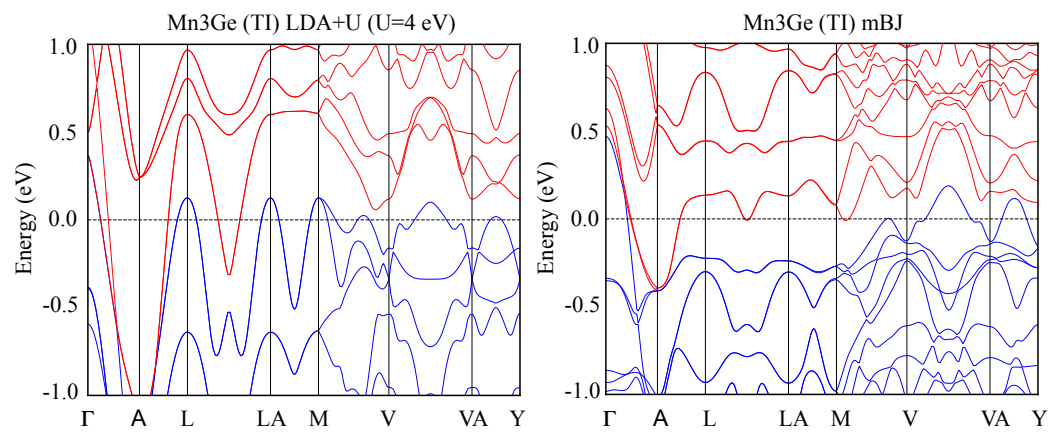

FIG. 20. Band structures of the TI $\mathrm{Mn}_{3} \mathrm{Ge}$ obtained from $\mathrm{LDA}+\mathrm{U}(\mathrm{U}=4 \mathrm{eV})$ and mBJ methods. 

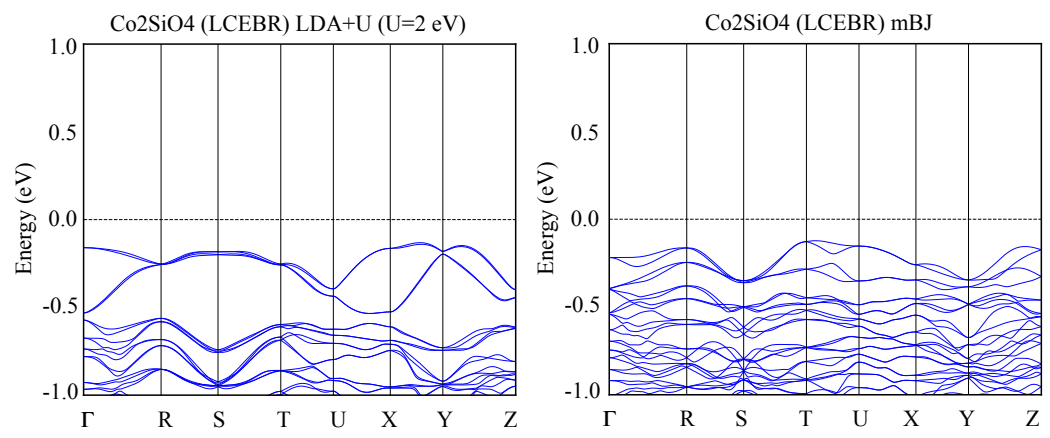

FIG. 21. Band structures of $\mathrm{Co}_{2} \mathrm{SiO}_{4}$ obtained from $\mathrm{LDA}+\mathrm{U}(U=2 \mathrm{eV})$ with LCEBR phase and mBJ methods with LCEBR phase.
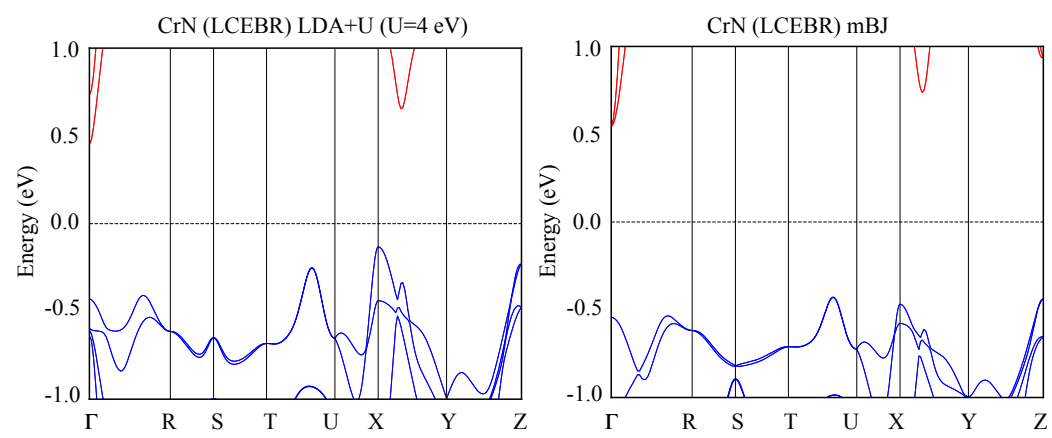

FIG. 22. Band structures of CrN obtained from LDA $+\mathrm{U}(U=2 \mathrm{eV})$ with LCEBR phase and mBJ methods with LCEBR phase.
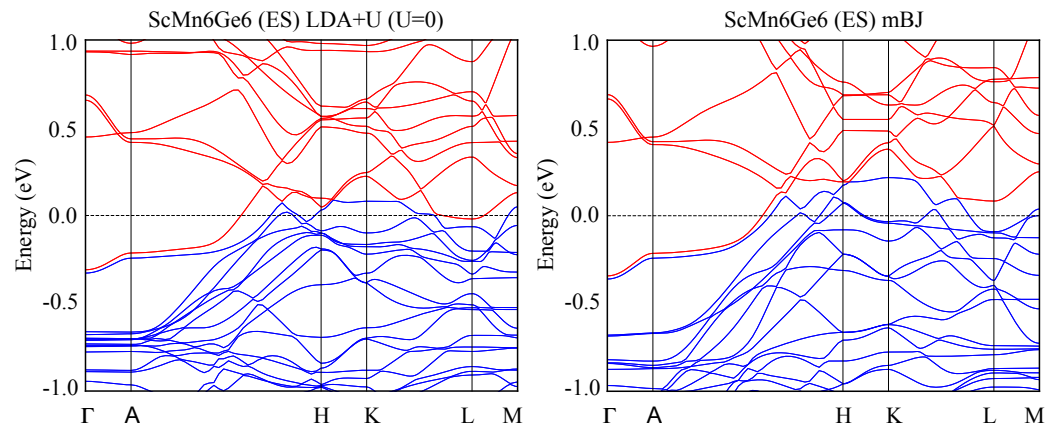

FIG. 23. Band structures of the $\mathrm{ES} \mathrm{ScMn}_{6} \mathrm{Ge}_{6}$ obtained from $\mathrm{LDA}+\mathrm{U}(U=0)$ and mBJ methods.
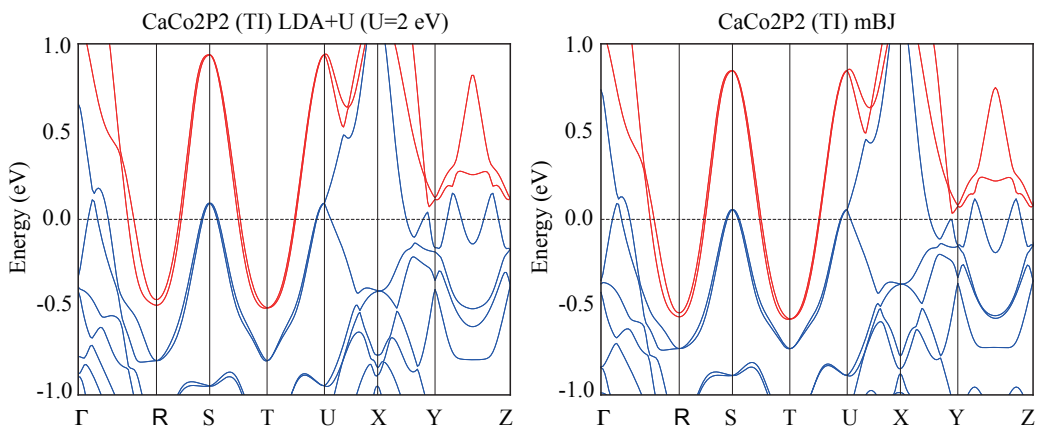

FIG. 24. Band structures of the TI $\mathrm{CaCo}_{2} \mathrm{P}_{2}$ obtained from $\mathrm{LDA}+\mathrm{U}(U=2 e V)$ and mBJ methods. 

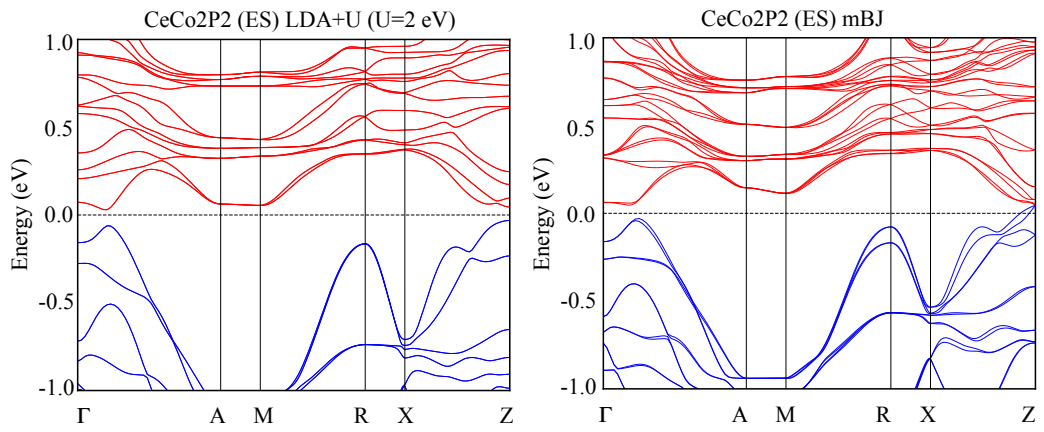

FIG. 25. Band structures of the ESFD $\mathrm{CeCo}_{2} \mathrm{P}_{2}$ obtained from $\mathrm{LDA}+\mathrm{U}(\mathrm{U}=2 \mathrm{eV})$ and mBJ methods.
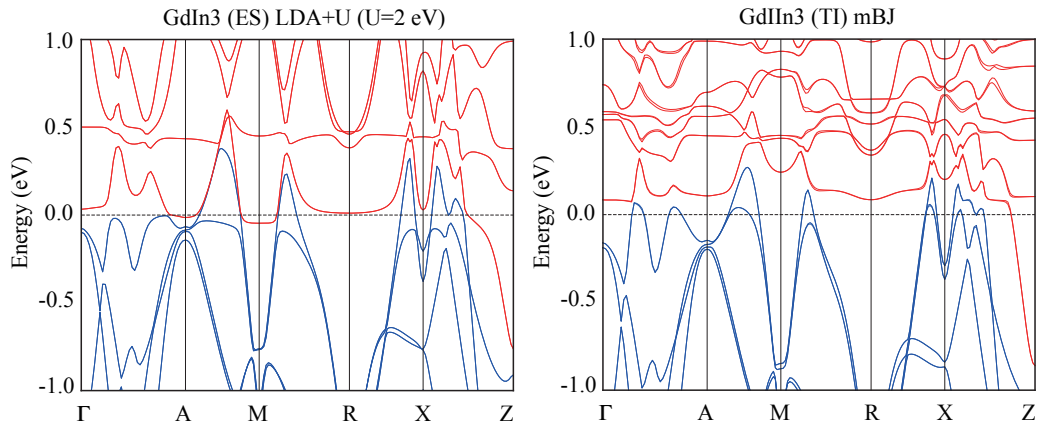

FIG. 26. Band structures of the ES GdIn 3 obtained from $\mathrm{LDA}+\mathrm{U}(U=2 \mathrm{eV})$ and mBJ methods.
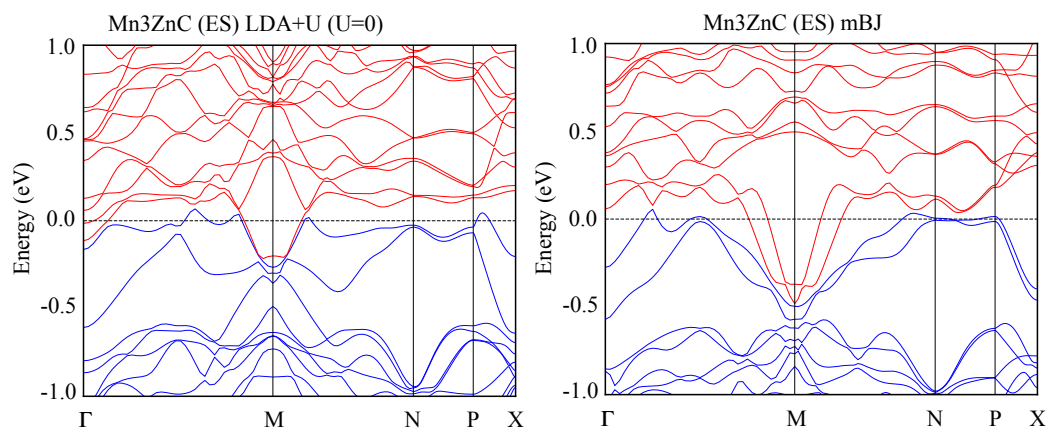

FIG. 27. Band structures of the $\mathrm{ES} \mathrm{Mn}_{3} \mathrm{ZnC}$ obtained from $\mathrm{LDA}+\mathrm{U}(U=2 \mathrm{eV})$ and $\mathrm{mBJ}$ methods.
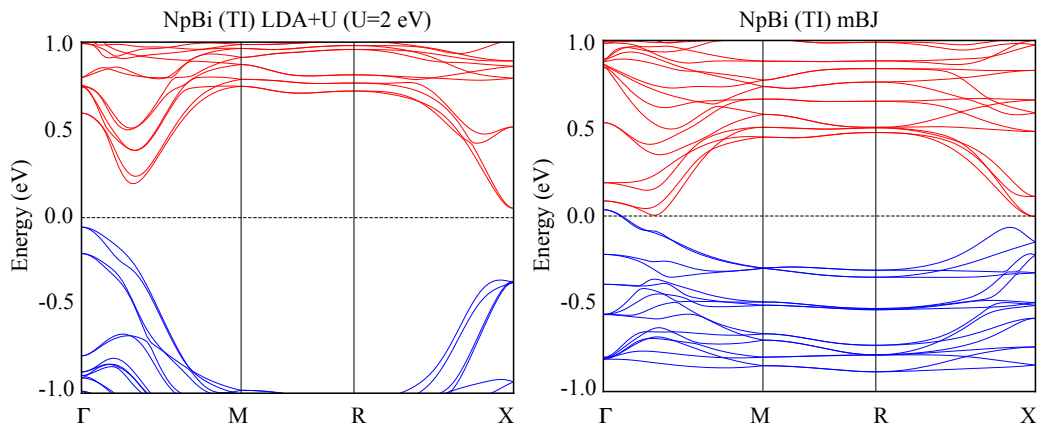

FIG. 28. Band structures of the TI NpBi obtained from LDA $+\mathrm{U}(U=2 \mathrm{eV})$ and mBJ methods. 

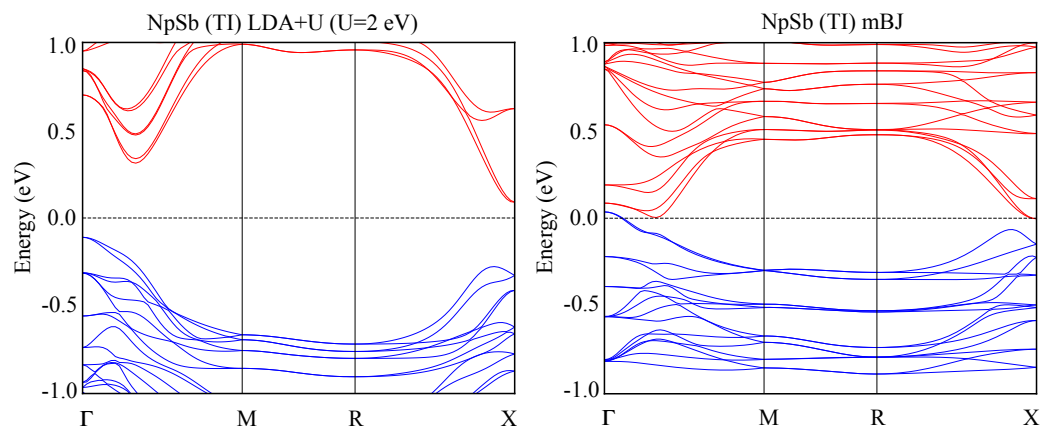

FIG. 29. Band structures of the TI NpSb obtained from LDA $+\mathrm{U}(U=2 e V)$ and mBJ methods. 

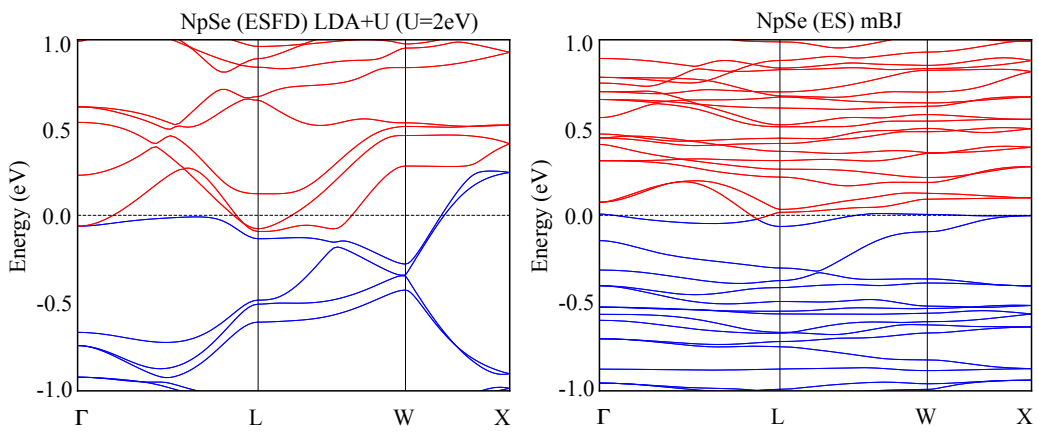

FIG. 30. Band structures of NpSe obtained from LDA $+\mathrm{U}(U=2 e V)$ with ESFD phase and mBJ methods with ES phase. In $\mathrm{LDA}+\mathrm{U}$ calculations, the irreps of the bands at $\Gamma$ point on the Fermi level is 2 -fold degenerate and half-filled. So, it's in ESFD phase. While, in mBJ calculations, the valence band that crossing the Fermi level is 1-dimentional. It's in the ES phase with a symmetry protected crossing point on the $\Gamma L$ path.

\section{Appendix F: Comparisons between LDA+U and LDA+Gutzwiller methods}

To further check the robustness of the results obtained from LDA $+\mathrm{U}$ calculations, we have performed the LDA + Gutzwiller calculations [74] for the two stable magnetic topological semimetals, $\mathrm{MnGeO}_{3}$ and $\mathrm{CeCo}_{2} \mathrm{P}_{2}$.

LDA+Gutzwiller is a many-body technics combined with DFT calculations, which has been successfully applied to predict correlated topological materials [95 97]. Similar to other post-LDA methods, i.e. LDA + U and LDA + DMFT, the total Hamiltonian adopt in LDA+Gutzwiller can be written as,

$$
H_{\text {total }}=H_{L D A}+H_{\text {int }}+H_{d c}
$$

with $H_{L D A}$ being the non-interacting Hamiltonian obtained by LDA+SOC, the atomic spin orbital coupling and $H_{\text {int }}$ being the interacting Hamiltonian. The last term in Eq. (F1) is the double counting Hamiltonian, which needs to be included to remove the local interaction energy treated by LDA already in the mean field manner. In the present study, the Kanamori-type interaction and the fully localized limit scheme for the double counting energy [98] are adopted.

In the LDA + Gutzwiller method, the Gutzwiller type wave function $\left|\psi_{G}\right\rangle=\hat{P}\left|\psi_{0}\right\rangle$ has been proposed for the trial wave function to minimize the ground state energy, where $\left|\psi_{0}\right\rangle$ is the non-interacting wave function and $\hat{P}$ is the local projector applied to adjust the probability of the local atomic configuration (in the many-particle Fock space). In addition, the Gutzwiller approximation is applied to evaluate the ground state energy and an effective Hamiltonian $H_{e f f} \approx \hat{P} H_{L D A} \hat{P}$ describing the quasi-particle dispersion can be obtained. For detailed description for the method please refer to references [74, 99, 100].

In the LDA+Gutzwiller calculation, we adopt the same parameter $\mathrm{U}$ as $\mathrm{LDA}+\mathrm{U}$ calculation. For $\mathrm{MnGeO}_{3}$, the quasi-particle weight of the $d$ electron on $\mathrm{Mn}$ is about 0.8 and the local magnetic moment on $\mathrm{Mn}$ is about $4.6 \mu_{B} / \mathrm{Mn}$ which is consistent with LDA $+\mathrm{U}$ calculation $\left(4.3 \mu_{B} / \mathrm{Mn}\right)$. Compared with the band structure obtained from LDA $+\mathrm{U}$ calculations in Figure 31(a), the quasi-particle band structure in LDA+Gutzwiller are renormalized by a factor of 0.86, as shown in Figure 31(b).

For $\mathrm{CeCo}_{2} \mathrm{P}_{2}$, the quasi-particle weight of the $f$ electron on Ce is about 0.25 and the occupation of $f$ electron is about 1.0/Ce. Compared with the band structure obtained from LDA $+\mathrm{U}$ calculations in Figure 31(c), the quasi-particle band structure in LDA+Gutzwiller are strongly renormalized by a factor of 0.25 , as shown in Figure 31(d). Although the large-size renormalization on $f$ orbitals changes the quasi-particle bands a lot, the symmetry enforced band crossing along $Z A$ path is stable.

Both comparisons for $\mathrm{MnGeO}_{3}$ and $\mathrm{CeCo}_{2} \mathrm{P}_{2}$ indicate that strong correlations only renormalize the band width by a factor of quasi-particle weight but don't change the topologies for the stable topological materials $\mathrm{MnGeO}_{3}$ and $\mathrm{CeCo}_{2} \mathrm{P}_{2}$.

\section{Appendix G: Topological phase diagrams of the topological materials that predicted by MTQC}

We tabulate the topological categories at different $U$ 's for all the magnetic materials. In the Table VII and VIII, each material list is represented by different colors based on their phase transition trends with increasing $U$ The tables 

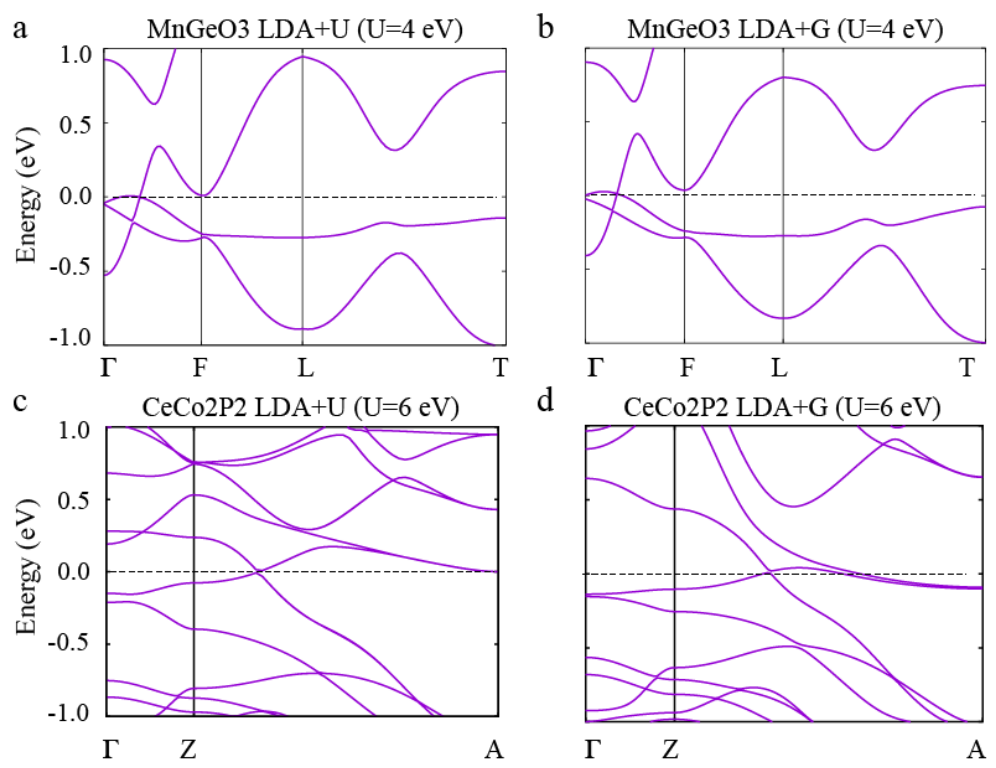

FIG. 31. (a) Electronic band structures of $\mathrm{MnGeO}_{3}$ obtained from LDA+U and (b) LDA+Gutzwiller (b) with the on-site Coulomb interaction $U=4 \mathrm{eV}$ and the Hund's coupling $J=0.8 \mathrm{eV}$. From LDA+Gutzwiller calculations, the quasi-particle weight of the $d$ electron on $\mathrm{Mn}$ is about 0.86 and the magnetic moments on $\mathrm{Mn}$ is about $4.8 \mu_{B}(\mathrm{c})$-(d) Electronic band structures of $\mathrm{CeCo}_{2} \mathrm{P}_{2}$ obtained from LDA+U and LDA+Gutzwiller, respectively. The on-site interaction of $f$ orbitals is taken as $U=6 e V$. From LDA+Gutzwiller calculations, the quasi-particle weight of the $f$ electron on Ce is about 0.25 . The magnetic moments on Ce and Co are 0.0 and $0.9 \mu_{B}$, respectively.

contain the material identification number in BCSMD (BCSID), chemical formula (Formula), magnetic space group (MSG), the correlated atoms (CA) that exhibit added $U$ in the $a b$ initio calculations, topological phases with different $U$ and the link to our plotted band structures (BS).

In Tables VII and VIII, the interaction parameter $U$ is varied in the range of $0,1,2,3,4 \mathrm{eV}$ for $d$ electron and 0 , 2, 4, $6 \mathrm{eV}$ for $f$ electron, respectively. Upon adding and increasing $\mathrm{U}$, there are 49 of the 130 topological materials that have stable topology (remain the same topology for all $U$ ), and 49 materials have nontrivial topology for weak correlation, while becoming topological trivial with strong correlations. There are 20 materials whose topologies are sensitive to the interaction and have topological phase transitions between TI and ES in a small interaction range. There are only 5 materials that belong to trivial class in the weak correlated case and become topological nontrivial when the correlation is strong enough. The trend of 'Topological $\rightarrow$ Trivial' upon large U is clear in our data.

The green color stands for nontrivial topology stable in the whole range of $U$ considered in the calculations. The blue color stands for topology nontrivial for weak correlation effect, but trivial with strong correlation. The color stands for topology sensitive to correlations and for topological phase transitions between TI and ES in a small interaction range. The red color stands for topology trivial in weak correlations, but nontrivial with increasing $U$. The grey color stands for the cases where the self consistent calculations are not converged and the topological phase diagrams are not completed. We have separated the material list into two parts: one for $d$ electron with Coulomb in the range of $0 \sim 4 \mathrm{eV}$, another for $f$ electron with Coulomb in the range of $0 \sim 6 \mathrm{eV}$. The materials are ordered by the MSG. We also tag the ESs/ESFDs with chiral MSGs, in which the crossing points carry nonzero chiral charges.

We emphasis that if a symmetry-data-vector cannot be written as an integer combination of EBRs (LCEBR) and the compatibility relations are satisfied, it is diagnosed as a TI in TableVII andVIII. This TI can be stable TI/SISM with stable topological index. In $\mathrm{H}$, we interpret all the TIs by their topological indices. We also find that some of the ES phases can be changed to TI/SISM phase by symmetry breaking. For example, the ES phase $(U=3,4 \mathrm{eV})$ of $\mathrm{Mn}_{3} \mathrm{Sn}$ (with BCSID-0.200) can be calssified as SISM phase (with indice $\eta_{4 I}=3$ ) if its MSG $63.464(\mathrm{Cm} / \mathrm{cm} /$ ) is subducted to the minimal subgroup MSG $2.4(P \overline{1})$.

TABLE VII: Topological phase diagram of the magnetic materials that have transition elements.. The interaction parameter $U$ of $d$ electrons on the correlated atoms have been set to 0,1,2, 3 and $4 \mathrm{eV}$. For the material EuFe $\mathrm{Es}_{2}$ (BCS-ID: 2.1), since the local magnetic moments on $\mathrm{Eu}$ are about $7.0 \mu_{B}$, which is fully spin-polarized, we take the $\mathrm{U}$ of $f$ electron on Eu as $4 \mathrm{eV}$ and the $\mathrm{U}$ of $d$ electron on $\mathrm{Fe}$ as $0,1,2,3,4 \mathrm{eV}$.

\begin{tabular}{|c|c|c|c|c|c|c|c|c|c|}
\hline BCS-ID & Formula & MSG & $\mathrm{CA}$ & $\mathrm{U}=0$ & $\mathrm{U}=1$ & $\mathrm{U}=2$ & $\mathrm{U}=3$ & $\mathrm{U}=4$ & BS \\
\hline 0.165 & $\mathrm{SrMn}(\mathrm{VO} 4)(\mathrm{OH})$ & $4.7\left(P 2_{1}\right)^{*}$ & $\mathrm{Mn}, \mathrm{V}$ & $\overline{\mathrm{ES}}$ & LCEBR & LCEBR & LCEBR & LCEBR & \begin{tabular}{l|l|} 
Table & LI
\end{tabular} \\
\hline
\end{tabular}




\begin{tabular}{|c|c|c|c|c|c|c|c|c|c|}
\hline 1.264 & CoPS3 & $11.57\left(P_{C} 2_{1} / m\right)$ & Co & TI & LCEBR & LCEBR & LCEBR & LCEBR & $\begin{array}{c}\text { Table } \\
\text { LXVI }\end{array}$ \\
\hline 0.203 & Mn3Ge & $12.62\left(C 2^{\prime} / \mathrm{m}^{\prime}\right)$ & $\mathrm{Mn}$ & $\mathrm{TI}$ & TI & $\mathrm{TI}$ & $\mathrm{TI}$ & $\mathrm{TI}$ & Table LXX \\
\hline 1.0 .13 & FeI2 & $12.62\left(C 2^{\prime} / m^{\prime}\right)$ & $\mathrm{Fe}$ & TI & LCEBR & LCEBR & LCEBR & LCEBR & $\begin{array}{c}\text { Table } \\
\text { [LXXI }\end{array}$ \\
\hline 1.201 & Cr2ReO6 & $14.80\left(P_{a} 2_{1} / c\right)$ & $\mathrm{Cr}$ & TI & LCEBR & LCEBR & TBD & TBD & $\begin{array}{c}\text { Table } \\
\text { XCVII }\end{array}$ \\
\hline 1.49 & $\mathrm{Ag} 2 \mathrm{NiO} 2$ & $15.90\left(C_{c} 2 / c\right)$ & $\mathrm{Ni}$ & $\mathrm{TI}$ & LCEBR & LCEBR & LCEBR & LCEBR & $\begin{array}{c}\text { Table } \\
\text { CXXXIV }\end{array}$ \\
\hline 1.50 & $\mathrm{AgNiO} 2$ & $18.22\left(P_{B} 2_{1} 2_{1} 2\right)^{*}$ & $\mathrm{Ni}$ & ES & ES & LCEBR & LCEBR & LCEBR & $\begin{array}{c}\text { Table } \\
\text { CXXXIX } \\
\end{array}$ \\
\hline 1.263 & Ca3Ru2O7 & $33.154\left(P_{C} n a 2_{1}\right)$ & $\mathrm{Ru}$ & ES & $\mathrm{ES}$ & LCEBR & LCEBR & LCEBR & Table CLV \\
\hline 0.85 & $\mathrm{KCo} 4(\mathrm{PO} 4) 3$ & $58.398\left(P n n^{\prime} m^{\prime}\right)$ & Co & ES & LCEBR & LCEBR & LCEBR & LCEBR & $\begin{array}{c}\text { Table } \\
\text { CLXXIX }\end{array}$ \\
\hline 0.140 & $\mathrm{LuFe} 4 \mathrm{Ge} 2$ & $58.399\left(P n^{\prime} n^{\prime} m^{\prime}\right)$ & $\mathrm{Fe}$ & ESFD & ESFD & ESFD & ESFD & ESFD & $\frac{\text { Table }}{\text { CLXXX }}$ \\
\hline 0.27 & YFe4Ge2 & $58.399\left(P n^{\prime} n^{\prime} m^{\prime}\right)$ & $\mathrm{Fe}$ & ESFD & ESFD & ESFD & ESFD & ESFD & $\begin{array}{c}\text { Table } \\
\text { CLXXXI }\end{array}$ \\
\hline 1.252 & $\mathrm{CaCo} 2 \mathrm{P} 2$ & $59.416\left(P_{I} m m n\right)$ & Co & LCEBR & TI & $\mathrm{TI}$ & $\mathrm{TI}$ & TI & Table \\
\hline 1.88 & $\mathrm{Mn} 5 \mathrm{Si} 3$ & $60.431\left(P_{C} b c n\right)$ & $\mathrm{Mn}$ & TI & ES & LCEBR & LCEBR & LCEBR & $\begin{array}{c}\text { Table } \\
\text { CLXXXVII }\end{array}$ \\
\hline 2.1 & EuFe2As2 & $61.439\left(P_{C} b c a\right)$ & $\mathrm{Eu}, \mathrm{Fe}$ & LCEBR & ES & $\mathrm{TI}$ & $\mathrm{TI}$ & LCEBR & $\begin{array}{c}\text { Table } \\
\text { CXCI }\end{array}$ \\
\hline 0.218 & $\mathrm{Co} 2 \mathrm{SiO} 4$ & $62.441($ Pnma $)$ & Co & $\mathrm{ES}$ & LCEBR & LCEBR & LCEBR & LCEBR & $\begin{array}{c}\text { Table } \\
\text { CXCV }\end{array}$ \\
\hline 0.219 & $\mathrm{Co} 2 \mathrm{SiO} 4$ & $62.441($ Pnma $)$ & Co & ES & ES & LCEBR & LCEBR & LCEBR & $\begin{array}{c}\text { Table } \\
\text { CXCVI }\end{array}$ \\
\hline 0.221 & $\mathrm{Fe} 2 \mathrm{SiO} 4$ & $62.441($ Pnma $)$ & $\mathrm{Fe}$ & ES & LCEBR & LCEBR & LCEBR & LCEBR & $\begin{array}{c}\text { Table } \\
\text { CXCVII }\end{array}$ \\
\hline 1.130 & $\mathrm{Cr} 2 \mathrm{As}$ & $62.450\left(P_{a} n m a\right)$ & $\mathrm{Cr}$ & LCEBR & TI & LCEBR & LCEBR & LCEBR & $\begin{array}{c}\text { Table } \\
\text { CCXIII }\end{array}$ \\
\hline 1.131 & $\mathrm{Fe} 2 \mathrm{As}$ & $62.450\left(P_{a} n m a\right)$ & $\mathrm{Fe}$ & $\mathrm{TI}$ & TI & ES & $\mathrm{TI}$ & TI & $\begin{array}{c}\text { Table } \\
\text { CCXIV }\end{array}$ \\
\hline 1.132 & $\mathrm{Mn} 2 \mathrm{As}$ & $62.450\left(P_{a} n m a\right)$ & $\mathrm{Mn}$ & ES & ES & ES & ES & ES & $\begin{array}{l}\text { Table } \\
\text { CCXV }\end{array}$ \\
\hline 1.28 & $\mathrm{CrN}$ & $62.450\left(P_{a} n m a\right)$ & $\mathrm{Cr}$ & TI & TI & LCEBR & LCEBR & LCEBR & $\begin{array}{c}\text { Table } \\
\text { CCXVI }\end{array}$ \\
\hline 0.199 & Mn3Sn & $63.463\left(C m c^{\prime} m^{\prime}\right)$ & $\mathrm{Mn}$ & ES & $\mathrm{ES}$ & ES & $\mathrm{ES}$ & ES & $\begin{array}{c}\text { Table } \\
\text { CCXIX }\end{array}$ \\
\hline 0.200 & Mn3Sn & $63.464\left(\mathrm{Cm}^{\prime} \mathrm{cm}^{\prime}\right)$ & $\mathrm{Mn}$ & TI & TI & TI & ES & ES & $\begin{array}{c}\text { Table } \\
\text { CCXX } \\
\end{array}$ \\
\hline 1.16 & $\mathrm{BaFe} 2 \mathrm{As} 2$ & $64.480\left(C_{A} m c a\right)$ & $\mathrm{Fe}$ & TI & TI & TI & TI & LCEBR & $\begin{array}{c}\text { Table } \\
\text { CCXXIV }\end{array}$ \\
\hline 1.52 & $\mathrm{CaFe} 2 \mathrm{As} 2$ & $64.480\left(C_{A} m c a\right)$ & $\mathrm{Fe}$ & TI & TI & TI & LCEBR & LCEBR & $\begin{array}{c}\text { Table } \\
\text { CCXXVI }\end{array}$ \\
\hline 2.15 & Mn3Ni20P6 & $65.486\left(\mathrm{Cmm}^{\prime} \mathrm{m}^{\prime}\right)$ & $\mathrm{Mn}, \mathrm{Ni}$ & TBD & ES & $\mathrm{ES}$ & TI & ES & $\frac{\text { Table }}{\text { CCXXVII }}$ \\
\hline 0.4 & $\mathrm{NiCr} 2 \mathrm{O} 4$ & $70.530\left(F d^{\prime} d^{\prime} d\right)$ & $\mathrm{Ni}, \mathrm{Cr}$ & ES & LCEBR & LCEBR & LCEBR & LCEBR & $\begin{array}{c}\text { Table } \\
\text { CCXXX }\end{array}$ \\
\hline 1.125 & $\mathrm{LaFeAsO}$ & $73.553\left(I_{c} b c a\right)$ & $\mathrm{Fe}$ & TI & LCEBR & LCEBR & LCEBR & LCEBR & $\begin{array}{c}\text { Table } \\
\text { CCXXXI }\end{array}$ \\
\hline 1.176 & YbCo2Si2 & $73.553\left(I_{c} b c a\right)$ & Co & LCEBR & TI & TI & TI & TI & $\begin{array}{c}\text { Table } \\
\text { CCXXXII }\end{array}$ \\
\hline 2.5 & $\mathrm{Mn} 3 \mathrm{CuN}$ & $85.59(P 4 / n)$ & $\mathrm{Mn}$ & ES & ES & $\mathrm{ES}$ & ES & ES & $\begin{array}{c}\text { Table } \\
\text { CCXXXIV }\end{array}$ \\
\hline
\end{tabular}




\begin{tabular}{|c|c|c|c|c|c|c|c|c|c|}
\hline 0.64 & $\mathrm{MnV} 2 \mathrm{O} 4$ & $88.81\left(I 4_{1} / a\right)$ & $\mathrm{Mn}, \mathrm{V}$ & $\mathrm{ES}$ & LCEBR & LCEBR & LCEBR & LCEBR & \begin{tabular}{c|} 
Table \\
CCXXXVII
\end{tabular} \\
\hline 1.85 & alpha-Mn & $114.282\left(P_{I} \overline{4} 2_{1} c\right)$ & $\mathrm{Mn}$ & ES & LCEBR & LCEBR & LCEBR & TBD & $\begin{array}{c}\text { Table } \\
\text { CCXLII }\end{array}$ \\
\hline 1.143 & $\mathrm{Mn} 3 \mathrm{Pt}$ & $132.456\left(P_{c} 4_{2} / \mathrm{mcm}\right)$ & $\mathrm{Mn}$ & ESFD & ESFD & ESFD & ESFD & ESFD & $\begin{array}{c}\text { Table } \\
\text { CCXLV] }\end{array}$ \\
\hline 1.146 & $\mathrm{LaCrAsO}$ & $138.528\left(P_{c} 4_{2} / n c m\right)$ & $\mathrm{Cr}$ & $\mathrm{TI}$ & $\mathrm{TI}$ & $\mathrm{TI}$ & $\mathrm{TI}$ & $\mathrm{TI}$ & $\begin{array}{c}\text { Table } \\
\text { CCXLIX }\end{array}$ \\
\hline 0.212 & Sr2Mn3As2O2 & $139.536\left(I 4^{\prime} / m^{\prime} m^{\prime} m\right)$ & $\mathrm{Mn}$ & ESFD & ESFD & ESFD & ESFD & ESFD & $\begin{array}{c}\text { Table } \\
\text { CCLI }\end{array}$ \\
\hline 2.19 & $\mathrm{Mn} 3 \mathrm{ZnC}$ & $139.537\left(I 4 / \mathrm{mm}^{\prime} \mathrm{m}^{\prime}\right)$ & $\mathrm{Mn}$ & $\mathrm{ES}$ & $\mathrm{ES}$ & $\overline{E S}$ & $\mathrm{ES}$ & $\mathrm{ES}$ & $\begin{array}{l}\text { Table } \\
\text { CCLIV }\end{array}$ \\
\hline 0.125 & $\mathrm{MnGeO} 3$ & $148.19\left(R \overline{3}^{\prime}\right)$ & $\mathrm{Mn}$ & $\mathrm{ES}$ & ES & $\mathrm{ES}$ & $\mathrm{ES}$ & ES & $\begin{array}{c}\text { Table } \\
\text { CCLXI }\end{array}$ \\
\hline 0.21 & $\mathrm{PbNiO} 3$ & $161.69(R 3 c)$ & $\mathrm{Ni}$ & ESFD & ESFD & ESFD & LCEBR & LCEBR & $\begin{array}{c}\text { Table } \\
\text { CCLXVII }\end{array}$ \\
\hline 1.0 .5 & Sr3CoIrO6 & $165.95\left(P \overline{3} c^{\prime} 1\right)$ & $\mathrm{Co}$ & $\mathrm{ES}$ & LCEBR & LCEBR & LCEBR & LCEBR & $\frac{\text { Table }}{\text { CCLXXIII }}$ \\
\hline 0.108 & Mn3Ir & $166.101\left(R \overline{3} m^{\prime}\right)$ & $\mathrm{Mn}$ & $\mathrm{ES}$ & $\mathrm{ES}$ & $\mathrm{ES}$ & $\mathrm{ES}$ & ES & $\begin{array}{c}\text { Table } \\
\text { CCLXXV }\end{array}$ \\
\hline 0.109 & $\mathrm{Mn} 3 \mathrm{Pt}$ & $166.101\left(R \overline{3} m^{\prime}\right)$ & $\mathrm{Mn}$ & $\mathrm{ES}$ & $\mathrm{ES}$ & $\mathrm{ES}$ & ES & $\mathrm{ES}$ & $\begin{array}{c}\text { Table } \\
\text { CCLXXVI }\end{array}$ \\
\hline 0.177 & $\mathrm{Mn} 3 \mathrm{GaN}$ & $166.97(R \overline{3} m)$ & $\mathrm{Mn}$ & $\mathrm{ES}$ & ES & $\mathrm{ES}$ & $\overline{\mathrm{ES}}$ & ESFD & $\begin{array}{c}\text { Table } \\
\text { CCLXXVII }\end{array}$ \\
\hline 1.153 & $\mathrm{Mn} 3 \mathrm{GaC}$ & $167.108\left(R_{I} \overline{3} c\right)$ & $\mathrm{Mn}$ & $\mathrm{ES}$ & LCEBR & LCEBR & LCEBR & LCEBR & $\begin{array}{c}\text { Table } \\
\text { CCLXXX }\end{array}$ \\
\hline 0.117 & LuFeO3 & $185.201\left(P 6_{3} c^{\prime} m^{\prime}\right)$ & $\mathrm{Fe}$ & TI & LCEBR & LCEBR & LCEBR & LCEBR & $\begin{array}{c}\text { Table } \\
\text { CCLXXXV }\end{array}$ \\
\hline 1.110 & ScMn6Ge6 & $192.252\left(P_{c} 6 / \mathrm{mcc}\right)$ & $\mathrm{Mn}$ & ES & $\mathrm{ES}$ & $\mathrm{ES}$ & ES & $\mathrm{ES}$ & \begin{tabular}{|c|} 
Table \\
CCLXXXVII
\end{tabular} \\
\hline 1.225 & ScMn6Ge6 & $192.252\left(P_{c} 6 / m c c\right)$ & $\mathrm{Mn}$ & $\mathrm{ES}$ & $\mathrm{ES}$ & ES & ES & $\mathrm{ES}$ & $\begin{array}{c}\text { Table } \\
\text { CCLXXXIX }\end{array}$ \\
\hline 0.118 & $\mathrm{Ba} 5 \mathrm{Co} 5 \mathrm{ClO} 13$ & $194.268\left(P 6_{3}^{\prime} / m^{\prime} m^{\prime} c\right)$ & $\mathrm{Co}$ & LCEBR & LCEBR & LCEBR & LCEBR & ES & $\begin{array}{c}\text { Table } \\
\text { CCXCI }\end{array}$ \\
\hline 0.150 & NiS2 & $205.33(P a \overline{3})$ & $\mathrm{Ni}$ & TI & LCEBR & LCEBR & LCEBR & LCEBR & $\begin{array}{c}\text { Table } \\
\text { CCXCIII }\end{array}$ \\
\hline 0.2 & $\mathrm{Cd} 2 \mathrm{Os} 2 \mathrm{O} 7$ & $227.131\left(F d \overline{3} m^{\prime}\right)$ & Os & $\mathrm{ES}$ & LCEBR & LCEBR & LCEBR & LCEBR & $\begin{array}{c}\text { Table } \\
\text { CCXCV }\end{array}$ \\
\hline
\end{tabular}

* Chiral MSG. The crossing points in the ES/ESFD phase with Chiral MSG must carry nonzero chiral charges. 90 91]

TABLE VIII: Topological phase diagram of the magnetic materials that have Rare-earth elements. The interaction parameter $U$ of $f$ electrons on Rare-earth elements have been set to $0,2,4$ and $6 \mathrm{eV}$. If the material also have transition elements, we take $U$ of $d$ electron as $2 \mathrm{eV}$.

\begin{tabular}{|c|c|c|c|c|c|c|c|c|}
\hline BCS-ID & Formula & MSG & $\mathrm{CA}$ & $\mathrm{U}=0$ & $\mathrm{U}=2$ & $\mathrm{U}=4$ & $\mathrm{U}=6$ & $\mathrm{BS}$ \\
\hline 1.206 & Dy2Fe2Si2C & $2.7\left(P_{S} \overline{1}\right)$ & $\mathrm{Dy}, \mathrm{Fe}$ & TI & $\mathrm{TI}$ & TI & TI & Table. CCXCVII \\
\hline 0.104 & ErVO3 & $11.54\left(P 2_{1}^{\prime} / m^{\prime}\right)$ & Er, V & LCEBR & $\mathrm{TI}$ & LCEBR & LCEBR & Table. [CCCII \\
\hline 0.106 & DyVO3 & $11.54\left(P 2_{1}^{\prime} / m^{\prime}\right)$ & $\mathrm{Dy}, \mathrm{V}$ & TI & LCEBR & LCEBR & LCEBR & Table. $\overline{\mathrm{CCCIII}}$ \\
\hline 1.22 & DyCu2Si2 & $12.63\left(C_{c} 2 / m\right)$ & $\mathrm{Dy}, \mathrm{Cu}$ & TBD & TBD & LCEBR & $\mathrm{ES}$ & Table. $\overline{\mathrm{CCCV}}$ \\
\hline 1.140 & $\mathrm{PrMgPb}$ & $13.73\left(P_{A} 2 / c\right)$ & $\operatorname{Pr}$ & $\mathrm{TI}$ & $\mathrm{TI}$ & $\mathrm{TI}$ & $\mathrm{TI}$ & Table. CCCVII \\
\hline 1.141 & $\mathrm{NdMgPb}$ & $13.73\left(P_{A} 2 / c\right)$ & $\mathrm{Nd}$ & TI & $\mathrm{TI}$ & LCEBR & LCEBR & Table. $\overline{\text { CCCVIII }}$ \\
\hline 0.105 & ErVO3 & $14.75\left(P 2_{1} / c\right)$ & Er, V & $\mathrm{ES}$ & LCEBR & LCEBR & LCEBR & Table. CCCX] \\
\hline 0.174 & Pr3Ru4Al12 & $15.89\left(C 2^{\prime} / c^{\prime}\right)$ & $\operatorname{Pr}, \mathrm{Ru}$ & TI & $\mathrm{TI}$ & $\mathrm{TI}$ & $\mathrm{TI}$ & Table. CCCXVI \\
\hline 0.226 & $\mathrm{NdCo} 2$ & $15.89\left(C 2^{\prime} / c^{\prime}\right)$ & $\mathrm{Nd}, \mathrm{Co}$ & $\mathrm{ES}$ & $\mathrm{TI}$ & $\mathrm{TI}$ & $\mathrm{TI}$ & Table. $\overline{\text { CCCXVII }}$ \\
\hline 2.10 & $\mathrm{HoP}$ & $15.89\left(C 2^{\prime} / c^{\prime}\right)$ & Ho & TI & TI & TI & TI & Table. $\overline{\text { CCCXVIII }}$ \\
\hline
\end{tabular}




\begin{tabular}{|c|c|c|c|c|c|c|c|c|}
\hline 1.211 & Dy2O2S & $15.90\left(C_{c} 2 / c\right)$ & Dy & $\mathrm{TI}$ & LCEBR & LCEBR & LCEBR & Table. CCCXXI \\
\hline 1.216 & $\mathrm{Nd} 2 \mathrm{BaNiO} 5$ & $15.90\left(C_{c} 2 / c\right)$ & $\mathrm{Nd}, \mathrm{Ni}$ & $\mathrm{TI}$ & LCEBR & LCEBR & LCEBR & Table. CCCXXIII \\
\hline 1.217 & Tb2BaNiO5 & $15.90\left(C_{c} 2 / c\right)$ & $\mathrm{Tb}, \mathrm{Ni}$ & TI & LCEBR & LCEBR & LCEBR & Table. $\overline{\overline{\text { CCCXXIV }}}$ \\
\hline 1.43 & $\mathrm{PrNiO} 3$ & $36.178\left(C_{a} m c 2_{1}\right)$ & $\mathrm{Pr}, \mathrm{Ni}$ & LCEBR & LCEBR & $\mathrm{ES}$ & LCEBR & Table. $\overline{\text { CCCXXIX }}$ \\
\hline 0.26 & TmAgGe & $38.191\left(A m^{\prime} m^{\prime} 2\right)$ & $\mathrm{Tm}$ & $\mathrm{ES}$ & TBD & TBD & TBD & Table. CCCXXX \\
\hline 2.12 & $\mathrm{TbMg}$ & $49.270\left(P c^{\prime} \mathrm{cm}^{\prime}\right)$ & $\mathrm{Tb}$ & $\mathrm{ES}$ & $\mathrm{ES}$ & $\mathrm{ES}$ & $\mathrm{ES}$ & Table. $\overline{\mathrm{CCCXXXI}}$ \\
\hline 1.139 & Ho2RhIn8 & $49.273\left(P_{c} c c m\right)$ & Но & $\mathrm{ES}$ & TI & $\mathrm{TI}$ & ES & Table. \\
\hline 2.11 & $\mathrm{TbMg}$ & $51.295\left(P m m^{\prime} a^{\prime}\right)$ & $\mathrm{Tb}$ & $\mathrm{ES}$ & ES & $\mathrm{ES}$ & ES & Table. CCCXXXIII \\
\hline 1.222 & Er2CoGa8 & $51.298\left(P_{a} m m a\right)$ & $\mathrm{Er}, \mathrm{Co}$ & $\mathrm{ES}$ & $\mathrm{ES}$ & $\mathrm{ES}$ & $\mathrm{ES}$ & Table. CCCXXXIV \\
\hline 1.150 & $\operatorname{PrAg}$ & $53.334\left(P_{B} m n a\right)$ & $\operatorname{Pr}$ & LCEBR & TBD & $\mathrm{TI}$ & TBD & Table. CCCXXXV \\
\hline 1.8 & CeRu2Al10 & $57.391\left(P_{C} b c m\right)$ & $\mathrm{Ce}, \mathrm{Ru}$ & TI & $\mathrm{TI}$ & $\mathrm{TI}$ & LCEBR & Table. CCCXXXVIII \\
\hline 0.187 & $\mathrm{CeMnAsO}$ & $59.407\left(P m^{\prime} m n\right)$ & $\mathrm{Ce}, \mathrm{Mn}$ & $\mathrm{ES}$ & LCEBR & LCEBR & LCEBR & Table. CCCXXXIX \\
\hline 0.185 & Nd5Ge4 & $62.447\left(P n m^{\prime} a^{\prime}\right)$ & $\mathrm{Nd}$ & $\mathrm{ES}$ & $\mathrm{ES}$ & TBD & ES & Table. CCCXLI \\
\hline 1.179 & $\mathrm{NdCoAsO}$ & $62.450\left(P_{a} n m a\right)$ & $\mathrm{Nd}, \mathrm{Co}$ & $\mathrm{ES}$ & TI & $\mathrm{ES}$ & $\mathrm{ES}$ & Table. $\overline{\mathrm{CCCXLV}}$ \\
\hline 0.149 & Nd3Ru4Al12 & $63.462\left(C m^{\prime} c^{\prime} m\right)$ & $\mathrm{Nd}, \mathrm{Ru}$ & $\mathrm{ES}$ & $\mathrm{ES}$ & $\mathrm{ES}$ & ES & Table. CCCXLVII \\
\hline 0.173 & Pr3Ru4Al12 & $63.462\left(C m^{\prime} c^{\prime} m\right)$ & $\operatorname{Pr}, \mathrm{Ru}$ & $\mathrm{ES}$ & $\mathrm{ES}$ & ES & $\mathrm{ES}$ & Table. CCCXLVIII \\
\hline 3.3 & Ho2RhIn8 & $63.464\left(\mathrm{Cm}^{\prime} \mathrm{cm}^{\prime}\right)$ & Ho & $\mathrm{ES}$ & $\mathrm{ES}$ & TBD & TBD & Table. CCCXLIX \\
\hline 1.200 & U2Ni2Sn & $63.466\left(C_{c} \mathrm{mcm}\right)$ & $\mathrm{U}, \mathrm{Ni}$ & LCEBR & $\mathrm{TI}$ & $\mathrm{TI}$ & $\mathrm{TI}$ & Table. CCCL \\
\hline 1.262 & NpRhGa5 & $63.466\left(C_{c} m c m\right)$ & $\mathrm{Np}$ & LCEBR & TI & $\mathrm{ES}$ & LCEBR & Table. $\overline{\text { CCCLI }}$ \\
\hline 1.195 & Er2Ni2In & $63.467\left(C_{a} m c m\right)$ & $\mathrm{Er}, \mathrm{Ni}$ & LCEBR & LCEBR & $\mathrm{TI}$ & LCEBR & Table. CCCLII \\
\hline 1.188 & CeRh2Si2 & $64.480\left(C_{A} m c a\right)$ & $\mathrm{Ce}$ & $\mathrm{TI}$ & LCEBR & $\mathrm{ES}$ & $\mathrm{TI}$ & Table. CCCLIV \\
\hline 1.223 & Tm2CoGa8 & $65.489\left(C_{a} \mathrm{mmm}\right)$ & $\mathrm{Tm}, \mathrm{Co}$ & TI & $\mathrm{ES}$ & $\mathrm{ES}$ & $\mathrm{ES}$ & Table. $\overline{\text { CCCLVI }}$ \\
\hline 1.142 & $\mathrm{CeMgPb}$ & $67.510\left(C_{A} m m a\right)$ & $\mathrm{Ce}$ & LCEBR & TI & TBD & TBD & Table. $\overline{\text { CCCLVIII }}$ \\
\hline 1.0 .12 & UAu2Si2 & $71.536\left(\operatorname{Im}^{\prime} m^{\prime} m\right)$ & $\mathrm{U}$ & $\mathrm{ES}$ & $\mathrm{ES}$ & ES & $\mathrm{ES}$ & Table. CCCLX \\
\hline 2.28 & NpNiGa5 & $74.559\left(\operatorname{Imm}^{\prime} a^{\prime}\right)$ & $\mathrm{Np}, \mathrm{Ni}$ & $\mathrm{ES}$ & $\mathrm{ES}$ & $\mathrm{ES}$ & $\mathrm{ES}$ & Table. $\overline{\text { CCCLXI }}$ \\
\hline 0.184 & Nd5Si4 & $92.114\left(P 4_{1} 2_{1}^{\prime} 2^{\prime}\right)^{*}$ & $\mathrm{Nd}$ & $\mathrm{ES}$ & $\mathrm{ES}$ & ES & $\mathrm{ES}$ & Table. CCCLXIV \\
\hline 1.0 .11 & CeCoGe3 & $107.231\left(I 4 m^{\prime} m^{\prime}\right)$ & $\mathrm{Ce}, \mathrm{Co}$ & LCEBR & $\mathrm{ES}$ & $\mathrm{ES}$ & TBD & Table. $\overline{C C C L X V}$ \\
\hline 2.26 & PrCo2P2 & $123.345\left(P 4 / \mathrm{mm}^{\prime} \mathrm{m}^{\prime}\right)$ & $\mathrm{Pr}, \mathrm{Co}$ & $\mathrm{ES}$ & $\mathrm{ES}$ & $\mathrm{ES}$ & $\mathrm{ES}$ & Table. CCCLXVI \\
\hline 1.162 & $\mathrm{NdMg}$ & $124.360\left(P_{c} 4 / m c c\right)$ & $\mathrm{Nd}$ & $\mathrm{ES}$ & $\mathrm{ES}$ & $\mathrm{ES}$ & ES & Table. CCCLXVII \\
\hline 1.251 & $\mathrm{NdCo} 2 \mathrm{P} 2$ & $\left(P_{c} 4 / m c c\right)$ & $\mathrm{Nd}, \mathrm{Co}$ & $\mathrm{ES}$ & $\mathrm{ES}$ & ES & TI & Table. CCCLXVIII \\
\hline 1.255 & UPtGa5 & $124.360\left(P_{c} 4 / m c c\right)$ & $\mathrm{U}$ & TI & $\mathrm{ES}$ & ES & ES & Table. CCCLXIX \\
\hline 1.261 & NpRhGa5 & $124.360\left(P_{c} 4 / m c c\right)$ & $\mathrm{Np}$ & $\mathrm{ES}$ & $\mathrm{ES}$ & $\mathrm{ES}$ & $\mathrm{ES}$ & Table. CCCLXX \\
\hline 2.14 & $\mathrm{NdMg}$ & $125.373\left(P_{C} 4 / n b m\right)$ & $\mathrm{Nd}$ & $\mathrm{ES}$ & ES & $\mathrm{ES}$ & ES & Table. CCCLXXII \\
\hline 1.253 & CeCo2P2 & $126.386\left(P_{I} 4 / n n c\right)$ & $\mathrm{Ce}, \mathrm{Co}$ & $\mathrm{ES}$ & $\mathrm{ES}$ & $\mathrm{ES}$ & ES & Table. CCCLXXIII \\
\hline 0.80 & U2Pd2In & $127.394\left(P 4^{\prime} / m^{\prime} b m^{\prime}\right)$ & $\mathrm{U}$ & ESFD & ESFD & ESFD & ESFD & Table. CCCLXXIV \\
\hline 0.81 & U2Pd2Sn & $127.394\left(P 4^{\prime} / m^{\prime} b m^{\prime}\right)$ & $\mathrm{U}$ & $\mathrm{TI}$ & $\mathrm{TI}$ & $\mathrm{TI}$ & $\mathrm{TI}$ & Table. CCCLXXV \\
\hline 1.81 & GdIn3 & $127.397\left(P_{C} 4 / \mathrm{mbm}\right)$ & $\mathrm{Gd}$ & $\mathrm{ES}$ & $\mathrm{ES}$ & ES & ES & Table. CCCLXXVII \\
\hline 1.102 & U2Ni2In & $128.408\left(P_{c} 4 / m n c\right)$ & $\mathrm{U}, \mathrm{Ni}$ & $\mathrm{ES}$ & $\mathrm{ES}$ & $\mathrm{ES}$ & $\mathrm{ES}$ & Table. $\overline{\text { CCCLXXVIII }}$ \\
\hline 1.160 & UP & $128.410\left(P_{I} 4 / m n c\right)$ & $\mathrm{U}$ & $\mathrm{ES}$ & ES & $\mathrm{ES}$ & $\mathrm{ES}$ & Table. CCCLXXIX \\
\hline 1.187 & TbRh2Si2 & $128.410\left(P_{I} 4 / m n c\right)$ & $\mathrm{Tb}$ & $\mathrm{ES}$ & $\mathrm{ES}$ & $\mathrm{ES}$ & $\mathrm{ES}$ & Table. CCCLXXX \\
\hline 1.208 & UAs & $128.410\left(P_{I} 4 / m n c\right)$ & $\mathrm{U}$ & $\mathrm{ES}$ & $\mathrm{ES}$ & $\mathrm{ES}$ & TI & Table. CCCLXXXI \\
\hline 1.21 & DyCo2Si2 & $128.410\left(P_{I} 4 / m n c\right)$ & Dy,Co & $\mathrm{ES}$ & ES & $\mathrm{ES}$ & $\mathrm{ES}$ & Table. CCCLXXXII \\
\hline 0.186 & $\mathrm{CeMnAsO}$ & $129.416\left(P 4^{\prime} / n^{\prime} m^{\prime} m\right)$ & $\mathrm{Ce}, \mathrm{Mn}$ & LCEBR & $\mathrm{TI}$ & $\mathrm{TI}$ & $\mathrm{TI}$ & Table. CCCLXXXIII \\
\hline 1.215 & UP2 & $130.432\left(P_{c} 4 / n c c\right)$ & $\mathrm{U}$ & $\mathrm{ES}$ & $\mathrm{ES}$ & $\mathrm{ES}$ & ES & Table. $\overline{\text { CCCLXXXV }}$ \\
\hline 2.13 & UP & $134.481\left(P_{C} 4_{2} / \mathrm{nnm}\right)$ & $\mathrm{U}$ & TI & TI & $\mathrm{TI}$ & TI & Table. CCCLXXXVII \\
\hline 2.20 & UAs & $134.481\left(P_{C} 4_{2} / n n m\right)$ & $\mathrm{U}$ & TI & $\mathrm{ES}$ & $\mathrm{TI}$ & TI & Table. CCCLXXXVIII \\
\hline 2.6 & $\mathrm{Nd} 2 \mathrm{CuO} 4$ & $134.481\left(P_{C} 4_{2} / \mathrm{nnm}\right)$ & $\mathrm{Nd}$ & TI & LCEBR & LCEBR & LCEBR & Table. CCCLXXXIX \\
\hline 1.103 & U2Rh2Sn & $135.492\left(P_{c} 4_{2} / m b c\right)$ & $\mathrm{U}$ & $\mathrm{ES}$ & $\mathrm{ES}$ & $\mathrm{ES}$ & $\mathrm{ES}$ & Table. $\overline{C C C X C}$ \\
\hline 1.207 & U2Rh2Sn & $135.492\left(P_{c} 4_{2} / m b c\right)$ & $\mathrm{U}$ & $\mathrm{ES}$ & $\mathrm{ES}$ & $\mathrm{ES}$ & $\mathrm{ES}$ & Table. $\overline{\text { CCCXCI }}$ \\
\hline 1.254 & UNiGa5 & $140.550\left(I_{c} 4 / \mathrm{mcm}\right)$ & $\mathrm{U}, \mathrm{Ni}$ & $\mathrm{ES}$ & TI & TI & LCEBR & Table. $\overline{\text { CCCXCII }}$ \\
\hline 1.82 & Nd2RhIn8 & $140.550\left(I_{c} 4 / \mathrm{mcm}\right)$ & $\mathrm{Nd}$ & LCEBR & $\mathrm{ES}$ & $\mathrm{ES}$ & $\mathrm{ES}$ & Table. CCCXCIII \\
\hline
\end{tabular}




\begin{tabular}{|c|c|c|c|c|c|c|c|c|}
\hline 1.87 & $\mathrm{TbCo} 2 \mathrm{Ga} 8$ & $140.550\left(I_{c} 4 / \mathrm{mcm}\right)$ & $\mathrm{Tb}, \mathrm{Co}$ & ES & $\mathrm{ES}$ & ES & ES & Table. CCCXCIV \\
\hline 0.126 & $\mathrm{NpCo} 2$ & $141.556\left(I 4_{1}^{\prime} / a^{\prime} m^{\prime} d\right)$ & $\mathrm{Np}, \mathrm{Co}$ & $\mathrm{ES}$ & TBD & $\mathrm{TI}$ & TI & Table. CCCXCVIII \\
\hline 0.151 & Tm2Mn2O7 & $141.557\left(I 4_{1} / a m^{\prime} d^{\prime}\right)$ & $\mathrm{Tm}, \mathrm{Mn}$ & ES & LCEBR & LCEBR & LCEBR & Table. CD \\
\hline 0.227 & $\mathrm{NdCo} 2$ & $141.557\left(I 4_{1} / a m^{\prime} d^{\prime}\right)$ & $\mathrm{Nd}, \mathrm{Co}$ & $\mathrm{ES}$ & ES & ES & ES & Table. $\overline{\mathrm{CDII}}$ \\
\hline 0.48 & $\mathrm{~Tb} 2 \mathrm{Sn} 2 \mathrm{O} 7$ & $141.557\left(I 4_{1} / a m^{\prime} d^{\prime}\right)$ & $\mathrm{Tb}$ & $\mathrm{ES}$ & LCEBR & LCEBR & LCEBR & Table. $\overline{\text { CDIII }}$ \\
\hline 0.49 & Ho2Ru2O7 & $141.557\left(I 4_{1} / a m^{\prime} d^{\prime}\right)$ & Ho,Ru & ES & LCEBR & LCEBR & $\mathrm{ES}$ & Table. CDIV \\
\hline 0.51 & Ho2Ru2O7 & $141.557\left(I 4_{1} / a m^{\prime} d^{\prime}\right)$ & Ho,Ru & $\mathrm{TI}$ & LCEBR & LCEBR & LCEBR & Table. CDV \\
\hline 1.161 & $\mathrm{PrFe} 3(\mathrm{BO} 3) 4$ & $155.48\left(R_{I} 32\right)^{*}$ & $\mathrm{Pr}, \mathrm{Fe}$ & LCEBR & TBD & ES & LCEBR & Table. $\overline{\mathrm{CDVI}}$ \\
\hline 0.169 & U3As4 & $161.71\left(R 3 c^{\prime}\right)$ & $\mathrm{U}$ & ES & $\mathrm{ES}$ & LCEBR & LCEBR & Table. CDVII \\
\hline 0.170 & U3P4 & $161.71\left(R 3 c^{\prime}\right)$ & $\mathrm{U}$ & ES & ES & LCEBR & LCEBR & Table. CDVIII \\
\hline 0.228 & $\mathrm{TbCo} 2$ & $166.101\left(R \overline{3} m^{\prime}\right)$ & $\mathrm{Tb}, \mathrm{Co}$ & $\mathrm{TI}$ & ES & TI & $\mathrm{TI}$ & Table. CDX \\
\hline 0.77 & Tb2Ti2O7 & $166.101\left(R \overline{3} m^{\prime}\right)$ & $\mathrm{Tb}, \mathrm{Ti}$ & ES & LCEBR & LCEBR & LCEBR & Table. $\overline{\mathrm{CDXI}}$ \\
\hline 3.8 & $\mathrm{NdZn}$ & $222.103\left(P_{I} n \overline{3} n\right)$ & $\mathrm{Nd}$ & ESFD & ES & $\mathrm{ES}$ & ESFD & Table. CDXV \\
\hline 3.12 & $\mathrm{NpSb}$ & $224.113\left(P n \overline{3} m^{\prime}\right)$ & $\mathrm{Np}$ & TI & $\mathrm{TI}$ & TI & TI & Table. CDXVI \\
\hline 3.2 & $\mathrm{UO} 2$ & $224.113\left(P n \overline{3} m^{\prime}\right)$ & $\mathrm{U}$ & ES & LCEBR & LCEBR & LCEBR & Table. CDXVII \\
\hline 3.7 & $\mathrm{NpBi}$ & $224.113\left(P n \overline{3} m^{\prime}\right)$ & $\mathrm{Np}$ & TI & TI & $\mathrm{TI}$ & $\mathrm{TI}$ & Table. CDXVIII \\
\hline 3.10 & NpSe & $228.139\left(F_{S} d \overline{3} c\right)$ & $\mathrm{Np}$ & $\mathrm{TI}$ & ESFD & ESFD & ESFD & Table. CDXIX \\
\hline 3.11 & $\mathrm{NpTe}$ & $228.139\left(F_{S} d \overline{3} c\right)$ & $\mathrm{Np}$ & $\mathrm{TI}$ & ESFD & ESFD & ESFD & Table. CDXX \\
\hline 3.9 & $\mathrm{NpS}$ & $228.139\left(F_{S} d \overline{3} c\right)$ & $\mathrm{Np}$ & ES & ESFD & ESFD & ESFD & Table. $\overline{\mathrm{CDXXI}}$ \\
\hline 3.6 & $\mathrm{DyCu}$ & $229.143\left(\operatorname{Im} \overline{3} m^{\prime}\right)$ & Dy & TBD & $\mathrm{ES}$ & $\mathrm{ES}$ & $\mathrm{ES}$ & Table. $\overline{\text { CDXXII }}$ \\
\hline
\end{tabular}

* Chiral MSG. The crossing points in the ES/ESFD phase with Chiral MSG must carry nonzero chiral charges. 90, 91]

Some of the topological comounds in Table VIII contain both $3 d$ element and $4 f / 5 f$ element, where we set the U value of $d$ electron as $2 \mathrm{eV}$. For comparisons, we have also considered the empirical U values for the $3 d$ electron and identified the topological phase diagram using MTQC theory. In the Table IX we chose 15 materials, each of which contains the $3 d$ element V, Co, Ni/Mn. The empirical U values of $\mathrm{V}, \mathrm{Co}, \mathrm{Ni}$ and $\mathrm{Mn}$ are set as 3.25, 3.7, 6.2 and $3.9 \mathrm{eV}$, respectively[? ]. As tabulated in Table IX] the results indicate that interaction of $3 d$ electron almost doesn't change the topological phase diagram for the compounds containing $4 f / 5 f$ element.

TABLE IX: Comparisions of the topological phase diagrams for some of the topological compounds containing both $3 d$ element and $4 f / 5 f$ element. For each material, the $\mathrm{U}$ value of $3 d$ element $\left(U_{3 d}\right)$ is set as $2 e V$ and an empirical value. The $\mathrm{U}$ value of $4 f / 5 f$ element $\left(U_{f}\right)$ is set as 2,4 and $6 \mathrm{eV}$. The cases that have different topologies when $U_{3 d}$ is taken $2 \mathrm{eV}$ and an empirical value are marked by red color.

\begin{tabular}{|c|c|c|c|c|c|c|c|}
\hline BCSID & Formula & MSG & $\mathrm{CA}$ & $U_{3 d}(e V)$ & $U_{f}=2 e V$ & $U_{f}=4 e V$ & $U_{f}=6 e \mathrm{~V}$ \\
\hline \multirow{2}{*}{2.26} & \multirow{2}{*}{$\mathrm{PrCo} 2 \mathrm{P} 2$} & \multirow{2}{*}{$123.345\left(P 4 / \mathrm{mm}^{\prime} \mathrm{m}^{\prime}\right)$} & \multirow{2}{*}{ Pr,Co } & 2 & $\mathrm{ES}$ & $\mathrm{ES}$ & $\mathrm{ES}$ \\
\hline & & & & 3.7 & ES & $\mathrm{ES}$ & $\mathrm{ES}$ \\
\hline \multirow{2}{*}{1.251} & \multirow{2}{*}{$\mathrm{NdCo} 2 \mathrm{P} 2$} & \multirow{2}{*}{$124.360\left(P_{c} 4 / m c c\right)$} & \multirow{2}{*}{$\mathrm{Nd}, \mathrm{Co}$} & 2 & $\mathrm{ES}$ & $\mathrm{ES}$ & TI \\
\hline & & & & 3.7 & $\mathrm{ES}$ & $\mathrm{ES}$ & $\mathrm{ES}$ \\
\hline \multirow{2}{*}{1.253} & \multirow{2}{*}{$\mathrm{CeCo} 2 \mathrm{P} 2$} & \multirow{2}{*}{$126.386\left(P_{I} 4 / n n c\right)$} & \multirow{2}{*}{$\mathrm{Ce}, \mathrm{Co}$} & 2 & $\mathrm{ES}$ & ES & ES \\
\hline & & & & 3.7 & $\mathrm{ES}$ & $\mathrm{ES}$ & $\mathrm{ES}$ \\
\hline \multirow{2}{*}{1.102} & \multirow{2}{*}{ U2Ni2In } & \multirow{2}{*}{$128.408\left(P_{c} 4 / m n c\right)$} & \multirow{2}{*}{$\mathrm{U}, \mathrm{Ni}$} & 2 & $\mathrm{ES}$ & ES & ES \\
\hline & & & & 6.2 & $\mathrm{ES}$ & $\mathrm{ES}$ & $\mathrm{ES}$ \\
\hline \multirow{2}{*}{1.21} & \multirow{2}{*}{ DyCo2Si2 } & \multirow{2}{*}{$128.410\left(P_{I} 4 / m n c\right)$} & \multirow{2}{*}{ Dy,Co } & 2 & ES & ES & $\mathrm{ES}$ \\
\hline & & & & 3.7 & $\mathrm{ES}$ & $\mathrm{ES}$ & $\mathrm{ES}$ \\
\hline \multirow{2}{*}{0.105} & \multirow{2}{*}{ ErVO3 } & \multirow{2}{*}{$14.75\left(P 2_{1} / c\right)$} & \multirow{2}{*}{ Er, V } & 2 & LCEBR & LCEBR & LCEBR \\
\hline & & & & 3.25 & LCEBR & LCEBR & LCEBR \\
\hline \multirow{2}{*}{1.254} & \multirow{2}{*}{ UNiGa5 } & \multirow{2}{*}{$140.550\left(I_{c} 4 / \mathrm{mcm}\right)$} & \multirow{2}{*}{$\mathrm{U}, \mathrm{Ni}$} & 2 & $\mathrm{TI}$ & $\mathrm{TI}$ & LCEBR \\
\hline & & & & 6.2 & TI & TI & TI \\
\hline \multirow{2}{*}{0.151} & \multirow{2}{*}{ Tm2Mn2O7 } & \multirow{2}{*}{$141.557\left(I 4_{1} / a m^{\prime} d^{\prime}\right)$} & \multirow{2}{*}{$\mathrm{Tm}, \mathrm{Mn}$} & 2 & LCEBR & LCEBR & LCEBR \\
\hline & & & & 3.9 & LCEBR & LCEBR & LCEBR \\
\hline \multirow{2}{*}{0.227} & \multirow{2}{*}{$\mathrm{NdCo} 2$} & \multirow{2}{*}{$141.557\left(I 4_{1} / a m^{\prime} d^{\prime}\right)$} & Nd $C$ & 2 & $\mathrm{ES}$ & $\mathrm{ES}$ & $\mathrm{ES}$ \\
\hline & & & $\mathrm{Nd}, \mathrm{CO}$ & 3.7 & $\mathrm{ES}$ & $\mathrm{ES}$ & $\mathrm{ES}$ \\
\hline 1.222 & Er2CoGa8 & $51.298\left(P_{a} m m a\right)$ & $\mathrm{Er}, \mathrm{Co}$ & 2 & ES & $\mathrm{ES}$ & $\mathrm{ES}$ \\
\hline
\end{tabular}




\begin{tabular}{|c|c|c|c|c|c|c|c|}
\hline & & & & 3.7 & ES & ES & ES \\
\hline \multirow{2}{*}{0.187} & \multirow{2}{*}{ CeMnAsO } & \multirow{2}{*}{ 59.407(Pm'mn) } & \multirow{2}{*}{$\mathrm{Ce}, \mathrm{Mn}$} & 2 & LCEBR & LCEBR & LCEBR \\
\hline & & & & 3.9 & LCEBR & LCEBR & LCEBR \\
\hline \multirow{2}{*}{1.200} & \multirow{2}{*}{$\mathrm{U} 2 \mathrm{Ni} 2 \mathrm{Sn}$} & \multirow{2}{*}{$63.466\left(C_{c} \mathrm{mcm}\right)$} & \multirow{2}{*}{$\mathrm{U}, \mathrm{Ni}$} & 2 & $\mathrm{TI}$ & $\mathrm{TI}$ & $\mathrm{TI}$ \\
\hline & & & & 6.2 & LCEBR & $\mathrm{TI}$ & $\mathrm{TI}$ \\
\hline \multirow{2}{*}{1.195} & \multirow{2}{*}{ Er2Ni2In } & \multirow{2}{*}{$63.467\left(C_{a} m c m\right)$} & \multirow{2}{*}{$\mathrm{Er}, \mathrm{Ni}$} & 2 & LCEBR & $\mathrm{TI}$ & LCEBR \\
\hline & & & & 6.2 & LCEBR & LCEBR & LCEBR \\
\hline \multirow{2}{*}{1.223} & \multirow{2}{*}{ Tm2CoGa 8} & \multirow{2}{*}{$65.489\left(C_{a} m m m\right)$} & \multirow{2}{*}{$\mathrm{Tm}, \mathrm{Co}$} & 2 & $\mathrm{ES}$ & ES & $\mathrm{ES}$ \\
\hline & & & & 3.7 & $\mathrm{ES}$ & $\mathrm{ES}$ & $\mathrm{ES}$ \\
\hline \multirow{2}{*}{2.28} & \multirow{2}{*}{ NpNiGa5 } & \multirow{2}{*}{$74.559\left(\operatorname{Imm}^{\prime} a^{\prime}\right)$} & \multirow{2}{*}{$\mathrm{Np}, \mathrm{Ni}$} & 2 & $\mathrm{ES}$ & $\mathrm{ES}$ & $\mathrm{ES}$ \\
\hline & & & & 6.2 & ES & ES & ES \\
\hline
\end{tabular}

\section{Appendix H: Physical interpretations for the TI classified by MTQC}

In MTQC theory [7], in order to have a physical interpretation for the SI, we reduce the SI of MSG to one of its subgroup, and then interpret the SI of the subgroup using the layer construction approach [26. In this work, all the involved SI are subduced onto MSG 2.4

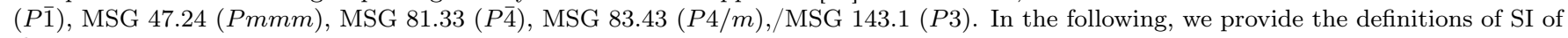
these groups.

\section{Definitions for the stable indices of MSG 2.4}

MSG 2.4 $(P \overline{1})$ has the SI group $\mathbb{Z}_{4} \times \mathbb{Z}_{2}^{3}$. The four SI $\left(\eta_{4 I}, z_{2 I, 1}, z_{2 I, 2}, z_{2 I, 3}\right)$ are defined as

$$
\begin{gathered}
\eta_{4 I}=\sum_{K} n_{K}^{-}=\sum_{K} \frac{1}{2}\left(n_{K}^{-}-n_{K}^{+}\right) \bmod 4, \\
z_{2 I, i=1,2,3}=C_{k_{i}=\pi} \quad \bmod 2=\sum_{K, K_{i}=\pi} n_{K}^{-} \bmod 2,
\end{gathered}
$$

where $K$ sums over the eight inversion-invariant momenta, and $n_{K}^{ \pm}$means the number of occupied even/odd states at the momentum $K$. $z_{2 I, i}$ is the parity of Chern number in the plane $k_{i}=\pi \cdot \eta_{4 I} \bmod 2$ is the parity of the Chern number difference between $k_{z}=0$ and $k_{z}=\pi$ planes. Thus $\eta_{4 I}=1,3$ correspond to Weyl semimetal (WSM) phase. For $\eta_{4 I}=2$ corresponds to an axion insulator phase/3D QAHI, which can not be distinguished from SI but from Wilson loop [? ]/surface state calculations.

Layer constructions Now make use of the layer constructions of the gapped states to build a mapping from the SI to topological invariants. Here we first give the SI of several layer constructions.

1. A Chern layer with $C= \pm 1$ at $x=0$ gives the SI (2100).

2. A Chern layer with $C= \pm 1$ at $x=\frac{1}{2}$ gives the SI (0100).

3. A Chern layer with $C= \pm 1$ at $y=0$ gives the SI (2010).

4. A Chern layer with $C= \pm 1$ at $y=\frac{1}{2}$ gives the SI (0010).

5. A Chern layer with $C= \pm 1$ at $z=0$ gives the SI (2001).

6. A Chern layer with $C= \pm 1$ at $z=\frac{1}{2}$ gives the SI (0001).

We take the Chern layers with $C=1$ at $z=0$ and $\frac{1}{2}$ as two examples to show how to calculate the SI of layer-constructed states. Since the SI indicate stable topological invariants (rather than fragile topological invariants), states having the same stable topological invariants must have the same SI. For layer-constructed states, the stable topological invariants are completely determined by the positions and directions of the layers [26] and the Chern numbers of layers. Other details about the layer constructions are irrelevant to determine the SI. We first consider a single layer with $C=1$ at $z=0$. The $z=0$ plane is inversion-invariant, and there are four momenta in the 2D Brillouin zone, i.e., $\left(k_{x}, k_{y}\right)=(0,0),(0, \pi),(\pi, 0),(\pi, \pi)$. The Bloch states at these four momenta are either even/odd under the inversion. According to the Fu-Kane formula

$$
(-1)^{C}=\prod_{K} \prod_{n \in \text { occ }} \lambda_{n}^{\prime}(K)
$$

where $K$ goes over the four inversion-invariant momenta and $\lambda_{K, n}^{\prime}$ is the parity of the $n$th occupied band at $K$, in a $C=1$ state, the total parity of the Bloch states at the four momenta must be odd. Here we assume the Chern layer has a single occupied band and the parities at $(0,0),(0, \pi),(\pi, 0),(\pi, \pi)$ are,,,-+++ , respectively. Then we copy the layer to all the integer $z$ positions, i.e., $z=0, \pm 1, \pm 2 \cdots$, to construct the 3D state. Supposing the 2D Bloch state of a single layer at $z$ is $\left|\psi_{k_{x}, k_{y}, z}\right\rangle$, then the 3D state Bloch state is given by

$$
\left|\psi_{\mathbf{k}}\right\rangle=\frac{1}{\sqrt{N_{z}}} \sum_{z=0, \pm 1 \ldots} e^{i z k_{z}}\left|\psi_{k_{x}, k_{y}, z}\right\rangle
$$


where $N_{z}$ is the period in the $z$-direction. Let us calculate the inversion eigenvalues of the 3D state. For $\left(k_{x}, k_{y}\right)=$ $(0,0),(0, \pi),(\pi, 0),(\pi, \pi)$, under the inversion operator $\hat{P}$, the 2D state at $z$ first gains an factor $\lambda^{\prime}\left(k_{x}, k_{y}\right)$ and then transforms to $-z$. Thus we obtain

$$
\hat{P}\left|\psi_{\mathbf{k}}\right\rangle=\lambda^{\prime}\left(k_{x}, k_{y}\right) \frac{1}{\sqrt{N_{z}}} \sum_{z=0, \pm 1 \ldots} e^{-i z k_{z}}\left|\psi_{k_{x}, k_{y}, z}\right\rangle
$$

For $k_{z}=0, \pi$, we further obtain

$$
\hat{P}\left|\psi_{\mathbf{k}}\right\rangle=\lambda^{\prime}\left(k_{x}, k_{y}\right) \frac{1}{\sqrt{N_{z}}} \sum_{z=0, \pm 1 \ldots} e^{i z k_{z}}\left|\psi_{k_{x}, k_{y}, z}\right\rangle=\lambda^{\prime}\left(k_{x}, k_{y}\right)\left|\psi_{\mathbf{k}}\right\rangle .
$$

Thus the parities of the 3D state are given by $\lambda\left(k_{x}, k_{y}, k_{z}\right)=\lambda^{\prime}\left(k_{x}, k_{y}\right)$. We obtain the parities of the 3D state at $\left(k_{x}, k_{y}, k_{z}\right)=$ $(0,0,0),(\pi, 0,0),(0, \pi, 0),(\pi, \pi, 0),(0,0, \pi),(\pi, 0, \pi),(0, \pi, \pi),(\pi, \pi, \pi)$ as,,,,,,,-+++-+++ , respectively. Substituting the parities into Eqs. (H1) and (H2), we obtain the SI as (2001).

Then we consider the same $2 \mathrm{D}$ layers locating at half-integer positions, i.e., $z= \pm \frac{1}{2}, \pm \frac{3}{2} \cdots$. The $3 \mathrm{D}$ Bloch state can be written as

$$
\left|\psi_{\mathbf{k}}\right\rangle=\frac{1}{\sqrt{N_{z}}} \sum_{z= \pm \frac{1}{2}, \pm \frac{3}{2} \cdots} e^{i z k_{z}}\left|\psi_{k_{x}, k_{y}, z}\right\rangle
$$

For $\left(k_{x}, k_{y}\right)=(0,0),(0, \pi),(\pi, 0),(\pi, \pi)$, we have the inversion action as

$$
\hat{P}\left|\psi_{\mathbf{k}}\right\rangle=\lambda^{\prime}\left(k_{x}, k_{y}\right) \frac{1}{\sqrt{N_{z}}} \sum_{z= \pm \frac{1}{2}, \pm \frac{3}{2} \cdots} e^{-i z k_{z}}\left|\psi_{k_{x}, k_{y}, z}\right\rangle .
$$

For $k_{z}=0, \pi$, we have $e^{-i z k_{z}}=e^{-2 i z k_{z}} \times e^{i z k_{z}}=e^{i k_{z}} \times e^{i z k_{z}}$ and hence

$$
\hat{P}\left|\psi_{\mathbf{k}}\right\rangle=\lambda^{\prime}\left(k_{x}, k_{y}\right) e^{i k_{z}} \frac{1}{\sqrt{N_{z}}} \sum_{z= \pm \frac{1}{2}, \pm \frac{3}{2} \cdots} e^{i z k_{z}}\left|\psi_{k_{x}, k_{y}, z}\right\rangle=\lambda^{\prime}\left(k_{x}, k_{y}\right) e^{i k_{z}}\left|\psi_{\mathbf{k}}\right\rangle .
$$

Thus the parities of the 3D state are given by $\lambda\left(k_{x}, k_{y}, k_{z}\right)=\lambda^{\prime}\left(k_{x}, k_{y}\right) e^{i k_{z}}$. We obtain the parities of the 3D state at $\left(k_{x}, k_{y}, k_{z}\right)=$ $(0,0,0),(\pi, 0,0),(0, \pi, 0),(\pi, \pi, 0),(0,0, \pi),(\pi, 0, \pi),(0, \pi, \pi),(\pi, \pi, \pi)$ as,,,,,,,,-++++--- respectively. Substituting the parities into Eqs. (H1) and (H2), we obtain the SI as (0001).

The SI of other layer constructions can be similarly calculated.

The inversion $\mathbb{Z}_{2}$ invariant and axion insulator We find that $\eta_{4 I}=2$ iff the origin point (000) is occupied by odd Chern layers. Hence, for gapped state, we define the inversion- $\mathbb{Z}_{2}$ invariant as

$$
\eta_{2 I}^{\prime}=\frac{1}{2} \eta_{4 I} \bmod 2
$$

When the total Chern number is zero, the axion $\theta$-angle is given by $\theta=\eta_{2 I} \pi$. For example, the state consists of $C=1$ layer at the $z=0$ plane and $C=-1$ Chern layer at the $z=\frac{1}{2}$ plane, which has the SI (2000), has zero total Chern number and $\eta_{2 I}^{\prime}=1$. This state is axion insulator. One can see this from the boundary state: for a finite centrosymmetric sample centered at the origin, the Chern layer at $z=0$ contributes to a chiral hinge mode, whereas the chiral modes from all the other layers cancel each pairwise. On the other hand, the state consists of $C=1$ Chern layers at both the $z=0, \frac{1}{2}$ planes, which also has the SI (2000), has total Chern number 2 and also $\eta_{2 I}^{\prime}=1$. This state is a 3D QAH, for which the $\theta$-angle is ill-defined. ( $\theta$ can still be constructed if the Chern numbers are non-zero, but will be origin-dependent 101 and loses the physical meaning of magnetoelectric response.)

In the rest, we will directly give the SI and the corresponding interpretations. Readers might refer to [7] for more details.

\section{Definitions for the stable indices of MSG 47.249}

MSG 47.249 $(P m m m)$ has the SI group $\mathbb{Z}_{4} \times \mathbb{Z}_{2}^{3}$. Because of the anti-commuting mirror symmetries, all states at the inversion-invariant momenta are doubly degenerate. The SI can be chosen as same as the MSG 47.250 Pmmm1' 26 because for this group TRS does not change the irreps. The $\mathbb{Z}_{4}$ factor is

$$
z_{4}=\sum_{K} \frac{1}{4}\left(n_{K}^{-}-n_{K}^{+}\right) \quad \bmod 4
$$

where $K$ sums over all inversion-invariant momenta and $n_{K}^{ \pm}$is the number of occupied even/odd states at $K$. $z_{4}$ can be thought as Eq. H1 except that $n_{K}^{-}$are replaced by the number of odd doublets. Odd $z_{4}$ corresponds to axion insulator and $z_{4}=2$ corresponds to a higher-order TI (HOTI) jointly protected by mirrors and $C_{2}$ rotations [7. The three $\mathbb{Z}_{2}$ factors are the mirror Chern number parities in the $k_{1,2,3}=\pi$ planes

$$
z_{2 w, i=1,2,3}=C_{m, k_{i}=\pi} \bmod 2=\sum_{K, K_{i}=\pi} \frac{1}{2} n_{K}^{-} \bmod 2 .
$$

Because of the anti-commuting mirror symmetries, net Chern numbers in all the directions vanishes.

\section{Definitions for the stable indices of MSG 81.33}

MSG 81.33(P $\overline{4})$ has the SI group $\mathbb{Z}_{4} \times \mathbb{Z}_{2}^{2}$. We choose the $\mathbb{Z}_{4}$ factor as the Chern number in the $k_{z}=\pi$ plane mod 4

$$
z_{4 S}=C_{\pi} \quad \bmod 4=2 N_{\mathrm{occ}}-\frac{1}{2} n_{Z}^{\frac{1}{2}}+\frac{1}{2} n_{Z}^{-\frac{1}{2}}-\frac{3}{2} n_{Z}^{\frac{3}{2}}+\frac{3}{2} n_{Z}^{-\frac{3}{2}}-\frac{1}{2} n_{A}^{\frac{1}{2}}+\frac{1}{2} n_{A}^{-\frac{1}{2}}-\frac{3}{2} n_{A}^{\frac{3}{2}}+\frac{3}{2} n_{A}^{-\frac{3}{2}}-n_{R}^{\frac{1}{2}}+n_{R}^{-\frac{1}{2}} \quad \bmod 4,
$$


where $n_{Z, A}^{\frac{1}{2},-\frac{1}{2}, \frac{3}{2},-\frac{3}{2}}$ are the numbers of occupied states with $S_{4}$ eigenvalues $e^{-i \frac{\pi}{4}}, e^{i \frac{\pi}{4}}, e^{-i \frac{3 \pi}{4}}, e^{i \frac{3 \pi}{4}}$, and $n_{R}^{\frac{1}{2},-\frac{1}{2}}$ are the numbers of occupied states with $C_{2}$ eigenvalues $e^{-i \frac{\pi}{4}}, e^{i \frac{\pi}{4}}$. We choose the first $\mathbb{Z}_{2}$ factor as the difference of Chern numbers in the $k_{z}=\pi$ plane and the $k_{z}=0$ plane over $2 \bmod 2$

$$
\delta_{2 S}=-n_{Z}^{\frac{3}{2}}+n_{Z}^{-\frac{3}{2}}-n_{A}^{\frac{3}{2}}+n_{A}^{-\frac{3}{2}}+n_{\Gamma}^{\frac{3}{2}}-n_{\Gamma}^{-\frac{3}{2}}+n_{M}^{\frac{3}{2}}-n_{M}^{-\frac{3}{2}} \bmod 2 .
$$

The $\delta_{2 S}=1$ phase is a WSM with 2 Weyl points between $k_{z}=0$ and $k_{z}=\pi$. One may be curious why $C_{\pi}-C_{0}$ is an even number. The answer is that it is enforced by the compatibility relations: the $k_{z}=0$ and $k_{z}=\pi$ planes must have the same $C_{2}$ eigenvalues and hence the same parity of Chern numbers. The second $\mathbb{Z}_{2}$ factor is

$$
z_{2}=\sum_{K=\Gamma, M, Z, A} \frac{n_{K}^{\frac{1}{2}}-n_{K}^{-\frac{3}{2}}}{2} \bmod 2 .
$$

For $z_{4 S}=0$ and $\delta_{2 S}=0, z_{2}$ corresponds to axion insulator/3D QAHIstate [7].

\section{Definitions for the stable indices of MSG $\mathbf{8 3 . 4 3}$}

MSG 83.43 $(P 4 / m)$ has the SI group $\mathbb{Z}_{4}^{3}$. We choose the three SI as

$$
\begin{aligned}
\delta_{4 m}=C_{\pi}^{+}-C_{0}^{-} \bmod 4 & =\sum_{K=Z, A}\left(-\frac{1}{2} n_{K}^{\frac{1}{2},+i}+\frac{1}{2} n_{K}^{-\frac{1}{2},+i}-\frac{3}{2} n_{K}^{\frac{3}{2},+i}+\frac{3}{2} n_{K}^{-\frac{3}{2},+i}\right)-n_{R}^{\frac{1}{2},+i}+n_{R}^{-\frac{1}{2},+i} \\
& -\sum_{K=\Gamma, M}\left(-\frac{1}{2} n_{K}^{\frac{1}{2},-i}+\frac{1}{2} n_{K}^{-\frac{1}{2},-i}-\frac{3}{2} n_{K}^{\frac{3}{2},-i}+\frac{3}{2} n_{K}^{-\frac{3}{2},-i}\right)+n_{X}^{\frac{1}{2},-i}+n_{X}^{-\frac{1}{2},-i} \bmod 4, \\
z_{4 m, \pi}^{+}=C_{\pi}^{+} \bmod 4=N_{o c c} & +\sum_{K=Z, A}\left(-\frac{1}{2} n_{K}^{\frac{1}{2},+i}+\frac{1}{2} n_{K}^{-\frac{1}{2},+i}-\frac{3}{2} n_{K}^{\frac{3}{2},+i}+\frac{3}{2} n_{K}^{-\frac{3}{2},+i}\right)-n_{R}^{\frac{1}{2},+i}+n_{R}^{-\frac{1}{2},+i} \bmod 4, \\
z_{4 m, \pi}^{-}=C_{\pi}^{-} \bmod 4=N_{o c c} & +\sum_{K=Z, A}\left(-\frac{1}{2} n_{K}^{\frac{1}{2},-i}+\frac{1}{2} n_{K}^{-\frac{1}{2},-i}-\frac{3}{2} n_{K}^{\frac{3}{2},-i}+\frac{3}{2} n_{K}^{-\frac{3}{2},-i}\right)-n_{R}^{\frac{1}{2},-i}+n_{R}^{-\frac{1}{2},-i} \bmod 4 .
\end{aligned}
$$

Here $C_{0, \pi}^{ \pm i}$ represents Chern number in the $\pm i$ mirror sector in the $k_{z}=0, \pi$ plane.

\section{Definitions for the stable indices of MSG 143.1}

MSG 143.1 (P3) has the SI group $\mathbb{Z}_{3}$. According to the Chern number formula, the Chern number $C$ is related to the $C_{3}$ eigenvalues

$$
e^{i \frac{2 \pi}{3} C}=(-1)^{N_{\text {occ }}} \prod_{n \in \text { occ }} \theta_{n}(\Gamma) \theta_{n}(K) \theta_{n}(K A),
$$

where $\theta_{n}\left(\Gamma, K, K^{\prime}\right)$ is the $C_{3}$ eigenvalue of the $n$th occupied state at the corresponding momentum. (One should not confuse the $C_{3}$ eigenvalues $\theta_{n}$ with the axion theta angle $\theta$ ). We define the SI as,

$$
z_{3 R}=\sum_{K=A, H, H A}\left(n_{K}^{-\frac{1}{2}}-n_{K}^{\frac{3}{2}}\right) \bmod 3 .
$$

\section{Stable indices of the magnetic TIs}

Using the SI defined above, we have explained the physical meaning for all of the TIs obtained from MTQC. We tabulate all of the TIs diagnosed by MTQC in Table X. For each material, we list the identification number in BCSMD (BCSID), chemical formula (Formula), Coulomb interaction strength in $\mathrm{LDA}+\mathrm{U}$ calculations (U), magnetic space group (MSG), SI of the MSG calculated by the machinery of MTQC (Indices(MTQC)), whose physical meaning is unclear, the SI and the corresponding subgroup (Min-sMSG), and the physical interpretation of the Indices (Interpretations).

TABLE X: Topological indices and the physical interpretations of the TIs predicted by MTQC.

\begin{tabular}{c|c|c|c|l|c|l|l}
\hline BCSID & Formula & $\mathrm{U}(\mathrm{eV})$ & MSG & Indices(MTQC) & Min-sMSG & Indices & Interpretations \\
\hline 1.206 & Dy2Fe2Si2C & 0 & $2.7\left(P_{S} \overline{1}\right)$ & $c 2=1$ & $2.4(P \overline{1})$ & $\begin{array}{l}z_{2 I, 1}=0, \\
z_{2 I, 2}=0, \\
z_{2 I, 3}=0, \\
\eta_{4 I}=2\end{array}$ & AXI/3D QAHI \\
& & & & & & $2.4(P \overline{1})$ & $\begin{array}{l}z_{2 I, 1}=0, \\
z_{2 I, 2}=0, \\
z_{2 I, 3}=0, \\
\eta_{4 I}=2\end{array}$ \\
\hline 1.206 & Dy2Fe2Si2C & 2 & $2.7\left(P_{S} \overline{1}\right)$ & $c 2=1$ & & & \\
& & & & & & & \\
\hline
\end{tabular}




\begin{tabular}{|c|c|c|c|c|c|c|c|}
\hline 1.206 & Dy2Fe2Si2C & 4 & $2.7\left(P_{S} \overline{1}\right)$ & $c 2=1$ & $2.4(P \overline{1})$ & $\begin{array}{l}z_{2 I, 1}=0 \\
z_{2 I, 2}=0 \\
z_{2 I, 3}=0 \\
\eta_{4 I}=2\end{array}$ & $\mathrm{AXI} / 3 \mathrm{D} \mathrm{QAHI}$ \\
\hline 1.206 & Dy2Fe2Si2C & 6 & $2.7\left(P_{S} \overline{1}\right)$ & $c 2=1$ & $2.4(P \overline{1})$ & $\begin{array}{l}z_{2 I, 1}=0 \\
z_{2 I, 2}=0 \\
z_{2 I, 3}=0 \\
\eta_{4 I}=2\end{array}$ & $\mathrm{AXI} / 3 \mathrm{D} \mathrm{QAHI}$ \\
\hline 0.104 & ErVO3 & 2 & $11.54\left(P 2_{1}^{\prime} / m^{\prime}\right)$ & $\begin{array}{l}c 2_{1}=0, c 2_{2}=0 \\
c 4=2\end{array}$ & $2.4(P \overline{1})$ & $\begin{array}{l}z_{2 I, 1}=0 \\
z_{2 I, 2}=0 \\
z_{2 I, 3}=0 \\
\eta_{4 I}=2\end{array}$ & $\overline{\mathrm{AXI} / 3 \mathrm{D} \mathrm{QAHI}}$ \\
\hline 0.106 & DyVO3 & 0 & $11.54\left(P 2_{1}^{\prime} / m^{\prime}\right)$ & $\begin{array}{l}c 2_{1}=1, c 2_{2}=1 \\
c 4=1\end{array}$ & $2.4(P \overline{1})$ & $\begin{array}{l}z_{2 I, 1}=1 \\
z_{2 I, 2}=0 \\
z_{2 I, 3}=1 \\
\eta_{4 I}=1\end{array}$ & SISM \\
\hline 1.264 & CoPS3 & 0 & $11.57\left(P_{C} 2_{1} / m\right)$ & $c 2=1$ & $2.4(P \overline{1})$ & $\begin{array}{l}z_{2 I, 1}=0 \\
z_{2 I, 2}=0 \\
z_{2 I, 3}=0 \\
\eta_{4 I}=2\end{array}$ & AXI/3D QAHI \\
\hline 0.203 & $\mathrm{Mn} 3 \mathrm{Ge}$ & 0 & $12.62\left(C 2^{\prime} / m^{\prime}\right)$ & $\begin{array}{l}c 2_{1}=0, c 2_{2}=0 \\
c 4=2\end{array}$ & $2.4(P \overline{1})$ & $\begin{array}{l}z_{2 I, 1}=0 \\
z_{2 I, 2}=0 \\
z_{2 I, 3}=0 \\
\eta_{4 I}=2\end{array}$ & AXI/3D QAHI \\
\hline 0.203 & $\mathrm{Mn} 3 \mathrm{Ge}$ & 1 & $12.62\left(C 2^{\prime} / m^{\prime}\right)$ & $\begin{array}{l}c 2_{1}=0, c 2_{2}=0 \\
c 4=2\end{array}$ & $2.4(P \overline{1})$ & $\begin{array}{l}z_{2 I, 1}=0 \\
z_{2 I, 2}=0 \\
z_{2 I, 3}=0 \\
\eta_{4 I}=2\end{array}$ & $\mathrm{AXI} / 3 \mathrm{D} \mathrm{QAHI}$ \\
\hline 0.203 & $\mathrm{Mn} 3 \mathrm{Ge}$ & 2 & $12.62\left(C 2^{\prime} / m^{\prime}\right)$ & $\begin{array}{l}c 2_{1}=0, c 2_{2}=0 \\
c 4=2\end{array}$ & $2.4(P \overline{1})$ & $\begin{array}{l}z_{2 I, 1}=0 \\
z_{2 I, 2}=0 \\
z_{2 I, 3}=0 \\
\eta_{4 I}=2\end{array}$ & $\mathrm{AXI} / 3 \mathrm{D} \mathrm{QAHI}$ \\
\hline 0.203 & $\mathrm{Mn} 3 \mathrm{Ge}$ & 3 & $12.62\left(C 2^{\prime} / m^{\prime}\right)$ & $\begin{array}{l}c 2_{1}=0, c 2_{2}=0 \\
c 4=2\end{array}$ & $2.4(P \overline{1})$ & $\begin{array}{l}z_{2 I, 1}=0 \\
z_{2 I, 2}=0 \\
z_{2 I, 3}=0 \\
\eta_{4 I}=2\end{array}$ & AXI/3D QAHI \\
\hline 0.203 & Mn3Ge & 4 & $12.62\left(C 2^{\prime} / m^{\prime}\right)$ & $\begin{array}{l}c 2_{1}=0, c 2_{2}=0 \\
c 4=2\end{array}$ & $2.4(P \overline{1})$ & $\begin{array}{l}z_{2 I, 1}=0 \\
z_{2 I, 2}=0 \\
z_{2 I, 3}=0 \\
\eta_{4 I}=2\end{array}$ & $\overline{\mathrm{AXI} / 3 \mathrm{D} \mathrm{QAHI}}$ \\
\hline 1.0 .13 & FeI2 & 0 & $12.62\left(C 2^{\prime} / m^{\prime}\right)$ & $\begin{array}{l}c 2_{1}=1, c 2_{2}=1 \\
c 4=3\end{array}$ & $2.4(P \overline{1})$ & $\begin{array}{l}z_{2 I, 1}=0 \\
z_{2 I, 2}=0 \\
z_{2 I, 3}=0 \\
\eta_{4 I}=1\end{array}$ & SISM \\
\hline 1.140 & $\mathrm{PrMgPb}$ & 0 & $13.73\left(P_{A} 2 / c\right)$ & $c 2=1$ & $2.4(P \overline{1})$ & $\begin{array}{l}z_{2 I, 1}=0 \\
z_{2 I, 2}=0 \\
z_{2 I, 3}=0 \\
\eta_{4 I}=2\end{array}$ & $\overline{\mathrm{AXI} / 3 \mathrm{D} \mathrm{QAHI}}$ \\
\hline 1.140 & $\mathrm{PrMgPb}$ & 2 & $13.73\left(P_{A} 2 / c\right)$ & $c 2=1$ & $2.4(P \overline{1})$ & $\begin{array}{l}z_{2 I, 1}=0 \\
z_{2 I, 2}=0 \\
z_{2 I, 3}=0 \\
\eta_{4 I}=2\end{array}$ & $\overline{\mathrm{AXI} / 3 \mathrm{D} \mathrm{QAHI}}$ \\
\hline 1.140 & $\mathrm{PrMgPb}$ & 4 & $13.73\left(P_{A} 2 / c\right)$ & $c 2=1$ & $2.4(P \overline{1})$ & $\begin{array}{l}z_{2 I, 1}=0 \\
z_{2 I, 2}=0 \\
z_{2 I, 3}=0 \\
\eta_{4 I}=2\end{array}$ & $\overline{\mathrm{AXI} / 3 \mathrm{D} \mathrm{QAHI}}$ \\
\hline
\end{tabular}




\begin{tabular}{|c|c|c|c|c|c|c|c|}
\hline 1.140 & $\mathrm{PrMgPb}$ & 6 & $13.73\left(P_{A} 2 / c\right)$ & $c 2=1$ & $2.4(P \overline{1})$ & \begin{tabular}{|l}
$z_{2 I, 1}=0$ \\
$z_{2 I, 2}=0$ \\
$z_{2 I, 3}=0$ \\
$\eta_{4 I}=2$
\end{tabular} & $\mathrm{AXI} / 3 \mathrm{D} \mathrm{QAHI}$ \\
\hline 1.141 & $\mathrm{NdMgPb}$ & 0 & $13.73\left(P_{A} 2 / c\right)$ & $c 2=1$ & $2.4(P \overline{1})$ & $\begin{array}{l}z_{2 I, 1}=0 \\
z_{2 I, 2}=0 \\
z_{2 I, 3}=0 \\
\eta_{4 I}=2\end{array}$ & $\mathrm{AXI} / 3 \mathrm{D} \mathrm{QAHI}$ \\
\hline 1.141 & $\mathrm{NdMgPb}$ & 2 & $13.73\left(P_{A} 2 / c\right)$ & $c 2=1$ & $2.4(P \overline{1})$ & \begin{tabular}{|l}
$z_{2 I, 1}=0$ \\
$z_{2 I, 2}=0$ \\
$z_{2 I, 3}=0$ \\
$\eta_{4 I}=2$
\end{tabular} & $\mathrm{AXI} / 3 \mathrm{D} \mathrm{QAHI}$ \\
\hline 1.201 & Cr2ReO6 & 0 & $14.80\left(P_{a} 2_{1} / c\right)$ & $c 2=1$ & $2.4(P \overline{1})$ & \begin{tabular}{|l}
$z_{2 I, 1}=0$ \\
$z_{2 I, 2}=0$ \\
$z_{2 I, 3}=0$ \\
$\eta_{4 I}=2$
\end{tabular} & $\mathrm{AXI} / 3 \mathrm{D} \mathrm{QAHI}$ \\
\hline 0.174 & Pr3Ru4Al12 & 0 & $15.89\left(C 2^{\prime} / c^{\prime}\right)$ & $c 2=1, c 4=1$ & $2.4(P \overline{1})$ & $\begin{array}{l}z_{2 I, 1}=0 \\
z_{2 I, 2}=0 \\
z_{2 I, 3}=0 \\
\eta_{4 I}=1\end{array}$ & SISM \\
\hline 0.174 & Pr3Ru4Al12 & 2 & $15.89\left(C 2^{\prime} / c^{\prime}\right)$ & $c 2=1, c 4=1$ & $2.4(P \overline{1})$ & $\begin{array}{l}z_{2 I, 1}=0 \\
z_{2 I, 2}=0 \\
z_{2 I, 3}=0 \\
\eta_{4 I}=1\end{array}$ & SISM \\
\hline 0.174 & Pr3Ru4Al12 & 4 & $15.89\left(C 2^{\prime} / c^{\prime}\right)$ & $c 2=1, c 4=1$ & $2.4(P \overline{1})$ & $\begin{array}{l}z_{2 I, 1}=0 \\
z_{2 I, 2}=0 \\
z_{2 I, 3}=0 \\
\eta_{4 I}=1\end{array}$ & SISM \\
\hline 0.174 & Pr3Ru4Al12 & 6 & $15.89\left(C 2^{\prime} / c^{\prime}\right)$ & $c 2=1, c 4=1$ & $2.4(P \overline{1})$ & $\begin{array}{l}z_{2 I, 1}=0 \\
z_{2 I, 2}=0 \\
z_{2 I, 3}=0 \\
\eta_{4 I}=1\end{array}$ & SISM \\
\hline 0.226 & $\mathrm{NdCo} 2$ & 2 & $15.89\left(C 2^{\prime} / c^{\prime}\right)$ & $c 2=1, c 4=3$ & $2.4(P \overline{1})$ & $\begin{array}{l}z_{2 I, 1}=0 \\
z_{2 I, 2}=0 \\
z_{2 I, 3}=0 \\
\eta_{4 I}=3\end{array}$ & SISM \\
\hline 0.226 & $\mathrm{NdCo} 2$ & 4 & $15.89\left(C 2^{\prime} / c^{\prime}\right)$ & $c 2=0, c 4=2$ & $2.4(P \overline{1})$ & $\begin{array}{l}z_{2 I, 1}=0 \\
z_{2 I, 2}=0 \\
z_{2 I, 3}=0 \\
\eta_{4 I}=2\end{array}$ & $\mathrm{AXI} / 3 \mathrm{D} \mathrm{QAHI}$ \\
\hline 0.226 & $\mathrm{NdCo} 2$ & 6 & $15.89\left(C 2^{\prime} / c^{\prime}\right)$ & $c 2=1, c 4=3$ & $2.4(P \overline{1})$ & $\begin{array}{l}z_{2 I, 1}=0 \\
z_{2 I, 2}=0 \\
z_{2 I, 3}=0 \\
\eta_{4 I}=3\end{array}$ & SISM \\
\hline 2.10 & HoP & 0 & $15.89\left(C 2^{\prime} / c^{\prime}\right)$ & $c 2=1, c 4=3$ & $2.4(P \overline{1})$ & $\begin{array}{l}z_{2 I, 1}=0 \\
z_{2 I, 2}=0 \\
z_{2 I, 3}=0 \\
\eta_{4 I}=3\end{array}$ & SISM \\
\hline 2.10 & HoP & 2 & $15.89\left(C 2^{\prime} / c^{\prime}\right)$ & $c 2=1, c 4=0$ & $2.4(P \overline{1})$ & $\begin{array}{l}z_{2 I, 1}=1 \\
z_{2 I, 2}=1 \\
z_{2 I, 3}=0 \\
\eta_{4 I}=0\end{array}$ & 3D QAHI \\
\hline 2.10 & $\mathrm{HoP}$ & 4 & $15.89\left(C 2^{\prime} / c^{\prime}\right)$ & $c 2=1, c 4=0$ & $2.4(P \overline{1})$ & $\begin{array}{l}z_{2 I, 1}=1 \\
z_{2 I, 2}=1 \\
z_{2 I, 3}=0 \\
\eta_{4 I}=0\end{array}$ & 3D QAHI \\
\hline
\end{tabular}




\begin{tabular}{|c|c|c|c|c|c|c|c|}
\hline 2.10 & $\mathrm{HoP}$ & 6 & $15.89\left(C 2^{\prime} / c^{\prime}\right)$ & $c 2=1, c 4=0$ & $2.4(P \overline{1})$ & $\begin{array}{l}z_{2 I, 1}=1 \\
z_{2 I, 2}=1, \\
z_{2 I, 3}=0 \\
\eta_{4 I}=0\end{array}$ & 3D QAHI \\
\hline 1.211 & Dy2O2S & 0 & $15.90\left(C_{c} 2 / c\right)$ & $c 2=1$ & $2.4(P \overline{1})$ & $\begin{array}{l}z_{2 I, 1}=0 \\
z_{2 I, 2}=0 \\
z_{2 I, 3}=0 \\
\eta_{4 I}=2\end{array}$ & $\mathrm{AXI} / 3 \mathrm{D} \mathrm{QAHI}$ \\
\hline 1.216 & $\mathrm{Nd} 2 \mathrm{BaNiO} 5$ & 0 & $15.90\left(C_{c} 2 / c\right)$ & $c 2=1$ & $2.4(P \overline{1})$ & $\begin{array}{l}z_{2 I, 1}=0 \\
z_{2 I, 2}=0 \\
z_{2 I, 3}=0 \\
\eta_{4 I}=2\end{array}$ & AXI/3D QAHI \\
\hline 1.217 & Tb2BaNiO5 & 0 & $15.90\left(C_{c} 2 / c\right)$ & $c 2=1$ & $2.4(P \overline{1})$ & $\begin{array}{l}z_{2 I, 1}=0, \\
z_{2 I, 2}=0, \\
z_{2 I, 3}=0, \\
\eta_{4 I}=2\end{array}$ & $\mathrm{AXI} / 3 \mathrm{D} \mathrm{QAHI}$ \\
\hline 1.49 & $\mathrm{Ag} 2 \mathrm{NiO} 2$ & 0 & $15.90\left(C_{c} 2 / c\right)$ & $c 2=1$ & $2.4(P \overline{1})$ & $\begin{array}{l}z_{2 I, 1}=0 \\
z_{2 I, 2}=0 \\
z_{2 I, 3}=0 \\
\eta_{4 I}=2\end{array}$ & $\mathrm{AXI} / 3 \mathrm{D} \mathrm{QAHI}$ \\
\hline 1.139 & Ho2RhIn8 & 2 & $49.273\left(P_{c} c c m\right)$ & $c 2=1$ & $2.4(P \overline{1})$ & $\begin{array}{l}z_{2 I, 1}=0 \\
z_{2 I, 2}=0 \\
z_{2 I, 3}=0 \\
\eta_{4 I}=2\end{array}$ & AXI \\
\hline 1.139 & Ho2RhIn8 & 4 & $49.273\left(P_{c} c c m\right)$ & $c 2=1$ & $2.4(P \overline{1})$ & $\begin{array}{l}z_{2 I, 1}=0 \\
z_{2 I, 2}=0 \\
z_{2 I, 3}=0 \\
\eta_{4 I}=2\end{array}$ & AXI \\
\hline 1.150 & $\operatorname{PrAg}$ & 4 & $53.334\left(P_{B} m n a\right)$ & $c 2=1$ & $2.4(P \overline{1})$ & $\begin{array}{l}z_{2 I, 1}=0 \\
z_{2 I, 2}=0 \\
z_{2 I, 3}=0 \\
\eta_{4 I}=2\end{array}$ & AXI \\
\hline 1.8 & CeRu2Al10 & 0 & $57.391\left(P_{C} b c m\right)$ & $c 2=1$ & $2.4(P \overline{1})$ & $\begin{array}{l}z_{2 I, 1}=0 \\
z_{2 I, 2}=0 \\
z_{2 I, 3}=0 \\
\eta_{4 I}=2\end{array}$ & AXI \\
\hline 1.8 & CeRu2Al10 & 2 & $57.391\left(P_{C} b c m\right)$ & $c 2=1$ & $2.4(P \overline{1})$ & $\begin{array}{l}z_{2 I, 1}=0 \\
z_{2 I, 2}=0 \\
z_{2 I, 3}=0 \\
\eta_{4 I}=2\end{array}$ & AXI \\
\hline 1.8 & CeRu2Al10 & 4 & $57.391\left(P_{C} b c m\right)$ & $c 2=1$ & $2.4(P \overline{1})$ & $\begin{array}{l}z_{2 I, 1}=0 \\
z_{2 I, 2}=0 \\
z_{2 I, 3}=0 \\
\eta_{4 I}=2\end{array}$ & AXI \\
\hline 1.252 & $\mathrm{CaCo} 2 \mathrm{P} 2$ & 1 & $59.416\left(P_{I} m m n\right)$ & $c 2=1$ & $2.4(P \overline{1})$ & $\begin{array}{l}z_{2 I, 1}=0 \\
z_{2 I, 2}=0 \\
z_{2 I, 3}=0 \\
\eta_{4 I}=2\end{array}$ & AXI \\
\hline 1.252 & $\mathrm{CaCo} 2 \mathrm{P} 2$ & 2 & $59.416\left(P_{I} m m n\right)$ & $c 2=1$ & $2.4(P \overline{1})$ & $\begin{array}{l}z_{2 I, 1}=0 \\
z_{2 I, 2}=0, \\
z_{2 I, 3}=0 \\
\eta_{4 I}=2\end{array}$ & AXI \\
\hline 1.252 & $\mathrm{CaCo} 2 \mathrm{P} 2$ & 3 & $59.416\left(P_{I} m m n\right)$ & $c 2=1$ & $2.4(P \overline{1})$ & $\begin{array}{l}z_{2 I, 1}=0 \\
z_{2 I, 2}=0 \\
z_{2 I, 3}=0 \\
\eta_{4 I}=2\end{array}$ & AXI \\
\hline
\end{tabular}




\begin{tabular}{|c|c|c|c|c|c|c|c|}
\hline 1.252 & $\mathrm{CaCo} 2 \mathrm{P} 2$ & 4 & $59.416\left(P_{I} m m n\right)$ & $c 2=1$ & $2.4(P \overline{1})$ & $\begin{array}{l}z_{2 I, 1}=0 \\
z_{2 I, 2}=0 \\
z_{2 I, 3}=0 \\
\eta_{4 I}=2\end{array}$ & AXI \\
\hline 1.88 & $\mathrm{Mn} 5 \mathrm{Si} 3$ & 0 & $60.431\left(P_{C} b c n\right)$ & $c 2=1$ & $2.4(P \overline{1})$ & $\begin{array}{l}z_{2 I, 1}=0 \\
z_{2 I, 2}=0 \\
z_{2 I, 3}=0 \\
\eta_{4 I}=2\end{array}$ & AXI \\
\hline 2.1 & EuFe2As2 & 2 & $61.439\left(P_{C} b c a\right)$ & $c 2=1$ & $2.4(P \overline{1})$ & $\begin{array}{l}z_{2 I, 1}=0 \\
z_{2 I, 2}=0 \\
z_{2 I, 3}=0 \\
\eta_{4 I}=2\end{array}$ & AXI \\
\hline 2.1 & EuFe2As2 & 3 & $61.439\left(P_{C} b c a\right)$ & $c 2=1$ & $2.4(P \overline{1})$ & $\begin{array}{l}z_{2 I, 1}=0 \\
z_{2 I, 2}=0 \\
z_{2 I, 3}=0 \\
\eta_{4 I}=2\end{array}$ & AXI \\
\hline 1.130 & $\mathrm{Cr} 2 \mathrm{As}$ & 1 & $62.450\left(P_{a} n m a\right)$ & $c 2=1$ & $2.4(P \overline{1})$ & $\begin{array}{l}z_{2 I, 1}=0 \\
z_{2 I, 2}=0 \\
z_{2 I, 3}=0 \\
\eta_{4 I}=2\end{array}$ & AXI \\
\hline 1.131 & $\mathrm{Fe} 2 \mathrm{As}$ & 0 & $62.450\left(P_{a} n m a\right)$ & $c 2=1$ & $2.4(P \overline{1})$ & $\begin{array}{l}z_{2 I, 1}=0 \\
z_{2 I, 2}=0 \\
z_{2 I, 3}=0 \\
\eta_{4 I}=2\end{array}$ & AXI \\
\hline 1.131 & $\mathrm{Fe} 2 \mathrm{As}$ & 1 & $62.450\left(P_{a} n m a\right)$ & $c 2=1$ & $2.4(P \overline{1})$ & $\begin{array}{l}z_{2 I, 1}=0 \\
z_{2 I, 2}=0 \\
z_{2 I, 3}=0 \\
\eta_{4 I}=2\end{array}$ & AXI \\
\hline 1.131 & $\mathrm{Fe} 2 \mathrm{As}$ & 3 & $62.450\left(P_{a} n m a\right)$ & $c 2=1$ & $2.4(P \overline{1})$ & $\begin{array}{l}z_{2 I, 1}=0 \\
z_{2 I, 2}=0 \\
z_{2 I, 3}=0 \\
\eta_{4 I}=2\end{array}$ & AXI \\
\hline 1.131 & $\mathrm{Fe} 2 \mathrm{As}$ & 4 & $62.450\left(P_{a} n m a\right)$ & $c 2=1$ & $2.4(P \overline{1})$ & $\begin{array}{l}z_{2 I, 1}=0 \\
z_{2 I, 2}=0 \\
z_{2 I, 3}=0 \\
\eta_{4 I}=2\end{array}$ & AXI \\
\hline 1.179 & $\mathrm{NdCoAsO}$ & 2 & $62.450\left(P_{a} n m a\right)$ & $c 2=1$ & $2.4(P \overline{1})$ & $\begin{array}{l}z_{2 I, 1}=0 \\
z_{2 I, 2}=0 \\
z_{2 I, 3}=0 \\
\eta_{4 I}=2\end{array}$ & $\overline{\mathrm{AXI}}$ \\
\hline 1.28 & $\mathrm{CrN}$ & 0 & $62.450\left(P_{a} n m a\right)$ & $c 2=1$ & $2.4(P \overline{1})$ & $\begin{array}{l}z_{2 I, 1}=0 \\
z_{2 I, 2}=0 \\
z_{2 I, 3}=0 \\
\eta_{4 I}=2\end{array}$ & AXI \\
\hline 1.28 & $\mathrm{CrN}$ & 1 & $62.450\left(P_{a} n m a\right)$ & $c 2=1$ & $2.4(P \overline{1})$ & $\begin{array}{l}\mid z_{2 I, 1}=0 \\
z_{2 I, 2}=0 \\
z_{2 I, 3}=0 \\
\eta_{4 I}=2\end{array}$ & AXI \\
\hline 0.200 & Mn3Sn & 0 & $63.464\left(\mathrm{Cm}^{\prime} \mathrm{cm}^{\prime}\right)$ & $c 2_{1}=1, c 2_{2}=0$ & $2.4(P \overline{1})$ & $\begin{array}{l}\mid z_{2 I, 1}=0 \\
z_{2 I, 2}=0 \\
z_{2 I, 3}=0 \\
\eta_{4 I}=2\end{array}$ & $\mathrm{AXI} / 3 \mathrm{D} \mathrm{QAHI}$ \\
\hline 0.200 & Mn3Sn & 1 & $63.464\left(\mathrm{Cm}^{\prime} \mathrm{cm}^{\prime}\right)$ & $c 2_{1}=1, c 2_{2}=0$ & $2.4(P \overline{1})$ & $\begin{array}{l}\mid z_{2 I, 1}=0 \\
z_{2 I, 2}=0 \\
z_{2 I, 3}=0 \\
\eta_{4 I}=2\end{array}$ & $\mathrm{AXI} / 3 \mathrm{D} \mathrm{QAHI}$ \\
\hline
\end{tabular}




\begin{tabular}{|c|c|c|c|c|c|c|c|}
\hline 0.200 & Mn3Sn & 2 & $63.464\left(\mathrm{Cm}^{\prime} \mathrm{cm}^{\prime}\right)$ & $c 2_{1}=1, c 2_{2}=0$ & $2.4(P \overline{1})$ & $\begin{array}{l}z_{2 I, 1}=0 \\
z_{2 I, 2}=0 \\
z_{2 I, 3}=0 \\
\eta_{4 I}=2\end{array}$ & $\mathrm{AXI} / 3 \mathrm{D} \mathrm{QAHI}$ \\
\hline 1.200 & U2Ni2Sn & 2 & $63.466\left(C_{c} m c m\right)$ & $c 2=1$ & $2.4(P \overline{1})$ & $\begin{array}{l}z_{2 I, 1}=0 \\
z_{2 I, 2}=0 \\
z_{2 I, 3}=0 \\
\eta_{4 I}=2\end{array}$ & AXI \\
\hline 1.200 & U2Ni2Sn & 4 & $63.466\left(C_{c} m c m\right)$ & $c 2=1$ & $2.4(P \overline{1})$ & $\begin{array}{l}z_{2 I, 1}=0 \\
z_{2 I, 2}=0 \\
z_{2 I, 3}=0 \\
\eta_{4 I}=2\end{array}$ & AXI \\
\hline 1.200 & U2Ni2Sn & 6 & $63.466\left(C_{c} m c m\right)$ & $c 2=1$ & $2.4(P \overline{1})$ & $\begin{array}{l}z_{2 I, 1}=0 \\
z_{2 I, 2}=0 \\
z_{2 I, 3}=0 \\
\eta_{4 I}=2\end{array}$ & AXI \\
\hline 1.262 & NpRhGa5 & 2 & $63.466\left(C_{c} m c m\right)$ & $c 2=1$ & $2.4(P \overline{1})$ & $\begin{array}{l}z_{2 I, 1}=0 \\
z_{2 I, 2}=0 \\
z_{2 I, 3}=0 \\
\eta_{4 I}=2\end{array}$ & AXI \\
\hline 1.195 & Er2Ni2In & 4 & $63.467\left(C_{a} m c m\right)$ & $c 2=1$ & $2.4(P \overline{1})$ & $\begin{array}{l}z_{2 I, 1}=0 \\
z_{2 I, 2}=0 \\
z_{2 I, 3}=0 \\
\eta_{4 I}=2\end{array}$ & AXI \\
\hline 1.16 & BaFe2As2 & 0 & $64.480\left(C_{A} m c a\right)$ & $c 2=1$ & $2.4(P \overline{1})$ & $\begin{array}{l}z_{2 I, 1}=0 \\
z_{2 I, 2}=0 \\
z_{2 I, 3}=0 \\
\eta_{4 I}=2\end{array}$ & AXI \\
\hline 1.16 & BaFe2As2 & 1 & $64.480\left(C_{A} m c a\right)$ & $c 2=1$ & $2.4(P \overline{1})$ & $\begin{array}{l}z_{2 I, 1}=0 \\
z_{2 I, 2}=0 \\
z_{2 I, 3}=0 \\
\eta_{4 I}=2\end{array}$ & AXI \\
\hline 1.16 & BaFe2As2 & 2 & $64.480\left(C_{A} m c a\right)$ & $c 2=1$ & $2.4(P \overline{1})$ & $\begin{array}{l}z_{2 I, 1}=0 \\
z_{2 I, 2}=0 \\
z_{2 I, 3}=0 \\
\eta_{4 I}=2\end{array}$ & AXI \\
\hline 1.16 & BaFe2As2 & 3 & $64.480\left(C_{A} m c a\right)$ & $c 2=1$ & $2.4(P \overline{1})$ & $\begin{array}{l}z_{2 I, 1}=0 \\
z_{2 I, 2}=0 \\
z_{2 I, 3}=0 \\
\eta_{4 I}=2\end{array}$ & AXI \\
\hline 1.188 & CeRh2Si2 & 0 & $64.480\left(C_{A} m c a\right)$ & $c 2=1$ & $2.4(P \overline{1})$ & $\begin{array}{l}z_{2 I, 1}=0 \\
z_{2 I, 2}=0 \\
z_{2 I, 3}=0 \\
\eta_{4 I}=2\end{array}$ & AXI \\
\hline 1.188 & CeRh2Si2 & 6 & $64.480\left(C_{A} m c a\right)$ & $c 2=1$ & $2.4(P \overline{1})$ & $\begin{array}{l}z_{2 I, 1}=0 \\
z_{2 I, 2}=0 \\
z_{2 I, 3}=0 \\
\eta_{4 I}=2\end{array}$ & AXI \\
\hline 1.52 & CaFe2As2 & 0 & $64.480\left(C_{A} m c a\right)$ & $c 2=1$ & $2.4(P \overline{1})$ & $\begin{array}{l}z_{2 I, 1}=0 \\
z_{2 I, 2}=0 \\
z_{2 I, 3}=0 \\
\eta_{4 I}=2\end{array}$ & AXI \\
\hline 1.52 & CaFe2As2 & 1 & $64.480\left(C_{A} m c a\right)$ & $c 2=1$ & $2.4(P \overline{1})$ & $\begin{array}{l}z_{2 I, 1}=0 \\
z_{2 I, 2}=0 \\
z_{2 I, 3}=0 \\
\eta_{4 I}=2\end{array}$ & AXI \\
\hline
\end{tabular}




\begin{tabular}{|c|c|c|c|c|c|c|c|}
\hline 1.52 & $\mathrm{CaFe} 2 \mathrm{As} 2$ & 2 & $64.480\left(C_{A} m c a\right)$ & $c 2=1$ & $2.4(P \overline{1})$ & $\begin{array}{l}z_{2 I, 1}=0 \\
z_{2 I, 2}=0 \\
z_{2 I, 3}=0 \\
\eta_{4 I}=2\end{array}$ & AXI \\
\hline 2.15 & Mn3Ni20P6 & 3 & $65.486\left(\mathrm{Cmm}^{\prime} \mathrm{m}^{\prime}\right)$ & $c 2_{1}=1, c 2_{2}=0$ & $2.4(P \overline{1})$ & $\begin{array}{l}z_{2 I, 1}=0 \\
z_{2 I, 2}=0 \\
z_{2 I, 3}=0 \\
\eta_{4 I}=2\end{array}$ & $\mathrm{AXI} / 3 \mathrm{D} \mathrm{QAHI}$ \\
\hline 1.142 & $\mathrm{CeMgPb}$ & 2 & $67.510\left(C_{A} m m a\right)$ & $c 2=1$ & $2.4(P \overline{1})$ & $\begin{array}{l}z_{2 I, 1}=0 \\
z_{2 I, 2}=0 \\
z_{2 I, 3}=0 \\
\eta_{4 I}=2\end{array}$ & AXI \\
\hline 1.125 & $\mathrm{LaFeAsO}$ & 0 & $73.553\left(I_{c} b c a\right)$ & $c 2=1$ & $2.4(P \overline{1})$ & $\begin{array}{l}z_{2 I, 1}=0 \\
z_{2 I, 2}=0 \\
z_{2 I, 3}=0 \\
\eta_{4 I}=2\end{array}$ & AXI \\
\hline 1.176 & YbCo2Si2 & 1 & $73.553\left(I_{c} b c a\right)$ & $c 2=1$ & $2.4(P \overline{1})$ & $\begin{array}{l}z_{2 I, 1}=0 \\
z_{2 I, 2}=0 \\
z_{2 I, 3}=0 \\
\eta_{4 I}=2\end{array}$ & AXI \\
\hline 1.176 & YbCo2Si2 & 2 & $73.553\left(I_{c} b c a\right)$ & $c 2=1$ & $2.4(P \overline{1})$ & $\begin{array}{l}z_{2 I, 1}=0 \\
z_{2 I, 2}=0 \\
z_{2 I, 3}=0 \\
\eta_{4 I}=2\end{array}$ & AXI \\
\hline 1.176 & YbCo2Si2 & 3 & $73.553\left(I_{c} b c a\right)$ & $c 2=1$ & $2.4(P \overline{1})$ & $\begin{array}{l}z_{2 I, 1}=0 \\
z_{2 I, 2}=0 \\
z_{2 I, 3}=0 \\
\eta_{4 I}=2\end{array}$ & AXI \\
\hline 1.176 & YbCo2Si2 & 4 & $73.553\left(I_{c} b c a\right)$ & $c 2=1$ & $2.4(P \overline{1})$ & $\begin{array}{l}z_{2 I, 1}=0 \\
z_{2 I, 2}=0 \\
z_{2 I, 3}=0 \\
\eta_{4 I}=2\end{array}$ & AXI \\
\hline 2.13 & UP & 0 & $134.481\left(P_{C} 4_{2} / n n m\right)$ & $c 2=1$ & $2.4(P \overline{1})$ & $\begin{array}{l}z_{2 I, 1}=0 \\
z_{2 I, 2}=0 \\
z_{2 I, 3}=0 \\
\eta_{4 I}=2\end{array}$ & AXI \\
\hline 2.13 & UP & 2 & $134.481\left(P_{C} 4_{2} / n n m\right)$ & $c 2=1$ & $2.4(P \overline{1})$ & $\begin{array}{l}z_{2 I, 1}=0 \\
z_{2 I, 2}=0 \\
z_{2 I, 3}=0 \\
\eta_{4 I}=2\end{array}$ & AXI \\
\hline 2.13 & UP & 4 & $134.481\left(P_{C} 4_{2} / n n m\right)$ & $c 2=1$ & $2.4(P \overline{1})$ & $\begin{array}{l}z_{2 I, 1}=0 \\
z_{2 I, 2}=0 \\
z_{2 I, 3}=0 \\
\eta_{4 I}=2\end{array}$ & AXI \\
\hline 2.13 & UP & 6 & $134.481\left(P_{C} 4_{2} / n n m\right)$ & $c 2=1$ & $2.4(P \overline{1})$ & $\begin{array}{l}z_{2 I, 1}=0 \\
z_{2 I, 2}=0 \\
z_{2 I, 3}=0 \\
\eta_{4 I}=2\end{array}$ & AXI \\
\hline 2.20 & UAs & 0 & $134.481\left(P_{C} 4_{2} / n n m\right)$ & $c 2=1$ & $2.4(P \overline{1})$ & $\begin{array}{l}z_{2 I, 1}=0 \\
z_{2 I, 2}=0 \\
z_{2 I, 3}=0 \\
\eta_{4 I}=2\end{array}$ & AXI \\
\hline 2.20 & UAs & 4 & $134.481\left(P_{C} 4_{2} / n n m\right)$ & $c 2=1$ & $2.4(P \overline{1})$ & $\begin{array}{l}z_{2 I, 1}=0 \\
z_{2 I, 2}=0 \\
z_{2 I, 3}=0 \\
\eta_{4 I}=2\end{array}$ & AXI \\
\hline
\end{tabular}




\begin{tabular}{|c|c|c|c|c|c|c|c|}
\hline 2.20 & UAs & 6 & $134.481\left(P_{C} 4_{2} / n n m\right)$ & $c 2=1$ & $2.4(P \overline{1})$ & $\begin{array}{l}z_{2 I, 1}=0 \\
z_{2 I, 2}=0 \\
z_{2 I, 3}=0 \\
\eta_{4 I}=2\end{array}$ & AXI \\
\hline 2.6 & $\mathrm{Nd} 2 \mathrm{CuO} 4$ & 0 & $134.481\left(P_{C} 4_{2} / n n m\right)$ & $c 2=1$ & $2.4(P \overline{1})$ & $\begin{array}{l}z_{2 I, 1}=0 \\
z_{2 I, 2}=0 \\
z_{2 I, 3}=0 \\
\eta_{4 I}=2\end{array}$ & AXI \\
\hline 1.146 & $\mathrm{LaCrAsO}$ & 0 & $138.528\left(P_{c} 4_{2} / \mathrm{ncm}\right)$ & $c 2=1$ & $2.4(P \overline{1})$ & $\begin{array}{l}z_{2 I, 1}=0 \\
z_{2 I, 2}=0 \\
z_{2 I, 3}=0 \\
\eta_{4 I}=2\end{array}$ & AXI \\
\hline 1.146 & $\mathrm{LaCrAsO}$ & 1 & $138.528\left(P_{c} 4_{2} / n c m\right)$ & $c 2=1$ & $2.4(P \overline{1})$ & $\begin{array}{l}z_{2 I, 1}=0 \\
z_{2 I, 2}=0 \\
z_{2 I, 3}=0 \\
\eta_{4 I}=2\end{array}$ & AXI \\
\hline 1.146 & $\mathrm{LaCrAsO}$ & 2 & $138.528\left(P_{c} 4_{2} / n \mathrm{~cm}\right)$ & $c 2=1$ & $2.4(P \overline{1})$ & $\begin{array}{l}z_{2 I, 1}=0 \\
z_{2 I, 2}=0 \\
z_{2 I, 3}=0 \\
\eta_{4 I}=2\end{array}$ & AXI \\
\hline 1.146 & $\mathrm{LaCrAsO}$ & 3 & $138.528\left(P_{c} 4_{2} / n c m\right)$ & $c 2=1$ & $2.4(P \overline{1})$ & $\begin{array}{l}z_{2 I, 1}=0 \\
z_{2 I, 2}=0 \\
z_{2 I, 3}=0 \\
\eta_{4 I}=2\end{array}$ & AXI \\
\hline 1.146 & $\mathrm{LaCrAsO}$ & 4 & $138.528\left(P_{c} 4_{2} / \mathrm{ncm}\right)$ & $c 2=1$ & $2.4(P \overline{1})$ & $\begin{array}{l}z_{2 I, 1}=0 \\
z_{2 I, 2}=0 \\
z_{2 I, 3}=0 \\
\eta_{4 I}=2\end{array}$ & AXI \\
\hline 0.228 & $\mathrm{TbCo} 2$ & 0 & $166.101\left(R \overline{3} m^{\prime}\right)$ & $c 2=1, c 4=1$ & $2.4(P \overline{1})$ & $\begin{array}{l}z_{2 I, 1}=0 \\
z_{2 I, 2}=0 \\
z_{2 I, 3}=0 \\
\eta_{4 I}=3\end{array}$ & SISM \\
\hline 0.228 & $\mathrm{TbCo} 2$ & 4 & $166.101\left(R \overline{3} m^{\prime}\right)$ & $c 2=1, c 4=3$ & $2.4(P \overline{1})$ & $\begin{array}{l}z_{2 I, 1}=0 \\
z_{2 I, 2}=0 \\
z_{2 I, 3}=0 \\
\eta_{4 I}=1\end{array}$ & SISM \\
\hline 0.228 & TbCo2 & 6 & $166.101\left(R \overline{3} m^{\prime}\right)$ & $c 2=1, c 4=1$ & $2.4(P \overline{1})$ & $\begin{array}{l}z_{2 I, 1}=0 \\
z_{2 I, 2}=0 \\
z_{2 I, 3}=0 \\
\eta_{4 I}=3\end{array}$ & SISM \\
\hline 0.150 & NiS2 & 0 & $205.33(P a \overline{3})$ & $c 2=1$ & $2.4(P \overline{1})$ & $\begin{array}{l}z_{2 I, 1}=0 \\
z_{2 I, 2}=0 \\
z_{2 I, 3}=0 \\
\eta_{4 I}=2\end{array}$ & $\overline{\mathrm{AXI} / 3 \mathrm{D} \mathrm{QAHI}}$ \\
\hline 3.12 & $\mathrm{NpSb}$ & 0 & $224.113\left(P n \overline{3} m^{\prime}\right)$ & $c 2=1$ & $2.4(P \overline{1})$ & $\begin{array}{l}z_{2 I, 1}=0 \\
z_{2 I, 2}=0 \\
z_{2 I, 3}=0 \\
\eta_{4 I}=2\end{array}$ & $\overline{\mathrm{AXI}}$ \\
\hline 3.12 & $\mathrm{NpSb}$ & 2 & $224.113\left(P n \overline{3} m^{\prime}\right)$ & $c 2=1$ & $2.4(P \overline{1})$ & $\begin{array}{l}z_{2 I, 1}=0 \\
z_{2 I, 2}=0 \\
z_{2 I, 3}=0 \\
\eta_{4 I}=2\end{array}$ & $\overline{\mathrm{AXI}}$ \\
\hline 3.12 & $\mathrm{NpSb}$ & 4 & $224.113\left(P n \overline{3} m^{\prime}\right)$ & $c 2=1$ & $2.4(P \overline{1})$ & $\begin{array}{l}z_{2 I, 1}=0 \\
z_{2 I, 2}=0 \\
z_{2 I, 3}=0 \\
\eta_{4 I}=2\end{array}$ & AXI \\
\hline
\end{tabular}




\begin{tabular}{|c|c|c|c|c|c|c|c|}
\hline 3.12 & $\mathrm{NpSb}$ & 6 & $224.113\left(\operatorname{Pn} \overline{3} m^{\prime}\right)$ & $c 2=1$ & $2.4(P \overline{1})$ & $\begin{array}{l}z_{2 I, 1}=0 \\
z_{2 I, 2}=0 \\
z_{2 I, 3}=0 \\
\eta_{4 I}=2\end{array}$ & AXI \\
\hline 3.7 & $\mathrm{NpBi}$ & 0 & $224.113\left(\operatorname{Pn} \overline{3} m^{\prime}\right)$ & $c 2=1$ & $2.4(P \overline{1})$ & $\begin{array}{l}z_{2 I, 1}=0 \\
z_{2 I, 2}=0 \\
z_{2 I, 3}=0 \\
\eta_{4 I}=2\end{array}$ & AXI \\
\hline 3.7 & $\mathrm{NpBi}$ & 2 & $224.113\left(P n \overline{3} m^{\prime}\right)$ & $c 2=1$ & $2.4(P \overline{1})$ & $\begin{array}{l}z_{2 I, 1}=0 \\
z_{2 I, 2}=0 \\
z_{2 I, 3}=0 \\
\eta_{4 I}=2\end{array}$ & AXI \\
\hline 3.7 & $\mathrm{NpBi}$ & 4 & $224.113\left(P n \overline{3} m^{\prime}\right)$ & $c 2=1$ & $2.4(P \overline{1})$ & $\begin{array}{l}z_{2 I, 1}=0 \\
z_{2 I, 2}=0 \\
z_{2 I, 3}=0 \\
\eta_{4 I}=2\end{array}$ & AXI \\
\hline 3.7 & $\mathrm{NpBi}$ & 6 & $224.113\left(\operatorname{Pn} \overline{3} m^{\prime}\right)$ & $c 2=1$ & $2.4(P \overline{1})$ & $\begin{array}{l}z_{2 I, 1}=0 \\
z_{2 I, 2}=0 \\
z_{2 I, 3}=0 \\
\eta_{4 I}=2\end{array}$ & AXI \\
\hline 3.10 & $\mathrm{NpSe}$ & 0 & $228.139\left(F_{S} d \overline{3} c\right)$ & $c 2=1$ & $2.4(P \overline{1})$ & $\begin{array}{l}z_{2 I, 1}=0 \\
z_{2 I, 2}=0 \\
z_{2 I, 3}=0 \\
\eta_{4 I}=2\end{array}$ & AXI \\
\hline 3.11 & $\mathrm{NpTe}$ & 0 & $228.139\left(F_{S} d \overline{3} c\right)$ & $c 2=1$ & $2.4(P \overline{1})$ & $\begin{array}{l}z_{2 I, 1}=0 \\
z_{2 I, 2}=0 \\
z_{2 I, 3}=0 \\
\eta_{4 I}=2\end{array}$ & AXI \\
\hline 1.223 & Tm2CoGa8 & 0 & $65.489\left(C_{a} m m m\right)$ & $c 2=0, c 4=1$ & $47.249(\mathrm{Pmmm})$ & $\begin{array}{l}z_{2 w, 1}=0 \\
z_{2 w, 2}=0 \\
z_{2 w, 3}=1 \\
z_{4}=3\end{array}$ & AXI/MTCI \\
\hline 1.254 & UNiGa5 & 2 & $140.550\left(I_{c} 4 / \mathrm{mcm}\right)$ & $c 4=3$ & $47.249($ Pmmm $)$ & $\begin{array}{l}z_{2 w, 1}=0 \\
z_{2 w, 2}=0 \\
z_{2 w, 3}=0 \\
z_{4}=3\end{array}$ & AXI \\
\hline 1.254 & UNiGa5 & 4 & $140.550\left(I_{c} 4 / \mathrm{mcm}\right)$ & $c 4=3$ & $47.249($ P $m m m)$ & $\begin{array}{l}z_{2 w, 1}=0 \\
z_{2 w, 2}=0 \\
z_{2 w, 3}=0 \\
z_{4}=3\end{array}$ & AXI \\
\hline 0.81 & $\mathrm{U} 2 \mathrm{Pd} 2 \mathrm{Sn}$ & 0 & $127.394\left(P 4^{\prime} / m^{\prime} b m^{\prime}\right)$ & $c 2=1$ & $81.33(P \overline{4})$ & $\begin{array}{l}\delta_{2 S}=1 \\
z_{2}=1 \\
z_{4 S}=0\end{array}$ & SISM $^{*}$ \\
\hline 0.81 & $\mathrm{U} 2 \mathrm{Pd} 2 \mathrm{Sn}$ & 2 & $127.394\left(P 4^{\prime} / m^{\prime} b m^{\prime}\right)$ & $c 2=1$ & $81.33(P \overline{4})$ & $\begin{array}{l}\delta_{2 S}=1 \\
z_{2}=1 \\
z_{4 S}=0\end{array}$ & SISM $^{*}$ \\
\hline 0.81 & $\mathrm{U} 2 \mathrm{Pd} 2 \mathrm{Sn}$ & 4 & $127.394\left(P 4^{\prime} / m^{\prime} b m^{\prime}\right)$ & $c 2=1$ & $81.33(P \overline{4})$ & $\begin{array}{l}\delta_{2 S}=1 \\
z_{2}=1 \\
z_{4 S}=0\end{array}$ & SISM $^{*}$ \\
\hline 0.81 & $\mathrm{U} 2 \mathrm{Pd} 2 \mathrm{Sn}$ & 6 & $127.394\left(P 4^{\prime} / m^{\prime} b m^{\prime}\right)$ & $c 2=1$ & $81.33(P \overline{4})$ & $\begin{array}{l}\delta_{2 S}=1 \\
z_{2}=1 \\
z_{4 S}=0\end{array}$ & SISM $^{*}$ \\
\hline 0.186 & $\mathrm{CeMnAsO}$ & 2 & $129.416\left(P 4^{\prime} / n^{\prime} m^{\prime} m\right)$ & $c 2=1$ & $81.33(P \overline{4})$ & $\begin{array}{l}\delta_{2 S}=1 \\
z_{2}=1 \\
z_{4 S}=0\end{array}$ & SISM $^{*}$ \\
\hline 0.186 & $\mathrm{CeMnAsO}$ & 4 & $129.416\left(P 4^{\prime} / n^{\prime} m^{\prime} m\right)$ & $c 2=1$ & $81.33(P \overline{4})$ & $\begin{array}{l}\delta_{2 S}=1 \\
z_{2}=1 \\
z_{4 S}=0\end{array}$ & SISM $^{*}$ \\
\hline
\end{tabular}




\begin{tabular}{c|c|c|c|l|c|l|c}
\hline 0.186 & CeMnAsO & 6 & $129.416\left(P 4^{\prime} / n^{\prime} m^{\prime} m\right)$ & $c 2=1$ & $81.33(P \overline{4})$ & $\begin{array}{l}\delta_{2 S}=1, \\
z_{2}=1, \\
z_{4 S}=0\end{array}$ & SISM $^{*}$ \\
\hline 0.126 & NpCo2 & 4 & $141.556\left(I 4_{1}^{\prime} / a^{\prime} m^{\prime} d\right)$ & $c 2=1$ & $81.33(P \overline{4})$ & $\begin{array}{l}\delta_{2 S}=0, \\
z_{2}=0, \\
z_{4 S}=2\end{array}$ & 3D QAHI \\
\hline 1.251 & NdCo2P2 & 6 & $124.360\left(P_{c} 4 / m c c\right)$ & $c 4=3$ & $83.43(P 4 / m)$ & $\begin{array}{l}z_{4 m, \pi}^{+}=0, \\
z_{4 m, \pi}^{-}=0, \\
\delta_{4 m}=3\end{array}$ & AXI/MTCI \\
\hline 1.255 & UPtGa5 & 0 & $124.360\left(P_{c} 4 / m c c\right)$ & $c 4=3$ & $83.43(P 4 / m)$ & $\begin{array}{l}z_{4 m, \pi}^{+}=0, \\
z_{4 m, \pi}^{-}=0, \\
\delta_{4 m}=3\end{array}$ & AXI/MTCI \\
\hline 1.208 & UAs & 6 & $128.410\left(P_{I} 4 / m n c\right)$ & $c 4=3$ & $83.43(P 4 / m)$ & $\begin{array}{l}z_{4 m, \pi}^{+}=0, \\
z_{4 m, \pi}^{-}=0, \\
\delta_{4 m}=3\end{array}$ & AXI/MTCI \\
\hline 0.51 & Ho2Ru2O7 & 0 & $141.557\left(I 4_{1} / a m^{\prime} d^{\prime}\right)$ & $c 2,=1, c 2_{2}=1$ & $88.81\left(I 4_{1} / a\right)$ & $\begin{array}{l}\eta_{2 I}^{\prime}=1, \\
z_{2}=0\end{array}$ & $3 \mathrm{D}$ QAHI \\
\hline 0.117 & LuFeO3 & 0 & $185.201\left(P 6_{3} c^{\prime} m^{\prime}\right)$ & $c 3=1$ & $143.1(P 3)$ & $z_{3 R}=2$ & 3D QAHI \\
\hline
\end{tabular}

* Compatible with SISM

\section{Appendix I: Compatibility-relations along high-symmetry paths of the symmetry enforced semimetals}

In TABLEXI we check all the compatibility relations between maximal $k$ vectors for the magnetic ESs in TABLE[VII VIII Once band structures break the compatibility relations between two maximal $k$ vectors, band crossings occur and form nodal points/nodal-lines.

TABLE XI: Compatibility-relations of the magnetic ESs. For each material, the first line lists BCSID, chemical formula, MSG and the value of $\mathrm{U}$ in $\mathrm{LDA}+\mathrm{U}$ calculations. The 1st-3rd coloumns are two maximal $k$ vectors $\left(k_{1}\right.$ and $\left.k_{2}\right)$ and the intermediate path between them. The 4th coloumn identifies whether compatibility-relations between $k_{1}$ and $k_{2}$ are satisfied. If the answer is 'no', there have symmetry enforced band crossings between $k_{1}$ and $k_{2}$. The 5 th coloumn gives the location of band crossings. 'Line' stands for the band crossings are protected by (screw-)rotational symmetries and there are isolated degenerate points on the line. 'Plane' stands for the band crossings are protected by (glide-)mirror symmetries and the crossing points form nodal-lines on the plane. The coordinates of $k$ vectors are written in the reciprocal conventional lattice, which are provided in the CoRepresentation subsection of BCS website.

\begin{tabular}{|c|c|c|c|c|}
\hline \multicolumn{5}{|c|}{ BCSID: 0.165; Formula: SrMn(VO4)(OH); MSG: $4.7\left(P 2_{1}\right)$; U=0 } \\
\hline maximal $k_{1}$ & intermediate path & maximal $k_{2}$ & satisfied? & Line/Plane \\
\hline$\Gamma:(0,0,0)$ & LD: $(0, \mathrm{v}, 0)$ & $\mathrm{Z}:(0,1 / 2,0)$ & no & Line \\
\hline $\mathrm{A}:(1 / 2,0,1 / 2)$ & $\mathrm{U}:(1 / 2, \mathrm{v}, 1 / 2)$ & $\mathrm{E}:(1 / 2,1 / 2,1 / 2)$ & yes & Line \\
\hline $\mathrm{B}:(0,0,1 / 2)$ & $\mathrm{V}:(0, \mathrm{v}, 1 / 2)$ & $\mathrm{D}:(0,1 / 2,1 / 2)$ & yes & Line \\
\hline $\mathrm{C}:(1 / 2,1 / 2,0)$ & $\mathrm{W}:(1 / 2, \mathrm{v}, 0)$ & $\mathrm{Y}:(1 / 2,0,0)$ & yes & Line \\
\hline \multicolumn{5}{|c|}{ BCSID: 1.22 ; Formula: DyCu2Si2; MSG: $12.63\left(C_{c} 2 / m\right)$; U=6eV } \\
\hline maximal $k_{1}$ & intermediate path & maximal $k_{2}$ & satisfied? & Line/Plane \\
\hline $\mathrm{A}:(0,0,1 / 2)$ & $\mathrm{U}:(0, \mathrm{v}, 1 / 2)$ & $\mathrm{M}:(0,1,1 / 2)$ & no & Line \\
\hline \multicolumn{5}{|c|}{ BCSID: 0.105; Formula: ErVO3; MSG: $14.75\left(P 2_{1} / c\right) ; \mathrm{U}=0$} \\
\hline maximal $k_{1}$ & intermediate path & maximal $k_{2}$ & satisfied? & Line/Plane \\
\hline$\Gamma:(0,0,0)$ & $\mathrm{LD}:(0, \mathrm{v}, 0)$ & $\mathrm{Z}:(0,1 / 2,0)$ & yes & Line \\
\hline $\mathrm{A}:(1 / 2,0,1 / 2)$ & $\mathrm{U}:(1 / 2, \mathrm{v}, 1 / 2)$ & $\mathrm{E}:(1 / 2,1 / 2,1 / 2)$ & yes & Line \\
\hline $\mathrm{B}:(0,0,1 / 2)$ & $\mathrm{V}:(0, \mathrm{v}, 1 / 2)$ & $\mathrm{D}:(0,1 / 2,1 / 2)$ & yes & Line \\
\hline $\mathrm{C}:(1 / 2,1 / 2,0)$ & $\mathrm{W}:(1 / 2, \mathrm{v}, 0)$ & $\mathrm{Y}:(1 / 2,0,0)$ & no & Line \\
\hline$\Gamma:(0,0,0)$ & $\mathrm{F}:(\mathrm{u}, 0, \mathrm{w})$ & $\mathrm{A}:(1 / 2,0,1 / 2)$ & yes & Plane \\
\hline$\Gamma:(0,0,0)$ & $\mathrm{F}:(\mathrm{u}, 0, \mathrm{w})$ & $\mathrm{B}:(0,0,1 / 2)$ & yes & Plane \\
\hline
\end{tabular}




\begin{tabular}{c|c|c|c|c}
\hline$\Gamma:(0,0,0)$ & $\mathrm{F}:(\mathrm{u}, 0, \mathrm{w})$ & $\mathrm{Y}:(1 / 2,0,0)$ & no & Plane \\
\hline $\mathrm{A}:(1 / 2,0,1 / 2)$ & $\mathrm{F}:(\mathrm{u}, 0, \mathrm{w})$ & $\mathrm{B}:(0,0,1 / 2)$ & yes & Plane \\
\hline $\mathrm{A}:(1 / 2,0,1 / 2)$ & $\mathrm{F}:(\mathrm{u}, 0, \mathrm{w})$ & $\mathrm{Y}:(1 / 2,0,0)$ & no & Plane \\
\hline $\mathrm{B}:(0,0,1 / 2)$ & $\mathrm{F}:(\mathrm{u}, 0, \mathrm{w})$ & $\mathrm{Y}:(1 / 2,0,0)$ & no & Plane \\
\hline $\mathrm{C}:(1 / 2,1 / 2,0)$ & $\mathrm{G}:(\mathrm{u}, 1 / 2, \mathrm{w})$ & $\mathrm{D}:(0,1 / 2,1 / 2)$ & yes & Plane \\
\hline $\mathrm{C}:(1 / 2,1 / 2,0)$ & $\mathrm{G}:(\mathrm{u}, 1 / 2, \mathrm{w})$ & $\mathrm{E}:(1 / 2,1 / 2,1 / 2)$ & yes & Plane \\
\hline $\mathrm{C}:(1 / 2,1 / 2,0)$ & $\mathrm{G}:(\mathrm{u}, 1 / 2, \mathrm{w})$ & $\mathrm{Z}:(0,1 / 2,0)$ & yes & Plane \\
\hline $\mathrm{D}:(0,1 / 2,1 / 2)$ & $\mathrm{G}:(\mathrm{u}, 1 / 2, \mathrm{w})$ & $\mathrm{E}:(1 / 2,1 / 2,1 / 2)$ & yes & Plane \\
\hline $\mathrm{D}:(0,1 / 2,1 / 2)$ & $\mathrm{G}:(\mathrm{u}, 1 / 2, \mathrm{w})$ & $\mathrm{Z}:(0,1 / 2,0)$ & yes & Plane \\
\hline $\mathrm{E}:(1 / 2,1 / 2,1 / 2)$ & $\mathrm{G}:(\mathrm{u}, 1 / 2, \mathrm{w})$ & $\mathrm{Z}:(0,1 / 2,0)$ & yes & Plane \\
\hline
\end{tabular}

BCSID: 1.263; Formula: Ca3Ru2O7; MSG: $33.154\left(P_{C} n a 2_{1}\right) ; \mathrm{U}=0$

\begin{tabular}{|c|c|c|c|c|}
\hline maximal $k_{1}$ & intermediate path & maximal $k_{2}$ & satisfied? & Line/Plane \\
\hline$\Gamma:(0,0,0)$ & SM:(u,0,0) & $\mathrm{X}:(1 / 2,0,0)$ & yes & Line \\
\hline$\Gamma:(0,0,0)$ & DT: $(0, \mathrm{v}, 0)$ & $\mathrm{Y}:(0,1 / 2,0)$ & yes & Line \\
\hline $\mathrm{R}:(1 / 2,1 / 2,1 / 2)$ & $\mathrm{E}:(\mathrm{u}, 1 / 2,1 / 2)$ & $\mathrm{T}:(0,1 / 2,1 / 2)$ & yes & Line \\
\hline $\mathrm{S}:(1 / 2,1 / 2,0)$ & $\mathrm{D}:(1 / 2, \mathrm{v}, 0)$ & $\mathrm{X}:(1 / 2,0,0)$ & yes & Line \\
\hline $\mathrm{S}:(1 / 2,1 / 2,0)$ & $\mathrm{C}:(\mathrm{u}, 1 / 2,0)$ & $\mathrm{Y}:(0,1 / 2,0)$ & yes & Line \\
\hline $\mathrm{T}:(0,1 / 2,1 / 2)$ & $\mathrm{H}:(0,1 / 2, \mathrm{w})$ & $\mathrm{Y}:(0,1 / 2,0)$ & yes & Line \\
\hline $\mathrm{U}:(1 / 2,0,1 / 2)$ & $\mathrm{G}:(1 / 2,0, \mathrm{w})$ & $\mathrm{X}:(1 / 2,0,0)$ & no & Line \\
\hline $\mathrm{U}:(1 / 2,0,1 / 2)$ & $\mathrm{A}:(\mathrm{u}, 0,1 / 2)$ & $\mathrm{Z}:(0,0,1 / 2)$ & yes & Line \\
\hline$\Gamma:(0,0,0)$ & $\mathrm{K}:(0, \mathrm{v}, \mathrm{w})$ & $\mathrm{T}:(0,1 / 2,1 / 2)$ & yes & Plane \\
\hline$\Gamma:(0,0,0)$ & $\mathrm{M}:(\mathrm{u}, 0, \mathrm{w})$ & $\mathrm{U}:(1 / 2,0,1 / 2)$ & yes & Plane \\
\hline$\Gamma:(0,0,0)$ & $\mathrm{M}:(\mathrm{u}, 0, \mathrm{w})$ & $\mathrm{X}:(1 / 2,0,0)$ & yes & Plane \\
\hline$\Gamma:(0,0,0)$ & $\mathrm{K}:(0, \mathrm{v}, \mathrm{w})$ & $\mathrm{Y}:(0,1 / 2,0)$ & yes & Plane \\
\hline$\Gamma:(0,0,0)$ & $\mathrm{K}:(0, \mathrm{v}, \mathrm{w})$ & $\mathrm{Z}:(0,0,1 / 2)$ & yes & Plane \\
\hline$\Gamma:(0,0,0)$ & $\mathrm{M}:(\mathrm{u}, 0, \mathrm{w})$ & $\mathrm{Z}:(0,0,1 / 2)$ & yes & Plane \\
\hline $\mathrm{R}:(1 / 2,1 / 2,1 / 2)$ & $\mathrm{L}:(1 / 2, \mathrm{v}, \mathrm{w})$ & $\mathrm{S}:(1 / 2,1 / 2,0)$ & yes & Plane \\
\hline $\mathrm{R}:(1 / 2,1 / 2,1 / 2)$ & $\mathrm{N}:(\mathrm{u}, 1 / 2, \mathrm{w})$ & $\mathrm{S}:(1 / 2,1 / 2,0)$ & yes & Plane \\
\hline $\mathrm{R}:(1 / 2,1 / 2,1 / 2)$ & $\mathrm{N}:(\mathrm{u}, 1 / 2, \mathrm{w})$ & $\mathrm{T}:(0,1 / 2,1 / 2)$ & yes & Plane \\
\hline $\mathrm{R}:(1 / 2,1 / 2,1 / 2)$ & $\mathrm{L}:(1 / 2, \mathrm{v}, \mathrm{w})$ & $\mathrm{U}:(1 / 2,0,1 / 2)$ & yes & Plane \\
\hline $\mathrm{R}:(1 / 2,1 / 2,1 / 2)$ & $\mathrm{L}:(1 / 2, \mathrm{v}, \mathrm{w})$ & $\mathrm{X}:(1 / 2,0,0)$ & yes & Plane \\
\hline $\mathrm{R}:(1 / 2,1 / 2,1 / 2)$ & $\mathrm{N}:(\mathrm{u}, 1 / 2, \mathrm{w})$ & $\mathrm{Y}:(0,1 / 2,0)$ & yes & Plane \\
\hline $\mathrm{S}:(1 / 2,1 / 2,0)$ & $\mathrm{N}:(\mathrm{u}, 1 / 2, \mathrm{w})$ & $\mathrm{T}:(0,1 / 2,1 / 2)$ & yes & Plane \\
\hline $\mathrm{S}:(1 / 2,1 / 2,0)$ & $\mathrm{L}:(1 / 2, \mathrm{v}, \mathrm{w})$ & $\mathrm{U}:(1 / 2,0,1 / 2)$ & yes & Plane \\
\hline $\mathrm{S}:(1 / 2,1 / 2,0)$ & $\mathrm{L}:(1 / 2, \mathrm{v}, \mathrm{w})$ & $\mathrm{X}:(1 / 2,0,0)$ & yes & Plane \\
\hline $\mathrm{S}:(1 / 2,1 / 2,0)$ & $\mathrm{N}:(\mathrm{u}, 1 / 2, \mathrm{w})$ & $\mathrm{Y}:(0,1 / 2,0)$ & yes & Plane \\
\hline $\mathrm{T}:(0,1 / 2,1 / 2)$ & $\mathrm{K}:(0, \mathrm{v}, \mathrm{w})$ & $\mathrm{Y}:(0,1 / 2,0)$ & yes & Plane \\
\hline $\mathrm{T}:(0,1 / 2,1 / 2)$ & $\mathrm{N}:(\mathrm{u}, 1 / 2, \mathrm{w})$ & $\mathrm{Y}:(0,1 / 2,0)$ & yes & Plane \\
\hline $\mathrm{T}:(0,1 / 2,1 / 2)$ & $\mathrm{K}:(0, \mathrm{v}, \mathrm{w})$ & $\mathrm{Z}:(0,0,1 / 2)$ & yes & Plane \\
\hline $\mathrm{U}:(1 / 2,0,1 / 2)$ & $\mathrm{L}:(1 / 2, \mathrm{v}, \mathrm{w})$ & $\mathrm{X}:(1 / 2,0,0)$ & yes & Plane \\
\hline $\mathrm{U}:(1 / 2,0,1 / 2)$ & $\mathrm{M}:(\mathrm{u}, 0, \mathrm{w})$ & $\mathrm{X}:(1 / 2,0,0)$ & yes & Plane \\
\hline $\mathrm{U}:(1 / 2,0,1 / 2)$ & $\mathrm{M}:(\mathrm{u}, 0, \mathrm{w})$ & $\mathrm{Z}:(0,0,1 / 2)$ & yes & Plane \\
\hline $\mathrm{X}:(1 / 2,0,0)$ & $\mathrm{M}:(\mathrm{u}, 0, \mathrm{w})$ & $\mathrm{Z}:(0,0,1 / 2)$ & yes & Plane \\
\hline $\mathrm{Y}:(0,1 / 2,0)$ & $\mathrm{K}:(0, \mathrm{v}, \mathrm{w})$ & $\mathrm{Z}:(0,0,1 / 2)$ & yes & Plane \\
\hline
\end{tabular}

BCSID: 1.263; Formula: Ca3Ru2O7; MSG: $33.154\left(P_{C} n a 2_{1}\right) ; \mathrm{U}=1 \mathrm{eV}$

\begin{tabular}{c|c|c|c|c}
\hline maximal $k_{1}$ & intermediate path & maximal $k_{2}$ & satisfied? & Line/Plane \\
\hline$\Gamma:(0,0,0)$ & $\mathrm{SM}:(\mathrm{u}, 0,0)$ & $\mathrm{X}:(1 / 2,0,0)$ & yes & Line \\
\hline$\Gamma:(0,0,0)$ & $\mathrm{DT}:(0, \mathrm{v}, 0)$ & $\mathrm{Y}:(0,1 / 2,0)$ & yes & Line \\
\hline $\mathrm{R}:(1 / 2,1 / 2,1 / 2)$ & $\mathrm{E}:(\mathrm{u}, 1 / 2,1 / 2)$ & $\mathrm{T}:(0,1 / 2,1 / 2)$ & yes & Line
\end{tabular}




\begin{tabular}{|c|c|c|c|c|}
\hline $\mathrm{S}:(1 / 2,1 / 2,0)$ & $\mathrm{D}:(1 / 2, \mathrm{v}, 0)$ & $\mathrm{X}:(1 / 2,0,0)$ & yes & Line \\
\hline $\mathrm{S}:(1 / 2,1 / 2,0)$ & $\mathrm{C}:(\mathrm{u}, 1 / 2,0)$ & $\mathrm{Y}:(0,1 / 2,0)$ & yes & Line \\
\hline $\mathrm{T}:(0,1 / 2,1 / 2)$ & $\mathrm{H}:(0,1 / 2, \mathrm{w})$ & $\mathrm{Y}:(0,1 / 2,0)$ & yes & Line \\
\hline $\mathrm{U}:(1 / 2,0,1 / 2)$ & $\mathrm{G}:(1 / 2,0, \mathrm{w})$ & $\mathrm{X}:(1 / 2,0,0)$ & no & Line \\
\hline $\mathrm{U}:(1 / 2,0,1 / 2)$ & $\mathrm{A}:(\mathrm{u}, 0,1 / 2)$ & $\mathrm{Z}:(0,0,1 / 2)$ & yes & Line \\
\hline$\Gamma:(0,0,0)$ & $\mathrm{K}:(0, \mathrm{v}, \mathrm{w})$ & $\mathrm{T}:(0,1 / 2,1 / 2)$ & yes & Plane \\
\hline$\Gamma:(0,0,0)$ & $\mathrm{M}:(\mathrm{u}, 0, \mathrm{w})$ & $\mathrm{U}:(1 / 2,0,1 / 2)$ & yes & Plane \\
\hline$\Gamma:(0,0,0)$ & $\mathrm{M}:(\mathrm{u}, 0, \mathrm{w})$ & $\mathrm{X}:(1 / 2,0,0)$ & yes & Plane \\
\hline$\Gamma:(0,0,0)$ & $\mathrm{K}:(0, \mathrm{v}, \mathrm{w})$ & $\mathrm{Y}:(0,1 / 2,0)$ & yes & Plane \\
\hline$\Gamma:(0,0,0)$ & $\mathrm{K}:(0, \mathrm{v}, \mathrm{w})$ & $\mathrm{Z}:(0,0,1 / 2)$ & yes & Plane \\
\hline$\Gamma:(0,0,0)$ & $\mathrm{M}:(\mathrm{u}, 0, \mathrm{w})$ & $\mathrm{Z}:(0,0,1 / 2)$ & yes & Plane \\
\hline $\mathrm{R}:(1 / 2,1 / 2,1 / 2)$ & $\mathrm{L}:(1 / 2, \mathrm{v}, \mathrm{w})$ & $\mathrm{S}:(1 / 2,1 / 2,0)$ & yes & Plane \\
\hline $\mathrm{R}:(1 / 2,1 / 2,1 / 2)$ & $\mathrm{N}:(\mathrm{u}, 1 / 2, \mathrm{w})$ & $\mathrm{S}:(1 / 2,1 / 2,0)$ & yes & Plane \\
\hline $\mathrm{R}:(1 / 2,1 / 2,1 / 2)$ & $\mathrm{N}:(\mathrm{u}, 1 / 2, \mathrm{w})$ & $\mathrm{T}:(0,1 / 2,1 / 2)$ & yes & Plane \\
\hline $\mathrm{R}:(1 / 2,1 / 2,1 / 2)$ & $\mathrm{L}:(1 / 2, \mathrm{v}, \mathrm{w})$ & $\mathrm{U}:(1 / 2,0,1 / 2)$ & yes & Plane \\
\hline $\mathrm{R}:(1 / 2,1 / 2,1 / 2)$ & $\mathrm{L}:(1 / 2, \mathrm{v}, \mathrm{w})$ & $\mathrm{X}:(1 / 2,0,0)$ & yes & Plane \\
\hline $\mathrm{R}:(1 / 2,1 / 2,1 / 2)$ & $\mathrm{N}:(\mathrm{u}, 1 / 2, \mathrm{w})$ & $\mathrm{Y}:(0,1 / 2,0)$ & yes & Plane \\
\hline $\mathrm{S}:(1 / 2,1 / 2,0)$ & $\mathrm{N}:(\mathrm{u}, 1 / 2, \mathrm{w})$ & $\mathrm{T}:(0,1 / 2,1 / 2)$ & yes & Plane \\
\hline $\mathrm{S}:(1 / 2,1 / 2,0)$ & $\mathrm{L}:(1 / 2, \mathrm{v}, \mathrm{w})$ & $\mathrm{U}:(1 / 2,0,1 / 2)$ & yes & Plane \\
\hline $\mathrm{S}:(1 / 2,1 / 2,0)$ & $\mathrm{L}:(1 / 2, \mathrm{v}, \mathrm{w})$ & $\mathrm{X}:(1 / 2,0,0)$ & yes & Plane \\
\hline $\mathrm{S}:(1 / 2,1 / 2,0)$ & $\mathrm{N}:(\mathrm{u}, 1 / 2, \mathrm{w})$ & $\mathrm{Y}:(0,1 / 2,0)$ & yes & Plane \\
\hline $\mathrm{T}:(0,1 / 2,1 / 2)$ & $\mathrm{K}:(0, \mathrm{v}, \mathrm{w})$ & $\mathrm{Y}:(0,1 / 2,0)$ & yes & Plane \\
\hline $\mathrm{T}:(0,1 / 2,1 / 2)$ & $\mathrm{N}:(\mathrm{u}, 1 / 2, \mathrm{w})$ & $\mathrm{Y}:(0,1 / 2,0)$ & yes & Plane \\
\hline $\mathrm{T}:(0,1 / 2,1 / 2)$ & $\mathrm{K}:(0, \mathrm{v}, \mathrm{w})$ & $\mathrm{Z}:(0,0,1 / 2)$ & yes & Plane \\
\hline $\mathrm{U}:(1 / 2,0,1 / 2)$ & $\mathrm{L}:(1 / 2, \mathrm{v}, \mathrm{w})$ & $\mathrm{X}:(1 / 2,0,0)$ & yes & Plane \\
\hline $\mathrm{U}:(1 / 2,0,1 / 2)$ & $\mathrm{M}:(\mathrm{u}, 0, \mathrm{w})$ & $\mathrm{X}:(1 / 2,0,0)$ & yes & Plane \\
\hline $\mathrm{U}:(1 / 2,0,1 / 2)$ & $\mathrm{M}:(\mathrm{u}, 0, \mathrm{w})$ & $\mathrm{Z}:(0,0,1 / 2)$ & yes & Plane \\
\hline $\mathrm{X}:(1 / 2,0,0)$ & $\mathrm{M}:(\mathrm{u}, 0, \mathrm{w})$ & $\mathrm{Z}:(0,0,1 / 2)$ & yes & Plane \\
\hline $\mathrm{Y}:(0,1 / 2,0)$ & $\mathrm{K}:(0, \mathrm{v}, \mathrm{w})$ & $\mathrm{Z}:(0,0,1 / 2)$ & yes & Plane \\
\hline \multicolumn{5}{|c|}{ BCSID: 1.43 ; Formula: PrNiO3; MSG: $36.178\left(C_{a} m c 2_{1}\right) ; \mathrm{U}=4 \mathrm{eV}$} \\
\hline maximal $k_{1}$ & intermediate path & maximal $k_{2}$ & satisfied? & Line/Plane \\
\hline$\Gamma:(0,0,0)$ & DT: $(0, \mathrm{v}, 0)$ & $\mathrm{Y}:(0,1,0)$ & yes & Line \\
\hline$\Gamma:(0,0,0)$ & SM:(u,0,0) & $\mathrm{Y}:(1,0,0)$ & yes & Line \\
\hline $\mathrm{R}:(1 / 2,1 / 2,1 / 2)$ & $\mathrm{D}:(1 / 2,1 / 2, \mathrm{w})$ & $\mathrm{S}:(1 / 2,1 / 2,0)$ & no & Line \\
\hline $\mathrm{T}:(0,1,1 / 2)$ & $\mathrm{B}:(0, \mathrm{v}, 1 / 2)$ & $\mathrm{Z}:(0,0,1 / 2)$ & yes & Line \\
\hline$\Gamma:(0,0,0)$ & $\mathrm{K}:(0, \mathrm{v}, \mathrm{w})$ & $\mathrm{T}:(0,1,1 / 2)$ & yes & Plane \\
\hline$\Gamma:(0,0,0)$ & $\mathrm{M}:(\mathrm{u}, 0, \mathrm{w})$ & $\mathrm{T}:(1,0,1 / 2)$ & yes & Plane \\
\hline$\Gamma:(0,0,0)$ & $\mathrm{K}:(0, \mathrm{v}, \mathrm{w})$ & $\mathrm{Y}:(0,1,0)$ & yes & Plane \\
\hline$\Gamma:(0,0,0)$ & $\mathrm{M}:(\mathrm{u}, 0, \mathrm{w})$ & $\mathrm{Y}:(1,0,0)$ & yes & Plane \\
\hline$\Gamma:(0,0,0)$ & $\mathrm{K}:(0, \mathrm{v}, \mathrm{w})$ & $\mathrm{Z}:(0,0,1 / 2)$ & yes & Plane \\
\hline$\Gamma:(0,0,0)$ & $\mathrm{M}:(\mathrm{u}, 0, \mathrm{w})$ & $\mathrm{Z}:(0,0,1 / 2)$ & yes & Plane \\
\hline $\mathrm{T}:(0,1,1 / 2)$ & $\mathrm{K}:(0, \mathrm{v}, \mathrm{w})$ & $\mathrm{Y}:(0,1,0)$ & yes & Plane \\
\hline $\mathrm{T}:(1,0,1 / 2)$ & $\mathrm{M}:(\mathrm{u}, 0, \mathrm{w})$ & $\mathrm{Y}:(1,0,0)$ & yes & Plane \\
\hline $\mathrm{T}:(0,1,1 / 2)$ & $\mathrm{K}:(0, \mathrm{v}, \mathrm{w})$ & $\mathrm{Z}:(0,0,1 / 2)$ & yes & Plane \\
\hline $\mathrm{T}:(1,0,1 / 2)$ & $\mathrm{M}:(\mathrm{u}, 0, \mathrm{w})$ & $\mathrm{Z}:(0,0,1 / 2)$ & yes & Plane \\
\hline $\mathrm{Y}:(0,1,0)$ & $\mathrm{K}:(0, \mathrm{v}, \mathrm{w})$ & $\mathrm{Z}:(0,0,1 / 2)$ & yes & Plane \\
\hline $\mathrm{Y}:(1,0,0)$ & $\mathrm{M}:(\mathrm{u}, 0, \mathrm{w})$ & $\mathrm{Z}:(0,0,1 / 2)$ & yes & Plane \\
\hline
\end{tabular}

BCSID: 0.26; Formula: TmAgGe; MSG: $38.191\left(A m^{\prime} m^{\prime} 2\right)$; U=0 \begin{tabular}{l|l|l|l|l} 
maximal $k_{1}$ & intermediate path & maximal $k_{2}$ & satisfied? & Line/Plane
\end{tabular} 


\begin{tabular}{|c|c|c|c|c|}
\hline$\Gamma:(0,0,0)$ & SM: $(0,0, w)$ & $Y:(0,0,1)$ & no & Line \\
\hline $\mathrm{T}:(1 / 2,0,-1)$ & $\mathrm{A}:(1 / 2,0, \mathrm{w})$ & $\mathrm{Z}:(1 / 2,0,0)$ & yes & Line \\
\hline \multicolumn{5}{|c|}{ BCSID: 2.12; Formula: TbMg; MSG: $49.270\left(P c^{\prime} \mathrm{cm}^{\prime}\right) ; \mathrm{U}=0$} \\
\hline maximal $k_{1}$ & intermediate path & maximal $k_{2}$ & satisfied? & Line/Plane \\
\hline$\Gamma:(0,0,0)$ & SM:(u,0,0) & $\mathrm{X}:(1 / 2,0,0)$ & no & Line \\
\hline$\Gamma:(0,0,0)$ & DT: $(0, \mathrm{v}, 0)$ & $\mathrm{Y}:(0,1 / 2,0)$ & yes & Line \\
\hline$\Gamma:(0,0,0)$ & $\mathrm{LD}:(0,0, \mathrm{w})$ & $\mathrm{Z}:(0,0,1 / 2)$ & no & Line \\
\hline $\mathrm{R}:(1 / 2,1 / 2,1 / 2)$ & $\mathrm{Q}:(1 / 2,1 / 2, \mathrm{w})$ & $\mathrm{S}:(1 / 2,1 / 2,0)$ & yes & Line \\
\hline $\mathrm{R}:(1 / 2,1 / 2,1 / 2)$ & $\mathrm{P}:(1 / 2, \mathrm{v}, 1 / 2)$ & $\mathrm{U}:(1 / 2,0,1 / 2)$ & yes & Line \\
\hline $\mathrm{S}:(1 / 2,1 / 2,0)$ & $\mathrm{D}:(1 / 2, \mathrm{v}, 0)$ & $\mathrm{X}:(1 / 2,0,0)$ & yes & Line \\
\hline $\mathrm{S}:(1 / 2,1 / 2,0)$ & $\mathrm{C}:(\mathrm{u}, 1 / 2,0)$ & $\mathrm{Y}:(0,1 / 2,0)$ & yes & Line \\
\hline $\mathrm{T}:(0,1 / 2,1 / 2)$ & $\mathrm{H}:(0,1 / 2, \mathrm{w})$ & $\mathrm{Y}:(0,1 / 2,0)$ & yes & Line \\
\hline $\mathrm{T}:(0,1 / 2,1 / 2)$ & $\mathrm{B}:(0, \mathrm{v}, 1 / 2)$ & $\mathrm{Z}:(0,0,1 / 2)$ & yes & Line \\
\hline $\mathrm{U}:(1 / 2,0,1 / 2)$ & $\mathrm{G}:(1 / 2,0, \mathrm{w})$ & $\mathrm{X}:(1 / 2,0,0)$ & yes & Line \\
\hline$\Gamma:(0,0,0)$ & $\mathrm{M}:(\mathrm{u}, 0, \mathrm{w})$ & $\mathrm{U}:(1 / 2,0,1 / 2)$ & no & Plane \\
\hline$\Gamma:(0,0,0)$ & $\mathrm{M}:(\mathrm{u}, 0, \mathrm{w})$ & $\mathrm{X}:(1 / 2,0,0)$ & no & Plane \\
\hline$\Gamma:(0,0,0)$ & $\mathrm{M}:(\mathrm{u}, 0, \mathrm{w})$ & $\mathrm{Z}:(0,0,1 / 2)$ & no & Plane \\
\hline $\mathrm{R}:(1 / 2,1 / 2,1 / 2)$ & $\mathrm{N}:(\mathrm{u}, 1 / 2, \mathrm{w})$ & $\mathrm{S}:(1 / 2,1 / 2,0)$ & yes & Plane \\
\hline $\mathrm{R}:(1 / 2,1 / 2,1 / 2)$ & $\mathrm{N}:(\mathrm{u}, 1 / 2, \mathrm{w})$ & $\mathrm{T}:(0,1 / 2,1 / 2)$ & yes & Plane \\
\hline $\mathrm{R}:(1 / 2,1 / 2,1 / 2)$ & $\mathrm{N}:(\mathrm{u}, 1 / 2, \mathrm{w})$ & $\mathrm{Y}:(0,1 / 2,0)$ & yes & Plane \\
\hline $\mathrm{S}:(1 / 2,1 / 2,0)$ & $\mathrm{N}:(\mathrm{u}, 1 / 2, \mathrm{w})$ & $\mathrm{T}:(0,1 / 2,1 / 2)$ & yes & Plane \\
\hline $\mathrm{S}:(1 / 2,1 / 2,0)$ & $\mathrm{N}:(\mathrm{u}, 1 / 2, \mathrm{w})$ & $\mathrm{Y}:(0,1 / 2,0)$ & yes & Plane \\
\hline $\mathrm{T}:(0,1 / 2,1 / 2)$ & $\mathrm{N}:(\mathrm{u}, 1 / 2, \mathrm{w})$ & $\mathrm{Y}:(0,1 / 2,0)$ & yes & Plane \\
\hline $\mathrm{U}:(1 / 2,0,1 / 2)$ & $\mathrm{M}:(\mathrm{u}, 0, \mathrm{w})$ & $\mathrm{X}:(1 / 2,0,0)$ & yes & Plane \\
\hline $\mathrm{U}:(1 / 2,0,1 / 2)$ & $\mathrm{M}:(\mathrm{u}, 0, \mathrm{w})$ & $\mathrm{Z}:(0,0,1 / 2)$ & yes & Plane \\
\hline $\mathrm{X}:(1 / 2,0,0)$ & $\mathrm{M}:(\mathrm{u}, 0, \mathrm{w})$ & $\mathrm{Z}:(0,0,1 / 2)$ & yes & Plane \\
\hline \multicolumn{5}{|c|}{ BCSID: 2.12 ; Formula: TbMg; MSG: $49.270\left(P c^{\prime} \mathrm{cm}^{\prime}\right) ; \mathrm{U}=2 \mathrm{eV}$} \\
\hline maximal $k_{1}$ & intermediate path & maximal $k_{2}$ & \begin{tabular}{|l|} 
satisfied? \\
\end{tabular} & Line/Plane \\
\hline$\Gamma:(0,0,0)$ & SM:(u,0,0) & $\mathrm{X}:(1 / 2,0,0)$ & no & Line \\
\hline$\Gamma:(0,0,0)$ & DT: $(0, \mathrm{v}, 0)$ & $\mathrm{Y}:(0,1 / 2,0)$ & yes & Line \\
\hline$\Gamma:(0,0,0)$ & LD: $(0,0, \mathrm{w})$ & $\mathrm{Z}:(0,0,1 / 2)$ & no & Line \\
\hline $\mathrm{R}:(1 / 2,1 / 2,1 / 2)$ & $\mathrm{Q}:(1 / 2,1 / 2, \mathrm{w})$ & $\mathrm{S}:(1 / 2,1 / 2,0)$ & yes & Line \\
\hline $\mathrm{R}:(1 / 2,1 / 2,1 / 2)$ & $\mathrm{P}:(1 / 2, \mathrm{v}, 1 / 2)$ & $\mathrm{U}:(1 / 2,0,1 / 2)$ & yes & Line \\
\hline $\mathrm{S}:(1 / 2,1 / 2,0)$ & $\mathrm{D}:(1 / 2, \mathrm{v}, 0)$ & $\mathrm{X}:(1 / 2,0,0)$ & yes & Line \\
\hline $\mathrm{S}:(1 / 2,1 / 2,0)$ & $\mathrm{C}:(\mathrm{u}, 1 / 2,0)$ & $\mathrm{Y}:(0,1 / 2,0)$ & yes & Line \\
\hline $\mathrm{T}:(0,1 / 2,1 / 2)$ & $\mathrm{H}:(0,1 / 2, \mathrm{w})$ & $\mathrm{Y}:(0,1 / 2,0)$ & yes & Line \\
\hline $\mathrm{T}:(0,1 / 2,1 / 2)$ & $\mathrm{B}:(0, \mathrm{v}, 1 / 2)$ & $\mathrm{Z}:(0,0,1 / 2)$ & yes & Line \\
\hline $\mathrm{U}:(1 / 2,0,1 / 2)$ & $\mathrm{G}:(1 / 2,0, \mathrm{w})$ & $\mathrm{X}:(1 / 2,0,0)$ & yes & Line \\
\hline$\Gamma:(0,0,0)$ & $\mathrm{M}:(\mathrm{u}, 0, \mathrm{w})$ & $\mathrm{U}:(1 / 2,0,1 / 2)$ & no & Plane \\
\hline$\Gamma:(0,0,0)$ & $\mathrm{M}:(\mathrm{u}, 0, \mathrm{w})$ & $\mathrm{X}:(1 / 2,0,0)$ & no & Plane \\
\hline$\Gamma:(0,0,0)$ & $\mathrm{M}:(\mathrm{u}, 0, \mathrm{w})$ & $\mathrm{Z}:(0,0,1 / 2)$ & no & Plane \\
\hline $\mathrm{R}:(1 / 2,1 / 2,1 / 2)$ & $\mathrm{N}:(\mathrm{u}, 1 / 2, \mathrm{w})$ & $\mathrm{S}:(1 / 2,1 / 2,0)$ & yes & Plane \\
\hline $\mathrm{R}:(1 / 2,1 / 2,1 / 2)$ & $\mathrm{N}:(\mathrm{u}, 1 / 2, \mathrm{w})$ & $\mathrm{T}:(0,1 / 2,1 / 2)$ & yes & Plane \\
\hline $\mathrm{R}:(1 / 2,1 / 2,1 / 2)$ & $\mathrm{N}:(\mathrm{u}, 1 / 2, \mathrm{w})$ & $\mathrm{Y}:(0,1 / 2,0)$ & yes & Plane \\
\hline $\mathrm{S}:(1 / 2,1 / 2,0)$ & $\mathrm{N}:(\mathrm{u}, 1 / 2, \mathrm{w})$ & $\mathrm{T}:(0,1 / 2,1 / 2)$ & yes & Plane \\
\hline $\mathrm{S}:(1 / 2,1 / 2,0)$ & $\mathrm{N}:(\mathrm{u}, 1 / 2, \mathrm{w})$ & $\mathrm{Y}:(0,1 / 2,0)$ & yes & Plane \\
\hline $\mathrm{T}:(0,1 / 2,1 / 2)$ & $\mathrm{N}:(\mathrm{u}, 1 / 2, \mathrm{w})$ & $\mathrm{Y}:(0,1 / 2,0)$ & yes & Plane \\
\hline $\mathrm{U}:(1 / 2,0,1 / 2)$ & $\mathrm{M}:(\mathrm{u}, 0, \mathrm{w})$ & $\mathrm{X}:(1 / 2,0,0)$ & yes & Plane \\
\hline $\mathrm{U}:(1 / 2,0,1 / 2)$ & $\mathrm{M}:(\mathrm{u}, 0, \mathrm{w})$ & $\mathrm{Z}:(0,0,1 / 2)$ & yes & Plane \\
\hline
\end{tabular}




\begin{tabular}{|c|c|c|c|c|}
\hline $\mathrm{X}:(1 / 2,0,0)$ & $\mathrm{M}:(\mathrm{u}, 0, \mathrm{w})$ & $\mathrm{Z}:(0,0,1 / 2)$ & yes & Plane \\
\hline \multicolumn{5}{|c|}{ BCSID: 2.12; Formula: TbMg; MSG: $49.270\left(P c^{\prime} \mathrm{cm}^{\prime}\right) ; \mathrm{U}=4 \mathrm{eV}$} \\
\hline maximal $k_{1}$ & intermediate path & maximal $k_{2}$ & satisfied? & Line/Plane \\
\hline$\Gamma:(0,0,0)$ & SM:(u,0,0) & $\mathrm{X}:(1 / 2,0,0)$ & yes & Line \\
\hline$\Gamma:(0,0,0)$ & DT: $(0, \mathrm{v}, 0)$ & $\mathrm{Y}:(0,1 / 2,0)$ & yes & Line \\
\hline$\Gamma:(0,0,0)$ & LD: $(0,0, w)$ & $\mathrm{Z}:(0,0,1 / 2)$ & yes & Line \\
\hline $\mathrm{R}:(1 / 2,1 / 2,1 / 2)$ & $\mathrm{Q}:(1 / 2,1 / 2, \mathrm{w})$ & $\mathrm{S}:(1 / 2,1 / 2,0)$ & no & Line \\
\hline $\mathrm{R}:(1 / 2,1 / 2,1 / 2)$ & $\mathrm{P}:(1 / 2, \mathrm{v}, 1 / 2)$ & $\mathrm{U}:(1 / 2,0,1 / 2)$ & yes & Line \\
\hline $\mathrm{S}:(1 / 2,1 / 2,0)$ & $\mathrm{D}:(1 / 2, \mathrm{v}, 0)$ & $\mathrm{X}:(1 / 2,0,0)$ & yes & Line \\
\hline $\mathrm{S}:(1 / 2,1 / 2,0)$ & $\mathrm{C}:(\mathrm{u}, 1 / 2,0)$ & $\mathrm{Y}:(0,1 / 2,0)$ & no & Line \\
\hline $\mathrm{T}:(0,1 / 2,1 / 2)$ & $\mathrm{H}:(0,1 / 2, \mathrm{w})$ & $\mathrm{Y}:(0,1 / 2,0)$ & yes & Line \\
\hline $\mathrm{T}:(0,1 / 2,1 / 2)$ & $\mathrm{B}:(0, \mathrm{v}, 1 / 2)$ & $\mathrm{Z}:(0,0,1 / 2)$ & yes & Line \\
\hline $\mathrm{U}:(1 / 2,0,1 / 2)$ & G: $(1 / 2,0, w)$ & $\mathrm{X}:(1 / 2,0,0)$ & yes & Line \\
\hline$\Gamma:(0,0,0)$ & $\mathrm{M}:(\mathrm{u}, 0, \mathrm{w})$ & $\mathrm{U}:(1 / 2,0,1 / 2)$ & yes & Plane \\
\hline$\Gamma:(0,0,0)$ & $\mathrm{M}:(\mathrm{u}, 0, \mathrm{w})$ & $\mathrm{X}:(1 / 2,0,0)$ & yes & Plane \\
\hline$\Gamma:(0,0,0)$ & $\mathrm{M}:(\mathrm{u}, 0, \mathrm{w})$ & $\mathrm{Z}:(0,0,1 / 2)$ & yes & Plane \\
\hline $\mathrm{R}:(1 / 2,1 / 2,1 / 2)$ & $\mathrm{N}:(\mathrm{u}, 1 / 2, \mathrm{w})$ & $\mathrm{S}:(1 / 2,1 / 2,0)$ & no & Plane \\
\hline $\mathrm{R}:(1 / 2,1 / 2,1 / 2)$ & $\mathrm{N}:(\mathrm{u}, 1 / 2, \mathrm{w})$ & $\mathrm{T}:(0,1 / 2,1 / 2)$ & yes & Plane \\
\hline $\mathrm{R}:(1 / 2,1 / 2,1 / 2)$ & $\mathrm{N}:(\mathrm{u}, 1 / 2, \mathrm{w})$ & $Y:(0,1 / 2,0)$ & yes & Plane \\
\hline $\mathrm{S}:(1 / 2,1 / 2,0)$ & $\mathrm{N}:(\mathrm{u}, 1 / 2, \mathrm{w})$ & $\mathrm{T}:(0,1 / 2,1 / 2)$ & no & Plane \\
\hline $\mathrm{S}:(1 / 2,1 / 2,0)$ & $\mathrm{N}:(\mathrm{u}, 1 / 2, \mathrm{w})$ & $\mathrm{Y}:(0,1 / 2,0)$ & no & Plane \\
\hline $\mathrm{T}:(0,1 / 2,1 / 2)$ & $\mathrm{N}:(\mathrm{u}, 1 / 2, \mathrm{w})$ & $\mathrm{Y}:(0,1 / 2,0)$ & yes & Plane \\
\hline $\mathrm{U}:(1 / 2,0,1 / 2)$ & $\mathrm{M}:(\mathrm{u}, 0, \mathrm{w})$ & $\mathrm{X}:(1 / 2,0,0)$ & yes & Plane \\
\hline $\mathrm{U}:(1 / 2,0,1 / 2)$ & $\mathrm{M}:(\mathrm{u}, 0, \mathrm{w})$ & $\mathrm{Z}:(0,0,1 / 2)$ & yes & Plane \\
\hline $\mathrm{X}:(1 / 2,0,0)$ & $\mathrm{M}:(\mathrm{u}, 0, \mathrm{w})$ & $\mathrm{Z}:(0,0,1 / 2)$ & yes & Plane \\
\hline \multicolumn{5}{|c|}{ BCSID: 2.12; Formula: TbMg; MSG: $49.270\left(P c^{\prime} \mathrm{cm}^{\prime}\right) ; \mathrm{U}=6 \mathrm{eV}$} \\
\hline maximal $k_{1}$ & intermediate path & maximal $k_{2}$ & satisfied? & Line/Plane \\
\hline$\Gamma:(0,0,0)$ & SM:(u,0,0) & $\mathrm{X}:(1 / 2,0,0)$ & no & Line \\
\hline$\Gamma:(0,0,0)$ & DT: $(0, \mathrm{v}, 0)$ & $\mathrm{Y}:(0,1 / 2,0)$ & yes & Line \\
\hline$\Gamma:(0,0,0)$ & $\mathrm{LD}:(0,0, \mathrm{w})$ & $\mathrm{Z}:(0,0,1 / 2)$ & yes & Line \\
\hline $\mathrm{R}:(1 / 2,1 / 2,1 / 2)$ & $\mathrm{Q}:(1 / 2,1 / 2, \mathrm{w})$ & $\mathrm{S}:(1 / 2,1 / 2,0)$ & yes & Line \\
\hline $\mathrm{R}:(1 / 2,1 / 2,1 / 2)$ & $\mathrm{P}:(1 / 2, \mathrm{v}, 1 / 2)$ & $\mathrm{U}:(1 / 2,0,1 / 2)$ & yes & Line \\
\hline $\mathrm{S}:(1 / 2,1 / 2,0)$ & $\mathrm{D}:(1 / 2, \mathrm{v}, 0)$ & $\mathrm{X}:(1 / 2,0,0)$ & no & Line \\
\hline $\mathrm{S}:(1 / 2,1 / 2,0)$ & $\mathrm{C}:(\mathrm{u}, 1 / 2,0)$ & $Y:(0,1 / 2,0)$ & yes & Line \\
\hline $\mathrm{T}:(0,1 / 2,1 / 2)$ & $\mathrm{H}:(0,1 / 2, \mathrm{w})$ & $Y:(0,1 / 2,0)$ & yes & Line \\
\hline $\mathrm{T}:(0,1 / 2,1 / 2)$ & $\mathrm{B}:(0, \mathrm{v}, 1 / 2)$ & $\mathrm{Z}:(0,0,1 / 2)$ & yes & Line \\
\hline $\mathrm{U}:(1 / 2,0,1 / 2)$ & $\mathrm{G}:(1 / 2,0, \mathrm{w})$ & $\mathrm{X}:(1 / 2,0,0)$ & no & Line \\
\hline$\Gamma:(0,0,0)$ & $\mathrm{M}:(\mathrm{u}, 0, \mathrm{w})$ & $\mathrm{U}:(1 / 2,0,1 / 2)$ & yes & Plane \\
\hline$\Gamma:(0,0,0)$ & $\mathrm{M}:(\mathrm{u}, 0, \mathrm{w})$ & $\mathrm{X}:(1 / 2,0,0)$ & no & Plane \\
\hline$\Gamma:(0,0,0)$ & $\mathrm{M}:(\mathrm{u}, 0, \mathrm{w})$ & $\mathrm{Z}:(0,0,1 / 2)$ & yes & Plane \\
\hline $\mathrm{R}:(1 / 2,1 / 2,1 / 2)$ & $\mathrm{N}:(\mathrm{u}, 1 / 2, \mathrm{w})$ & $\mathrm{S}:(1 / 2,1 / 2,0)$ & yes & Plane \\
\hline $\mathrm{R}:(1 / 2,1 / 2,1 / 2)$ & $\mathrm{N}:(\mathrm{u}, 1 / 2, \mathrm{w})$ & $\mathrm{T}:(0,1 / 2,1 / 2)$ & yes & Plane \\
\hline $\mathrm{R}:(1 / 2,1 / 2,1 / 2)$ & $\mathrm{N}:(\mathrm{u}, 1 / 2, \mathrm{w})$ & $\mathrm{Y}:(0,1 / 2,0)$ & yes & Plane \\
\hline $\mathrm{S}:(1 / 2,1 / 2,0)$ & $\mathrm{N}:(\mathrm{u}, 1 / 2, \mathrm{w})$ & $\mathrm{T}:(0,1 / 2,1 / 2)$ & yes & Plane \\
\hline $\mathrm{S}:(1 / 2,1 / 2,0)$ & $\mathrm{N}:(\mathrm{u}, 1 / 2, \mathrm{w})$ & $\mathrm{Y}:(0,1 / 2,0)$ & yes & Plane \\
\hline $\mathrm{T}:(0,1 / 2,1 / 2)$ & $\mathrm{N}:(\mathrm{u}, 1 / 2, \mathrm{w})$ & $Y:(0,1 / 2,0)$ & yes & Plane \\
\hline $\mathrm{U}:(1 / 2,0,1 / 2)$ & $\mathrm{M}:(\mathrm{u}, 0, \mathrm{w})$ & $\mathrm{X}:(1 / 2,0,0)$ & no & Plane \\
\hline $\mathrm{U}:(1 / 2,0,1 / 2)$ & $\mathrm{M}:(\mathrm{u}, 0, \mathrm{w})$ & $\mathrm{Z}:(0,0,1 / 2)$ & yes & Plane \\
\hline $\mathrm{X}:(1 / 2,0,0)$ & $\mathrm{M}:(\mathrm{u}, 0, \mathrm{w})$ & $\mathrm{Z}:(0,0,1 / 2)$ & no & Plane \\
\hline
\end{tabular}




\begin{tabular}{|c|c|c|c|c|}
\hline \multicolumn{5}{|c|}{ BCSID: 1.139 ; Formula: Ho2RhIn8; MSG: $49.273\left(P_{c} c c m\right) ; \mathrm{U}=0$} \\
\hline maximal $k_{1}$ & intermediate path & $\operatorname{maximal} k_{2}$ & satisfied? & Line/Plane \\
\hline $\mathrm{R}:(1 / 2,1 / 2,1 / 2)$ & $\mathrm{E}:(\mathrm{u}, 1 / 2,1 / 2)$ & $\mathrm{T}:(0,1 / 2,1 / 2)$ & no & Line \\
\hline $\mathrm{R}:(1 / 2,1 / 2,1 / 2)$ & $\mathrm{P}:(1 / 2, \mathrm{v}, 1 / 2)$ & $\mathrm{U}:(1 / 2,0,1 / 2)$ & no & Line \\
\hline $\mathrm{T}:(0,1 / 2,1 / 2)$ & $\mathrm{B}:(0, \mathrm{v}, 1 / 2)$ & $\mathrm{Z}:(0,0,1 / 2)$ & no & Line \\
\hline $\mathrm{U}:(1 / 2,0,1 / 2)$ & $\mathrm{A}:(\mathrm{u}, 0,1 / 2)$ & $\mathrm{Z}:(0,0,1 / 2)$ & no & Line \\
\hline $\mathrm{R}:(1 / 2,1 / 2,1 / 2)$ & $\mathrm{W}:(\mathrm{u}, \mathrm{v}, 1 / 2)$ & $\mathrm{T}:(0,1 / 2,1 / 2)$ & no & Plane \\
\hline $\mathrm{R}:(1 / 2,1 / 2,1 / 2)$ & $\mathrm{W}:(\mathrm{u}, \mathrm{v}, 1 / 2)$ & $\mathrm{U}:(1 / 2,0,1 / 2)$ & no & Plane \\
\hline $\mathrm{R}:(1 / 2,1 / 2,1 / 2)$ & $\mathrm{W}:(\mathrm{u}, \mathrm{v}, 1 / 2)$ & $\mathrm{Z}:(0,0,1 / 2)$ & yes & Plane \\
\hline $\mathrm{T}:(0,1 / 2,1 / 2)$ & $\mathrm{W}:(\mathrm{u}, \mathrm{v}, 1 / 2)$ & $\mathrm{U}:(1 / 2,0,1 / 2)$ & yes & Plane \\
\hline $\mathrm{T}:(0,1 / 2,1 / 2)$ & $\mathrm{W}:(\mathrm{u}, \mathrm{v}, 1 / 2)$ & $\mathrm{Z}:(0,0,1 / 2)$ & no & Plane \\
\hline $\mathrm{U}:(1 / 2,0,1 / 2)$ & $\mathrm{W}:(\mathrm{u}, \mathrm{v}, 1 / 2)$ & $\mathrm{Z}:(0,0,1 / 2)$ & no & Plane \\
\hline \multicolumn{5}{|c|}{ BCSID: 2.11; Formula: TbMg; MSG: $51.295\left(P m m^{\prime} a^{\prime}\right) ; \mathrm{U}=0$} \\
\hline $\operatorname{maximal} k_{1}$ & intermediate path & maximal $k_{2}$ & satisfied? & Line/Plane \\
\hline$\Gamma:(0,0,0)$ & SM: $(u, 0,0)$ & $\mathrm{X}:(1 / 2,0,0)$ & no & Line \\
\hline$\Gamma:(0,0,0)$ & DT: $(0, \mathrm{v}, 0)$ & $\mathrm{Y}:(0,1 / 2,0)$ & yes & Line \\
\hline$\Gamma:(0,0,0)$ & LD: $(0,0, \mathrm{w})$ & $\mathrm{Z}:(0,0,1 / 2)$ & yes & Line \\
\hline $\mathrm{R}:(1 / 2,1 / 2,1 / 2)$ & $\mathrm{E}:(\mathrm{u}, 1 / 2,1 / 2)$ & $\mathrm{T}:(0,1 / 2,1 / 2)$ & no & Line \\
\hline $\mathrm{R}:(1 / 2,1 / 2,1 / 2)$ & $\mathrm{P}:(1 / 2, \mathrm{v}, 1 / 2)$ & $\mathrm{U}:(1 / 2,0,1 / 2)$ & yes & Line \\
\hline $\mathrm{S}:(1 / 2,1 / 2,0)$ & $\mathrm{D}:(1 / 2, \mathrm{v}, 0)$ & $\mathrm{X}:(1 / 2,0,0)$ & yes & Line \\
\hline $\mathrm{S}:(1 / 2,1 / 2,0)$ & $\mathrm{C}:(\mathrm{u}, 1 / 2,0)$ & $\mathrm{Y}:(0,1 / 2,0)$ & yes & Line \\
\hline $\mathrm{T}:(0,1 / 2,1 / 2)$ & $\mathrm{H}:(0,1 / 2, \mathrm{w})$ & $\mathrm{Y}:(0,1 / 2,0)$ & no & Line \\
\hline $\mathrm{T}:(0,1 / 2,1 / 2)$ & $\mathrm{B}:(0, \mathrm{v}, 1 / 2)$ & $\mathrm{Z}:(0,0,1 / 2)$ & no & Line \\
\hline $\mathrm{U}:(1 / 2,0,1 / 2)$ & $\mathrm{A}:(\mathrm{u}, 0,1 / 2)$ & $\mathrm{Z}:(0,0,1 / 2)$ & yes & Line \\
\hline$\Gamma:(0,0,0)$ & $\mathrm{K}:(0, \mathrm{v}, \mathrm{w})$ & $\mathrm{T}:(0,1 / 2,1 / 2)$ & no & Plane \\
\hline$\Gamma:(0,0,0)$ & $\mathrm{K}:(0, \mathrm{v}, \mathrm{w})$ & $\mathrm{Y}:(0,1 / 2,0)$ & yes & Plane \\
\hline$\Gamma:(0,0,0)$ & $\mathrm{K}:(0, \mathrm{v}, \mathrm{w})$ & $\mathrm{Z}:(0,0,1 / 2)$ & yes & Plane \\
\hline $\mathrm{R}:(1 / 2,1 / 2,1 / 2)$ & $\mathrm{L}:(1 / 2, \mathrm{v}, \mathrm{w})$ & $\mathrm{S}:(1 / 2,1 / 2,0)$ & yes & Plane \\
\hline $\mathrm{R}:(1 / 2,1 / 2,1 / 2)$ & $\mathrm{L}:(1 / 2, \mathrm{v}, \mathrm{w})$ & $\mathrm{U}:(1 / 2,0,1 / 2)$ & yes & Plane \\
\hline $\mathrm{R}:(1 / 2,1 / 2,1 / 2)$ & $\mathrm{L}:(1 / 2, \mathrm{v}, \mathrm{w})$ & $\mathrm{X}:(1 / 2,0,0)$ & yes & Plane \\
\hline $\mathrm{S}:(1 / 2,1 / 2,0)$ & $\mathrm{L}:(1 / 2, \mathrm{v}, \mathrm{w})$ & $\mathrm{U}:(1 / 2,0,1 / 2)$ & yes & Plane \\
\hline $\mathrm{S}:(1 / 2,1 / 2,0)$ & $\mathrm{L}:(1 / 2, \mathrm{v}, \mathrm{w})$ & $\mathrm{X}:(1 / 2,0,0)$ & yes & Plane \\
\hline $\mathrm{T}:(0,1 / 2,1 / 2)$ & $\mathrm{K}:(0, \mathrm{v}, \mathrm{w})$ & $\mathrm{Y}:(0,1 / 2,0)$ & no & Plane \\
\hline $\mathrm{T}:(0,1 / 2,1 / 2)$ & $\mathrm{K}:(0, \mathrm{v}, \mathrm{w})$ & $\mathrm{Z}:(0,0,1 / 2)$ & no & Plane \\
\hline $\mathrm{U}:(1 / 2,0,1 / 2)$ & $\mathrm{L}:(1 / 2, \mathrm{v}, \mathrm{w})$ & $\mathrm{X}:(1 / 2,0,0)$ & yes & Plane \\
\hline $\mathrm{Y}:(0,1 / 2,0)$ & $\mathrm{K}:(0, \mathrm{v}, \mathrm{w})$ & $\mathrm{Z}:(0,0,1 / 2)$ & yes & Plane \\
\hline \multicolumn{5}{|c|}{ BCSID: 2.11; Formula: TbMg; MSG: $51.295\left(P m m^{\prime} a^{\prime}\right) ; \mathrm{U}=2 \mathrm{eV}$} \\
\hline maximal $k_{1}$ & intermediate path & maximal $k_{2}$ & satisfied? & Line/Plane \\
\hline$\Gamma:(0,0,0)$ & SM: $(u, 0,0)$ & $\mathrm{X}:(1 / 2,0,0)$ & no & Line \\
\hline$\Gamma:(0,0,0)$ & DT: $(0, \mathrm{v}, 0)$ & $\mathrm{Y}:(0,1 / 2,0)$ & yes & Line \\
\hline$\Gamma:(0,0,0)$ & LD: $(0,0, \mathrm{w})$ & $\mathrm{Z}:(0,0,1 / 2)$ & yes & Line \\
\hline $\mathrm{R}:(1 / 2,1 / 2,1 / 2)$ & $\mathrm{E}:(\mathrm{u}, 1 / 2,1 / 2)$ & $\mathrm{T}:(0,1 / 2,1 / 2)$ & yes & Line \\
\hline $\mathrm{R}:(1 / 2,1 / 2,1 / 2)$ & $\mathrm{P}:(1 / 2, \mathrm{v}, 1 / 2)$ & $\mathrm{U}:(1 / 2,0,1 / 2)$ & yes & Line \\
\hline $\mathrm{S}:(1 / 2,1 / 2,0)$ & $\mathrm{D}:(1 / 2, \mathrm{v}, 0)$ & $\mathrm{X}:(1 / 2,0,0)$ & yes & Line \\
\hline $\mathrm{S}:(1 / 2,1 / 2,0)$ & $\mathrm{C}:(\mathrm{u}, 1 / 2,0)$ & $\mathrm{Y}:(0,1 / 2,0)$ & yes & Line \\
\hline $\mathrm{T}:(0,1 / 2,1 / 2)$ & $\mathrm{H}:(0,1 / 2, \mathrm{w})$ & $\mathrm{Y}:(0,1 / 2,0)$ & no & Line \\
\hline $\mathrm{T}:(0,1 / 2,1 / 2)$ & $\mathrm{B}:(0, \mathrm{v}, 1 / 2)$ & $\mathrm{Z}:(0,0,1 / 2)$ & no & Line \\
\hline $\mathrm{U}:(1 / 2,0,1 / 2)$ & $\mathrm{A}:(\mathrm{u}, 0,1 / 2)$ & $\mathrm{Z}:(0,0,1 / 2)$ & yes & Line \\
\hline
\end{tabular}




\begin{tabular}{c|c|c|c|c}
\hline$\Gamma:(0,0,0)$ & $\mathrm{K}:(0, \mathrm{v}, \mathrm{w})$ & $\mathrm{T}:(0,1 / 2,1 / 2)$ & no & Plane \\
\hline$\Gamma:(0,0,0)$ & $\mathrm{K}:(0, \mathrm{v}, \mathrm{w})$ & $\mathrm{Y}:(0,1 / 2,0)$ & yes & Plane \\
\hline$\Gamma:(0,0,0)$ & $\mathrm{K}:(0, \mathrm{v}, \mathrm{w})$ & $\mathrm{Z}:(0,0,1 / 2)$ & yes & Plane \\
\hline $\mathrm{R}:(1 / 2,1 / 2,1 / 2)$ & $\mathrm{L}:(1 / 2, \mathrm{v}, \mathrm{w})$ & $\mathrm{S}:(1 / 2,1 / 2,0)$ & yes & Plane \\
\hline $\mathrm{R}:(1 / 2,1 / 2,1 / 2)$ & $\mathrm{L}:(1 / 2, \mathrm{v}, \mathrm{w})$ & $\mathrm{U}:(1 / 2,0,1 / 2)$ & yes & Plane \\
\hline $\mathrm{R}:(1 / 2,1 / 2,1 / 2)$ & $\mathrm{L}:(1 / 2, \mathrm{v}, \mathrm{w})$ & $\mathrm{X}:(1 / 2,0,0)$ & yes & Plane \\
\hline $\mathrm{S}:(1 / 2,1 / 2,0)$ & $\mathrm{L}:(1 / 2, \mathrm{v}, \mathrm{w})$ & $\mathrm{U}:(1 / 2,0,1 / 2)$ & yes & Plane \\
\hline $\mathrm{S}:(1 / 2,1 / 2,0)$ & $\mathrm{L}:(1 / 2, \mathrm{v}, \mathrm{w})$ & $\mathrm{X}:(1 / 2,0,0)$ & yes & Plane \\
\hline $\mathrm{T}:(0,1 / 2,1 / 2)$ & $\mathrm{K}:(0, \mathrm{v}, \mathrm{w})$ & $\mathrm{Y}:(0,1 / 2,0)$ & no & Plane \\
\hline $\mathrm{T}:(0,1 / 2,1 / 2)$ & $\mathrm{K}:(0, \mathrm{v}, \mathrm{w})$ & $\mathrm{Z}:(0,0,1 / 2)$ & no & Plane \\
\hline $\mathrm{U}:(1 / 2,0,1 / 2)$ & $\mathrm{L}:(1 / 2, \mathrm{v}, \mathrm{w})$ & $\mathrm{X}:(1 / 2,0,0)$ & yes & Plane \\
\hline $\mathrm{Y}:(0,1 / 2,0)$ & $\mathrm{K}:(0, \mathrm{v}, \mathrm{w})$ & $\mathrm{Z}:(0,0,1 / 2)$ & yes & Plane \\
\hline
\end{tabular}

\begin{tabular}{c|c|c|c|c}
\hline \multicolumn{5}{c}{ BCSID: $2.11 ;$ Formula: TbMg; MSG: $51.295\left(P m m^{\prime} a^{\prime}\right) \mathrm{U}=4 \mathrm{eV}$} \\
\hline maximal $k_{1}$ & intermediate path & maximal $k_{2}$ & satisfied? & Line/Plane \\
\hline$\Gamma:(0,0,0)$ & $\mathrm{SM}:(\mathrm{u}, 0,0)$ & $\mathrm{X}:(1 / 2,0,0)$ & yes & Line \\
\hline$\Gamma:(0,0,0)$ & $\mathrm{DT}:(0, \mathrm{v}, 0)$ & $\mathrm{Y}:(0,1 / 2,0)$ & yes & Line \\
\hline$\Gamma:(0,0,0)$ & $\mathrm{LD}:(0,0, \mathrm{w})$ & $\mathrm{Z}:(0,0,1 / 2)$ & yes & Line \\
\hline $\mathrm{R}:(1 / 2,1 / 2,1 / 2)$ & $\mathrm{E}:(\mathrm{u}, 1 / 2,1 / 2)$ & $\mathrm{T}:(0,1 / 2,1 / 2)$ & yes & Line \\
\hline $\mathrm{R}:(1 / 2,1 / 2,1 / 2)$ & $\mathrm{P}:(1 / 2, \mathrm{v}, 1 / 2)$ & $\mathrm{U}:(1 / 2,0,1 / 2)$ & yes & Line \\
\hline $\mathrm{S}:(1 / 2,1 / 2,0)$ & $\mathrm{D}:(1 / 2, \mathrm{v}, 0)$ & $\mathrm{X}:(1 / 2,0,0)$ & yes & Line \\
\hline $\mathrm{S}:(1 / 2,1 / 2,0)$ & $\mathrm{C}:(\mathrm{u}, 1 / 2,0)$ & $\mathrm{Y}:(0,1 / 2,0)$ & yes & Line \\
\hline $\mathrm{T}:(0,1 / 2,1 / 2)$ & $\mathrm{H}:(0,1 / 2, \mathrm{w})$ & $\mathrm{Y}:(0,1 / 2,0)$ & no & Line \\
\hline $\mathrm{T}:(0,1 / 2,1 / 2)$ & $\mathrm{B}:(0, \mathrm{v}, 1 / 2)$ & $\mathrm{Z}:(0,0,1 / 2)$ & no & Line \\
\hline $\mathrm{U}:(1 / 2,0,1 / 2)$ & $\mathrm{A}:(\mathrm{u}, 0,1 / 2)$ & $\mathrm{Z}:(0,0,1 / 2)$ & yes & Line \\
\hline$\Gamma:(0,0,0)$ & $\mathrm{K}:(0, \mathrm{v}, \mathrm{w})$ & $\mathrm{T}:(0,1 / 2,1 / 2)$ & no & Plane \\
\hline$\Gamma:(0,0,0)$ & $\mathrm{K}:(0, \mathrm{v}, \mathrm{w})$ & $\mathrm{Y}:(0,1 / 2,0)$ & yes & Plane \\
\hline$\Gamma:(0,0,0)$ & $\mathrm{K}:(0, \mathrm{v}, \mathrm{w})$ & $\mathrm{Z}:(0,0,1 / 2)$ & yes & Plane \\
\hline $\mathrm{R}:(1 / 2,1 / 2,1 / 2)$ & $\mathrm{L}:(1 / 2, \mathrm{v}, \mathrm{w})$ & $\mathrm{S}:(1 / 2,1 / 2,0)$ & yes & Plane \\
\hline $\mathrm{R}:(1 / 2,1 / 2,1 / 2)$ & $\mathrm{L}:(1 / 2, \mathrm{v}, \mathrm{w})$ & $\mathrm{U}:(1 / 2,0,1 / 2)$ & yes & Plane \\
\hline $\mathrm{R}:(1 / 2,1 / 2,1 / 2)$ & $\mathrm{L}:(1 / 2, \mathrm{v}, \mathrm{w})$ & $\mathrm{X}:(1 / 2,0,0)$ & yes & Plane \\
\hline $\mathrm{S}:(1 / 2,1 / 2,0)$ & $\mathrm{L}:(1 / 2, \mathrm{v}, \mathrm{w})$ & $\mathrm{U}:(1 / 2,0,1 / 2)$ & yes & Plane \\
\hline $\mathrm{S}:(1 / 2,1 / 2,0)$ & $\mathrm{L}:(1 / 2, \mathrm{v}, \mathrm{w})$ & $\mathrm{X}:(1 / 2,0,0)$ & yes & Plane \\
\hline $\mathrm{T}:(0,1 / 2,1 / 2)$ & $\mathrm{K}:(0, \mathrm{v}, \mathrm{w})$ & $\mathrm{Y}:(0,1 / 2,0)$ & no & Plane \\
\hline $\mathrm{T}:(0,1 / 2,1 / 2)$ & $\mathrm{K}:(0, \mathrm{v}, \mathrm{w})$ & $\mathrm{Z}:(0,0,1 / 2)$ & no & Plane \\
\hline $\mathrm{U}:(1 / 2,0,1 / 2)$ & $\mathrm{L}:(1 / 2, \mathrm{v}, \mathrm{w})$ & $\mathrm{X}:(1 / 2,0,0)$ & yes & Plane \\
\hline $\mathrm{Y}:(0,1 / 2,0)$ & $\mathrm{K}:(0, \mathrm{v}, \mathrm{w})$ & $\mathrm{Z}:(0,0,1 / 2)$ & yes & Plane \\
\hline
\end{tabular}

\begin{tabular}{c|c|c|c|c}
\hline \multicolumn{6}{c}{ BCSID: 2.11 Formula: TbMg; MSG: $51.295\left(P m^{\prime} a^{\prime}\right) ; \mathrm{U}=6 \mathrm{eV}$} \\
\hline maximal $k_{1}$ & intermediate path & maximal $k_{2}$ & satisfied? & Line/Plane \\
\hline$\Gamma:(0,0,0)$ & $\mathrm{SM}:(\mathrm{u}, 0,0)$ & $\mathrm{X}:(1 / 2,0,0)$ & yes & Line \\
\hline$\Gamma:(0,0,0)$ & $\mathrm{DT}:(0, \mathrm{v}, 0)$ & $\mathrm{Y}:(0,1 / 2,0)$ & yes & Line \\
\hline$\Gamma:(0,0,0)$ & $\mathrm{LD}:(0,0, \mathrm{w})$ & $\mathrm{Z}:(0,0,1 / 2)$ & yes & Line \\
\hline $\mathrm{R}:(1 / 2,1 / 2,1 / 2)$ & $\mathrm{E}:(\mathrm{u}, 1 / 2,1 / 2)$ & $\mathrm{T}:(0,1 / 2,1 / 2)$ & yes & Line \\
\hline $\mathrm{R}:(1 / 2,1 / 2,1 / 2)$ & $\mathrm{P}:(1 / 2, \mathrm{v}, 1 / 2)$ & $\mathrm{U}:(1 / 2,0,1 / 2)$ & yes & Line \\
\hline $\mathrm{S}:(1 / 2,1 / 2,0)$ & $\mathrm{D}:(1 / 2, \mathrm{v}, 0)$ & $\mathrm{X}:(1 / 2,0,0)$ & yes & Line \\
\hline $\mathrm{S}:(1 / 2,1 / 2,0)$ & $\mathrm{C}:(\mathrm{u}, 1 / 2,0)$ & $\mathrm{Y}:(0,1 / 2,0)$ & yes & Line \\
\hline $\mathrm{T}:(0,1 / 2,1 / 2)$ & $\mathrm{H}:(0,1 / 2, \mathrm{w})$ & $\mathrm{Y}:(0,1 / 2,0)$ & no & Line \\
\hline $\mathrm{T}:(0,1 / 2,1 / 2)$ & $\mathrm{B}:(0, \mathrm{v}, 1 / 2)$ & $\mathrm{Z}:(0,0,1 / 2)$ & no & Line \\
\hline $\mathrm{U}:(1 / 2,0,1 / 2)$ & $\mathrm{A}:(\mathrm{u}, 0,1 / 2)$ & $\mathrm{Z}:(0,0,1 / 2)$ & yes & Line \\
\hline$\Gamma:(0,0,0)$ & $\mathrm{K}:(0, \mathrm{v}, \mathrm{w})$ & $\mathrm{T}:(0,1 / 2,1 / 2)$ & no & Plane
\end{tabular}




\begin{tabular}{c|c|c|c|c}
\hline$\Gamma:(0,0,0)$ & $\mathrm{K}:(0, \mathrm{v}, \mathrm{w})$ & $\mathrm{Y}:(0,1 / 2,0)$ & yes & Plane \\
\hline$\Gamma:(0,0,0)$ & $\mathrm{K}:(0, \mathrm{v}, \mathrm{w})$ & $\mathrm{Z}:(0,0,1 / 2)$ & yes & Plane \\
\hline $\mathrm{R}:(1 / 2,1 / 2,1 / 2)$ & $\mathrm{L}:(1 / 2, \mathrm{v}, \mathrm{w})$ & $\mathrm{S}:(1 / 2,1 / 2,0)$ & yes & Plane \\
\hline $\mathrm{R}:(1 / 2,1 / 2,1 / 2)$ & $\mathrm{L}:(1 / 2, \mathrm{v}, \mathrm{w})$ & $\mathrm{U}:(1 / 2,0,1 / 2)$ & yes & Plane \\
\hline $\mathrm{R}:(1 / 2,1 / 2,1 / 2)$ & $\mathrm{L}:(1 / 2, \mathrm{v}, \mathrm{w})$ & $\mathrm{X}:(1 / 2,0,0)$ & yes & Plane \\
\hline $\mathrm{S}:(1 / 2,1 / 2,0)$ & $\mathrm{L}:(1 / 2, \mathrm{v}, \mathrm{w})$ & $\mathrm{U}:(1 / 2,0,1 / 2)$ & yes & Plane \\
\hline $\mathrm{S}:(1 / 2,1 / 2,0)$ & $\mathrm{L}:(1 / 2, \mathrm{v}, \mathrm{w})$ & $\mathrm{X}:(1 / 2,0,0)$ & yes & Plane \\
\hline $\mathrm{T}:(0,1 / 2,1 / 2)$ & $\mathrm{K}:(0, \mathrm{v}, \mathrm{w})$ & $\mathrm{Y}:(0,1 / 2,0)$ & no & Plane \\
\hline $\mathrm{T}:(0,1 / 2,1 / 2)$ & $\mathrm{K}:(0, \mathrm{v}, \mathrm{w})$ & $\mathrm{Z}:(0,0,1 / 2)$ & no & Plane \\
\hline $\mathrm{U}:(1 / 2,0,1 / 2)$ & $\mathrm{L}:(1 / 2, \mathrm{v}, \mathrm{w})$ & $\mathrm{X}:(1 / 2,0,0)$ & yes & Plane \\
\hline $\mathrm{Y}:(0,1 / 2,0)$ & $\mathrm{K}:(0, \mathrm{v}, \mathrm{w})$ & $\mathrm{Z}:(0,0,1 / 2)$ & yes & Plane \\
\hline
\end{tabular}

BCSID: 1.222; Formula: Er2CoGa8; MSG: $51.298\left(P_{a} m m a\right)$; U=0

\begin{tabular}{c|c|c|c|c}
\hline maximal $k_{1}$ & intermediate path & maximal $k_{2}$ & satisfied? & Line/Plane \\
\hline $\mathrm{R}:(1 / 2,1 / 2,1 / 2)$ & $\mathrm{P}:(1 / 2, \mathrm{v}, 1 / 2)$ & $\mathrm{U}:(1 / 2,0,1 / 2)$ & yes & Line \\
\hline $\mathrm{S}:(1 / 2,1 / 2,0)$ & $\mathrm{D}:(1 / 2, \mathrm{v}, 0)$ & $\mathrm{X}:(1 / 2,0,0)$ & no & Line \\
\hline
\end{tabular}

BCSID: 1.222; Formula: Er2CoGa8; MSG: $51.298\left(P_{a} m m a\right)$; U=2eV maximal $k_{1} \quad$ intermediate path $\quad$ maximal $k_{2}$ satisfied? $\mid$ Line/Plane

\begin{tabular}{c|c|c|c|c}
\hline $\mathrm{R}:(1 / 2,1 / 2,1 / 2)$ & $\mathrm{P}:(1 / 2, \mathrm{v}, 1 / 2)$ & $\mathrm{U}:(1 / 2,0,1 / 2)$ & no & Line \\
\hline $\mathrm{S}:(1 / 2,1 / 2,0)$ & $\mathrm{D}:(1 / 2, \mathrm{v}, 0)$ & $\mathrm{X}:(1 / 2,0,0)$ & no & Line \\
\hline
\end{tabular}

BCSID: 1.3; Formula: Sr2IrO4; MSG: $54.352\left(P_{I} c c a\right) ; \mathrm{U}=1 \mathrm{eV}$

\begin{tabular}{c|c|c|c|c} 
maximal $k_{1}$ & intermediate path & maximal $k_{2}$ & satisfied? & Line/Plane \\
\hline $\mathrm{R}:(1 / 2,1 / 2,1 / 2)$ & $\mathrm{E}:(\mathrm{u}, 1 / 2,1 / 2)$ & $\mathrm{T}:(0,1 / 2,1 / 2)$ & yes & Line \\
\hline $\mathrm{S}:(1 / 2,1 / 2,0)$ & $\mathrm{D}:(1 / 2, \mathrm{v}, 0)$ & $\mathrm{X}:(1 / 2,0,0)$ & yes & Line \\
\hline $\mathrm{T}:(0,1 / 2,1 / 2)$ & $\mathrm{B}:(0, \mathrm{v}, 1 / 2)$ & $\mathrm{Z}:(0,0,1 / 2)$ & no & Line \\
\hline $\mathrm{U}:(1 / 2,0,1 / 2)$ & $\mathrm{A}:(\mathrm{u}, 0,1 / 2)$ & $\mathrm{Z}:(0,0,1 / 2)$ & no & Line \\
\hline $\mathrm{R}:(1 / 2,1 / 2,1 / 2)$ & $\mathrm{N}:(\mathrm{u}, 1 / 2, \mathrm{w})$ & $\mathrm{S}:(1 / 2,1 / 2,0)$ & yes & Plane \\
\hline $\mathrm{R}:(1 / 2,1 / 2,1 / 2)$ & $\mathrm{N}:(\mathrm{u}, 1 / 2, \mathrm{w})$ & $\mathrm{T}:(0,1 / 2,1 / 2)$ & yes & Plane \\
\hline $\mathrm{R}:(1 / 2,1 / 2,1 / 2)$ & $\mathrm{W}:(\mathrm{u}, \mathrm{v}, 1 / 2)$ & $\mathrm{T}:(0,1 / 2,1 / 2)$ & yes & Plane \\
\hline $\mathrm{R}:(1 / 2,1 / 2,1 / 2)$ & $\mathrm{W}:(\mathrm{u}, \mathrm{v}, 1 / 2)$ & $\mathrm{U}:(1 / 2,0,1 / 2)$ & yes & Plane \\
\hline $\mathrm{R}:(1 / 2,1 / 2,1 / 2)$ & $\mathrm{N}:(\mathrm{u}, 1 / 2, \mathrm{w})$ & $\mathrm{Y}:(0,1 / 2,0)$ & yes & Plane \\
\hline $\mathrm{R}:(1 / 2,1 / 2,1 / 2)$ & $\mathrm{W}:(\mathrm{u}, \mathrm{v}, 1 / 2)$ & $\mathrm{Z}:(0,0,1 / 2)$ & no & Plane \\
\hline $\mathrm{S}:(1 / 2,1 / 2,0)$ & $\mathrm{N}:(\mathrm{u}, 1 / 2, \mathrm{w})$ & $\mathrm{T}:(0,1 / 2,1 / 2)$ & yes & Plane \\
\hline $\mathrm{S}:(1 / 2,1 / 2,0)$ & $\mathrm{N}:(\mathrm{u}, 1 / 2, \mathrm{w})$ & $\mathrm{Y}:(0,1 / 2,0)$ & yes & Plane \\
\hline $\mathrm{T}:(0,1 / 2,1 / 2)$ & $\mathrm{W}:(\mathrm{u}, \mathrm{v}, 1 / 2)$ & $\mathrm{U}:(1 / 2,0,1 / 2)$ & yes & Plane \\
\hline $\mathrm{T}:(0,1 / 2,1 / 2)$ & $\mathrm{N}:(\mathrm{u}, 1 / 2, \mathrm{w})$ & $\mathrm{Y}:(0,1 / 2,0)$ & yes & Plane \\
\hline $\mathrm{T}:(0,1 / 2,1 / 2)$ & $\mathrm{W}:(\mathrm{u}, \mathrm{v}, 1 / 2)$ & $\mathrm{Z}:(0,0,1 / 2)$ & no & Plane \\
\hline $\mathrm{U}:(1 / 2,0,1 / 2)$ & $\mathrm{W}:(\mathrm{u}, \mathrm{v}, 1 / 2)$ & $\mathrm{Z}:(0,0,1 / 2)$ & no & Plane \\
\hline
\end{tabular}

\begin{tabular}{c|c|c|c|c}
\hline \multicolumn{6}{c}{ BCSID: 0.85; Formula: KCo4(PO4)3; MSG: $58.398\left(P n n^{\prime} m^{\prime}\right) ; \mathrm{U}=0$} \\
\hline maximal $k_{1}$ & intermediate path & maximal $k_{2}$ & satisfied? & Line/Plane \\
\hline$\Gamma:(0,0,0)$ & SM:(u,0,0) & $\mathrm{X}:(1 / 2,0,0)$ & yes & Line \\
\hline$\Gamma:(0,0,0)$ & $\mathrm{DT}:(0, \mathrm{v}, 0)$ & $\mathrm{Y}:(0,1 / 2,0)$ & yes & Line \\
\hline$\Gamma:(0,0,0)$ & $\mathrm{LD}:(0,0, \mathrm{w})$ & $\mathrm{Z}:(0,0,1 / 2)$ & yes & Line \\
\hline $\mathrm{R}:(1 / 2,1 / 2,1 / 2)$ & $\mathrm{P}:(1 / 2, \mathrm{v}, 1 / 2)$ & $\mathrm{U}:(1 / 2,0,1 / 2)$ & yes & Line \\
\hline $\mathrm{U}:(1 / 2,0,1 / 2)$ & $\mathrm{G}:(1 / 2,0, \mathrm{w})$ & $\mathrm{X}:(1 / 2,0,0)$ & yes & Line \\
\hline $\mathrm{U}:(1 / 2,0,1 / 2)$ & $\mathrm{A}:(\mathrm{u}, 0,1 / 2)$ & $\mathrm{Z}:(0,0,1 / 2)$ & no & Line \\
\hline$\Gamma:(0,0,0)$ & $\mathrm{K}:(0, \mathrm{v}, \mathrm{w})$ & $\mathrm{T}:(0,1 / 2,1 / 2)$ & yes & Plane \\
\hline$\Gamma:(0,0,0)$ & $\mathrm{K}:(0, \mathrm{v}, \mathrm{w})$ & $\mathrm{Y}:(0,1 / 2,0)$ & yes & Plane
\end{tabular}




\begin{tabular}{c|c|c|c|c}
\hline$\Gamma:(0,0,0)$ & $\mathrm{K}:(0, \mathrm{v}, \mathrm{w})$ & $\mathrm{Z}:(0,0,1 / 2)$ & yes & Plane \\
\hline $\mathrm{R}:(1 / 2,1 / 2,1 / 2)$ & $\mathrm{L}:(1 / 2, \mathrm{v}, \mathrm{w})$ & $\mathrm{S}:(1 / 2,1 / 2,0)$ & yes & Plane \\
\hline $\mathrm{R}:(1 / 2,1 / 2,1 / 2)$ & $\mathrm{L}:(1 / 2, \mathrm{v}, \mathrm{w})$ & $\mathrm{U}:(1 / 2,0,1 / 2)$ & yes & Plane \\
\hline $\mathrm{R}:(1 / 2,1 / 2,1 / 2)$ & $\mathrm{L}:(1 / 2, \mathrm{v}, \mathrm{w})$ & $\mathrm{X}:(1 / 2,0,0)$ & yes & Plane \\
\hline $\mathrm{S}:(1 / 2,1 / 2,0)$ & $\mathrm{L}:(1 / 2, \mathrm{v}, \mathrm{w})$ & $\mathrm{U}:(1 / 2,0,1 / 2)$ & yes & Plane \\
\hline $\mathrm{S}:(1 / 2,1 / 2,0)$ & $\mathrm{L}:(1 / 2, \mathrm{v}, \mathrm{w})$ & $\mathrm{X}:(1 / 2,0,0)$ & yes & Plane \\
\hline $\mathrm{T}:(0,1 / 2,1 / 2)$ & $\mathrm{K}:(0, \mathrm{v}, \mathrm{w})$ & $\mathrm{Y}:(0,1 / 2,0)$ & yes & Plane \\
\hline $\mathrm{T}:(0,1 / 2,1 / 2)$ & $\mathrm{K}:(0, \mathrm{v}, \mathrm{w})$ & $\mathrm{Z}:(0,0,1 / 2)$ & yes & Plane \\
\hline $\mathrm{U}:(1 / 2,0,1 / 2)$ & $\mathrm{L}:(1 / 2, \mathrm{v}, \mathrm{w})$ & $\mathrm{X}:(1 / 2,0,0)$ & yes & Plane \\
\hline $\mathrm{Y}:(0,1 / 2,0)$ & $\mathrm{K}:(0, \mathrm{v}, \mathrm{w})$ & $\mathrm{Z}:(0,0,1 / 2)$ & yes & Plane \\
\hline
\end{tabular}

BCSID: 0.187; Formula: CeMnAsO; MSG: 59.407 (Pm'mn); U=0

\begin{tabular}{c|c|c|c|c}
\hline maximal $k_{1}$ & intermediate path & maximal $k_{2}$ & satisfied? & Line/Plane \\
\hline $\mathrm{R}:(1 / 2,1 / 2,1 / 2)$ & $\mathrm{Q}:(1 / 2,1 / 2, \mathrm{w})$ & $\mathrm{S}:(1 / 2,1 / 2,0)$ & yes & Line \\
\hline $\mathrm{R}:(1 / 2,1 / 2,1 / 2)$ & $\mathrm{E}:(\mathrm{u}, 1 / 2,1 / 2)$ & $\mathrm{T}:(0,1 / 2,1 / 2)$ & no & Line \\
\hline $\mathrm{S}:(1 / 2,1 / 2,0)$ & $\mathrm{C}:(\mathrm{u}, 1 / 2,0)$ & $\mathrm{Y}:(0,1 / 2,0)$ & no & Line \\
\hline $\mathrm{T}:(0,1 / 2,1 / 2)$ & $\mathrm{H}:(0,1 / 2, \mathrm{w})$ & $\mathrm{Y}:(0,1 / 2,0)$ & yes & Line \\
\hline $\mathrm{R}:(1 / 2,1 / 2,1 / 2)$ & $\mathrm{N}:(\mathrm{u}, 1 / 2, \mathrm{w})$ & $\mathrm{S}:(1 / 2,1 / 2,0)$ & yes & Plane \\
\hline $\mathrm{R}:(1 / 2,1 / 2,1 / 2)$ & $\mathrm{N}:(\mathrm{u}, 1 / 2, \mathrm{w})$ & $\mathrm{T}:(0,1 / 2,1 / 2)$ & no & Plane \\
\hline $\mathrm{R}:(1 / 2,1 / 2,1 / 2)$ & $\mathrm{N}:(\mathrm{u}, 1 / 2, \mathrm{w})$ & $\mathrm{Y}:(0,1 / 2,0)$ & no & Plane \\
\hline $\mathrm{S}:(1 / 2,1 / 2,0)$ & $\mathrm{N}:(\mathrm{u}, 1 / 2, \mathrm{w})$ & $\mathrm{T}:(0,1 / 2,1 / 2)$ & no & Plane \\
\hline $\mathrm{S}:(1 / 2,1 / 2,0)$ & $\mathrm{N}:(\mathrm{u}, 1 / 2, \mathrm{w})$ & $\mathrm{Y}:(0,1 / 2,0)$ & no & Plane \\
\hline $\mathrm{T}:(0,1 / 2,1 / 2)$ & $\mathrm{N}:(\mathrm{u}, 1 / 2, \mathrm{w})$ & $\mathrm{Y}:(0,1 / 2,0)$ & yes & Plane \\
\hline \multicolumn{5}{|l}{}
\end{tabular}

\begin{tabular}{c|c|c|c|c}
\hline \multicolumn{3}{c}{ BCSID $1.88 ;$ Formula: Mn5Si3; MSG: $60.431\left(P_{C} b c n\right) ; \mathrm{U}=1 \mathrm{eV}$} \\
\hline maximal $k_{1}$ & intermediate path & maximal $k_{2}$ & satisfied? & Line/Plane \\
\hline $\mathrm{R}:(1 / 2,1 / 2,1 / 2)$ & $\mathrm{Q}:(1 / 2,1 / 2, \mathrm{w})$ & $\mathrm{S}:(1 / 2,1 / 2,0)$ & no & Line \\
\hline $\mathrm{R}:(1 / 2,1 / 2,1 / 2)$ & $\mathrm{P}:(1 / 2, \mathrm{v}, 1 / 2)$ & $\mathrm{U}:(1 / 2,0,1 / 2)$ & yes & Line \\
\hline $\mathrm{S}:(1 / 2,1 / 2,0)$ & $\mathrm{D}:(1 / 2, \mathrm{v}, 0)$ & $\mathrm{X}:(1 / 2,0,0)$ & yes & Line \\
\hline $\mathrm{S}:(1 / 2,1 / 2,0)$ & $\mathrm{C}:(\mathrm{u}, 1 / 2,0)$ & $\mathrm{Y}:(0,1 / 2,0)$ & no & Line \\
\hline $\mathrm{T}:(0,1 / 2,1 / 2)$ & $\mathrm{H}:(0,1 / 2, \mathrm{w})$ & $\mathrm{Y}:(0,1 / 2,0)$ & yes & Line \\
\hline $\mathrm{U}:(1 / 2,0,1 / 2)$ & $\mathrm{A}:(\mathrm{u}, 0,1 / 2)$ & $\mathrm{Z}:(0,0,1 / 2)$ & yes & Line \\
\hline $\mathrm{R}:(1 / 2,1 / 2,1 / 2)$ & $\mathrm{N}:(\mathrm{u}, 1 / 2, \mathrm{w})$ & $\mathrm{S}:(1 / 2,1 / 2,0)$ & no & Plane \\
\hline $\mathrm{R}:(1 / 2,1 / 2,1 / 2)$ & $\mathrm{N}:(\mathrm{u}, 1 / 2, \mathrm{w})$ & $\mathrm{T}:(0,1 / 2,1 / 2)$ & yes & Plane \\
\hline $\mathrm{R}:(1 / 2,1 / 2,1 / 2)$ & $\mathrm{W}:(\mathrm{u}, \mathrm{v}, 1 / 2)$ & $\mathrm{T}:(0,1 / 2,1 / 2)$ & yes & Plane \\
\hline $\mathrm{R}:(1 / 2,1 / 2,1 / 2)$ & $\mathrm{W}:(\mathrm{u}, \mathrm{v}, 1 / 2)$ & $\mathrm{U}:(1 / 2,0,1 / 2)$ & yes & Plane \\
\hline $\mathrm{R}:(1 / 2,1 / 2,1 / 2)$ & $\mathrm{N}:(\mathrm{u}, 1 / 2, \mathrm{w})$ & $\mathrm{Y}:(0,1 / 2,0)$ & yes & Plane \\
\hline $\mathrm{R}:(1 / 2,1 / 2,1 / 2)$ & $\mathrm{W}:(\mathrm{u}, \mathrm{v}, 1 / 2)$ & $\mathrm{Z}:(0,0,1 / 2)$ & yes & Plane \\
\hline $\mathrm{S}:(1 / 2,1 / 2,0)$ & $\mathrm{N}:(\mathrm{u}, 1 / 2, \mathrm{w})$ & $\mathrm{T}:(0,1 / 2,1 / 2)$ & no & Plane \\
\hline $\mathrm{S}:(1 / 2,1 / 2,0)$ & $\mathrm{N}:(\mathrm{u}, 1 / 2, \mathrm{w})$ & $\mathrm{Y}:(0,1 / 2,0)$ & no & Plane \\
\hline $\mathrm{T}:(0,1 / 2,1 / 2)$ & $\mathrm{W}:(\mathrm{u}, \mathrm{v}, 1 / 2)$ & $\mathrm{U}:(1 / 2,0,1 / 2)$ & yes & Plane \\
\hline $\mathrm{T}:(0,1 / 2,1 / 2)$ & $\mathrm{N}:(\mathrm{u}, 1 / 2, \mathrm{w})$ & $\mathrm{Y}:(0,1 / 2,0)$ & yes & Plane \\
\hline $\mathrm{T}:(0,1 / 2,1 / 2)$ & $\mathrm{W}:(\mathrm{u}, \mathrm{v}, 1 / 2)$ & $\mathrm{Z}:(0,0,1 / 2)$ & yes & Plane \\
\hline $\mathrm{U}:(1 / 2,0,1 / 2)$ & $\mathrm{W}:(\mathrm{u}, \mathrm{v}, 1 / 2)$ & $\mathrm{Z}:(0,0,1 / 2)$ & yes & Plane \\
\hline
\end{tabular}

BCSID: 2.1; Formula: EuFe2As2; MSG: $61.439\left(P_{C} b c a\right) ; \mathrm{U}=1 \mathrm{eV}$

\begin{tabular}{c|c|c|c|c}
\hline maximal $k_{1}$ & intermediate path & maximal $k_{2}$ & satisfied? & Line/Plane \\
\hline $\mathrm{R}:(1 / 2,1 / 2,1 / 2)$ & $\mathrm{Q}:(1 / 2,1 / 2, \mathrm{w})$ & $\mathrm{S}:(1 / 2,1 / 2,0)$ & yes & Line \\
\hline $\mathrm{R}:(1 / 2,1 / 2,1 / 2)$ & $\mathrm{E}:(\mathrm{u}, 1 / 2,1 / 2)$ & $\mathrm{T}:(0,1 / 2,1 / 2)$ & yes & Line \\
\hline $\mathrm{R}:(1 / 2,1 / 2,1 / 2)$ & $\mathrm{P}:(1 / 2, \mathrm{v}, 1 / 2)$ & $\mathrm{U}:(1 / 2,0,1 / 2)$ & yes & Line \\
\hline $\mathrm{S}:(1 / 2,1 / 2,0)$ & $\mathrm{D}:(1 / 2, \mathrm{v}, 0)$ & $\mathrm{X}:(1 / 2,0,0)$ & yes & Line
\end{tabular}




\begin{tabular}{c|c|c|c|c}
\hline $\mathrm{T}:(0,1 / 2,1 / 2)$ & $\mathrm{H}:(0,1 / 2, \mathrm{w})$ & $\mathrm{Y}:(0,1 / 2,0)$ & no & Line \\
\hline $\mathrm{U}:(1 / 2,0,1 / 2)$ & $\mathrm{A}:(\mathrm{u}, 0,1 / 2)$ & $\mathrm{Z}:(0,0,1 / 2)$ & yes & Line \\
\hline $\mathrm{R}:(1 / 2,1 / 2,1 / 2)$ & $\mathrm{W}:(\mathrm{u}, \mathrm{v}, 1 / 2)$ & $\mathrm{T}:(0,1 / 2,1 / 2)$ & yes & Plane \\
\hline $\mathrm{R}:(1 / 2,1 / 2,1 / 2)$ & $\mathrm{W}:(\mathrm{u}, \mathrm{v}, 1 / 2)$ & $\mathrm{U}:(1 / 2,0,1 / 2)$ & yes & Plane \\
\hline $\mathrm{R}:(1 / 2,1 / 2,1 / 2)$ & $\mathrm{W}:(\mathrm{u}, \mathrm{v}, 1 / 2)$ & $\mathrm{Z}:(0,0,1 / 2)$ & yes & Plane \\
\hline $\mathrm{T}:(0,1 / 2,1 / 2)$ & $\mathrm{W}:(\mathrm{u}, \mathrm{v}, 1 / 2)$ & $\mathrm{U}:(1 / 2,0,1 / 2)$ & yes & Plane \\
\hline $\mathrm{T}:(0,1 / 2,1 / 2)$ & $\mathrm{W}:(\mathrm{u}, \mathrm{v}, 1 / 2)$ & $\mathrm{Z}:(0,0,1 / 2)$ & yes & Plane \\
\hline $\mathrm{U}:(1 / 2,0,1 / 2)$ & $\mathrm{W}:(\mathrm{u}, \mathrm{v}, 1 / 2)$ & $\mathrm{Z}:(0,0,1 / 2)$ & yes & Plane \\
\hline
\end{tabular}

\begin{tabular}{|c|c|c|c|c|}
\hline \multicolumn{5}{|c|}{ BCSID: 0.218; Formula: Co2SiO4; MSG: 62.441 (Pnma); U=0 } \\
\hline maximal $k_{1}$ & intermediate path & maximal $k_{2}$ & satisfied? & Line/Plane \\
\hline $\mathrm{R}:(1 / 2,1 / 2,1 / 2)$ & $\mathrm{Q}:(1 / 2,1 / 2, \mathrm{w})$ & $\mathrm{S}:(1 / 2,1 / 2,0)$ & yes & Line \\
\hline $\mathrm{S}:(1 / 2,1 / 2,0)$ & $\mathrm{D}:(1 / 2, \mathrm{v}, 0)$ & $\mathrm{X}:(1 / 2,0,0)$ & no & Line \\
\hline $\mathrm{T}:(0,1 / 2,1 / 2)$ & $\mathrm{H}:(0,1 / 2, \mathrm{w})$ & $\mathrm{Y}:(0,1 / 2,0)$ & yes & Line \\
\hline $\mathrm{T}:(0,1 / 2,1 / 2)$ & $\mathrm{B}:(0, \mathrm{v}, 1 / 2)$ & $\mathrm{Z}:(0,0,1 / 2)$ & yes & Line \\
\hline$\Gamma:(0,0,0)$ & $\mathrm{V}:(\mathrm{u}, \mathrm{v}, 0)$ & $\mathrm{S}:(1 / 2,1 / 2,0)$ & yes & Plane \\
\hline$\Gamma:(0,0,0)$ & $\mathrm{K}:(0, \mathrm{v}, \mathrm{w})$ & $\mathrm{T}:(0,1 / 2,1 / 2)$ & yes & Plane \\
\hline$\Gamma:(0,0,0)$ & $\mathrm{M}:(\mathrm{u}, 0, \mathrm{w})$ & $\mathrm{U}:(1 / 2,0,1 / 2)$ & yes & Plane \\
\hline$\Gamma:(0,0,0)$ & $\mathrm{M}:(\mathrm{u}, 0, \mathrm{w})$ & $\mathrm{X}:(1 / 2,0,0)$ & yes & Plane \\
\hline$\Gamma:(0,0,0)$ & $\mathrm{V}:(\mathrm{u}, \mathrm{v}, 0)$ & $\mathrm{X}:(1 / 2,0,0)$ & yes & Plane \\
\hline$\Gamma:(0,0,0)$ & $\mathrm{K}:(0, \mathrm{v}, \mathrm{w})$ & $\mathrm{Y}:(0,1 / 2,0)$ & yes & Plane \\
\hline$\Gamma:(0,0,0)$ & $\mathrm{V}:(\mathrm{u}, \mathrm{v}, 0)$ & $\mathrm{Y}:(0,1 / 2,0)$ & yes & Plane \\
\hline$\Gamma:(0,0,0)$ & $\mathrm{K}:(0, \mathrm{v}, \mathrm{w})$ & $\mathrm{Z}:(0,0,1 / 2)$ & yes & Plane \\
\hline$\Gamma:(0,0,0)$ & $\mathrm{M}:(\mathrm{u}, 0, \mathrm{w})$ & $\mathrm{Z}:(0,0,1 / 2)$ & yes & Plane \\
\hline $\mathrm{R}:(1 / 2,1 / 2,1 / 2)$ & $\mathrm{L}:(1 / 2, \mathrm{v}, \mathrm{w})$ & $\mathrm{S}:(1 / 2,1 / 2,0)$ & yes & Plane \\
\hline $\mathrm{R}:(1 / 2,1 / 2,1 / 2)$ & $\mathrm{N}:(\mathrm{u}, 1 / 2, \mathrm{w})$ & $\mathrm{S}:(1 / 2,1 / 2,0)$ & yes & Plane \\
\hline $\mathrm{R}:(1 / 2,1 / 2,1 / 2)$ & $\mathrm{N}:(\mathrm{u}, 1 / 2, \mathrm{w})$ & $\mathrm{T}:(0,1 / 2,1 / 2)$ & yes & Plane \\
\hline $\mathrm{R}:(1 / 2,1 / 2,1 / 2)$ & $\mathrm{W}:(\mathrm{u}, \mathrm{v}, 1 / 2)$ & $\mathrm{T}:(0,1 / 2,1 / 2)$ & yes & Plane \\
\hline $\mathrm{R}:(1 / 2,1 / 2,1 / 2)$ & $\mathrm{L}:(1 / 2, \mathrm{v}, \mathrm{w})$ & $\mathrm{U}:(1 / 2,0,1 / 2)$ & yes & Plane \\
\hline $\mathrm{R}:(1 / 2,1 / 2,1 / 2)$ & $\mathrm{W}:(\mathrm{u}, \mathrm{v}, 1 / 2)$ & $\mathrm{U}:(1 / 2,0,1 / 2)$ & yes & Plane \\
\hline $\mathrm{R}:(1 / 2,1 / 2,1 / 2)$ & $\mathrm{L}:(1 / 2, \mathrm{v}, \mathrm{w})$ & $\mathrm{X}:(1 / 2,0,0)$ & yes & Plane \\
\hline $\mathrm{R}:(1 / 2,1 / 2,1 / 2)$ & $\mathrm{N}:(\mathrm{u}, 1 / 2, \mathrm{w})$ & $\mathrm{Y}:(0,1 / 2,0)$ & yes & Plane \\
\hline $\mathrm{R}:(1 / 2,1 / 2,1 / 2)$ & $\mathrm{W}:(\mathrm{u}, \mathrm{v}, 1 / 2)$ & $\mathrm{Z}:(0,0,1 / 2)$ & yes & Plane \\
\hline $\mathrm{S}:(1 / 2,1 / 2,0)$ & $\mathrm{N}:(\mathrm{u}, 1 / 2, \mathrm{w})$ & $\mathrm{T}:(0,1 / 2,1 / 2)$ & yes & Plane \\
\hline $\mathrm{S}:(1 / 2,1 / 2,0)$ & $\mathrm{L}:(1 / 2, \mathrm{v}, \mathrm{w})$ & $\mathrm{U}:(1 / 2,0,1 / 2)$ & yes & Plane \\
\hline $\mathrm{S}:(1 / 2,1 / 2,0)$ & $\mathrm{L}:(1 / 2, \mathrm{v}, \mathrm{w})$ & $\mathrm{X}:(1 / 2,0,0)$ & yes & Plane \\
\hline $\mathrm{S}:(1 / 2,1 / 2,0)$ & $\mathrm{V}:(\mathrm{u}, \mathrm{v}, 0)$ & $\mathrm{X}:(1 / 2,0,0)$ & yes & Plane \\
\hline $\mathrm{S}:(1 / 2,1 / 2,0)$ & $\mathrm{N}:(\mathrm{u}, 1 / 2, \mathrm{w})$ & $\mathrm{Y}:(0,1 / 2,0)$ & yes & Plane \\
\hline $\mathrm{S}:(1 / 2,1 / 2,0)$ & $\mathrm{V}:(\mathrm{u}, \mathrm{v}, 0)$ & $\mathrm{Y}:(0,1 / 2,0)$ & yes & Plane \\
\hline $\mathrm{T}:(0,1 / 2,1 / 2)$ & $\mathrm{W}:(\mathrm{u}, \mathrm{v}, 1 / 2)$ & $\mathrm{U}:(1 / 2,0,1 / 2)$ & yes & Plane \\
\hline $\mathrm{T}:(0,1 / 2,1 / 2)$ & $\mathrm{K}:(0, \mathrm{v}, \mathrm{w})$ & $\mathrm{Y}:(0,1 / 2,0)$ & yes & Plane \\
\hline $\mathrm{T}:(0,1 / 2,1 / 2)$ & $\mathrm{N}:(\mathrm{u}, 1 / 2, \mathrm{w})$ & $Y:(0,1 / 2,0)$ & yes & Plane \\
\hline $\mathrm{T}:(0,1 / 2,1 / 2)$ & $\mathrm{K}:(0, \mathrm{v}, \mathrm{w})$ & $\mathrm{Z}:(0,0,1 / 2)$ & yes & Plane \\
\hline $\mathrm{T}:(0,1 / 2,1 / 2)$ & $\mathrm{W}:(\mathrm{u}, \mathrm{v}, 1 / 2)$ & $\mathrm{Z}:(0,0,1 / 2)$ & yes & Plane \\
\hline $\mathrm{U}:(1 / 2,0,1 / 2)$ & $\mathrm{L}:(1 / 2, \mathrm{v}, \mathrm{w})$ & $\mathrm{X}:(1 / 2,0,0)$ & yes & Plane \\
\hline $\mathrm{U}:(1 / 2,0,1 / 2)$ & $\mathrm{M}:(\mathrm{u}, 0, \mathrm{w})$ & $\mathrm{X}:(1 / 2,0,0)$ & yes & Plane \\
\hline $\mathrm{U}:(1 / 2,0,1 / 2)$ & $\mathrm{M}:(\mathrm{u}, 0, \mathrm{w})$ & $\mathrm{Z}:(0,0,1 / 2)$ & yes & Plane \\
\hline $\mathrm{U}:(1 / 2,0,1 / 2)$ & $\mathrm{W}:(\mathrm{u}, \mathrm{v}, 1 / 2)$ & $\mathrm{Z}:(0,0,1 / 2)$ & yes & Plane \\
\hline $\mathrm{X}:(1 / 2,0,0)$ & $\mathrm{V}:(\mathrm{u}, \mathrm{v}, 0)$ & $\mathrm{Y}:(0,1 / 2,0)$ & yes & Plane \\
\hline $\mathrm{X}:(1 / 2,0,0)$ & $\mathrm{M}:(\mathrm{u}, 0, \mathrm{w})$ & $\mathrm{Z}:(0,0,1 / 2)$ & yes & Plane \\
\hline$Y:(0,1 / 2,0)$ & $\mathrm{K}:(0, \mathrm{v}, \mathrm{w})$ & $\mathrm{Z}:(0,0,1 / 2)$ & yes & Plane \\
\hline
\end{tabular}




\begin{tabular}{|c|c|c|c|c|}
\hline \multicolumn{5}{|c|}{ BCSID: 0.219; Formula: Co2SiO4; MSG: 62.441 (Pnma); U=0 } \\
\hline maximal $k_{1}$ & intermediate path & maximal $k_{2}$ & satisfied? & Line/Plane \\
\hline $\mathrm{R}:(1 / 2,1 / 2,1 / 2)$ & $\mathrm{Q}:(1 / 2,1 / 2, \mathrm{w})$ & $\mathrm{S}:(1 / 2,1 / 2,0)$ & yes & Line \\
\hline $\mathrm{S}:(1 / 2,1 / 2,0)$ & $\mathrm{D}:(1 / 2, \mathrm{v}, 0)$ & $\mathrm{X}:(1 / 2,0,0)$ & no & Line \\
\hline $\mathrm{T}:(0,1 / 2,1 / 2)$ & $\mathrm{H}:(0,1 / 2, \mathrm{w})$ & $\mathrm{Y}:(0,1 / 2,0)$ & yes & Line \\
\hline $\mathrm{T}:(0,1 / 2,1 / 2)$ & $\mathrm{B}:(0, \mathrm{v}, 1 / 2)$ & $\mathrm{Z}:(0,0,1 / 2)$ & yes & Line \\
\hline$\Gamma:(0,0,0)$ & $\mathrm{V}:(\mathrm{u}, \mathrm{v}, 0)$ & $\mathrm{S}:(1 / 2,1 / 2,0)$ & yes & Plane \\
\hline$\Gamma:(0,0,0)$ & $\mathrm{K}:(0, \mathrm{v}, \mathrm{w})$ & $\mathrm{T}:(0,1 / 2,1 / 2)$ & yes & Plane \\
\hline$\Gamma:(0,0,0)$ & $\mathrm{M}:(\mathrm{u}, 0, \mathrm{w})$ & $\mathrm{U}:(1 / 2,0,1 / 2)$ & yes & Plane \\
\hline$\Gamma:(0,0,0)$ & $\mathrm{M}:(\mathrm{u}, 0, \mathrm{w})$ & $\mathrm{X}:(1 / 2,0,0)$ & yes & Plane \\
\hline$\Gamma:(0,0,0)$ & $\mathrm{V}:(\mathrm{u}, \mathrm{v}, 0)$ & $\mathrm{X}:(1 / 2,0,0)$ & yes & Plane \\
\hline$\Gamma:(0,0,0)$ & $\mathrm{K}:(0, \mathrm{v}, \mathrm{w})$ & $\mathrm{Y}:(0,1 / 2,0)$ & yes & Plane \\
\hline$\Gamma:(0,0,0)$ & $\mathrm{V}:(\mathrm{u}, \mathrm{v}, 0)$ & $Y:(0,1 / 2,0)$ & yes & Plane \\
\hline$\Gamma:(0,0,0)$ & $\mathrm{K}:(0, \mathrm{v}, \mathrm{w})$ & $\mathrm{Z}:(0,0,1 / 2)$ & yes & Plane \\
\hline$\Gamma:(0,0,0)$ & $\mathrm{M}:(\mathrm{u}, 0, \mathrm{w})$ & $\mathrm{Z}:(0,0,1 / 2)$ & yes & Plane \\
\hline $\mathrm{R}:(1 / 2,1 / 2,1 / 2)$ & $\mathrm{L}:(1 / 2, \mathrm{v}, \mathrm{w})$ & $\mathrm{S}:(1 / 2,1 / 2,0)$ & yes & Plane \\
\hline $\mathrm{R}:(1 / 2,1 / 2,1 / 2)$ & $\mathrm{N}:(\mathrm{u}, 1 / 2, \mathrm{w})$ & $\mathrm{S}:(1 / 2,1 / 2,0)$ & yes & Plane \\
\hline $\mathrm{R}:(1 / 2,1 / 2,1 / 2)$ & $\mathrm{N}:(\mathrm{u}, 1 / 2, \mathrm{w})$ & $\mathrm{T}:(0,1 / 2,1 / 2)$ & yes & Plane \\
\hline $\mathrm{R}:(1 / 2,1 / 2,1 / 2)$ & $\mathrm{W}:(\mathrm{u}, \mathrm{v}, 1 / 2)$ & $\mathrm{T}:(0,1 / 2,1 / 2)$ & yes & Plane \\
\hline $\mathrm{R}:(1 / 2,1 / 2,1 / 2)$ & $\mathrm{L}:(1 / 2, \mathrm{v}, \mathrm{w})$ & $\mathrm{U}:(1 / 2,0,1 / 2)$ & yes & Plane \\
\hline $\mathrm{R}:(1 / 2,1 / 2,1 / 2)$ & $\mathrm{W}:(\mathrm{u}, \mathrm{v}, 1 / 2)$ & $\mathrm{U}:(1 / 2,0,1 / 2)$ & yes & Plane \\
\hline $\mathrm{R}:(1 / 2,1 / 2,1 / 2)$ & $\mathrm{L}:(1 / 2, \mathrm{v}, \mathrm{w})$ & $\mathrm{X}:(1 / 2,0,0)$ & yes & Plane \\
\hline $\mathrm{R}:(1 / 2,1 / 2,1 / 2)$ & $\mathrm{N}:(\mathrm{u}, 1 / 2, \mathrm{w})$ & $\mathrm{Y}:(0,1 / 2,0)$ & yes & Plane \\
\hline $\mathrm{R}:(1 / 2,1 / 2,1 / 2)$ & $\mathrm{W}:(\mathrm{u}, \mathrm{v}, 1 / 2)$ & $\mathrm{Z}:(0,0,1 / 2)$ & yes & Plane \\
\hline $\mathrm{S}:(1 / 2,1 / 2,0)$ & $\mathrm{N}:(\mathrm{u}, 1 / 2, \mathrm{w})$ & $\mathrm{T}:(0,1 / 2,1 / 2)$ & yes & Plane \\
\hline $\mathrm{S}:(1 / 2,1 / 2,0)$ & $\mathrm{L}:(1 / 2, \mathrm{v}, \mathrm{w})$ & $\mathrm{U}:(1 / 2,0,1 / 2)$ & yes & Plane \\
\hline $\mathrm{S}:(1 / 2,1 / 2,0)$ & $\mathrm{L}:(1 / 2, \mathrm{v}, \mathrm{w})$ & $\mathrm{X}:(1 / 2,0,0)$ & yes & Plane \\
\hline $\mathrm{S}:(1 / 2,1 / 2,0)$ & $\mathrm{V}:(\mathrm{u}, \mathrm{v}, 0)$ & $\mathrm{X}:(1 / 2,0,0)$ & yes & Plane \\
\hline $\mathrm{S}:(1 / 2,1 / 2,0)$ & $\mathrm{N}:(\mathrm{u}, 1 / 2, \mathrm{w})$ & $\mathrm{Y}:(0,1 / 2,0)$ & yes & Plane \\
\hline $\mathrm{S}:(1 / 2,1 / 2,0)$ & $\mathrm{V}:(\mathrm{u}, \mathrm{v}, 0)$ & $\mathrm{Y}:(0,1 / 2,0)$ & yes & Plane \\
\hline $\mathrm{T}:(0,1 / 2,1 / 2)$ & $\mathrm{W}:(\mathrm{u}, \mathrm{v}, 1 / 2)$ & $\mathrm{U}:(1 / 2,0,1 / 2)$ & yes & Plane \\
\hline $\mathrm{T}:(0,1 / 2,1 / 2)$ & $\mathrm{K}:(0, \mathrm{v}, \mathrm{w})$ & $Y:(0,1 / 2,0)$ & yes & Plane \\
\hline $\mathrm{T}:(0,1 / 2,1 / 2)$ & $\mathrm{N}:(\mathrm{u}, 1 / 2, \mathrm{w})$ & $\mathrm{Y}:(0,1 / 2,0)$ & yes & Plane \\
\hline $\mathrm{T}:(0,1 / 2,1 / 2)$ & $\mathrm{K}:(0, \mathrm{v}, \mathrm{w})$ & $\mathrm{Z}:(0,0,1 / 2)$ & yes & Plane \\
\hline $\mathrm{T}:(0,1 / 2,1 / 2)$ & $\mathrm{W}:(\mathrm{u}, \mathrm{v}, 1 / 2)$ & $\mathrm{Z}:(0,0,1 / 2)$ & yes & Plane \\
\hline $\mathrm{U}:(1 / 2,0,1 / 2)$ & $\mathrm{L}:(1 / 2, \mathrm{v}, \mathrm{w})$ & $\mathrm{X}:(1 / 2,0,0)$ & yes & Plane \\
\hline $\mathrm{U}:(1 / 2,0,1 / 2)$ & $\mathrm{M}:(\mathrm{u}, 0, \mathrm{w})$ & $\mathrm{X}:(1 / 2,0,0)$ & yes & Plane \\
\hline $\mathrm{U}:(1 / 2,0,1 / 2)$ & $\mathrm{M}:(\mathrm{u}, 0, \mathrm{w})$ & $\mathrm{Z}:(0,0,1 / 2)$ & yes & Plane \\
\hline $\mathrm{U}:(1 / 2,0,1 / 2)$ & $\mathrm{W}:(\mathrm{u}, \mathrm{v}, 1 / 2)$ & $\mathrm{Z}:(0,0,1 / 2)$ & yes & Plane \\
\hline $\mathrm{X}:(1 / 2,0,0)$ & $\mathrm{V}:(\mathrm{u}, \mathrm{v}, 0)$ & $\mathrm{Y}:(0,1 / 2,0)$ & yes & Plane \\
\hline $\mathrm{X}:(1 / 2,0,0)$ & $\mathrm{M}:(\mathrm{u}, 0, \mathrm{w})$ & $\mathrm{Z}:(0,0,1 / 2)$ & yes & Plane \\
\hline $\mathrm{Y}:(0,1 / 2,0)$ & $\mathrm{K}:(0, \mathrm{v}, \mathrm{w})$ & $\mathrm{Z}:(0,0,1 / 2)$ & yes & Plane \\
\hline
\end{tabular}

BCSID: 0.219; Formula: Co2SiO4; MSG: 62.441 (Pnma); $\mathrm{U}=1 \mathrm{eV}$

\begin{tabular}{c|c|c|c|c}
\hline maximal $k_{1}$ & intermediate path & maximal $k_{2}$ & satisfied? & Line/Plane \\
\hline $\mathrm{R}:(1 / 2,1 / 2,1 / 2)$ & $\mathrm{Q}:(1 / 2,1 / 2, \mathrm{w})$ & $\mathrm{S}:(1 / 2,1 / 2,0)$ & yes & Line \\
\hline $\mathrm{S}:(1 / 2,1 / 2,0)$ & $\mathrm{D}:(1 / 2, \mathrm{v}, 0)$ & $\mathrm{X}:(1 / 2,0,0)$ & no & Line \\
\hline $\mathrm{T}:(0,1 / 2,1 / 2)$ & $\mathrm{H}:(0,1 / 2, \mathrm{w})$ & $\mathrm{Y}:(0,1 / 2,0)$ & yes & Line \\
\hline $\mathrm{T}:(0,1 / 2,1 / 2)$ & $\mathrm{B}:(0, \mathrm{v}, 1 / 2)$ & $\mathrm{Z}:(0,0,1 / 2)$ & yes & Line \\
\hline$\Gamma:(0,0,0)$ & $\mathrm{V}:(\mathrm{u}, \mathrm{v}, 0)$ & $\mathrm{S}:(1 / 2,1 / 2,0)$ & yes & Plane
\end{tabular}




\begin{tabular}{|c|c|c|c|c|}
\hline$\Gamma:(0,0,0)$ & $\mathrm{K}:(0, \mathrm{v}, \mathrm{w})$ & $\mathrm{T}:(0,1 / 2,1 / 2)$ & yes & Plane \\
\hline$\Gamma:(0,0,0)$ & $\mathrm{M}:(\mathrm{u}, 0, \mathrm{w})$ & $\mathrm{U}:(1 / 2,0,1 / 2)$ & yes & Plane \\
\hline$\Gamma:(0,0,0)$ & $\mathrm{M}:(\mathrm{u}, 0, \mathrm{w})$ & $\mathrm{X}:(1 / 2,0,0)$ & yes & Plane \\
\hline$\Gamma:(0,0,0)$ & $\mathrm{V}:(\mathrm{u}, \mathrm{v}, 0)$ & $\mathrm{X}:(1 / 2,0,0)$ & yes & Plane \\
\hline$\Gamma:(0,0,0)$ & $\mathrm{K}:(0, \mathrm{v}, \mathrm{w})$ & $Y:(0,1 / 2,0)$ & yes & Plane \\
\hline$\Gamma:(0,0,0)$ & $\mathrm{V}:(\mathrm{u}, \mathrm{v}, 0)$ & $Y:(0,1 / 2,0)$ & yes & Plane \\
\hline$\Gamma:(0,0,0)$ & $\mathrm{K}:(0, \mathrm{v}, \mathrm{w})$ & $\mathrm{Z}:(0,0,1 / 2)$ & yes & Plane \\
\hline$\Gamma:(0,0,0)$ & $\mathrm{M}:(\mathrm{u}, 0, \mathrm{w})$ & $\mathrm{Z}:(0,0,1 / 2)$ & yes & Plane \\
\hline $\mathrm{R}:(1 / 2,1 / 2,1 / 2)$ & $\mathrm{L}:(1 / 2, \mathrm{v}, \mathrm{w})$ & $\mathrm{S}:(1 / 2,1 / 2,0)$ & yes & Plane \\
\hline $\mathrm{R}:(1 / 2,1 / 2,1 / 2)$ & $\mathrm{N}:(\mathrm{u}, 1 / 2, \mathrm{w})$ & $\mathrm{S}:(1 / 2,1 / 2,0)$ & yes & Plane \\
\hline $\mathrm{R}:(1 / 2,1 / 2,1 / 2)$ & $\mathrm{N}:(\mathrm{u}, 1 / 2, \mathrm{w})$ & $\mathrm{T}:(0,1 / 2,1 / 2)$ & yes & Plane \\
\hline $\mathrm{R}:(1 / 2,1 / 2,1 / 2)$ & $\mathrm{W}:(\mathrm{u}, \mathrm{v}, 1 / 2)$ & $\mathrm{T}:(0,1 / 2,1 / 2)$ & yes & Plane \\
\hline $\mathrm{R}:(1 / 2,1 / 2,1 / 2)$ & $\mathrm{L}:(1 / 2, \mathrm{v}, \mathrm{w})$ & $\mathrm{U}:(1 / 2,0,1 / 2)$ & yes & Plane \\
\hline $\mathrm{R}:(1 / 2,1 / 2,1 / 2)$ & $\mathrm{W}:(\mathrm{u}, \mathrm{v}, 1 / 2)$ & $\mathrm{U}:(1 / 2,0,1 / 2)$ & yes & Plane \\
\hline $\mathrm{R}:(1 / 2,1 / 2,1 / 2)$ & $\mathrm{L}:(1 / 2, \mathrm{v}, \mathrm{w})$ & $\mathrm{X}:(1 / 2,0,0)$ & yes & Plane \\
\hline $\mathrm{R}:(1 / 2,1 / 2,1 / 2)$ & $\mathrm{N}:(\mathrm{u}, 1 / 2, \mathrm{w})$ & $Y:(0,1 / 2,0)$ & yes & Plane \\
\hline $\mathrm{R}:(1 / 2,1 / 2,1 / 2)$ & $\mathrm{W}:(\mathrm{u}, \mathrm{v}, 1 / 2)$ & $\mathrm{Z}:(0,0,1 / 2)$ & yes & Plane \\
\hline $\mathrm{S}:(1 / 2,1 / 2,0)$ & $\mathrm{N}:(\mathrm{u}, 1 / 2, \mathrm{w})$ & $\mathrm{T}:(0,1 / 2,1 / 2)$ & yes & Plane \\
\hline $\mathrm{S}:(1 / 2,1 / 2,0)$ & $\mathrm{L}:(1 / 2, \mathrm{v}, \mathrm{w})$ & $\mathrm{U}:(1 / 2,0,1 / 2)$ & yes & Plane \\
\hline $\mathrm{S}:(1 / 2,1 / 2,0)$ & $\mathrm{L}:(1 / 2, \mathrm{v}, \mathrm{w})$ & $\mathrm{X}:(1 / 2,0,0)$ & yes & Plane \\
\hline $\mathrm{S}:(1 / 2,1 / 2,0)$ & $\mathrm{V}:(\mathrm{u}, \mathrm{v}, 0)$ & $\mathrm{X}:(1 / 2,0,0)$ & yes & Plane \\
\hline $\mathrm{S}:(1 / 2,1 / 2,0)$ & $\mathrm{N}:(\mathrm{u}, 1 / 2, \mathrm{w})$ & $\mathrm{Y}:(0,1 / 2,0)$ & yes & Plane \\
\hline $\mathrm{S}:(1 / 2,1 / 2,0)$ & $\mathrm{V}:(\mathrm{u}, \mathrm{v}, 0)$ & $\mathrm{Y}:(0,1 / 2,0)$ & yes & Plane \\
\hline $\mathrm{T}:(0,1 / 2,1 / 2)$ & $\mathrm{W}:(\mathrm{u}, \mathrm{v}, 1 / 2)$ & $\mathrm{U}:(1 / 2,0,1 / 2)$ & yes & Plane \\
\hline $\mathrm{T}:(0,1 / 2,1 / 2)$ & $\mathrm{K}:(0, \mathrm{v}, \mathrm{w})$ & $\mathrm{Y}:(0,1 / 2,0)$ & yes & Plane \\
\hline $\mathrm{T}:(0,1 / 2,1 / 2)$ & $\mathrm{N}:(\mathrm{u}, 1 / 2, \mathrm{w})$ & $\mathrm{Y}:(0,1 / 2,0)$ & yes & Plane \\
\hline $\mathrm{T}:(0,1 / 2,1 / 2)$ & $\mathrm{K}:(0, \mathrm{v}, \mathrm{w})$ & $\mathrm{Z}:(0,0,1 / 2)$ & yes & Plane \\
\hline $\mathrm{T}:(0,1 / 2,1 / 2)$ & $\mathrm{W}:(\mathrm{u}, \mathrm{v}, 1 / 2)$ & $\mathrm{Z}:(0,0,1 / 2)$ & yes & Plane \\
\hline $\mathrm{U}:(1 / 2,0,1 / 2)$ & $\mathrm{L}:(1 / 2, \mathrm{v}, \mathrm{w})$ & $\mathrm{X}:(1 / 2,0,0)$ & yes & Plane \\
\hline $\mathrm{U}:(1 / 2,0,1 / 2)$ & $\mathrm{M}:(\mathrm{u}, 0, \mathrm{w})$ & $\mathrm{X}:(1 / 2,0,0)$ & yes & Plane \\
\hline $\mathrm{U}:(1 / 2,0,1 / 2)$ & $\mathrm{M}:(\mathrm{u}, 0, \mathrm{w})$ & $\mathrm{Z}:(0,0,1 / 2)$ & yes & Plane \\
\hline $\mathrm{U}:(1 / 2,0,1 / 2)$ & $\mathrm{W}:(\mathrm{u}, \mathrm{v}, 1 / 2)$ & $\mathrm{Z}:(0,0,1 / 2)$ & yes & Plane \\
\hline $\mathrm{X}:(1 / 2,0,0)$ & $\mathrm{V}:(\mathrm{u}, \mathrm{v}, 0)$ & $\mathrm{Y}:(0,1 / 2,0)$ & yes & Plane \\
\hline $\mathrm{X}:(1 / 2,0,0)$ & $\mathrm{M}:(\mathrm{u}, 0, \mathrm{w})$ & $\mathrm{Z}:(0,0,1 / 2)$ & yes & Plane \\
\hline $\mathrm{Y}:(0,1 / 2,0)$ & $\mathrm{K}:(0, \mathrm{v}, \mathrm{w})$ & $\mathrm{Z}:(0,0,1 / 2)$ & yes & Plane \\
\hline
\end{tabular}

BCSID: 0.221; Formula: Fe2SiO4; MSG: 62.441 (Pnma); U=0

\begin{tabular}{c|c|c|c|c}
\hline maximal $k_{1}$ & intermediate path & maximal $k_{2}$ & satisfied? & Line/Plane \\
\hline $\mathrm{R}:(1 / 2,1 / 2,1 / 2)$ & $\mathrm{Q}:(1 / 2,1 / 2, \mathrm{w})$ & $\mathrm{S}:(1 / 2,1 / 2,0)$ & yes & Line \\
\hline $\mathrm{S}:(1 / 2,1 / 2,0)$ & $\mathrm{D}:(1 / 2, \mathrm{v}, 0)$ & $\mathrm{X}:(1 / 2,0,0)$ & no & Line \\
\hline $\mathrm{T}:(0,1 / 2,1 / 2)$ & $\mathrm{H}:(0,1 / 2, \mathrm{w})$ & $\mathrm{Y}:(0,1 / 2,0)$ & yes & Line \\
\hline $\mathrm{T}:(0,1 / 2,1 / 2)$ & $\mathrm{B}:(0, \mathrm{v}, 1 / 2)$ & $\mathrm{Z}:(0,0,1 / 2)$ & no & Line \\
\hline$\Gamma:(0,0,0)$ & $\mathrm{V}:(\mathrm{u}, \mathrm{v}, 0)$ & $\mathrm{S}:(1 / 2,1 / 2,0)$ & yes & Plane \\
\hline$\Gamma:(0,0,0)$ & $\mathrm{K}:(0, \mathrm{v}, \mathrm{w})$ & $\mathrm{T}:(0,1 / 2,1 / 2)$ & yes & Plane \\
\hline$\Gamma:(0,0,0)$ & $\mathrm{M}:(\mathrm{u}, 0, \mathrm{w})$ & $\mathrm{U}:(1 / 2,0,1 / 2)$ & yes & Plane \\
\hline$\Gamma:(0,0,0)$ & $\mathrm{M}:(\mathrm{u}, 0, \mathrm{w})$ & $\mathrm{X}:(1 / 2,0,0)$ & yes & Plane \\
\hline$\Gamma:(0,0,0)$ & $\mathrm{V}:(\mathrm{u}, \mathrm{v}, 0)$ & $\mathrm{X}:(1 / 2,0,0)$ & yes & Plane \\
\hline$\Gamma:(0,0,0)$ & $\mathrm{K}:(0, \mathrm{v}, \mathrm{w})$ & $\mathrm{Y}:(0,1 / 2,0)$ & yes & Plane \\
\hline$\Gamma:(0,0,0)$ & $\mathrm{V}:(\mathrm{u}, \mathrm{v}, 0)$ & $\mathrm{Y}:(0,1 / 2,0)$ & yes & Plane \\
\hline$\Gamma:(0,0,0)$ & $\mathrm{K}:(0, \mathrm{v}, \mathrm{w})$ & $\mathrm{Z}:(0,0,1 / 2)$ & yes & Plane \\
\hline$\Gamma:(0,0,0)$ & $\mathrm{M}:(\mathrm{u}, 0, \mathrm{w})$ & $\mathrm{Z}:(0,0,1 / 2)$ & yes & Plane
\end{tabular}




\begin{tabular}{|c|c|c|c|c|}
\hline $\mathrm{R}:(1 / 2,1 / 2,1 / 2)$ & $\mathrm{L}:(1 / 2, \mathrm{v}, \mathrm{w})$ & $\mathrm{S}:(1 / 2,1 / 2,0)$ & yes & Plane \\
\hline $\mathrm{R}:(1 / 2,1 / 2,1 / 2)$ & $\mathrm{N}:(\mathrm{u}, 1 / 2, \mathrm{w})$ & $\mathrm{S}:(1 / 2,1 / 2,0)$ & yes & Plane \\
\hline $\mathrm{R}:(1 / 2,1 / 2,1 / 2)$ & $\mathrm{N}:(\mathrm{u}, 1 / 2, \mathrm{w})$ & $\mathrm{T}:(0,1 / 2,1 / 2)$ & yes & Plane \\
\hline $\mathrm{R}:(1 / 2,1 / 2,1 / 2)$ & $\mathrm{W}:(\mathrm{u}, \mathrm{v}, 1 / 2)$ & $\mathrm{T}:(0,1 / 2,1 / 2)$ & yes & Plane \\
\hline $\mathrm{R}:(1 / 2,1 / 2,1 / 2)$ & $\mathrm{L}:(1 / 2, \mathrm{v}, \mathrm{w})$ & $\mathrm{U}:(1 / 2,0,1 / 2)$ & yes & Plane \\
\hline $\mathrm{R}:(1 / 2,1 / 2,1 / 2)$ & $\mathrm{W}:(\mathrm{u}, \mathrm{v}, 1 / 2)$ & $\mathrm{U}:(1 / 2,0,1 / 2)$ & yes & Plane \\
\hline $\mathrm{R}:(1 / 2,1 / 2,1 / 2)$ & $\mathrm{L}:(1 / 2, \mathrm{v}, \mathrm{w})$ & $\mathrm{X}:(1 / 2,0,0)$ & yes & Plane \\
\hline $\mathrm{R}:(1 / 2,1 / 2,1 / 2)$ & $\mathrm{N}:(\mathrm{u}, 1 / 2, \mathrm{w})$ & $\mathrm{Y}:(0,1 / 2,0)$ & yes & Plane \\
\hline $\mathrm{R}:(1 / 2,1 / 2,1 / 2)$ & $\mathrm{W}:(\mathrm{u}, \mathrm{v}, 1 / 2)$ & $\mathrm{Z}:(0,0,1 / 2)$ & yes & Plane \\
\hline $\mathrm{S}:(1 / 2,1 / 2,0)$ & $\mathrm{N}:(\mathrm{u}, 1 / 2, \mathrm{w})$ & $\mathrm{T}:(0,1 / 2,1 / 2)$ & yes & Plane \\
\hline $\mathrm{S}:(1 / 2,1 / 2,0)$ & $\mathrm{L}:(1 / 2, \mathrm{v}, \mathrm{w})$ & $\mathrm{U}:(1 / 2,0,1 / 2)$ & yes & Plane \\
\hline $\mathrm{S}:(1 / 2,1 / 2,0)$ & $\mathrm{L}:(1 / 2, \mathrm{v}, \mathrm{w})$ & $\mathrm{X}:(1 / 2,0,0)$ & yes & Plane \\
\hline $\mathrm{S}:(1 / 2,1 / 2,0)$ & $\mathrm{V}:(\mathrm{u}, \mathrm{v}, 0)$ & $\mathrm{X}:(1 / 2,0,0)$ & yes & Plane \\
\hline $\mathrm{S}:(1 / 2,1 / 2,0)$ & $\mathrm{N}:(\mathrm{u}, 1 / 2, \mathrm{w})$ & $\mathrm{Y}:(0,1 / 2,0)$ & yes & Plane \\
\hline $\mathrm{S}:(1 / 2,1 / 2,0)$ & $\mathrm{V}:(\mathrm{u}, \mathrm{v}, 0)$ & $\mathrm{Y}:(0,1 / 2,0)$ & yes & Plane \\
\hline $\mathrm{T}:(0,1 / 2,1 / 2)$ & $\mathrm{W}:(\mathrm{u}, \mathrm{v}, 1 / 2)$ & $\mathrm{U}:(1 / 2,0,1 / 2)$ & yes & Plane \\
\hline $\mathrm{T}:(0,1 / 2,1 / 2)$ & $\mathrm{K}:(0, \mathrm{v}, \mathrm{w})$ & $\mathrm{Y}:(0,1 / 2,0)$ & yes & Plane \\
\hline $\mathrm{T}:(0,1 / 2,1 / 2)$ & $\mathrm{N}:(\mathrm{u}, 1 / 2, \mathrm{w})$ & $Y:(0,1 / 2,0)$ & yes & Plane \\
\hline $\mathrm{T}:(0,1 / 2,1 / 2)$ & $\mathrm{K}:(0, \mathrm{v}, \mathrm{w})$ & $\mathrm{Z}:(0,0,1 / 2)$ & yes & Plane \\
\hline $\mathrm{T}:(0,1 / 2,1 / 2)$ & $\mathrm{W}:(\mathrm{u}, \mathrm{v}, 1 / 2)$ & $\mathrm{Z}:(0,0,1 / 2)$ & yes & Plane \\
\hline $\mathrm{U}:(1 / 2,0,1 / 2)$ & $\mathrm{L}:(1 / 2, \mathrm{v}, \mathrm{w})$ & $\mathrm{X}:(1 / 2,0,0)$ & yes & Plane \\
\hline $\mathrm{U}:(1 / 2,0,1 / 2)$ & $\mathrm{M}:(\mathrm{u}, 0, \mathrm{w})$ & $\mathrm{X}:(1 / 2,0,0)$ & yes & Plane \\
\hline $\mathrm{U}:(1 / 2,0,1 / 2)$ & $\mathrm{M}:(\mathrm{u}, 0, \mathrm{w})$ & $\mathrm{Z}:(0,0,1 / 2)$ & yes & Plane \\
\hline $\mathrm{U}:(1 / 2,0,1 / 2)$ & $\mathrm{W}:(\mathrm{u}, \mathrm{v}, 1 / 2)$ & $\mathrm{Z}:(0,0,1 / 2)$ & yes & Plane \\
\hline $\mathrm{X}:(1 / 2,0,0)$ & $\mathrm{V}:(\mathrm{u}, \mathrm{v}, 0)$ & $\mathrm{Y}:(0,1 / 2,0)$ & yes & Plane \\
\hline $\mathrm{X}:(1 / 2,0,0)$ & $\mathrm{M}:(\mathrm{u}, 0, \mathrm{w})$ & $\mathrm{Z}:(0,0,1 / 2)$ & yes & Plane \\
\hline $\mathrm{Y}:(0,1 / 2,0)$ & $\mathrm{K}:(0, \mathrm{v}, \mathrm{w})$ & $\mathrm{Z}:(0,0,1 / 2)$ & yes & Plane \\
\hline \multicolumn{5}{|c|}{ BCSID: 0.185; Formula: Nd5Ge4; MSG: $62.447\left(P n m^{\prime} a^{\prime}\right) ; \mathrm{U}=0$} \\
\hline maximal $k_{1}$ & intermediate path & maximal $k_{2}$ & satisfied? & Line/Plane \\
\hline$\Gamma:(0,0,0)$ & SM:(u,0,0) & $\mathrm{X}:(1 / 2,0,0)$ & no & Line \\
\hline$\Gamma:(0,0,0)$ & DT: $(0, \mathrm{v}, 0)$ & $\mathrm{Y}:(0,1 / 2,0)$ & yes & Line \\
\hline$\Gamma:(0,0,0)$ & $\mathrm{LD}:(0,0, \mathrm{w})$ & $\mathrm{Z}:(0,0,1 / 2)$ & yes & Line \\
\hline $\mathrm{R}:(1 / 2,1 / 2,1 / 2)$ & $\mathrm{Q}:(1 / 2,1 / 2, \mathrm{w})$ & $\mathrm{S}:(1 / 2,1 / 2,0)$ & yes & Line \\
\hline $\mathrm{R}:(1 / 2,1 / 2,1 / 2)$ & $\mathrm{E}:(\mathrm{u}, 1 / 2,1 / 2)$ & $\mathrm{T}:(0,1 / 2,1 / 2)$ & yes & Line \\
\hline $\mathrm{S}:(1 / 2,1 / 2,0)$ & $\mathrm{D}:(1 / 2, \mathrm{v}, 0)$ & $\mathrm{X}:(1 / 2,0,0)$ & yes & Line \\
\hline$\Gamma:(0,0,0)$ & $\mathrm{K}:(0, \mathrm{v}, \mathrm{w})$ & $\mathrm{T}:(0,1 / 2,1 / 2)$ & yes & Plane \\
\hline$\Gamma:(0,0,0)$ & $\mathrm{K}:(0, \mathrm{v}, \mathrm{w})$ & $\mathrm{Y}:(0,1 / 2,0)$ & yes & Plane \\
\hline$\Gamma:(0,0,0)$ & $\mathrm{K}:(0, \mathrm{v}, \mathrm{w})$ & $\mathrm{Z}:(0,0,1 / 2)$ & yes & Plane \\
\hline $\mathrm{R}:(1 / 2,1 / 2,1 / 2)$ & $\mathrm{L}:(1 / 2, \mathrm{v}, \mathrm{w})$ & $\mathrm{S}:(1 / 2,1 / 2,0)$ & yes & Plane \\
\hline $\mathrm{R}:(1 / 2,1 / 2,1 / 2)$ & $\mathrm{L}:(1 / 2, \mathrm{v}, \mathrm{w})$ & $\mathrm{U}:(1 / 2,0,1 / 2)$ & yes & Plane \\
\hline $\mathrm{R}:(1 / 2,1 / 2,1 / 2)$ & $\mathrm{L}:(1 / 2, \mathrm{v}, \mathrm{w})$ & $\mathrm{X}:(1 / 2,0,0)$ & yes & Plane \\
\hline $\mathrm{S}:(1 / 2,1 / 2,0)$ & $\mathrm{L}:(1 / 2, \mathrm{v}, \mathrm{w})$ & $\mathrm{U}:(1 / 2,0,1 / 2)$ & yes & Plane \\
\hline $\mathrm{S}:(1 / 2,1 / 2,0)$ & $\mathrm{L}:(1 / 2, \mathrm{v}, \mathrm{w})$ & $\mathrm{X}:(1 / 2,0,0)$ & yes & Plane \\
\hline $\mathrm{T}:(0,1 / 2,1 / 2)$ & $\mathrm{K}:(0, \mathrm{v}, \mathrm{w})$ & $\mathrm{Y}:(0,1 / 2,0)$ & yes & Plane \\
\hline $\mathrm{T}:(0,1 / 2,1 / 2)$ & $\mathrm{K}:(0, \mathrm{v}, \mathrm{w})$ & $\mathrm{Z}:(0,0,1 / 2)$ & yes & Plane \\
\hline $\mathrm{U}:(1 / 2,0,1 / 2)$ & $\mathrm{L}:(1 / 2, \mathrm{v}, \mathrm{w})$ & $\mathrm{X}:(1 / 2,0,0)$ & yes & Plane \\
\hline $\mathrm{Y}:(0,1 / 2,0)$ & $\mathrm{K}:(0, \mathrm{v}, \mathrm{w})$ & $\mathrm{Z}:(0,0,1 / 2)$ & yes & Plane \\
\hline
\end{tabular}

BCSID: 1.131; Formula: Fe2As; MSG: $62.450\left(P_{a} n m a\right) ; \mathrm{U}=2 \mathrm{eV}$

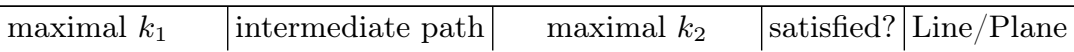




\begin{tabular}{c|c|c|c|c}
\hline $\mathrm{R}:(1 / 2,1 / 2,1 / 2)$ & $\mathrm{Q}:(1 / 2,1 / 2, \mathrm{w})$ & $\mathrm{S}:(1 / 2,1 / 2,0)$ & yes & Line \\
\hline $\mathrm{S}:(1 / 2,1 / 2,0)$ & $\mathrm{D}:(1 / 2, \mathrm{v}, 0)$ & $\mathrm{X}:(1 / 2,0,0)$ & no & Line \\
\hline $\mathrm{T}:(0,1 / 2,1 / 2)$ & $\mathrm{H}:(0,1 / 2, \mathrm{w})$ & $\mathrm{Y}:(0,1 / 2,0)$ & yes & Line \\
\hline $\mathrm{T}:(0,1 / 2,1 / 2)$ & $\mathrm{B}:(0, \mathrm{v}, 1 / 2)$ & $\mathrm{Z}:(0,0,1 / 2)$ & yes & Line \\
\hline $\mathrm{R}:(1 / 2,1 / 2,1 / 2)$ & $\mathrm{N}:(\mathrm{u}, 1 / 2, \mathrm{w})$ & $\mathrm{S}:(1 / 2,1 / 2,0)$ & yes & Plane \\
\hline $\mathrm{R}:(1 / 2,1 / 2,1 / 2)$ & $\mathrm{N}:(\mathrm{u}, 1 / 2, \mathrm{w})$ & $\mathrm{T}:(0,1 / 2,1 / 2)$ & yes & Plane \\
\hline $\mathrm{R}:(1 / 2,1 / 2,1 / 2)$ & $\mathrm{W}:(\mathrm{u}, \mathrm{v}, 1 / 2)$ & $\mathrm{T}:(0,1 / 2,1 / 2)$ & yes & Plane \\
\hline $\mathrm{R}:(1 / 2,1 / 2,1 / 2)$ & $\mathrm{W}:(\mathrm{u}, \mathrm{v}, 1 / 2)$ & $\mathrm{U}:(1 / 2,0,1 / 2)$ & yes & Plane \\
\hline $\mathrm{R}:(1 / 2,1 / 2,1 / 2)$ & $\mathrm{N}:(\mathrm{u}, 1 / 2, \mathrm{w})$ & $\mathrm{Y}:(0,1 / 2,0)$ & yes & Plane \\
\hline $\mathrm{R}:(1 / 2,1 / 2,1 / 2)$ & $\mathrm{W}:(\mathrm{u}, \mathrm{v}, 1 / 2)$ & $\mathrm{Z}:(0,0,1 / 2)$ & yes & Plane \\
\hline $\mathrm{S}:(1 / 2,1 / 2,0)$ & $\mathrm{N}:(\mathrm{u}, 1 / 2, \mathrm{w})$ & $\mathrm{T}:(0,1 / 2,1 / 2)$ & yes & Plane \\
\hline $\mathrm{S}:(1 / 2,1 / 2,0)$ & $\mathrm{N}:(\mathrm{u}, 1 / 2, \mathrm{w})$ & $\mathrm{Y}:(0,1 / 2,0)$ & yes & Plane \\
\hline $\mathrm{T}:(0,1 / 2,1 / 2)$ & $\mathrm{W}:(\mathrm{u}, \mathrm{v}, 1 / 2)$ & $\mathrm{U}:(1 / 2,0,1 / 2)$ & yes & Plane \\
\hline $\mathrm{T}:(0,1 / 2,1 / 2)$ & $\mathrm{N}:(\mathrm{u}, 1 / 2, \mathrm{w})$ & $\mathrm{Y}:(0,1 / 2,0)$ & yes & Plane \\
\hline $\mathrm{T}:(0,1 / 2,1 / 2)$ & $\mathrm{W}:(\mathrm{u}, \mathrm{v}, 1 / 2)$ & $\mathrm{Z}:(0,0,1 / 2)$ & yes & Plane \\
\hline $\mathrm{U}:(1 / 2,0,1 / 2)$ & $\mathrm{W}:(\mathrm{u}, \mathrm{v}, 1 / 2)$ & $\mathrm{Z}:(0,0,1 / 2)$ & yes & Plane \\
\hline
\end{tabular}

\begin{tabular}{c|c|c|c|c}
\hline \multicolumn{5}{c}{ BCSID: 1.132; Formula: Mn2As; MSG: $62.450\left(P_{a} n m a\right) ; \mathrm{U}=0$} \\
\hline maximal $k_{1}$ & intermediate path & maximal $k_{2}$ & satisfied? & Line/Plane \\
\hline $\mathrm{R}:(1 / 2,1 / 2,1 / 2)$ & $\mathrm{Q}:(1 / 2,1 / 2, \mathrm{w})$ & $\mathrm{S}:(1 / 2,1 / 2,0)$ & yes & Line \\
\hline $\mathrm{S}:(1 / 2,1 / 2,0)$ & $\mathrm{D}:(1 / 2, \mathrm{v}, 0)$ & $\mathrm{X}:(1 / 2,0,0)$ & no & Line \\
\hline $\mathrm{T}:(0,1 / 2,1 / 2)$ & $\mathrm{H}:(0,1 / 2, \mathrm{w})$ & $\mathrm{Y}:(0,1 / 2,0)$ & yes & Line \\
\hline $\mathrm{T}:(0,1 / 2,1 / 2)$ & $\mathrm{B}:(0, \mathrm{v}, 1 / 2)$ & $\mathrm{Z}:(0,0,1 / 2)$ & yes & Line \\
\hline $\mathrm{R}:(1 / 2,1 / 2,1 / 2)$ & $\mathrm{N}:(\mathrm{u}, 1 / 2, \mathrm{w})$ & $\mathrm{S}:(1 / 2,1 / 2,0)$ & yes & Plane \\
\hline $\mathrm{R}:(1 / 2,1 / 2,1 / 2)$ & $\mathrm{N}:(\mathrm{u}, 1 / 2, \mathrm{w})$ & $\mathrm{T}:(0,1 / 2,1 / 2)$ & yes & Plane \\
\hline $\mathrm{R}:(1 / 2,1 / 2,1 / 2)$ & $\mathrm{W}:(\mathrm{u}, \mathrm{v}, 1 / 2)$ & $\mathrm{T}:(0,1 / 2,1 / 2)$ & yes & Plane \\
\hline $\mathrm{R}:(1 / 2,1 / 2,1 / 2)$ & $\mathrm{W}:(\mathrm{u}, \mathrm{v}, 1 / 2)$ & $\mathrm{U}:(1 / 2,0,1 / 2)$ & yes & Plane \\
\hline $\mathrm{R}:(1 / 2,1 / 2,1 / 2)$ & $\mathrm{N}:(\mathrm{u}, 1 / 2, \mathrm{w})$ & $\mathrm{Y}:(0,1 / 2,0)$ & yes & Plane \\
\hline $\mathrm{R}:(1 / 2,1 / 2,1 / 2)$ & $\mathrm{W}:(\mathrm{u}, \mathrm{v}, 1 / 2)$ & $\mathrm{Z}:(0,0,1 / 2)$ & yes & Plane \\
\hline $\mathrm{S}:(1 / 2,1 / 2,0)$ & $\mathrm{N}:(\mathrm{u}, 1 / 2, \mathrm{w})$ & $\mathrm{T}:(0,1 / 2,1 / 2)$ & yes & Plane \\
\hline $\mathrm{S}:(1 / 2,1 / 2,0)$ & $\mathrm{N}:(\mathrm{u}, 1 / 2, \mathrm{w})$ & $\mathrm{Y}:(0,1 / 2,0)$ & yes & Plane \\
\hline $\mathrm{T}:(0,1 / 2,1 / 2)$ & $\mathrm{W}:(\mathrm{u}, \mathrm{v}, 1 / 2)$ & $\mathrm{U}:(1 / 2,0,1 / 2)$ & yes & Plane \\
\hline $\mathrm{T}:(0,1 / 2,1 / 2)$ & $\mathrm{N}:(\mathrm{u}, 1 / 2, \mathrm{w})$ & $\mathrm{Y}:(0,1 / 2,0)$ & yes & Plane \\
\hline $\mathrm{T}:(0,1 / 2,1 / 2)$ & $\mathrm{W}:(\mathrm{u}, \mathrm{v}, 1 / 2)$ & $\mathrm{Z}:(0,0,1 / 2)$ & yes & Plane \\
\hline $\mathrm{U}:(1 / 2,0,1 / 2)$ & $\mathrm{W}:(\mathrm{u}, \mathrm{v}, 1 / 2)$ & $\mathrm{Z}:(0,0,1 / 2)$ & yes & Plane \\
\hline
\end{tabular}

BCSID: 1.132; Formula: Mn2As; MSG: $62.450\left(P_{a} n m a\right) ; \mathrm{U}=1 \mathrm{eV}$

\begin{tabular}{c|c|c|c|c}
\hline maximal $k_{1}$ & intermediate path & maximal $k_{2}$ & satisfied? & Line/Plane \\
\hline $\mathrm{R}:(1 / 2,1 / 2,1 / 2)$ & $\mathrm{Q}:(1 / 2,1 / 2, \mathrm{w})$ & $\mathrm{S}:(1 / 2,1 / 2,0)$ & yes & Line \\
\hline $\mathrm{S}:(1 / 2,1 / 2,0)$ & $\mathrm{D}:(1 / 2, \mathrm{v}, 0)$ & $\mathrm{X}:(1 / 2,0,0)$ & no & Line \\
\hline $\mathrm{T}:(0,1 / 2,1 / 2)$ & $\mathrm{H}:(0,1 / 2, \mathrm{w})$ & $\mathrm{Y}:(0,1 / 2,0)$ & yes & Line \\
\hline $\mathrm{T}:(0,1 / 2,1 / 2)$ & $\mathrm{B}:(0, \mathrm{v}, 1 / 2)$ & $\mathrm{Z}:(0,0,1 / 2)$ & yes & Line \\
\hline $\mathrm{R}:(1 / 2,1 / 2,1 / 2)$ & $\mathrm{N}:(\mathrm{u}, 1 / 2, \mathrm{w})$ & $\mathrm{S}:(1 / 2,1 / 2,0)$ & yes & Plane \\
\hline $\mathrm{R}:(1 / 2,1 / 2,1 / 2)$ & $\mathrm{N}:(\mathrm{u}, 1 / 2, \mathrm{w})$ & $\mathrm{T}:(0,1 / 2,1 / 2)$ & yes & Plane \\
\hline $\mathrm{R}:(1 / 2,1 / 2,1 / 2)$ & $\mathrm{W}:(\mathrm{u}, \mathrm{v}, 1 / 2)$ & $\mathrm{T}:(0,1 / 2,1 / 2)$ & yes & Plane \\
\hline $\mathrm{R}:(1 / 2,1 / 2,1 / 2)$ & $\mathrm{W}:(\mathrm{u}, \mathrm{v}, 1 / 2)$ & $\mathrm{U}:(1 / 2,0,1 / 2)$ & yes & Plane \\
\hline $\mathrm{R}:(1 / 2,1 / 2,1 / 2)$ & $\mathrm{N}:(\mathrm{u}, 1 / 2, \mathrm{w})$ & $\mathrm{Y}:(0,1 / 2,0)$ & yes & Plane \\
\hline $\mathrm{R}:(1 / 2,1 / 2,1 / 2)$ & $\mathrm{W}:(\mathrm{u}, \mathrm{v}, 1 / 2)$ & $\mathrm{Z}:(0,0,1 / 2)$ & yes & Plane \\
\hline $\mathrm{S}:(1 / 2,1 / 2,0)$ & $\mathrm{N}:(\mathrm{u}, 1 / 2, \mathrm{w})$ & $\mathrm{T}:(0,1 / 2,1 / 2)$ & yes & Plane \\
\hline $\mathrm{S}:(1 / 2,1 / 2,0)$ & $\mathrm{N}:(\mathrm{u}, 1 / 2, \mathrm{w})$ & $\mathrm{Y}:(0,1 / 2,0)$ & yes & Plane \\
\hline $\mathrm{T}:(0,1 / 2,1 / 2)$ & $\mathrm{W}:(\mathrm{u}, \mathrm{v}, 1 / 2)$ & $\mathrm{U}:(1 / 2,0,1 / 2)$ & yes & Plane
\end{tabular}




\begin{tabular}{c|c|c|c|c}
\hline $\mathrm{T}:(0,1 / 2,1 / 2)$ & $\mathrm{N}:(\mathrm{u}, 1 / 2, \mathrm{w})$ & $\mathrm{Y}:(0,1 / 2,0)$ & yes & Plane \\
\hline $\mathrm{T}:(0,1 / 2,1 / 2)$ & $\mathrm{W}:(\mathrm{u}, \mathrm{v}, 1 / 2)$ & $\mathrm{Z}:(0,0,1 / 2)$ & yes & Plane \\
\hline $\mathrm{U}:(1 / 2,0,1 / 2)$ & $\mathrm{W}:(\mathrm{u}, \mathrm{v}, 1 / 2)$ & $\mathrm{Z}:(0,0,1 / 2)$ & yes & Plane \\
\hline
\end{tabular}

BCSID: 1.132; Formula: Mn2As; MSG: $62.450\left(P_{a} n m a\right)$; $\mathrm{U}=2 \mathrm{eV}$

\begin{tabular}{c|c|c|c|c}
\hline maximal $k_{1}$ & intermediate path & maximal $k_{2}$ & satisfied? & Line/Plane \\
\hline $\mathrm{R}:(1 / 2,1 / 2,1 / 2)$ & $\mathrm{Q}:(1 / 2,1 / 2, \mathrm{w})$ & $\mathrm{S}:(1 / 2,1 / 2,0)$ & yes & Line \\
\hline $\mathrm{S}:(1 / 2,1 / 2,0)$ & $\mathrm{D}:(1 / 2, \mathrm{v}, 0)$ & $\mathrm{X}:(1 / 2,0,0)$ & no & Line \\
\hline $\mathrm{T}:(0,1 / 2,1 / 2)$ & $\mathrm{H}:(0,1 / 2, \mathrm{w})$ & $\mathrm{Y}:(0,1 / 2,0)$ & yes & Line \\
\hline $\mathrm{T}:(0,1 / 2,1 / 2)$ & $\mathrm{B}:(0, \mathrm{v}, 1 / 2)$ & $\mathrm{Z}:(0,0,1 / 2)$ & yes & Line \\
\hline $\mathrm{R}:(1 / 2,1 / 2,1 / 2)$ & $\mathrm{N}:(\mathrm{u}, 1 / 2, \mathrm{w})$ & $\mathrm{S}:(1 / 2,1 / 2,0)$ & yes & Plane \\
\hline $\mathrm{R}:(1 / 2,1 / 2,1 / 2)$ & $\mathrm{N}:(\mathrm{u}, 1 / 2, \mathrm{w})$ & $\mathrm{T}:(0,1 / 2,1 / 2)$ & yes & Plane \\
\hline $\mathrm{R}:(1 / 2,1 / 2,1 / 2)$ & $\mathrm{W}:(\mathrm{u}, \mathrm{v}, 1 / 2)$ & $\mathrm{T}:(0,1 / 2,1 / 2)$ & yes & Plane \\
\hline $\mathrm{R}:(1 / 2,1 / 2,1 / 2)$ & $\mathrm{W}:(\mathrm{u}, \mathrm{v}, 1 / 2)$ & $\mathrm{U}:(1 / 2,0,1 / 2)$ & yes & Plane \\
\hline $\mathrm{R}:(1 / 2,1 / 2,1 / 2)$ & $\mathrm{N}:(\mathrm{u}, 1 / 2, \mathrm{w})$ & $\mathrm{Y}:(0,1 / 2,0)$ & yes & Plane \\
\hline $\mathrm{R}:(1 / 2,1 / 2,1 / 2)$ & $\mathrm{W}:(\mathrm{u}, \mathrm{v}, 1 / 2)$ & $\mathrm{Z}:(0,0,1 / 2)$ & yes & Plane \\
\hline $\mathrm{S}:(1 / 2,1 / 2,0)$ & $\mathrm{N}:(\mathrm{u}, 1 / 2, \mathrm{w})$ & $\mathrm{T}:(0,1 / 2,1 / 2)$ & yes & Plane \\
\hline $\mathrm{S}:(1 / 2,1 / 2,0)$ & $\mathrm{N}:(\mathrm{u}, 1 / 2, \mathrm{w})$ & $\mathrm{Y}:(0,1 / 2,0)$ & yes & Plane \\
\hline $\mathrm{T}:(0,1 / 2,1 / 2)$ & $\mathrm{W}:(\mathrm{u}, \mathrm{v}, 1 / 2)$ & $\mathrm{U}:(1 / 2,0,1 / 2)$ & yes & Plane \\
\hline $\mathrm{T}:(0,1 / 2,1 / 2)$ & $\mathrm{N}:(\mathrm{u}, 1 / 2, \mathrm{w})$ & $\mathrm{Y}:(0,1 / 2,0)$ & yes & Plane \\
\hline $\mathrm{T}:(0,1 / 2,1 / 2)$ & $\mathrm{W}:(\mathrm{u}, \mathrm{v}, 1 / 2)$ & $\mathrm{Z}:(0,0,1 / 2)$ & yes & Plane \\
\hline $\mathrm{U}:(1 / 2,0,1 / 2)$ & $\mathrm{W}:(\mathrm{u}, \mathrm{v}, 1 / 2)$ & $\mathrm{Z}:(0,0,1 / 2)$ & yes & Plane \\
\hline
\end{tabular}

BCSID: 1.132; Formula: Mn2As; MSG: $62.450\left(P_{a} n m a\right) ; \mathrm{U}=3 \mathrm{eV}$

\begin{tabular}{c|c|c|c|c}
\hline maximal $k_{1}$ & intermediate path & maximal $k_{2}$ & satisfied? & Line/Plane \\
\hline $\mathrm{R}:(1 / 2,1 / 2,1 / 2)$ & $\mathrm{Q}:(1 / 2,1 / 2, \mathrm{w})$ & $\mathrm{S}:(1 / 2,1 / 2,0)$ & yes & Line \\
\hline $\mathrm{S}:(1 / 2,1 / 2,0)$ & $\mathrm{D}:(1 / 2, \mathrm{v}, 0)$ & $\mathrm{X}:(1 / 2,0,0)$ & no & Line \\
\hline $\mathrm{T}:(0,1 / 2,1 / 2)$ & $\mathrm{H}:(0,1 / 2, \mathrm{w})$ & $\mathrm{Y}:(0,1 / 2,0)$ & yes & Line \\
\hline $\mathrm{T}:(0,1 / 2,1 / 2)$ & $\mathrm{B}:(0, \mathrm{v}, 1 / 2)$ & $\mathrm{Z}:(0,0,1 / 2)$ & yes & Line \\
\hline $\mathrm{R}:(1 / 2,1 / 2,1 / 2)$ & $\mathrm{N}:(\mathrm{u}, 1 / 2, \mathrm{w})$ & $\mathrm{S}:(1 / 2,1 / 2,0)$ & yes & Plane \\
\hline $\mathrm{R}:(1 / 2,1 / 2,1 / 2)$ & $\mathrm{N}:(\mathrm{u}, 1 / 2, \mathrm{w})$ & $\mathrm{T}:(0,1 / 2,1 / 2)$ & yes & Plane \\
\hline $\mathrm{R}:(1 / 2,1 / 2,1 / 2)$ & $\mathrm{W}:(\mathrm{u}, \mathrm{v}, 1 / 2)$ & $\mathrm{T}:(0,1 / 2,1 / 2)$ & yes & Plane \\
\hline $\mathrm{R}:(1 / 2,1 / 2,1 / 2)$ & $\mathrm{W}:(\mathrm{u}, \mathrm{v}, 1 / 2)$ & $\mathrm{U}:(1 / 2,0,1 / 2)$ & yes & Plane \\
\hline $\mathrm{R}:(1 / 2,1 / 2,1 / 2)$ & $\mathrm{N}:(\mathrm{u}, 1 / 2, \mathrm{w})$ & $\mathrm{Y}:(0,1 / 2,0)$ & yes & Plane \\
\hline $\mathrm{R}:(1 / 2,1 / 2,1 / 2)$ & $\mathrm{W}:(\mathrm{u}, \mathrm{v}, 1 / 2)$ & $\mathrm{Z}:(0,0,1 / 2)$ & yes & Plane \\
\hline $\mathrm{S}:(1 / 2,1 / 2,0)$ & $\mathrm{N}:(\mathrm{u}, 1 / 2, \mathrm{w})$ & $\mathrm{T}:(0,1 / 2,1 / 2)$ & yes & Plane \\
\hline $\mathrm{S}:(1 / 2,1 / 2,0)$ & $\mathrm{N}:(\mathrm{u}, 1 / 2, \mathrm{w})$ & $\mathrm{Y}:(0,1 / 2,0)$ & yes & Plane \\
\hline $\mathrm{T}:(0,1 / 2,1 / 2)$ & $\mathrm{W}:(\mathrm{u}, \mathrm{v}, 1 / 2)$ & $\mathrm{U}:(1 / 2,0,1 / 2)$ & yes & Plane \\
\hline $\mathrm{T}:(0,1 / 2,1 / 2)$ & $\mathrm{N}:(\mathrm{u}, 1 / 2, \mathrm{w})$ & $\mathrm{Y}:(0,1 / 2,0)$ & yes & Plane \\
\hline $\mathrm{T}:(0,1 / 2,1 / 2)$ & $\mathrm{W}:(\mathrm{u}, \mathrm{v}, 1 / 2)$ & $\mathrm{Z}:(0,0,1 / 2)$ & yes & Plane \\
\hline $\mathrm{U}:(1 / 2,0,1 / 2)$ & $\mathrm{W}:(\mathrm{u}, \mathrm{v}, 1 / 2)$ & $\mathrm{Z}:(0,0,1 / 2)$ & yes & Plane \\
\hline
\end{tabular}

BCSID: 1.179; Formula: NdCoAsO; MSG: $62.450\left(P_{a} n m a\right)$; U=4eV

\begin{tabular}{c|c|c|c|c}
\hline maximal $k_{1}$ & intermediate path & maximal $k_{2}$ & satisfied? & Line/Plane \\
\hline $\mathrm{R}:(1 / 2,1 / 2,1 / 2)$ & $\mathrm{Q}:(1 / 2,1 / 2, \mathrm{w})$ & $\mathrm{S}:(1 / 2,1 / 2,0)$ & yes & Line \\
\hline $\mathrm{S}:(1 / 2,1 / 2,0)$ & $\mathrm{D}:(1 / 2, \mathrm{v}, 0)$ & $\mathrm{X}:(1 / 2,0,0)$ & no & Line \\
\hline $\mathrm{T}:(0,1 / 2,1 / 2)$ & $\mathrm{H}:(0,1 / 2, \mathrm{w})$ & $\mathrm{Y}:(0,1 / 2,0)$ & yes & Line \\
\hline $\mathrm{T}:(0,1 / 2,1 / 2)$ & $\mathrm{B}:(0, \mathrm{v}, 1 / 2)$ & $\mathrm{Z}:(0,0,1 / 2)$ & yes & Line \\
\hline $\mathrm{R}:(1 / 2,1 / 2,1 / 2)$ & $\mathrm{N}:(\mathrm{u}, 1 / 2, \mathrm{w})$ & $\mathrm{S}:(1 / 2,1 / 2,0)$ & yes & Plane \\
\hline $\mathrm{R}:(1 / 2,1 / 2,1 / 2)$ & $\mathrm{N}:(\mathrm{u}, 1 / 2, \mathrm{w})$ & $\mathrm{T}:(0,1 / 2,1 / 2)$ & yes & Plane \\
\hline $\mathrm{R}:(1 / 2,1 / 2,1 / 2)$ & $\mathrm{W}:(\mathrm{u}, \mathrm{v}, 1 / 2)$ & $\mathrm{T}:(0,1 / 2,1 / 2)$ & yes & Plane
\end{tabular}




\begin{tabular}{c|c|c|c|c}
\hline $\mathrm{R}:(1 / 2,1 / 2,1 / 2)$ & $\mathrm{W}:(\mathrm{u}, \mathrm{v}, 1 / 2)$ & $\mathrm{U}:(1 / 2,0,1 / 2)$ & yes & Plane \\
\hline $\mathrm{R}:(1 / 2,1 / 2,1 / 2)$ & $\mathrm{N}:(\mathrm{u}, 1 / 2, \mathrm{w})$ & $\mathrm{Y}:(0,1 / 2,0)$ & yes & Plane \\
\hline $\mathrm{R}:(1 / 2,1 / 2,1 / 2)$ & $\mathrm{W}:(\mathrm{u}, \mathrm{v}, 1 / 2)$ & $\mathrm{Z}:(0,0,1 / 2)$ & yes & Plane \\
\hline $\mathrm{S}:(1 / 2,1 / 2,0)$ & $\mathrm{N}:(\mathrm{u}, 1 / 2, \mathrm{w})$ & $\mathrm{T}:(0,1 / 2,1 / 2)$ & yes & Plane \\
\hline $\mathrm{S}:(1 / 2,1 / 2,0)$ & $\mathrm{N}:(\mathrm{u}, 1 / 2, \mathrm{w})$ & $\mathrm{Y}:(0,1 / 2,0)$ & yes & Plane \\
\hline $\mathrm{T}:(0,1 / 2,1 / 2)$ & $\mathrm{W}:(\mathrm{u}, \mathrm{v}, 1 / 2)$ & $\mathrm{U}:(1 / 2,0,1 / 2)$ & yes & Plane \\
\hline $\mathrm{T}:(0,1 / 2,1 / 2)$ & $\mathrm{N}:(\mathrm{u}, 1 / 2, \mathrm{w})$ & $\mathrm{Y}:(0,1 / 2,0)$ & yes & Plane \\
\hline $\mathrm{T}:(0,1 / 2,1 / 2)$ & $\mathrm{W}:(\mathrm{u}, \mathrm{v}, 1 / 2)$ & $\mathrm{Z}:(0,0,1 / 2)$ & yes & Plane \\
\hline $\mathrm{U}:(1 / 2,0,1 / 2)$ & $\mathrm{W}:(\mathrm{u}, \mathrm{v}, 1 / 2)$ & $\mathrm{Z}:(0,0,1 / 2)$ & yes & Plane \\
\hline
\end{tabular}

\begin{tabular}{|c|c|c|c|c|}
\hline \multicolumn{5}{|c|}{ BCSID: 1.179; Formula: NdCoAsO; MSG: $62.450\left(P_{a} n m a\right) ; \mathrm{U}=6 \mathrm{eV}$} \\
\hline maximal $k_{1}$ & intermediate path & maximal $k_{2}$ & satisfied? & Line/Plane \\
\hline $\mathrm{R}:(1 / 2,1 / 2,1 / 2)$ & $\mathrm{Q}:(1 / 2,1 / 2, \mathrm{w})$ & $\mathrm{S}:(1 / 2,1 / 2,0)$ & yes & Line \\
\hline $\mathrm{S}:(1 / 2,1 / 2,0)$ & $\mathrm{D}:(1 / 2, \mathrm{v}, 0)$ & $\mathrm{X}:(1 / 2,0,0)$ & no & Line \\
\hline $\mathrm{T}:(0,1 / 2,1 / 2)$ & $\mathrm{H}:(0,1 / 2, \mathrm{w})$ & $\mathrm{Y}:(0,1 / 2,0)$ & yes & Line \\
\hline $\mathrm{T}:(0,1 / 2,1 / 2)$ & $\mathrm{B}:(0, \mathrm{v}, 1 / 2)$ & $\mathrm{Z}:(0,0,1 / 2)$ & yes & Line \\
\hline $\mathrm{R}:(1 / 2,1 / 2,1 / 2)$ & $\mathrm{N}:(\mathrm{u}, 1 / 2, \mathrm{w})$ & $\mathrm{S}:(1 / 2,1 / 2,0)$ & yes & Plane \\
\hline $\mathrm{R}:(1 / 2,1 / 2,1 / 2)$ & $\mathrm{N}:(\mathrm{u}, 1 / 2, \mathrm{w})$ & $\mathrm{T}:(0,1 / 2,1 / 2)$ & yes & Plane \\
\hline $\mathrm{R}:(1 / 2,1 / 2,1 / 2)$ & $\mathrm{W}:(\mathrm{u}, \mathrm{v}, 1 / 2)$ & $\mathrm{T}:(0,1 / 2,1 / 2)$ & yes & Plane \\
\hline $\mathrm{R}:(1 / 2,1 / 2,1 / 2)$ & $\mathrm{W}:(\mathrm{u}, \mathrm{v}, 1 / 2)$ & $\mathrm{U}:(1 / 2,0,1 / 2)$ & yes & Plane \\
\hline $\mathrm{R}:(1 / 2,1 / 2,1 / 2)$ & $\mathrm{N}:(\mathrm{u}, 1 / 2, \mathrm{w})$ & $\mathrm{Y}:(0,1 / 2,0)$ & yes & Plane \\
\hline $\mathrm{R}:(1 / 2,1 / 2,1 / 2)$ & $\mathrm{W}:(\mathrm{u}, \mathrm{v}, 1 / 2)$ & $\mathrm{Z}:(0,0,1 / 2)$ & yes & Plane \\
\hline $\mathrm{S}:(1 / 2,1 / 2,0)$ & $\mathrm{N}:(\mathrm{u}, 1 / 2, \mathrm{w})$ & $\mathrm{T}:(0,1 / 2,1 / 2)$ & yes & Plane \\
\hline $\mathrm{S}:(1 / 2,1 / 2,0)$ & $\mathrm{N}:(\mathrm{u}, 1 / 2, \mathrm{w})$ & $\mathrm{Y}:(0,1 / 2,0)$ & yes & Plane \\
\hline $\mathrm{T}:(0,1 / 2,1 / 2)$ & $\mathrm{W}:(\mathrm{u}, \mathrm{v}, 1 / 2)$ & $\mathrm{U}:(1 / 2,0,1 / 2)$ & yes & Plane \\
\hline $\mathrm{T}:(0,1 / 2,1 / 2)$ & $\mathrm{N}:(\mathrm{u}, 1 / 2, \mathrm{w})$ & $\mathrm{Y}:(0,1 / 2,0)$ & yes & Plane \\
\hline $\mathrm{T}:(0,1 / 2,1 / 2)$ & $\mathrm{W}:(\mathrm{u}, \mathrm{v}, 1 / 2)$ & $\mathrm{Z}:(0,0,1 / 2)$ & yes & Plane \\
\hline $\mathrm{U}:(1 / 2,0,1 / 2)$ & $\mathrm{W}:(\mathrm{u}, \mathrm{v}, 1 / 2)$ & $\mathrm{Z}:(0,0,1 / 2)$ & yes & Plane \\
\hline \multicolumn{5}{|c|}{ BCSID: 0.149; Formula: Nd3Ru4Al12; MSG: $63.462\left(C m^{\prime} c^{\prime} m\right) ; \mathrm{U}=2 \mathrm{eV}$} \\
\hline maximal $k_{1}$ & intermediate path & maximal $k_{2}$ & satisfied? & Line/Plane \\
\hline$\Gamma:(0,0,0)$ & DT: $(0, \mathrm{v}, 0)$ & $\mathrm{Y}:(0,1,0)$ & yes & Line \\
\hline$\Gamma:(0,0,0)$ & SM:(u,0,0) & $\mathrm{Y}:(1,0,0)$ & yes & Line \\
\hline$\Gamma:(0,0,0)$ & LD: $(0,0, w)$ & $\mathrm{Z}:(0,0,1 / 2)$ & no & Line \\
\hline $\mathrm{T}:(1,0,1 / 2)$ & $\mathrm{H}:(1,0, \mathrm{w})$ & $\mathrm{Y}:(1,0,0)$ & yes & Line \\
\hline $\mathrm{T}:(1,0,1 / 2)$ & $\mathrm{A}:(\mathrm{u}, 0,1 / 2)$ & $\mathrm{Z}:(0,0,1 / 2)$ & yes & Line \\
\hline $\mathrm{R}:(1 / 2,1 / 2,-1 / 2)$ & $\mathrm{D}:(1 / 2,1 / 2, \mathrm{w})$ & $\mathrm{S}:(1 / 2,1 / 2,0)$ & yes & Line \\
\hline $\mathrm{RA}:(1 / 2,-1 / 2,-1 / 2)$ & $\mathrm{DA}:(1 / 2,-1 / 2, \mathrm{w})$ & $\mathrm{SA}:(1 / 2,-1 / 2,0)$ & yes & Line \\
\hline$\Gamma:(0,0,0)$ & $\mathrm{P}:(\mathrm{u}, \mathrm{v}, 0)$ & $\mathrm{Y}:(0,1,0)$ & yes & Plane \\
\hline$\Gamma:(0,0,0)$ & $\mathrm{P}:(\mathrm{u}, \mathrm{v}, 0)$ & $\mathrm{S}:(1 / 2,1 / 2,0)$ & yes & Plane \\
\hline$\Gamma:(0,0,0)$ & $\mathrm{P}:(\mathrm{u}, \mathrm{v}, 0)$ & $\mathrm{SA}:(-1 / 2,1 / 2,0)$ & yes & Plane \\
\hline $\mathrm{T}:(1,0,1 / 2)$ & $\mathrm{Q}:(\mathrm{u}, \mathrm{v}, 1 / 2)$ & $\mathrm{Z}:(0,0,1 / 2)$ & yes & Plane \\
\hline $\mathrm{T}:(1,0,1 / 2)$ & $\mathrm{Q}:(\mathrm{u}, \mathrm{v}, 1 / 2)$ & $\mathrm{R}:(1 / 2,1 / 2,1 / 2)$ & yes & Plane \\
\hline $\mathrm{T}:(1,0,1 / 2)$ & $\mathrm{Q}:(\mathrm{u}, \mathrm{v}, 1 / 2)$ & RA:(1/2,-1/2,1/2) & yes & Plane \\
\hline$Y:(1,0,0)$ & $\mathrm{P}:(\mathrm{u}, \mathrm{v}, 0)$ & $\mathrm{S}:(1 / 2,1 / 2,0)$ & yes & Plane \\
\hline$Y:(1,0,0)$ & $\mathrm{P}:(\mathrm{u}, \mathrm{v}, 0)$ & $\mathrm{SA}:(1 / 2,-1 / 2,0)$ & yes & Plane \\
\hline $\mathrm{Z}:(0,0,1 / 2)$ & $\mathrm{Q}:(\mathrm{u}, \mathrm{v}, 1 / 2)$ & $\mathrm{R}:(1 / 2,1 / 2,1 / 2)$ & yes & Plane \\
\hline $\mathrm{Z}:(0,0,1 / 2)$ & $\mathrm{Q}:(\mathrm{u}, \mathrm{v}, 1 / 2)$ & RA:(-1/2,1/2,1/2) & yes & Plane \\
\hline $\mathrm{R}:(1 / 2,1 / 2,1 / 2)$ & $\mathrm{Q}:(\mathrm{u}, \mathrm{v}, 1 / 2)$ & RA:(-1/2,1/2,1/2) & yes & Plane \\
\hline $\mathrm{S}:(1 / 2,1 / 2,0)$ & $\mathrm{P}:(\mathrm{u}, \mathrm{v}, 0)$ & $\mathrm{SA}:(-1 / 2,1 / 2,0)$ & yes & Plane \\
\hline
\end{tabular}




\begin{tabular}{c|c|c|c|c}
\hline \multicolumn{6}{c}{ BCSID: 0.149; Formula: Nd3Ru4Al12; MSG: 63.462} & $\left(\mathrm{Cm}^{\prime} \mathrm{c}^{\prime} m\right)$ & $\mathrm{U}=6 \mathrm{eV}$ \\
\hline maximal $k_{1}$ & intermediate path & maximal $k_{2}$ & satisfied? & Line/Plane \\
\hline$\Gamma:(0,0,0)$ & $\mathrm{DT}:(0, \mathrm{v}, 0)$ & $\mathrm{Y}:(0,1,0)$ & yes & Line \\
\hline$\Gamma:(0,0,0)$ & $\mathrm{SM}:(\mathrm{u}, 0,0)$ & $\mathrm{Y}:(1,0,0)$ & yes & Line \\
\hline$\Gamma:(0,0,0)$ & $\mathrm{LD}:(0,0, \mathrm{w})$ & $\mathrm{Z}:(0,0,1 / 2)$ & no & Line \\
\hline $\mathrm{T}:(1,0,1 / 2)$ & $\mathrm{H}:(1,0, \mathrm{w})$ & $\mathrm{Y}:(1,0,0)$ & yes & Line \\
\hline $\mathrm{T}:(1,0,1 / 2)$ & $\mathrm{A}:(\mathrm{u}, 0,1 / 2)$ & $\mathrm{Z}:(0,0,1 / 2)$ & yes & Line \\
\hline $\mathrm{R}:(1 / 2,1 / 2,-1 / 2)$ & $\mathrm{D}:(1 / 2,1 / 2, \mathrm{w})$ & $\mathrm{S}:(1 / 2,1 / 2,0)$ & yes & Line \\
\hline $\mathrm{RA}:(1 / 2,-1 / 2,-1 / 2)$ & $\mathrm{DA}:(1 / 2,-1 / 2, \mathrm{w})$ & $\mathrm{SA}:(1 / 2,-1 / 2,0)$ & yes & Line \\
\hline$\Gamma:(0,0,0)$ & $\mathrm{P}:(\mathrm{u}, \mathrm{v}, 0)$ & $\mathrm{Y}:(0,1,0)$ & yes & Plane \\
\hline$\Gamma:(0,0,0)$ & $\mathrm{P}:(\mathrm{u}, \mathrm{v}, 0)$ & $\mathrm{S}:(1 / 2,1 / 2,0)$ & yes & Plane \\
\hline$\Gamma:(0,0,0)$ & $\mathrm{P}:(\mathrm{u}, \mathrm{v}, 0)$ & $\mathrm{SA}:(-1 / 2,1 / 2,0)$ & yes & Plane \\
\hline $\mathrm{T}:(1,0,1 / 2)$ & $\mathrm{Q}:(\mathrm{u}, \mathrm{v}, 1 / 2)$ & $\mathrm{Z}:(0,0,1 / 2)$ & yes & Plane \\
\hline $\mathrm{T}:(1,0,1 / 2)$ & $\mathrm{Q}:(\mathrm{u}, \mathrm{v}, 1 / 2)$ & $\mathrm{R}:(1 / 2,1 / 2,1 / 2)$ & yes & Plane \\
\hline $\mathrm{T}:(1,0,1 / 2)$ & $\mathrm{Q}:(\mathrm{u}, \mathrm{v}, 1 / 2)$ & $\mathrm{RA}:(1 / 2,-1 / 2,1 / 2)$ & yes & Plane \\
\hline $\mathrm{Y}:(1,0,0)$ & $\mathrm{P}:(\mathrm{u}, \mathrm{v}, 0)$ & $\mathrm{S}:(1 / 2,1 / 2,0)$ & yes & Plane \\
\hline $\mathrm{Y}:(1,0,0)$ & $\mathrm{P}:(\mathrm{u}, \mathrm{v}, 0)$ & $\mathrm{SA}:(1 / 2,-1 / 2,0)$ & yes & Plane \\
\hline $\mathrm{Z}:(0,0,1 / 2)$ & $\mathrm{Q}:(\mathrm{u}, \mathrm{v}, 1 / 2)$ & $\mathrm{R}:(1 / 2,1 / 2,1 / 2)$ & yes & Plane \\
\hline $\mathrm{Z}:(0,0,1 / 2)$ & $\mathrm{Q}:(\mathrm{u}, \mathrm{v}, 1 / 2)$ & $\mathrm{RA}:(-1 / 2,1 / 2,1 / 2)$ & yes & Plane \\
\hline $\mathrm{R}:(1 / 2,1 / 2,1 / 2)$ & $\mathrm{Q}:(\mathrm{u}, \mathrm{v}, 1 / 2)$ & $\mathrm{RA}:(-1 / 2,1 / 2,1 / 2)$ & yes & Plane \\
\hline $\mathrm{S}:(1 / 2,1 / 2,0)$ & $\mathrm{P}:(\mathrm{u}, \mathrm{v}, 0)$ & $\mathrm{SA}:(-1 / 2,1 / 2,0)$ & yes & Plane \\
\hline & & & & \\
\hline
\end{tabular}

\begin{tabular}{c|c|c|c|c}
\hline \multicolumn{4}{c}{ BCSID: 0.173; Formula: Pr3Ru4Al12; MSG: $63.462\left(\mathrm{Cm}^{\prime} \mathrm{c}^{\prime} m\right)$} & $\mathrm{U}=2 \mathrm{eV}$ \\
\hline maximal $k_{1}$ & intermediate path & maximal $k_{2}$ & satisfied? & Line/Plane \\
\hline$\Gamma:(0,0,0)$ & $\mathrm{DT}:(0, \mathrm{v}, 0)$ & $\mathrm{Y}:(0,1,0)$ & yes & Line \\
\hline$\Gamma:(0,0,0)$ & $\mathrm{SM}:(\mathrm{u}, 0,0)$ & $\mathrm{Y}:(1,0,0)$ & yes & Line \\
\hline$\Gamma:(0,0,0)$ & $\mathrm{LD}:(0,0, \mathrm{w})$ & $\mathrm{Z}:(0,0,1 / 2)$ & no & Line \\
\hline $\mathrm{T}:(1,0,1 / 2)$ & $\mathrm{H}:(1,0, \mathrm{w})$ & $\mathrm{Y}:(1,0,0)$ & yes & Line \\
\hline $\mathrm{T}:(1,0,1 / 2)$ & $\mathrm{A}:(\mathrm{u}, 0,1 / 2)$ & $\mathrm{Z}:(0,0,1 / 2)$ & yes & Line \\
\hline $\mathrm{R}:(1 / 2,1 / 2,-1 / 2)$ & $\mathrm{D}:(1 / 2,1 / 2, \mathrm{w})$ & $\mathrm{S}:(1 / 2,1 / 2,0)$ & yes & Line \\
\hline $\mathrm{RA}:(1 / 2,-1 / 2,-1 / 2)$ & $\mathrm{DA}:(1 / 2,-1 / 2, \mathrm{w})$ & $\mathrm{SA}:(1 / 2,-1 / 2,0)$ & yes & Line \\
\hline$\Gamma:(0,0,0)$ & $\mathrm{P}:(\mathrm{u}, \mathrm{v}, 0)$ & $\mathrm{Y}:(0,1,0)$ & yes & Plane \\
\hline$\Gamma:(0,0,0)$ & $\mathrm{P}:(\mathrm{u}, \mathrm{v}, 0)$ & $\mathrm{S}:(1 / 2,1 / 2,0)$ & yes & Plane \\
\hline$\Gamma:(0,0,0)$ & $\mathrm{P}:(\mathrm{u}, \mathrm{v}, 0)$ & $\mathrm{SA}:(-1 / 2,1 / 2,0)$ & yes & Plane \\
\hline $\mathrm{T}:(1,0,1 / 2)$ & $\mathrm{Q}:(\mathrm{u}, \mathrm{v}, 1 / 2)$ & $\mathrm{Z}:(0,0,1 / 2)$ & yes & Plane \\
\hline $\mathrm{T}:(1,0,1 / 2)$ & $\mathrm{Q}:(\mathrm{u}, \mathrm{v}, 1 / 2)$ & $\mathrm{R}:(1 / 2,1 / 2,1 / 2)$ & yes & Plane \\
\hline $\mathrm{T}:(1,0,1 / 2)$ & $\mathrm{Q}:(\mathrm{u}, \mathrm{v}, 1 / 2)$ & $\mathrm{RA}:(1 / 2,-1 / 2,1 / 2)$ & yes & Plane \\
\hline $\mathrm{Y}:(1,0,0)$ & $\mathrm{P}:(\mathrm{u}, \mathrm{v}, 0)$ & $\mathrm{S}:(1 / 2,1 / 2,0)$ & yes & Plane \\
\hline $\mathrm{Y}:(1,0,0)$ & $\mathrm{P}:(\mathrm{u}, \mathrm{v}, 0)$ & $\mathrm{SA}:(1 / 2,-1 / 2,0)$ & yes & Plane \\
\hline $\mathrm{Z}:(0,0,1 / 2)$ & $\mathrm{Q}:(\mathrm{u}, \mathrm{v}, 1 / 2)$ & $\mathrm{R}:(1 / 2,1 / 2,1 / 2)$ & yes & Plane \\
\hline $\mathrm{Z}:(0,0,1 / 2)$ & $\mathrm{Q}:(\mathrm{u}, \mathrm{v}, 1 / 2)$ & $\mathrm{RA}:(-1 / 2,1 / 2,1 / 2)$ & yes & Plane \\
\hline $\mathrm{R}:(1 / 2,1 / 2,1 / 2)$ & $\mathrm{Q}:(\mathrm{u}, \mathrm{v}, 1 / 2)$ & $\mathrm{RA}:(-1 / 2,1 / 2,1 / 2)$ & yes & Plane \\
\hline $\mathrm{S}:(1 / 2,1 / 2,0)$ & $\mathrm{P}:(\mathrm{u}, \mathrm{v}, 0)$ & $\mathrm{SA}:(-1 / 2,1 / 2,0)$ & yes & Plane \\
\hline & & & & \\
\hline
\end{tabular}

\begin{tabular}{c|c|c|c|c}
\hline \multicolumn{4}{c}{ BCSID: 0.173; Formula: Pr3Ru4Al12; MSG: 63.462 } & $\left(\mathrm{Cm}^{\prime} \mathrm{c}^{\prime} m\right) ; \mathrm{U}=4 \mathrm{eV}$ \\
\hline maximal $k_{1}$ & intermediate path & maximal $k_{2}$ & satisfied? & Line/Plane \\
\hline$\Gamma:(0,0,0)$ & $\mathrm{DT}:(0, \mathrm{v}, 0)$ & $\mathrm{Y}:(0,1,0)$ & yes & Line \\
\hline$\Gamma:(0,0,0)$ & $\mathrm{SM}:(\mathrm{u}, 0,0)$ & $\mathrm{Y}:(1,0,0)$ & yes & Line \\
\hline$\Gamma:(0,0,0)$ & $\mathrm{LD}:(0,0, \mathrm{w})$ & $\mathrm{Z}:(0,0,1 / 2)$ & no & Line \\
\hline $\mathrm{T}:(1,0,1 / 2)$ & $\mathrm{H}:(1,0, \mathrm{w})$ & $\mathrm{Y}:(1,0,0)$ & yes & Line \\
\hline $\mathrm{T}:(1,0,1 / 2)$ & $\mathrm{A}:(\mathrm{u}, 0,1 / 2)$ & $\mathrm{Z}:(0,0,1 / 2)$ & yes & Line
\end{tabular}




\begin{tabular}{c|c|c|c|c}
\hline $\mathrm{R}:(1 / 2,1 / 2,-1 / 2)$ & $\mathrm{D}:(1 / 2,1 / 2, \mathrm{w})$ & $\mathrm{S}:(1 / 2,1 / 2,0)$ & yes & Line \\
\hline $\mathrm{RA}:(1 / 2,-1 / 2,-1 / 2)$ & $\mathrm{DA}:(1 / 2,-1 / 2, \mathrm{w})$ & $\mathrm{SA}:(1 / 2,-1 / 2,0)$ & yes & Line \\
\hline$\Gamma:(0,0,0)$ & $\mathrm{P}:(\mathrm{u}, \mathrm{v}, 0)$ & $\mathrm{Y}:(0,1,0)$ & yes & Plane \\
\hline$\Gamma:(0,0,0)$ & $\mathrm{P}:(\mathrm{u}, \mathrm{v}, 0)$ & $\mathrm{S}:(1 / 2,1 / 2,0)$ & yes & Plane \\
\hline$\Gamma:(0,0,0)$ & $\mathrm{P}:(\mathrm{u}, \mathrm{v}, 0)$ & $\mathrm{SA}:(-1 / 2,1 / 2,0)$ & yes & Plane \\
\hline $\mathrm{T}:(1,0,1 / 2)$ & $\mathrm{Q}:(\mathrm{u}, \mathrm{v}, 1 / 2)$ & $\mathrm{Z}:(0,0,1 / 2)$ & yes & Plane \\
\hline $\mathrm{T}:(1,0,1 / 2)$ & $\mathrm{Q}:(\mathrm{u}, \mathrm{v}, 1 / 2)$ & $\mathrm{R}:(1 / 2,1 / 2,1 / 2)$ & yes & Plane \\
\hline $\mathrm{T}:(1,0,1 / 2)$ & $\mathrm{Q}:(\mathrm{u}, \mathrm{v}, 1 / 2)$ & $\mathrm{RA}:(1 / 2,-1 / 2,1 / 2)$ & yes & Plane \\
\hline $\mathrm{Y}:(1,0,0)$ & $\mathrm{P}:(\mathrm{u}, \mathrm{v}, 0)$ & $\mathrm{S}:(1 / 2,1 / 2,0)$ & yes & Plane \\
\hline $\mathrm{Y}:(1,0,0)$ & $\mathrm{P}:(\mathrm{u}, \mathrm{v}, 0)$ & $\mathrm{SA}:(1 / 2,-1 / 2,0)$ & yes & Plane \\
\hline $\mathrm{Z}:(0,0,1 / 2)$ & $\mathrm{Q}:(\mathrm{u}, \mathrm{v}, 1 / 2)$ & $\mathrm{R}:(1 / 2,1 / 2,1 / 2)$ & yes & Plane \\
\hline $\mathrm{Z}:(0,0,1 / 2)$ & $\mathrm{Q}:(\mathrm{u}, \mathrm{v}, 1 / 2)$ & $\mathrm{RA}:(-1 / 2,1 / 2,1 / 2)$ & yes & Plane \\
\hline $\mathrm{R}:(1 / 2,1 / 2,1 / 2)$ & $\mathrm{Q}:(\mathrm{u}, \mathrm{v}, 1 / 2)$ & $\mathrm{RA}:(-1 / 2,1 / 2,1 / 2)$ & yes & Plane \\
\hline $\mathrm{S}:(1 / 2,1 / 2,0)$ & $\mathrm{P}:(\mathrm{u}, \mathrm{v}, 0)$ & $\mathrm{SA}:(-1 / 2,1 / 2,0)$ & yes & Plane \\
\hline
\end{tabular}

\begin{tabular}{c|c|c|c|c}
\hline \multicolumn{5}{c}{ BCSID: 0.173; Formula: Pr3Ru4Al12; MSG: 63.462 $\left(\mathrm{Cm}^{\prime} \mathrm{c}^{\prime} m\right) ; \mathrm{U}=6 \mathrm{eV}$} \\
\hline maximal $k_{1}$ & intermediate path & maximal $k_{2}$ & satisfied? & Line/Plane \\
\hline$\Gamma:(0,0,0)$ & $\mathrm{DT}:(0, \mathrm{v}, 0)$ & $\mathrm{Y}:(0,1,0)$ & yes & Line \\
\hline$\Gamma:(0,0,0)$ & $\mathrm{SM}:(\mathrm{u}, 0,0)$ & $\mathrm{Y}:(1,0,0)$ & yes & Line \\
\hline$\Gamma:(0,0,0)$ & $\mathrm{LD}:(0,0, \mathrm{w})$ & $\mathrm{Z}:(0,0,1 / 2)$ & no & Line \\
\hline $\mathrm{T}:(1,0,1 / 2)$ & $\mathrm{H}:(1,0, \mathrm{w})$ & $\mathrm{Y}:(1,0,0)$ & yes & Line \\
\hline $\mathrm{T}:(1,0,1 / 2)$ & $\mathrm{A}:(\mathrm{u}, 0,1 / 2)$ & $\mathrm{Z}:(0,0,1 / 2)$ & yes & Line \\
\hline $\mathrm{R}:(1 / 2,1 / 2,-1 / 2)$ & $\mathrm{D}:(1 / 2,1 / 2, \mathrm{w})$ & $\mathrm{S}:(1 / 2,1 / 2,0)$ & yes & Line \\
\hline $\mathrm{RA}:(1 / 2,-1 / 2,-1 / 2)$ & $\mathrm{DA}:(1 / 2,-1 / 2, \mathrm{w})$ & $\mathrm{SA}:(1 / 2,-1 / 2,0)$ & yes & Line \\
\hline$\Gamma:(0,0,0)$ & $\mathrm{P}:(\mathrm{u}, \mathrm{v}, 0)$ & $\mathrm{Y}:(0,1,0)$ & yes & Plane \\
\hline$\Gamma:(0,0,0)$ & $\mathrm{P}:(\mathrm{u}, \mathrm{v}, 0)$ & $\mathrm{S}:(1 / 2,1 / 2,0)$ & yes & Plane \\
\hline$\Gamma:(0,0,0)$ & $\mathrm{P}:(\mathrm{u}, \mathrm{v}, 0)$ & $\mathrm{SA}:(-1 / 2,1 / 2,0)$ & yes & Plane \\
\hline $\mathrm{T}:(1,0,1 / 2)$ & $\mathrm{Q}:(\mathrm{u}, \mathrm{v}, 1 / 2)$ & $\mathrm{Z}:(0,0,1 / 2)$ & yes & Plane \\
\hline $\mathrm{T}:(1,0,1 / 2)$ & $\mathrm{Q}:(\mathrm{u}, \mathrm{v}, 1 / 2)$ & $\mathrm{R}:(1 / 2,1 / 2,1 / 2)$ & yes & Plane \\
\hline $\mathrm{T}:(1,0,1 / 2)$ & $\mathrm{Q}:(\mathrm{u}, \mathrm{v}, 1 / 2)$ & $\mathrm{RA}:(1 / 2,-1 / 2,1 / 2)$ & yes & Plane \\
\hline $\mathrm{Y}:(1,0,0)$ & $\mathrm{P}:(\mathrm{u}, \mathrm{v}, 0)$ & $\mathrm{S}:(1 / 2,1 / 2,0)$ & yes & Plane \\
\hline $\mathrm{Y}:(1,0,0)$ & $\mathrm{P}:(\mathrm{u}, \mathrm{v}, 0)$ & $\mathrm{SA}:(1 / 2,-1 / 2,0)$ & yes & Plane \\
\hline $\mathrm{Z}:(0,0,1 / 2)$ & $\mathrm{Q}:(\mathrm{u}, \mathrm{v}, 1 / 2)$ & $\mathrm{R}:(1 / 2,1 / 2,1 / 2)$ & yes & Plane \\
\hline $\mathrm{Z}:(0,0,1 / 2)$ & $\mathrm{Q}:(\mathrm{u}, \mathrm{v}, 1 / 2)$ & $\mathrm{RA}:(-1 / 2,1 / 2,1 / 2)$ & yes & Plane \\
\hline $\mathrm{R}:(1 / 2,1 / 2,1 / 2)$ & $\mathrm{Q}:(\mathrm{u}, \mathrm{v}, 1 / 2)$ & $\mathrm{RA}:(-1 / 2,1 / 2,1 / 2)$ & yes & Plane \\
\hline $\mathrm{S}:(1 / 2,1 / 2,0)$ & $\mathrm{P}:(\mathrm{u}, \mathrm{v}, 0)$ & $\mathrm{SA}:(-1 / 2,1 / 2,0)$ & yes & Plane \\
\hline & & & & \\
\hline
\end{tabular}

\begin{tabular}{c|c|c|c|c}
\hline \multicolumn{6}{c}{ BCSID: 0.199; Formula: Mn3Sn; MSG: $63.463\left(\mathrm{Cm}^{\prime} \mathrm{m}^{\prime}\right) ; \mathrm{U}=0$} \\
\hline maximal $k_{1}$ & intermediate path & maximal $k_{2}$ & satisfied? & Line/Plane \\
\hline$\Gamma:(0,0,0)$ & $\mathrm{DT}:(0, \mathrm{v}, 0)$ & $\mathrm{Y}:(0,1,0)$ & yes & Line \\
\hline$\Gamma:(0,0,0)$ & $\mathrm{SM}:(\mathrm{u}, 0,0)$ & $\mathrm{Y}:(1,0,0)$ & no & Line \\
\hline$\Gamma:(0,0,0)$ & $\mathrm{LD}:(0,0, \mathrm{w})$ & $\mathrm{Z}:(0,0,1 / 2)$ & no & Line \\
\hline $\mathrm{T}:(1,0,1 / 2)$ & $\mathrm{H}:(1,0, \mathrm{w})$ & $\mathrm{Y}:(1,0,0)$ & no & Line \\
\hline $\mathrm{T}:(0,1,1 / 2)$ & $\mathrm{B}:(0, \mathrm{v}, 1 / 2)$ & $\mathrm{Z}:(0,0,1 / 2)$ & no & Line \\
\hline$\Gamma:(0,0,0)$ & $\mathrm{K}:(0, \mathrm{v}, \mathrm{w})$ & $\mathrm{T}:(0,1,1 / 2)$ & no & Plane \\
\hline$\Gamma:(0,0,0)$ & $\mathrm{K}:(0, \mathrm{v}, \mathrm{w})$ & $\mathrm{Y}:(0,1,0)$ & yes & Plane \\
\hline$\Gamma:(0,0,0)$ & $\mathrm{K}:(0, \mathrm{v}, \mathrm{w})$ & $\mathrm{Z}:(0,0,1 / 2)$ & no & Plane \\
\hline $\mathrm{T}:(0,1,1 / 2)$ & $\mathrm{K}:(0, \mathrm{v}, \mathrm{w})$ & $\mathrm{Y}:(0,1,0)$ & no & Plane \\
\hline $\mathrm{T}:(0,1,1 / 2)$ & $\mathrm{K}:(0, \mathrm{v}, \mathrm{w})$ & $\mathrm{Z}:(0,0,1 / 2)$ & no & Plane \\
\hline $\mathrm{Y}:(0,1,0)$ & $\mathrm{K}:(0, \mathrm{v}, \mathrm{w})$ & $\mathrm{Z}:(0,0,1 / 2)$ & no & Plane \\
\hline
\end{tabular}




\begin{tabular}{|c|c|c|c|c|}
\hline \multicolumn{5}{|c|}{ BCSID: 3.3; Formula: Ho2RhIn8; MSG: $63.464\left(\mathrm{Cm}^{\prime} \mathrm{cm}^{\prime}\right) ; \mathrm{U}=0$} \\
\hline maximal $k_{1}$ & intermediate path & maximal $k_{2}$ & satisfied? & Line/Plane \\
\hline$\Gamma:(0,0,0)$ & DT: $(0, \mathrm{v}, 0)$ & $\mathrm{Y}:(0,1,0)$ & no & Line \\
\hline$\Gamma:(0,0,0)$ & SM:(u,0,0) & $Y:(1,0,0)$ & yes & Line \\
\hline$\Gamma:(0,0,0)$ & $\mathrm{LD}:(0,0, \mathrm{w})$ & $\mathrm{Z}:(0,0,1 / 2)$ & yes & Line \\
\hline $\mathrm{T}:(1,0,1 / 2)$ & $\mathrm{H}:(1,0, \mathrm{w})$ & $\mathrm{Y}:(1,0,0)$ & yes & Line \\
\hline$\Gamma:(0,0,0)$ & $\mathrm{M}:(\mathrm{u}, 0, \mathrm{w})$ & $\mathrm{T}:(1,0,1 / 2)$ & yes & Plane \\
\hline$\Gamma:(0,0,0)$ & $\mathrm{M}:(\mathrm{u}, 0, \mathrm{w})$ & $\mathrm{Y}:(1,0,0)$ & yes & Plane \\
\hline$\Gamma:(0,0,0)$ & $\mathrm{M}:(\mathrm{u}, 0, \mathrm{w})$ & $\mathrm{Z}:(0,0,1 / 2)$ & yes & Plane \\
\hline $\mathrm{T}:(1,0,1 / 2)$ & $\mathrm{M}:(\mathrm{u}, 0, \mathrm{w})$ & $Y:(1,0,0)$ & yes & Plane \\
\hline $\mathrm{T}:(1,0,1 / 2)$ & $\mathrm{M}:(\mathrm{u}, 0, \mathrm{w})$ & $\mathrm{Z}:(0,0,1 / 2)$ & yes & Plane \\
\hline$Y:(1,0,0)$ & $\mathrm{M}:(\mathrm{u}, 0, \mathrm{w})$ & $\mathrm{Z}:(0,0,1 / 2)$ & yes & Plane \\
\hline \multicolumn{5}{|c|}{ BCSID: 3.3 ; Formula: Ho2RhIn8; MSG: $63.464\left(\mathrm{Cm}^{\prime} \mathrm{cm}^{\prime}\right)$; U=1eV } \\
\hline maximal $k_{1}$ & intermediate path & maximal $k_{2}$ & satisfied? & Line/Plane \\
\hline$\Gamma:(0,0,0)$ & DT: $(0, \mathrm{v}, 0)$ & $\mathrm{Y}:(0,1,0)$ & yes & Line \\
\hline$\Gamma:(0,0,0)$ & SM:(u,0,0) & $\mathrm{Y}:(1,0,0)$ & no & Line \\
\hline$\Gamma:(0,0,0)$ & LD: $(0,0, w)$ & $\mathrm{Z}:(0,0,1 / 2)$ & yes & Line \\
\hline $\mathrm{T}:(1,0,1 / 2)$ & $\mathrm{H}:(1,0, \mathrm{w})$ & $\mathrm{Y}:(1,0,0)$ & no & Line \\
\hline$\Gamma:(0,0,0)$ & $\mathrm{M}:(\mathrm{u}, 0, \mathrm{w})$ & $\mathrm{T}:(1,0,1 / 2)$ & yes & Plane \\
\hline$\Gamma:(0,0,0)$ & $\mathrm{M}:(\mathrm{u}, 0, \mathrm{w})$ & $Y:(1,0,0)$ & no & Plane \\
\hline$\Gamma:(0,0,0)$ & $\mathrm{M}:(\mathrm{u}, 0, \mathrm{w})$ & $\mathrm{Z}:(0,0,1 / 2)$ & yes & Plane \\
\hline $\mathrm{T}:(1,0,1 / 2)$ & $\mathrm{M}:(\mathrm{u}, 0, \mathrm{w})$ & $\mathrm{Y}:(1,0,0)$ & no & Plane \\
\hline $\mathrm{T}:(1,0,1 / 2)$ & $\mathrm{M}:(\mathrm{u}, 0, \mathrm{w})$ & $\mathrm{Z}:(0,0,1 / 2)$ & yes & Plane \\
\hline$Y:(1,0,0)$ & $\mathrm{M}:(\mathrm{u}, 0, \mathrm{w})$ & $\mathrm{Z}:(0,0,1 / 2)$ & no & Plane \\
\hline \multicolumn{5}{|c|}{ BCSID: 3.3; Formula: Ho2RhIn8; MSG: $63.464\left(C m^{\prime} \mathrm{cm}^{\prime}\right) ; \mathrm{U}=2 \mathrm{eV}$} \\
\hline maximal $k_{1}$ & intermediate path & maximal $k_{2}$ & satisfied? & Line/Plane \\
\hline$\Gamma:(0,0,0)$ & DT: $(0, \mathrm{v}, 0)$ & $\mathrm{Y}:(0,1,0)$ & yes & Line \\
\hline$\Gamma:(0,0,0)$ & SM:(u,0,0) & $Y:(1,0,0)$ & no & Line \\
\hline$\Gamma:(0,0,0)$ & LD: $(0,0, \mathrm{w})$ & $\mathrm{Z}:(0,0,1 / 2)$ & yes & Line \\
\hline $\mathrm{T}:(1,0,1 / 2)$ & $\mathrm{H}:(1,0, \mathrm{w})$ & $Y:(1,0,0)$ & no & Line \\
\hline$\Gamma:(0,0,0)$ & $\mathrm{M}:(\mathrm{u}, 0, \mathrm{w})$ & $\mathrm{T}:(1,0,1 / 2)$ & yes & Plane \\
\hline$\Gamma:(0,0,0)$ & $\mathrm{M}:(\mathrm{u}, 0, \mathrm{w})$ & $\mathrm{Y}:(1,0,0)$ & no & Plane \\
\hline$\Gamma:(0,0,0)$ & $\mathrm{M}:(\mathrm{u}, 0, \mathrm{w})$ & $\mathrm{Z}:(0,0,1 / 2)$ & yes & Plane \\
\hline $\mathrm{T}:(1,0,1 / 2)$ & $\mathrm{M}:(\mathrm{u}, 0, \mathrm{w})$ & $\mathrm{Y}:(1,0,0)$ & no & Plane \\
\hline $\mathrm{T}:(1,0,1 / 2)$ & $\mathrm{M}:(\mathrm{u}, 0, \mathrm{w})$ & $\mathrm{Z}:(0,0,1 / 2)$ & yes & Plane \\
\hline $\mathrm{Y}:(1,0,0)$ & $\mathrm{M}:(\mathrm{u}, 0, \mathrm{w})$ & $\mathrm{Z}:(0,0,1 / 2)$ & no & Plane \\
\hline \multicolumn{5}{|c|}{ BCSID: $1.262 ;$ Formula: NpRhGa5; MSG: $63.466\left(C_{c} m c m\right) ; \mathrm{U}=4 \mathrm{eV}$} \\
\hline maximal $k_{1}$ & intermediate path & maximal $k_{2}$ & satisfied? & Line/Plane \\
\hline $\mathrm{T}:(1,0,1 / 2)$ & $\mathrm{A}:(\mathrm{u}, 0,1 / 2)$ & $\mathrm{Z}:(0,0,1 / 2)$ & no & Line \\
\hline
\end{tabular}

BCSID: 1.145; Formula: Mn3Ni20P6; MSG: $64.480\left(C_{A} m c a\right) ; \mathrm{U}=0$ \begin{tabular}{l|l|l|l|l} 
maximal $k_{1}$ & intermediate path & maximal $k_{2}$ & satisfied? & Line/Plane
\end{tabular}

\begin{tabular}{l|l|l|l|l}
$\mathrm{T}:(1,0,1 / 2)$ & $\mathrm{A}:(\mathrm{u}, 0,1 / 2)$ & $\mathrm{Z}:(0,0,1 / 2)$ & no & Line \\
\hline
\end{tabular}

BCSID: 1.188; Formula: CeRh2Si2; MSG: $64.480\left(C_{A} m c a\right) ; \mathrm{U}=4 \mathrm{eV}$ \begin{tabular}{l|l|l|l|l} 
maximal $k_{1}$ & intermediate path & maximal $k_{2}$ & satisfied? & Line/Plane
\end{tabular}

\begin{tabular}{c|c|c|c|c}
$\mathrm{T}:(1,0,1 / 2)$ & $\mathrm{A}:(\mathrm{u}, 0,1 / 2)$ & $\mathrm{Z}:(0,0,1 / 2)$ & no & Line \\
\hline
\end{tabular}




\begin{tabular}{c|c|c|c|c}
\hline \multicolumn{5}{c}{ BCSID: $2.15 ;$ Formula: Mn3Ni20P6; MSG: 65.486 $\left(\mathrm{Cm}^{\prime} \mathrm{m}^{\prime}\right) ; \mathrm{U}=1 \mathrm{eV}$} \\
\hline maximal $k_{1}$ & intermediate path & maximal $k_{2}$ & satisfied? & Line/Plane \\
\hline$\Gamma:(0,0,0)$ & $\mathrm{DT}:(0, \mathrm{v}, 0)$ & $\mathrm{Y}:(0,1,0)$ & yes & Line \\
\hline$\Gamma:(0,0,0)$ & $\mathrm{SM}:(\mathrm{u}, 0,0)$ & $\mathrm{Y}:(1,0,0)$ & no & Line \\
\hline$\Gamma:(0,0,0)$ & $\mathrm{LD}:(0,0, \mathrm{w})$ & $\mathrm{Z}:(0,0,1 / 2)$ & yes & Line \\
\hline $\mathrm{T}:(1,0,1 / 2)$ & $\mathrm{H}:(1,0, \mathrm{w})$ & $\mathrm{Y}:(1,0,0)$ & no & Line \\
\hline $\mathrm{T}:(1,0,1 / 2)$ & $\mathrm{A}:(\mathrm{u}, 0,1 / 2)$ & $\mathrm{Z}:(0,0,1 / 2)$ & no & Line \\
\hline $\mathrm{T}:(0,1,1 / 2)$ & $\mathrm{B}:(0, \mathrm{v}, 1 / 2)$ & $\mathrm{Z}:(0,0,1 / 2)$ & no & Line \\
\hline$\Gamma:(0,0,0)$ & $\mathrm{K}:(0, \mathrm{v}, \mathrm{w})$ & $\mathrm{T}:(0,1,1 / 2)$ & no & Plane \\
\hline$\Gamma:(0,0,0)$ & $\mathrm{K}:(0, \mathrm{v}, \mathrm{w})$ & $\mathrm{Y}:(0,1,0)$ & yes & Plane \\
\hline$\Gamma:(0,0,0)$ & $\mathrm{K}:(0, \mathrm{v}, \mathrm{w})$ & $\mathrm{Z}:(0,0,1 / 2)$ & yes & Plane \\
\hline $\mathrm{T}:(0,1,1 / 2)$ & $\mathrm{K}:(0, \mathrm{v}, \mathrm{w})$ & $\mathrm{Y}:(0,1,0)$ & no & Plane \\
\hline $\mathrm{T}:(0,1,1 / 2)$ & $\mathrm{K}:(0, \mathrm{v}, \mathrm{w})$ & $\mathrm{Z}:(0,0,1 / 2)$ & no & Plane \\
\hline $\mathrm{Y}:(0,1,0)$ & $\mathrm{K}:(0, \mathrm{v}, \mathrm{w})$ & $\mathrm{Z}:(0,0,1 / 2)$ & yes & Plane \\
\hline
\end{tabular}

\begin{tabular}{|c|c|c|c|c|}
\hline \multicolumn{5}{|c|}{ BCSID: 2.15 ; Formula: Mn3Ni20P6; MSG: $65.486\left(\mathrm{Cm}^{\prime} \mathrm{m}^{\prime}\right) ; \mathrm{U}=2 \mathrm{eV}$} \\
\hline maximal $k_{1}$ & intermediate path & maximal $k_{2}$ & satisfied? & Line/Plane \\
\hline$\Gamma:(0,0,0)$ & DT: $(0, \mathrm{v}, 0)$ & $\mathrm{Y}:(0,1,0)$ & yes & Line \\
\hline$\Gamma:(0,0,0)$ & SM:(u,0,0) & $\mathrm{Y}:(1,0,0)$ & no & Line \\
\hline$\Gamma:(0,0,0)$ & $\mathrm{LD}:(0,0, \mathrm{w})$ & $\mathrm{Z}:(0,0,1 / 2)$ & yes & Line \\
\hline $\mathrm{T}:(1,0,1 / 2)$ & $\mathrm{H}:(1,0, \mathrm{w})$ & $\mathrm{Y}:(1,0,0)$ & yes & Line \\
\hline $\mathrm{T}:(1,0,1 / 2)$ & $\mathrm{A}:(\mathrm{u}, 0,1 / 2)$ & $\mathrm{Z}:(0,0,1 / 2)$ & yes & Line \\
\hline $\mathrm{T}:(0,1,1 / 2)$ & $\mathrm{B}:(0, \mathrm{v}, 1 / 2)$ & $\mathrm{Z}:(0,0,1 / 2)$ & yes & Line \\
\hline$\Gamma:(0,0,0)$ & $\mathrm{K}:(0, \mathrm{v}, \mathrm{w})$ & $\mathrm{T}:(0,1,1 / 2)$ & yes & Plane \\
\hline$\Gamma:(0,0,0)$ & $\mathrm{K}:(0, \mathrm{v}, \mathrm{w})$ & $\mathrm{Y}:(0,1,0)$ & yes & Plane \\
\hline$\Gamma:(0,0,0)$ & $\mathrm{K}:(0, \mathrm{v}, \mathrm{w})$ & $\mathrm{Z}:(0,0,1 / 2)$ & yes & Plane \\
\hline $\mathrm{T}:(0,1,1 / 2)$ & $\mathrm{K}:(0, \mathrm{v}, \mathrm{w})$ & $\mathrm{Y}:(0,1,0)$ & yes & Plane \\
\hline $\mathrm{T}:(0,1,1 / 2)$ & $\mathrm{K}:(0, \mathrm{v}, \mathrm{w})$ & $\mathrm{Z}:(0,0,1 / 2)$ & yes & Plane \\
\hline $\mathrm{Y}:(0,1,0)$ & $\mathrm{K}:(0, \mathrm{v}, \mathrm{w})$ & $\mathrm{Z}:(0,0,1 / 2)$ & yes & Plane \\
\hline \multicolumn{5}{|c|}{ BCSID: 2.15; Formula: Mn3Ni20P6; MSG: $65.486\left(\mathrm{Cm}^{\prime} \mathrm{m}^{\prime}\right) ; \mathrm{U}=4 \mathrm{eV}$} \\
\hline maximal $k_{1}$ & intermediate path & maximal $k_{2}$ & satisfied? & Line/Plane \\
\hline$\Gamma:(0,0,0)$ & DT: $(0, \mathrm{v}, 0)$ & $\mathrm{Y}:(0,1,0)$ & yes & Line \\
\hline$\Gamma:(0,0,0)$ & SM:(u,0,0) & $\mathrm{Y}:(1,0,0)$ & no & Line \\
\hline$\Gamma:(0,0,0)$ & $\mathrm{LD}:(0,0, \mathrm{w})$ & $\mathrm{Z}:(0,0,1 / 2)$ & yes & Line \\
\hline $\mathrm{T}:(1,0,1 / 2)$ & $\mathrm{H}:(1,0, \mathrm{w})$ & $\mathrm{Y}:(1,0,0)$ & yes & Line \\
\hline $\mathrm{T}:(1,0,1 / 2)$ & $\mathrm{A}:(\mathrm{u}, 0,1 / 2)$ & $\mathrm{Z}:(0,0,1 / 2)$ & yes & Line \\
\hline $\mathrm{T}:(0,1,1 / 2)$ & $\mathrm{B}:(0, \mathrm{v}, 1 / 2)$ & $\mathrm{Z}:(0,0,1 / 2)$ & yes & Line \\
\hline$\Gamma:(0,0,0)$ & $\mathrm{K}:(0, \mathrm{v}, \mathrm{w})$ & $\mathrm{T}:(0,1,1 / 2)$ & yes & Plane \\
\hline$\Gamma:(0,0,0)$ & $\mathrm{K}:(0, \mathrm{v}, \mathrm{w})$ & $Y:(0,1,0)$ & yes & Plane \\
\hline$\Gamma:(0,0,0)$ & $\mathrm{K}:(0, \mathrm{v}, \mathrm{w})$ & $\mathrm{Z}:(0,0,1 / 2)$ & yes & Plane \\
\hline $\mathrm{T}:(0,1,1 / 2)$ & $\mathrm{K}:(0, \mathrm{v}, \mathrm{w})$ & $Y:(0,1,0)$ & yes & Plane \\
\hline $\mathrm{T}:(0,1,1 / 2)$ & $\mathrm{K}:(0, \mathrm{v}, \mathrm{w})$ & $\mathrm{Z}:(0,0,1 / 2)$ & yes & Plane \\
\hline $\mathrm{Y}:(0,1,0)$ & $\mathrm{K}:(0, \mathrm{v}, \mathrm{w})$ & $\mathrm{Z}:(0,0,1 / 2)$ & yes & Plane \\
\hline
\end{tabular}

BCSID: 1.223; Formula: Tm2CoGa8; MSG: $65.489\left(C_{a} m m m\right) ; \mathrm{U}=2 \mathrm{eV}$ \begin{tabular}{l|l|l|l|l|} 
maximal $k_{1}$ & intermediate path & maximal $k_{2}$ & satisfied? & Line/Plane \\
\hline
\end{tabular}

\begin{tabular}{l|l|l|l|l}
$\mathrm{R}:(1 / 2,1 / 2,1 / 2)$ & $\mathrm{D}:(1 / 2,1 / 2, \mathrm{w})$ & $\mathrm{S}:(1 / 2,1 / 2,0)$ & no & Line \\
\hline
\end{tabular}

BCSID: 1.223; Formula: Tm2CoGa8; MSG: $65.489\left(C_{a} m m m\right) ; \mathrm{U}=4 \mathrm{eV}$ maximal $k_{1} \quad \mid$ intermediate path $\mid$ maximal $k_{2} \quad$ satisfied? $\mid$ Line/Plane 


\begin{tabular}{|c|c|c|c|c|}
\hline $\mathrm{R}:(1 / 2,1 / 2,1 / 2)$ & $\mathrm{D}:(1 / 2,1 / 2, \mathrm{w})$ & $\mathrm{S}:(1 / 2,1 / 2,0)$ & no & Line \\
\hline \multicolumn{5}{|c|}{ BCSID: 0.4; Formula: NiCr2O4; MSG: $70.530\left(F d^{\prime} d^{\prime} d\right) ; \mathrm{U}=0$} \\
\hline maximal $k_{1}$ & intermediate path & maximal $k_{2}$ & satisfied? & Line/Plane \\
\hline$\Gamma:(0,0,0)$ & SM:(u,0,0) & $\mathrm{T}:(1,0,0)$ & yes & Line \\
\hline$\Gamma:(0,0,0)$ & DT: $(0, \mathrm{v}, 0)$ & $Y:(0,1,0)$ & yes & Line \\
\hline$\Gamma:(0,0,0)$ & LD: $(0,0, \mathrm{w})$ & $\mathrm{Z}:(0,0,1)$ & no & Line \\
\hline $\mathrm{T}:(0,1,1)$ & $\mathrm{H}:(0,1, \mathrm{w})$ & $\mathrm{Y}:(0,1,0)$ & yes & Line \\
\hline$\Gamma:(0,0,0)$ & $\mathrm{M}:(\mathrm{u}, \mathrm{v}, 0)$ & $\mathrm{T}:(1,0,0)$ & yes & Plane \\
\hline$\Gamma:(0,0,0)$ & $\mathrm{M}:(\mathrm{u}, \mathrm{v}, 0)$ & $\mathrm{Y}:(0,1,0)$ & yes & Plane \\
\hline$\Gamma:(0,0,0)$ & $\mathrm{M}:(\mathrm{u}, \mathrm{v}, 0)$ & $\mathrm{Z}:(1,1,0)$ & yes & Plane \\
\hline $\mathrm{T}:(1,0,0)$ & $\mathrm{M}:(\mathrm{u}, \mathrm{v}, 0)$ & $Y:(0,1,0)$ & yes & Plane \\
\hline $\mathrm{T}:(1,0,0)$ & $\mathrm{M}:(\mathrm{u}, \mathrm{v}, 0)$ & $\mathrm{Z}:(1,1,0)$ & yes & Plane \\
\hline$Y:(0,1,0)$ & $\mathrm{M}:(\mathrm{u}, \mathrm{v}, 0)$ & $\mathrm{Z}:(1,1,0)$ & yes & Plane \\
\hline
\end{tabular}

BCSID: 1.0.12; Formula: UAu2Si2; MSG: $71.536\left(\mathrm{Im}^{\prime} \mathrm{m}^{\prime} \mathrm{m}\right)$; $\mathrm{U}=0$

\begin{tabular}{|c|c|c|c|c|}
\hline maximal $k_{1}$ & intermediate path & maximal $k_{2}$ & satisfied? & Line/Plane \\
\hline$\Gamma:(0,0,0)$ & DT: $(0, \mathrm{v}, 0)$ & $\mathrm{X}:(0,1,0)$ & no & Line \\
\hline$\Gamma:(0,0,0)$ & LD: $(0,0, w)$ & $\mathrm{X}:(0,0,1)$ & no & Line \\
\hline$\Gamma:(0,0,0)$ & SM:(u,0,0) & $\mathrm{X}:(1,0,0)$ & no & Line \\
\hline $\mathrm{W}:(1 / 2,1 / 2,1 / 2)$ & $\mathrm{P}:(1 / 2,1 / 2, \mathrm{w})$ & $\mathrm{T}:(1 / 2,1 / 2,0)$ & yes & Line \\
\hline $\mathrm{W}:(1 / 2,1 / 2,1 / 2)$ & $\mathrm{P}:(1 / 2,1 / 2, \mathrm{w})$ & TA: $(1 / 2,1 / 2,1)$ & yes & Line \\
\hline $\mathrm{T}:(1 / 2,1 / 2,2)$ & $\mathrm{P}:(1 / 2,1 / 2, \mathrm{w})$ & TA: $(1 / 2,1 / 2,1)$ & yes & Line \\
\hline$\Gamma:(0,0,0)$ & $\mathrm{C}:(\mathrm{u}, \mathrm{v}, 0)$ & $\mathrm{X}:(0,1,0)$ & no & Plane \\
\hline$\Gamma:(0,0,0)$ & $\mathrm{C}:(\mathrm{u}, \mathrm{v}, 0)$ & $\mathrm{T}:(1 / 2,1 / 2,0)$ & no & Plane \\
\hline$\Gamma:(0,0,0)$ & $\mathrm{C}:(\mathrm{u}, \mathrm{v}, 0)$ & TA:(-1/2,1/2,0) & no & Plane \\
\hline $\mathrm{X}:(2,1,0)$ & $\mathrm{C}:(\mathrm{u}, \mathrm{v}, 0)$ & $\mathrm{T}:(3 / 2,3 / 2,0)$ & no & Plane \\
\hline $\mathrm{X}:(2,1,0)$ & $\mathrm{C}:(\mathrm{u}, \mathrm{v}, 0)$ & TA: $(3 / 2,1 / 2,0)$ & no & Plane \\
\hline $\mathrm{T}:(3 / 2,-1 / 2,0)$ & $\mathrm{C}:(\mathrm{u}, \mathrm{v}, 0)$ & TA: $(1 / 2,-1 / 2,0)$ & yes & Plane \\
\hline \multicolumn{5}{|c|}{ BCSID: 1.0.12; Formula: UAu2Si2; MSG: $71.536\left(\operatorname{Im}^{\prime} m^{\prime} m\right) ; \mathrm{U}=2 \mathrm{eV}$} \\
\hline $\operatorname{maximal} k_{1}$ & intermediate path & maximal $k_{2}$ & satisfied? & Line/Plane \\
\hline$\Gamma:(0,0,0)$ & DT: $(0, \mathrm{v}, 0)$ & $\mathrm{X}:(0,1,0)$ & no & Line \\
\hline$\Gamma:(0,0,0)$ & LD: $(0,0, w)$ & $\mathrm{X}:(0,0,1)$ & yes & Line \\
\hline$\Gamma:(0,0,0)$ & SM:(u,0,0) & $\mathrm{X}:(1,0,0)$ & no & Line \\
\hline $\mathrm{W}:(1 / 2,1 / 2,1 / 2)$ & $\mathrm{P}:(1 / 2,1 / 2, \mathrm{w})$ & $\mathrm{T}:(1 / 2,1 / 2,0)$ & yes & Line \\
\hline $\mathrm{W}:(1 / 2,1 / 2,1 / 2)$ & $\mathrm{P}:(1 / 2,1 / 2, \mathrm{w})$ & TA: $(1 / 2,1 / 2,1)$ & yes & Line \\
\hline $\mathrm{T}:(1 / 2,1 / 2,2)$ & $\mathrm{P}:(1 / 2,1 / 2, \mathrm{w})$ & TA: $(1 / 2,1 / 2,1)$ & yes & Line \\
\hline$\Gamma:(0,0,0)$ & $\mathrm{C}:(\mathrm{u}, \mathrm{v}, 0)$ & $\mathrm{X}:(0,1,0)$ & no & Plane \\
\hline$\Gamma:(0,0,0)$ & $\mathrm{C}:(\mathrm{u}, \mathrm{v}, 0)$ & $\mathrm{T}:(1 / 2,1 / 2,0)$ & no & Plane \\
\hline$\Gamma:(0,0,0)$ & $\mathrm{C}:(\mathrm{u}, \mathrm{v}, 0)$ & TA: $(-1 / 2,1 / 2,0)$ & no & Plane \\
\hline $\mathrm{X}:(2,1,0)$ & $\mathrm{C}:(\mathrm{u}, \mathrm{v}, 0)$ & $\mathrm{T}:(3 / 2,3 / 2,0)$ & yes & Plane \\
\hline $\mathrm{X}:(2,1,0)$ & $\mathrm{C}:(\mathrm{u}, \mathrm{v}, 0)$ & TA: $(3 / 2,1 / 2,0)$ & yes & Plane \\
\hline $\mathrm{T}:(3 / 2,-1 / 2,0)$ & $\mathrm{C}:(\mathrm{u}, \mathrm{v}, 0)$ & TA: $(1 / 2,-1 / 2,0)$ & yes & Plane \\
\hline
\end{tabular}

BCSID: 1.0.12; Formula: UAu2Si2; MSG: $71.536\left(\mathrm{Im}^{\prime} \mathrm{m}^{\prime} \mathrm{m}\right)$; U=6eV

\begin{tabular}{c|c|c|c|c}
\hline maximal $k_{1}$ & intermediate path & maximal $k_{2}$ & satisfied? & Line/Plane \\
\hline$\Gamma:(0,0,0)$ & DT: $(0, \mathrm{v}, 0)$ & $\mathrm{X}:(0,1,0)$ & no & Line \\
\hline$\Gamma:(0,0,0)$ & LD: $(0,0, \mathrm{w})$ & $\mathrm{X}:(0,0,1)$ & yes & Line \\
\hline$\Gamma:(0,0,0)$ & SM: $(\mathrm{u}, 0,0)$ & $\mathrm{X}:(1,0,0)$ & no & Line \\
\hline
\end{tabular}




\begin{tabular}{c|c|c|c|c}
\hline $\mathrm{W}:(1 / 2,1 / 2,1 / 2)$ & $\mathrm{P}:(1 / 2,1 / 2, \mathrm{w})$ & $\mathrm{T}:(1 / 2,1 / 2,0)$ & yes & Line \\
\hline $\mathrm{W}:(1 / 2,1 / 2,1 / 2)$ & $\mathrm{P}:(1 / 2,1 / 2, \mathrm{w})$ & $\mathrm{TA}:(1 / 2,1 / 2,1)$ & yes & Line \\
\hline $\mathrm{T}:(1 / 2,1 / 2,2)$ & $\mathrm{P}:(1 / 2,1 / 2, \mathrm{w})$ & $\mathrm{TA}:(1 / 2,1 / 2,1)$ & yes & Line \\
\hline$\Gamma:(0,0,0)$ & $\mathrm{C}:(\mathrm{u}, \mathrm{v}, 0)$ & $\mathrm{X}:(0,1,0)$ & no & Plane \\
\hline$\Gamma:(0,0,0)$ & $\mathrm{C}:(\mathrm{u}, \mathrm{v}, 0)$ & $\mathrm{T}:(1 / 2,1 / 2,0)$ & no & Plane \\
\hline$\Gamma:(0,0,0)$ & $\mathrm{C}:(\mathrm{u}, \mathrm{v}, 0)$ & $\mathrm{TA}:(-1 / 2,1 / 2,0)$ & no & Plane \\
\hline $\mathrm{X}:(2,1,0)$ & $\mathrm{C}:(\mathrm{u}, \mathrm{v}, 0)$ & $\mathrm{T}:(3 / 2,3 / 2,0)$ & yes & Plane \\
\hline $\mathrm{X}:(2,1,0)$ & $\mathrm{C}:(\mathrm{u}, \mathrm{v}, 0)$ & $\mathrm{TA}:(3 / 2,1 / 2,0)$ & yes & Plane \\
\hline $\mathrm{T}:(3 / 2,-1 / 2,0)$ & $\mathrm{C}:(\mathrm{u}, \mathrm{v}, 0)$ & $\mathrm{TA}:(1 / 2,-1 / 2,0)$ & yes & Plane \\
\hline
\end{tabular}

\begin{tabular}{|c|c|c|c|c|}
\hline \multicolumn{5}{|c|}{ BCSID: 2.28; Formula: NpNiGa5; MSG: $74.559\left(\right.$ Im $\left.^{\prime} a^{\prime}\right) ; \mathrm{U}=0$} \\
\hline maximal $k_{1}$ & intermediate path & maximal $k_{2}$ & satisfied? & Line/Plane \\
\hline$\Gamma:(0,0,0)$ & $\mathrm{DT}:(0, \mathrm{v}, 0)$ & $\mathrm{X}:(0,1,0)$ & yes & Line \\
\hline$\Gamma:(0,0,0)$ & $\mathrm{LD}:(0,0, \mathrm{w})$ & $\mathrm{X}:(0,0,1)$ & yes & Line \\
\hline$\Gamma:(0,0,0)$ & SM:(u,0,0) & $\mathrm{X}:(1,0,0)$ & no & Line \\
\hline $\mathrm{W}:(1 / 2,1 / 2,1 / 2)$ & $\mathrm{D}:(\mathrm{u}, 1 / 2,1 / 2)$ & $\mathrm{S}:(0,1 / 2,1 / 2)$ & yes & Line \\
\hline $\mathrm{W}:(1 / 2,1 / 2,1 / 2)$ & $\mathrm{D}:(\mathrm{u}, 1 / 2,1 / 2)$ & $\mathrm{SA}:(1,1 / 2,1 / 2)$ & yes & Line \\
\hline $\mathrm{S}:(0,1 / 2,1 / 2)$ & $\mathrm{D}:(\mathrm{u}, 1 / 2,1 / 2)$ & SA: $(1,1 / 2,1 / 2)$ & yes & Line \\
\hline$\Gamma:(0,0,0)$ & $\mathrm{A}:(0, \mathrm{v}, \mathrm{w})$ & $\mathrm{X}:(0,0,1)$ & yes & Plane \\
\hline$\Gamma:(0,0,0)$ & $\mathrm{A}:(0, \mathrm{v}, \mathrm{w})$ & $\mathrm{S}:(0,1 / 2,1 / 2)$ & yes & Plane \\
\hline$\Gamma:(0,0,0)$ & $\mathrm{A}:(0, \mathrm{v}, \mathrm{w})$ & SA: $(0,-1 / 2,1 / 2)$ & yes & Plane \\
\hline $\mathrm{X}:(0,2,1)$ & $\mathrm{A}:(0, \mathrm{v}, \mathrm{w})$ & $\mathrm{S}:(0,3 / 2,3 / 2)$ & yes & Plane \\
\hline $\mathrm{X}:(0,2,1)$ & $\mathrm{A}:(0, \mathrm{v}, \mathrm{w})$ & $\mathrm{SA}:(0,3 / 2,1 / 2)$ & yes & Plane \\
\hline $\mathrm{S}:(0,1 / 2,1 / 2)$ & $\mathrm{A}:(0, \mathrm{v}, \mathrm{w})$ & $\mathrm{SA}:(0,-1 / 2,1 / 2)$ & yes & Plane \\
\hline \multicolumn{5}{|c|}{ BCSID: 2.28; Formula: NpNiGa5; MSG: $74.559\left(\operatorname{Im}^{\prime} a^{\prime}\right) ; \mathrm{U}=2 \mathrm{eV}$} \\
\hline maximal $k_{1}$ & intermediate path & maximal $k_{2}$ & satisfied? & Line/Plane \\
\hline$\Gamma:(0,0,0)$ & DT: $(0, \mathrm{v}, 0)$ & $\mathrm{X}:(0,1,0)$ & no & Line \\
\hline$\Gamma:(0,0,0)$ & $\mathrm{LD}:(0,0, \mathrm{w})$ & $\mathrm{X}:(0,0,1)$ & no & Line \\
\hline$\Gamma:(0,0,0)$ & $\mathrm{SM}:(\mathrm{u}, 0,0)$ & $\mathrm{X}:(1,0,0)$ & yes & Line \\
\hline $\mathrm{W}:(1 / 2,1 / 2,1 / 2)$ & $\mathrm{D}:(\mathrm{u}, 1 / 2,1 / 2)$ & $\mathrm{S}:(0,1 / 2,1 / 2)$ & yes & Line \\
\hline $\mathrm{W}:(1 / 2,1 / 2,1 / 2)$ & $\mathrm{D}:(\mathrm{u}, 1 / 2,1 / 2)$ & SA: $(1,1 / 2,1 / 2)$ & yes & Line \\
\hline $\mathrm{S}:(0,1 / 2,1 / 2)$ & $\mathrm{D}:(\mathrm{u}, 1 / 2,1 / 2)$ & $\mathrm{SA}:(1,1 / 2,1 / 2)$ & yes & Line \\
\hline$\Gamma:(0,0,0)$ & $\mathrm{A}:(0, \mathrm{v}, \mathrm{w})$ & $\mathrm{X}:(0,0,1)$ & no & Plane \\
\hline$\Gamma:(0,0,0)$ & $\mathrm{A}:(0, \mathrm{v}, \mathrm{w})$ & $\mathrm{S}:(0,1 / 2,1 / 2)$ & no & Plane \\
\hline$\Gamma:(0,0,0)$ & $\mathrm{A}:(0, \mathrm{v}, \mathrm{w})$ & $\mathrm{SA}:(0,-1 / 2,1 / 2)$ & no & Plane \\
\hline $\mathrm{X}:(0,2,1)$ & $\mathrm{A}:(0, \mathrm{v}, \mathrm{w})$ & $\mathrm{S}:(0,3 / 2,3 / 2)$ & yes & Plane \\
\hline $\mathrm{X}:(0,2,1)$ & $\mathrm{A}:(0, \mathrm{v}, \mathrm{w})$ & $\mathrm{SA}:(0,3 / 2,1 / 2)$ & yes & Plane \\
\hline $\mathrm{S}:(0,1 / 2,1 / 2)$ & $\mathrm{A}:(0, \mathrm{v}, \mathrm{w})$ & $\mathrm{SA}:(0,-1 / 2,1 / 2)$ & yes & Plane \\
\hline
\end{tabular}

\begin{tabular}{c|c|c|c|c}
\hline \multicolumn{5}{c}{ BCSID: $2.28 ;$ Formula: NpNiGa5; MSG: 74.559 $\left(\mathrm{Im}^{\prime} a^{\prime}\right) ; \mathrm{U}=6 \mathrm{eV}$} \\
\hline maximal $k_{1}$ & intermediate path & maximal $k_{2}$ & satisfied? & Line/Plane \\
\hline$\Gamma:(0,0,0)$ & $\mathrm{DT}:(0, \mathrm{v}, 0)$ & $\mathrm{X}:(0,1,0)$ & no & Line \\
\hline$\Gamma:(0,0,0)$ & $\mathrm{LD}:(0,0, \mathrm{w})$ & $\mathrm{X}:(0,0,1)$ & no & Line \\
\hline$\Gamma:(0,0,0)$ & $\mathrm{SM}:(\mathrm{u}, 0,0)$ & $\mathrm{X}:(1,0,0)$ & yes & Line \\
\hline $\mathrm{W}:(1 / 2,1 / 2,1 / 2)$ & $\mathrm{D}:(\mathrm{u}, 1 / 2,1 / 2)$ & $\mathrm{S}:(0,1 / 2,1 / 2)$ & yes & Line \\
\hline $\mathrm{W}:(1 / 2,1 / 2,1 / 2)$ & $\mathrm{D}:(\mathrm{u}, 1 / 2,1 / 2)$ & $\mathrm{SA}:(1,1 / 2,1 / 2)$ & yes & Line \\
\hline $\mathrm{S}:(0,1 / 2,1 / 2)$ & $\mathrm{D}:(\mathrm{u}, 1 / 2,1 / 2)$ & $\mathrm{SA}:(1,1 / 2,1 / 2)$ & yes & Line \\
\hline$\Gamma:(0,0,0)$ & $\mathrm{A}:(0, \mathrm{v}, \mathrm{w})$ & $\mathrm{X}:(0,0,1)$ & no & Plane \\
\hline$\Gamma:(0,0,0)$ & $\mathrm{A}:(0, \mathrm{v}, \mathrm{w})$ & $\mathrm{S}:(0,1 / 2,1 / 2)$ & no & Plane \\
\hline$\Gamma:(0,0,0)$ & $\mathrm{A}:(0, \mathrm{v}, \mathrm{w})$ & $\mathrm{SA}:(0,-1 / 2,1 / 2)$ & no & Plane
\end{tabular}




\begin{tabular}{c|c|c|c|c}
\hline $\mathrm{X}:(0,2,1)$ & $\mathrm{A}:(0, \mathrm{v}, \mathrm{w})$ & $\mathrm{S}:(0,3 / 2,3 / 2)$ & yes & Plane \\
\hline $\mathrm{X}:(0,2,1)$ & $\mathrm{A}:(0, \mathrm{v}, \mathrm{w})$ & $\mathrm{SA}:(0,3 / 2,1 / 2)$ & yes & Plane \\
\hline $\mathrm{S}:(0,1 / 2,1 / 2)$ & $\mathrm{A}:(0, \mathrm{v}, \mathrm{w})$ & $\mathrm{SA}:(0,-1 / 2,1 / 2)$ & yes & Plane \\
\hline
\end{tabular}

BCSID: 2.5; Formula: Mn3CuN; MSG: $85.59(P 4 / n)$; U=0

\begin{tabular}{c|c|c|c|c}
\hline maximal $k_{1}$ & intermediate path & maximal $k_{2}$ & satisfied? & Line/Plane \\
\hline$\Gamma:(0,0,0)$ & SM: $(\mathrm{u}, \mathrm{u}, 0)$ & $\mathrm{M}:(1 / 2,1 / 2,0)$ & yes & Line \\
\hline$\Gamma:(0,0,0)$ & $\mathrm{DT}:(0, \mathrm{v}, 0)$ & $\mathrm{X}:(0,1 / 2,0)$ & yes & Line \\
\hline$\Gamma:(0,0,0)$ & $\mathrm{LD}:(0,0, \mathrm{w})$ & $\mathrm{Z}:(0,0,1 / 2)$ & no & Line \\
\hline $\mathrm{A}:(1 / 2,1 / 2,1 / 2)$ & $\mathrm{V}:(1 / 2,1 / 2, \mathrm{w})$ & $\mathrm{M}:(1 / 2,1 / 2,0)$ & yes & Line \\
\hline $\mathrm{A}:(1 / 2,1 / 2,1 / 2)$ & $\mathrm{T}:(\mathrm{u}, 1 / 2,1 / 2)$ & $\mathrm{R}:(0,1 / 2,1 / 2)$ & yes & Line \\
\hline $\mathrm{A}:(1 / 2,1 / 2,1 / 2)$ & $\mathrm{S}:(\mathrm{u}, \mathrm{u}, 1 / 2)$ & $\mathrm{Z}:(0,0,1 / 2)$ & yes & Line \\
\hline $\mathrm{M}:(1 / 2,1 / 2,0)$ & $\mathrm{Y}:(\mathrm{u}, 1 / 2,0)$ & $\mathrm{X}:(0,1 / 2,0)$ & yes & Line \\
\hline $\mathrm{R}:(0,1 / 2,1 / 2)$ & $\mathrm{W}:(0,1 / 2, \mathrm{w})$ & $\mathrm{X}:(0,1 / 2,0)$ & yes & Line \\
\hline $\mathrm{R}:(0,1 / 2,1 / 2)$ & $\mathrm{U}:(0, \mathrm{v}, 1 / 2)$ & $\mathrm{Z}:(0,0,1 / 2)$ & yes & Line \\
\hline$\Gamma:(0,0,0)$ & $\mathrm{D}:(\mathrm{u}, \mathrm{v}, 0)$ & $\mathrm{M}:(1 / 2,1 / 2,0)$ & yes & Plane \\
\hline$\Gamma:(0,0,0)$ & $\mathrm{D}:(\mathrm{u}, \mathrm{v}, 0)$ & $\mathrm{X}:(0,1 / 2,0)$ & yes & Plane \\
\hline $\mathrm{A}:(1 / 2,1 / 2,1 / 2)$ & $\mathrm{E}:(\mathrm{u}, \mathrm{v}, 1 / 2)$ & $\mathrm{R}:(0,1 / 2,1 / 2)$ & yes & Plane \\
\hline $\mathrm{A}:(1 / 2,1 / 2,1 / 2)$ & $\mathrm{E}:(\mathrm{u}, \mathrm{v}, 1 / 2)$ & $\mathrm{Z}:(0,0,1 / 2)$ & yes & Plane \\
\hline $\mathrm{M}:(1 / 2,1 / 2,0)$ & $\mathrm{D}:(\mathrm{u}, \mathrm{v}, 0)$ & $\mathrm{X}:(0,1 / 2,0)$ & yes & Plane \\
\hline $\mathrm{R}:(0,1 / 2,1 / 2)$ & $\mathrm{E}:(\mathrm{u}, \mathrm{v}, 1 / 2)$ & $\mathrm{Z}:(0,0,1 / 2)$ & yes & Plane \\
\hline
\end{tabular}

BCSID: 2.5; Formula: Mn3CuN; MSG: $85.59(P 4 / n)$; U=1eV

\begin{tabular}{c|c|c|c|c}
\hline maximal $k_{1}$ & intermediate path & maximal $k_{2}$ & satisfied? & Line/Plane \\
\hline$\Gamma:(0,0,0)$ & $\mathrm{SM}:(\mathrm{u}, \mathrm{u}, 0)$ & $\mathrm{M}:(1 / 2,1 / 2,0)$ & yes & Line \\
\hline$\Gamma:(0,0,0)$ & $\mathrm{DT}:(0, \mathrm{v}, 0)$ & $\mathrm{X}:(0,1 / 2,0)$ & yes & Line \\
\hline$\Gamma:(0,0,0)$ & $\mathrm{LD}:(0,0, \mathrm{w})$ & $\mathrm{Z}:(0,0,1 / 2)$ & no & Line \\
\hline $\mathrm{A}:(1 / 2,1 / 2,1 / 2)$ & $\mathrm{V}:(1 / 2,1 / 2, \mathrm{w})$ & $\mathrm{M}:(1 / 2,1 / 2,0)$ & yes & Line \\
\hline $\mathrm{A}:(1 / 2,1 / 2,1 / 2)$ & $\mathrm{T}:(\mathrm{u}, 1 / 2,1 / 2)$ & $\mathrm{R}:(0,1 / 2,1 / 2)$ & yes & Line \\
\hline $\mathrm{A}:(1 / 2,1 / 2,1 / 2)$ & $\mathrm{S}:(\mathrm{u}, \mathrm{u}, 1 / 2)$ & $\mathrm{Z}:(0,0,1 / 2)$ & yes & Line \\
\hline $\mathrm{M}:(1 / 2,1 / 2,0)$ & $\mathrm{Y}:(\mathrm{u}, 1 / 2,0)$ & $\mathrm{X}:(0,1 / 2,0)$ & yes & Line \\
\hline $\mathrm{R}:(0,1 / 2,1 / 2)$ & $\mathrm{W}:(0,1 / 2, \mathrm{w})$ & $\mathrm{X}:(0,1 / 2,0)$ & yes & Line \\
\hline $\mathrm{R}:(0,1 / 2,1 / 2)$ & $\mathrm{U}:(0, \mathrm{v}, 1 / 2)$ & $\mathrm{Z}:(0,0,1 / 2)$ & yes & Line \\
\hline$\Gamma:(0,0,0)$ & $\mathrm{D}:(\mathrm{u}, \mathrm{v}, 0)$ & $\mathrm{M}:(1 / 2,1 / 2,0)$ & yes & Plane \\
\hline$\Gamma:(0,0,0)$ & $\mathrm{D}:(\mathrm{u}, \mathrm{v}, 0)$ & $\mathrm{X}:(0,1 / 2,0)$ & yes & Plane \\
\hline $\mathrm{A}:(1 / 2,1 / 2,1 / 2)$ & $\mathrm{E}:(\mathrm{u}, \mathrm{v}, 1 / 2)$ & $\mathrm{R}:(0,1 / 2,1 / 2)$ & yes & Plane \\
\hline $\mathrm{A}:(1 / 2,1 / 2,1 / 2)$ & $\mathrm{E}:(\mathrm{u}, \mathrm{v}, 1 / 2)$ & $\mathrm{Z}:(0,0,1 / 2)$ & yes & Plane \\
\hline $\mathrm{M}:(1 / 2,1 / 2,0)$ & $\mathrm{D}:(\mathrm{u}, \mathrm{v}, 0)$ & $\mathrm{X}:(0,1 / 2,0)$ & yes & Plane \\
\hline $\mathrm{R}:(0,1 / 2,1 / 2)$ & $\mathrm{E}:(\mathrm{u}, \mathrm{v}, 1 / 2)$ & $\mathrm{Z}:(0,0,1 / 2)$ & yes & Plane \\
\hline
\end{tabular}

BCSID: 2.5; Formula: Mn3CuN; MSG: $85.59(P 4 / n)$; U= 2eV

\begin{tabular}{c|c|c|c|c}
\hline maximal $k_{1}$ & intermediate path & maximal $k_{2}$ & satisfied? & Line/Plane \\
\hline$\Gamma:(0,0,0)$ & $\mathrm{SM}:(\mathrm{u}, \mathrm{u}, 0)$ & $\mathrm{M}:(1 / 2,1 / 2,0)$ & yes & Line \\
\hline$\Gamma:(0,0,0)$ & $\mathrm{DT}:(0, \mathrm{v}, 0)$ & $\mathrm{X}:(0,1 / 2,0)$ & yes & Line \\
\hline$\Gamma:(0,0,0)$ & $\mathrm{LD}:(0,0, \mathrm{w})$ & $\mathrm{Z}:(0,0,1 / 2)$ & no & Line \\
\hline $\mathrm{A}:(1 / 2,1 / 2,1 / 2)$ & $\mathrm{V}:(1 / 2,1 / 2, \mathrm{w})$ & $\mathrm{M}:(1 / 2,1 / 2,0)$ & yes & Line \\
\hline $\mathrm{A}:(1 / 2,1 / 2,1 / 2)$ & $\mathrm{T}:(\mathrm{u}, 1 / 2,1 / 2)$ & $\mathrm{R}:(0,1 / 2,1 / 2)$ & yes & Line \\
\hline $\mathrm{A}:(1 / 2,1 / 2,1 / 2)$ & $\mathrm{S}:(\mathrm{u}, \mathrm{u}, 1 / 2)$ & $\mathrm{Z}:(0,0,1 / 2)$ & yes & Line \\
\hline $\mathrm{M}:(1 / 2,1 / 2,0)$ & $\mathrm{Y}:(\mathrm{u}, 1 / 2,0)$ & $\mathrm{X}:(0,1 / 2,0)$ & yes & Line \\
\hline $\mathrm{R}:(0,1 / 2,1 / 2)$ & $\mathrm{W}:(0,1 / 2, \mathrm{w})$ & $\mathrm{X}:(0,1 / 2,0)$ & yes & Line \\
\hline $\mathrm{R}:(0,1 / 2,1 / 2)$ & $\mathrm{U}:(0, \mathrm{v}, 1 / 2)$ & $\mathrm{Z}:(0,0,1 / 2)$ & yes & Line
\end{tabular}




\begin{tabular}{c|c|c|c|c}
\hline$\Gamma:(0,0,0)$ & $\mathrm{D}:(\mathrm{u}, \mathrm{v}, 0)$ & $\mathrm{M}:(1 / 2,1 / 2,0)$ & yes & Plane \\
\hline$\Gamma:(0,0,0)$ & $\mathrm{D}:(\mathrm{u}, \mathrm{v}, 0)$ & $\mathrm{X}:(0,1 / 2,0)$ & yes & Plane \\
\hline $\mathrm{A}:(1 / 2,1 / 2,1 / 2)$ & $\mathrm{E}:(\mathrm{u}, \mathrm{v}, 1 / 2)$ & $\mathrm{R}:(0,1 / 2,1 / 2)$ & yes & Plane \\
\hline $\mathrm{A}:(1 / 2,1 / 2,1 / 2)$ & $\mathrm{E}:(\mathrm{u}, \mathrm{v}, 1 / 2)$ & $\mathrm{Z}:(0,0,1 / 2)$ & yes & Plane \\
\hline $\mathrm{M}:(1 / 2,1 / 2,0)$ & $\mathrm{D}:(\mathrm{u}, \mathrm{v}, 0)$ & $\mathrm{X}:(0,1 / 2,0)$ & yes & Plane \\
\hline $\mathrm{R}:(0,1 / 2,1 / 2)$ & $\mathrm{E}:(\mathrm{u}, \mathrm{v}, 1 / 2)$ & $\mathrm{Z}:(0,0,1 / 2)$ & yes & Plane \\
\hline
\end{tabular}

BCSID: 2.5; Formula: Mn3CuN; MSG: $85.59(P 4 / n)$; U=3eV

\begin{tabular}{c|c|c|c|c}
\hline maximal $k_{1}$ & intermediate path & maximal $k_{2}$ & satisfied? & Line/Plane \\
\hline$\Gamma:(0,0,0)$ & $\mathrm{SM}:(\mathrm{u}, \mathrm{u}, 0)$ & $\mathrm{M}:(1 / 2,1 / 2,0)$ & yes & Line \\
\hline$\Gamma:(0,0,0)$ & $\mathrm{DT}:(0, \mathrm{v}, 0)$ & $\mathrm{X}:(0,1 / 2,0)$ & yes & Line \\
\hline$\Gamma:(0,0,0)$ & $\mathrm{LD}:(0,0, \mathrm{w})$ & $\mathrm{Z}:(0,0,1 / 2)$ & no & Line \\
\hline $\mathrm{A}:(1 / 2,1 / 2,1 / 2)$ & $\mathrm{V}:(1 / 2,1 / 2, \mathrm{w})$ & $\mathrm{M}:(1 / 2,1 / 2,0)$ & yes & Line \\
\hline $\mathrm{A}:(1 / 2,1 / 2,1 / 2)$ & $\mathrm{T}:(\mathrm{u}, 1 / 2,1 / 2)$ & $\mathrm{R}:(0,1 / 2,1 / 2)$ & yes & Line \\
\hline $\mathrm{A}:(1 / 2,1 / 2,1 / 2)$ & $\mathrm{S}:(\mathrm{u}, \mathrm{u}, 1 / 2)$ & $\mathrm{Z}:(0,0,1 / 2)$ & yes & Line \\
\hline $\mathrm{M}:(1 / 2,1 / 2,0)$ & $\mathrm{Y}:(\mathrm{u}, 1 / 2,0)$ & $\mathrm{X}:(0,1 / 2,0)$ & yes & Line \\
\hline $\mathrm{R}:(0,1 / 2,1 / 2)$ & $\mathrm{W}:(0,1 / 2, \mathrm{w})$ & $\mathrm{X}:(0,1 / 2,0)$ & yes & Line \\
\hline $\mathrm{R}:(0,1 / 2,1 / 2)$ & $\mathrm{U}:(0, \mathrm{v}, 1 / 2)$ & $\mathrm{Z}:(0,0,1 / 2)$ & yes & Line \\
\hline$\Gamma:(0,0,0)$ & $\mathrm{D}:(\mathrm{u}, \mathrm{v}, 0)$ & $\mathrm{M}:(1 / 2,1 / 2,0)$ & yes & Plane \\
\hline$\Gamma:(0,0,0)$ & $\mathrm{D}:(\mathrm{u}, \mathrm{v}, 0)$ & $\mathrm{X}:(0,1 / 2,0)$ & yes & Plane \\
\hline $\mathrm{A}:(1 / 2,1 / 2,1 / 2)$ & $\mathrm{E}:(\mathrm{u}, \mathrm{v}, 1 / 2)$ & $\mathrm{R}:(0,1 / 2,1 / 2)$ & yes & Plane \\
\hline $\mathrm{A}:(1 / 2,1 / 2,1 / 2)$ & $\mathrm{E}:(\mathrm{u}, \mathrm{v}, 1 / 2)$ & $\mathrm{Z}:(0,0,1 / 2)$ & yes & Plane \\
\hline $\mathrm{M}:(1 / 2,1 / 2,0)$ & $\mathrm{D}:(\mathrm{u}, \mathrm{v}, 0)$ & $\mathrm{X}:(0,1 / 2,0)$ & yes & Plane \\
\hline $\mathrm{R}:(0,1 / 2,1 / 2)$ & $\mathrm{E}:(\mathrm{u}, \mathrm{v}, 1 / 2)$ & $\mathrm{Z}:(0,0,1 / 2)$ & yes & Plane \\
\hline
\end{tabular}

BCSID: 2.5; Formula: Mn3CuN; MSG: $85.59(P 4 / n)$; $\mathrm{U}=4 \mathrm{eV}$

\begin{tabular}{c|c|c|c|c}
\hline maximal $k_{1}$ & intermediate path & maximal $k_{2}$ & satisfied? & Line/Plane \\
\hline$\Gamma:(0,0,0)$ & $\mathrm{SM}:(\mathrm{u}, \mathrm{u}, 0)$ & $\mathrm{M}:(1 / 2,1 / 2,0)$ & yes & Line \\
\hline$\Gamma:(0,0,0)$ & $\mathrm{DT}:(0, \mathrm{v}, 0)$ & $\mathrm{X}:(0,1 / 2,0)$ & yes & Line \\
\hline$\Gamma:(0,0,0)$ & $\mathrm{LD}:(0,0, \mathrm{w})$ & $\mathrm{Z}:(0,0,1 / 2)$ & no & Line \\
\hline $\mathrm{A}:(1 / 2,1 / 2,1 / 2)$ & $\mathrm{V}:(1 / 2,1 / 2, \mathrm{w})$ & $\mathrm{M}:(1 / 2,1 / 2,0)$ & no & Line \\
\hline $\mathrm{A}:(1 / 2,1 / 2,1 / 2)$ & $\mathrm{T}:(\mathrm{u}, 1 / 2,1 / 2)$ & $\mathrm{R}:(0,1 / 2,1 / 2)$ & yes & Line \\
\hline $\mathrm{A}:(1 / 2,1 / 2,1 / 2)$ & $\mathrm{S}:(\mathrm{u}, \mathrm{u}, 1 / 2)$ & $\mathrm{Z}:(0,0,1 / 2)$ & yes & Line \\
\hline $\mathrm{M}:(1 / 2,1 / 2,0)$ & $\mathrm{Y}:(\mathrm{u}, 1 / 2,0)$ & $\mathrm{X}:(0,1 / 2,0)$ & yes & Line \\
\hline $\mathrm{R}:(0,1 / 2,1 / 2)$ & $\mathrm{W}:(0,1 / 2, \mathrm{w})$ & $\mathrm{X}:(0,1 / 2,0)$ & yes & Line \\
\hline $\mathrm{R}:(0,1 / 2,1 / 2)$ & $\mathrm{U}:(0, \mathrm{v}, 1 / 2)$ & $\mathrm{Z}:(0,0,1 / 2)$ & yes & Line \\
\hline$\Gamma:(0,0,0)$ & $\mathrm{D}:(\mathrm{u}, \mathrm{v}, 0)$ & $\mathrm{M}:(1 / 2,1 / 2,0)$ & yes & Plane \\
\hline$\Gamma:(0,0,0)$ & $\mathrm{D}:(\mathrm{u}, \mathrm{v}, 0)$ & $\mathrm{X}:(0,1 / 2,0)$ & yes & Plane \\
\hline $\mathrm{A}:(1 / 2,1 / 2,1 / 2)$ & $\mathrm{E}:(\mathrm{u}, \mathrm{v}, 1 / 2)$ & $\mathrm{R}:(0,1 / 2,1 / 2)$ & yes & Plane \\
\hline $\mathrm{A}:(1 / 2,1 / 2,1 / 2)$ & $\mathrm{E}:(\mathrm{u}, \mathrm{v}, 1 / 2)$ & $\mathrm{Z}:(0,0,1 / 2)$ & yes & Plane \\
\hline $\mathrm{M}:(1 / 2,1 / 2,0)$ & $\mathrm{D}:(\mathrm{u}, \mathrm{v}, 0)$ & $\mathrm{X}:(0,1 / 2,0)$ & yes & Plane \\
\hline $\mathrm{R}:(0,1 / 2,1 / 2)$ & $\mathrm{E}:(\mathrm{u}, \mathrm{v}, 1 / 2)$ & $\mathrm{Z}:(0,0,1 / 2)$ & yes & Plane \\
\hline
\end{tabular}

BCSID: 0.207; Formula: TlFe1.6Se2; MSG: $87.75(I 4 / m)$; U=1eV

\begin{tabular}{c|c|c|c|c}
\hline maximal $k_{1}$ & intermediate path & maximal $k_{2}$ & satisfied? & Line/Plane \\
\hline$\Gamma:(0,0,0)$ & $\mathrm{LD}:(0,0, \mathrm{w})$ & $\mathrm{M}:(0,0,1)$ & yes & Line \\
\hline$\Gamma:(0,0,0)$ & $\mathrm{SM}:(\mathrm{u}, 0,0)$ & $\mathrm{M}:(1,0,0)$ & no & Line \\
\hline$\Gamma:(0,0,0)$ & $\mathrm{DT}:(\mathrm{u}, \mathrm{u}, 0)$ & $\mathrm{X}:(1 / 2,1 / 2,0)$ & no & Line \\
\hline $\mathrm{M}:(1,0,0)$ & $\mathrm{Y}:(\mathrm{u}, 1-\mathrm{u}, 0)$ & $\mathrm{X}:(1 / 2,1 / 2,0)$ & yes & Line \\
\hline $\mathrm{P}:(1 / 2,1 / 2,1 / 2)$ & $\mathrm{W}:(1 / 2,1 / 2, \mathrm{w})$ & $\mathrm{X}:(1 / 2,1 / 2,0)$ & yes & Line \\
\hline$\Gamma:(0,0,0)$ & $\mathrm{C}:(\mathrm{u}, \mathrm{v}, 0)$ & $\mathrm{M}:(0,1,0)$ & no & Plane
\end{tabular}




\begin{tabular}{|c|c|c|c|c|}
\hline$\Gamma:(0,0,0)$ & $\mathrm{C}:(\mathrm{u}, \mathrm{v}, 0)$ & $\mathrm{X}:(1 / 2,1 / 2,0)$ & no & Plane \\
\hline $\mathrm{M}:(2,1,0)$ & $\mathrm{C}:(\mathrm{u}, \mathrm{v}, 0)$ & $\mathrm{X}:(3 / 2,3 / 2,0)$ & yes & Plane \\
\hline \multicolumn{5}{|c|}{ BCSID: 0.207; Formula: TlFe1.6Se2; MSG: $87.75(I 4 / m)$; U=2eV } \\
\hline maximal $k_{1}$ & intermediate path & maximal $k_{2}$ & satisfied? & Line/Plane \\
\hline$\Gamma:(0,0,0)$ & $\mathrm{LD}:(0,0, \mathrm{w})$ & $\mathrm{M}:(0,0,1)$ & yes & Line \\
\hline$\Gamma:(0,0,0)$ & SM:(u,0,0) & $\mathrm{M}:(1,0,0)$ & yes & Line \\
\hline$\Gamma:(0,0,0)$ & $\mathrm{DT}:(\mathrm{u}, \mathrm{u}, 0)$ & $\mathrm{X}:(1 / 2,1 / 2,0)$ & no & Line \\
\hline $\mathrm{M}:(1,0,0)$ & $\mathrm{Y}:(\mathrm{u}, 1-\mathrm{u}, 0)$ & $\mathrm{X}:(1 / 2,1 / 2,0)$ & no & Line \\
\hline $\mathrm{P}:(1 / 2,1 / 2,1 / 2)$ & $\mathrm{W}:(1 / 2,1 / 2, \mathrm{w})$ & $\mathrm{X}:(1 / 2,1 / 2,0)$ & yes & Line \\
\hline$\Gamma:(0,0,0)$ & $\mathrm{C}:(\mathrm{u}, \mathrm{v}, 0)$ & $\mathrm{M}:(0,1,0)$ & yes & Plane \\
\hline$\Gamma:(0,0,0)$ & $\mathrm{C}:(\mathrm{u}, \mathrm{v}, 0)$ & $\mathrm{X}:(1 / 2,1 / 2,0)$ & no & Plane \\
\hline $\mathrm{M}:(2,1,0)$ & $\mathrm{C}:(\mathrm{u}, \mathrm{v}, 0)$ & $\mathrm{X}:(3 / 2,3 / 2,0)$ & no & Plane \\
\hline \multicolumn{5}{|c|}{ BCSID: 0.207; Formula: TlFe1.6Se2; MSG: $87.75(I 4 / m) ; \mathrm{U}=3 \mathrm{eV}$} \\
\hline maximal $k_{1}$ & intermediate path & maximal $k_{2}$ & satisfied? & Line/Plane \\
\hline$\Gamma:(0,0,0)$ & LD: $(0,0, w)$ & $\mathrm{M}:(0,0,1)$ & yes & Line \\
\hline$\Gamma:(0,0,0)$ & SM:(u,0,0) & $\mathrm{M}:(1,0,0)$ & no & Line \\
\hline$\Gamma:(0,0,0)$ & DT:(u,u,0) & $\mathrm{X}:(1 / 2,1 / 2,0)$ & no & Line \\
\hline $\mathrm{M}:(1,0,0)$ & $\mathrm{Y}:(\mathrm{u}, 1-\mathrm{u}, 0)$ & $\mathrm{X}:(1 / 2,1 / 2,0)$ & yes & Line \\
\hline $\mathrm{P}:(1 / 2,1 / 2,1 / 2)$ & $\mathrm{W}:(1 / 2,1 / 2, \mathrm{w})$ & $\mathrm{X}:(1 / 2,1 / 2,0)$ & yes & Line \\
\hline$\Gamma:(0,0,0)$ & $\mathrm{C}:(\mathrm{u}, \mathrm{v}, 0)$ & $\mathrm{M}:(0,1,0)$ & no & Plane \\
\hline$\Gamma:(0,0,0)$ & $\mathrm{C}:(\mathrm{u}, \mathrm{v}, 0)$ & $\mathrm{X}:(1 / 2,1 / 2,0)$ & no & Plane \\
\hline $\mathrm{M}:(2,1,0)$ & $\mathrm{C}:(\mathrm{u}, \mathrm{v}, 0)$ & $\mathrm{X}:(3 / 2,3 / 2,0)$ & yes & Plane \\
\hline \multicolumn{5}{|c|}{ BCSID: 0.64; Formula: MnV2O4; MSG: $88.81\left(I 4_{1} / a\right)$; U=0 } \\
\hline $\operatorname{maximal} k_{1}$ & intermediate path & maximal $k_{2}$ & satisfied? & Line/Plane \\
\hline$\Gamma:(0,0,0)$ & LD: $(0,0, w)$ & $\mathrm{M}:(0,0,1)$ & no & Line \\
\hline$\Gamma:(0,0,0)$ & SM:(u,0,0) & $\mathrm{M}:(1,0,0)$ & yes & Line \\
\hline$\Gamma:(0,0,0)$ & DT:(u,u,0) & $\mathrm{X}:(1 / 2,1 / 2,0)$ & yes & Line \\
\hline $\mathrm{M}:(1,0,0)$ & $\mathrm{Y}:(\mathrm{u}, 1-\mathrm{u}, 0)$ & $\mathrm{X}:(1 / 2,1 / 2,0)$ & yes & Line \\
\hline $\mathrm{P}:(1 / 2,1 / 2,1 / 2)$ & $\mathrm{W}:(1 / 2,1 / 2, \mathrm{w})$ & $\mathrm{X}:(1 / 2,1 / 2,0)$ & yes & Line \\
\hline$\Gamma:(0,0,0)$ & $\mathrm{C}:(\mathrm{u}, \mathrm{v}, 0)$ & $\mathrm{M}:(0,1,0)$ & yes & Plane \\
\hline$\Gamma:(0,0,0)$ & $\mathrm{C}:(\mathrm{u}, \mathrm{v}, 0)$ & $\mathrm{X}:(1 / 2,1 / 2,0)$ & yes & Plane \\
\hline $\mathrm{M}:(2,1,0)$ & $\mathrm{C}:(\mathrm{u}, \mathrm{v}, 0)$ & $\mathrm{X}:(3 / 2,3 / 2,0)$ & yes & Plane \\
\hline
\end{tabular}

BCSID: 1.0.11; Formula: CeCoGe3; MSG: $107.231\left(I 4 m^{\prime} m^{\prime}\right) ; \mathrm{U}=4 \mathrm{eV}$

\begin{tabular}{c|c|c|c|c}
\hline maximal $k_{1}$ & intermediate path & maximal $k_{2}$ & satisfied? & Line/Plane \\
\hline$\Gamma:(0,0,0)$ & $\mathrm{LD}:(0,0, \mathrm{w})$ & $\mathrm{M}:(0,0,1)$ & no & Line \\
\hline $\mathrm{P}:(1 / 2,1 / 2,1 / 2)$ & $\mathrm{W}:(1 / 2,1 / 2, \mathrm{w})$ & $\mathrm{X}:(1 / 2,1 / 2,0)$ & yes & Line \\
\hline
\end{tabular}

BCSID: 1.85; Formula: alpha-Mn; MSG: $114.282\left(P_{I}-42_{1} c\right) ; \mathrm{U}=0$

\begin{tabular}{c|c|c|c|c}
\hline maximal $k_{1}$ & intermediate path & maximal $k_{2}$ & satisfied? & Line/Plane \\
\hline$\Gamma:(0,0,0)$ & $\mathrm{SM}:(\mathrm{u}, \mathrm{u}, 0)$ & $\mathrm{M}:(1 / 2,1 / 2,0)$ & yes & Line \\
\hline$\Gamma:(0,0,0)$ & $\mathrm{DT}:(0, \mathrm{v}, 0)$ & $\mathrm{X}:(0,1 / 2,0)$ & yes & Line \\
\hline $\mathrm{M}:(1 / 2,1 / 2,0)$ & $\mathrm{Y}:(\mathrm{u}, 1 / 2,0)$ & $\mathrm{X}:(0,1 / 2,0)$ & yes & Line \\
\hline $\mathrm{R}:(0,1 / 2,1 / 2)$ & $\mathrm{W}:(0,1 / 2, \mathrm{w})$ & $\mathrm{X}:(0,1 / 2,0)$ & no & Line \\
\hline$\Gamma:(0,0,0)$ & $\mathrm{C}:(\mathrm{u}, \mathrm{u}, \mathrm{w})$ & $\mathrm{A}:(1 / 2,1 / 2,1 / 2)$ & yes & Plane \\
\hline$\Gamma:(0,0,0)$ & $\mathrm{C}:(\mathrm{u}, \mathrm{u}, \mathrm{w})$ & $\mathrm{M}:(1 / 2,1 / 2,0)$ & yes & Plane \\
\hline$\Gamma:(0,0,0)$ & $\mathrm{C}:(\mathrm{u}, \mathrm{u}, \mathrm{w})$ & $\mathrm{Z}:(0,0,1 / 2)$ & yes & Plane \\
\hline $\mathrm{A}:(1 / 2,1 / 2,1 / 2)$ & $\mathrm{C}:(\mathrm{u}, \mathrm{u}, \mathrm{w})$ & $\mathrm{M}:(1 / 2,1 / 2,0)$ & yes & Plane
\end{tabular}




\begin{tabular}{|c|c|c|c|c|}
\hline $\mathrm{A}:(1 / 2,1 / 2,1 / 2)$ & $\mathrm{C}:(\mathrm{u}, \mathrm{u}, \mathrm{w})$ & $\mathrm{Z}:(0,0,1 / 2)$ & yes & Plane \\
\hline $\mathrm{M}:(1 / 2,1 / 2,0)$ & $\mathrm{C}:(\mathrm{u}, \mathrm{u}, \mathrm{w})$ & $\mathrm{Z}:(0,0,1 / 2)$ & yes & Plane \\
\hline \multicolumn{5}{|c|}{ BCSID: 2.26 ; Formula: PrCo2P2; MSG: $123.345\left(P 4 / \mathrm{mm}^{\prime} \mathrm{m}^{\prime}\right) ; \mathrm{U}=0$} \\
\hline maximal $k_{1}$ & intermediate path & maximal $k_{2}$ & satisfied? & Line/Plane \\
\hline$\Gamma:(0,0,0)$ & SM:(u,u,0) & $\mathrm{M}:(1 / 2,1 / 2,0)$ & no & Line \\
\hline$\Gamma:(0,0,0)$ & DT: $(0, \mathrm{v}, 0)$ & $\mathrm{X}:(0,1 / 2,0)$ & no & Line \\
\hline$\Gamma:(0,0,0)$ & LD: $(0,0, \mathrm{w})$ & $\mathrm{Z}:(0,0,1 / 2)$ & yes & Line \\
\hline $\mathrm{A}:(1 / 2,1 / 2,1 / 2)$ & $\mathrm{V}:(1 / 2,1 / 2, \mathrm{w})$ & $\mathrm{M}:(1 / 2,1 / 2,0)$ & no & Line \\
\hline $\mathrm{A}:(1 / 2,1 / 2,1 / 2)$ & $\mathrm{T}:(\mathrm{u}, 1 / 2,1 / 2)$ & $\mathrm{R}:(0,1 / 2,1 / 2)$ & yes & Line \\
\hline $\mathrm{A}:(1 / 2,1 / 2,1 / 2)$ & $\mathrm{S}:(\mathrm{u}, \mathrm{u}, 1 / 2)$ & $\mathrm{Z}:(0,0,1 / 2)$ & yes & Line \\
\hline $\mathrm{M}:(1 / 2,1 / 2,0)$ & $\mathrm{Y}:(\mathrm{u}, 1 / 2,0)$ & $\mathrm{X}:(0,1 / 2,0)$ & no & Line \\
\hline $\mathrm{R}:(0,1 / 2,1 / 2)$ & $\mathrm{W}:(0,1 / 2, \mathrm{w})$ & $\mathrm{X}:(0,1 / 2,0)$ & yes & Line \\
\hline $\mathrm{R}:(0,1 / 2,1 / 2)$ & $\mathrm{U}:(0, \mathrm{v}, 1 / 2)$ & $\mathrm{Z}:(0,0,1 / 2)$ & yes & Line \\
\hline$\Gamma:(0,0,0)$ & $\mathrm{D}:(\mathrm{u}, \mathrm{v}, 0)$ & $\mathrm{M}:(1 / 2,1 / 2,0)$ & no & Plane \\
\hline$\Gamma:(0,0,0)$ & $\mathrm{D}:(\mathrm{u}, \mathrm{v}, 0)$ & $\mathrm{X}:(0,1 / 2,0)$ & no & Plane \\
\hline $\mathrm{A}:(1 / 2,1 / 2,1 / 2)$ & $\mathrm{E}:(\mathrm{u}, \mathrm{v}, 1 / 2)$ & $\mathrm{R}:(0,1 / 2,1 / 2)$ & yes & Plane \\
\hline $\mathrm{A}:(1 / 2,1 / 2,1 / 2)$ & $\mathrm{E}:(\mathrm{u}, \mathrm{v}, 1 / 2)$ & $\mathrm{Z}:(0,0,1 / 2)$ & yes & Plane \\
\hline $\mathrm{M}:(1 / 2,1 / 2,0)$ & $\mathrm{D}:(\mathrm{u}, \mathrm{v}, 0)$ & $\mathrm{X}:(0,1 / 2,0)$ & no & Plane \\
\hline $\mathrm{R}:(0,1 / 2,1 / 2)$ & $\mathrm{E}:(\mathrm{u}, \mathrm{v}, 1 / 2)$ & $\mathrm{Z}:(0,0,1 / 2)$ & yes & Plane \\
\hline
\end{tabular}

\begin{tabular}{c|c|c|c|c}
\hline \multicolumn{5}{c}{ BCSID: $2.26 ;$ Formula: PrCo2P2; MSG: $123.345\left(P 4 / m^{\prime} m^{\prime}\right) ; \mathrm{U}=2 \mathrm{eV}$} \\
\hline maximal $k_{1}$ & intermediate path & maximal $k_{2}$ & satisfied? & Line/Plane \\
\hline$\Gamma:(0,0,0)$ & $\mathrm{SM}:(\mathrm{u}, \mathrm{u}, 0)$ & $\mathrm{M}:(1 / 2,1 / 2,0)$ & yes & Line \\
\hline$\Gamma:(0,0,0)$ & $\mathrm{DT}:(0, \mathrm{v}, 0)$ & $\mathrm{X}:(0,1 / 2,0)$ & yes & Line \\
\hline$\Gamma:(0,0,0)$ & $\mathrm{LD}:(0,0, \mathrm{w})$ & $\mathrm{Z}:(0,0,1 / 2)$ & yes & Line \\
\hline $\mathrm{A}:(1 / 2,1 / 2,1 / 2)$ & $\mathrm{V}:(1 / 2,1 / 2, \mathrm{w})$ & $\mathrm{M}:(1 / 2,1 / 2,0)$ & yes & Line \\
\hline $\mathrm{A}:(1 / 2,1 / 2,1 / 2)$ & $\mathrm{T}:(\mathrm{u}, 1 / 2,1 / 2)$ & $\mathrm{R}:(0,1 / 2,1 / 2)$ & no & Line \\
\hline $\mathrm{A}:(1 / 2,1 / 2,1 / 2)$ & $\mathrm{S}:(\mathrm{u}, \mathrm{u}, 1 / 2)$ & $\mathrm{Z}:(0,0,1 / 2)$ & yes & Line \\
\hline $\mathrm{M}:(1 / 2,1 / 2,0)$ & $\mathrm{Y}:(\mathrm{u}, 1 / 2,0)$ & $\mathrm{X}:(0,1 / 2,0)$ & yes & Line \\
\hline $\mathrm{R}:(0,1 / 2,1 / 2)$ & $\mathrm{W}:(0,1 / 2, \mathrm{w})$ & $\mathrm{X}:(0,1 / 2,0)$ & yes & Line \\
\hline $\mathrm{R}:(0,1 / 2,1 / 2)$ & $\mathrm{U}:(0, \mathrm{v}, 1 / 2)$ & $\mathrm{Z}:(0,0,1 / 2)$ & no & Line \\
\hline$\Gamma:(0,0,0)$ & $\mathrm{D}:(\mathrm{u}, \mathrm{v}, 0)$ & $\mathrm{M}:(1 / 2,1 / 2,0)$ & yes & Plane \\
\hline$\Gamma:(0,0,0)$ & $\mathrm{D}:(\mathrm{u}, \mathrm{v}, 0)$ & $\mathrm{X}:(0,1 / 2,0)$ & yes & Plane \\
\hline $\mathrm{A}:(1 / 2,1 / 2,1 / 2)$ & $\mathrm{E}:(\mathrm{u}, \mathrm{v}, 1 / 2)$ & $\mathrm{R}:(0,1 / 2,1 / 2)$ & no & Plane \\
\hline $\mathrm{A}:(1 / 2,1 / 2,1 / 2)$ & $\mathrm{E}:(\mathrm{u}, \mathrm{v}, 1 / 2)$ & $\mathrm{Z}:(0,0,1 / 2)$ & yes & Plane \\
\hline $\mathrm{M}:(1 / 2,1 / 2,0)$ & $\mathrm{D}:(\mathrm{u}, \mathrm{v}, 0)$ & $\mathrm{X}:(0,1 / 2,0)$ & yes & Plane \\
\hline $\mathrm{R}:(0,1 / 2,1 / 2)$ & $\mathrm{E}:(\mathrm{u}, \mathrm{v}, 1 / 2)$ & $\mathrm{Z}:(0,0,1 / 2)$ & no & Plane \\
\hline
\end{tabular}

\begin{tabular}{c|c|c|c|c}
\multicolumn{4}{c}{ BCSID: $2.26 ;$ Formula: PrCo2P2; MSG: $123.345\left(P 4 / m^{\prime} m^{\prime}\right) ; \mathrm{U}=4 \mathrm{eV}$} \\
\hline maximal $k_{1}$ & intermediate path & maximal $k_{2}$ & satisfied? & Line/Plane \\
\hline$\Gamma:(0,0,0)$ & $\mathrm{SM}:(\mathrm{u}, \mathrm{u}, 0)$ & $\mathrm{M}:(1 / 2,1 / 2,0)$ & yes & Line \\
\hline$\Gamma:(0,0,0)$ & $\mathrm{DT}:(0, \mathrm{v}, 0)$ & $\mathrm{X}:(0,1 / 2,0)$ & no & Line \\
\hline$\Gamma:(0,0,0)$ & $\mathrm{LD}:(0,0, \mathrm{w})$ & $\mathrm{Z}:(0,0,1 / 2)$ & yes & Line \\
\hline $\mathrm{A}:(1 / 2,1 / 2,1 / 2)$ & $\mathrm{V}:(1 / 2,1 / 2, \mathrm{w})$ & $\mathrm{M}:(1 / 2,1 / 2,0)$ & yes & Line \\
\hline $\mathrm{A}:(1 / 2,1 / 2,1 / 2)$ & $\mathrm{T}:(\mathrm{u}, 1 / 2,1 / 2)$ & $\mathrm{R}:(0,1 / 2,1 / 2)$ & no & Line \\
\hline $\mathrm{A}:(1 / 2,1 / 2,1 / 2)$ & $\mathrm{S}:(\mathrm{u}, \mathrm{u}, 1 / 2)$ & $\mathrm{Z}:(0,0,1 / 2)$ & no & Line \\
\hline $\mathrm{M}:(1 / 2,1 / 2,0)$ & $\mathrm{Y}:(\mathrm{u}, 1 / 2,0)$ & $\mathrm{X}:(0,1 / 2,0)$ & no & Line \\
\hline $\mathrm{R}:(0,1 / 2,1 / 2)$ & $\mathrm{W}:(0,1 / 2, \mathrm{w})$ & $\mathrm{X}:(0,1 / 2,0)$ & no & Line \\
\hline $\mathrm{R}:(0,1 / 2,1 / 2)$ & $\mathrm{U}:(0, \mathrm{v}, 1 / 2)$ & $\mathrm{Z}:(0,0,1 / 2)$ & no & Line \\
\hline$\Gamma:(0,0,0)$ & $\mathrm{D}:(\mathrm{u}, \mathrm{v}, 0)$ & $\mathrm{M}:(1 / 2,1 / 2,0)$ & yes & Plane
\end{tabular}




\begin{tabular}{c|c|c|c|c}
\hline$\Gamma:(0,0,0)$ & $\mathrm{D}:(\mathrm{u}, \mathrm{v}, 0)$ & $\mathrm{X}:(0,1 / 2,0)$ & no & Plane \\
\hline $\mathrm{A}:(1 / 2,1 / 2,1 / 2)$ & $\mathrm{E}:(\mathrm{u}, \mathrm{v}, 1 / 2)$ & $\mathrm{R}:(0,1 / 2,1 / 2)$ & no & Plane \\
\hline $\mathrm{A}:(1 / 2,1 / 2,1 / 2)$ & $\mathrm{E}:(\mathrm{u}, \mathrm{v}, 1 / 2)$ & $\mathrm{Z}:(0,0,1 / 2)$ & no & Plane \\
\hline $\mathrm{M}:(1 / 2,1 / 2,0)$ & $\mathrm{D}:(\mathrm{u}, \mathrm{v}, 0)$ & $\mathrm{X}:(0,1 / 2,0)$ & no & Plane \\
\hline $\mathrm{R}:(0,1 / 2,1 / 2)$ & $\mathrm{E}:(\mathrm{u}, \mathrm{v}, 1 / 2)$ & $\mathrm{Z}:(0,0,1 / 2)$ & no & Plane \\
\hline
\end{tabular}

BCSID: 2.26; Formula: PrCo2P2; MSG: $123.345\left(P 4 / \mathrm{mm}^{\prime} \mathrm{m}^{\prime}\right) ; \mathrm{U}=6 \mathrm{eV}$

\begin{tabular}{c|c|c|c|c}
\hline maximal $k_{1}$ & intermediate path & maximal $k_{2}$ & satisfied? & Line/Plane \\
\hline$\Gamma:(0,0,0)$ & $\mathrm{SM}:(\mathrm{u}, \mathrm{u}, 0)$ & $\mathrm{M}:(1 / 2,1 / 2,0)$ & no & Line \\
\hline$\Gamma:(0,0,0)$ & $\mathrm{DT}:(0, \mathrm{v}, 0)$ & $\mathrm{X}:(0,1 / 2,0)$ & no & Line \\
\hline$\Gamma:(0,0,0)$ & $\mathrm{LD}:(0,0, \mathrm{w})$ & $\mathrm{Z}:(0,0,1 / 2)$ & no & Line \\
\hline $\mathrm{A}:(1 / 2,1 / 2,1 / 2)$ & $\mathrm{V}:(1 / 2,1 / 2, \mathrm{w})$ & $\mathrm{M}:(1 / 2,1 / 2,0)$ & yes & Line \\
\hline $\mathrm{A}:(1 / 2,1 / 2,1 / 2)$ & $\mathrm{T}:(\mathrm{u}, 1 / 2,1 / 2)$ & $\mathrm{R}:(0,1 / 2,1 / 2)$ & no & Line \\
\hline $\mathrm{A}:(1 / 2,1 / 2,1 / 2)$ & $\mathrm{S}:(\mathrm{u}, \mathrm{u}, 1 / 2)$ & $\mathrm{Z}:(0,0,1 / 2)$ & yes & Line \\
\hline $\mathrm{M}:(1 / 2,1 / 2,0)$ & $\mathrm{Y}:(\mathrm{u}, 1 / 2,0)$ & $\mathrm{X}:(0,1 / 2,0)$ & yes & Line \\
\hline $\mathrm{R}:(0,1 / 2,1 / 2)$ & $\mathrm{W}:(0,1 / 2, \mathrm{w})$ & $\mathrm{X}:(0,1 / 2,0)$ & yes & Line \\
\hline $\mathrm{R}:(0,1 / 2,1 / 2)$ & $\mathrm{U}:(0, \mathrm{v}, 1 / 2)$ & $\mathrm{Z}:(0,0,1 / 2)$ & no & Line \\
\hline$\Gamma:(0,0,0)$ & $\mathrm{D}:(\mathrm{u}, \mathrm{v}, 0)$ & $\mathrm{M}:(1 / 2,1 / 2,0)$ & no & Plane \\
\hline$\Gamma:(0,0,0)$ & $\mathrm{D}:(\mathrm{u}, \mathrm{v}, 0)$ & $\mathrm{X}:(0,1 / 2,0)$ & no & Plane \\
\hline $\mathrm{A}:(1 / 2,1 / 2,1 / 2)$ & $\mathrm{E}:(\mathrm{u}, \mathrm{v}, 1 / 2)$ & $\mathrm{R}:(0,1 / 2,1 / 2)$ & no & Plane \\
\hline $\mathrm{A}:(1 / 2,1 / 2,1 / 2)$ & $\mathrm{E}:(\mathrm{u}, \mathrm{v}, 1 / 2)$ & $\mathrm{Z}:(0,0,1 / 2)$ & yes & Plane \\
\hline $\mathrm{M}:(1 / 2,1 / 2,0)$ & $\mathrm{D}:(\mathrm{u}, \mathrm{v}, 0)$ & $\mathrm{X}:(0,1 / 2,0)$ & yes & Plane \\
\hline $\mathrm{R}:(0,1 / 2,1 / 2)$ & $\mathrm{E}:(\mathrm{u}, \mathrm{v}, 1 / 2)$ & $\mathrm{Z}:(0,0,1 / 2)$ & no & Plane \\
\hline
\end{tabular}

\begin{tabular}{c|c|c|c|c}
\hline \multicolumn{3}{c}{ BCSID: $1.162 ;$ Formula: NdMg; MSG: $124.360\left(P_{c} 4 / m c c\right) ; \mathrm{U}=0$} \\
\hline maximal $k_{1}$ & intermediate path & maximal $k_{2}$ & satisfied? & Line/Plane \\
\hline$\Gamma:(0,0,0)$ & $\mathrm{LD}:(0,0, \mathrm{w})$ & $\mathrm{Z}:(0,0,1 / 2)$ & yes & Line \\
\hline $\mathrm{A}:(1 / 2,1 / 2,1 / 2)$ & $\mathrm{V}:(1 / 2,1 / 2, \mathrm{w})$ & $\mathrm{M}:(1 / 2,1 / 2,0)$ & yes & Line \\
\hline $\mathrm{A}:(1 / 2,1 / 2,1 / 2)$ & $\mathrm{T}:(\mathrm{u}, 1 / 2,1 / 2)$ & $\mathrm{R}:(0,1 / 2,1 / 2)$ & no & Line \\
\hline $\mathrm{A}:(1 / 2,1 / 2,1 / 2)$ & $\mathrm{S}:(\mathrm{u}, \mathrm{u}, 1 / 2)$ & $\mathrm{Z}:(0,0,1 / 2)$ & no & Line \\
\hline $\mathrm{R}:(0,1 / 2,1 / 2)$ & $\mathrm{U}:(0, \mathrm{v}, 1 / 2)$ & $\mathrm{Z}:(0,0,1 / 2)$ & yes & Line \\
\hline $\mathrm{A}:(1 / 2,1 / 2,1 / 2)$ & $\mathrm{E}:(\mathrm{u}, \mathrm{v}, 1 / 2)$ & $\mathrm{R}:(0,1 / 2,1 / 2)$ & no & Plane \\
\hline $\mathrm{A}:(1 / 2,1 / 2,1 / 2)$ & $\mathrm{E}:(\mathrm{u}, \mathrm{v}, 1 / 2)$ & $\mathrm{Z}:(0,0,1 / 2)$ & no & Plane \\
\hline $\mathrm{R}:(0,1 / 2,1 / 2)$ & $\mathrm{E}:(\mathrm{u}, \mathrm{v}, 1 / 2)$ & $\mathrm{Z}:(0,0,1 / 2)$ & yes & Plane \\
\hline
\end{tabular}

\section{BCSID: 1.162; Formula: NdMg; MSG: $124.360\left(P_{c} 4 / m c c\right)$; $=2 \mathrm{eV}$}

\begin{tabular}{c|c|c|c|c}
\hline maximal $k_{1}$ & intermediate path & maximal $k_{2}$ & satisfied? & Line/Plane \\
\hline$\Gamma:(0,0,0)$ & $\mathrm{LD}:(0,0, \mathrm{w})$ & $\mathrm{Z}:(0,0,1 / 2)$ & yes & Line \\
\hline $\mathrm{A}:(1 / 2,1 / 2,1 / 2)$ & $\mathrm{V}:(1 / 2,1 / 2, \mathrm{w})$ & $\mathrm{M}:(1 / 2,1 / 2,0)$ & no & Line \\
\hline $\mathrm{A}:(1 / 2,1 / 2,1 / 2)$ & $\mathrm{T}:(\mathrm{u}, 1 / 2,1 / 2)$ & $\mathrm{R}:(0,1 / 2,1 / 2)$ & yes & Line \\
\hline $\mathrm{A}:(1 / 2,1 / 2,1 / 2)$ & $\mathrm{S}:(\mathrm{u}, \mathrm{u}, 1 / 2)$ & $\mathrm{Z}:(0,0,1 / 2)$ & yes & Line \\
\hline $\mathrm{R}:(0,1 / 2,1 / 2)$ & $\mathrm{U}:(0, \mathrm{v}, 1 / 2)$ & $\mathrm{Z}:(0,0,1 / 2)$ & yes & Line \\
\hline $\mathrm{A}:(1 / 2,1 / 2,1 / 2)$ & $\mathrm{E}:(\mathrm{u}, \mathrm{v}, 1 / 2)$ & $\mathrm{R}:(0,1 / 2,1 / 2)$ & yes & Plane \\
\hline $\mathrm{A}:(1 / 2,1 / 2,1 / 2)$ & $\mathrm{E}:(\mathrm{u}, \mathrm{v}, 1 / 2)$ & $\mathrm{Z}:(0,0,1 / 2)$ & yes & Plane \\
\hline $\mathrm{R}:(0,1 / 2,1 / 2)$ & $\mathrm{E}:(\mathrm{u}, \mathrm{v}, 1 / 2)$ & $\mathrm{Z}:(0,0,1 / 2)$ & yes & Plane \\
\hline
\end{tabular}

BCSID: 1.162; Formula: NdMg; MSG: $124.360\left(P_{c} 4 / m c c\right) ; \mathrm{U}=6 \mathrm{eV}$

\begin{tabular}{c|c|c|c|c}
\hline maximal $k_{1}$ & intermediate path & maximal $k_{2}$ & satisfied? & Line/Plane \\
\hline$\Gamma:(0,0,0)$ & $\mathrm{LD}:(0,0, \mathrm{w})$ & $\mathrm{Z}:(0,0,1 / 2)$ & yes & Line \\
\hline $\mathrm{A}:(1 / 2,1 / 2,1 / 2)$ & $\mathrm{V}:(1 / 2,1 / 2, \mathrm{w})$ & $\mathrm{M}:(1 / 2,1 / 2,0)$ & yes & Line \\
\hline $\mathrm{A}:(1 / 2,1 / 2,1 / 2)$ & $\mathrm{T}:(\mathrm{u}, 1 / 2,1 / 2)$ & $\mathrm{R}:(0,1 / 2,1 / 2)$ & no & Line
\end{tabular}




\begin{tabular}{c|c|c|c|c}
\hline $\mathrm{A}:(1 / 2,1 / 2,1 / 2)$ & $\mathrm{S}:(\mathrm{u}, \mathrm{u}, 1 / 2)$ & $\mathrm{Z}:(0,0,1 / 2)$ & no & Line \\
\hline $\mathrm{R}:(0,1 / 2,1 / 2)$ & $\mathrm{U}:(0, \mathrm{v}, 1 / 2)$ & $\mathrm{Z}:(0,0,1 / 2)$ & yes & Line \\
\hline $\mathrm{A}:(1 / 2,1 / 2,1 / 2)$ & $\mathrm{E}:(\mathrm{u}, \mathrm{v}, 1 / 2)$ & $\mathrm{R}:(0,1 / 2,1 / 2)$ & no & Plane \\
\hline $\mathrm{A}:(1 / 2,1 / 2,1 / 2)$ & $\mathrm{E}:(\mathrm{u}, \mathrm{v}, 1 / 2)$ & $\mathrm{Z}:(0,0,1 / 2)$ & no & Plane \\
\hline $\mathrm{R}:(0,1 / 2,1 / 2)$ & $\mathrm{E}:(\mathrm{u}, \mathrm{v}, 1 / 2)$ & $\mathrm{Z}:(0,0,1 / 2)$ & yes & Plane \\
\hline
\end{tabular}

BCSID: 1.251; Formula: NdCo2P2; MSG: $124.360\left(P_{c} 4 / m c c\right)$; U=0

\begin{tabular}{c|c|c|c|c}
\hline maximal $k_{1}$ & intermediate path & maximal $k_{2}$ & satisfied? & Line/Plane \\
\hline$\Gamma:(0,0,0)$ & $\mathrm{LD}:(0,0, \mathrm{w})$ & $\mathrm{Z}:(0,0,1 / 2)$ & no & Line \\
\hline $\mathrm{A}:(1 / 2,1 / 2,1 / 2)$ & $\mathrm{V}:(1 / 2,1 / 2, \mathrm{w})$ & $\mathrm{M}:(1 / 2,1 / 2,0)$ & yes & Line \\
\hline $\mathrm{A}:(1 / 2,1 / 2,1 / 2)$ & $\mathrm{T}:(\mathrm{u}, 1 / 2,1 / 2)$ & $\mathrm{R}:(0,1 / 2,1 / 2)$ & yes & Line \\
\hline $\mathrm{A}:(1 / 2,1 / 2,1 / 2)$ & $\mathrm{S}:(\mathrm{u}, \mathrm{u}, 1 / 2)$ & $\mathrm{Z}:(0,0,1 / 2)$ & no & Line \\
\hline $\mathrm{R}:(0,1 / 2,1 / 2)$ & $\mathrm{U}:(0, \mathrm{v}, 1 / 2)$ & $\mathrm{Z}:(0,0,1 / 2)$ & no & Line \\
\hline $\mathrm{A}:(1 / 2,1 / 2,1 / 2)$ & $\mathrm{E}:(\mathrm{u}, \mathrm{v}, 1 / 2)$ & $\mathrm{R}:(0,1 / 2,1 / 2)$ & yes & Plane \\
\hline $\mathrm{A}:(1 / 2,1 / 2,1 / 2)$ & $\mathrm{E}:(\mathrm{u}, \mathrm{v}, 1 / 2)$ & $\mathrm{Z}:(0,0,1 / 2)$ & no & Plane \\
\hline $\mathrm{R}:(0,1 / 2,1 / 2)$ & $\mathrm{E}:(\mathrm{u}, \mathrm{v}, 1 / 2)$ & $\mathrm{Z}:(0,0,1 / 2)$ & no & Plane \\
\hline
\end{tabular}

\begin{tabular}{|c|c|c|c|c|}
\hline \multicolumn{5}{|c|}{ BCSID: 1.251 ; Formula: NdCo2P2; MSG: $124.360\left(P_{c} 4 / m c c\right) ; \mathrm{U}=2 \mathrm{eV}$} \\
\hline maximal $k_{1}$ & intermediate path & maximal $k_{2}$ & satisfied? & Line/Plane \\
\hline$\Gamma:(0,0,0)$ & $\mathrm{LD}:(0,0, \mathrm{w})$ & $\mathrm{Z}:(0,0,1 / 2)$ & yes & Line \\
\hline $\mathrm{A}:(1 / 2,1 / 2,1 / 2)$ & $\mathrm{V}:(1 / 2,1 / 2, \mathrm{w})$ & $\mathrm{M}:(1 / 2,1 / 2,0)$ & yes & Line \\
\hline $\mathrm{A}:(1 / 2,1 / 2,1 / 2)$ & $\mathrm{T}:(\mathrm{u}, 1 / 2,1 / 2)$ & $\mathrm{R}:(0,1 / 2,1 / 2)$ & no & Line \\
\hline $\mathrm{A}:(1 / 2,1 / 2,1 / 2)$ & $\mathrm{S}:(\mathrm{u}, \mathrm{u}, 1 / 2)$ & $\mathrm{Z}:(0,0,1 / 2)$ & no & Line \\
\hline $\mathrm{R}:(0,1 / 2,1 / 2)$ & $\mathrm{U}:(0, \mathrm{v}, 1 / 2)$ & $\mathrm{Z}:(0,0,1 / 2)$ & yes & Line \\
\hline $\mathrm{A}:(1 / 2,1 / 2,1 / 2)$ & $\mathrm{E}:(\mathrm{u}, \mathrm{v}, 1 / 2)$ & $\mathrm{R}:(0,1 / 2,1 / 2)$ & no & Plane \\
\hline $\mathrm{A}:(1 / 2,1 / 2,1 / 2)$ & $\mathrm{E}:(\mathrm{u}, \mathrm{v}, 1 / 2)$ & $\mathrm{Z}:(0,0,1 / 2)$ & no & Plane \\
\hline $\mathrm{R}:(0,1 / 2,1 / 2)$ & $\mathrm{E}:(\mathrm{u}, \mathrm{v}, 1 / 2)$ & $\mathrm{Z}:(0,0,1 / 2)$ & yes & Plane \\
\hline \multicolumn{5}{|c|}{ BCSID: 1.251 ; Formula: NdCo2P2; MSG: $124.360\left(P_{c} 4 / m c c\right) ; \mathrm{U}=4 \mathrm{eV}$} \\
\hline maximal $k_{1}$ & intermediate path & maximal $k_{2}$ & satisfied? & Line/Plane \\
\hline$\Gamma:(0,0,0)$ & LD: $(0,0, w)$ & $\mathrm{Z}:(0,0,1 / 2)$ & no & Line \\
\hline $\mathrm{A}:(1 / 2,1 / 2,1 / 2)$ & $\mathrm{V}:(1 / 2,1 / 2, \mathrm{w})$ & $\mathrm{M}:(1 / 2,1 / 2,0)$ & yes & Line \\
\hline $\mathrm{A}:(1 / 2,1 / 2,1 / 2)$ & $\mathrm{T}:(\mathrm{u}, 1 / 2,1 / 2)$ & $\mathrm{R}:(0,1 / 2,1 / 2)$ & yes & Line \\
\hline $\mathrm{A}:(1 / 2,1 / 2,1 / 2)$ & $\mathrm{S}:(\mathrm{u}, \mathrm{u}, 1 / 2)$ & $\mathrm{Z}:(0,0,1 / 2)$ & yes & Line \\
\hline $\mathrm{R}:(0,1 / 2,1 / 2)$ & $\mathrm{U}:(0, \mathrm{v}, 1 / 2)$ & $\mathrm{Z}:(0,0,1 / 2)$ & yes & Line \\
\hline $\mathrm{A}:(1 / 2,1 / 2,1 / 2)$ & $\mathrm{E}:(\mathrm{u}, \mathrm{v}, 1 / 2)$ & $\mathrm{R}:(0,1 / 2,1 / 2)$ & yes & Plane \\
\hline $\mathrm{A}:(1 / 2,1 / 2,1 / 2)$ & $\mathrm{E}:(\mathrm{u}, \mathrm{v}, 1 / 2)$ & $\mathrm{Z}:(0,0,1 / 2)$ & yes & Plane \\
\hline $\mathrm{R}:(0,1 / 2,1 / 2)$ & $\mathrm{E}:(\mathrm{u}, \mathrm{v}, 1 / 2)$ & $\mathrm{Z}:(0,0,1 / 2)$ & yes & Plane \\
\hline
\end{tabular}

\begin{tabular}{c|c|c|c|c}
\multicolumn{5}{c}{ BCSID: 1.255 ; Formula: UPtGa5; MSG: $124.360\left(P_{c} 4 / m c c\right) ; \mathrm{U}=2 \mathrm{eV}$} \\
\hline maximal $k_{1}$ & intermediate path & maximal $k_{2}$ & satisfied? & Line/Plane \\
\hline$\Gamma:(0,0,0)$ & $\mathrm{LD}:(0,0, \mathrm{w})$ & $\mathrm{Z}:(0,0,1 / 2)$ & no & Line \\
\hline $\mathrm{A}:(1 / 2,1 / 2,1 / 2)$ & $\mathrm{V}:(1 / 2,1 / 2, \mathrm{w})$ & $\mathrm{M}:(1 / 2,1 / 2,0)$ & yes & Line \\
\hline $\mathrm{A}:(1 / 2,1 / 2,1 / 2)$ & $\mathrm{T}:(\mathrm{u}, 1 / 2,1 / 2)$ & $\mathrm{R}:(0,1 / 2,1 / 2)$ & yes & Line \\
\hline $\mathrm{A}:(1 / 2,1 / 2,1 / 2)$ & $\mathrm{S}:(\mathrm{u}, \mathrm{u}, 1 / 2)$ & $\mathrm{Z}:(0,0,1 / 2)$ & yes & Line \\
\hline $\mathrm{R}:(0,1 / 2,1 / 2)$ & $\mathrm{U}:(0, \mathrm{v}, 1 / 2)$ & $\mathrm{Z}:(0,0,1 / 2)$ & yes & Line \\
\hline $\mathrm{A}:(1 / 2,1 / 2,1 / 2)$ & $\mathrm{E}:(\mathrm{u}, \mathrm{v}, 1 / 2)$ & $\mathrm{R}:(0,1 / 2,1 / 2)$ & yes & Plane \\
\hline $\mathrm{A}:(1 / 2,1 / 2,1 / 2)$ & $\mathrm{E}:(\mathrm{u}, \mathrm{v}, 1 / 2)$ & $\mathrm{Z}:(0,0,1 / 2)$ & yes & Plane \\
\hline $\mathrm{R}:(0,1 / 2,1 / 2)$ & $\mathrm{E}:(\mathrm{u}, \mathrm{v}, 1 / 2)$ & $\mathrm{Z}:(0,0,1 / 2)$ & yes & Plane \\
\hline
\end{tabular}




\begin{tabular}{c|c|c|c|c}
\hline maximal $k_{1}$ & intermediate path & maximal $k_{2}$ & satisfied? & Line/Plane \\
\hline$\Gamma:(0,0,0)$ & $\mathrm{LD}:(0,0, \mathrm{w})$ & $\mathrm{Z}:(0,0,1 / 2)$ & no & Line \\
\hline $\mathrm{A}:(1 / 2,1 / 2,1 / 2)$ & $\mathrm{V}:(1 / 2,1 / 2, \mathrm{w})$ & $\mathrm{M}:(1 / 2,1 / 2,0)$ & yes & Line \\
\hline $\mathrm{A}:(1 / 2,1 / 2,1 / 2)$ & $\mathrm{T}:(\mathrm{u}, 1 / 2,1 / 2)$ & $\mathrm{R}:(0,1 / 2,1 / 2)$ & yes & Line \\
\hline $\mathrm{A}:(1 / 2,1 / 2,1 / 2)$ & $\mathrm{S}:(\mathrm{u}, \mathrm{u}, 1 / 2)$ & $\mathrm{Z}:(0,0,1 / 2)$ & yes & Line \\
\hline $\mathrm{R}:(0,1 / 2,1 / 2)$ & $\mathrm{U}:(0, \mathrm{v}, 1 / 2)$ & $\mathrm{Z}:(0,0,1 / 2)$ & yes & Line \\
\hline $\mathrm{A}:(1 / 2,1 / 2,1 / 2)$ & $\mathrm{E}:(\mathrm{u}, \mathrm{v}, 1 / 2)$ & $\mathrm{R}:(0,1 / 2,1 / 2)$ & yes & Plane \\
\hline $\mathrm{A}:(1 / 2,1 / 2,1 / 2)$ & $\mathrm{E}:(\mathrm{u}, \mathrm{v}, 1 / 2)$ & $\mathrm{Z}:(0,0,1 / 2)$ & yes & Plane \\
\hline $\mathrm{R}:(0,1 / 2,1 / 2)$ & $\mathrm{E}:(\mathrm{u}, \mathrm{v}, 1 / 2)$ & $\mathrm{Z}:(0,0,1 / 2)$ & yes & Plane \\
\hline
\end{tabular}

BCSID: 1.255; Formula: UPtGa5; MSG: $124.360\left(P_{c} 4 / m c c\right) ; \mathrm{U}=6 \mathrm{eV}$

\begin{tabular}{c|c|c|c|c}
\hline maximal $k_{1}$ & intermediate path & maximal $k_{2}$ & satisfied? & Line/Plane \\
\hline$\Gamma:(0,0,0)$ & $\mathrm{LD}:(0,0, \mathrm{w})$ & $\mathrm{Z}:(0,0,1 / 2)$ & no & Line \\
\hline $\mathrm{A}:(1 / 2,1 / 2,1 / 2)$ & $\mathrm{V}:(1 / 2,1 / 2, \mathrm{w})$ & $\mathrm{M}:(1 / 2,1 / 2,0)$ & no & Line \\
\hline $\mathrm{A}:(1 / 2,1 / 2,1 / 2)$ & $\mathrm{T}:(\mathrm{u}, 1 / 2,1 / 2)$ & $\mathrm{R}:(0,1 / 2,1 / 2)$ & yes & Line \\
\hline $\mathrm{A}:(1 / 2,1 / 2,1 / 2)$ & $\mathrm{S}:(\mathrm{u}, \mathrm{u}, 1 / 2)$ & $\mathrm{Z}:(0,0,1 / 2)$ & yes & Line \\
\hline $\mathrm{R}:(0,1 / 2,1 / 2)$ & $\mathrm{U}:(0, \mathrm{v}, 1 / 2)$ & $\mathrm{Z}:(0,0,1 / 2)$ & yes & Line \\
\hline $\mathrm{A}:(1 / 2,1 / 2,1 / 2)$ & $\mathrm{E}:(\mathrm{u}, \mathrm{v}, 1 / 2)$ & $\mathrm{R}:(0,1 / 2,1 / 2)$ & yes & Plane \\
\hline $\mathrm{A}:(1 / 2,1 / 2,1 / 2)$ & $\mathrm{E}:(\mathrm{u}, \mathrm{v}, 1 / 2)$ & $\mathrm{Z}:(0,0,1 / 2)$ & yes & Plane \\
\hline $\mathrm{R}:(0,1 / 2,1 / 2)$ & $\mathrm{E}:(\mathrm{u}, \mathrm{v}, 1 / 2)$ & $\mathrm{Z}:(0,0,1 / 2)$ & yes & Plane \\
\hline
\end{tabular}

BCSID: 1.261; Formula: NpRhGa5; MSG: $124.360\left(P_{c} 4 / m c c\right) ; \mathrm{U}=0$

\begin{tabular}{c|c|c|c|c}
\hline maximal $k_{1}$ & intermediate path & maximal $k_{2}$ & satisfied? & Line/Plane \\
\hline$\Gamma:(0,0,0)$ & $\mathrm{LD}:(0,0, \mathrm{w})$ & $\mathrm{Z}:(0,0,1 / 2)$ & no & Line \\
\hline $\mathrm{A}:(1 / 2,1 / 2,1 / 2)$ & $\mathrm{V}:(1 / 2,1 / 2, \mathrm{w})$ & $\mathrm{M}:(1 / 2,1 / 2,0)$ & yes & Line \\
\hline $\mathrm{A}:(1 / 2,1 / 2,1 / 2)$ & $\mathrm{T}:(\mathrm{u}, 1 / 2,1 / 2)$ & $\mathrm{R}:(0,1 / 2,1 / 2)$ & yes & Line \\
\hline $\mathrm{A}:(1 / 2,1 / 2,1 / 2)$ & $\mathrm{S}:(\mathrm{u}, \mathrm{u}, 1 / 2)$ & $\mathrm{Z}:(0,0,1 / 2)$ & yes & Line \\
\hline $\mathrm{R}:(0,1 / 2,1 / 2)$ & $\mathrm{U}:(0, \mathrm{v}, 1 / 2)$ & $\mathrm{Z}:(0,0,1 / 2)$ & yes & Line \\
\hline $\mathrm{A}:(1 / 2,1 / 2,1 / 2)$ & $\mathrm{E}:(\mathrm{u}, \mathrm{v}, 1 / 2)$ & $\mathrm{R}:(0,1 / 2,1 / 2)$ & yes & Plane \\
\hline $\mathrm{A}:(1 / 2,1 / 2,1 / 2)$ & $\mathrm{E}:(\mathrm{u}, \mathrm{v}, 1 / 2)$ & $\mathrm{Z}:(0,0,1 / 2)$ & yes & Plane \\
\hline $\mathrm{R}:(0,1 / 2,1 / 2)$ & $\mathrm{E}:(\mathrm{u}, \mathrm{v}, 1 / 2)$ & $\mathrm{Z}:(0,0,1 / 2)$ & yes & Plane \\
\hline
\end{tabular}

\begin{tabular}{c|c|c|c|c}
\hline \multicolumn{2}{c}{ BCSID: $1.261 ;$ Formula: NpRhGa5; MSG: 124.360} & $\left(P_{c} 4 / m c c\right) ; \mathrm{U}=2 \mathrm{eV}$ \\
\hline maximal $k_{1}$ & intermediate path & maximal $k_{2}$ & satisfied? & Line/Plane \\
\hline$\Gamma:(0,0,0)$ & $\mathrm{LD}:(0,0, \mathrm{w})$ & $\mathrm{Z}:(0,0,1 / 2)$ & no & Line \\
\hline $\mathrm{A}:(1 / 2,1 / 2,1 / 2)$ & $\mathrm{V}:(1 / 2,1 / 2, \mathrm{w})$ & $\mathrm{M}:(1 / 2,1 / 2,0)$ & no & Line \\
\hline $\mathrm{A}:(1 / 2,1 / 2,1 / 2)$ & $\mathrm{T}:(\mathrm{u}, 1 / 2,1 / 2)$ & $\mathrm{R}:(0,1 / 2,1 / 2)$ & no & Line \\
\hline $\mathrm{A}:(1 / 2,1 / 2,1 / 2)$ & $\mathrm{S}:(\mathrm{u}, \mathrm{u}, 1 / 2)$ & $\mathrm{Z}:(0,0,1 / 2)$ & yes & Line \\
\hline $\mathrm{R}:(0,1 / 2,1 / 2)$ & $\mathrm{U}:(0, \mathrm{v}, 1 / 2)$ & $\mathrm{Z}:(0,0,1 / 2)$ & no & Line \\
\hline $\mathrm{A}:(1 / 2,1 / 2,1 / 2)$ & $\mathrm{E}:(\mathrm{u}, \mathrm{v}, 1 / 2)$ & $\mathrm{R}:(0,1 / 2,1 / 2)$ & no & Plane \\
\hline $\mathrm{A}:(1 / 2,1 / 2,1 / 2)$ & $\mathrm{E}:(\mathrm{u}, \mathrm{v}, 1 / 2)$ & $\mathrm{Z}:(0,0,1 / 2)$ & yes & Plane \\
\hline $\mathrm{R}:(0,1 / 2,1 / 2)$ & $\mathrm{E}:(\mathrm{u}, \mathrm{v}, 1 / 2)$ & $\mathrm{Z}:(0,0,1 / 2)$ & no & Plane \\
\hline
\end{tabular}

\begin{tabular}{c|c|c|c|c}
\hline \multicolumn{2}{c}{ BCSID: $1.261 ;$ Formula: NpRhGa5; MSG: 124.360} & $\left(P_{c} 4 / m c c\right) ; \mathrm{U}=4 \mathrm{eV}$ \\
\hline maximal $k_{1}$ & intermediate path & maximal $k_{2}$ & satisfied? & Line/Plane \\
\hline$\Gamma:(0,0,0)$ & $\mathrm{LD}:(0,0, \mathrm{w})$ & $\mathrm{Z}:(0,0,1 / 2)$ & yes & Line \\
\hline $\mathrm{A}:(1 / 2,1 / 2,1 / 2)$ & $\mathrm{V}:(1 / 2,1 / 2, \mathrm{w})$ & $\mathrm{M}:(1 / 2,1 / 2,0)$ & no & Line \\
\hline $\mathrm{A}:(1 / 2,1 / 2,1 / 2)$ & $\mathrm{T}:(\mathrm{u}, 1 / 2,1 / 2)$ & $\mathrm{R}:(0,1 / 2,1 / 2)$ & no & Line \\
\hline $\mathrm{A}:(1 / 2,1 / 2,1 / 2)$ & $\mathrm{S}:(\mathrm{u}, \mathrm{u}, 1 / 2)$ & $\mathrm{Z}:(0,0,1 / 2)$ & no & Line \\
\hline $\mathrm{R}:(0,1 / 2,1 / 2)$ & $\mathrm{U}:(0, \mathrm{v}, 1 / 2)$ & $\mathrm{Z}:(0,0,1 / 2)$ & yes & Line \\
\hline $\mathrm{A}:(1 / 2,1 / 2,1 / 2)$ & $\mathrm{E}:(\mathrm{u}, \mathrm{v}, 1 / 2)$ & $\mathrm{R}:(0,1 / 2,1 / 2)$ & no & Plane
\end{tabular}




\begin{tabular}{|c|c|c|c|c|}
\hline $\mathrm{A}:(1 / 2,1 / 2,1 / 2)$ & $\mathrm{E}:(\mathrm{u}, \mathrm{v}, 1 / 2)$ & $\mathrm{Z}:(0,0,1 / 2)$ & no & Plane \\
\hline $\mathrm{R}:(0,1 / 2,1 / 2)$ & $\mathrm{E}:(\mathrm{u}, \mathrm{v}, 1 / 2)$ & $\mathrm{Z}:(0,0,1 / 2)$ & yes & Plane \\
\hline \multicolumn{5}{|c|}{ BCSID: 1.261; Formula: NpRhGa5; MSG: $124.360\left(P_{c} 4 / m c c\right) ; \mathrm{U}=6 \mathrm{eV}$} \\
\hline maximal $k_{1}$ & intermediate path & maximal $k_{2}$ & satisfied? & Line/Plane \\
\hline$\Gamma:(0,0,0)$ & $\mathrm{LD}:(0,0, \mathrm{w})$ & $\mathrm{Z}:(0,0,1 / 2)$ & no & Line \\
\hline $\mathrm{A}:(1 / 2,1 / 2,1 / 2)$ & $\mathrm{V}:(1 / 2,1 / 2, \mathrm{w})$ & $\mathrm{M}:(1 / 2,1 / 2,0)$ & no & Line \\
\hline $\mathrm{A}:(1 / 2,1 / 2,1 / 2)$ & $\mathrm{T}:(\mathrm{u}, 1 / 2,1 / 2)$ & $\mathrm{R}:(0,1 / 2,1 / 2)$ & no & Line \\
\hline $\mathrm{A}:(1 / 2,1 / 2,1 / 2)$ & $\mathrm{S}:(\mathrm{u}, \mathrm{u}, 1 / 2)$ & $\mathrm{Z}:(0,0,1 / 2)$ & yes & Line \\
\hline $\mathrm{R}:(0,1 / 2,1 / 2)$ & $\mathrm{U}:(0, \mathrm{v}, 1 / 2)$ & $\mathrm{Z}:(0,0,1 / 2)$ & no & Line \\
\hline $\mathrm{A}:(1 / 2,1 / 2,1 / 2)$ & $\mathrm{E}:(\mathrm{u}, \mathrm{v}, 1 / 2)$ & $\mathrm{R}:(0,1 / 2,1 / 2)$ & no & Plane \\
\hline $\mathrm{A}:(1 / 2,1 / 2,1 / 2)$ & $\mathrm{E}:(\mathrm{u}, \mathrm{v}, 1 / 2)$ & $\mathrm{Z}:(0,0,1 / 2)$ & yes & Plane \\
\hline $\mathrm{R}:(0,1 / 2,1 / 2)$ & $\mathrm{E}:(\mathrm{u}, \mathrm{v}, 1 / 2)$ & $\mathrm{Z}:(0,0,1 / 2)$ & no & Plane \\
\hline
\end{tabular}

\begin{tabular}{|c|c|c|c|c|}
\hline \multicolumn{5}{|c|}{ BCSID: 2.14; Formula: NdMg; MSG: $125.373\left(P_{C} 4 / n b m\right) ; \mathrm{U}=0$} \\
\hline maximal $k_{1}$ & intermediate path & maximal $k_{2}$ & satisfied? & Line/Plane \\
\hline$\Gamma:(0,0,0)$ & $\mathrm{LD}:(0,0, \mathrm{w})$ & $\mathrm{Z}:(0,0,1 / 2)$ & no & Line \\
\hline $\mathrm{A}:(1 / 2,1 / 2,1 / 2)$ & $\mathrm{T}:(\mathrm{u}, 1 / 2,1 / 2)$ & $\mathrm{R}:(0,1 / 2,1 / 2)$ & yes & Line \\
\hline $\mathrm{M}:(1 / 2,1 / 2,0)$ & $\mathrm{Y}:(\mathrm{u}, 1 / 2,0)$ & $\mathrm{X}:(0,1 / 2,0)$ & yes & Line \\
\hline $\mathrm{R}:(0,1 / 2,1 / 2)$ & $\mathrm{W}:(0,1 / 2, \mathrm{w})$ & $\mathrm{X}:(0,1 / 2,0)$ & yes & Line \\
\hline $\mathrm{A}:(1 / 2,1 / 2,1 / 2)$ & $\mathrm{F}:(\mathrm{u}, 1 / 2, \mathrm{w})$ & $\mathrm{M}:(1 / 2,1 / 2,0)$ & yes & Plane \\
\hline $\mathrm{A}:(1 / 2,1 / 2,1 / 2)$ & $\mathrm{F}:(\mathrm{u}, 1 / 2, \mathrm{w})$ & $\mathrm{R}:(0,1 / 2,1 / 2)$ & yes & Plane \\
\hline $\mathrm{A}:(1 / 2,1 / 2,1 / 2)$ & $\mathrm{F}:(\mathrm{u}, 1 / 2, \mathrm{w})$ & $\mathrm{X}:(0,1 / 2,0)$ & yes & Plane \\
\hline $\mathrm{M}:(1 / 2,1 / 2,0)$ & $\mathrm{F}:(\mathrm{u}, 1 / 2, \mathrm{w})$ & $\mathrm{R}:(0,1 / 2,1 / 2)$ & yes & Plane \\
\hline $\mathrm{M}:(1 / 2,1 / 2,0)$ & $\mathrm{F}:(\mathrm{u}, 1 / 2, \mathrm{w})$ & $\mathrm{X}:(0,1 / 2,0)$ & yes & Plane \\
\hline $\mathrm{R}:(0,1 / 2,1 / 2)$ & $\mathrm{F}:(\mathrm{u}, 1 / 2, \mathrm{w})$ & $\mathrm{X}:(0,1 / 2,0)$ & yes & Plane \\
\hline \multicolumn{5}{|c|}{ BCSID: 2.14; Formula: NdMg; MSG: $125.373\left(P_{C} 4 / n b m\right) ; \mathrm{U}=2 \mathrm{eV}$} \\
\hline maximal $k_{1}$ & intermediate path & maximal $k_{2}$ & satisfied? & Line/Plane \\
\hline$\Gamma:(0,0,0)$ & LD: $(0,0, \mathrm{w})$ & $\mathrm{Z}:(0,0,1 / 2)$ & no & Line \\
\hline $\mathrm{A}:(1 / 2,1 / 2,1 / 2)$ & $\mathrm{T}:(\mathrm{u}, 1 / 2,1 / 2)$ & $\mathrm{R}:(0,1 / 2,1 / 2)$ & yes & Line \\
\hline $\mathrm{M}:(1 / 2,1 / 2,0)$ & $\mathrm{Y}:(\mathrm{u}, 1 / 2,0)$ & $\mathrm{X}:(0,1 / 2,0)$ & yes & Line \\
\hline $\mathrm{R}:(0,1 / 2,1 / 2)$ & $\mathrm{W}:(0,1 / 2, \mathrm{w})$ & $\mathrm{X}:(0,1 / 2,0)$ & yes & Line \\
\hline $\mathrm{A}:(1 / 2,1 / 2,1 / 2)$ & $\mathrm{F}:(\mathrm{u}, 1 / 2, \mathrm{w})$ & $\mathrm{M}:(1 / 2,1 / 2,0)$ & yes & Plane \\
\hline $\mathrm{A}:(1 / 2,1 / 2,1 / 2)$ & $\mathrm{F}:(\mathrm{u}, 1 / 2, \mathrm{w})$ & $\mathrm{R}:(0,1 / 2,1 / 2)$ & yes & Plane \\
\hline $\mathrm{A}:(1 / 2,1 / 2,1 / 2)$ & $\mathrm{F}:(\mathrm{u}, 1 / 2, \mathrm{w})$ & $\mathrm{X}:(0,1 / 2,0)$ & yes & Plane \\
\hline $\mathrm{M}:(1 / 2,1 / 2,0)$ & $\mathrm{F}:(\mathrm{u}, 1 / 2, \mathrm{w})$ & $\mathrm{R}:(0,1 / 2,1 / 2)$ & yes & Plane \\
\hline $\mathrm{M}:(1 / 2,1 / 2,0)$ & $\mathrm{F}:(\mathrm{u}, 1 / 2, \mathrm{w})$ & $\mathrm{X}:(0,1 / 2,0)$ & yes & Plane \\
\hline $\mathrm{R}:(0,1 / 2,1 / 2)$ & $\mathrm{F}:(\mathrm{u}, 1 / 2, \mathrm{w})$ & $\mathrm{X}:(0,1 / 2,0)$ & yes & Plane \\
\hline
\end{tabular}

\begin{tabular}{c|c|c|c|c}
\hline \multicolumn{6}{c}{ BCSID: $2.14 ;$ Formula: NdMg; MSG: $125.373\left(P_{C} 4 / n b m\right) ; \mathrm{U}=4 \mathrm{eV}$} \\
\hline maximal $k_{1}$ & intermediate path & maximal $k_{2}$ & satisfied? & Line $/$ Plane \\
\hline$\Gamma:(0,0,0)$ & $\mathrm{LD}:(0,0, \mathrm{w})$ & $\mathrm{Z}:(0,0,1 / 2)$ & no & Line \\
\hline $\mathrm{A}:(1 / 2,1 / 2,1 / 2)$ & $\mathrm{T}:(\mathrm{u}, 1 / 2,1 / 2)$ & $\mathrm{R}:(0,1 / 2,1 / 2)$ & yes & Line \\
\hline $\mathrm{M}:(1 / 2,1 / 2,0)$ & $\mathrm{Y}:(\mathrm{u}, 1 / 2,0)$ & $\mathrm{X}:(0,1 / 2,0)$ & yes & Line \\
\hline $\mathrm{R}:(0,1 / 2,1 / 2)$ & $\mathrm{W}:(0,1 / 2, \mathrm{w})$ & $\mathrm{X}:(0,1 / 2,0)$ & yes & Line \\
\hline $\mathrm{A}:(1 / 2,1 / 2,1 / 2)$ & $\mathrm{F}:(\mathrm{u}, 1 / 2, \mathrm{w})$ & $\mathrm{M}:(1 / 2,1 / 2,0)$ & yes & Plane \\
\hline $\mathrm{A}:(1 / 2,1 / 2,1 / 2)$ & $\mathrm{F}:(\mathrm{u}, 1 / 2, \mathrm{w})$ & $\mathrm{R}:(0,1 / 2,1 / 2)$ & yes & Plane \\
\hline $\mathrm{A}:(1 / 2,1 / 2,1 / 2)$ & $\mathrm{F}:(\mathrm{u}, 1 / 2, \mathrm{w})$ & $\mathrm{X}:(0,1 / 2,0)$ & yes & Plane \\
\hline $\mathrm{M}:(1 / 2,1 / 2,0)$ & $\mathrm{F}:(\mathrm{u}, 1 / 2, \mathrm{w})$ & $\mathrm{R}:(0,1 / 2,1 / 2)$ & yes & Plane \\
\hline $\mathrm{M}:(1 / 2,1 / 2,0)$ & $\mathrm{F}:(\mathrm{u}, 1 / 2, \mathrm{w})$ & $\mathrm{X}:(0,1 / 2,0)$ & yes & Plane
\end{tabular}




\begin{tabular}{c|c|c|c|c}
\hline $\mathrm{R}:(0,1 / 2,1 / 2)$ & $\mathrm{F}:(\mathrm{u}, 1 / 2, \mathrm{w})$ & $\mathrm{X}:(0,1 / 2,0)$ & yes & Plane \\
\hline \multicolumn{5}{|l}{} \\
\hline \multicolumn{5}{c}{ BCSID: $2.14 ;$ Formula: NdMg; MSG: $125.373\left(P_{C} 4 / n b m\right) ; \mathrm{U}=6 \mathrm{eV}$} \\
\hline maximal $k_{1}$ & intermediate path & maximal $k_{2}$ & satisfied? & Line/Plane \\
\hline$\Gamma:(0,0,0)$ & $\mathrm{LD}:(0,0, \mathrm{w})$ & $\mathrm{Z}:(0,0,1 / 2)$ & no & Line \\
\hline $\mathrm{A}:(1 / 2,1 / 2,1 / 2)$ & $\mathrm{T}:(\mathrm{u}, 1 / 2,1 / 2)$ & $\mathrm{R}:(0,1 / 2,1 / 2)$ & yes & Line \\
\hline $\mathrm{M}:(1 / 2,1 / 2,0)$ & $\mathrm{Y}:(\mathrm{u}, 1 / 2,0)$ & $\mathrm{X}:(0,1 / 2,0)$ & yes & Line \\
\hline $\mathrm{R}:(0,1 / 2,1 / 2)$ & $\mathrm{W}:(0,1 / 2, \mathrm{w})$ & $\mathrm{X}:(0,1 / 2,0)$ & yes & Line \\
\hline $\mathrm{A}:(1 / 2,1 / 2,1 / 2)$ & $\mathrm{F}:(\mathrm{u}, 1 / 2, \mathrm{w})$ & $\mathrm{M}:(1 / 2,1 / 2,0)$ & yes & Plane \\
\hline $\mathrm{A}:(1 / 2,1 / 2,1 / 2)$ & $\mathrm{F}:(\mathrm{u}, 1 / 2, \mathrm{w})$ & $\mathrm{R}:(0,1 / 2,1 / 2)$ & yes & Plane \\
\hline $\mathrm{A}:(1 / 2,1 / 2,1 / 2)$ & $\mathrm{F}:(\mathrm{u}, 1 / 2, \mathrm{w})$ & $\mathrm{X}:(0,1 / 2,0)$ & yes & Plane \\
\hline $\mathrm{M}:(1 / 2,1 / 2,0)$ & $\mathrm{F}:(\mathrm{u}, 1 / 2, \mathrm{w})$ & $\mathrm{R}:(0,1 / 2,1 / 2)$ & yes & Plane \\
\hline $\mathrm{M}:(1 / 2,1 / 2,0)$ & $\mathrm{F}:(\mathrm{u}, 1 / 2, \mathrm{w})$ & $\mathrm{X}:(0,1 / 2,0)$ & yes & Plane \\
\hline $\mathrm{R}:(0,1 / 2,1 / 2)$ & $\mathrm{F}:(\mathrm{u}, 1 / 2, \mathrm{w})$ & $\mathrm{X}:(0,1 / 2,0)$ & yes & Plane \\
\hline
\end{tabular}

\begin{tabular}{c|c|c|c|c}
\hline \multicolumn{5}{c}{ BCSID: 1.253; Formula: CeCo2P2; MSG: 126.386 $\left(P_{I} 4 / n n c\right) ; \mathrm{U}=0$} \\
\hline maximal $k_{1}$ & intermediate path & maximal $k_{2}$ & satisfied? & Line/Plane \\
\hline$\Gamma:(0,0,0)$ & $\mathrm{LD}:(0,0, \mathrm{w})$ & $\mathrm{Z}:(0,0,1 / 2)$ & no & Line \\
\hline $\mathrm{A}:(1 / 2,1 / 2,1 / 2)$ & $\mathrm{S}:(\mathrm{u}, \mathrm{u}, 1 / 2)$ & $\mathrm{Z}:(0,0,1 / 2)$ & yes & Line \\
\hline $\mathrm{M}:(1 / 2,1 / 2,0)$ & $\mathrm{Y}:(\mathrm{u}, 1 / 2,0)$ & $\mathrm{X}:(0,1 / 2,0)$ & yes & Line \\
\hline $\mathrm{R}:(0,1 / 2,1 / 2)$ & $\mathrm{W}:(0,1 / 2, \mathrm{w})$ & $\mathrm{X}:(0,1 / 2,0)$ & yes & Line \\
\hline $\mathrm{R}:(0,1 / 2,1 / 2)$ & $\mathrm{U}:(0, \mathrm{v}, 1 / 2)$ & $\mathrm{Z}:(0,0,1 / 2)$ & yes & Line \\
\hline $\mathrm{A}:(1 / 2,1 / 2,1 / 2)$ & $\mathrm{F}:(\mathrm{u}, 1 / 2, \mathrm{w})$ & $\mathrm{M}:(1 / 2,1 / 2,0)$ & yes & Plane \\
\hline $\mathrm{A}:(1 / 2,1 / 2,1 / 2)$ & $\mathrm{E}:(\mathrm{u}, \mathrm{v}, 1 / 2)$ & $\mathrm{R}:(0,1 / 2,1 / 2)$ & yes & Plane \\
\hline $\mathrm{A}:(1 / 2,1 / 2,1 / 2)$ & $\mathrm{F}:(\mathrm{u}, 1 / 2, \mathrm{w})$ & $\mathrm{R}:(0,1 / 2,1 / 2)$ & yes & Plane \\
\hline $\mathrm{A}:(1 / 2,1 / 2,1 / 2)$ & $\mathrm{F}:(\mathrm{u}, 1 / 2, \mathrm{w})$ & $\mathrm{X}:(0,1 / 2,0)$ & yes & Plane \\
\hline $\mathrm{A}:(1 / 2,1 / 2,1 / 2)$ & $\mathrm{E}:(\mathrm{u}, \mathrm{v}, 1 / 2)$ & $\mathrm{Z}:(0,0,1 / 2)$ & yes & Plane \\
\hline $\mathrm{M}:(1 / 2,1 / 2,0)$ & $\mathrm{F}:(\mathrm{u}, 1 / 2, \mathrm{w})$ & $\mathrm{R}:(0,1 / 2,1 / 2)$ & yes & Plane \\
\hline $\mathrm{M}:(1 / 2,1 / 2,0)$ & $\mathrm{F}:(\mathrm{u}, 1 / 2, \mathrm{w})$ & $\mathrm{X}:(0,1 / 2,0)$ & yes & Plane \\
\hline $\mathrm{R}:(0,1 / 2,1 / 2)$ & $\mathrm{F}:(\mathrm{u}, 1 / 2, \mathrm{w})$ & $\mathrm{X}:(0,1 / 2,0)$ & yes & Plane \\
\hline $\mathrm{R}:(0,1 / 2,1 / 2)$ & $\mathrm{E}:(\mathrm{u}, \mathrm{v}, 1 / 2)$ & $\mathrm{Z}:(0,0,1 / 2)$ & yes & Plane \\
\hline
\end{tabular}

\begin{tabular}{c|c|c|c|c}
\hline \multicolumn{2}{c}{ BCSID: $1.253 ;$ Formula: CeCo2P2; MSG: $126.386\left(P_{I} 4 / n n c\right) ; \mathrm{U}=2 \mathrm{eV}$} \\
\hline maximal $k_{1}$ & intermediate path & maximal $k_{2}$ & satisfied? & Line/Plane \\
\hline$\Gamma:(0,0,0)$ & $\mathrm{LD}:(0,0, \mathrm{w})$ & $\mathrm{Z}:(0,0,1 / 2)$ & no & Line \\
\hline $\mathrm{A}:(1 / 2,1 / 2,1 / 2)$ & $\mathrm{S}:(\mathrm{u}, \mathrm{u}, 1 / 2)$ & $\mathrm{Z}:(0,0,1 / 2)$ & yes & Line \\
\hline $\mathrm{M}:(1 / 2,1 / 2,0)$ & $\mathrm{Y}:(\mathrm{u}, 1 / 2,0)$ & $\mathrm{X}:(0,1 / 2,0)$ & yes & Line \\
\hline $\mathrm{R}:(0,1 / 2,1 / 2)$ & $\mathrm{W}:(0,1 / 2, \mathrm{w})$ & $\mathrm{X}:(0,1 / 2,0)$ & yes & Line \\
\hline $\mathrm{R}:(0,1 / 2,1 / 2)$ & $\mathrm{U}:(0, \mathrm{v}, 1 / 2)$ & $\mathrm{Z}:(0,0,1 / 2)$ & yes & Line \\
\hline $\mathrm{A}:(1 / 2,1 / 2,1 / 2)$ & $\mathrm{F}:(\mathrm{u}, 1 / 2, \mathrm{w})$ & $\mathrm{M}:(1 / 2,1 / 2,0)$ & yes & Plane \\
\hline $\mathrm{A}:(1 / 2,1 / 2,1 / 2)$ & $\mathrm{E}:(\mathrm{u}, \mathrm{v}, 1 / 2)$ & $\mathrm{R}:(0,1 / 2,1 / 2)$ & yes & Plane \\
\hline $\mathrm{A}:(1 / 2,1 / 2,1 / 2)$ & $\mathrm{F}:(\mathrm{u}, 1 / 2, \mathrm{w})$ & $\mathrm{R}:(0,1 / 2,1 / 2)$ & yes & Plane \\
\hline $\mathrm{A}:(1 / 2,1 / 2,1 / 2)$ & $\mathrm{F}:(\mathrm{u}, 1 / 2, \mathrm{w})$ & $\mathrm{X}:(0,1 / 2,0)$ & yes & Plane \\
\hline $\mathrm{A}:(1 / 2,1 / 2,1 / 2)$ & $\mathrm{E}:(\mathrm{u}, \mathrm{v}, 1 / 2)$ & $\mathrm{Z}:(0,0,1 / 2)$ & yes & Plane \\
\hline $\mathrm{M}:(1 / 2,1 / 2,0)$ & $\mathrm{F}:(\mathrm{u}, 1 / 2, \mathrm{w})$ & $\mathrm{R}:(0,1 / 2,1 / 2)$ & yes & Plane \\
\hline $\mathrm{M}:(1 / 2,1 / 2,0)$ & $\mathrm{F}:(\mathrm{u}, 1 / 2, \mathrm{w})$ & $\mathrm{X}:(0,1 / 2,0)$ & yes & Plane \\
\hline $\mathrm{R}:(0,1 / 2,1 / 2)$ & $\mathrm{F}:(\mathrm{u}, 1 / 2, \mathrm{w})$ & $\mathrm{X}:(0,1 / 2,0)$ & yes & Plane \\
\hline $\mathrm{R}:(0,1 / 2,1 / 2)$ & $\mathrm{E}:(\mathrm{u}, \mathrm{v}, 1 / 2)$ & $\mathrm{Z}:(0,0,1 / 2)$ & yes & Plane \\
\hline
\end{tabular}

BCSID: 1.253; Formula: CeCo2P2; MSG: $126.386\left(P_{I} 4 / n n c\right)$; $=4 \mathrm{eV}$ maximal $k_{1} \quad \mid$ intermediate path $\mid$ maximal $k_{2} \quad$ satisfied? $\mid$ Line/Plane 


\begin{tabular}{c|c|c|c|c}
\hline$\Gamma:(0,0,0)$ & $\mathrm{LD}:(0,0, \mathrm{w})$ & $\mathrm{Z}:(0,0,1 / 2)$ & yes & Line \\
\hline $\mathrm{A}:(1 / 2,1 / 2,1 / 2)$ & $\mathrm{S}:(\mathrm{u}, \mathrm{u}, 1 / 2)$ & $\mathrm{Z}:(0,0,1 / 2)$ & no & Line \\
\hline $\mathrm{M}:(1 / 2,1 / 2,0)$ & $\mathrm{Y}:(\mathrm{u}, 1 / 2,0)$ & $\mathrm{X}:(0,1 / 2,0)$ & yes & Line \\
\hline $\mathrm{R}:(0,1 / 2,1 / 2)$ & $\mathrm{W}:(0,1 / 2, \mathrm{w})$ & $\mathrm{X}:(0,1 / 2,0)$ & yes & Line \\
\hline $\mathrm{R}:(0,1 / 2,1 / 2)$ & $\mathrm{U}:(0, \mathrm{v}, 1 / 2)$ & $\mathrm{Z}:(0,0,1 / 2)$ & no & Line \\
\hline $\mathrm{A}:(1 / 2,1 / 2,1 / 2)$ & $\mathrm{F}:(\mathrm{u}, 1 / 2, \mathrm{w})$ & $\mathrm{M}:(1 / 2,1 / 2,0)$ & yes & Plane \\
\hline $\mathrm{A}:(1 / 2,1 / 2,1 / 2)$ & $\mathrm{E}:(\mathrm{u}, \mathrm{v}, 1 / 2)$ & $\mathrm{R}:(0,1 / 2,1 / 2)$ & yes & Plane \\
\hline $\mathrm{A}:(1 / 2,1 / 2,1 / 2)$ & $\mathrm{F}:(\mathrm{u}, 1 / 2, \mathrm{w})$ & $\mathrm{R}:(0,1 / 2,1 / 2)$ & yes & Plane \\
\hline $\mathrm{A}:(1 / 2,1 / 2,1 / 2)$ & $\mathrm{F}:(\mathrm{u}, 1 / 2, \mathrm{w})$ & $\mathrm{X}:(0,1 / 2,0)$ & yes & Plane \\
\hline $\mathrm{A}:(1 / 2,1 / 2,1 / 2)$ & $\mathrm{E}:(\mathrm{u}, \mathrm{v}, 1 / 2)$ & $\mathrm{Z}:(0,0,1 / 2)$ & no & Plane \\
\hline $\mathrm{M}:(1 / 2,1 / 2,0)$ & $\mathrm{F}:(\mathrm{u}, 1 / 2, \mathrm{w})$ & $\mathrm{R}:(0,1 / 2,1 / 2)$ & yes & Plane \\
\hline $\mathrm{M}:(1 / 2,1 / 2,0)$ & $\mathrm{F}:(\mathrm{u}, 1 / 2, \mathrm{w})$ & $\mathrm{X}:(0,1 / 2,0)$ & yes & Plane \\
\hline $\mathrm{R}:(0,1 / 2,1 / 2)$ & $\mathrm{F}:(\mathrm{u}, 1 / 2, \mathrm{w})$ & $\mathrm{X}:(0,1 / 2,0)$ & yes & Plane \\
\hline $\mathrm{R}:(0,1 / 2,1 / 2)$ & $\mathrm{E}:(\mathrm{u}, \mathrm{v}, 1 / 2)$ & $\mathrm{Z}:(0,0,1 / 2)$ & no & Plane \\
\hline
\end{tabular}

\begin{tabular}{c|c|c|c|c}
\hline \multicolumn{5}{c}{ BCSID: 1.253; Formula: CeCo2P2; MSG: $126.386\left(P_{I} 4 / n n c\right) ; \mathrm{U}=6 \mathrm{eV}$} \\
\hline maximal $k_{1}$ & intermediate path & maximal $k_{2}$ & satisfied? & Line/Plane \\
\hline$\Gamma:(0,0,0)$ & $\mathrm{LD}:(0,0, \mathrm{w})$ & $\mathrm{Z}:(0,0,1 / 2)$ & yes & Line \\
\hline $\mathrm{A}:(1 / 2,1 / 2,1 / 2)$ & $\mathrm{S}:(\mathrm{u}, \mathrm{u}, 1 / 2)$ & $\mathrm{Z}:(0,0,1 / 2)$ & no & Line \\
\hline $\mathrm{M}:(1 / 2,1 / 2,0)$ & $\mathrm{Y}:(\mathrm{u}, 1 / 2,0)$ & $\mathrm{X}:(0,1 / 2,0)$ & yes & Line \\
\hline $\mathrm{R}:(0,1 / 2,1 / 2)$ & $\mathrm{W}:(0,1 / 2, \mathrm{w})$ & $\mathrm{X}:(0,1 / 2,0)$ & yes & Line \\
\hline $\mathrm{R}:(0,1 / 2,1 / 2)$ & $\mathrm{U}:(0, \mathrm{v}, 1 / 2)$ & $\mathrm{Z}:(0,0,1 / 2)$ & no & Line \\
\hline $\mathrm{A}:(1 / 2,1 / 2,1 / 2)$ & $\mathrm{F}:(\mathrm{u}, 1 / 2, \mathrm{w})$ & $\mathrm{M}:(1 / 2,1 / 2,0)$ & yes & Plane \\
\hline $\mathrm{A}:(1 / 2,1 / 2,1 / 2)$ & $\mathrm{E}:(\mathrm{u}, \mathrm{v}, 1 / 2)$ & $\mathrm{R}:(0,1 / 2,1 / 2)$ & yes & Plane \\
\hline $\mathrm{A}:(1 / 2,1 / 2,1 / 2)$ & $\mathrm{F}:(\mathrm{u}, 1 / 2, \mathrm{w})$ & $\mathrm{R}:(0,1 / 2,1 / 2)$ & yes & Plane \\
\hline $\mathrm{A}:(1 / 2,1 / 2,1 / 2)$ & $\mathrm{F}:(\mathrm{u}, 1 / 2, \mathrm{w})$ & $\mathrm{X}:(0,1 / 2,0)$ & yes & Plane \\
\hline $\mathrm{A}:(1 / 2,1 / 2,1 / 2)$ & $\mathrm{E}:(\mathrm{u}, \mathrm{v}, 1 / 2)$ & $\mathrm{Z}:(0,0,1 / 2)$ & no & Plane \\
\hline $\mathrm{M}:(1 / 2,1 / 2,0)$ & $\mathrm{F}:(\mathrm{u}, 1 / 2, \mathrm{w})$ & $\mathrm{R}:(0,1 / 2,1 / 2)$ & yes & Plane \\
\hline $\mathrm{M}:(1 / 2,1 / 2,0)$ & $\mathrm{F}:(\mathrm{u}, 1 / 2, \mathrm{w})$ & $\mathrm{X}:(0,1 / 2,0)$ & yes & Plane \\
\hline $\mathrm{R}:(0,1 / 2,1 / 2)$ & $\mathrm{F}:(\mathrm{u}, 1 / 2, \mathrm{w})$ & $\mathrm{X}:(0,1 / 2,0)$ & yes & Plane \\
\hline $\mathrm{R}:(0,1 / 2,1 / 2)$ & $\mathrm{E}:(\mathrm{u}, \mathrm{v}, 1 / 2)$ & $\mathrm{Z}:(0,0,1 / 2)$ & no & Plane \\
\hline
\end{tabular}

BCSID: 1.81; Formula: GdIn3; MSG: $127.397\left(P_{C} 4 / m b m\right) ; \mathrm{U}=0$

\begin{tabular}{c|c|c|c|c}
\hline maximal $k_{1}$ & intermediate path & maximal $k_{2}$ & satisfied? & Line/Plane \\
\hline$\Gamma:(0,0,0)$ & $\mathrm{LD}:(0,0, \mathrm{w})$ & $\mathrm{Z}:(0,0,1 / 2)$ & no & Line \\
\hline $\mathrm{A}:(1 / 2,1 / 2,1 / 2)$ & $\mathrm{V}:(1 / 2,1 / 2, \mathrm{w})$ & $\mathrm{M}:(1 / 2,1 / 2,0)$ & no & Line \\
\hline $\mathrm{R}:(0,1 / 2,1 / 2)$ & $\mathrm{W}:(0,1 / 2, \mathrm{w})$ & $\mathrm{X}:(0,1 / 2,0)$ & yes & Line \\
\hline
\end{tabular}

BCSID: 1.81; Formula: GdIn3; MSG: $127.397\left(P_{C} 4 / m b m\right) ; \mathrm{U}=2 \mathrm{eV}$

\begin{tabular}{c|c|c|c|c}
\hline maximal $k_{1}$ & intermediate path & maximal $k_{2}$ & satisfied? & Line/Plane \\
\hline$\Gamma:(0,0,0)$ & $\mathrm{LD}:(0,0, \mathrm{w})$ & $\mathrm{Z}:(0,0,1 / 2)$ & no & Line \\
\hline $\mathrm{A}:(1 / 2,1 / 2,1 / 2)$ & $\mathrm{V}:(1 / 2,1 / 2, \mathrm{w})$ & $\mathrm{M}:(1 / 2,1 / 2,0)$ & yes & Line \\
\hline $\mathrm{R}:(0,1 / 2,1 / 2)$ & $\mathrm{W}:(0,1 / 2, \mathrm{w})$ & $\mathrm{X}:(0,1 / 2,0)$ & yes & Line \\
\hline
\end{tabular}

BCSID: 1.81; Formula: GdIn3; MSG: $127.397\left(P_{C} 4 / m b m\right) ; \mathrm{U}=4 \mathrm{eV}$

\begin{tabular}{c|c|c|c|c}
\hline maximal $k_{1}$ & intermediate path & maximal $k_{2}$ & satisfied? & Line/Plane \\
\hline$\Gamma:(0,0,0)$ & $\mathrm{LD}:(0,0, \mathrm{w})$ & $\mathrm{Z}:(0,0,1 / 2)$ & no & Line \\
\hline $\mathrm{A}:(1 / 2,1 / 2,1 / 2)$ & $\mathrm{V}:(1 / 2,1 / 2, \mathrm{w})$ & $\mathrm{M}:(1 / 2,1 / 2,0)$ & no & Line \\
\hline $\mathrm{R}:(0,1 / 2,1 / 2)$ & $\mathrm{W}:(0,1 / 2, \mathrm{w})$ & $\mathrm{X}:(0,1 / 2,0)$ & yes & Line \\
\hline
\end{tabular}




\begin{tabular}{c|c|c|c|c}
\hline \multicolumn{4}{c}{ BCSID: $1.81 ;$ Formula: GdIn3; MSG: $127.397\left(P_{C} 4 / m b m\right) ; \mathrm{U}=6 \mathrm{eV}$} \\
\hline maximal $k_{1}$ & intermediate path & maximal $k_{2}$ & satisfied? & Line/Plane \\
\hline$\Gamma:(0,0,0)$ & $\mathrm{LD}:(0,0, \mathrm{w})$ & $\mathrm{Z}:(0,0,1 / 2)$ & no & Line \\
\hline $\mathrm{A}:(1 / 2,1 / 2,1 / 2)$ & $\mathrm{V}:(1 / 2,1 / 2, \mathrm{w})$ & $\mathrm{M}:(1 / 2,1 / 2,0)$ & yes & Line \\
\hline $\mathrm{R}:(0,1 / 2,1 / 2)$ & $\mathrm{W}:(0,1 / 2, \mathrm{w})$ & $\mathrm{X}:(0,1 / 2,0)$ & yes & Line \\
\hline
\end{tabular}

BCSID: 1.102; Formula: U2Ni2In; MSG: $128.408\left(P_{c} 4 / m n c\right) ; \mathrm{U}=0$

\begin{tabular}{c|c|c|c|c}
\hline maximal $k_{1}$ & intermediate path & maximal $k_{2}$ & satisfied? & Line/Plane \\
\hline$\Gamma:(0,0,0)$ & $\mathrm{LD}:(0,0, \mathrm{w})$ & $\mathrm{Z}:(0,0,1 / 2)$ & no & Line \\
\hline $\mathrm{A}:(1 / 2,1 / 2,1 / 2)$ & $\mathrm{T}:(\mathrm{u}, 1 / 2,1 / 2)$ & $\mathrm{R}:(0,1 / 2,1 / 2)$ & no & Line \\
\hline $\mathrm{A}:(1 / 2,1 / 2,1 / 2)$ & $\mathrm{S}:(\mathrm{u}, \mathrm{u}, 1 / 2)$ & $\mathrm{Z}:(0,0,1 / 2)$ & no & Line \\
\hline $\mathrm{R}:(0,1 / 2,1 / 2)$ & $\mathrm{W}:(0,1 / 2, \mathrm{w})$ & $\mathrm{X}:(0,1 / 2,0)$ & yes & Line \\
\hline $\mathrm{R}:(0,1 / 2,1 / 2)$ & $\mathrm{U}:(0, \mathrm{v}, 1 / 2)$ & $\mathrm{Z}:(0,0,1 / 2)$ & yes & Line \\
\hline $\mathrm{A}:(1 / 2,1 / 2,1 / 2)$ & $\mathrm{F}:(\mathrm{u}, 1 / 2, \mathrm{w})$ & $\mathrm{M}:(1 / 2,1 / 2,0)$ & yes & Plane \\
\hline $\mathrm{A}:(1 / 2,1 / 2,1 / 2)$ & $\mathrm{E}:(\mathrm{u}, \mathrm{v}, 1 / 2)$ & $\mathrm{R}:(0,1 / 2,1 / 2)$ & no & Plane \\
\hline $\mathrm{A}:(1 / 2,1 / 2,1 / 2)$ & $\mathrm{F}:(\mathrm{u}, 1 / 2, \mathrm{w})$ & $\mathrm{R}:(0,1 / 2,1 / 2)$ & yes & Plane \\
\hline $\mathrm{A}:(1 / 2,1 / 2,1 / 2)$ & $\mathrm{F}:(\mathrm{u}, 1 / 2, \mathrm{w})$ & $\mathrm{X}:(0,1 / 2,0)$ & yes & Plane \\
\hline $\mathrm{A}:(1 / 2,1 / 2,1 / 2)$ & $\mathrm{E}:(\mathrm{u}, \mathrm{v}, 1 / 2)$ & $\mathrm{Z}:(0,0,1 / 2)$ & no & Plane \\
\hline $\mathrm{M}:(1 / 2,1 / 2,0)$ & $\mathrm{F}:(\mathrm{u}, 1 / 2, \mathrm{w})$ & $\mathrm{R}:(0,1 / 2,1 / 2)$ & yes & Plane \\
\hline $\mathrm{M}:(1 / 2,1 / 2,0)$ & $\mathrm{F}:(\mathrm{u}, 1 / 2, \mathrm{w})$ & $\mathrm{X}:(0,1 / 2,0)$ & yes & Plane \\
\hline $\mathrm{R}:(0,1 / 2,1 / 2)$ & $\mathrm{F}:(\mathrm{u}, 1 / 2, \mathrm{w})$ & $\mathrm{X}:(0,1 / 2,0)$ & yes & Plane \\
\hline $\mathrm{R}:(0,1 / 2,1 / 2)$ & $\mathrm{E}:(\mathrm{u}, \mathrm{v}, 1 / 2)$ & $\mathrm{Z}:(0,0,1 / 2)$ & yes & Plane \\
\hline
\end{tabular}

BCSID: 1.102 ; Formula: U2Ni2In; MSG: $128.408\left(P_{c} 4 / m n c\right) ; \mathrm{U}=2 \mathrm{eV}$

\begin{tabular}{c|c|c|c|c}
\hline maximal $k_{1}$ & intermediate path & maximal $k_{2}$ & satisfied? & Line/Plane \\
\hline$\Gamma:(0,0,0)$ & $\mathrm{LD}:(0,0, \mathrm{w})$ & $\mathrm{Z}:(0,0,1 / 2)$ & yes & Line \\
\hline $\mathrm{A}:(1 / 2,1 / 2,1 / 2)$ & $\mathrm{T}:(\mathrm{u}, 1 / 2,1 / 2)$ & $\mathrm{R}:(0,1 / 2,1 / 2)$ & no & Line \\
\hline $\mathrm{A}:(1 / 2,1 / 2,1 / 2)$ & $\mathrm{S}:(\mathrm{u}, \mathrm{u}, 1 / 2)$ & $\mathrm{Z}:(0,0,1 / 2)$ & no & Line \\
\hline $\mathrm{R}:(0,1 / 2,1 / 2)$ & $\mathrm{W}:(0,1 / 2, \mathrm{w})$ & $\mathrm{X}:(0,1 / 2,0)$ & yes & Line \\
\hline $\mathrm{R}:(0,1 / 2,1 / 2)$ & $\mathrm{U}:(0, \mathrm{v}, 1 / 2)$ & $\mathrm{Z}:(0,0,1 / 2)$ & yes & Line \\
\hline $\mathrm{A}:(1 / 2,1 / 2,1 / 2)$ & $\mathrm{F}:(\mathrm{u}, 1 / 2, \mathrm{w})$ & $\mathrm{M}:(1 / 2,1 / 2,0)$ & yes & Plane \\
\hline $\mathrm{A}:(1 / 2,1 / 2,1 / 2)$ & $\mathrm{E}:(\mathrm{u}, \mathrm{v}, 1 / 2)$ & $\mathrm{R}:(0,1 / 2,1 / 2)$ & no & Plane \\
\hline $\mathrm{A}:(1 / 2,1 / 2,1 / 2)$ & $\mathrm{F}:(\mathrm{u}, 1 / 2, \mathrm{w})$ & $\mathrm{R}:(0,1 / 2,1 / 2)$ & yes & Plane \\
\hline $\mathrm{A}:(1 / 2,1 / 2,1 / 2)$ & $\mathrm{F}:(\mathrm{u}, 1 / 2, \mathrm{w})$ & $\mathrm{X}:(0,1 / 2,0)$ & yes & Plane \\
\hline $\mathrm{A}:(1 / 2,1 / 2,1 / 2)$ & $\mathrm{E}:(\mathrm{u}, \mathrm{v}, 1 / 2)$ & $\mathrm{Z}:(0,0,1 / 2)$ & no & Plane \\
\hline $\mathrm{M}:(1 / 2,1 / 2,0)$ & $\mathrm{F}:(\mathrm{u}, 1 / 2, \mathrm{w})$ & $\mathrm{R}:(0,1 / 2,1 / 2)$ & yes & Plane \\
\hline $\mathrm{M}:(1 / 2,1 / 2,0)$ & $\mathrm{F}:(\mathrm{u}, 1 / 2, \mathrm{w})$ & $\mathrm{X}:(0,1 / 2,0)$ & yes & Plane \\
\hline $\mathrm{R}:(0,1 / 2,1 / 2)$ & $\mathrm{F}:(\mathrm{u}, 1 / 2, \mathrm{w})$ & $\mathrm{X}:(0,1 / 2,0)$ & yes & Plane \\
\hline $\mathrm{R}:(0,1 / 2,1 / 2)$ & $\mathrm{E}:(\mathrm{u}, \mathrm{v}, 1 / 2)$ & $\mathrm{Z}:(0,0,1 / 2)$ & yes & Plane \\
\hline
\end{tabular}

BCSID: 1.102; Formula: U2Ni2In; MSG: $128.408\left(P_{c} 4 / m n c\right) ; \mathrm{U}=4 \mathrm{eV}$

\begin{tabular}{c|c|c|c|c}
\hline maximal $k_{1}$ & intermediate path & maximal $k_{2}$ & satisfied? & Line/Plane \\
\hline$\Gamma:(0,0,0)$ & $\mathrm{LD}:(0,0, \mathrm{w})$ & $\mathrm{Z}:(0,0,1 / 2)$ & yes & Line \\
\hline $\mathrm{A}:(1 / 2,1 / 2,1 / 2)$ & $\mathrm{T}:(\mathrm{u}, 1 / 2,1 / 2)$ & $\mathrm{R}:(0,1 / 2,1 / 2)$ & yes & Line \\
\hline $\mathrm{A}:(1 / 2,1 / 2,1 / 2)$ & $\mathrm{S}:(\mathrm{u}, \mathrm{u}, 1 / 2)$ & $\mathrm{Z}:(0,0,1 / 2)$ & no & Line \\
\hline $\mathrm{R}:(0,1 / 2,1 / 2)$ & $\mathrm{W}:(0,1 / 2, \mathrm{w})$ & $\mathrm{X}:(0,1 / 2,0)$ & yes & Line \\
\hline $\mathrm{R}:(0,1 / 2,1 / 2)$ & $\mathrm{U}:(0, \mathrm{v}, 1 / 2)$ & $\mathrm{Z}:(0,0,1 / 2)$ & no & Line \\
\hline $\mathrm{A}:(1 / 2,1 / 2,1 / 2)$ & $\mathrm{F}:(\mathrm{u}, 1 / 2, \mathrm{w})$ & $\mathrm{M}:(1 / 2,1 / 2,0)$ & yes & Plane \\
\hline $\mathrm{A}:(1 / 2,1 / 2,1 / 2)$ & $\mathrm{E}:(\mathrm{u}, \mathrm{v}, 1 / 2)$ & $\mathrm{R}:(0,1 / 2,1 / 2)$ & yes & Plane \\
\hline $\mathrm{A}:(1 / 2,1 / 2,1 / 2)$ & $\mathrm{F}:(\mathrm{u}, 1 / 2, \mathrm{w})$ & $\mathrm{R}:(0,1 / 2,1 / 2)$ & yes & Plane \\
\hline $\mathrm{A}:(1 / 2,1 / 2,1 / 2)$ & $\mathrm{F}:(\mathrm{u}, 1 / 2, \mathrm{w})$ & $\mathrm{X}:(0,1 / 2,0)$ & yes & Plane
\end{tabular}




\begin{tabular}{c|c|c|c|c}
\hline $\mathrm{A}:(1 / 2,1 / 2,1 / 2)$ & $\mathrm{E}:(\mathrm{u}, \mathrm{v}, 1 / 2)$ & $\mathrm{Z}:(0,0,1 / 2)$ & no & Plane \\
\hline $\mathrm{M}:(1 / 2,1 / 2,0)$ & $\mathrm{F}:(\mathrm{u}, 1 / 2, \mathrm{w})$ & $\mathrm{R}:(0,1 / 2,1 / 2)$ & yes & Plane \\
\hline $\mathrm{M}:(1 / 2,1 / 2,0)$ & $\mathrm{F}:(\mathrm{u}, 1 / 2, \mathrm{w})$ & $\mathrm{X}:(0,1 / 2,0)$ & yes & Plane \\
\hline $\mathrm{R}:(0,1 / 2,1 / 2)$ & $\mathrm{F}:(\mathrm{u}, 1 / 2, \mathrm{w})$ & $\mathrm{X}:(0,1 / 2,0)$ & yes & Plane \\
\hline $\mathrm{R}:(0,1 / 2,1 / 2)$ & $\mathrm{E}:(\mathrm{u}, \mathrm{v}, 1 / 2)$ & $\mathrm{Z}:(0,0,1 / 2)$ & no & Plane \\
\hline
\end{tabular}

BCSID: 1.102; Formula: U2Ni2In; MSG: $128.408\left(P_{c} 4 / m n c\right) ; \mathrm{U}=6 \mathrm{eV}$

\begin{tabular}{c|c|c|c|c}
\hline maximal $k_{1}$ & intermediate path & maximal $k_{2}$ & satisfied? & Line/Plane \\
\hline$\Gamma:(0,0,0)$ & $\mathrm{LD}:(0,0, \mathrm{w})$ & $\mathrm{Z}:(0,0,1 / 2)$ & no & Line \\
\hline $\mathrm{A}:(1 / 2,1 / 2,1 / 2)$ & $\mathrm{T}:(\mathrm{u}, 1 / 2,1 / 2)$ & $\mathrm{R}:(0,1 / 2,1 / 2)$ & no & Line \\
\hline $\mathrm{A}:(1 / 2,1 / 2,1 / 2)$ & $\mathrm{S}:(\mathrm{u}, \mathrm{u}, 1 / 2)$ & $\mathrm{Z}:(0,0,1 / 2)$ & no & Line \\
\hline $\mathrm{R}:(0,1 / 2,1 / 2)$ & $\mathrm{W}:(0,1 / 2, \mathrm{w})$ & $\mathrm{X}:(0,1 / 2,0)$ & no & Line \\
\hline $\mathrm{R}:(0,1 / 2,1 / 2)$ & $\mathrm{U}:(0, \mathrm{v}, 1 / 2)$ & $\mathrm{Z}:(0,0,1 / 2)$ & yes & Line \\
\hline $\mathrm{A}:(1 / 2,1 / 2,1 / 2)$ & $\mathrm{F}:(\mathrm{u}, 1 / 2, \mathrm{w})$ & $\mathrm{M}:(1 / 2,1 / 2,0)$ & yes & Plane \\
\hline $\mathrm{A}:(1 / 2,1 / 2,1 / 2)$ & $\mathrm{E}:(\mathrm{u}, \mathrm{v}, 1 / 2)$ & $\mathrm{R}:(0,1 / 2,1 / 2)$ & no & Plane \\
\hline $\mathrm{A}:(1 / 2,1 / 2,1 / 2)$ & $\mathrm{F}:(\mathrm{u}, 1 / 2, \mathrm{w})$ & $\mathrm{R}:(0,1 / 2,1 / 2)$ & no & Plane \\
\hline $\mathrm{A}:(1 / 2,1 / 2,1 / 2)$ & $\mathrm{F}:(\mathrm{u}, 1 / 2, \mathrm{w})$ & $\mathrm{X}:(0,1 / 2,0)$ & yes & Plane \\
\hline $\mathrm{A}:(1 / 2,1 / 2,1 / 2)$ & $\mathrm{E}:(\mathrm{u}, \mathrm{v}, 1 / 2)$ & $\mathrm{Z}:(0,0,1 / 2)$ & no & Plane \\
\hline $\mathrm{M}:(1 / 2,1 / 2,0)$ & $\mathrm{F}:(\mathrm{u}, 1 / 2, \mathrm{w})$ & $\mathrm{R}:(0,1 / 2,1 / 2)$ & no & Plane \\
\hline $\mathrm{M}:(1 / 2,1 / 2,0)$ & $\mathrm{F}:(\mathrm{u}, 1 / 2, \mathrm{w})$ & $\mathrm{X}:(0,1 / 2,0)$ & yes & Plane \\
\hline $\mathrm{R}:(0,1 / 2,1 / 2)$ & $\mathrm{F}:(\mathrm{u}, 1 / 2, \mathrm{w})$ & $\mathrm{X}:(0,1 / 2,0)$ & no & Plane \\
\hline $\mathrm{R}:(0,1 / 2,1 / 2)$ & $\mathrm{E}:(\mathrm{u}, \mathrm{v}, 1 / 2)$ & $\mathrm{Z}:(0,0,1 / 2)$ & yes & Plane \\
\hline
\end{tabular}

BCSID: 1.160; Formula: UP; MSG: $128.410\left(P_{I} 4 / m n c\right) ; \mathrm{U}=0$

\begin{tabular}{c|c|c|c|c}
\hline maximal $k_{1}$ & intermediate path & maximal $k_{2}$ & satisfied? & Line/Plane \\
\hline$\Gamma:(0,0,0)$ & $\mathrm{LD}:(0,0, \mathrm{w})$ & $\mathrm{Z}:(0,0,1 / 2)$ & yes & Line \\
\hline $\mathrm{A}:(1 / 2,1 / 2,1 / 2)$ & $\mathrm{V}:(1 / 2,1 / 2, \mathrm{w})$ & $\mathrm{M}:(1 / 2,1 / 2,0)$ & yes & Line \\
\hline $\mathrm{A}:(1 / 2,1 / 2,1 / 2)$ & $\mathrm{T}:(\mathrm{u}, 1 / 2,1 / 2)$ & $\mathrm{R}:(0,1 / 2,1 / 2)$ & no & Line \\
\hline $\mathrm{A}:(1 / 2,1 / 2,1 / 2)$ & $\mathrm{S}:(\mathrm{u}, \mathrm{u}, 1 / 2)$ & $\mathrm{Z}:(0,0,1 / 2)$ & no & Line \\
\hline $\mathrm{R}:(0,1 / 2,1 / 2)$ & $\mathrm{W}:(0,1 / 2, \mathrm{w})$ & $\mathrm{X}:(0,1 / 2,0)$ & no & Line \\
\hline $\mathrm{R}:(0,1 / 2,1 / 2)$ & $\mathrm{U}:(0, \mathrm{v}, 1 / 2)$ & $\mathrm{Z}:(0,0,1 / 2)$ & yes & Line \\
\hline $\mathrm{A}:(1 / 2,1 / 2,1 / 2)$ & $\mathrm{E}:(\mathrm{u}, \mathrm{v}, 1 / 2)$ & $\mathrm{R}:(0,1 / 2,1 / 2)$ & no & Plane \\
\hline $\mathrm{A}:(1 / 2,1 / 2,1 / 2)$ & $\mathrm{E}:(\mathrm{u}, \mathrm{v}, 1 / 2)$ & $\mathrm{Z}:(0,0,1 / 2)$ & no & Plane \\
\hline $\mathrm{R}:(0,1 / 2,1 / 2)$ & $\mathrm{E}:(\mathrm{u}, \mathrm{v}, 1 / 2)$ & $\mathrm{Z}:(0,0,1 / 2)$ & yes & Plane \\
\hline
\end{tabular}

BCSID: 1.160; Formula: UP; MSG: $128.410\left(P_{I} 4 / m n c\right) ; \mathrm{U}=2 \mathrm{eV}$

\begin{tabular}{c|c|c|c|c}
\hline maximal $k_{1}$ & intermediate path & maximal $k_{2}$ & satisfied? & Line/Plane \\
\hline$\Gamma:(0,0,0)$ & $\mathrm{LD}:(0,0, \mathrm{w})$ & $\mathrm{Z}:(0,0,1 / 2)$ & no & Line \\
\hline $\mathrm{A}:(1 / 2,1 / 2,1 / 2)$ & $\mathrm{V}:(1 / 2,1 / 2, \mathrm{w})$ & $\mathrm{M}:(1 / 2,1 / 2,0)$ & no & Line \\
\hline $\mathrm{A}:(1 / 2,1 / 2,1 / 2)$ & $\mathrm{T}:(\mathrm{u}, 1 / 2,1 / 2)$ & $\mathrm{R}:(0,1 / 2,1 / 2)$ & yes & Line \\
\hline $\mathrm{A}:(1 / 2,1 / 2,1 / 2)$ & $\mathrm{S}:(\mathrm{u}, \mathrm{u}, 1 / 2)$ & $\mathrm{Z}:(0,0,1 / 2)$ & no & Line \\
\hline $\mathrm{R}:(0,1 / 2,1 / 2)$ & $\mathrm{W}:(0,1 / 2, \mathrm{w})$ & $\mathrm{X}:(0,1 / 2,0)$ & yes & Line \\
\hline $\mathrm{R}:(0,1 / 2,1 / 2)$ & $\mathrm{U}:(0, \mathrm{v}, 1 / 2)$ & $\mathrm{Z}:(0,0,1 / 2)$ & no & Line \\
\hline $\mathrm{A}:(1 / 2,1 / 2,1 / 2)$ & $\mathrm{E}:(\mathrm{u}, \mathrm{v}, 1 / 2)$ & $\mathrm{R}:(0,1 / 2,1 / 2)$ & yes & Plane \\
\hline $\mathrm{A}:(1 / 2,1 / 2,1 / 2)$ & $\mathrm{E}:(\mathrm{u}, \mathrm{v}, 1 / 2)$ & $\mathrm{Z}:(0,0,1 / 2)$ & no & Plane \\
\hline $\mathrm{R}:(0,1 / 2,1 / 2)$ & $\mathrm{E}:(\mathrm{u}, \mathrm{v}, 1 / 2)$ & $\mathrm{Z}:(0,0,1 / 2)$ & no & Plane \\
\hline
\end{tabular}

BCSID: 1.160; Formula: UP; MSG: $128.410\left(P_{I} 4 / m n c\right) ; \mathrm{U}=4 \mathrm{eV}$

\begin{tabular}{c|c|c|c|c}
\hline maximal $k_{1}$ & intermediate path & maximal $k_{2}$ & satisfied? & Line/Plane \\
\hline$\Gamma:(0,0,0)$ & $\mathrm{LD}:(0,0, \mathrm{w})$ & $\mathrm{Z}:(0,0,1 / 2)$ & yes & Line \\
\hline $\mathrm{A}:(1 / 2,1 / 2,1 / 2)$ & $\mathrm{V}:(1 / 2,1 / 2, \mathrm{w})$ & $\mathrm{M}:(1 / 2,1 / 2,0)$ & no & Line
\end{tabular}




\begin{tabular}{c|c|c|c|c}
\hline $\mathrm{A}:(1 / 2,1 / 2,1 / 2)$ & $\mathrm{T}:(\mathrm{u}, 1 / 2,1 / 2)$ & $\mathrm{R}:(0,1 / 2,1 / 2)$ & yes & Line \\
\hline $\mathrm{A}:(1 / 2,1 / 2,1 / 2)$ & $\mathrm{S}:(\mathrm{u}, \mathrm{u}, 1 / 2)$ & $\mathrm{Z}:(0,0,1 / 2)$ & yes & Line \\
\hline $\mathrm{R}:(0,1 / 2,1 / 2)$ & $\mathrm{W}:(0,1 / 2, \mathrm{w})$ & $\mathrm{X}:(0,1 / 2,0)$ & yes & Line \\
\hline $\mathrm{R}:(0,1 / 2,1 / 2)$ & $\mathrm{U}:(0, \mathrm{v}, 1 / 2)$ & $\mathrm{Z}:(0,0,1 / 2)$ & yes & Line \\
\hline $\mathrm{A}:(1 / 2,1 / 2,1 / 2)$ & $\mathrm{E}:(\mathrm{u}, \mathrm{v}, 1 / 2)$ & $\mathrm{R}:(0,1 / 2,1 / 2)$ & yes & Plane \\
\hline $\mathrm{A}:(1 / 2,1 / 2,1 / 2)$ & $\mathrm{E}:(\mathrm{u}, \mathrm{v}, 1 / 2)$ & $\mathrm{Z}:(0,0,1 / 2)$ & yes & Plane \\
\hline $\mathrm{R}:(0,1 / 2,1 / 2)$ & $\mathrm{E}:(\mathrm{u}, \mathrm{v}, 1 / 2)$ & $\mathrm{Z}:(0,0,1 / 2)$ & yes & Plane \\
\hline
\end{tabular}

BCSID: 1.160; Formula: UP; MSG: $128.410\left(P_{I} 4 / m n c\right) ; \mathrm{U}=6 \mathrm{eV}$

\begin{tabular}{c|c|c|c|c}
\hline maximal $k_{1}$ & intermediate path & maximal $k_{2}$ & satisfied? & Line/Plane \\
\hline$\Gamma:(0,0,0)$ & $\mathrm{LD}:(0,0, \mathrm{w})$ & $\mathrm{Z}:(0,0,1 / 2)$ & no & Line \\
\hline $\mathrm{A}:(1 / 2,1 / 2,1 / 2)$ & $\mathrm{V}:(1 / 2,1 / 2, \mathrm{w})$ & $\mathrm{M}:(1 / 2,1 / 2,0)$ & yes & Line \\
\hline $\mathrm{A}:(1 / 2,1 / 2,1 / 2)$ & $\mathrm{T}:(\mathrm{u}, 1 / 2,1 / 2)$ & $\mathrm{R}:(0,1 / 2,1 / 2)$ & no & Line \\
\hline $\mathrm{A}:(1 / 2,1 / 2,1 / 2)$ & $\mathrm{S}:(\mathrm{u}, \mathrm{u}, 1 / 2)$ & $\mathrm{Z}:(0,0,1 / 2)$ & no & Line \\
\hline $\mathrm{R}:(0,1 / 2,1 / 2)$ & $\mathrm{W}:(0,1 / 2, \mathrm{w})$ & $\mathrm{X}:(0,1 / 2,0)$ & yes & Line \\
\hline $\mathrm{R}:(0,1 / 2,1 / 2)$ & $\mathrm{U}:(0, \mathrm{v}, 1 / 2)$ & $\mathrm{Z}:(0,0,1 / 2)$ & yes & Line \\
\hline $\mathrm{A}:(1 / 2,1 / 2,1 / 2)$ & $\mathrm{E}:(\mathrm{u}, \mathrm{v}, 1 / 2)$ & $\mathrm{R}:(0,1 / 2,1 / 2)$ & no & Plane \\
\hline $\mathrm{A}:(1 / 2,1 / 2,1 / 2)$ & $\mathrm{E}:(\mathrm{u}, \mathrm{v}, 1 / 2)$ & $\mathrm{Z}:(0,0,1 / 2)$ & no & Plane \\
\hline $\mathrm{R}:(0,1 / 2,1 / 2)$ & $\mathrm{E}:(\mathrm{u}, \mathrm{v}, 1 / 2)$ & $\mathrm{Z}:(0,0,1 / 2)$ & yes & Plane \\
\hline
\end{tabular}

BCSID: 1.187; Formula: TbRh2Si2; MSG: $128.410\left(P_{I} 4 / m n c\right) ; \mathrm{U}=0$

\begin{tabular}{c|c|c|c|c}
\hline maximal $k_{1}$ & intermediate path & maximal $k_{2}$ & satisfied? & Line/Plane \\
\hline$\Gamma:(0,0,0)$ & $\mathrm{LD}:(0,0, \mathrm{w})$ & $\mathrm{Z}:(0,0,1 / 2)$ & no & Line \\
\hline $\mathrm{A}:(1 / 2,1 / 2,1 / 2)$ & $\mathrm{V}:(1 / 2,1 / 2, \mathrm{w})$ & $\mathrm{M}:(1 / 2,1 / 2,0)$ & yes & Line \\
\hline $\mathrm{A}:(1 / 2,1 / 2,1 / 2)$ & $\mathrm{T}:(\mathrm{u}, 1 / 2,1 / 2)$ & $\mathrm{R}:(0,1 / 2,1 / 2)$ & yes & Line \\
\hline $\mathrm{A}:(1 / 2,1 / 2,1 / 2)$ & $\mathrm{S}:(\mathrm{u}, \mathrm{u}, 1 / 2)$ & $\mathrm{Z}:(0,0,1 / 2)$ & yes & Line \\
\hline $\mathrm{R}:(0,1 / 2,1 / 2)$ & $\mathrm{W}:(0,1 / 2, \mathrm{w})$ & $\mathrm{X}:(0,1 / 2,0)$ & no & Line \\
\hline $\mathrm{R}:(0,1 / 2,1 / 2)$ & $\mathrm{U}:(0, \mathrm{v}, 1 / 2)$ & $\mathrm{Z}:(0,0,1 / 2)$ & yes & Line \\
\hline $\mathrm{A}:(1 / 2,1 / 2,1 / 2)$ & $\mathrm{E}:(\mathrm{u}, \mathrm{v}, 1 / 2)$ & $\mathrm{R}:(0,1 / 2,1 / 2)$ & yes & Plane \\
\hline $\mathrm{A}:(1 / 2,1 / 2,1 / 2)$ & $\mathrm{E}:(\mathrm{u}, \mathrm{v}, 1 / 2)$ & $\mathrm{Z}:(0,0,1 / 2)$ & yes & Plane \\
\hline $\mathrm{R}:(0,1 / 2,1 / 2)$ & $\mathrm{E}:(\mathrm{u}, \mathrm{v}, 1 / 2)$ & $\mathrm{Z}:(0,0,1 / 2)$ & yes & Plane \\
\hline
\end{tabular}

\begin{tabular}{c|c|c|c|c}
\hline \multicolumn{5}{c}{ BCSID: $1.187 ;$ Formula: TbRh2Si2; MSG: $128.410\left(P_{I} 4 / m n c\right) ; \mathrm{U}=2 \mathrm{eV}$} \\
\hline maximal $k_{1}$ & intermediate path & maximal $k_{2}$ & satisfied? & Line/Plane \\
\hline$\Gamma:(0,0,0)$ & $\mathrm{LD}:(0,0, \mathrm{w})$ & $\mathrm{Z}:(0,0,1 / 2)$ & no & Line \\
\hline $\mathrm{A}:(1 / 2,1 / 2,1 / 2)$ & $\mathrm{V}:(1 / 2,1 / 2, \mathrm{w})$ & $\mathrm{M}:(1 / 2,1 / 2,0)$ & yes & Line \\
\hline $\mathrm{A}:(1 / 2,1 / 2,1 / 2)$ & $\mathrm{T}:(\mathrm{u}, 1 / 2,1 / 2)$ & $\mathrm{R}:(0,1 / 2,1 / 2)$ & yes & Line \\
\hline $\mathrm{A}:(1 / 2,1 / 2,1 / 2)$ & $\mathrm{S}:(\mathrm{u}, \mathrm{u}, 1 / 2)$ & $\mathrm{Z}:(0,0,1 / 2)$ & yes & Line \\
\hline $\mathrm{R}:(0,1 / 2,1 / 2)$ & $\mathrm{W}:(0,1 / 2, \mathrm{w})$ & $\mathrm{X}:(0,1 / 2,0)$ & yes & Line \\
\hline $\mathrm{R}:(0,1 / 2,1 / 2)$ & $\mathrm{U}:(0, \mathrm{v}, 1 / 2)$ & $\mathrm{Z}:(0,0,1 / 2)$ & yes & Line \\
\hline $\mathrm{A}:(1 / 2,1 / 2,1 / 2)$ & $\mathrm{E}:(\mathrm{u}, \mathrm{v}, 1 / 2)$ & $\mathrm{R}:(0,1 / 2,1 / 2)$ & yes & Plane \\
\hline $\mathrm{A}:(1 / 2,1 / 2,1 / 2)$ & $\mathrm{E}:(\mathrm{u}, \mathrm{v}, 1 / 2)$ & $\mathrm{Z}:(0,0,1 / 2)$ & yes & Plane \\
\hline $\mathrm{R}:(0,1 / 2,1 / 2)$ & $\mathrm{E}:(\mathrm{u}, \mathrm{v}, 1 / 2)$ & $\mathrm{Z}:(0,0,1 / 2)$ & yes & Plane \\
\hline
\end{tabular}

\begin{tabular}{c|c|c|c|c}
\hline \multicolumn{4}{c}{ BCSID: $1.187 ;$ Formula: TbRh2Si2; MSG: $128.410\left(P_{I} 4 / m n c\right) ; \mathrm{U}=4 \mathrm{eV}$} \\
\hline maximal $k_{1}$ & intermediate path & maximal $k_{2}$ & satisfied? & Line/Plane \\
\hline$\Gamma:(0,0,0)$ & $\mathrm{LD}:(0,0, \mathrm{w})$ & $\mathrm{Z}:(0,0,1 / 2)$ & no & Line \\
\hline $\mathrm{A}:(1 / 2,1 / 2,1 / 2)$ & $\mathrm{V}:(1 / 2,1 / 2, \mathrm{w})$ & $\mathrm{M}:(1 / 2,1 / 2,0)$ & yes & Line \\
\hline $\mathrm{A}:(1 / 2,1 / 2,1 / 2)$ & $\mathrm{T}:(\mathrm{u}, 1 / 2,1 / 2)$ & $\mathrm{R}:(0,1 / 2,1 / 2)$ & no & Line \\
\hline $\mathrm{A}:(1 / 2,1 / 2,1 / 2)$ & $\mathrm{S}:(\mathrm{u}, \mathrm{u}, 1 / 2)$ & $\mathrm{Z}:(0,0,1 / 2)$ & no & Line \\
\hline $\mathrm{R}:(0,1 / 2,1 / 2)$ & $\mathrm{W}:(0,1 / 2, \mathrm{w})$ & $\mathrm{X}:(0,1 / 2,0)$ & yes & Line
\end{tabular}




\begin{tabular}{c|c|c|c|c}
\hline $\mathrm{R}:(0,1 / 2,1 / 2)$ & $\mathrm{U}:(0, \mathrm{v}, 1 / 2)$ & $\mathrm{Z}:(0,0,1 / 2)$ & yes & Line \\
\hline $\mathrm{A}:(1 / 2,1 / 2,1 / 2)$ & $\mathrm{E}:(\mathrm{u}, \mathrm{v}, 1 / 2)$ & $\mathrm{R}:(0,1 / 2,1 / 2)$ & no & Plane \\
\hline $\mathrm{A}:(1 / 2,1 / 2,1 / 2)$ & $\mathrm{E}:(\mathrm{u}, \mathrm{v}, 1 / 2)$ & $\mathrm{Z}:(0,0,1 / 2)$ & no & Plane \\
\hline $\mathrm{R}:(0,1 / 2,1 / 2)$ & $\mathrm{E}:(\mathrm{u}, \mathrm{v}, 1 / 2)$ & $\mathrm{Z}:(0,0,1 / 2)$ & yes & Plane \\
\hline
\end{tabular}

BCSID: 1.187; Formula: TbRh2Si2; MSG: $128.410\left(P_{I} 4 / m n c\right) ; \mathrm{U}=6 \mathrm{eV}$

\begin{tabular}{c|c|c|c|c}
\hline maximal $k_{1}$ & intermediate path & maximal $k_{2}$ & satisfied? & Line/Plane \\
\hline$\Gamma:(0,0,0)$ & $\mathrm{LD}:(0,0, \mathrm{w})$ & $\mathrm{Z}:(0,0,1 / 2)$ & no & Line \\
\hline $\mathrm{A}:(1 / 2,1 / 2,1 / 2)$ & $\mathrm{V}:(1 / 2,1 / 2, \mathrm{w})$ & $\mathrm{M}:(1 / 2,1 / 2,0)$ & yes & Line \\
\hline $\mathrm{A}:(1 / 2,1 / 2,1 / 2)$ & $\mathrm{T}:(\mathrm{u}, 1 / 2,1 / 2)$ & $\mathrm{R}:(0,1 / 2,1 / 2)$ & no & Line \\
\hline $\mathrm{A}:(1 / 2,1 / 2,1 / 2)$ & $\mathrm{S}:(\mathrm{u}, \mathrm{u}, 1 / 2)$ & $\mathrm{Z}:(0,0,1 / 2)$ & no & Line \\
\hline $\mathrm{R}:(0,1 / 2,1 / 2)$ & $\mathrm{W}:(0,1 / 2, \mathrm{w})$ & $\mathrm{X}:(0,1 / 2,0)$ & yes & Line \\
\hline $\mathrm{R}:(0,1 / 2,1 / 2)$ & $\mathrm{U}:(0, \mathrm{v}, 1 / 2)$ & $\mathrm{Z}:(0,0,1 / 2)$ & yes & Line \\
\hline $\mathrm{A}:(1 / 2,1 / 2,1 / 2)$ & $\mathrm{E}:(\mathrm{u}, \mathrm{v}, 1 / 2)$ & $\mathrm{R}:(0,1 / 2,1 / 2)$ & no & Plane \\
\hline $\mathrm{A}:(1 / 2,1 / 2,1 / 2)$ & $\mathrm{E}:(\mathrm{u}, \mathrm{v}, 1 / 2)$ & $\mathrm{Z}:(0,0,1 / 2)$ & no & Plane \\
\hline $\mathrm{R}:(0,1 / 2,1 / 2)$ & $\mathrm{E}:(\mathrm{u}, \mathrm{v}, 1 / 2)$ & $\mathrm{Z}:(0,0,1 / 2)$ & yes & Plane \\
\hline
\end{tabular}

\begin{tabular}{|c|c|c|c|c|}
\hline \multicolumn{5}{|c|}{ BCSID: 1.208; Formula: UAs; MSG: $128.410\left(P_{I} 4 / m n c\right) ; \mathrm{U}=0$} \\
\hline maximal $k_{1}$ & intermediate path & maximal $k_{2}$ & satisfied? & Line/Plane \\
\hline$\Gamma:(0,0,0)$ & $\mathrm{LD}:(0,0, \mathrm{w})$ & $\mathrm{Z}:(0,0,1 / 2)$ & yes & Line \\
\hline $\mathrm{A}:(1 / 2,1 / 2,1 / 2)$ & $\mathrm{V}:(1 / 2,1 / 2, \mathrm{w})$ & $\mathrm{M}:(1 / 2,1 / 2,0)$ & yes & Line \\
\hline $\mathrm{A}:(1 / 2,1 / 2,1 / 2)$ & $\mathrm{T}:(\mathrm{u}, 1 / 2,1 / 2)$ & $\mathrm{R}:(0,1 / 2,1 / 2)$ & no & Line \\
\hline $\mathrm{A}:(1 / 2,1 / 2,1 / 2)$ & $\mathrm{S}:(\mathrm{u}, \mathrm{u}, 1 / 2)$ & $\mathrm{Z}:(0,0,1 / 2)$ & no & Line \\
\hline $\mathrm{R}:(0,1 / 2,1 / 2)$ & $\mathrm{W}:(0,1 / 2, \mathrm{w})$ & $\mathrm{X}:(0,1 / 2,0)$ & no & Line \\
\hline $\mathrm{R}:(0,1 / 2,1 / 2)$ & $\mathrm{U}:(0, \mathrm{v}, 1 / 2)$ & $\mathrm{Z}:(0,0,1 / 2)$ & yes & Line \\
\hline $\mathrm{A}:(1 / 2,1 / 2,1 / 2)$ & $\mathrm{E}:(\mathrm{u}, \mathrm{v}, 1 / 2)$ & $\mathrm{R}:(0,1 / 2,1 / 2)$ & no & Plane \\
\hline $\mathrm{A}:(1 / 2,1 / 2,1 / 2)$ & $\mathrm{E}:(\mathrm{u}, \mathrm{v}, 1 / 2)$ & $\mathrm{Z}:(0,0,1 / 2)$ & no & Plane \\
\hline $\mathrm{R}:(0,1 / 2,1 / 2)$ & $\mathrm{E}:(\mathrm{u}, \mathrm{v}, 1 / 2)$ & $\mathrm{Z}:(0,0,1 / 2)$ & yes & Plane \\
\hline \multicolumn{5}{|c|}{ BCSID: 1.208; Formula: UAs; MSG: $128.410\left(P_{I} 4 / m n c\right) ; \mathrm{U}=2 \mathrm{eV}$} \\
\hline maximal $k_{1}$ & intermediate path & maximal $k_{2}$ & satisfied? & Line/Plane \\
\hline$\Gamma:(0,0,0)$ & LD: $(0,0, w)$ & $\mathrm{Z}:(0,0,1 / 2)$ & yes & Line \\
\hline $\mathrm{A}:(1 / 2,1 / 2,1 / 2)$ & $\mathrm{V}:(1 / 2,1 / 2, \mathrm{w})$ & $\mathrm{M}:(1 / 2,1 / 2,0)$ & no & Line \\
\hline $\mathrm{A}:(1 / 2,1 / 2,1 / 2)$ & $\mathrm{T}:(\mathrm{u}, 1 / 2,1 / 2)$ & $\mathrm{R}:(0,1 / 2,1 / 2)$ & yes & Line \\
\hline $\mathrm{A}:(1 / 2,1 / 2,1 / 2)$ & $\mathrm{S}:(\mathrm{u}, \mathrm{u}, 1 / 2)$ & $\mathrm{Z}:(0,0,1 / 2)$ & yes & Line \\
\hline $\mathrm{R}:(0,1 / 2,1 / 2)$ & $\mathrm{W}:(0,1 / 2, \mathrm{w})$ & $\mathrm{X}:(0,1 / 2,0)$ & yes & Line \\
\hline $\mathrm{R}:(0,1 / 2,1 / 2)$ & $\mathrm{U}:(0, \mathrm{v}, 1 / 2)$ & $\mathrm{Z}:(0,0,1 / 2)$ & yes & Line \\
\hline $\mathrm{A}:(1 / 2,1 / 2,1 / 2)$ & $\mathrm{E}:(\mathrm{u}, \mathrm{v}, 1 / 2)$ & $\mathrm{R}:(0,1 / 2,1 / 2)$ & yes & Plane \\
\hline $\mathrm{A}:(1 / 2,1 / 2,1 / 2)$ & $\mathrm{E}:(\mathrm{u}, \mathrm{v}, 1 / 2)$ & $\mathrm{Z}:(0,0,1 / 2)$ & yes & Plane \\
\hline $\mathrm{R}:(0,1 / 2,1 / 2)$ & $\mathrm{E}:(\mathrm{u}, \mathrm{v}, 1 / 2)$ & $\mathrm{Z}:(0,0,1 / 2)$ & yes & Plane \\
\hline
\end{tabular}

\begin{tabular}{c|c|c|c|c}
\hline \multicolumn{5}{c}{ BCSID: 1.208; Formula: UAs; MSG: $128.410\left(P_{I} 4 / m n c\right) ; \mathrm{U}=4 \mathrm{eV}$} \\
\hline maximal $k_{1}$ & intermediate path & maximal $k_{2}$ & satisfied? & Line/Plane \\
\hline$\Gamma:(0,0,0)$ & $\mathrm{LD}:(0,0, \mathrm{w})$ & $\mathrm{Z}:(0,0,1 / 2)$ & yes & Line \\
\hline $\mathrm{A}:(1 / 2,1 / 2,1 / 2)$ & $\mathrm{V}:(1 / 2,1 / 2, \mathrm{w})$ & $\mathrm{M}:(1 / 2,1 / 2,0)$ & no & Line \\
\hline $\mathrm{A}:(1 / 2,1 / 2,1 / 2)$ & $\mathrm{T}:(\mathrm{u}, 1 / 2,1 / 2)$ & $\mathrm{R}:(0,1 / 2,1 / 2)$ & yes & Line \\
\hline $\mathrm{A}:(1 / 2,1 / 2,1 / 2)$ & $\mathrm{S}:(\mathrm{u}, \mathrm{u}, 1 / 2)$ & $\mathrm{Z}:(0,0,1 / 2)$ & yes & Line \\
\hline $\mathrm{R}:(0,1 / 2,1 / 2)$ & $\mathrm{W}:(0,1 / 2, \mathrm{w})$ & $\mathrm{X}:(0,1 / 2,0)$ & yes & Line \\
\hline $\mathrm{R}:(0,1 / 2,1 / 2)$ & $\mathrm{U}:(0, \mathrm{v}, 1 / 2)$ & $\mathrm{Z}:(0,0,1 / 2)$ & yes & Line \\
\hline $\mathrm{A}:(1 / 2,1 / 2,1 / 2)$ & $\mathrm{E}:(\mathrm{u}, \mathrm{v}, 1 / 2)$ & $\mathrm{R}:(0,1 / 2,1 / 2)$ & yes & Plane \\
\hline $\mathrm{A}:(1 / 2,1 / 2,1 / 2)$ & $\mathrm{E}:(\mathrm{u}, \mathrm{v}, 1 / 2)$ & $\mathrm{Z}:(0,0,1 / 2)$ & yes & Plane
\end{tabular}




\begin{tabular}{|c|c|c|c|c|}
\hline $\mathrm{R}:(0,1 / 2,1 / 2)$ & $\mathrm{E}:(\mathrm{u}, \mathrm{v}, 1 / 2)$ & $\mathrm{Z}:(0,0,1 / 2)$ & yes & Plane \\
\hline \multicolumn{5}{|c|}{ BCSID: 1.21; Formula: DyCo2Si2; MSG: $128.410\left(P_{I} 4 / m n c\right) ; \mathrm{U}=2 \mathrm{eV}$} \\
\hline maximal $k_{1}$ & intermediate path & maximal $k_{2}$ & satisfied? & Line/Plane \\
\hline$\Gamma:(0,0,0)$ & $\mathrm{LD}:(0,0, \mathrm{w})$ & $\mathrm{Z}:(0,0,1 / 2)$ & no & Line \\
\hline $\mathrm{A}:(1 / 2,1 / 2,1 / 2)$ & $\mathrm{V}:(1 / 2,1 / 2, \mathrm{w})$ & $\mathrm{M}:(1 / 2,1 / 2,0)$ & yes & Line \\
\hline $\mathrm{A}:(1 / 2,1 / 2,1 / 2)$ & $\mathrm{T}:(\mathrm{u}, 1 / 2,1 / 2)$ & $\mathrm{R}:(0,1 / 2,1 / 2)$ & yes & Line \\
\hline $\mathrm{A}:(1 / 2,1 / 2,1 / 2)$ & $\mathrm{S}:(\mathrm{u}, \mathrm{u}, 1 / 2)$ & $\mathrm{Z}:(0,0,1 / 2)$ & yes & Line \\
\hline $\mathrm{R}:(0,1 / 2,1 / 2)$ & $\mathrm{W}:(0,1 / 2, \mathrm{w})$ & $\mathrm{X}:(0,1 / 2,0)$ & yes & Line \\
\hline $\mathrm{R}:(0,1 / 2,1 / 2)$ & $\mathrm{U}:(0, \mathrm{v}, 1 / 2)$ & $\mathrm{Z}:(0,0,1 / 2)$ & yes & Line \\
\hline $\mathrm{A}:(1 / 2,1 / 2,1 / 2)$ & $\mathrm{E}:(\mathrm{u}, \mathrm{v}, 1 / 2)$ & $\mathrm{R}:(0,1 / 2,1 / 2)$ & yes & Plane \\
\hline $\mathrm{A}:(1 / 2,1 / 2,1 / 2)$ & $\mathrm{E}:(\mathrm{u}, \mathrm{v}, 1 / 2)$ & $\mathrm{Z}:(0,0,1 / 2)$ & yes & Plane \\
\hline $\mathrm{R}:(0,1 / 2,1 / 2)$ & $\mathrm{E}:(\mathrm{u}, \mathrm{v}, 1 / 2)$ & $\mathrm{Z}:(0,0,1 / 2)$ & yes & Plane \\
\hline \multicolumn{5}{|c|}{ BCSID: 1.21 ; Formula: DyCo2Si2; MSG: $128.410\left(P_{I} 4 / m n c\right) ; \mathrm{U}=4 \mathrm{eV}$} \\
\hline maximal $k_{1}$ & intermediate path & $\operatorname{maximal} k_{2}$ & satisfied? & Line/Plane \\
\hline$\Gamma:(0,0,0)$ & $\mathrm{LD}:(0,0, \mathrm{w})$ & $\mathrm{Z}:(0,0,1 / 2)$ & no & Line \\
\hline $\mathrm{A}:(1 / 2,1 / 2,1 / 2)$ & $\mathrm{V}:(1 / 2,1 / 2, \mathrm{w})$ & $\mathrm{M}:(1 / 2,1 / 2,0)$ & yes & Line \\
\hline $\mathrm{A}:(1 / 2,1 / 2,1 / 2)$ & $\mathrm{T}:(\mathrm{u}, 1 / 2,1 / 2)$ & $\mathrm{R}:(0,1 / 2,1 / 2)$ & yes & Line \\
\hline $\mathrm{A}:(1 / 2,1 / 2,1 / 2)$ & $\mathrm{S}:(\mathrm{u}, \mathrm{u}, 1 / 2)$ & $\mathrm{Z}:(0,0,1 / 2)$ & yes & Line \\
\hline $\mathrm{R}:(0,1 / 2,1 / 2)$ & $\mathrm{W}:(0,1 / 2, \mathrm{w})$ & $\mathrm{X}:(0,1 / 2,0)$ & yes & Line \\
\hline $\mathrm{R}:(0,1 / 2,1 / 2)$ & $\mathrm{U}:(0, \mathrm{v}, 1 / 2)$ & $\mathrm{Z}:(0,0,1 / 2)$ & yes & Line \\
\hline $\mathrm{A}:(1 / 2,1 / 2,1 / 2)$ & $\mathrm{E}:(\mathrm{u}, \mathrm{v}, 1 / 2)$ & $\mathrm{R}:(0,1 / 2,1 / 2)$ & yes & Plane \\
\hline $\mathrm{A}:(1 / 2,1 / 2,1 / 2)$ & $\mathrm{E}:(\mathrm{u}, \mathrm{v}, 1 / 2)$ & $\mathrm{Z}:(0,0,1 / 2)$ & yes & Plane \\
\hline $\mathrm{R}:(0,1 / 2,1 / 2)$ & $\mathrm{E}:(\mathrm{u}, \mathrm{v}, 1 / 2)$ & $\mathrm{Z}:(0,0,1 / 2)$ & yes & Plane \\
\hline \multicolumn{5}{|c|}{ BCSID: 1.215 ; Formula: UP2; MSG: $130.432\left(P_{c} 4 / n c c\right) ; \mathrm{U}=0$} \\
\hline maximal $k_{1}$ & intermediate path & maximal $k_{2}$ & satisfied? & Line/Plane \\
\hline$\Gamma:(0,0,0)$ & $\mathrm{LD}:(0,0, \mathrm{w})$ & $\mathrm{Z}:(0,0,1 / 2)$ & yes & Line \\
\hline $\mathrm{A}:(1 / 2,1 / 2,1 / 2)$ & $\mathrm{S}:(\mathrm{u}, \mathrm{u}, 1 / 2)$ & $\mathrm{Z}:(0,0,1 / 2)$ & no & Line \\
\hline $\mathrm{M}:(1 / 2,1 / 2,0)$ & $\mathrm{Y}:(\mathrm{u}, 1 / 2,0)$ & $\mathrm{X}:(0,1 / 2,0)$ & yes & Line \\
\hline $\mathrm{R}:(0,1 / 2,1 / 2)$ & $\mathrm{U}:(0, \mathrm{v}, 1 / 2)$ & $\mathrm{Z}:(0,0,1 / 2)$ & no & Line \\
\hline $\mathrm{A}:(1 / 2,1 / 2,1 / 2)$ & $\mathrm{F}:(\mathrm{u}, 1 / 2, \mathrm{w})$ & $\mathrm{M}:(1 / 2,1 / 2,0)$ & yes & Plane \\
\hline $\mathrm{A}:(1 / 2,1 / 2,1 / 2)$ & $\mathrm{E}:(\mathrm{u}, \mathrm{v}, 1 / 2)$ & $\mathrm{R}:(0,1 / 2,1 / 2)$ & yes & Plane \\
\hline $\mathrm{A}:(1 / 2,1 / 2,1 / 2)$ & $\mathrm{F}:(\mathrm{u}, 1 / 2, \mathrm{w})$ & $\mathrm{R}:(0,1 / 2,1 / 2)$ & yes & Plane \\
\hline $\mathrm{A}:(1 / 2,1 / 2,1 / 2)$ & $\mathrm{F}:(\mathrm{u}, 1 / 2, \mathrm{w})$ & $\mathrm{X}:(0,1 / 2,0)$ & yes & Plane \\
\hline $\mathrm{A}:(1 / 2,1 / 2,1 / 2)$ & $\mathrm{E}:(\mathrm{u}, \mathrm{v}, 1 / 2)$ & $\mathrm{Z}:(0,0,1 / 2)$ & no & Plane \\
\hline $\mathrm{M}:(1 / 2,1 / 2,0)$ & $\mathrm{F}:(\mathrm{u}, 1 / 2, \mathrm{w})$ & $\mathrm{R}:(0,1 / 2,1 / 2)$ & yes & Plane \\
\hline $\mathrm{M}:(1 / 2,1 / 2,0)$ & $\mathrm{F}:(\mathrm{u}, 1 / 2, \mathrm{w})$ & $\mathrm{X}:(0,1 / 2,0)$ & yes & Plane \\
\hline $\mathrm{R}:(0,1 / 2,1 / 2)$ & $\mathrm{F}:(\mathrm{u}, 1 / 2, \mathrm{w})$ & $\mathrm{X}:(0,1 / 2,0)$ & yes & Plane \\
\hline $\mathrm{R}:(0,1 / 2,1 / 2)$ & $\mathrm{E}:(\mathrm{u}, \mathrm{v}, 1 / 2)$ & $\mathrm{Z}:(0,0,1 / 2)$ & no & Plane \\
\hline
\end{tabular}

BCSID: 1.215; Formula: UP2; MSG: $130.432\left(P_{c} 4 / n c c\right)$; $\mathrm{U}=2 \mathrm{eV}$

\begin{tabular}{c|c|c|c|c}
\hline maximal $k_{1}$ & intermediate path & maximal $k_{2}$ & satisfied? & Line/Plane \\
\hline$\Gamma:(0,0,0)$ & $\mathrm{LD}:(0,0, \mathrm{w})$ & $\mathrm{Z}:(0,0,1 / 2)$ & no & Line \\
\hline $\mathrm{A}:(1 / 2,1 / 2,1 / 2)$ & $\mathrm{S}:(\mathrm{u}, \mathrm{u}, 1 / 2)$ & $\mathrm{Z}:(0,0,1 / 2)$ & yes & Line \\
\hline $\mathrm{M}:(1 / 2,1 / 2,0)$ & $\mathrm{Y}:(\mathrm{u}, 1 / 2,0)$ & $\mathrm{X}:(0,1 / 2,0)$ & yes & Line \\
\hline $\mathrm{R}:(0,1 / 2,1 / 2)$ & $\mathrm{U}:(0, \mathrm{v}, 1 / 2)$ & $\mathrm{Z}:(0,0,1 / 2)$ & yes & Line \\
\hline $\mathrm{A}:(1 / 2,1 / 2,1 / 2)$ & $\mathrm{F}:(\mathrm{u}, 1 / 2, \mathrm{w})$ & $\mathrm{M}:(1 / 2,1 / 2,0)$ & yes & Plane \\
\hline $\mathrm{A}:(1 / 2,1 / 2,1 / 2)$ & $\mathrm{E}:(\mathrm{u}, \mathrm{v}, 1 / 2)$ & $\mathrm{R}:(0,1 / 2,1 / 2)$ & yes & Plane \\
\hline $\mathrm{A}:(1 / 2,1 / 2,1 / 2)$ & $\mathrm{F}:(\mathrm{u}, 1 / 2, \mathrm{w})$ & $\mathrm{R}:(0,1 / 2,1 / 2)$ & yes & Plane
\end{tabular}




\begin{tabular}{c|c|c|c|c}
\hline $\mathrm{A}:(1 / 2,1 / 2,1 / 2)$ & $\mathrm{F}:(\mathrm{u}, 1 / 2, \mathrm{w})$ & $\mathrm{X}:(0,1 / 2,0)$ & yes & Plane \\
\hline $\mathrm{A}:(1 / 2,1 / 2,1 / 2)$ & $\mathrm{E}:(\mathrm{u}, \mathrm{v}, 1 / 2)$ & $\mathrm{Z}:(0,0,1 / 2)$ & yes & Plane \\
\hline $\mathrm{M}:(1 / 2,1 / 2,0)$ & $\mathrm{F}:(\mathrm{u}, 1 / 2, \mathrm{w})$ & $\mathrm{R}:(0,1 / 2,1 / 2)$ & yes & Plane \\
\hline $\mathrm{M}:(1 / 2,1 / 2,0)$ & $\mathrm{F}:(\mathrm{u}, 1 / 2, \mathrm{w})$ & $\mathrm{X}:(0,1 / 2,0)$ & yes & Plane \\
\hline $\mathrm{R}:(0,1 / 2,1 / 2)$ & $\mathrm{F}:(\mathrm{u}, 1 / 2, \mathrm{w})$ & $\mathrm{X}:(0,1 / 2,0)$ & yes & Plane \\
\hline $\mathrm{R}:(0,1 / 2,1 / 2)$ & $\mathrm{E}:(\mathrm{u}, \mathrm{v}, 1 / 2)$ & $\mathrm{Z}:(0,0,1 / 2)$ & yes & Plane \\
\hline
\end{tabular}

\begin{tabular}{c|c|c|c|c}
\hline \multicolumn{6}{c}{ BCSID: 1.215; Formula: UP2; MSG: $130.432\left(P_{c} 4 / n c c\right) ; \mathrm{U}=4 \mathrm{eV}$} \\
\hline maximal $k_{1}$ & intermediate path & maximal $k_{2}$ & satisfied? & Line/Plane \\
\hline$\Gamma:(0,0,0)$ & $\mathrm{LD}:(0,0, \mathrm{w})$ & $\mathrm{Z}:(0,0,1 / 2)$ & yes & Line \\
\hline $\mathrm{A}:(1 / 2,1 / 2,1 / 2)$ & $\mathrm{S}:(\mathrm{u}, \mathrm{u}, 1 / 2)$ & $\mathrm{Z}:(0,0,1 / 2)$ & no & Line \\
\hline $\mathrm{M}:(1 / 2,1 / 2,0)$ & $\mathrm{Y}:(\mathrm{u}, 1 / 2,0)$ & $\mathrm{X}:(0,1 / 2,0)$ & yes & Line \\
\hline $\mathrm{R}:(0,1 / 2,1 / 2)$ & $\mathrm{U}:(0, \mathrm{v}, 1 / 2)$ & $\mathrm{Z}:(0,0,1 / 2)$ & no & Line \\
\hline $\mathrm{A}:(1 / 2,1 / 2,1 / 2)$ & $\mathrm{F}:(\mathrm{u}, 1 / 2, \mathrm{w})$ & $\mathrm{M}:(1 / 2,1 / 2,0)$ & yes & Plane \\
\hline $\mathrm{A}:(1 / 2,1 / 2,1 / 2)$ & $\mathrm{E}:(\mathrm{u}, \mathrm{v}, 1 / 2)$ & $\mathrm{R}:(0,1 / 2,1 / 2)$ & yes & Plane \\
\hline $\mathrm{A}:(1 / 2,1 / 2,1 / 2)$ & $\mathrm{F}:(\mathrm{u}, 1 / 2, \mathrm{w})$ & $\mathrm{R}:(0,1 / 2,1 / 2)$ & yes & Plane \\
\hline $\mathrm{A}:(1 / 2,1 / 2,1 / 2)$ & $\mathrm{F}:(\mathrm{u}, 1 / 2, \mathrm{w})$ & $\mathrm{X}:(0,1 / 2,0)$ & yes & Plane \\
\hline $\mathrm{A}:(1 / 2,1 / 2,1 / 2)$ & $\mathrm{E}:(\mathrm{u}, \mathrm{v}, 1 / 2)$ & $\mathrm{Z}:(0,0,1 / 2)$ & no & Plane \\
\hline $\mathrm{M}:(1 / 2,1 / 2,0)$ & $\mathrm{F}:(\mathrm{u}, 1 / 2, \mathrm{w})$ & $\mathrm{R}:(0,1 / 2,1 / 2)$ & yes & Plane \\
\hline $\mathrm{M}:(1 / 2,1 / 2,0)$ & $\mathrm{F}:(\mathrm{u}, 1 / 2, \mathrm{w})$ & $\mathrm{X}:(0,1 / 2,0)$ & yes & Plane \\
\hline $\mathrm{R}:(0,1 / 2,1 / 2)$ & $\mathrm{F}:(\mathrm{u}, 1 / 2, \mathrm{w})$ & $\mathrm{X}:(0,1 / 2,0)$ & yes & Plane \\
\hline $\mathrm{R}:(0,1 / 2,1 / 2)$ & $\mathrm{E}:(\mathrm{u}, \mathrm{v}, 1 / 2)$ & $\mathrm{Z}:(0,0,1 / 2)$ & no & Plane \\
\hline
\end{tabular}

\begin{tabular}{c|c|c|c|c}
\hline \multicolumn{6}{c}{ BCSID: $1.215 ;$ Formula: UP2; MSG: $130.432\left(P_{c} 4 / n c c\right) ; \mathrm{U}=6 \mathrm{eV}$} \\
\hline maximal $k_{1}$ & intermediate path & maximal $k_{2}$ & satisfied? & Line/Plane \\
\hline$\Gamma:(0,0,0)$ & $\mathrm{LD}:(0,0, \mathrm{w})$ & $\mathrm{Z}:(0,0,1 / 2)$ & yes & Line \\
\hline $\mathrm{A}:(1 / 2,1 / 2,1 / 2)$ & $\mathrm{S}:(\mathrm{u}, \mathrm{u}, 1 / 2)$ & $\mathrm{Z}:(0,0,1 / 2)$ & no & Line \\
\hline $\mathrm{M}:(1 / 2,1 / 2,0)$ & $\mathrm{Y}:(\mathrm{u}, 1 / 2,0)$ & $\mathrm{X}:(0,1 / 2,0)$ & yes & Line \\
\hline $\mathrm{R}:(0,1 / 2,1 / 2)$ & $\mathrm{U}:(0, \mathrm{v}, 1 / 2)$ & $\mathrm{Z}:(0,0,1 / 2)$ & no & Line \\
\hline $\mathrm{A}:(1 / 2,1 / 2,1 / 2)$ & $\mathrm{F}:(\mathrm{u}, 1 / 2, \mathrm{w})$ & $\mathrm{M}:(1 / 2,1 / 2,0)$ & yes & Plane \\
\hline $\mathrm{A}:(1 / 2,1 / 2,1 / 2)$ & $\mathrm{E}:(\mathrm{u}, \mathrm{v}, 1 / 2)$ & $\mathrm{R}:(0,1 / 2,1 / 2)$ & yes & Plane \\
\hline $\mathrm{A}:(1 / 2,1 / 2,1 / 2)$ & $\mathrm{F}:(\mathrm{u}, 1 / 2, \mathrm{w})$ & $\mathrm{R}:(0,1 / 2,1 / 2)$ & yes & Plane \\
\hline $\mathrm{A}:(1 / 2,1 / 2,1 / 2)$ & $\mathrm{F}:(\mathrm{u}, 1 / 2, \mathrm{w})$ & $\mathrm{X}:(0,1 / 2,0)$ & yes & Plane \\
\hline $\mathrm{A}:(1 / 2,1 / 2,1 / 2)$ & $\mathrm{E}:(\mathrm{u}, \mathrm{v}, 1 / 2)$ & $\mathrm{Z}:(0,0,1 / 2)$ & no & Plane \\
\hline $\mathrm{M}:(1 / 2,1 / 2,0)$ & $\mathrm{F}:(\mathrm{u}, 1 / 2, \mathrm{w})$ & $\mathrm{R}:(0,1 / 2,1 / 2)$ & yes & Plane \\
\hline $\mathrm{M}:(1 / 2,1 / 2,0)$ & $\mathrm{F}:(\mathrm{u}, 1 / 2, \mathrm{w})$ & $\mathrm{X}:(0,1 / 2,0)$ & yes & Plane \\
\hline $\mathrm{R}:(0,1 / 2,1 / 2)$ & $\mathrm{F}:(\mathrm{u}, 1 / 2, \mathrm{w})$ & $\mathrm{X}:(0,1 / 2,0)$ & yes & Plane \\
\hline $\mathrm{R}:(0,1 / 2,1 / 2)$ & $\mathrm{E}:(\mathrm{u}, \mathrm{v}, 1 / 2)$ & $\mathrm{Z}:(0,0,1 / 2)$ & no & Plane \\
\hline
\end{tabular}

\begin{tabular}{c|c|c|c|c}
\hline \multicolumn{6}{c}{ BCSID: $2.20 ;$ Formula: UAs; MSG: $134.481\left(P_{C} 4_{2} / n n m\right) ; \mathrm{U}=2 \mathrm{eV}$} \\
\hline maximal $k_{1}$ & intermediate path & maximal $k_{2}$ & satisfied? & Line/Plane \\
\hline$\Gamma:(0,0,0)$ & $\mathrm{LD}:(0,0, \mathrm{w})$ & $\mathrm{Z}:(0,0,1 / 2)$ & yes & Line \\
\hline $\mathrm{M}:(1 / 2,1 / 2,0)$ & $\mathrm{Y}:(\mathrm{u}, 1 / 2,0)$ & $\mathrm{X}:(0,1 / 2,0)$ & no & Line \\
\hline $\mathrm{R}:(0,1 / 2,1 / 2)$ & $\mathrm{W}:(0,1 / 2, \mathrm{w})$ & $\mathrm{X}:(0,1 / 2,0)$ & no & Line \\
\hline $\mathrm{R}:(0,1 / 2,1 / 2)$ & $\mathrm{U}:(0, \mathrm{v}, 1 / 2)$ & $\mathrm{Z}:(0,0,1 / 2)$ & yes & Line \\
\hline $\mathrm{A}:(1 / 2,1 / 2,1 / 2)$ & $\mathrm{F}:(\mathrm{u}, 1 / 2, \mathrm{w})$ & $\mathrm{M}:(1 / 2,1 / 2,0)$ & yes & Plane \\
\hline $\mathrm{A}:(1 / 2,1 / 2,1 / 2)$ & $\mathrm{F}:(\mathrm{u}, 1 / 2, \mathrm{w})$ & $\mathrm{R}:(0,1 / 2,1 / 2)$ & yes & Plane \\
\hline $\mathrm{A}:(1 / 2,1 / 2,1 / 2)$ & $\mathrm{F}:(\mathrm{u}, 1 / 2, \mathrm{w})$ & $\mathrm{X}:(0,1 / 2,0)$ & no & Plane \\
\hline $\mathrm{M}:(1 / 2,1 / 2,0)$ & $\mathrm{F}:(\mathrm{u}, 1 / 2, \mathrm{w})$ & $\mathrm{R}:(0,1 / 2,1 / 2)$ & yes & Plane \\
\hline $\mathrm{M}:(1 / 2,1 / 2,0)$ & $\mathrm{F}:(\mathrm{u}, 1 / 2, \mathrm{w})$ & $\mathrm{X}:(0,1 / 2,0)$ & no & Plane \\
\hline $\mathrm{R}:(0,1 / 2,1 / 2)$ & $\mathrm{F}:(\mathrm{u}, 1 / 2, \mathrm{w})$ & $\mathrm{X}:(0,1 / 2,0)$ & no & Plane
\end{tabular}




\begin{tabular}{c|c|c|c|c}
\hline \multicolumn{6}{c}{ BCSID: $1.103 ;$ Formula: U2Rh2Sn; MSG: 135.492} & $\left(P_{c} 4_{2} / m b c\right) ; \mathrm{U}=0$ \\
\hline maximal $k_{1}$ & intermediate path & maximal $k_{2}$ & satisfied? & Line/Plane \\
\hline$\Gamma:(0,0,0)$ & $\mathrm{LD}:(0,0, \mathrm{w})$ & $\mathrm{Z}:(0,0,1 / 2)$ & no & Line \\
\hline $\mathrm{A}:(1 / 2,1 / 2,1 / 2)$ & $\mathrm{S}:(\mathrm{u}, \mathrm{u}, 1 / 2)$ & $\mathrm{Z}:(0,0,1 / 2)$ & yes & Line \\
\hline $\mathrm{R}:(0,1 / 2,1 / 2)$ & $\mathrm{W}:(0,1 / 2, \mathrm{w})$ & $\mathrm{X}:(0,1 / 2,0)$ & yes & Line \\
\hline $\mathrm{A}:(1 / 2,1 / 2,1 / 2)$ & $\mathrm{F}:(\mathrm{u}, 1 / 2, \mathrm{w})$ & $\mathrm{M}:(1 / 2,1 / 2,0)$ & yes & Plane \\
\hline $\mathrm{A}:(1 / 2,1 / 2,1 / 2)$ & $\mathrm{E}:(\mathrm{u}, \mathrm{v}, 1 / 2)$ & $\mathrm{R}:(0,1 / 2,1 / 2)$ & yes & Plane \\
\hline $\mathrm{A}:(1 / 2,1 / 2,1 / 2)$ & $\mathrm{F}:(\mathrm{u}, 1 / 2, \mathrm{w})$ & $\mathrm{R}:(0,1 / 2,1 / 2)$ & yes & Plane \\
\hline $\mathrm{A}:(1 / 2,1 / 2,1 / 2)$ & $\mathrm{F}:(\mathrm{u}, 1 / 2, \mathrm{w})$ & $\mathrm{X}:(0,1 / 2,0)$ & yes & Plane \\
\hline $\mathrm{A}:(1 / 2,1 / 2,1 / 2)$ & $\mathrm{E}:(\mathrm{u}, \mathrm{v}, 1 / 2)$ & $\mathrm{Z}:(0,0,1 / 2)$ & yes & Plane \\
\hline $\mathrm{M}:(1 / 2,1 / 2,0)$ & $\mathrm{F}:(\mathrm{u}, 1 / 2, \mathrm{w})$ & $\mathrm{R}:(0,1 / 2,1 / 2)$ & yes & Plane \\
\hline $\mathrm{M}:(1 / 2,1 / 2,0)$ & $\mathrm{F}:(\mathrm{u}, 1 / 2, \mathrm{w})$ & $\mathrm{X}:(0,1 / 2,0)$ & yes & Plane \\
\hline $\mathrm{R}:(0,1 / 2,1 / 2)$ & $\mathrm{F}:(\mathrm{u}, 1 / 2, \mathrm{w})$ & $\mathrm{X}:(0,1 / 2,0)$ & yes & Plane \\
\hline $\mathrm{R}:(0,1 / 2,1 / 2)$ & $\mathrm{E}:(\mathrm{u}, \mathrm{v}, 1 / 2)$ & $\mathrm{Z}:(0,0,1 / 2)$ & yes & Plane \\
\hline
\end{tabular}

\begin{tabular}{c|c|c|c|c}
\hline \multicolumn{5}{c}{ BCSID: 1.103; Formula: U2Rh2Sn; MSG: $135.492\left(P_{c} 4_{2} / m b c\right) ; \mathrm{U}=2 \mathrm{eV}$} \\
\hline maximal $k_{1}$ & intermediate path & maximal $k_{2}$ & satisfied? & Line/Plane \\
\hline$\Gamma:(0,0,0)$ & $\mathrm{LD}:(0,0, \mathrm{w})$ & $\mathrm{Z}:(0,0,1 / 2)$ & no & Line \\
\hline $\mathrm{A}:(1 / 2,1 / 2,1 / 2)$ & $\mathrm{S}:(\mathrm{u}, \mathrm{u}, 1 / 2)$ & $\mathrm{Z}:(0,0,1 / 2)$ & yes & Line \\
\hline $\mathrm{R}:(0,1 / 2,1 / 2)$ & $\mathrm{W}:(0,1 / 2, \mathrm{w})$ & $\mathrm{X}:(0,1 / 2,0)$ & yes & Line \\
\hline $\mathrm{A}:(1 / 2,1 / 2,1 / 2)$ & $\mathrm{F}:(\mathrm{u}, 1 / 2, \mathrm{w})$ & $\mathrm{M}:(1 / 2,1 / 2,0)$ & yes & Plane \\
\hline $\mathrm{A}:(1 / 2,1 / 2,1 / 2)$ & $\mathrm{E}:(\mathrm{u}, \mathrm{v}, 1 / 2)$ & $\mathrm{R}:(0,1 / 2,1 / 2)$ & yes & Plane \\
\hline $\mathrm{A}:(1 / 2,1 / 2,1 / 2)$ & $\mathrm{F}:(\mathrm{u}, 1 / 2, \mathrm{w})$ & $\mathrm{R}:(0,1 / 2,1 / 2)$ & yes & Plane \\
\hline $\mathrm{A}:(1 / 2,1 / 2,1 / 2)$ & $\mathrm{F}:(\mathrm{u}, 1 / 2, \mathrm{w})$ & $\mathrm{X}:(0,1 / 2,0)$ & yes & Plane \\
\hline $\mathrm{A}:(1 / 2,1 / 2,1 / 2)$ & $\mathrm{E}:(\mathrm{u}, \mathrm{v}, 1 / 2)$ & $\mathrm{Z}:(0,0,1 / 2)$ & yes & Plane \\
\hline $\mathrm{M}:(1 / 2,1 / 2,0)$ & $\mathrm{F}:(\mathrm{u}, 1 / 2, \mathrm{w})$ & $\mathrm{R}:(0,1 / 2,1 / 2)$ & yes & Plane \\
\hline $\mathrm{M}:(1 / 2,1 / 2,0)$ & $\mathrm{F}:(\mathrm{u}, 1 / 2, \mathrm{w})$ & $\mathrm{X}:(0,1 / 2,0)$ & yes & Plane \\
\hline $\mathrm{R}:(0,1 / 2,1 / 2)$ & $\mathrm{F}:(\mathrm{u}, 1 / 2, \mathrm{w})$ & $\mathrm{X}:(0,1 / 2,0)$ & yes & Plane \\
\hline $\mathrm{R}:(0,1 / 2,1 / 2)$ & $\mathrm{E}:(\mathrm{u}, \mathrm{v}, 1 / 2)$ & $\mathrm{Z}:(0,0,1 / 2)$ & yes & Plane \\
\hline
\end{tabular}

\begin{tabular}{c|c|c|c|c}
\hline \multicolumn{5}{c}{ BCSID: 1.103; Formula: U2Rh2Sn; MSG: $135.492\left(P_{c} 4_{2} / m b c\right) ; \mathrm{U}=4 \mathrm{eV}$} \\
\hline maximal $k_{1}$ & intermediate path & maximal $k_{2}$ & satisfied? & Line/Plane \\
\hline$\Gamma:(0,0,0)$ & $\mathrm{LD}:(0,0, \mathrm{w})$ & $\mathrm{Z}:(0,0,1 / 2)$ & no & Line \\
\hline $\mathrm{A}:(1 / 2,1 / 2,1 / 2)$ & $\mathrm{S}:(\mathrm{u}, \mathrm{u}, 1 / 2)$ & $\mathrm{Z}:(0,0,1 / 2)$ & yes & Line \\
\hline $\mathrm{R}:(0,1 / 2,1 / 2)$ & $\mathrm{W}:(0,1 / 2, \mathrm{w})$ & $\mathrm{X}:(0,1 / 2,0)$ & yes & Line \\
\hline $\mathrm{A}:(1 / 2,1 / 2,1 / 2)$ & $\mathrm{F}:(\mathrm{u}, 1 / 2, \mathrm{w})$ & $\mathrm{M}:(1 / 2,1 / 2,0)$ & yes & Plane \\
\hline $\mathrm{A}:(1 / 2,1 / 2,1 / 2)$ & $\mathrm{E}:(\mathrm{u}, \mathrm{v}, 1 / 2)$ & $\mathrm{R}:(0,1 / 2,1 / 2)$ & yes & Plane \\
\hline $\mathrm{A}:(1 / 2,1 / 2,1 / 2)$ & $\mathrm{F}:(\mathrm{u}, 1 / 2, \mathrm{w})$ & $\mathrm{R}:(0,1 / 2,1 / 2)$ & yes & Plane \\
\hline $\mathrm{A}:(1 / 2,1 / 2,1 / 2)$ & $\mathrm{F}:(\mathrm{u}, 1 / 2, \mathrm{w})$ & $\mathrm{X}:(0,1 / 2,0)$ & yes & Plane \\
\hline $\mathrm{A}:(1 / 2,1 / 2,1 / 2)$ & $\mathrm{E}:(\mathrm{u}, \mathrm{v}, 1 / 2)$ & $\mathrm{Z}:(0,0,1 / 2)$ & yes & Plane \\
\hline $\mathrm{M}:(1 / 2,1 / 2,0)$ & $\mathrm{F}:(\mathrm{u}, 1 / 2, \mathrm{w})$ & $\mathrm{R}:(0,1 / 2,1 / 2)$ & yes & Plane \\
\hline $\mathrm{M}:(1 / 2,1 / 2,0)$ & $\mathrm{F}:(\mathrm{u}, 1 / 2, \mathrm{w})$ & $\mathrm{X}:(0,1 / 2,0)$ & yes & Plane \\
\hline $\mathrm{R}:(0,1 / 2,1 / 2)$ & $\mathrm{F}:(\mathrm{u}, 1 / 2, \mathrm{w})$ & $\mathrm{X}:(0,1 / 2,0)$ & yes & Plane \\
\hline $\mathrm{R}:(0,1 / 2,1 / 2)$ & $\mathrm{E}:(\mathrm{u}, \mathrm{v}, 1 / 2)$ & $\mathrm{Z}:(0,0,1 / 2)$ & yes & Plane \\
\hline
\end{tabular}

\begin{tabular}{c|c|c|c|c}
\multicolumn{4}{c}{ BCSID: 1.103 ; Formula: U2Rh2Sn; MSG: $135.492\left(P_{c} 4_{2} / m b c\right) ; \mathrm{U}=6 \mathrm{eV}$} \\
\hline maximal $k_{1}$ & intermediate path & maximal $k_{2}$ & satisfied? & Line/Plane \\
\hline$\Gamma:(0,0,0)$ & $\mathrm{LD}:(0,0, \mathrm{w})$ & $\mathrm{Z}:(0,0,1 / 2)$ & no & Line \\
\hline $\mathrm{A}:(1 / 2,1 / 2,1 / 2)$ & $\mathrm{S}:(\mathrm{u}, \mathrm{u}, 1 / 2)$ & $\mathrm{Z}:(0,0,1 / 2)$ & yes & Line \\
\hline $\mathrm{R}:(0,1 / 2,1 / 2)$ & $\mathrm{W}:(0,1 / 2, \mathrm{w})$ & $\mathrm{X}:(0,1 / 2,0)$ & yes & Line
\end{tabular}




\begin{tabular}{c|c|c|c|c}
\hline $\mathrm{A}:(1 / 2,1 / 2,1 / 2)$ & $\mathrm{F}:(\mathrm{u}, 1 / 2, \mathrm{w})$ & $\mathrm{M}:(1 / 2,1 / 2,0)$ & yes & Plane \\
\hline $\mathrm{A}:(1 / 2,1 / 2,1 / 2)$ & $\mathrm{E}:(\mathrm{u}, \mathrm{v}, 1 / 2)$ & $\mathrm{R}:(0,1 / 2,1 / 2)$ & yes & Plane \\
\hline $\mathrm{A}:(1 / 2,1 / 2,1 / 2)$ & $\mathrm{F}:(\mathrm{u}, 1 / 2, \mathrm{w})$ & $\mathrm{R}:(0,1 / 2,1 / 2)$ & yes & Plane \\
\hline $\mathrm{A}:(1 / 2,1 / 2,1 / 2)$ & $\mathrm{F}:(\mathrm{u}, 1 / 2, \mathrm{w})$ & $\mathrm{X}:(0,1 / 2,0)$ & yes & Plane \\
\hline $\mathrm{A}:(1 / 2,1 / 2,1 / 2)$ & $\mathrm{E}:(\mathrm{u}, \mathrm{v}, 1 / 2)$ & $\mathrm{Z}:(0,0,1 / 2)$ & yes & Plane \\
\hline $\mathrm{M}:(1 / 2,1 / 2,0)$ & $\mathrm{F}:(\mathrm{u}, 1 / 2, \mathrm{w})$ & $\mathrm{R}:(0,1 / 2,1 / 2)$ & yes & Plane \\
\hline $\mathrm{M}:(1 / 2,1 / 2,0)$ & $\mathrm{F}:(\mathrm{u}, 1 / 2, \mathrm{w})$ & $\mathrm{X}:(0,1 / 2,0)$ & yes & Plane \\
\hline $\mathrm{R}:(0,1 / 2,1 / 2)$ & $\mathrm{F}:(\mathrm{u}, 1 / 2, \mathrm{w})$ & $\mathrm{X}:(0,1 / 2,0)$ & yes & Plane \\
\hline $\mathrm{R}:(0,1 / 2,1 / 2)$ & $\mathrm{E}:(\mathrm{u}, \mathrm{v}, 1 / 2)$ & $\mathrm{Z}:(0,0,1 / 2)$ & yes & Plane \\
\hline
\end{tabular}

BCSID: 1.207; Formula: U2Rh2Sn; MSG: $135.492\left(P_{c} 4_{2} / m b c\right) ; \mathrm{U}=0$

\begin{tabular}{c|c|c|c|c}
\hline maximal $k_{1}$ & intermediate path & maximal $k_{2}$ & satisfied? & Line/Plane \\
\hline$\Gamma:(0,0,0)$ & $\mathrm{LD}:(0,0, \mathrm{w})$ & $\mathrm{Z}:(0,0,1 / 2)$ & no & Line \\
\hline $\mathrm{A}:(1 / 2,1 / 2,1 / 2)$ & $\mathrm{S}:(\mathrm{u}, \mathrm{u}, 1 / 2)$ & $\mathrm{Z}:(0,0,1 / 2)$ & yes & Line \\
\hline $\mathrm{R}:(0,1 / 2,1 / 2)$ & $\mathrm{W}:(0,1 / 2, \mathrm{w})$ & $\mathrm{X}:(0,1 / 2,0)$ & yes & Line \\
\hline $\mathrm{A}:(1 / 2,1 / 2,1 / 2)$ & $\mathrm{F}:(\mathrm{u}, 1 / 2, \mathrm{w})$ & $\mathrm{M}:(1 / 2,1 / 2,0)$ & yes & Plane \\
\hline $\mathrm{A}:(1 / 2,1 / 2,1 / 2)$ & $\mathrm{E}:(\mathrm{u}, \mathrm{v}, 1 / 2)$ & $\mathrm{R}:(0,1 / 2,1 / 2)$ & yes & Plane \\
\hline $\mathrm{A}:(1 / 2,1 / 2,1 / 2)$ & $\mathrm{F}:(\mathrm{u}, 1 / 2, \mathrm{w})$ & $\mathrm{R}:(0,1 / 2,1 / 2)$ & yes & Plane \\
\hline $\mathrm{A}:(1 / 2,1 / 2,1 / 2)$ & $\mathrm{F}:(\mathrm{u}, 1 / 2, \mathrm{w})$ & $\mathrm{X}:(0,1 / 2,0)$ & yes & Plane \\
\hline $\mathrm{A}:(1 / 2,1 / 2,1 / 2)$ & $\mathrm{E}:(\mathrm{u}, \mathrm{v}, 1 / 2)$ & $\mathrm{Z}:(0,0,1 / 2)$ & yes & Plane \\
\hline $\mathrm{M}:(1 / 2,1 / 2,0)$ & $\mathrm{F}:(\mathrm{u}, 1 / 2, \mathrm{w})$ & $\mathrm{R}:(0,1 / 2,1 / 2)$ & yes & Plane \\
\hline $\mathrm{M}:(1 / 2,1 / 2,0)$ & $\mathrm{F}:(\mathrm{u}, 1 / 2, \mathrm{w})$ & $\mathrm{X}:(0,1 / 2,0)$ & yes & Plane \\
\hline $\mathrm{R}:(0,1 / 2,1 / 2)$ & $\mathrm{F}:(\mathrm{u}, 1 / 2, \mathrm{w})$ & $\mathrm{X}:(0,1 / 2,0)$ & yes & Plane \\
\hline $\mathrm{R}:(0,1 / 2,1 / 2)$ & $\mathrm{E}:(\mathrm{u}, \mathrm{v}, 1 / 2)$ & $\mathrm{Z}:(0,0,1 / 2)$ & yes & Plane \\
\hline
\end{tabular}

\begin{tabular}{c|c|c|c|c}
\multicolumn{5}{c}{ BCSID: 1.207; Formula: U2Rh2Sn; MSG: $135.492\left(P_{c} 4_{2} / m b c\right) ; \mathrm{U}=2 \mathrm{eV}$} \\
\hline maximal $k_{1}$ & intermediate path & maximal $k_{2}$ & satisfied? & Line/Plane \\
\hline$\Gamma:(0,0,0)$ & $\mathrm{LD}:(0,0, \mathrm{w})$ & $\mathrm{Z}:(0,0,1 / 2)$ & no & Line \\
\hline $\mathrm{A}:(1 / 2,1 / 2,1 / 2)$ & $\mathrm{S}:(\mathrm{u}, \mathrm{u}, 1 / 2)$ & $\mathrm{Z}:(0,0,1 / 2)$ & yes & Line \\
\hline $\mathrm{R}:(0,1 / 2,1 / 2)$ & $\mathrm{W}:(0,1 / 2, \mathrm{w})$ & $\mathrm{X}:(0,1 / 2,0)$ & yes & Line \\
\hline $\mathrm{A}:(1 / 2,1 / 2,1 / 2)$ & $\mathrm{F}:(\mathrm{u}, 1 / 2, \mathrm{w})$ & $\mathrm{M}:(1 / 2,1 / 2,0)$ & yes & Plane \\
\hline $\mathrm{A}:(1 / 2,1 / 2,1 / 2)$ & $\mathrm{E}:(\mathrm{u}, \mathrm{v}, 1 / 2)$ & $\mathrm{R}:(0,1 / 2,1 / 2)$ & yes & Plane \\
\hline $\mathrm{A}:(1 / 2,1 / 2,1 / 2)$ & $\mathrm{F}:(\mathrm{u}, 1 / 2, \mathrm{w})$ & $\mathrm{R}:(0,1 / 2,1 / 2)$ & yes & Plane \\
\hline $\mathrm{A}:(1 / 2,1 / 2,1 / 2)$ & $\mathrm{F}:(\mathrm{u}, 1 / 2, \mathrm{w})$ & $\mathrm{X}:(0,1 / 2,0)$ & yes & Plane \\
\hline $\mathrm{A}:(1 / 2,1 / 2,1 / 2)$ & $\mathrm{E}:(\mathrm{u}, \mathrm{v}, 1 / 2)$ & $\mathrm{Z}:(0,0,1 / 2)$ & yes & Plane \\
\hline $\mathrm{M}:(1 / 2,1 / 2,0)$ & $\mathrm{F}:(\mathrm{u}, 1 / 2, \mathrm{w})$ & $\mathrm{R}:(0,1 / 2,1 / 2)$ & yes & Plane \\
\hline $\mathrm{M}:(1 / 2,1 / 2,0)$ & $\mathrm{F}:(\mathrm{u}, 1 / 2, \mathrm{w})$ & $\mathrm{X}:(0,1 / 2,0)$ & yes & Plane \\
\hline $\mathrm{R}:(0,1 / 2,1 / 2)$ & $\mathrm{F}:(\mathrm{u}, 1 / 2, \mathrm{w})$ & $\mathrm{X}:(0,1 / 2,0)$ & yes & Plane \\
\hline $\mathrm{R}:(0,1 / 2,1 / 2)$ & $\mathrm{E}:(\mathrm{u}, \mathrm{v}, 1 / 2)$ & $\mathrm{Z}:(0,0,1 / 2)$ & yes & Plane \\
\hline
\end{tabular}

\begin{tabular}{c|c|c|c|c}
\hline \multicolumn{4}{c}{ BCSID: 1.207; Formula: U2Rh2Sn; MSG: $135.492\left(P_{c} 4_{2} / m b c\right) ; \mathrm{U}=4 \mathrm{eV}$} \\
\hline maximal $k_{1}$ & intermediate path & maximal $k_{2}$ & satisfied? & Line/Plane \\
\hline$\Gamma:(0,0,0)$ & $\mathrm{LD}:(0,0, \mathrm{w})$ & $\mathrm{Z}:(0,0,1 / 2)$ & no & Line \\
\hline $\mathrm{A}:(1 / 2,1 / 2,1 / 2)$ & $\mathrm{S}:(\mathrm{u}, \mathrm{u}, 1 / 2)$ & $\mathrm{Z}:(0,0,1 / 2)$ & yes & Line \\
\hline $\mathrm{R}:(0,1 / 2,1 / 2)$ & $\mathrm{W}:(0,1 / 2, \mathrm{w})$ & $\mathrm{X}:(0,1 / 2,0)$ & yes & Line \\
\hline $\mathrm{A}:(1 / 2,1 / 2,1 / 2)$ & $\mathrm{F}:(\mathrm{u}, 1 / 2, \mathrm{w})$ & $\mathrm{M}:(1 / 2,1 / 2,0)$ & yes & Plane \\
\hline $\mathrm{A}:(1 / 2,1 / 2,1 / 2)$ & $\mathrm{E}:(\mathrm{u}, \mathrm{v}, 1 / 2)$ & $\mathrm{R}:(0,1 / 2,1 / 2)$ & yes & Plane \\
\hline $\mathrm{A}:(1 / 2,1 / 2,1 / 2)$ & $\mathrm{F}:(\mathrm{u}, 1 / 2, \mathrm{w})$ & $\mathrm{R}:(0,1 / 2,1 / 2)$ & yes & Plane \\
\hline $\mathrm{A}:(1 / 2,1 / 2,1 / 2)$ & $\mathrm{F}:(\mathrm{u}, 1 / 2, \mathrm{w})$ & $\mathrm{X}:(0,1 / 2,0)$ & yes & Plane \\
\hline $\mathrm{A}:(1 / 2,1 / 2,1 / 2)$ & $\mathrm{E}:(\mathrm{u}, \mathrm{v}, 1 / 2)$ & $\mathrm{Z}:(0,0,1 / 2)$ & yes & Plane \\
\hline $\mathrm{M}:(1 / 2,1 / 2,0)$ & $\mathrm{F}:(\mathrm{u}, 1 / 2, \mathrm{w})$ & $\mathrm{R}:(0,1 / 2,1 / 2)$ & yes & Plane
\end{tabular}




\begin{tabular}{c|c|c|c|c}
\hline $\mathrm{M}:(1 / 2,1 / 2,0)$ & $\mathrm{F}:(\mathrm{u}, 1 / 2, \mathrm{w})$ & $\mathrm{X}:(0,1 / 2,0)$ & yes & Plane \\
\hline $\mathrm{R}:(0,1 / 2,1 / 2)$ & $\mathrm{F}:(\mathrm{u}, 1 / 2, \mathrm{w})$ & $\mathrm{X}:(0,1 / 2,0)$ & yes & Plane \\
\hline $\mathrm{R}:(0,1 / 2,1 / 2)$ & $\mathrm{E}:(\mathrm{u}, \mathrm{v}, 1 / 2)$ & $\mathrm{Z}:(0,0,1 / 2)$ & yes & Plane \\
\hline
\end{tabular}

\begin{tabular}{c|c|c|c|c}
\hline \multicolumn{5}{c}{ BCSID: 1.207; Formula: U2Rh2Sn; MSG: $135.492\left(P_{c} 4_{2} / m b c\right) ; \mathrm{U}=6 \mathrm{eV}$} \\
\hline maximal $k_{1}$ & intermediate path & maximal $k_{2}$ & satisfied? & Line/Plane \\
\hline$\Gamma:(0,0,0)$ & $\mathrm{LD}:(0,0, \mathrm{w})$ & $\mathrm{Z}:(0,0,1 / 2)$ & no & Line \\
\hline $\mathrm{A}:(1 / 2,1 / 2,1 / 2)$ & $\mathrm{S}:(\mathrm{u}, \mathrm{u}, 1 / 2)$ & $\mathrm{Z}:(0,0,1 / 2)$ & yes & Line \\
\hline $\mathrm{R}:(0,1 / 2,1 / 2)$ & $\mathrm{W}:(0,1 / 2, \mathrm{w})$ & $\mathrm{X}:(0,1 / 2,0)$ & yes & Line \\
\hline $\mathrm{A}:(1 / 2,1 / 2,1 / 2)$ & $\mathrm{F}:(\mathrm{u}, 1 / 2, \mathrm{w})$ & $\mathrm{M}:(1 / 2,1 / 2,0)$ & yes & Plane \\
\hline $\mathrm{A}:(1 / 2,1 / 2,1 / 2)$ & $\mathrm{E}:(\mathrm{u}, \mathrm{v}, 1 / 2)$ & $\mathrm{R}:(0,1 / 2,1 / 2)$ & yes & Plane \\
\hline $\mathrm{A}:(1 / 2,1 / 2,1 / 2)$ & $\mathrm{F}:(\mathrm{u}, 1 / 2, \mathrm{w})$ & $\mathrm{R}:(0,1 / 2,1 / 2)$ & yes & Plane \\
\hline $\mathrm{A}:(1 / 2,1 / 2,1 / 2)$ & $\mathrm{F}:(\mathrm{u}, 1 / 2, \mathrm{w})$ & $\mathrm{X}:(0,1 / 2,0)$ & yes & Plane \\
\hline $\mathrm{A}:(1 / 2,1 / 2,1 / 2)$ & $\mathrm{E}:(\mathrm{u}, \mathrm{v}, 1 / 2)$ & $\mathrm{Z}:(0,0,1 / 2)$ & yes & Plane \\
\hline $\mathrm{M}:(1 / 2,1 / 2,0)$ & $\mathrm{F}:(\mathrm{u}, 1 / 2, \mathrm{w})$ & $\mathrm{R}:(0,1 / 2,1 / 2)$ & yes & Plane \\
\hline $\mathrm{M}:(1 / 2,1 / 2,0)$ & $\mathrm{F}:(\mathrm{u}, 1 / 2, \mathrm{w})$ & $\mathrm{X}:(0,1 / 2,0)$ & yes & Plane \\
\hline $\mathrm{R}:(0,1 / 2,1 / 2)$ & $\mathrm{F}:(\mathrm{u}, 1 / 2, \mathrm{w})$ & $\mathrm{X}:(0,1 / 2,0)$ & yes & Plane \\
\hline $\mathrm{R}:(0,1 / 2,1 / 2)$ & $\mathrm{E}:(\mathrm{u}, \mathrm{v}, 1 / 2)$ & $\mathrm{Z}:(0,0,1 / 2)$ & yes & Plane \\
\hline
\end{tabular}

\begin{tabular}{|c|c|c|c|c|}
\hline \multicolumn{5}{|c|}{ BCSID: 2.19 ; Formula: Mn3ZnC; MSG: $139.537\left(I 4 / \mathrm{mm}^{\prime} \mathrm{m}^{\prime}\right) ; \mathrm{U}=0$} \\
\hline maximal $k_{1}$ & intermediate path & maximal $k_{2}$ & satisfied? & Line/Plane \\
\hline$\Gamma:(0,0,0)$ & $\mathrm{LD}:(0,0, \mathrm{w})$ & $\mathrm{M}:(0,0,1)$ & no & Line \\
\hline$\Gamma:(0,0,0)$ & SM:(u,0,0) & $\mathrm{M}:(1,0,0)$ & no & Line \\
\hline$\Gamma:(0,0,0)$ & DT: $(\mathrm{u}, \mathrm{u}, 0)$ & $\mathrm{X}:(1 / 2,1 / 2,0)$ & no & Line \\
\hline $\mathrm{M}:(1,0,0)$ & $\mathrm{Y}:(\mathrm{u}, 1-\mathrm{u}, 0)$ & $\mathrm{X}:(1 / 2,1 / 2,0)$ & no & Line \\
\hline $\mathrm{P}:(1 / 2,1 / 2,1 / 2)$ & $\mathrm{W}:(1 / 2,1 / 2, \mathrm{w})$ & $\mathrm{X}:(1 / 2,1 / 2,0)$ & yes & Line \\
\hline$\Gamma:(0,0,0)$ & $\mathrm{C}:(\mathrm{u}, \mathrm{v}, 0)$ & $\mathrm{M}:(0,1,0)$ & no & Plane \\
\hline$\Gamma:(0,0,0)$ & $\mathrm{C}:(\mathrm{u}, \mathrm{v}, 0)$ & $\mathrm{X}:(1 / 2,1 / 2,0)$ & no & Plane \\
\hline M: $(2,1,0)$ & $\mathrm{C}:(\mathrm{u}, \mathrm{v}, 0)$ & $\mathrm{X}:(3 / 2,3 / 2,0)$ & no & Plane \\
\hline \multicolumn{5}{|c|}{ BCSID: 2.19; Formula: Mn3ZnC; MSG: $139.537\left(I 4 / \mathrm{mm}^{\prime} \mathrm{m}^{\prime}\right) ; \mathrm{U}=2 \mathrm{eV}$} \\
\hline $\operatorname{maximal} k_{1}$ & intermediate path & maximal $k_{2}$ & satisfied? & Line/Plane \\
\hline$\Gamma:(0,0,0)$ & $\mathrm{LD}:(0,0, \mathrm{w})$ & $\mathrm{M}:(0,0,1)$ & no & Line \\
\hline$\Gamma:(0,0,0)$ & SM:(u,0,0) & $\mathrm{M}:(1,0,0)$ & no & Line \\
\hline$\Gamma:(0,0,0)$ & DT:(u,u,0) & $\mathrm{X}:(1 / 2,1 / 2,0)$ & no & Line \\
\hline $\mathrm{M}:(1,0,0)$ & $\mathrm{Y}:(\mathrm{u}, 1-\mathrm{u}, 0)$ & $\mathrm{X}:(1 / 2,1 / 2,0)$ & yes & Line \\
\hline $\mathrm{P}:(1 / 2,1 / 2,1 / 2)$ & $\mathrm{W}:(1 / 2,1 / 2, \mathrm{w})$ & $\mathrm{X}:(1 / 2,1 / 2,0)$ & yes & Line \\
\hline$\Gamma:(0,0,0)$ & $\mathrm{C}:(\mathrm{u}, \mathrm{v}, 0)$ & $\mathrm{M}:(0,1,0)$ & no & Plane \\
\hline$\Gamma:(0,0,0)$ & $\mathrm{C}:(\mathrm{u}, \mathrm{v}, 0)$ & $\mathrm{X}:(1 / 2,1 / 2,0)$ & no & Plane \\
\hline $\mathrm{M}:(2,1,0)$ & $\mathrm{C}:(\mathrm{u}, \mathrm{v}, 0)$ & $\mathrm{X}:(3 / 2,3 / 2,0)$ & yes & Plane \\
\hline
\end{tabular}

\begin{tabular}{c|c|c|c|c}
\hline \multicolumn{5}{c}{ BCSID: $2.19 ;$ Formula: Mn3ZnC; MSG: $139.537\left(I 4 / \mathrm{mm}^{\prime} \mathrm{m}^{\prime}\right) ; \mathrm{U}=3 \mathrm{eV}$} \\
\hline maximal $k_{1}$ & intermediate path & maximal $k_{2}$ & satisfied? & Line/Plane \\
\hline$\Gamma:(0,0,0)$ & $\mathrm{LD}:(0,0, \mathrm{w})$ & $\mathrm{M}:(0,0,1)$ & no & Line \\
\hline$\Gamma:(0,0,0)$ & $\mathrm{SM}:(\mathrm{u}, 0,0)$ & $\mathrm{M}:(1,0,0)$ & no & Line \\
\hline$\Gamma:(0,0,0)$ & $\mathrm{DT}:(\mathrm{u}, \mathrm{u}, 0)$ & $\mathrm{X}:(1 / 2,1 / 2,0)$ & no & Line \\
\hline $\mathrm{M}:(1,0,0)$ & $\mathrm{Y}:(\mathrm{u}, 1-\mathrm{u}, 0)$ & $\mathrm{X}:(1 / 2,1 / 2,0)$ & yes & Line \\
\hline $\mathrm{P}:(1 / 2,1 / 2,1 / 2)$ & $\mathrm{W}:(1 / 2,1 / 2, \mathrm{w})$ & $\mathrm{X}:(1 / 2,1 / 2,0)$ & yes & Line \\
\hline$\Gamma:(0,0,0)$ & $\mathrm{C}:(\mathrm{u}, \mathrm{v}, 0)$ & $\mathrm{M}:(0,1,0)$ & no & Plane \\
\hline$\Gamma:(0,0,0)$ & $\mathrm{C}:(\mathrm{u}, \mathrm{v}, 0)$ & $\mathrm{X}:(1 / 2,1 / 2,0)$ & no & Plane \\
\hline $\mathrm{M}:(2,1,0)$ & $\mathrm{C}:(\mathrm{u}, \mathrm{v}, 0)$ & $\mathrm{X}:(3 / 2,3 / 2,0)$ & yes & Plane
\end{tabular}




\begin{tabular}{|c|c|c|c|c|}
\hline \multicolumn{5}{|c|}{ BCSID: 2.19 ; Formula: Mn3ZnC; MSG: $139.537\left(I 4 / \mathrm{mm}^{\prime} \mathrm{m}^{\prime}\right) ; \mathrm{U}=4 \mathrm{eV}$} \\
\hline $\operatorname{maximal} k_{1}$ & intermediate path & $\operatorname{maximal} k_{2}$ & satisfied? & Line/Plane \\
\hline$\Gamma:(0,0,0)$ & LD: $(0,0, w)$ & $\mathrm{M}:(0,0,1)$ & no & Line \\
\hline$\Gamma:(0,0,0)$ & SM:(u,0,0) & $\mathrm{M}:(1,0,0)$ & no & Line \\
\hline$\Gamma:(0,0,0)$ & DT:(u,u,0) & $\mathrm{X}:(1 / 2,1 / 2,0)$ & no & Line \\
\hline $\mathrm{M}:(1,0,0)$ & $\mathrm{Y}:(\mathrm{u}, 1-\mathrm{u}, 0)$ & $\mathrm{X}:(1 / 2,1 / 2,0)$ & yes & Line \\
\hline $\mathrm{P}:(1 / 2,1 / 2,1 / 2)$ & $\mathrm{W}:(1 / 2,1 / 2, \mathrm{w})$ & $\mathrm{X}:(1 / 2,1 / 2,0)$ & yes & Line \\
\hline$\Gamma:(0,0,0)$ & $\mathrm{C}:(\mathrm{u}, \mathrm{v}, 0)$ & $\mathrm{M}:(0,1,0)$ & no & Plane \\
\hline$\Gamma:(0,0,0)$ & $\mathrm{C}:(\mathrm{u}, \mathrm{v}, 0)$ & $\mathrm{X}:(1 / 2,1 / 2,0)$ & no & Plane \\
\hline $\mathrm{M}:(2,1,0)$ & $\mathrm{C}:(\mathrm{u}, \mathrm{v}, 0)$ & $\mathrm{X}:(3 / 2,3 / 2,0)$ & yes & Plane \\
\hline \multicolumn{5}{|c|}{ BCSID: 1.80; Formula: DyCo2Ga8; MSG: $140.550\left(I_{c} 4 / m c m\right) ; \mathrm{U}=2 \mathrm{eV}$} \\
\hline maximal $k_{1}$ & intermediate path & maximal $k_{2}$ & satisfied? & Line/Plane \\
\hline$\Gamma:(0,0,0)$ & LD: $(0,0, \mathrm{w})$ & $\mathrm{M}:(0,0,1)$ & no & Line \\
\hline \multicolumn{5}{|c|}{ BCSID: 1.80; Formula: DyCo2Ga8; MSG: $140.550\left(I_{c} 4 / \mathrm{mcm}\right) ; \mathrm{U}=4 \mathrm{eV}$} \\
\hline maximal $k_{1}$ & intermediate path & maximal $k_{2}$ & satisfied? & Line/Plane \\
\hline$\Gamma:(0,0,0)$ & LD: $(0,0, \mathrm{w})$ & $\mathrm{M}:(0,0,1)$ & no & Line \\
\hline \multicolumn{5}{|c|}{ BCSID: 1.80; Formula: DyCo2Ga8; MSG: $140.550\left(I_{c} 4 / m c m\right) ; \mathrm{U}=6 \mathrm{eV}$} \\
\hline maximal $k_{1}$ & intermediate path & maximal $k_{2}$ & satisfied? & Line/Plane \\
\hline$\Gamma:(0,0,0)$ & LD: $(0,0, \mathrm{w})$ & $\mathrm{M}:(0,0,1)$ & no & Line \\
\hline \multicolumn{5}{|c|}{ BCSID: 1.82 ; Formula: Nd2RhIn8; MSG: $140.550\left(I_{c} 4 / m c m\right) ; \mathrm{U}=4 \mathrm{eV}$} \\
\hline maximal $k_{1}$ & intermediate path & maximal $k_{2}$ & satisfied? & Line/Plane \\
\hline$\Gamma:(0,0,0)$ & $\mathrm{LD}:(0,0, \mathrm{w})$ & $\mathrm{M}:(0,0,1)$ & no & Line \\
\hline \multicolumn{5}{|c|}{ BCSID: 1.82; Formula: Nd2RhIn8; MSG: $140.550\left(I_{c} 4 / \mathrm{mcm}\right) ; \mathrm{U}=6 \mathrm{eV}$} \\
\hline maximal $k_{1}$ & intermediate path & maximal $k_{2}$ & satisfied? & Line/Plane \\
\hline$\Gamma:(0,0,0)$ & LD: $(0,0, w)$ & $\mathrm{M}:(0,0,1)$ & no & Line \\
\hline \multicolumn{5}{|c|}{ BCSID: 1.87; Formula: TbCo2Ga8; MSG: $140.550\left(I_{c} 4 / m c m\right) ; \mathrm{U}=2 \mathrm{eV}$} \\
\hline maximal $k_{1}$ & intermediate path & maximal $k_{2}$ & satisfied? & Line/Plane \\
\hline$\Gamma:(0,0,0)$ & $\mathrm{LD}:(0,0, \mathrm{w})$ & $\mathrm{M}:(0,0,1)$ & no & Line \\
\hline \multicolumn{5}{|c|}{ BCSID: 1.87; Formula: TbCo2Ga8; MSG: $140.550\left(I_{c} 4 / m c m\right) ; \mathrm{U}=4 \mathrm{eV}$} \\
\hline maximal $k_{1}$ & intermediate path & maximal $k_{2}$ & satisfied? & Line/Plane \\
\hline$\Gamma:(0,0,0)$ & LD: $(0,0, w)$ & $\mathrm{M}:(0,0,1)$ & no & Line \\
\hline \multicolumn{5}{|c|}{ BCSID: 0.151; Formula: Tm2Mn2O7; MSG: $141.557\left(I 4_{1} / a m^{\prime} d^{\prime}\right) ; \mathrm{U}=0$} \\
\hline maximal $k_{1}$ & intermediate path & maximal $k_{2}$ & satisfied? & Line/Plane \\
\hline$\Gamma:(0,0,0)$ & $\mathrm{LD}:(0,0, \mathrm{w})$ & $\mathrm{M}:(0,0,1)$ & no & Line \\
\hline$\Gamma:(0,0,0)$ & SM: $(u, 0,0)$ & $\mathrm{M}:(1,0,0)$ & no & Line \\
\hline$\Gamma:(0,0,0)$ & DT: $(\mathrm{u}, \mathrm{u}, 0)$ & $\mathrm{X}:(1 / 2,1 / 2,0)$ & no & Line \\
\hline $\mathrm{P}:(1 / 2,1 / 2,1 / 2)$ & $\mathrm{W}:(1 / 2,1 / 2, \mathrm{w})$ & $\mathrm{X}:(1 / 2,1 / 2,0)$ & yes & Line \\
\hline$\Gamma:(0,0,0)$ & $\mathrm{C}:(\mathrm{u}, \mathrm{v}, 0)$ & $\mathrm{M}:(0,1,0)$ & no & Plane \\
\hline$\Gamma:(0,0,0)$ & $\mathrm{C}:(\mathrm{u}, \mathrm{v}, 0)$ & $\mathrm{X}:(1 / 2,1 / 2,0)$ & no & Plane \\
\hline $\mathrm{M}:(2,1,0)$ & $\mathrm{C}:(\mathrm{u}, \mathrm{v}, 0)$ & $\mathrm{X}:(3 / 2,3 / 2,0)$ & yes & Plane \\
\hline
\end{tabular}

BCSID: 0.158; Formula: Yb2Ti2O7; MSG: $141.557\left(I_{1} / a m^{\prime} d^{\prime}\right) ; \mathrm{U}=1 \mathrm{eV}$ 


\begin{tabular}{c|c|c|c|c}
\hline maximal $k_{1}$ & intermediate path & maximal $k_{2}$ & satisfied? & Line/Plane \\
\hline$\Gamma:(0,0,0)$ & $\mathrm{LD}:(0,0, \mathrm{w})$ & $\mathrm{M}:(0,0,1)$ & no & Line \\
\hline$\Gamma:(0,0,0)$ & $\mathrm{SM}:(\mathrm{u}, 0,0)$ & $\mathrm{M}:(1,0,0)$ & yes & Line \\
\hline$\Gamma:(0,0,0)$ & $\mathrm{DT}:(\mathrm{u}, \mathrm{u}, 0)$ & $\mathrm{X}:(1 / 2,1 / 2,0)$ & yes & Line \\
\hline $\mathrm{P}:(1 / 2,1 / 2,1 / 2)$ & $\mathrm{W}:(1 / 2,1 / 2, \mathrm{w})$ & $\mathrm{X}:(1 / 2,1 / 2,0)$ & yes & Line \\
\hline$\Gamma:(0,0,0)$ & $\mathrm{C}:(\mathrm{u}, \mathrm{v}, 0)$ & $\mathrm{M}:(0,1,0)$ & yes & Plane \\
\hline$\Gamma:(0,0,0)$ & $\mathrm{C}:(\mathrm{u}, \mathrm{v}, 0)$ & $\mathrm{X}:(1 / 2,1 / 2,0)$ & yes & Plane \\
\hline $\mathrm{M}:(2,1,0)$ & $\mathrm{C}:(\mathrm{u}, \mathrm{v}, 0)$ & $\mathrm{X}:(3 / 2,3 / 2,0)$ & yes & Plane \\
\hline
\end{tabular}

BCSID: 0.227; Formula: NdCo2; MSG: $141.557\left(I 4_{1} / a m^{\prime} d^{\prime}\right) ; \mathrm{U}=0$

\begin{tabular}{c|c|c|c|c}
\hline maximal $k_{1}$ & intermediate path & maximal $k_{2}$ & satisfied? & Line/Plane \\
\hline$\Gamma:(0,0,0)$ & $\mathrm{LD}:(0,0, \mathrm{w})$ & $\mathrm{M}:(0,0,1)$ & no & Line \\
\hline$\Gamma:(0,0,0)$ & $\mathrm{SM}:(\mathrm{u}, 0,0)$ & $\mathrm{M}:(1,0,0)$ & yes & Line \\
\hline$\Gamma:(0,0,0)$ & $\mathrm{DT}:(\mathrm{u}, \mathrm{u}, 0)$ & $\mathrm{X}:(1 / 2,1 / 2,0)$ & yes & Line \\
\hline $\mathrm{P}:(1 / 2,1 / 2,1 / 2)$ & $\mathrm{W}:(1 / 2,1 / 2, \mathrm{w})$ & $\mathrm{X}:(1 / 2,1 / 2,0)$ & yes & Line \\
\hline$\Gamma:(0,0,0)$ & $\mathrm{C}:(\mathrm{u}, \mathrm{v}, 0)$ & $\mathrm{M}:(0,1,0)$ & yes & Plane \\
\hline$\Gamma:(0,0,0)$ & $\mathrm{C}:(\mathrm{u}, \mathrm{v}, 0)$ & $\mathrm{X}:(1 / 2,1 / 2,0)$ & yes & Plane \\
\hline $\mathrm{M}:(2,1,0)$ & $\mathrm{C}:(\mathrm{u}, \mathrm{v}, 0)$ & $\mathrm{X}:(3 / 2,3 / 2,0)$ & yes & Plane \\
\hline
\end{tabular}

BCSID: 0.227; Formula: NdCo2; MSG: $141.557\left(I 4_{1} / a m^{\prime} d^{\prime}\right) ; \mathrm{U}=2 \mathrm{eV}$

\begin{tabular}{c|c|c|c|c}
\hline maximal $k_{1}$ & intermediate path & maximal $k_{2}$ & satisfied? & Line/Plane \\
\hline$\Gamma:(0,0,0)$ & $\mathrm{LD}:(0,0, \mathrm{w})$ & $\mathrm{M}:(0,0,1)$ & no & Line \\
\hline$\Gamma:(0,0,0)$ & $\mathrm{SM}:(\mathrm{u}, 0,0)$ & $\mathrm{M}:(1,0,0)$ & yes & Line \\
\hline$\Gamma:(0,0,0)$ & $\mathrm{DT}:(\mathrm{u}, \mathrm{u}, 0)$ & $\mathrm{X}:(1 / 2,1 / 2,0)$ & yes & Line \\
\hline $\mathrm{P}:(1 / 2,1 / 2,1 / 2)$ & $\mathrm{W}:(1 / 2,1 / 2, \mathrm{w})$ & $\mathrm{X}:(1 / 2,1 / 2,0)$ & yes & Line \\
\hline$\Gamma:(0,0,0)$ & $\mathrm{C}:(\mathrm{u}, \mathrm{v}, 0)$ & $\mathrm{M}:(0,1,0)$ & yes & Plane \\
\hline$\Gamma:(0,0,0)$ & $\mathrm{C}:(\mathrm{u}, \mathrm{v}, 0)$ & $\mathrm{X}:(1 / 2,1 / 2,0)$ & yes & Plane \\
\hline $\mathrm{M}:(2,1,0)$ & $\mathrm{C}:(\mathrm{u}, \mathrm{v}, 0)$ & $\mathrm{X}:(3 / 2,3 / 2,0)$ & yes & Plane \\
\hline
\end{tabular}

\begin{tabular}{c|c|c|c|c}
\hline \multicolumn{5}{c}{ BCSID: 0.227; Formula: NdCo2; MSG: $141.557\left(I 4_{1} / \mathrm{am}^{\prime} d^{\prime}\right) ; \mathrm{U}=4 \mathrm{eV}$} \\
\hline maximal $k_{1}$ & intermediate path & maximal $k_{2}$ & satisfied? & Line $/$ Plane \\
\hline$\Gamma:(0,0,0)$ & $\mathrm{LD}:(0,0, \mathrm{w})$ & $\mathrm{M}:(0,0,1)$ & no & Line \\
\hline$\Gamma:(0,0,0)$ & $\mathrm{SM}:(\mathrm{u}, 0,0)$ & $\mathrm{M}:(1,0,0)$ & yes & Line \\
\hline$\Gamma:(0,0,0)$ & $\mathrm{DT}:(\mathrm{u}, \mathrm{u}, 0)$ & $\mathrm{X}:(1 / 2,1 / 2,0)$ & yes & Line \\
\hline $\mathrm{P}:(1 / 2,1 / 2,1 / 2)$ & $\mathrm{W}:(1 / 2,1 / 2, \mathrm{w})$ & $\mathrm{X}:(1 / 2,1 / 2,0)$ & yes & Line \\
\hline$\Gamma:(0,0,0)$ & $\mathrm{C}:(\mathrm{u}, \mathrm{v}, 0)$ & $\mathrm{M}:(0,1,0)$ & yes & Plane \\
\hline$\Gamma:(0,0,0)$ & $\mathrm{C}:(\mathrm{u}, \mathrm{v}, 0)$ & $\mathrm{X}:(1 / 2,1 / 2,0)$ & yes & Plane \\
\hline $\mathrm{M}:(2,1,0)$ & $\mathrm{C}:(\mathrm{u}, \mathrm{v}, 0)$ & $\mathrm{X}:(3 / 2,3 / 2,0)$ & yes & Plane \\
\hline
\end{tabular}

\begin{tabular}{c|c|c|c|c}
\hline \multicolumn{5}{c}{ BCSID: 0.227 Formula: NdCo2; MSG: $141.557\left(I 4_{1} / \mathrm{am}^{\prime} d^{\prime}\right) ; \mathrm{U}=6 \mathrm{eV}$} \\
\hline maximal $k_{1}$ & intermediate path & maximal $k_{2}$ & satisfied? & Line/Plane \\
\hline$\Gamma:(0,0,0)$ & $\mathrm{LD}:(0,0, \mathrm{w})$ & $\mathrm{M}:(0,0,1)$ & no & Line \\
\hline$\Gamma:(0,0,0)$ & $\mathrm{SM}:(\mathrm{u}, 0,0)$ & $\mathrm{M}:(1,0,0)$ & no & Line \\
\hline$\Gamma:(0,0,0)$ & $\mathrm{DT}:(\mathrm{u}, \mathrm{u}, 0)$ & $\mathrm{X}:(1 / 2,1 / 2,0)$ & no & Line \\
\hline $\mathrm{P}:(1 / 2,1 / 2,1 / 2)$ & $\mathrm{W}:(1 / 2,1 / 2, \mathrm{w})$ & $\mathrm{X}:(1 / 2,1 / 2,0)$ & yes & Line \\
\hline$\Gamma:(0,0,0)$ & $\mathrm{C}:(\mathrm{u}, \mathrm{v}, 0)$ & $\mathrm{M}:(0,1,0)$ & no & Plane \\
\hline$\Gamma:(0,0,0)$ & $\mathrm{C}:(\mathrm{u}, \mathrm{v}, 0)$ & $\mathrm{X}:(1 / 2,1 / 2,0)$ & no & Plane \\
\hline $\mathrm{M}:(2,1,0)$ & $\mathrm{C}:(\mathrm{u}, \mathrm{v}, 0)$ & $\mathrm{X}:(3 / 2,3 / 2,0)$ & yes & Plane \\
\hline
\end{tabular}

BCSID: 0.48; Formula: Tb2Sn2O7; MSG: $141.557\left(I 4_{1} / a m^{\prime} d^{\prime}\right) ; \mathrm{U}=0$ 


\begin{tabular}{c|c|c|c|c}
\hline maximal $k_{1}$ & intermediate path & maximal $k_{2}$ & satisfied? & Line/Plane \\
\hline$\Gamma:(0,0,0)$ & $\mathrm{LD}:(0,0, \mathrm{w})$ & $\mathrm{M}:(0,0,1)$ & yes & Line \\
\hline$\Gamma:(0,0,0)$ & $\mathrm{SM}:(\mathrm{u}, 0,0)$ & $\mathrm{M}:(1,0,0)$ & yes & Line \\
\hline$\Gamma:(0,0,0)$ & $\mathrm{DT}:(\mathrm{u}, \mathrm{u}, 0)$ & $\mathrm{X}:(1 / 2,1 / 2,0)$ & yes & Line \\
\hline $\mathrm{P}:(1 / 2,1 / 2,1 / 2)$ & $\mathrm{W}:(1 / 2,1 / 2, \mathrm{w})$ & $\mathrm{X}:(1 / 2,1 / 2,0)$ & no & Line \\
\hline$\Gamma:(0,0,0)$ & $\mathrm{C}:(\mathrm{u}, \mathrm{v}, 0)$ & $\mathrm{M}:(0,1,0)$ & yes & Plane \\
\hline$\Gamma:(0,0,0)$ & $\mathrm{C}:(\mathrm{u}, \mathrm{v}, 0)$ & $\mathrm{X}:(1 / 2,1 / 2,0)$ & yes & Plane \\
\hline $\mathrm{M}:(2,1,0)$ & $\mathrm{C}:(\mathrm{u}, \mathrm{v}, 0)$ & $\mathrm{X}:(3 / 2,3 / 2,0)$ & yes & Plane \\
\hline
\end{tabular}

BCSID: 0.49; Formula: Ho2Ru2O7; MSG: $141.557\left(I 4_{1} / a m^{\prime} d^{\prime}\right)$; U=0

\begin{tabular}{|c|c|c|c|c|}
\hline maximal $k_{1}$ & intermediate path & maximal $k_{2}$ & satisfied? & Line/Plane \\
\hline$\Gamma:(0,0,0)$ & $\mathrm{LD}:(0,0, \mathrm{w})$ & $\mathrm{M}:(0,0,1)$ & no & Line \\
\hline$\Gamma:(0,0,0)$ & SM:(u,0,0) & $\mathrm{M}:(1,0,0)$ & no & Line \\
\hline$\Gamma:(0,0,0)$ & DT: $(u, u, 0)$ & $\mathrm{X}:(1 / 2,1 / 2,0)$ & no & Line \\
\hline $\mathrm{P}:(1 / 2,1 / 2,1 / 2)$ & $\mathrm{W}:(1 / 2,1 / 2, \mathrm{w})$ & $\mathrm{X}:(1 / 2,1 / 2,0)$ & yes & Line \\
\hline$\Gamma:(0,0,0)$ & $\mathrm{C}:(\mathrm{u}, \mathrm{v}, 0)$ & $\mathrm{M}:(0,1,0)$ & no & Plane \\
\hline$\Gamma:(0,0,0)$ & $\mathrm{C}:(\mathrm{u}, \mathrm{v}, 0)$ & $\mathrm{X}:(1 / 2,1 / 2,0)$ & no & Plane \\
\hline $\mathrm{M}:(2,1,0)$ & $\mathrm{C}:(\mathrm{u}, \mathrm{v}, 0)$ & $\mathrm{X}:(3 / 2,3 / 2,0)$ & yes & Plane \\
\hline \multicolumn{5}{|c|}{ BCSID: 0.51; Formula: Ho2Ru2O7; MSG: $141.557\left(I 4_{1} / a m^{\prime} d^{\prime}\right)$; U=6eV } \\
\hline maximal $k_{1}$ & intermediate path & maximal $k_{2}$ & satisfied? & Line/Plane \\
\hline$\Gamma:(0,0,0)$ & $\mathrm{LD}:(0,0, \mathrm{w})$ & $\mathrm{M}:(0,0,1)$ & no & Line \\
\hline$\Gamma:(0,0,0)$ & SM:(u,0,0) & $\mathrm{M}:(1,0,0)$ & no & Line \\
\hline$\Gamma:(0,0,0)$ & $\mathrm{DT}:(\mathrm{u}, \mathrm{u}, 0)$ & $\mathrm{X}:(1 / 2,1 / 2,0)$ & no & Line \\
\hline $\mathrm{P}:(1 / 2,1 / 2,1 / 2)$ & $\mathrm{W}:(1 / 2,1 / 2, \mathrm{w})$ & $\mathrm{X}:(1 / 2,1 / 2,0)$ & yes & Line \\
\hline$\Gamma:(0,0,0)$ & $\mathrm{C}:(\mathrm{u}, \mathrm{v}, 0)$ & $\mathrm{M}:(0,1,0)$ & no & Plane \\
\hline$\Gamma:(0,0,0)$ & $\mathrm{C}:(\mathrm{u}, \mathrm{v}, 0)$ & $\mathrm{X}:(1 / 2,1 / 2,0)$ & no & Plane \\
\hline $\mathrm{M}:(2,1,0)$ & $\mathrm{C}:(\mathrm{u}, \mathrm{v}, 0)$ & $\mathrm{X}:(3 / 2,3 / 2,0)$ & yes & Plane \\
\hline \multicolumn{5}{|c|}{ BCSID: 0.125; Formula: MnGeO3; MSG: $148.19\left(R-3^{\prime}\right)$; U=3eV } \\
\hline maximal $k_{1}$ & intermediate path & maximal $k_{2}$ & satisfied? & Line/Plane \\
\hline$\Gamma:(0,0,0)$ & LD: $(0,0, w)$ & $\mathrm{T}:(0,0,3 / 2)$ & no & Line \\
\hline
\end{tabular}

BCSID: 0.125; Formula: MnGeO3; MSG: $148.19\left(R-3^{\prime}\right)$; $\mathrm{U}=4 \mathrm{eV}$ \begin{tabular}{l|l|l|l|l} 
maximal $k_{1}$ & intermediate path & maximal $k_{2}$ & satisfied? & Line/Plane
\end{tabular}

\begin{tabular}{c|c|c|c|c}
$\Gamma:(0,0,0)$ & LD: $(0,0, \mathrm{w})$ & $\mathrm{T}:(0,0,3 / 2)$ & no & Line
\end{tabular}

BCSID: 1.161; Formula: PrFe3(BO3)4; MSG: $155.48\left(R_{I} 32\right) ; \mathrm{U}=4 \mathrm{eV}$

\begin{tabular}{c|c|c|c|c}
\hline maximal $k_{1}$ & intermediate path & maximal $k_{2}$ & satisfied? & Line/Plane \\
\hline$\Gamma:(0,0,0)$ & SM: $\left(\mathrm{u},-2^{*} \mathrm{u}, 0\right)$ & $\mathrm{F}:(-1 / 2,1,0)$ & yes & Line \\
\hline$\Gamma:(0,0,0)$ & $\mathrm{LD}:(0,0, \mathrm{w})$ & $\mathrm{T}:(0,0,3 / 2)$ & no & Line \\
\hline $\mathrm{L}:(1 / 2,1 / 2,3 / 2)$ & $\mathrm{Y}:(\mathrm{u}, \mathrm{u}, 3 / 2)$ & $\mathrm{T}:(0,0,3 / 2)$ & yes & Line \\
\hline
\end{tabular}

BCSID: 0.169; Formula: U3As4; MSG: $161.71\left(R 3 c^{\prime}\right)$; U=0

\begin{tabular}{c|c|c|c|c}
\hline maximal $k_{1}$ & intermediate path & maximal $k_{2}$ & satisfied? & Line/Plane \\
\hline$\Gamma:(0,0,0)$ & LD: $(0,0, \mathrm{w})$ & $\mathrm{T}:(0,0,3 / 2)$ & no & Line \\
\hline
\end{tabular}

BCSID: 0.169; Formula: U3As4; MSG: $161.71\left(R 3 c^{\prime}\right)$; U=2eV

\begin{tabular}{c|c|c|c|c} 
maximal $k_{1}$ & intermediate path & maximal $k_{2}$ & satisfied? & Line/Plane \\
\hline$\Gamma:(0,0,0)$ & LD: $(0,0, \mathrm{w})$ & $\mathrm{T}:(0,0,3 / 2)$ & no & Line \\
\hline
\end{tabular}




\begin{tabular}{|c|c|c|c|c|}
\hline \multicolumn{5}{|c|}{ BCSID: 0.170; Formula: U3P4; MSG: $161.71\left(R 3 c^{\prime}\right) ; \mathrm{U}=0$} \\
\hline maximal $k_{1}$ & intermediate path & maximal $k_{2}$ & satisfied? & Line/Plane \\
\hline$\Gamma:(0,0,0)$ & LD: $(0,0, \mathrm{w})$ & $\mathrm{T}:(0,0,3 / 2)$ & no & Line \\
\hline \multicolumn{5}{|c|}{ BCSID: 0.170; Formula: U3P4; MSG: $161.71\left(R 3 c^{\prime}\right) ; \mathrm{U}=2 \mathrm{eV}$} \\
\hline maximal $k_{1}$ & intermediate path & maximal $k_{2}$ & satisfied? & Line/Plane \\
\hline$\Gamma:(0,0,0)$ & $\mathrm{LD}:(0,0, \mathrm{w})$ & $\mathrm{T}:(0,0,3 / 2)$ & no & Line \\
\hline \multicolumn{5}{|c|}{ BCSID: 1.0 .5 ; Formula: Sr3CoIrO6; MSG: $165.95\left(P-3 c^{\prime} 1\right) ; \mathrm{U}=0$} \\
\hline maximal $k_{1}$ & intermediate path & maximal $k_{2}$ & satisfied? & Line/Plane \\
\hline$\Gamma:(0,0,0)$ & $\mathrm{DT}:(0,0, \mathrm{w})$ & $\mathrm{A}:(0,0,1 / 2)$ & no & Line \\
\hline $\mathrm{H}:(1 / 3,1 / 3,1 / 2)$ & $\mathrm{P}:(1 / 3,1 / 3, \mathrm{w})$ & $\mathrm{K}:(1 / 3,1 / 3,0)$ & yes & Line \\
\hline \multicolumn{5}{|c|}{ BCSID: 0.177; Formula: Mn3GaN; MSG: $166.97(R-3 m) ; \mathrm{U}=0$} \\
\hline maximal $k_{1}$ & intermediate path & maximal $k_{2}$ & satisfied? & Line/Plane \\
\hline$\Gamma:(0,0,0)$ & SM:(u,-2*u,0) & $\mathrm{F}:(-1 / 2,1,0)$ & no & Line \\
\hline$\Gamma:(0,0,0)$ & $\mathrm{LD}:(0,0, \mathrm{w})$ & $\mathrm{T}:(0,0,3 / 2)$ & yes & Line \\
\hline $\mathrm{L}:(1 / 2,1 / 2,3 / 2)$ & $\mathrm{Y}:(\mathrm{u}, \mathrm{u}, 3 / 2)$ & $\mathrm{T}:(0,0,3 / 2)$ & yes & Line \\
\hline$\Gamma:(0,0,0)$ & $\mathrm{C}:(\mathrm{u},-\mathrm{u}, \mathrm{w})$ & $\mathrm{F}:(1 / 2,-1 / 2,1)$ & no & Plane \\
\hline$\Gamma:(0,0,0)$ & $\mathrm{C}:(\mathrm{u},-\mathrm{u}, \mathrm{w})$ & $\mathrm{L}:(-1 / 2,1 / 2,1 / 2)$ & yes & Plane \\
\hline$\Gamma:(0,0,0)$ & $\mathrm{C}:(\mathrm{u},-\mathrm{u}, \mathrm{w})$ & $\mathrm{T}:(0,0,3 / 2)$ & yes & Plane \\
\hline $\mathrm{F}:(0,1 / 2,1)$ & $\mathrm{C}:(0, \mathrm{u}, \mathrm{w})$ & $\mathrm{L}:(0,-1 / 2,1 / 2)$ & no & Plane \\
\hline $\mathrm{F}:(0,1 / 2,1)$ & $\mathrm{C}:(0, \mathrm{u}, \mathrm{w})$ & $\mathrm{T}:(0,1,1 / 2)$ & no & Plane \\
\hline $\mathrm{L}:(-1 / 2,1 / 2,1 / 2)$ & $\mathrm{C}:(\mathrm{u},-\mathrm{u}, \mathrm{w})$ & $\mathrm{T}:(0,0,3 / 2)$ & yes & Plane \\
\hline \multicolumn{5}{|c|}{ BCSID: 0.177; Formula: Mn3GaN; MSG: $166.97(R-3 m)$; U=2eV } \\
\hline maximal $k_{1}$ & intermediate path & $\operatorname{maximal} k_{2}$ & satisfied? & Line/Plane \\
\hline$\Gamma:(0,0,0)$ & SM:(u,-2*u,0) & $\mathrm{F}:(-1 / 2,1,0)$ & no & Line \\
\hline$\Gamma:(0,0,0)$ & $\mathrm{LD}:(0,0, \mathrm{w})$ & $\mathrm{T}:(0,0,3 / 2)$ & no & Line \\
\hline $\mathrm{L}:(1 / 2,1 / 2,3 / 2)$ & $\mathrm{Y}:(\mathrm{u}, \mathrm{u}, 3 / 2)$ & $\mathrm{T}:(0,0,3 / 2)$ & yes & Line \\
\hline$\Gamma:(0,0,0)$ & $\mathrm{C}:(\mathrm{u},-\mathrm{u}, \mathrm{w})$ & $\mathrm{F}:(1 / 2,-1 / 2,1)$ & no & Plane \\
\hline$\Gamma:(0,0,0)$ & $C:(u,-u, w)$ & $\mathrm{L}:(-1 / 2,1 / 2,1 / 2)$ & no & Plane \\
\hline$\Gamma:(0,0,0)$ & $\mathrm{C}:(\mathrm{u},-\mathrm{u}, \mathrm{w})$ & $\mathrm{T}:(0,0,3 / 2)$ & no & Plane \\
\hline $\mathrm{F}:(0,1 / 2,1)$ & $\mathrm{C}:(0, \mathrm{u}, \mathrm{w})$ & $\mathrm{L}:(0,-1 / 2,1 / 2)$ & yes & Plane \\
\hline $\mathrm{F}:(0,1 / 2,1)$ & $\mathrm{C}:(0, \mathrm{u}, \mathrm{w})$ & $\mathrm{T}:(0,1,1 / 2)$ & yes & Plane \\
\hline $\mathrm{L}:(-1 / 2,1 / 2,1 / 2)$ & $\mathrm{C}:(\mathrm{u},-\mathrm{u}, \mathrm{w})$ & $\mathrm{T}:(0,0,3 / 2)$ & yes & Plane \\
\hline \multicolumn{5}{|c|}{ BCSID: 0.108; Formula: Mn3Ir; MSG: $166.101\left(R-3 m^{\prime}\right) ; \mathrm{U}=0$} \\
\hline $\operatorname{maximal} k_{1}$ & intermediate path & maximal $k_{2}$ & satisfied? & Line/Plane \\
\hline$\Gamma:(0,0,0)$ & $\mathrm{LD}:(0,0, \mathrm{w})$ & $\mathrm{T}:(0,0,3 / 2)$ & no & Line \\
\hline \multicolumn{5}{|c|}{ BCSID: 0.108; Formula: Mn3Ir; MSG: $166.101\left(R-3 m^{\prime}\right)$; U=1eV } \\
\hline $\operatorname{maximal} k_{1}$ & intermediate path & maximal $k_{2}$ & satisfied? & Line/Plane \\
\hline$\Gamma:(0,0,0)$ & LD: $(0,0, \mathrm{w})$ & $\mathrm{T}:(0,0,3 / 2)$ & no & Line \\
\hline \multicolumn{5}{|c|}{ BCSID: 0.108; Formula: Mn3Ir; MSG: $166.101\left(R-3 m^{\prime}\right)$; U=2eV } \\
\hline maximal $k_{1}$ & intermediate path & maximal $k_{2}$ & satisfied? & Line/Plane \\
\hline$\Gamma:(0,0,0)$ & $\mathrm{LD}:(0,0, \mathrm{w})$ & $\mathrm{T}:(0,0,3 / 2)$ & no & Line \\
\hline
\end{tabular}

BCSID: 0.108; Formula: Mn3Ir; MSG: $166.101\left(R-3 m^{\prime}\right)$; $\mathrm{U}=3 \mathrm{eV}$ maximal $k_{1} \quad \mid$ intermediate path $\quad$ maximal $k_{2} \quad$ satisfied? $\mid$ Line/Plane 


\begin{tabular}{|c|c|c|c|c|}
\hline$\Gamma:(0,0,0)$ & LD: $(0,0, w)$ & $\mathrm{T}:(0,0,3 / 2)$ & no & Line \\
\hline \multicolumn{5}{|c|}{ BCSID: 0.108; Formula: Mn3Ir; MSG: $166.101\left(R-3 m^{\prime}\right) ; \mathrm{U}=4 \mathrm{eV}$} \\
\hline maximal $k_{1}$ & intermediate path & maximal $k_{2}$ & satisfied? & Line/Plane \\
\hline$\Gamma:(0,0,0)$ & LD: $(0,0, w)$ & $\mathrm{T}:(0,0,3 / 2)$ & no & Line \\
\hline \multicolumn{5}{|c|}{ BCSID: 0.109; Formula: Mn3Pt; MSG: $166.101\left(R-3 m^{\prime}\right) ; \mathrm{U}=0$} \\
\hline maximal $k_{1}$ & intermediate path & maximal $k_{2}$ & satisfied? & Line/Plane \\
\hline$\Gamma:(0,0,0)$ & LD: $(0,0, w)$ & $\mathrm{T}:(0,0,3 / 2)$ & no & Line \\
\hline \multicolumn{5}{|c|}{ BCSID: 0.109; Formula: Mn3Pt; MSG: $166.101\left(R-3 m^{\prime}\right) ; \mathrm{U}=2 \mathrm{eV}$} \\
\hline maximal $k_{1}$ & intermediate path & maximal $k_{2}$ & satisfied? & Line/Plane \\
\hline$\Gamma:(0,0,0)$ & $\mathrm{LD}:(0,0, \mathrm{w})$ & $\mathrm{T}:(0,0,3 / 2)$ & no & Line \\
\hline \multicolumn{5}{|c|}{ BCSID: 0.109; Formula: Mn3Pt; MSG: $166.101\left(R-3 m^{\prime}\right) ; \mathrm{U}=3 \mathrm{eV}$} \\
\hline maximal $k_{1}$ & intermediate path & maximal $k_{2}$ & satisfied? & Line/Plane \\
\hline$\Gamma:(0,0,0)$ & LD: $(0,0, \mathrm{w})$ & $\mathrm{T}:(0,0,3 / 2)$ & no & Line \\
\hline \multicolumn{5}{|c|}{ BCSID: 0.109; Formula: Mn3Pt; MSG: $166.101\left(R-3 m^{\prime}\right) ; \mathrm{U}=4 \mathrm{eV}$} \\
\hline maximal $k_{1}$ & intermediate path & maximal $k_{2}$ & satisfied? & Line/Plane \\
\hline$\Gamma:(0,0,0)$ & LD: $(0,0, \mathrm{w})$ & $\mathrm{T}:(0,0,3 / 2)$ & no & Line \\
\hline \multicolumn{5}{|c|}{ BCSID: 0.228; Formula: TbCo2; MSG: $166.101\left(R-3 m^{\prime}\right) ; \mathrm{U}=2 \mathrm{eV}$} \\
\hline maximal $k_{1}$ & intermediate path & maximal $k_{2}$ & satisfied? & Line/Plane \\
\hline$\Gamma:(0,0,0)$ & LD: $(0,0, \mathrm{w})$ & $\mathrm{T}:(0,0,3 / 2)$ & no & Line \\
\hline \multicolumn{5}{|c|}{ BCSID: 0.77; Formula: Tb2Ti2O7; MSG: $166.101\left(R-3 m^{\prime}\right) ; \mathrm{U}=0$} \\
\hline maximal $k_{1}$ & intermediate path & maximal $k_{2}$ & satisfied? & Line/Plane \\
\hline$\Gamma:(0,0,0)$ & LD: $(0,0, \mathrm{w})$ & $\mathrm{T}:(0,0,3 / 2)$ & no & Line \\
\hline \multicolumn{5}{|c|}{ BCSID: 1.153; Formula: Mn3GaC; MSG: $167.108\left(R_{I}-3 c\right) ; \mathrm{U}=0$} \\
\hline maximal $k_{1}$ & intermediate path & maximal $k_{2}$ & satisfied? & Line/Plane \\
\hline$\Gamma:(0,0,0)$ & LD: $(0,0, \mathrm{w})$ & $\mathrm{T}:(0,0,3 / 2)$ & no & Line \\
\hline \multicolumn{5}{|c|}{ BCSID: 0.33; Formula: HoMnO3; MSG: $185.197\left(P 6_{3} \mathrm{~cm}\right) ; \mathrm{U}=4 \mathrm{eV}$} \\
\hline maximal $k_{1}$ & intermediate path & maximal $k_{2}$ & satisfied? & Line/Plane \\
\hline$\Gamma:(0,0,0)$ & DT: $(0,0, \mathrm{w})$ & $\mathrm{A}:(0,0,1 / 2)$ & no & Line \\
\hline$\Gamma:(0,0,0)$ & $\mathrm{LD}:(\mathrm{u}, \mathrm{u}, 0)$ & $\mathrm{K}:(1 / 3,1 / 3,0)$ & yes & Line \\
\hline$\Gamma:(0,0,0)$ & $\mathrm{LD}:(\mathrm{u}, \mathrm{u}, 0)$ & $\mathrm{M}:(1 / 2,1 / 2,0)$ & yes & Line \\
\hline$\Gamma:(0,0,0)$ & SM:(u,0,0) & $\mathrm{M}:(1 / 2,0,0)$ & yes & Line \\
\hline $\mathrm{A}:(0,0,1 / 2)$ & $\mathrm{Q}:(\mathrm{u}, \mathrm{u}, 1 / 2)$ & $\mathrm{H}:(1 / 3,1 / 3,1 / 2)$ & yes & Line \\
\hline $\mathrm{A}:(0,0,1 / 2)$ & $\mathrm{Q}:(\mathrm{u}, \mathrm{u}, 1 / 2)$ & $\mathrm{L}:(1 / 2,1 / 2,1 / 2)$ & yes & Line \\
\hline $\mathrm{A}:(0,0,1 / 2)$ & $\mathrm{R}:(\mathrm{u}, 0,1 / 2)$ & $\mathrm{L}:(1 / 2,0,1 / 2)$ & yes & Line \\
\hline $\mathrm{H}:(1 / 3,1 / 3,1 / 2)$ & $\mathrm{P}:(1 / 3,1 / 3, \mathrm{w})$ & $\mathrm{K}:(1 / 3,1 / 3,0)$ & yes & Line \\
\hline $\mathrm{H}:(1 / 3,1 / 3,1 / 2)$ & $\mathrm{Q}:(\mathrm{u}, \mathrm{u}, 1 / 2)$ & $\mathrm{L}:(1 / 2,1 / 2,1 / 2)$ & yes & Line \\
\hline $\mathrm{K}:(1 / 3,1 / 3,0)$ & $\mathrm{LD}:(\mathrm{u}, \mathrm{u}, 0)$ & $\mathrm{M}:(1 / 2,1 / 2,0)$ & yes & Line \\
\hline$\Gamma:(0,0,0)$ & $\mathrm{C}:(\mathrm{u}, \mathrm{u}, \mathrm{w})$ & $\mathrm{A}:(0,0,1 / 2)$ & yes & Plane \\
\hline$\Gamma:(0,0,0)$ & $\mathrm{D}:(\mathrm{u}, 0, \mathrm{w})$ & $\mathrm{A}:(0,0,1 / 2)$ & yes & Plane \\
\hline$\Gamma:(0,0,0)$ & $\mathrm{C}:(\mathrm{u}, \mathrm{u}, \mathrm{w})$ & $\mathrm{H}:(1 / 3,1 / 3,1 / 2)$ & yes & Plane \\
\hline$\Gamma:(0,0,0)$ & $\mathrm{C}:(\mathrm{u}, \mathrm{u}, \mathrm{w})$ & $\mathrm{K}:(1 / 3,1 / 3,0)$ & yes & Plane \\
\hline$\Gamma:(0,0,0)$ & $\mathrm{C}:(\mathrm{u}, \mathrm{u}, \mathrm{w})$ & $\mathrm{L}:(1 / 2,1 / 2,1 / 2)$ & yes & Plane \\
\hline
\end{tabular}




\begin{tabular}{c|c|c|c|c}
\hline$\Gamma:(0,0,0)$ & $\mathrm{D}:(\mathrm{u}, 0, \mathrm{w})$ & $\mathrm{L}:(1 / 2,0,1 / 2)$ & yes & Plane \\
\hline$\Gamma:(0,0,0)$ & $\mathrm{C}:(\mathrm{u}, \mathrm{u}, \mathrm{w})$ & $\mathrm{M}:(1 / 2,1 / 2,0)$ & yes & Plane \\
\hline$\Gamma:(0,0,0)$ & $\mathrm{D}:(\mathrm{u}, 0, \mathrm{w})$ & $\mathrm{M}:(1 / 2,0,0)$ & yes & Plane \\
\hline $\mathrm{A}:(0,0,1 / 2)$ & $\mathrm{C}:(\mathrm{u}, \mathrm{u}, \mathrm{w})$ & $\mathrm{H}:(1 / 3,1 / 3,1 / 2)$ & yes & Plane \\
\hline $\mathrm{A}:(0,0,1 / 2)$ & $\mathrm{C}:(\mathrm{u}, \mathrm{u}, \mathrm{w})$ & $\mathrm{K}:(1 / 3,1 / 3,0)$ & yes & Plane \\
\hline $\mathrm{A}:(0,0,1 / 2)$ & $\mathrm{C}:(\mathrm{u}, \mathrm{u}, \mathrm{w})$ & $\mathrm{L}:(1 / 2,1 / 2,1 / 2)$ & yes & Plane \\
\hline $\mathrm{A}:(0,0,1 / 2)$ & $\mathrm{D}:(\mathrm{u}, 0, \mathrm{w})$ & $\mathrm{L}:(1 / 2,0,1 / 2)$ & yes & Plane \\
\hline $\mathrm{A}:(0,0,1 / 2)$ & $\mathrm{C}:(\mathrm{u}, \mathrm{u}, \mathrm{w})$ & $\mathrm{M}:(1 / 2,1 / 2,0)$ & yes & Plane \\
\hline $\mathrm{A}:(0,0,1 / 2)$ & $\mathrm{D}:(\mathrm{u}, 0, \mathrm{w})$ & $\mathrm{M}:(1 / 2,0,0)$ & yes & Plane \\
\hline $\mathrm{H}:(1 / 3,1 / 3,1 / 2)$ & $\mathrm{C}:(\mathrm{u}, \mathrm{u}, \mathrm{w})$ & $\mathrm{K}:(1 / 3,1 / 3,0)$ & yes & Plane \\
\hline $\mathrm{H}:(1 / 3,1 / 3,1 / 2)$ & $\mathrm{C}:(\mathrm{u}, \mathrm{u}, \mathrm{w})$ & $\mathrm{K}:(2 / 3,2 / 3,0)$ & yes & Plane \\
\hline $\mathrm{H}:(1 / 3,1 / 3,1 / 2)$ & $\mathrm{C}:(\mathrm{u}, \mathrm{u}, \mathrm{w})$ & $\mathrm{L}:(1 / 2,1 / 2,1 / 2)$ & yes & Plane \\
\hline $\mathrm{H}:(1 / 3,1 / 3,1 / 2)$ & $\mathrm{C:}(\mathrm{u}, \mathrm{u}, \mathrm{w})$ & $\mathrm{M}:(1 / 2,1 / 2,0)$ & yes & Plane \\
\hline $\mathrm{K}:(1 / 3,1 / 3,0)$ & $\mathrm{C}:(\mathrm{u}, \mathrm{u}, \mathrm{w})$ & $\mathrm{L}:(1 / 2,1 / 2,1 / 2)$ & yes & Plane \\
\hline $\mathrm{K}:(1 / 3,1 / 3,0)$ & $\mathrm{C}:(\mathrm{u}, \mathrm{u}, \mathrm{w})$ & $\mathrm{M}:(1 / 2,1 / 2,0)$ & yes & Plane \\
\hline $\mathrm{L}:(1 / 2,-1,1 / 2)$ & $\mathrm{C}:(\mathrm{u},-2 * \mathrm{u}, \mathrm{w})$ & $\mathrm{M}:(1 / 2,-1,0)$ & yes & Plane \\
\hline $\mathrm{L}:(1 / 2,0,1 / 2)$ & $\mathrm{D}:(\mathrm{u}, 0, \mathrm{w})$ & $\mathrm{M}:(1 / 2,0,0)$ & yes & Plane \\
\hline
\end{tabular}

BCSID: 1.110; Formula: ScMn6Ge6; MSG: $192.252\left(P_{c} 6 / m c c\right) ; \mathrm{U}=0$

\begin{tabular}{|c|c|c|c|c|}
\hline maximal $k_{1}$ & intermediate path & maximal $k_{2}$ & satisfied? & Line/Plane \\
\hline$\Gamma:(0,0,0)$ & DT: $(0,0, \mathrm{w})$ & $\mathrm{A}:(0,0,1 / 2)$ & yes & Line \\
\hline $\mathrm{A}:(0,0,1 / 2)$ & $\mathrm{Q}:(\mathrm{u}, \mathrm{u}, 1 / 2)$ & $\mathrm{H}:(1 / 3,1 / 3,1 / 2)$ & no & Line \\
\hline $\mathrm{A}:(0,0,1 / 2)$ & $\mathrm{Q}:(\mathrm{u}, \mathrm{u}, 1 / 2)$ & $\mathrm{L}:(1 / 2,1 / 2,1 / 2)$ & yes & Line \\
\hline $\mathrm{A}:(0,0,1 / 2)$ & $\mathrm{R}:(\mathrm{u}, 0,1 / 2)$ & $\mathrm{L}:(1 / 2,0,1 / 2)$ & yes & Line \\
\hline $\mathrm{H}:(1 / 3,1 / 3,1 / 2)$ & $\mathrm{P}:(1 / 3,1 / 3, \mathrm{w})$ & $\mathrm{K}:(1 / 3,1 / 3,0)$ & yes & Line \\
\hline $\mathrm{H}:(1 / 3,1 / 3,1 / 2)$ & $\mathrm{Q}:(\mathrm{u}, \mathrm{u}, 1 / 2)$ & $\mathrm{L}:(1 / 2,1 / 2,1 / 2)$ & no & Line \\
\hline $\mathrm{A}:(0,0,1 / 2)$ & $\mathrm{E}:(\mathrm{u}, \mathrm{v}, 1 / 2)$ & $\mathrm{H}:(1 / 3,1 / 3,1 / 2)$ & no & Plane \\
\hline $\mathrm{A}:(0,0,1 / 2)$ & $\mathrm{E}:(\mathrm{u}, \mathrm{v}, 1 / 2)$ & $\mathrm{L}:(1 / 2,0,1 / 2)$ & yes & Plane \\
\hline $\mathrm{H}:(1 / 3,1 / 3,1 / 2)$ & $\mathrm{E}:(\mathrm{u}, \mathrm{v}, 1 / 2)$ & $\mathrm{L}:(1 / 2,0,1 / 2)$ & no & Plane \\
\hline \multicolumn{5}{|c|}{ BCSID: 1.225; Formula: ScMn6Ge6; MSG: $192.252\left(P_{c} 6 / m c c\right) ; \mathrm{U}=0$} \\
\hline maximal $k_{1}$ & intermediate path & maximal $k_{2}$ & satisfied? & Line/Plane \\
\hline$\Gamma:(0,0,0)$ & DT: $(0,0, \mathrm{w})$ & $\mathrm{A}:(0,0,1 / 2)$ & yes & Line \\
\hline $\mathrm{A}:(0,0,1 / 2)$ & $\mathrm{Q}:(\mathrm{u}, \mathrm{u}, 1 / 2)$ & $\mathrm{H}:(1 / 3,1 / 3,1 / 2)$ & no & Line \\
\hline $\mathrm{A}:(0,0,1 / 2)$ & $\mathrm{Q}:(\mathrm{u}, \mathrm{u}, 1 / 2)$ & $\mathrm{L}:(1 / 2,1 / 2,1 / 2)$ & yes & Line \\
\hline $\mathrm{A}:(0,0,1 / 2)$ & $\mathrm{R}:(\mathrm{u}, 0,1 / 2)$ & $\mathrm{L}:(1 / 2,0,1 / 2)$ & yes & Line \\
\hline $\mathrm{H}:(1 / 3,1 / 3,1 / 2)$ & $\mathrm{P}:(1 / 3,1 / 3, \mathrm{w})$ & $\mathrm{K}:(1 / 3,1 / 3,0)$ & yes & Line \\
\hline $\mathrm{H}:(1 / 3,1 / 3,1 / 2)$ & $\mathrm{Q}:(\mathrm{u}, \mathrm{u}, 1 / 2)$ & $\mathrm{L}:(1 / 2,1 / 2,1 / 2)$ & no & Line \\
\hline $\mathrm{A}:(0,0,1 / 2)$ & $\mathrm{E}:(\mathrm{u}, \mathrm{v}, 1 / 2)$ & $\mathrm{H}:(1 / 3,1 / 3,1 / 2)$ & no & Plane \\
\hline $\mathrm{A}:(0,0,1 / 2)$ & $\mathrm{E}:(\mathrm{u}, \mathrm{v}, 1 / 2)$ & $\mathrm{L}:(1 / 2,0,1 / 2)$ & yes & Plane \\
\hline $\mathrm{H}:(1 / 3,1 / 3,1 / 2)$ & $\mathrm{E}:(\mathrm{u}, \mathrm{v}, 1 / 2)$ & $\mathrm{L}:(1 / 2,0,1 / 2)$ & no & Plane \\
\hline
\end{tabular}

\begin{tabular}{c|c|c|c|c}
\hline \multicolumn{5}{c}{ BCSID: $1.225 ;$ Formula: ScMn6Ge6; MSG: $192.252\left(P_{c} 6 / m c c\right) ; \mathrm{U}=1 \mathrm{eV}$} \\
\hline maximal $k_{1}$ & intermediate path & maximal $k_{2}$ & satisfied? & Line/Plane \\
\hline$\Gamma:(0,0,0)$ & $\mathrm{DT}:(0,0, \mathrm{w})$ & $\mathrm{A}:(0,0,1 / 2)$ & yes & Line \\
\hline $\mathrm{A}:(0,0,1 / 2)$ & $\mathrm{Q}:(\mathrm{u}, \mathrm{u}, 1 / 2)$ & $\mathrm{H}:(1 / 3,1 / 3,1 / 2)$ & no & Line \\
\hline $\mathrm{A}:(0,0,1 / 2)$ & $\mathrm{Q}:(\mathrm{u}, \mathrm{u}, 1 / 2)$ & $\mathrm{L}:(1 / 2,1 / 2,1 / 2)$ & yes & Line \\
\hline $\mathrm{A}:(0,0,1 / 2)$ & $\mathrm{R}:(\mathrm{u}, 0,1 / 2)$ & $\mathrm{L}:(1 / 2,0,1 / 2)$ & yes & Line \\
\hline $\mathrm{H}:(1 / 3,1 / 3,1 / 2)$ & $\mathrm{P}:(1 / 3,1 / 3, \mathrm{w})$ & $\mathrm{K}:(1 / 3,1 / 3,0)$ & yes & Line \\
\hline $\mathrm{H}:(1 / 3,1 / 3,1 / 2)$ & $\mathrm{Q}:(\mathrm{u}, \mathrm{u}, 1 / 2)$ & $\mathrm{L}:(1 / 2,1 / 2,1 / 2)$ & no & Line \\
\hline $\mathrm{A}:(0,0,1 / 2)$ & $\mathrm{E}:(\mathrm{u}, \mathrm{v}, 1 / 2)$ & $\mathrm{H}:(1 / 3,1 / 3,1 / 2)$ & no & Plane
\end{tabular}




\begin{tabular}{c|c|c|c|c}
\hline $\mathrm{A}:(0,0,1 / 2)$ & $\mathrm{E}:(\mathrm{u}, \mathrm{v}, 1 / 2)$ & $\mathrm{L}:(1 / 2,0,1 / 2)$ & yes & Plane \\
\hline $\mathrm{H}:(1 / 3,1 / 3,1 / 2)$ & $\mathrm{E}:(\mathrm{u}, \mathrm{v}, 1 / 2)$ & $\mathrm{L}:(1 / 2,0,1 / 2)$ & no & Plane \\
\hline \multicolumn{5}{|l}{} \\
\hline \multicolumn{5}{l}{ BCSID: $1.225 ;$ Formula: ScMn6Ge6; MSG: $192.252\left(P_{c} 6 / m c c\right) ; \mathrm{U}=2 \mathrm{eV}$} \\
\hline maximal $k_{1}$ & intermediate path & maximal $k_{2}$ & satisfied? & Line/Plane \\
\hline$\Gamma:(0,0,0)$ & $\mathrm{DT}:(0,0, \mathrm{w})$ & $\mathrm{A}:(0,0,1 / 2)$ & yes & Line \\
\hline $\mathrm{A}:(0,0,1 / 2)$ & $\mathrm{Q}:(\mathrm{u}, \mathrm{u}, 1 / 2)$ & $\mathrm{H}:(1 / 3,1 / 3,1 / 2)$ & no & Line \\
\hline $\mathrm{A}:(0,0,1 / 2)$ & $\mathrm{Q}:(\mathrm{u}, \mathrm{u}, 1 / 2)$ & $\mathrm{L}:(1 / 2,1 / 2,1 / 2)$ & no & Line \\
\hline $\mathrm{A}:(0,0,1 / 2)$ & $\mathrm{R}:(\mathrm{u}, 0,1 / 2)$ & $\mathrm{L}:(1 / 2,0,1 / 2)$ & no & Line \\
\hline $\mathrm{H}:(1 / 3,1 / 3,1 / 2)$ & $\mathrm{P}:(1 / 3,1 / 3, \mathrm{w})$ & $\mathrm{K}:(1 / 3,1 / 3,0)$ & yes & Line \\
\hline $\mathrm{H}:(1 / 3,1 / 3,1 / 2)$ & $\mathrm{Q}:(\mathrm{u}, \mathrm{u}, 1 / 2)$ & $\mathrm{L}:(1 / 2,1 / 2,1 / 2)$ & no & Line \\
\hline $\mathrm{A}:(0,0,1 / 2)$ & $\mathrm{E}:(\mathrm{u}, \mathrm{v}, 1 / 2)$ & $\mathrm{H}:(1 / 3,1 / 3,1 / 2)$ & no & Plane \\
\hline $\mathrm{A}:(0,0,1 / 2)$ & $\mathrm{E}:(\mathrm{u}, \mathrm{v}, 1 / 2)$ & $\mathrm{L}:(1 / 2,0,1 / 2)$ & no & Plane \\
\hline $\mathrm{H}:(1 / 3,1 / 3,1 / 2)$ & $\mathrm{E}:(\mathrm{u}, \mathrm{v}, 1 / 2)$ & $\mathrm{L}:(1 / 2,0,1 / 2)$ & no & Plane \\
\hline
\end{tabular}

\begin{tabular}{|c|c|c|c|c|}
\hline \multicolumn{5}{|c|}{ BCSID: 1.225; Formula: ScMn6Ge6; MSG: $192.252\left(P_{c} 6 / m c c\right) ; \mathrm{U}=3 \mathrm{eV}$} \\
\hline maximal $k_{1}$ & intermediate path & maximal $k_{2}$ & satisfied? & Line/Plane \\
\hline$\Gamma:(0,0,0)$ & $\mathrm{DT}:(0,0, \mathrm{w})$ & $\mathrm{A}:(0,0,1 / 2)$ & no & Line \\
\hline $\mathrm{A}:(0,0,1 / 2)$ & $\mathrm{Q}:(\mathrm{u}, \mathrm{u}, 1 / 2)$ & $\mathrm{H}:(1 / 3,1 / 3,1 / 2)$ & no & Line \\
\hline $\mathrm{A}:(0,0,1 / 2)$ & $\mathrm{Q}:(\mathrm{u}, \mathrm{u}, 1 / 2)$ & $\mathrm{L}:(1 / 2,1 / 2,1 / 2)$ & yes & Line \\
\hline $\mathrm{A}:(0,0,1 / 2)$ & $\mathrm{R}:(\mathrm{u}, 0,1 / 2)$ & $\mathrm{L}:(1 / 2,0,1 / 2)$ & yes & Line \\
\hline $\mathrm{H}:(1 / 3,1 / 3,1 / 2)$ & $\mathrm{P}:(1 / 3,1 / 3, \mathrm{w})$ & $\mathrm{K}:(1 / 3,1 / 3,0)$ & no & Line \\
\hline $\mathrm{H}:(1 / 3,1 / 3,1 / 2)$ & $\mathrm{Q}:(\mathrm{u}, \mathrm{u}, 1 / 2)$ & $\mathrm{L}:(1 / 2,1 / 2,1 / 2)$ & no & Line \\
\hline $\mathrm{A}:(0,0,1 / 2)$ & $\mathrm{E}:(\mathrm{u}, \mathrm{v}, 1 / 2)$ & $\mathrm{H}:(1 / 3,1 / 3,1 / 2)$ & no & Plane \\
\hline $\mathrm{A}:(0,0,1 / 2)$ & $\mathrm{E}:(\mathrm{u}, \mathrm{v}, 1 / 2)$ & $\mathrm{L}:(1 / 2,0,1 / 2)$ & yes & Plane \\
\hline $\mathrm{H}:(1 / 3,1 / 3,1 / 2)$ & $\mathrm{E}:(\mathrm{u}, \mathrm{v}, 1 / 2)$ & $\mathrm{L}:(1 / 2,0,1 / 2)$ & no & Plane \\
\hline \multicolumn{5}{|c|}{ BCSID: 0.118; Formula: Ba5Co5ClO13; MSG: $194.268\left(P 6_{3}^{\prime} / m^{\prime} m^{\prime} c\right) ; \mathrm{U}=4 \mathrm{eV}$} \\
\hline maximal $k_{1}$ & intermediate path & maximal $k_{2}$ & satisfied? & Line/Plane \\
\hline$\Gamma:(0,0,0)$ & DT: $(0,0, w)$ & $\mathrm{A}:(0,0,1 / 2)$ & yes & Line \\
\hline$\Gamma:(0,0,0)$ & $\mathrm{LD}:(\mathrm{u}, \mathrm{u}, 0)$ & $\mathrm{K}:(1 / 3,1 / 3,0)$ & yes & Line \\
\hline$\Gamma:(0,0,0)$ & $\mathrm{LD}:(\mathrm{u}, \mathrm{u}, 0)$ & $\mathrm{M}:(1 / 2,1 / 2,0)$ & yes & Line \\
\hline$\Gamma:(0,0,0)$ & SM:(u,0,0) & $\mathrm{M}:(1 / 2,0,0)$ & no & Line \\
\hline $\mathrm{H}:(1 / 3,1 / 3,1 / 2)$ & $\mathrm{P}:(1 / 3,1 / 3, \mathrm{w})$ & $\mathrm{K}:(1 / 3,1 / 3,0)$ & yes & Line \\
\hline $\mathrm{K}:(1 / 3,1 / 3,0)$ & $\mathrm{LD}:(\mathrm{u}, \mathrm{u}, 0)$ & $\mathrm{M}:(1 / 2,1 / 2,0)$ & yes & Line \\
\hline $\mathrm{L}:(1 / 2,0,1 / 2)$ & $\mathrm{U}:(1 / 2,0, \mathrm{w})$ & $\mathrm{M}:(1 / 2,0,0)$ & yes & Line \\
\hline$\Gamma:(0,0,0)$ & $\mathrm{C}:(\mathrm{u}, \mathrm{u}, \mathrm{w})$ & $\mathrm{A}:(0,0,1 / 2)$ & yes & Plane \\
\hline$\Gamma:(0,0,0)$ & $\mathrm{C}:(\mathrm{u}, \mathrm{u}, \mathrm{w})$ & $\mathrm{H}:(1 / 3,1 / 3,1 / 2)$ & yes & Plane \\
\hline$\Gamma:(0,0,0)$ & $\mathrm{C}:(\mathrm{u}, \mathrm{u}, \mathrm{w})$ & $\mathrm{K}:(1 / 3,1 / 3,0)$ & yes & Plane \\
\hline$\Gamma:(0,0,0)$ & $\mathrm{C}:(\mathrm{u}, \mathrm{u}, \mathrm{w})$ & $\mathrm{L}:(1 / 2,1 / 2,1 / 2)$ & yes & Plane \\
\hline$\Gamma:(0,0,0)$ & $\mathrm{C}:(\mathrm{u}, \mathrm{u}, \mathrm{w})$ & $\mathrm{M}:(1 / 2,1 / 2,0)$ & yes & Plane \\
\hline $\mathrm{A}:(0,0,1 / 2)$ & $\mathrm{C}:(\mathrm{u}, \mathrm{u}, \mathrm{w})$ & $\mathrm{H}:(1 / 3,1 / 3,1 / 2)$ & yes & Plane \\
\hline $\mathrm{A}:(0,0,1 / 2)$ & $\mathrm{C}:(\mathrm{u}, \mathrm{u}, \mathrm{w})$ & $\mathrm{K}:(1 / 3,1 / 3,0)$ & yes & Plane \\
\hline $\mathrm{A}:(0,0,1 / 2)$ & $\mathrm{C}:(\mathrm{u}, \mathrm{u}, \mathrm{w})$ & $\mathrm{L}:(1 / 2,1 / 2,1 / 2)$ & yes & Plane \\
\hline $\mathrm{A}:(0,0,1 / 2)$ & $\mathrm{C}:(\mathrm{u}, \mathrm{u}, \mathrm{w})$ & $\mathrm{M}:(1 / 2,1 / 2,0)$ & yes & Plane \\
\hline $\mathrm{H}:(1 / 3,1 / 3,1 / 2)$ & $\mathrm{C}:(\mathrm{u}, \mathrm{u}, \mathrm{w})$ & $\mathrm{K}:(1 / 3,1 / 3,0)$ & yes & Plane \\
\hline $\mathrm{H}:(1 / 3,1 / 3,1 / 2)$ & $\mathrm{C}:(\mathrm{u}, \mathrm{u}, \mathrm{w})$ & $\mathrm{L}:(1 / 2,1 / 2,1 / 2)$ & yes & Plane \\
\hline $\mathrm{H}:(1 / 3,1 / 3,1 / 2)$ & $\mathrm{C}:(\mathrm{u}, \mathrm{u}, \mathrm{w})$ & $\mathrm{M}:(1 / 2,1 / 2,0)$ & yes & Plane \\
\hline $\mathrm{K}:(1 / 3,1 / 3,0)$ & $\mathrm{C}:(\mathrm{u}, \mathrm{u}, \mathrm{w})$ & $\mathrm{L}:(1 / 2,1 / 2,1 / 2)$ & yes & Plane \\
\hline $\mathrm{K}:(1 / 3,1 / 3,0)$ & $\mathrm{C}:(\mathrm{u}, \mathrm{u}, \mathrm{w})$ & $\mathrm{M}:(1 / 2,1 / 2,0)$ & yes & Plane \\
\hline $\mathrm{L}:(1 / 2,-1,1 / 2)$ & $\mathrm{C}:\left(\mathrm{u},-2^{*} \mathrm{u}, \mathrm{w}\right)$ & $\mathrm{M}:(1 / 2,-1,0)$ & yes & Plane \\
\hline
\end{tabular}




\begin{tabular}{|c|c|c|c|c|}
\hline \multicolumn{5}{|c|}{ BCSID: 3.8; Formula: NdZn; MSG: $222.103\left(P_{I} n-3 n\right)$; U=2eV } \\
\hline maximal $k_{1}$ & intermediate path & maximal $k_{2}$ & satisfied? & Line/Plane \\
\hline$\Gamma:(0,0,0)$ & LD:(u,u,u) & $\mathrm{R}:(1 / 2,1 / 2,1 / 2)$ & yes & Line \\
\hline$\Gamma:(0,0,0)$ & DT: $(0, \mathrm{v}, 0)$ & $\mathrm{X}:(0,1 / 2,0)$ & no & Line \\
\hline $\mathrm{M}:(1 / 2,1 / 2,0)$ & $\mathrm{Z}:(\mathrm{u}, 1 / 2,0)$ & $\mathrm{X}:(0,1 / 2,0)$ & no & Line \\
\hline $\mathrm{R}:(1 / 2,1 / 2,1 / 2)$ & $\mathrm{S}:(\mathrm{u}, 1 / 2, \mathrm{u})$ & $\mathrm{X}:(0,1 / 2,0)$ & no & Line \\
\hline $\mathrm{M}:(1 / 2,1 / 2,0)$ & $\mathrm{B}:(\mathrm{u}, 1 / 2, \mathrm{w})$ & $\mathrm{R}:(1 / 2,1 / 2,1 / 2)$ & yes & Plane \\
\hline $\mathrm{M}:(1 / 2,1 / 2,0)$ & $\mathrm{B}:(\mathrm{u}, 1 / 2, \mathrm{w})$ & $\mathrm{X}:(0,1 / 2,0)$ & no & Plane \\
\hline $\mathrm{R}:(1 / 2,1 / 2,1 / 2)$ & $\mathrm{B}:(\mathrm{u}, 1 / 2, \mathrm{w})$ & $\mathrm{X}:(0,1 / 2,0)$ & no & Plane \\
\hline \multicolumn{5}{|c|}{ BCSID: 3.8; Formula: NdZn; MSG: $222.103\left(P_{I} n-3 n\right)$; U=4eV } \\
\hline maximal $k_{1}$ & intermediate path & maximal $k_{2}$ & satisfied? & Line/Plane \\
\hline$\Gamma:(0,0,0)$ & LD: $(\mathrm{u}, \mathrm{u}, \mathrm{u})$ & $\mathrm{R}:(1 / 2,1 / 2,1 / 2)$ & yes & Line \\
\hline$\Gamma:(0,0,0)$ & DT: $(0, \mathrm{v}, 0)$ & $\mathrm{X}:(0,1 / 2,0)$ & no & Line \\
\hline $\mathrm{M}:(1 / 2,1 / 2,0)$ & $\mathrm{Z}:(\mathrm{u}, 1 / 2,0)$ & $\mathrm{X}:(0,1 / 2,0)$ & yes & Line \\
\hline $\mathrm{R}:(1 / 2,1 / 2,1 / 2)$ & $\mathrm{S}:(\mathrm{u}, 1 / 2, \mathrm{u})$ & $\mathrm{X}:(0,1 / 2,0)$ & yes & Line \\
\hline $\mathrm{M}:(1 / 2,1 / 2,0)$ & $\mathrm{B}:(\mathrm{u}, 1 / 2, \mathrm{w})$ & $\mathrm{R}:(1 / 2,1 / 2,1 / 2)$ & yes & Plane \\
\hline $\mathrm{M}:(1 / 2,1 / 2,0)$ & $\mathrm{B}:(\mathrm{u}, 1 / 2, \mathrm{w})$ & $\mathrm{X}:(0,1 / 2,0)$ & yes & Plane \\
\hline $\mathrm{R}:(1 / 2,1 / 2,1 / 2)$ & $\mathrm{B}:(\mathrm{u}, 1 / 2, \mathrm{w})$ & $\mathrm{X}:(0,1 / 2,0)$ & yes & Plane \\
\hline \multicolumn{5}{|c|}{ BCSID: 3.2 ; Formula: UO2; MSG: $224.113\left(P n-3 m^{\prime}\right) ; \mathrm{U}=0$} \\
\hline maximal $k_{1}$ & intermediate path & maximal $k_{2}$ & satisfied? & Line/Plane \\
\hline$\Gamma:(0,0,0)$ & SM: $(u, u, 0)$ & $\mathrm{M}:(1 / 2,1 / 2,0)$ & yes & Line \\
\hline$\Gamma:(0,0,0)$ & $\mathrm{LD}:(\mathrm{u}, \mathrm{u}, \mathrm{u})$ & $\mathrm{R}:(1 / 2,1 / 2,1 / 2)$ & no & Line \\
\hline $\mathrm{M}:(1 / 2,1 / 2,0)$ & $\mathrm{Z}:(\mathrm{u}, 1 / 2,0)$ & $\mathrm{X}:(0,1 / 2,0)$ & yes & Line \\
\hline $\mathrm{M}:(-1 / 2,1 / 2,0)$ & $\mathrm{ZA}:(-1 / 2,-\mathrm{u}, 0)$ & $\mathrm{X}:(-1 / 2,0,0)$ & yes & Line \\
\hline $\mathrm{R}:(1 / 2,1 / 2,1 / 2)$ & $\mathrm{S}:(\mathrm{u}, 1 / 2, \mathrm{u})$ & $\mathrm{X}:(0,1 / 2,0)$ & yes & Line \\
\hline$\Gamma:(0,0,0)$ & $\mathrm{A}:(\mathrm{u}, \mathrm{v}, 0)$ & $\mathrm{M}:(1 / 2,1 / 2,0)$ & yes & Plane \\
\hline$\Gamma:(0,0,0)$ & $\mathrm{A}:(\mathrm{u}, \mathrm{v}, 0)$ & $\mathrm{X}:(0,1 / 2,0)$ & yes & Plane \\
\hline $\mathrm{M}:(1 / 2,1 / 2,0)$ & $\mathrm{B}:(\mathrm{u}, 1 / 2, \mathrm{w})$ & $\mathrm{R}:(1 / 2,1 / 2,1 / 2)$ & yes & Plane \\
\hline $\mathrm{M}:(1 / 2,1 / 2,0)$ & $\mathrm{A}:(\mathrm{u}, \mathrm{v}, 0)$ & $\mathrm{X}:(0,1 / 2,0)$ & yes & Plane \\
\hline $\mathrm{M}:(1 / 2,1 / 2,0)$ & $\mathrm{B}:(\mathrm{u}, 1 / 2, \mathrm{w})$ & $\mathrm{X}:(0,1 / 2,0)$ & yes & Plane \\
\hline $\mathrm{R}:(1 / 2,1 / 2,1 / 2)$ & $\mathrm{B}:(\mathrm{u}, 1 / 2, \mathrm{w})$ & $\mathrm{X}:(0,1 / 2,0)$ & yes & Plane \\
\hline
\end{tabular}

BCSID: 3.9; Formula: NpS; MSG: $228.139\left(F_{S} d-3 c\right)$; U=0

\begin{tabular}{c|c|c|c|c}
\hline maximal $k_{1}$ & intermediate path & maximal $k_{2}$ & satisfied? & Line/Plane \\
\hline$\Gamma:(0,0,0)$ & $\mathrm{LD}:(\mathrm{u}, \mathrm{u}, \mathrm{u})$ & $\mathrm{L}:(1 / 2,1 / 2,1 / 2)$ & no & Line \\
\hline$\Gamma:(0,0,0)$ & $\mathrm{DT}:(0, \mathrm{v}, 0)$ & $\mathrm{X}:(0,1,0)$ & yes & Line \\
\hline $\mathrm{W}:(1 / 2,1,0)$ & $\mathrm{V}:(\mathrm{u}, 1,0)$ & $\mathrm{X}:(0,1,0)$ & yes & Line \\
\hline
\end{tabular}

\begin{tabular}{c|c|c|c|c}
\hline \multicolumn{5}{c}{ BCSID: 0.127; Formula: Dy3A15O12; MSG: $230.148\left(I a-3 d^{\prime}\right) ; \mathrm{U}=0$} \\
\hline maximal $k_{1}$ & intermediate path & maximal $k_{2}$ & satisfied? & Line/Plane \\
\hline$\Gamma:(0,0,0)$ & LD: $(\mathrm{u}, \mathrm{u}, \mathrm{u})$ & $\mathrm{H}:(1,1,1)$ & no & Line \\
\hline$\Gamma:(0,0,0)$ & $\mathrm{SM}:(\mathrm{u}, \mathrm{u}, 0)$ & $\mathrm{N}:(1 / 2,1 / 2,0)$ & yes & Line \\
\hline$\Gamma:(0,0,0)$ & $\mathrm{LD}:(\mathrm{u}, \mathrm{u}, \mathrm{u})$ & $\mathrm{P}:(1 / 2,1 / 2,1 / 2)$ & no & Line \\
\hline $\mathrm{H}:(1,1,1)$ & $\mathrm{LD}:(\mathrm{u}, \mathrm{u}, \mathrm{u})$ & $\mathrm{P}:(1 / 2,1 / 2,1 / 2)$ & no & Line \\
\hline $\mathrm{N}:(1 / 2,1 / 2,0)$ & $\mathrm{D}:(1 / 2,1 / 2, \mathrm{w})$ & $\mathrm{P}:(1 / 2,1 / 2,1 / 2)$ & yes & Line \\
\hline$\Gamma:(0,0,0)$ & $\mathrm{A}:(\mathrm{u}, \mathrm{v}, 0)$ & $\mathrm{H}:(0,1,0)$ & yes & Plane \\
\hline$\Gamma:(0,0,0)$ & $\mathrm{A}:(\mathrm{u}, \mathrm{v}, 0)$ & $\mathrm{N}:(1 / 2,1 / 2,0)$ & yes & Plane \\
\hline $\mathrm{H}:(2,1,0)$ & $\mathrm{A}:(\mathrm{u}, \mathrm{v}, 0)$ & $\mathrm{N}:(3 / 2,3 / 2,0)$ & yes & Plane
\end{tabular}




\section{Appendix J: Detailed discussion of the ideal magnetic TI and SMs}

\section{Higher-order topology of the ideal Axion insulator $\mathrm{NpBi}$}

The crystal structure of $\mathrm{NpBi}$ adopts a face-centered cubic lattice with space group $F m \overline{3} m$ (No. 225) in the high temperature paramagnetic phase. Below $192.5 \mathrm{~K}$, it undergoes a phase transition to the antiferromagnetic phase and the magnetic unit cell adopts simple cubic lattice with MSG 224.113(Pn $\left.\overline{3} m^{\prime}\right)$, as shown in FIG. 32 (a). The generators of this MSG include two-fold screw rotation along the [100] direction $\tilde{C}_{2 x}=\left\{C_{2 x} \mid(0,0.5,0.5)\right\}$, three-fold rotation along the [111] direction $C_{3 x y z}$, inversion $I$ and an anti-unitary symmetry $C_{2 \bar{x} z} \cdot T\left(C_{2 \bar{x} z}\right.$ is the two-fold rotation along the [110] direction and $T$ is the time reversal symmetry). We use the experimental lattice constant $a=6.37 \AA$ and the magnetic momentum of $\mathrm{Np}$ is initialized as $2.42 \mu_{B}$ along the (111) direction in our first-principle calculations. In order to consider the interaction of the $5 \mathrm{f}$ electron on $\mathrm{Np}$, we apply the $\mathrm{LDA}+\mathrm{U}$ calculation with $\mathrm{U}=0,2,4,6 \mathrm{eV}$. We find that the band structures at the four Us have the same topology. In the main text, we have calculated the parity-based stable topological indices and find $Z_{4}=2$, which corresponds to the axion insulator phase. Here we only chose $U=2 \mathrm{eV}$ to analyze the material's topological surface states. It has been proved in Ref. 43 that the $C_{2} \cdot T$ symmetry protects the gapless surface states of axion insulators, on the plane perpendicular to the two-fold rotation axis. Such an axion insulator also exhibits chiral hinge states 61, 65, 102 between the two gapped surfaces related by $C_{2} \cdot T$ symmetry.

There are three equivalent first Miller planes, $\langle\overline{1} 10\rangle,\langle 0 \overline{1} 1\rangle$ and $\langle 10 \overline{1}\rangle$ that preserve $C_{2} \cdot T$ symmetry. We perform the surface states calculations on the $\langle\overline{1} 10\rangle$ plane where the $C_{2} \cdot T$ symmetry is preserved. As shown in FIG. 32 (e), the surface Dirac cone emerges and strictly on the $\tilde{\Gamma} \tilde{Z}$ path constrained by the glide mirror symmetry $\tilde{M}_{z}=\left\{M_{z} \mid(0.5,0.5,0)\right\}$. For the $\langle 001\rangle$ surface plane, it has no $C_{2} \cdot T$ symmetry and the surface states are fully gaped as shown in FIG. 32 (f). Considering the $I$ and $C_{3 x y z}$ symmetries, the chiral flow of charge on the hinges of a cubic NpBi sample is schematically shown in FIG. 32, d).

a

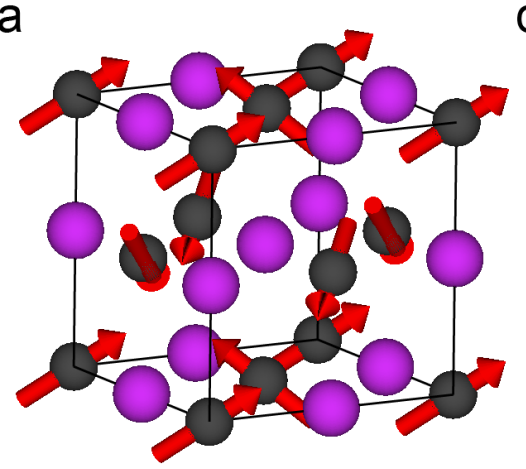

d

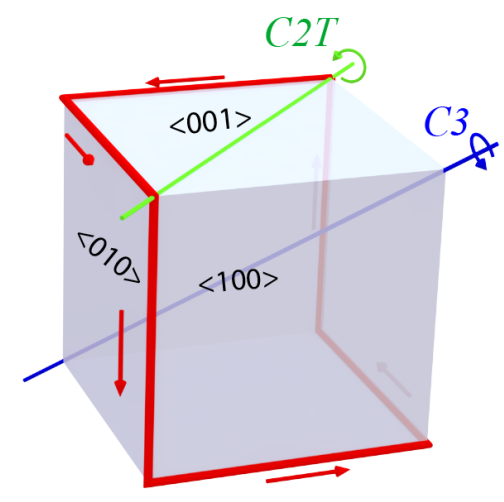

d

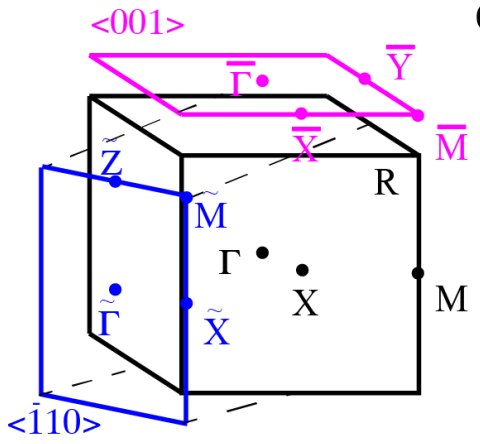

e
C

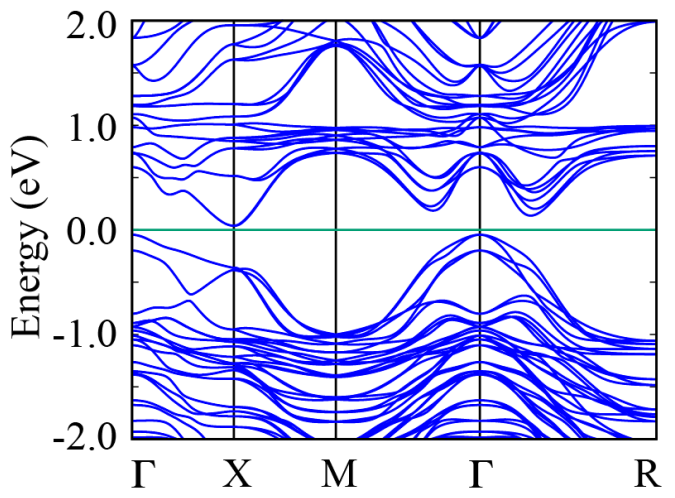

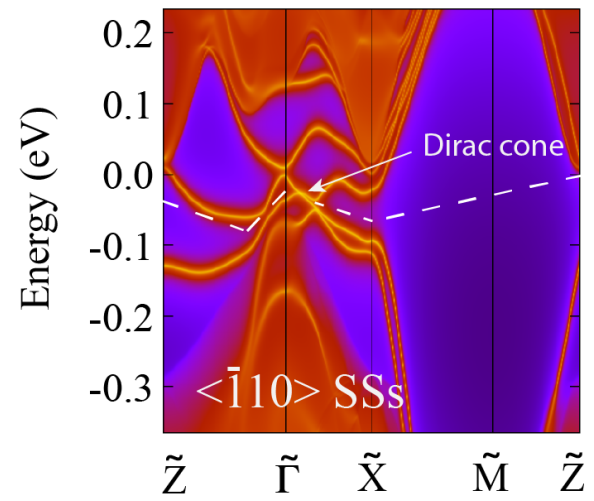

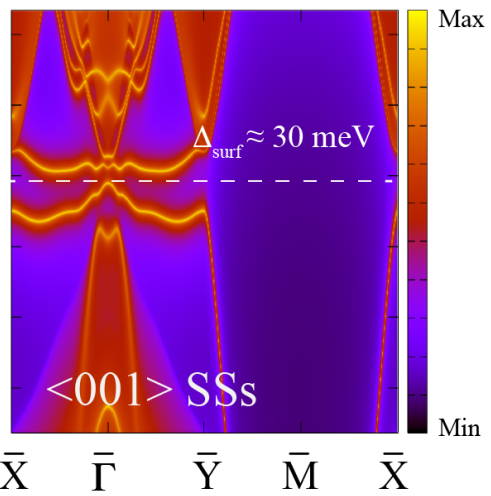

FIG. 32. (a) The crystal and magnetic structures of NpBi with MSG 224.113(Pn $\left.\overline{3} m^{\prime}\right)$. (b) 3D bulk BZ and the projected 2D surface BZ on the $\langle 001\rangle$ (blue) and $\langle\overline{1} 10\rangle$ (pink) surfaces. (c) Electronic band structures of NpBi along the high symmetry paths in BZ with the interaction parameter $U=2 \mathrm{eV}$. (d) Schematic of the chiral flow of charge on the hinge of NpBi. The 1D chiral modes (red) on the six hinges are related to each other by $I$ and $C_{3 x y z}$ symmetries. (e) Surface states calculation on the $C_{2 \bar{x} y} \cdot T$ symmetric $\langle\overline{1} 10\rangle$ surface plane. The Dirac cone surface states is along the path $\tilde{\Gamma} \tilde{X}$ constrained by the glide mirror symmetry $\tilde{M}_{z}=\left\{M_{z} \mid(0.5,0.5,0)\right\}$ and the dashed line indicates the "curved Fermi level", the band number below which equals that of electrons. (f) The surface states of $\langle 001\rangle$ surface plane have a full gap in the whole BZ. 


\begin{tabular}{c|c|c|c|c|c|c}
\hline \hline$\Lambda_{\alpha}$ & MLCG & Irreps & $\left\{C_{4 z}^{+} \mid(0.5,0,0)\right\}$ & $I$ & $\left\{M_{z} \mid(0.5,0.5,0)\right\}$ & $\left\{M_{y} \mid(0.5,0,0.5)\right\}$ \\
\hline \multirow{2}{*}{$\Gamma$} & \multirow{2}{*}{$4 / m m m 1^{\prime}$} & $\bar{\Gamma}_{6}(2)$ & $-\sqrt{2}$ & 2 & 0 & 0 \\
\cline { 3 - 7 } & $\bar{\Gamma}_{9}(2)$ & $\sqrt{2}$ & -2 & 0 & 0 \\
\hline \multirow{2}{*}{$\mathrm{Z}$} & \multirow{2}{*}{$4 / m m m 1^{\prime}$} & $\bar{Z}_{5}(2)$ & $-\sqrt{2}$ & 0 & $-2 i$ & 0 \\
\cline { 3 - 6 } & $\bar{Z}_{7}(2)$ & $-\sqrt{2}$ & 0 & $2 i$ & 0 \\
\hline \hline
\end{tabular}

TABLE XII. Character table of the irreducible co-representations $\bar{\Gamma}_{6}, \bar{\Gamma}_{9}, \bar{Z}_{5}$ and $\bar{Z}_{7}$ of MSG $126.386\left(P_{I} 4 / n n c\right)$ at $\Gamma$ and $\mathrm{Z}$ points, respectively. The first two columns are the momenta and their magnetic little co-group(MLCG); the third column is the irreps of the MLCG; the 4th-7th columns are the characters of the symmetry generators of the unitary subgroup. The $\bar{\Gamma}_{6}$ and $\bar{\Gamma}_{9}$ irreps have different eigenvalues of $C_{4 z}$ and $I$, and $\bar{Z}_{5}$ and $\bar{Z}_{7}$ irreps have different eigenvalues of $M_{z}$.

\section{Topological phase diagram of the ideal antiferromagnetic nodal-line semimetal $\mathrm{CeCo}_{2} \mathbf{P}_{2}$}

The space group(SG) of $\mathrm{CeCo}_{2} \mathrm{P}_{2}$ in the paramagnetic phase is $I 4 / \mathrm{mmm}$. The antiferromagnetic phase transition occurs at $440 \mathrm{~K}$ below which the magnetic structure, as shown in FIG. 33.a), is characterized by the MSG 126.386( $\left.P_{I} 4 / n n c\right)$. The generators of this MSG include inversion $I$, four-fold rotation $\tilde{C}_{4 z}=\left\{C_{4 z} \mid(0.5,0,0)\right\}$, two-fold rotation $\tilde{C}_{2 x}=\left\{C_{2 x} \mid(0,0.5,0.5)\right\}$ and $\tilde{C}_{2 x y}=\left\{C_{2 x y} \mid(0,0,0.5)\right\}$, and the anti-unitary translation $T \tau(\tau=(0.5,0.5,0.5))$.

Considering that the correlation effect of the $f$ electron on Ce is very strong, we take the Coulomb interaction strength of the $4 f$ electron in the range $0 \sim 6 \mathrm{eV}$. For convenience, we set $\mathrm{U}=2 \mathrm{eV}$ for the $3 d$ electron of the Co atom. As shown in the phase diagram Table VIII $\mathrm{CeCo}_{2} \mathrm{P}_{2}$ is $\mathrm{ES}$ for all $\mathrm{U}$ values. But we find that there is a topological phase transition between DSM and NLSM around $U=3.85$ eV.

The band structures along $\Gamma-Z-R$ path have been plotted in the FIG. 33(c), with the Coulomb interaction $U=0,2,3.5,4$ and 6 eV. When $U<3.85 \mathrm{eV}$, both the band inversion between the highest occupied valence bands(HOVB) and the lowest unoccupied conduction bands(LUCB) occurs around $\Gamma$ point. The irreps of HOVB and LUCB are $\bar{\Gamma}_{6}$ and $\bar{\Gamma}_{9}$, which have different $C_{4 z}$ eigenvalues (See the characters in Table XII. Thus the band inversion between them creates two crossing points protected by $C_{4 z}$ on the $k_{x}=k_{y}=0$ line. As the Dirac points are symmetry protected on the $k_{x}=k_{y}=0$ line with co-little group $4 / m m m 1^{\prime}, \mathrm{CeCo}_{2} \mathrm{P}_{2}$ is also a higher-order topological SM. 41] With $U>3.85 \mathrm{eV}$, the band inversion around $\Gamma$ point is removed and the two Dirac points annihilate each other. However, a new band inversion between HOVB and LUCB appears around $Z$ point. As the irreps of the two bands at $Z$ point are $\bar{Z}_{5}$ and $\bar{Z}_{7}$, which have different $M_{z}$ eigenvalues, the band crossing between them form a nodal line on the $M_{z}$ invariant $k_{z}=\pi$ plane. We also find that for $\mathrm{U}$ equal to the critical point of $3.85 \mathrm{eV}$, band inversions occur both around $\Gamma$ and $Z$ points. So the Dirac points and nodal line coexist at the critical point $U=3.85 \mathrm{eV}$.

When we take the Coulomb interaction of $f$ electron on Ce to be $6 \mathrm{eV}$ and $d$ electron interaction on Co to be $2 \mathrm{eV}$, the calculated magnetic moments on Co is $0.94 \mu_{B}$, which matches the experimental magnetic moments. Then the band structure in FIG. 33.d) has a clean Fermi surface and forms an ideal antiferromagnetic NLSM. The surface states on the $\langle 001\rangle$ surface plane are calculated with the Wannier tight-binding Hamiltonian. As shown in the FIG. 33(b), there are two branches of surface states connecting to the nodal points near the Fermi level; These are distinguishable from the bulk states and can be observed by the ARPES/STM experiments.

\section{Topological phase diagram of the antiferromagnetic Dirac semimetal $\mathrm{MnGeO}_{3}$}

$\mathrm{MnGeO}_{3}$ with ilmenite structure was reported to be an antiferromagnet with Néel temperature $T_{N}=120 \mathrm{~K}$. The magnetic moment on the divalent cation $\mathrm{Mn}^{2+}$ is about $5 \mu_{B}$, which is realized by the fully polarized high-spin configuration. It has a hexagonal lattice with SG $R \overline{3}$ and MSG $148.19\left(R \overline{3}^{\prime}\right)$ in the paramagnetic and antiferromagnetic phase, respectively. The MSG have two symmetry generators, three-fold rotation $C_{3 z}$ and the combination of inversion and time reversal symmetries $P \cdot T$. The experimental lattice constants $a=5.012$ $\AA$ and $b=14.2986 \AA$ are used in the first principle calculations. Because of the absence of time reversal symmetry, a new situation not possible in the time reversal invariant space groups arises in the MSG $148.19\left(R \overline{3}^{\prime}\right)$. In this situation, all the k points on the $C_{3 z}$ rotational axis are maximal k points. If the eigenvalues of $C_{3 z}$ between any two points on the axis are changed, the system is predicted to be an ES.

We take the Coulomb interaction strength of $3 d$ electron of $\mathrm{Mn}$ from 0 to $4 \mathrm{eV}$ and plot the band structures along the $C_{3 z}$ symmetric path $\bar{F}-\Gamma-F$, as shown in the FIG. 34 where the green and red lines represent the bands that have different eigenvalues of $C_{3 z}$ symmetry. The little co-group(LCG) of the momenta along $\Gamma \mathrm{F}$ path is $\overline{3}^{\prime}$, which have two irreducible co-representations $\bar{\Gamma}_{5} \bar{\Gamma}_{6}$ with $C_{3}=e^{ \pm i \pi / 3}$ doublet and $\bar{\Gamma}_{4} \bar{\Gamma}_{4}$ with $C_{3}=-1,-1$ doublet, where the two doublets are stabilized by the $P \cdot T$ symmetry. Without taking into account the correlation effect, the bands H1 with irrep $\bar{\Gamma}_{5} \bar{\Gamma}_{6}$ and L1 with irrep $\bar{\Gamma}_{4} \bar{\Gamma}_{4}$ have an anti-crossing between the $\bar{F}$ and $\Gamma$ points, which generate two Dirac points (DPs). Upon adding U, the second highest occupied band H2 moves up and there exist 2 pairs of DPs with $U=2 \mathrm{eV}$, as shown in FIG. 34.c). With increasing $U$ to 3 and $4 \mathrm{eV}$, one out of the two pairs DPs annihilates, leaving one pair of DPs locating on the two sides of $\Gamma$ point. In the FIG. 2(c)-(d) of the main text, we have calculated the surface states with $U=4 \mathrm{eV}$, on the $\langle 010\rangle$ surface plane. The surface states connecting to the Dirac cone on $\Gamma F$ path can be distinguished from the bulk states.

In symmorphic groups, Dirac nodes always appear in pairs. Since the irrep of one band is changed when it traverses though a symmetry protected DP, there must be even number of DPs for the irrep to go back to itself after a period of the BZ. It indicates that the crystalline Nielsen-Ninomiya theorem, which guarantees the doubling of the number of Dirac nodes in a crystal is still valid for the Dirac fermions in magnetic crystals. 
a

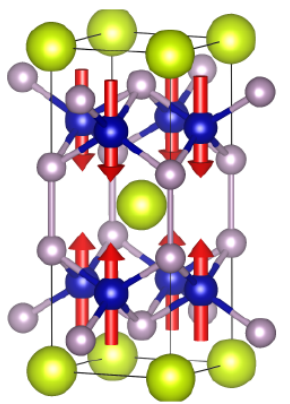

b

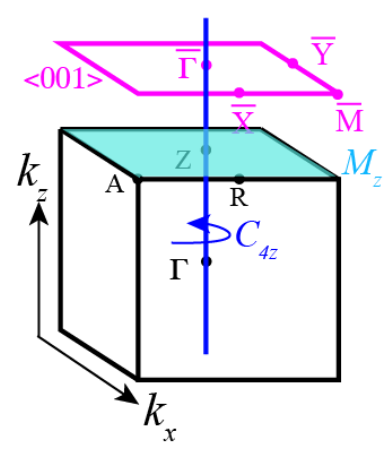

c

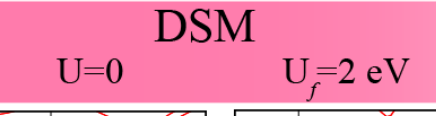

DSM\&NLSM $\mathrm{U}_{f}=3.85 \mathrm{eV}$
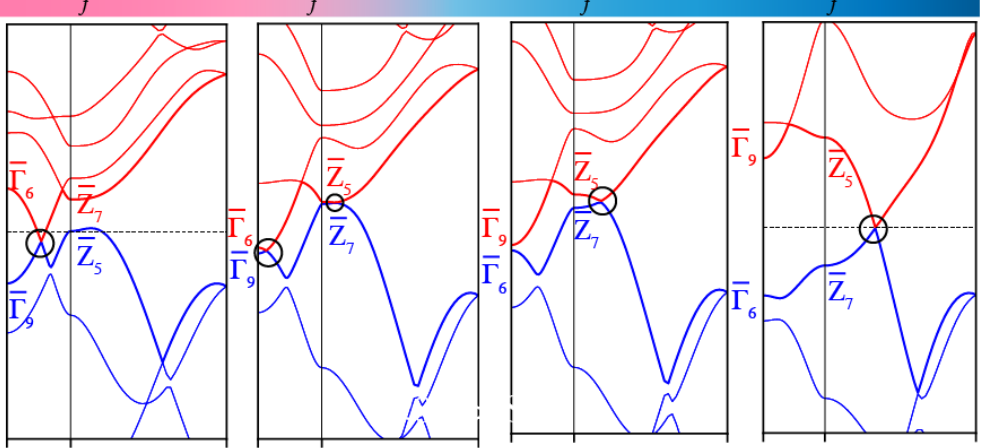

d
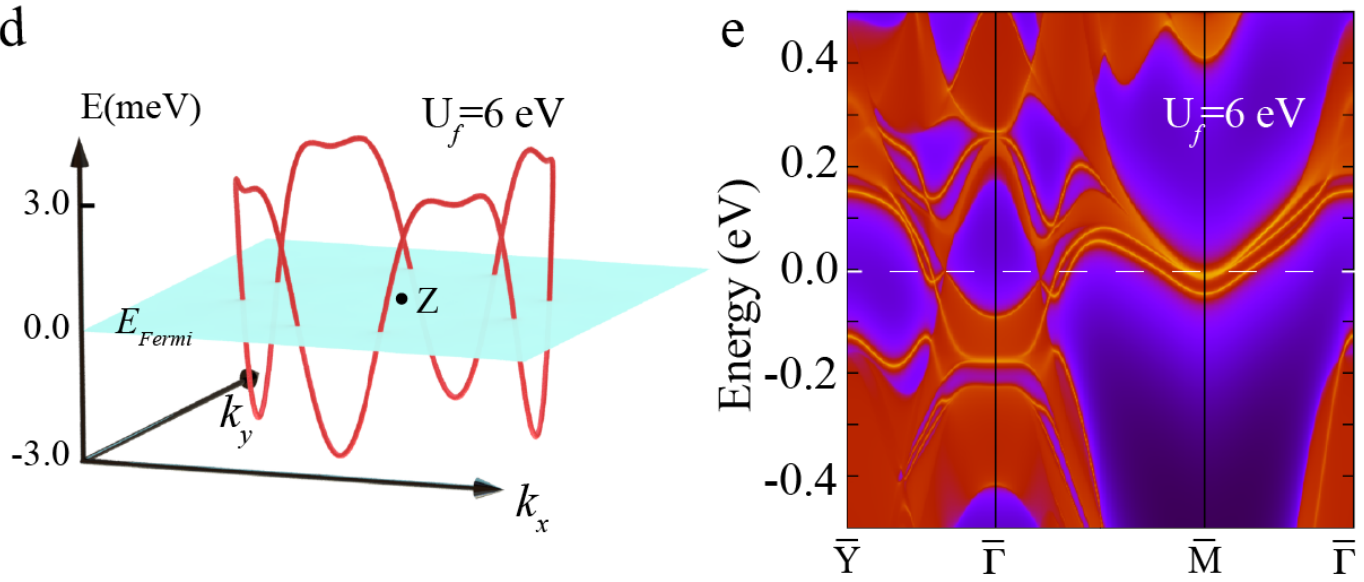

FIG. 33. (a) The crystal and magnetic structures of the antiferromagnetic $\mathrm{CeCo}_{2} \mathrm{P}_{2}$ with MSG $126.386\left(P_{I} 4 / n n c\right)$. (b) The 3D BZ with high symmetry momenta and the surface BZ projected on the $\langle 001\rangle$ surface plane. The $C_{4 z}$ rotation axis along $\Gamma Z$ and the $M_{z}$ plane $k_{z}=\pi$ are represented by the blue line and green plane, respectively. (c) The topological phase diagrams of $\mathrm{CeCo}_{2} \mathrm{P}_{2}$ with different Coulomb interaction strength applied. The irreps of the HOVB and LUCB are blue-colored and red-colored at both $\Gamma$ and $Z$ points. The topology with $U \leq 3 \mathrm{eV}$ belongs to DSM and is transformed to NLSM once $U \geq 4$ $\mathrm{eV}$. All of the nodal points along the high symmetry path are indicated by the black circles. (d) The energy dispersion of the nodal line on the $k_{z}=\pi$ plane, which is protected by $M_{z}$ symmetry. (e) The drumhead-like surface states that connect the nodal line on the $\langle 001\rangle$ surface. The Hubbard $\mathrm{U}$ of $f$ electron on $\mathrm{Ce}$ is equal to $6 \mathrm{eV}$ in (d) and (e).

\section{Weyl nodes, Nodal-lines and Anomalous Hall effect in $\mathrm{Mn}_{3} \mathrm{ZnC}$}

$\mathrm{Mn}_{3} \mathrm{ZnC}$ adopts a cubic lattice with anti-perovskite structure in the paramagnetic phase and undergoes a phase transition to ferromagnetic phase with Currie temperature $T_{c}=470 \mathrm{~K}$. At $215 \mathrm{~K}$, there is a second order phase transition from ferromagnetic to non-collinear ferrimagnetic phase accompanied with a structural transformation from cubic lattice to a body-centered tetragonal lattice, as shown in FIG. 35 In this article, we focus on the low-temperature non-collinear ferrimagnetic phase of $\mathrm{Mn}_{3} \mathrm{ZnC}$

The MSG of ferrimagnetic $\mathrm{Mn}_{3} \mathrm{ZnC}$ is MSG 139.537( $\left.I_{4} / \mathrm{mm}^{\prime} \mathrm{m}^{\prime}\right)$, which is generated by the four-fold rotational symmetry along [001] direction $C_{4 z}$, inversion symmetry $I$ and the anti-unitary symmetry $C_{2 x} \cdot T\left(C_{2 x}\right.$ is the two-fold rotational symmetry along [100] direction and $T$ is time reversal symmetry). There are two non-equivalent Mn atoms occupy the Wyckoff position $4 c(0,1 / 2,0)$ and $8 f(1 / 4,1 / 4,1 / 4)$, respectively. The $\mathrm{Mn}$ atoms on $4 c$ site form the non-collinear antiferromagnetic structure with ferromagnetic canting along [001] direction. While the Mn atoms on $8 f$ site are ferromagnetically polarized along [001] direction. In the ab initio calculations, non-collinear ferrimagnetic $\mathrm{Mn}_{3} \mathrm{ZnC}$ is diagnosed as $\mathrm{ES}$ for all $U$ values (i.e. $U=0,1,2,3,4 \mathrm{eV}$ for $3 d$ electron on $\mathrm{Mn}$ ). Considering the strong correlations on $3 d$ electron, we take $U=4 \mathrm{eV}$ and explain the band structure's topology in detail.

When $U=4 \mathrm{eV}$, compatibility-relations of the band representations are not satisfied along $\Gamma X$ and $\Gamma T$ paths. So there have symmetry enforced band crossings between the HOVB and LUCB along $\Gamma X$ and $\Gamma T$. In FIG. 35;, we plot the band structures along $T-\Gamma-X$ and $S_{0} \Gamma$ near the Fermi level. The irreps of HOVB and LUCB have been indicated by different colors. (See TABLE XIII for the characters of the irreps.) Along $T \Gamma$ path, one can easily find two crossing points, WP3 and WP4, between the HOVB and LUCB, which have different $C_{4 z}$ eigenvalues. So WP3 and WP4 are $C_{4 z}$ protected Weyl points (WPs). On the $\Gamma X$ and $S_{0} \Gamma$ paths, there are many band crossings, which have different $M_{z}$ eigenvalues. So these crossing points on the $k_{z}=0$ plane form the Nodal-lines (NLs) protected by mirror symmetry, $M_{z}$. Using WannierTools package, we find 10 pairs of WPs and 5 NLs in the first BZ. The positions of them are plotted in the BZ, as shown in FIG. 35 The 10 pairs of WPs can be classified to four non-equivalent types, among which WP3 and WP4 are 

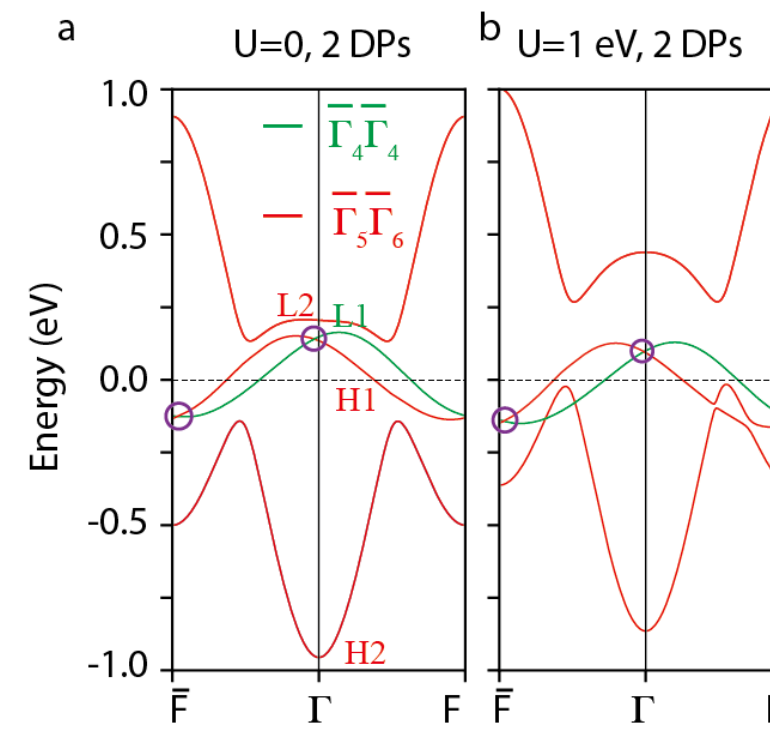

C $\mathrm{U}=2 \mathrm{eV}, 4 \mathrm{DPs}$

d $\mathrm{U}=3 \mathrm{eV}, 2 \mathrm{DPs}$

e $\quad \mathrm{U}=4 \mathrm{eV}, 2 \mathrm{DPs}$
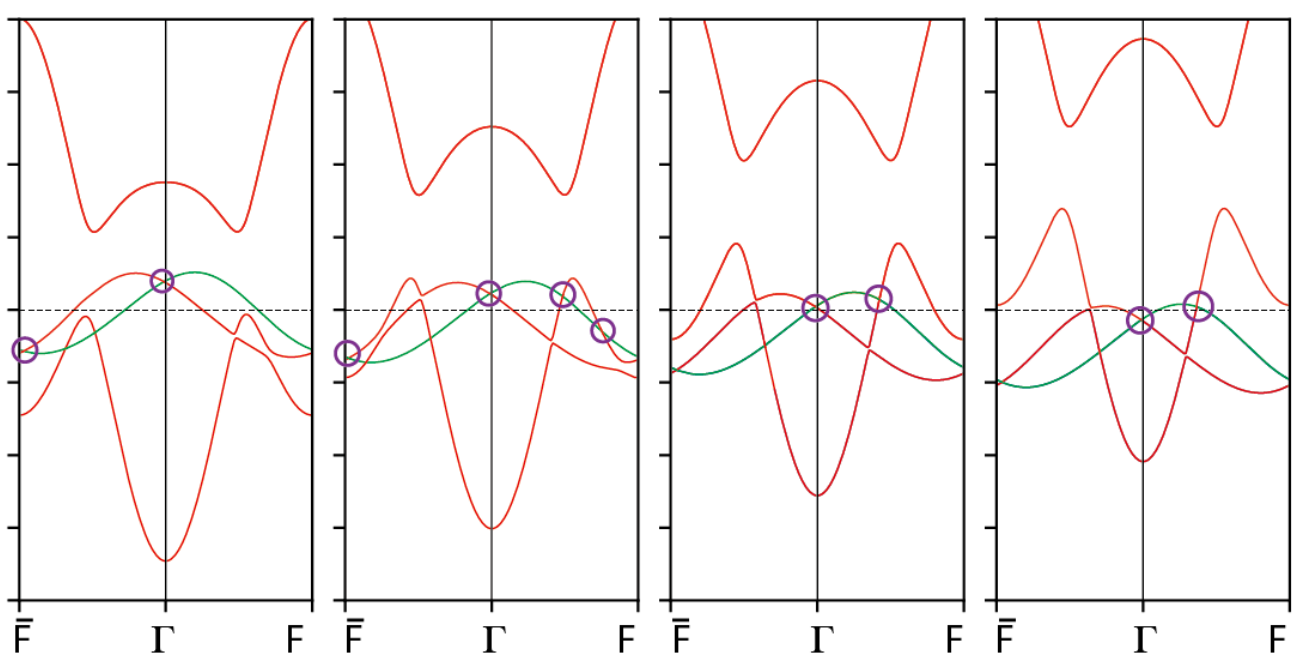

FIG. 34. The band structures and topologies evolution of $\mathrm{MnGeO}_{3}\left(\mathrm{MSG} R \overline{3}^{\prime}\right)$ with the Hubbard $U$ parameter increasing from 0 to $4 \mathrm{eV}$. The $\left(N_{e}-1\right)$ th to $\left(N_{e}+2\right)$ th bands are plotted, with $N_{e}$ being the number of electrons per unit cell. All of the points on the $\bar{F}-\Gamma-F$ path respect the same LCG $\overline{3}^{\prime}$, which have two different 2-dimensional irreps named as $\bar{\Gamma}_{4} \bar{\Gamma}_{4}$ and $\bar{\Gamma}_{5} \bar{\Gamma}_{6}$. The three lines H1, H2 and L2 have the same $\bar{\Gamma}_{5} \bar{\Gamma}_{6}$ representation and are red-colored; the line L1 is the $\bar{\Gamma}_{4} \bar{\Gamma}_{4}$ representation and is blue-colored. (a) For $U=0, \mathrm{~L} 1$ and H1 have an anti-crossing along the $\bar{F} \Gamma$ path and generate two DPs. (b)-(e) With increasing the Coulomb interaction, the lines L2 and $\mathrm{H} 2$ move to higher energy and much more crossing generate between $\mathrm{H} 2$ and the branch of (L1, H1). The DPs formed by the HOVB and LUCB are indicated by the purple circles.

symmetry enforced WPs, WP1 and WP2 are generated by accidental band crossings. WPs in each type are related by $C_{4 z}$ and $M_{z}$ symmetries. Using Wilson loop method [? ], we have identified the chiralities of the WPs, as shown in FIG. 35. We tabulated the exact positions and chiralities of them in TABLE XIV The $5 \mathrm{NLs}$ are classified to two types. The first type (NL1) is symmetry enforced and localized around the $\Gamma$ point. The other four NLs (NL2) are generated by accidental band crossings near $S_{0} \Gamma$ path and they are related by $C_{4 z}$ symmetry.

In time reversal breaking (TRB) systems, nonzero Berry curvatures of occupied Bloch states contribute the intrinsic anomalous Hall conductivities (AHC). In non-collinear ferrimagnetic $\mathrm{Mn}_{3} \mathrm{ZnC}$, the Berry curvatures of bands near the WPs and NLs are very large, which will contribute giant $\mathrm{AHC}$. We calculate the $\mathrm{AHC}$ of ferrimagnetic $\mathrm{Mn}_{3} \mathrm{ZnC}$ by the sum of Berry curvatures over all occupied bands,

$$
\sigma_{x y}=-\frac{2 \pi e^{2}}{h} \int_{B Z} \frac{d^{3} \vec{k}}{(2 \pi)^{3}} \sum_{n} f_{n}(\vec{k}) \Omega_{n}^{z}(\vec{k})
$$

where $f_{n}(\vec{k})$ is the Fermi-Dirac distribution function, and $n$ is the index of the occupied bands. The Berry curvature is arisen from Kubo-formula derivation,

$$
\Omega_{n}^{z}(\vec{k})=-2 \operatorname{Im} \sum_{m \neq n} \frac{\left\langle\Psi_{n \vec{k}}\left|v_{x}\right| \Psi_{m \vec{k}}\right\rangle\left\langle\Psi_{m \vec{k}}\left|v_{y}\right| \Psi_{n \vec{k}}\right\rangle}{\left(E_{m}(\vec{k})-E_{n}(\vec{k})\right)^{2}}
$$

where $v_{x(y)}$ is the velocity operator. We calculate the intrinsic AHC with a $101 \times 101 \times 101 k$-point grid in the first BZ based on the Wannier tight-binding Hamiltonian.

In FIG. 35; , we plot the energy dependence of intrinsic AHC near the Fermi level. On the Fermi level, the AHC is about $-123 \Omega^{-1} \cdot \mathrm{cm}^{-1}$, which can be observed in transport experiments.

\begin{tabular}{c|c|c|c|c|c|c|c}
\hline \hline$\Lambda_{\alpha}$ & MLCG & Irreps & $C_{4 z}^{+}$ & $\Lambda_{\alpha}$ & MLCG & Irreps & $M_{z}$ \\
\hline \multirow{4}{*}{$\operatorname{LD}(0,0, \mathrm{w})$} & \multirow{3}{*}{$42^{\prime} 2^{\prime}$} & $\overline{L D}_{5}$ & $\frac{-1+i}{\sqrt{2}}$ & \multirow{2}{*}{$\mathrm{DT}(\mathrm{u}, \mathrm{u}, 0)$} & $m^{\prime} m 2^{\prime}$ & $\overline{D T}_{3}$ & $-\mathrm{i}$ \\
\cline { 3 - 6 } & & $\overline{L D}_{6}$ & $\frac{1-i}{\sqrt{2}}$ & & $\overline{D T}_{4}$ & $\mathrm{i}$ \\
\cline { 3 - 8 } & & $\overline{L D}_{7}$ & $\frac{-1-i}{\sqrt{2}}$ & \multirow{2}{*}{$\mathrm{SM}(\mathrm{u}, 0,0)$} & $m^{\prime} m 2^{\prime}$ & $\overline{S M}_{3}$ & $-\mathrm{i}$ \\
\cline { 3 - 4 } & & $\overline{L D}_{8}$ & $\frac{1+i}{\sqrt{2}}$ & & & $\overline{S M}_{4}$ & $\mathrm{i}$ \\
\hline \hline
\end{tabular}

TABLE XIII. Character table of the irreducible co-representations of MSG $P_{I} 4 / n n c$ at $L D(0,0, w), D T(u, u, 0)$ and $S M(u, 0,0)$ points. The first two(5th-6th) columns are the momenta and their magnetic little co-groups(MLCG); the third(7th) column are the irreps of the MLCG; the $4 \mathrm{th}(8 \mathrm{th})$ columns are the characters of the symmetry generators of the unitary subgroup. 

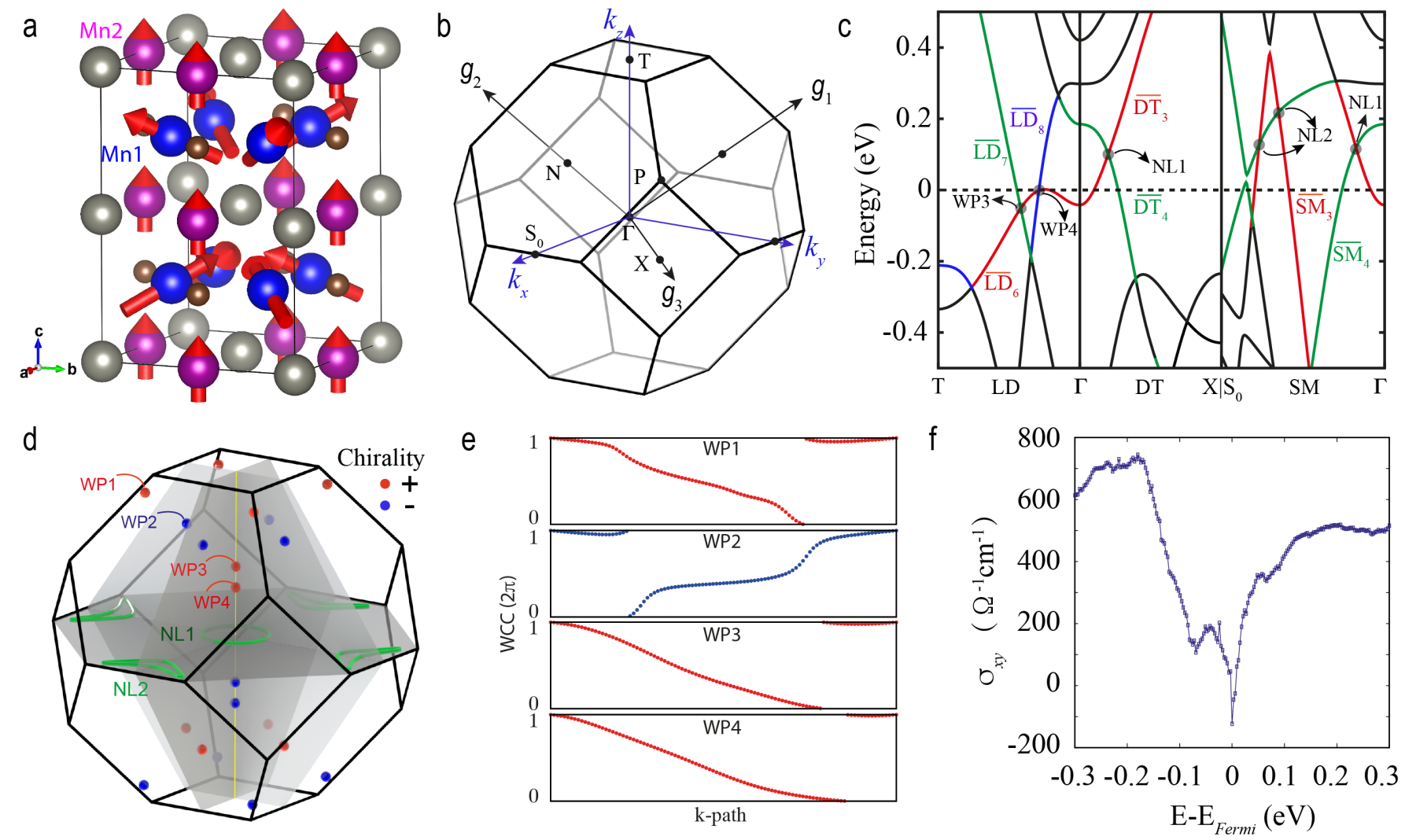

FIG. 35. Weyl nodes and Nodal-lines in ferrimagnetic $\mathrm{Mn}_{3} \mathrm{ZnC}$ (MSG $I_{4} / \mathrm{mm}^{\prime} m^{\prime}$ ) with $U=4 \mathrm{eV}$. (a) The magnetic crystal structure and (b) the corresponding Brillouin zone (BZ) of non-collinear ferrimagnetic $\mathrm{Mn}_{3} \mathrm{ZnC}$. (c) Band structures along the high-symmetry paths $T-\Gamma-X$ and $S_{0} \Gamma$. The irreps of HOVB and LUCB are tagged by different colors. Band crossings on $T \Gamma$ path form the two WPs, WP3 and WP4. Band crossings on the $k_{z}=0$ plane generate 5 NLs, which are protected by $M_{z}$ symmetry. (d) Locations of WPs and NLs in the first BZ. The red(blue) balls stand for WPs with chirality $+1(-1)$. (e) The evolution of Wannier charge centers (WCCs) on the manifold that enclosing WP1, WP2, WP3 and WP4, respectively. (f) Energy dependence of the intrinsic $\mathrm{AHC}$ in the non-collinear ferrimagnetic $\mathrm{Mn}_{3} \mathrm{ZnC}$. The Fermi energy is set to 0.

\section{Appendix K: Fragile bands in the magnetic materials}

In Table $\mathrm{XV}$ we tabulate all of the magnetic materials that have fragile bands below the Fermi level. For the materials having more than one fragile brach, we provide the irreducible co-representations at high symmetry momenta of the fragile branches closest to the Fermi level.

TABLE XV: The fragile occupied bands in magnetic materials. In the first four columns the chemical formulae (Formula), the material identification number on BCSMD (BCSID), the magnetic space group number (MSG) and the Coulomb interaction strength $(\mathrm{U})$ are tabulated. The fifth column gives the number of fragile branches (NF) in the band structure of the corresponding material. In the sixth and seventh columns, the band indices (Bands) and the irreducible representations (Irreps) at high symmetry momenta of the fragile branch closest to the Fermi level are tabulated.

\begin{tabular}{c|c|c|c|c|c|l}
\hline Formula & BCSID & MSG & $\mathrm{U}(\mathrm{eV})$ & $\mathrm{NF}$ & Bands & Irreps \\
\hline CeCoGe3 & 1.0 .11 & 107.231 & 4 & 1 & $-83 \sim-81$ & $\bar{\Gamma}_{6}+\bar{\Gamma}_{8}+\bar{M}_{6}+\bar{M}_{8}+\bar{N}_{2}+\bar{P}_{4}+\bar{X}_{4}$ \\
\hline alpha-Mn & 1.85 & 114.282 & 0 & 5 & $-43 \sim-40$ & $\bar{A}_{6}+\bar{A}_{7}+\bar{\Gamma}_{6}+\bar{M}_{7}+\bar{R}_{2} \bar{R}_{4}+\bar{R}_{3} \bar{R}_{5}+\bar{X}_{2}+\bar{X}_{3}+\bar{X}_{4}+\bar{X}_{5}+\bar{Z}_{6}+\bar{Z}_{7}$ \\
\hline PrCo2P2 & 2.26 & 123.345 & 0 & 1 & $-115 \sim-108$ & $\begin{array}{l}\bar{A}_{10}+\bar{A}_{11}+\bar{A}_{12}+\bar{A}_{5}+\bar{A}_{6}+\bar{A}_{7}+\bar{A}_{8}+\bar{A}_{9}+\bar{\Gamma}_{10}+\bar{\Gamma}_{12}+\bar{\Gamma}_{6}+ \\
\bar{\Gamma}_{8}+\bar{M}_{11}+\bar{M}_{5}+\bar{M}_{6}+\bar{M}_{7}+\bar{M}_{8}+\bar{M}_{9}+\bar{R}_{3}+\bar{R}_{4}+\bar{R}_{5}+\bar{R}_{6}+ \\
\bar{X}_{3}+\bar{X}_{4}+\bar{X}_{5}+\bar{X}_{6}+\bar{Z}_{10}+\bar{Z}_{12}+\bar{Z}_{6}+\bar{Z}_{8}\end{array}$ \\
\hline NdCo2P2 & 1.251 & 124.360 & 0 & 2 & $-119 \sim-112$ & $\begin{array}{l}\bar{A}_{5}+\bar{A}_{6}+\bar{A}_{7}+\bar{A}_{8}+\bar{\Gamma}_{7}+\bar{\Gamma}_{9}+\bar{M}_{6}+\bar{M}_{7}+\bar{M}_{8}+\bar{R}_{3}+\bar{R}_{4}+ \\
\bar{X}_{5}+\bar{X}_{6}+\bar{Z}_{6}+\bar{Z}_{8} \\
\bar{A}_{5}+\bar{A}_{6}+\bar{A}_{7}+\bar{A}_{8}+\bar{\Gamma}_{7}+\bar{\Gamma}_{9}+\bar{M}_{6}+\bar{M}_{7}+\bar{M}_{8}+\bar{R}_{3}+\bar{R}_{4}+ \\
\bar{X}_{5}+\bar{X}_{6}+\bar{Z}_{6}+\bar{Z}_{8}\end{array}$ \\
\hline
\end{tabular}




\begin{tabular}{c|c|c|c}
\hline \hline WPs & Position $\left(k_{x}, k_{y}, k_{z}\right)\left(\AA^{-1}\right)$ & Chirality & $E-E_{\text {Fermi }}(\mathrm{eV})$ \\
\hline \hline \multirow{4}{*}{ WP1 } & $(0.3101,0.3101,0.7154)$ & +1 & -0.0767 \\
\cline { 2 - 4 } & $(-0.3101,-0.3101,0.7154)$ & +1 & -0.0767 \\
\cline { 2 - 4 } & $(-0.3101,0.3101,0.7154)$ & +1 & -0.0767 \\
\cline { 2 - 4 } & $(0.3101,-0.3101,0.7154)$ & +1 & -0.0767 \\
\cline { 2 - 4 } & $(-0.3101,0.3101,-0.7154)$ & -1 & -0.0767 \\
\cline { 2 - 4 } & $(-0.3101,-0.3101,-0.7154)$ & -1 & -0.0767 \\
\cline { 2 - 4 } & $(0.3101,0.3101,-0.7154)$ & -1 & -0.0767 \\
\hline \multirow{4}{*}{ WP2 } & $(0.0,-0.2798,0.5009)$ & -1 & -0.0767 \\
\cline { 2 - 4 } & $(0.0,0.2798,0.5009)$ & -1 & -0.2310 \\
\cline { 2 - 4 } & $(0.2798,0.0,0.5009)$ & -1 & -0.2310 \\
\cline { 2 - 4 } & $(-0.2798,0.0,0.5009)$ & -1 & -0.2310 \\
\cline { 2 - 4 } & $(0.0,0.2798,-0.5009)$ & +1 & -0.2310 \\
\cline { 2 - 4 } & $(0.0,-0.2798,-0.5009)$ & +1 & -0.2310 \\
\hline \multirow{4}{*}{ WP3 } & $(0.2798,0.0,-0.5009)$ & +1 & -0.2310 \\
\cline { 2 - 4 } & $(0.2798,0.0,-0.5009)$ & +1 & -0.2310 \\
\hline \multirow{2}{*}{ WP4 } & $(0.0,0.0,0.3343)$ & -1 & -0.14 \\
\cline { 2 - 4 } & $(0.0,0.0,0.3343)$ & -1 & -0.14 \\
\hline
\end{tabular}

TABLE XIV. The 10 pairs of WPs in the first BZ. The first column is the type of WPs. In each type, the WPs are related by $C_{4 z}$ and $M_{z}$ symmetries. The second column is the position of each WP in the cartesian coordinate system of BZ. The 4th-5th columns are the chirality and energy related to the Fermi level of each WP.

\begin{tabular}{|c|c|c|c|c|c|c|}
\hline UPtGa5 & 1.255 & 124.360 & 6 & 1 & $-37 \sim-30$ & $\begin{array}{l}\bar{A}_{5}+\bar{A}_{7}+\bar{\Gamma}_{7}+\bar{\Gamma}_{8}+\bar{\Gamma}_{9}+\bar{M}_{6}+\bar{M}_{8}+\bar{R}_{3}+\bar{R}_{4}+\bar{X}_{5}+\bar{X}_{6}+\bar{Z}_{5}+ \\
\bar{Z}_{6}+\bar{Z}_{7}+\bar{Z}_{8}\end{array}$ \\
\hline NpRhGa5 & 1.261 & 124.360 & 0 & 4 & $-69 \sim-66$ & $\bar{A}_{5}+\bar{A}_{7}+\bar{\Gamma}_{6}+\bar{M}_{6}+\bar{R}_{3}+\bar{R}_{4}+\bar{X}_{6}+\bar{Z}_{5}+\bar{Z}_{7}$ \\
\hline NpRhGa5 & 1.261 & 124.360 & 4 & 1 & $-167 \sim-164$ & $\bar{A}_{5}+\bar{A}_{8}+\bar{\Gamma}_{8}+\bar{\Gamma}_{9}+\bar{M}_{8}+\bar{M}_{9}+\bar{R}_{3}+\bar{R}_{4}+\bar{X}_{6}+\bar{Z}_{6}+\bar{Z}_{7}$ \\
\hline Fe4Si2Sn7O16 & .197 & 12.63 & 1 & 1 & $-163 \sim-160$ & $\bar{A}_{3} \bar{A}_{5}+\bar{A}_{4} \bar{A}_{6}+\bar{\Gamma}_{3} \bar{\Gamma}_{4}+\bar{L}_{2} \bar{L}_{3}+\bar{M}_{3} \bar{M}_{5}+\bar{M}_{4} \bar{M}_{6}+\bar{V}_{3} \bar{V}_{3}+\bar{Y}_{5} \bar{Y}_{6}$ \\
\hline $\mathrm{MnBr} 2$ & 1.239 & 12.63 & 2 & 1 & $-11 \sim-8$ & $\bar{A}_{3} \bar{A}_{5}+\bar{A}_{4} \bar{A}_{6}+\bar{\Gamma}_{5} \bar{\Gamma}_{6}+\bar{L}_{2} \bar{L}_{3}+\bar{M}_{3} \bar{M}_{5}+\bar{M}_{4} \bar{M}_{6}+\bar{V}_{3} \bar{V}_{3}+\bar{Y}_{3} \bar{Y}_{4}$ \\
\hline $\mathrm{Li} 2 \mathrm{MnO} 3$ & 1.97 & 12.63 & 0 & 2 & $-119 \sim-116$ & $\bar{A}_{3} \bar{A}_{5}+\bar{A}_{4} \bar{A}_{6}+\bar{\Gamma}_{5} \bar{\Gamma}_{6}+\bar{L}_{2} \bar{L}_{3}+\bar{M}_{3} \bar{M}_{5}+\bar{M}_{4} \bar{M}_{6}+\bar{V}_{3} \bar{V}_{3}+\bar{Y}_{3} \bar{Y}_{4}$ \\
\hline U2Ni2In & 1.102 & 128.408 & 0 & 4 & $-155 \sim-140$ & $\begin{array}{l}\bar{A}_{5} \bar{A}_{6}+\bar{A}_{7} \bar{A}_{8}+\bar{\Gamma}_{6}+\bar{\Gamma}_{7}+\bar{M}_{6} \bar{M}_{7}+\bar{R}_{2} \bar{R}_{7}+\bar{R}_{3} \bar{R}_{6}+\bar{R}_{4} \bar{R}_{9}+ \\
\bar{R}_{5} \bar{R}_{8}+\bar{X}_{3} \bar{X}_{4}+\bar{Z}_{5}+\bar{Z}_{6}+\bar{Z}_{7}+\bar{Z}_{8}\end{array}$ \\
\hline U2Ni2In & 1.102 & 128.408 & 2 & 2 & $-155 \sim-140$ & $\begin{array}{l}\bar{A}_{5} \bar{A}_{6}+\bar{A}_{7} \bar{A}_{8}+\bar{\Gamma}_{6}+\bar{\Gamma}_{7}+\bar{M}_{6} \bar{M}_{7}+\bar{R}_{2} \bar{R}_{7}+\bar{R}_{3} \bar{R}_{6}+\bar{R}_{4} \bar{R}_{9}+ \\
\bar{R}_{5} \bar{R}_{8}+\bar{X}_{3} \bar{X}_{4}+\bar{Z}_{5}+\bar{Z}_{6}+\bar{Z}_{7}+\bar{Z}_{8}\end{array}$ \\
\hline U2Ni2In & 1.102 & 128.408 & 4 & 2 & $-155 \sim-140$ & $\begin{array}{l}\bar{A}_{5} \bar{A}_{6}+\bar{A}_{7} \bar{A}_{8}+\bar{\Gamma}_{6}+\bar{\Gamma}_{7}+\bar{M}_{6} \bar{M}_{7}+\bar{R}_{2} \bar{R}_{7}+\bar{R}_{3} \bar{R}_{6}+\bar{R}_{4} \bar{R}_{9}+ \\
\bar{R}_{5} \bar{R}_{8}+\bar{X}_{3} \bar{X}_{4}+\bar{Z}_{5}+\bar{Z}_{6}+\bar{Z}_{7}+\bar{Z}_{8}\end{array}$ \\
\hline U2Ni2In & 1.102 & 128.408 & 6 & 2 & $-171 \sim-164$ & $\begin{array}{l}\bar{A}_{5} \bar{A}_{6}+\bar{A}_{7} \bar{A}_{8}+\bar{\Gamma}_{6}+\bar{\Gamma}_{7}+\bar{M}_{6} \bar{M}_{7}+\bar{R}_{2} \bar{R}_{7}+\bar{R}_{3} \bar{R}_{6}+\bar{R}_{4} \bar{R}_{9}+ \\
\bar{R}_{5} \bar{R}_{8}+\bar{X}_{3} \bar{X}_{4}+\bar{Z}_{5}+\bar{Z}_{6}+\bar{Z}_{7}+\bar{Z}_{8}\end{array}$ \\
\hline TbRh2Si2 & 187 & 28.410 & 0 & & $-65 \sim-62$ & $\bar{A}_{5}+\bar{A}_{7}+\bar{\Gamma}_{7}+\bar{M}_{6}+\bar{M}_{7}+\bar{R}_{2} \bar{R}_{4}+\bar{R}_{3} \bar{R}_{5}+\bar{X}_{3}+\bar{X}_{4}+\bar{Z}_{6}+\bar{Z}_{8}$ \\
\hline TbRh2Si2 & 1.187 & 128.410 & 4 & & $-69 \sim-66$ & $\bar{A}_{5}+\bar{A}_{7}+\bar{\Gamma}_{9}+\bar{M}_{8}+\bar{M}_{9}+\bar{R}_{2} \bar{R}_{4}+\bar{R}_{3} \bar{R}_{5}+\bar{X}_{3}+\bar{X}_{4}+\bar{Z}_{6}+\bar{Z}_{8}$ \\
\hline TbRh2Si2 & 1.187 & 128.410 & 6 & 1 & $-51 \sim-48$ & $\bar{A}_{5}+\bar{A}_{7}+\bar{\Gamma}_{7}+\bar{M}_{6}+\bar{M}_{7}+\bar{R}_{2} \bar{R}_{4}+\bar{R}_{3} \bar{R}_{5}+\bar{X}_{3}+\bar{X}_{4}+\bar{Z}_{6}+\bar{Z}_{8}$ \\
\hline DyCo2Si2 & 1.21 & 128.410 & 0 & 2 & $-63 \sim-58$ & $\begin{array}{l}\bar{A}_{6}+\bar{A}_{7}+\bar{A}_{8}+\bar{\Gamma}_{8}+\bar{\Gamma}_{9}+\bar{M}_{8}+\bar{M}_{9}+\bar{R}_{6} \bar{R}_{8}+\bar{R}_{7} \bar{R}_{9}+\bar{X}_{3}+\bar{X}_{4}+ \\
\bar{Z}_{5}+\bar{Z}_{7}+\bar{Z}_{8}\end{array}$ \\
\hline DyCo2Si2 & 1.21 & 128.410 & 2 & 1 & & $\begin{array}{l}\bar{A}_{5}+\bar{A}_{6}+\bar{A}_{7}+\bar{A}_{8}+\bar{\Gamma}_{7}+\bar{\Gamma}_{8}+\bar{\Gamma}_{9}+\bar{M}_{6}+\bar{M}_{7}+\bar{M}_{8}+\bar{M}_{9}+ \\
\bar{R}_{2} \bar{R}_{4}+\bar{R}_{3} \bar{R}_{5}+\bar{R}_{6} \bar{R}_{8}+\bar{R}_{7} \bar{R}_{9}+\bar{X}_{3}+\bar{X}_{4}+\bar{Z}_{5}+\bar{Z}_{6}+\bar{Z}_{7}+\bar{Z}_{8}\end{array}$ \\
\hline DyCo2Si2 & 1.21 & 128.410 & 4 & 3 & $-9 \sim-6$ & $\bar{A}_{6}+\bar{A}_{7}+\bar{\Gamma}_{6}+\bar{\Gamma}_{9}+\bar{M}_{7}+\bar{M}_{9}+\bar{R}_{6} \bar{R}_{8}+\bar{R}_{7} \bar{R}_{9}+\bar{X}_{3}+\bar{X}_{4}+\bar{Z}_{6}+\bar{Z}_{7}$ \\
\hline $\mathrm{CeMnAsO}$ & 0.186 & 129.416 & 0 & ( & $-1 \sim 0$ & $\bar{A}_{7}+\bar{\Gamma}_{7}+\bar{M}_{6}+\bar{R}_{2} \bar{R}_{4}+\bar{X}_{2} \bar{X}_{4}+\bar{Z}_{6}$ \\
\hline $\mathrm{CeMnAsO}$ & 36 & 129.416 & 3 & & $-9 \sim-8$ & $\bar{A}_{6}+\bar{\Gamma}_{6}+\bar{M}_{7}+\bar{R}_{2} \bar{R}_{4}+\bar{X}_{2} \bar{X}_{4}+\bar{Z}_{7}$ \\
\hline $\mathrm{CeMnAsO}$ & 0.186 & 129.416 & 4 & 6 & $-1 \sim 0$ & $\bar{A}_{7}+\bar{\Gamma}_{7}+\bar{M}_{7}+\bar{R}_{3} \bar{R}_{5}+\bar{X}_{3} \bar{X}_{5}+\bar{Z}_{7}$ \\
\hline CaMnBi2 & 0.72 & 129.416 & 0 & 4 & $-7 \sim-6$ & $\bar{A}_{6}+\bar{\Gamma}_{6}+\bar{M}_{6}+\bar{R}_{3} \bar{R}_{5}+\bar{X}_{3} \bar{X}_{5}+\bar{Z}_{6}$ \\
\hline
\end{tabular}




\begin{tabular}{|c|c|c|c|c|c|c|}
\hline $\mathrm{CaMnBi} 2$ & 0.72 & $\mid 129.416$ & 1 & 4 & $-7 \sim-6$ & $\bar{A}_{6}+\bar{\Gamma}_{6}+\bar{M}_{6}+\bar{R}_{3} \bar{R}_{5}+\bar{X}_{3} \bar{X}_{5}+\bar{Z}_{6}$ \\
\hline $\mathrm{CaMnBi} 2$ & 0.72 & 129.416 & 2 & 8 & $-1 \sim 0$ & $\bar{A}_{7}+\bar{\Gamma}_{6}+\bar{M}_{6}+\bar{R}_{3} \bar{R}_{5}+\bar{X}_{3} \bar{X}_{5}+\bar{Z}_{7}$ \\
\hline $\mathrm{CaMnBi} 2$ & 0.72 & 129.416 & 3 & 5 & $-1 \sim 0$ & $\bar{A}_{7}+\bar{\Gamma}_{6}+\bar{M}_{6}+\bar{R}_{3} \bar{R}_{5}+\bar{X}_{3} \bar{X}_{5}+\bar{Z}_{7}$ \\
\hline $\mathrm{CaMnBi} 2$ & 0.72 & 129.416 & 4 & 5 & $-1 \sim 0$ & $\bar{A}_{7}+\bar{\Gamma}_{6}+\bar{M}_{6}+\bar{R}_{3} \bar{R}_{5}+\bar{X}_{3} \bar{X}_{5}+\bar{Z}_{7}$ \\
\hline UPt2Si2 & 0.194 & 129.419 & 0 & 6 & $-19 \sim-18$ & $\bar{A}_{7}+\bar{\Gamma}_{6}+\bar{M}_{7}+\bar{R}_{2} \bar{R}_{4}$ \\
\hline UPt2Si2 & 0.194 & 129.419 & 2 & 5 & $-39 \sim-38$ & $\bar{A}_{7}+\bar{\Gamma}_{7}+\bar{M}_{7}+\bar{R}_{3} \bar{R}_{5}$ \\
\hline UPt2Si2 & 0.194 & 129.419 & 4 & 9 & $-13 \sim-12$ & $\bar{A}_{7}+\bar{\Gamma}_{7}+\bar{M}_{7}+\bar{R}_{3} \bar{R}_{5}$ \\
\hline UPt2Si2 & 0.194 & 129.419 & 6 & 8 & $-31 \sim-28$ & $\bar{A}_{6}+\bar{A}_{7}+\bar{\Gamma}_{7}+\bar{M}_{6}+\bar{M}_{7}+\bar{R}_{3} \bar{R}_{5}+\bar{X}_{3} \bar{X}_{5}+\bar{Z}_{7}$ \\
\hline $\mathrm{Nd} 2 \mathrm{CuO} 4$ & 2.6 & 134.481 & 2 & 1 & $-71 \sim-56$ & $\bar{A}_{6} \bar{A}_{7}+\bar{\Gamma}_{6}+\bar{\Gamma}_{7}+\bar{\Gamma}_{8}+\bar{\Gamma}_{9}+\bar{M}_{5}+\bar{R}_{3} \bar{R}_{4}+\bar{X}_{3}+\bar{X}_{4}+\bar{Z}_{5}$ \\
\hline UNiGa5 & 1.254 & 140.550 & 6 & 2 & $-43 \sim-40$ & $\bar{\Gamma}_{8}+\bar{\Gamma}_{9}+\bar{M}_{6}+\bar{M}_{7}+\bar{N}_{2}+\bar{P}_{7}+\bar{X}_{5}+\bar{X}_{6}$ \\
\hline Nd2RhIn8 & 1.82 & 140.550 & 4 & 1 & $-97 \sim-90$ & $\bar{\Gamma}_{6}+\bar{\Gamma}_{7}+\bar{\Gamma}_{8}+\bar{M}_{6}+\bar{M}_{7}+\bar{N}_{2}+\bar{P}_{6}+\bar{P}_{7}+\bar{X}_{5}+\bar{X}_{6}$ \\
\hline TbCo2Ga8 & 1.87 & 140.550 & 2 & 1 & $-63 \sim-60$ & $\bar{\Gamma}_{8}+\bar{\Gamma}_{9}+\bar{M}_{6}+\bar{M}_{7}+\bar{N}_{2}+\bar{P}_{6}+\bar{X}_{5}+\bar{X}_{6}$ \\
\hline Er2Ru2O7 & 0.154 & 141.554 & 0 & 1 & $-115 \sim-112$ & $\bar{Z}_{3}+\bar{Z}_{4}+\bar{L}_{3}+\bar{H}_{2} \bar{H}_{4}+\bar{H}_{3}+\bar{H}_{5}+\bar{Y}_{3}+\bar{Y}_{4}+\bar{T}_{3}+\bar{T}_{4}$ \\
\hline $\mathrm{Er} 2 \mathrm{Ru} 2 \mathrm{O} 7$ & 0.154 & 141.554 & 2 & 1 & $-103 \sim-100$ & $\bar{Z}_{3}+\bar{Z}_{4}+\bar{L}_{3}+\bar{H}_{2} \bar{H}_{4}+\bar{H}_{3}+\bar{H}_{5}+\bar{Y}_{3}+\bar{Y}_{4}+\bar{T}_{3}+\bar{T}_{4}+\bar{\Gamma}_{5}+\bar{\Gamma}_{6}$ \\
\hline Er2Ru2O7 & 0.154 & 141.554 & 4 & 1 & $-87 \sim-84$ & $\bar{Z}_{3}+\bar{Z}_{4}+\bar{L}_{3}+\bar{H}_{2} \bar{H}_{4}+\bar{H}_{3}+\bar{H}_{5}+\bar{Y}_{3}+\bar{Y}_{4}+\bar{T}_{3}+\bar{T}_{4}+\bar{\Gamma}_{5}+\bar{\Gamma}_{6}$ \\
\hline Gd2Sn2O7 & 0.47 & 141.555 & 2 & 3 & $-99 \sim-96$ & $\bar{X}_{5} \bar{X}_{6}+\bar{R}_{3}+\bar{R}_{4}+\bar{S}_{3}+\bar{S}_{4}+\bar{W}_{2} \bar{W}_{4}+\bar{W}_{3}+\bar{W}_{5}+\bar{T}_{2}+\bar{\Gamma}_{5}+\bar{\Gamma}_{6}$ \\
\hline $\mathrm{Gd} 2 \mathrm{Sn} 2 \mathrm{O} 7$ & 0.47 & 141.555 & 4 & 1 & $-139 \sim-136$ & $\bar{X}_{5} \bar{X}_{6}+\bar{R}_{5}+\bar{R}_{6}+\bar{S}_{5}+\bar{S}_{6}+\bar{W}_{2} \bar{W}_{4}+\bar{W}_{3}+\bar{W}_{5}+\bar{T}_{2}+\bar{\Gamma}_{5}+\bar{\Gamma}_{6}$ \\
\hline Gd2Sn2O7 & 0.47 & 141.555 & 6 & 1 & $-139 \sim-136$ & $\bar{X}_{5} \bar{X}_{6}+\bar{R}_{5}+\bar{R}_{6}+\bar{S}_{5}+\bar{S}_{6}+\bar{W}_{2} \bar{W}_{4}+\bar{W}_{3}+\bar{W}_{5}+\bar{T}_{2}+\bar{\Gamma}_{5}+\bar{\Gamma}_{6}$ \\
\hline $\mathrm{Tm} 2 \mathrm{Mn} 2 \mathrm{O} 7$ & 0.151 & 141.557 & 0 & 1 & $-25 \sim-12$ & $\begin{array}{l}\bar{\Gamma}_{10}+\bar{\Gamma}_{11}+\bar{\Gamma}_{12}+\bar{\Gamma}_{5}+\bar{\Gamma}_{6}+\bar{\Gamma}_{7}+\bar{\Gamma}_{8}+\bar{\Gamma}_{9}+\bar{M}_{3}+\bar{M}_{4}+\bar{N}_{2}+ \\
\bar{N}_{3}+\bar{P}_{5}+\bar{P}_{6}+\bar{P}_{7}+\bar{P}_{8}+\bar{X}_{2}\end{array}$ \\
\hline $\mathrm{Tb} 2 \mathrm{Sn} 2 \mathrm{O} 7$ & 0.48 & 141.557 & 2 & 1 & $-119 \sim-116$ & $\bar{\Gamma}_{11}+\bar{\Gamma}_{6}+\bar{\Gamma}_{8}+\bar{\Gamma}_{9}+\bar{M}_{3}+\bar{M}_{4}+\bar{N}_{2}+\bar{P}_{5}+\bar{P}_{6}+\bar{P}_{7}+\bar{X}_{2}$ \\
\hline $\mathrm{Tb} 2 \mathrm{Sn} 2 \mathrm{O} 7$ & 0.48 & 141.557 & 4 & 1 & $-119 \sim-116$ & $\bar{\Gamma}_{11}+\bar{\Gamma}_{6}+\bar{\Gamma}_{8}+\bar{\Gamma}_{9}+\bar{M}_{3}+\bar{M}_{4}+\bar{N}_{2}+\bar{P}_{5}+\bar{P}_{6}+\bar{P}_{7}$ \\
\hline Ho2Ru2O7 & 0.51 & 141.557 & 0 & 2 & $-19 \sim-16$ & $\bar{\Gamma}_{5}+\bar{\Gamma}_{6}+\bar{\Gamma}_{7}+\bar{\Gamma}_{8}+\bar{M}_{3}+\bar{M}_{4}+\bar{N}_{2}+\bar{N}_{3}+\bar{P}_{5}+\bar{P}_{7}+\bar{X}_{2}$ \\
\hline $\mathrm{Li} 2 \mathrm{Mn}(\mathrm{SO} 4) 2$ & 0.122 & 14.75 & 0 & 1 & $-121 \sim-118$ & $\bar{A}_{2}+\bar{B}_{2}+\bar{C}_{2}+\bar{D}_{3}+\bar{D}_{4}+\bar{E}_{3}+\bar{E}_{4}+\bar{\Gamma}_{5}+\bar{\Gamma}_{6}+\bar{Y}_{3}+\bar{Y}_{4}+\bar{Z}_{2}$ \\
\hline $\mathrm{Li} 2 \mathrm{Mn}(\mathrm{SO} 4) 2$ & 0.122 & 14.75 & 1 & 2 & $-101 \sim-98$ & $\bar{A}_{2}+\bar{B}_{2}+\bar{C}_{2}+\bar{D}_{5}+\bar{D}_{6}+\bar{E}_{3}+\bar{E}_{4}+\bar{\Gamma}_{5}+\bar{\Gamma}_{6}+\bar{Y}_{5}+\bar{Y}_{6}+\bar{Z}_{2}$ \\
\hline $\mathrm{Li} 2 \mathrm{Mn}(\mathrm{SO} 4) 2$ & 0.122 & 14.75 & 2 & 1 & $-121 \sim-118$ & $\bar{A}_{2}+\bar{B}_{2}+\bar{C}_{2}+\bar{D}_{3}+\bar{D}_{4}+\bar{E}_{3}+\bar{E}_{4}+\bar{\Gamma}_{5}+\bar{\Gamma}_{6}+\bar{Y}_{3}+\bar{Y}_{4}+\bar{Z}_{2}$ \\
\hline $\mathrm{Li} 2 \mathrm{Mn}(\mathrm{SO} 4) 2$ & 0.122 & 14.75 & 3 & 1 & $-121 \sim-118$ & $\bar{A}_{2}+\bar{B}_{2}+\bar{C}_{2}+\bar{D}_{3}+\bar{D}_{4}+\bar{E}_{3}+\bar{E}_{4}+\bar{\Gamma}_{5}+\bar{\Gamma}_{6}+\bar{Y}_{3}+\bar{Y}_{4}+\bar{Z}_{2}$ \\
\hline $\mathrm{Li} 2 \mathrm{Co}(\mathrm{SO} 4) 2$ & 0.121 & 14.79 & 0 & 2 & $-109 \sim-106$ & $\bar{U}_{2} \bar{U}_{3}+\bar{Z}_{2} \bar{Z}_{3}+\bar{V}_{2} \bar{V}_{3}+\bar{T}_{3} \bar{T}_{3}+\bar{R}_{2} \bar{R}_{2}+\bar{X}_{3}+\bar{Y}_{2} \bar{Y}_{3}+\bar{\Gamma}_{3}$ \\
\hline $\mathrm{Li} 2 \mathrm{Co}(\mathrm{SO} 4) 2$ & 0.121 & 14.79 & 1 & 1 & $-125 \sim-122$ & $\bar{U}_{2} \bar{U}_{3}+\bar{Z}_{2} \bar{Z}_{3}+\bar{V}_{2} \bar{V}_{3}+\bar{T}_{3} \bar{T}_{3}+\bar{R}_{2} \bar{R}_{2}+\bar{X}_{2}+\bar{Y}_{2} \bar{Y}_{3}+\bar{\Gamma}_{2}$ \\
\hline $\mathrm{Li} 2 \mathrm{Co}(\mathrm{SO} 4) 2$ & 0.121 & 14.79 & 3 & 1 & $-125 \sim-122$ & $\bar{U}_{2} \bar{U}_{3}+\bar{Z}_{2} \bar{Z}_{3}+\bar{V}_{2} \bar{V}_{3}+\bar{T}_{3} \bar{T}_{3}+\bar{R}_{2} \bar{R}_{2}+\bar{X}_{2}+\bar{Y}_{2} \bar{Y}_{3}+\bar{\Gamma}_{2}$ \\
\hline $\mathrm{Li} 2 \mathrm{Co}(\mathrm{SO} 4) 2$ & 0.121 & 14.79 & 4 & 1 & $-125 \sim-122$ & $\bar{U}_{2} \bar{U}_{3}+\bar{Z}_{2} \bar{Z}_{3}+\bar{V}_{2} \bar{V}_{3}+\bar{T}_{3} \bar{T}_{3}+\bar{R}_{2} \bar{R}_{2}+\bar{X}_{2}+\bar{Y}_{2} \bar{Y}_{3}+\bar{\Gamma}_{2}$ \\
\hline $\mathrm{NiN} 2 \mathrm{O} 6$ & 0.78 & 148.17 & 0 & 1 & $-87 \sim-86$ & $\bar{F}_{2}+\bar{\Gamma}_{5}+\bar{\Gamma}_{6}+\bar{L}_{3}+\bar{T}_{8}+\bar{T}_{9}$ \\
\hline $\mathrm{NiN} 2 \mathrm{O} 6$ & 0.78 & 148.17 & 2 & 1 & $-153 \sim-151$ & $\bar{F}_{2}+\bar{F}_{3}+\bar{\Gamma}_{7}+\bar{\Gamma}_{8}+\bar{\Gamma}_{9}+\bar{L}_{2}+\bar{T}_{5}+\bar{T}_{6}+\bar{T}_{7}$ \\
\hline $\mathrm{NiN} 2 \mathrm{O} 6$ & 0.78 & 148.17 & 3 & 1 & $-153 \sim-151$ & $\bar{F}_{2}+\bar{F}_{3}+\bar{\Gamma}_{7}+\bar{\Gamma}_{8}+\bar{\Gamma}_{9}+\bar{L}_{2}+\bar{T}_{5}+\bar{T}_{6}+\bar{T}_{7}$ \\
\hline $\mathrm{NiCO} 3$ & 0.113 & 15.85 & 1 & 1 & $-23 \sim-20$ & $\bar{A}_{2}+\bar{\Gamma}_{5}+\bar{\Gamma}_{6}+\bar{L}_{3}+\bar{M}_{2}+\bar{V}_{2}+\bar{V}_{3}+\bar{Y}_{5}+\bar{Y}_{6}$ \\
\hline $\mathrm{CoCO} 3$ & 0.114 & 15.85 & 2 & 1 & $-27 \sim-22$ & $\bar{A}_{2}+\bar{\Gamma}_{3}+\bar{\Gamma}_{4}+\bar{\Gamma}_{5}+\bar{\Gamma}_{6}+\bar{L}_{2}+\bar{M}_{2}+\bar{V}_{2}+\bar{V}_{3}+\bar{Y}_{3}+\bar{Y}_{4}$ \\
\hline $\mathrm{CoCO} 3$ & 0.114 & 15.85 & 3 & 1 & $-11 \sim-8$ & $\bar{A}_{2}+\bar{\Gamma}_{5}+\bar{\Gamma}_{6}+\bar{L}_{3}+\bar{M}_{2}+\bar{V}_{2}+\bar{V}_{3}+\bar{Y}_{5}+\bar{Y}_{6}$ \\
\hline $\mathrm{CoCO} 3$ & 0.114 & 15.85 & 4 & 1 & $-11 \sim-8$ & $\bar{A}_{2}+\bar{\Gamma}_{5}+\bar{\Gamma}_{6}+\bar{L}_{3}+\bar{M}_{2}+\bar{V}_{2}+\bar{V}_{3}+\bar{Y}_{5}+\bar{Y}_{6}$ \\
\hline $\mathrm{MnCO} 3$ & 0.115 & 15.85 & 0 & 1 & $-19 \sim-16$ & $\bar{A}_{2}+\bar{\Gamma}_{3}+\bar{\Gamma}_{4}+\bar{L}_{2}+\bar{M}_{2}+\bar{V}_{2}+\bar{V}_{3}+\bar{Y}_{3}+\bar{Y}_{4}$ \\
\hline $\mathrm{MnCO} 3$ & 0.115 & 15.85 & 1 & 1 & $-13 \sim-10$ & $\bar{A}_{2}+\bar{\Gamma}_{5}+\bar{\Gamma}_{6}+\bar{L}_{3}+\bar{M}_{2}+\bar{V}_{2}+\bar{V}_{3}+\bar{Y}_{5}+\bar{Y}_{6}$ \\
\hline $\mathrm{MnCO} 3$ & 0.115 & 15.85 & 4 & 1 & $-9 \sim-6$ & $\bar{A}_{2}+\bar{\Gamma}_{5}+\bar{\Gamma}_{6}+\bar{L}_{3}+\bar{M}_{2}+\bar{V}_{2}+\bar{V}_{3}+\bar{Y}_{5}+\bar{Y}_{6}$ \\
\hline FeBO3 & 0.112 & 15.89 & 1 & 1 & $-19 \sim-16$ & $\bar{Z}_{2} \bar{Z}_{3}+\bar{U}_{3}+\bar{T}_{2}+\bar{R}_{2} \bar{R}_{3}+\bar{X}_{2}+\bar{X}_{3}+\bar{Y}_{2}+\bar{Y}_{3}+\bar{V}_{3}+\bar{\Gamma}_{3}$ \\
\hline $\mathrm{FeBO} 3$ & 0.112 & 15.89 & 2 & 1 & $-19 \sim-16$ & $\bar{Z}_{2} \bar{Z}_{3}+\bar{U}_{3}+\bar{T}_{2}+\bar{R}_{2} \bar{R}_{3}+\bar{X}_{2}+\bar{X}_{3}+\bar{Y}_{2}+\bar{Y}_{3}+\bar{V}_{3}+\bar{\Gamma}_{3}$ \\
\hline $\mathrm{FeBO} 3$ & 0.112 & 15.89 & 3 & 1 & $-3 \sim 0$ & $\bar{Z}_{2} \bar{Z}_{3}+\bar{U}_{2}+\bar{T}_{3}+\bar{R}_{2} \bar{R}_{3}+\bar{X}_{2}+\bar{X}_{3}+\bar{Y}_{2}+\bar{Y}_{3}+\bar{V}_{2}+\bar{\Gamma}_{2}$ \\
\hline $\mathrm{FeBO} 3$ & 0.112 & 15.89 & 4 & 3 & $-3 \sim 0$ & $\bar{Z}_{2} \bar{Z}_{3}+\bar{U}_{2}+\bar{T}_{3}+\bar{R}_{2} \bar{R}_{3}+\bar{X}_{2}+\bar{X}_{3}+\bar{Y}_{2}+\bar{Y}_{3}+\bar{V}_{2}+\bar{\Gamma}_{2}$ \\
\hline Pr3Ru4Al12 & 0.174 & 15.89 & 0 & 1 & $-145 \sim-140$ & $\bar{Z}_{2} \bar{Z}_{3}+\bar{U}_{2}+\bar{U}_{3}+\bar{T}_{2}+\bar{T}_{3}+\bar{R}_{2} \bar{R}_{3}+\bar{X}_{2}+\bar{Y}_{2}+\bar{V}_{2}+\bar{\Gamma}_{2}+\bar{\Gamma}_{3}$ \\
\hline Fe2O3-alpha & 0.65 & 15.89 & 0 & 1 & $-23 \sim-20$ & $\bar{Z}_{2} \bar{Z}_{3}+\bar{U}_{3}+\bar{T}_{2}+\bar{R}_{2} \bar{R}_{3}+\bar{X}_{2}+\bar{X}_{3}+\bar{Y}_{2}+\bar{Y}_{3}+\bar{V}_{2}+\bar{\Gamma}_{2}$ \\
\hline $\mathrm{Yb} 2 \mathrm{O} 2 \mathrm{Se}$ & 1.214 & 15.90 & 2 & 1 & $-103 \sim-100$ & $\bar{A}_{2}+\bar{\Gamma}_{5} \bar{\Gamma}_{6}+\bar{L}_{2} \bar{L}_{3}+\bar{M}_{2}+\bar{V}_{2} \bar{V}_{2}+\bar{Y}_{3} \bar{Y}_{4}$ \\
\hline $\mathrm{CoBr} 2$ & 1.245 & 15.90 & 0 & 1 & $-11 \sim-8$ & $\bar{A}_{2}+\bar{\Gamma}_{5} \bar{\Gamma}_{6}+\bar{L}_{2} \bar{L}_{3}+\bar{M}_{2}+\bar{V}_{2} \bar{V}_{2}+\bar{Y}_{3} \bar{Y}_{4}$ \\
\hline $\mathrm{CoBr} 2$ & 1.245 & 15.90 & 1 & 1 & $-7 \sim-4$ & $\bar{A}_{2}+\bar{\Gamma}_{5} \bar{\Gamma}_{6}+\bar{L}_{2} \bar{L}_{3}+\bar{M}_{2}+\bar{V}_{2} \bar{V}_{2}+\bar{Y}_{3} \bar{Y}_{4}$ \\
\hline $\mathrm{CoBr} 2$ & 1.245 & 15.90 & 2 & 1 & $-7 \sim-4$ & $\bar{A}_{2}+\bar{\Gamma}_{5} \bar{\Gamma}_{6}+\bar{L}_{2} \bar{L}_{3}+\bar{M}_{2}+\bar{V}_{2} \bar{V}_{2}+\bar{Y}_{3} \bar{Y}_{4}$ \\
\hline $\mathrm{CoBr} 2$ & 1.245 & 15.90 & 3 & 1 & $-27 \sim-24$ & $\bar{A}_{2}+\bar{\Gamma}_{3} \bar{\Gamma}_{4}+\bar{L}_{2} \bar{L}_{3}+\bar{M}_{2}+\bar{V}_{3} \bar{V}_{3}+\bar{Y}_{5} \bar{Y}_{6}$ \\
\hline $\mathrm{CoCl} 2$ & 1.246 & 15.90 & 0 & 1 & $-33 \sim-30$ & $\bar{A}_{2}+\bar{\Gamma}_{3} \bar{\Gamma}_{4}+\bar{L}_{2} \bar{L}_{3}+\bar{M}_{2}+\bar{V}_{3} \bar{V}_{3}+\bar{Y}_{5} \bar{Y}_{6}$ \\
\hline
\end{tabular}




\begin{tabular}{|c|c|c|c|c|c|c|}
\hline $\mathrm{CoCl} 2$ & 1.246 & 15.90 & 1 & 1 & $-11 \sim-8$ & $\bar{A}_{2}+\bar{\Gamma}_{5} \bar{\Gamma}_{6}+\bar{L}_{2} \bar{L}_{3}+\bar{M}_{2}+\bar{V}_{2} \bar{V}_{2}+\bar{Y}_{3} \bar{Y}_{4}$ \\
\hline $\mathrm{CoCl} 2$ & 1.246 & 15.90 & 3 & 1 & $-7 \sim-4$ & $\bar{A}_{2}+\bar{\Gamma}_{5} \bar{\Gamma}_{6}+\bar{L}_{2} \bar{L}_{3}+\bar{M}_{2}+\bar{V}_{2} \bar{V}_{2}+\bar{Y}_{3} \bar{Y}_{4}$ \\
\hline $\mathrm{NiCl} 2$ & 1.247 & 15.90 & 0 & 1 & $-35 \sim-32$ & $\bar{A}_{2}+\bar{\Gamma}_{3} \bar{\Gamma}_{4}+\bar{L}_{2} \bar{L}_{3}+\bar{M}_{2}+\bar{V}_{3} \bar{V}_{3}+\bar{Y}_{5} \bar{Y}_{6}$ \\
\hline $\mathrm{NiCl} 2$ & 1.247 & 15.90 & 1 & 1 & $-35 \sim-32$ & $\bar{A}_{2}+\bar{\Gamma}_{3} \bar{\Gamma}_{4}+\bar{L}_{2} \bar{L}_{3}+\bar{M}_{2}+\bar{V}_{3} \bar{V}_{3}+\bar{Y}_{5} \bar{Y}_{6}$ \\
\hline $\mathrm{NiCl} 2$ & 1.247 & 15.90 & 2 & 1 & $-7 \sim-4$ & $\bar{A}_{2}+\bar{\Gamma}_{5} \bar{\Gamma}_{6}+\bar{L}_{2} \bar{L}_{3}+\bar{M}_{2}+\bar{V}_{2} \bar{V}_{2}+\bar{Y}_{3} \bar{Y}_{4}$ \\
\hline NiBr2 & 1.248 & 15.90 & 0 & 1 & $-35 \sim-32$ & $\bar{A}_{2}+\bar{\Gamma}_{3} \bar{\Gamma}_{4}+\bar{L}_{2} \bar{L}_{3}+\bar{M}_{2}+\bar{V}_{3} \bar{V}_{3}+\bar{Y}_{5} \bar{Y}_{6}$ \\
\hline $\mathrm{NiBr} 2$ & 1.248 & 15.90 & 1 & 2 & $-7 \sim-4$ & $\bar{A}_{2}+\bar{\Gamma}_{5} \bar{\Gamma}_{6}+\bar{L}_{2} \bar{L}_{3}+\bar{M}_{2}+\bar{V}_{2} \bar{V}_{2}+\bar{Y}_{3} \bar{Y}_{4}$ \\
\hline $\mathrm{NiBr} 2$ & 1.248 & 15.90 & 2 & 1 & $-7 \sim-4$ & $\bar{A}_{2}+\bar{\Gamma}_{5} \bar{\Gamma}_{6}+\bar{L}_{2} \bar{L}_{3}+\bar{M}_{2}+\bar{V}_{2} \bar{V}_{2}+\bar{Y}_{3} \bar{Y}_{4}$ \\
\hline $\mathrm{NiBr} 2$ & 1.248 & 15.90 & 3 & 1 & $-7 \sim-4$ & $\bar{A}_{2}+\bar{\Gamma}_{5} \bar{\Gamma}_{6}+\bar{L}_{2} \bar{L}_{3}+\bar{M}_{2}+\bar{V}_{2} \bar{V}_{2}+\bar{Y}_{3} \bar{Y}_{4}$ \\
\hline $\mathrm{Ag} 2 \mathrm{NiO} 2$ & 1.49 & 15.90 & 1 & 1 & $-87 \sim-84$ & $\bar{A}_{2}+\bar{\Gamma}_{5} \bar{\Gamma}_{6}+\bar{L}_{2} \bar{L}_{3}+\bar{M}_{2}+\bar{V}_{3} \bar{V}_{3}+\bar{Y}_{3} \bar{Y}_{4}$ \\
\hline $\mathrm{Ag} 2 \mathrm{NiO} 2$ & 1.49 & 15.90 & 2 & 1 & $-29 \sim-26$ & $\bar{A}_{2}+\bar{\Gamma}_{5} \bar{\Gamma}_{6}+\bar{L}_{2} \bar{L}_{3}+\bar{M}_{2}+\bar{V}_{2} \bar{V}_{2}+\bar{Y}_{3} \bar{Y}_{4}$ \\
\hline $\mathrm{Sr} 2 \mathrm{CoOsO} 6$ & 1.72 & 15.90 & 0 & 5 & $-69 \sim-66$ & $\bar{A}_{2}+\bar{\Gamma}_{3} \bar{\Gamma}_{4}+\bar{L}_{2} \bar{L}_{3}+\bar{M}_{2}+\bar{V}_{2} \bar{V}_{2}+\bar{Y}_{5} \bar{Y}_{6}$ \\
\hline $\mathrm{Sr} 2 \mathrm{CoOsO} 6$ & 1.72 & 15.90 & 1 & 5 & $-69 \sim-66$ & $\bar{A}_{2}+\bar{\Gamma}_{3} \bar{\Gamma}_{4}+\bar{L}_{2} \bar{L}_{3}+\bar{M}_{2}+\bar{V}_{2} \bar{V}_{2}+\bar{Y}_{5} \bar{Y}_{6}$ \\
\hline $\mathrm{Sr} 2 \mathrm{CoOsO} 6$ & 1.72 & 15.90 & 2 & 4 & $-97 \sim-94$ & $\bar{A}_{2}+\bar{\Gamma}_{5} \bar{\Gamma}_{6}+\bar{L}_{2} \bar{L}_{3}+\bar{M}_{2}+\bar{V}_{3} \bar{V}_{3}+\bar{Y}_{3} \bar{Y}_{4}$ \\
\hline $\mathrm{Sr} 2 \mathrm{CoOsO} 6$ & 1.72 & 15.90 & 3 & 6 & $-65 \sim-62$ & $\bar{A}_{2}+\bar{\Gamma}_{3} \bar{\Gamma}_{4}+\bar{L}_{2} \bar{L}_{3}+\bar{M}_{2}+\bar{V}_{3} \bar{V}_{3}+\bar{Y}_{5} \bar{Y}_{6}$ \\
\hline $\mathrm{Sr} 2 \mathrm{CoOsO} 6$ & 1.72 & 15.90 & 4 & 5 & $-65 \sim-62$ & $\bar{A}_{2}+\bar{\Gamma}_{3} \bar{\Gamma}_{4}+\bar{L}_{2} \bar{L}_{3}+\bar{M}_{2}+\bar{V}_{2} \bar{V}_{2}+\bar{Y}_{5} \bar{Y}_{6}$ \\
\hline $\mathrm{VCl} 2$ & 1.237 & 159.64 & 1 & 2 & $-115 \sim-114$ & $\bar{A}_{4}+\bar{A}_{5}+\bar{\Gamma}_{4} \bar{\Gamma}_{5}+\bar{H} A_{6}+\bar{K} A_{6}+\bar{L}_{3}+\bar{L}_{4}+\bar{M}_{3} \bar{M}_{4}$ \\
\hline $\mathrm{VCl} 2$ & 1.237 & 159.64 & 2 & 2 & $-115 \sim-114$ & $\bar{A}_{4}+\bar{A}_{5}+\bar{\Gamma}_{4} \bar{\Gamma}_{5}+\bar{H} A_{6}+\bar{K} A_{6}+\bar{L}_{3}+\bar{L}_{4}+\bar{M}_{3} \bar{M}_{4}$ \\
\hline $\mathrm{VCl} 2$ & 1.237 & 159.64 & 3 & 2 & $-115 \sim-114$ & $\bar{A}_{4}+\bar{A}_{5}+\bar{\Gamma}_{4} \bar{\Gamma}_{5}+H A_{6}+\bar{K} A_{6}+\bar{L}_{3}+\bar{L}_{4}+\bar{M}_{3} \bar{M}_{4}$ \\
\hline $\mathrm{VCl} 2$ & 1.237 & 159.64 & 4 & 2 & $-115 \sim-114$ & $\bar{A}_{4}+\bar{A}_{5}+\bar{\Gamma}_{4} \bar{\Gamma}_{5}+\bar{H} A_{6}+\bar{K} A_{6}+\bar{L}_{3}+\bar{L}_{4}+\bar{M}_{3} \bar{M}_{4}$ \\
\hline $\mathrm{SrRu} 2 \mathrm{O} 6$ & 1.186 & 162.78 & 0 & 3 & $-31 \sim-24$ & $\begin{array}{l}\bar{A}_{4} \bar{A}_{6}+\bar{A}_{5} \bar{A}_{7}+\bar{A}_{8} \bar{A}_{9}+\bar{\Gamma}_{4} \bar{\Gamma}_{5}+\bar{\Gamma}_{8}+\bar{H}_{4} \bar{H}_{5}+\bar{H}_{6}+\bar{K}_{4} \bar{K}_{5}+\bar{K}_{6}+ \\
\bar{L}_{3} \bar{L}_{5}+\bar{L}_{4} \bar{L}_{6}+\bar{M}_{3} \bar{M}_{4}+\bar{M}_{5} \bar{M}_{6}\end{array}$ \\
\hline $\mathrm{SrRu} 2 \mathrm{O} 6$ & 1.186 & 162.78 & 1 & 3 & $-31 \sim-24$ & $\begin{array}{l}\bar{A}_{4} \bar{A}_{6}+\bar{A}_{5} \bar{A}_{7}+\bar{A}_{8} \bar{A}_{9}+\bar{\Gamma}_{4} \bar{\Gamma}_{5}+\bar{\Gamma}_{8}+\bar{H}_{4} \bar{H}_{5}+\bar{H}_{6}+\bar{K}_{4} \bar{K}_{5}+\bar{K}_{6}+ \\
\bar{L}_{3} \bar{L}_{5}+\bar{L}_{4} \bar{L}_{6}+\bar{M}_{3} \bar{M}_{4}+\bar{M}_{5} \bar{M}_{6}\end{array}$ \\
\hline $\mathrm{SrRu} 2 \mathrm{O} 6$ & 1.186 & 162.78 & 2 & 3 & $-31 \sim-24$ & $\begin{array}{l}\bar{A}_{4} \bar{A}_{6}+\bar{A}_{5} \bar{A}_{7}+\bar{A}_{8} \bar{A}_{9}+\bar{\Gamma}_{4} \bar{\Gamma}_{5}+\bar{\Gamma}_{8}+\bar{H}_{4} \bar{H}_{5}+\bar{H}_{6}+\bar{K}_{4} \bar{K}_{5}+\bar{K}_{6}+ \\
\bar{L}_{3} \bar{L}_{5}+\bar{L}_{4} \bar{L}_{6}+\bar{M}_{3} \bar{M}_{4}+\bar{M}_{5} \bar{M}_{6}\end{array}$ \\
\hline $\mathrm{SrRu} 2 \mathrm{O} 6$ & 1.186 & 162.78 & 3 & 3 & $-31 \sim-24$ & $\begin{array}{l}\bar{A}_{4} \bar{A}_{6}+\bar{A}_{5} \bar{A}_{7}+\bar{A}_{8} \bar{A}_{9}+\bar{\Gamma}_{4} \bar{\Gamma}_{5}+\bar{\Gamma}_{8}+\bar{H}_{4} \bar{H}_{5}+\bar{H}_{6}+\bar{K}_{4} \bar{K}_{5}+\bar{K}_{6}+ \\
\bar{L}_{3} \bar{L}_{5}+\bar{L}_{4} \bar{L}_{6}+\bar{M}_{3} \bar{M}_{4}+\bar{M}_{5} \bar{M}_{6}\end{array}$ \\
\hline $\mathrm{SrRu} 2 \mathrm{O} 6$ & 1.186 & 162.78 & 4 & 3 & $-31 \sim-20$ & $\begin{array}{l}\bar{A}_{4} \bar{A}_{6}+\bar{A}_{5} \bar{A}_{7}+\bar{A}_{8} \bar{A}_{9}+\bar{\Gamma}_{4} \bar{\Gamma}_{5}+\bar{\Gamma}_{8}+\bar{\Gamma}_{9}+\bar{H}_{4} \bar{H}_{5}+\bar{H}_{6}+\bar{K}_{4} \bar{K}_{5}+ \\
\bar{K}_{6}+\bar{L}_{3} \bar{L}_{5}+\bar{L}_{4} \bar{L}_{6}+\bar{M}_{3} \bar{M}_{4}+\bar{M}_{5} \bar{M}_{6}\end{array}$ \\
\hline FeBr2 & 1.242 & 165.96 & 0 & 5 & $-3 \sim-2$ & $\bar{A}_{4}+\bar{\Gamma}_{4} \bar{\Gamma}_{5}+\bar{H}_{6}+\bar{K}_{6}+\bar{L}_{2}+\bar{M}_{3} \bar{M}_{4}$ \\
\hline $\mathrm{FeBr} 2$ & 1.242 & 165.96 & 1 & 5 & $-3 \sim-2$ & $\bar{A}_{4}+\bar{\Gamma}_{4} \bar{\Gamma}_{5}+\bar{H}_{6}+\bar{K}_{6}+\bar{L}_{2}+\bar{M}_{3} \bar{M}_{4}$ \\
\hline FeBr2 & 1.242 & 165.96 & 2 & 7 & $-3 \sim-2$ & $\bar{A}_{4}+\bar{\Gamma}_{4} \bar{\Gamma}_{5}+\bar{H}_{6}+\bar{K}_{6}+\bar{L}_{2}+\bar{M}_{3} \bar{M}_{4}$ \\
\hline $\mathrm{FeBr} 2$ & 1.242 & 165.96 & 3 & 7 & $-3 \sim-2$ & $\bar{A}_{4}+\bar{\Gamma}_{4} \bar{\Gamma}_{5}+\bar{H}_{6}+\bar{K}_{6}+\bar{L}_{2}+\bar{M}_{3} \bar{M}_{4}$ \\
\hline $\mathrm{FeBr} 2$ & 1.242 & 165.96 & 4 & 7 & $-11 \sim-4$ & $\begin{array}{l}\bar{A}_{4}+\bar{A}_{6}+\bar{\Gamma}_{4} \bar{\Gamma}_{5}+\bar{\Gamma}_{6} \bar{\Gamma}_{7}+\bar{\Gamma}_{9}+\bar{H}_{4} \bar{H}_{5}+\bar{H}_{6}+\bar{K}_{4} \bar{K}_{5}+\bar{K}_{6}+\bar{L}_{2}+ \\
\bar{M}_{3} \bar{M}_{4}+\bar{M}_{5} \bar{M}_{6}\end{array}$ \\
\hline Mn3Ir & 0.108 & 166.101 & 3 & 1 & $-15 \sim-11$ & $\bar{F}_{2}+\bar{F}_{3}+\bar{\Gamma}_{4}+\bar{\Gamma}_{5}+\bar{\Gamma}_{6}+\bar{L}_{2}+\bar{L}_{3}+\bar{T}_{4}+\bar{T}_{5}+\bar{T}_{6}$ \\
\hline $\mathrm{Mn} 3 \mathrm{Pt}$ & 0.109 & 166.101 & 1 & 1 & $-6 \sim-4$ & $\bar{F}_{2}+\bar{F}_{3}+\bar{\Gamma}_{4}+\bar{\Gamma}_{5}+\bar{\Gamma}_{6}+\bar{L}_{3}+\bar{T}_{4}+\bar{T}_{8}+\bar{T}_{9}$ \\
\hline Nd3Sb3Mg2O14 & 0.167 & 166.101 & 0 & 5 & $-4 \sim-3$ & $\bar{F}_{3}+\bar{\Gamma}_{8}+\bar{\Gamma}_{9}+\bar{L}_{3}+\bar{T}_{8}+\bar{T}_{9}$ \\
\hline Nd3Sb3Mg2O14 & 0.167 & 166.101 & 2 & 4 & $-1 \sim 0$ & $\bar{F}_{3}+\bar{\Gamma}_{7}+\bar{\Gamma}_{8}+\bar{L}_{3}+\bar{T}_{7}+\bar{T}_{8}$ \\
\hline $\mathrm{TbCo} 2$ & 0.228 & 166.101 & 0 & 2 & $-57 \sim-56$ & $\bar{F}_{2}+\bar{\Gamma}_{5}+\bar{\Gamma}_{6}+\bar{L}_{2}+\bar{T}_{5}+\bar{T}_{6}$ \\
\hline $\mathrm{TbCo} 2$ & 0.228 & 166.101 & 2 & 1 & $-36 \sim-35$ & $\bar{F}_{2}+\bar{\Gamma}_{5}+\bar{\Gamma}_{6}+\bar{L}_{2}+\bar{T}_{5}+\bar{T}_{6}$ \\
\hline Tb2Ti2O7 & 0.77 & 166.101 & 6 & 7 & $-14 \sim-10$ & $\bar{F}_{2}+\bar{F}_{3}+\bar{\Gamma}_{7}+\bar{\Gamma}_{8}+\bar{\Gamma}_{9}+\bar{L}_{2}+\bar{L}_{3}+\bar{T}_{4}+\bar{T}_{5}+\bar{T}_{8}+\bar{T}_{9}$ \\
\hline Gd2Ti2O7 & 1.56 & 166.102 & 0 & 3 & $-121 \sim-118$ & $\bar{F}_{3} \bar{F}_{4}+\bar{\Gamma}_{9}+\bar{L}_{3} \bar{L}_{5}+\bar{L}_{4} \bar{L}_{6}+\bar{T}_{8} \bar{T}_{9}$ \\
\hline Gd2Ti2O7 & 1.56 & 166.102 & 4 & 1 & $-197 \sim-194$ & $\bar{F}_{3} \bar{F}_{4}+\bar{\Gamma}_{9}+\bar{L}_{3} \bar{L}_{5}+\bar{L}_{4} \bar{L}_{6}+\bar{T}_{8} \bar{T}_{9}$ \\
\hline $\mathrm{Gd} 2 \mathrm{Ti} 2 \mathrm{O} 7$ & 1.56 & 166.102 & 6 & 1 & $-197 \sim-194$ & $\bar{F}_{3} \bar{F}_{4}+\bar{\Gamma}_{9}+\bar{L}_{3} \bar{L}_{5}+\bar{L}_{4} \bar{L}_{6}+\bar{T}_{8} \bar{T}_{9}$ \\
\hline $\mathrm{Mn} 3 \mathrm{Cu} 0.5 \mathrm{Ge} 0.5 \mathrm{~N}$ & 0.74 & 166.97 & 0 & 2 & $-12 \sim-11$ & $\bar{F}_{5}+\bar{F}_{6}+\bar{\Gamma}_{8}+\bar{L}_{5}+\bar{L}_{6}+\bar{T}_{8}$ \\
\hline $\mathrm{FeCO} 3$ & 0.116 & 167.103 & 1 & 1 & $-13 \sim-10$ & $\bar{F}_{3}+\bar{F}_{4}+\bar{\Gamma}_{6}+\bar{\Gamma}_{7}+\bar{\Gamma}_{9}+\bar{L}_{2}+\bar{T}_{4}+\bar{T}_{5}$ \\
\hline $\mathrm{FeCO} 3$ & 0.116 & 167.103 & 3 & 1 & $-25 \sim-22$ & $\bar{F}_{5}+\bar{F}_{6}+\bar{\Gamma}_{4}+\bar{\Gamma}_{5}+\bar{\Gamma}_{8}+\bar{L}_{2}+\bar{T}_{4}+\bar{T}_{5}$ \\
\hline $\mathrm{AgFe} 3(\mathrm{SO} 4) 2(\mathrm{OD}) 6$ & 1.129 & 167.108 & 0 & 4 & $-55 \sim-50$ & $\bar{F}_{3} \bar{F}_{4}+\bar{\Gamma}_{6} \bar{\Gamma}_{7}+\bar{\Gamma}_{8}+\bar{\Gamma}_{9}+\bar{L}_{2}+\bar{T}_{4}+\bar{T}_{5}+\bar{T}_{6}$ \\
\hline $\mathrm{AgFe} 3(\mathrm{SO} 4) 2(\mathrm{OD}) 6$ & 1.129 & 167.108 & 1 & 4 & $-65 \sim-62$ & $\bar{F}_{5} \bar{F}_{6}+\bar{\Gamma}_{4} \bar{\Gamma}_{5}+\bar{\Gamma}_{8}+\bar{L}_{2}+\bar{T}_{4}+\bar{T}_{5}$ \\
\hline $\mathrm{AgFe} 3(\mathrm{SO} 4) 2(\mathrm{OD}) 6$ & 1.129 & 167.108 & 2 & 2 & $-143 \sim-140$ & $\bar{F}_{5} \bar{F}_{6}+\bar{\Gamma}_{8}+\bar{L}_{2}+\bar{T}_{5}+\bar{T}_{6}$ \\
\hline $\mathrm{AgFe} 3(\mathrm{SO} 4) 2(\mathrm{OD}) 6$ & 1.129 & 167.108 & 3 & 1 & $-241 \sim-238$ & $\bar{F}_{5} \bar{F}_{6}+\bar{\Gamma}_{8}+\bar{L}_{2}+\bar{T}_{5}+\bar{T}_{6}$ \\
\hline $\mathrm{AgFe} 3(\mathrm{SO} 4) 2(\mathrm{OD}) 6$ & 1.129 & $\mid 167.108$ & 4 & 1 & $|-241 \sim-238|$ & $\bar{F}_{5} \bar{F}_{6}+\bar{\Gamma}_{8}+\bar{L}_{2}+\bar{T}_{5}+\bar{T}_{6}$ \\
\hline
\end{tabular}




\begin{tabular}{|c|c|c|c|c|c|c|}
\hline $\mathrm{Mn} 3 \mathrm{GaC}$ & 1.153 & 167.108 & 0 & 1 & $-29 \sim-24$ & $\bar{F}_{5} \bar{F}_{6}+\bar{\Gamma}_{4} \bar{\Gamma}_{5}+\bar{\Gamma}_{8}+\bar{\Gamma}_{9}+\bar{L}_{2}+\bar{T}_{4}+\bar{T}_{6}$ \\
\hline $\mathrm{Mn} 3 \mathrm{GaC}$ & 1.153 & 167.108 & 1 & 1 & $-3 \sim 0$ & $\bar{F}_{5} \bar{F}_{6}+\bar{\Gamma}_{4} \bar{\Gamma}_{5}+\bar{\Gamma}_{8}+\bar{L}_{2}+\bar{T}_{4}+\bar{T}_{6}$ \\
\hline $\mathrm{Mn} 3 \mathrm{GaC}$ & 1.153 & 167.108 & 2 & 1 & $-47 \sim-44$ & $\bar{F}_{5} \bar{F}_{6}+\bar{\Gamma}_{4} \bar{\Gamma}_{5}+\bar{\Gamma}_{8}+\bar{L}_{2}+\bar{T}_{4}+\bar{T}_{6}$ \\
\hline $\mathrm{Mn} 3 \mathrm{GaC}$ & 1.153 & 167.108 & 3 & 2 & $-23 \sim-20$ & $\bar{F}_{5} \bar{F}_{6}+\bar{\Gamma}_{4} \bar{\Gamma}_{5}+\bar{\Gamma}_{8}+\bar{L}_{2}+\bar{T}_{4}+\bar{T}_{6}$ \\
\hline $\mathrm{Mn} 3 \mathrm{GaC}$ & 1.153 & 167.108 & 4 & 1 & $-29 \sim-26$ & $\bar{F}_{5} \bar{F}_{6}+\bar{\Gamma}_{4} \bar{\Gamma}_{5}+\bar{\Gamma}_{8}+\bar{L}_{2}+\bar{T}_{4}+\bar{T}_{6}$ \\
\hline $\mathrm{FeCl} 2$ & 1.241 & 167.108 & 0 & 2 & $-9 \sim-6$ & $\bar{F}_{3} \bar{F}_{4}+\bar{\Gamma}_{6} \bar{\Gamma}_{7}+\bar{\Gamma}_{9}+\bar{L}_{2}+\bar{T}_{4}+\bar{T}_{6}$ \\
\hline $\mathrm{FeCl} 2$ & 1.241 & 167.108 & 1 & 1 & $-9 \sim-6$ & $\bar{F}_{3} \bar{F}_{4}+\bar{\Gamma}_{6} \bar{\Gamma}_{7}+\bar{\Gamma}_{9}+\bar{L}_{2}+\bar{T}_{4}+\bar{T}_{6}$ \\
\hline YMnO3 & 0.44 & 173.131 & 4 & 1 & $-199 \sim-196$ & $\bar{A}_{5} \bar{A}_{6}+\bar{\Gamma}_{5} \bar{\Gamma}_{6}+\bar{H}_{4} \bar{H}_{4}+\bar{H} A_{4} \bar{H} A_{4}+\bar{K}_{4}+l$ \\
\hline HoMnO3 & 0.32 & 185.197 & 6 & 8 & $-15 \sim-14$ & $\bar{A}_{7}+\bar{\Gamma}_{7}+\bar{H}_{6}+\bar{K}_{6}$ \\
\hline HoMnO3 & 0.33 & 185.197 & 4 & 8 & $-21 \sim-20$ & $\bar{A}_{7}+\bar{\Gamma}_{7}+\bar{H}_{6}+\bar{K}_{6}$ \\
\hline YMnO3 & 0.6 & 185.197 & 3 & 1 & $-95 \sim-92$ & $\bar{A}_{7}+\bar{\Gamma}_{7}+\bar{H}_{4}+\bar{H}_{5}+\bar{H}_{6}+\bar{K}_{4}+\bar{K}_{5}+\bar{K}_{6}+\bar{L}_{5}+\bar{M}_{5}$ \\
\hline $\mathrm{LuFeO} 3$ & 0.117 & 185.201 & 2 & 1 & $-181 \sim-178$ & $\begin{array}{l}\bar{A}_{10} \bar{A}_{9}+\bar{A}_{11} \bar{A}_{12}+\bar{\Gamma}_{10}+\bar{\Gamma}_{11}+\bar{\Gamma}_{12}+\bar{\Gamma}_{9}+\bar{H}_{4} \bar{H}_{4}+\bar{K}_{4}+\bar{L}_{3} \bar{L}_{4}+ \\
\bar{M}_{3}+\bar{M}_{4}\end{array}$ \\
\hline $\mathrm{LuFeO} 3$ & 0.117 & 185.201 & 3 & 1 & $-181 \sim-178$ & $\begin{array}{l}\bar{A}_{10} \bar{A}_{9}+\bar{A}_{11} \bar{A}_{12}+\bar{\Gamma}_{10}+\bar{\Gamma}_{11}+\bar{\Gamma}_{12}+\bar{\Gamma}_{9}+\bar{H}_{4} \bar{H}_{4}+\bar{K}_{4}+\bar{L}_{3} \bar{L}_{4}+ \\
\bar{M}_{3}+\bar{M}_{4}\end{array}$ \\
\hline $\mathrm{LuFeO} 3$ & 0.117 & 185.201 & 4 & 1 & $-181 \sim-178$ & $\begin{array}{l}\bar{A}_{10} \bar{A}_{9}+\bar{A}_{11} \bar{A}_{12}+\bar{\Gamma}_{10}+\bar{\Gamma}_{11}+\bar{\Gamma}_{12}+\bar{\Gamma}_{9}+\bar{H}_{4} \bar{H}_{4}+\bar{K}_{4}+\bar{L}_{3} \bar{L}_{4}+ \\
\bar{M}_{3}+\bar{M}_{4}\end{array}$ \\
\hline $\mathrm{ScMnO} 3$ & 0.7 & 185.201 & 0 & 1 & -199 & $\begin{array}{l}\bar{A}_{10} \bar{A}_{9}+\bar{A}_{11} \bar{A}_{12}+\bar{\Gamma}_{10}+\bar{\Gamma}_{11}+\bar{\Gamma}_{12}+\bar{\Gamma}_{9}+\bar{H}_{4} \bar{H}_{4}+\bar{K}_{4}+\bar{L}_{3} \bar{L}_{4}+ \\
\bar{M}_{3}+\bar{M}_{4}\end{array}$ \\
\hline ScMn6Ge6 & 110 & 192.252 & 0 & 3 & $-167 \sim-164$ & $\bar{A}_{11}+\bar{A}_{8}+\bar{\Gamma}_{11}+\bar{\Gamma}_{9}+\bar{H}_{4} \bar{H}_{5}+\bar{H}_{9}+\bar{K}_{7}+\bar{K}_{\varepsilon}$ \\
\hline ScMn6Ge6 & 1.110 & 192.252 & 4 & 3 & $-189 \sim-186$ & $\bar{A}_{12}+\bar{A}_{8}+\bar{\Gamma}_{11}+\bar{\Gamma}_{8}+\bar{H}_{4} \bar{H}_{5}+\bar{H}_{6} \bar{H}_{7}+\bar{K}_{7}+\bar{L}_{3}+\bar{L}_{4}+\bar{M}_{5}+\bar{M}_{6}$ \\
\hline ScMn6Ge6 & 1.225 & 192.252 & 0 & 6 & $-31 \sim-22$ & $\begin{array}{l}\bar{A}_{10}+\bar{A}_{11}+\bar{A}_{7}+\bar{A}_{8}+\bar{\Gamma}_{12}+\bar{\Gamma}_{7}+\bar{\Gamma}_{8}+\bar{\Gamma}_{9}+\bar{H}_{4} \bar{H}_{5}+\bar{H}_{6} \bar{H}_{7}+ \\
\bar{H}_{8}+\bar{H}_{9}+\bar{K}_{7}+\bar{K}_{8}+\bar{K}_{9}+\bar{L}_{3}+\bar{L}_{4}+\bar{M}_{5}+\bar{M}_{6}\end{array}$ \\
\hline ScMn6Ge6 & 1.225 & 192.252 & 1 & 3 & $-281 \sim-278$ & $\bar{A}_{11}+\bar{A}_{9}+\bar{\Gamma}_{12}+\bar{\Gamma}_{9}+\bar{H}_{4} \bar{H}_{5}+\bar{H}_{6} \bar{H}_{7}+\bar{K}_{7}+\bar{L}_{3}+\bar{L}_{4}+\bar{M}_{5}+\bar{M}_{6}$ \\
\hline ScMn6Ge6 & 1.225 & 192.252 & 2 & 4 & $-163 \sim-152$ & $\begin{array}{l}\bar{A}_{10}+\bar{A}_{11}+\bar{A}_{12}+\bar{A}_{7}+\bar{A}_{8}+\bar{\Gamma}_{10}+\bar{\Gamma}_{11}+\bar{\Gamma}_{7}+\bar{\Gamma}_{8}+\bar{\Gamma}_{9}+\bar{H}_{4} \bar{H}_{5}+ \\
\bar{H}_{6} \bar{H}_{7}+\bar{H}_{8}+\bar{H}_{9}+\bar{K}_{7}+\bar{K}_{8}+\bar{K}_{9}+\bar{L}_{3}+\bar{L}_{4}+\bar{M}_{5}+\bar{M}_{6}\end{array}$ \\
\hline ScMn6Ge6 & 1.225 & 192.252 & 3 & 2 & $-281 \sim-278$ & $\bar{A}_{11}+\bar{A}_{9}+\bar{\Gamma}_{12}+\bar{\Gamma}_{9}+\bar{H}_{4} \bar{H}_{5}+\bar{H}_{6} \bar{H}_{7}+\bar{K}_{7}+\bar{L}_{3}+\bar{L}_{4}+\bar{M}_{5}+\bar{M}_{6}$ \\
\hline ScMn6Ge6 & 1.225 & 192.252 & 4 & 2 & $-281 \sim-278$ & $\bar{A}_{11}+\bar{A}_{9}+\bar{\Gamma}_{12}+\bar{\Gamma}_{9}+\bar{H}_{4} \bar{H}_{5}+\bar{H}_{6} \bar{H}_{7}+\bar{K}_{7}+\bar{L}_{3}+\bar{L}_{4}+\bar{M}_{5}+\bar{M}_{6}$ \\
\hline $\mathrm{Ba} 5 \mathrm{Co} 5 \mathrm{ClO} 13$ & 0.118 & 194.268 & 1 & 28 & $-13 \sim-10$ & $\bar{A}_{5}+\bar{\Gamma}_{8}+\bar{\Gamma}_{9}+\bar{H}_{4} \bar{H}_{5}+\bar{H}_{6}+\bar{K}_{4}+\bar{K}_{5}+\bar{K}_{6}+\bar{L}_{2}+\bar{M}_{3}+\bar{M}_{4}+\bar{M}_{5}+\bar{M}_{6}$ \\
\hline $\mathrm{Ba} 5 \mathrm{Co} 5 \mathrm{ClO} 13$ & 0.118 & 194.268 & 2 & 21 & $-11 \sim-10$ & $\bar{A}_{4}+\bar{\Gamma}_{6}+\bar{\Gamma}_{7}+\bar{H}_{6}+\bar{K}_{6}+\bar{L}_{2}+\bar{M}_{5}+\bar{M}_{6}$ \\
\hline $\mathrm{Ba} 5 \mathrm{Co} 5 \mathrm{ClO} 13$ & 0.118 & 194.268 & 3 & 29 & $-3 \sim-2$ & $\bar{A}_{4}+\bar{\Gamma}_{4}+\bar{\Gamma}_{5}+\bar{H}_{6}+\bar{K}_{6}+\bar{L}_{2}+\bar{M}_{3}+\bar{M}_{4}$ \\
\hline $\mathrm{Ba} 5 \mathrm{Co} 5 \mathrm{ClO} 13$ & 0.118 & 194.268 & 4 & 27 & $-5 \sim-4$ & $\bar{A}_{4}+\bar{\Gamma}_{6}+\bar{\Gamma}_{7}+\bar{H}_{6}+\bar{K}_{6}+\bar{L}_{2}+\bar{M}_{5}+\bar{M}_{6}$ \\
\hline $\mathrm{Na} 3 \mathrm{Co}(\mathrm{CO} 3) 2 \mathrm{Cl}$ & 0.70 & 203.26 & 1 & 2 & $-83 \sim-80$ & $\bar{\Gamma}_{8}+\bar{L}_{6}+\bar{L}_{8}+\bar{L}_{9}+\bar{W}_{2}$ \\
\hline $\mathrm{Na} 3 \mathrm{Co}(\mathrm{CO} 3) 2 \mathrm{Cl}$ & 0.70 & 203.26 & 2 & 1 & $-15 \sim-8$ & $\begin{array}{l}\bar{\Gamma}_{5}+\bar{\Gamma}_{7}+\bar{\Gamma}_{9}+\bar{L}_{4}+\bar{L}_{5}+\bar{L}_{6}+\bar{L}_{7}+\bar{L}_{8}+\bar{L}_{9}+\bar{W}_{2}+\bar{W}_{3}+\bar{W}_{4}+ \\
\bar{W}_{5}+\bar{X}_{3}+\bar{X}_{4}\end{array}$ \\
\hline $\mathrm{Na} 3 \mathrm{Co}(\mathrm{CO} 3) 2 \mathrm{Cl}$ & 0.70 & 203.26 & 3 & 4 & $-11 \sim-8$ & $\bar{\Gamma}_{7}+\bar{\Gamma}_{9}+\bar{L}_{4}+\bar{L}_{5}+\bar{L}_{7}+\bar{L}_{9}+\bar{W}_{2}+\bar{W}_{3}+\bar{W}_{4}+\bar{W}_{5}+\bar{X}_{3}+\bar{X}_{4}$ \\
\hline NiS2 & 0.150 & 205.33 & 0 & 1 & $-63 \sim-52$ & $\bar{\Gamma}_{10}+\bar{\Gamma}_{5}+\bar{\Gamma}_{9}+\bar{M}_{3}+\bar{M}_{4}+\bar{R}_{10}+\bar{R}_{11}+\bar{R}_{4}+\bar{R}_{8}+\bar{R}_{9}+\bar{X}_{3}+\bar{X}_{4}$ \\
\hline $\mathrm{NiS} 2$ & 0.150 & 205.33 & 1 & 1 & $-63 \sim-52$ & $\bar{\Gamma}_{10}+\bar{\Gamma}_{5}+\bar{\Gamma}_{9}+\bar{M}_{3}+\bar{M}_{4}+\bar{R}_{10}+\bar{R}_{11}+\bar{R}_{4}+\bar{R}_{8}+\bar{R}_{9}+\bar{X}_{3}+\bar{X}_{4}$ \\
\hline Gd2Ti2O7 & 3.16 & 216.77 & 0 & 6 & $-115 \sim-112$ & $\bar{\Gamma}_{6}+\bar{\Gamma}_{7}+\bar{L}_{6} \bar{L}_{6}+\bar{W}_{5} \bar{W}_{6}+\bar{W}_{7} \bar{W}_{8}+\bar{X}_{6}+\bar{X}_{7}$ \\
\hline NdZn & 3.8 & 222.103 & 2 & 1 & $-59 \sim-28$ & $\bar{\Gamma}_{10}+\bar{\Gamma}_{11}+\bar{\Gamma}_{6}+\bar{\Gamma}_{8}+\bar{\Gamma}_{9}+\bar{M}_{5}+\bar{R}_{5}+\bar{R}_{6}+\bar{R}_{7}+\bar{X}_{5}+\bar{X}_{6}+\bar{X}_{7}+\bar{X}_{8}$ \\
\hline $\mathrm{USb}$ & 3.12 & 224.113 & 4 & 1 & $-11 \sim 0$ & $\bar{\Gamma}_{10}+\bar{\Gamma}_{5}+\bar{\Gamma}_{8}+\bar{\Gamma}_{9}+\bar{M}_{3}+\bar{M}_{4}+\bar{R}_{10}+\bar{R}_{7}+\bar{R}_{8}+\bar{R}_{9}+\bar{X}_{3}+\bar{X}_{4}$ \\
\hline $\mathrm{Cd} 2 \mathrm{Os} 2 \mathrm{O} 7$ & 0.2 & 227.131 & 1 & 3 & $-23 \sim-12$ & $\begin{array}{l}\bar{\Gamma}_{10}+\bar{\Gamma}_{5}+\bar{\Gamma}_{6}+\bar{\Gamma}_{7}+\bar{\Gamma}_{8}+\bar{\Gamma}_{9}+\bar{L}_{4}+\bar{L}_{5}+\bar{L}_{6}+\bar{W}_{2}+\bar{W}_{3} \bar{W}_{5}+ \\
\bar{W}_{4}+\bar{X}_{3}+\bar{X}_{4}\end{array}$ \\
\hline $\mathrm{Cd} 2 \mathrm{Os} 2 \mathrm{O} 7$ & 0.2 & 227.131 & 2 & 1 & $-23 \sim-12$ & $\begin{array}{l}\bar{\Gamma}_{10}+\bar{\Gamma}_{5}+\bar{\Gamma}_{6}+\bar{\Gamma}_{7}+\bar{\Gamma}_{8}+\bar{\Gamma}_{9}+\bar{L}_{4}+\bar{L}_{5}+\bar{L}_{6}+\bar{W}_{2}+\bar{W}_{3} \bar{W}_{5}+ \\
\bar{W}_{4}+\bar{X}_{3}+\bar{X}_{4}\end{array}$ \\
\hline NpSe & 10 & 228.139 & 0 & 2 & $35 \sim-8$ & $\bar{\Gamma}_{11}+\bar{\Gamma}_{6}+\bar{\Gamma}_{8}+\bar{\Gamma}_{9}+\bar{L}_{4}+\bar{L}_{5}+\bar{L}_{6}+\bar{W}_{3} \bar{W}_{4}$ \\
\hline NpSe & 3.10 & 228.139 & 2 & 1 & $-67 \sim-52$ & $\bar{\Gamma}_{10}+\bar{\Gamma}_{11}+\bar{\Gamma}_{8}+\bar{\Gamma}_{9}+\bar{L}_{4}+\bar{L}_{5}+\bar{L}_{6}+\bar{W}_{3} \bar{W}_{4}+V$ \\
\hline $\mathrm{NpTe}$ & 3.11 & 228.139 & 0 & 1 & $-115 \sim-108$ & $\bar{\Gamma}_{11}+\bar{L}_{4}+\bar{L}_{6}+\bar{W}_{3} \bar{W}_{4}+\bar{W}_{5} \bar{W}_{6}+\bar{W}_{7}+\bar{X}_{5}$ \\
\hline $\mathrm{NpTe}$ & 3.11 & 228.139 & 2 & 1 & $-31 \sim-12$ & $\bar{\Gamma}_{11}+\bar{\Gamma}_{6}+\bar{\Gamma}_{8}+\bar{\Gamma}_{9}+\bar{L}_{4}+\bar{L}_{5}+\bar{L}_{6}+\bar{W}_{3} \bar{W}_{4}+\bar{W}_{5} \bar{W}_{6}+\bar{W}_{7}+\bar{X}_{5}$ \\
\hline $\mathrm{NpTe}$ & 3.11 & 228.139 & 6 & 1 & $-67 \sim-60$ & $\bar{\Gamma}_{11}+\bar{\Gamma}_{8}+\bar{\Gamma}_{9}+\bar{L}_{4}+\bar{L}_{5}+\bar{L}_{6}+\bar{W}_{5} \bar{W}_{6}+\bar{W}_{7}+\bar{X}_{5}$ \\
\hline $\mathrm{NpS}$ & 3.9 & 228.139 & 0 & 1 & $-35 \sim-8$ & $\bar{\Gamma}_{11}+\bar{\Gamma}_{6}+\bar{\Gamma}_{8}+\bar{\Gamma}_{9}+\bar{L}_{4}+\bar{L}_{5}+\bar{L}_{6}+\bar{W}_{3} \bar{W}_{4}+\bar{W}_{5} \bar{W}_{6}+\bar{W}_{7}+\bar{X}_{5}$ \\
\hline Fe2O3-alpha & 0.66 & 2.4 & 0 & 1 & $-53 \sim-50$ & $\bar{\Gamma}_{3}+\bar{R}_{2}+\bar{T}_{2}+\bar{U}_{2}+\bar{U}_{3}+\bar{V}_{2}+\bar{V}_{3}+\bar{X}_{2}+\bar{Y}_{2}+\bar{Y}_{3}+\bar{Z}_{2}+\bar{Z}_{3}$ \\
\hline NiS2 & 1.167 & 2.7 & 3 & 1 & $-101 \sim-98$ & $\bar{\Gamma}_{2} \bar{\Gamma}_{2}+\bar{R}_{2} \bar{R}_{3}+\bar{T}_{2} \bar{T}_{3}+\bar{U}_{2} \bar{U}_{3}+\bar{V}_{3} \bar{V}_{3}+\bar{X}_{2} \bar{X}_{2}+\bar{Y}_{2} \bar{Y}_{2}+\bar{Z}_{2} \bar{Z}_{3}$ \\
\hline
\end{tabular}




\begin{tabular}{|c|c|c|c|c|c|c|}
\hline NiS2 & 1.167 & 2.7 & 4 & 1 & $-109 \sim-106$ & $\mid \bar{\Gamma}_{2} \bar{\Gamma}_{2}+\bar{R}_{2} \bar{R}_{3}+\bar{T}_{2} \bar{T}_{3}+\bar{U}_{2} \bar{U}_{3}+\bar{V}_{3} \bar{V}_{3}+\bar{X}_{2} \bar{X}_{2}+\bar{Y}_{2} \bar{Y}_{2}+\bar{Z}_{2} \bar{Z}_{3}$ \\
\hline FePSe3 & 1.210 & 2.7 & 1 & 1 & $-75 \sim-72$ & $\bar{\Gamma}_{2} \bar{\Gamma}_{2}+\bar{R}_{2} \bar{R}_{3}+\bar{T}_{2} \bar{T}_{3}+\bar{U}_{2} \bar{U}_{3}+\bar{V}_{3} \bar{V}_{3}+\bar{X}_{3} \bar{X}_{3}+\bar{Y}_{3} \bar{Y}_{3}+\bar{Z}_{2} \bar{Z}_{3}$ \\
\hline $\mathrm{CrCl} 3$ & 1.244 & 2.7 & 0 & & $-15 \sim-12$ & $\bar{\Gamma}_{2} \bar{\Gamma}_{2}+\bar{R}_{2} \bar{R}_{3}+\bar{T}_{2} \bar{T}_{3}+\bar{U}_{2} \bar{U}_{3}+\bar{V}_{3} \bar{V}_{3}+\bar{X}_{3} \bar{X}_{3}+\bar{Y}_{3} \bar{Y}_{3}+\bar{Z}_{2} \bar{Z}_{3}$ \\
\hline $\mathrm{CrCl} 3$ & 244 & 2.7 & 1 & & $-87 \sim-84$ & $\bar{\Gamma}_{2} \bar{\Gamma}_{2}+\bar{R}_{2} \bar{R}_{3}+\bar{T}_{2} \bar{T}_{3}+\bar{U}_{2} \bar{U}_{3}+\bar{V}_{3} \bar{V}_{3}+\bar{X}_{3} \bar{X}_{3}+\bar{Y}_{3} \bar{Y}_{3}+\bar{Z}_{2} \bar{Z}_{3}$ \\
\hline $\mathrm{CrCl} 3$ & 1.244 & 2.7 & 2 & 2 & $-87 \sim-84$ & $\bar{\Gamma}_{2} \bar{\Gamma}_{2}+\bar{R}_{2} \bar{R}_{3}+\bar{T}_{2} \bar{T}_{3}+\bar{U}_{2} \bar{U}_{3}+\bar{V}_{3} \bar{V}_{3}+\bar{X}_{3} \bar{X}_{3}+\bar{Y}_{3} \bar{Y}_{3}+\bar{Z}_{2} \bar{Z}_{3}$ \\
\hline $\mathrm{CrCl} 3$ & 1.244 & 2.7 & 3 & 3 & $-87 \sim-84$ & $\bar{\Gamma}_{2} \bar{\Gamma}_{2}+\bar{R}_{2} \bar{R}_{3}+\bar{T}_{2} \bar{T}_{3}+\bar{U}_{2} \bar{U}_{3}+\bar{V}_{3} \bar{V}_{3}+\bar{X}_{3} \bar{X}_{3}+\bar{Y}_{3} \bar{Y}_{3}+\bar{Z}_{2} \bar{Z}_{3}$ \\
\hline $\mathrm{CrCl} 3$ & 1.244 & 2.7 & 4 & 4 & $-43 \sim-40$ & $\bar{\Gamma}_{3} \bar{\Gamma}_{3}+\bar{R}_{2} \bar{R}_{3}+\bar{T}_{2} \bar{T}_{3}+\bar{U}_{2} \bar{U}_{3}+\bar{V}_{2} \bar{V}_{2}+\bar{X}_{2} \bar{X}_{2}+\bar{Y}_{2} \bar{Y}_{2}+\bar{Z}_{2} \bar{Z}_{3}$ \\
\hline $\mathrm{BaNi} 2 \mathrm{~V} 2 \mathrm{O} 8$ & 1.256 & 2.7 & 0 & 2 & $-39 \sim-36$ & $\bar{\Gamma}_{2} \bar{\Gamma}_{2}+\bar{R}_{2} \bar{R}_{3}+\bar{T}_{2} \bar{T}_{3}+\bar{U}_{2} \bar{U}_{3}+\bar{V}_{3} \bar{V}_{3}+\bar{X}_{3} \bar{X}_{3}+\bar{Y}_{3} \bar{Y}_{3}+\bar{Z}_{2} \bar{Z}_{3}$ \\
\hline $\mathrm{BaNi} 2 \mathrm{~V} 2 \mathrm{O} 8$ & 256 & 2.7 & 1 & & $-39 \sim-36$ & $\bar{\Gamma}_{2} \bar{\Gamma}_{2}+\bar{R}_{2} \bar{R}_{3}+\bar{T}_{2} \bar{T}_{3}+\bar{U}_{2} \bar{U}_{3}+\bar{V}_{3} \bar{V}_{3}+\bar{X}_{3} \bar{X}_{3}+\bar{Y}_{3} \bar{Y}_{3}+\bar{Z}_{2} \bar{Z}_{3}$ \\
\hline $\mathrm{BaNi} 2 \mathrm{~V} 2 \mathrm{O} 8$ & 1.256 & 2.7 & 2 & & $-63 \sim-60$ & $\bar{\Gamma}_{2} \bar{\Gamma}_{2}+\bar{R}_{2} \bar{R}_{3}+\bar{T}_{2} \bar{T}_{3}+\bar{U}_{2} \bar{U}_{3}+\bar{V}_{3} \bar{V}_{3}+\bar{X}_{3} \bar{X}_{3}+\bar{Y}_{3} \bar{Y}_{3}+\bar{Z}_{2} \bar{Z}_{3}$ \\
\hline $\mathrm{BaNi} 2 \mathrm{~V} 2 \mathrm{O} 8$ & 1.256 & 2.7 & 3 & 2 & $-63 \sim-60$ & $\bar{\Gamma}_{2} \bar{\Gamma}_{2}+\bar{R}_{2} \bar{R}_{3}+\bar{T}_{2} \bar{T}_{3}+\bar{U}_{2} \bar{U}_{3}+\bar{V}_{3} \bar{V}_{3}+\bar{X}_{3} \bar{X}_{3}+\bar{Y}_{3} \bar{Y}_{3}+\bar{Z}_{2} \bar{Z}_{3}$ \\
\hline $\mathrm{BaNi} 2 \mathrm{~V} 2 \mathrm{O} 8$ & 1.256 & 2.7 & 4 & 2 & $-15 \sim-12$ & $\bar{\Gamma}_{2} \bar{\Gamma}_{2}+\bar{R}_{2} \bar{R}_{3}+\bar{T}_{2} \bar{T}_{3}+\bar{U}_{2} \bar{U}_{3}+\bar{V}_{3} \bar{V}_{3}+\bar{X}_{3} \bar{X}_{3}+\bar{Y}_{3} \bar{Y}_{3}+\bar{Z}_{2} \bar{Z}_{3}$ \\
\hline Ho2RhIn8 & 1.139 & 49.273 & 0 & & $-97 \sim-94$ & $\bar{\Gamma}_{5}+\bar{R}_{3}+\bar{R}_{4}+\bar{S}_{6}+\bar{T}_{3}+\bar{T}_{4}+\bar{U}_{3}+\bar{U}_{4}+\bar{X}_{5}+\bar{Y}_{5}+\bar{Z}_{3}+\bar{Z}_{4}$ \\
\hline Er2CoGa8 & 1.222 & 51.298 & 0 & 1 & $-109 \sim-106$ & $\bar{\Gamma}_{6}+\bar{R}_{3}+\bar{R}_{4}+\bar{S}_{3}+\bar{S}_{4}+\bar{T}_{5}+\bar{U}_{3}+\bar{U}_{4}+\bar{X}_{3}+$ \\
\hline Er2CoGa8 & 1.222 & 51.298 & 2 & 1 & $-109 \sim-106$ & $\bar{\Gamma}_{6}+\bar{R}_{3}+\bar{R}_{4}+\bar{S}_{3}+\bar{S}_{4}+\bar{T}_{5}+\bar{U}_{3}+\bar{U}_{4}+\bar{X}_{3}+\bar{X}_{4}+\bar{Y}_{5}+\bar{Z}_{5}$ \\
\hline $\operatorname{PrAg}$ & 1.150 & 53.334 & 0 & 1 & $-7 \sim-4$ & $\begin{array}{l}\bar{\Gamma}_{6}+\bar{R}_{6} \bar{R}_{7}+\bar{R}_{8} \bar{R}_{9}+\bar{S}_{3}+\bar{S}_{4}+\bar{T}_{3}+\bar{T}_{4}+\bar{U}_{2} \bar{U}_{3}+\bar{U}_{4} \bar{U}_{5}+\bar{X}_{3}+ \\
\bar{X}_{4}+\bar{Y}_{6}+\bar{Z}_{3}+\bar{Z}_{4}\end{array}$ \\
\hline YMn3Al4O12 & 1.158 & 58.404 & 3 & 2 & $-151 \sim-148$ & $\begin{array}{l}\bar{\Gamma}_{5}+\bar{R}_{3}+\bar{R}_{4}+\bar{S}_{6}+\bar{T}_{6} \bar{T}_{8}+\bar{T}_{7} \bar{T}_{9}+\bar{U}_{6} \bar{U}_{8}+\bar{U}_{7} \bar{U}_{9}+\bar{X}_{3}+\bar{X}_{4}+ \\
\bar{Y}_{3}+\bar{Y}_{4}+\bar{Z}_{3}+\bar{Z}_{4}\end{array}$ \\
\hline YMn3Al4O12 & 1.158 & 58.404 & 4 & 2 & $-151 \sim-148$ & $\begin{array}{l}\bar{\Gamma}_{5}+\bar{R}_{3}+\bar{R}_{4}+\bar{S}_{6}+\bar{T}_{6} \bar{T}_{8}+\bar{T}_{7} \bar{T}_{9}+\bar{U}_{6} \bar{U}_{8}+\bar{U}_{7} \bar{U}_{9}+\bar{X}_{3}+\bar{X}_{4}+ \\
\bar{Y}_{3}+\bar{Y}_{4}+\bar{Z}_{3}+\bar{Z}_{4}\end{array}$ \\
\hline Nd3Ru4Al12 & 149 & 3.462 & 6 & & $-93 \sim-90$ & $\bar{C}_{2}+\bar{D}_{2}+\bar{Y}_{5}+\bar{Y}_{6}+\bar{B}_{5}+\bar{B}_{6}+\bar{E}_{2}+\bar{A}_{5}+\bar{A}_{6}+\bar{\Gamma}_{3}+\bar{\Gamma}_{4}+\bar{Z}_{2}$ \\
\hline Pr3Ru4Al12 & 173 & 63.462 & 2 & & $-51 \sim-48$ & $\bar{C}_{2}+\bar{D}_{2}+\bar{Y}_{5}+\bar{Y}_{6}+\bar{B}_{5}+\bar{B}_{6}+\bar{E}_{2}+\bar{A}_{5}+\bar{A}_{6}$ \\
\hline Pr3Ru4Al12 & 0.173 & 63.462 & 4 & 1 & $-143 \sim-140$ & $\bar{C}_{2}+\bar{D}_{2}+\bar{Y}_{5}+\bar{Y}_{6}+\bar{B}_{5}+\bar{B}_{6}+\bar{E}_{2}+\bar{A}_{5}+\bar{A}_{6}+\bar{\Gamma}_{3}+\bar{\Gamma}_{4}+\bar{Z}_{2}$ \\
\hline Pr3Ru4Al12 & 0.173 & 63.462 & 6 & 1 & $-143 \sim-140$ & $\bar{C}_{2}+\bar{D}_{2}+\bar{Y}_{5}+\bar{Y}_{6}+\bar{B}_{5}+\bar{B}_{6}+\bar{E}_{2}+\bar{A}_{5}+\bar{A}_{6}+\bar{\Gamma}_{3}+\bar{\Gamma}_{4}+\bar{Z}_{2}$ \\
\hline Mn3Ni20P6 & 145 & 4.480 & 0 & & $-361 \sim-358$ & $\bar{\Gamma}_{6}+\bar{R}_{3} \bar{R}_{4}+\bar{S}_{2}+\bar{T}_{3}+\bar{T}_{4}+\bar{Y}_{5}+\bar{Z}_{3}+\bar{Z}_{4}$ \\
\hline Mn3Ni20P6 & 1.145 & 64.480 & 4 & & $-5 \sim 0$ & $\bar{\Gamma}_{6}+\bar{R}_{5} \bar{R}_{6}+\bar{S}_{2}+\bar{T}_{3}+\bar{T}_{4}+\bar{Y}_{5}+\bar{Y}_{6}+\bar{Z}_{3}+\bar{Z}_{4}$ \\
\hline CeRh2Si2 & 1.188 & 64.480 & 0 & & $-7 \sim-4$ & $\bar{\Gamma}_{5}+\bar{R}_{3} \bar{R}_{4}+\bar{S}_{2}+\bar{T}_{3}+\bar{T}_{4}+\bar{Y}_{6}+\bar{Z}_{3}+\bar{Z}_{4}$ \\
\hline CeRh2Si2 & 1.188 & 64.480 & 2 & & $-11 \sim-8$ & $\bar{\Gamma}_{5}+\bar{R}_{5} \bar{R}_{6}+\bar{S}_{2}+\bar{T}_{3}+\bar{T}_{4}+\bar{Y}_{6}+\bar{Z}_{3}+\bar{Z}_{4}$ \\
\hline CeRh2Si2 & 1.188 & 64.480 & 4 & & $-11 \sim-8$ & $\bar{\Gamma}_{5}+\bar{R}_{5} \bar{R}_{6}+\bar{S}_{2}+\bar{T}_{3}+\bar{T}_{4}+\bar{Y}_{6}+\bar{Z}_{3}+\bar{Z}_{4}$ \\
\hline $\mathrm{CaFe} 2 \mathrm{As} 2$ & .52 & 1.480 & & & $-43 \sim-40$ & $\bar{\Gamma}_{6}+\bar{R}_{5} \bar{R}_{6}+\bar{S}_{2}+\bar{T}_{3}+\bar{T}_{4}+\bar{Y}_{5}+\bar{Z}_{3}+\bar{Z}_{4}$ \\
\hline $\mathrm{CaFe} 2 \mathrm{As} 2$ & 1.52 & 64.480 & 3 & & $3 \sim-40$ & $\bar{\Gamma}_{6}+\bar{R}_{5} \bar{R}_{6}+\bar{S}_{2}+\bar{T}_{3}+\bar{T}_{4}+\bar{Y}_{5}+\bar{Z}_{3}+\bar{Z}_{4}$ \\
\hline $\mathrm{CaFe} 2 \mathrm{As} 2$ & 1.52 & 64.480 & 4 & 1 & $-43 \sim-40$ & $\bar{\Gamma}_{6}+\bar{R}_{5} \bar{R}_{6}+\bar{S}_{2}+\bar{T}_{3}+\bar{T}_{4}+\bar{Y}_{5}+\bar{Z}_{3}+\bar{Z}_{4}$ \\
\hline Mn3Ni20P6 & 2.15 & 65.486 & 1 & 1 & $-831 \sim-830$ & $\bar{L}_{2}+\bar{V}_{3}+\bar{M}_{3}+\bar{M}_{4}+\bar{A}_{5}+\bar{A}_{6}+\bar{\Gamma}_{5}+\bar{\Gamma}_{6}+\bar{Y}_{3}+\bar{Y}_{4}$ \\
\hline $\mathrm{Gd} 2 \mathrm{CuO} 4$ & 1.104 & 66.500 & 0 & & $-17 \sim-14$ & $\bar{\Gamma}_{5}+\bar{R}_{3} \bar{R}_{3}+\bar{R}_{4} \bar{R}_{4}+\bar{S}_{3} \bar{S}_{5}+\bar{S}_{4} \bar{S}_{6}+\bar{T}_{3}+\bar{T}_{4}+\bar{Y}_{5}+\bar{Z}_{3}+\bar{Z}_{4}$ \\
\hline $\mathrm{Gd} 2 \mathrm{CuO} 4$ & 1.104 & 66.500 & 1 & & $-5 \sim-2$ & $\bar{\Gamma}_{6}+\bar{R}_{3} \bar{R}_{3}+\bar{R}_{4} \bar{R}_{4}+\bar{S}_{3} \bar{S}_{5}+\bar{S}_{4} \bar{S}_{6}+\bar{T}_{3}+\bar{T}_{4}+\bar{Y}_{6}+\bar{Z}_{3}+\bar{Z}_{4}$ \\
\hline $\mathrm{NiCr} 2 \mathrm{O} 4$ & 0.4 & 70.530 & 4 & & $-59 \sim-50$ & $\bar{V}_{2}+\bar{V}_{3}+\bar{M}_{2}+\bar{A}_{2}+\bar{Y}_{3} \bar{Y}_{5}+\bar{Y}_{4} \bar{Y}_{6}+\bar{\Gamma}_{3}+\bar{\Gamma}_{4}+\bar{\Gamma}_{5}+\bar{\Gamma}_{6}+\bar{L}_{2}+\bar{L}_{3}$ \\
\hline NpNiGa5 & 2.28 & 74.559 & 2 & & $-67 \sim-64$ & $\bar{L}_{2}+\bar{L}_{3}+\bar{A}_{5}+\bar{A}_{6}+\bar{M}_{3}+\bar{M}_{4}+\bar{V}_{2} \bar{V}_{3}+\bar{U}_{3} \bar{U}_{4}+\bar{Y}_{5}+\bar{Y}_{6}+\bar{\Gamma}_{5}+\bar{\Gamma}_{6}$ \\
\hline $\mathrm{Sr} 2 \mathrm{FeOsO} 6$ & 1.47 & 83.50 & 0 & 1 & $-87 \sim-84$ & $\begin{array}{l}\bar{A}_{11} \bar{A}_{6}+\bar{A}_{8} \bar{A}_{9}+\bar{\Gamma}_{6} \bar{\Gamma}_{8}+\bar{M}_{5} \bar{M}_{8}+\bar{M}_{6} \bar{M}_{7}+\bar{R}_{5} \bar{R}_{5}+\bar{R}_{6} \bar{R}_{6}+\bar{X}_{3} \bar{X}_{5}+ \\
\bar{X}_{4} \bar{X}_{6}+\bar{Z}_{10} \bar{Z}_{8}+\bar{Z}_{12} \bar{Z}_{6}\end{array}$ \\
\hline $\mathrm{a} 2 \mathrm{CoO} 2 \mathrm{Ag} 2 \mathrm{Se} 2$ & 2.24 & 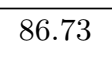 & & & $-179 \sim-172$ & $\bar{A}_{10} \bar{A}_{11}+\bar{A}_{12} \bar{A}_{9}+\bar{\Gamma}_{10} \bar{\Gamma}_{12}+\bar{\Gamma}_{5} \bar{\Gamma}_{7}+\bar{M}_{3} \bar{M}_{4}+\bar{R}_{2}+\bar{X}_{2}+\bar{Z}_{3} \bar{Z}_{4}$ \\
\hline $\mathrm{a} 2 \mathrm{CoO} 2 \mathrm{Ag} 2 \mathrm{Se} 2$ & 2.24 & 86.73 & 1 & & $-179 \sim-172$ & ${ }_{9}+\bar{\Gamma}_{10} \bar{\Gamma}_{12}+\bar{\Gamma}_{5} \bar{\Gamma}_{7}+\bar{M}_{3} \bar{M}_{4}+\bar{R}_{2}+\bar{X}_{2}+\bar{Z}_{3} \bar{Z}_{4}$ \\
\hline $\mathrm{Ba} 2 \mathrm{CoO} 2 \mathrm{Ag} 2 \mathrm{Se} 2$ & 2.24 & 86.73 & J & 2 & $-95 \sim-88$ & $\bar{A}_{5} \bar{A}_{8}+\bar{A}_{6} \bar{A}_{7}+\bar{\Gamma}_{10} \bar{\Gamma}_{12}+\bar{\Gamma}_{5} \bar{\Gamma}_{7}+\bar{M}_{3} \bar{M}_{4}+\bar{R}_{2}+\bar{X}_{2}+\bar{Z}_{3} \bar{Z}_{4}$ \\
\hline $\mathrm{Ba} 2 \mathrm{CoO} 2 \mathrm{Ag} 2 \mathrm{Se} 2$ & 2.24 & 86.73 & 4 & 1 & $-111 \sim-104$ & $\bar{A}_{5} \bar{A}_{8}+\bar{A}_{6} \bar{A}_{7}+\bar{\Gamma}_{11} \bar{\Gamma}_{9}+\bar{\Gamma}_{6} \bar{\Gamma}_{8}+\bar{M}_{3} \bar{M}_{4}+\bar{R}_{2}+\bar{X}_{2}+\bar{Z}_{3} \bar{Z}_{4}$ \\
\hline
\end{tabular}

\section{Appendix L: Magnetic moments for each materials with different Coulomb interactions}

The experimental and calculated magnetic moments on the nonequivalent magnetic atoms of each material have been listed below. As it only consider the moments that contributed by spin component in the VASP, it may have an estimate for those materials that have large orbital magnetic moments. For all of the magnetic materials, we define the average error of the calculated magnetic moments as $\frac{1}{N} \sum_{i=1}^{N} \frac{\left|M_{i}^{E x p}-M_{i}^{T}\right|}{M_{i}^{E x p}} \times 100 \%$, where $N$ is the number of nonequivalent magnetic atoms, $M^{E x p}$ is the experimental magnetic moments and $M^{T}$ is the calculated magnetic moments. We label the magnetic moments that are closest to the experimental value by red color and 


\begin{tabular}{c|c|c|c|c|c}
\hline \hline Average error & $0 \sim 10 \%$ & $10 \% \sim 30 \%$ & $30 \% \sim 50 \%$ & $>50 \%$ & Total \\
\hline Count & 139 & 117 & 68 & 79 & 403 \\
\hline \hline
\end{tabular}

TABLE XVI. Statistics of the materials with the average error of calculated magnetic moments margin in $0 \sim 10 \%, 10 \% \sim 30 \%$, $30 \% \sim 50 \%$ and $>50 \%$.

tabulate the number of materials with the error margin in $010 \%, 10 \% 30 \%, 30 \% 50 \%$ and $>50 \%$ in Table XVI

TABLE XVII: Magnetic moments of the magnetic materials with $3 d / 4 d$ electrons. The first four column give the chemical formulae (Formula), BCSID, MSG for each materials. The 5th to 11th column give the magnetic moments of each nonequivalent magnetic atom, including the experimental (Exp.) magnetic moments and the calculated magnetic moments with Coulomb interaction $U=0,1,2,3,4 \mathrm{eV}$. The calculated magnetic moments closest to the experimental value are tagged by red color.

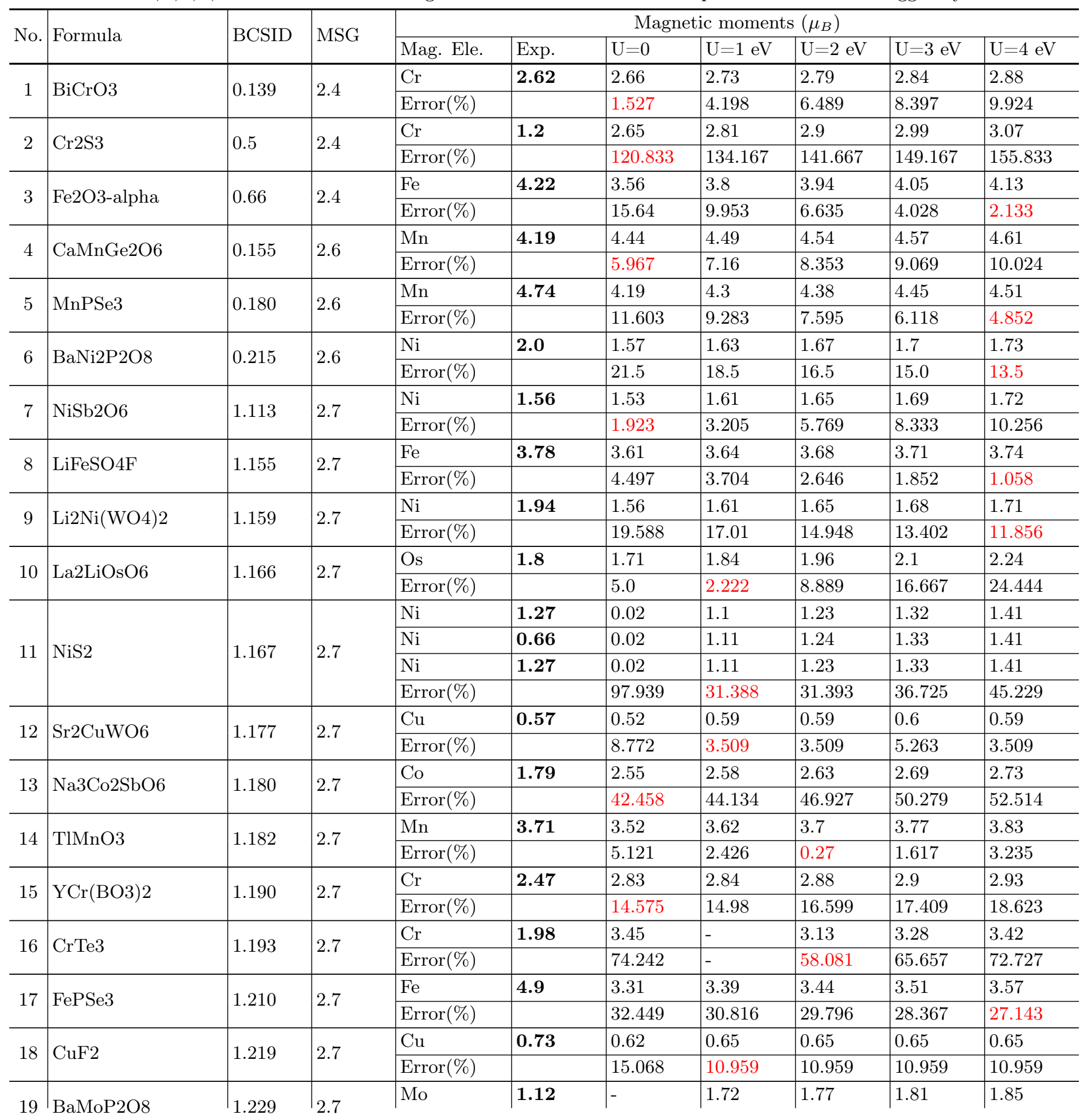




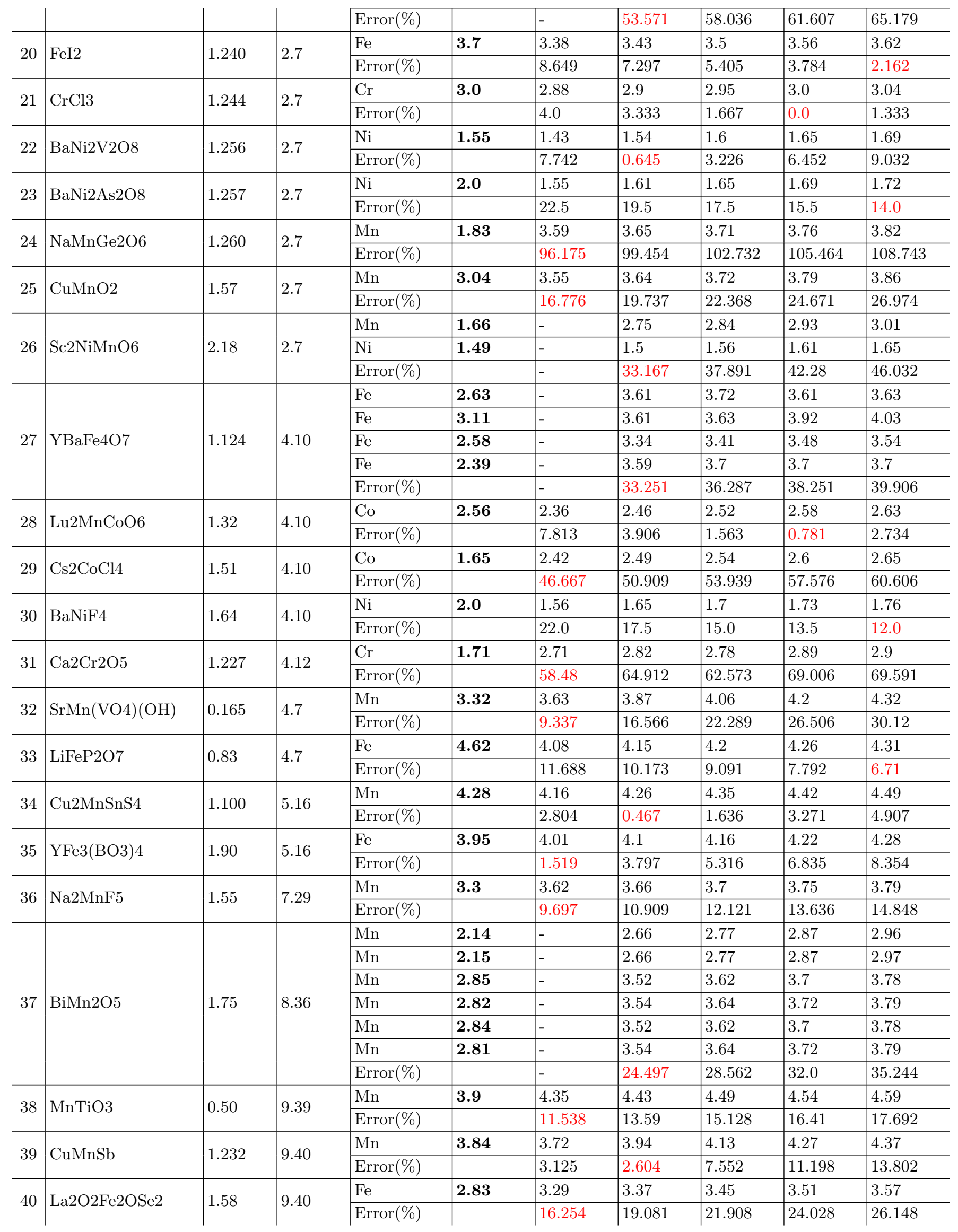




\begin{tabular}{|c|c|c|c|c|c|c|c|c|c|c|}
\hline \multirow{2}{*}{41} & \multirow{2}{*}{$\mathrm{BaFe} 2 \mathrm{Se} 3$} & \multirow{2}{*}{1.120} & \multirow{2}{*}{9.41} & $\mathrm{Fe}$ & 2.8 & - & 3.06 & 3.2 & 3.3 & - \\
\hline & & & & Error(\%) & & - & 9.286 & 14.286 & 17.857 & - \\
\hline \multirow{2}{*}{42} & \multirow{2}{*}{$\mathrm{Li} 2 \mathrm{CoSiO} 4$} & \multirow{2}{*}{1.79} & \multirow{2}{*}{9.41} & Co & 2.92 & 2.51 & 2.57 & 2.62 & 2.67 & 2.71 \\
\hline & & & & Error(\%) & & 14.041 & 11.986 & 10.274 & 8.562 & 7.192 \\
\hline \multirow{2}{*}{43} & \multirow{2}{*}{$\mathrm{RuCl} 3$} & \multirow{2}{*}{1.228} & \multirow{2}{*}{10.49} & $\mathrm{Ru}$ & 0.44 & 0.66 & 0.66 & 0.22 & 0.58 & 0.43 \\
\hline & & & & Error(\%) & & 50.0 & 50.0 & 50.0 & 31.818 & 2.273 \\
\hline \multirow{2}{*}{44} & \multirow{2}{*}{$\mathrm{AgMnVO} 4$} & \multirow{2}{*}{1.116} & \multirow{2}{*}{11.55} & $\mathrm{Mn}$ & 4.0 & 4.41 & 4.48 & 4.53 & 4.57 & 4.61 \\
\hline & & & & Error(\%) & & 10.25 & 12.0 & 13.25 & 14.25 & 15.25 \\
\hline \multirow{2}{*}{45} & \multirow{2}{*}{$\mathrm{Co} 2 \mathrm{C} 10 \mathrm{O} 8 \mathrm{H} 2$} & 1134 & 11.57 & Co & 2.98 & 2.6 & 2.65 & 2.69 & 2.73 & 2.77 \\
\hline & & 1.134 & 11.08 & \begin{tabular}{|l|} 
Error(\%) \\
\end{tabular} & & 12.752 & 11.074 & 9.732 & 8.389 & 7.047 \\
\hline 46 & NiPS3 & 1230 & 1157 & $\mathrm{Ni}$ & 1.0 & 1.13 & 1.27 & 1.36 & 1.43 & 1.49 \\
\hline 40 & NiPS3 & 1.230 & 11.58 & Error(\%) & & 13.0 & 27.0 & 36.0 & 43.0 & 49.0 \\
\hline 47 & $\mathrm{CoPS} 3$ & 1264 & 1157 & Co & 3.36 & 2.24 & 2.36 & 2.44 & 2.51 & 2.57 \\
\hline $4 r$ & WOFSS & 1.204 & 11.08 & \begin{tabular}{|l|} 
Error $(\%)$ \\
\end{tabular} & & 33.333 & 29.762 & 27.381 & 25.298 & 23.512 \\
\hline 48 & & 0163 & & Mn & 4.43 & 4.25 & 4.34 & 4.41 & 4.47 & 4.25 \\
\hline 48 & MnPS3 & 0.163 & 12.60 & Error(\%) & & 4.063 & 2.032 & 0.451 & 0.903 & 4.063 \\
\hline 49 & $\mathrm{CaMn} 2 \mathrm{Sb} 2$ & 092 & 1260 & $\mathrm{Mn}$ & 2.8 & 3.83 & 4.04 & 4.2 & 4.32 & 4.41 \\
\hline 49 & CaMn2sb2 & 0.92 & 12.60 & \begin{tabular}{|l|} 
Error(\%) \\
\end{tabular} & & 36.786 & 44.286 & 50.0 & 54.286 & 57.5 \\
\hline 50 & $\mathrm{~A} \rho 2 \mathrm{CrO} 2$ & 101 & 1260 & $\mathrm{Cr}$ & 2.9 & 2.59 & 2.73 & 2.82 & 2.88 & - \\
\hline 50 & Agzoruz & 1.0 .1 & 12.00 & Error(\%) & & 10.69 & 5.862 & 2.759 & 0.69 & - \\
\hline 51 & Mn3Ge & 0203 & 1262 & $\mathrm{Mn}$ & 1.7 & 2.78 & 3.24 & 3.58 & 3.82 & 4.01 \\
\hline 0 & tintere & 0.200 & 12.02 & Error(\%) & & 63.529 & 90.588 & 110.588 & 124.706 & 135.882 \\
\hline 52 & & 1013 & 1262 & $\mathrm{Fe}$ & 1.0 & 3.39 & 3.45 & 3.51 & 3.57 & 3.62 \\
\hline 52 & Fel2 & 1.0 .13 & 12.62 & Error(\%) & & 239.0 & 245.0 & 251.0 & 257.0 & 262.0 \\
\hline 53 & CoV2O6 & 106 & 1262 & Co & 4.4 & 2.53 & 2.59 & 2.65 & 2.7 & 2.74 \\
\hline 53 & $\operatorname{cov} 200$ & 1.0 .0 & 12.02 & Error(\%) & & 42.5 & 41.136 & 39.773 & 38.636 & 37.727 \\
\hline 54 & I & 107 & 1262 & $\mathrm{Fe}$ & 4.5 & 3.65 & 3.7 & 3.77 & - & 3.9 \\
\hline 34 & Lure 204 & 1.0 .8 & 12.02 & \begin{tabular}{|l|} 
Error(\%) \\
\end{tabular} & & 18.889 & 17.778 & 16.222 & - & 13.333 \\
\hline 55 & FeI? & 314 & 1262 & $\mathrm{Fe}$ & 1.0 & 3.4 & 3.41 & 3.5 & 3.56 & - \\
\hline 50 & | Fel2 & 3.14 & 12.62 & \begin{tabular}{|l|} 
Error(\%) \\
\end{tabular} & & 240.0 & 241.0 & 250.0 & 256.0 & - \\
\hline 56 & FePS3 & 183 & 1263 & $\mathrm{Fe}$ & 4.52 & 3.22 & 3.32 & 3.4 & 3.47 & 3.53 \\
\hline 56 & FePS3 & 1.183 & 12.63 & \begin{tabular}{|l|} 
Error(\%) \\
\end{tabular} & & 28.761 & 26.549 & 24.779 & 23.23 & 21.903 \\
\hline 57 & FA1Si2Sn7O16 & 197 & 1262 & $\mathrm{Fe}$ & 2.52 & 3.6 & 3.63 & 3.66 & 3.7 & 3.73 \\
\hline $5 r$ & Fe4S12Sn & 1.198 & 12.63 & Error(\%) & & 42.857 & 44.048 & 45.238 & 46.825 & 48.016 \\
\hline 58 & $\mathrm{MnBr} 2$ & 1239 & 1263 & Mn & 1.0 & 4.42 & 4.48 & 4.52 & 4.57 & 4.6 \\
\hline & INIIDTL & 1.239 & 12.03 & \begin{tabular}{|l|} 
Error $(\%)$ \\
\end{tabular} & & 342.0 & 348.0 & 352.0 & 357.0 & 360.0 \\
\hline 59 & Li2MnO3 & 197 & 1263 & Mn & 2.35 & 2.68 & 2.74 & 2.82 & 2.9 & 2.99 \\
\hline 59 & L12MnOS & 1.98 & 12.03 & Error(\%) & & 14.043 & 16.596 & 20.0 & 23.404 & 27.234 \\
\hline 60 & SraF2Fe?OS? & 22 & 1264 & $\mathrm{Fe}$ & 3.3 & 0.1 & 0.09 & 0.08 & 0.07 & 0.06 \\
\hline 60 & $\mathrm{Sr} 2 \mathrm{~F} 2 \mathrm{Fe} 2 \mathrm{OS} 2$ & 2.2 & 12.64 & \begin{tabular}{|l|} 
Error(\%) \\
\end{tabular} & & 96.97 & 97.273 & 97.576 & 97.879 & 98.182 \\
\hline 61 & NiWO4 & 194 & 1370 & $\mathrm{Ni}$ & 2.25 & 1.5 & 1.56 & 1.62 & 1.56 & 1.7 \\
\hline 01 & N1WVO 4 & 1.194 & 13.10 & \begin{tabular}{|l|} 
Error(\%) \\
\end{tabular} & & 33.333 & 30.667 & 28.0 & 30.667 & 24.444 \\
\hline 62 & Ca4IrO6 & 1114 & 1374 & Ir & 0.42 & 0.3 & 0.39 & 0.44 & 0.33 & 0.52 \\
\hline 02 & |Ca4lrub & 1.114 & $13.4^{2}$ & Error(\%) & & 28.571 & 7.143 & 4.762 & 21.429 & 23.81 \\
\hline 62 & $\mathrm{~N}_{\mathrm{F}} \mathrm{SO} \mathrm{SO}$ & & & $\mathrm{Fe}$ & 3.85 & 3.59 & 3.63 & 3.67 & 3.7 & 3.73 \\
\hline 63 & NaFeSO4F & 1.121 & 13.74 & \begin{tabular}{|l|} 
Error(\%) \\
\end{tabular} & & 6.753 & 5.714 & 4.675 & 3.896 & 3.117 \\
\hline 64 & $\mathrm{NaCOSO} 4 \mathrm{~F}$ & 1126 & 1374 & Co & 4.06 & 2.61 & 2.67 & 2.71 & 2.75 & 2.79 \\
\hline 64 & Nacosu4F & 1.126 & $13.4^{2}$ & Error(\%) & & 35.714 & 34.236 & 33.251 & 32.266 & 31.281 \\
\hline & & & & Mn & 3.4 & 2.95 & 2.87 & 2.92 & 2.98 & 4.2 \\
\hline 65 & & & & Mn & 2.96 & 2.92 & 2.86 & 2.91 & 2.97 & 3.06 \\
\hline 65 & Mn2GeO4 & 0.103 & 14.75 & Mn & 4.47 & 3.97 & 4.22 & 4.33 & 4.41 & 4.45 \\
\hline & & & & \begin{tabular}{|l|} 
Error(\%) \\
\end{tabular} & & 8.591 & 8.186 & 6.313 & 4.678 & 9.118 \\
\hline
\end{tabular}




\begin{tabular}{|c|c|c|c|c|c|c|c|c|c|c|}
\hline \multirow{2}{*}{66} & \multirow{2}{*}{ Li2Mn(SO4)2 } & \multirow{2}{*}{0.122} & \multirow{2}{*}{14.75} & Mn & 4.59 & 4.52 & 4.56 & 4.6 & 4.63 & 4.65 \\
\hline & & & & Error(\%) & & 1.525 & 0.654 & 0.218 & 0.871 & 1.307 \\
\hline \multirow{2}{*}{67} & \multirow{2}{*}{ La2LiRuO6 } & \multirow{2}{*}{0.148} & \multirow{2}{*}{14.75} & $\mathrm{Ru}$ & 2.2 & 1.84 & 1.92 & 2.0 & 2.07 & 2.15 \\
\hline & & & & Error(\%) & & 16.364 & 12.727 & 9.091 & 5.909 & 2.273 \\
\hline \multirow{3}{*}{68} & \multirow{3}{*}{ Ca2MnReO6 } & \multirow{3}{*}{0.204} & \multirow{3}{*}{14.75} & Mn & 4.34 & 4.16 & - & - & 4.47 & - \\
\hline & & & & $\operatorname{Re}$ & 0.22 & 0.41 & - & - & 0.7 & - \\
\hline & & & & Error(\%) & & 45.256 & - & - & 110.589 & - \\
\hline \multirow{2}{*}{69} & \multirow{2}{*}{$\mathrm{LiCrGe} 2 \mathrm{O} 6$} & \multirow{2}{*}{0.217} & \multirow{2}{*}{14.77} & $\mathrm{Cr}$ & 2.33 & 2.77 & 2.8 & 2.84 & 2.87 & 2.9 \\
\hline & & & & Error(\%) & & 18.884 & 20.172 & 21.888 & 23.176 & 24.464 \\
\hline \multirow{2}{*}{70} & \multirow{2}{*}{$\mathrm{LiFePO} 4$} & 0152 & 1478 & $\mathrm{Fe}$ & 4.09 & 3.55 & 3.6 & 3.64 & 3.68 & 3.71 \\
\hline & & 0.152 & 14.78 & Error(\%) & & 13.203 & 11.98 & 11.002 & 10.024 & 9.291 \\
\hline 71 & I iFeSi2OG & 028 & 1478 & $\mathrm{Fe}$ & 4.68 & 4.06 & 4.12 & 4.17 & 4.23 & 4.28 \\
\hline 71 & $\mathrm{LiFeS12O6}$ & 0.28 & 14.78 & \begin{tabular}{|l|} 
Error(\%) \\
\end{tabular} & & 13.248 & 11.966 & 10.897 & 9.615 & 8.547 \\
\hline 72 & I i i2 & 0121 & 1479 & Co & 3.33 & 2.63 & 2.68 & 2.72 & 2.76 & 2.79 \\
\hline$r 2$ & $\mathrm{Ll} 2 \mathrm{CO}(\mathrm{SU} 4) 2$ & 0.121 & 14.19 & Error(\%) & & 21.021 & 19.52 & 18.318 & 17.117 & 16.216 \\
\hline 73 & & & 1479 & Co & 2.92 & 2.46 & 2.5 & 2.56 & 2.6 & 2.65 \\
\hline 73 & YZMnCoOb & 0.164 & 14.79 & \begin{tabular}{|l|} 
Error(\%) \\
\end{tabular} & & 15.753 & 14.384 & 12.329 & 10.959 & 9.247 \\
\hline & & & & $\mathrm{Cu}$ & 0.51 & - & 0.66 & 0.7 & 0.73 & 0.76 \\
\hline 74 & CuSb2O6 & 1.133 & 14.80 & Error(\%) & & - & 29.412 & 37.255 & \begin{tabular}{|l}
43.137 \\
\end{tabular} & 49.02 \\
\hline 75 & $\mathrm{Li} 2 \mathrm{Fe}(\mathrm{SO} 4)_{2}$ & 1147 & 1480 & $\mathrm{Fe}$ & 3.22 & 3.6 & 3.63 & 3.66 & - & 3.73 \\
\hline 75 & $\mathrm{Li2Fe}(\mathrm{SO} 4) 2$ & 1.147 & 14.80 & Error(\%) & & 11.801 & 12.733 & 13.665 & - & 15.839 \\
\hline & & & & Mn & 3.75 & 4.08 & 4.2 & 4.3 & 4.38 & 4.46 \\
\hline 76 & MnV206 & 1.196 & 14.80 & \begin{tabular}{|l|} 
Error(\%) \\
\end{tabular} & & 8.8 & 12.0 & 14.667 & 16.8 & 18.933 \\
\hline 77 & ScaNiMnOG & 1100 & 1480 & Mn & 1.66 & 2.61 & 2.74 & 2.97 & 3.15 & 3.18 \\
\hline 77 & Sc2NiMnO6 & 1.199 & 14.80 & Error(\%) & & 57.229 & 65.06 & 78.916 & 89.759 & 91.566 \\
\hline & & & & $\mathrm{Cr}$ & 1.62 & 0.97 & 1.87 & - & 2.63 & - \\
\hline 78 & Cr2ReO6 & 1.201 & 14.80 & Re & 0.26 & 0.09 & 0.23 & - & 0.47 & - \\
\hline & & & & Error(\%) & & 52.754 & 13.485 & - & 71.558 & - \\
\hline & & & & $\mathrm{Fe}$ & 4.27 & 3.99 & 4.07 & 4.13 & 4.19 & 4.25 \\
\hline 79 & LiFeGe2O6 & 1.39 & 14.80 & \begin{tabular}{|l|} 
Error(\%) \\
\end{tabular} & & 6.557 & 4.684 & 3.279 & 1.874 & 0.468 \\
\hline 80 & $\mathrm{CuO}$ & 1.62 & 1480 & $\mathrm{Cu}$ & 0.65 & - & 0.49 & 0.55 & 0.59 & 0.63 \\
\hline 80 & CuO & 1.62 & 14.80 & Error(\%) & & - & 24.615 & 15.385 & 9.231 & 3.077 \\
\hline 81 & $\mathrm{MnPb} 4 \mathrm{Sb} 6 \mathrm{~S} 14$ & 163 & 1480 & $\mathrm{Mn}$ & 3.2 & - & - & 4.39 & 4.45 & 4.51 \\
\hline 81 & MnPb4Sb6s14 & 1.63 & 14.80 & \begin{tabular}{|l|} 
Error(\%) \\
\end{tabular} & & - & - & 37.187 & 39.063 & 40.937 \\
\hline & & & & $\mathrm{Ni}$ & 1.93 & 1.55 & 1.61 & 1.65 & - & - \\
\hline 82 & NiTa2O6 & 1.112 & 14.82 & Error(\%) & & 19.689 & 16.58 & 14.508 & - & - \\
\hline 83 & $\mathrm{NaFePO} 4$ & 1117 & 1482 & $\mathrm{Fe}$ & 3.89 & 3.58 & 3.62 & 3.66 & 3.69 & 3.72 \\
\hline 83 & | NaFerO4 & 1.117 & 14.82 & Error(\%) & & 7.969 & 6.941 & 5.913 & 5.141 & 4.37 \\
\hline & Ni2SiO4 & 1202 & 1482 & $\mathrm{Ni}$ & 1.41 & 1.52 & 1.59 & 1.63 & 1.67 & 1.7 \\
\hline 84 & Ni2SiO4 & 1.203 & 14.82 & \begin{tabular}{|l|} 
Error(\%) \\
\end{tabular} & & 7.801 & 12.766 & 15.603 & 18.44 & 20.567 \\
\hline & & & & $\mathrm{Ni}$ & 2.03 & 1.53 & 1.6 & 1.64 & 1.67 & 1.71 \\
\hline 85 & $\mathrm{Ni} 2 \mathrm{SiO} 4$ & 1.204 & 14.82 & $\mathrm{Ni}$ & 2.08 & 1.54 & 1.62 & 1.67 & 1.7 & 1.74 \\
\hline & & & & \begin{tabular}{|l|} 
Error(\%) \\
\end{tabular} & & 25.296 & 21.649 & 19.462 & 18.002 & 16.055 \\
\hline & & & & $\mathrm{Fe}$ & 1.77 & 2.81 & 3.07 & 3.25 & 3.37 & 3.48 \\
\hline 86 & CsFe2Se3 & 1.26 & 14.82 & Error(\%) & & 58.757 & 73.446 & 83.616 & 90.395 & 96.61 \\
\hline & & 1154 & 1484 & $\mathrm{Fe}$ & 1.73 & 4.06 & 4.12 & 4.18 & 4.23 & 4.28 \\
\hline 87 & NaFeSi2O6 & 1.154 & 14.84 & \begin{tabular}{|l|} 
Error(\%) \\
\end{tabular} & & 134.682 & 138.15 & 141.618 & 144.509 & 147.399 \\
\hline 88 & $\mathrm{CaCoGe} 2 \mathrm{O} 6$ & 1169 & 14.84 & Co & 2.93 & 2.59 & 2.63 & 2.67 & 2.71 & 2.76 \\
\hline 88 & |CacoGezUb & 1.169 & 14.84 & Error(\%) & & 11.604 & 10.239 & 8.874 & 7.509 & 5.802 \\
\hline & & & & $\mathrm{Cu}$ & 0.52 & - & 0.58 & 0.61 & 0.64 & 0.67 \\
\hline 89 & CuSe2O5 & 1.2 & 14.84 & \begin{tabular}{|l} 
Error(\%) \\
\end{tabular} & & - & 11.538 & 17.308 & \begin{tabular}{|l|}
23.077 \\
\end{tabular} & 28.846 \\
\hline 90 & Ca3Co2O6 & 1.60 & 14.84 & Co & 1.0 & 0.13 & 2.73 & 2.86 & 2.93 & 3.01 \\
\hline
\end{tabular}




\begin{tabular}{|c|c|c|c|c|c|c|c|c|c|c|}
\hline & & & & Error(\%) & & 87.0 & 173.0 & 186.0 & 193.0 & 201.0 \\
\hline \multirow{2}{*}{91} & \multirow{2}{*}{ Ba3LaRu2O9 } & \multirow{2}{*}{1.94} & \multirow{2}{*}{14.84} & $\mathrm{Ru}$ & 1.43 & 0.98 & 1.72 & 1.8 & 1.86 & 1.92 \\
\hline & & & & Error(\%) & & \begin{tabular}{|l|}
31.469 \\
\end{tabular} & 20.28 & 25.874 & 30.07 & 34.266 \\
\hline \multirow{2}{*}{92} & \multirow{2}{*}{ NiCO3 } & \multirow{2}{*}{0.113} & \multirow{2}{*}{15.85} & $\mathrm{Ni}$ & 1.73 & 1.54 & 1.62 & 1.66 & 1.7 & 1.73 \\
\hline & & & & Error(\%) & & 10.983 & 6.358 & 4.046 & 1.734 & 0.0 \\
\hline \multirow{2}{*}{93} & \multirow{2}{*}{$\mathrm{CoCO} 3$} & \multirow{2}{*}{0.114} & \multirow{2}{*}{15.85} & Co & 1.73 & 2.57 & 2.63 & 2.68 & 2.72 & 2.76 \\
\hline & & & & Error(\%) & & 48.555 & 52.023 & 54.913 & 57.225 & 59.538 \\
\hline 94 & $\mathrm{MnCO} 3$ & 0.115 & 15.85 & Error(\%) & & 159.538 & 162.428 & 164.74 & 166.474 & 168.208 \\
\hline \multirow{2}{*}{95} & \multirow{2}{*}{$\mathrm{BiCrO} 3$} & & & $\mathrm{Cr}$ & 2.04 & 2.66 & 2.73 & 2.8 & 2.84 & 2.89 \\
\hline & & 0.138 & 15.80 & Error(\%) & & \begin{tabular}{|l|}
30.392 \\
\end{tabular} & 33.824 & \begin{tabular}{|l|}
37.255 \\
\end{tabular} & 39.216 & 41.667 \\
\hline & & & & $\mathrm{Co}$ & 1.57 & 2.61 & 2.69 & 2.77 & 2.92 & 2.98 \\
\hline 96 & Sr2CoOsO6 & 0.210 & 15.85 & Os & 0.7 & 0.71 & 1.08 & 0.17 & 1.65 & 0.3 \\
\hline & & & & $\mathrm{Co}$ & 1.03 & 0.87 & 0.94 & 2.65 & 2.7 & 2.74 \\
\hline & & & & $\mathrm{Co}$ & 2.73 & 0.79 & 2.54 & 2.61 & 2.67 & 2.72 \\
\hline 08 & CO2T०O6 & 0.145 & 15.87 & $\mathrm{Co}$ & 2.84 & 0.71 & 2.52 & 2.59 & 2.65 & 2.7 \\
\hline 90 & 6031000 & 0.140 & 10.01 & $\mathrm{Co}$ & 1.1 & 1.02 & 0.4 & 2.62 & 2.67 & 2.72 \\
\hline & & & & $\mathrm{Co}$ & 2.85 & 2.33 & 2.44 & 2.52 & 2.59 & 2.65 \\
\hline & & & & Error(\%) & & 37.423 & 20.998 & \begin{tabular}{|l|}
64.048 \\
\end{tabular} & 64.575 & 65.122 \\
\hline 99 & $\mathrm{CaMnGe} 2 \mathrm{O} 6$ & 0.156 & 15.87 & $\mathrm{Mn}$ & 4.17 & 4.46 & 4.51 & 4.55 & 4.58 & 4.62 \\
\hline 99 & Calmngezu & 0.150 & 15.88 & Error(\%) & & 6.954 & 8.153 & 9.113 & 9.832 & 10.791 \\
\hline & & & & $\mathrm{Co}$ & 3.5 & 2.49 & 2.45 & 2.62 & 2.67 & 2.71 \\
\hline 100 & $\mathrm{Co} 4 \mathrm{Nb} 2 \mathrm{O} 9$ & 0.196 & 15.88 & $\mathrm{Co}$ & 2.6 & 2.46 & 0.84 & 2.63 & 2.67 & 2.73 \\
\hline 100 & FENU4F & 0.120 & 10.09 & Error(\%) & & 6.25 & 4.63 & 3.241 & 1.852 & \begin{tabular}{|l|}
0.463 \\
\end{tabular} \\
\hline 104 & Mn3Ti2Te6 & 0.176 & 1589 & $\mathrm{Mn}$ & 4.2 & 4.2 & 4.3 & 4.39 & 4.46 & 4.52 \\
\hline & N1110 $1121 \mathrm{eO}$ & & 10.09 & Error(\%) & & 0.0 & 2.381 & 4.524 & 6.19 & 7.619 \\
\hline 105 & $\mathrm{Ca}_{3} \mathrm{Li} \mathrm{OsO6}_{\mathrm{s}}$ & 03 & 1589 & Os & 2.2 & 1.69 & 1.83 & 1.96 & 2.09 & 2.23 \\
\hline 100 & 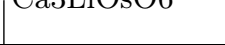 & 0.0 & 10.03 & Error(\%) & & 23.182 & 16.818 & 10.909 & 5.0 & 1.364 \\
\hline 106 & FerO3_alnh & 065 & 1580 & $\mathrm{Fe}$ & 4.12 & 3.58 & 3.81 & 3.95 & 4.05 & 4.13 \\
\hline 100 & геz-u-dipia & 0.00 & 10.09 & Error(\%) & & 13.107 & 7.524 & 4.126 & 1.699 & 0.243 \\
\hline 107 & $\mathrm{Sr} 2 \mathrm{CuTeO} 6$ & 1.168 & 15.90 & $\mathrm{Cu}$ & \begin{tabular}{|l|}
0.69 \\
\end{tabular} & - & 0.62 & 0.65 & 0.69 & 0.72 \\
\hline & & & & Error(\%) & & - & 10.145 & 5.797 & 0.0 & 4.348 \\
\hline 108 & CoV2O6-alpha & 1.17 & 15.90 & $\mathrm{Co}$ & 5.08 & 2.51 & 2.59 & 2.65 & 2.7 & 2.74 \\
\hline 100 & 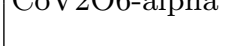 & 1.16 & 10.90 & Error(\%) & & 50.591 & 49.016 & 47.835 & 46.85 & 46.063 \\
\hline 100 & FeI? & 1209 & 1500 & $\mathrm{Fe}$ & 3.7 & 3.37 & 3.43 & 3.49 & 3.56 & 3.61 \\
\hline 109 & Fel 2 & 1.209 & 10.90 & Error(\%) & & 8.919 & 7.297 & 5.676 & 3.784 & 2.432 \\
\hline 110 & $\mathrm{CoBr} 2$ & 1245 & 15.90 & $\mathrm{Co}$ & \begin{tabular}{|l|}
2.77 \\
\end{tabular} & 2.42 & 2.49 & 2.55 & 2.6 & 2.65 \\
\hline 110 & CoDr 2 & 1.240 & 10.90 & Error(\%) & & 12.635 & 10.108 & 7.942 & 6.137 & \begin{tabular}{|l|}
4.332 \\
\end{tabular} \\
\hline 111 & $\mathrm{CoCl} 2$ & 1246 & 15.90 & Co & 3.0 & 2.48 & 2.55 & 2.59 & 2.64 & 2.68 \\
\hline 111 & Coviz & 1.240 & 10.90 & \begin{tabular}{|l|} 
Error $(\%)$ \\
\end{tabular} & & 17.333 & 15.0 & \begin{tabular}{|l|}
13.667 \\
\end{tabular} & 12.0 & 10.667 \\
\hline 112 & $\mathrm{NiCl} 2$ & 1.247 & 15.90 & $\mathrm{Ni}$ & \begin{tabular}{|l|}
2.11 \\
\end{tabular} & 1.43 & 1.5 & 1.54 & 1.59 & 1.63 \\
\hline 112 & 101012 & 1.246 & 10.90 & \begin{tabular}{|l|} 
Error(\%) \\
\end{tabular} & & 32.227 & 28.91 & 27.014 & 24.645 & 22.749 \\
\hline
\end{tabular}




\begin{tabular}{|c|c|c|c|c|c|c|c|c|c|c|}
\hline \multirow{2}{*}{113} & \multirow{2}{*}{$\mathrm{NiBr} 2$} & \multirow{2}{*}{1.248} & \multirow{2}{*}{15.90} & $\mathrm{Ni}$ & 2.0 & 1.35 & 1.42 & 1.47 & 1.53 & 1.58 \\
\hline & & & & Error(\%) & & 32.5 & 29.0 & 26.5 & 23.5 & 21.0 \\
\hline \multirow{2}{*}{114} & \multirow{2}{*}{$\mathrm{MnO}$} & \multirow{2}{*}{1.31} & \multirow{2}{*}{15.90} & $\mathrm{Mn}$ & 5.66 & 4.33 & 4.41 & 4.47 & 4.52 & 4.57 \\
\hline & & & & \begin{tabular}{|l|} 
Error(\%) \\
\end{tabular} & & 23.498 & 22.085 & 21.025 & 20.141 & 19.258 \\
\hline \multirow{3}{*}{115} & \multirow{3}{*}{$\mathrm{Ag} 2 \mathrm{NiO} 2$} & \multirow{3}{*}{1.49} & \multirow{3}{*}{15.90} & $\mathrm{Ni}$ & 0.66 & 0.53 & 0.66 & 0.9 & 1.09 & 1.25 \\
\hline & & & & $\mathrm{Ni}$ & 0.33 & 0.54 & 0.87 & 0.98 & 1.08 & 1.22 \\
\hline & & & & Error(\%) & & 41.666 & 81.818 & 116.667 & 146.212 & 179.545 \\
\hline \multirow{2}{*}{116} & \multirow{2}{*}{$\mathrm{NiO}$} & \multirow{2}{*}{1.6} & \multirow{2}{*}{15.90} & $\mathrm{Ni}$ & 2.45 & 1.34 & 1.44 & 1.52 & 1.58 & 1.63 \\
\hline & & & & \begin{tabular}{|l|} 
Error(\%) \\
\end{tabular} & & 45.306 & 41.224 & 37.959 & 35.51 & 33.469 \\
\hline \multirow{2}{*}{117} & \multirow{2}{*}{$\mathrm{CoO}$} & 160 & 1500 & Co & 3.98 & 2.4 & - & 2.55 & 2.63 & 2.69 \\
\hline & & 1.69 & 15.90 & Error(\%) & & 39.698 & - & 35.93 & 33.92 & 32.412 \\
\hline 118 & CoV2O6 & 170 & 1500 & Co & 3.98 & 2.53 & 2.59 & 2.65 & 2.7 & 2.74 \\
\hline 110 & 608200 & 1.10 & 10.90 & \begin{tabular}{|l} 
Error(\%) \\
\end{tabular} & & 36.432 & 34.925 & 33.417 & 32.161 & 31.156 \\
\hline 110 & $\mathrm{~V} O \mathrm{Cl}$ & 1.27 & 1501 & V & 1.3 & 1.74 & 1.8 & 1.84 & 1.87 & 1.89 \\
\hline 119 & 12001 & 1.37 & 15.91 & Error(\%) & & 33.846 & 38.462 & 41.538 & 43.846 & 45.385 \\
\hline 100 & A Ni:O & 150 & 18 20 & $\mathrm{Ni}$ & 1.55 & 1.31 & 1.42 & 1.54 & 1.6 & - \\
\hline 120 & AgNiO2 & 1.50 & 18.22 & \begin{tabular}{|l|} 
Error(\%) \\
\end{tabular} & & 15.484 & 8.387 & 0.645 & 3.226 & - \\
\hline 121 & $\mathrm{FePO} 4$ & 017 & 1925 & $\mathrm{Fe}$ & 4.16 & 3.97 & 4.06 & 4.13 & 4.19 & 4.25 \\
\hline 121 & | & 0.18 & 19.25 & Error(\%) & & 4.567 & 2.404 & 0.721 & 0.721 & 2.163 \\
\hline 122 & C & 0129 & 1927 & $\mathrm{Cu}$ & 0.99 & 0.37 & 0.03 & 0.45 & 0.45 & 0.45 \\
\hline 122 & CuзMого9 & 0.129 & 19.27 & Error(\%) & & 62.626 & 96.97 & 54.545 & 54.545 & 54.545 \\
\hline 123 & Conth2O6 & 1224 & 1028 & $\mathrm{Co}$ & 3.05 & 2.55 & 2.61 & 2.66 & 2.71 & 2.75 \\
\hline 123 & CoNb206 & 1.224 & 19.28 & \begin{tabular}{|l|} 
Error(\%) \\
\end{tabular} & & 16.393 & 14.426 & 12.787 & 11.148 & 9.836 \\
\hline & & & & $\mathrm{Ni}$ & 0.6 & 1.42 & 1.5 & 1.55 & 1.59 & 1.64 \\
\hline 124 & $\mathrm{CsNiCl} 3$ & 1.0 .4 & 20.34 & $\mathrm{Ni}$ & 0.4 & 1.42 & 1.5 & 1.55 & 1.59 & 1.64 \\
\hline & & & & \begin{tabular}{|l|} 
Error(\%) \\
\end{tabular} & & 195.833 & 212.5 & 222.917 & 231.25 & 241.667 \\
\hline & & & & V & 0.47 & 1.6 & 1.71 & 1.78 & 1.84 & 1.88 \\
\hline 125 & $\mathrm{Mg} \vee 2 \mathrm{O} 4$ & 1.138 & 20.37 & Error(\%) & & 240.426 & 263.83 & 278.723 & 291.489 & 300.0 \\
\hline 120 & $\mathrm{FaCh}_{\mathrm{C}}$ & 007 & 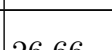 & $\mathrm{Fe}$ & 3.68 & 3.56 & 3.56 & 3.61 & 3.65 & 3.68 \\
\hline 126 & FeSb2O4 & 0.97 & 26.66 & \begin{tabular}{|l|} 
Error(\%) \\
\end{tabular} & & 3.261 & 3.261 & 1.902 & 0.815 & 0.0 \\
\hline & & & & $\mathrm{Cu}$ & 0.09 & - & 0.02 & 0.6 & 0.65 & 0.69 \\
\hline 127 & $\mathrm{Cu} 3 \mathrm{Mo} 2 \mathrm{O} 9$ & 0.130 & 26.68 & $\mathrm{Cu}$ & 0.62 & - & 0.01 & 0.66 & 0.68 & 0.71 \\
\hline & & & & Error(\%) & & - & 88.083 & 286.559 & 315.95 & 340.591 \\
\hline & & & & $\mathrm{Ni}$ & 1.65 & 1.6 & 1.63 & 1.67 & 1.71 & 1.75 \\
\hline 128 & $\mathrm{Ni3B} 7 \mathrm{O} 13 \mathrm{Cl}$ & 0.133 & 29.101 & $\mathrm{Ni}$ & \begin{tabular}{|l|}
0.79 \\
\end{tabular} & 1.58 & 1.63 & 1.67 & 1.71 & 1.74 \\
\hline & & & & \begin{tabular}{|l|} 
Error $(\%)$ \\
\end{tabular} & & 51.515 & 53.771 & 56.302 & 60.046 & 63.157 \\
\hline & & & & $\mathrm{Ni}$ & 3.56 & 1.54 & 1.62 & 1.66 & 1.7 & 1.74 \\
\hline & & & & $\mathrm{Ni}$ & 1.33 & 1.5 & 1.61 & 1.65 & 1.69 & 1.73 \\
\hline 129 & Ni3B7O13Br & 0.135 & 29.101 & $\mathrm{Ni}$ & 1.4 & 1.51 & 1.61 & 1.66 & \begin{tabular}{|l|}
1.7 \\
\end{tabular} & 1.73 \\
\hline & & & & \begin{tabular}{|l|} 
Error(\%) \\
\end{tabular} & & 25.794 & 30.183 & 32.0 & 33.582 & 34.923 \\
\hline 120 & & & & Mn & 0.5 & 0.22 & 1.44 & 1.1 & 2.9 & 1.54 \\
\hline 130 & MnS2 & 1.18 & 29.105 & \begin{tabular}{|l} 
Error(\%) \\
\end{tabular} & & 56.0 & 188.0 & 120.0 & 480.0 & 208.0 \\
\hline 131 & $\mathrm{SrCO} 2 \mathrm{~V} 2 \mathrm{O}$ & 171 & 29110 & Co & 2.25 & - & - & 2.63 & 2.68 & 2.73 \\
\hline 131 & SrCo2V 208 & 1.71 & 29.110 & \begin{tabular}{|l} 
Error(\%) \\
\end{tabular} & & - & - & 16.889 & 19.111 & 21.333 \\
\hline 120 & 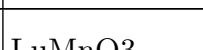 & 1 101 & 21120 & Mn & 3.37 & 3.44 & 3.55 & 3.63 & 3.7 & 3.76 \\
\hline 132 & LuMnO3 & 1.101 & 31.129 & Error(\%) & & 2.077 & 5.341 & 7.715 & 9.792 & 11.573 \\
\hline & & & & $\mathrm{Ga}$ & 4.7 & 0.02 & 0.02 & 0.02 & 0.01 & 0.01 \\
\hline 133 & $\mathrm{CaFeO} 3$ & 038 & 22147 & $\mathrm{Fe}$ & 3.9 & 3.72 & 3.89 & 4.0 & 4.09 & 4.16 \\
\hline 133 & | GaFeO3 & 0.38 & 33.147 & $\mathrm{Fe}$ & 4.5 & 3.73 & 3.89 & 4.0 & 4.09 & 4.16 \\
\hline & & & & \begin{tabular}{|l|} 
Error(\%) \\
\end{tabular} & & 40.433 & 37.795 & 37.75 & 37.923 & 38.003 \\
\hline & & & & Co & 2.83 & - & 2.7 & 2.81 & 2.9 & 2.98 \\
\hline & & & & \begin{tabular}{|l}
$\mathrm{Co}$ \\
\end{tabular} & 2.04 & - & 2.32 & 2.43 & 2.52 & 2.6 \\
\hline
\end{tabular}




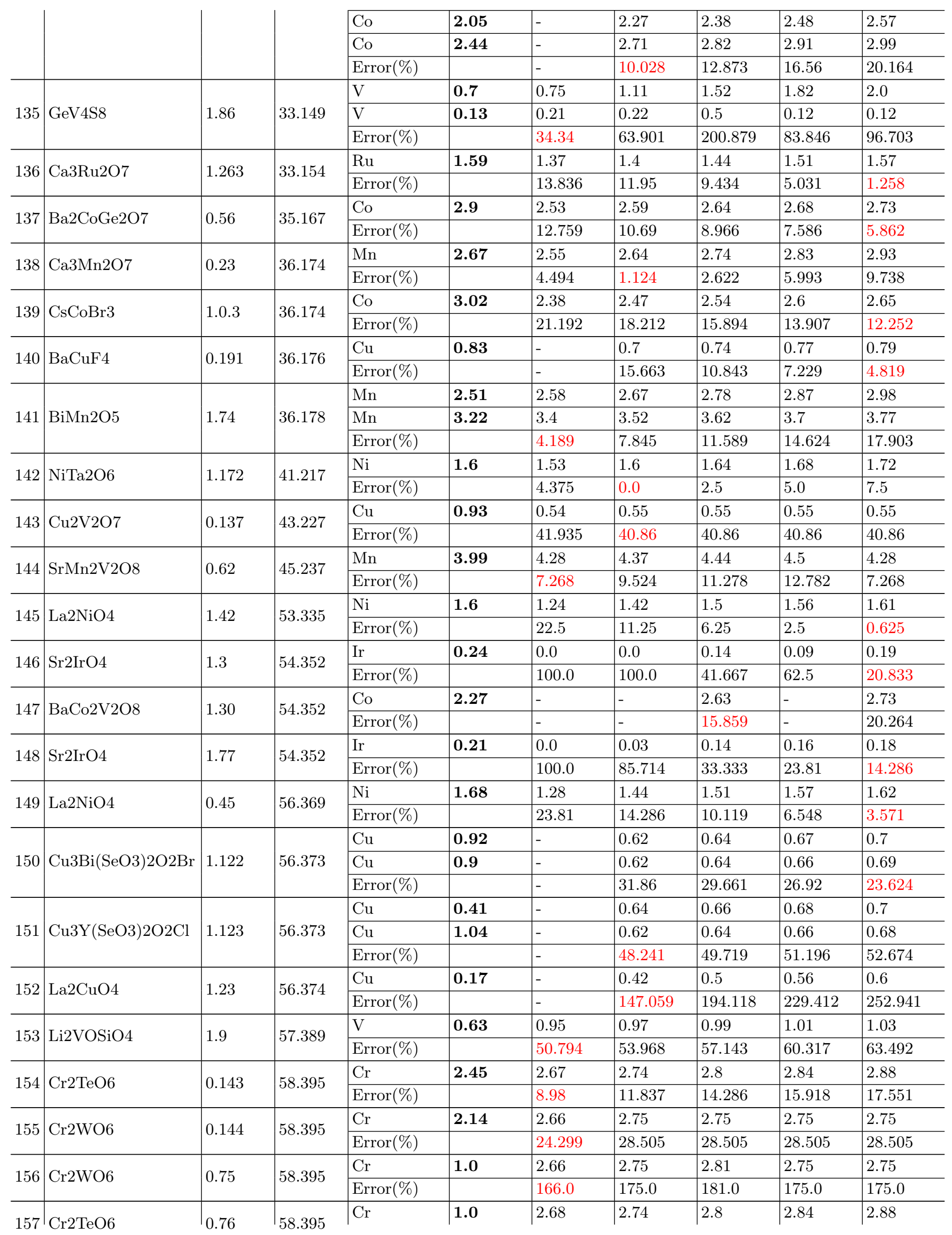




\begin{tabular}{|c|c|c|c|c|c|c|c|c|c|c|}
\hline & & & & Error(\%) & & 168.0 & 174.0 & 180.0 & 184.0 & 188.0 \\
\hline \multirow{2}{*}{158} & \multirow{2}{*}{$\mathrm{Mn}(\mathrm{N}(\mathrm{CN} 2)) 2$} & \multirow{2}{*}{0.131} & \multirow{2}{*}{58.398} & Mn & 5.01 & 4.42 & 4.48 & 4.52 & 4.56 & 4.6 \\
\hline & & & & Error(\%) & & 11.776 & 10.579 & 9.78 & 8.982 & 8.184 \\
\hline \multirow{2}{*}{159} & \multirow{2}{*}{$\mathrm{NiF} 2$} & \multirow{2}{*}{0.36} & \multirow{2}{*}{58.398} & $\mathrm{Ni}$ & 2.0 & 1.61 & 1.69 & 1.73 & 1.76 & 1.78 \\
\hline & & & & Error(\%) & & 19.5 & 15.5 & 13.5 & 12.0 & 11.0 \\
\hline \multirow{4}{*}{160} & \multirow{4}{*}{$\mathrm{KCo} 4(\mathrm{PO} 4) 3$} & \multirow{4}{*}{0.85} & \multirow{4}{*}{58.398} & Co & 2.16 & 2.53 & 2.59 & 2.66 & 2.7 & 2.74 \\
\hline & & & & Co & 3.18 & 2.64 & 2.67 & 2.7 & 2.73 & 2.76 \\
\hline & & & & Co & 3.14 & 2.45 & 2.5 & 2.57 & 2.64 & 2.69 \\
\hline & & & & Error(\%) & & 18.695 & 18.776 & 18.798 & 18.358 & 18.131 \\
\hline \multirow{2}{*}{161} & \multirow{2}{*}{ LuFe4Ge2 } & \multirow{2}{*}{0.140} & \multirow{2}{*}{58.399} & $\mathrm{Fe}$ & 0.45 & 1.83 & 2.27 & 2.54 & 2.73 & 2.79 \\
\hline & & & & Error(\%) & & 306.667 & 404.444 & 464.444 & 506.667 & 520.0 \\
\hline \multirow{2}{*}{162} & $\mathrm{VEn} A \mathrm{C} 2$ & 027 & 58,200 & $\mathrm{Fe}$ & 0.64 & 1.94 & 2.31 & 2.56 & 2.75 & 2.83 \\
\hline & Yrequez & 0.21 & 30.099 & Error(\%) & & 203.125 & 260.938 & 300.0 & 329.687 & 342.188 \\
\hline 163 & YMn3Al4O12 & 1158 & 58.404 & $\mathrm{Mn}$ & 2.92 & 3.65 & 3.71 & 3.77 & 3.82 & 3.88 \\
\hline 100 & 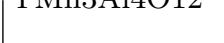 & 1.100 & 00.404 & Error(\%) & & 25.0 & 27.055 & 29.11 & 30.822 & 32.877 \\
\hline 164 & $\mathrm{CuMnAs}_{\mathrm{B}}$ & 0222 & 59.407 & $\mathrm{Mn}$ & 3.6 & 3.54 & 3.83 & 4.05 & 4.21 & 4.34 \\
\hline 104 & TUIMIIAs & 0.222 & 59.406 & Error(\%) & & 1.667 & 6.389 & 12.5 & 16.944 & 20.556 \\
\hline 165 & |CPQP & 1052 & 50416 & Co & 0.32 & 0.49 & 0.57 & 0.64 & 0.71 & 0.79 \\
\hline 100 & $6 a c 0212$ & 1.202 & | & Error(\%) & & 53.125 & 78.125 & 100.0 & 121.875 & 146.875 \\
\hline 166 & $\mathrm{CoSe} 2 \mathrm{O} 5$ & 0.119 & 60.419 & Co & 3.2 & 2.57 & 2.63 & 2.68 & 2.72 & 2.76 \\
\hline 100 & JONELU & 0.119 & 00.419 & \begin{tabular}{|l|} 
Error(\%) \\
\end{tabular} & & 19.688 & 17.813 & 16.25 & 15.0 & 13.75 \\
\hline 167 & CoSe2O5 & 0161 & 60.419 & Co & 3.0 & - & - & 2.68 & - & 2.68 \\
\hline $10 r$ & fosezos & 0.101 & 00.419 & Error(\%) & & - & - & 10.667 & - & 10.667 \\
\hline 168 & Mn5Si3 & 1.88 & 60.431 & $\mathrm{Mn}$ & 1.89 & 2.54 & 3.1 & 3.48 & 3.72 & 3.91 \\
\hline 100 & & 1.00 & & \begin{tabular}{|l|} 
Error(\%) \\
\end{tabular} & & 34.392 & 64.021 & 84.127 & 96.825 & 106.878 \\
\hline & & & & $\mathrm{Mn}$ & 3.05 & 3.42 & 3.55 & 3.64 & 3.72 & 3.78 \\
\hline & & & & $\mathrm{Mn}$ & 3.47 & 3.52 & 3.62 & 3.7 & 3.77 & 3.83 \\
\hline 160 & Mp & 040 & 61422 & $\mathrm{Mn}$ & 3.49 & 3.52 & 3.61 & 3.69 & 3.76 & 3.82 \\
\hline 107 & 101112- v-aippia & 0.40 & 01.400 & $\mathrm{Mn}$ & 3.27 & 3.48 & 3.59 & 3.67 & 3.75 & 3.82 \\
\hline & & & & $\mathrm{Mn}$ & 4.19 & 3.48 & 3.58 & 3.66 & 3.74 & 3.8 \\
\hline & & & & Error(\%) & & 7.559 & 9.701 & 11.317 & 12.753 & 13.979 \\
\hline & & & & Mn & 2.69 & - & 3.54 & 3.63 & 3.71 & 3.77 \\
\hline & & & & $\mathrm{Mn}$ & 3.1 & - & 3.63 & 3.71 & 3.77 & 3.83 \\
\hline 170 & Mn203-alnha & 041 & 61433 & Mn & 3.01 & - & 3.61 & 3.69 & 3.76 & 3.82 \\
\hline 170 & MnzU3-alpna & 0.41 & 01.433 & Mn & 2.92 & - & 3.59 & 3.67 & 3.75 & 3.82 \\
\hline & & & & $\mathrm{Mn}$ & 3.54 & - & 3.58 & 3.67 & 3.74 & 3.8 \\
\hline & & & & \begin{tabular}{|l|} 
Error(\%) \\
\end{tabular} & & - & 18.541 & 21.313 & 23.705 & 25.755 \\
\hline 171 & $\mathrm{Li} 2 \mathrm{Ni}(\mathrm{SO} 4) 2$ & 0.71 & 61.437 & $\mathrm{Ni}$ & 2.15 & - & - & 1.38 & 1.46 & 1.53 \\
\hline 111 & |LIZNI(SU4)Z & 0.11 & | & Error(\%) & & - & - & 35.814 & 32.093 & 28.837 \\
\hline & & & & $\mathrm{Eu}$ & 6.8 & 6.73 & 6.77 & 6.9 & 6.9 & 6.9 \\
\hline 172 & EuFe2As2 & 2.1 & 61.439 & $\mathrm{Fe}$ & 0.98 & 0.0 & 2.38 & 2.69 & 2.88 & 3.01 \\
\hline & & & & Error(\%) & & 50.515 & 71.65 & 87.98 & 97.674 & 104.306 \\
\hline & & & & $\mathrm{Mn}$ & 4.09 & 4.44 & 4.5 & 4.54 & 4.58 & 4.61 \\
\hline 173 & $\mathrm{Mn} 2 \mathrm{GeO} 4$ & 0.102 & 62.441 & $\mathrm{Mn}$ & 4.9 & 4.45 & 4.5 & 4.55 & 4.59 & 4.62 \\
\hline & & & & Error(\%) & & 8.871 & 9.094 & 9.072 & 9.153 & 9.214 \\
\hline & & & & $\mathrm{Fe}$ & 4.13 & 3.99 & 4.1 & 4.19 & 4.28 & 4.34 \\
\hline 174 & $\mathrm{NH} 4 \mathrm{Fe} 2 \mathrm{O} 6$ & 0.168 & 62.441 & $\mathrm{Fe}$ & 3.12 & 3.77 & 3.77 & 3.75 & 3.73 & 3.74 \\
\hline & & & & Error(\%) & & 12.112 & 10.78 & 10.822 & 11.592 & 12.478 \\
\hline & & & & $\mathrm{Fe}$ & 3.99 & 3.79 & 3.79 & 3.77 & 3.73 & 3.75 \\
\hline 175 & RbFe2F6 & 0.192 & 62.441 & $\mathrm{Fe}$ & 4.29 & 3.99 & 4.1 & 4.19 & 4.28 & 4.34 \\
\hline & & & & Error(\%) & & 6.003 & 4.72 & 3.923 & 3.375 & 3.591 \\
\hline & & & & Co & 3.88 & 2.44 & 2.6 & 2.65 & 2.7 & 2.74 \\
\hline 176 & $\mathrm{Co} 2 \mathrm{SiO} 4$ & 0.218 & 62.441 & & & & & & & \\
\hline
\end{tabular}




\begin{tabular}{|c|c|c|c|c|c|c|c|c|c|c|}
\hline & & & & Co & 3.64 & 2.53 & 2.63 & 2.68 & 2.72 & 2.76 \\
\hline & & & & Error(\%) & & 33.804 & 30.369 & 29.038 & 27.843 & 26.779 \\
\hline \multirow{2}{*}{177} & \multirow{2}{*}{$\mathrm{Co} 2 \mathrm{SiO} 4$} & \multirow{2}{*}{0.219} & \multirow{2}{*}{62.441} & $\mathrm{Co}$ & 3.87 & 2.44 & 2.57 & 2.65 & 2.7 & 2.73 \\
\hline & & & & Error $(\%)$ & & 30.714 & 28.289 & 26.21 & 24.519 & 23.535 \\
\hline \multirow[b]{2}{*}{178} & \multirow[b]{2}{*}{$\mathrm{Fe} 2 \mathrm{SiO} 4$} & \multirow[b]{2}{*}{0.221} & \multirow[b]{2}{*}{62.441} & $\mathrm{Fe}$ & 4.44 & 3.49 & 3.58 & 3.62 & 3.67 & 3.7 \\
\hline & & & & $\mathrm{Fe}$ & 4.4 & 3.57 & 3.59 & 3.63 & 3.67 & 3.7 \\
\hline \multirow{3}{*}{179} & \multirow{3}{*}{$\mathrm{Rb} 2 \mathrm{Fe} 2 \mathrm{O}(\mathrm{AsO} 4) 2$} & \multirow{3}{*}{0.90} & \multirow{3}{*}{62.441} & $\mathrm{Fe}$ & 3.64 & 3.89 & 4.0 & 4.09 & 4.16 & 4.22 \\
\hline & & & & $\mathrm{Fe}$ & 3.19 & 3.75 & 3.97 & 4.06 & 4.15 & 4.21 \\
\hline & & & & Error(\%) & & 12.211 & 17.171 & 19.817 & 22.19 & 23.954 \\
\hline \multirow{2}{*}{180} & \multirow{2}{*}{$\mathrm{CoSO} 4$} & \multirow{2}{*}{0.96} & \multirow{2}{*}{62.441} & $\mathrm{Co}$ & 3.22 & 2.61 & 2.67 & 2.71 & 2.75 & 2.78 \\
\hline & & & & Error $(\%)$ & & 18.944 & 17.081 & 15.839 & 14.596 & 13.665 \\
\hline 181 & $\mathrm{KCrF} 4$ & & & Error(\%) & & 34.596 & - & - & - & - \\
\hline 182 & I iNiPOA & 088 & 62444 & $\mathrm{Ni}$ & 2.22 & 1.56 & 1.64 & 1.68 & 1.71 & 1.74 \\
\hline 102 & LINII U & 0.00 & 02.444 & Error(\%) & & 29.73 & 26.126 & 24.324 & 22.973 & 21.622 \\
\hline 183 & $\mathrm{ILiCOPO4}$ & 0193 & 62445 & $\mathrm{Co}$ & 1.0 & 2.54 & 2.62 & 2.67 & 2.71 & 2.75 \\
\hline 100 & Dicor & 0.190 & 02.440 & Error(\%) & & 154.0 & 162.0 & 167.0 & 171.0 & 175.0 \\
\hline 181 & SrFrrou & 0.216 & 62445 & Er & 4.5 & 2.74 & - & 2.81 & - & 2.85 \\
\hline 104 & N121204 & 0.210 & 02.440 & Error(\%) & & 39.111 & - & 37.556 & - & 36.667 \\
\hline & & & & Mn & 4.43 & 4.5 & 4.55 & 4.58 & 4.62 & 4.5 \\
\hline 185 & KMn4(PO4) 3 & 086 & 62445 & Mn & 4.21 & 4.46 & 4.51 & 4.55 & 4.59 & 4.46 \\
\hline 100 & 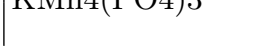 & 0.00 & 02.440 & Mn & 4.57 & 4.49 & 4.53 & 4.57 & 4.6 & 4.49 \\
\hline & & & & Error(\%) & & 24.099 & 24.257 & 25.473 & 26.578 & 27.406 \\
\hline & & & & $\mathrm{Mn}$ & 3.85 & 4.44 & 4.5 & 4.54 & 4.58 & 4.62 \\
\hline 189 & $\mathrm{Mn} 2 \mathrm{SiO} 4$ & 0.220 & 62.446 & $\mathrm{Mn}$ & 4.68 & 4.46 & 4.51 & 4.56 & 4.6 & 4.63 \\
\hline & & & & Error(\%) & & 10.012 & 10.258 & 10.243 & 10.336 & 10.534 \\
\hline 190 & LaMnO3 & 01 & 62448 & Mn & 3.87 & 3.55 & 3.63 & 3.71 & 3.77 & 3.83 \\
\hline 190 & Larrnos & 0.1 & 02.440 & Error(\%) & & 8.269 & 6.202 & 4.134 & 2.584 & 1.034 \\
\hline 101 & $\mathrm{Na}_{\mathrm{s}} \mathrm{O}_{3}$ & 025 & 62448 & Os & 1.0 & 0.97 & 1.37 & 1.65 & 1.88 & 2.08 \\
\hline 191 & IVAUSus & 0.20 & 02.440 & Error(\%) & & 3.0 & 37.0 & 65.0 & 88.0 & 108.0 \\
\hline & & & & $\mathrm{Fe}$ & 3.1 & 3.88 & 4.01 & 4.09 & 4.16 & 4.22 \\
\hline 192 & $\mathrm{Rb} 2 \mathrm{Fe} 2 \mathrm{O}(\mathrm{AsO} 4) 2$ & 0.91 & 62.448 & $\mathrm{Fe}$ & 3.09 & 3.74 & 3.94 & 4.06 & 4.14 & 4.21 \\
\hline & & & & Error(\%) & & 23.099 & 28.431 & 31.664 & 34.087 & 36.188 \\
\hline 193 & $\mathrm{IiMnPO4}$ & 024 & 62449 & Mn & 3.9 & 4.5 & 4.54 & 4.58 & 4.61 & 4.64 \\
\hline 100 & 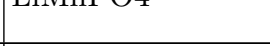 & 0.24 & 02.49 & Error(\%) & & 15.385 & 16.41 & 17.436 & 18.205 & 18.974 \\
\hline & & & & $\mathrm{Cr}$ & 0.4 & 0.01 & 1.76 & 0.01 & 0.6 & 0.05 \\
\hline 194 & $\mathrm{Cr} 2 \mathrm{As}$ & 1.130 & 62.450 & $\mathrm{Cr}$ & 1.34 & 2.1 & 2.63 & 0.25 & 0.57 & 1.05 \\
\hline & & & & Error(\%) & & 77.108 & 218.134 & 89.422 & 53.731 & 54.571 \\
\hline & & & & $\mathrm{Fe}$ & 0.95 & 1.27 & 2.09 & 2.48 & 2.72 & 2.89 \\
\hline 195 & $\mathrm{Fe} 2 \mathrm{As}$ & 1.131 & 62.450 & $\mathrm{Fe}$ & 1.52 & 2.34 & 2.61 & 2.82 & 3.04 & 3.07 \\
\hline & & & & Error(\%) & & 43.816 & 95.855 & 123.289 & 143.158 & 153.092 \\
\hline
\end{tabular}




\begin{tabular}{|c|c|c|c|c|c|c|c|c|c|c|}
\hline \multirow{3}{*}{196} & \multirow{3}{*}{ Mn2As } & \multirow{3}{*}{1.132} & \multirow{3}{*}{62.450} & $\mathrm{Mn}$ & 3.7 & 2.03 & 2.59 & 3.35 & 3.53 & 3.68 \\
\hline & & & & $\mathrm{Mn}$ & 3.5 & 3.52 & 3.8 & 4.04 & 4.2 & 4.32 \\
\hline & & & & Error(\%) & & 22.854 & 19.286 & 12.444 & \begin{tabular}{|l|}
12.297 \\
\end{tabular} & 11.984 \\
\hline \multirow{2}{*}{197} & \multirow{2}{*}{$\mathrm{CrN}$} & \multirow{2}{*}{1.28} & \multirow{2}{*}{62.450} & $\mathrm{Cr}$ & 2.4 & 2.46 & 2.65 & 2.76 & 2.83 & 2.89 \\
\hline & & & & Error(\%) & & 2.5 & 10.417 & 15.0 & 17.917 & 20.417 \\
\hline \multirow{2}{*}{198} & \multirow{2}{*}{ Mn3O4 } & \multirow{2}{*}{1.1} & \multirow{2}{*}{62.452} & $\mathrm{Mn}$ & 3.49 & 3.56 & 3.7 & 3.85 & 3.83 & 3.9 \\
\hline & & & & Error(\%) & & 2.006 & 6.017 & 10.315 & 9.742 & 11.748 \\
\hline \multirow[t]{2}{*}{199} & \multirow[t]{2}{*}{ Sr2Mn3Sb2O2 } & \multirow[t]{2}{*}{2.27} & \multirow[t]{2}{*}{63.459} & $\mathrm{Mn}$ & 3.5 & 3.7 & 3.97 & 4.15 & 4.28 & 4.37 \\
\hline & & & & Error(\%) & & 5.714 & 7.428 & 11.31 & 14.0 & 16.0 \\
\hline \multirow{2}{*}{200} & \multirow{2}{*}{ Mn3Sn } & & & $\mathrm{Mn}$ & 3.0 & 3.34 & 3.48 & 3.74 & 3.92 & 4.07 \\
\hline & & 0.199 & 03.403 & Error(\%) & & 11.333 & 16.0 & 24.667 & 30.667 & 35.667 \\
\hline 201 & $\mathrm{Mn} 3 \mathrm{Sn}$ & 0200 & 63464 & $\mathrm{Mn}$ & 3.0 & 3.16 & 3.51 & 3.77 & 3.97 & 4.13 \\
\hline 203 & Gd2C10 & 082 & 64476 & $\mathrm{Cu}$ & 1.02 & - & 0.25 & 0.42 & 0.48 & 0.53 \\
\hline 200 & Guz & 0.02 & 04.410 & Error(\%) & & - & 75.49 & 58.824 & 52.941 & 48.039 \\
\hline 204 & Mn3Ni20P6 & 1.145 & 64.480 & Mn & 2.4 & 3.71 & 3.93 & 4.11 & 4.25 & 4.36 \\
\hline 207 & 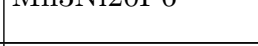 & 1.170 & 0 & Error(\%) & & 54.583 & 63.75 & 71.25 & 77.083 & 81.667 \\
\hline 205 & BaFe2As2 & 1.16 & 64.480 & $\mathrm{Fe}$ & 0.87 & 2.02 & 2.55 & 2.8 & 2.97 & 3.09 \\
\hline 200 & DarezAsZ & 1.10 & 04.400 & Error(\%) & & 132.184 & 193.103 & 221.839 & 241.379 & 255.172 \\
\hline 206 & K2NiF4 & 1240 & 64.480 & $\mathrm{Ni}$ & 1.0 & 1.55 & 1.65 & 1.69 & 1.73 & 1.76 \\
\hline 200 & N2ANA & 1.249 & 04.400 & Error $(\%)$ & & 55.0 & 65.0 & 69.0 & 73.0 & 76.0 \\
\hline & & & 64.480 & $\mathrm{Fe}$ & 0.8 & 1.83 & 2.35 & 2.67 & 2.86 & 3.0 \\
\hline 207 & CaFezAsz & 1.52 & 04.480 & Error(\%) & & 128.75 & 193.75 & 233.75 & 257.5 & 275.0 \\
\hline & & & & $\mathrm{Ni}$ & 1.64 & 0.66 & 0.43 & 1.54 & 1.62 & 1.67 \\
\hline 211 & $\mathrm{NiCr} 2 \mathrm{O} 4$ & 0.4 & 70.530 & $\mathrm{Cr}$ & 1.4 & 2.6 & 2.75 & 2.84 & 2.89 & 2.93 \\
\hline & & & & Error(\%) & & 72.735 & 85.104 & 54.478 & 53.824 & 55.558 \\
\hline 212 & IaFeAsO & 1125 & 73553 & $\mathrm{Fe}$ & 0.63 & 1.91 & 2.46 & 2.74 & 2.93 & 3.07 \\
\hline 212 & Lareasu & 1.120 & 10.000 & Error(\%) & & 203.175 & 290.476 & 334.921 & \begin{tabular}{|l|}
365.079 \\
\end{tabular} & 387.302 \\
\hline 213 & YbCo2Si2 & 1.176 & 73.553 & $\mathrm{Yb}$ & 1.41 & 0.0 & 0.0 & 0.0 & 3.65 & - \\
\hline 210 & & 1.160 & 10.003 & Error(\%) & & 100.0 & 100.0 & 100.0 & 158.865 & - \\
\hline & & & & $\mathrm{Fe}$ & 1.83 & 3.82 & 3.96 & 4.05 & 4.13 & 4.2 \\
\hline 214 & $\mathrm{Sr} 2 \mathrm{FeOsO} 6$ & 1.47 & 83.50 & Os & 0.48 & 1.36 & 1.64 & 1.83 & 1.99 & 2.15 \\
\hline & & & & Error(\%) & & \begin{tabular}{|l|}
146.039 \\
\end{tabular} & 179.03 & 201.281 & 220.134 & 238.712 \\
\hline & & & & $\mathrm{Mn}$ & 2.86 & 2.81 & 3.2 & 3.48 & 3.68 & 3.9 \\
\hline 215 & $\mathrm{Mn} 3 \mathrm{CuN}$ & 2.5 & 85.59 & $\mathrm{Mn}$ & 0.65 & 3.13 & 3.43 & 3.63 & \begin{tabular}{|l|}
3.78 \\
\end{tabular} & 3.95 \\
\hline & & & & Error(\%) & & 191.643 & \begin{tabular}{|l|}
219.79 \\
\end{tabular} & 240.07 & 255.105 & \begin{tabular}{|l|}
272.028 \\
\end{tabular} \\
\hline 216 & Sr2CoO2A 2 (Se? & 223 & 8673 & Co & 3.77 & 2.21 & 2.45 & 2.52 & 2.58 & 2.63 \\
\hline 210 & srzeouzAgzsez & 2.23 & 80.13 & Error(\%) & & \begin{tabular}{|l|}
41.379 \\
\end{tabular} & 35.013 & 33.156 & 31.565 & \begin{tabular}{|l|}
30.239 \\
\end{tabular} \\
\hline 217 & Ba2CoO2A 2 -Se? & 224 & 8673 & $\mathrm{Co}$ & 3.97 & 2.38 & 2.47 & 2.54 & 2.61 & 2.66 \\
\hline 216 & DaZCOUZAgZsez & 2.24 & 00.13 & Error(\%) & & 40.05 & \begin{tabular}{|l|}
37.783 \\
\end{tabular} & 36.02 & 34.257 & \begin{tabular}{|l|}
32.997 \\
\end{tabular} \\
\hline & & & & $\mathrm{Mn}$ & 4.2 & 4.2 & 4.33 & 4.42 & 4.49 & 4.55 \\
\hline 218 & MnV2O4 & 0.64 & 88.81 & $\mathrm{~V}$ & 1.3 & 1.66 & 1.73 & 1.78 & 1.82 & 1.86 \\
\hline
\end{tabular}




\begin{tabular}{|c|c|c|c|c|c|c|c|c|c|c|}
\hline & & & & Error(\%) & & 13.846 & 18.086 & 21.081 & 23.452 & 25.705 \\
\hline \multirow{2}{*}{219} & \multirow{2}{*}{ FeTa2O6 } & \multirow{2}{*}{2.22} & \multirow{2}{*}{88.86} & $\mathrm{Fe}$ & 3.69 & 3.55 & 3.58 & 3.62 & 3.66 & 3.69 \\
\hline & & & & Error(\%) & & 3.794 & 2.981 & 1.897 & 0.813 & 0.0 \\
\hline \multirow{2}{*}{220} & \multirow{2}{*}{$\mathrm{Ba}(\mathrm{TiO}) \mathrm{Cu} 4(\mathrm{PO} 4) 4$} & \multirow{2}{*}{1.235} & \multirow{2}{*}{94.132} & $\mathrm{Cu}$ & 0.8 & - & 0.65 & 0.68 & 0.7 & 0.72 \\
\hline & & & & \begin{tabular}{|l|} 
Error(\%) \\
\end{tabular} & & - & 18.75 & 15.0 & 12.5 & 10.0 \\
\hline \multirow{2}{*}{221} & \multirow{2}{*}{$\mathrm{ZnV} 2 \mathrm{O} 4$} & \multirow{2}{*}{1.24} & \multirow{2}{*}{96.150} & V & 0.65 & 1.57 & 1.66 & 1.81 & 1.86 & 1.89 \\
\hline & & & & Error(\%) & & 141.538 & 155.385 & 178.462 & 186.154 & 190.769 \\
\hline \multirow{2}{*}{222} & \multirow{2}{*}{$\mathrm{MgCr} 2 \mathrm{O} 4$} & \multirow{2}{*}{3.4} & \multirow{2}{*}{111.255} & $\mathrm{Cr}$ & 0.69 & 0.01 & 2.88 & 2.98 & 3.07 & 3.17 \\
\hline & & & & Error(\%) & & 98.551 & 317.391 & 331.884 & 344.928 & 359.42 \\
\hline \multirow{7}{*}{223} & \multirow{7}{*}{ alpha-Mn } & & & $\mathrm{Mn}$ & 2.83 & 0.01 & 3.52 & 3.81 & 4.0 & - \\
\hline & & & & $\mathrm{Mn}$ & 1.83 & 0.05 & 0.34 & 0.11 & 0.22 & - \\
\hline & & & & $\mathrm{Mn}$ & 0.74 & 0.84 & 0.11 & 0.05 & 0.21 & - \\
\hline & & 1.85 & 114.282 & Mn & 0.48 & 0.26 & 0.13 & 0.07 & 0.08 & - \\
\hline & & & & $\mathrm{Mn}$ & 0.59 & 0.24 & 1.18 & 0.2 & 0.08 & - \\
\hline & & & & $\mathrm{Mn}$ & 0.66 & 0.18 & 0.25 & 0.17 & 0.11 & - \\
\hline & & & & Error(\%) & & 64.718 & 70.997 & 74.604 & 75.675 & - \\
\hline ل & I & 1105 & 10 & $\mathrm{Cu}$ & 0.89 & - & 0.62 & 0.65 & 0.68 & 0.7 \\
\hline 224 & Gecu2 24 & 1.185 & 122.338 & \begin{tabular}{|l|} 
Error(\%) \\
\end{tabular} & & - & 30.337 & 26.966 & 23.596 & 21.348 \\
\hline 225 & CaMnRi2 & 072 & 129416 & Mn & 3.73 & 3.87 & 4.08 & 4.23 & 4.35 & 4.45 \\
\hline 225 & CaMnB12 & 0.72 & 129.416 & Error(\%) & & 3.753 & 9.383 & 13.405 & 16.622 & 19.303 \\
\hline 226 & $\mathrm{Mn} 3 \mathrm{Pt}$ & 1143 & 132456 & Mn & 3.4 & 3.04 & 3.56 & 3.98 & 4.18 & 4.31 \\
\hline 220 & MnsPt & 1.143 & 132.456 & Error(\%) & & 10.588 & 4.706 & 17.059 & 22.941 & 26.765 \\
\hline 227 & MnF2 & 015 & 136499 & Mn & 4.6 & 4.54 & 4.58 & 4.61 & 4.64 & 4.67 \\
\hline 227 & $\mathrm{MnF} 2$ & 0.15 & 136.499 & Error(\%) & & 1.304 & 0.435 & 0.217 & 0.87 & 1.522 \\
\hline 208 & COF? & 0178 & 136,409 & Co & 2.6 & 2.64 & 2.69 & 2.73 & 2.77 & 2.8 \\
\hline 228 & $\mathrm{CoF}^{2} 2$ & 0.178 & 136.499 & Error(\%) & & 1.538 & 3.462 & 5.0 & 6.538 & 7.692 \\
\hline 229 & Fe2TeO6 & 0.142 & 136.503 & $\mathrm{Fe}$ & 4.19 & 3.8 & 3.97 & 4.08 & 4.15 & 4.22 \\
\hline 229 & Fez 1eub & 0.142 & 136.503 & \begin{tabular}{|l|} 
Error(\%) \\
\end{tabular} & & 9.308 & 5.251 & 2.625 & 0.955 & 0.716 \\
\hline 230 & $\mathrm{I} \Omega \mathrm{rr} \Delta \mathrm{s} O$ & 1146 & 138528 & $\mathrm{Cr}$ & 1.57 & 2.63 & 3.05 & 3.35 & 3.56 & 3.71 \\
\hline 250 & LaCrAsU & 1.140 & 138.528 & Error(\%) & & 67.516 & 94.268 & 113.376 & 126.752 & 136.306 \\
\hline 231 & RaMn2As2 & 018 & 139536 & $\mathrm{Mn}$ & 3.88 & 3.68 & 3.93 & 4.11 & 4.24 & 4.35 \\
\hline 201 & DОМIILASL & 0.10 & 109.030 & \begin{tabular}{|l|} 
Error(\%) \\
\end{tabular} & & 5.155 & 1.289 & 5.928 & 9.278 & 12.113 \\
\hline 222 & SrOMn 240 & 0212 & 130536 & Mn & 3.4 & 3.56 & 3.83 & 4.04 & 4.21 & 4.31 \\
\hline 232 & STZMnJASZU2 & 0.212 & 139.530 & Error(\%) & & 4.706 & 12.647 & 18.824 & 23.824 & 26.765 \\
\hline 233 & SrMnBi2 & 073 & 139536 & Mn & 3.75 & 3.98 & 4.16 & 4.29 & 4.4 & 4.48 \\
\hline 233 & SrMnB12 | & 0.33 & 139.536 & \begin{tabular}{|l|} 
Error(\%) \\
\end{tabular} & & 6.133 & 10.933 & 14.4 & 17.333 & 19.467 \\
\hline 234 & BaMn2Bi2 & 0.89 & 139.536 & $\mathrm{Mn}$ & 3.83 & 3.97 & 4.15 & 4.29 & 4.39 & 4.48 \\
\hline 234 & | BalMn2B12 & 0.89 & 139.530 & Error(\%) & & 3.655 & 8.355 & 12.01 & 14.621 & 16.971 \\
\hline & & & & Mn & 2.73 & 2.58 & 3.0 & 3.28 & 3.5 & 3.67 \\
\hline 235 & Mn3ZnC & 2.19 & 139.537 & \begin{tabular}{|l}
$\mathrm{Mn}$ \\
\end{tabular} & \begin{tabular}{|l|}
1.6 \\
\end{tabular} & \begin{tabular}{|l|}
2.48 \\
\end{tabular} & 3.08 & 3.43 & 3.63 & 3.78 \\
\hline & & & & Error(\%) & & 30.247 & 51.195 & 67.261 & 77.54 & 85.341 \\
\hline & & & & $\mathrm{Ni}$ & 2.22 & 1.52 & 1.64 & 1.69 & 1.73 & 1.76 \\
\hline 236 & KNiF3 & 1.250 & 140.550 & \begin{tabular}{|l} 
Error(\%) \\
\end{tabular} & & 31.532 & 26.126 & 23.874 & 22.072 & 20.721 \\
\hline 237 & COA1204 & 0.58 & 141556 & Co & 1.9 & 2.53 & 2.59 & 2.64 & 2.69 & 2.73 \\
\hline 237 & COAI2O4 & 0.58 & 141.556 & Error(\%) & & 33.158 & 36.316 & 38.947 & 41.579 & 43.684 \\
\hline 238 & $\mathrm{Ca} 2 \mathrm{MnO} 4$ & 0.211 & 142.568 & Mn & 2.4 & 2.54 & 2.63 & 2.72 & 2.82 & 2.91 \\
\hline 238 & Ca2MnO4 & 0.211 & 142.568 & \begin{tabular}{|l|} 
Error $(\%)$ \\
\end{tabular} & & 5.833 & 9.583 & 13.333 & 17.5 & 21.25 \\
\hline & & & & $\mathrm{Mn}$ & 3.39 & 3.64 & 3.7 & 3.76 & 3.82 & 3.87 \\
\hline 239 & LaMn3Cr4O12 & 1.156 & 146.12 & $\mathrm{Cr}$ & 2.89 & 2.57 & 2.67 & 2.75 & 2.81 & 2.85 \\
\hline & & & & Error(\%) & & 9.223 & 8.378 & 7.879 & 7.726 & 7.772 \\
\hline & & & & $\mathrm{Ni}$ & 2.03 & 1.48 & 1.57 & 1.62 & 1.66 & 1.7 \\
\hline 240 & Ni3'TeO6 & 1.165 & 146.12 & \begin{tabular}{|l|} 
Error(\%) \\
\end{tabular} & & 27.094 & 22.66 & 20.197 & 18.227 & 16.256 \\
\hline
\end{tabular}




\begin{tabular}{|c|c|c|c|c|c|c|c|c|c|c|}
\hline \multirow{3}{*}{241} & \multirow{3}{*}{ NiN2O6 } & \multirow{3}{*}{0.78} & \multirow{3}{*}{148.17} & $\mathrm{Ni}$ & 1.34 & 1.56 & 1.62 & 1.66 & 1.7 & 1.73 \\
\hline & & & & $\mathrm{Ni}$ & 1.33 & 1.56 & 1.62 & 1.66 & 1.7 & 1.73 \\
\hline & & & & Error(\%) & & 16.856 & 21.35 & 24.346 & 27.343 & 29.59 \\
\hline \multirow{2}{*}{242} & \multirow{2}{*}{ MnGeO3 } & \multirow{2}{*}{0.125} & \multirow{2}{*}{148.19} & $\mathrm{Mn}$ & 4.6 & 3.55 & 3.78 & 3.98 & 4.13 & 4.27 \\
\hline & & & & Error(\%) & & 22.826 & 17.826 & 13.478 & 10.217 & 7.174 \\
\hline \multirow{2}{*}{243} & \multirow{2}{*}{ MnTiO3 } & \multirow{2}{*}{0.19} & \multirow{2}{*}{148.19} & $\mathrm{Mn}$ & 4.55 & 4.36 & 4.43 & 4.5 & 4.55 & 4.59 \\
\hline & & & & Error(\%) & & 4.176 & 2.637 & 1.099 & 0.0 & 0.879 \\
\hline \multirow{2}{*}{244} & \multirow{2}{*}{ Ba3MnNb2O9 } & \multirow{2}{*}{1.0 .8} & \multirow{2}{*}{157.53} & Mn & 4.91 & 4.34 & 4.41 & 4.47 & 4.52 & 4.56 \\
\hline & & & & Error(\%) & & 11.609 & 10.183 & 8.961 & 7.943 & 7.128 \\
\hline \multirow{2}{*}{245} & \multirow{2}{*}{ Ba3Nb2NiO9 } & 113 & & $\mathrm{Ni}$ & 1.47 & 1.58 & 1.62 & 1.66 & 1.7 & 1.73 \\
\hline & & 1.13 & $\mid 159.64$ & Error(\%) & & 7.483 & 10.204 & 12.925 & 15.646 & 17.687 \\
\hline 246 & VCl2 & 1237 & 15964 & $\mathrm{~V}$ & 0.93 & 2.51 & 2.58 & 2.63 & 2.67 & 2.51 \\
\hline 246 & VCl2 & 1.237 & 159.64 & \begin{tabular}{|l|} 
Error(\%) \\
\end{tabular} & & 169.892 & 177.419 & 182.796 & 187.097 & 169.892 \\
\hline 247 & VBr? & 1238 & 15964 & V & 2.48 & 2.53 & 2.59 & 2.64 & 2.68 & 2.53 \\
\hline 246 & VBrz & 1.238 & 159.04 & Error(\%) & & 2.016 & 4.435 & 6.452 & 8.065 & 2.016 \\
\hline 248 & $\mathrm{PbNiO3}$ & 021 & 16160 & $\mathrm{Ni}$ & 1.69 & - & 1.53 & 1.59 & - & 1.68 \\
\hline 248 & |FDNIOS & 0.21 & $\mid$ & Error(\%) & & - & 9.467 & 5.917 & - & 0.592 \\
\hline & & & & Mn & 2.94 & 3.76 & 3.97 & 4.13 & 4.26 & 4.24 \\
\hline 249 & $\mathrm{CuMnSb}$ & 1.233 & 161.72 & Error(\%) & & 27.891 & 35.034 & 40.476 & 44.898 & 44.218 \\
\hline 250 & $\mathrm{C}_{112 \mathrm{Mn}}$ & 1265 & 16172 & Mn & 3.9 & 3.75 & 3.96 & 4.13 & 4.26 & 4.36 \\
\hline 200 & | CUMIIID & 1.200 & 101.12 & \begin{tabular}{|l|} 
Error(\%) \\
\end{tabular} & & 3.846 & 1.538 & 5.897 & 9.231 & 11.795 \\
\hline 251 & SrRu2O6 & 1186 & 16278 & $\mathrm{Ru}$ & 1.3 & 1.43 & 1.69 & 1.85 & 1.98 & 2.09 \\
\hline 251 & | SrRüvo & 1.180 & 102.18 & \begin{tabular}{|l|} 
Error $(\%)$ \\
\end{tabular} & & 10.0 & 30.0 & 42.308 & 52.308 & 60.769 \\
\hline 252 & Co4Nb2OQ & 0111 & 16594 & Co & 3.0 & 2.41 & 2.56 & 2.62 & 2.67 & 2.72 \\
\hline 202 & CO4ND2U9 & 0.111 & 105.94 & Error(\%) & & 19.667 & 14.667 & 12.667 & 11.0 & 9.333 \\
\hline & & & & Ir & 0.5 & 0.15 & 0.05 & 0.14 & 0.2 & 0.26 \\
\hline & & & & \begin{tabular}{|l|}
$\mathrm{Ir}$ \\
\end{tabular} & 0.25 & 0.14 & 0.05 & 0.14 & 0.2 & 0.26 \\
\hline 253 & Sr3NiIrO6 & 1.0.10 & 165.95 & $\mathrm{Ni}$ & 1.5 & 1.4 & 1.5 & 1.57 & 1.63 & 1.68 \\
\hline & & & & $\mathrm{Ni}$ & 0.75 & 1.4 & 1.5 & 1.57 & 1.63 & 1.68 \\
\hline & & & & Error(\%) & & 51.834 & 67.5 & 57.5 & 51.5 & 47.0 \\
\hline & & & & Co & 3.6 & 2.53 & 2.57 & 2.61 & 2.65 & 2.7 \\
\hline & & & & Co & 1.8 & 2.53 & 2.57 & 2.61 & 2.66 & 2.7 \\
\hline 254 & Sr3CoIrO6 & 1.0 .5 & 165.95 & Ir & 0.6 & 0.38 & 0.17 & 0.01 & 0.1 & 0.18 \\
\hline & & & & Ir & 0.3 & 0.38 & 0.17 & 0.01 & 0.1 & 0.18 \\
\hline & & & & \begin{tabular}{|l|} 
Error(\%) \\
\end{tabular} & & 33.404 & 46.597 & 66.875 & 56.041 & 46.25 \\
\hline & & & & $\mathrm{Fe}$ & 3.9 & 3.48 & 3.52 & 3.57 & 3.61 & 3.65 \\
\hline 255 & FeBr2 & 1.242 & 165.96 & Error(\%) & & 10.769 & 9.744 & 8.462 & 7.436 & 6.41 \\
\hline 256 & $\mathrm{Mn} 3 \mathrm{Ir}$ & 0108 & 166101 & Mn & 2.45 & 2.87 & 3.34 & 3.69 & 3.94 & 4.13 \\
\hline 256 & Mn3ir & 0.108 & 106.101 & \begin{tabular}{|l|} 
Error(\%) \\
\end{tabular} & & 17.143 & 36.327 & 50.612 & 60.816 & 68.571 \\
\hline 257 & $\mathrm{Mn} 3 \mathrm{Pt}$ & 0100 & 166101 & Mn & 2.94 & 3.07 & 3.49 & 3.77 & 3.99 & 4.18 \\
\hline 257 & Mn3Pt & 0.109 & 166.101 & Error(\%) & & 4.422 & 18.707 & 28.231 & 35.714 & 42.177 \\
\hline & & & & $\mathrm{Mn}$ & 1.17 & 2.57 & 3.01 & 3.3 & 3.51 & 3.72 \\
\hline 258 & $\mathrm{Mn} 3 \mathrm{GaN}$ & 0.177 & 166.97 & \begin{tabular}{|l} 
Error(\%) \\
\end{tabular} & & 119.658 & 157.265 & 182.051 & 200.0 & 217.949 \\
\hline 250 & $\mathrm{FoCO} 3$ & 0116 & 167103 & $\mathrm{Fe}$ & 1.0 & 3.57 & 3.61 & 3.65 & 3.68 & 3.72 \\
\hline 259 & $\mathrm{FeCO} 3$ & 0.116 & 167.103 & Error(\%) & & 257.0 & 261.0 & 265.0 & 268.0 & 272.0 \\
\hline 260 & $\mathrm{Cr} 2 \mathrm{O} 3$ & 0.59 & 167.106 & $\mathrm{Cr}$ & 2.48 & 2.67 & 2.75 & 2.81 & 2.86 & 2.9 \\
\hline 260 & $\mathrm{Cr} 2 \mathrm{O} 3$ & 0.59 & 167.106 & \begin{tabular}{|l|} 
Error $(\%)$ \\
\end{tabular} & & 7.661 & 10.887 & 13.306 & 15.323 & 16.935 \\
\hline 261 & $\mathrm{Mn} 3 \mathrm{CaC}$ & 1153 & 167108 & $\mathrm{Mn}$ & 1.82 & 1.78 & 2.78 & 3.24 & 3.46 & 3.64 \\
\hline 261 & |MnsGac & 1.153 & 167.108 & \begin{tabular}{|l|} 
Error(\%) \\
\end{tabular} & & 2.198 & 52.747 & 78.022 & 90.11 & 100.0 \\
\hline & $\mathrm{FeCl} 2$ & 1241 & 167108 & $\mathrm{Fe}$ & 4.5 & 3.52 & 3.56 & 3.6 & 3.59 & 3.67 \\
\hline 262 & FeCl2 & 1.241 & 167.108 & Error(\%) & & 21.778 & 20.889 & 20.0 & 20.222 & 18.444 \\
\hline 263 & ScMnO3 & 0.8 & | 173.129 & Mn & 3.54 & 3.4 & 3.49 & 3.58 & 3.66 & 3.73 \\
\hline
\end{tabular}




\begin{tabular}{|c|c|c|c|c|c|c|c|c|c|c|}
\hline & & & & Error(\%) & & 3.955 & 1.412 & 1.13 & 3.39 & 5.367 \\
\hline \multirow{2}{*}{264} & \multirow{2}{*}{ YMnO3 } & \multirow{2}{*}{0.44} & \multirow{2}{*}{173.131} & $\mathrm{Mn}$ & 3.14 & 3.51 & 3.59 & 3.67 & 3.74 & 3.8 \\
\hline & & & & Error $(\%)$ & & 11.783 & 14.331 & 16.879 & \begin{tabular}{|l|}
19.108 \\
\end{tabular} & 21.019 \\
\hline \multirow{2}{*}{265} & \multirow{2}{*}{ YMnO3 } & \multirow{2}{*}{0.6} & \multirow{2}{*}{185.197} & $\mathrm{Mn}$ & 2.91 & 3.47 & 3.56 & 3.64 & 3.72 & 3.78 \\
\hline & & & & \begin{tabular}{|l} 
Error $(\%)$ \\
\end{tabular} & & 19.244 & 22.337 & 25.086 & 27.835 & 29.897 \\
\hline \multirow{2}{*}{266} & \multirow{2}{*}{$\mathrm{LuFeO} 3$} & \multirow{2}{*}{0.117} & \multirow{2}{*}{185.201} & $\mathrm{Fe}$ & 2.9 & 3.58 & 3.85 & 3.97 & 4.06 & 4.14 \\
\hline & & & & Error $(\%)$ & & 23.448 & 32.759 & 36.897 & 40.0 & 42.759 \\
\hline \multirow{2}{*}{267} & \multirow{2}{*}{$\mathrm{ScMnO} 3$} & \multirow{2}{*}{0.7} & \multirow{2}{*}{185.201} & $\mathrm{Mn}$ & 3.03 & 3.4 & 3.49 & 3.58 & 3.66 & 3.73 \\
\hline & & & & Error(\%) & & 12.211 & 15.182 & 18.152 & 20.792 & 23.102 \\
\hline \multirow{2}{*}{268} & \multirow{2}{*}{$\mathrm{CsFeCl} 3$} & \multirow{2}{*}{1.0 .14} & \multirow{2}{*}{189.223} & $\mathrm{Fe}$ & 3.16 & 3.5 & 3.5 & 3.58 & 3.63 & 3.66 \\
\hline & & & & Error(\%) & & 10.759 & 10.759 & 13.291 & \begin{tabular}{|l|}
14.873 \\
\end{tabular} & 15.823 \\
\hline \multirow{2}{*}{269} & \multirow{2}{*}{ ScMn6Ge6 } & \multirow{2}{*}{1.110} & \multirow{2}{*}{192.252} & $\mathrm{Mn}$ & 1.96 & 2.08 & 2.41 & 2.76 & 3.5 & 3.82 \\
\hline & & & & Error(\%) & & 6.122 & 22.959 & 40.816 & 78.571 & 94.898 \\
\hline \multirow{2}{*}{270} & \multirow{2}{*}{ ScMn6Ge6 } & \multirow{2}{*}{1.225} & \multirow{2}{*}{192.252} & $\mathrm{Mn}$ & 2.08 & 2.04 & 2.39 & 2.73 & 3.48 & 3.83 \\
\hline & & & & Error(\%) & & 1.923 & 14.904 & 31.25 & 67.308 & 84.135 \\
\hline \multirow{3}{*}{271} & & & & Co & 2.8 & 2.43 & 2.53 & 2.58 & 2.63 & 2.68 \\
\hline & $\mathrm{CsCoCl3}$ & 1.0 .9 & 193.259 & $\mathrm{Co}$ & 2.66 & 2.43 & 2.54 & 2.58 & 2.63 & 2.68 \\
\hline & & & & Error(\%) & & 10.93 & 7.077 & 5.433 & 3.6 & 2.519 \\
\hline & & & & $\mathrm{Co}$ & 0.61 & 0.64 & 0.53 & 0.42 & 0.31 & 0.27 \\
\hline 272 & Ba.5Co.5ClO13 & 0.118 & 194268 & Co & 2.21 & 2.48 & 2.62 & 2.71 & 2.82 & 2.88 \\
\hline 212 & Dascosulvis & 0.110 & 194.200 & Co & 0.35 & 0.39 & 0.36 & 0.44 & 0.46 & 0.97 \\
\hline & & & & Error(\%) & & 9.521 & 11.508 & 26.495 & 36.07 & 87.733 \\
\hline 273 & $\mathrm{Na} 3 \mathrm{Co}(\mathrm{CO} 3)_{2 \mathrm{Cl}}$ & 070 & |20326 & $\mathrm{Co}$ & 1.73 & 2.6 & 2.65 & 2.7 & 2.74 & 2.77 \\
\hline 263 & Nasuo(c)osul & 0.10 & 203.20 & \begin{tabular}{|l|} 
Error $(\%)$ \\
\end{tabular} & & 50.289 & 53.179 & 56.069 & 58.382 & 60.116 \\
\hline 274 & $\mathrm{NiS} 2$ & 0.150 & 205.33 & $\mathrm{Ni}$ & 0.99 & 0.59 & 1.09 & 1.24 & 1.37 & 1.45 \\
\hline 214 & IVINZ & 0.100 & 200.00 & Error $(\%)$ & & 40.404 & 10.101 & 25.253 & 38.384 & 46.465 \\
\hline 275 & MnTe2 & 0.20 & 205.33 & $\mathrm{Mn}$ & 4.28 & 4.06 & 4.22 & 4.33 & 4.41 & 4.48 \\
\hline 260 & 10111 $1 \mathrm{e} 2$ & 0.20 & 200.03 & \begin{tabular}{|l|} 
Error $(\%)$ \\
\end{tabular} & & 5.14 & 1.402 & 1.168 & 3.037 & 4.673 \\
\hline 276 & $\mathrm{Cd} 2 \mathrm{Os} 2 \mathrm{O} 7$ & 0.2 & 227.131 & Os & 1.04 & 0.73 & 1.31 & 1.66 & 1.92 & 2.14 \\
\hline 210 & UazOsZO & 0.2 & 226.151 & Error(\%) & & 29.808 & 25.962 & 59.615 & 84.615 & \begin{tabular}{|l}
105.769 \\
\end{tabular} \\
\hline
\end{tabular}

TABLE XVIII: Magnetic moments of the magnetic materials with $4 f / 5 f$ electron. The first four column give the chemical formulae (Formula), BCSID, MSG for each materials. The 5th to 10th column give the magnetic moments of each nonequivalent magnetic atom, including the experimental (Exp.) magnetic moments and the calculated magnetic moments with Coulomb interaction $U=0,2,4,6 \mathrm{eV}$ for $4 f / 5 f$ electron. If the materials have transition metal elements at the same time, we take the Coulomb interactions of $3 d / 4 d$ electron as $2 \mathrm{eV}$. The calculated magnetic moments closest to the experimental value are labeled by red color.

\begin{tabular}{|c|c|c|c|c|c|c|c|c|c|}
\hline \multirow{2}{*}{ No. } & \multirow{2}{*}{ Formula } & \multirow{2}{*}{ BCSID } & \multirow{2}{*}{ MSG } & \multicolumn{6}{|c|}{ Magnetic moments $\left(\mu_{B}\right)$} \\
\hline & & & & Mag. Ele. & Exp. & $\mathrm{U}=0$ & $\mathrm{U}=2 \mathrm{eV}$ & $\mathrm{U}=4 \mathrm{eV}$ & $\mathrm{U}=6 \mathrm{eV}$ \\
\hline 277 & $\mathrm{HoCr}(\mathrm{BO} 3) 2$ & 1.191 & 2.7 & $\mathrm{Cr}$ & 3.07 & 2.84 & - & 2.82 & - \\
\hline \multirow[t]{2}{*}{278} & \multirow[t]{2}{*}{ Dy $2 \mathrm{Fe} 2 \mathrm{Si} 2 \mathrm{C}$} & \multirow[t]{2}{*}{1.206} & \multirow[t]{2}{*}{2.7} & Dy & 12.11 & 4.81 & 4.87 & 4.99 & 5.14 \\
\hline & & & & Error(\%) & & 60.281 & 59.785 & 58.794 & 57.556 \\
\hline \multirow{2}{*}{279} & \multirow{2}{*}{$\mathrm{Tm} 2 \mathrm{BaNiO} 5$} & \multirow{2}{*}{1.218} & \multirow{2}{*}{2.7} & Tm & 3.33 & 1.45 & 1.73 & 1.8 & 1.83 \\
\hline & & & & Error $(\%)$ & & 77.793 & 33.589 & 34.277 & 46.871 \\
\hline \multirow{3}{*}{280} & \multirow{3}{*}{$\mathrm{Nd} 2 \mathrm{NaOsO} 6$} & \multirow{3}{*}{1.38} & \multirow{3}{*}{2.7} & $\mathrm{Nd}$ & 1.61 & - & - & 2.94 & - \\
\hline & & & & Os & 0.9 & - & - & 1.79 & - \\
\hline & & & & Error(\%) & & - & - & 90.748 & - \\
\hline 281 & U3Al2Si3 & 0.37 & 5.15 & $\mathrm{U}$ & 0.16 & 0.1 & 1.69 & 0.56 & 1.61 \\
\hline
\end{tabular}




\begin{tabular}{|c|c|c|c|c|c|c|c|c|c|}
\hline \multirow[t]{2}{*}{282} & \multirow[t]{2}{*}{ GdBiPt } & \multirow[t]{2}{*}{1.111} & \multirow[t]{2}{*}{9.40} & Gd & \begin{tabular}{|l|}
6.61 \\
\end{tabular} & 6.87 & 6.98 & 7.03 & 7.06 \\
\hline & & & & Error(\%) & & 3.933 & 5.598 & 6.354 & 6.808 \\
\hline \multirow[t]{2}{*}{283} & \multirow[t]{2}{*}{ ErVO3 } & \multirow[t]{2}{*}{0.104} & \multirow[t]{2}{*}{11.54} & V & 1.19 & 0.05 & 0.01 & 0.59 & 0.5 \\
\hline & & & & \begin{tabular}{|l|} 
Error(\%) \\
\end{tabular} & & 95.798 & 99.16 & 50.42 & 57.983 \\
\hline \multirow{3}{*}{284} & \multirow{3}{*}{ DyVO3 } & \multirow{3}{*}{0.106} & \multirow{3}{*}{11.54} & Dy & 7.76 & - & 4.79 & 4.91 & 4.99 \\
\hline & & & & $\mathrm{V}$ & 1.45 & - & 1.81 & 1.77 & 0.42 \\
\hline & & & & \begin{tabular}{|l|} 
Error(\%) \\
\end{tabular} & & - & 31.551 & 29.397 & 53.365 \\
\hline \multirow[t]{2}{*}{285} & \multirow[t]{2}{*}{ Tb2Fe2Si2C } & \multirow[t]{2}{*}{1.171} & \multirow[t]{2}{*}{12.63} & $\mathrm{~Tb}$ & 8.0 & 5.88 & 5.97 & 6.01 & - \\
\hline & & & & Error(\%) & & 26.5 & 25.375 & 24.875 & - \\
\hline \multirow[t]{2}{*}{286} & \multirow[t]{2}{*}{ DyCu2Si2 } & 1.22 & 12.63 & Dy & 8.3 & 4.31 & 4.56 & 4.85 & 4.94 \\
\hline & & & & Error(\%) & & 48.072 & 45.06 & 41.566 & 40.482 \\
\hline 287 & 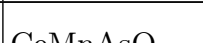 & 0.180 & 1267 & $\mathrm{Ce}$ & 0.76 & 0.64 & 0.3 & 0.36 & - \\
\hline 281 & UEMTASU & 0.188 & $13.0 \%$ & $\mathrm{Mn}$ & 3.32 & 3.47 & 4.0 & 4.28 & - \\
\hline & & & & Error(\%) & & 10.154 & 40.504 & 40.774 & - \\
\hline 288 & PrMgPb & 1.140 & 13.73 & Pr & 1.8 & 2.15 & 2.0 & 2.26 & 2.28 \\
\hline & & & & Error(\%) & & 19.444 & 11.111 & 25.556 & 26.667 \\
\hline 289 & $\mathrm{NdMgPb}$ & 1.141 & 13.73 & $\operatorname{Pr}$ & 3.38 & 2.13 & 2.01 & 1.86 & 1.82 \\
\hline & & & & Error(\%) & & 36.982 & 40.533 & 44.97 & 46.154 \\
\hline 290 & $\mathrm{Ho} 2 \mathrm{O} 2 \mathrm{Se}$ & 1.213 & 13.73 & Ho & 9.3 & 3.68 & 3.79 & 3.87 & 3.93 \\
\hline & & & & Error(\%) & & 60.43 & 59.247 & 58.387 & 57.742 \\
\hline 291 & ErVO3 & 0.105 & 1475 & Er & 8.2 & 2.47 & 2.78 & 2.82 & 2.87 \\
\hline & Ditro & 0.100 & 17.10 & $\mathrm{~V}$ & 1.47 & 0.15 & 1.7 & 0.18 & 0.3 \\
\hline & & & & Error(\%) & & 79.837 & 40.872 & 76.683 & 72.296 \\
\hline ل & NdaNTPOC & 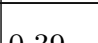 & 1475 & $\mathrm{Nd}$ & 2.25 & 2.93 & 2.92 & 2.93 & 2.94 \\
\hline 202 & 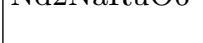 & & 17.10 & $\mathrm{Ru}$ & 1.62 & 1.57 & 0.76 & 2.04 & 2.04 \\
\hline & & & & Error(\%) & & 16.654 & 41.432 & 28.074 & 28.296 \\
\hline 293 & TbOOH & 2.21 & 14.78 & $\mathrm{~Tb}$ & 8.02 & 5.79 & 5.88 & 5.94 & - \\
\hline & & & & Error(\%) & & 27.805 & 26.683 & 25.935 & - \\
\hline 204 & SrHo 204 & 28 & 1478 & Ho & 6.08 & 3.75 & - & 3.9 & 3.97 \\
\hline 294 & DInO204 & 2.0 & 14.10 & Ho & 7.74 & 3.54 & - & 3.89 & 3.96 \\
\hline & & & & Error(\%) & & 46.293 & - & 42.799 & 41.771 \\
\hline 295 & BaNd2O4 & 1.95 & 14.80 & $\mathrm{Nd}$ & 2.64 & - & - & - & 2.94 \\
\hline & & & & \begin{tabular}{|l|} 
Error(\%) \\
\end{tabular} & & - & - & - & 11.364 \\
\hline 296 & LiErF4 & 1.35 & 14.84 & Er & 2.2 & 2.8 & 2.83 & 2.9 & 2.92 \\
\hline & & & & Error(\%) & & 27.273 & 28.636 & 31.818 & 32.727 \\
\hline 297 & Pr3Bu4Al12 & 0174 & 1589 & $\mathrm{Pr}$ & 3.2 & 1.96 & 2.06 & 2.18 & 1.71 \\
\hline 20 & 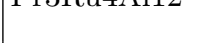 & 0.174 & 10.00 & $\mathrm{Pr}$ & \begin{tabular}{|l|l|}
1.39 \\
\end{tabular} & 1.95 & 2.04 & 2.04 & 1.01 \\
\hline & & & & Error(\%) & & 39.519 & 41.194 & 39.318 & 36.95 \\
\hline 298 & $\mathrm{NdCl} 2$ & 0.226 & 1589 & $\mathrm{Nd}$ & 2.8 & 3.5 & 0.44 & 0.97 & 3.17 \\
\hline & & & 10.05 & Co & 0.74 & 1.3 & 0.4 & 1.3 & 1.32 \\
\hline & & & & Error(\%) & & 50.338 & 65.116 & 70.517 & 45.796 \\
\hline 299 & HoP & 2.10 & 15.89 & Ho & 8.8 & 3.5 & 3.61 & 3.77 & 3.88 \\
\hline & & & & Error(\%) & & 60.227 & 58.977 & 57.159 & 55.909 \\
\hline 300 & $\mathrm{Ho} 2 \mathrm{BaNiO} 5$ & 114 & 15.90 & Ho & 9.06 & 3.65 & 3.85 & 3.92 & 3.99 \\
\hline & & & & $\mathrm{Ni}$ & 1.39 & 0.05 & 1.42 & 1.43 & 1.43 \\
\hline & & & & Error(\%) & & 78.058 & 29.832 & 29.805 & 29.419 \\
\hline 201 ل & FroponiO5 & 115 & 1500 & Ho & 7.89 & 3.65 & 3.82 & 3.92 & 3.99 \\
\hline 301 & | ErZBanivo & 1.10 & 10.90 & $\mathrm{Ni}$ & 1.54 & 0.06 & 0.18 & 1.43 & 1.43 \\
\hline & & & & Error(\%) & & 74.921 & 69.948 & 28.729 & 28.286 \\
\hline 302 & Dy2O2S & 1.211 & 15.90 & Dy & 7.2 & 4.85 & 4.85 & 4.89 & - \\
\hline & & & & \begin{tabular}{|l|} 
Error(\%) \\
\end{tabular} & & 32.639 & 32.639 & 32.083 & - \\
\hline 303 & Dy $2 \mathrm{O} 2 \mathrm{Se}$ & 1.212 & 15.90 & Dy & 9.0 & 4.84 & 4.85 & 4.89 & 4.96 \\
\hline
\end{tabular}




\begin{tabular}{|c|c|c|c|c|c|c|c|c|c|}
\hline & & & & Error(\%) & & 46.222 & 46.111 & 45.667 & 44.889 \\
\hline \multirow{3}{*}{304} & \multirow{3}{*}{ Nd2BaNiO5 } & \multirow{3}{*}{1.216} & \multirow{3}{*}{15.90} & $\mathrm{Nd}$ & 2.65 & 2.91 & 2.92 & 2.96 & 2.96 \\
\hline & & & & $\mathrm{Ni}$ & 1.58 & 0.14 & 0.23 & 1.55 & 1.55 \\
\hline & & & & \begin{tabular}{|l|} 
Error(\%) \\
\end{tabular} & & 50.476 & 47.816 & 6.798 & 6.798 \\
\hline \multirow{3}{*}{305} & \multirow{3}{*}{$\mathrm{Tb} 2 \mathrm{BaNiO} 5$} & \multirow{3}{*}{1.217} & \multirow{3}{*}{15.90} & $\mathrm{Ni}$ & 2.02 & - & 0.17 & 0.31 & 0.04 \\
\hline & & & & $\mathrm{Tb}$ & 8.03 & - & 6.01 & 6.05 & 6.15 \\
\hline & & & & Error(\%) & & - & 58.37 & 54.656 & 60.716 \\
\hline \multirow{3}{*}{306} & \multirow{3}{*}{ Dy2BaNiO5 } & \multirow{3}{*}{1.36} & \multirow{3}{*}{15.90} & Dy & 7.7 & 4.63 & 4.84 & 4.91 & 5.01 \\
\hline & & & & $\mathrm{Ni}$ & 1.35 & 0.15 & 1.55 & 0.04 & 0.04 \\
\hline & & & & Error(\%) & & 64.379 & 25.978 & 66.636 & 65.987 \\
\hline \multirow{3}{*}{307} & \multirow{3}{*}{$\mathrm{Er} 2 \mathrm{BaNiO} 5$} & \multirow{3}{*}{1.53} & \multirow{3}{*}{15.90} & Er & 7.24 & 2.66 & 2.82 & 2.84 & - \\
\hline & & & & $\mathrm{Ni}$ & 1.39 & 1.27 & 1.43 & 0.02 & - \\
\hline & & & & Error(\%) & & 35.947 & 31.964 & 79.668 & - \\
\hline \multirow[t]{2}{*}{308} & \multirow[t]{2}{*}{ HoMnO3 } & \multirow[t]{2}{*}{1.20} & 31.129 & Mn & 3.87 & 0.0 & - & - & - \\
\hline & & & & Error(\%) & & 100.0 & - & - & - \\
\hline 309 & ErAuGe & 1.33 & 33.154 & Er & 8.8 & 2.52 & 2.69 & 2.71 & 2.88 \\
\hline & & & & Error(\%) & & 71.364 & 69.432 & 69.205 & 67.273 \\
\hline 310 & PrNiO3 & 1.43 & 36.178 & $\mathrm{Ni}$ & 0.93 & - & 0.89 & 0.79 & 0.9 \\
\hline & & & & Error(\%) & & - & 4.301 & 15.054 & 3.226 \\
\hline 311 & TmAgGe & 0.26 & 38.191 & $\mathrm{Tm}$ & 6.44 & 1.49 & 1.6 & 1.55 & 1.51 \\
\hline & & & & Error(\%) & & 76.863 & 75.155 & 75.932 & 76.553 \\
\hline 312 & TbMg & 2.12 & 49.270 & $\mathrm{~Tb}$ & 7.34 & 5.9 & 5.86 & 6.06 & 6.18 \\
\hline & & & & Error(\%) & & 19.619 & 20.163 & 17.439 & 15.804 \\
\hline 313 & Ho2RhIn8 & 1.139 & 49.273 & Ho & 6.9 & 3.61 & 3.66 & 3.76 & 3.94 \\
\hline & & & & Error(\%) & & 47.681 & 46.957 & 45.507 & 42.899 \\
\hline 314 & $\mathrm{TbMg}$ & 2.11 & 51.295 & $\mathrm{~Tb}$ & 7.34 & 5.92 & 6.06 & 6.16 & 6.18 \\
\hline & & & & Error(\%) & & 19.346 & 17.439 & 16.076 & 15.804 \\
\hline 315 & Er2CoGa8 & 1.222 & 51.298 & Er & 4.71 & 2.46 & 2.69 & 2.81 & 2.91 \\
\hline & & & & Error(\%) & & 47.771 & 42.887 & 40.34 & 38.217 \\
\hline 316 & $\mathrm{PrAg}$ & 1.150 & 53.334 & $\operatorname{Pr}$ & 2.12 & 2.11 & 2.13 & 2.1 & 2.36 \\
\hline & & & & Error(\%) & & 0.472 & 0.472 & 0.943 & 11.321 \\
\hline 317 & DyB4 & 0.22 & 55.355 & Dy & 9.8 & 4.72 & 4.81 & 4.86 & 4.98 \\
\hline & & & & Error(\%) & & 51.837 & 50.918 & 50.408 & 49.184 \\
\hline 318 & $\mathrm{Gd} 2 \mathrm{CuO} 4$ & 1.105 & 56.374 & $\mathrm{Cu}$ & 1.22 & - & 0.45 & 0.56 & - \\
\hline & & & & \begin{tabular}{|l|} 
Error $(\%)$ \\
\end{tabular} & & - & 63.115 & 54.098 & - \\
\hline 319 & CeRu2Al10 & 1.8 & 57.391 & $\mathrm{Ce}$ & 0.34 & - & 0.02 & 0.17 & 0.22 \\
\hline & & & & Error(\%) & & - & 94.118 & 50.0 & 35.294 \\
\hline & & & & $\mathrm{Ce}$ & 0.7 & - & 0.77 & 0.44 & 0.05 \\
\hline 320 & CeMnAsO & 0.187 & 59.407 & $\mathrm{Mn}$ & 3.3 & - & 4.01 & 4.03 & 4.03 \\
\hline & & & & Error(\%) & & - & 15.758 & 29.632 & 57.49 \\
\hline 321 & EuZrO3 & 0.146 & 62.444 & $\mathrm{Eu}$ & 7.3 & 6.73 & 6.82 & 6.88 & 6.92 \\
\hline & & & & Error(\%) & & 7.808 & 6.575 & 5.753 & 5.205 \\
\hline & & & & $\mathrm{Nd}$ & 1.85 & 3.24 & 3.15 & - & 3.14 \\
\hline 322 & Nd5Ge4 & 0.185 & 62.447 & $\mathrm{Nd}$ & 3.17 & 3.15 & 3.05 & - & 3.03 \\
\hline & & & & $\mathrm{Nd}$ & 2.75 & 3.21 & 3.08 & - & 3.03 \\
\hline & & & & Error(\%) & & 30.831 & 28.685 & - & 28.109 \\
\hline 323 & EuZrO3 & 0.147 & 62.449 & $\mathrm{Eu}$ & 6.4 & 6.75 & 6.82 & 6.88 & 6.92 \\
\hline & & & & \begin{tabular}{|l|} 
Error(\%) \\
\end{tabular} & & 5.469 & 6.562 & 7.5 & 8.125 \\
\hline 324 & DyCoO3 & 0.159 & 62.449 & Dy & 9.08 & 4.83 & 4.86 & 4.93 & 5.0 \\
\hline & & & & Error(\%) & & 46.806 & 46.476 & 45.705 & 44.934 \\
\hline 325 & DyScO3 & 0.171 & 62.449 & Dy & 9.47 & 4.8 & 4.86 & 4.92 & 5.0 \\
\hline
\end{tabular}




\begin{tabular}{|c|c|c|c|c|c|c|c|c|c|}
\hline & & & & Error(\%) & & $\mid 49.314$ & $\mid 48.68$ & $\mid 48.046$ & |47.202 \\
\hline \multirow{3}{*}{326} & \multirow{3}{*}{$\mathrm{NdCoAsO}$} & \multirow{3}{*}{1.179} & \multirow{3}{*}{62.450} & $\mathrm{Nd}$ & 1.39 & 3.04 & 2.93 & 2.95 & 2.95 \\
\hline & & & & Co & 0.32 & $\mid 0.48$ & 0.56 & 0.57 & 0.56 \\
\hline & & & & Error(\%) & & 84.353 & 92.896 & 95.177 & 93.615 \\
\hline \multirow[t]{2}{*}{327} & \multirow[t]{2}{*}{ U3Ru4Al12 } & \multirow[t]{2}{*}{0.12} & \multirow[t]{2}{*}{63.461} & $\mathrm{U}$ & 2.5 & 1.49 & - & 1.63 & 1.43 \\
\hline & & & & Error(\%) & & 40.4 & - & 34.8 & 42.8 \\
\hline \multirow{3}{*}{328} & \multirow{3}{*}{ Nd3Ru4Al12 } & \multirow{3}{*}{0.149} & \multirow{3}{*}{63.462} & $\mathrm{Nd}$ & 0.95 & 0.07 & 0.23 & 0.07 & - \\
\hline & & & & $\mathrm{Nd}$ & 2.66 & 3.21 & 3.11 & 3.07 & - \\
\hline & & & & \begin{tabular}{|l|} 
Error(\%) \\
\end{tabular} & & 56.654 & 46.354 & 54.023 & - \\
\hline \multirow{3}{*}{329} & \multirow{3}{*}{ Pr3Ru4Al12 } & \multirow{3}{*}{0.173} & \multirow{3}{*}{63.462} & $\operatorname{Pr}$ & 3.1 & 1.85 & 2.04 & 2.04 & 2.03 \\
\hline & & & & $\operatorname{Pr}$ & 1.4 & 0.07 & 0.1 & 2.04 & 1.03 \\
\hline & & & & Error(\%) & & \begin{tabular}{|l|}
67.661 \\
\end{tabular} & 63.526 & 39.954 & 30.472 \\
\hline \multirow[t]{2}{*}{330} & \multirow[t]{2}{*}{ Ho2RhIn8 } & 3.3 & 63.464 & Ho & 7.5 & 3.41 & 3.55 & - & - \\
\hline & & & & Error(\%) & & 54.533 & 52.667 & - & - \\
\hline 331 & U2Ni2Sn & 1.200 & 63.466 & $\mathrm{U}$ & 1.41 & 1.44 & 1.74 & 1.69 & 1.3 \\
\hline & & & & Error(\%) & & 2.128 & 23.404 & 19.858 & 7.801 \\
\hline 332 & NpRhGa5 & 1.262 & 63.466 & $\mathrm{~Np}$ & 0.89 & 2.86 & 3.39 & 3.59 & 3.73 \\
\hline & & & & Error(\%) & & 221.348 & 280.899 & 303.371 & 319.101 \\
\hline 333 & Er2Ni2In & 1.195 & 63.467 & Er & 7.71 & - & 2.7 & 2.78 & 2.91 \\
\hline & & & & Error(\%) & & - & 64.981 & 63.943 & 62.257 \\
\hline 334 & CeB6 & 3.13 & 64.479 & $\mathrm{Ce}$ & 0.28 & 0.57 & - & - & - \\
\hline & & & & Error(\%) & & 103.571 & - & - & - \\
\hline 335 & CeRh2Si2 & 1.188 & 64.480 & $\mathrm{Ce}$ & 1.5 & 0.22 & 0.87 & 0.94 & 0.15 \\
\hline & & & & Error(\%) & & 85.333 & 42.0 & 37.333 & 90.0 \\
\hline & & & & $\mathrm{Tb}$ & 9.45 & 5.81 & 5.92 & 5.99 & - \\
\hline 336 & TbGe2 & 0.141 & 65.483 & $\mathrm{~Tb}$ & 7.55 & 5.69 & 5.73 & 5.98 & - \\
\hline & & & & Error(\%) & & 31.577 & 30.73 & 28.704 & - \\
\hline 337 & Tm2CoGa8 & 1.223 & 65.489 & $\mathrm{Tm}$ & 2.35 & 1.23 & 1.4 & 1.48 & 1.78 \\
\hline & & & & Error(\%) & & 47.66 & 40.426 & 37.021 & 24.255 \\
\hline & & & & $\operatorname{Pr}$ & 0.08 & - & 1.85 & 0.16 & 1.95 \\
\hline 338 & $\mathrm{Pr} 2 \mathrm{CuO} 4$ & 1.106 & 66.500 & $\mathrm{Cu}$ & \begin{tabular}{|l|}
0.4 \\
\end{tabular} & - & \begin{tabular}{|l|}
0.04 \\
\end{tabular} & \begin{tabular}{|l|}
0.02 \\
\end{tabular} & \begin{tabular}{|l|}
0.37 \\
\end{tabular} \\
\hline & & & & Error(\%) & & - & 1151.25 & 97.5 & 1172.5 \\
\hline 339 & $\mathrm{CeMgPb}$ & 1.142 & 67.510 & $\mathrm{Ce}$ & 1.39 & 0.66 & 0.89 & 0.02 & 0.03 \\
\hline & & & & Error(\%) & & 52.518 & 35.971 & 98.561 & 97.842 \\
\hline 340 & EuTiO3 & 0.16 & 69.523 & $\mathrm{Eu}$ & 6.93 & - & - & 6.87 & - \\
\hline & & & & \begin{tabular}{|l|} 
Error $(\%)$ \\
\end{tabular} & & - & - & 0.866 & - \\
\hline 341 & UAu2Si2 & 1.0 .12 & 71.536 & $\mathrm{U}$ & 0.9 & 0.36 & 2.32 & 2.51 & 2.65 \\
\hline & & & & \begin{tabular}{|l|} 
Error(\%) \\
\end{tabular} & & 60.0 & 157.778 & 178.889 & 194.444 \\
\hline 342 & NpNiGa5 & 2.28 & 74.559 & $\mathrm{~Np}$ & 0.84 & 0.18 & 3.45 & 3.59 & 3.74 \\
\hline & & & & Error(\%) & & 78.571 & 310.714 & 327.381 & 345.238 \\
\hline 343 & KTb3F12 & 1.59 & 84.58 & $\mathrm{~Tb}$ & 6.95 & 6.23 & 6.41 & 6.63 & - \\
\hline & & & & Error(\%) & & 10.36 & 7.77 & 4.604 & - \\
\hline 344 & Ho2Ge2O7 & 0.107 & 92.111 & Ho & \begin{tabular}{|l|}
9.06 \\
\end{tabular} & 3.75 & 3.84 & 3.88 & 3.94 \\
\hline & & & & Error(\%) & & 58.609 & 57.616 & 57.174 & 56.512 \\
\hline & & & & $\mathrm{Nd}$ & 2.39 & 3.32 & 3.31 & 3.25 & - \\
\hline 345 & Nd5Si4 & 0.184 & 92.114 & $\mathrm{Nd}$ & 2.47 & 3.22 & 3.17 & 3.12 & - \\
\hline & & & & $\mathrm{Nd}$ & 2.79 & 3.29 & 3.21 & 3.16 & - \\
\hline & & & & \begin{tabular}{|l|} 
Error $(\%)$ \\
\end{tabular} & & 29.066 & 27.296 & 25.187 & - \\
\hline 346 & CeCoGe3 & 1.0 .11 & 107.231 & $\mathrm{Ce}$ & 0.36 & 0.07 & 0.46 & 0.06 & - \\
\hline & & & & Error(\%) & & 80.556 & 27.778 & 83.333 & - \\
\hline 347 & $\mathrm{PrCo} 2 \mathrm{P} 2$ & 2.26 & 123345 & $\mathrm{Pr}$ & 3.08 & 1.76 & 1.76 & 1.97 & 1.96 \\
\hline 341 & PTCOLYZ & 2.20 & 120.040 & Co & 0.9 & 0.73 & 0.88 & 0.86 & 0.87 \\
\hline
\end{tabular}




\begin{tabular}{|c|c|c|c|c|c|c|c|c|c|}
\hline & & & & Error(\%) & & 30.873 & 22.54 & $\mid 20.241$ & 19.849 \\
\hline \multirow[t]{2}{*}{$\overline{348}$} & \multirow[t]{2}{*}{$\mathrm{NdMg}$} & \multirow[t]{2}{*}{1.162} & \multirow[t]{2}{*}{124.360} & $\mathrm{Nd}$ & 2.6 & 3.42 & 3.49 & 3.26 & 3.13 \\
\hline & & & & Error(\%) & & 31.538 & 34.231 & 25.385 & 20.385 \\
\hline \multirow{3}{*}{349} & \multirow{3}{*}{$\mathrm{NdCo} 2 \mathrm{P} 2$} & \multirow{3}{*}{1.251} & \multirow{3}{*}{124.360} & $\mathrm{Nd}$ & 0.74 & 0.71 & 2.99 & 2.96 & 0.38 \\
\hline & & & & Co & 0.64 & 0.75 & 0.91 & 0.01 & 0.87 \\
\hline & & & & Error $(\%)$ & & 10.621 & 173.121 & 199.219 & 42.293 \\
\hline \multirow[t]{2}{*}{350} & \multirow[t]{2}{*}{ UPtGa5 } & \multirow[t]{2}{*}{1.255} & \multirow[t]{2}{*}{124.360} & $\mathrm{U}$ & 0.32 & 0.94 & 1.67 & 2.35 & 1.57 \\
\hline & & & & Error(\%) & & 193.75 & 421.875 & 634.375 & 390.625 \\
\hline \multirow[t]{2}{*}{351} & \multirow[t]{2}{*}{ NpRhGa5 } & \multirow[t]{2}{*}{1.261} & \multirow[t]{2}{*}{124.360} & $\mathrm{~Np}$ & 0.32 & 0.05 & 3.39 & 0.35 & 2.79 \\
\hline & & & & Error(\%) & & 84.375 & 959.375 & 9.375 & 771.875 \\
\hline \multirow[t]{2}{*}{352} & \multirow[t]{2}{*}{ CeMn2Ge4O12 } & 0.189 & 125.367 & $\mathrm{Mn}$ & 4.61 & 4.41 & 4.42 & 4.43 & 4.45 \\
\hline & & & & Error(\%) & & 4.338 & 4.121 & 3.905 & 3.471 \\
\hline 353 & $\mathrm{NdMg}$ & 2.14 & 125.373 & $\mathrm{Nd}$ & 2.56 & 3.41 & 3.28 & 3.2 & 3.16 \\
\hline & & & & Error(\%) & & 33.203 & 28.125 & 25.0 & 23.438 \\
\hline 354 & CeCo2P2 & 1.253 & 126.386 & Co & 0.94 & 0.78 & 0.96 & 0.95 & 0.93 \\
\hline & & & & Error(\%) & & 17.021 & 2.128 & 1.064 & 1.064 \\
\hline 355 & U2Pd2In & 0.80 & 127.394 & $\mathrm{U}$ & 1.4 & 1.53 & 1.55 & 2.39 & 2.54 \\
\hline & & & & Error(\%) & & 9.286 & 10.714 & 70.714 & 81.429 \\
\hline 356 & U2Pd2Sn & 0.81 & 127.394 & $\mathrm{U}$ & 1.9 & 1.59 & 1.5 & 2.44 & 2.57 \\
\hline & & & & Error(\%) & & 16.316 & \begin{tabular}{|l|}
21.053 \\
\end{tabular} & \begin{tabular}{|l|}
28.421 \\
\end{tabular} & \begin{tabular}{|l|}
35.263 \\
\end{tabular} \\
\hline 357 & GdB4 & 0.9 & 127.395 & $\mathrm{Gd}$ & 7.14 & 6.84 & 6.93 & 6.99 & 7.04 \\
\hline & & & & Error(\%) & & 4.202 & 2.941 & 2.101 & 1.401 \\
\hline 358 & GdIn3 & 1.81 & 127.397 & Gd & 1.0 & 0.36 & 0.09 & 1.32 & 1.13 \\
\hline & & & & Error(\%) & & 64.0 & 91.0 & 32.0 & 13.0 \\
\hline 250 & & & & $\mathrm{U}$ & 0.59 & 1.43 & 1.5 & 1.67 & 2.56 \\
\hline 359 & U2N12In & 1.102 & 128.408 & $\mathrm{Ni}$ & 0.37 & 0.01 & 0.01 & 0.01 & 0.0 \\
\hline & & & & Error(\%) & & 119.835 & 125.768 & 140.174 & 216.949 \\
\hline 360 & UP & 1.160 & 128.410 & $\mathrm{U}$ & 1.7 & 1.58 & 1.64 & 1.77 & 1.4 \\
\hline & & & & Error(\%) & & 7.059 & 3.529 & 4.118 & 17.647 \\
\hline 361 & TbRh2Si2 & 1.187 & 128.410 & $\mathrm{~Tb}$ & 8.5 & 6.07 & 5.93 & 6.0 & 6.04 \\
\hline & & & & Error(\%) & & 28.588 & 30.235 & 29.412 & 28.941 \\
\hline 362 & UAs & 1.208 & 128.410 & $\mathrm{U}$ & 1.9 & 1.79 & 1.85 & 2.09 & 2.29 \\
\hline & & & & Error(\%) & & 5.789 & 2.632 & 10.0 & 20.526 \\
\hline 363 & DyCo2Si2 & 1.21 & 128.410 & Dy & 9.5 & 4.83 & \begin{tabular}{|l|}
4.87 \\
\end{tabular} & 4.9 & 4.95 \\
\hline & & & & Error(\%) & & 49.158 & 48.737 & 48.421 & 47.895 \\
\hline 364 & CeMnAsO & 0.186 & 129.416 & $\mathrm{Mn}$ & 2.78 & - & 3.98 & 3.99 & 3.99 \\
\hline & & & & Error(\%) & & - & 43.165 & 43.525 & 43.525 \\
\hline 365 & UPt2Si2 & 0.194 & 129.419 & $\mathrm{U}$ & 1.67 & 1.54 & 1.78 & 1.72 & 1.55 \\
\hline & & & & Error(\%) & & 7.784 & 6.587 & 2.994 & 7.186 \\
\hline 366 & UP2 & 1.215 & 130.432 & $\mathrm{U}$ & 1.0 & 1.05 & 1.26 & 1.4 & 1.46 \\
\hline & & & & Error(\%) & & 5.0 & 26.0 & 40.0 & 46.0 \\
\hline 367 & CeSbTe & 1.271 & 130.432 & $\mathrm{Ce}$ & 0.38 & 0.44 & 0.88 & 0.08 & 0.13 \\
\hline & & & & \begin{tabular}{|l|} 
Error(\%) \\
\end{tabular} & & 15.789 & 131.579 & 78.947 & \begin{tabular}{|l}
65.789 \\
\end{tabular} \\
\hline 368 & UP & 2.13 & 134.481 & U & 1.91 & 1.62 & 1.91 & 2.19 & 2.34 \\
\hline & & & & Error(\%) & & 15.183 & 0.0 & 14.66 & 22.513 \\
\hline 369 & UAs & 2.20 & 134.481 & $\mathrm{U}$ & 2.26 & 1.74 & 2.07 & 2.26 & 2.43 \\
\hline & & & & \begin{tabular}{|l|} 
Error(\%) \\
\end{tabular} & & 23.009 & 8.407 & 0.0 & 7.522 \\
\hline 370 & $\mathrm{Nd} 2 \mathrm{CuO} 4$ & 2.6 & 134.481 & $\mathrm{Cu}$ & 1.0 & - & 0.3 & 0.36 & 0.27 \\
\hline & & & & Error(\%) & & - & 70.0 & 64.0 & 73.0 \\
\hline 371 & U2Rh2Sn & 1.103 & 135.492 & $\mathrm{U}$ & 0.53 & 1.17 & 1.42 & 1.58 & 1.53 \\
\hline & & & & Error(\%) & & 120.755 & 167.925 & 198.113 & 188.679 \\
\hline 372 & U2Rh2Sn & 1.207 & 135.492 & $\mathrm{U}$ & 0.5 & 1.1 & 1.4 & 1.57 & 1.57 \\
\hline
\end{tabular}




\begin{tabular}{|c|c|c|c|c|c|c|c|c|c|}
\hline & & & & $\mathrm{Rh}$ & 0.06 & 0.0 & 0.01 & 0.01 & 0.01 \\
\hline & & & & Error(\%) & & 110.0 & 131.667 & 148.667 & 148.667 \\
\hline \multirow[t]{2}{*}{373} & \multirow{2}{*}{ UNiGa5 } & \multirow{2}{*}{1.254} & \multirow{2}{*}{140.550} & $\mathrm{U}$ & 0.75 & 0.0 & 2.17 & 2.31 & 2.58 \\
\hline & & & & Error(\%) & & 100.0 & 189.333 & 208.0 & 244.0 \\
\hline \multirow[t]{2}{*}{374} & \multirow[t]{2}{*}{ Nd2RhIn8 } & \multirow[t]{2}{*}{1.82} & \multirow[t]{2}{*}{140.550} & $\mathrm{Nd}$ & 2.53 & 3.5 & 3.3 & 3.14 & 3.03 \\
\hline & & & & Error(\%) & & 38.34 & 30.435 & 24.111 & 19.763 \\
\hline \multirow[t]{2}{*}{375} & \multirow[t]{2}{*}{ TbCo2Ga8 } & \multirow[t]{2}{*}{1.87} & \multirow[t]{2}{*}{140.550} & $\mathrm{~Tb}$ & 9.6 & 5.54 & 5.85 & 5.98 & 6.05 \\
\hline & & & & Error(\%) & & 42.292 & 39.063 & 37.708 & 36.979 \\
\hline \multirow{3}{*}{376} & \multirow{3}{*}{ Er2Ru2O7 } & \multirow{3}{*}{0.154} & \multirow{3}{*}{141.554} & Er & 4.5 & 2.55 & 2.79 & 2.84 & 2.87 \\
\hline & & & & $\mathrm{Ru}$ & 2.0 & 0.13 & 1.22 & 1.24 & 0.02 \\
\hline & & & & Error(\%) & & 68.417 & 38.5 & 37.444 & 67.611 \\
\hline \multirow[t]{2}{*}{$\overline{377}$} & \multirow[t]{2}{*}{ Er2Ti2O7 } & 0.29 & 141.554 & Er & 3.25 & 2.74 & 2.8 & 2.84 & 2.91 \\
\hline & & & & Error(\%) & & 15.692 & 13.846 & 12.615 & 10.462 \\
\hline 378 & Gd2Sn2O7 & 0.47 & 141.555 & $\mathrm{Gd}$ & 6.08 & 6.83 & 6.89 & 6.95 & 7.0 \\
\hline & & & & Error(\%) & & 12.336 & 13.322 & 14.309 & 15.132 \\
\hline 379 & $\mathrm{NpCo} 2$ & 0.126 & 141.556 & $\mathrm{~Np}$ & 0.5 & 0.0 & 0.08 & 0.03 & 0.02 \\
\hline & & & & Error(\%) & & 100.0 & 84.0 & 94.0 & 96.0 \\
\hline 380 & GdVO4 & 0.198 & 141.556 & $\mathrm{Gd}$ & 7.0 & 6.83 & 6.89 & 6.93 & 6.97 \\
\hline & & & & Error(\%) & & 2.429 & 1.571 & 1.0 & 0.429 \\
\hline 381 & & 0151 & & $\mathrm{Mn}$ & 1.85 & - & - & - & 2.93 \\
\hline 381 & $1 \mathrm{mzMn} z \mathrm{O}$ & 0.151 & 141.556 & $\mathrm{Tm}$ & 2.31 & - & - & - & 1.8 \\
\hline & & & & Error(\%) & & - & - & - & 40.228 \\
\hline 382 & Yb2Sn2O7 & 0.157 & 141.557 & $\mathrm{Yb}$ & 1.05 & - & 0.61 & 0.68 & 0.75 \\
\hline & & & & Error(\%) & & - & 41.905 & 35.238 & 28.571 \\
\hline 383 & $\mathrm{NdCO} 2$ & م 2277 & 141557 & $\mathrm{Nd}$ & 2.43 & 0.09 & 0.21 & 3.12 & 2.68 \\
\hline 383 & NaCo & 0.221 & 141.530 & Co & 0.59 & 0.24 & 1.18 & 1.7 & 1.07 \\
\hline & & & & Error(\%) & & 77.809 & 95.679 & 108.266 & 45.822 \\
\hline 384 & $\mathrm{~Tb} 2 \mathrm{Sn} 2 \mathrm{O} 7$ & 0.48 & 141.557 & $\mathrm{~Tb}$ & 5.87 & 5.8 & 5.87 & 5.94 & 6.02 \\
\hline & & & & Error(\%) & & 1.193 & 0.0 & 1.193 & 2.555 \\
\hline 385 & Ho2Ru2O7 & 0.49 & 141.557 & $\mathrm{Ru}$ & 1.2 & 1.27 & 1.57 & 0.97 & 0.08 \\
\hline & & & & Error(\%) & & 5.833 & 30.833 & 19.167 & 93.333 \\
\hline 386 & & 0.51 & 141557 & Ho & 6.3 & 3.67 & 3.84 & 3.9 & 3.97 \\
\hline 386 & HozRuzOr & 0.51 & 141.557 & $\mathrm{Ru}$ & 1.8 & 0.46 & 1.57 & 1.63 & 1.81 \\
\hline & & & & Error(\%) & & 58.095 & 25.913 & 23.77 & 18.77 \\
\hline 387 & $\mathrm{PrFe} 3(\mathrm{BO} 3)_{4}$ & 1.161 & 155,48 & $\mathrm{Pr}$ & 0.79 & - & - & 2.58 & - \\
\hline 301 & | ГТГЕग(DUS 4 & 1.101 & 153.40 & $\mathrm{Fe}$ & 4.32 & - & - & 3.94 & - \\
\hline & & & & Error(\%) & & - & - & 117.689 & - \\
\hline 388 & U3As4 & 0.169 & 161.71 & $\mathrm{U}$ & 1.9 & 1.69 & 1.73 & 1.59 & 1.54 \\
\hline & & & & \begin{tabular}{|l|} 
Error(\%) \\
\end{tabular} & & 11.053 & 8.947 & 16.316 & 18.947 \\
\hline 389 & U3P4 & 0.170 & 161.71 & $\mathrm{U}$ & 1.48 & 1.62 & 1.66 & 1.49 & 1.5 \\
\hline & & & & Error(\%) & & 9.459 & 12.162 & 0.676 & 1.351 \\
\hline 390 & Nd3Sb3Mg2O14 & 0.167 & 166.101 & $\mathrm{Nd}$ & 1.79 & 2.95 & 2.95 & 2.95 & 2.95 \\
\hline & & & & Error(\%) & & 64.804 & 64.804 & 64.804 & 64.804 \\
\hline & & & & $\mathrm{Tb}$ & 8.3 & 0.29 & 5.89 & 6.01 & 5.24 \\
\hline 391 & $\mathrm{TbCo} 2$ & 0.228 & 166.101 & Co & 1.3 & 0.03 & 0.06 & 0.37 & 1.41 \\
\hline & & & & Co & 1.19 & 0.04 & 0.06 & 0.37 & 1.49 \\
\hline & & & & \begin{tabular}{|l} 
Error(\%) \\
\end{tabular} & & 96.946 & 73.127 & 56.012 & 23.513 \\
\hline & Th2Тi2О7 & 077 & 166101 & $\mathrm{~Tb}$ & 5.54 & - & - & - & 6.06 \\
\hline 392 & 10211201 & 0.18 & 106.101 & $\mathrm{~Tb}$ & 3.54 & - & - & - & 6.06 \\
\hline & & & & Error(\%) & & - & - & - & 40.286 \\
\hline 393 & TbMg3 & 1.189 & 167.108 & $\mathrm{~Tb}$ & 9.6 & 5.89 & - & 6.08 & - \\
\hline & & & & Error(\%) & & 38.646 & - & 36.667 & - \\
\hline
\end{tabular}




\begin{tabular}{|c|c|c|c|c|c|c|c|c|c|}
\hline \multirow[t]{2}{*}{394} & \multirow{2}{*}{ HoMnO3 } & \multirow[t]{2}{*}{0.32} & \multirow[t]{2}{*}{185.197} & $\mathrm{Mn}$ & \begin{tabular}{|l|}
2.98 \\
\end{tabular} & 3.48 & 0.03 & 0.55 & - \\
\hline & & & & Error(\%) & & 16.779 & 98.993 & 81.544 & - \\
\hline \multirow{3}{*}{395} & \multirow{3}{*}{ HoMnO3 } & \multirow{3}{*}{0.33} & \multirow{3}{*}{185.197} & Ho & 2.87 & 0.09 & 2.31 & 3.9 & - \\
\hline & & & & $\mathrm{Mn}$ & 3.05 & 0.05 & 0.07 & 0.05 & - \\
\hline & & & & Error(\%) & & 97.612 & 58.608 & 67.124 & - \\
\hline \multirow[t]{2}{*}{396} & \multirow[t]{2}{*}{ NdZn } & \multirow[t]{2}{*}{3.8} & \multirow[t]{2}{*}{222.103} & $\mathrm{Nd}$ & 2.51 & 3.37 & 3.33 & 3.17 & 3.14 \\
\hline & & & & Error(\%) & & 34.263 & 32.669 & 26.295 & 25.1 \\
\hline \multirow[t]{2}{*}{397} & \multirow[t]{2}{*}{$\mathrm{NpSb}$} & \multirow[t]{2}{*}{3.12} & \multirow[t]{2}{*}{224.113} & $\mathrm{~Np}$ & 2.86 & 3.46 & 3.61 & 3.71 & 3.79 \\
\hline & & & & Error(\%) & & 20.979 & 26.224 & 29.72 & 32.517 \\
\hline \multirow[t]{2}{*}{398} & \multirow[t]{2}{*}{ UO2 } & \multirow[t]{2}{*}{3.2} & \multirow[t]{2}{*}{224.113} & $\mathrm{U}$ & 1.73 & 0.96 & 1.49 & 1.53 & 1.56 \\
\hline & & & & Error(\%) & & 44.509 & 13.873 & 11.561 & 9.827 \\
\hline \multirow[t]{2}{*}{399} & \multirow[t]{2}{*}{$\mathrm{NpBi}$} & \multirow[t]{2}{*}{3.7} & \multirow[t]{2}{*}{224.113} & $\mathrm{~Np}$ & 2.42 & 3.59 & 3.69 & 3.76 & 3.83 \\
\hline & & & & Error(\%) & & 48.347 & 52.479 & 55.372 & 58.264 \\
\hline \multirow[t]{2}{*}{400} & \multirow[t]{2}{*}{$\mathrm{NpSe}$} & \multirow[t]{2}{*}{3.10} & \multirow[t]{2}{*}{228.139} & $\mathrm{~Np}$ & 1.3 & 3.42 & 3.64 & 3.77 & 3.86 \\
\hline & & & & Error(\%) & & 163.077 & 180.0 & 190.0 & 196.923 \\
\hline \multirow[t]{2}{*}{401} & \multirow[t]{2}{*}{ NpTe } & \multirow[t]{2}{*}{3.11} & \multirow[t]{2}{*}{228.139} & $\mathrm{~Np}$ & 1.4 & 3.69 & 3.76 & 3.85 & 3.92 \\
\hline & & & & Error(\%) & & 163.571 & 168.571 & 175.0 & 180.0 \\
\hline \multirow[t]{2}{*}{402} & \multirow[t]{2}{*}{$\mathrm{NpS}$} & 3.9 & 228.139 & $\mathrm{~Np}$ & 0.69 & 3.22 & 3.51 & 3.69 & 3.81 \\
\hline & & & & Error $(\%)$ & & 366.667 & 408.696 & 434.783 & 452.174 \\
\hline 403 & $\mathrm{DyCu}$ & 3.6 & 229.143 & Dy & 8.63 & 4.4 & 4.41 & 4.65 & 5.04 \\
\hline & & & & Error(\%) & & 49.015 & 48.899 & 46.118 & 41.599 \\
\hline
\end{tabular}




\section{Ferro(Ferri)magnetic materials}

Among the 403 materials, there have 51 magnetic materials with net moments/ferromagnetic canting. All of them are tabulated in Table XIX with the BCSID, chemical formula and the MSG. All of the MSGs of them are Type-I/Type-III MSG, which are compatible with ferro(ferri)magnets.

TABLE XIX: Magnetic materials with non-zero net moments or ferromagnetic canting.

\begin{tabular}{|c|c|c||c|c|c||c|c|c|}
\hline BCSID & Formula & MSG & BCSID & Formula & MSG & BCSID & Formula & MSG \\
\hline 0.26 & TmAgGe & 38.191 & 0.36 & NiF2 & 58.398 & 0.37 & U3Al2Si3 & 5.15 \\
\hline 0.48 & Tb2Sn2O7 & 141.557 & 0.49 & Ho2Ru2O7 & 141.557 & 0.64 & MnV2O4 & 88.81 \\
\hline 0.77 & Tb2Ti2O7 & 166.101 & 0.78 & NiN2O6 & 148.17 & 0.85 & KCo4(PO4)3 & 58.398 \\
\hline 0.91 & Rb2Fe2O(AsO4)2 & 62.448 & 0.121 & Li2Co(SO4)2 & 14.79 & 0.122 & Li2Mn(SO4)2 & 14.75 \\
\hline 0.149 & Nd3Ru4l12 & 63.462 & 0.151 & Tm2Mn2O7 & 141.557 & 0.157 & Yb2Sn2O7 & 141.557 \\
\hline 0.165 & SrMn(VO4)(OH) & 4.7 & 0.169 & U3As4 & 161.71 & 0.170 & U3P4 & 161.71 \\
\hline 0.173 & Pr3Ru4Al12 & 63.462 & 0.174 & Pr3Ru4Al12 & 15.89 & 0.176 & Mn3Ti2Te6 & 15.89 \\
\hline 0.184 & Nd5Si4 & 92.114 & 0.185 & Nd5Ge4 & 62.447 & 0.191 & BaCuF4 & 36.176 \\
\hline 0.203 & Mn3Ge & 12.62 & 0.220 & Mn2SiO4 & 62.446 & 0.226 & NdCo2 & 15.89 \\
\hline 0.227 & NdCo2 & 141.557 & 0.228 & TbCo2 & 166.101 & 1.0 .11 & CeCoGe3 & 107.231 \\
\hline 1.0 .12 & UAu2Si2 & 71.536 & 1.0 .13 & FeI2 & 12.62 & 2.5 & Mn3CuN & 85.59 \\
\hline 2.10 & HoP & 15.89 & 2.11 & TbMg & 51.295 & 2.12 & TbMg & 49.270 \\
\hline 2.19 & Mn3ZnC & 139.537 & 2.28 & NpNiGa5 & 74.559 & 3.3 & Ho2RhIn8 & 63.464 \\
\hline
\end{tabular}

\section{Appendix M: Band structures and detailed information}

We list the band structures for all of the 403 magnetic materials in the following tables, with the energy range set between $-1.0 \sim 1.0 \mathrm{eV}$ relative to the Fermi level. Apart from the crystal and band structures for each material, the tables also include the material identification number on BCSMD (BCSID), chemical formula (Formula), the ICSD number if available, the magnetic space group (MSG) and stable topological classification of the MSG (T.C.). For the magnetic TIs diagnosed by MTQC, see $\mathrm{H}$ for the stable topological indices and the

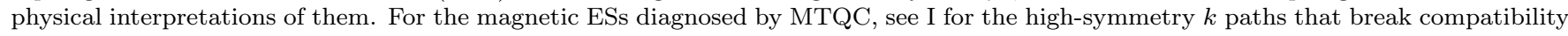
relations. 


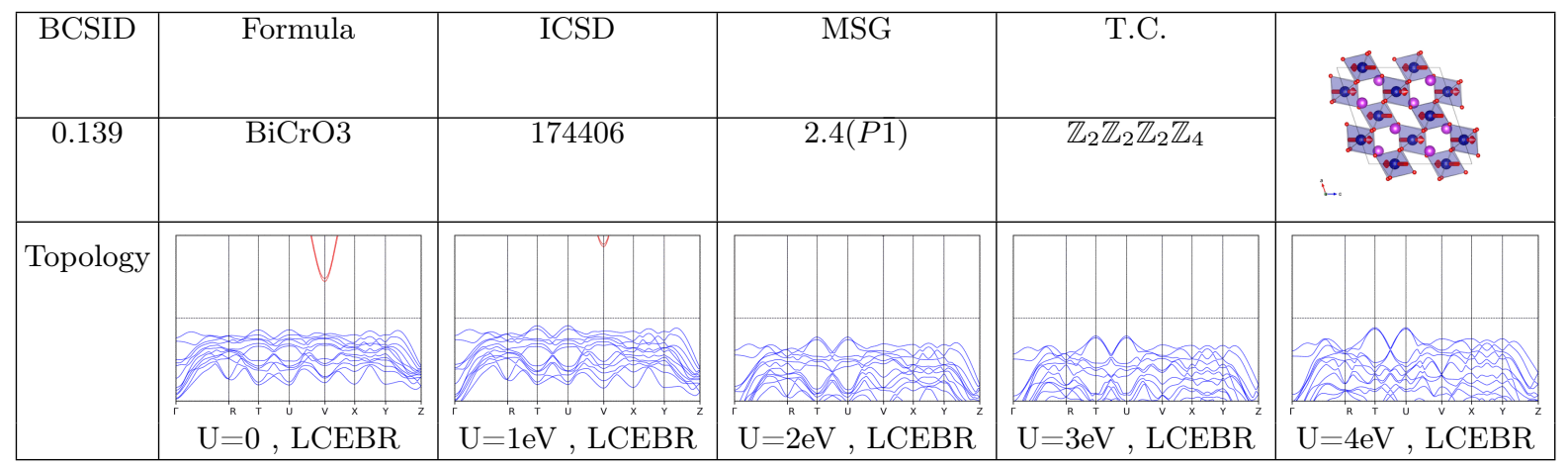

TABLE XX. Topology phase diagram of BiCrO3.

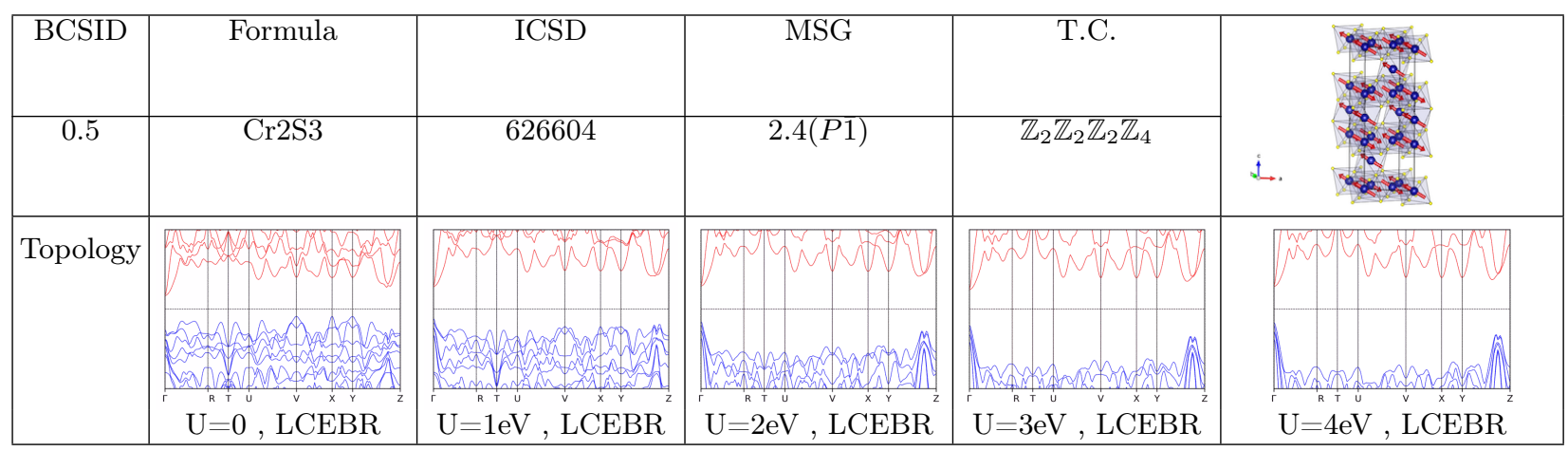

TABLE XXI. Topology phase diagram of Cr2S3.

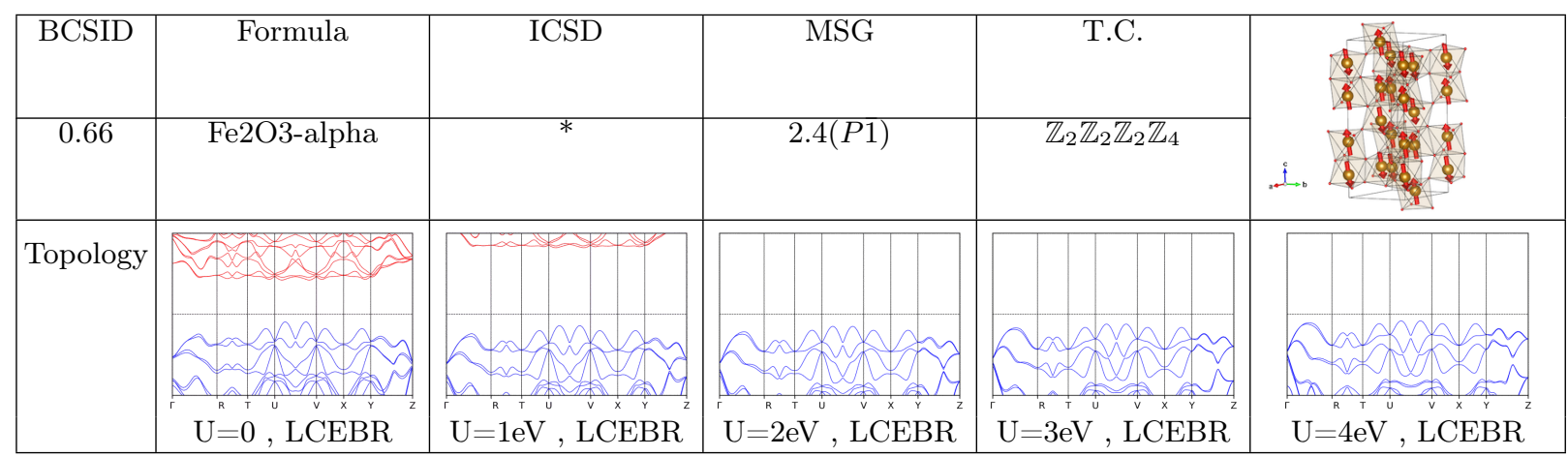

TABLE XXII. Topology phase diagram of Fe2O3-alpha.

\begin{tabular}{|c|c|c|c|c|c|}
\hline BCSID & Formula & ICSD & MSG & T.C. & \\
\hline 0.155 & CaMnGe2O6 & * & $2.6\left(P 1^{\prime}\right)$ & $\mathrm{w} / \mathrm{o}$ & 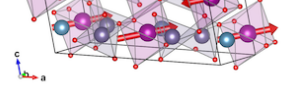 \\
\hline \multicolumn{6}{|l|}{ Topology } \\
\hline & $\mathrm{U}^{\mathrm{R}}=0, \mathrm{~T}$ & $\mathrm{U}=1 \mathrm{eV}, \mathrm{LCEBR}$ & $\mathrm{U}=2 \mathrm{eV}, \mathrm{LCEBR}^{\mathrm{R}}$ & $\mathrm{U}=3 \mathrm{eV}, \mathrm{LCEBR}^{\mathrm{R}}$ & $\mathrm{U}=4 \mathrm{eV}, \mathrm{LCEBR}$ \\
\hline
\end{tabular}

TABLE XXIII. Topology phase diagram of CaMnGe2O6. 


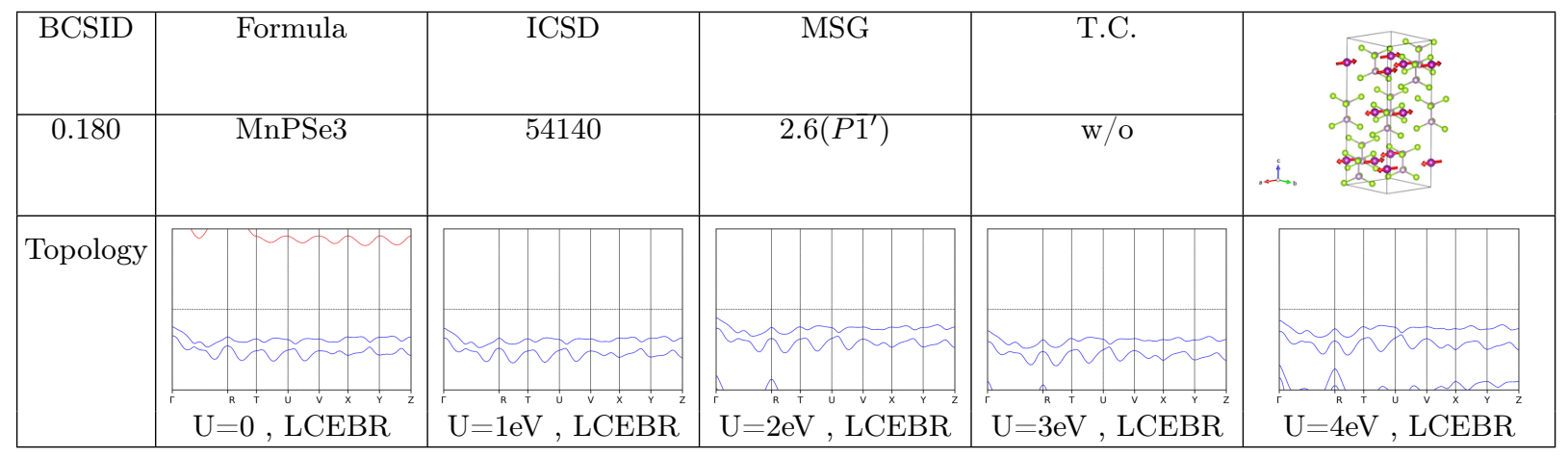

TABLE XXIV. Topology phase diagram of MnPSe3.

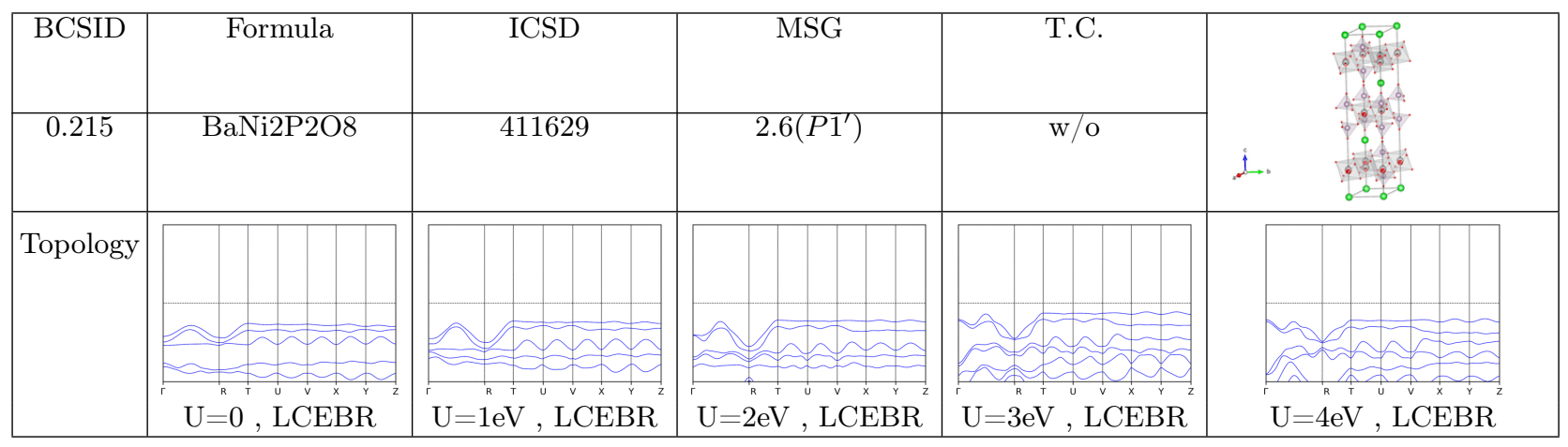

TABLE XXV. Topology phase diagram of BaNi2P2O8.

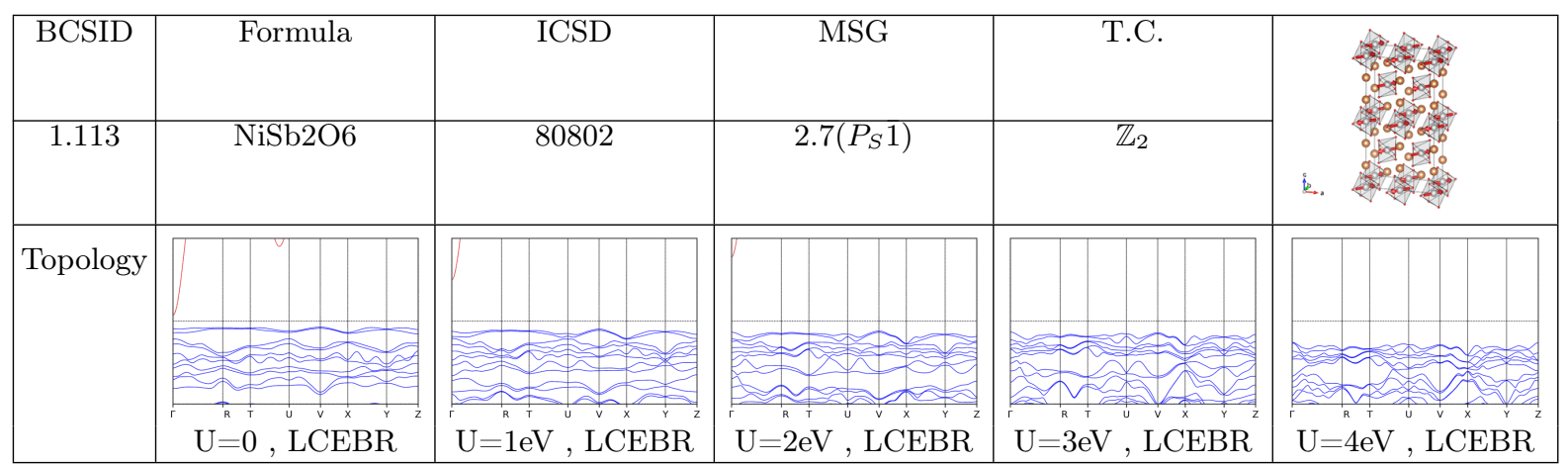

TABLE XXVI. Topology phase diagram of NiSb2O6.

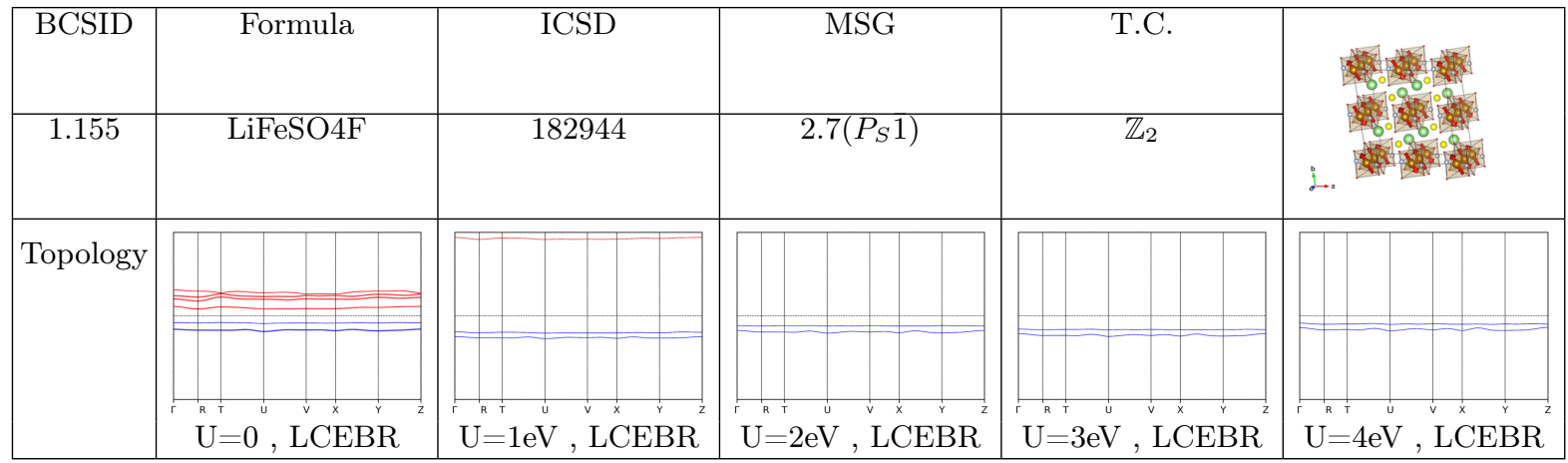

TABLE XXVII. Topology phase diagram of LiFeSO4F. 




TABLE XXVIII. Topology phase diagram of Li2Ni(WO4)2.

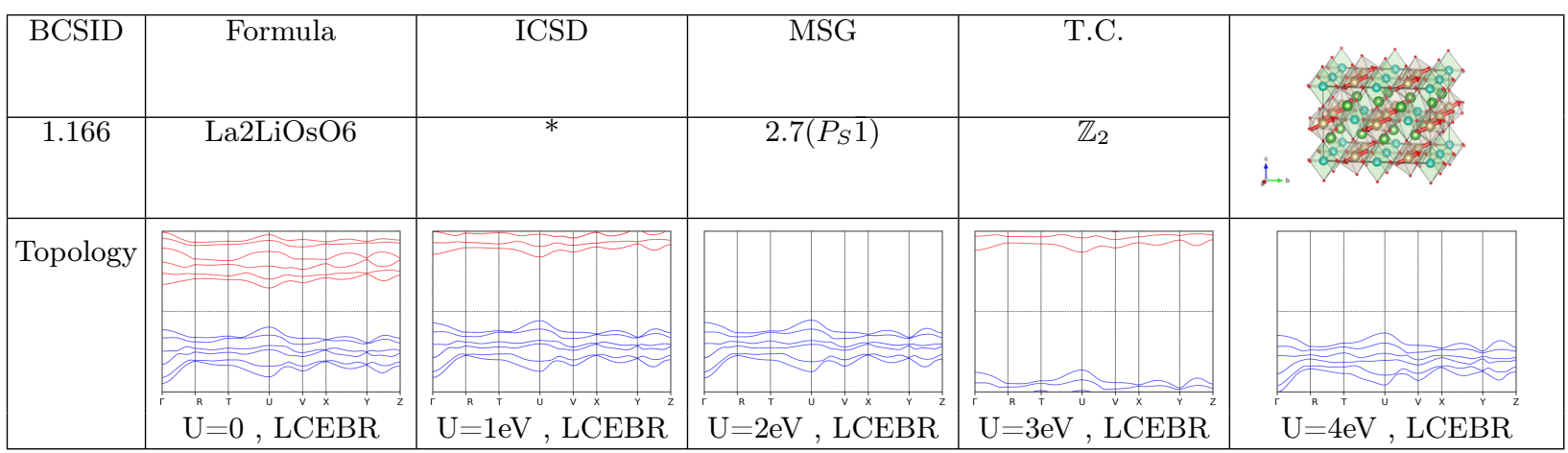

TABLE XXIX. Topology phase diagram of La2LiOsO6.

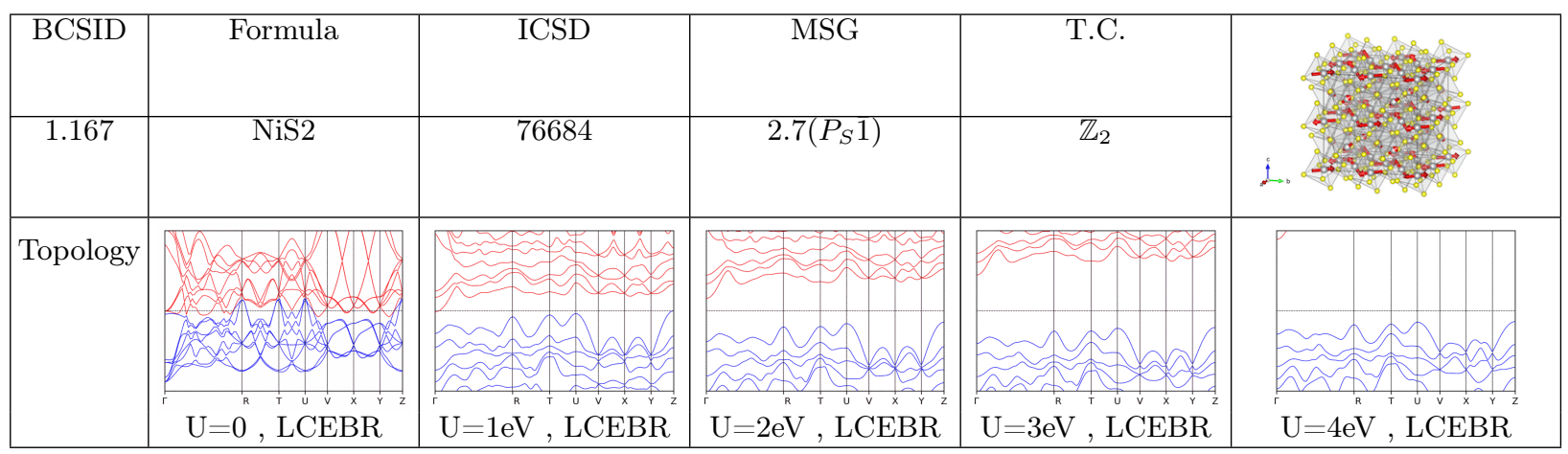

TABLE XXX. Topology phase diagram of NiS2.

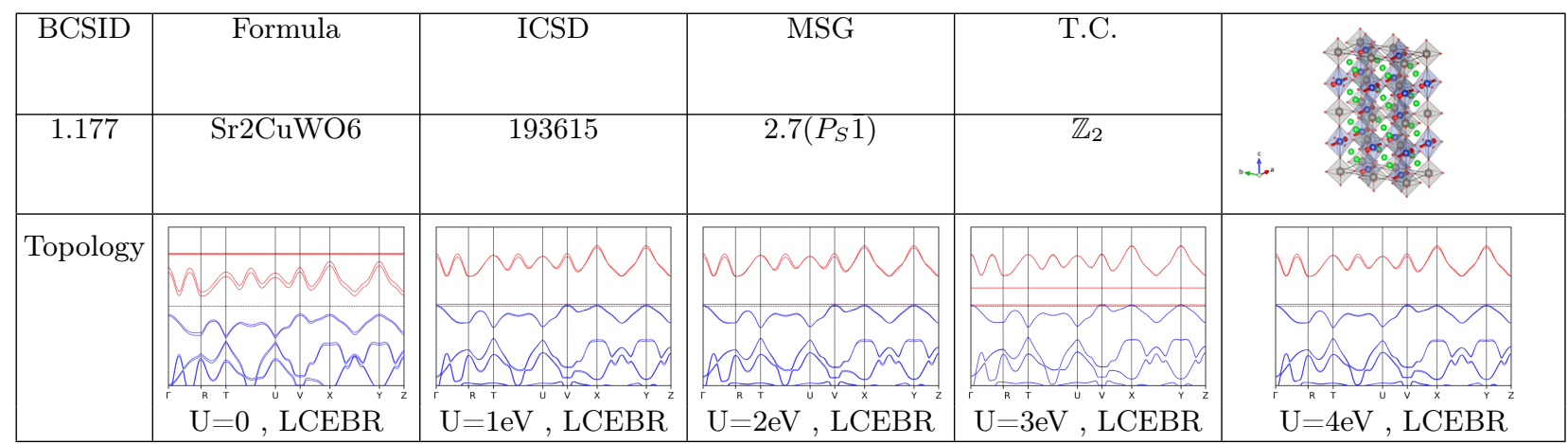

TABLE XXXI. Topology phase diagram of Sr2CuWO6. 


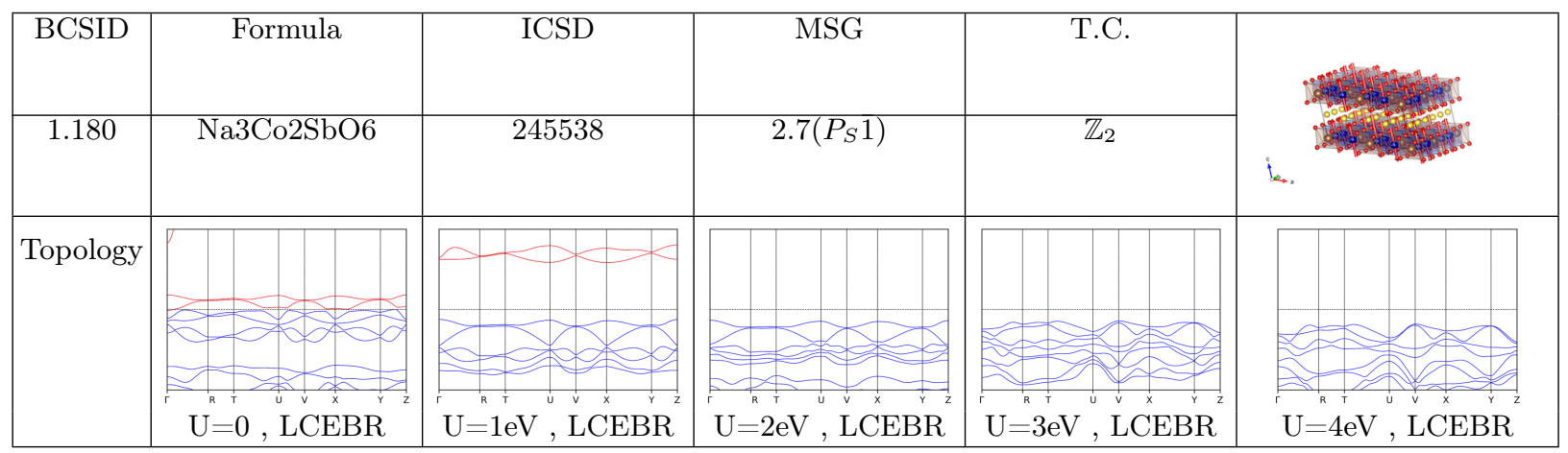

TABLE XXXII. Topology phase diagram of Na3Co2SbO6.

\begin{tabular}{|c|c|c|c|c|c|}
\hline BCSID & Formula & ICSD & MSG & T.C. & \\
\hline 1.182 & TlMnO3 & * & $2.7\left(P_{S} 1\right)$ & $\mathbb{Z}_{2}$ & 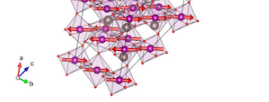 \\
\hline Topology & & $(7 y$ & & W & \\
\hline & $\mathrm{U}=0, \mathrm{LCEBR}$ & $\mathrm{U}^{\mathrm{R}}=1 \mathrm{eV}, \mathrm{LCEBR}$ & $\mathrm{U}^{\mathrm{R}}=2^{\top} \mathrm{eV}, \mathrm{LCEBR}$ & $\mathrm{U}^{\mathrm{R}}=3 \mathrm{eV}^{\top}, \mathrm{LCEBR}^{2}$ & $\mathrm{U}=4 \mathrm{eV}^{\mathrm{r}}, \mathrm{LCEBR}$ \\
\hline
\end{tabular}

TABLE XXXIII. Topology phase diagram of TIMnO3.

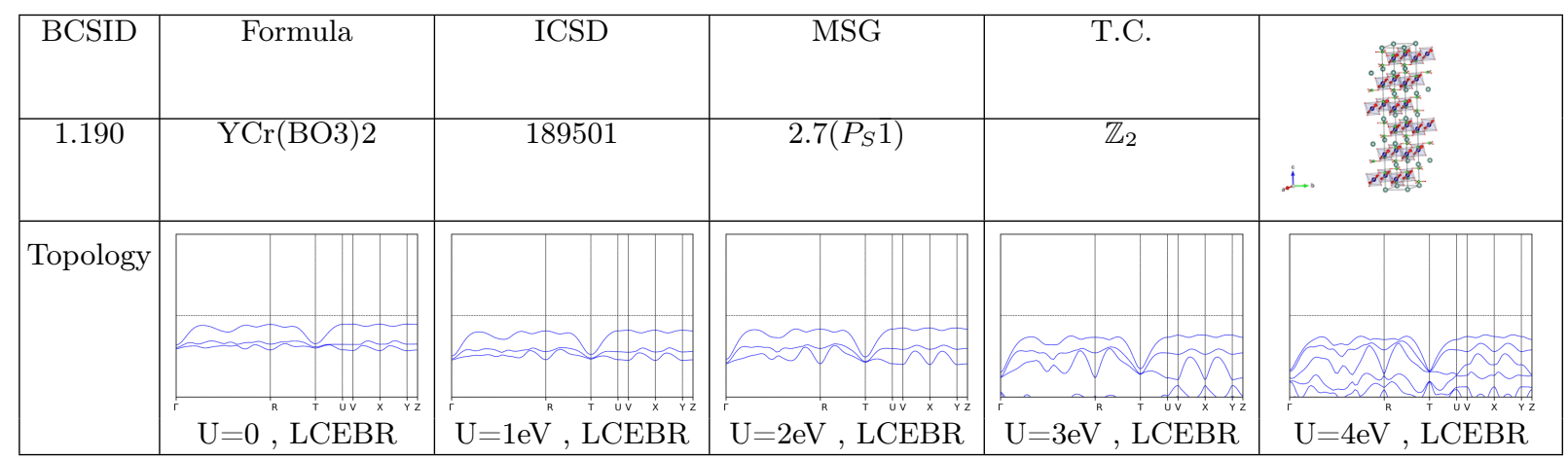

TABLE XXXIV. Topology phase diagram of $\mathrm{YCr}(\mathrm{BO} 3) 2$.

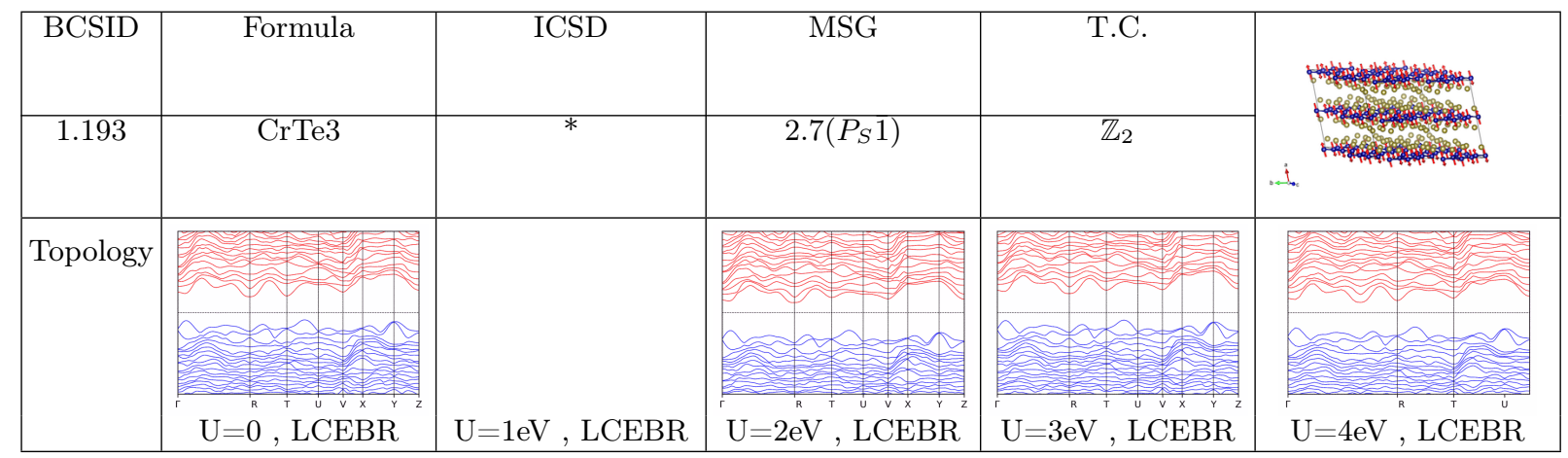

TABLE XXXV. Topology phase diagram of CrTe3. 


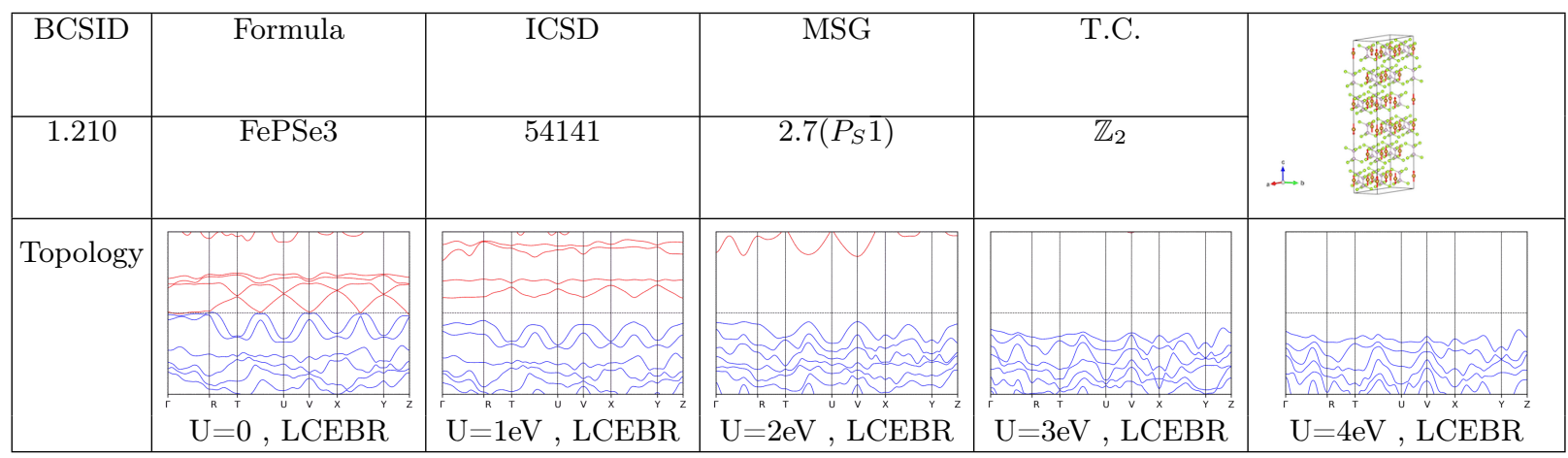

TABLE XXXVI. Topology phase diagram of FePSe3.

\begin{tabular}{|c|c|c|c|c|c|}
\hline BCSID & Formula & ICSD & MSG & T.C. & \\
\hline 1.219 & $\mathrm{CuF} 2$ & 9790 & $2.7\left(P_{S} 1\right)$ & $\mathbb{Z}_{2}$ & \\
\hline Topology & & & & & \\
\hline & $\mathrm{U}^{\mathrm{r}}=0, \mathrm{LCEBR}$ & $\mathrm{U}=1^{\top} \mathrm{eV}, \mathrm{LCEBR}$ & $\mathrm{U}^{\mathrm{n}}=2 \mathrm{eV}, \mathrm{LCEBR}$ & $\mathrm{U}=3 \mathrm{eV}, \mathrm{LCEBR}^{*}$ & $\mathrm{U}^{n}=4 \mathrm{eV}^{i}, \mathrm{LCEBR}$ \\
\hline
\end{tabular}

TABLE XXXVII. Topology phase diagram of CuF2.

\begin{tabular}{|c|c|c|c|c|c|}
\hline BCSID & Formula & ICSD & MSG & T.C. & \\
\hline 1.229 & BaMoP2O8 & 79507 & $2.7\left(P_{S} 1\right)$ & $\mathbb{Z}_{2}$ & \\
\hline Topology & & & & & \\
\hline & $\mathrm{U}^{\mathrm{n}}=0, \mathrm{~T}, \mathrm{LEBF}$ & $\mathrm{U}=1 \mathrm{eV}, \mathrm{LCEBR}$ & $\mathrm{U}=2 \mathrm{eV}, \mathrm{LCEBR}$ & $\mathrm{U}=3 \mathrm{eV}^{\mathrm{r}}, \mathrm{LCEBR}$ & $\mathrm{U}=4 \mathrm{eV}, \mathrm{LCEBR}$ \\
\hline
\end{tabular}

TABLE XXXVIII. Topology phase diagram of BaMoP2O8.

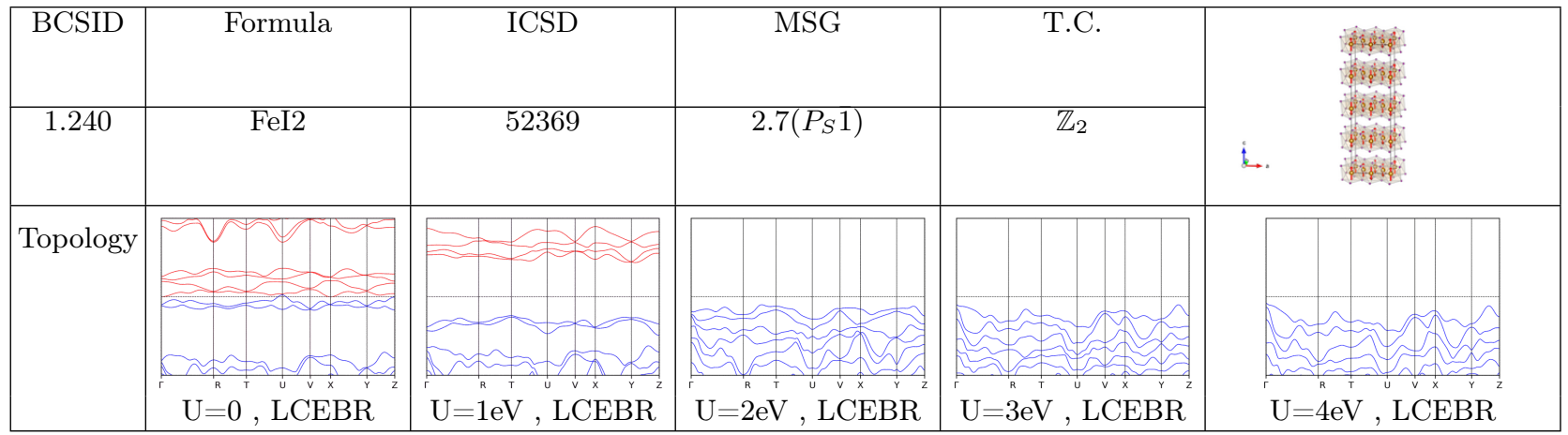

TABLE XXXIX. Topology phase diagram of FeI2. 


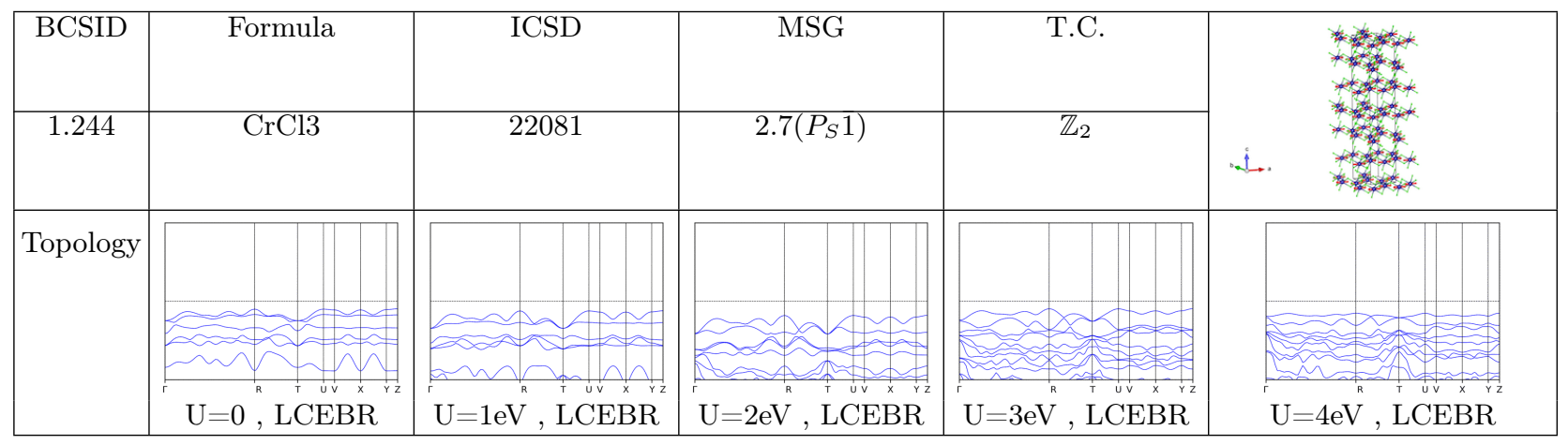

TABLE XL. Topology phase diagram of $\mathrm{CrCl} 3$.

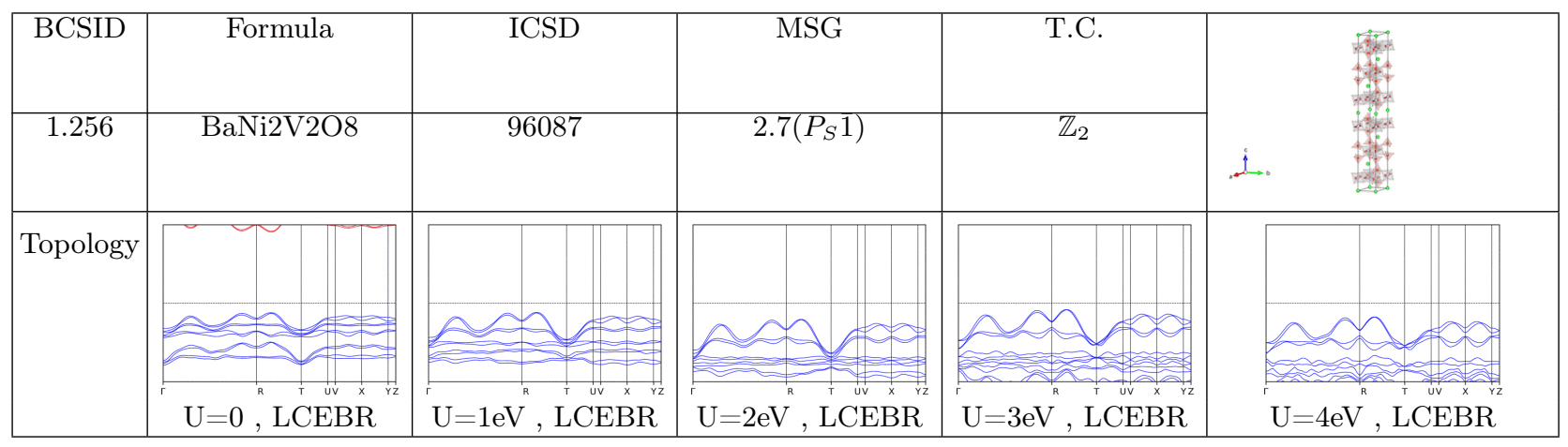

TABLE XLI. Topology phase diagram of BaNi2V2O8.

\begin{tabular}{|c|c|c|c|c|c|}
\hline BCSID & Formula & ICSD & MSG & T.C. & \\
\hline 1.257 & BaNi2As2O8 & 27014 & $2.7\left(P_{S} 1\right)$ & $\overline{\mathbb{Z}_{2}}$ & Wy \\
\hline Topology & & & & & \\
\hline & $\mathrm{U}^{\mathrm{n}}=0, \mathrm{LCE}$ & 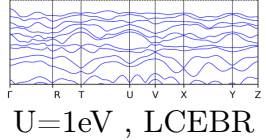 & 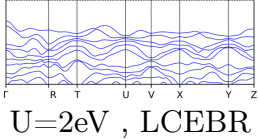 & $\underbrace{}_{\mathrm{U}=3 \mathrm{eV}, \mathrm{LCEBR}}$ & $\mathrm{U}_{\mathrm{U}=4 \mathrm{eV}, \mathrm{LCEBR}}^{\mathrm{V} \times}$ \\
\hline
\end{tabular}

TABLE XLII. Topology phase diagram of BaNi2As2O8.

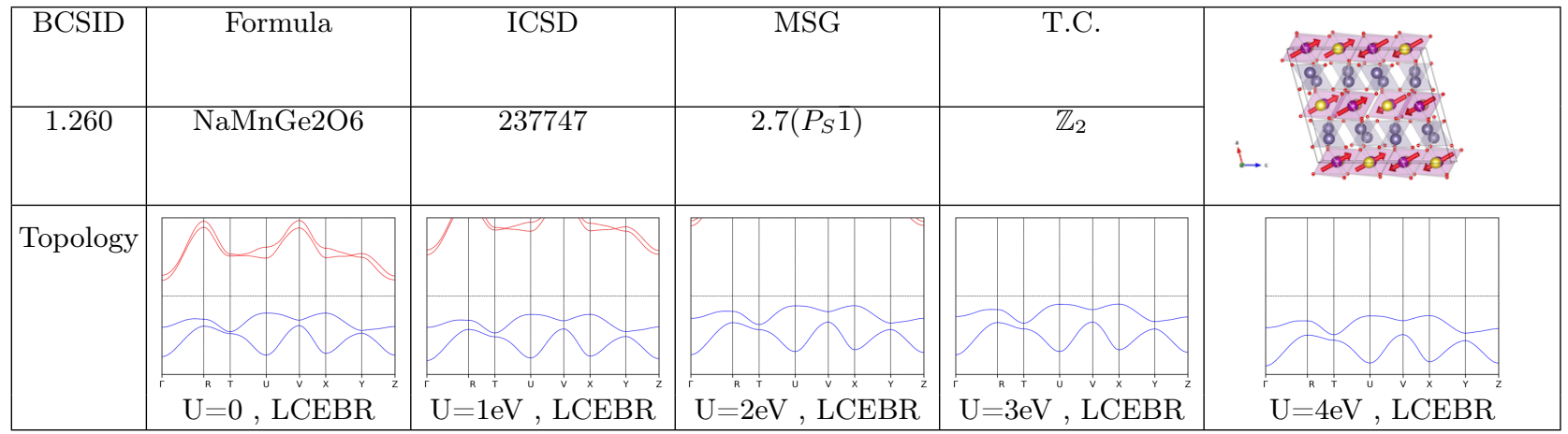

TABLE XLIII. Topology phase diagram of NaMnGe2O6. 


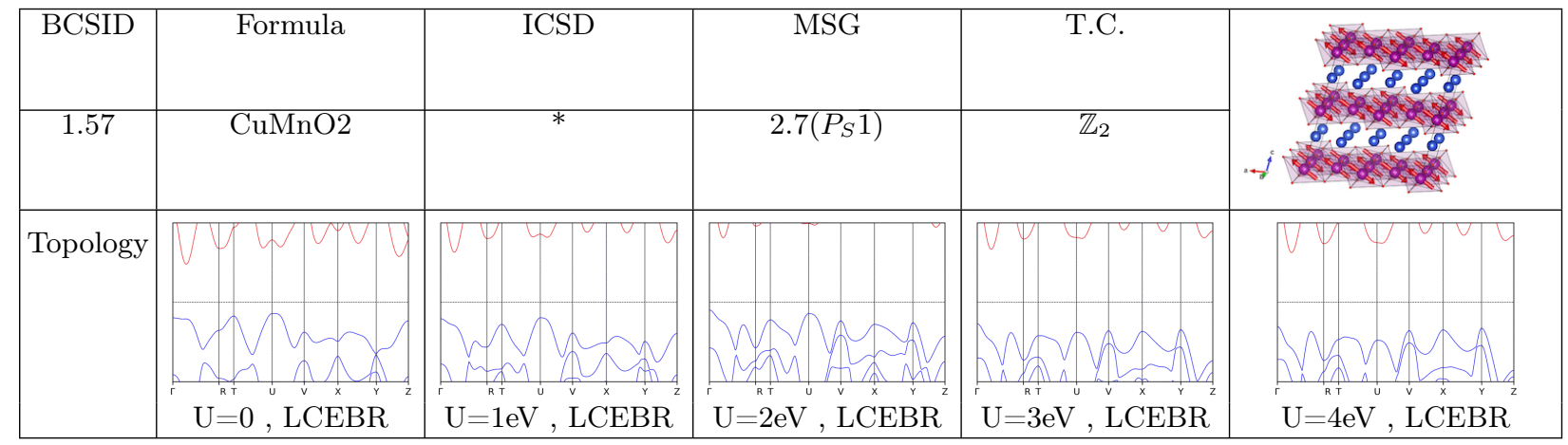

TABLE XLIV. Topology phase diagram of CuMnO2.

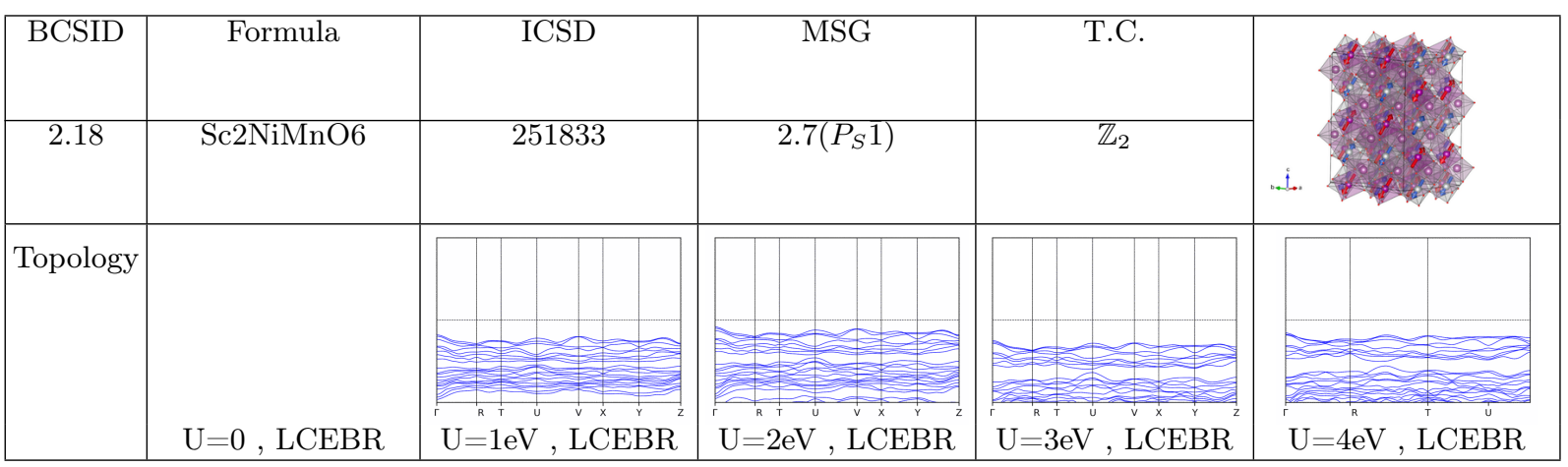

TABLE XLV. Topology phase diagram of Sc2NiMnO6.

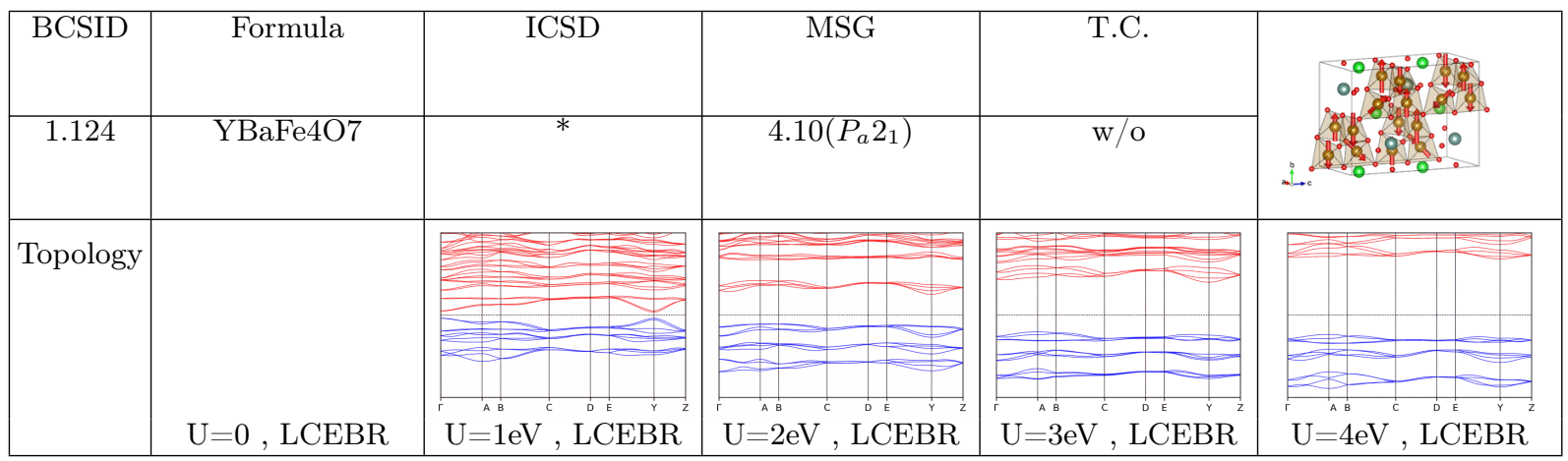

TABLE XLVI. Topology phase diagram of YBaFe4O7.

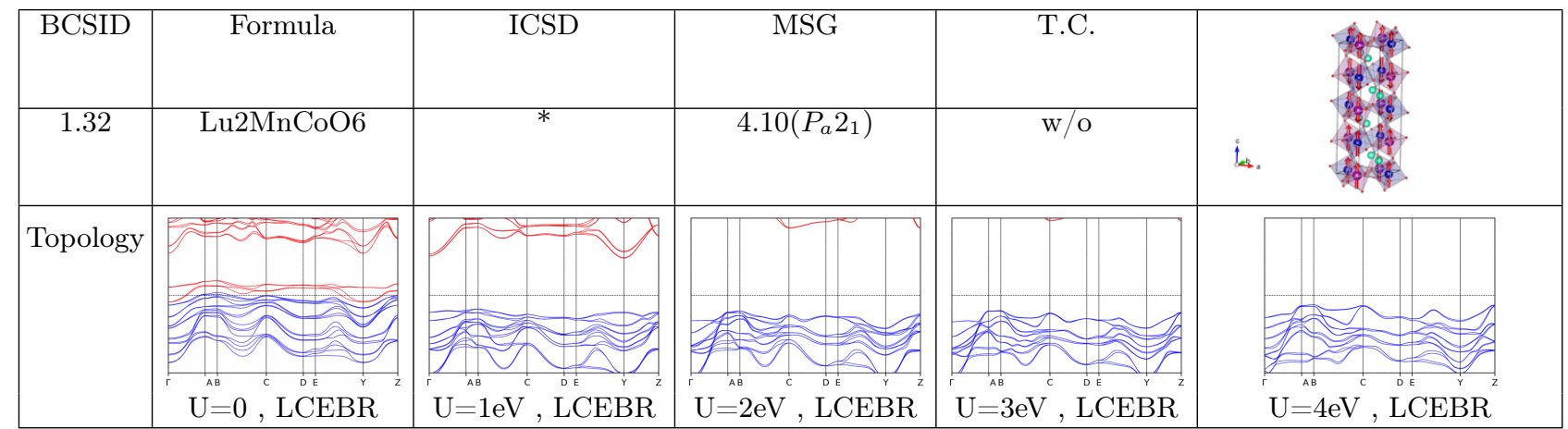

TABLE XLVII. Topology phase diagram of Lu2MnCoO6. 


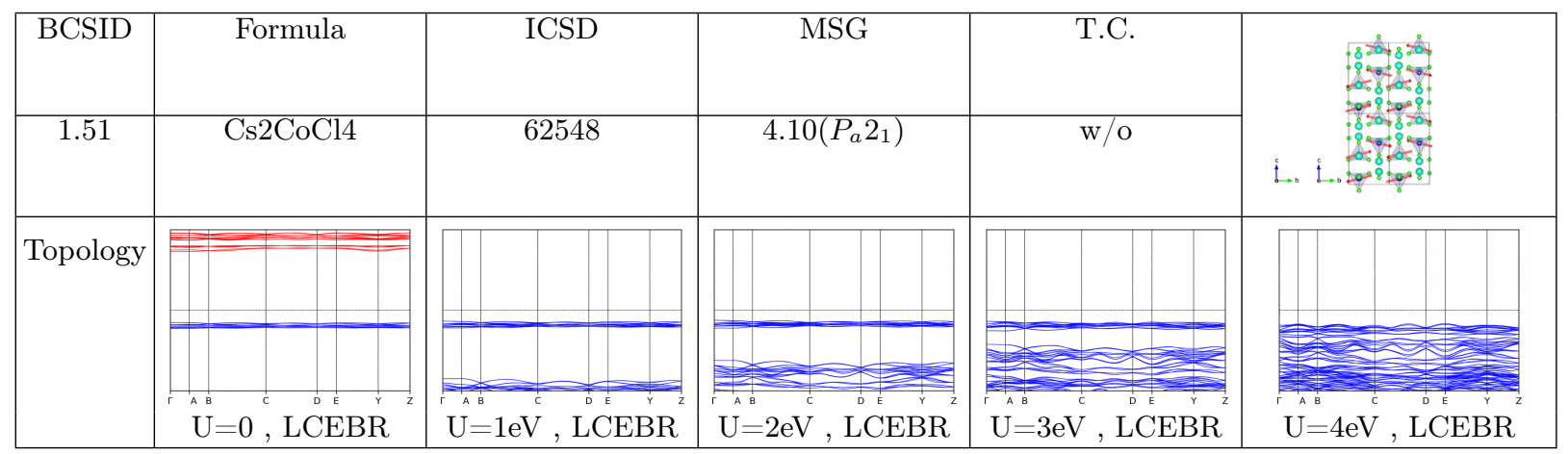

TABLE XLVIII. Topology phase diagram of Cs2CoCl4.

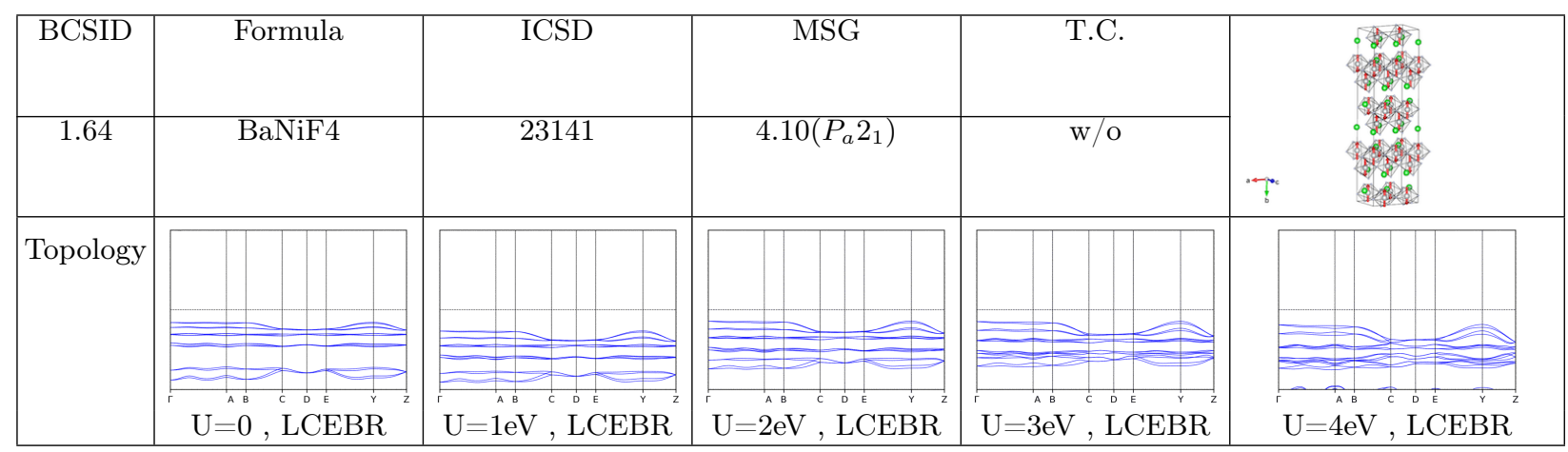

TABLE XLIX. Topology phase diagram of BaNiF4.

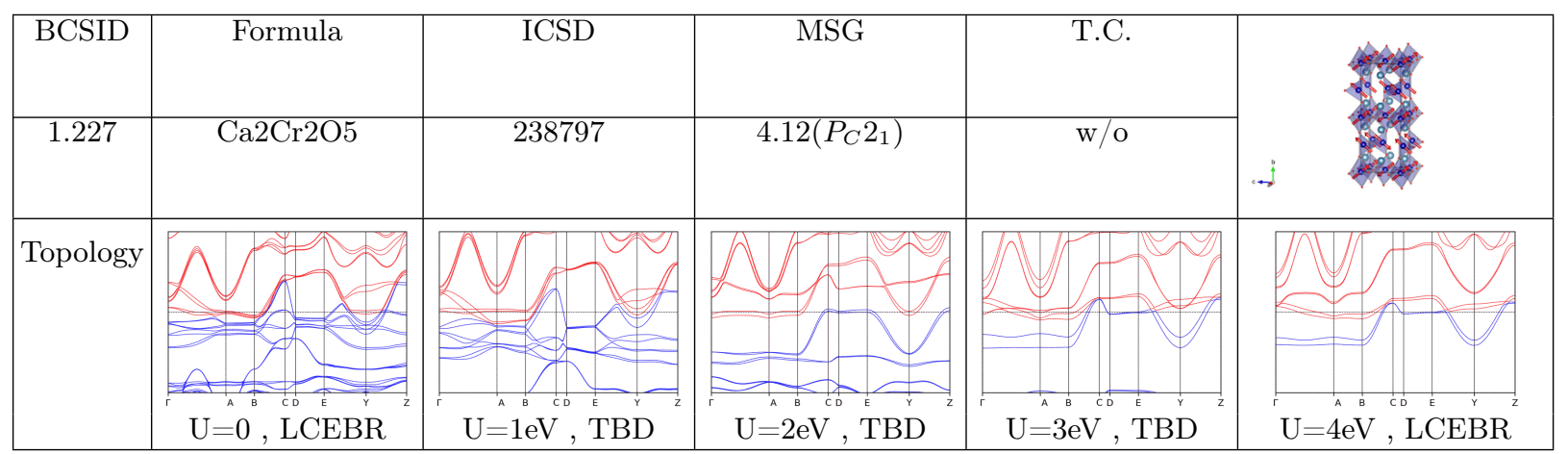

TABLE L. Topology phase diagram of Ca2Cr2O5.

\begin{tabular}{|c|c|c|c|c|c|}
\hline BCSID & Formula & ICSD & MSG & T.C. & \\
\hline 0.165 & $\mathrm{SrMn}(\mathrm{VO} 4)(\mathrm{OH})$ & * & $4.7\left(P 2_{1}\right)$ & $\mathrm{w} / \mathrm{o}$ & $\therefore \quad \frac{808-8}{0}$ \\
\hline Topology & (1) & U ${ }^{A}=1 \mathrm{eV}^{B}, \mathrm{LCEBR}^{C^{C}}$ & 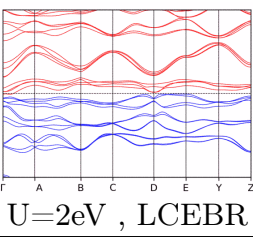 & 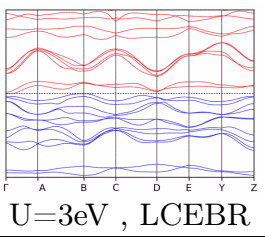 & ( \\
\hline
\end{tabular}

TABLE LI. Topology phase diagram of $\mathrm{SrMn}(\mathrm{VO} 4)(\mathrm{OH})$. 


\begin{tabular}{|c|c|c|c|c|c|}
\hline BCSID & Formula & ICSD & MSG & T.C. & \\
\hline 0.83 & LiFeP2O7 & 95751 & $4.7\left(P 2_{1}\right)$ & $\mathrm{w} / \mathrm{o}$ & i \\
\hline Topology & & & & & \\
\hline & $\hat{\mathrm{U}}=0,{ }^{\mathrm{B}}{ }^{\mathrm{C}} \mathrm{LCEBR}$ & $\mathrm{U}^{\mathrm{A}}=1 \mathrm{eV}, \mathrm{LCEBR}^{\mathrm{c}}$ & $\mathrm{U}^{A}=2 \mathrm{eV}^{\mathrm{B}}, \mathrm{LCEBR}^{\mathrm{C}}$ & $\mathrm{U}^{A}=3 \mathrm{eV}^{\mathrm{c}}, \mathrm{LCEBR}^{\mathrm{E}}$ & $\mathrm{U}^{\mathrm{A}}=4 \mathrm{eV}^{\mathrm{B}}, \mathrm{LCEBR}^{\mathrm{c}}$ \\
\hline
\end{tabular}

TABLE LII. Topology phase diagram of LiFeP2O7.

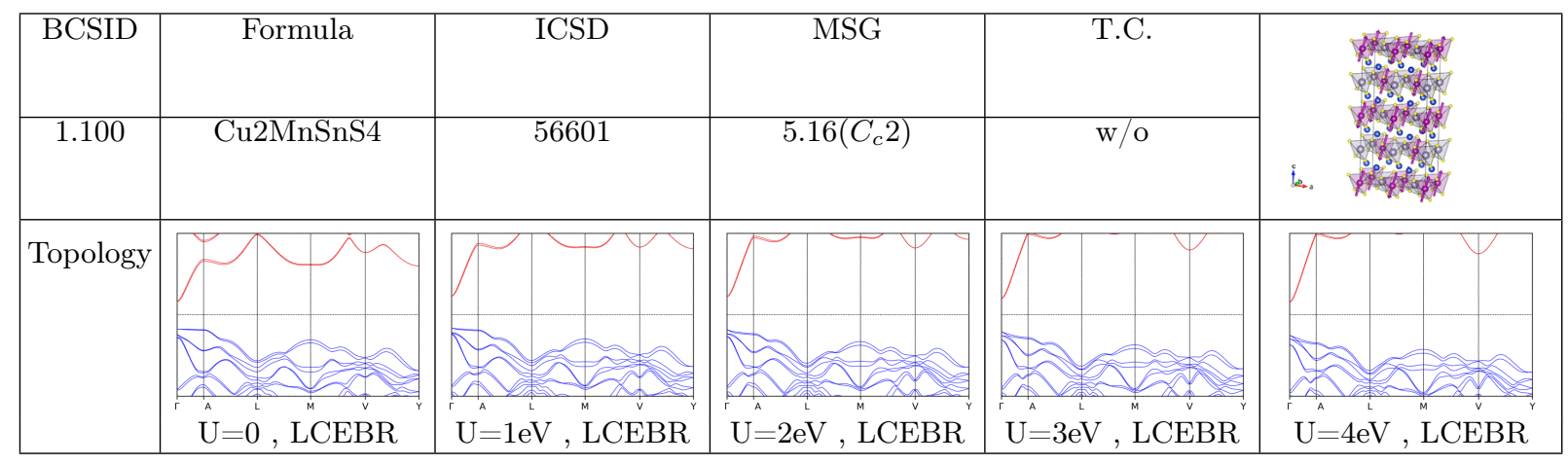

TABLE LIII. Topology phase diagram of Cu2MnSnS4.

\begin{tabular}{|c|c|c|c|c|c|}
\hline BCSID & Formula & ICSD & MSG & T.C. & \\
\hline 1.90 & $\overline{\mathrm{YFe}}(\mathrm{BO} 3) 4$ & $*$ & $5.16\left(C_{c} 2\right)$ & $\mathrm{w} / \mathrm{O}$ & f. \\
\hline \multicolumn{6}{|l|}{ Topology } \\
\hline & $\mathrm{U}=0, \mathrm{LCEBR}$ & $\mathrm{U}=1 \mathrm{eV}, \mathrm{TBD}$ & $\mathrm{U}=2 \mathrm{eV}, \mathrm{LCEBR}$ & $\mathrm{U}=3 \mathrm{eV}, \mathrm{TBD}$ & $\mathrm{U}=4 \mathrm{eV}, \mathrm{LCEBR}$ \\
\hline
\end{tabular}

TABLE LIV. Topology phase diagram of YFe3(BO3)4.

\begin{tabular}{|c|c|c|c|c|c|}
\hline BCSID & Formula & ICSD & MSG & T.C. & \\
\hline 1.55 & Na2MnF5 & * & $7.29\left(P_{b} c\right)$ & $\mathrm{w} / \mathrm{o}$ & 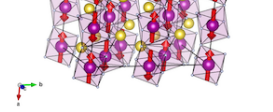 \\
\hline Topology & + & & & & \\
\hline & $\mathrm{U}^{A}=0, \quad \mathrm{LCEBR}^{\mathrm{E}}$ & $\mathrm{U}=1 \mathrm{eV}, \mathrm{LCEBR}^{\mathrm{E}}$ & $\mathrm{U}=2 \mathrm{eV}, \mathrm{LCEB}^{\mathrm{E}}$ & $\mathrm{I}={ }^{\mathrm{A}}{ }^{\mathrm{B}} \mathrm{eV}, \quad \mathrm{LCEBR}^{\mathrm{E}}$ & $\mathrm{U}=4 \mathrm{eV}, \mathrm{LCEBR}^{\mathrm{E}}$ \\
\hline
\end{tabular}

TABLE LV. Topology phase diagram of Na2MnF5. 


\begin{tabular}{|c|c|c|c|c|c|}
\hline BCSID & Formula & ICSD & MSG & T.C. & 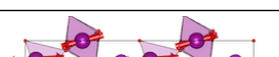 \\
\hline 1.75 & BiMn2O5 & * & $8.36\left(C_{a} m\right)$ & $\mathrm{w} / \mathrm{o}$ & \\
\hline \multicolumn{6}{|l|}{ Topology } \\
\hline & & 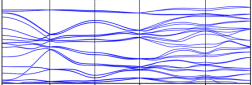 & $\mathrm{n} \times \mathrm{n}$ & $\ldots$ & se \\
\hline & $\mathrm{U}=0, \mathrm{LCEBR}$ & $\mathrm{U}=1 \mathrm{eV}, \mathrm{LCEBR}$ & $\mathrm{U}=2 \mathrm{eV}, \mathrm{LCEBR}$ & $\mathrm{U}=3 \mathrm{eV}, \mathrm{LCEBR}$ & $\mathrm{U}=4 \mathrm{eV}, \mathrm{LCEBR}$ \\
\hline
\end{tabular}

TABLE LVI. Topology phase diagram of BiMn2O5.

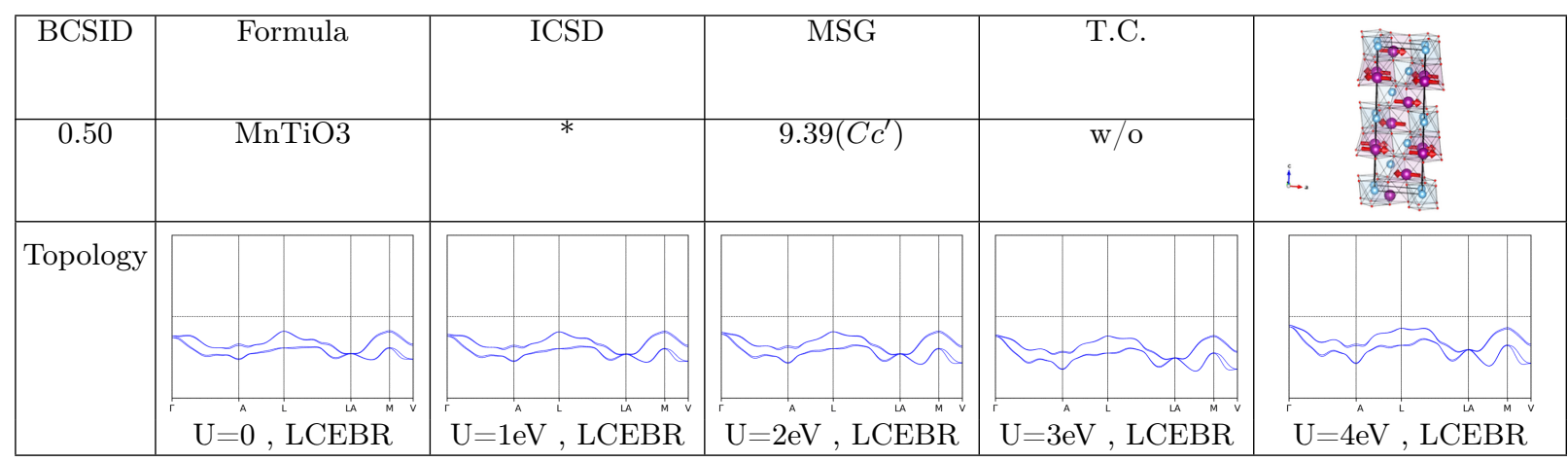

TABLE LVII. Topology phase diagram of MnTiO3.

\begin{tabular}{|c|c|c|c|c|c|}
\hline BCSID & Formula & ICSD & MSG & T.C. & \\
\hline 1.232 & CuMnSb & 628385 & $9.40\left(C_{c} c\right)$ & $\mathrm{w} / \mathrm{o}$ & \\
\hline Topology & $w_{m} m a$ & $x_{2}$ & & $V, V$ & \\
\hline & $\hat{\mathrm{U}}=0, \stackrel{\mathrm{L}}{\mathrm{L}} \mathrm{CEBR}^{\mathrm{i}}$ & $\mathrm{U}=1 \mathrm{eV}, \mathrm{LCEBR}$ & $\mathrm{U}=2 \mathrm{eV}, \mathrm{LCEBR}$ & $\hat{\mathrm{U}}=3 \mathrm{eV}, \mathrm{LCEBR}$ & $\mathrm{U}^{A}=4 \mathrm{eV}, \mathrm{LCEBR}$ \\
\hline
\end{tabular}

TABLE LVIII. Topology phase diagram of CuMnSb.

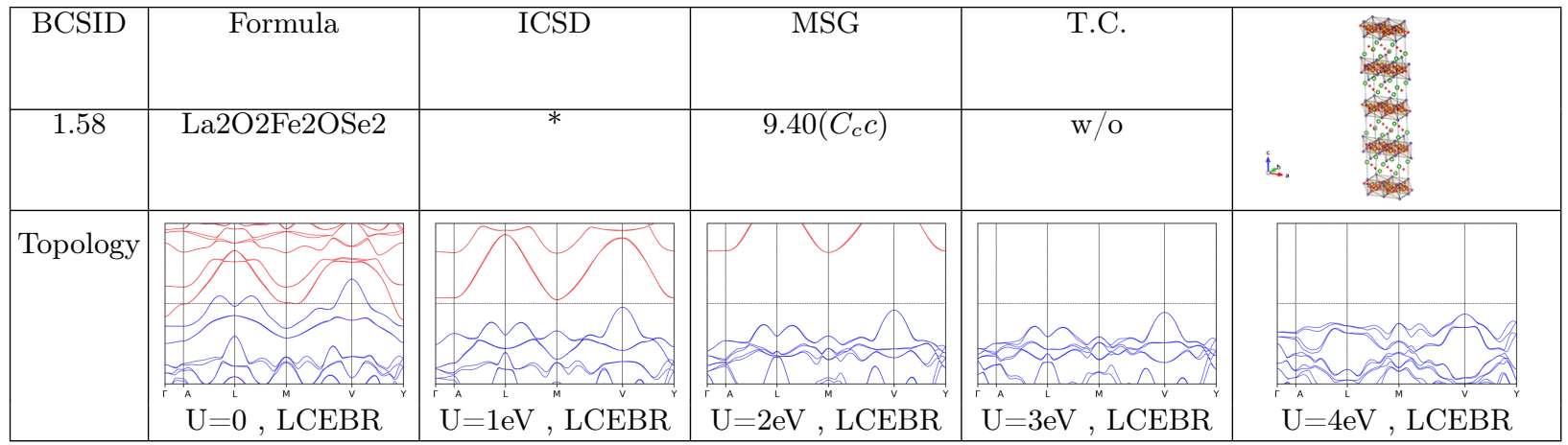

TABLE LIX. Topology phase diagram of La2O2Fe2OSe2. 


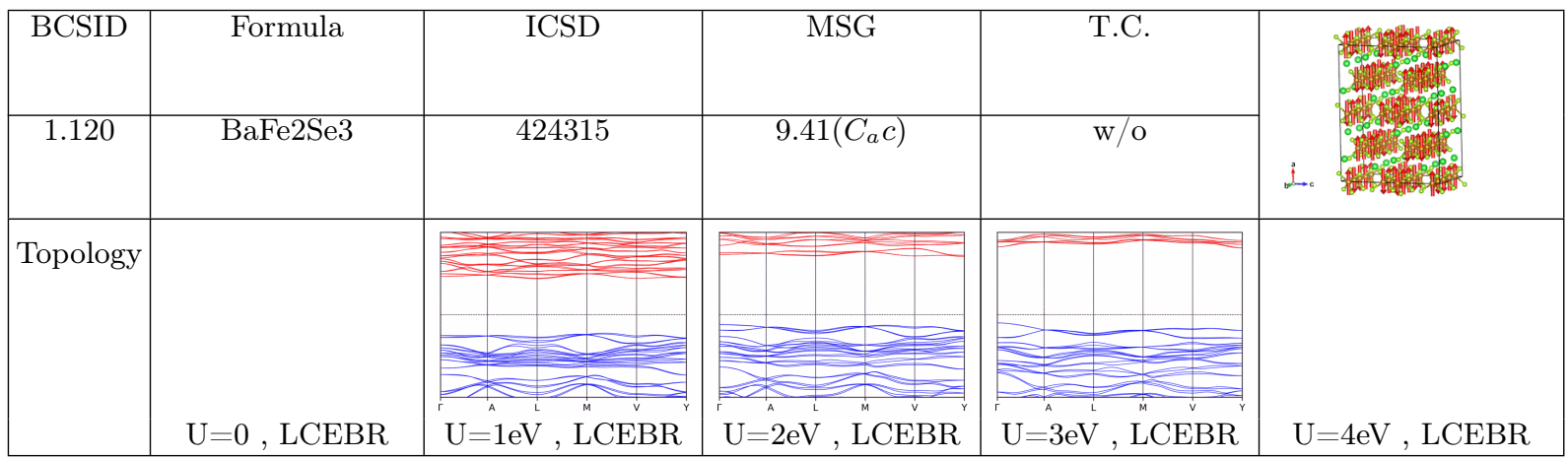

TABLE LX. Topology phase diagram of BaFe2Se3.

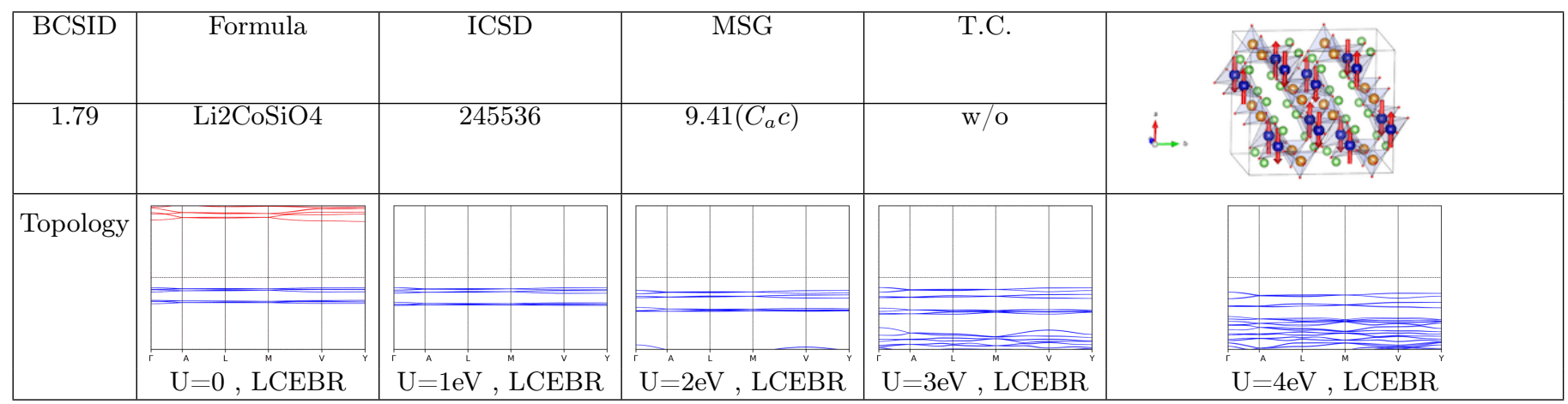

TABLE LXI. Topology phase diagram of Li2CoSiO4.

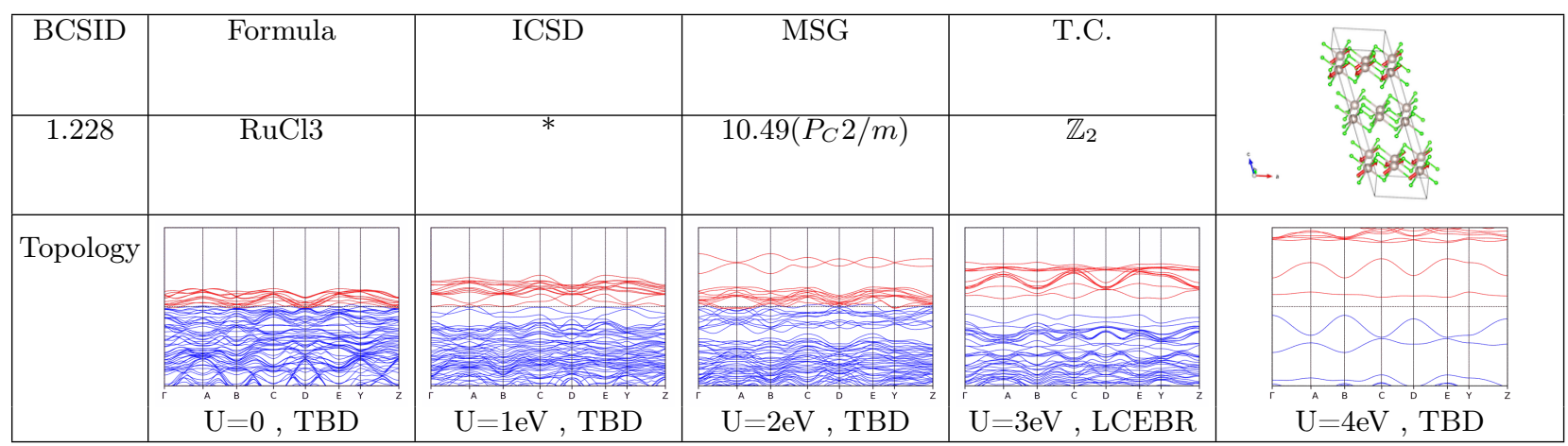

TABLE LXII. Topology phase diagram of RuCl3.

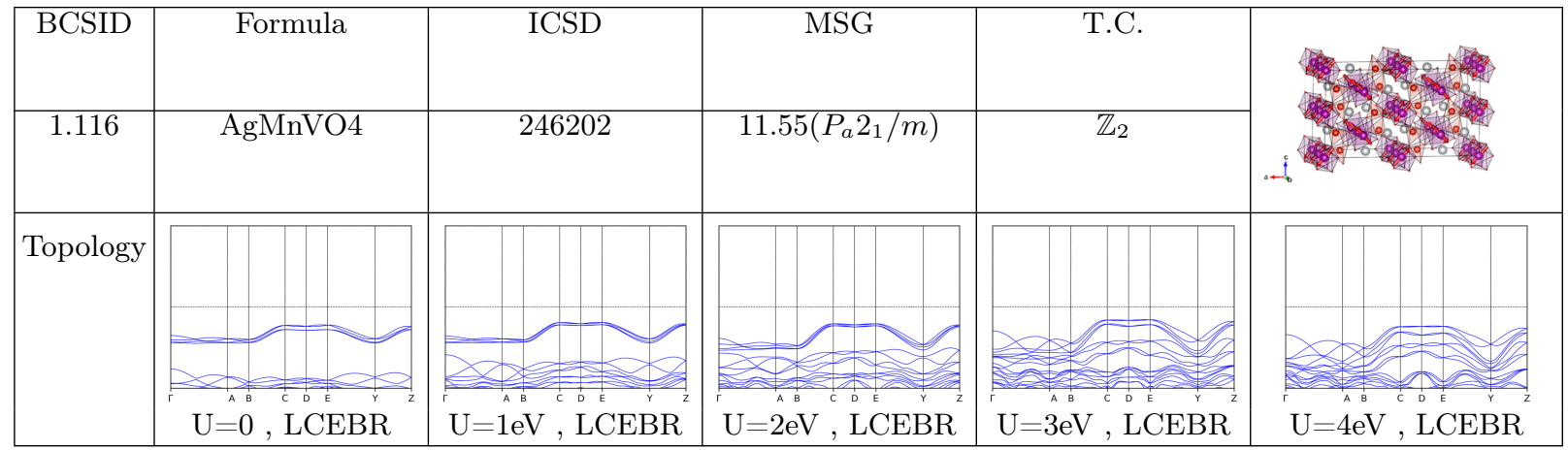

TABLE LXIII. Topology phase diagram of AgMnVO4. 


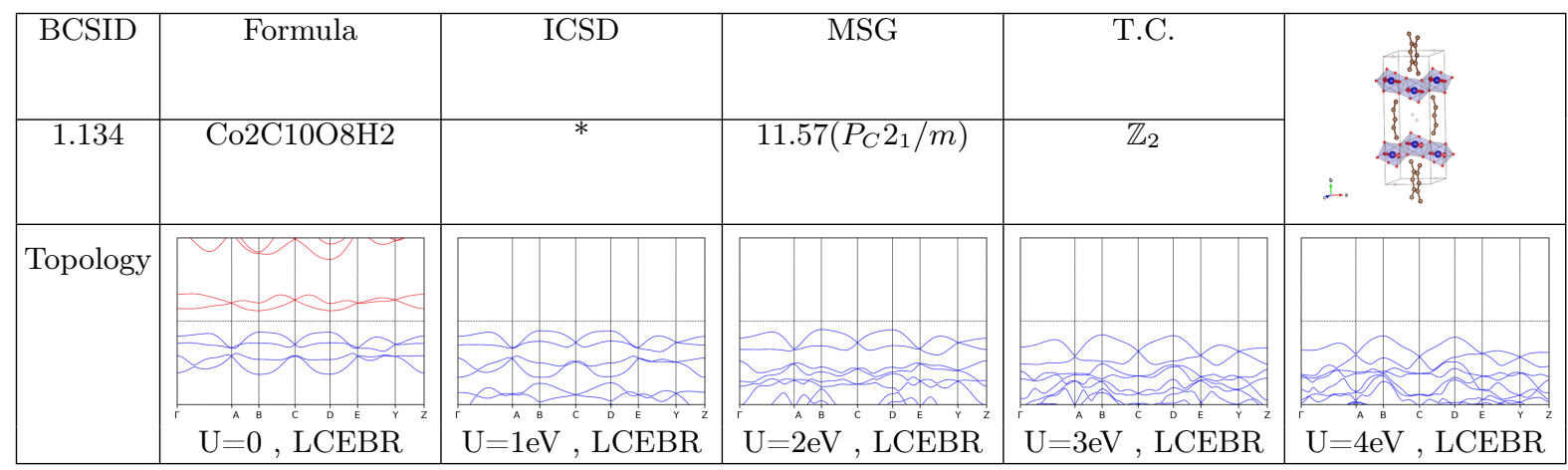

TABLE LXIV. Topology phase diagram of Co2C10O8H2.

\begin{tabular}{|c|c|c|c|c|c|}
\hline BCSID & Formula & $\overline{\mathrm{ICSD}}$ & $\overline{\mathrm{MSG}}$ & T.C. & \\
\hline 1.230 & NiPS3 & 646133 & $11.57\left(P_{C} 2_{1} / m\right)$ & $\mathbb{Z}_{2}$ & \\
\hline Topology & ase & & & & \\
\hline & $\mathrm{U}^{\mathrm{A}}=0^{\mathrm{b}}, \mathrm{LCEBR}$ & $\mathrm{U}=1 \mathrm{eV}, \mathrm{LCEBR}^{\mathrm{c}}$ & $\mathrm{U}=2 \mathrm{eV}^{\mathrm{s}}, \mathrm{LCEBR}^{\mathrm{c}}$ & $\mathrm{U}=3 \mathrm{eV}^{\mathrm{a}}, \mathrm{LCEBR}$ & $\mathrm{U}=4 \mathrm{eV}^{\mathrm{B}}, \mathrm{LCEBR}^{\circ}$ \\
\hline
\end{tabular}

TABLE LXV. Topology phase diagram of NiPS3.

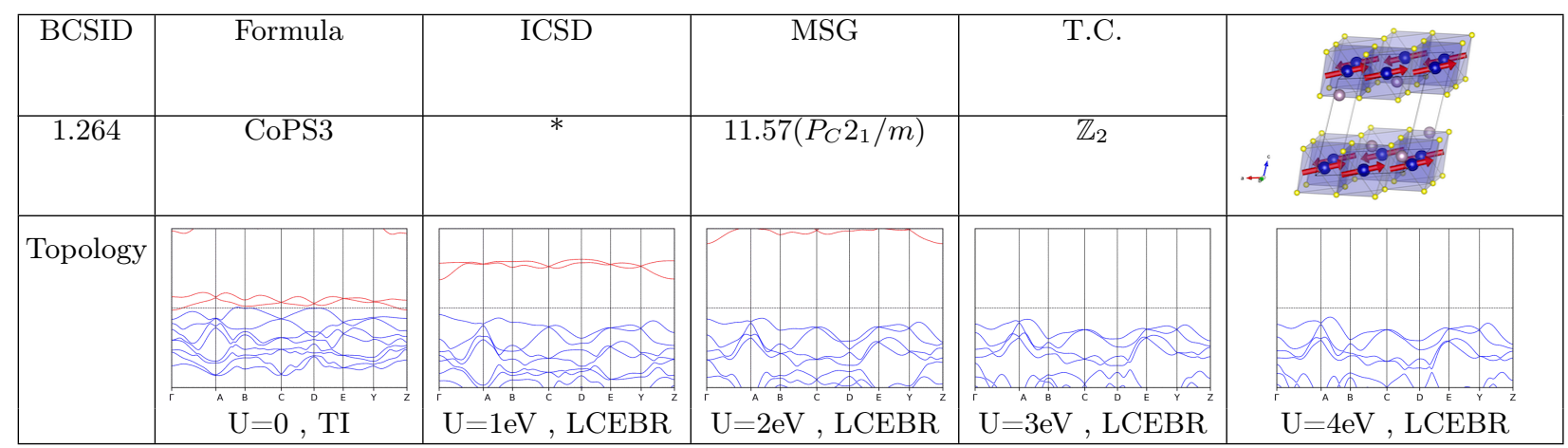

TABLE LXVI. Topology phase diagram of CoPS3.

\begin{tabular}{|c|c|c|c|c|c|}
\hline BCSID & Formula & ICSD & MSG & T.C. & \multirow{2}{*}{ 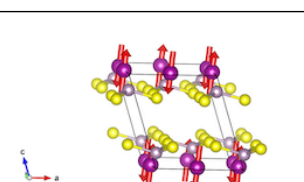 } \\
\hline 0.163 & MnPS3 & 61391 & $12.60\left(C 2^{\prime} / m\right)$ & $\mathrm{w} / \mathrm{o}$ & \\
\hline \multirow[t]{2}{*}{ Topology } & & & & & \\
\hline & $\mathrm{U}=0, \mathrm{LCEBR}$ & $\mathrm{U}=1 \mathrm{eV}, \mathrm{LCEBR}$ & $\mathrm{U}=2 \mathrm{eV}, \mathrm{LCEBR}$ & $\mathrm{U}=3 \mathrm{eV}, \mathrm{LCEBR}$ & $=4 \mathrm{eV}, \mathrm{LCEBR}$ \\
\hline
\end{tabular}

TABLE LXVII. Topology phase diagram of MnPS3. 


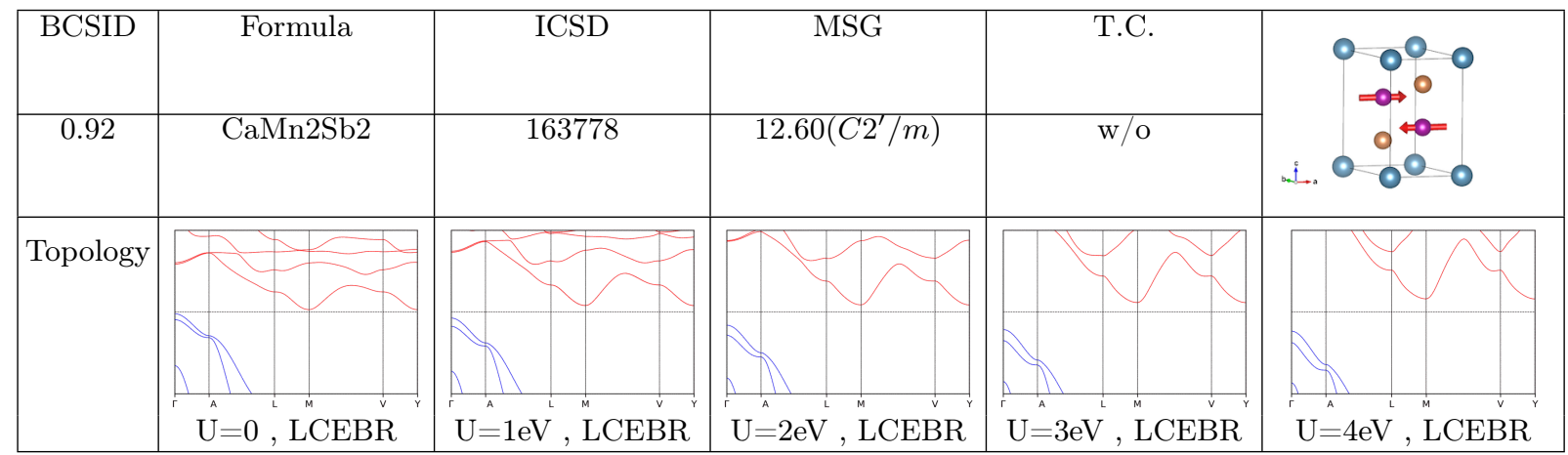

TABLE LXVIII. Topology phase diagram of CaMn2Sb2.

\begin{tabular}{|c|c|c|c|c|c|}
\hline BCSID & Formula & ICSD & MSG & T.C. & \\
\hline 1.0 .1 & $\mathrm{Ag} 2 \mathrm{CrO} 2$ & * & $12.60\left(C 2^{\prime} / m\right)$ & $\mathrm{w} / \mathrm{o}$ & 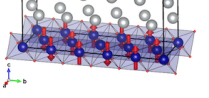 \\
\hline \multicolumn{6}{|l|}{ Topology } \\
\hline & $\hat{\mathrm{U}}=0, \mathrm{LCEBR}^{\mathrm{m}}$ & $\mathrm{U}^{\mathrm{A}}=1 \mathrm{eV}, \mathrm{LCEBR}^{\mathrm{M}}$ & $\mathrm{U}^{\mathrm{A}}=2 \mathrm{eV}, \mathrm{LCEBR}^{\prime \prime}$ & $\mathrm{U}^{\wedge}=3 \mathrm{eV}, \mathrm{LCEBR}^{\prime \prime}$ & $\mathrm{U}=4 \mathrm{eV}, \mathrm{TBD}$ \\
\hline
\end{tabular}

TABLE LXIX. Topology phase diagram of Ag2CrO2.

\begin{tabular}{|c|c|c|c|c|c|}
\hline BCSID & Formula & ICSD & MSG & T.C. & \\
\hline 0.203 & Mn3Ge & 603343 & $12.62\left(C 2^{\prime} / \mathrm{m}^{\prime}\right)$ & $\mathbb{Z}_{2} \mathbb{Z}_{2} \mathbb{Z}_{4}$ & \\
\hline \multirow[t]{2}{*}{ Topology } & & & & & \\
\hline & ${ }^{\wedge} \mathrm{U}=0, \mathrm{i}, \mathrm{TI}$ & $\hat{\mathrm{U}}=1 \mathrm{eV}, \mathrm{I}$ & $\hat{\mathrm{U}}=2 \mathrm{eV}, \mathrm{Ti}$ & $\hat{\mathrm{U}}=3 \mathrm{eV}, \mathrm{i}$ & $\hat{U}=4 \mathrm{eV}^{\mathrm{i}}, \mathrm{TI}$ \\
\hline
\end{tabular}

TABLE LXX. Topology phase diagram of Mn3Ge.

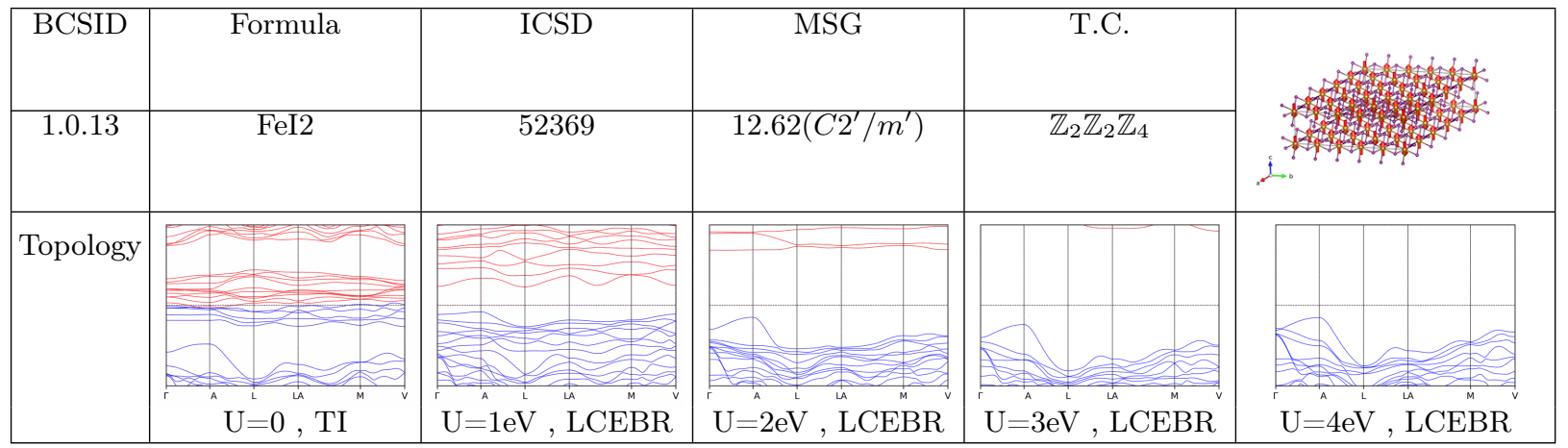

TABLE LXXI. Topology phase diagram of FeI2. 


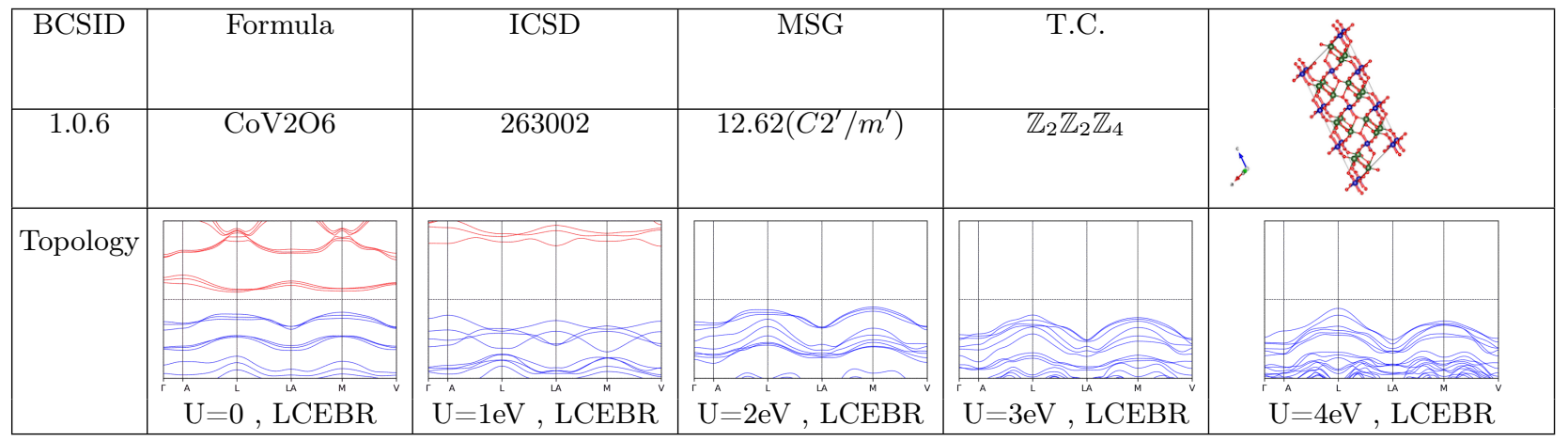

TABLE LXXII. Topology phase diagram of CoV2O6.

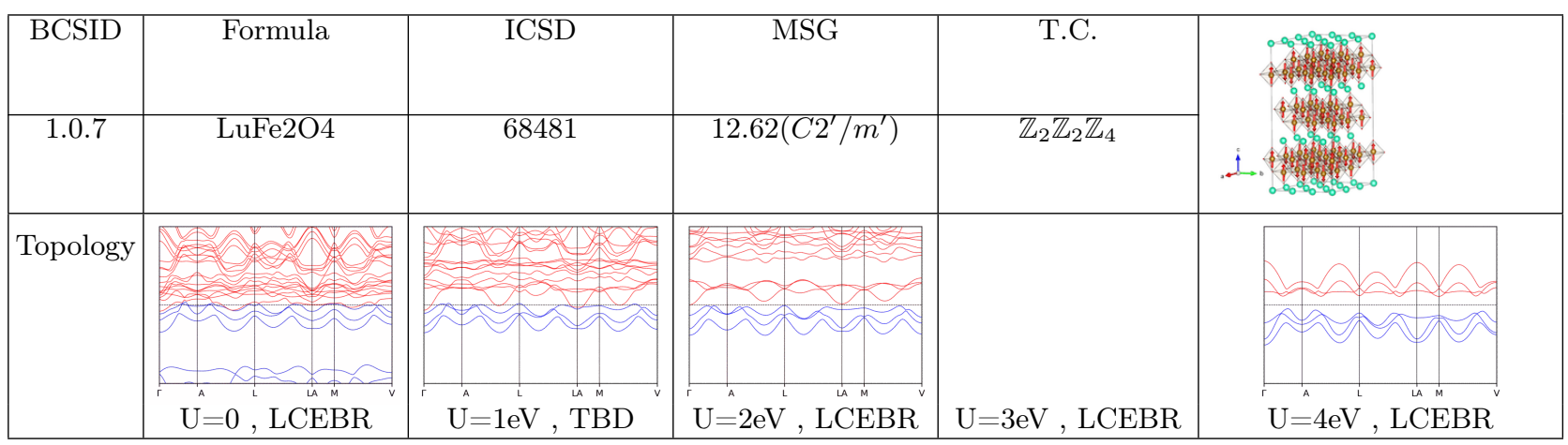

TABLE LXXIII. Topology phase diagram of LuFe2O4.

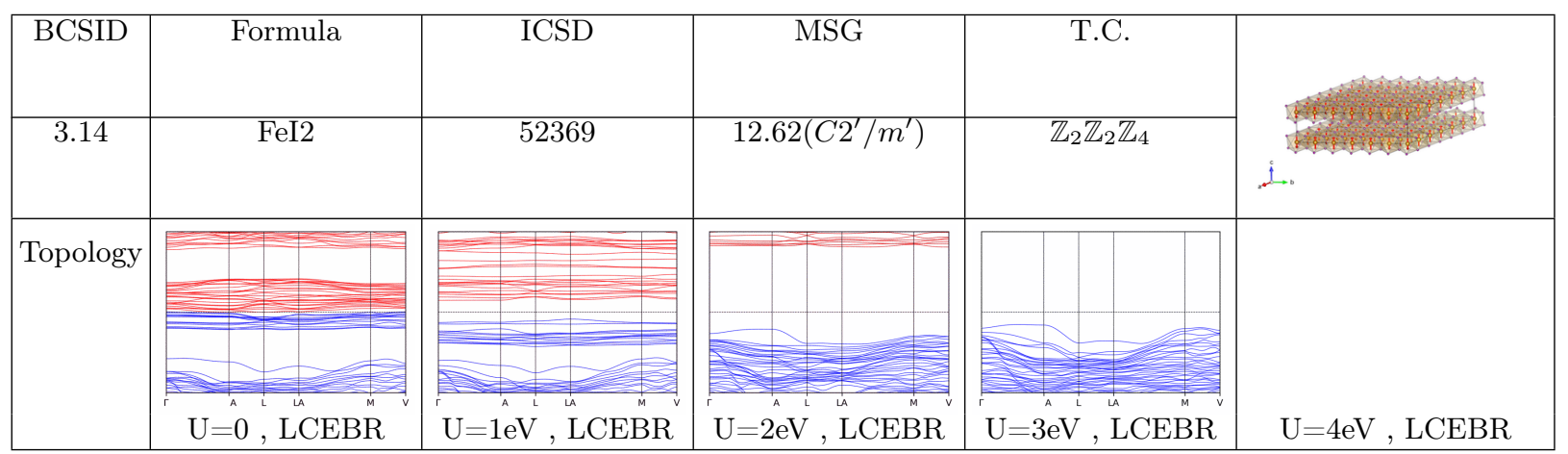

TABLE LXXIV. Topology phase diagram of FeI2.

\begin{tabular}{|c|c|c|c|c|c|}
\hline BCSID & Formula & ICSD & MSG & T.C. & \\
\hline 1.183 & FePS3 & * & $12.63\left(C_{c} 2 / m\right)$ & $\mathbb{Z}_{2}$ & L. Pis \\
\hline Topology & & & & & \\
\hline & $\mathrm{U}^{\mathrm{A}}=0^{\mathrm{L}}, \mathrm{L}^{\mathrm{M}} \mathrm{CEBR}$ & $\mathrm{U}=1 \mathrm{eV}, \mathrm{LCEBR}$ & $\mathrm{U}=2 \mathrm{eV}, \mathrm{LCEBR}$ & $\mathrm{U}=3 \mathrm{eV}, \mathrm{LCEBR}$ & $\mathrm{U}=4 \mathrm{eV}, \mathrm{M}$ \\
\hline
\end{tabular}

TABLE LXXV. Topology phase diagram of FePS3. 


\begin{tabular}{|c|c|c|c|c|c|c|c|}
\hline BCSID & Formula & ICSD & \multicolumn{2}{|c|}{ MSG } & \multicolumn{2}{|c|}{ T.C. } & \multirow{2}{*}{ 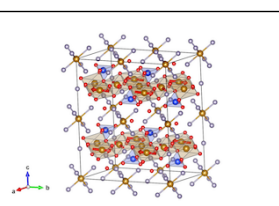 } \\
\hline 1.197 & Fe4Si2Sn7O16 & 407506 & \multicolumn{2}{|c|}{$12.63\left(C_{c} 2 / m\right)$} & \multicolumn{2}{|c|}{$\mathbb{Z}_{2}$} & \\
\hline \multirow[t]{4}{*}{ Topology } & $\begin{array}{rll} & \\
& \\
\end{array}$ & & & & & & \\
\hline & \begin{tabular}{|l} 
\\
\end{tabular} & & & & & & E \\
\hline & 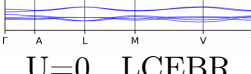 & \begin{tabular}{|c|c|c|c|} 
& \\
\end{tabular} & A & 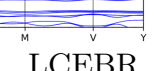 & A & " is & 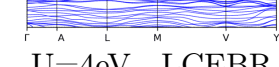 \\
\hline & $\mathrm{U}=0, \mathrm{LCEBR}$ & $U=1 \mathrm{eV}, \mathrm{LCEBR}$ & & , LCEBR & $\mathrm{U}=3 \mathrm{eV}$ & , LCEBR & $\mathrm{U}=4 \mathrm{eV}, \mathrm{LCEBR}$ \\
\hline
\end{tabular}

TABLE LXXVI. Topology phase diagram of Fe4Si2Sn7O16.

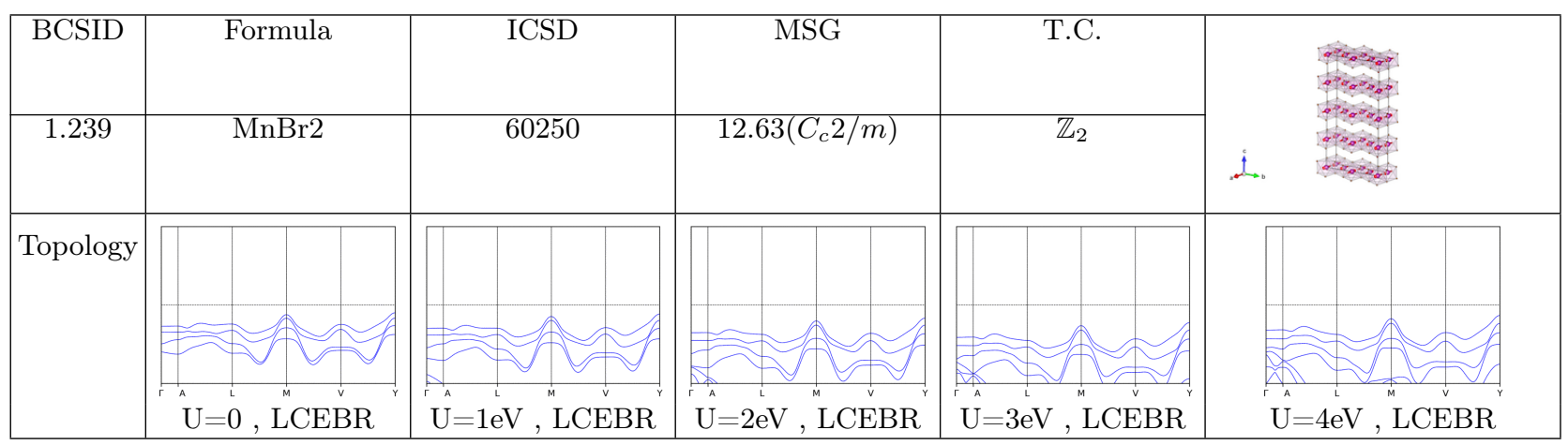

TABLE LXXVII. Topology phase diagram of MnBr2.

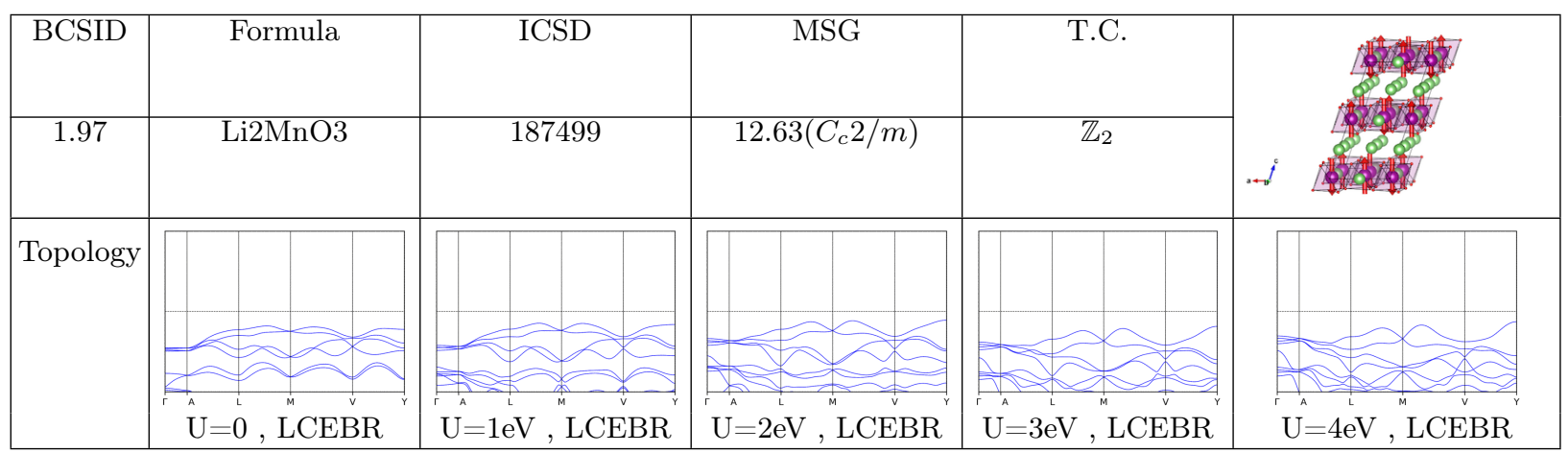

TABLE LXXVIII. Topology phase diagram of Li2MnO3.

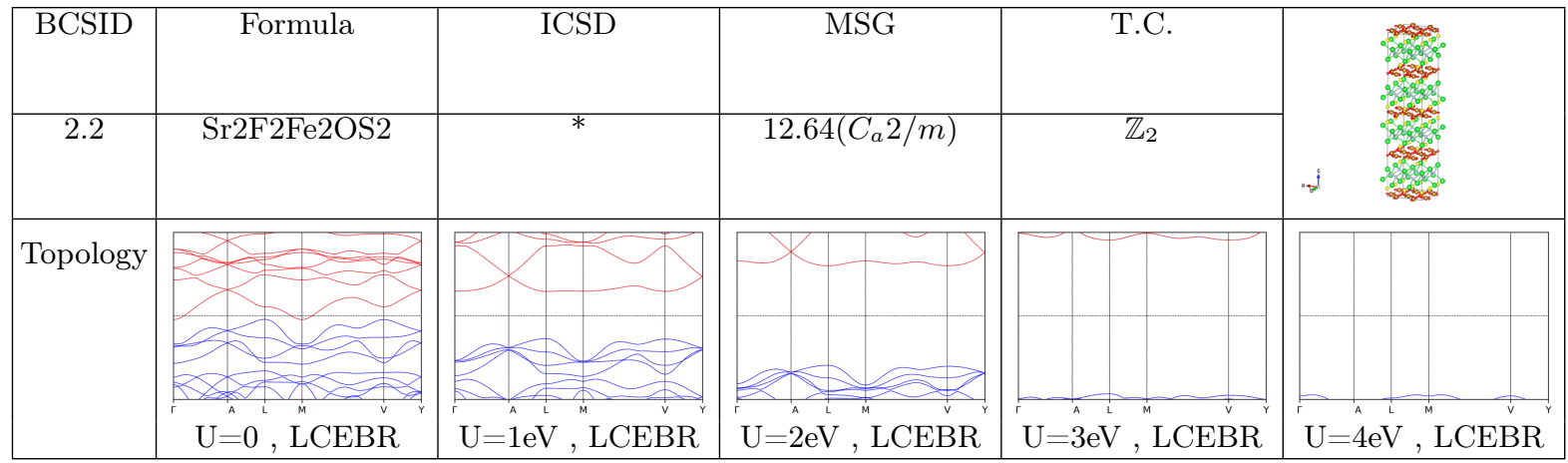

TABLE LXXIX. Topology phase diagram of Sr2F2Fe2OS2. 


\begin{tabular}{|c|c|c|c|c|c|}
\hline BCSID & Formula & ICSD & MSG & T.C. & \\
\hline 1.194 & NiWO4 & 15852 & $13.70\left(P_{a} 2 / c\right)$ & $\mathbb{Z}_{2}$ & \\
\hline Topology & 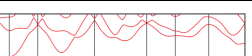 & PV & & PV & t \\
\hline & $\mathrm{U}^{\mathrm{B}}=0, \mathrm{LCEBR}$ & $\mathrm{U}^{\mathrm{B}}=1 \mathrm{eV}, \mathrm{LCEBR}$ & $\mathrm{A}^{\mathrm{B}}=2 \mathrm{eV}, \mathrm{LCEBF}$ & $\begin{array}{l}\mathrm{A} \\
\mathrm{U}=3 \mathrm{eV}, \mathrm{LCEBR}^{\mathrm{E}}\end{array}$ & $\begin{array}{l}\mathrm{A}^{\mathrm{B}} \\
\mathrm{U}=4 \mathrm{eV}, \mathrm{LCEBR}\end{array}$ \\
\hline
\end{tabular}

TABLE LXXX. Topology phase diagram of NiWO4.

\begin{tabular}{|c|c|c|c|c|c|}
\hline BCSID & Formula & ICSD & MSG & T.C. & \\
\hline 1.114 & Ca4IrO6 & 280873 & $13.74\left(P_{C} 2 / c\right)$ & $\mathbb{Z}_{2}$ & P. \\
\hline \multirow[t]{2}{*}{ Topology } & & & & & \\
\hline & $\mathrm{U}^{\mathrm{B}}=0, \mathrm{LCEBR}^{\mathrm{c}}$ & $\mathrm{U}=1 \mathrm{eV}, \mathrm{LCEBR}^{\mathrm{c}}$ & $\mathrm{U}=2 \mathrm{eV}, \mathrm{LCEBR}^{2}$ & $\mathrm{U}=3 \mathrm{eV}, \mathrm{LCEBR}$ & $\mathrm{U}=4 \mathrm{eV}, \mathrm{LCEBR}$ \\
\hline
\end{tabular}

TABLE LXXXI. Topology phase diagram of Ca4IrO6.

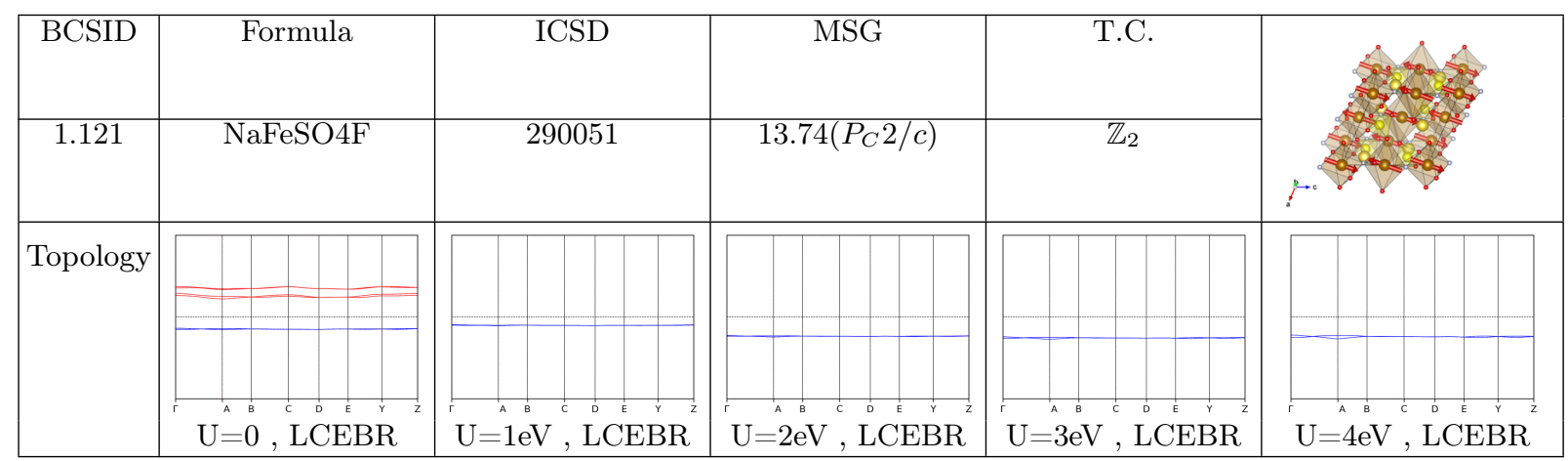

TABLE LXXXII. Topology phase diagram of NaFeSO4F.

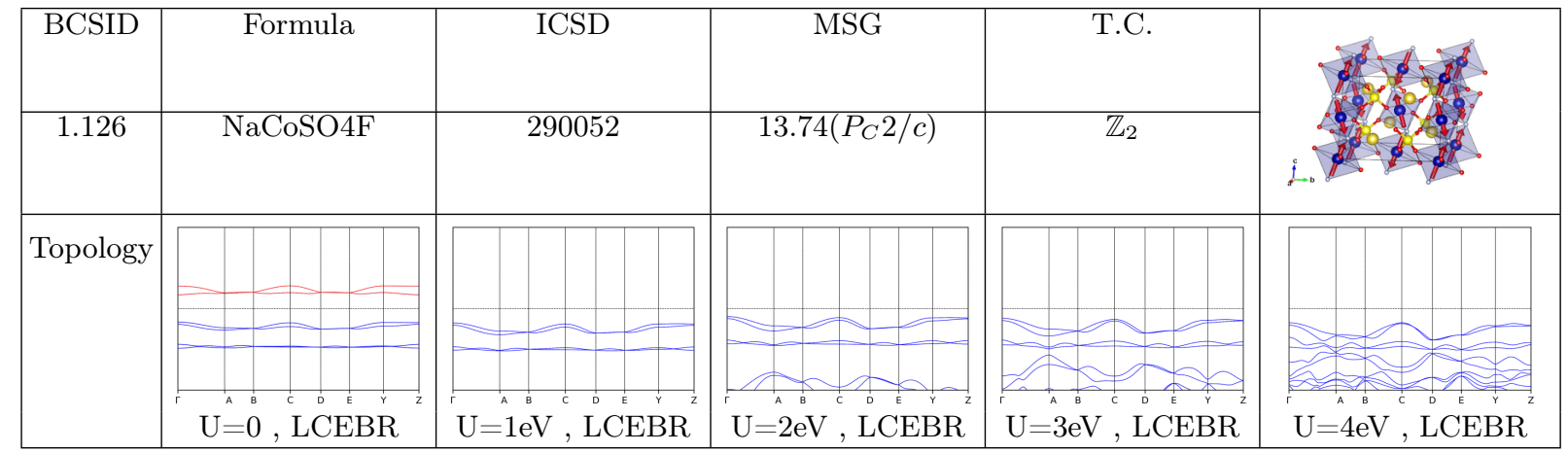

TABLE LXXXIII. Topology phase diagram of NaCoSO4F. 


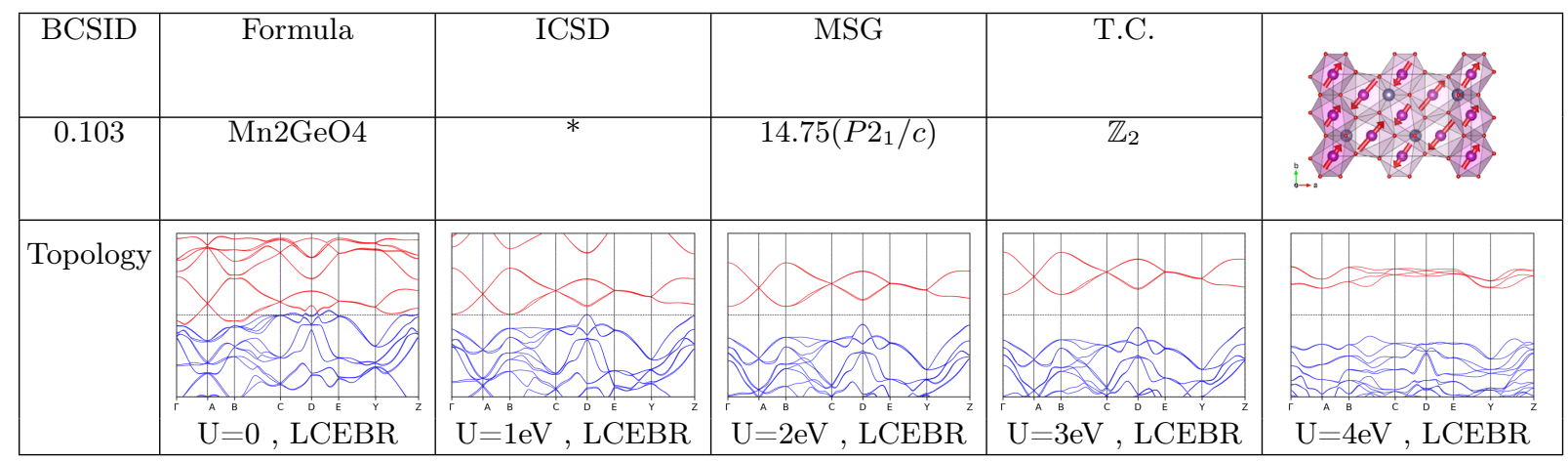

TABLE LXXXIV. Topology phase diagram of Mn2GeO4.

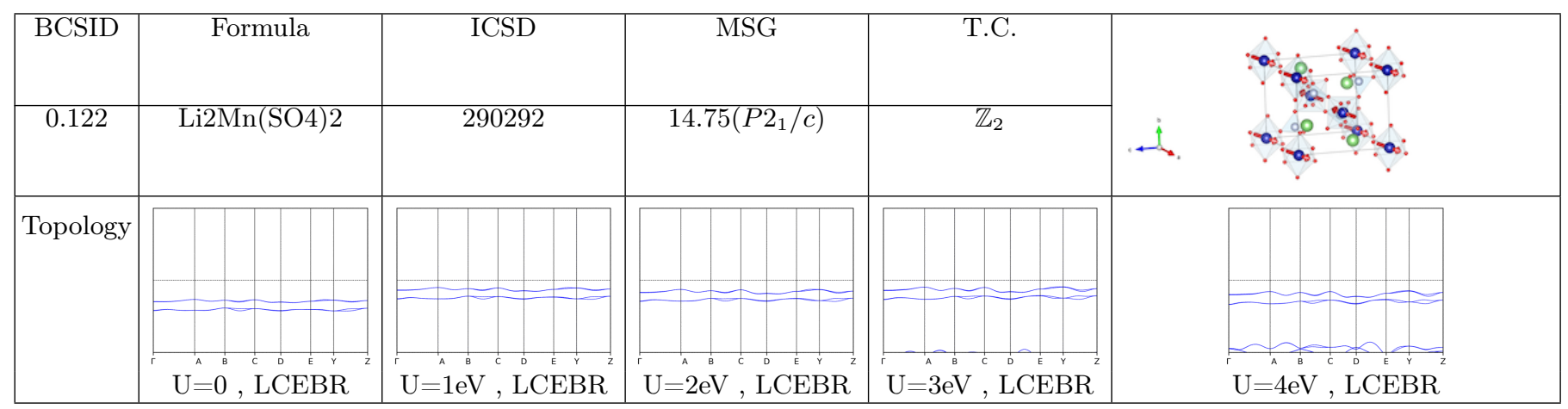

TABLE LXXXV. Topology phase diagram of Li2Mn(SO4)2.

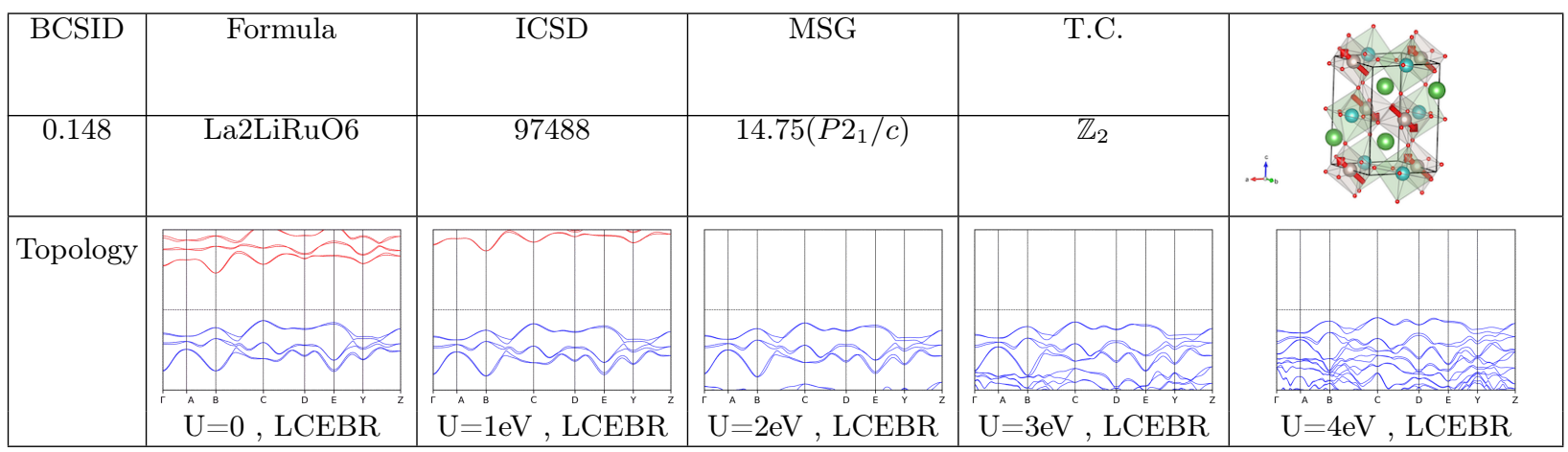

TABLE LXXXVI. Topology phase diagram of La2LiRuO6.

\begin{tabular}{|c|c|c|c|c|c|}
\hline BCSID & Formula & ICSD & MSG & T.C. & \\
\hline 0.204 & Ca2MnReO6 & * & $14.75\left(P 2_{1} / c\right)$ & $\mathbb{Z}_{2}$ & \\
\hline \multirow[t]{2}{*}{ Topology } & & & & & \\
\hline & $\mathrm{U}=0, \mathrm{LCEBR}$ & $\mathrm{U}=1 \mathrm{eV}, \mathrm{TBD}$ & $\mathrm{U}=2 \mathrm{eV}, \mathrm{TBD}$ & $\mathrm{U}=3 \mathrm{eV}, \mathrm{LCEBR}$ & $\mathrm{U}=4 \mathrm{eV}, \mathrm{LCEBR}$ \\
\hline
\end{tabular}

TABLE LXXXVII. Topology phase diagram of Ca2MnReO6. 


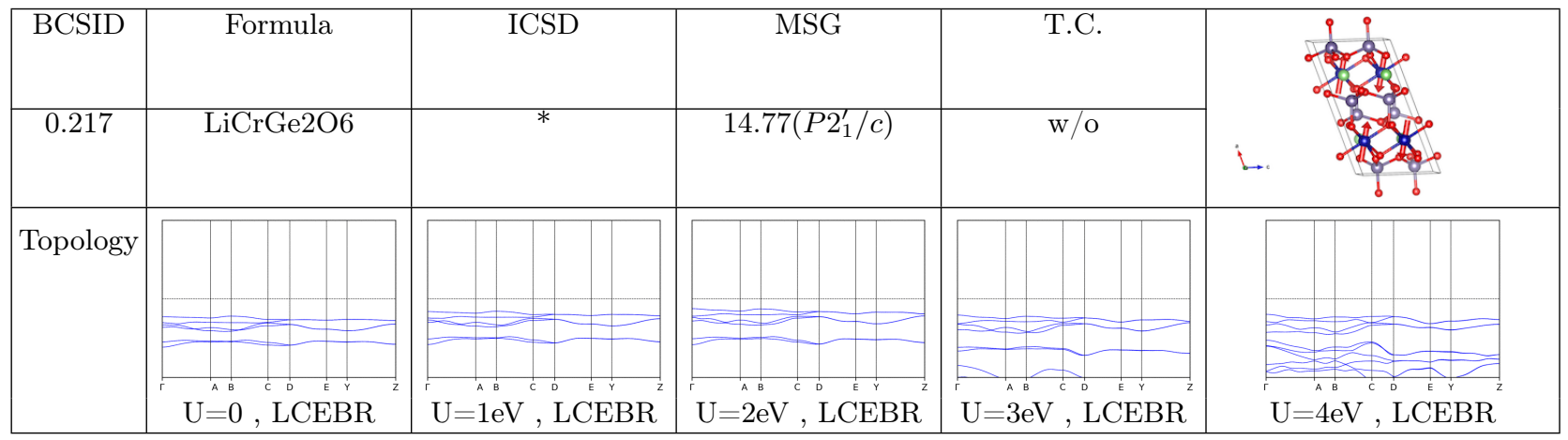

TABLE LXXXVIII. Topology phase diagram of LiCrGe2O6.

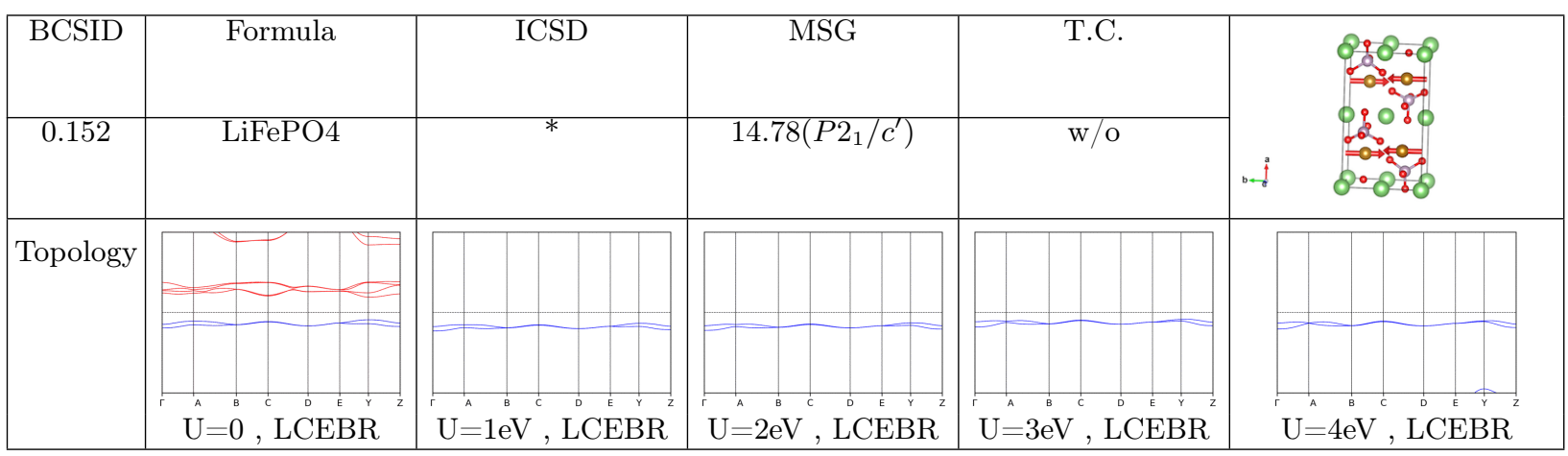

TABLE LXXXIX. Topology phase diagram of LiFePO4.

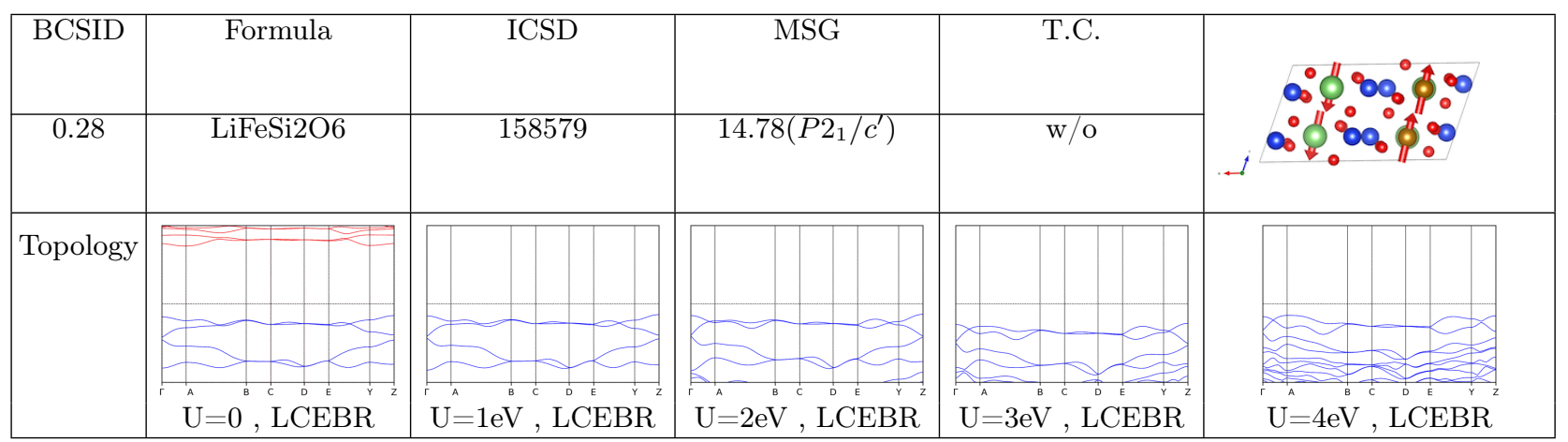

TABLE XC. Topology phase diagram of LiFeSi2O6.

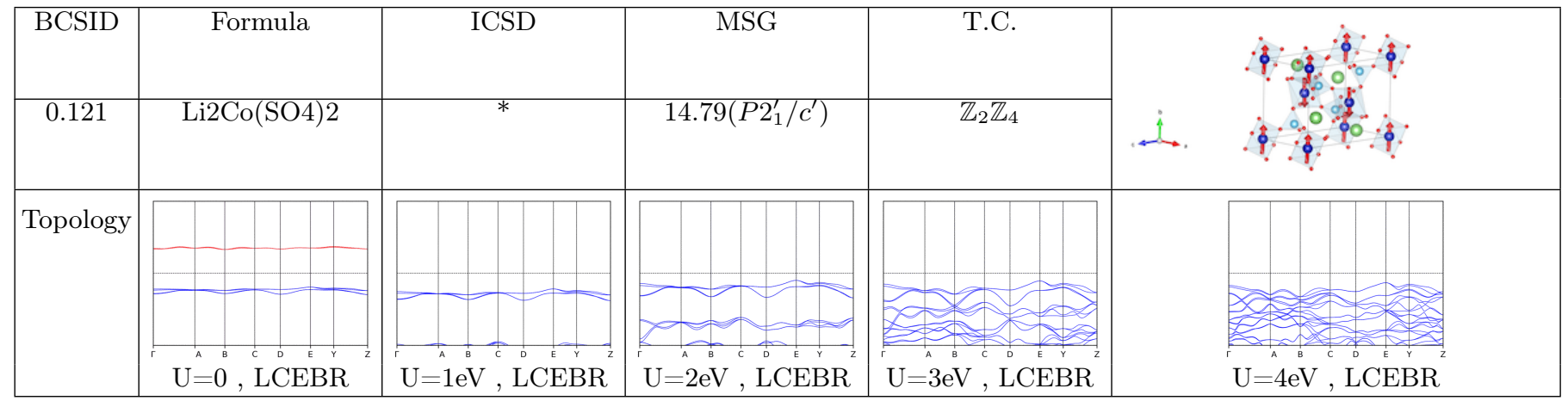

TABLE XCI. Topology phase diagram of Li2Co(SO4)2. 


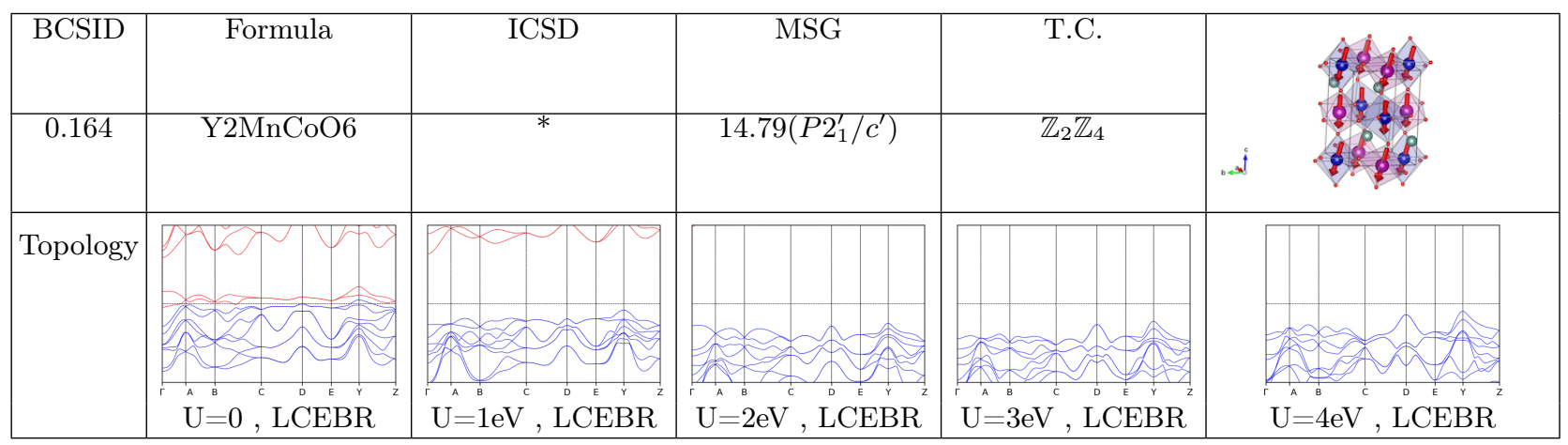

TABLE XCII. Topology phase diagram of Y2MnCoO6.

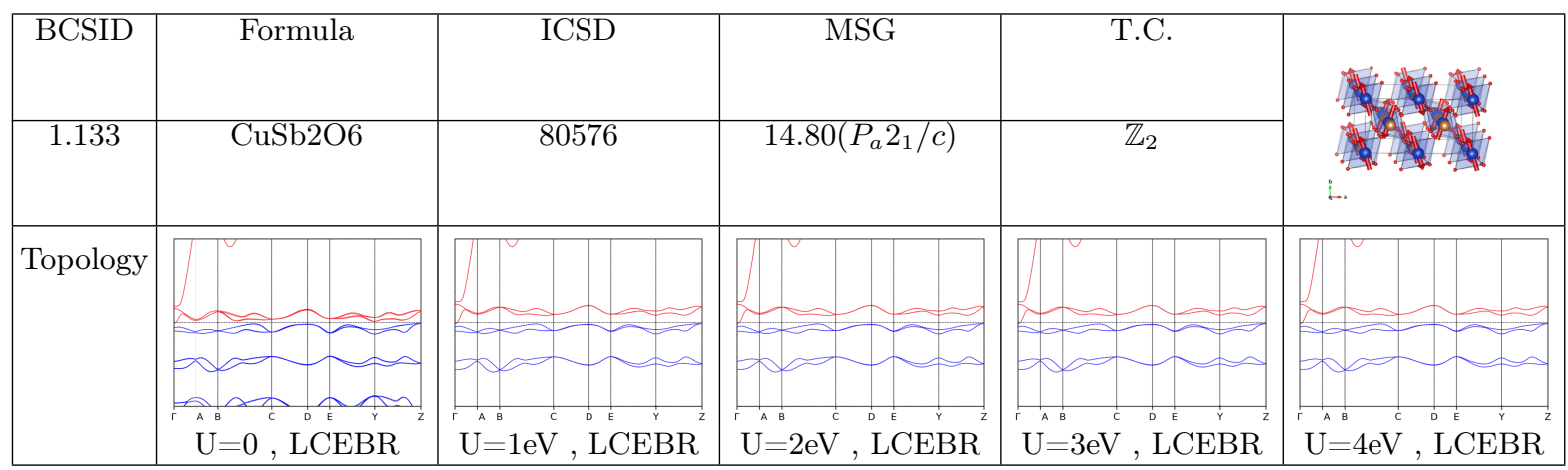

TABLE XCIII. Topology phase diagram of CuSb2O6.

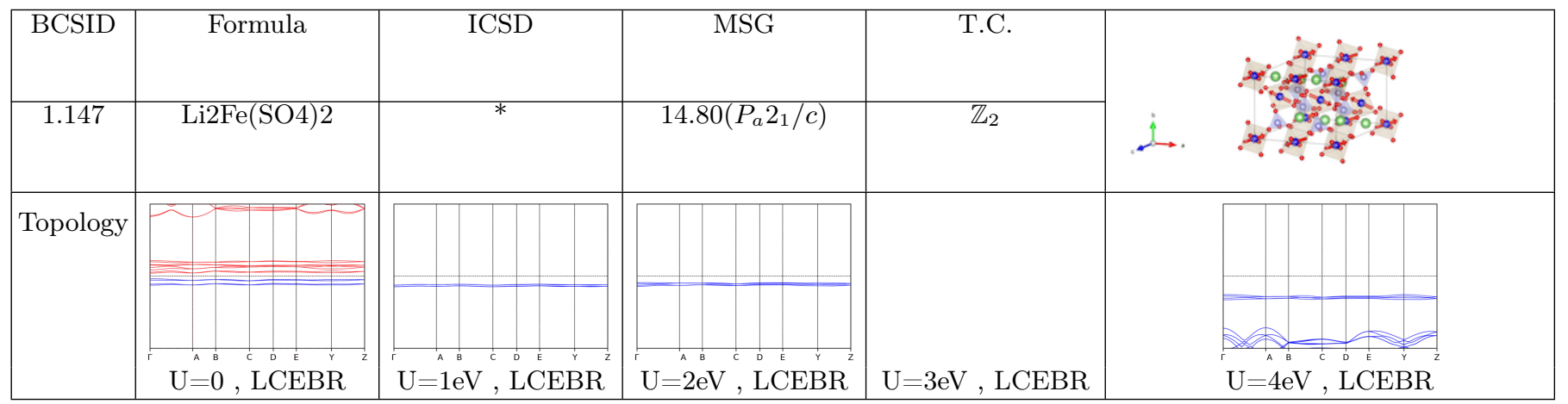

TABLE XCIV. Topology phase diagram of Li2Fe(SO4)2.

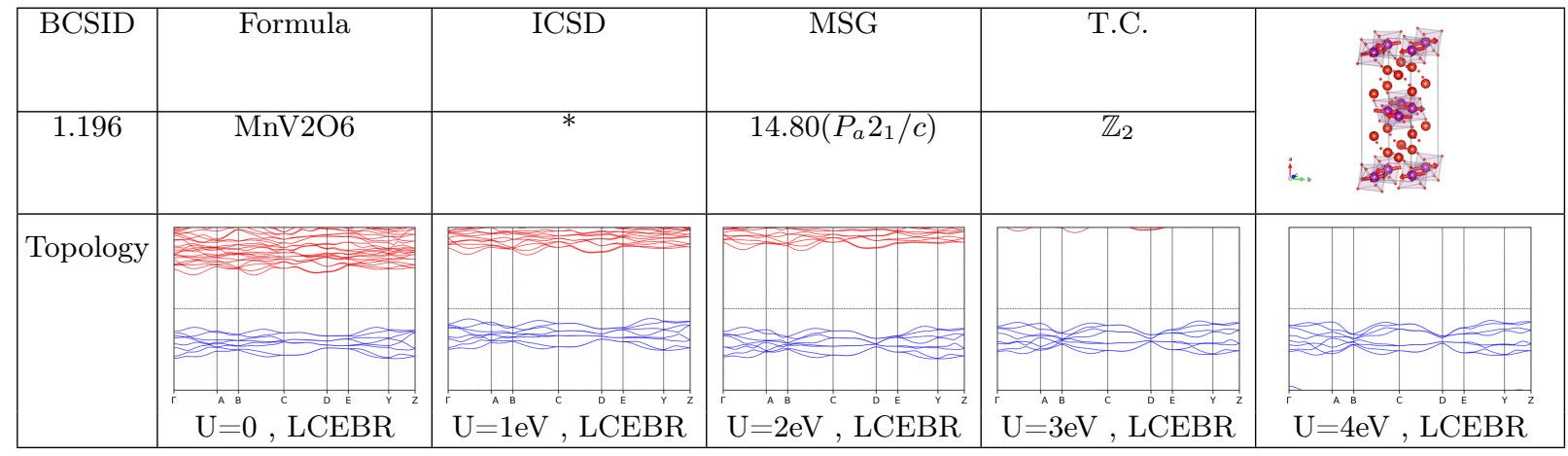

TABLE XCV. Topology phase diagram of MnV2O6. 


\begin{tabular}{|c|c|c|c|c|c|}
\hline BCSID & Formula & ICSD & MSG & T.C. & \\
\hline 1.199 & Sc2NiMnO6 & 251833 & $14.80\left(P_{a} 2_{1} / c\right)$ & $\mathbb{Z}_{2}$ & \\
\hline \multicolumn{6}{|l|}{ Topology } \\
\hline & $\begin{array}{l}\hat{A}=0, L C E B R \\
\mathrm{U}=0, \mathrm{~L}\end{array}$ & $\begin{array}{l}\mathrm{A} \\
\mathrm{U}=1 \mathrm{eV},\end{array}$ & $\mathrm{U}=2 \mathrm{eV}, \stackrel{L}{\mathrm{~L} C E B R}$ & $\mathrm{U}=3 \mathrm{eV}, \stackrel{\mathrm{LCEBR}}{\mathrm{L}}$ & $\mathrm{U}=4 \mathrm{eV}, \mathrm{TBD}$ \\
\hline
\end{tabular}

TABLE XCVI. Topology phase diagram of Sc2NiMnO6.

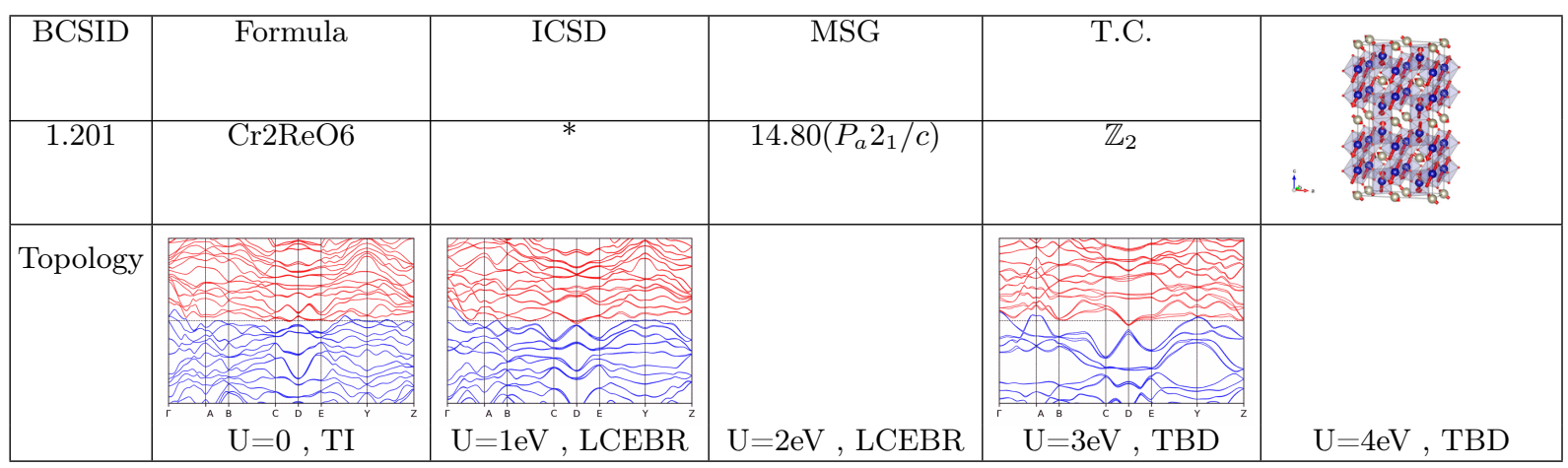

TABLE XCVII. Topology phase diagram of Cr2ReO6.

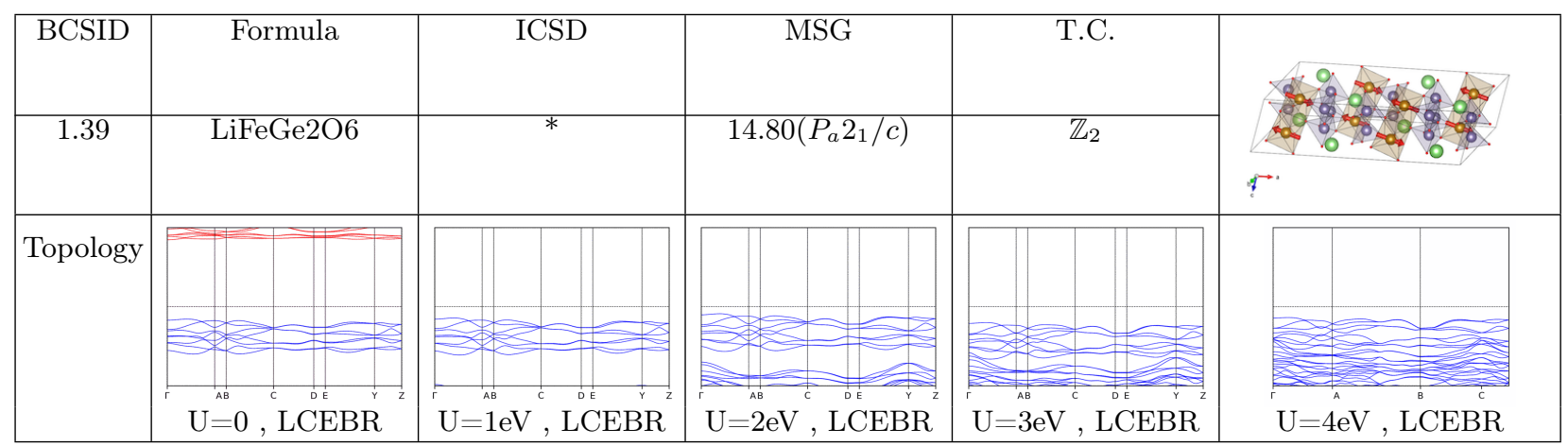

TABLE XCVIII. Topology phase diagram of LiFeGe2O6.

\begin{tabular}{|c|c|c|c|c|c|}
\hline BCSID & Formula & ICSD & MSG & T.C. \\
\hline 1.62 & $\mathrm{CuO}$ & $*$ & $14.80\left(P_{a} 2_{1} / c\right)$ & \\
\hline Topology & & & \\
\hline
\end{tabular}

TABLE XCIX. Topology phase diagram of $\mathrm{CuO}$. 


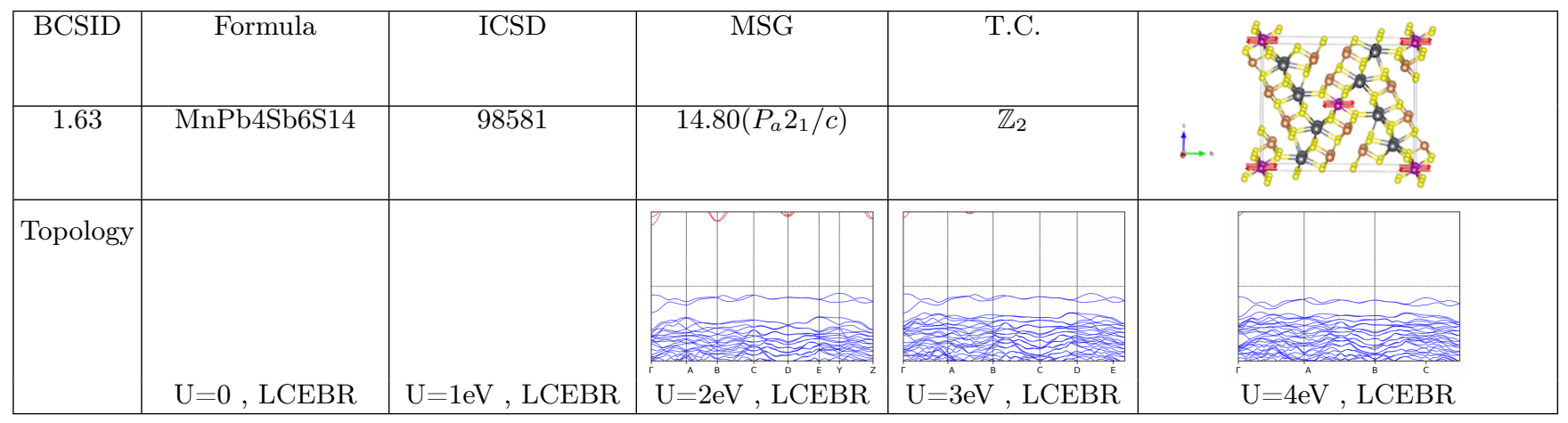

TABLE C. Topology phase diagram of MnPb4Sb6S14.

\begin{tabular}{|c|c|c|c|c|c|}
\hline BCSID & Formula & ICSD & MSG & T.C. & \\
\hline 1.112 & NiTa2O6 & 247807 & $14.82\left(P_{c} 2_{1} / c\right)$ & $\mathbb{Z}_{2}$ & \\
\hline \multirow{2}{*}{ Topology } & $\nabla$ & & & & \\
\hline & 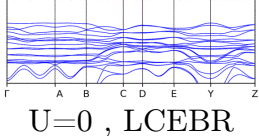 & 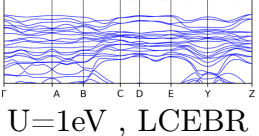 & $\begin{array}{l}\mathrm{A}{ }_{\mathrm{B}}^{\mathrm{B}} \mathrm{C}_{\mathrm{D}}^{\mathrm{D}} \mathrm{E}, \mathrm{V} \\
\mathrm{U}=2 \mathrm{LCEBR}\end{array}$ & 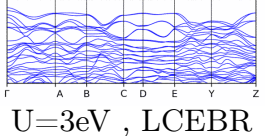 & $\mathrm{U}=4 \mathrm{eV}, \mathrm{LCEBR}$ \\
\hline
\end{tabular}

TABLE CI. Topology phase diagram of NiTa2O6.

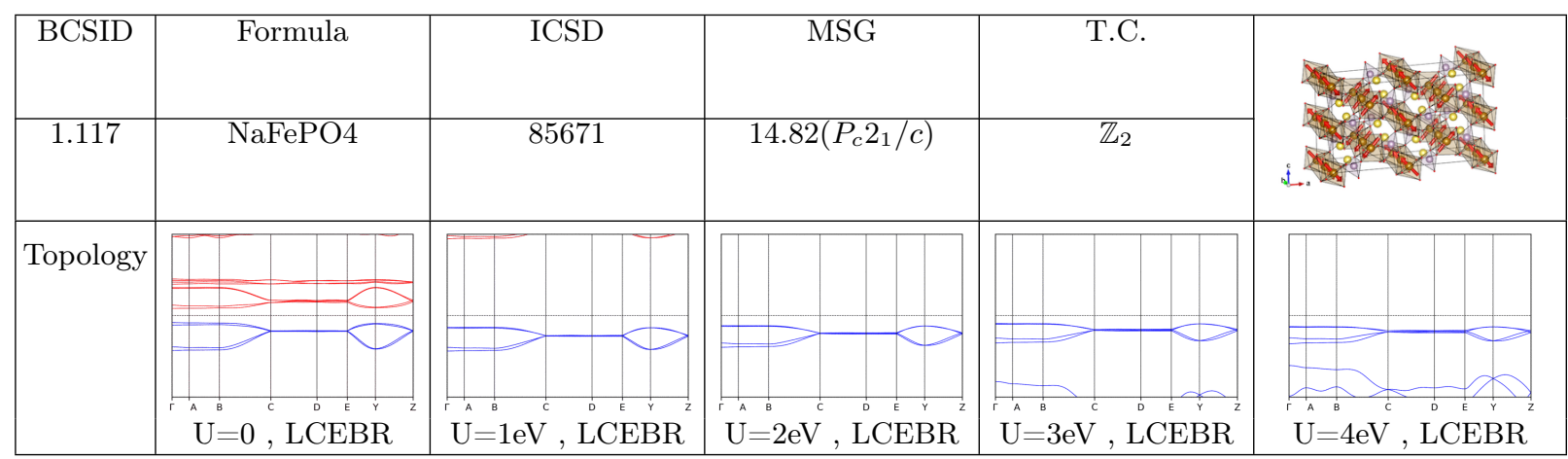

TABLE CII. Topology phase diagram of NaFePO4.

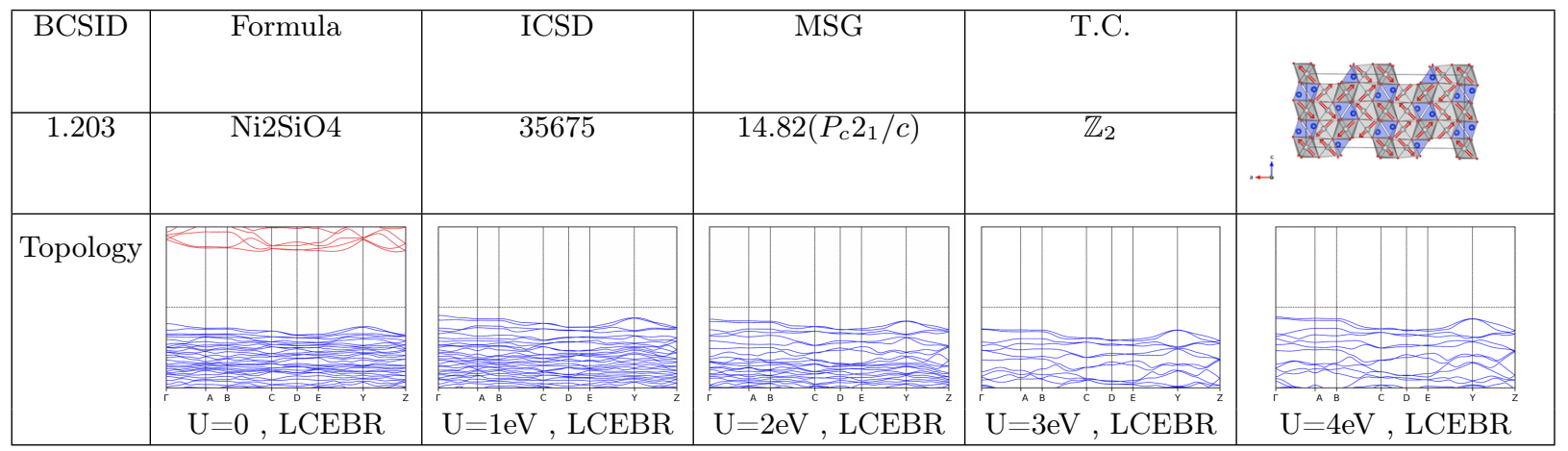

TABLE CIII. Topology phase diagram of Ni2SiO4. 


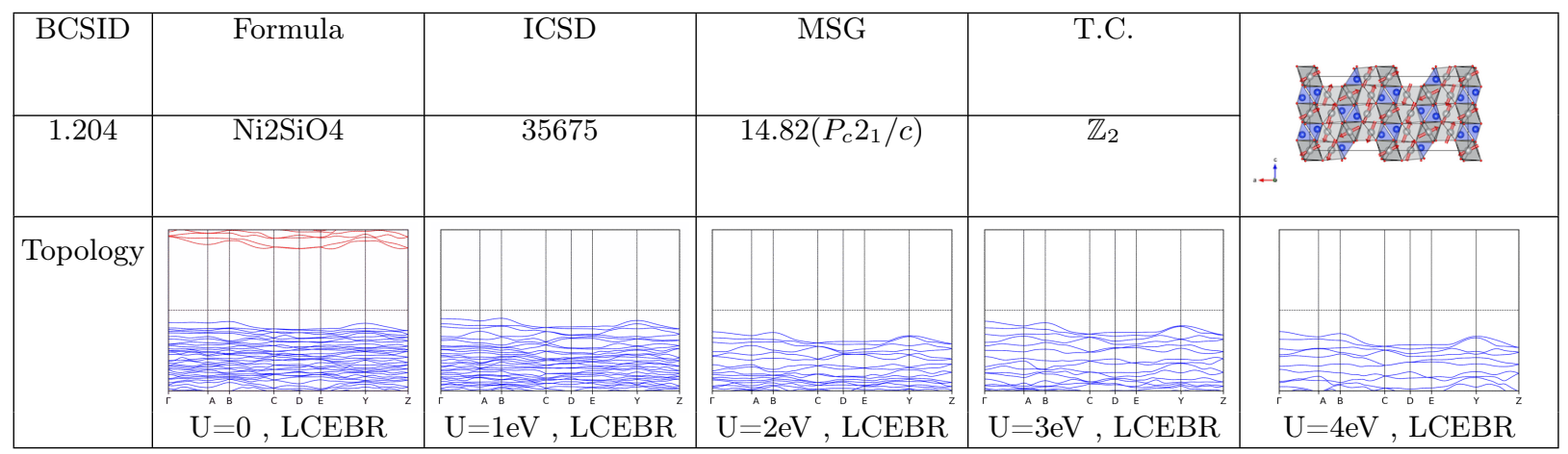

TABLE CIV. Topology phase diagram of Ni2SiO4.

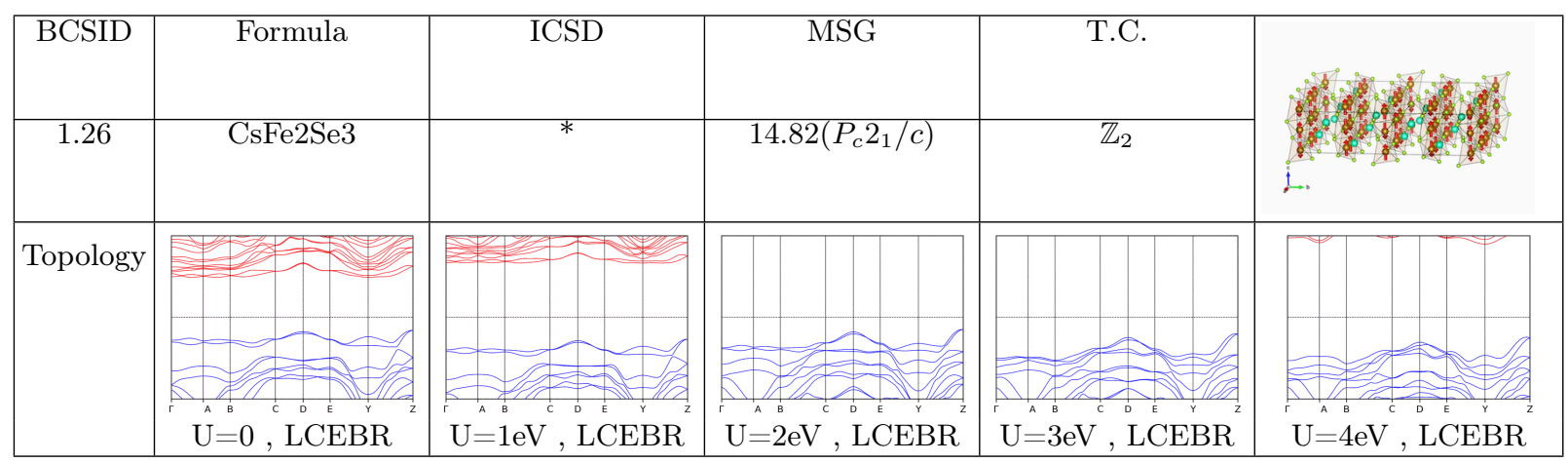

TABLE CV. Topology phase diagram of CsFe2Se3.

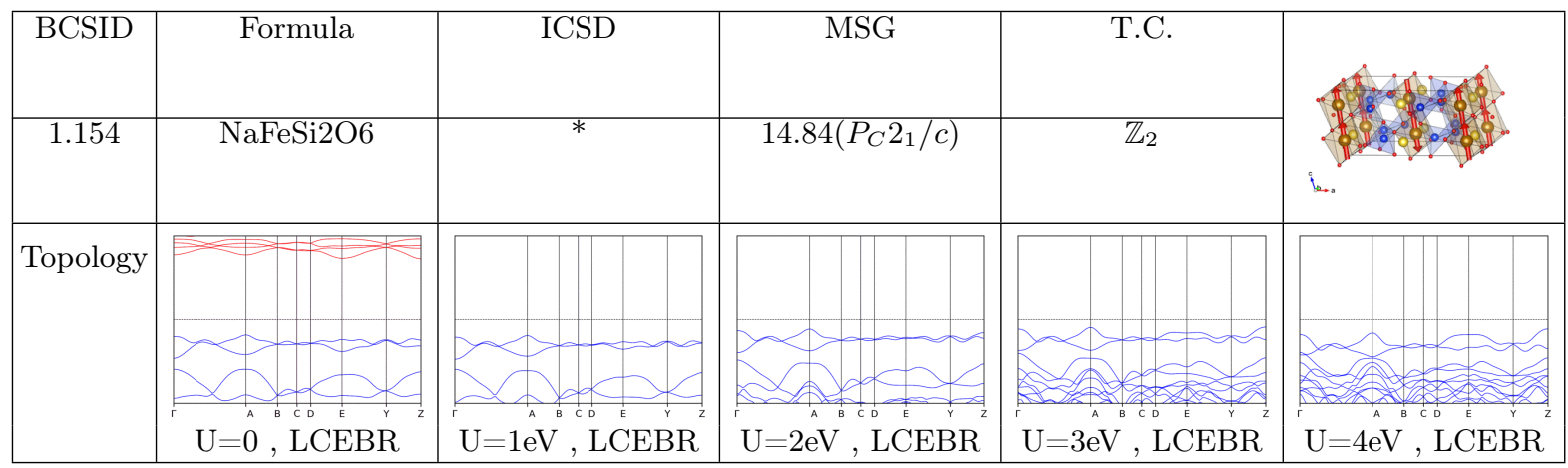

TABLE CVI. Topology phase diagram of NaFeSi2O6.

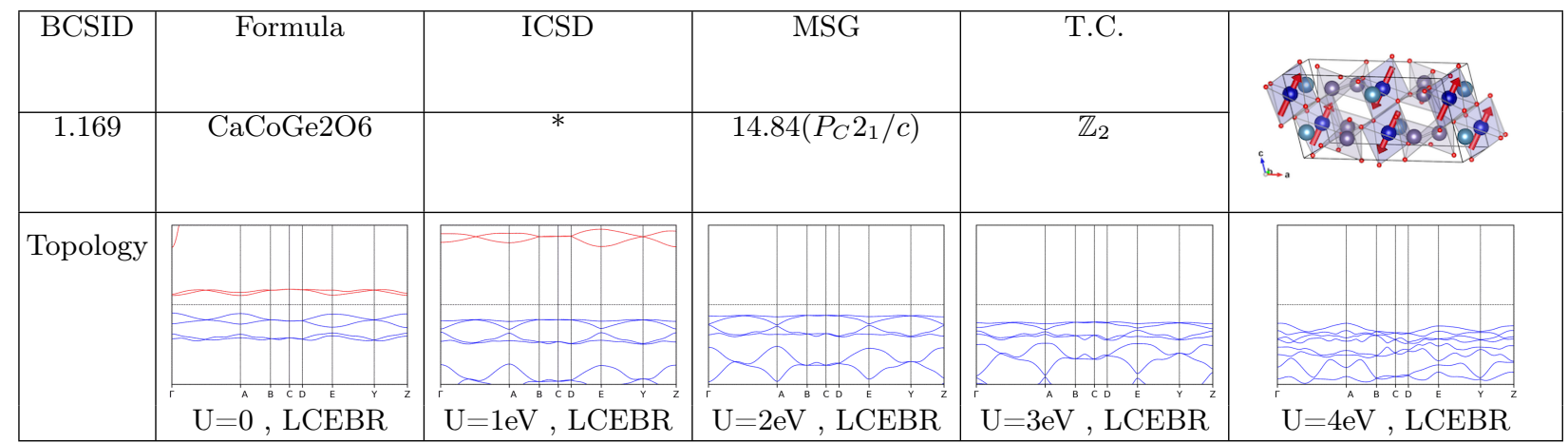

TABLE CVII. Topology phase diagram of CaCoGe2O6. 


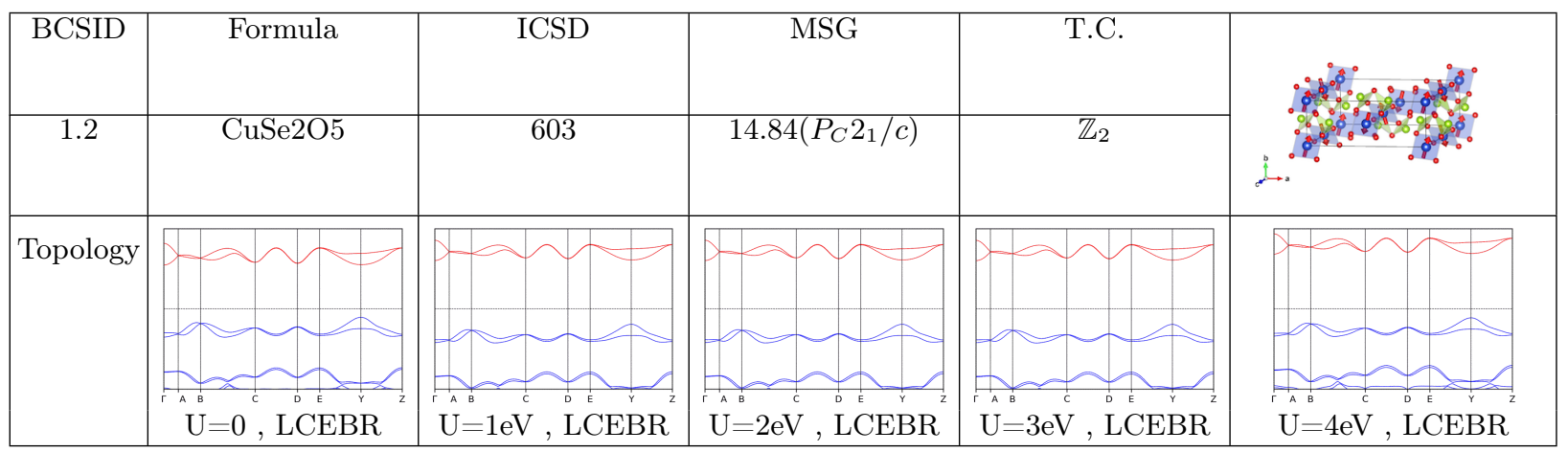

TABLE CVIII. Topology phase diagram of CuSe2O5.

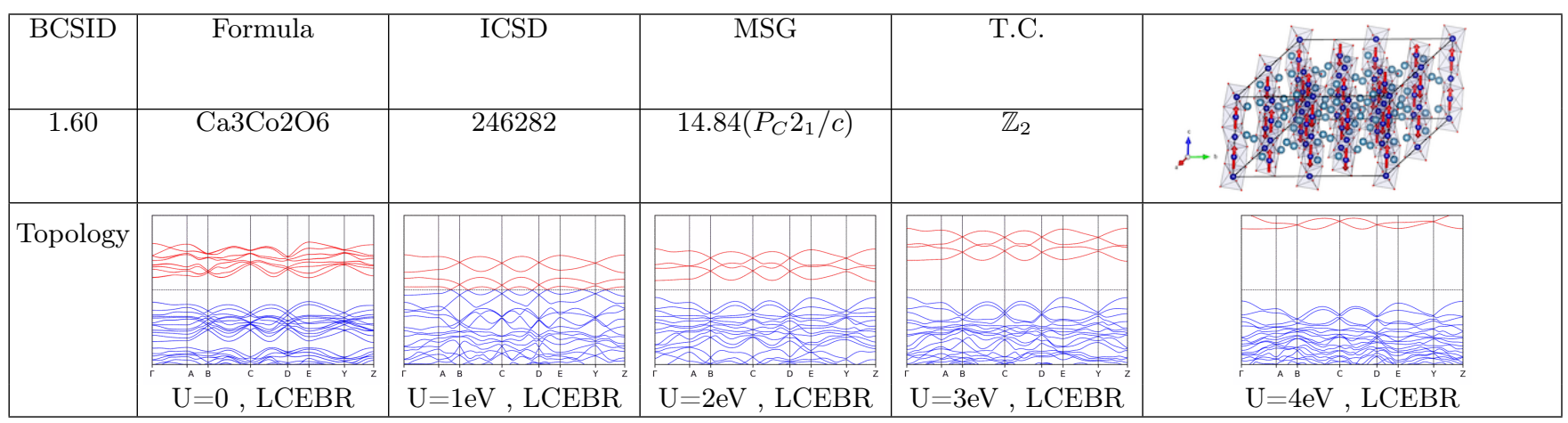

TABLE CIX. Topology phase diagram of Ca3Co2O6.

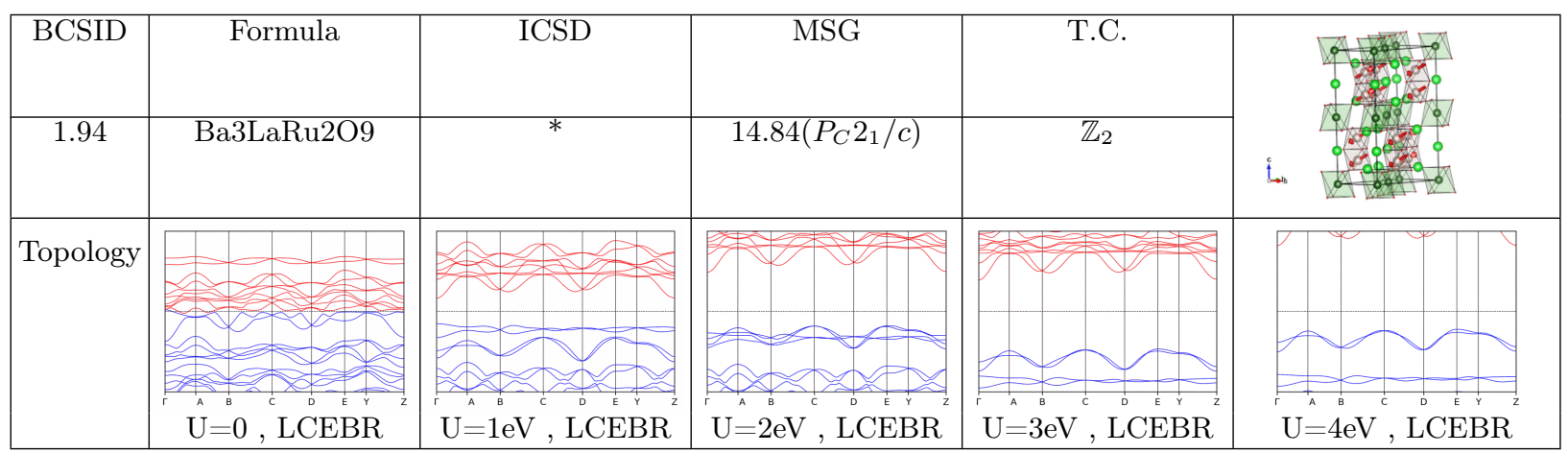

TABLE CX. Topology phase diagram of Ba3LaRu2O9.

\begin{tabular}{|c|c|c|c|c|c|}
\hline BCSID & Formula & ICSD & MSG & T.C. & \\
\hline 0.113 & NiCO3 & 173985 & $15.85(C 2 / c)$ & $\mathbb{Z}_{2} \mathbb{Z}_{2}$ & In \\
\hline Topology & & & & & \\
\hline & $\mathrm{U}=0^{\mathrm{A}}{ }^{\mathrm{i}},{ }^{\mathrm{m}} \mathrm{LCEBR}$ & $\mathrm{U}=1 \mathrm{eV}, \mathrm{LCEBR}$ & $\mathrm{U}=2 \mathrm{eV}, \mathrm{LCEBR}$ & $\mathrm{U}=3 \mathrm{eV}, \mathrm{LCEBR}$ & $\mathrm{U}=4 \mathrm{eV}, \mathrm{LCEBR}$ \\
\hline
\end{tabular}

TABLE CXI. Topology phase diagram of NiCO3. 


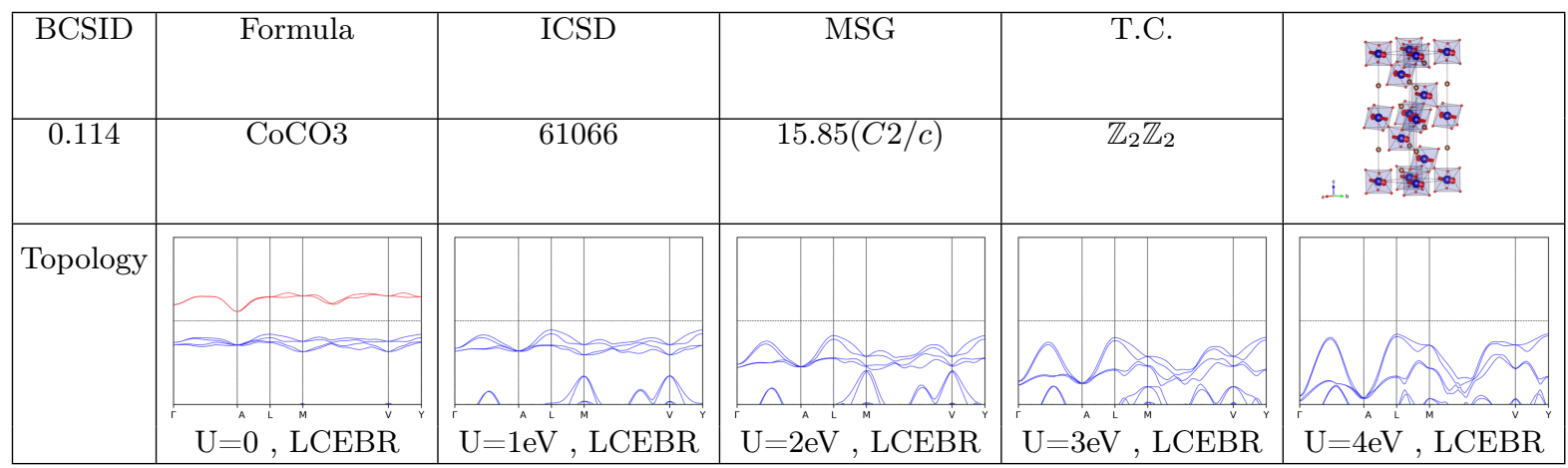

TABLE CXII. Topology phase diagram of CoCO3.

\begin{tabular}{|c|c|c|c|c|c|c|c|c|}
\hline BCSID & Formula & \multicolumn{2}{|c|}{$\overline{\text { ICSD }}$} & \multicolumn{2}{|c|}{ MSG } & \multicolumn{2}{|c|}{ T.C. } & \multirow{2}{*}{ 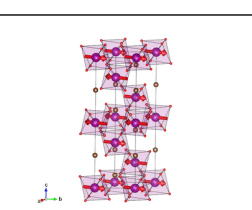 } \\
\hline 0.115 & $\mathrm{MnCO} 3$ & \multicolumn{2}{|c|}{$*$} & \multicolumn{2}{|c|}{$15.85(C 2 / c)$} & \multicolumn{2}{|c|}{$\mathbb{Z}_{2} \mathbb{Z}_{2}$} & \\
\hline \multirow[t]{2}{*}{ Topology } & & & & & & & & \\
\hline & $\mathrm{U}=0, \mathrm{LCEBR}$ & \multicolumn{2}{|c|}{$\mathrm{U}=1 \mathrm{eV}, \mathrm{LCEBR}$} & \multicolumn{2}{|c|}{$\mathrm{U}=2 \mathrm{eV}, \mathrm{LCEBR}$} & \multicolumn{2}{|c|}{$\mathrm{U}=3 \mathrm{eV}, \mathrm{LCEBR}$} & $\mathrm{U}=4 \mathrm{eV}, \mathrm{LCEBR}$ \\
\hline
\end{tabular}

TABLE CXIII. Topology phase diagram of MnCO3.

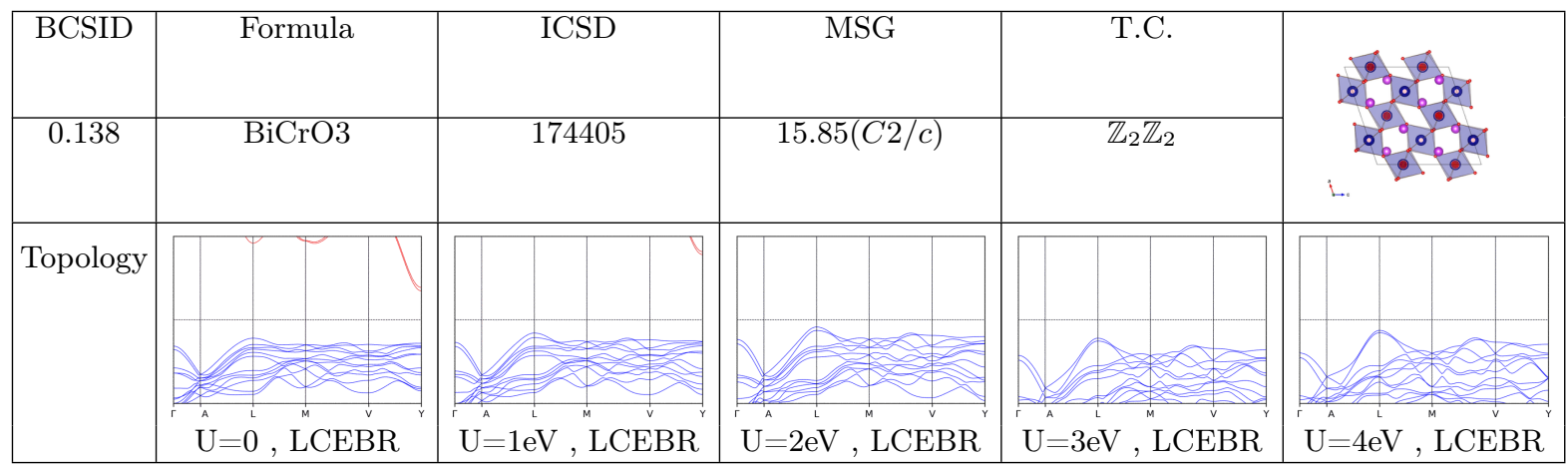

TABLE CXIV. Topology phase diagram of BiCrO3.

\begin{tabular}{|c|c|c|c|c|c|}
\hline BCSID & Formula & ICSD & MSG & T.C. & \\
\hline 0.210 & $\mathrm{Sr} 2 \mathrm{CoOsO} 6$ & * & $15.85(C 2 / c)$ & $\mathbb{Z}_{2} \mathbb{Z}_{2}$ & \\
\hline Topology & E & T & & & \\
\hline & $\hat{\mathrm{U}}=0, \mathrm{LCEBF}$ & $\mathrm{U}=1 \mathrm{eV}, \mathrm{LCEBR}$ & $\mathrm{U}=2 \mathrm{eV}, \mathrm{LCEBR}$ & $\mathrm{U}=3 \mathrm{eV}, \mathrm{LCEBR}$ & $\mathrm{U}=4 \mathrm{eV}, \mathrm{LCEBR}$ \\
\hline
\end{tabular}

TABLE CXV. Topology phase diagram of Sr2CoOsO6. 


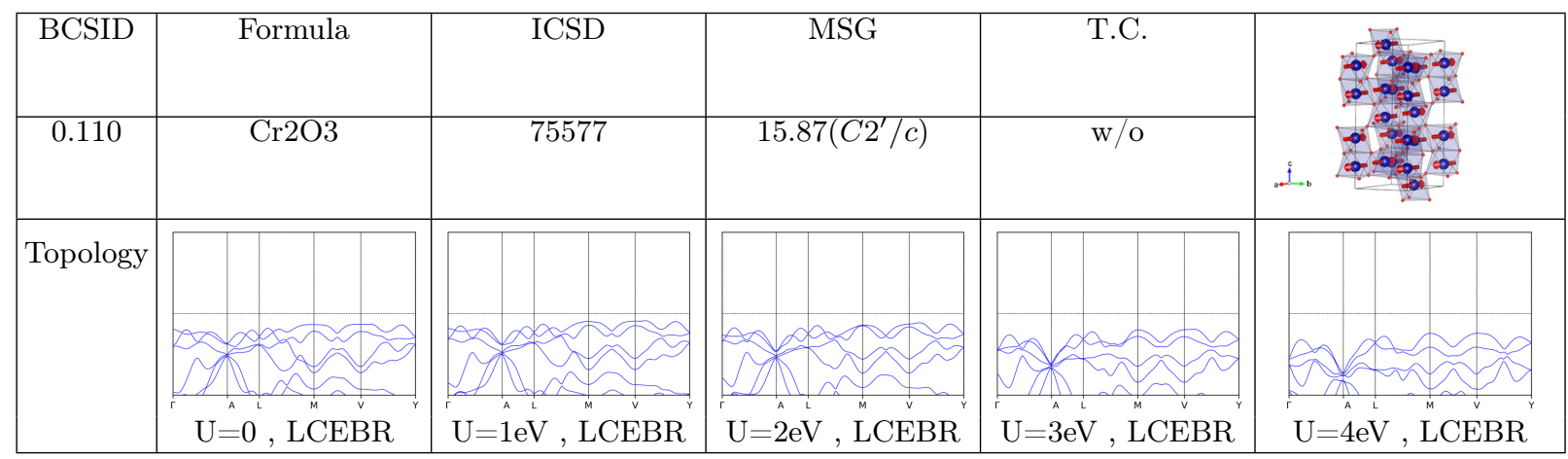

TABLE CXVI. Topology phase diagram of Cr2O3.

\begin{tabular}{|c|c|c|c|c|c|c|c|}
\hline BCSID & Formula & ICSD & $\mathrm{M}_{\mathrm{s}}$ & & & .C. & \\
\hline 0.145 & Co3TeO6 & 183805 & 15.87( & $\left.C 2^{\prime} / c\right)$ & & 10 & $10.08^{9}$ \\
\hline \multirow[t]{4}{*}{ Topology } & 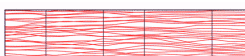 & \begin{tabular}{|l|l|} 
& $=13$ \\
\end{tabular} & & & & & \\
\hline & S & 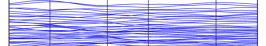 & & & & & \\
\hline & 10 & $=1$ & 1 & 10 & 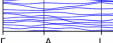 & & 要 \\
\hline & $\hat{\mathrm{U}}=0, \mathrm{LCEBR}$ & $\mathrm{U}=1 \mathrm{eV}, \mathrm{LCEBR}$ & $\mathrm{U}=2 \mathrm{eV}$, & LCEBR & $\mathrm{U}=3 \mathrm{eV}$ & LCEBR & $\mathrm{U}=4 \mathrm{eV}, \mathrm{LCEBR}$ \\
\hline
\end{tabular}

TABLE CXVII. Topology phase diagram of Co3TeO6.

\begin{tabular}{|c|c|c|c|c|c|c|c|c|c|}
\hline BCSID & Formula & & SD & & & T. & C. & \multirow{2}{*}{\multicolumn{2}{|c|}{ 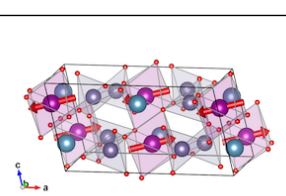 }} \\
\hline 0.156 & CaMnGe2O6 & & & \multicolumn{2}{|c|}{$15.87\left(C 2^{\prime} / c\right)$} & \multicolumn{2}{|c|}{$\mathrm{w} / \mathrm{o}$} & & \\
\hline \multirow[t]{2}{*}{ Topology } & 7 & & & & & & & & \\
\hline & $\mathrm{U}^{\wedge}=0, \mathrm{LCEBR}$ & \multicolumn{2}{|c|}{$\mathrm{U}=1 \mathrm{eV}, \mathrm{LCEBR}$} & \multicolumn{2}{|c|}{$\mathrm{U}=2 \mathrm{eV}, \mathrm{LCEBR}$} & \multicolumn{2}{|c|}{$\mathrm{U}=3 \mathrm{eV}, \mathrm{LCEBR}$} & \multicolumn{2}{|c|}{$\mathrm{U}=4 \mathrm{eV}, \mathrm{LCEBR}$} \\
\hline
\end{tabular}

TABLE CXVIII. Topology phase diagram of CaMnGe2O6.

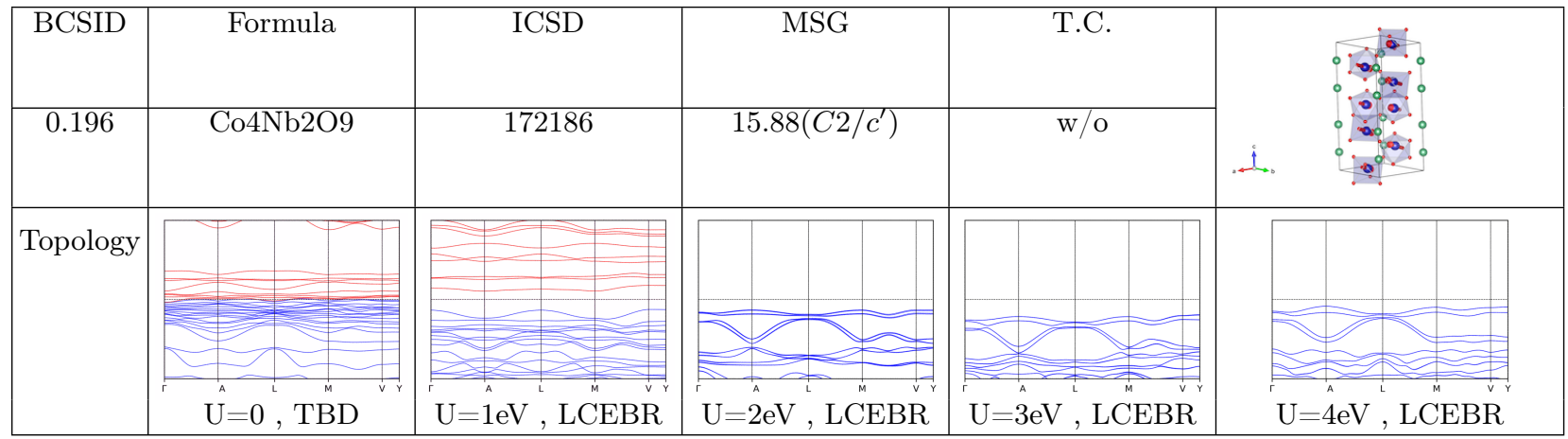

TABLE CXIX. Topology phase diagram of Co4Nb2O9. 


\begin{tabular}{|c|c|c|c|c|c|}
\hline BCSID & Formula & ICSD & MSG & T.C. & \\
\hline 0.197 & Co4Nb2O9 & & $15.88\left(C 2 / c^{\prime}\right)$ & $\mathrm{w} / \mathrm{o}$ & L. \\
\hline \multirow[t]{3}{*}{ Topology } & & $1 \%$ 男 & & & \\
\hline & 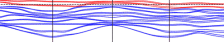 & 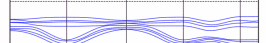 & & & \\
\hline & $\mathrm{U}=0, \mathrm{LCEBR}$ & $\mathrm{U}=1 \mathrm{eV}, \mathrm{LCEBR}$ & $\mathrm{U}=2 \mathrm{e} \mathrm{eV}, \mathrm{LCEBR}$ & $\mathrm{U}=\hat{3} \mathrm{eV}, \mathrm{LCEBR}$ & $\mathrm{U}=4 \mathrm{eV}, \mathrm{LCEBR}$ \\
\hline
\end{tabular}

TABLE CXX. Topology phase diagram of Co4Nb2O9.

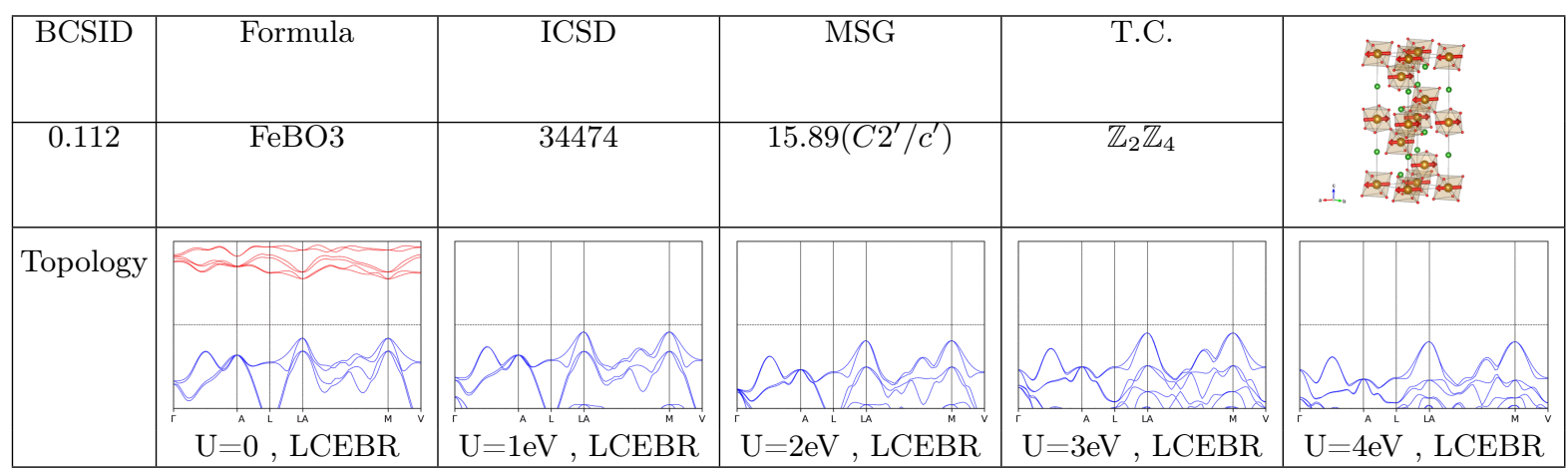

TABLE CXXI. Topology phase diagram of FeBO3.

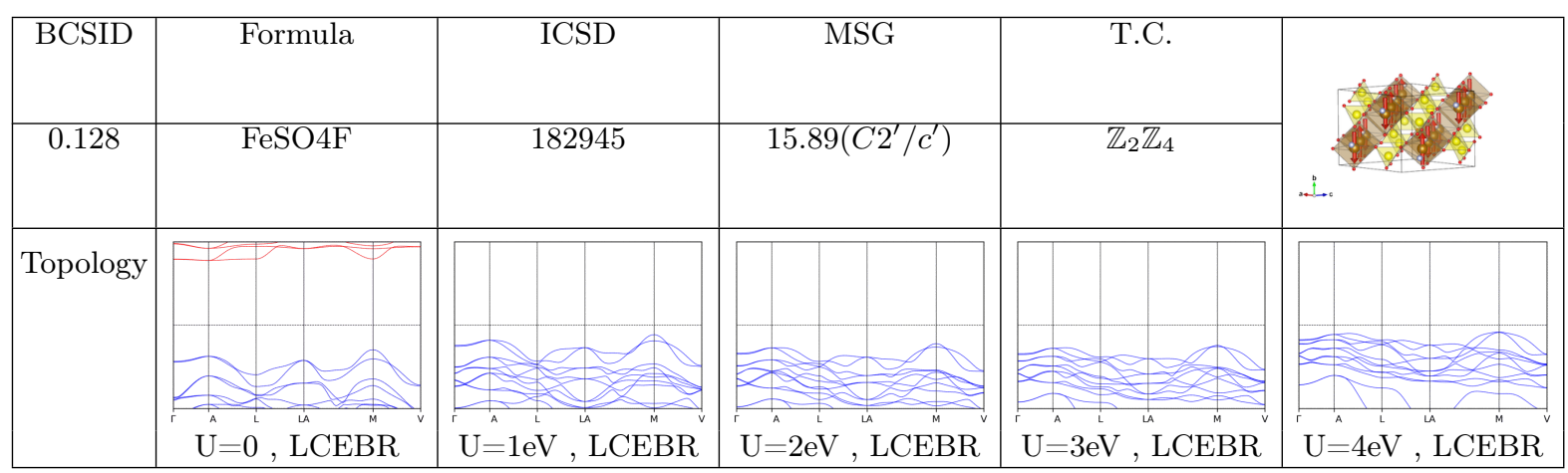

TABLE CXXII. Topology phase diagram of FeSO4F.

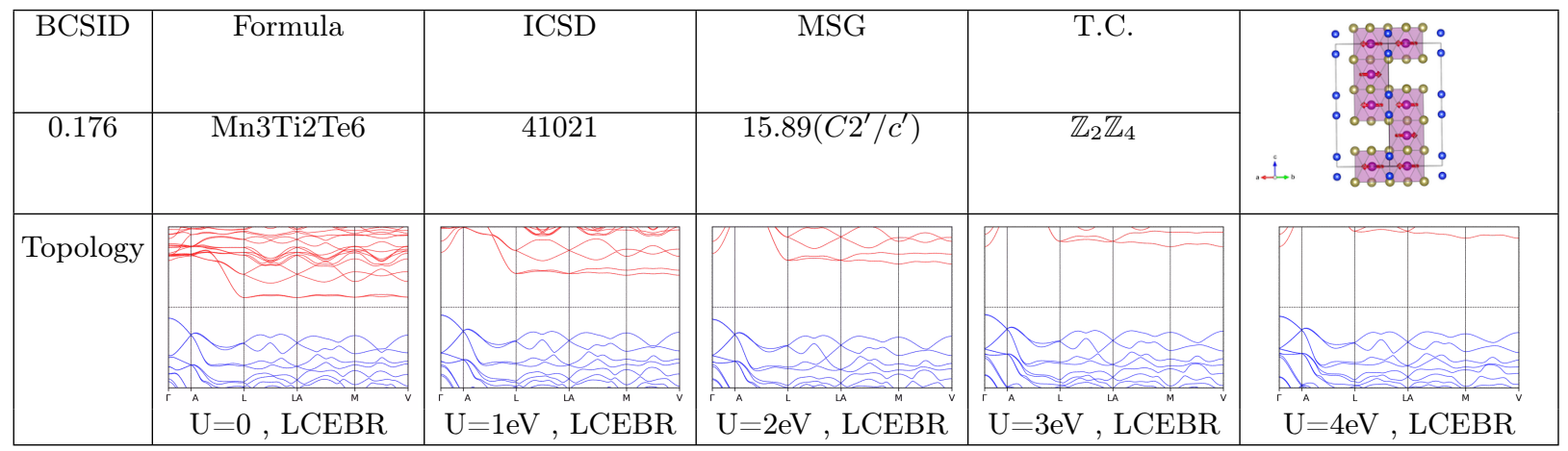

TABLE CXXIII. Topology phase diagram of Mn3Ti2Te6. 


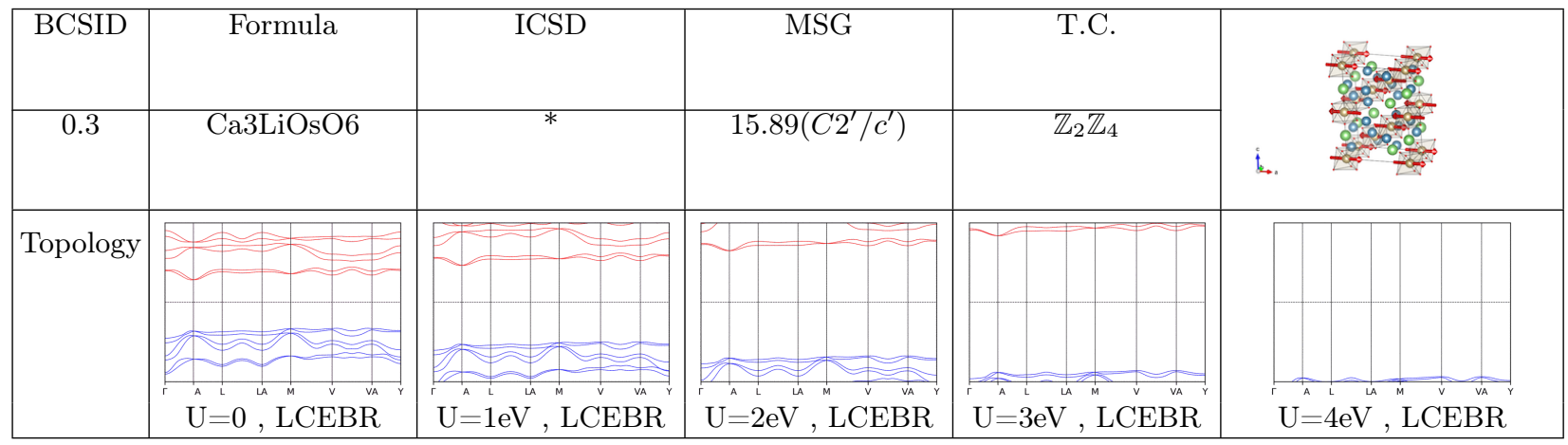

TABLE CXXIV. Topology phase diagram of Ca3LiOsO6.

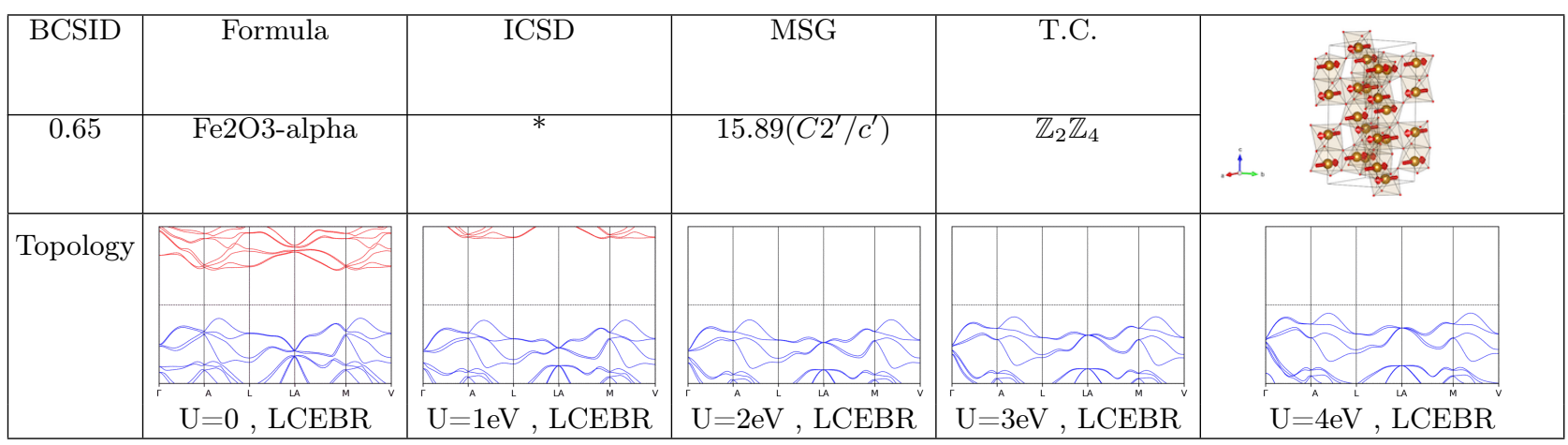

TABLE CXXV. Topology phase diagram of Fe2O3-alpha.

\begin{tabular}{|c|c|c|c|c|c|}
\hline BCSID & Formula & ICSD & MSG & T.C. & \\
\hline 1.168 & Sr2CuTeO6 & $*$ & $15.90\left(C_{c} 2 / c\right)$ & $\mathbb{Z}_{2}$ & 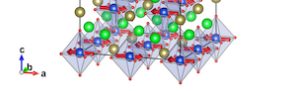 \\
\hline \multicolumn{6}{|l|}{ Topology } \\
\hline & $\mathrm{U}^{\mathrm{A}}=0, \mathrm{~L}^{\mathrm{m}} \mathrm{LEBR}$ & $\mathrm{U}=1 \mathrm{eV}, \mathrm{LCEBR}$ & $\mathrm{U}^{\mathrm{A}}=2 \mathrm{eV}, \mathrm{LCEBR}$ & $\mathrm{U}^{\mathrm{A}}=3 \mathrm{eV}, \mathrm{LCEBR}$ & $\mathrm{U}^{\mathrm{A}}=4 \mathrm{eV}, \mathrm{LCEBR}$ \\
\hline
\end{tabular}

TABLE CXXVI. Topology phase diagram of Sr2CuTeO6.

\begin{tabular}{|c|c|c|c|c|c|}
\hline BCSID & Formula & ICSD & MSG & T.C. & \\
\hline 1.17 & CoV2O6-alpha & * & $15.90\left(C_{c} 2 / c\right)$ & $\mathbb{Z}_{2}$ & \\
\hline Topology & & & & & \\
\hline & $\mathrm{U}=0, \mathrm{LCEBR}$ & $\mathrm{U}=1 \mathrm{eV}, \mathrm{LCEBR}$ & $\mathrm{U}=2 \mathrm{eV}, \mathrm{LCEBR}$ & $\mathrm{U}=3 \mathrm{eV}, \mathrm{LCEBR}$ & $\mathrm{U}=4 \mathrm{eV}, \mathrm{LCEBR}$ \\
\hline
\end{tabular}

TABLE CXXVII. Topology phase diagram of CoV2O6-alpha. 


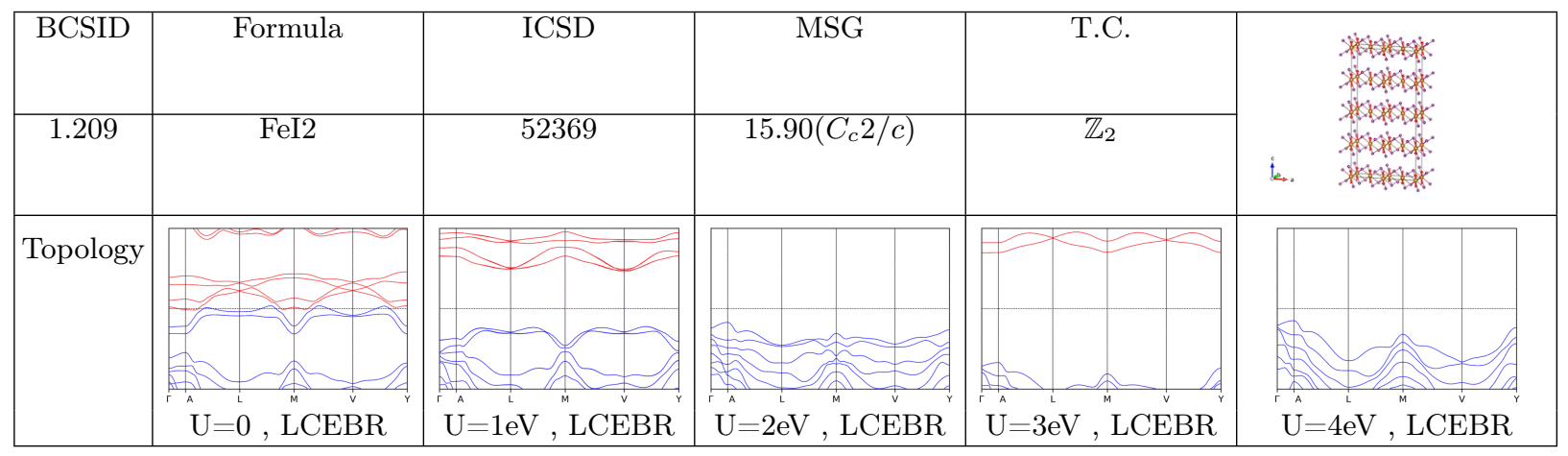

TABLE CXXVIII. Topology phase diagram of FeI2.

\begin{tabular}{|c|c|c|c|c|c|}
\hline BCSID & Formula & ICSD & MSG & T.C. & \\
\hline 1.245 & $\mathrm{CoBr} 2$ & 52364 & $15.90\left(C_{c} 2 / c\right)$ & $\mathbb{Z}_{2}$ & 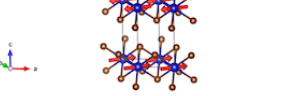 \\
\hline Topology & & & & & \\
\hline & $\mathrm{U}=0, \mathrm{LCEBR}$ & $\mathrm{U}=1 \mathrm{eV}, \mathrm{LCEBR}$ & $\mathrm{U}=2 \mathrm{eV}, \mathrm{LCEBR}$ & $\mathrm{U}=3 \mathrm{eV}, \mathrm{LCEBR}$ & $\mathrm{U}=4 \mathrm{eV}, \mathrm{LCEBR}$ \\
\hline
\end{tabular}

TABLE CXXIX. Topology phase diagram of CoBr2.

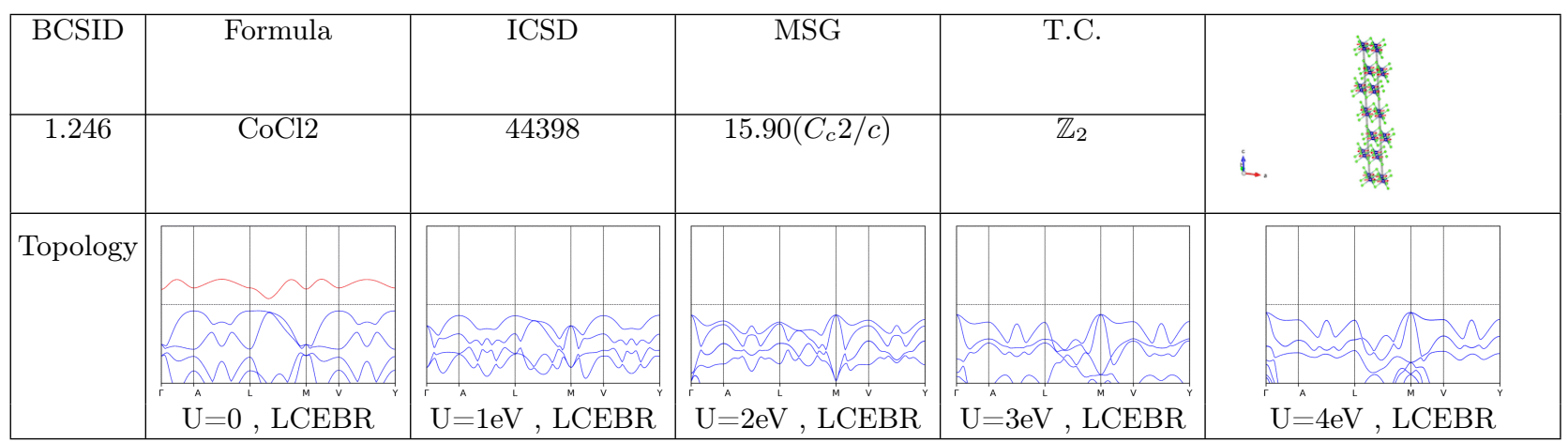

TABLE CXXX. Topology phase diagram of $\mathrm{CoCl} 2$.

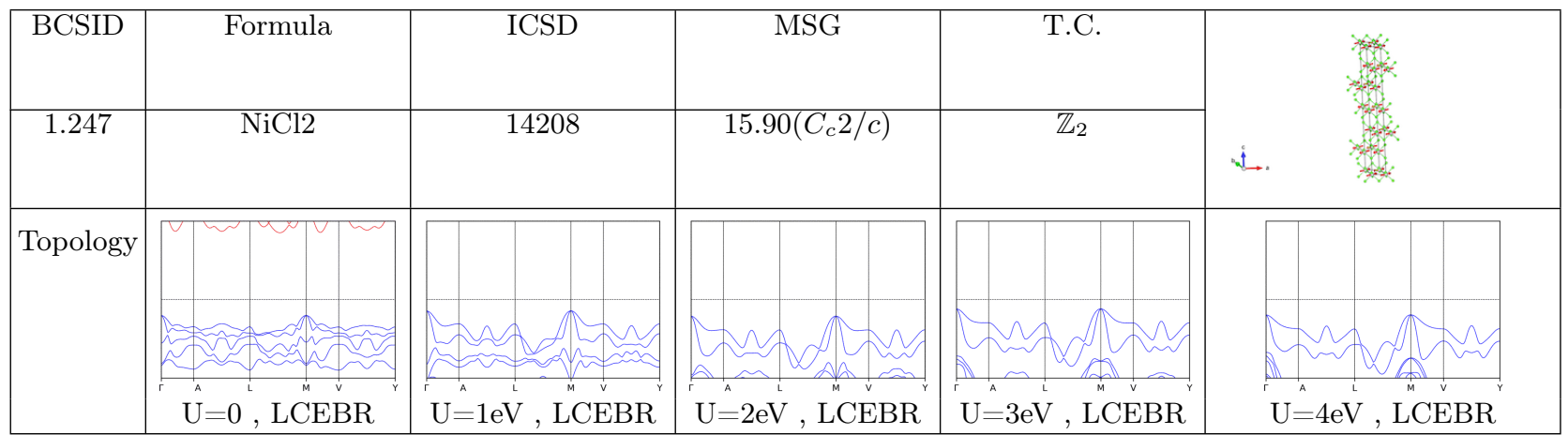

TABLE CXXXI. Topology phase diagram of NiCl2. 


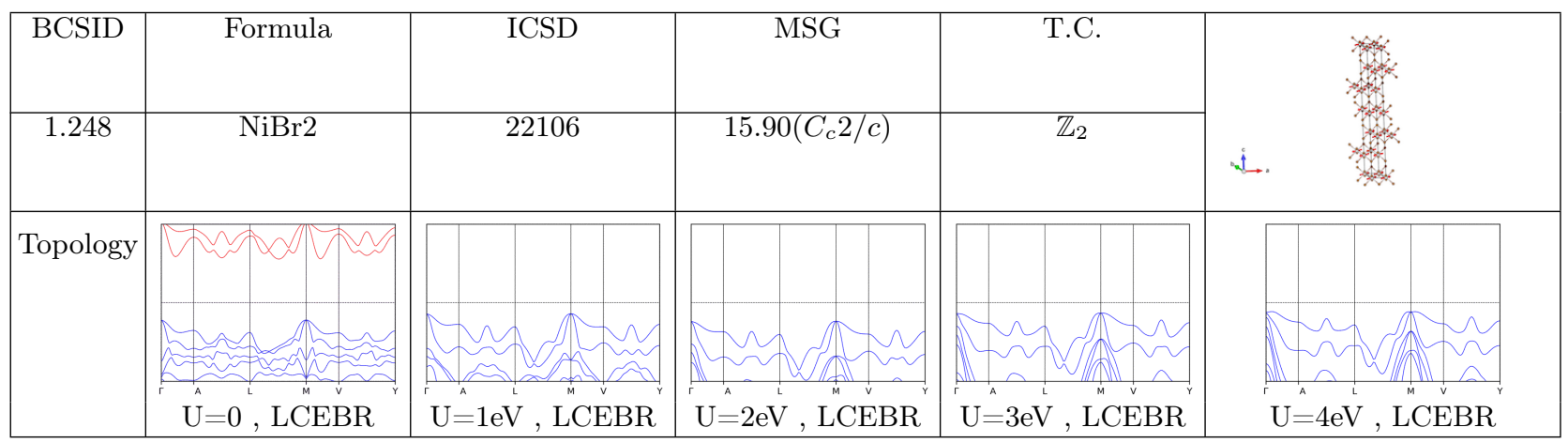

TABLE CXXXII. Topology phase diagram of NiBr2.

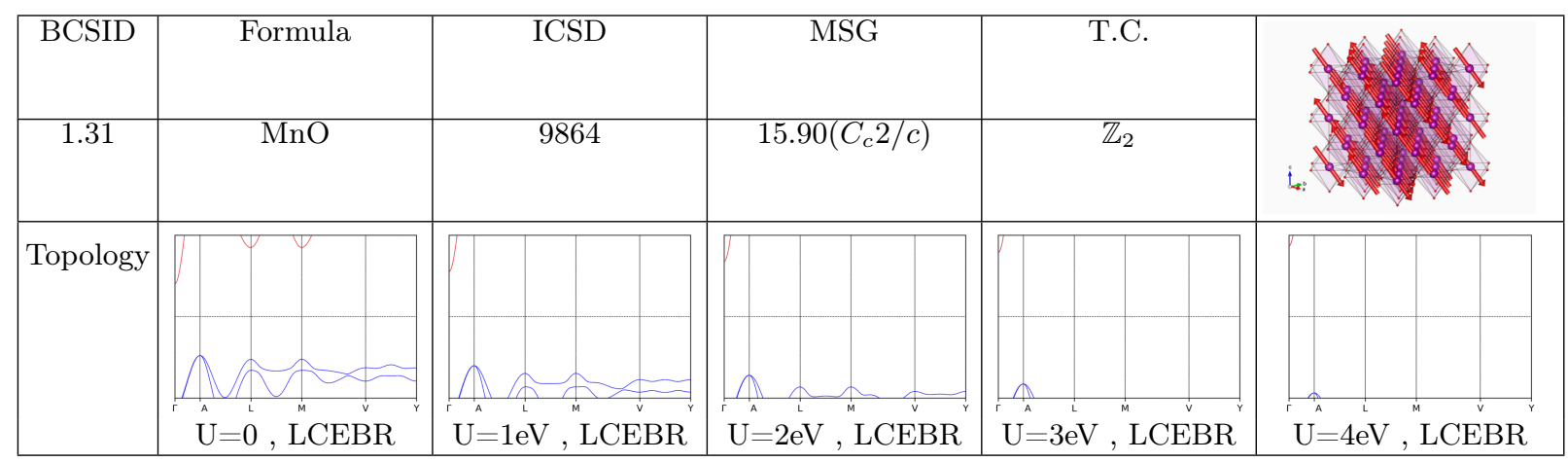

TABLE CXXXIII. Topology phase diagram of $\mathrm{MnO}$.

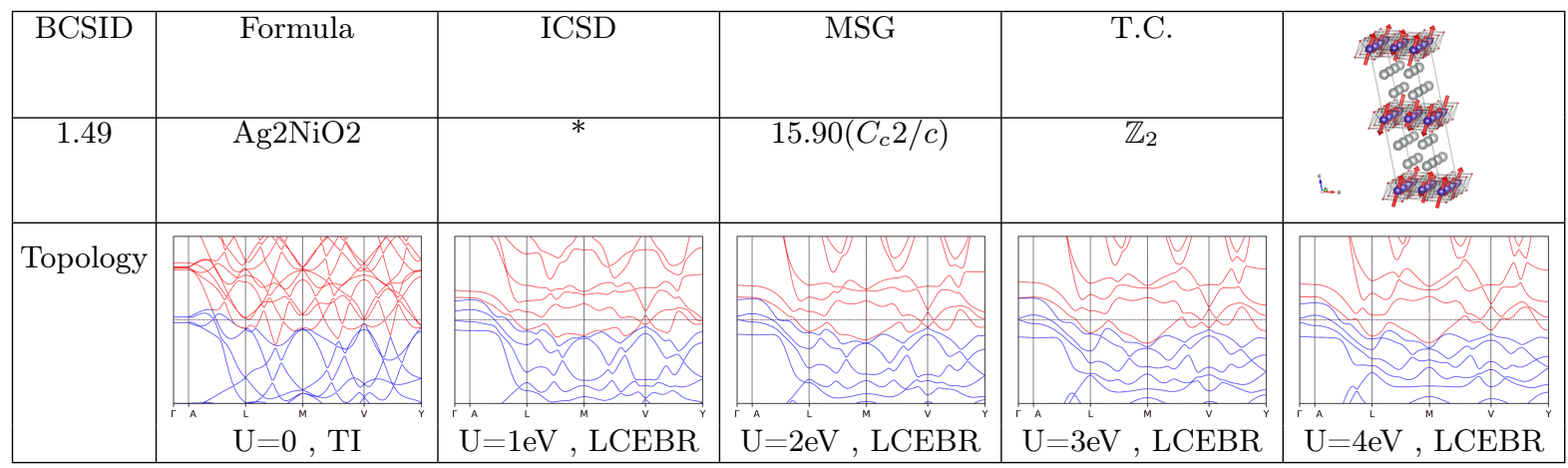

TABLE CXXXIV. Topology phase diagram of Ag2NiO2.

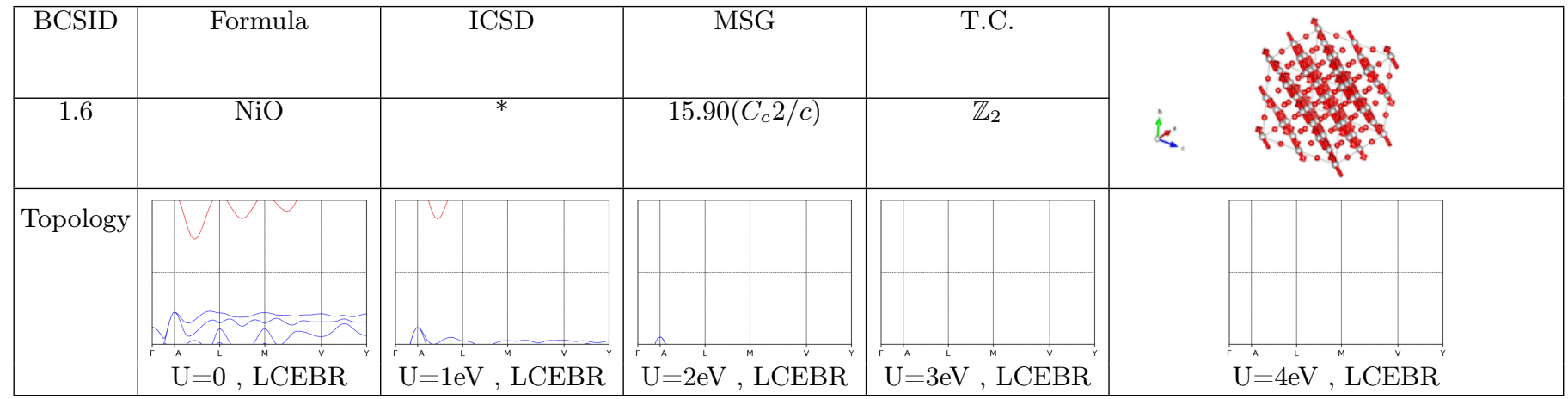

TABLE CXXXV. Topology phase diagram of NiO. 


\begin{tabular}{|c|c|c|c|c|c|}
\hline BCSID & Formula & ICSD & MSG & T.C. & \& \\
\hline 1.69 & $\mathrm{CoO}$ & * & $15.90\left(C_{c} 2 / c\right)$ & $\mathbb{Z}_{2}$ & \\
\hline \multirow[t]{2}{*}{ Topology } & V & & & & \\
\hline & $\hat{\mathrm{U}}^{\mathrm{A}}=\mathrm{C}^{\mathrm{L}}, \mathrm{LCEBR}^{\mathrm{M}}$ & $\mathrm{U}=1 \mathrm{eV}, \mathrm{LCEBR}$ & $\mathrm{U}^{\mathrm{A}}=2 \mathrm{eV}, \mathrm{LCEBR}$ & $\mathrm{U}^{\mathrm{A}}=3 \mathrm{eV},{ }^{\mathrm{M}} \mathrm{LCEBR}$ & $\mathrm{U}^{\mathrm{A}}=4 \mathrm{eV}, \mathrm{L}, \mathrm{LCEBR}$ \\
\hline
\end{tabular}

TABLE CXXXVI. Topology phase diagram of CoO.

\begin{tabular}{|c|c|c|c|c|c|}
\hline BCSID & Formula & $\overline{\mathrm{ICSD}}$ & $\overline{M S G}$ & T.C. & \\
\hline 1.70 & CoV2O6 & 263002 & $15.90\left(C_{c} 2 / c\right)$ & $\mathbb{Z}_{2}$ & \\
\hline \multicolumn{6}{|l|}{ Topology } \\
\hline & $\hat{\mathrm{U}}=0^{\mathrm{i}}, \mathrm{LCEBR}^{\mathrm{m}}$ & $\mathrm{U}^{\mathrm{A}}=1 \mathrm{eV}^{i}, \mathrm{LCEBR}$ & $\mathrm{U}^{\mathrm{A}}=2 \mathrm{eV}, \mathrm{LCEBR}$ & $\mathrm{U}^{\mathrm{A}}=3 \mathrm{eV}, \mathrm{LCEBR}$ & $\mathrm{U}^{\mathrm{A}}=4 \mathrm{eV}, \mathrm{LCEBR}$ \\
\hline
\end{tabular}

TABLE CXXXVII. Topology phase diagram of CoV2O6.

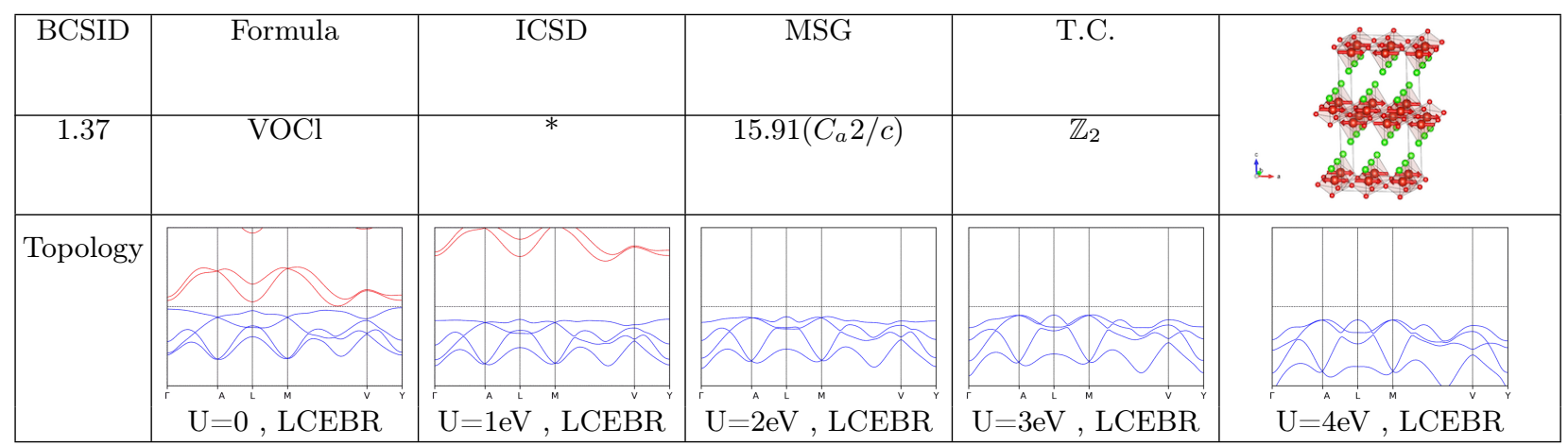

TABLE CXXXVIII. Topology phase diagram of VOCl.

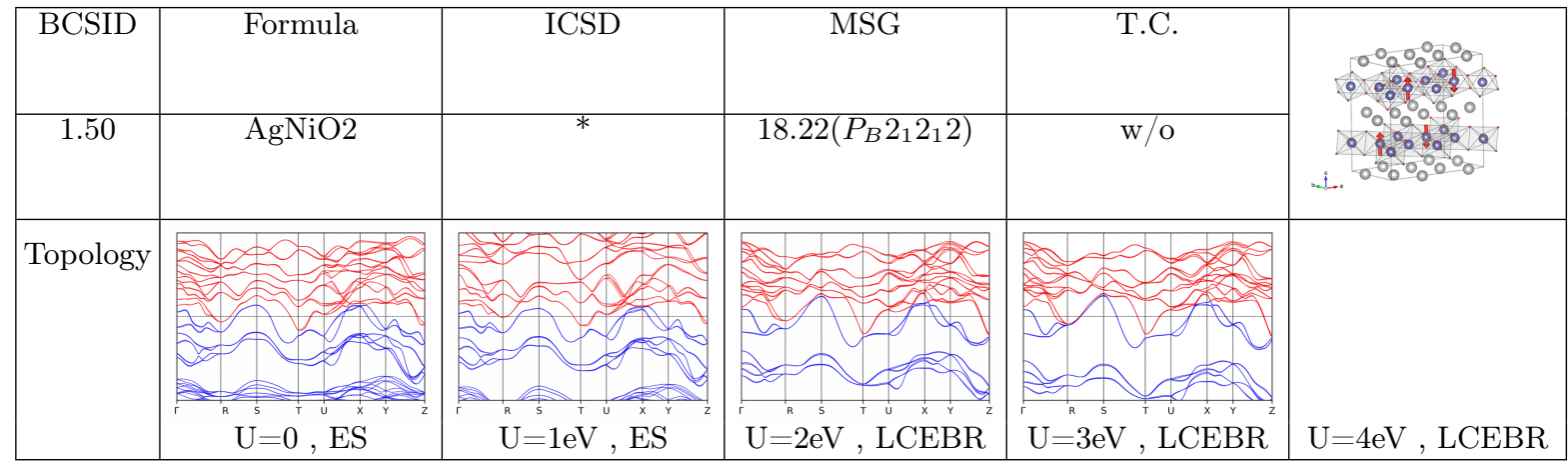

TABLE CXXXIX. Topology phase diagram of AgNiO2. 


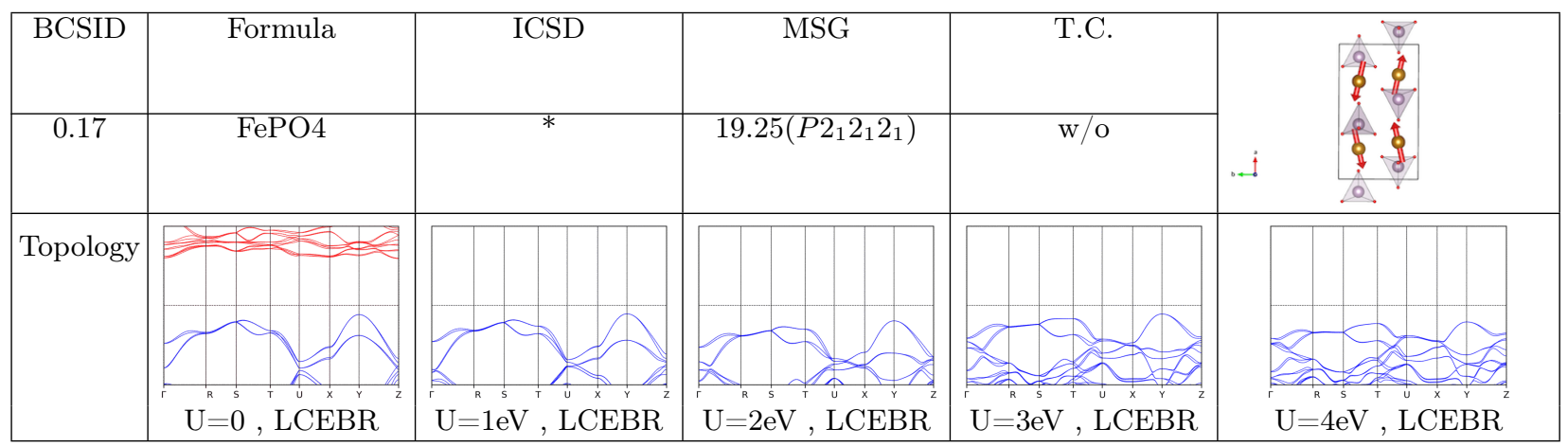

TABLE CXL. Topology phase diagram of FePO4.

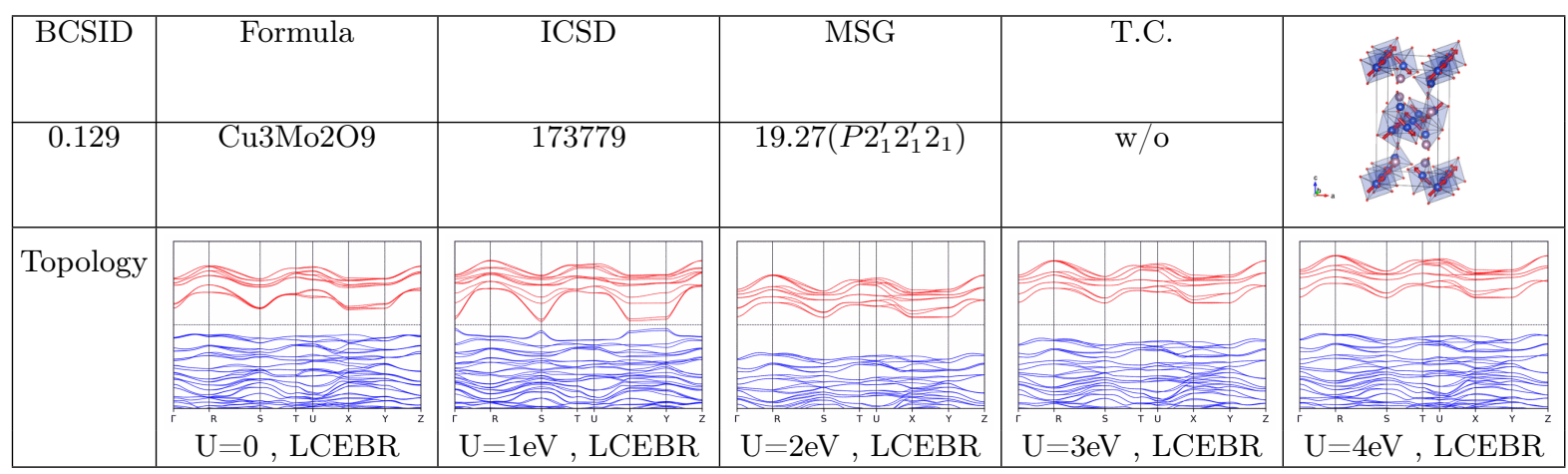

TABLE CXLI. Topology phase diagram of Cu3Mo2O9.

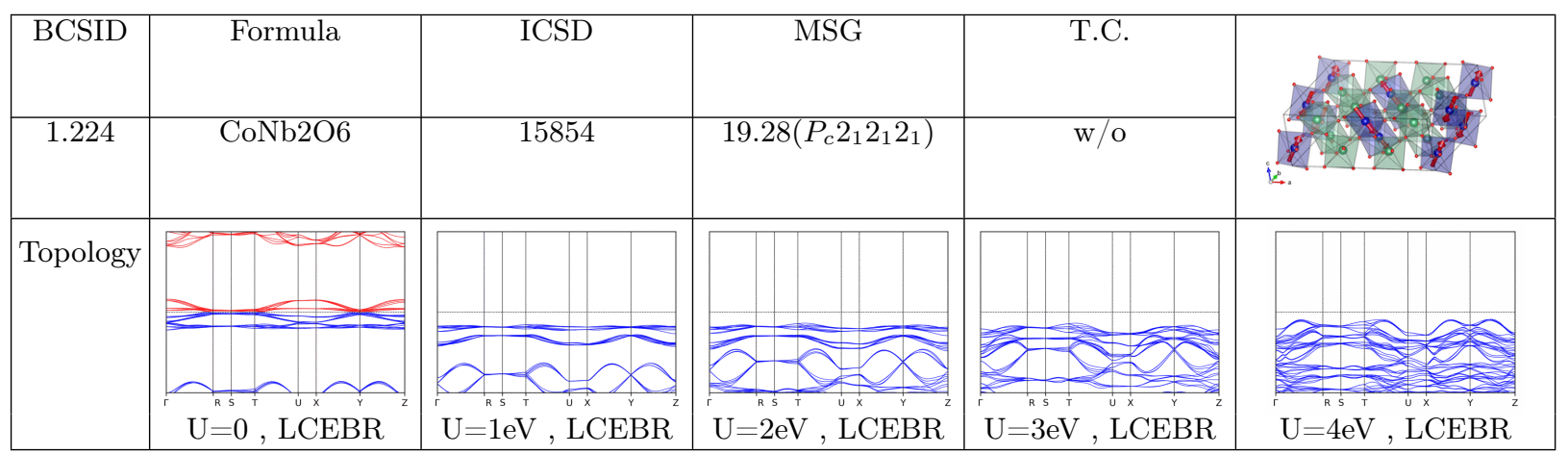

TABLE CXLII. Topology phase diagram of CoNb2O6.

\begin{tabular}{|c|c|c|c|c|c|}
\hline BCSID & Formula & ICSD & MSG & T.C. & \\
\hline 1.0 .4 & $\mathrm{CsNiCl3}$ & 59371 & $20.34\left(C 22^{\prime} 2_{1}^{\prime}\right)$ & $\mathrm{w} / \mathrm{o}$ & $\therefore \quad i \& \frac{9}{8} 8 \frac{9}{8}$ \\
\hline Topology & \begin{tabular}{l|l|l|} 
& & \\
\end{tabular} & & & & \\
\hline
\end{tabular}

TABLE CXLIII. Topology phase diagram of CsNiCl3. 


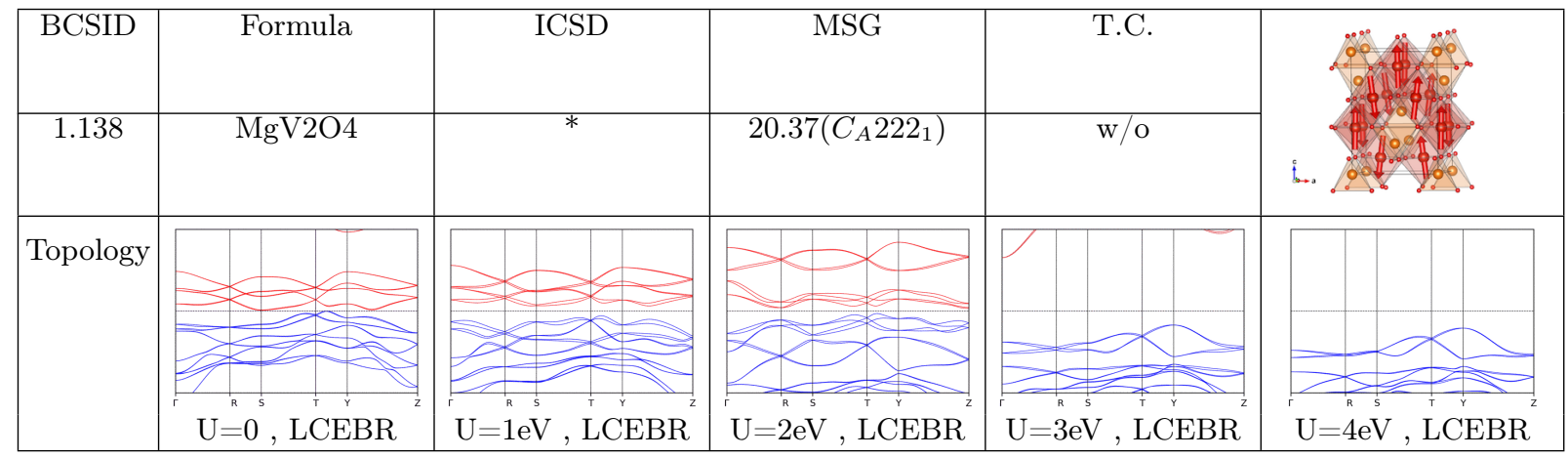

TABLE CXLIV. Topology phase diagram of MgV2O4.

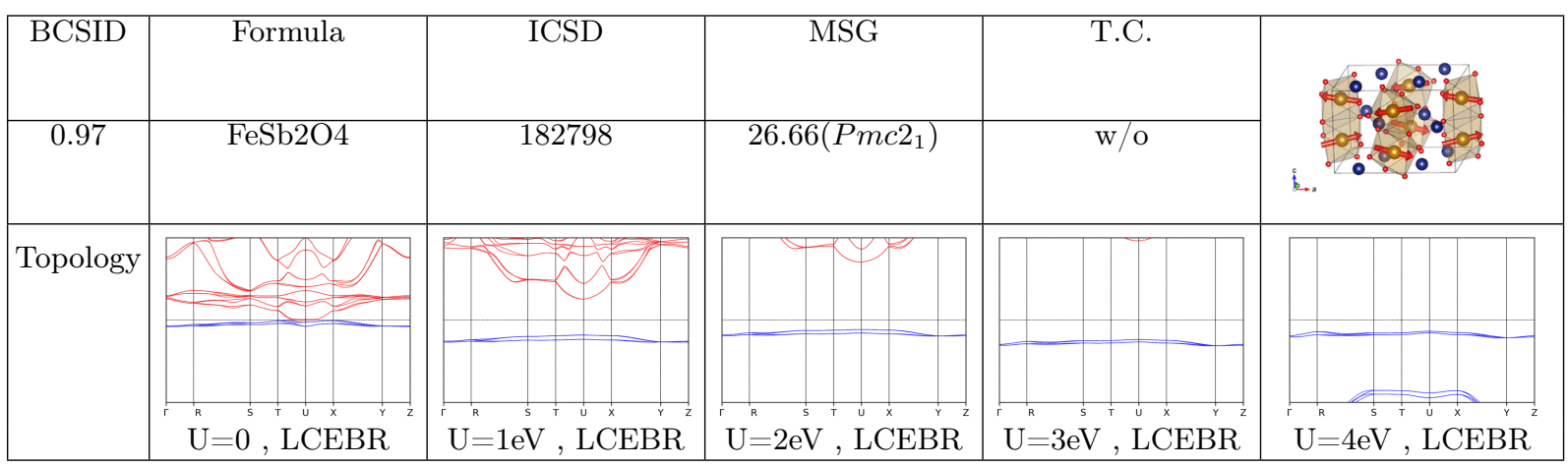

TABLE CXLV. Topology phase diagram of FeSb2O4.

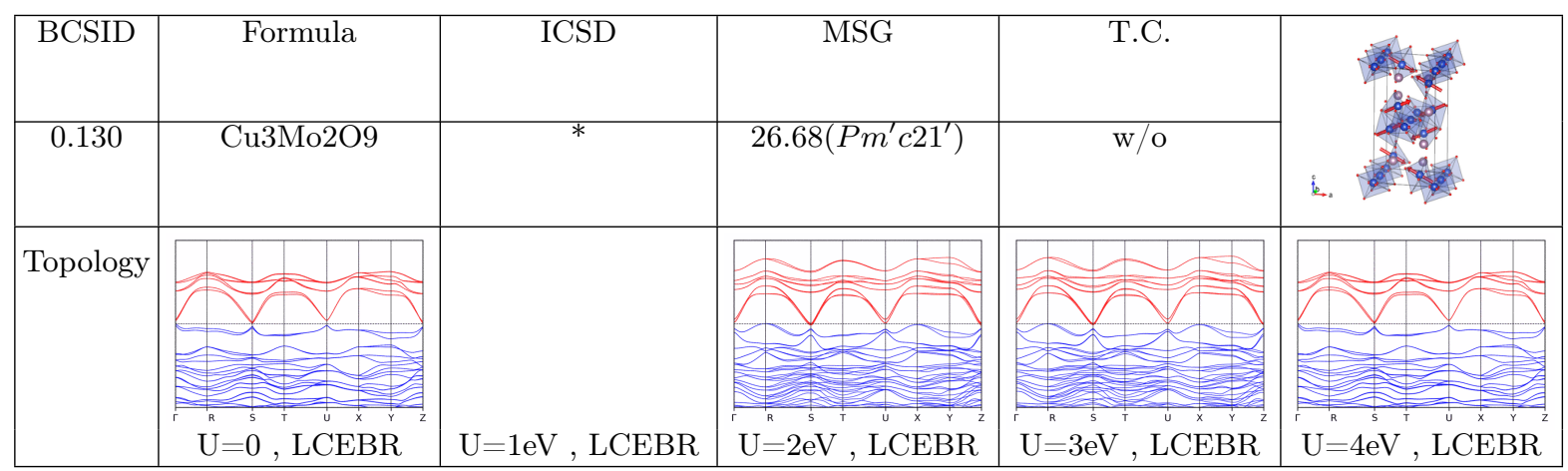

TABLE CXLVI. Topology phase diagram of Cu3Mo2O9.

\begin{tabular}{|c|c|c|c|c|c|}
\hline BCSID & Formula & ICSD & MSG & T.C. & \\
\hline 0.133 & Ni3B7O13Cl & * & $29.101\left(P c^{\prime} a 2_{1}^{\prime}\right)$ & $\mathrm{w} / \mathrm{o}$ & -10 \\
\hline \multicolumn{6}{|l|}{ Topology } \\
\hline & $\mathrm{U}=0, \mathrm{LCEBR}$ & $\mathrm{U}=1 \mathrm{eV}, \mathrm{LCEBR}$ & $\mathrm{U}=2 \mathrm{eV}, \mathrm{LCEBR}$ & $\mathrm{U}=3 \mathrm{eV}, \mathrm{LCEBR}$ & $\mathrm{U}=4 \mathrm{eV}, \mathrm{LCEBR}$ \\
\hline
\end{tabular}

TABLE CXLVII. Topology phase diagram of Ni3B7O13Cl. 


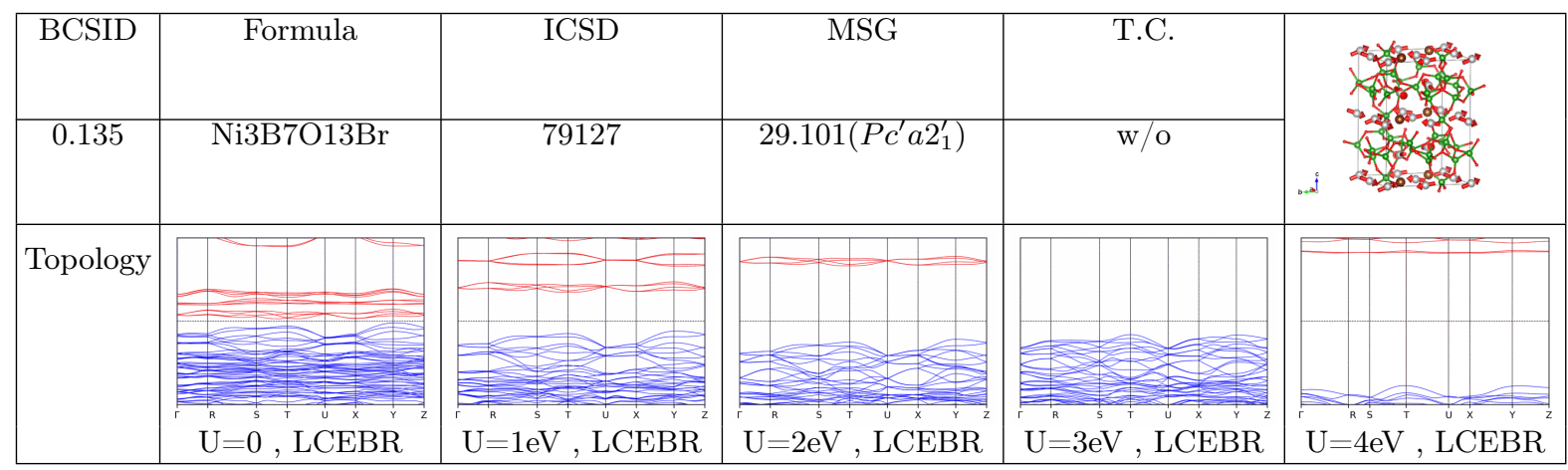

TABLE CXLVIII. Topology phase diagram of Ni3B7O13Br.

\begin{tabular}{|c|c|c|c|c|c|}
\hline BCSID & Formula & ICSD & $\overline{\mathrm{MSG}}$ & T.C. & tᄒ \\
\hline 1.18 & MnS2 & 36545 & $29.105\left(P_{b} c a 2_{1}\right)$ & $\mathrm{w} / \mathrm{o}$ & 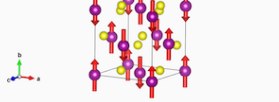 \\
\hline \multicolumn{6}{|l|}{ Topology } \\
\hline & $\mathrm{U}=0, \mathrm{LCEBR}$ & $\mathrm{U}=1 \mathrm{eV}, \mathrm{LCEBR}$ & $\mathrm{U}=2 \mathrm{eV}, \mathrm{LCEBR}$ & $\mathrm{U}=3 \mathrm{eV}, \mathrm{LCEBR}$ & $\mathrm{U}=4 \mathrm{eV}, \mathrm{LCEBR}$ \\
\hline
\end{tabular}

TABLE CXLIX. Topology phase diagram of MnS2.

\begin{tabular}{|c|c|c|c|c|c|}
\hline BCSID & Formula & ICSD & MSG & T.C. & \\
\hline 1.71 & SrCo2V2O8 & 400765 & $29.110\left(P_{I} c a 2_{1}\right)$ & $\mathrm{w} / \mathrm{o}$ & \& \& $89 ?$ \\
\hline \multirow[t]{3}{*}{ Topology } & \multirow[b]{3}{*}{$\mathrm{U}=0, \mathrm{LCEBR}$} & \multirow[b]{3}{*}{$\mathrm{U}=1 \mathrm{eV}, \mathrm{LCEBR}$} & & & \\
\hline & & & 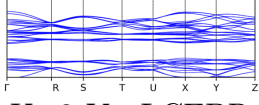 & $\sum_{h=4}$ & $\sum_{s_{i}}$ \\
\hline & & & $\mathrm{U}=2 \mathrm{eV}, \mathrm{LCEBR}$ & $\mathrm{U}=3 \mathrm{eV}, \mathrm{LCEBR}$ & $\mathrm{U}=4 \mathrm{eV}, \mathrm{LCEBR}$ \\
\hline
\end{tabular}

TABLE CL. Topology phase diagram of SrCo2V2O8.

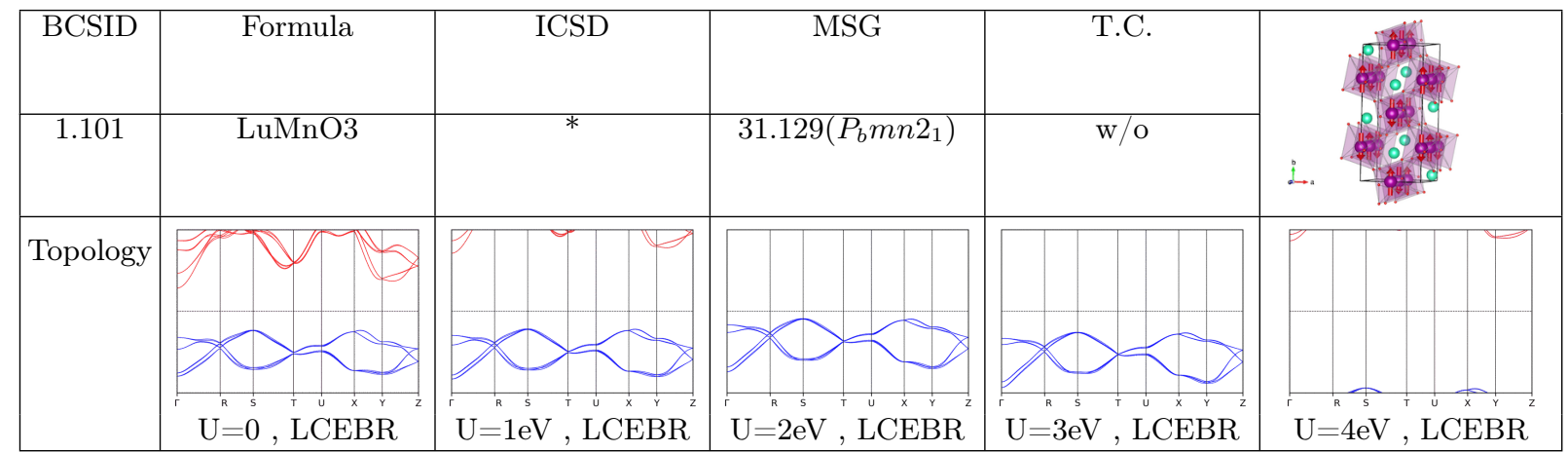

TABLE CLI. Topology phase diagram of LuMnO3. 


\begin{tabular}{|c|c|c|c|c|c|}
\hline BCSID & Formula & ICSD & MSG & T.C. & 0.0 \\
\hline 0.38 & GaFeO3 & 151722 & $33.147\left(P n a^{\prime} 2_{1}^{\prime}\right)$ & $\mathrm{w} / \mathrm{O}$ & \\
\hline Topology & IISt & & & & \\
\hline & 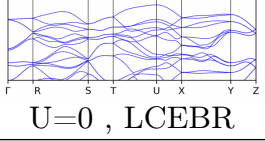 & $\begin{array}{l}\mathrm{s}_{\mathrm{r}}^{\mathrm{T}} \mathrm{U}^{\mathrm{x}} \\
\mathrm{U}=1 \mathrm{eV}, \mathrm{LCEBR}\end{array}$ & $\begin{array}{l}\mathrm{U}^{\mathrm{R}}=2 \mathrm{eV}, \mathrm{LCEBR}^{\mathrm{x}} \\
\mathrm{L}\end{array}$ & $\mathrm{U}_{\mathrm{U}^{\mathrm{R}} \mathrm{s}=\mathrm{eV}^{\mathrm{T}}, \mathrm{LCEBR}^{\mathrm{x}}}$ & $\underbrace{}_{\mathrm{r}_{\mathrm{s}}^{\mathrm{T}} \mathrm{U}_{\mathrm{U}}^{\mathrm{x}}=4 \mathrm{eV}, \mathrm{LCEBR}}$ \\
\hline
\end{tabular}

TABLE CLII. Topology phase diagram of GaFeO3.

\begin{tabular}{|c|c|c|c|c|c|}
\hline BCSID & Formula & ICSD & MSG & T.C. & \\
\hline 0.46 & $\mathrm{CaBaCo} 4 \mathrm{O} 7$ & * & $33.147\left(\right.$ Pna $\left.^{\prime} 2_{1}^{\prime}\right)$ & $\mathrm{w} / \mathrm{o}$ & 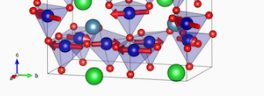 \\
\hline Topology & $\mathrm{U}=0, \mathrm{LCEBR}$ & 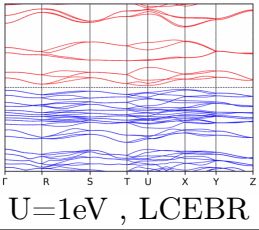 & $\mathrm{U}^{\mathrm{R}=2 \mathrm{eV}^{\mathrm{s}}, \mathrm{LCEBR}^{\mathrm{T}} \mathrm{LCH}^{\mathrm{r}}}$ & 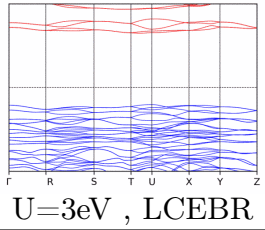 & 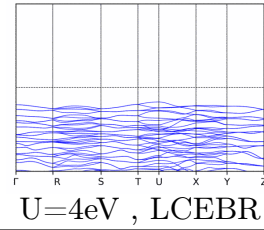 \\
\hline
\end{tabular}

TABLE CLIII. Topology phase diagram of CaBaCo4O7.

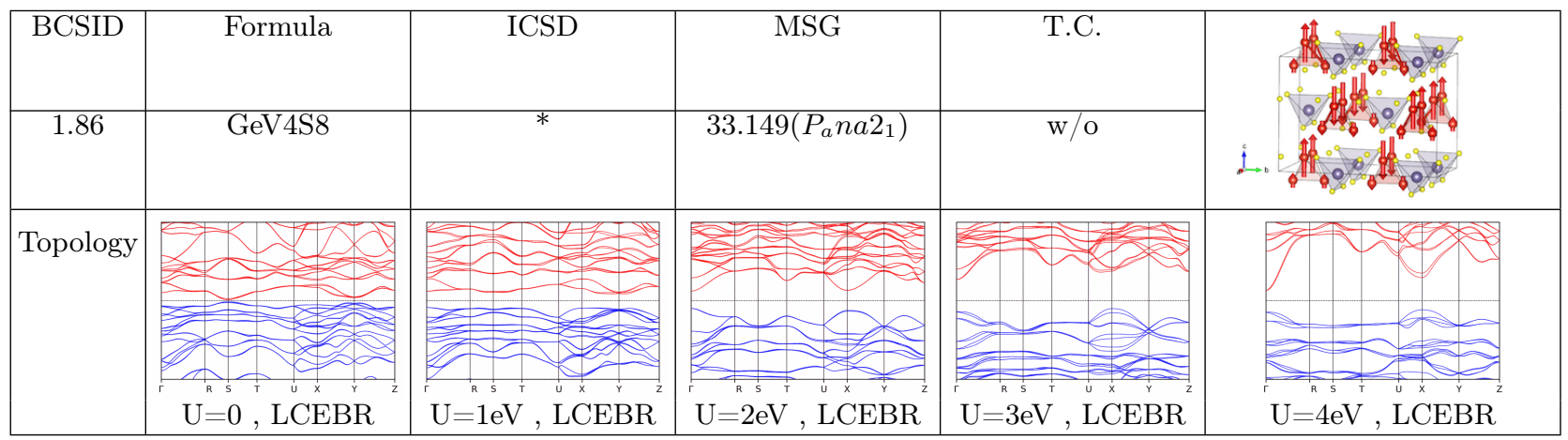

TABLE CLIV. Topology phase diagram of GeV4S8.

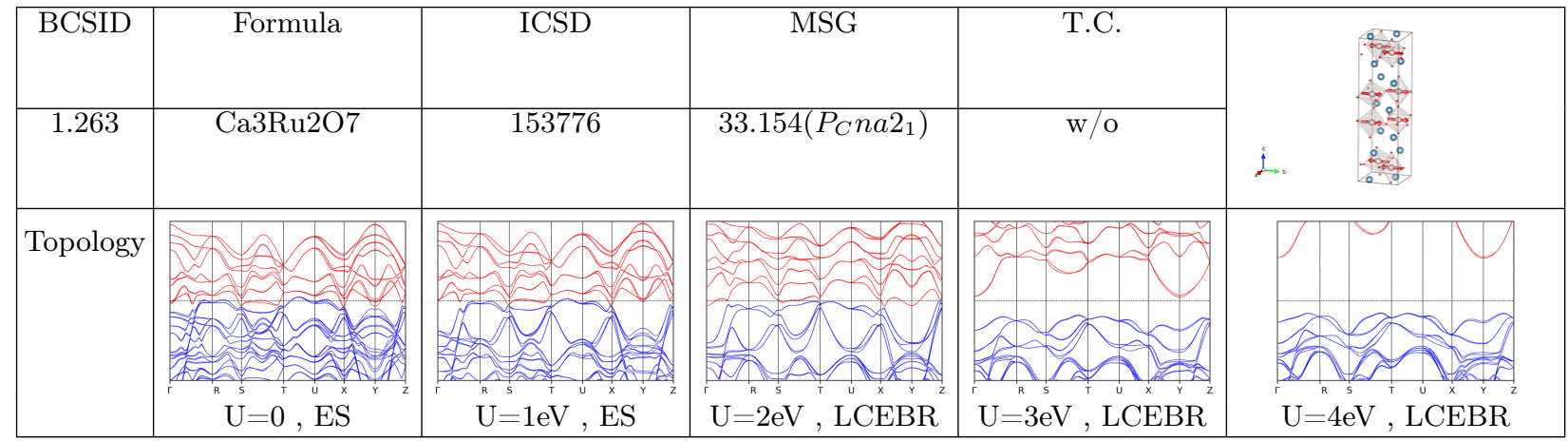

TABLE CLV. Topology phase diagram of Ca3Ru2O7. 


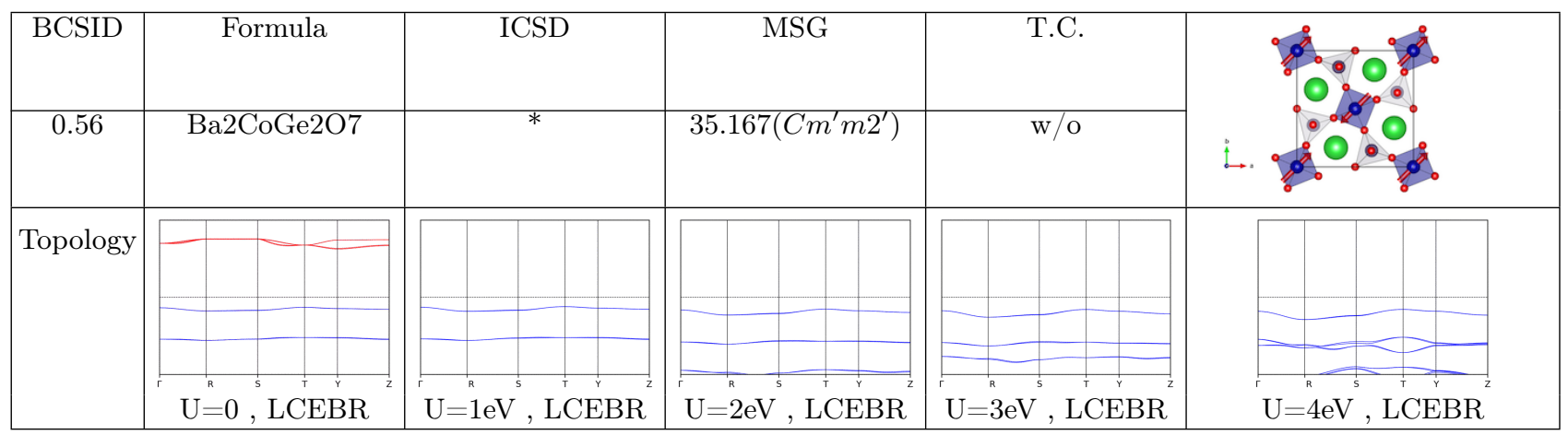

TABLE CLVI. Topology phase diagram of Ba2CoGe2O7.

\begin{tabular}{|c|c|c|c|c|c|}
\hline BCSID & Formula & ICSD & MSG & T.C. & 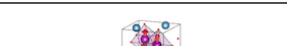 \\
\hline 0.23 & Ca3Mn2O7 & 55666 & $36.174\left(C m^{\prime} c 2_{1}^{\prime}\right)$ & $\mathrm{w} / \mathrm{O}$ & 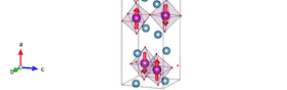 \\
\hline Topology & & $\forall$ & & & \\
\hline & $\mathrm{U}=0,{ }^{\mathrm{s}}, \mathrm{LCEBR}$ & $\mathrm{U}^{\mathrm{R}}=1 \mathrm{eV}, \mathrm{LCEBR}$ & $\begin{array}{l}\mathrm{S}_{\mathrm{T}}{ }^{\mathrm{r}}=2 \mathrm{eV}, \mathrm{LCEBR} \\
\mathrm{r}\end{array}$ & ${ }_{\mathrm{U}^{\mathrm{R}} \mathrm{S}_{\mathrm{T}}^{\mathrm{T}} \mathrm{eV}, \mathrm{LCEBR}}$ & $\underbrace{\mathrm{T}}_{\mathrm{U}^{\mathrm{R}}=4 \mathrm{eV}, \mathrm{LCEBR}}$ \\
\hline
\end{tabular}

TABLE CLVII. Topology phase diagram of Ca3Mn2O7.

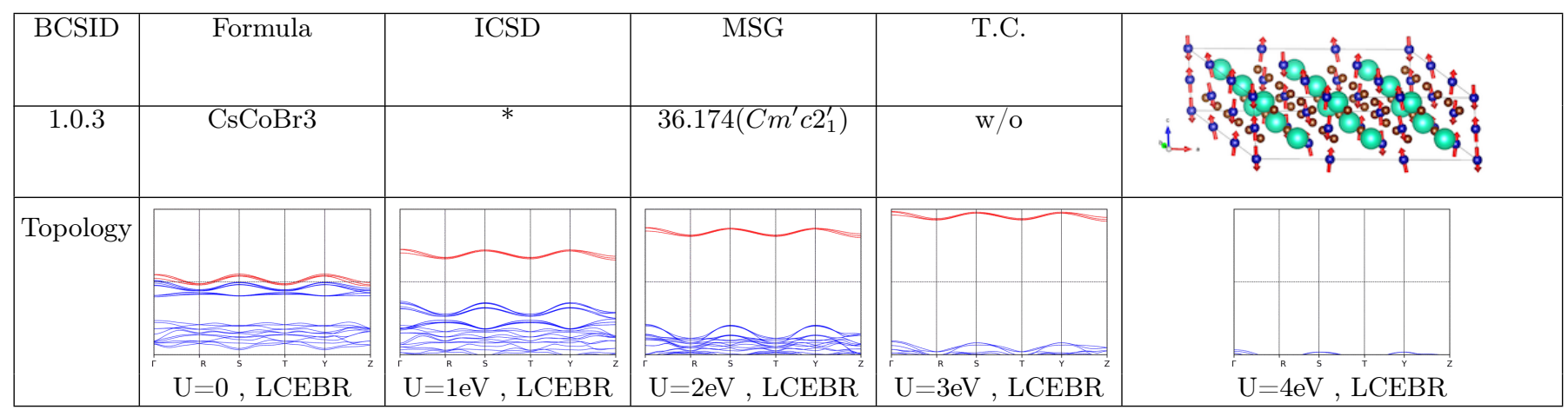

TABLE CLVIII. Topology phase diagram of CsCoBr3.

\begin{tabular}{|c|c|c|c|c|c|}
\hline BCSID & Formula & ICSD & MSG & T.C. & \\
\hline 0.191 & $\mathrm{BaCuF} 4$ & 9930 & $36.176\left(C m^{\prime} c^{\prime} 2_{1}\right)$ & $\mathrm{w} / \mathrm{O}$ & $\begin{array}{l}\text { ho } \\
i \$ 0^{\circ}\end{array}$ \\
\hline Topology & & & & & \\
\hline & $\mathrm{U}=0, \mathrm{LCEBR}$ & $\mathrm{U}=1 \mathrm{eV}, \mathrm{LCEBR}$ & $\mathrm{U}=2 \mathrm{eV}, \mathrm{LCEBR}$ & $\mathrm{U}=3 \mathrm{eV}, \mathrm{LCEBR}$ & $\mathrm{U}=4 \mathrm{eV}, \mathrm{LCEBR}$ \\
\hline
\end{tabular}

TABLE CLIX. Topology phase diagram of BaCuF4. 


\begin{tabular}{|c|c|c|c|c|c|}
\hline BCSID & Formula & ICSD & MSG & T.C. & \\
\hline 1.74 & BiMn2O5 & & $36.178\left(C_{a} m c 2_{1}\right)$ & $\mathrm{w} / \mathrm{o}$ & \\
\hline \multirow[t]{3}{*}{ Topology } & & & & & \\
\hline & 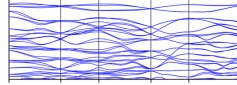 & 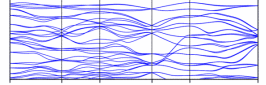 & r & $\ldots$. & 2 \\
\hline & $\mathrm{U}^{\mathrm{R}}=0^{\mathrm{s}}, \mathrm{LCEBR}$ & $\mathrm{U}=1 \mathrm{eV}^{\mathrm{s}}, \mathrm{LCEBR}^{\mathrm{z}}$ & $\mathrm{U}=2 \mathrm{eV}^{\mathrm{s}}, \mathrm{LCEBR}$ & $\mathrm{U}=3 \mathrm{eV}^{\mathrm{s}}, \mathrm{LCEBR}^{\mathrm{z}}$ & $\mathrm{U}=4 \mathrm{eV}^{\mathrm{s}}, \mathrm{LCEBR}$ \\
\hline
\end{tabular}

TABLE CLX. Topology phase diagram of BiMn2O5.

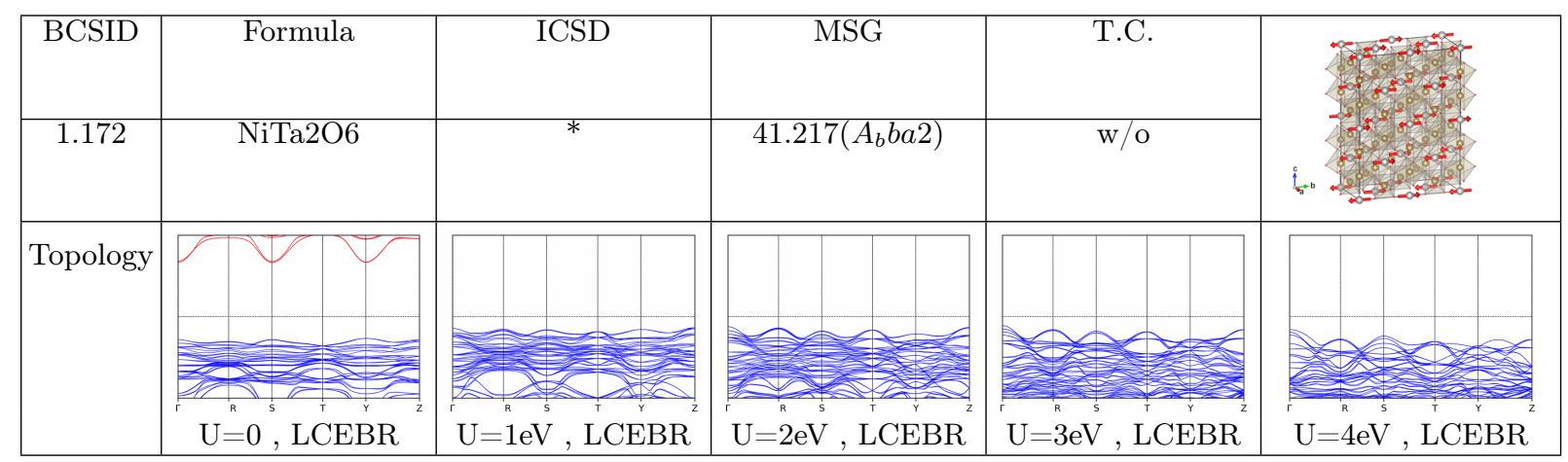

TABLE CLXI. Topology phase diagram of NiTa2O6.

\begin{tabular}{|c|c|c|c|c|c|}
\hline BCSID & Formula & ICSD & MSG & T.C. & \\
\hline 0.137 & Cu2V2O7 & $*$ & $43.227\left(F d^{\prime} d^{\prime} 2\right)$ & w/o & \\
\cline { 1 - 5 } Topology & & & & & \\
& & & & & \\
& & & & & \\
& & & & & \\
$\mathrm{U}=0, \mathrm{LCEBR}$ & $\mathrm{U}=1 \mathrm{eV}, \mathrm{LCEBR}$ & $\mathrm{U}=2 \mathrm{eV}, \mathrm{LCEBR}$ & $\mathrm{U}=3 \mathrm{eV}, \mathrm{LCEBR}$ & $\mathrm{U}=4 \mathrm{eV}, \mathrm{LCEBR}$ \\
\hline
\end{tabular}

TABLE CLXII. Topology phase diagram of Cu2V2O7.

\begin{tabular}{|c|c|c|c|c|c|}
\hline BCSID & Formula & ICSD & MSG & T.C. & \\
\hline 0.62 & SrMn2V2O8 & 182224 & $45.237\left(I b^{\prime} a 2^{\prime}\right)$ & $\mathrm{w} / \mathrm{o}$ & $I_{1.0}-0.07000$ \\
\hline \multirow[t]{3}{*}{ Topology } & & & & & \\
\hline & $\Leftrightarrow \ll$ & $\sum x$ & $\infty$ & 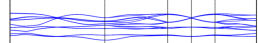 & $\ll<$ \\
\hline & $\mathrm{U}=0^{k}, \mathrm{LCEBR}$ & $\mathrm{U}=1 \mathrm{eV}^{\mathrm{R}}, \mathrm{LCEBR}$ & $\mathrm{U}=2 \mathrm{eV}^{\mathrm{n}}, \mathrm{LCEBH}$ & $\mathrm{U}=3 \mathrm{eV}, \mathrm{LCEBR}$ & $\mathrm{U}=4 \mathrm{eV}^{\circ}, \mathrm{LCEBR}$ \\
\hline
\end{tabular}

TABLE CLXIII. Topology phase diagram of SrMn2V2O8. 


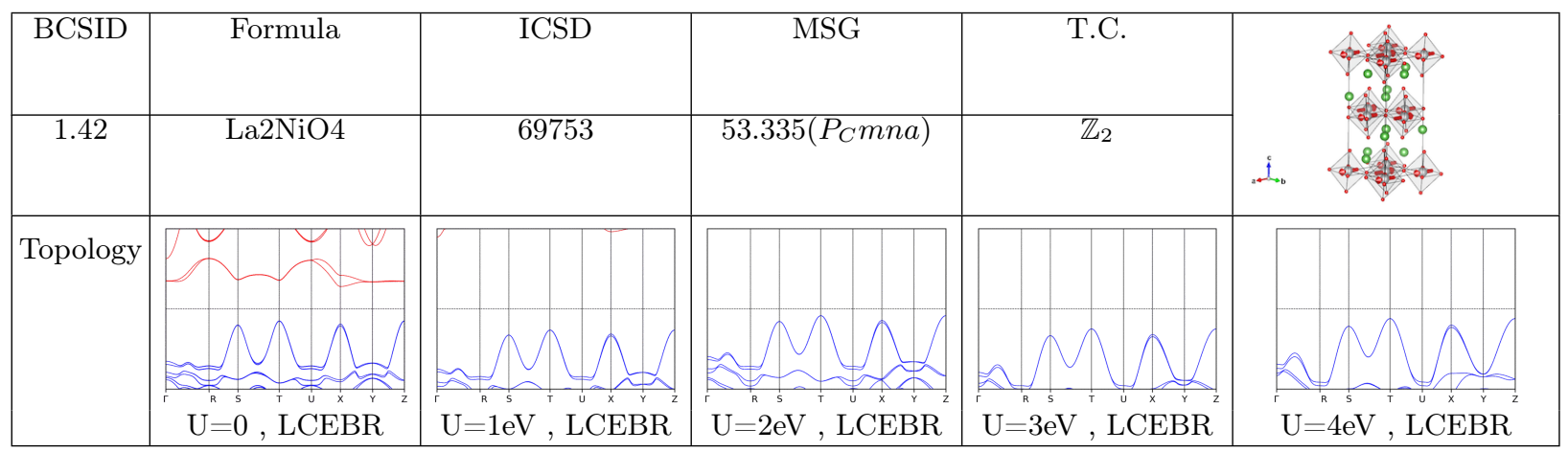

TABLE CLXIV. Topology phase diagram of La2NiO4.

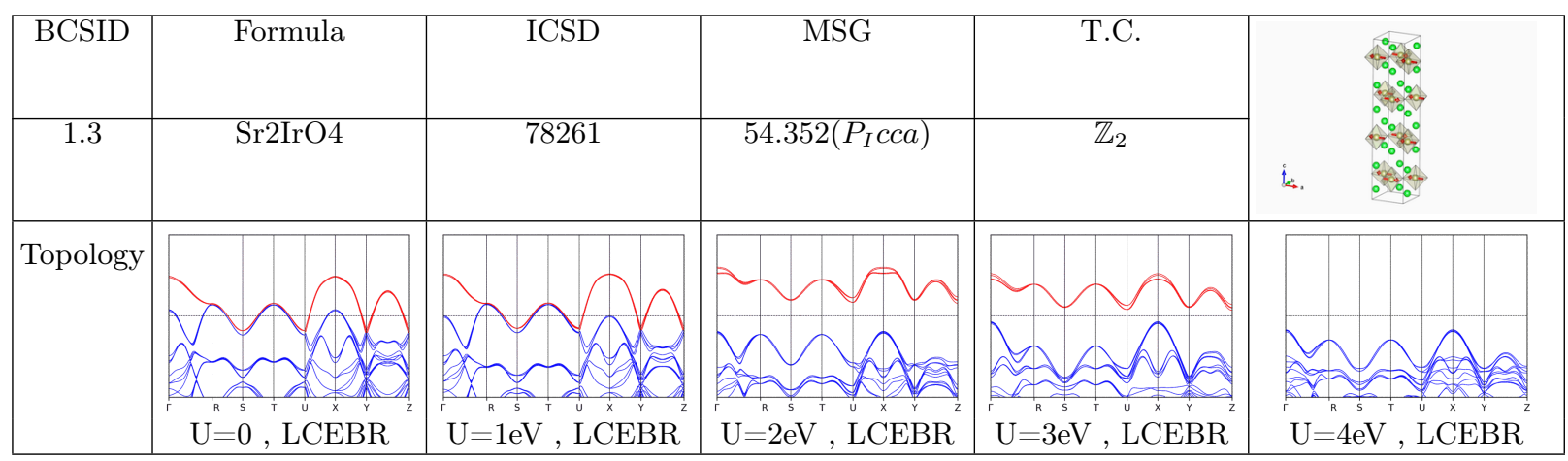

TABLE CLXV. Topology phase diagram of Sr2IrO4.

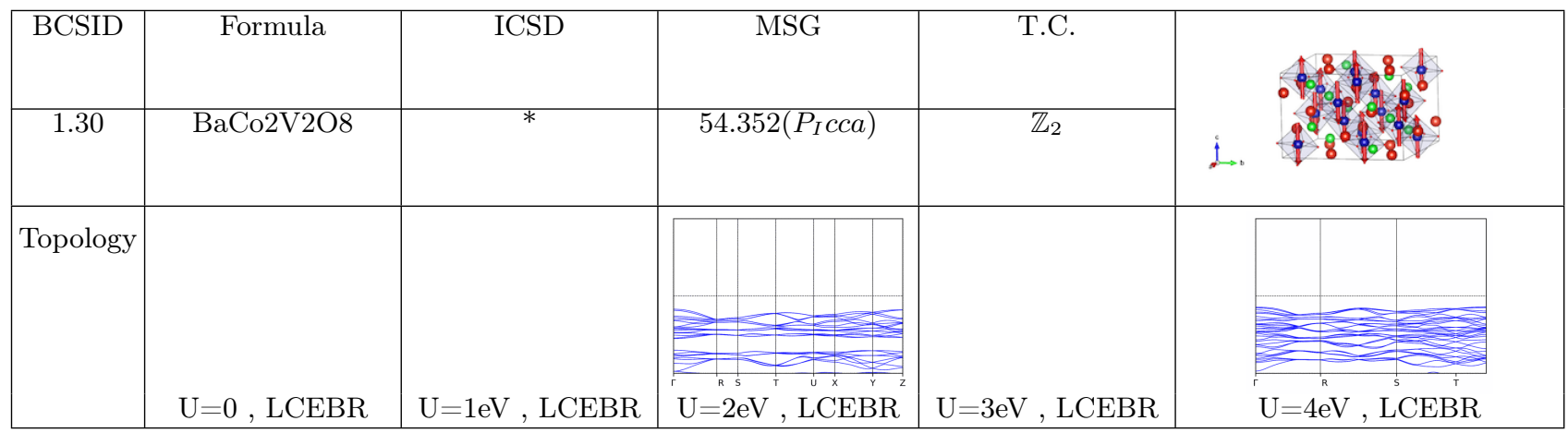

TABLE CLXVI. Topology phase diagram of BaCo2V2O8.

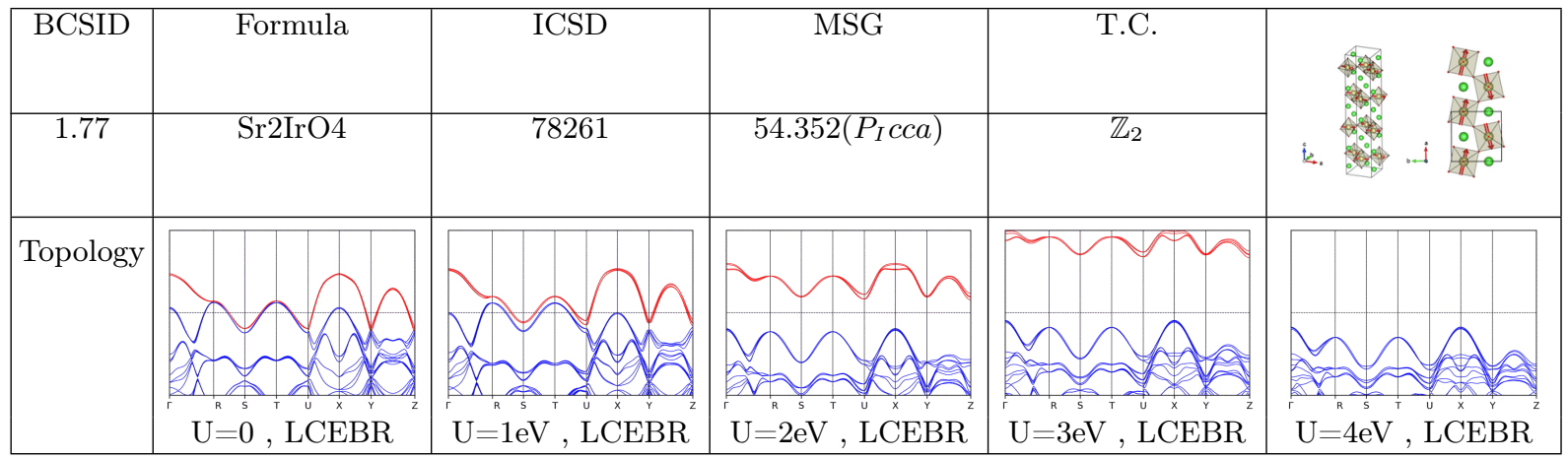

TABLE CLXVII. Topology phase diagram of Sr2IrO4. 


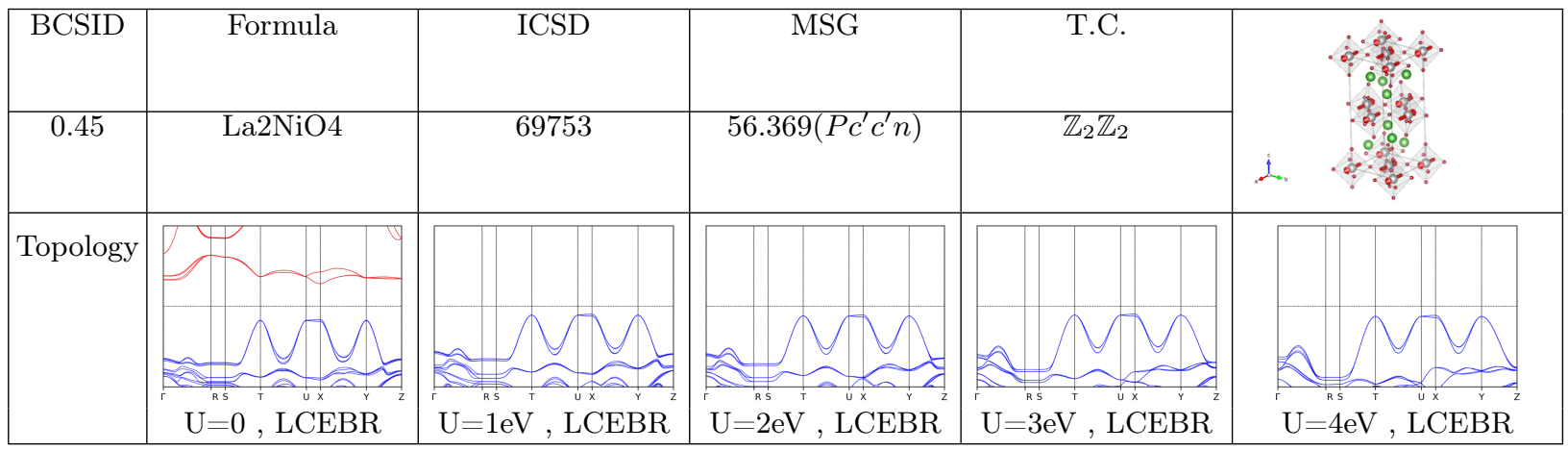

TABLE CLXVIII. Topology phase diagram of La2NiO4.

\begin{tabular}{|c|c|c|c|c|c|}
\hline BCSID & Formula & ICSD & MSG & T.C. & \\
\hline 1.122 & $\mathrm{Cu} 3 \mathrm{Bi}(\mathrm{SeO} 3) 2 \mathrm{O} 2 \mathrm{Br}$ & 280759 & $56.373\left(P_{c} c c n\right)$ & $\mathbb{Z}_{2}$ & 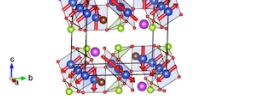 \\
\hline Topology & & & & & \\
\hline & 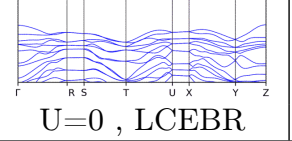 & $\begin{array}{l}\mathrm{R}_{\mathrm{rs}}^{\mathrm{T}} \\
\mathrm{U}=1 \mathrm{eV}, \mathrm{LCEBR}\end{array}$ & 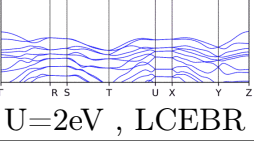 & 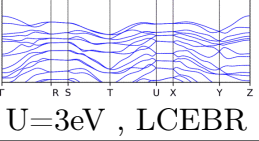 & 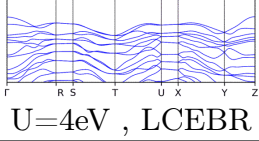 \\
\hline
\end{tabular}

TABLE CLXIX. Topology phase diagram of Cu3Bi(SeO3)2O2Br.

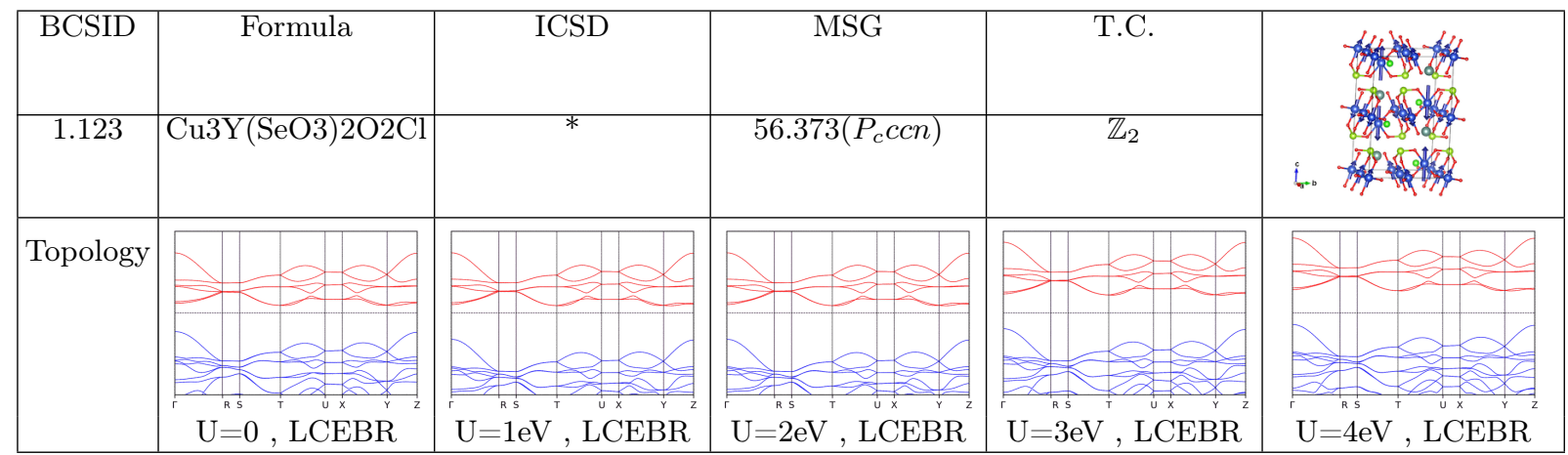

TABLE CLXX. Topology phase diagram of Cu3Y(SeO3)2O2Cl.

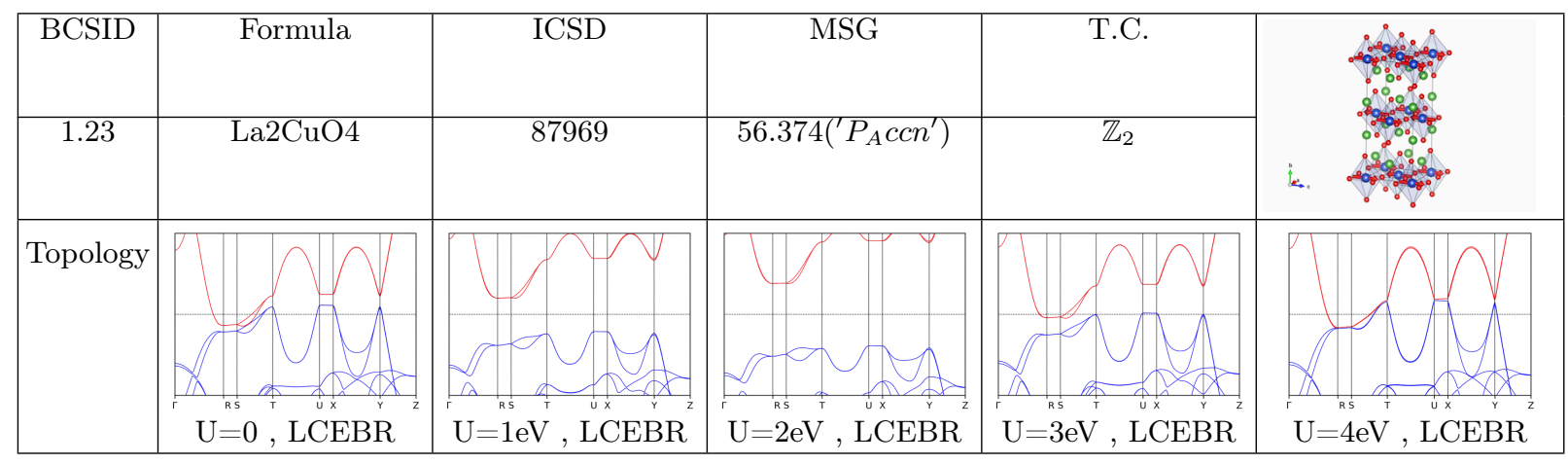

TABLE CLXXI. Topology phase diagram of La2CuO4. 


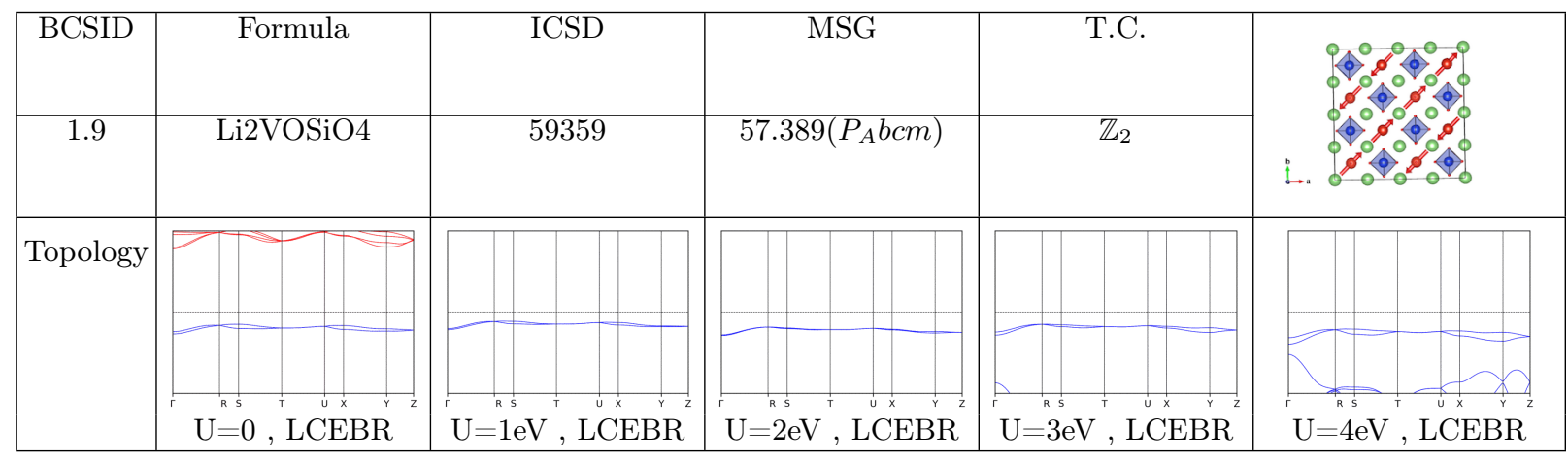

TABLE CLXXII. Topology phase diagram of Li2VOSiO4.

\begin{tabular}{|c|c|c|c|c|c|}
\hline BCSID & Formula & ICSD & MSG & T.C. & \\
\hline 0.143 & Cr2TeO6 & 24794 & $58.395\left(P n^{\prime} n m\right)$ & $\mathrm{w} / \mathrm{o}$ & \& $\quad \frac{0.000}{0}$ \\
\hline Topology & $\nabla$ & & & & \\
\hline & $\mathrm{U}^{\mathrm{R}}=0^{\mathrm{s}}, \mathrm{L}^{\mathrm{T}} \mathrm{LEBR}^{\mathrm{v}}$ & $\mathrm{U}^{\mathrm{R}}=1 \mathrm{eV}^{\mathrm{s}}, \mathrm{LCEBR}^{\mathrm{v}}$ & $\mathrm{U}^{\mathrm{R}}=2 \mathrm{eV}^{\mathrm{s}}, \mathrm{LCEBR}^{\mathrm{x}}$ & $\mathrm{U}^{\mathrm{R}}=3 \mathrm{eV}^{\mathrm{s}}, \mathrm{LCEBR}^{\mathrm{z}}$ & $\mathrm{U}^{\mathrm{R}}=4 \mathrm{eV}^{\mathrm{s}}, \mathrm{LCEBR}^{\mathrm{z}}$ \\
\hline
\end{tabular}

TABLE CLXXIII. Topology phase diagram of Cr2TeO6.

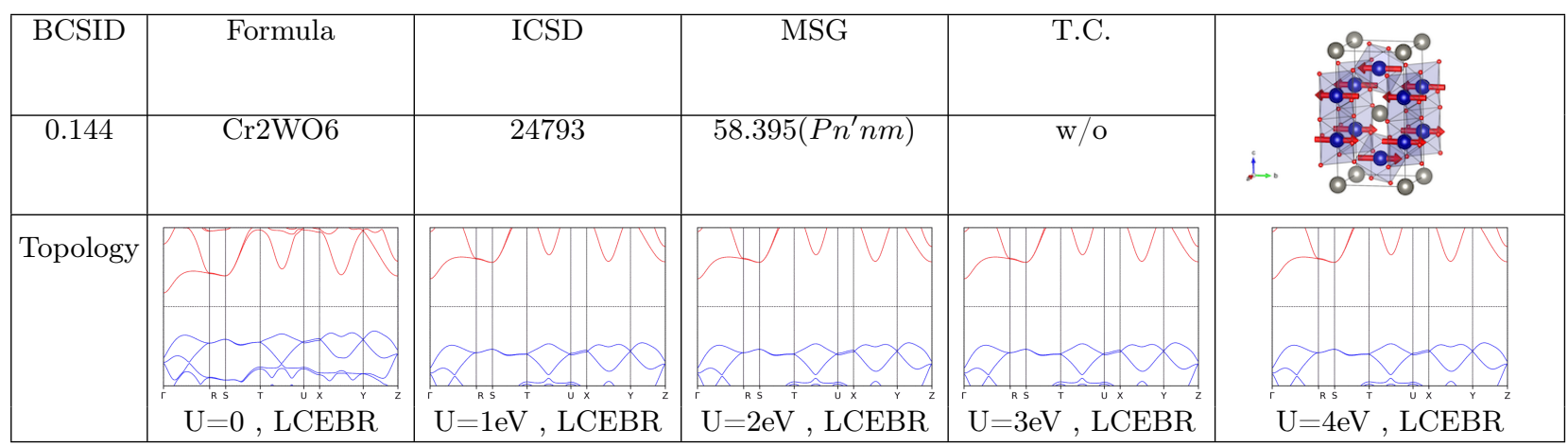

TABLE CLXXIV. Topology phase diagram of Cr2WO6.

\begin{tabular}{|c|c|c|c|c|c|}
\hline BCSID & Formula & ICSD & MSG & T.C. & \\
\hline 0.75 & Cr2WO6 & 24793 & $58.395\left(P n^{\prime} n m\right)$ & $\mathrm{w} / \mathrm{o}$ & \\
\hline Topology & & $\nabla$ & $W$ & V & V \\
\hline & $\mathrm{U}^{\mathrm{n}=} 0, \mathrm{LCEBH}$ & $\mathrm{U}=1 \mathrm{eV}, \mathrm{LCEBR}$ & $\mathrm{U}=2 \mathrm{eV}^{\mathrm{T}}, \mathrm{LCEBR}$ & $\mathrm{U}=3 \mathrm{eV}^{\mathrm{R} .5}, \mathrm{LCEBR}^{2}$ & $\mathrm{U}=4 \mathrm{eV}^{\top}, \mathrm{LCEBR}$ \\
\hline
\end{tabular}

TABLE CLXXV. Topology phase diagram of Cr2WO6. 


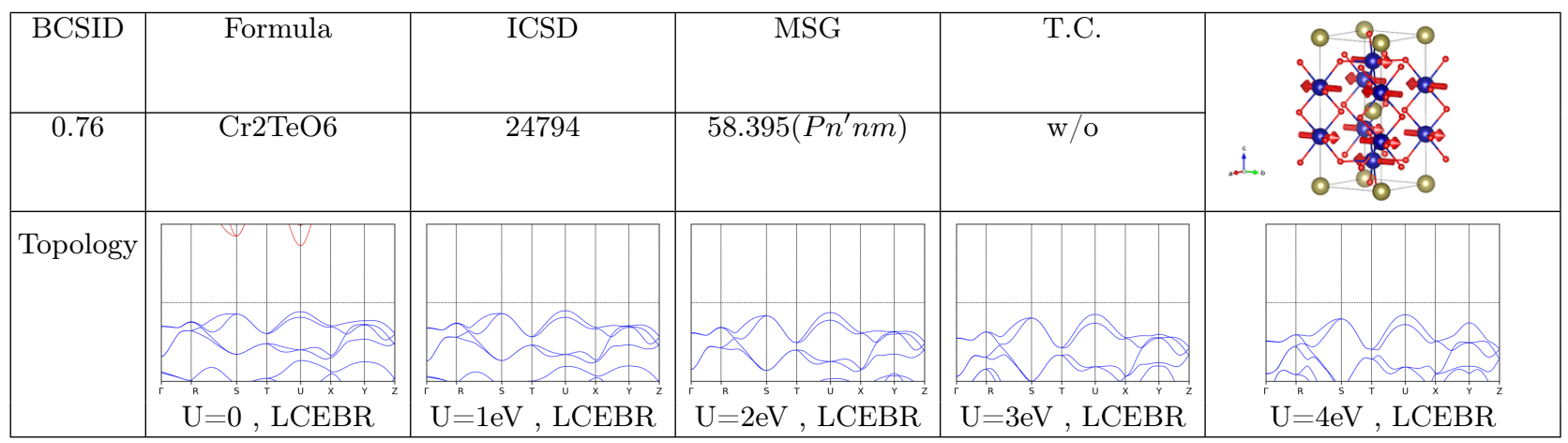

TABLE CLXXVI. Topology phase diagram of $\mathrm{Cr} 2 \mathrm{TeO} 6$.

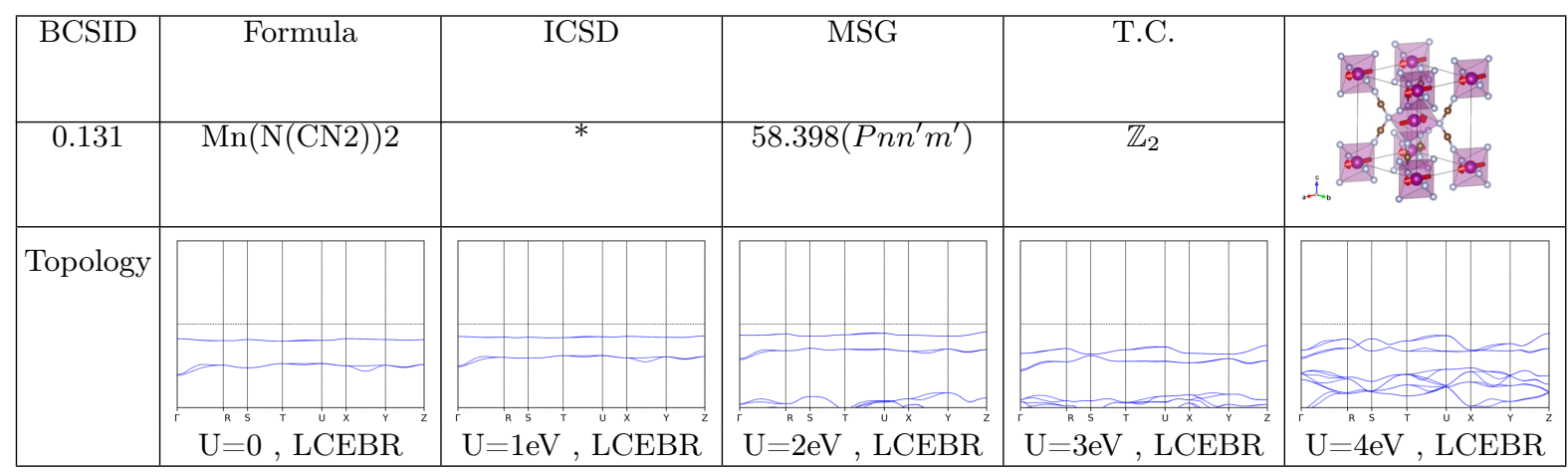

TABLE CLXXVII. Topology phase diagram of $\mathrm{Mn}(\mathrm{N}(\mathrm{CN} 2)) 2$.

\begin{tabular}{|c|c|c|c|c|c|}
\hline BCSID & Formula & $\overline{\mathrm{ICSD}}$ & $\overline{\mathrm{MSG}}$ & T.C. & $000^{\circ}$ \\
\hline 0.36 & $\mathrm{NiF} 2$ & * & $58.398\left(P n n^{\prime} m^{\prime}\right)$ & $\mathbb{Z}_{2}$ & \\
\hline Topology & $\nabla$ & & & & \\
\hline & $\mathrm{U}=0, \mathrm{LCEBR}$ & $\mathrm{U}=1 \mathrm{eV}, \mathrm{LCEBR}$ & $\mathrm{U}=2 \mathrm{eV}, \mathrm{LCEBR}$ & $\mathrm{U}=3 \mathrm{eV}, \mathrm{LCEBR}$ & $\mathrm{U}=4 \mathrm{eV}, \mathrm{LCEBR}$ \\
\hline
\end{tabular}

TABLE CLXXVIII. Topology phase diagram of NiF2.

\begin{tabular}{|c|c|c|c|c|c|}
\hline BCSID & Formula & ICSD & MSG & T.C. & \\
\hline 0.85 & $\overline{\mathrm{KCo}} 4(\mathrm{PO} 4) 3$ & & $58.398\left(P n n^{\prime} m^{\prime}\right)$ & $\mathbb{Z}_{2}$ & 2k \\
\hline Topology & 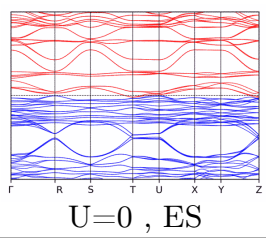 & 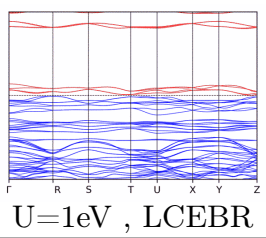 & 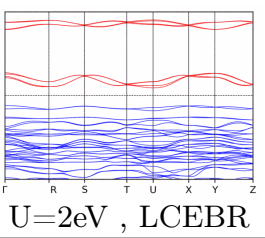 & $\mathrm{U}_{\mathrm{U}=3 \mathrm{eV}, \mathrm{LCEBR}}^{\mathrm{n}}$ & ${\mathrm{U}=4 \mathrm{eV}, \mathrm{LCEBR}^{\mathrm{r}}}^{\mathrm{i}}$ \\
\hline
\end{tabular}

TABLE CLXXIX. Topology phase diagram of KCo4(PO4)3. 


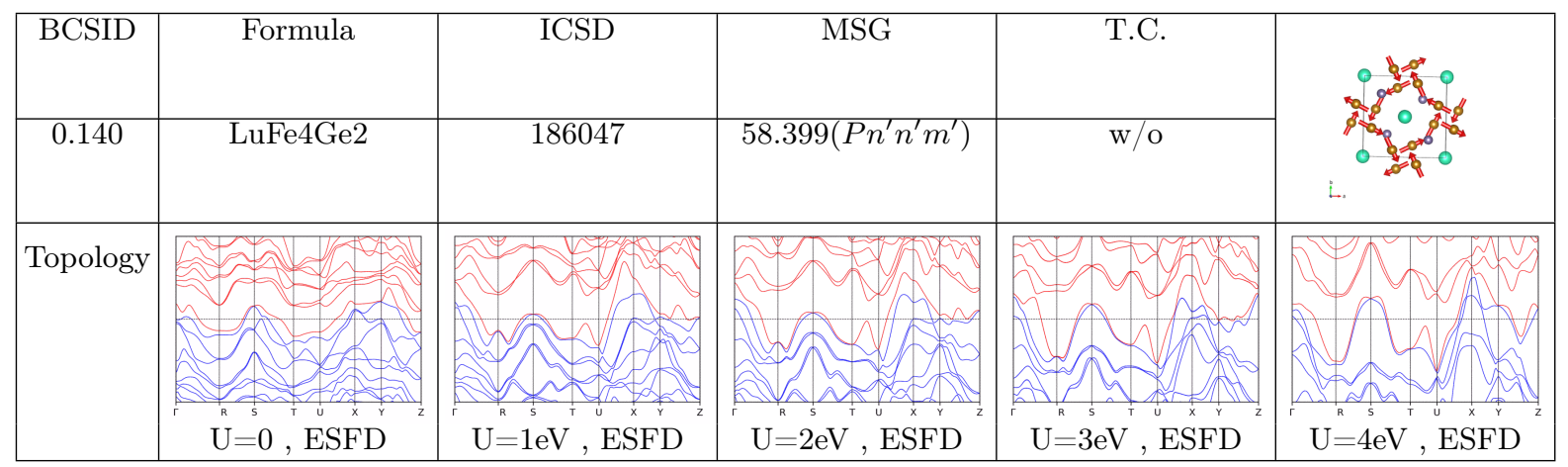

TABLE CLXXX. Topology phase diagram of LuFe4Ge2.

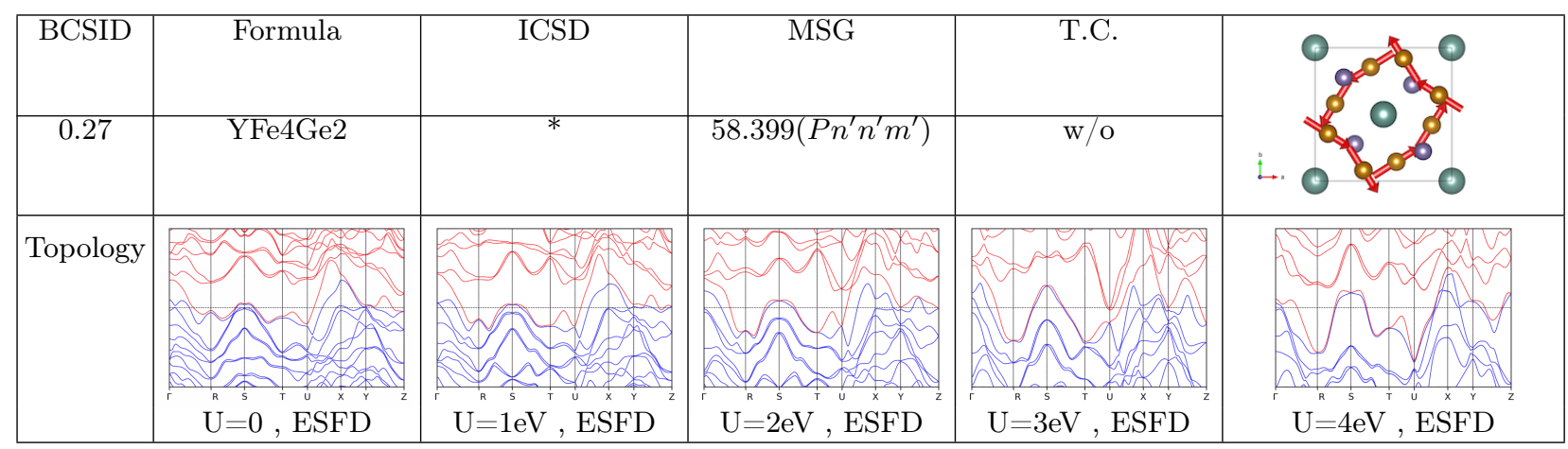

TABLE CLXXXI. Topology phase diagram of YFe4Ge2.

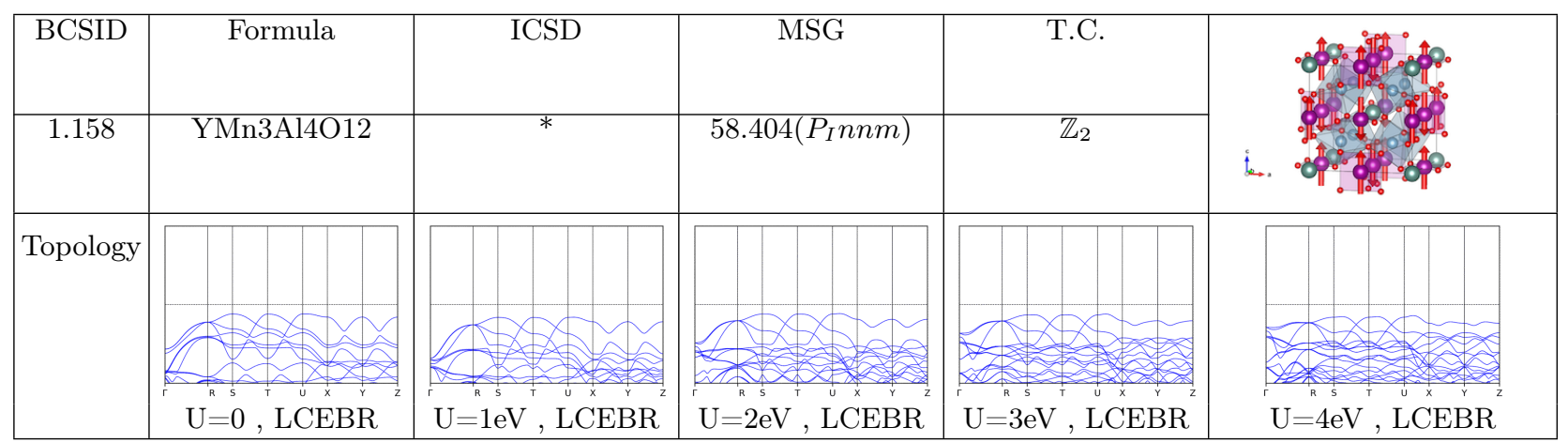

TABLE CLXXXII. Topology phase diagram of YMn3Al4O12.

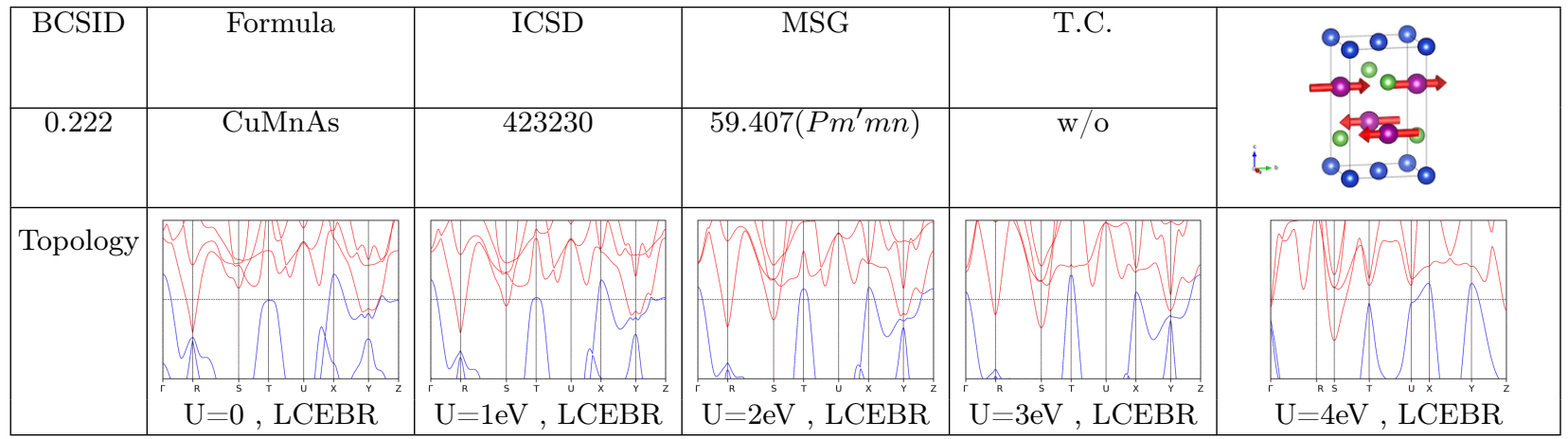

TABLE CLXXXIII. Topology phase diagram of CuMnAs. 


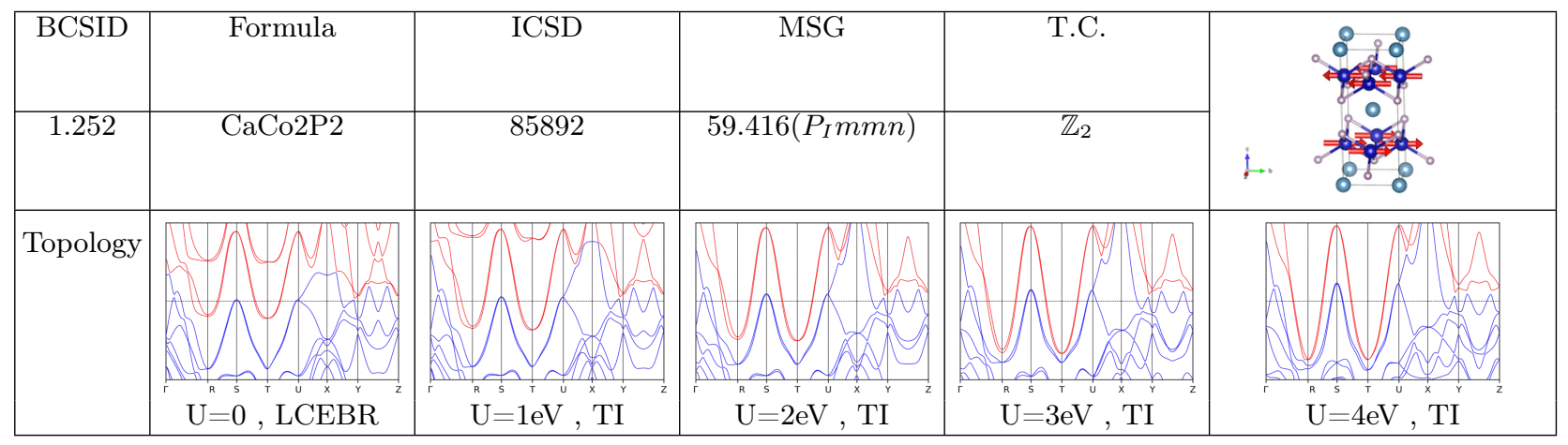

TABLE CLXXXIV. Topology phase diagram of CaCo2P2.

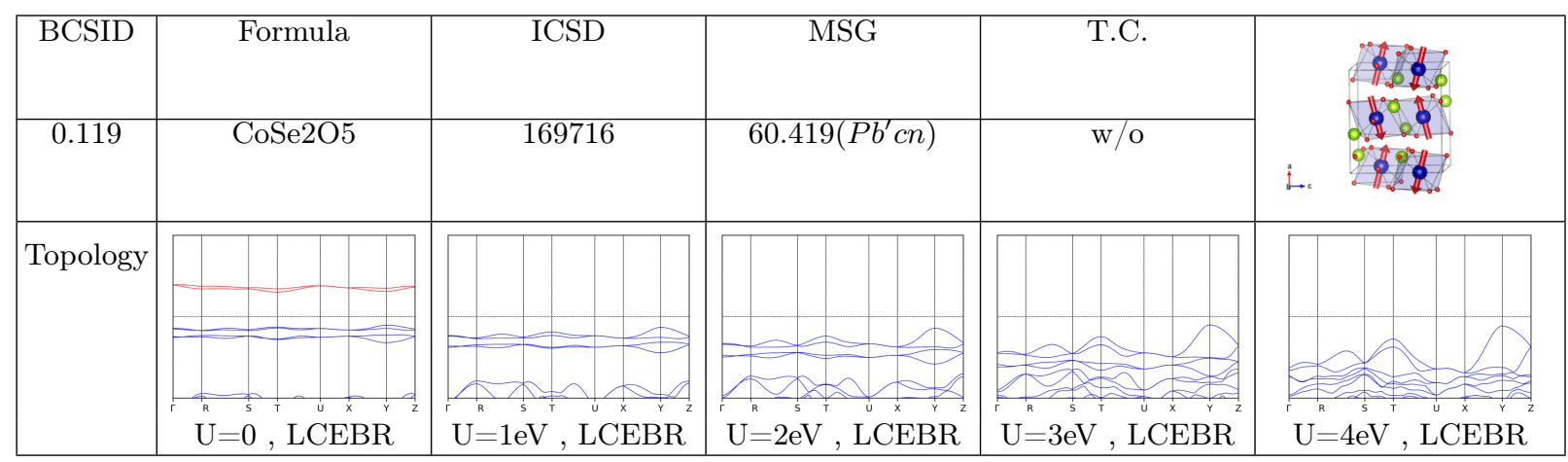

TABLE CLXXXV. Topology phase diagram of CoSe2O5.

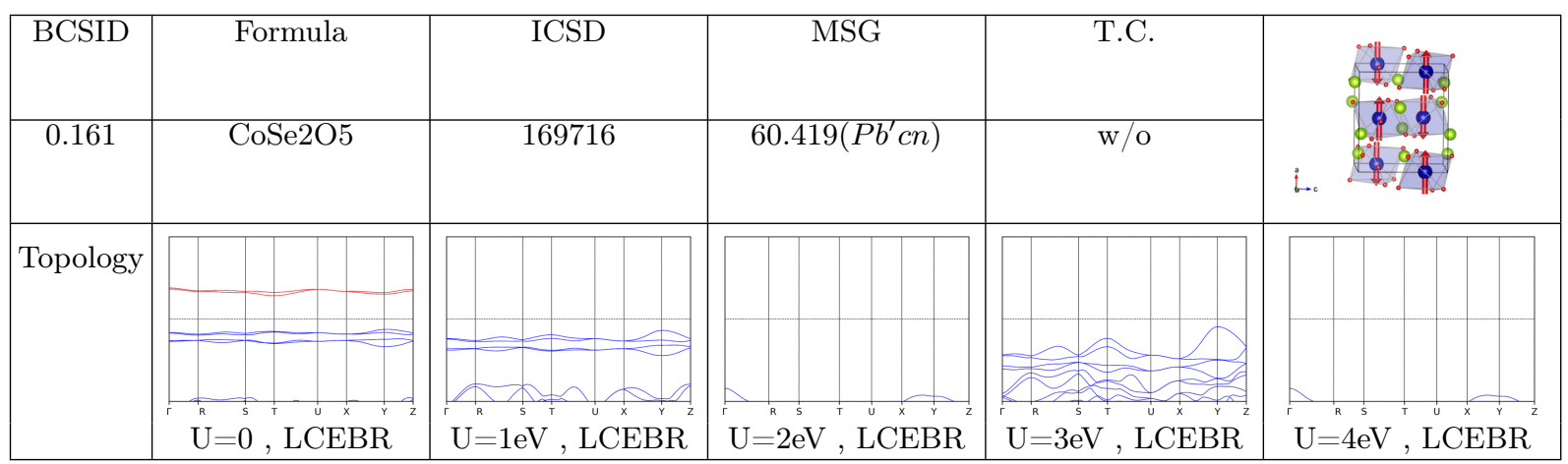

TABLE CLXXXVI. Topology phase diagram of CoSe2O5.

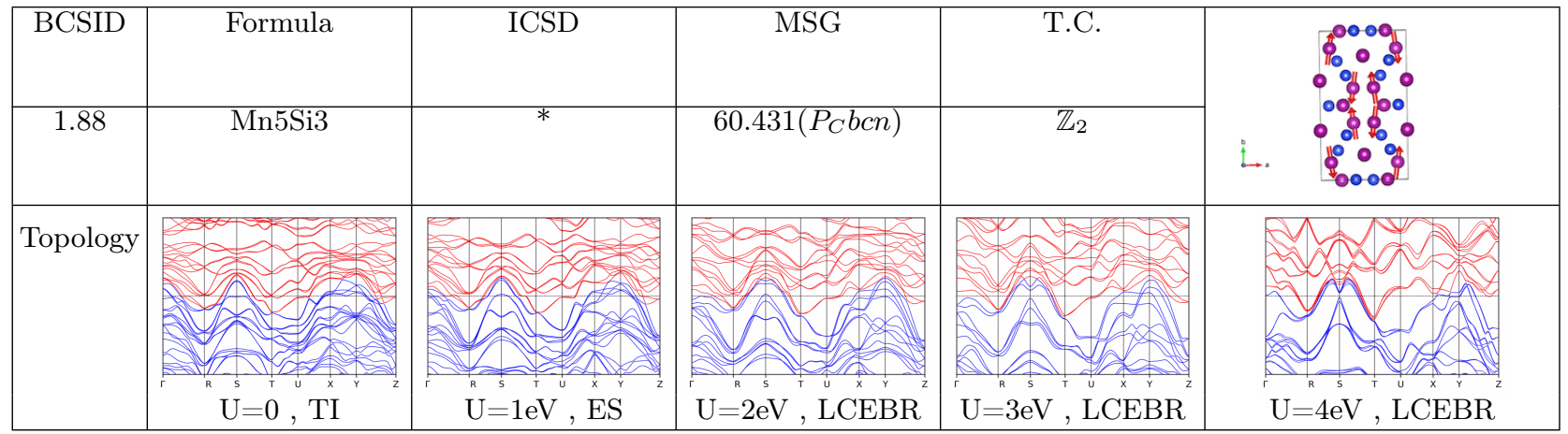

TABLE CLXXXVII. Topology phase diagram of Mn5Si3. 


\begin{tabular}{|c|c|c|c|c|c|}
\hline BCSID & Formula & ICSD & MSG & T.C. & \\
\hline 0.40 & Mn2O3-alpha & & $61.433(\mathrm{Pbca})$ & $\mathbb{Z}_{2}$ & \\
\hline \multirow[t]{5}{*}{ Topology } & 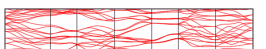 & 2 & $\geqslant$ & $+\infty \times 27$ & F \\
\hline & 8 & & & & \\
\hline & 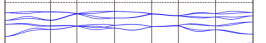 & $\Leftrightarrow$ & 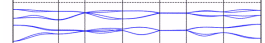 & 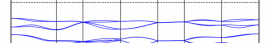 & $\Rightarrow$ \\
\hline & $\therefore$. & 121 & 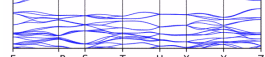 & $\checkmark x$ & 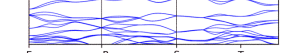 \\
\hline & $\mathrm{U}^{\mathrm{R}}=0, \mathrm{LCEBR}$ & $\mathrm{U}=1 \mathrm{eV}, \mathrm{LCEBR}$ & $\mathrm{U}=2 \mathrm{eV}, \mathrm{LCEBR}$ & $\mathrm{U}=3 \mathrm{eV}, \mathrm{LCEBR}$ & $\mathrm{U}=4 \mathrm{eV}^{\mathrm{n}}, \mathrm{LCEBR}^{\mathrm{s}}$ \\
\hline
\end{tabular}

TABLE CLXXXVIII. Topology phase diagram of Mn2O3-alpha.

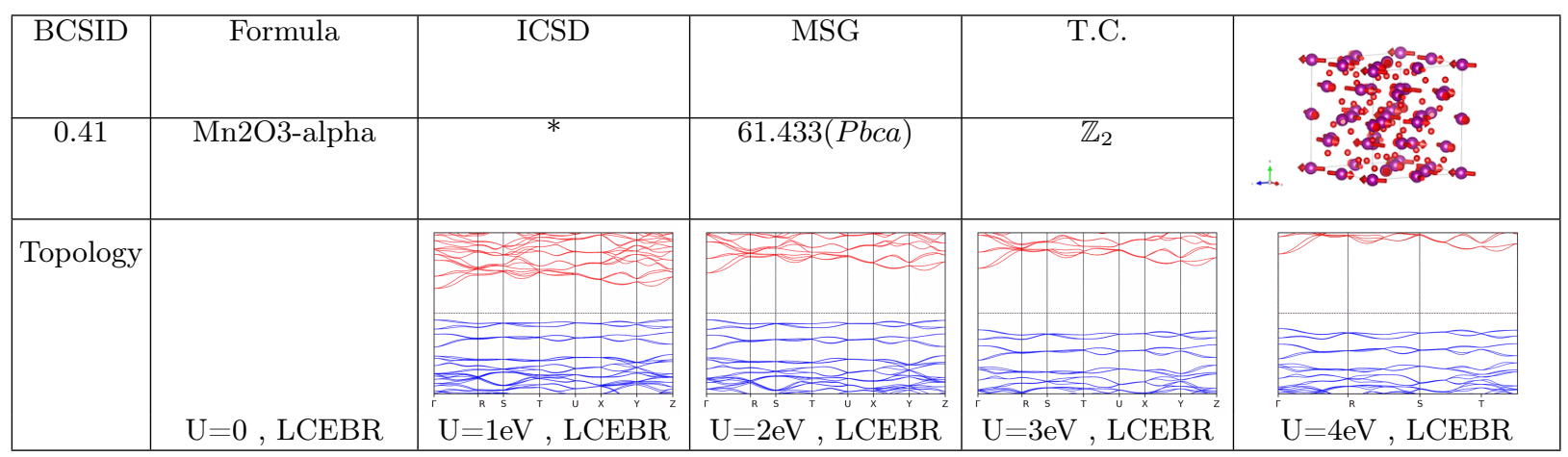

TABLE CLXXXIX. Topology phase diagram of Mn2O3-alpha.

\begin{tabular}{|c|c|c|c|c|c|}
\hline BCSID & Formula & ICSD & MSG & T.C. & \\
\hline 0.71 & $\overline{\mathrm{Li} 2 \mathrm{Ni}(\mathrm{SO} 4) 2}$ & * & $61.437\left(P b^{\prime} c^{\prime} a^{\prime}\right)$ & $\mathrm{w} / \mathrm{o}$ & $\begin{array}{r}10 \\
+\quad 801\end{array}$ \\
\hline \multirow[t]{2}{*}{ Topology } & \multirow[b]{2}{*}{$\mathrm{U}=0, \mathrm{LCEBR}$} & \multirow[b]{2}{*}{$\mathrm{U}=1 \mathrm{eV}, \mathrm{LCEBR}$} & & & 8 \\
\hline & & & 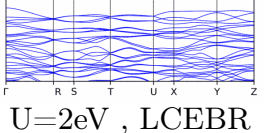 & 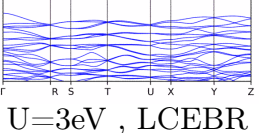 & ${\mathrm{U}=4 \mathrm{eV}, \mathrm{LCEBR}^{\mathrm{r}}}^{\mathrm{r}}$ \\
\hline
\end{tabular}

TABLE CXC. Topology phase diagram of Li2Ni(SO4)2.

\begin{tabular}{|c|c|c|c|c|c|}
\hline BCSID & Formula & ICSD & MSG & T.C. & $\leftarrow 0$ \\
\hline 2.1 & EuFe2As2 & & $61.439\left(P_{C} b c a\right)$ & $\mathbb{Z}_{2}$ & $\rightarrow \quad$ \\
\hline Topology & 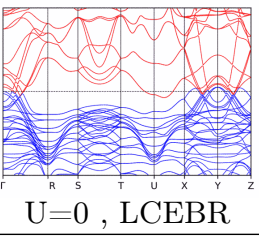 & 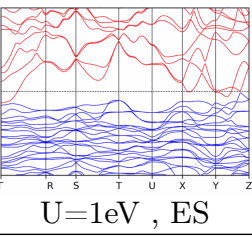 & $\mathrm{U}^{\mathrm{s}}=2 \mathrm{eV}, \mathrm{TI}$ & 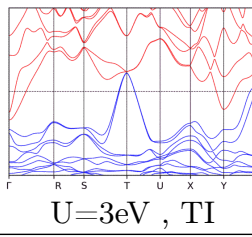 & $\underset{{\mathrm{U}=4 \mathrm{eV}^{\mathrm{r}}, \mathrm{LCEBR}}^{\mathrm{x}}}{\mathrm{C}_{\mathrm{r}}^{\mathrm{r}}}$ \\
\hline
\end{tabular}

TABLE CXCI. Topology phase diagram of EuFe2As2. 


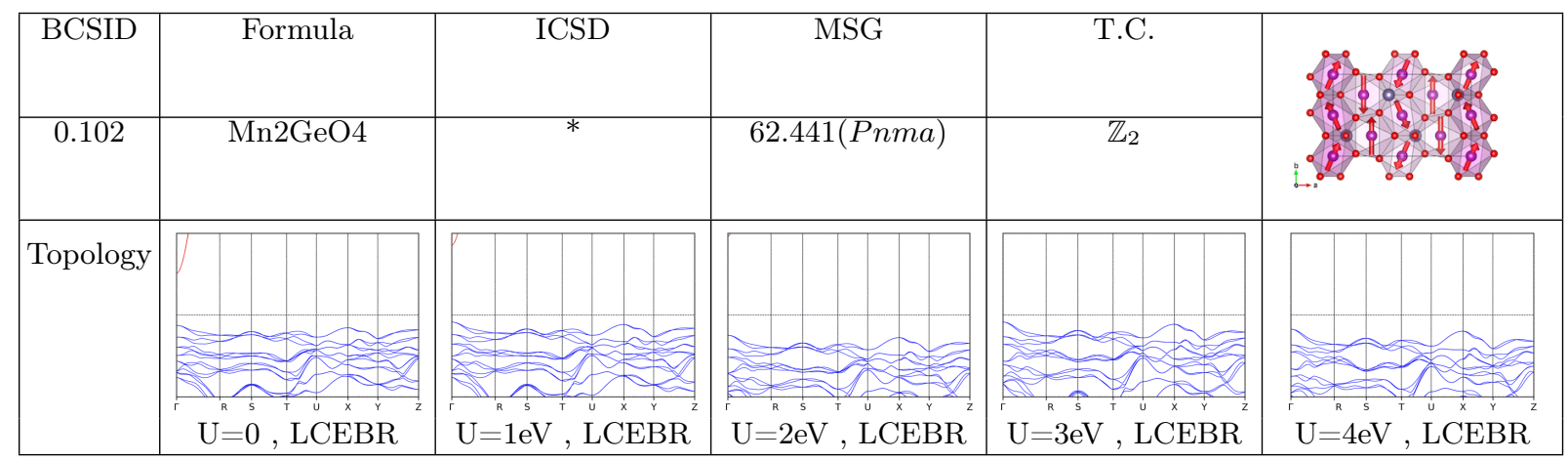

TABLE CXCII. Topology phase diagram of Mn2GeO4.

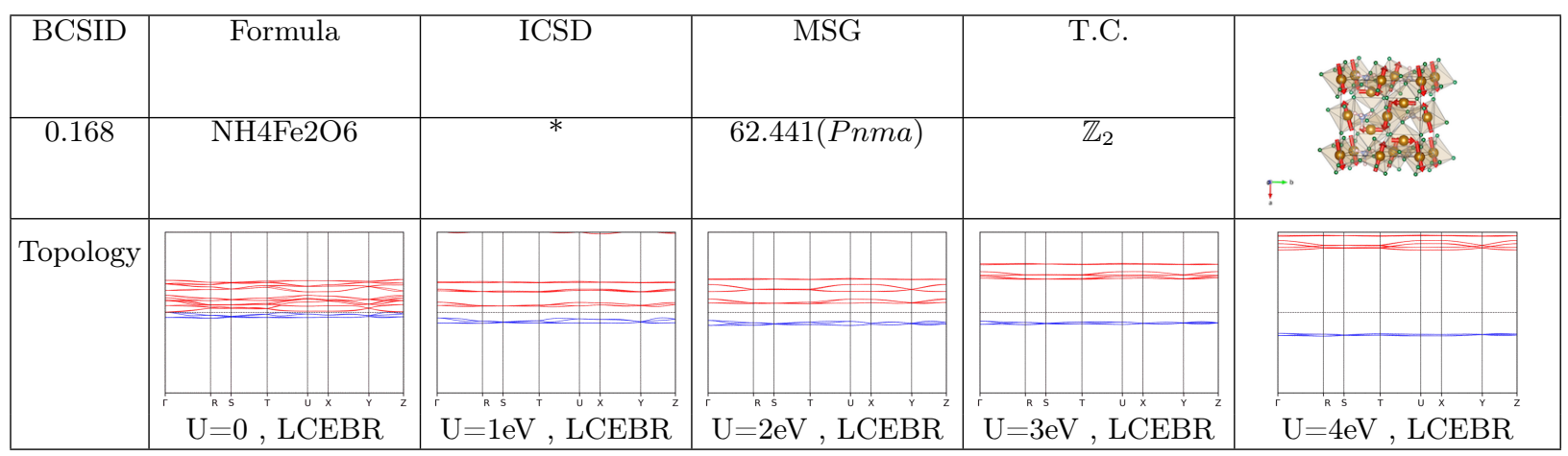

TABLE CXCIII. Topology phase diagram of NH4Fe2O6.

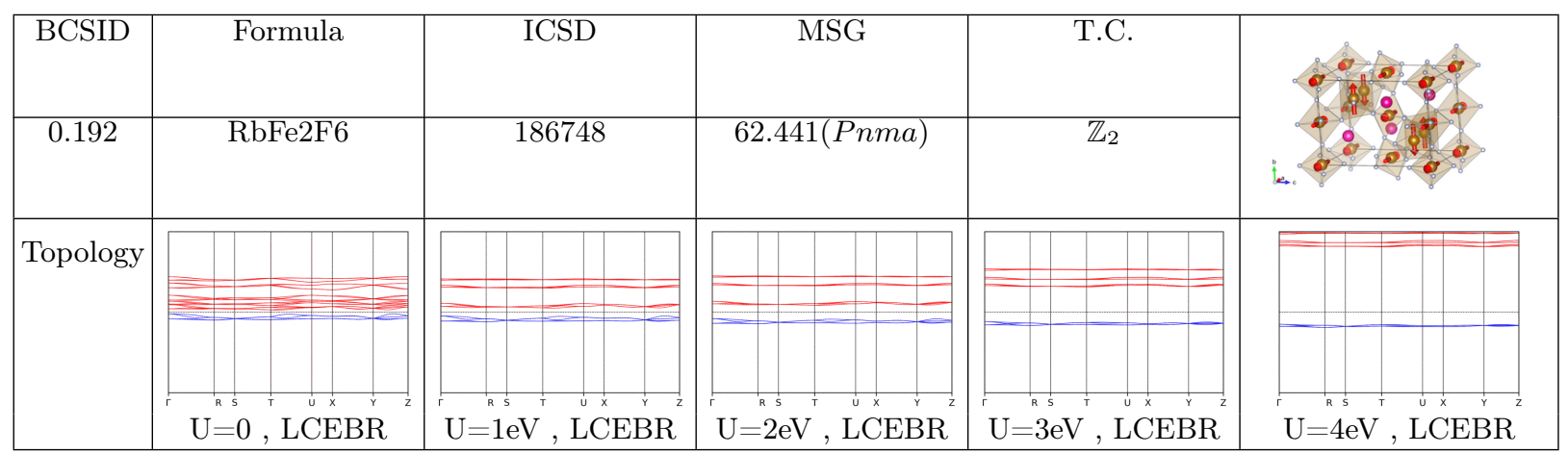

TABLE CXCIV. Topology phase diagram of RbFe2F6.

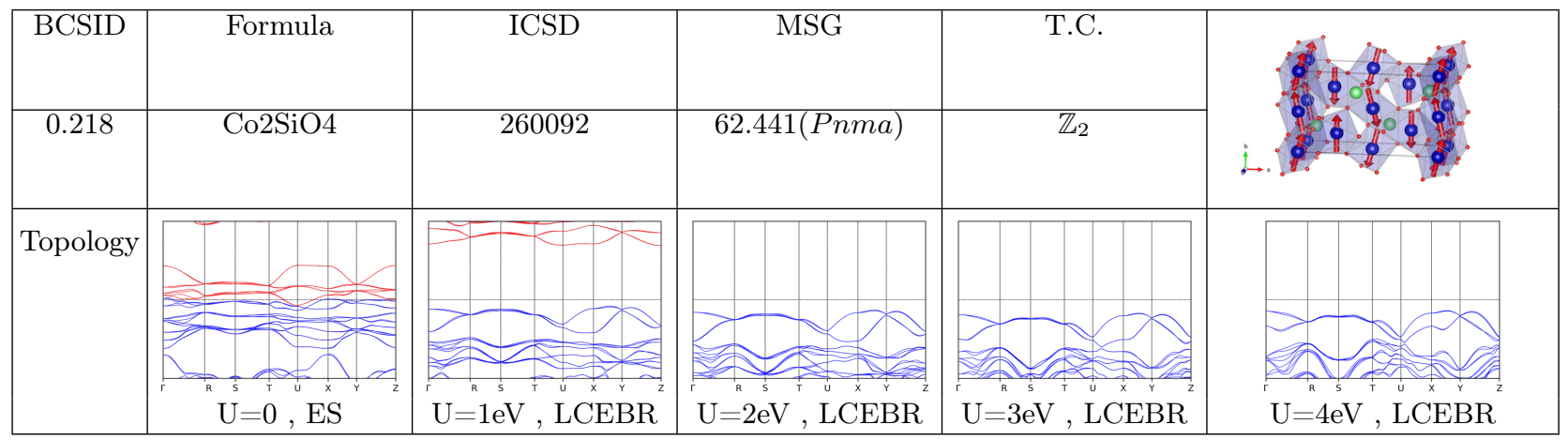

TABLE CXCV. Topology phase diagram of Co2SiO4. 


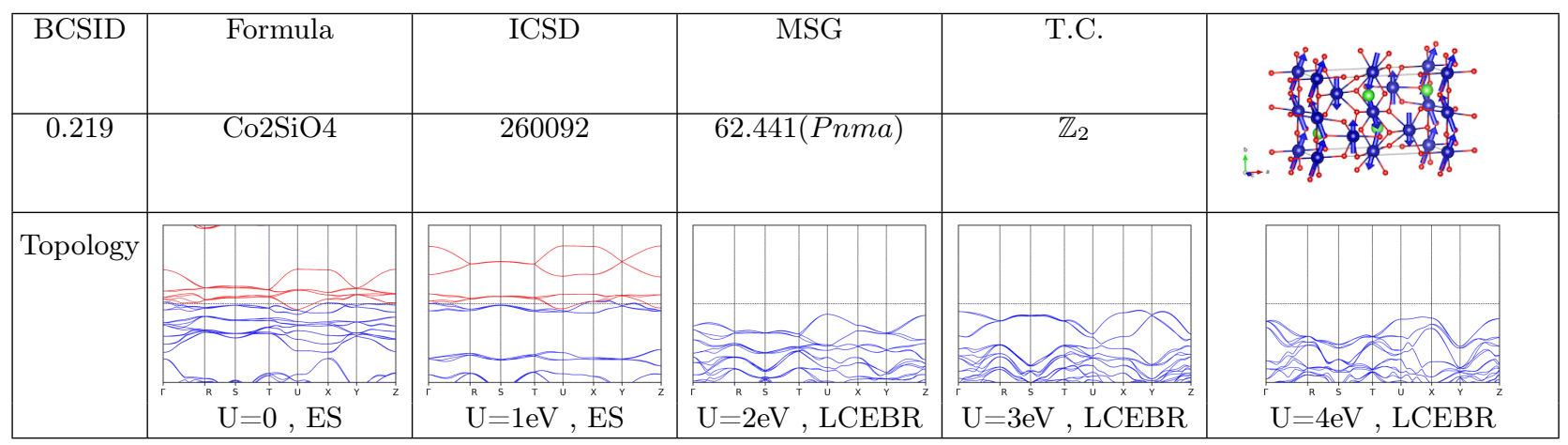

TABLE CXCVI. Topology phase diagram of Co2SiO4.

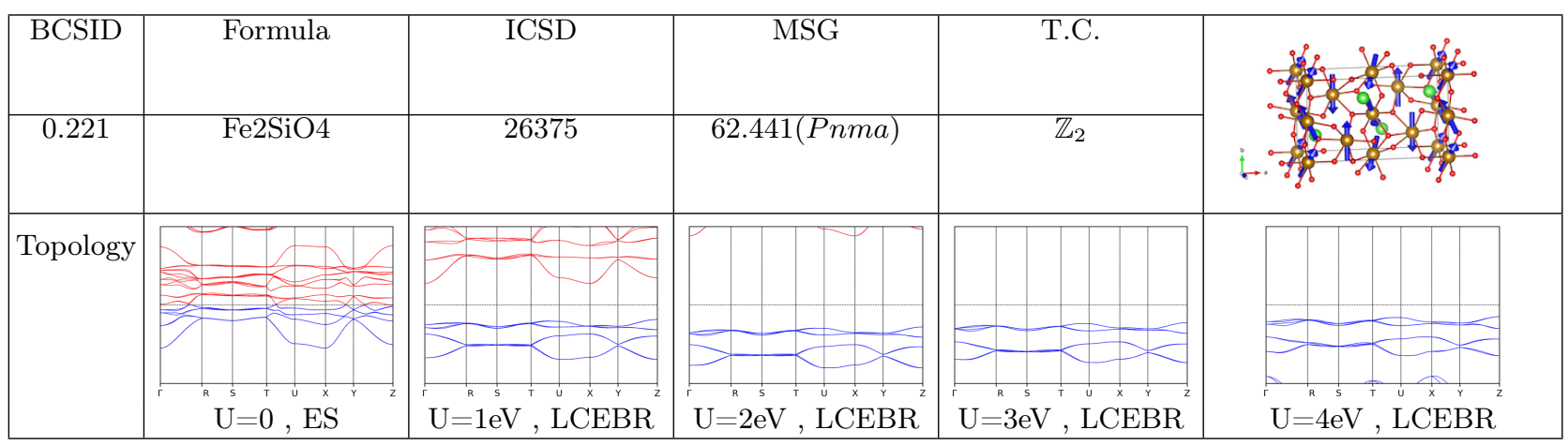

TABLE CXCVII. Topology phase diagram of Fe2SiO4.

\begin{tabular}{|c|c|c|c|c|c|}
\hline BCSID & Formula & ICSD & MSG & T.C. & \\
\hline 0.90 & $\mathrm{Rb} 2 \mathrm{Fe} 2 \mathrm{O}(\mathrm{AsO} 4) 2$ & * & $62.441($ Pnma $)$ & $\mathbb{Z}_{2}$ & 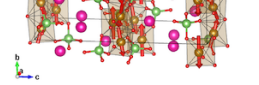 \\
\hline \multirow[t]{2}{*}{ Topology } & 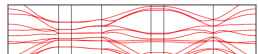 & ts & & & \\
\hline & $\mathrm{U}^{\mathrm{R}}=0^{\top}, \mathrm{LCEBR}$ & $\mathrm{U}=1 \mathrm{eV}, \mathrm{LCEBR}$ & $\mathrm{U}=2 \mathrm{eV}, \mathrm{LCEBR}$ & $\mathrm{U}=3 \mathrm{eV}, \mathrm{LCEBR}$ & $\mathrm{U}=4 \mathrm{eV}, \mathrm{LCEBR}$ \\
\hline
\end{tabular}

TABLE CXCVIII. Topology phase diagram of Rb2Fe2O(AsO4)2.

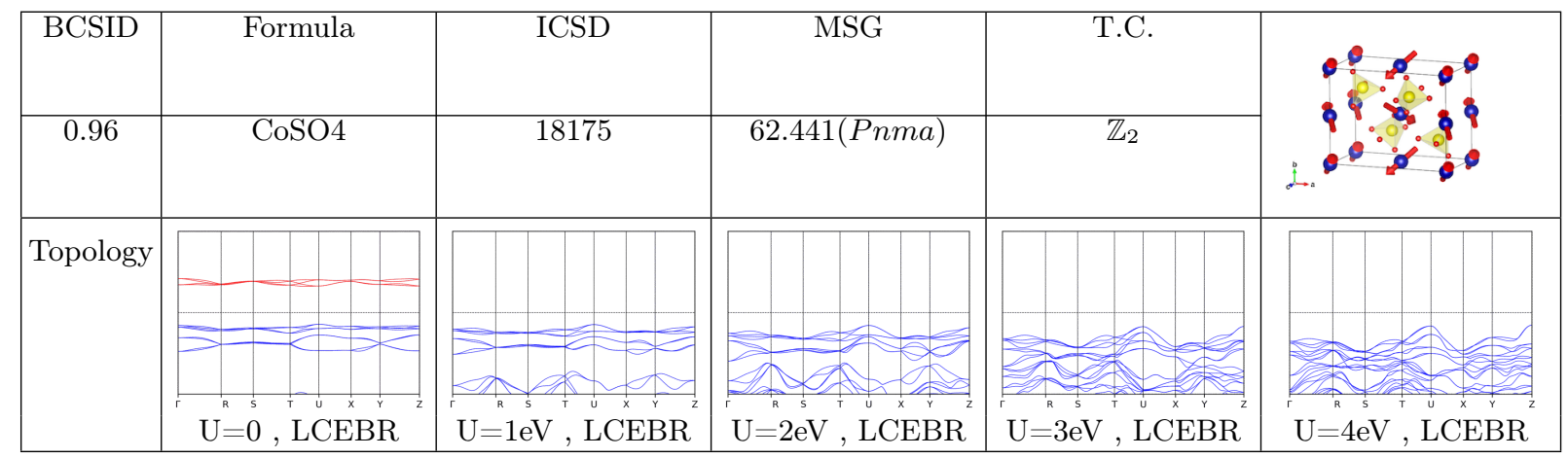

TABLE CXCIX. Topology phase diagram of CoSO4. 


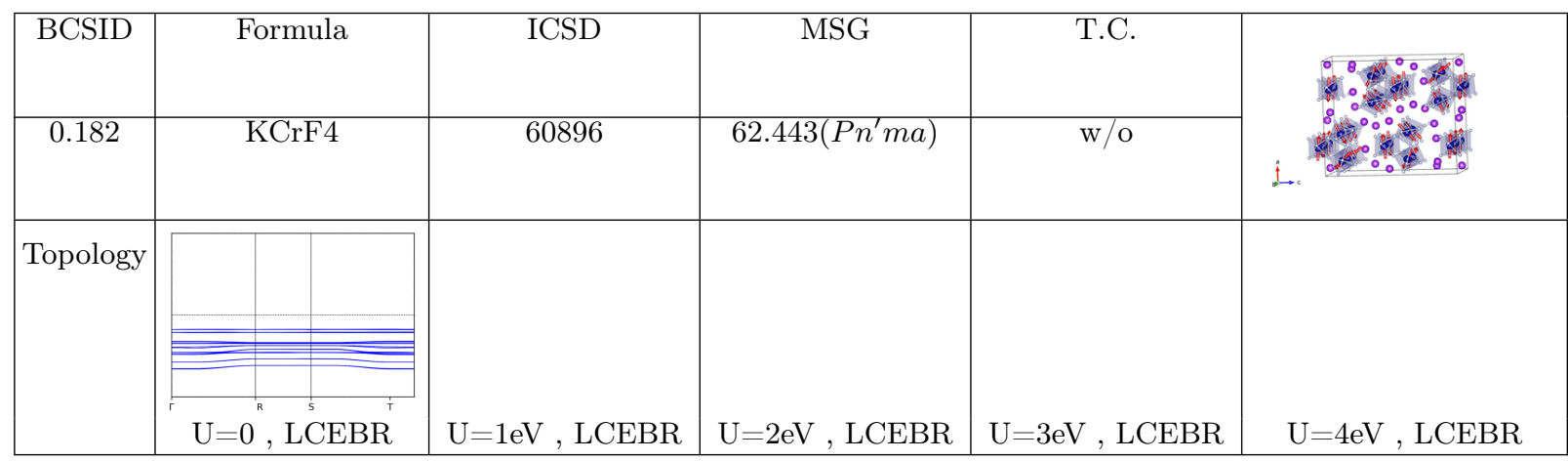

TABLE CC. Topology phase diagram of KCrF4.

\begin{tabular}{|c|c|c|c|c|c|}
\hline BCSID & Formula & ICSD & MSG & T.C. & \\
\hline 0.88 & LiNiPO4 & 402760 & $62.444\left(P n m^{\prime} a\right)$ & $\mathrm{w} / \mathrm{o}$ & L. \\
\hline Topology & & & & & \\
\hline & $\mathrm{U}^{\mathrm{s}}=0^{\mathrm{s}}, \mathrm{LCEBR}^{\mathrm{x}}$ & $\mathrm{U}^{\mathrm{R}}=1 \mathrm{eV}^{\mathrm{s}}, \mathrm{LCEBR}$ & 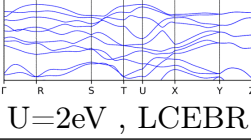 & $\mathrm{U}^{\mathrm{n}}=3 \mathrm{eV}^{\mathrm{s}}, \mathrm{LCEBR}$ & 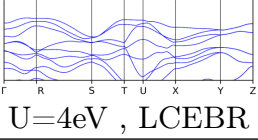 \\
\hline
\end{tabular}

TABLE CCI. Topology phase diagram of LiNiPO4.

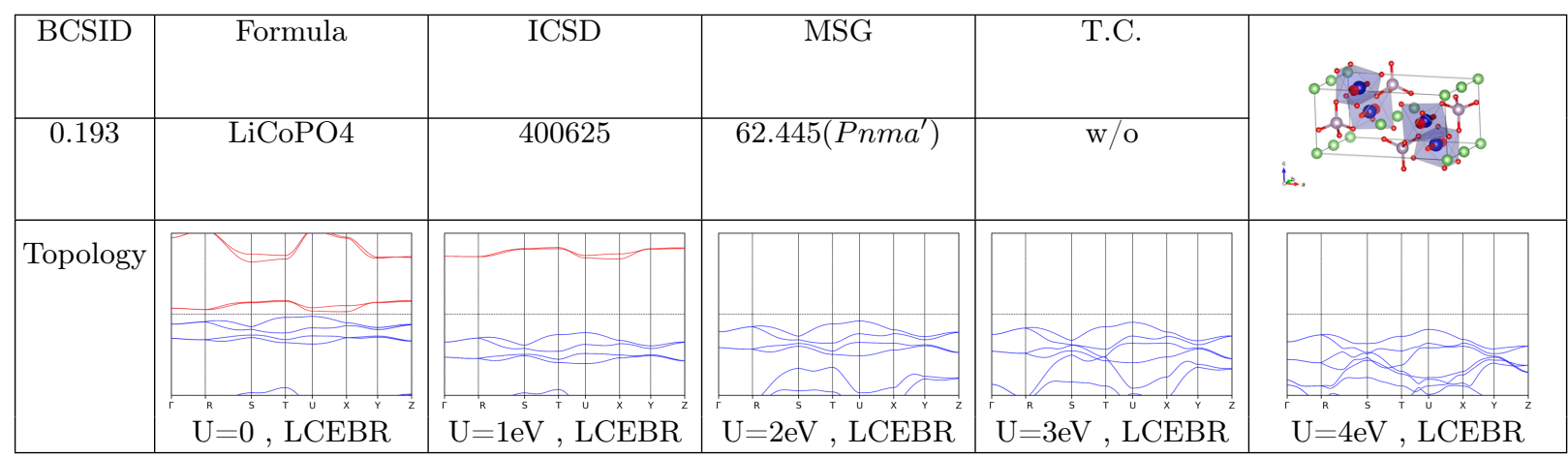

TABLE CCII. Topology phase diagram of LiCoPO4.

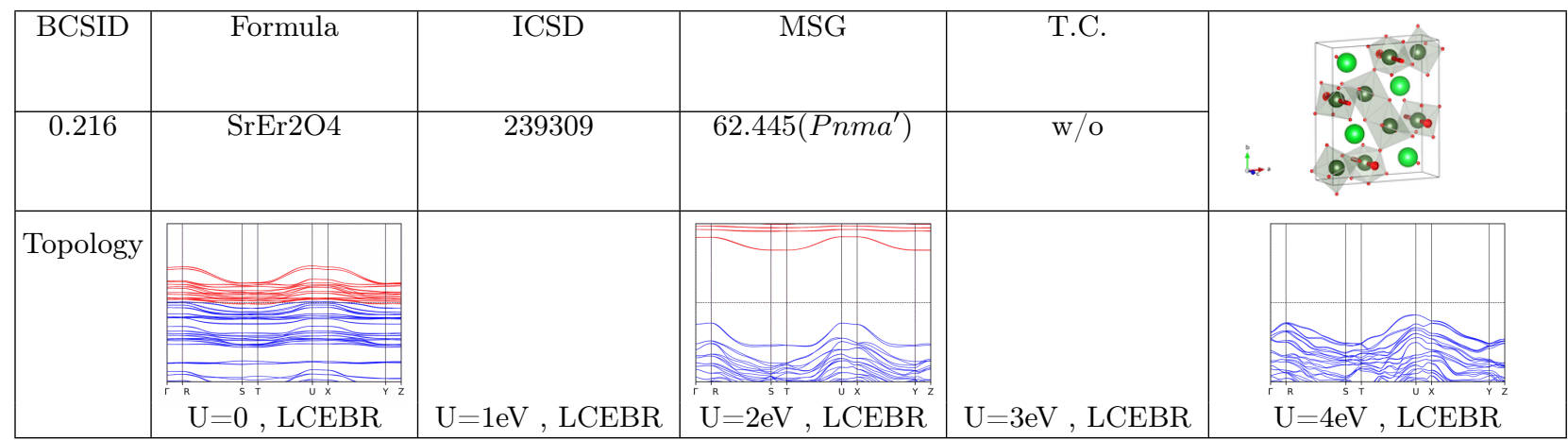

TABLE CCIII. Topology phase diagram of SrEr2O4. 


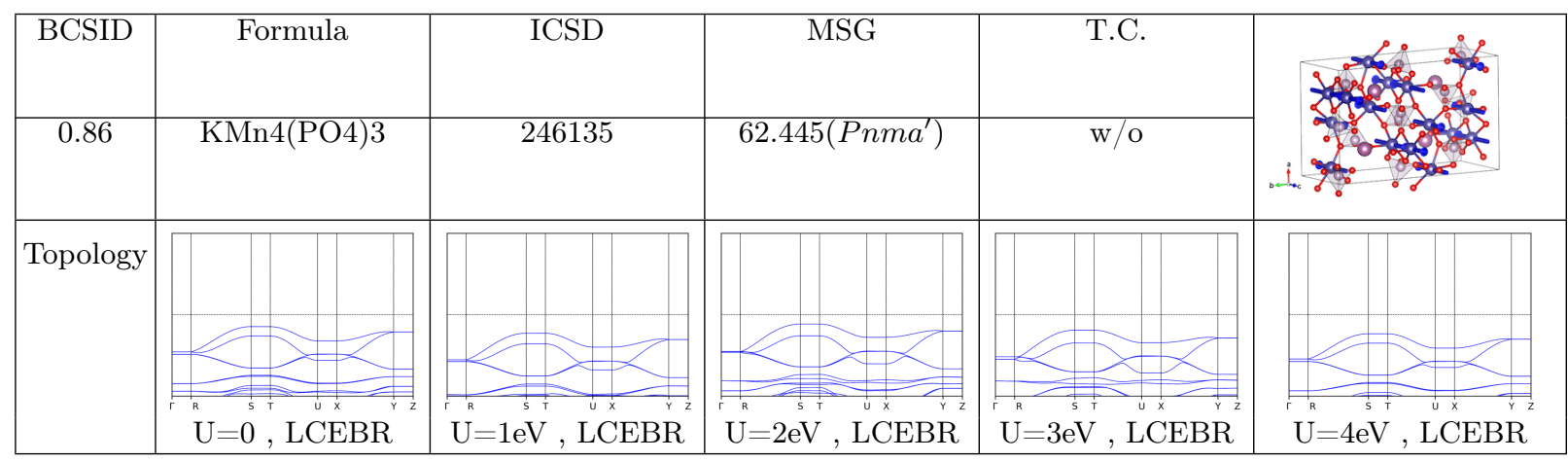

TABLE CCIV. Topology phase diagram of KMn4(PO4)3.

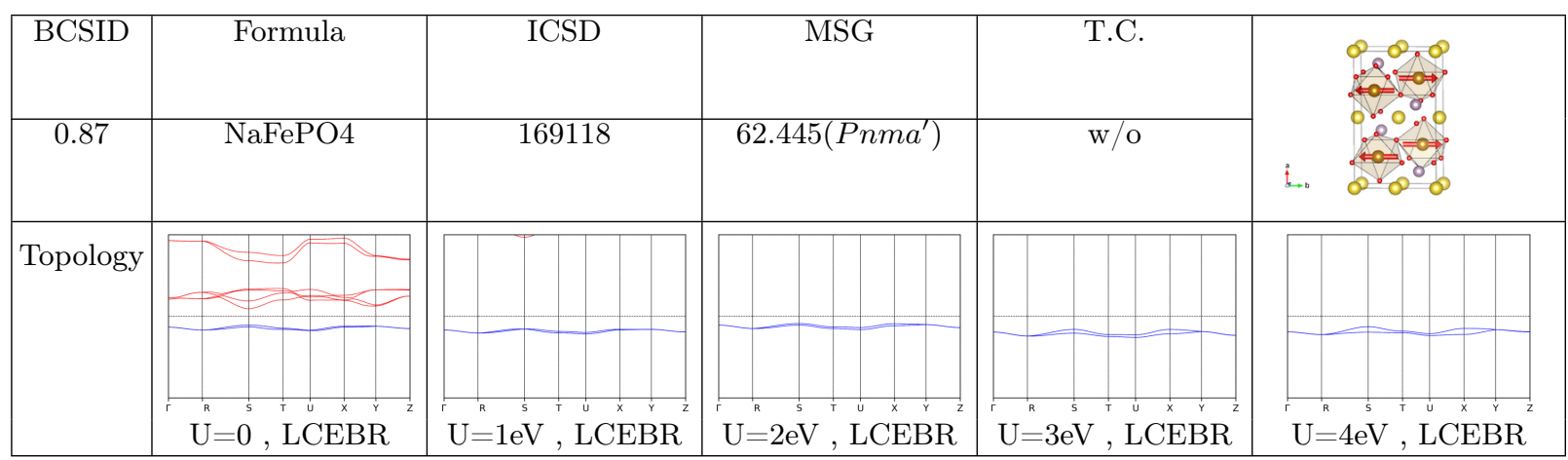

TABLE CCV. Topology phase diagram of NaFePO4.

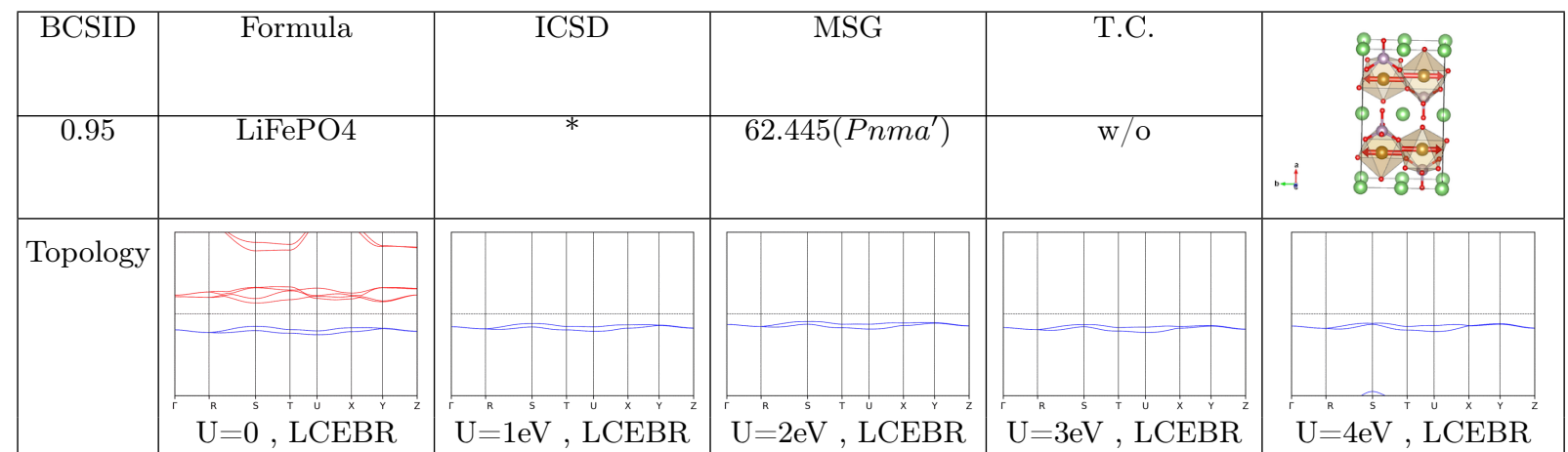

TABLE CCVI. Topology phase diagram of LiFePO4.

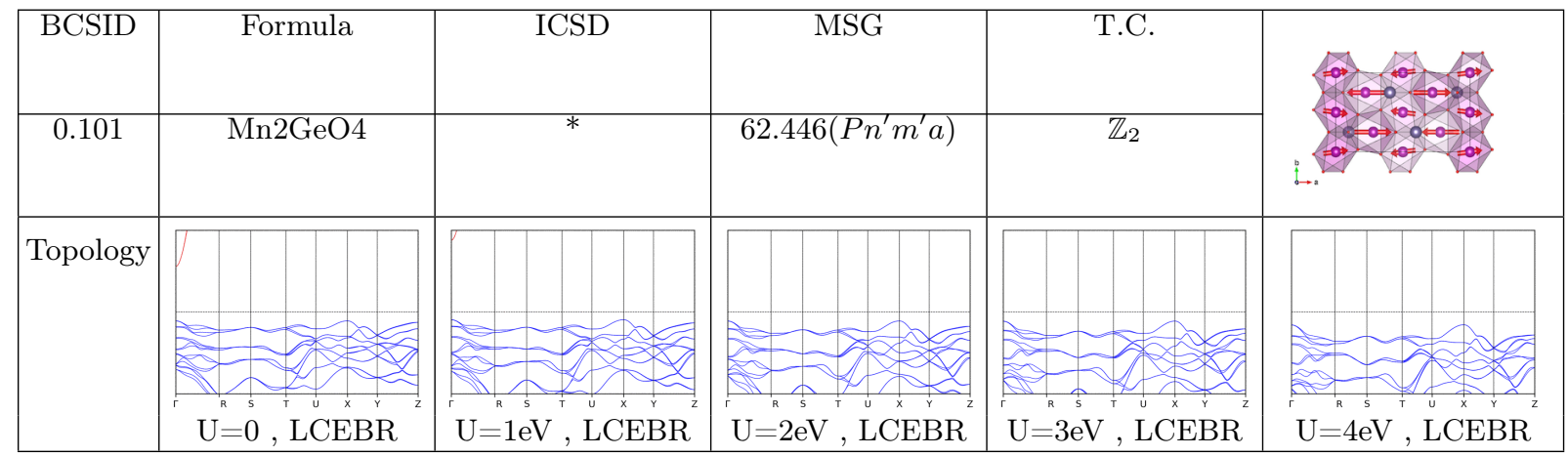

TABLE CCVII. Topology phase diagram of Mn2GeO4. 


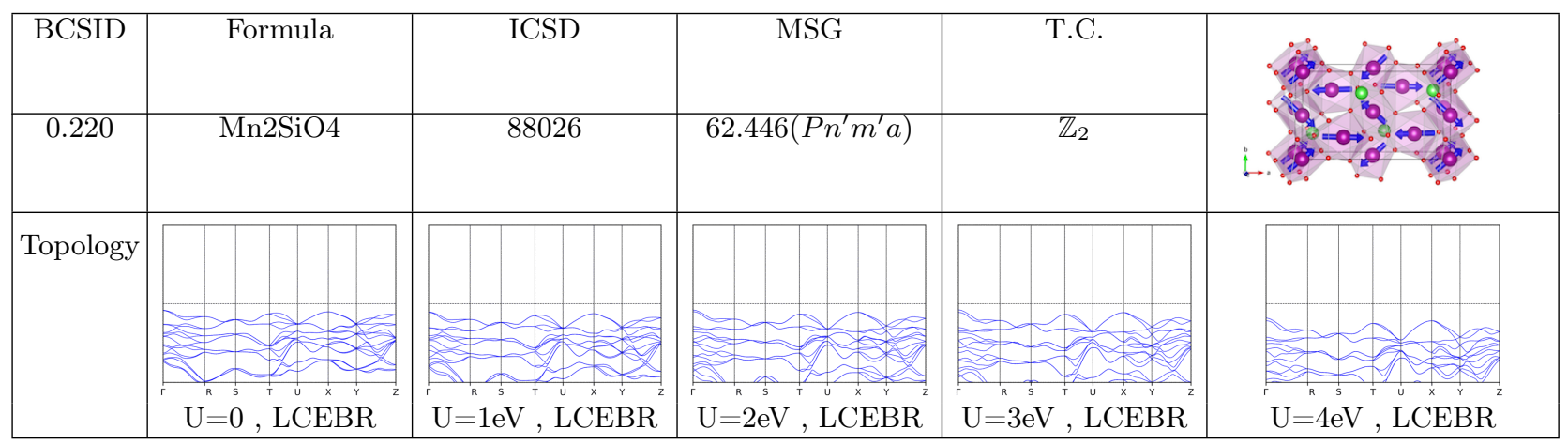

TABLE CCVIII. Topology phase diagram of Mn2SiO4.

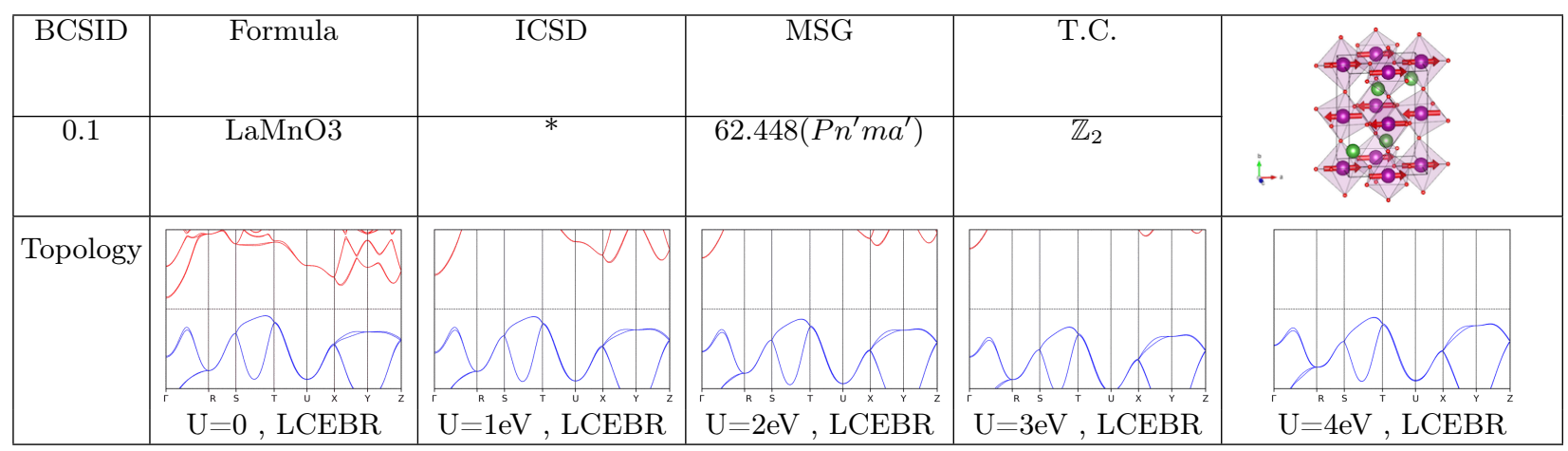

TABLE CCIX. Topology phase diagram of LaMnO3.

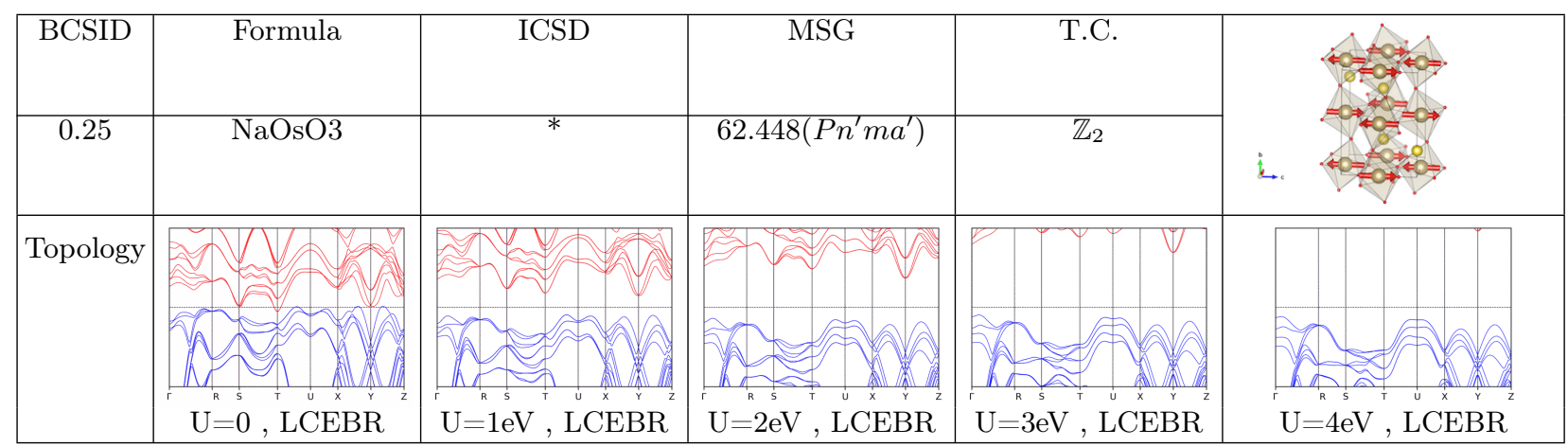

TABLE CCX. Topology phase diagram of NaOsO3.

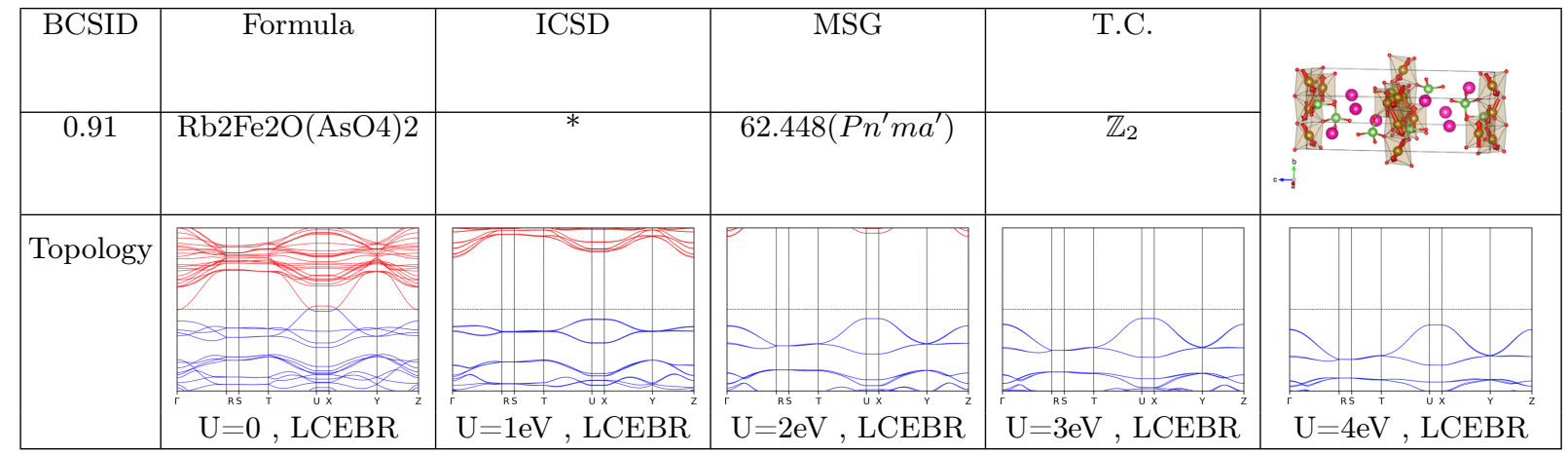

TABLE CCXI. Topology phase diagram of Rb2Fe2O(AsO4)2. 


\begin{tabular}{|c|c|c|c|c|c|}
\hline BCSID & Formula & ICSD & MSG & T.C. & \\
\hline 0.24 & LiMnPO4 & 25834 & $62.449\left(P n^{\prime} m^{\prime} a^{\prime}\right)$ & $\mathrm{w} / \mathrm{o}$ & $i_{\infty}$ \\
\hline Topology & & & & & \\
\hline & $\mathrm{U}=0, \mathrm{LCEBR}$ & $\mathrm{U}=1 \mathrm{eV}, \mathrm{LCEBR}$ & $\mathrm{U}=2 \mathrm{eV}, \mathrm{LCEBR}$ & $\mathrm{U}=3 \mathrm{eV}, \mathrm{LCEBR}$ & $\mathrm{U}=4 \mathrm{eV}, \mathrm{LCEBR}$ \\
\hline
\end{tabular}

TABLE CCXII. Topology phase diagram of LiMnPO4.

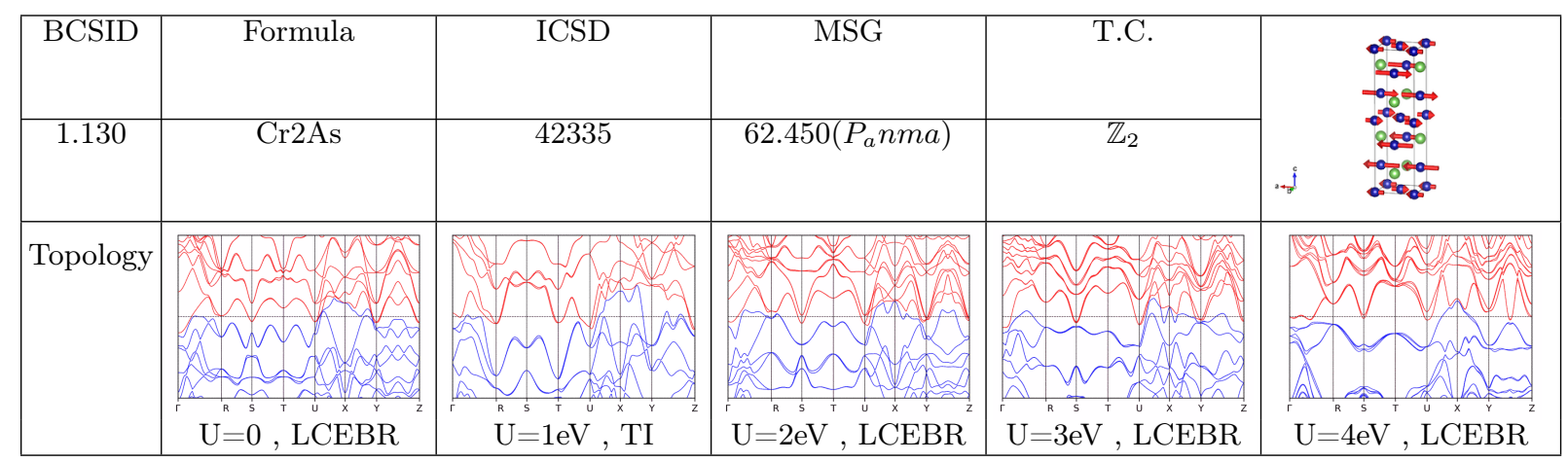

TABLE CCXIII. Topology phase diagram of Cr2As.

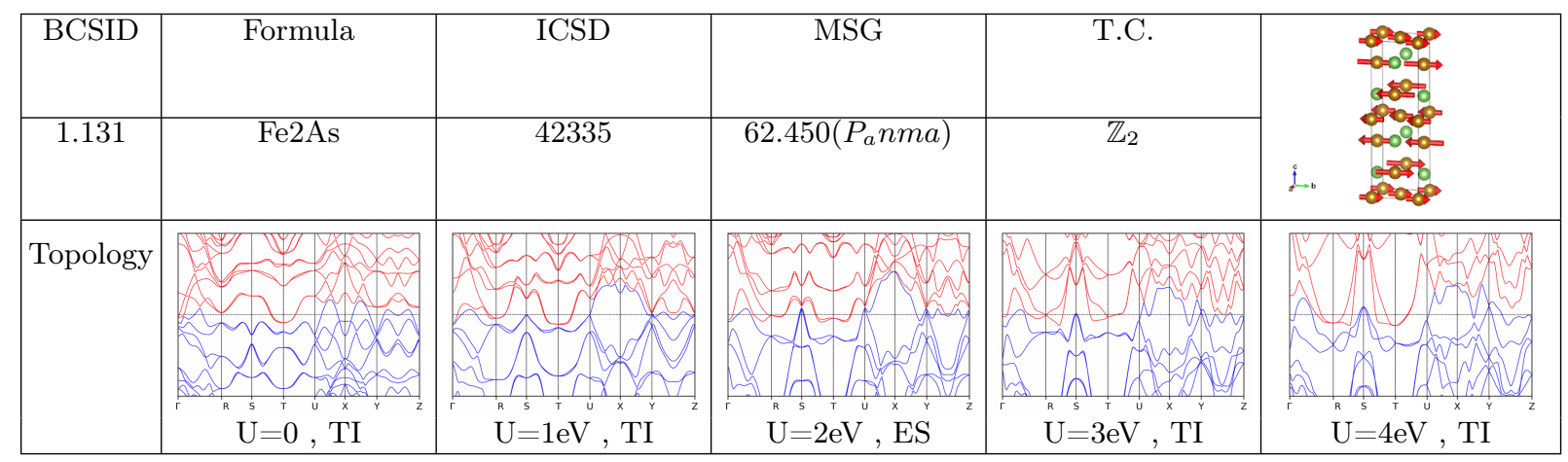

TABLE CCXIV. Topology phase diagram of Fe2As.

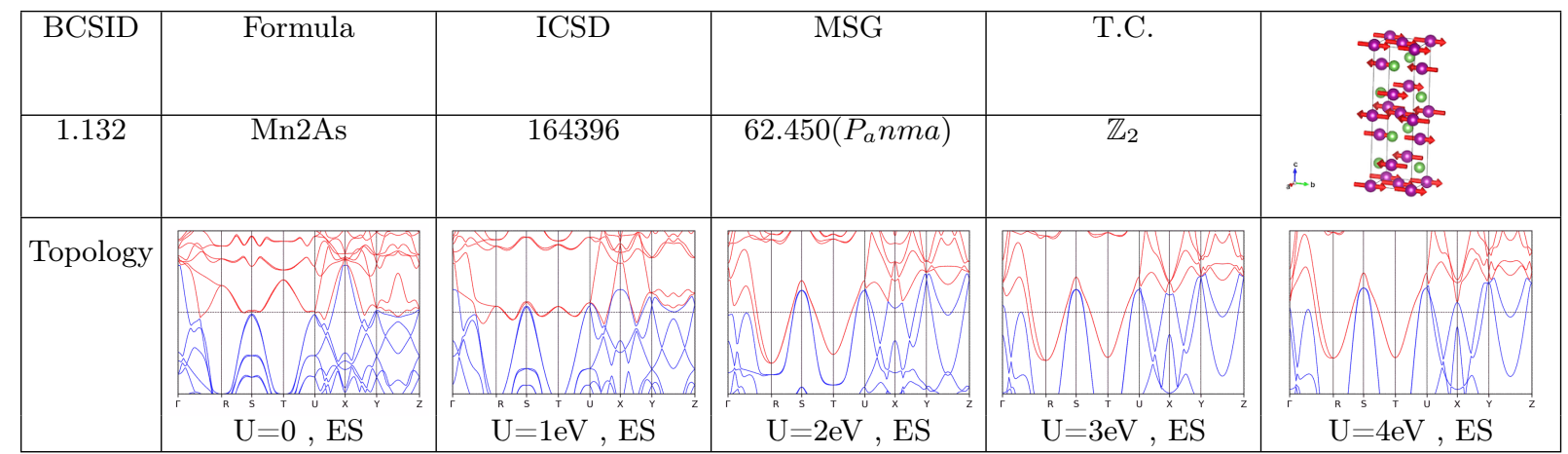

TABLE CCXV. Topology phase diagram of Mn2As. 


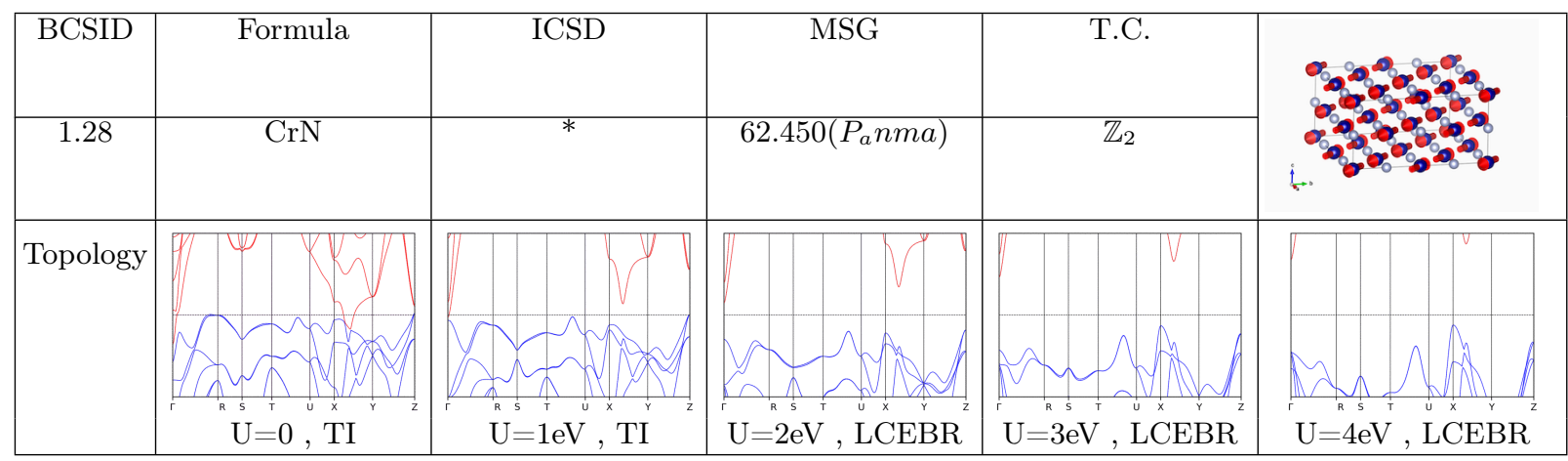

TABLE CCXVI. Topology phase diagram of CrN.

\begin{tabular}{|c|c|c|c|c|c|}
\hline BCSID & Formula & ICSD & MSG & T.C. & \\
\hline 1.1 & $\mathrm{Mn} 3 \mathrm{O} 4$ & * & $62.452\left(P_{c} n m a\right)$ & $\mathbb{Z}_{2}$ & t. \\
\hline Topology & 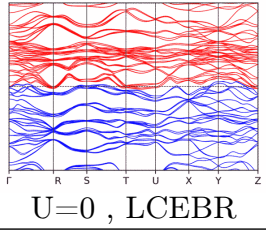 & 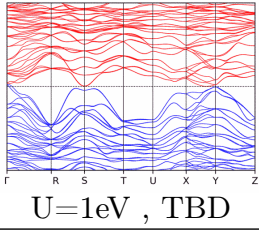 & $\mathrm{U}=2 \mathrm{eV}, \mathrm{TBD}$ & $\begin{array}{l}\text { R } \\
\mathrm{U}=3 \mathrm{eV}, \mathrm{LCEBR}\end{array}$ & $\begin{array}{l}\mathrm{U}^{\mathrm{5}} \mathrm{eV}^{\circ}, \mathrm{LCEBR} \\
\end{array}$ \\
\hline
\end{tabular}

TABLE CCXVII. Topology phase diagram of Mn3O4.

\begin{tabular}{|c|c|c|c|c|c|}
\hline BCSID & Formula & ICSD & MSG & T.C. & 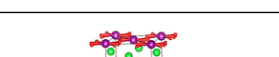 \\
\hline 2.27 & Sr2Mn3Sb2O2 & 81792 & $63.459\left(\mathrm{Cm}^{\prime} \mathrm{cm}\right)$ & $\mathrm{w} / \mathrm{o}$ & t. \\
\hline Topology & & & & & 8 \\
\hline & $\mathrm{U}^{\mathrm{R}}=0^{\mathrm{s}}, \mathrm{LCEBR}^{\top}$ & $\mathrm{U}^{\mathrm{R}}=1 \mathrm{eV}^{\mathrm{s}}, \mathrm{TCEBR}$ & $\mathrm{U}^{\mathrm{R}}=2 \mathrm{eV}^{\mathrm{s}}, \mathrm{LCEBR}^{\mathrm{T}}$ & $\mathrm{U}^{\mathrm{R}}=3 \mathrm{eV}^{\mathrm{s}}, \mathrm{TCEBR}$ & $\mathrm{U}=4^{\mathrm{R}} \mathrm{eV}^{\mathrm{s}}, \mathrm{LCEBR}^{\mathrm{z}}$ \\
\hline
\end{tabular}

TABLE CCXVIII. Topology phase diagram of Sr2Mn3Sb2O2.

\begin{tabular}{|c|c|c|c|c|c|}
\hline BCSID & Formula & ICSD & MSG & T.C. & \\
\hline 0.199 & $\mathrm{Mn} 3 \mathrm{Sn}$ & 643730 & $63.463\left(C m c^{\prime} m^{\prime}\right)$ & $\mathbb{Z}_{2} \mathbb{Z}_{2}$ & \\
\hline \multicolumn{6}{|l|}{ Topology } \\
\hline & $\mathrm{U}^{\mathrm{s}}=0, \mathrm{ES}$ & $\mathrm{U}^{\mathrm{R}}=1 \mathrm{eV}, \mathrm{E}^{\mathrm{s}}$ & $\begin{array}{l}\mathrm{B}^{\mathrm{s}} \\
\mathrm{S}^{2}\end{array}$ & $\mathrm{U}^{\mathrm{R}}=3 \mathrm{eV}^{\top}, \mathrm{ES}$ & $\mathrm{U}^{\mathrm{R}}=4 \mathrm{eV}^{\top}, \mathrm{ES}$ \\
\hline
\end{tabular}

TABLE CCXIX. Topology phase diagram of Mn3Sn. 


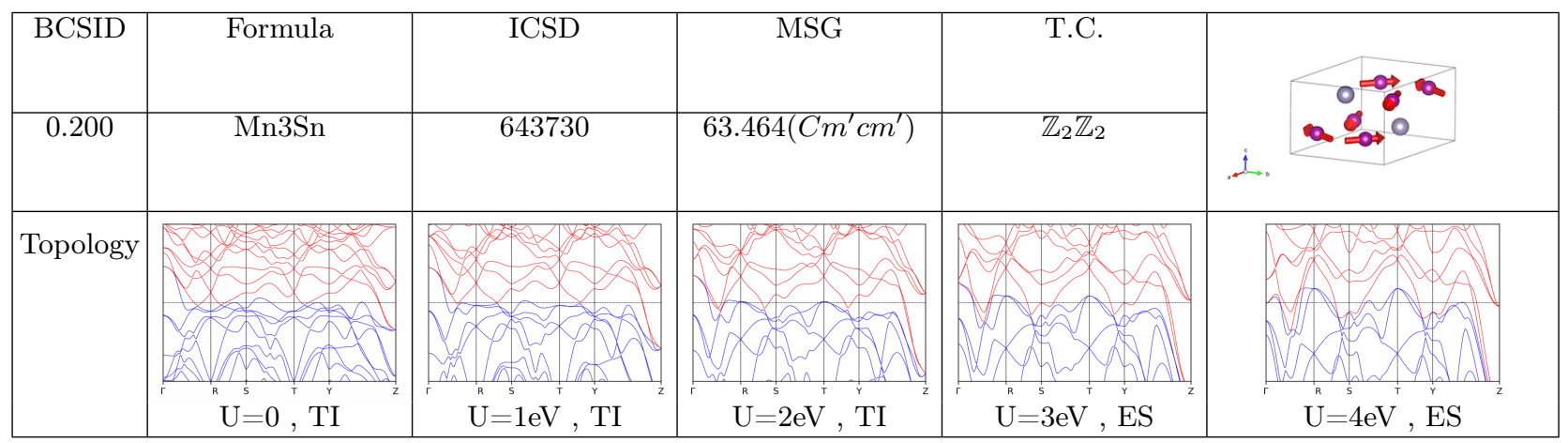

TABLE CCXX. Topology phase diagram of Mn3Sn.

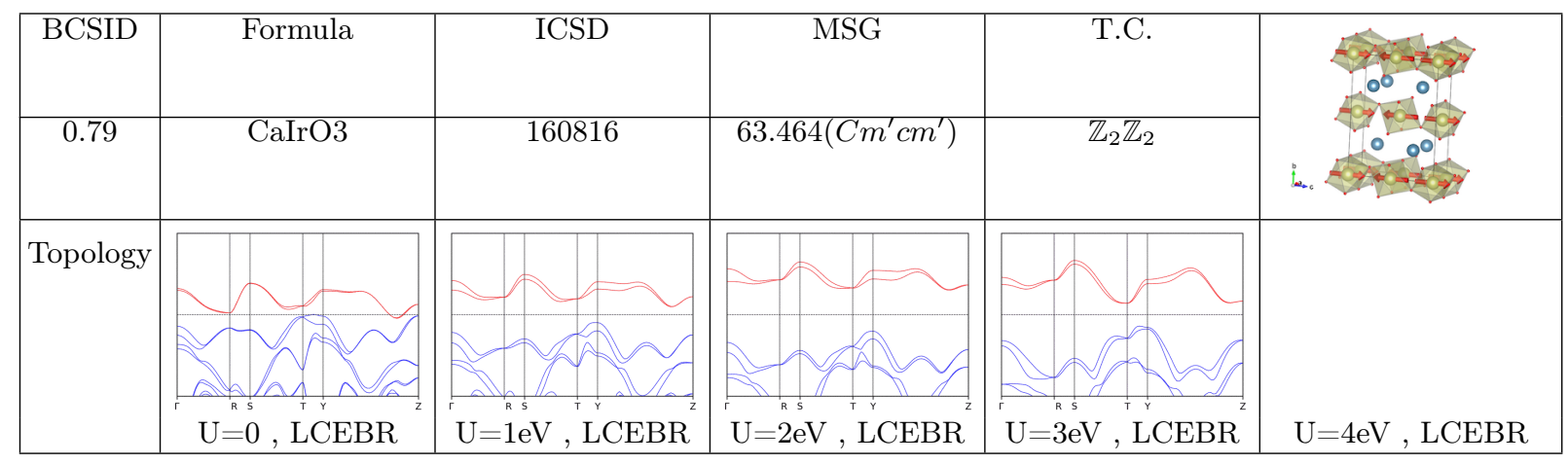

TABLE CCXXI. Topology phase diagram of CaIrO3.

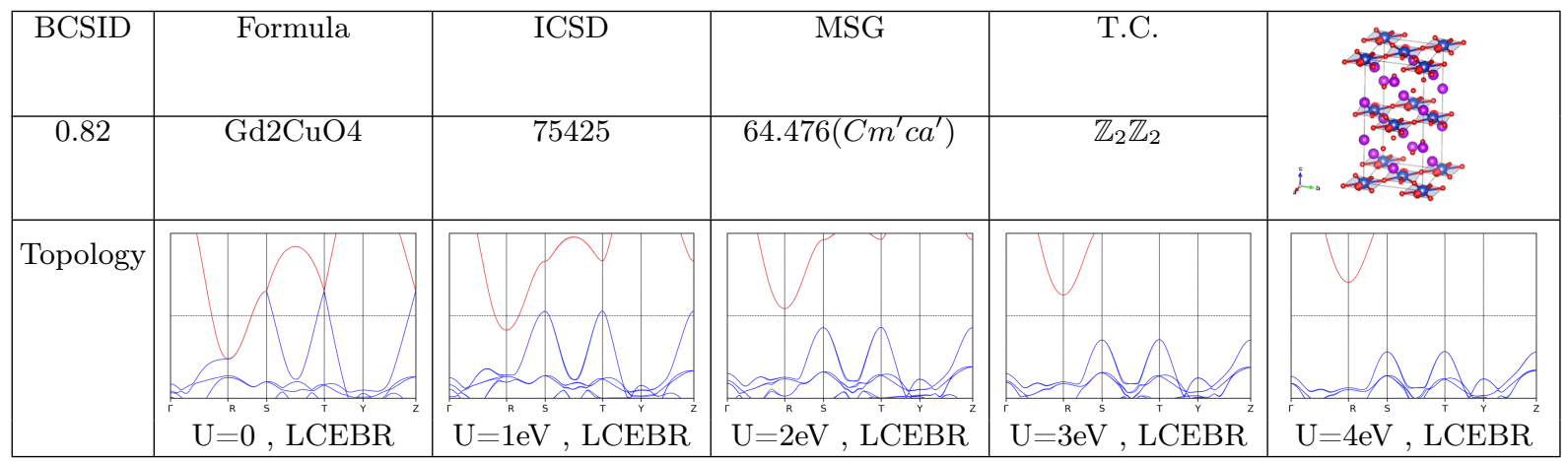

TABLE CCXXII. Topology phase diagram of $\mathrm{Gd} 2 \mathrm{CuO} 4$.

\begin{tabular}{|c|c|c|c|c|c|}
\hline BCSID & Formula & ICSD & MSG & T.C. & \\
\hline 1.145 & Mn3Ni20P6 & 72351 & $64.480\left(C_{A} m c a\right)$ & $\mathbb{Z}_{2}$ & 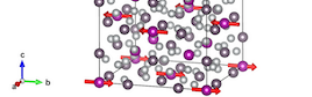 \\
\hline Topology & 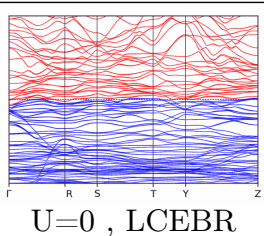 & $\mathrm{U}_{\mathrm{U}=1 \mathrm{eV}, \mathrm{TBD}^{\mathrm{h}}}$ & $\mathrm{U}^{n}=2 \mathrm{eV}, \mathrm{TBD}$ & ${ }_{\mathrm{U}^{\mathrm{R}}=3 \mathrm{eV}, \mathrm{TBD}^{\mathrm{T}}{ }^{\mathrm{r}}}$ & \\
\hline
\end{tabular}

TABLE CCXXIII. Topology phase diagram of Mn3Ni20P6. 


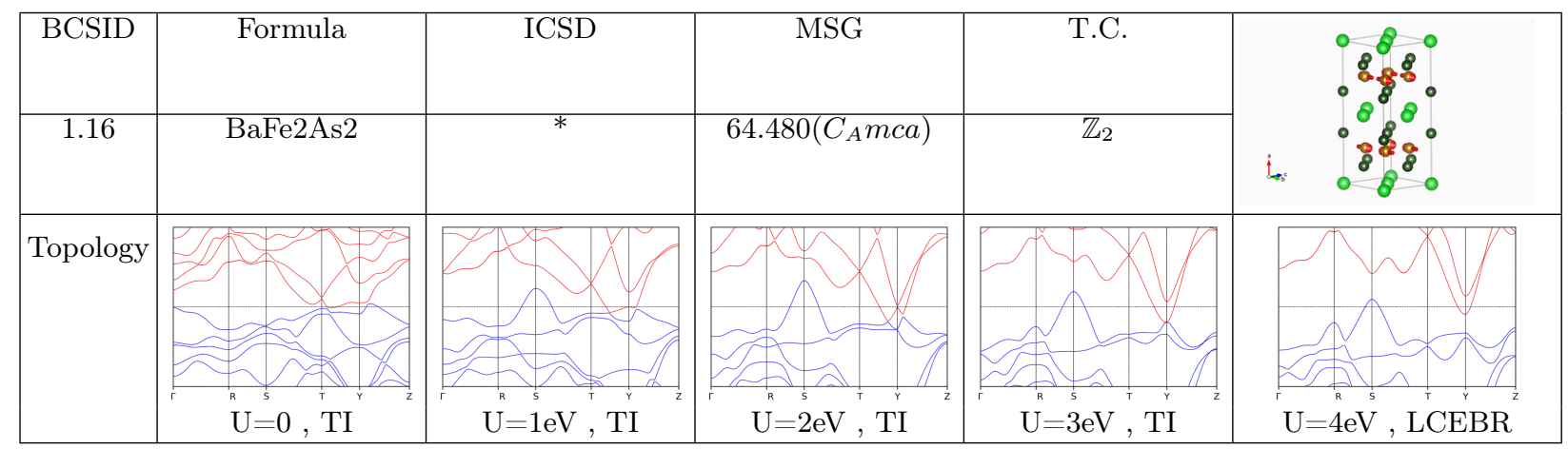

TABLE CCXXIV. Topology phase diagram of BaFe2As2.

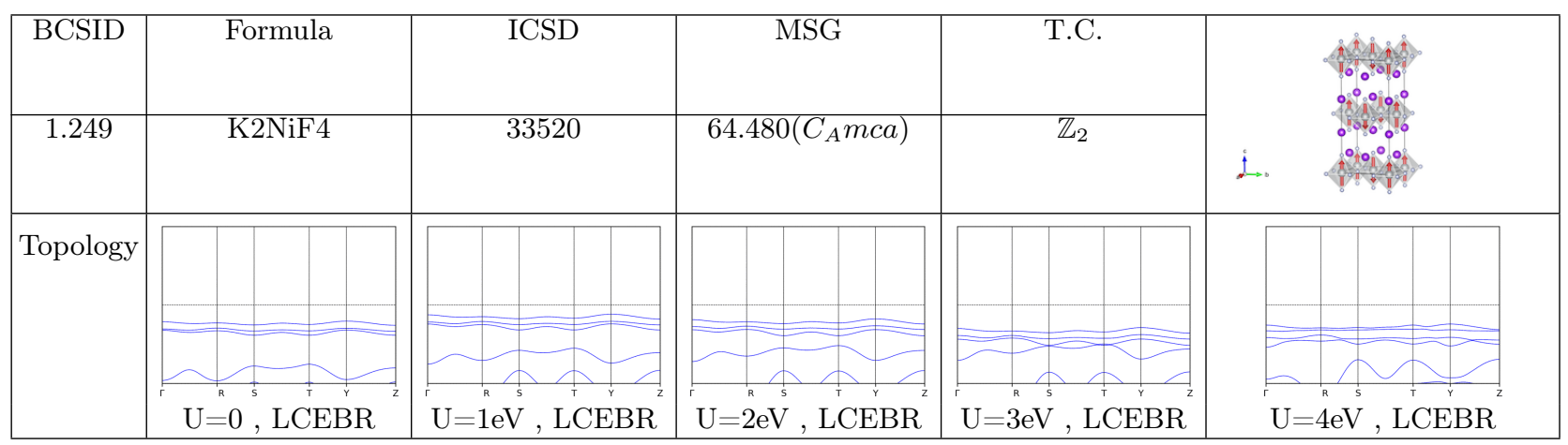

TABLE CCXXV. Topology phase diagram of K2NiF4.

\begin{tabular}{|c|c|c|c|c|c|}
\hline BCSID & Formula & ICSD & MSG & T.C. & \\
\hline 1.52 & CaFe2As2 & T & $64.480\left(C_{A} m c a\right)$ & $\mathbb{Z}_{2}$ & \\
\hline \multicolumn{6}{|l|}{ Topology } \\
\hline & $\mathrm{U}^{\mathrm{R}}=0, \mathrm{~T}, \mathrm{TI}$ & $\mathrm{U}^{\mathrm{R}}=1 \mathrm{eV}^{\mathrm{s}}, \mathrm{T}$ & $\mathrm{U}^{\mathrm{R}}=2 \mathrm{~s} \mathrm{eV}^{\top}, \mathrm{TI}^{\mathrm{r}}$ & $\mathrm{U}=3^{\mathrm{R}} \mathrm{eV}^{\mathrm{s}}, \mathrm{LCEBR}^{\top}$ & $\mathrm{U}=4^{\mathrm{R}} \mathrm{eV}^{\mathrm{s}}, \mathrm{LCEBR}^{\top}$ \\
\hline
\end{tabular}

TABLE CCXXVI. Topology phase diagram of CaFe2As2.

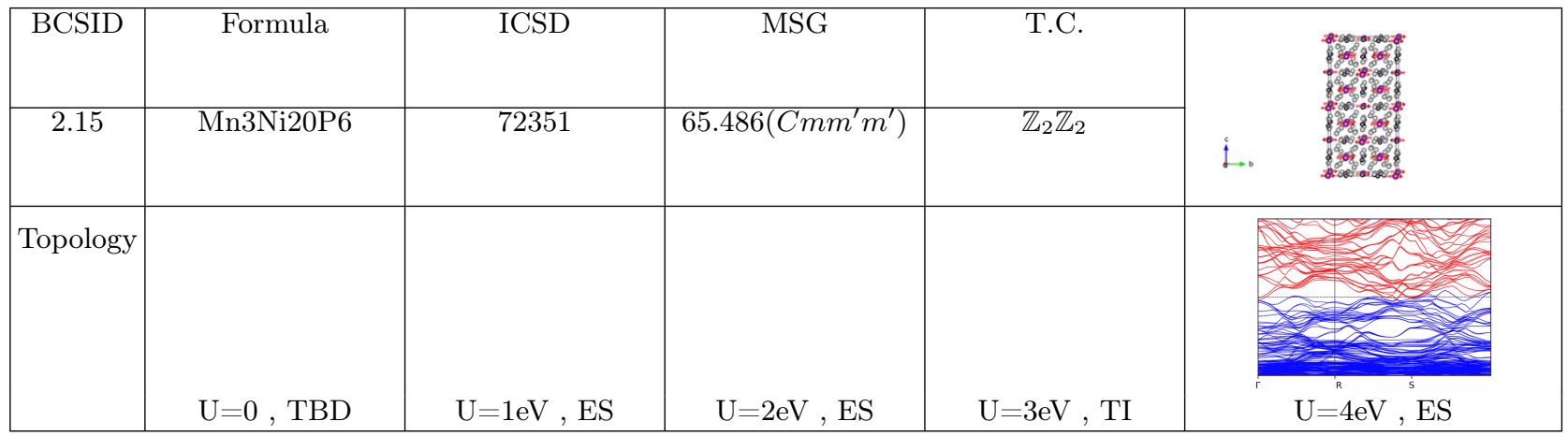

TABLE CCXXVII. Topology phase diagram of Mn3Ni20P6. 


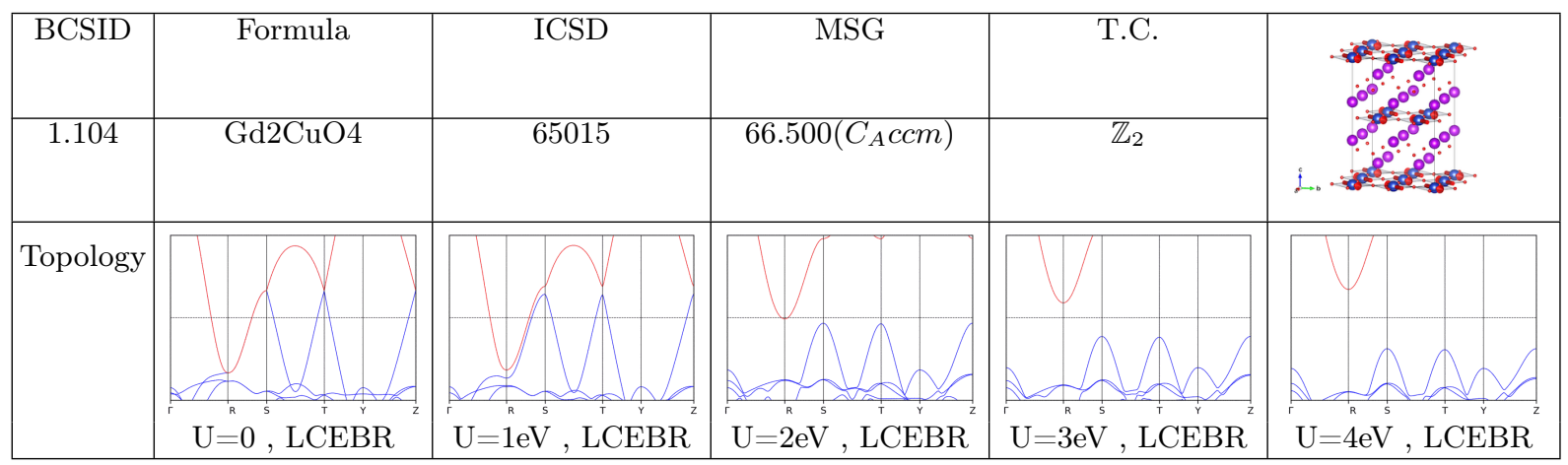

TABLE CCXXVIII. Topology phase diagram of Gd2CuO4.

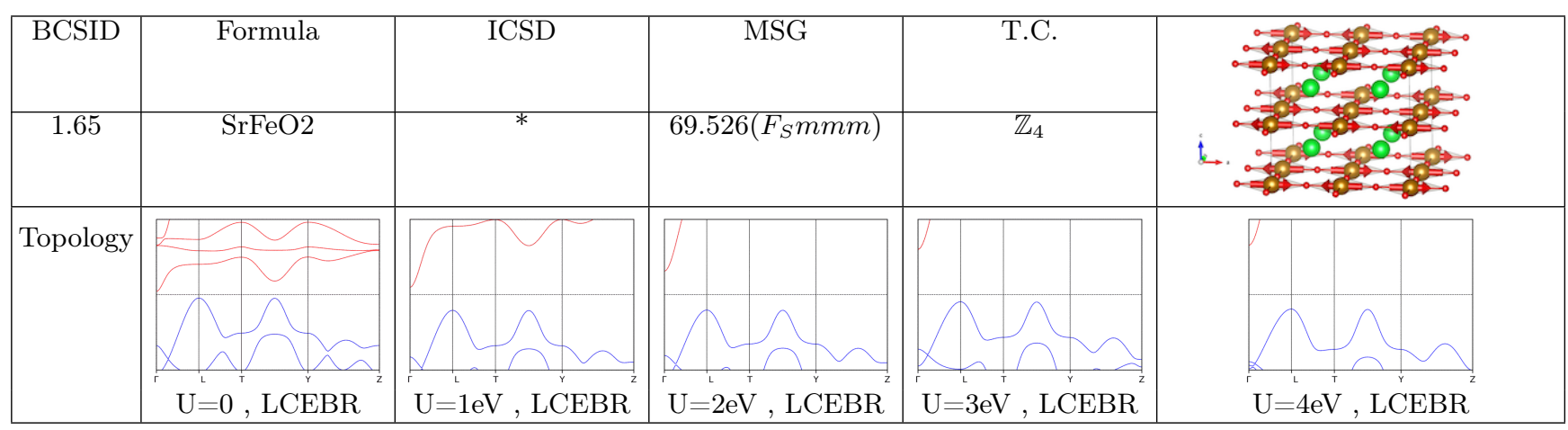

TABLE CCXXIX. Topology phase diagram of SrFeO2.

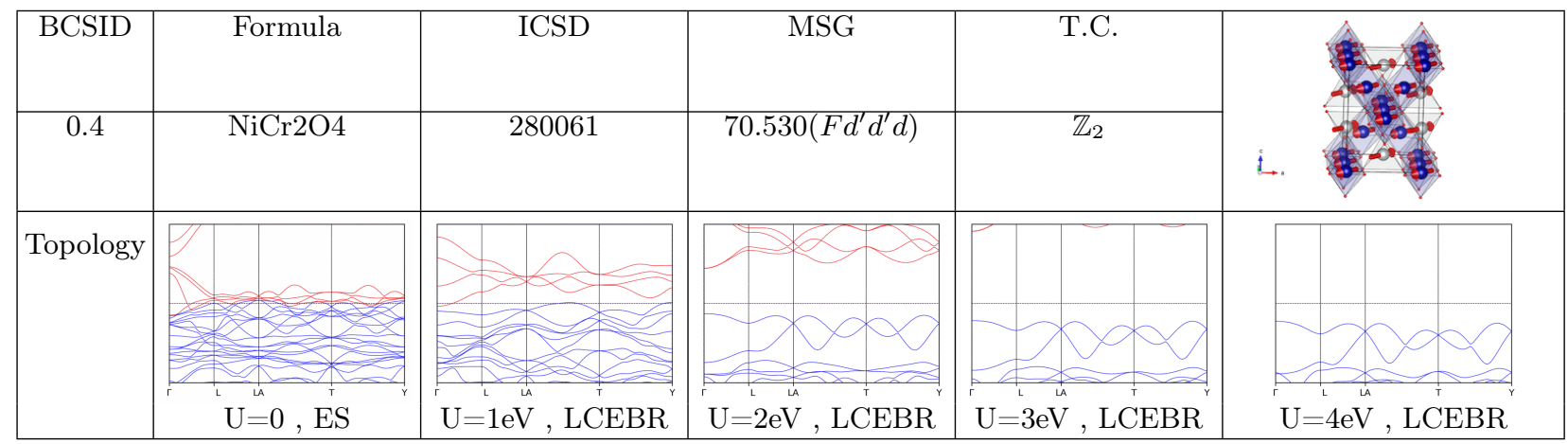

TABLE CCXXX. Topology phase diagram of NiCr2O4.

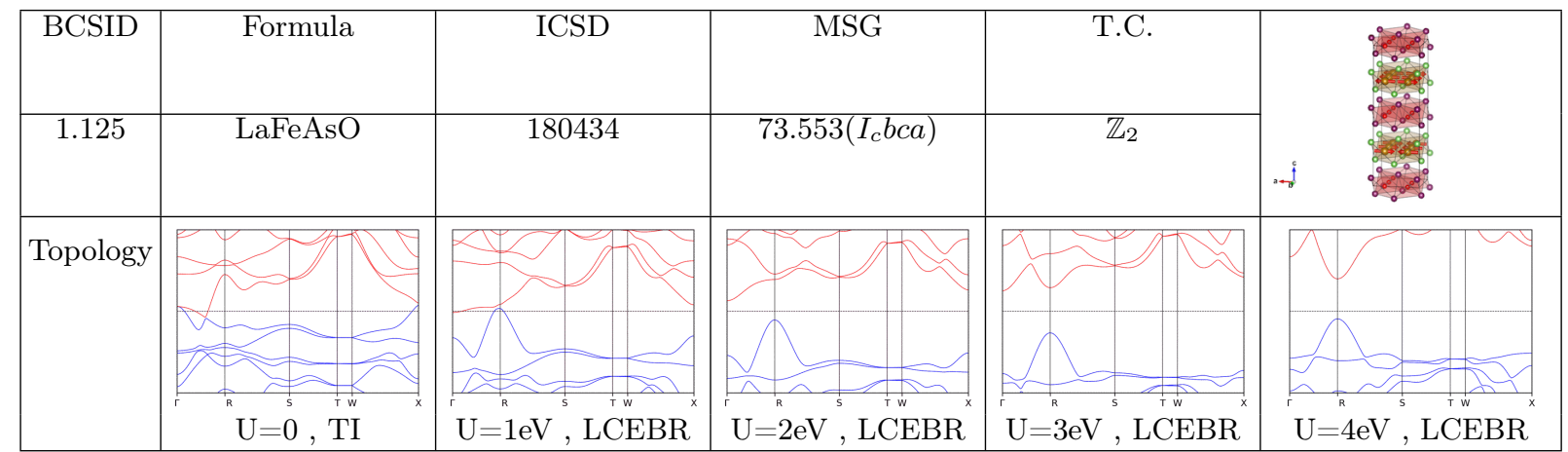

TABLE CCXXXI. Topology phase diagram of LaFeAsO. 


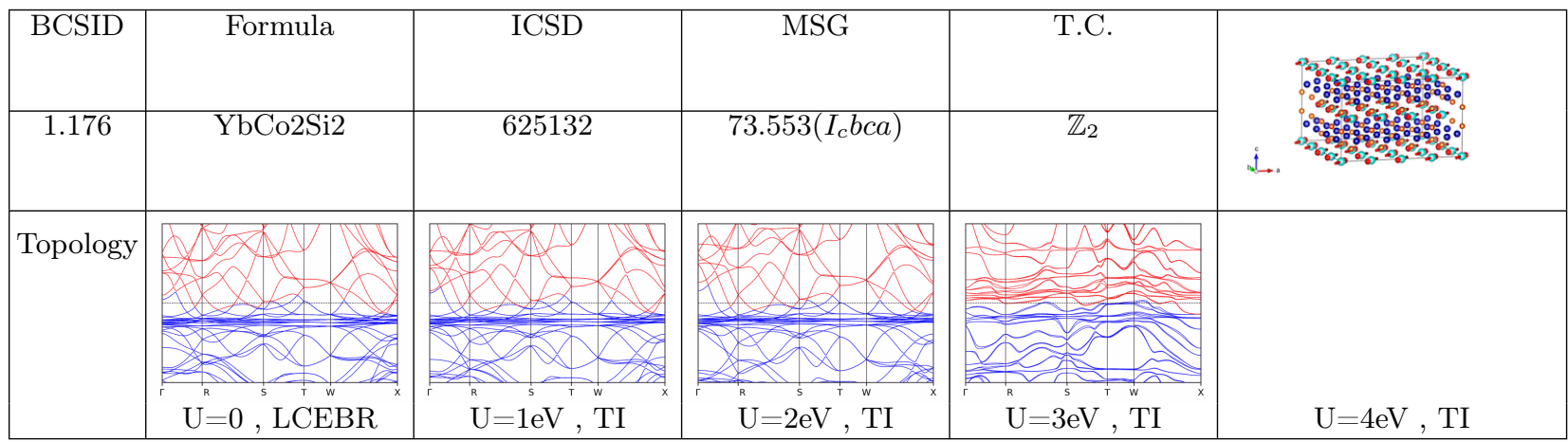

TABLE CCXXXII. Topology phase diagram of YbCo2Si2.

\begin{tabular}{|c|c|c|c|c|c|}
\hline \begin{tabular}{|l|l} 
BCSID \\
\end{tabular} & Formula & ICSD & MSG & T.C. & \\
\hline 1.47 & $\mathrm{Sr} 2 \mathrm{FeOsO} 6$ & * & $83.50\left(P_{I} 4 / m\right)$ & $\mathbb{Z}_{4}$ & \\
\hline Topology & & & 7 & & \\
\hline & $\mathrm{U}=0^{A}{ }^{M}, \mathrm{LCEBR}$ & $\mathrm{U}=1 \mathrm{eV}^{\mathrm{n}}, \mathrm{LCE}^{\mathrm{n}} \mathrm{CER}^{\mathrm{x}}$ & $\mathrm{U}=2 \mathrm{eV}^{\mathrm{m}}, \mathrm{LCE}^{\mathrm{n}} \mathrm{CER}^{\mathrm{x}}$ & $\mathrm{U}=3 \mathrm{eV}^{\mathrm{n}}, \mathrm{LCEBR}^{\mathrm{n}}$ & $\mathrm{U}=4 \mathrm{eV}^{\mathrm{n}}, \mathrm{I}$ \\
\hline
\end{tabular}

TABLE CCXXXIII. Topology phase diagram of $\mathrm{Sr} 2 \mathrm{FeOsO6.}$

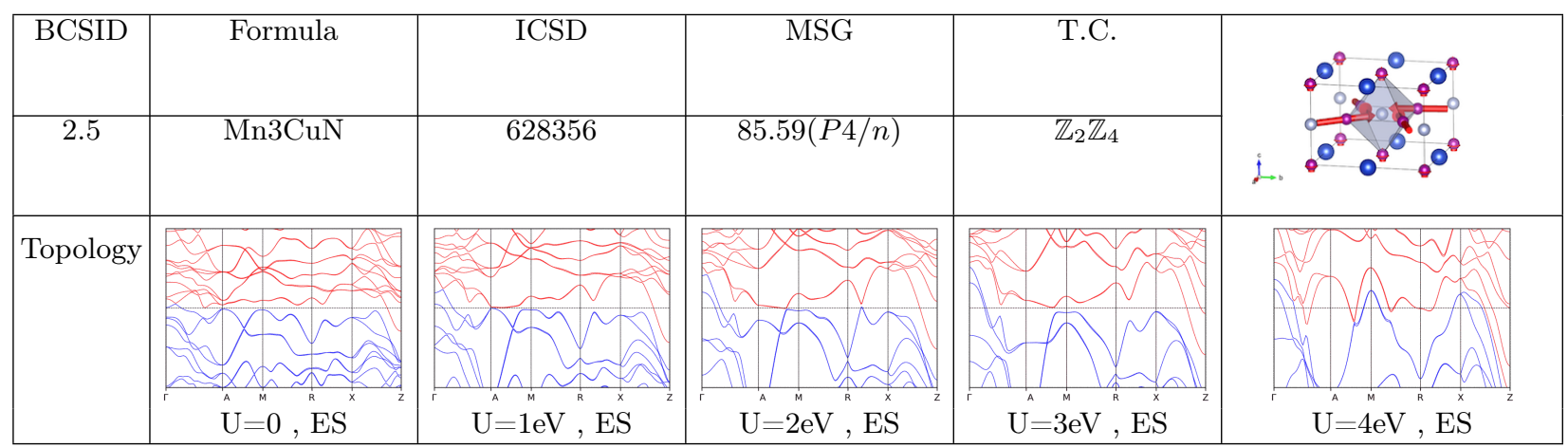

TABLE CCXXXIV. Topology phase diagram of Mn3CuN.

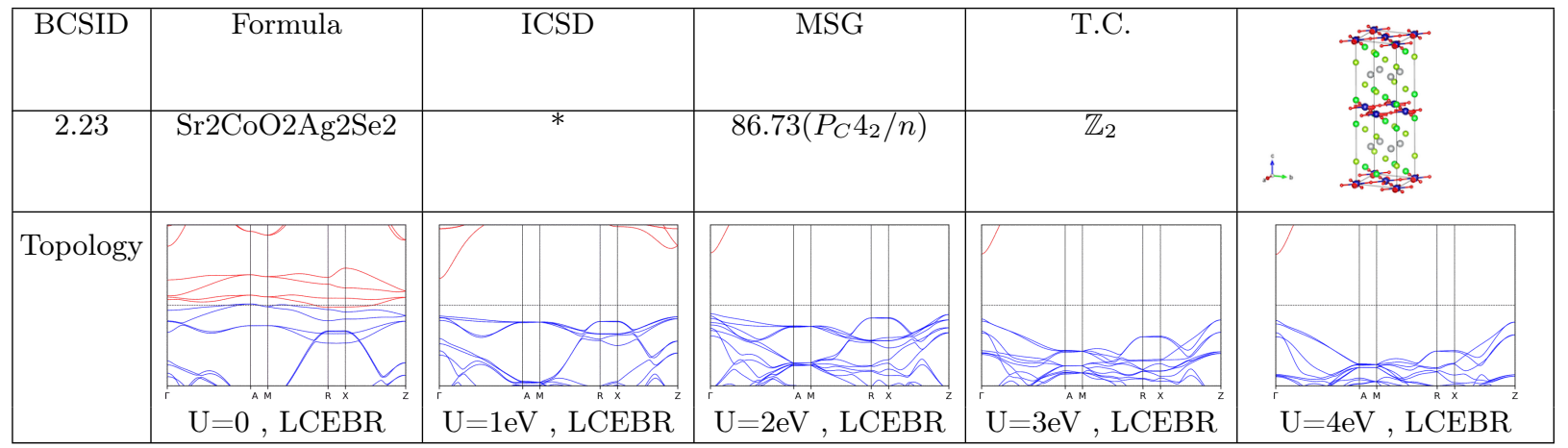

TABLE CCXXXV. Topology phase diagram of Sr2CoO2Ag2Se2. 


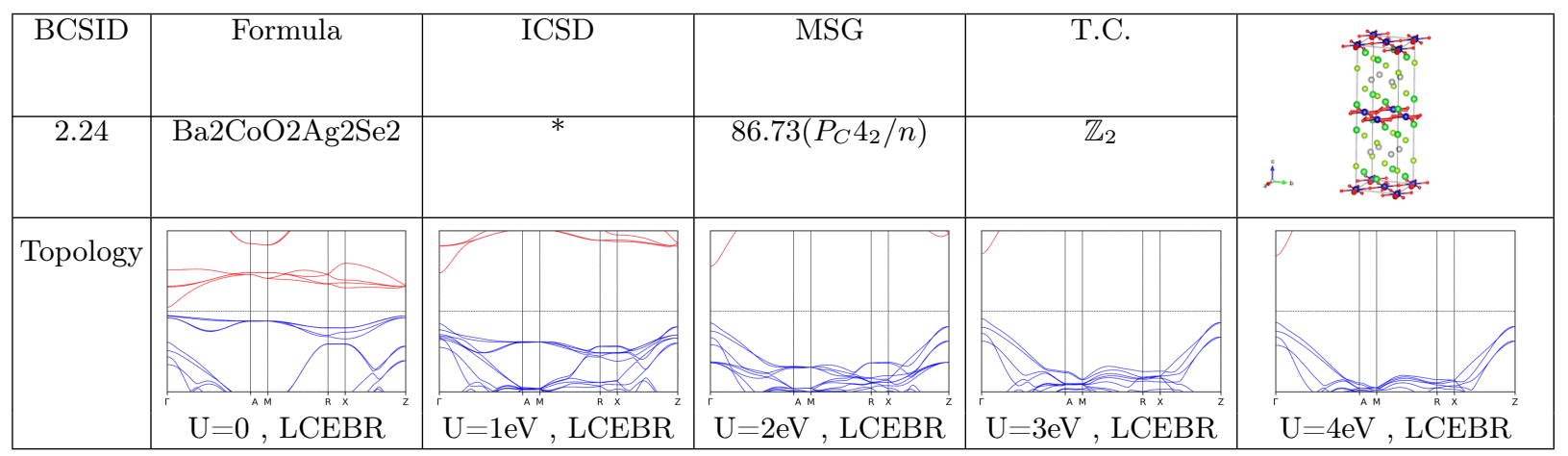

TABLE CCXXXVI. Topology phase diagram of Ba2CoO2Ag2Se2.

\begin{tabular}{|c|c|c|c|c|c|}
\hline BCSID & Formula & ICSD & $\overline{\mathrm{MSG}}$ & T.C. & \\
\hline 0.64 & $\mathrm{MnV} 2 \mathrm{O} 4$ & $*$ & $88.81\left(I 4_{1} / a\right)$ & $\mathbb{Z}_{2} \mathbb{Z}_{2}$ & i. $\quad 0-500$ \\
\hline Topology & & & & & \\
\hline & $\mathrm{U}=0^{M}, \mathrm{ES}$ & $\mathrm{U}=1 \mathrm{eV}, \mathrm{LCEBR}^{\mathrm{m}}$ & $\mathrm{U}=2 \mathrm{eV}^{\prime}, \mathrm{LCEBR}$ & $\mathrm{U}=3 \mathrm{eV}^{\prime \prime}, \mathrm{LCEBR}$ & $\mathrm{U}=4 \mathrm{eV}, \mathrm{LCEBR}$ \\
\hline
\end{tabular}

TABLE CCXXXVII. Topology phase diagram of MnV2O4.

\begin{tabular}{|c|c|c|c|c|c|}
\hline BCSID & Formula & ICSD & MSG & T.C. & \\
\hline 2.22 & FeTa2O6 & 201754 & $88.86\left(I_{c} 4_{1} / a\right)$ & $\mathbb{Z}_{2}$ & 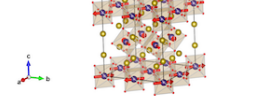 \\
\hline Topology & \begin{tabular}{|l|l|l|} 
& & \\
& & \\
\end{tabular} & Wos & & & \\
\hline & $\mathrm{U}=0, \mathrm{LCEBR}$ & $\mathrm{U}=1 \mathrm{eV}, \mathrm{LCEBR}$ & $\mathrm{U}=2 \mathrm{eV}, \mathrm{LCEBR}$ & $\mathrm{U}=3 \mathrm{eV}, \mathrm{LCEBR}$ & $\mathrm{U}=4 \mathrm{eV}, \mathrm{LCEBR}$ \\
\hline
\end{tabular}

TABLE CCXXXVIII. Topology phase diagram of FeTa2O6.

\begin{tabular}{|c|c|c|c|c|c|c|c|}
\hline BCSID & Formula & ICSD & \multicolumn{2}{|c|}{ MSG } & \multicolumn{2}{|c|}{ T.C. } & \multirow{2}{*}{ 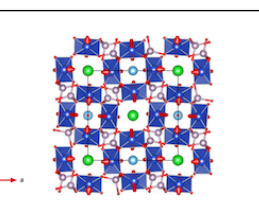 } \\
\hline 1.235 & $\mathrm{Ba}(\mathrm{TiO}) \mathrm{Cu} 4(\mathrm{PO} 4) 4$ & 239146 & \multicolumn{2}{|c|}{$94.132\left(P_{c} 4_{2} 2_{1} 2\right)$} & \multicolumn{2}{|r|}{$\mathrm{w} / \mathrm{O}$} & \\
\hline \multirow[t]{4}{*}{ Topology } & \multirow[b]{4}{*}{$\mathrm{U}=0, \mathrm{LCEBR}$} & \multirow[b]{4}{*}{$\mathrm{U}=1 \mathrm{eV}, \mathrm{LCEBR}$} & & 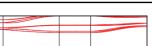 & 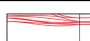 & 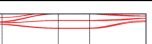 & \\
\hline & & & Es & $\sum=$ & 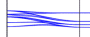 & $\Longrightarrow$ & $\sum$ \\
\hline & & & 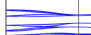 & 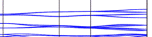 & E & 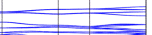 & 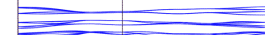 \\
\hline & & & $\mathrm{U}=2 \mathrm{e}^{\mathrm{A}}$ & , LCEBR & $\mathrm{U}=3 \mathrm{e}^{\mathrm{A}}$ & , LCEBR & $\mathrm{I} U=4 \mathrm{eV}^{\mathrm{A}}, \mathrm{LCEBR}$ \\
\hline
\end{tabular}

TABLE CCXXXIX. Topology phase diagram of $\mathrm{Ba}(\mathrm{TiO}) \mathrm{Cu} 4(\mathrm{PO} 4) 4$. 


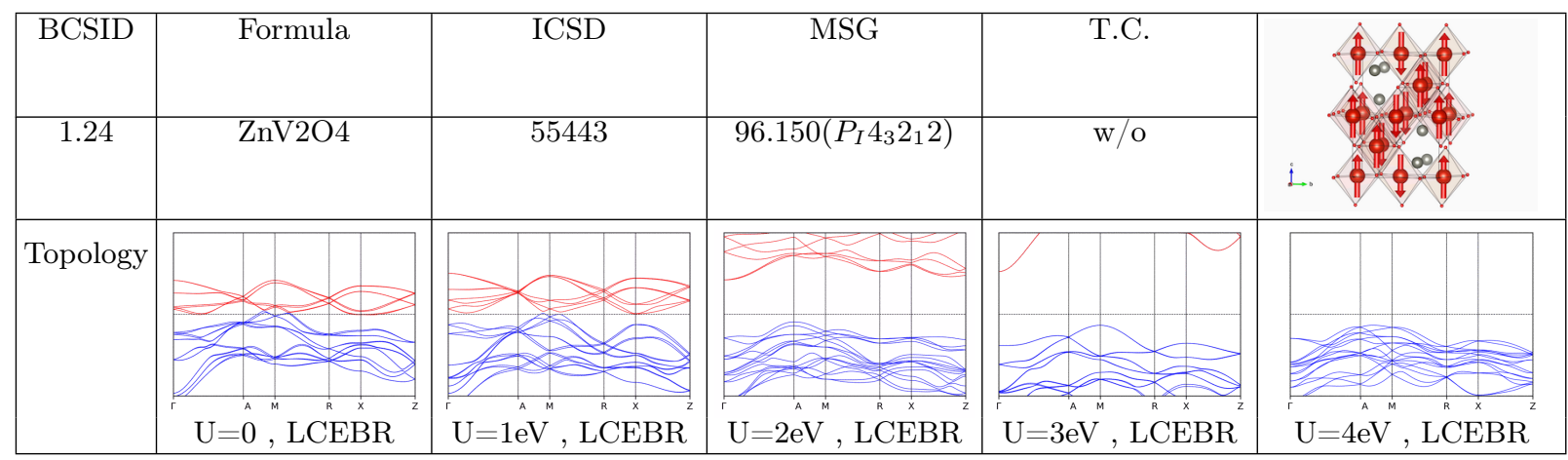

TABLE CCXL. Topology phase diagram of ZnV2O4.

\begin{tabular}{|c|c|c|c|c|c|}
\hline BCSID & Formula & ICSD & MSG & T.C. & \\
\hline 3.4 & $\mathrm{MgCr} 2 \mathrm{O} 4$ & * & $111.255\left(P 42^{\prime} m^{\prime}\right)$ & $\mathbb{Z}_{2} \mathbb{Z}_{2} \mathbb{Z}_{4}$ & \\
\hline Topology & N1\% & & & & \\
\hline & Y & +3 & 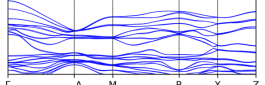 & $\frac{3}{25}$ & 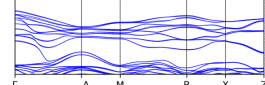 \\
\hline & $\mathrm{U}=0, \mathrm{LCEBR}$ & $\mathrm{U}=1 \mathrm{eV}, \mathrm{LCEBR}$ & $\mathrm{U}=2 \mathrm{eV}, \mathrm{LCEBR}$ & $\mathrm{U}=3 \mathrm{eV}, \mathrm{LCEBR}$ & $\mathrm{U}=4 \mathrm{eV}, \mathrm{LCEBR}$ \\
\hline
\end{tabular}

TABLE CCXLI. Topology phase diagram of MgCr2O4.

\begin{tabular}{|c|c|c|c|c|c|}
\hline BCSID & Formula & ICSD & MSG & T.C. & \\
\hline 1.85 & alpha-Mn & & $114.282\left(P_{I} 42_{1} c\right)$ & $\overline{\mathbb{Z}_{2}}$ & t. Thas \\
\hline Topology & $\frac{\mathrm{A}_{\mathrm{m}=0, \mathrm{ES}^{\mathrm{x}}}}{\mathrm{U}}$ & $\mathrm{U}_{\mathrm{U}=1 \mathrm{eV} \mathrm{V}^{\prime \prime}, \mathrm{LCEBR}^{\mathrm{n}}}^{\mathrm{x}}$ & $\mathrm{U}_{\mathrm{U}=2 \mathrm{eV}, \mathrm{LCEBR}}^{\mathrm{n}}$ & 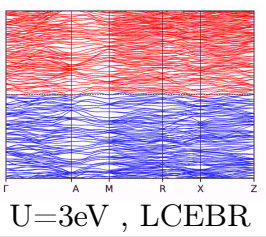 & $\mathrm{U}=4 \mathrm{eV}, \mathrm{TBD}$ \\
\hline
\end{tabular}

TABLE CCXLII. Topology phase diagram of alpha-Mn.

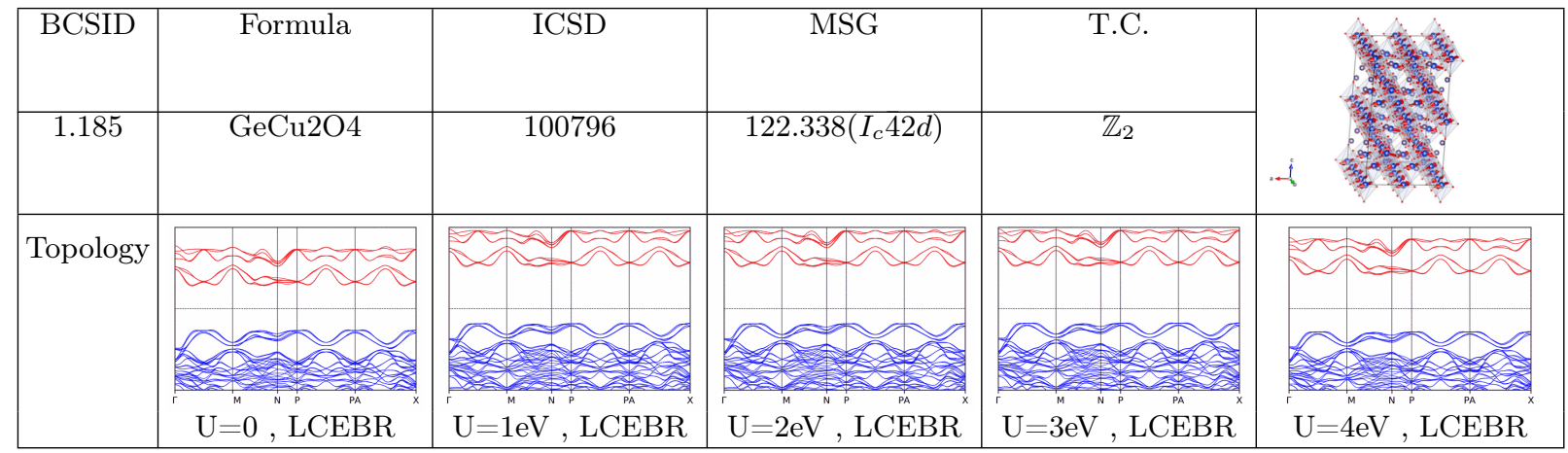

TABLE CCXLIII. Topology phase diagram of GeCu2O4. 


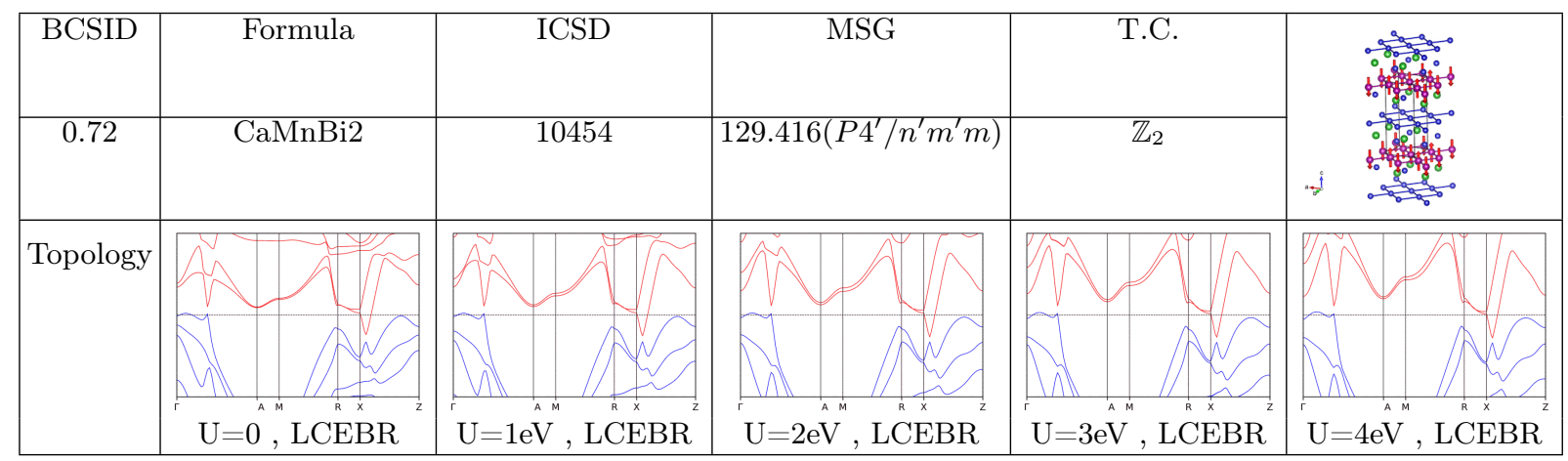

TABLE CCXLIV. Topology phase diagram of CaMnBi2.

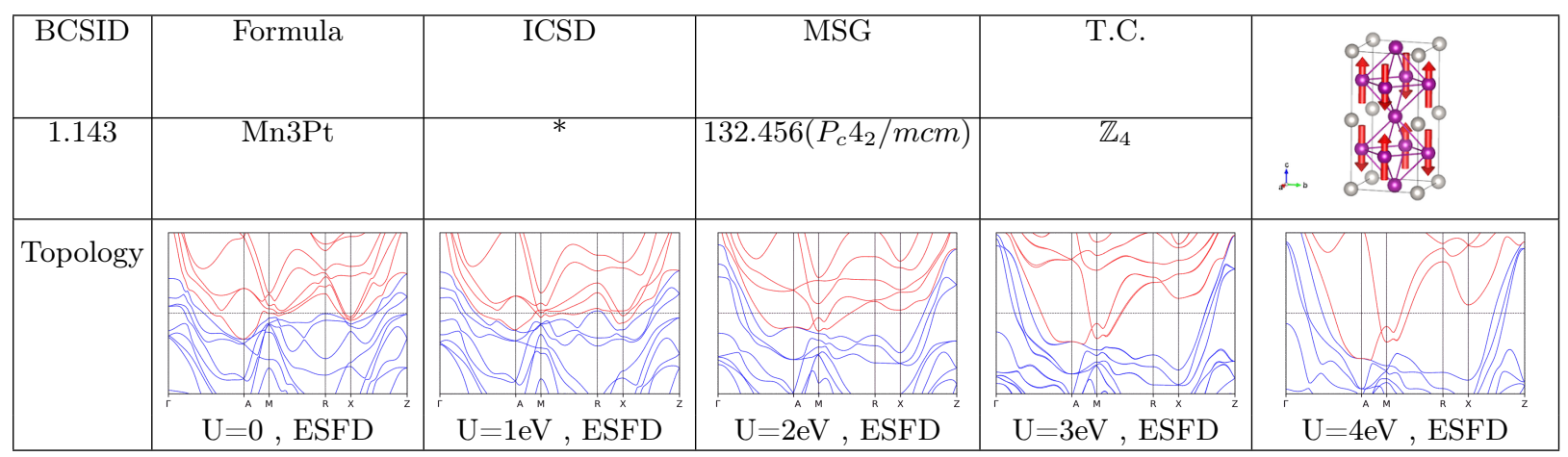

TABLE CCXLV. Topology phase diagram of Mn3Pt.

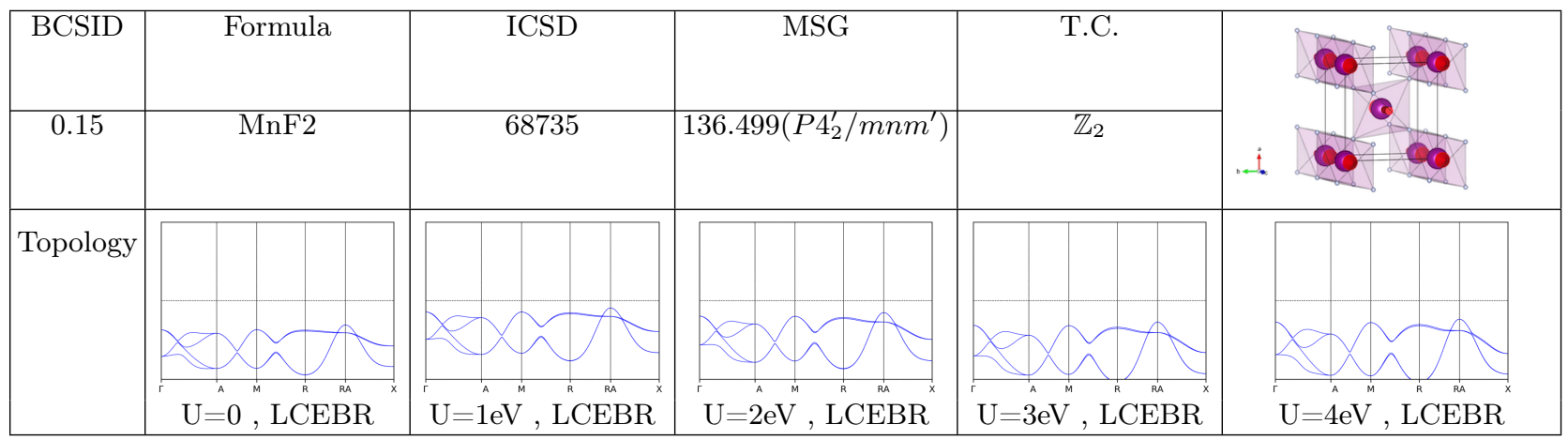

TABLE CCXLVI. Topology phase diagram of MnF2.

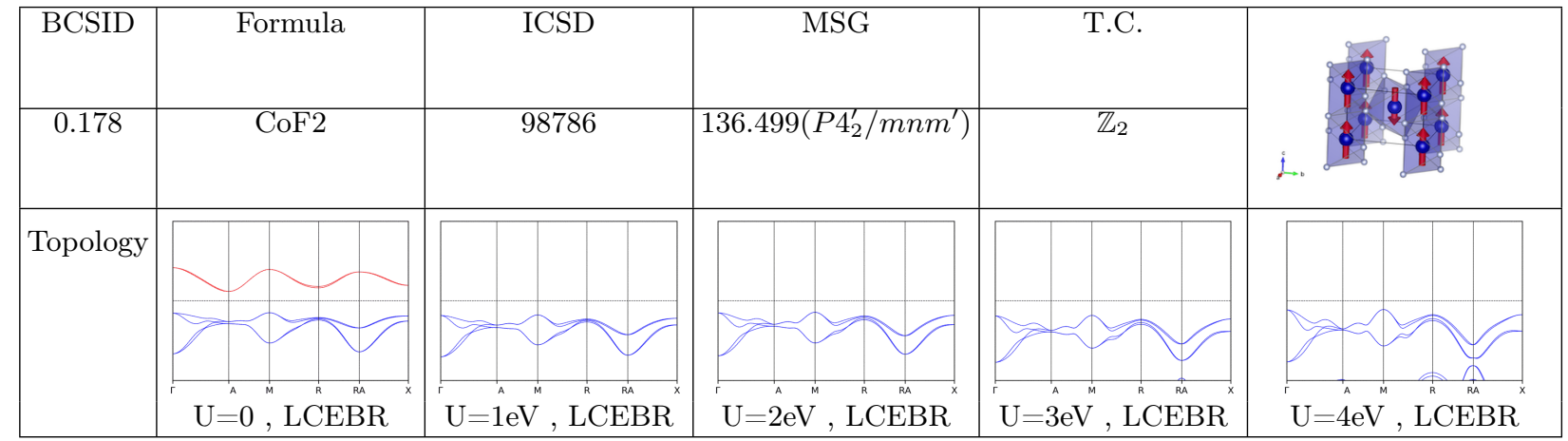

TABLE CCXLVII. Topology phase diagram of CoF2. 


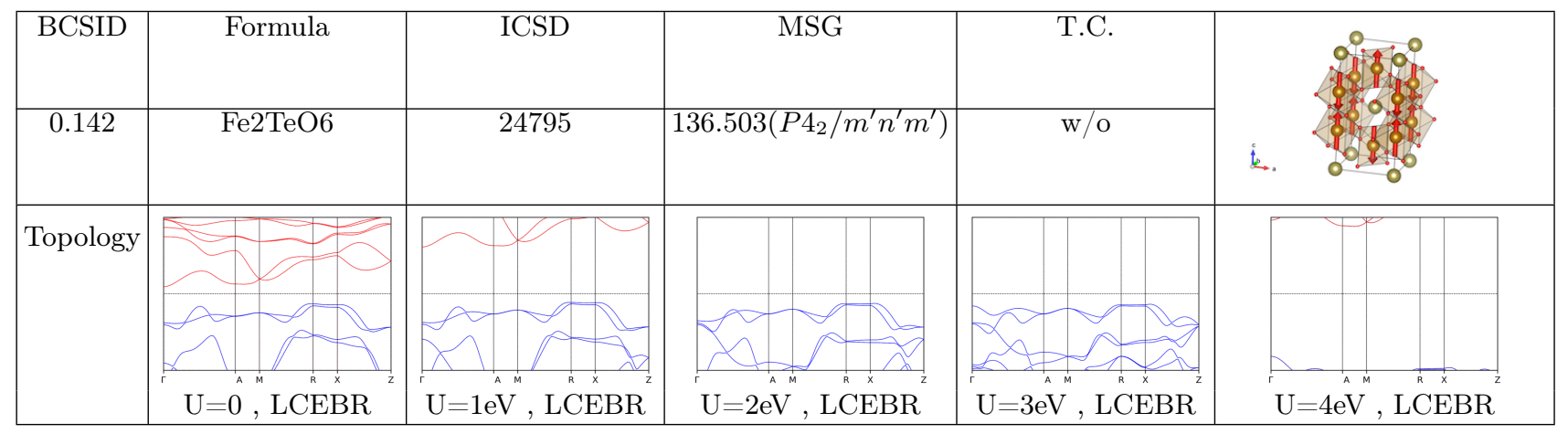

TABLE CCXLVIII. Topology phase diagram of Fe2TeO6.

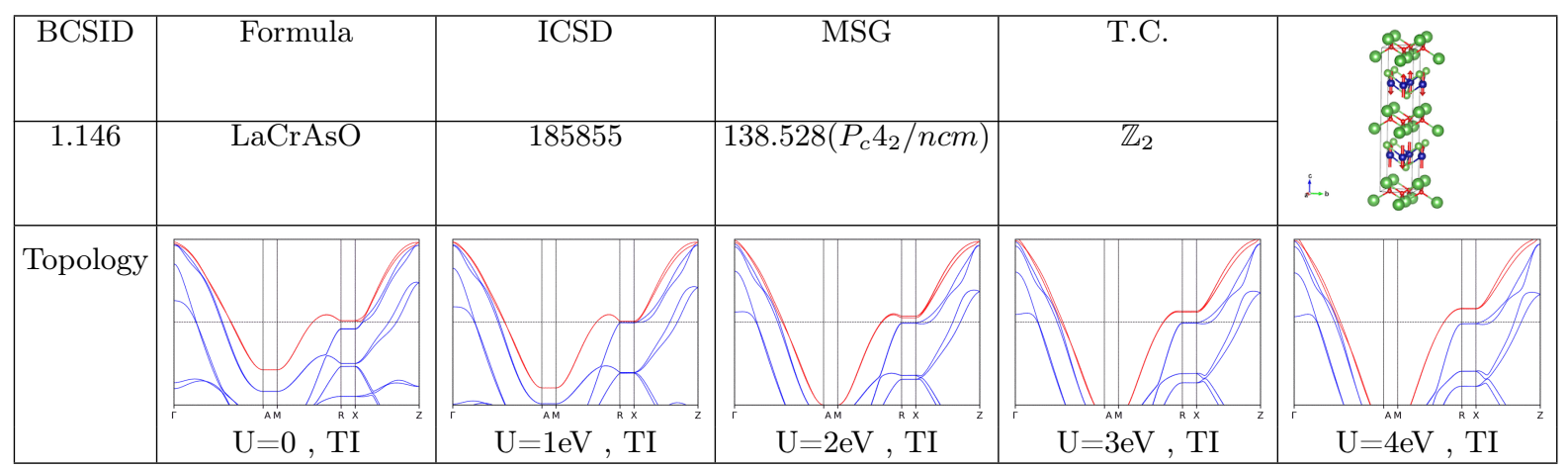

TABLE CCXLIX. Topology phase diagram of LaCrAsO.

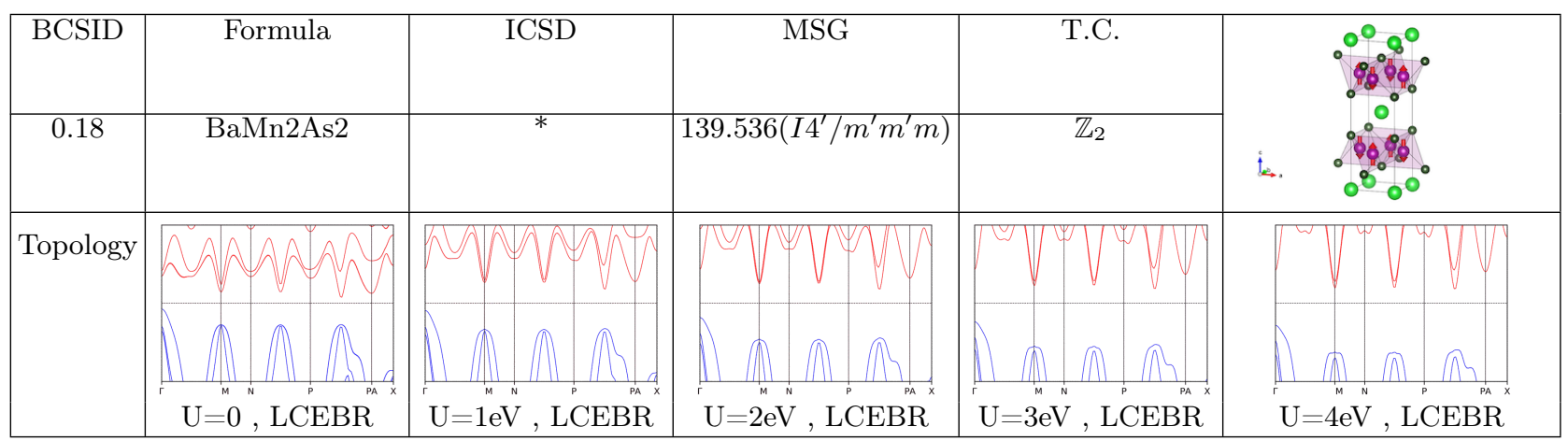

TABLE CCL. Topology phase diagram of BaMn2As2.

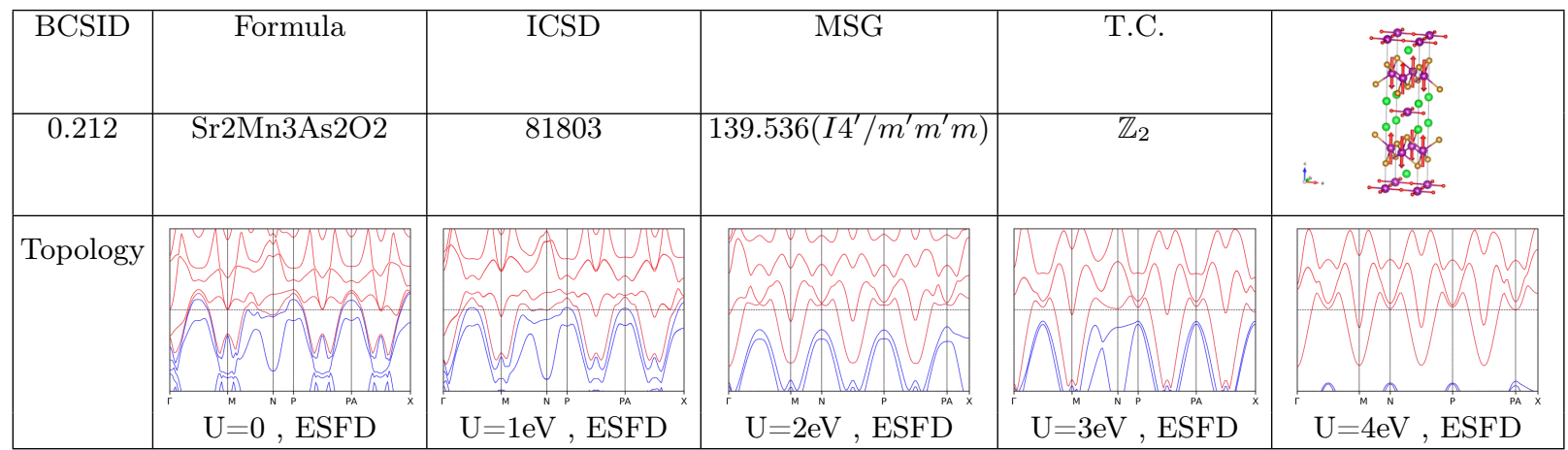

TABLE CCLI. Topology phase diagram of Sr2Mn3As2O2. 


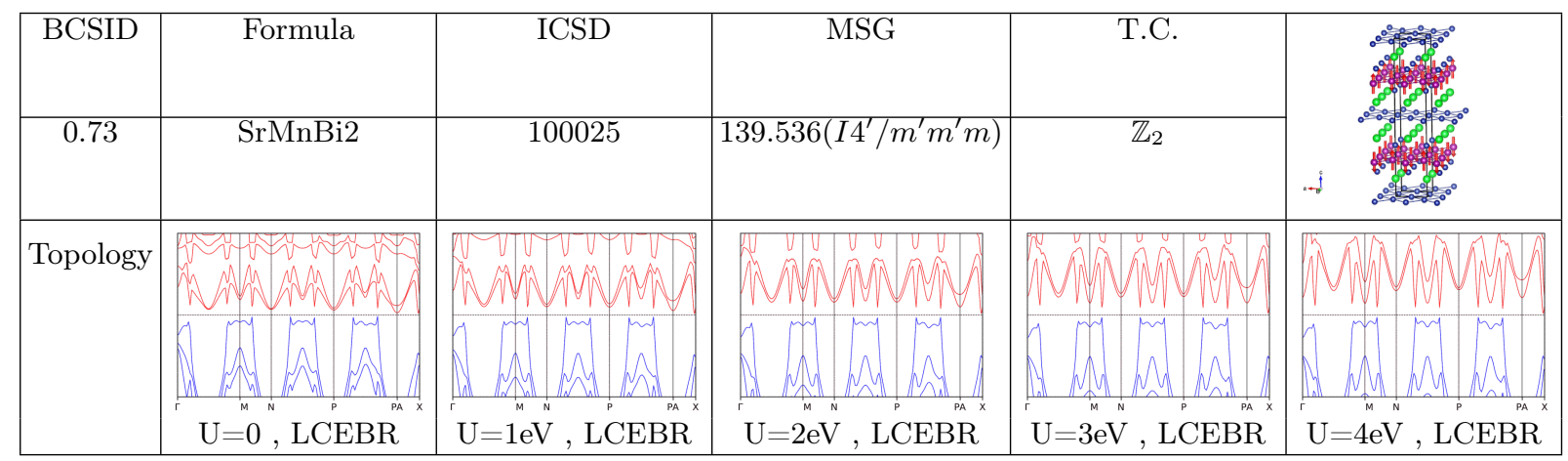

TABLE CCLII. Topology phase diagram of SrMnBi2.

\begin{tabular}{|c|c|c|c|c|c|}
\hline BCSID & Formula & ICSD & $\mathrm{MSG}$ & T.C. & $0^{\circ}$ \\
\hline 0.89 & BaMn2Bi2 & * & $139.536\left(I 4^{\prime} / m^{\prime} m^{\prime} m\right)$ & $\mathbb{Z}_{2}$ & i. $0_{0}^{0}$ \\
\hline Topology & & & & & \\
\hline & $\mathrm{U}=0, \mathrm{LCEB}$ & $\mathrm{U}=1 \mathrm{eV}, \mathrm{LCEBR}$ & $\mathrm{U}=2 \mathrm{eV}, \mathrm{LCEBR}$ & $\mathrm{U}=3 \mathrm{eV}, \mathrm{LCEBR}$ & $\mathrm{U}=4 \mathrm{eV}, \mathrm{LCEBR}$ \\
\hline
\end{tabular}

TABLE CCLIII. Topology phase diagram of BaMn2Bi2.

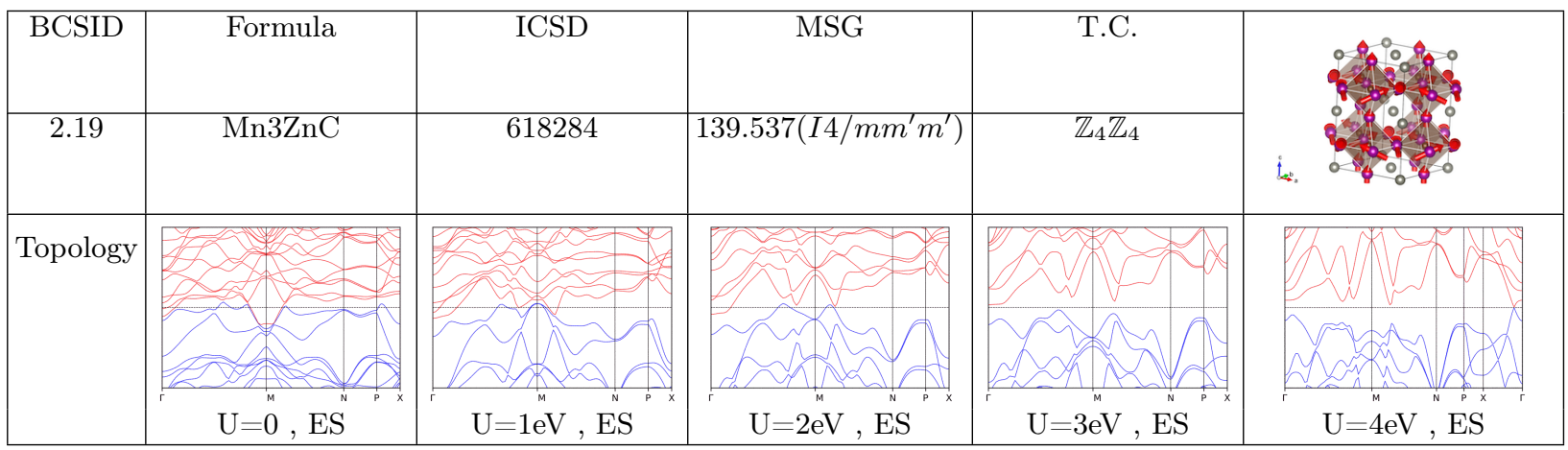

TABLE CCLIV. Topology phase diagram of Mn3ZnC.

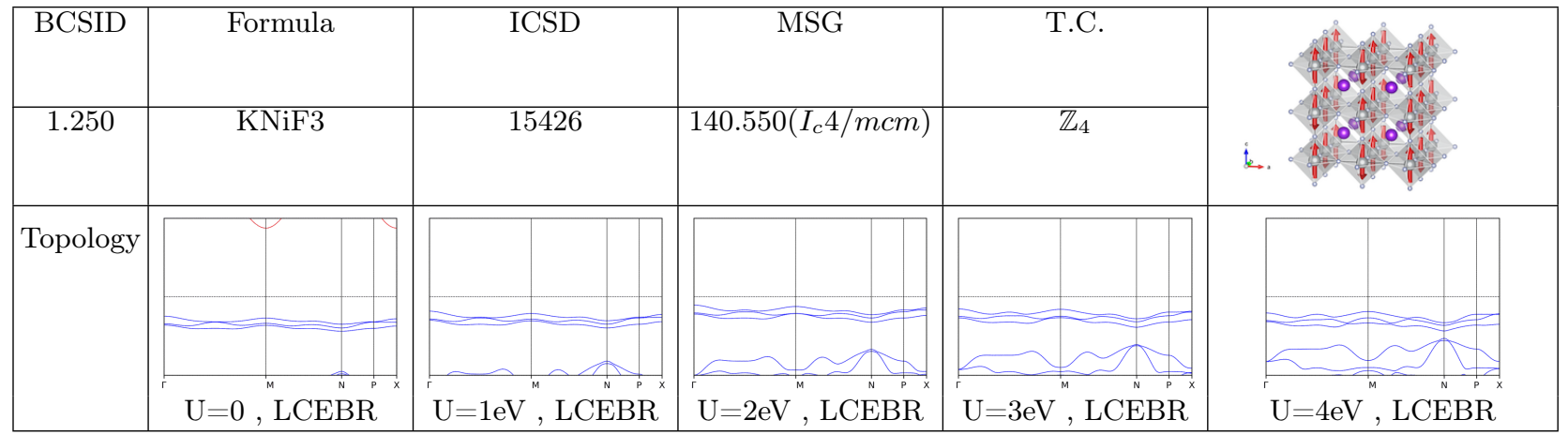

TABLE CCLV. Topology phase diagram of KNiF3. 


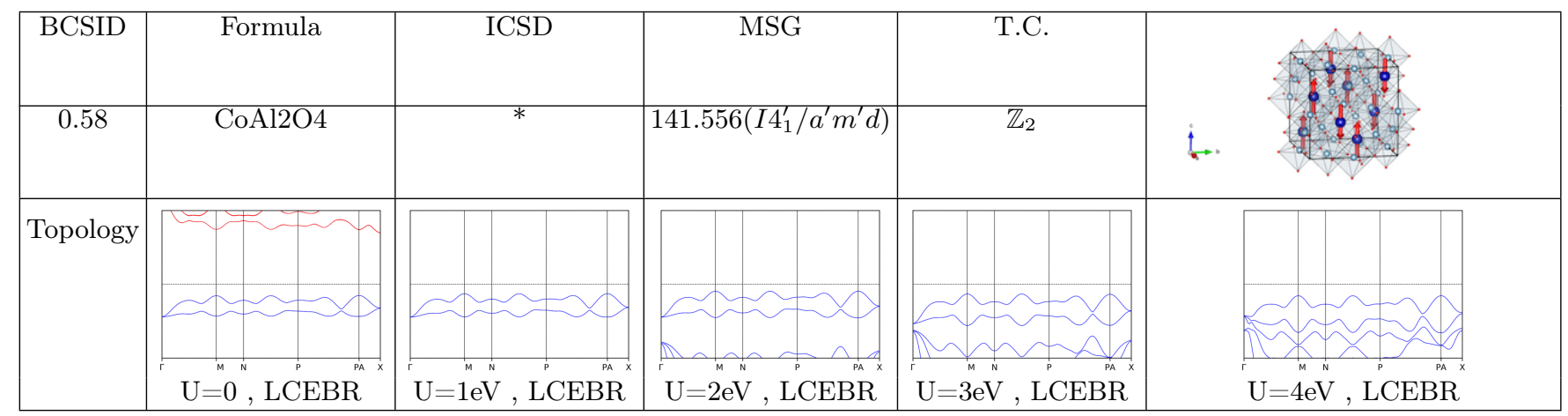

TABLE CCLVI. Topology phase diagram of CoAl2O4.

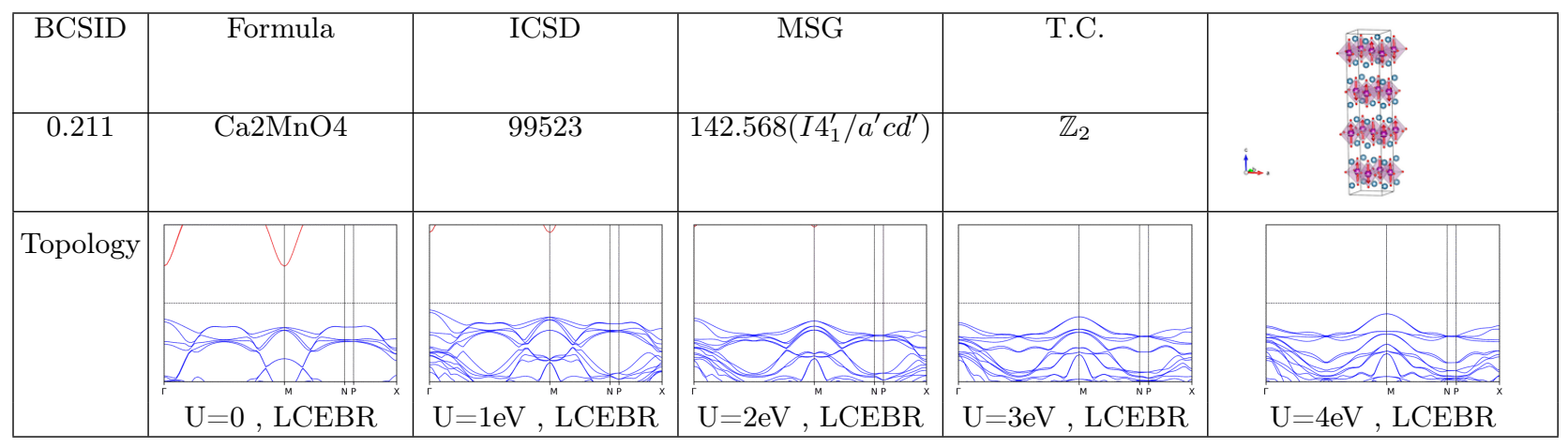

TABLE CCLVII. Topology phase diagram of Ca2MnO4.

\begin{tabular}{|c|c|c|c|c|c|}
\hline BCSID & Formula & ICSD & MSG & T.C. & \\
\hline 1.156 & LaMn3Cr4O12 & * & $146.12\left(R_{I} 3\right)$ & $\mathrm{w} / \mathrm{O}$ & \\
\hline \multirow[t]{3}{*}{ Topology } & 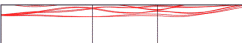 & & & & \\
\hline & 2 & 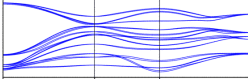 & & DE & \\
\hline & $\mathrm{U}=0, \mathrm{LCEBR}$ & $\mathrm{U}=1 \mathrm{eV}, \mathrm{LCEBR}$ & $\mathrm{U}=2 \mathrm{eV}, \mathrm{LCEBR}$ & $\mathrm{U}=3 \mathrm{eV}, \mathrm{LCEBR}$ & $\mathrm{U}=4 \mathrm{eV}, \mathrm{LCEBR}$ \\
\hline
\end{tabular}

TABLE CCLVIII. Topology phase diagram of LaMn3Cr4O12.

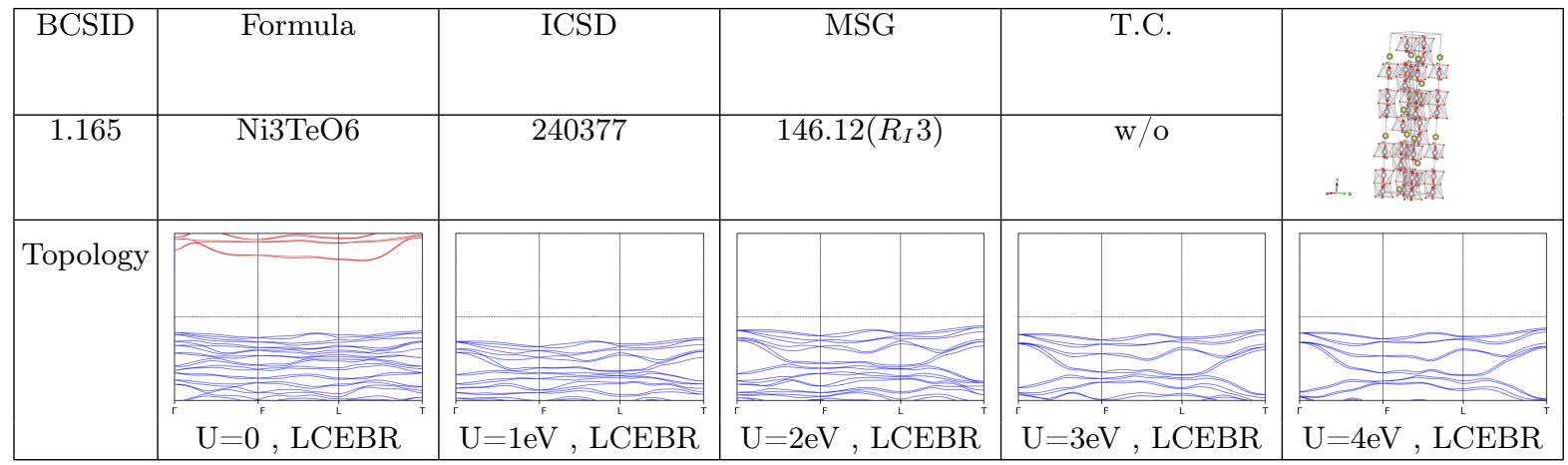

TABLE CCLIX. Topology phase diagram of Ni3TeO6. 


\begin{tabular}{|c|c|c|c|c|c|c|}
\hline BCSID & Formula & ICSD & MSG & \multicolumn{2}{|c|}{ T.C. } & sies it \\
\hline 0.78 & NiN2O6 & * & $148.17(R 3)$ & \multicolumn{2}{|c|}{$\mathbb{Z}_{2} \mathbb{Z}_{4}$} & \\
\hline \multicolumn{7}{|l|}{ Topology } \\
\hline & Elen & nes & 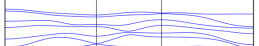 & $E$ & & 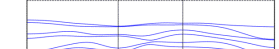 \\
\hline & 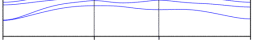 & Enara & $\sum N$ & $=$ & 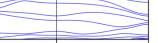 & $x_{S=n}$ \\
\hline & $\mathrm{U}=0, \mathrm{LCEBR}$ & $\mathrm{U}=1 \mathrm{eV}, \mathrm{LCEBR}$ & $\mathrm{U}=2 \mathrm{eV}, \mathrm{LCEBR}$ & $\mathrm{U}=3 \mathrm{eV}$ & , LCEBR & $\mathrm{U}=4 \mathrm{eV}, \mathrm{LCEBR}$ \\
\hline
\end{tabular}

TABLE CCLX. Topology phase diagram of NiN2O6.

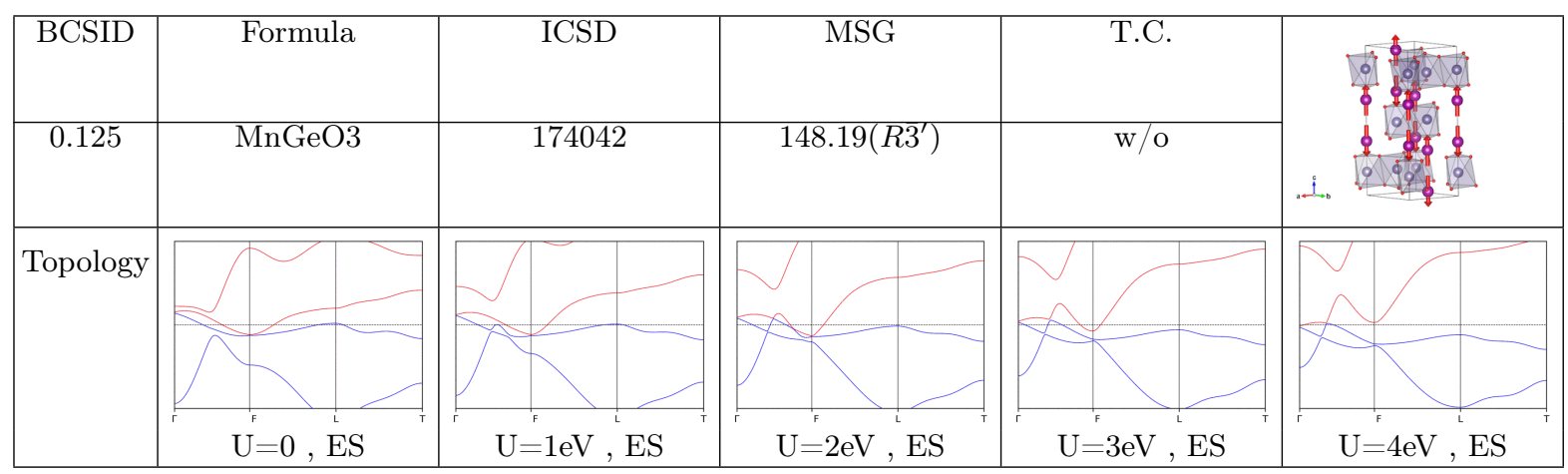

TABLE CCLXI. Topology phase diagram of MnGeO3.

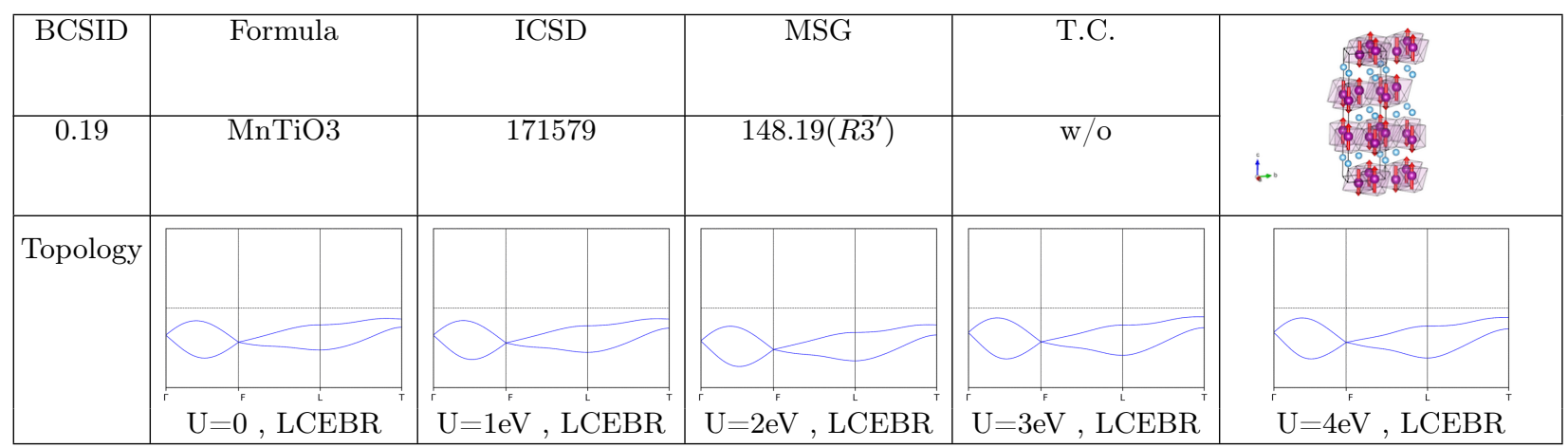

TABLE CCLXII. Topology phase diagram of MnTiO3.

\begin{tabular}{|c|c|c|c|c|c|}
\hline BCSID & Formula & ICSD & MSG & T.C. & \\
\hline 1.0 .8 & Ba3MnNb2O9 & $*$ & $157.53(P 31 \mathrm{~m})$ & $\mathrm{w} / \mathrm{o}$ & \\
\hline Topology & & & & & \\
& & & & & \\
& & & & & \\
$\mathrm{U}=0, \mathrm{LCEBR}$ & $\mathrm{U}=1 \mathrm{eV}, \mathrm{LCEBR}$ & $\mathrm{U}=2 \mathrm{eV}, \mathrm{TBD}$ & $\mathrm{U}=3 \mathrm{eV}, \mathrm{LCEBR}$ & $\mathrm{U}=4 \mathrm{eV}, \mathrm{LCEBR}$ \\
\hline
\end{tabular}

TABLE CCLXIII. Topology phase diagram of Ba3MnNb2O9. 


\begin{tabular}{|c|c|c|c|c|c|}
\hline BCSID & Formula & ICSD & MSG & T.C. & \\
\hline 1.13 & Ba3Nb2NiO9 & 240280 & $159.64\left(P_{c} 31 c\right)$ & $\mathrm{w} / \mathrm{O}$ & \\
\hline \multicolumn{6}{|l|}{ Topology } \\
\hline & $\mathrm{U}=0, \mathrm{LCEBR}$ & $\mathrm{U}=1 \mathrm{eV}, \mathrm{TBD}$ & $\mathrm{U}=2 \mathrm{eV}, \mathrm{LCEBR}$ & $\mathrm{U}=3 \mathrm{eV}, \mathrm{LCEBR}$ & $\mathrm{U}=4 \mathrm{eV}, \mathrm{TBD}$ \\
\hline
\end{tabular}

TABLE CCLXIV. Topology phase diagram of Ba3Nb2NiO9.

\begin{tabular}{|c|c|c|c|c|c|}
\hline BCSID & Formula & ICSD & $\mathrm{MSG}$ & T.C. & \\
\hline 1.237 & $\mathrm{VCl} 2$ & 246905 & $159.64\left(P_{c} 31 c\right)$ & $\mathrm{w} / \mathrm{o}$ & 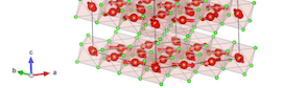 \\
\hline Topology & 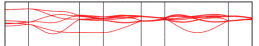 & & & & $E$ \\
\hline & $\mathrm{U}=0, \mathrm{LCEBR}$ & $\mathrm{U}^{\mathrm{A}}=1 \mathrm{eV}, \mathrm{LCEBR}^{\mathrm{K}}$ & $\mathrm{U}^{\mathrm{A}}=2 \mathrm{eV}^{\mathrm{HA}},{ }^{\mathrm{K}} \mathrm{LCEBR}^{\mathrm{KA}}$ & $\mathrm{U}^{\mathrm{A}}=3 \mathrm{eV}^{\mathrm{HA}}, \mathrm{LCEBR}^{\mathrm{KA}}$ & $\mathrm{U}^{\mathrm{A}}=4 \mathrm{eV},{ }^{\mathrm{KA}} \mathrm{LEBR}$ \\
\hline
\end{tabular}

TABLE CCLXV. Topology phase diagram of VCl2.

\begin{tabular}{|c|c|c|c|c|c|}
\hline BCSID & Formula & ICSD & MSG & T.C. & \\
\hline 1.238 & $\overline{\mathrm{VBr} 2}$ & 246906 & $159.64\left(P_{c} 31 c\right)$ & $\mathrm{w} / \mathrm{O}$ & I. $\sin _{0}$ \\
\hline Topology & & & & & \\
\hline & $\mathrm{U}^{\mathrm{A}}=0^{\mathrm{HA}}, \mathrm{LCEBR}^{\mathrm{K}} \mathrm{KCA}^{\mathrm{L}}$ & $\mathrm{U}^{\mathrm{A}}=1 \mathrm{eV}, \mathrm{KCER}^{\mathrm{K}} \mathrm{LCAR}^{\mathrm{M}}$ & $\mathrm{U}^{\mathrm{A}}=2 \mathrm{eV}^{\mathrm{H}}, \mathrm{KCEBR}^{\mathrm{KA}}$ & $\mathrm{U}^{\mathrm{A}}=3 \mathrm{eV}^{\mathrm{HA}}, \mathrm{LCEBR}^{\mathrm{K}^{\mathrm{KA}}}$ & $\mathrm{U}^{\mathrm{A}}=4 \mathrm{eV}^{\mathrm{HA}}, \mathrm{LCEBR}^{\mathrm{KA}^{\mathrm{A}}}$ \\
\hline
\end{tabular}

TABLE CCLXVI. Topology phase diagram of VBr2.

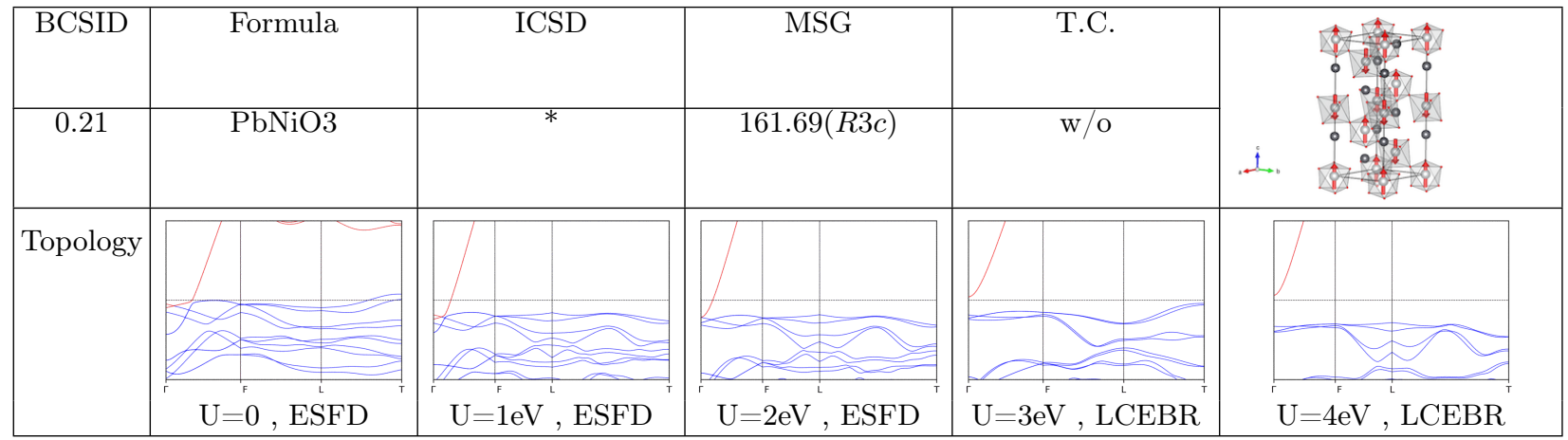

TABLE CCLXVII. Topology phase diagram of PbNiO3. 


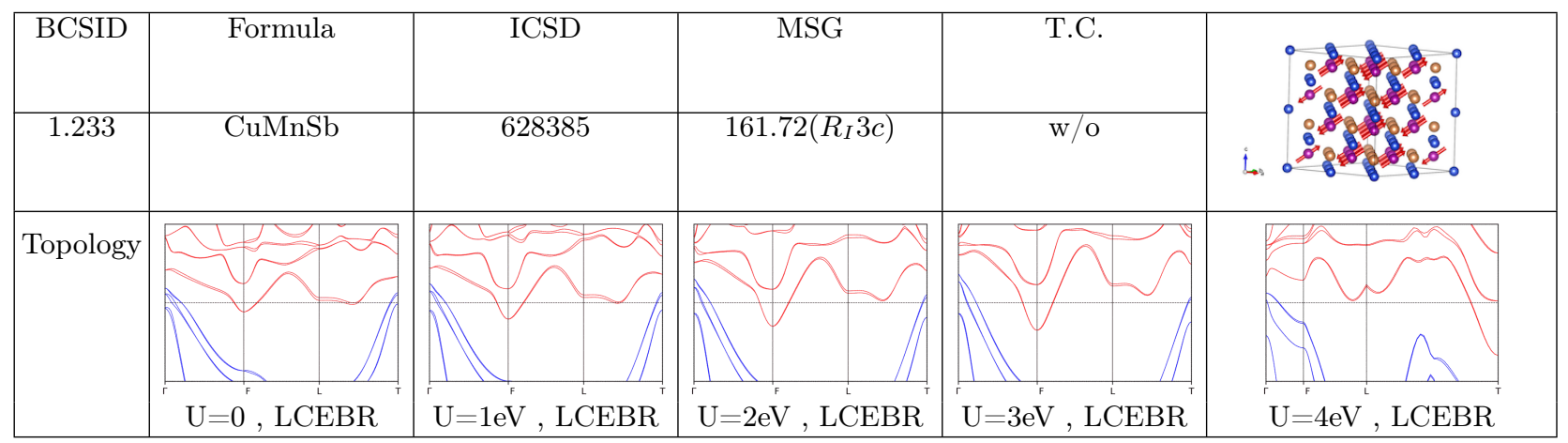

TABLE CCLXVIII. Topology phase diagram of CuMnSb.

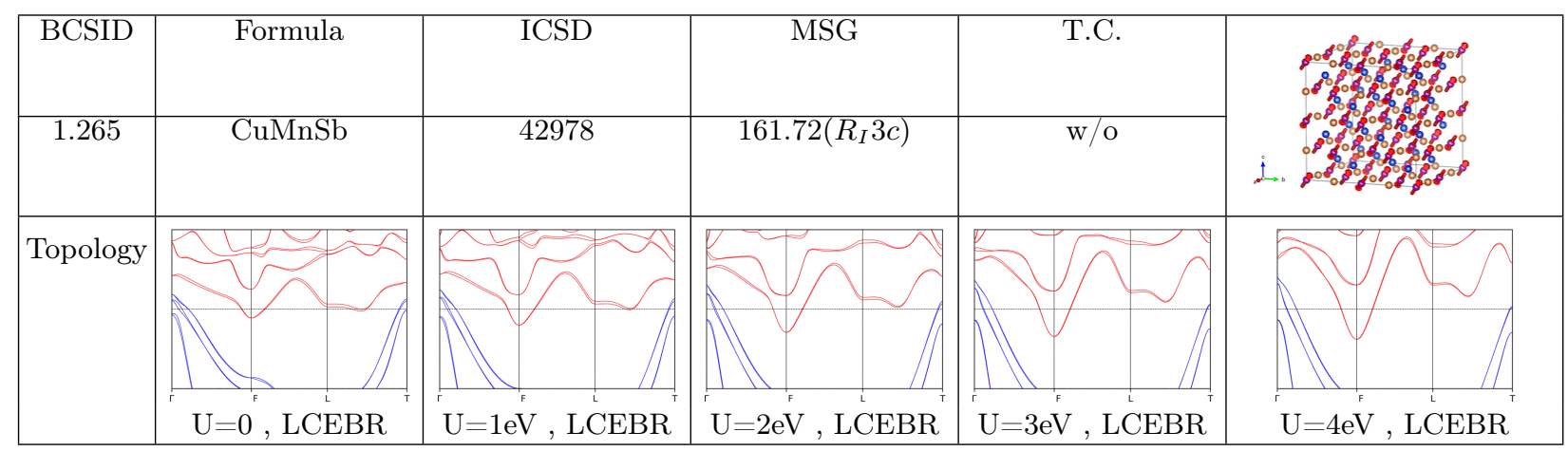

TABLE CCLXIX. Topology phase diagram of CuMnSb.

\begin{tabular}{|c|c|c|c|c|c|}
\hline BCSID & Formula & ICSD & MSG & T.C. & \\
\hline 1.186 & SrRu2O6 & 248351 & $162.78\left(P_{c} 31 m\right)$ & $\mathbb{Z}_{2}$ & . \\
\hline Topology & $\infty$ & 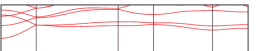 & & & \\
\hline & $\mathrm{U}^{\mathrm{A}}=0, \mathrm{LCEBR}^{\mathrm{k}}$ & $\mathrm{U}^{\mathrm{A}}=1 \mathrm{eV}, \mathrm{LCEBR}^{\mathrm{k}}$ & $\mathrm{U}^{\mathrm{A}}=2 \mathrm{eV}, \mathrm{LCEBR}^{\mathrm{k}}$ & $\mathrm{U}^{\mathrm{A}}=3 \mathrm{eV}, \mathrm{LCEBR}^{\mathrm{k}}$ & $\mathrm{U}^{\mathrm{A}}=4 \mathrm{eV}, \mathrm{LCEBR}^{\mathrm{k}}$ \\
\hline
\end{tabular}

TABLE CCLXX. Topology phase diagram of SrRu2O6.

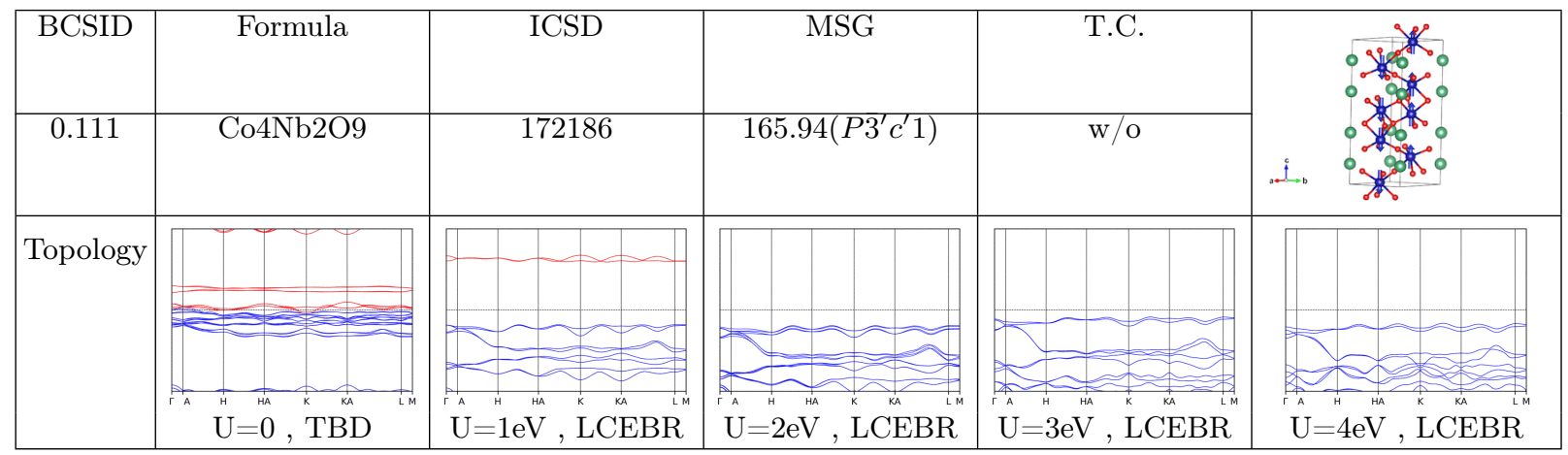

TABLE CCLXXI. Topology phase diagram of Co4Nb2O9. 


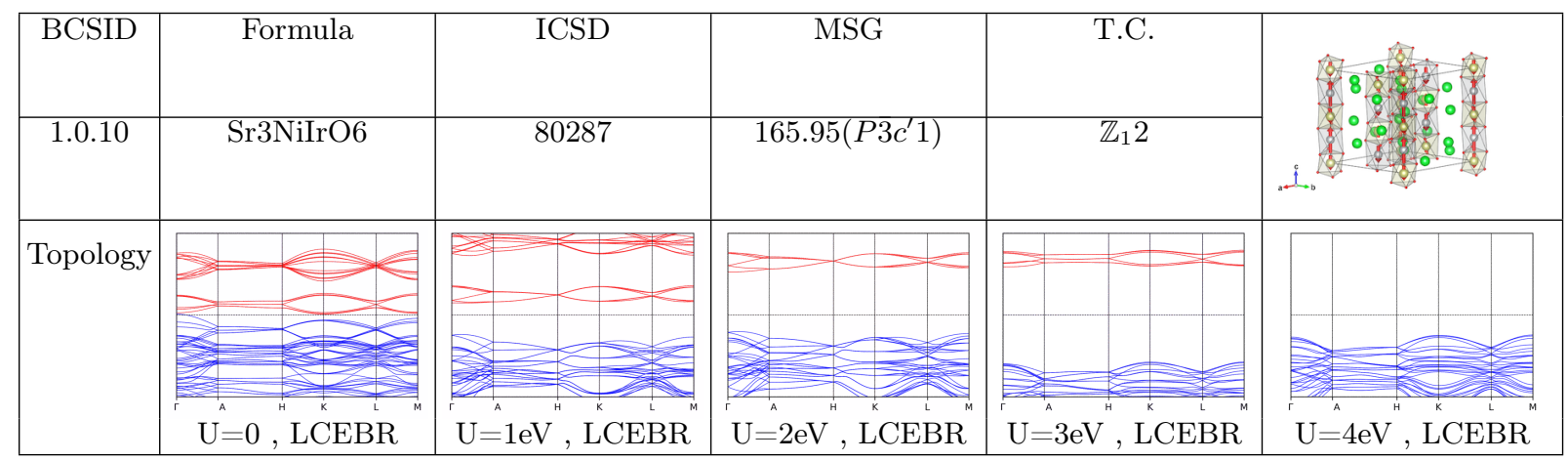

TABLE CCLXXII. Topology phase diagram of Sr3NiIrO6.

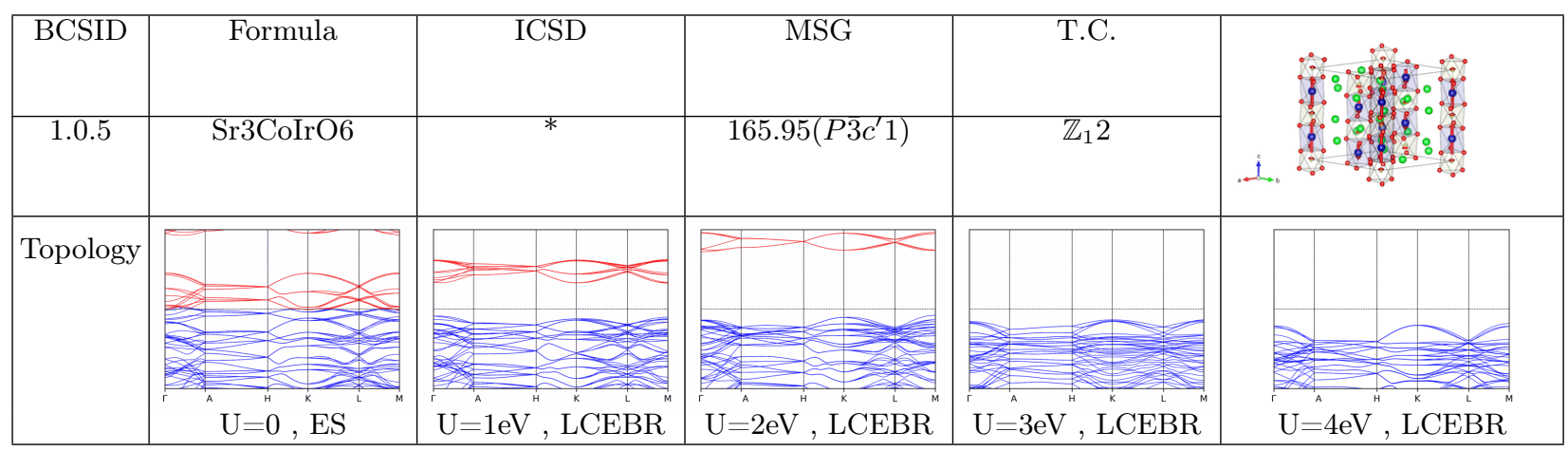

TABLE CCLXXIII. Topology phase diagram of Sr3CoIrO6.

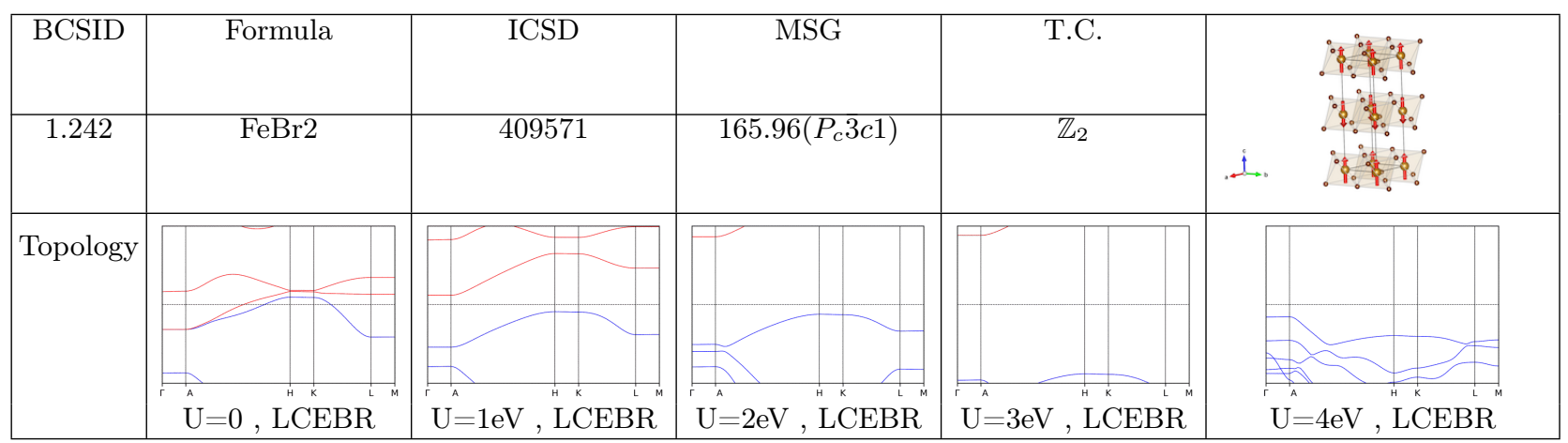

TABLE CCLXXIV. Topology phase diagram of FeBr2.

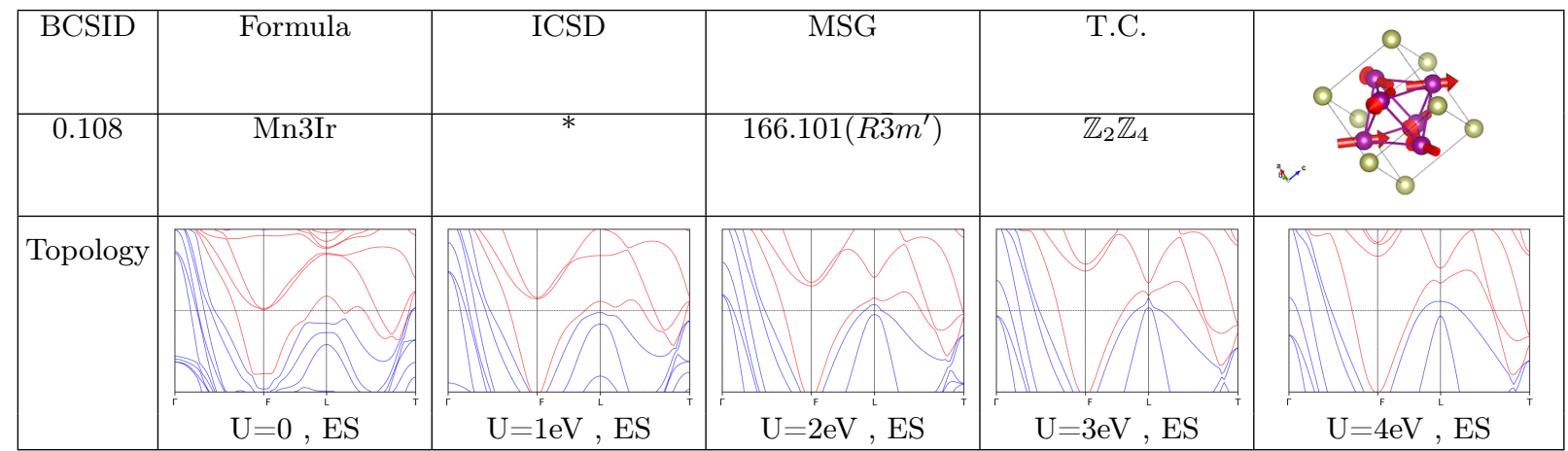

TABLE CCLXXV. Topology phase diagram of Mn3Ir. 


\begin{tabular}{|c|c|c|c|c|c|}
\hline BCSID & Formula & ICSD & MSG & T.C. \\
\hline 0.109 & Mn3Pt & $*$ & $166.101\left(R 3 m^{\prime}\right)$ & $\mathbb{Z}_{2} \mathbb{Z}_{4}$ \\
\hline Topology & & & & \\
& & &
\end{tabular}

TABLE CCLXXVI. Topology phase diagram of Mn3Pt.

\begin{tabular}{|c|c|c|c|c|c|}
\hline BCSID & Formula & ICSD & MSG & T.C. & \\
\hline 0.177 & Mn3GaN & 87399 & $166.97(R 3 m)$ & $\mathbb{Z}_{2}$ \\
\hline Topology & & & & \\
\hline
\end{tabular}

TABLE CCLXXVII. Topology phase diagram of Mn3GaN.

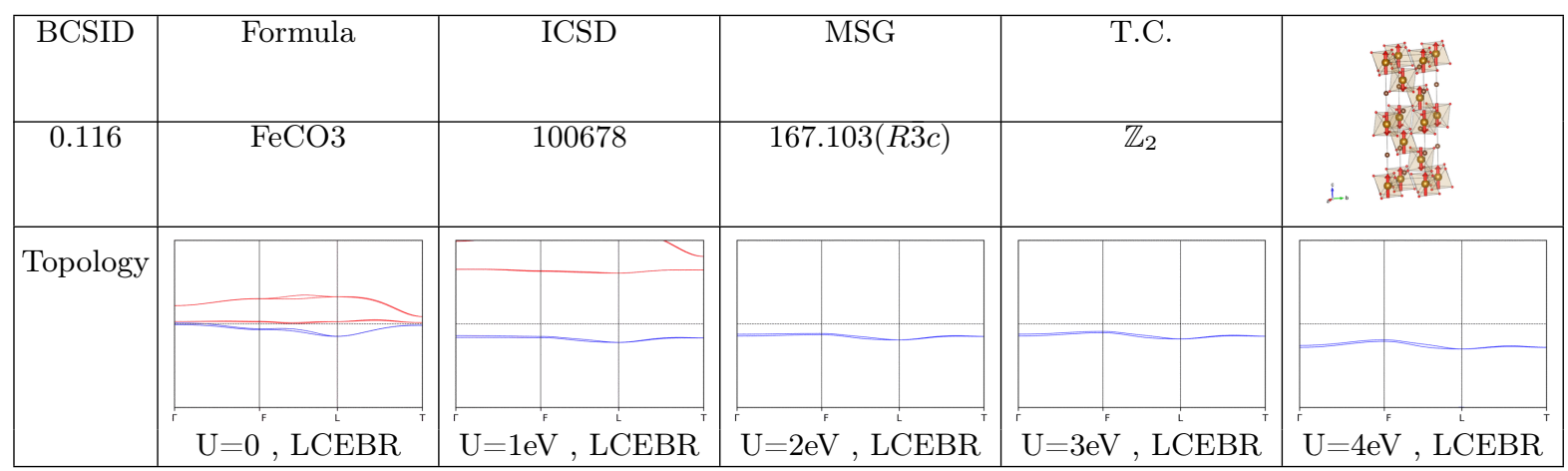

TABLE CCLXXVIII. Topology phase diagram of FeCO3.

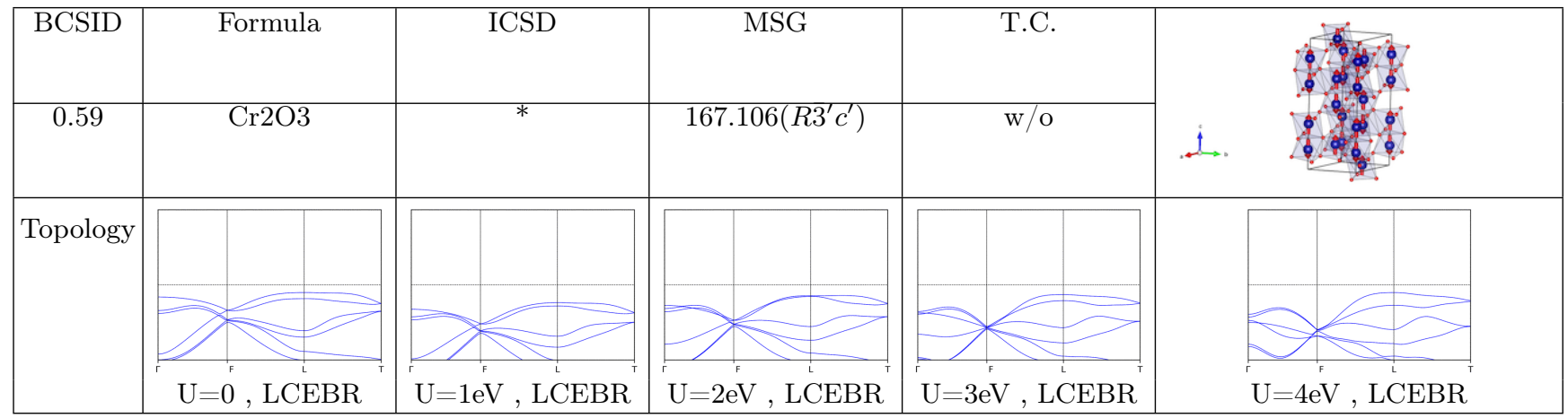

TABLE CCLXXIX. Topology phase diagram of Cr2O3. 


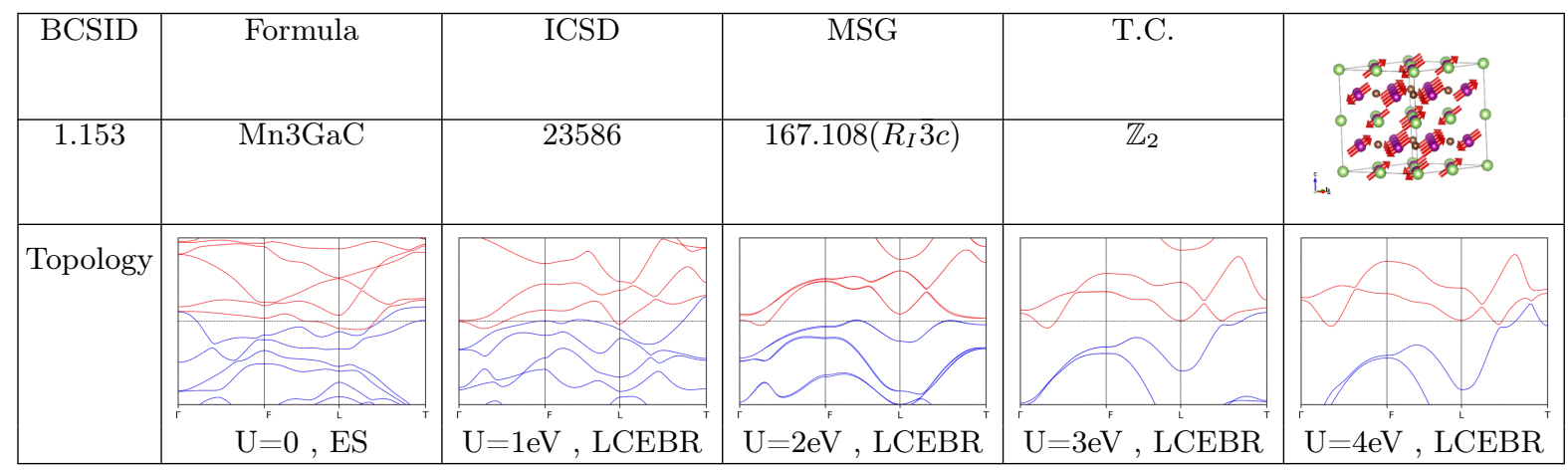

TABLE CCLXXX. Topology phase diagram of Mn3GaC.

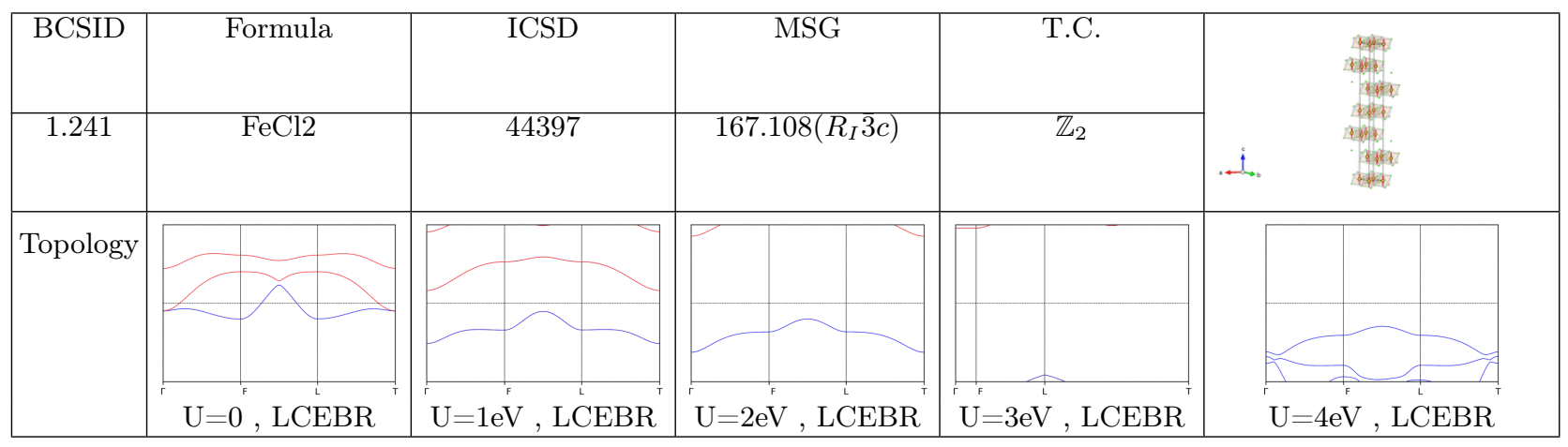

TABLE CCLXXXI. Topology phase diagram of FeCl2.

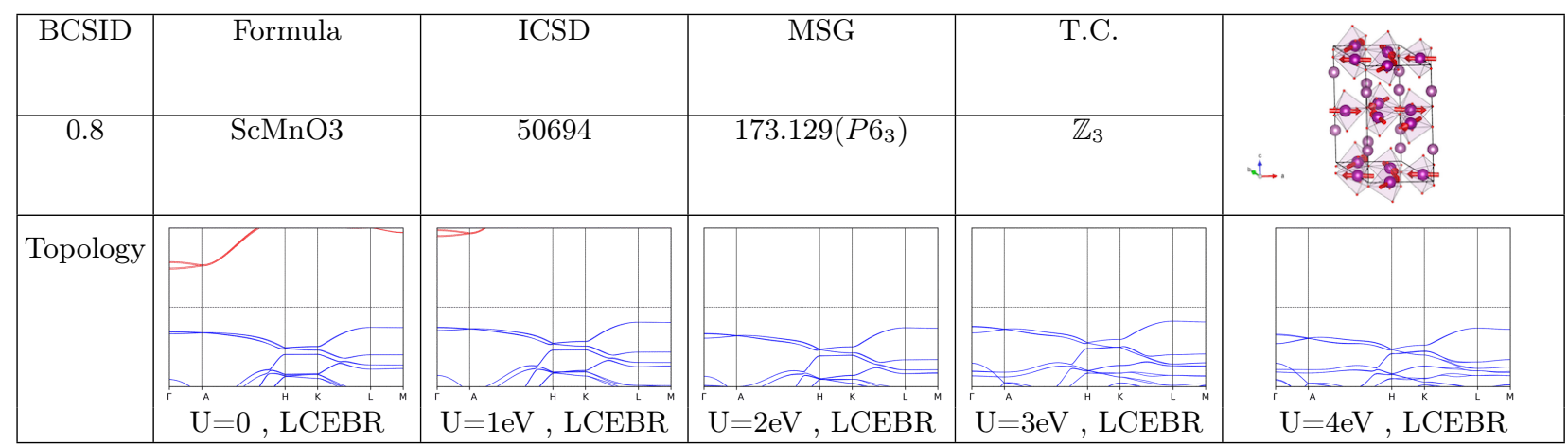

TABLE CCLXXXII. Topology phase diagram of ScMnO3.

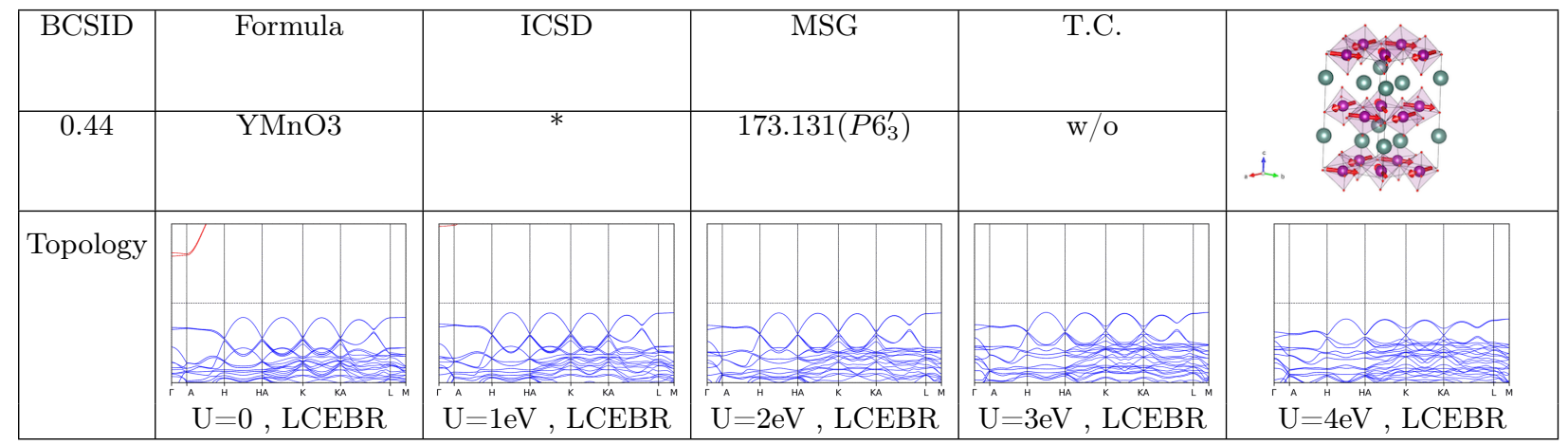

TABLE CCLXXXIII. Topology phase diagram of YMnO3. 


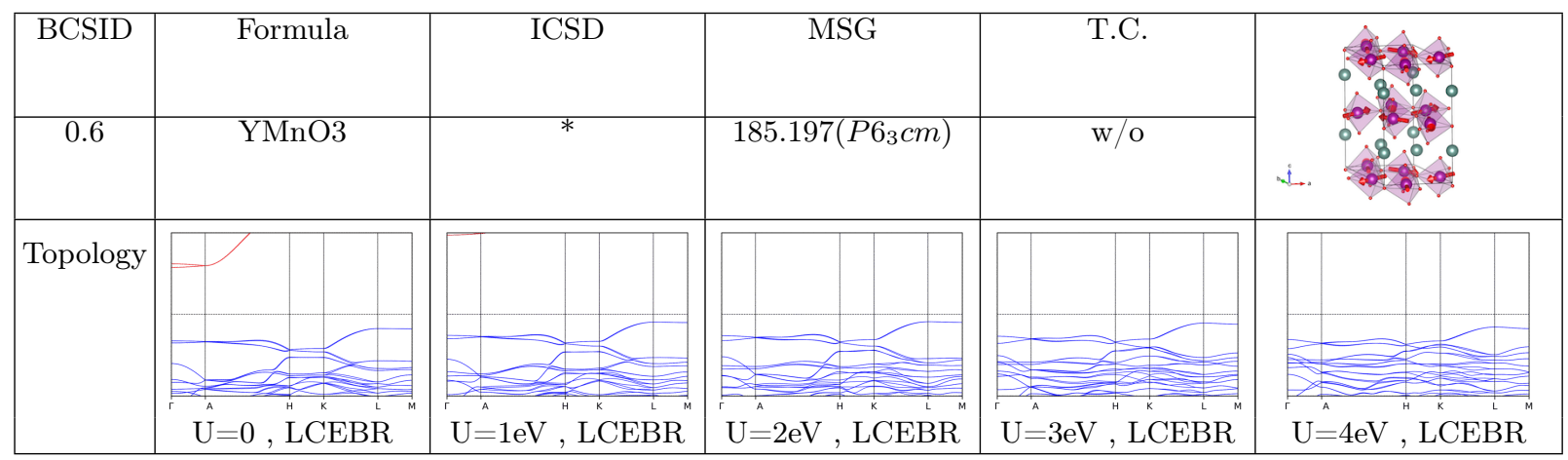

TABLE CCLXXXIV. Topology phase diagram of YMnO3.

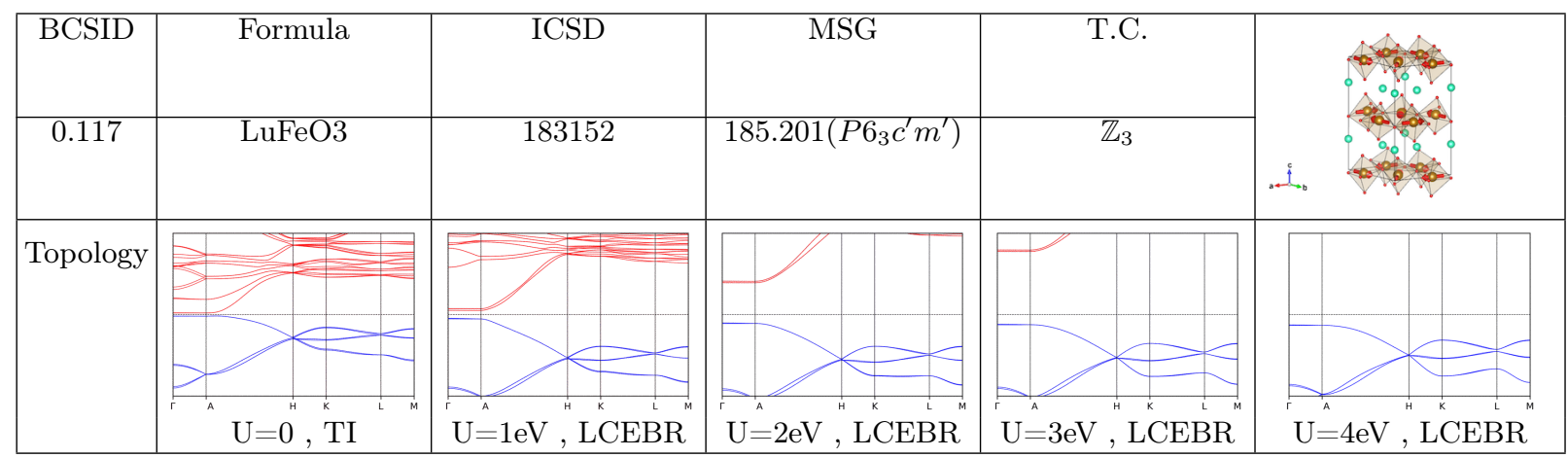

TABLE CCLXXXV. Topology phase diagram of LuFeO3.

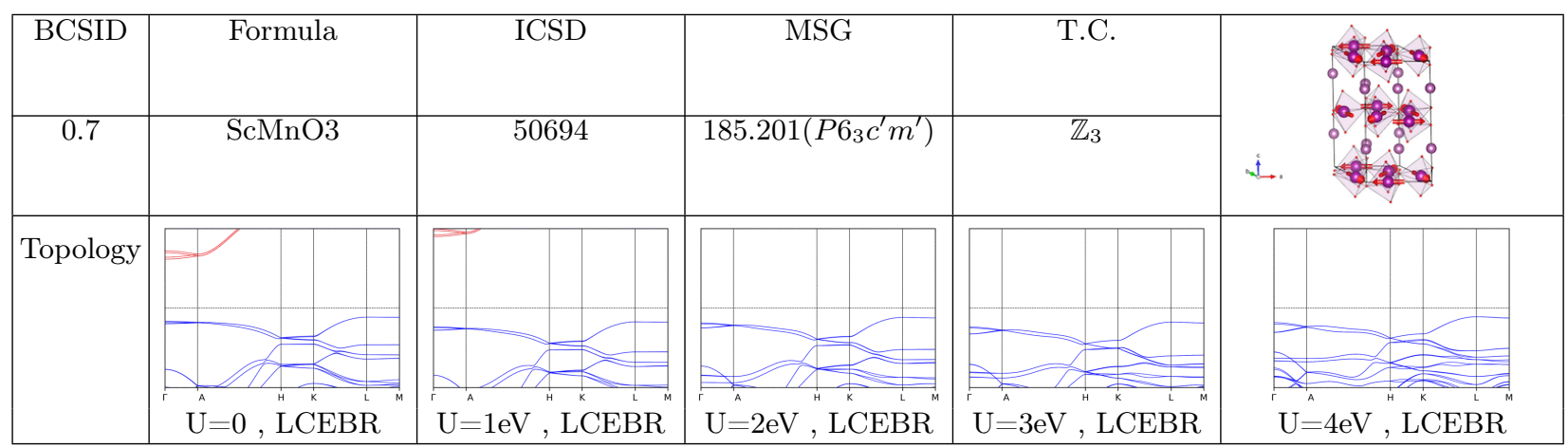

TABLE CCLXXXVI. Topology phase diagram of ScMnO3.

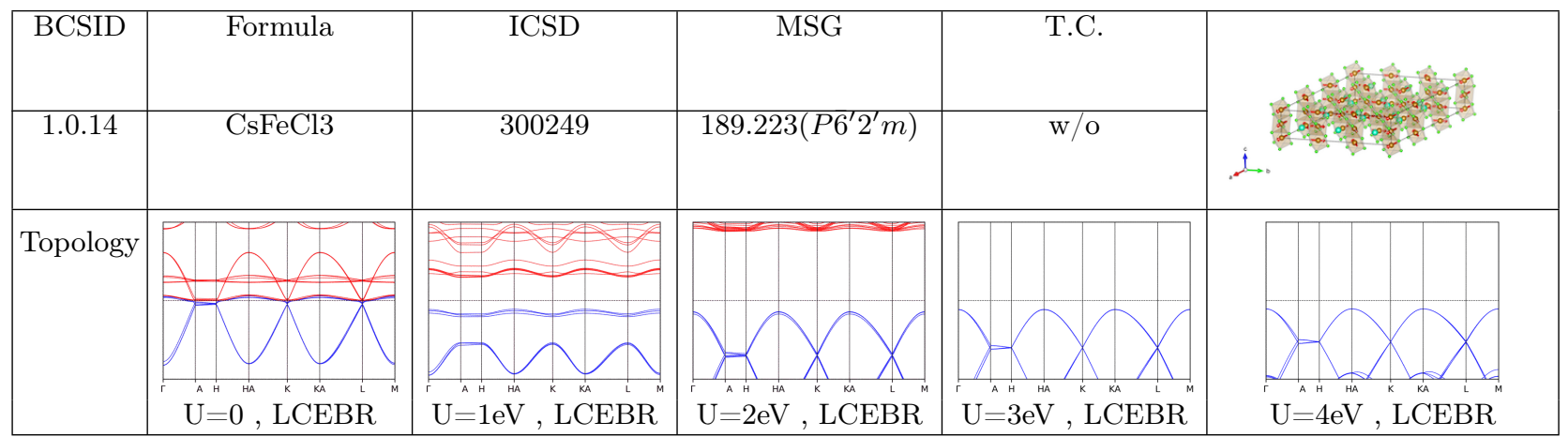

TABLE CCLXXXVII. Topology phase diagram of CsFeCl3. 


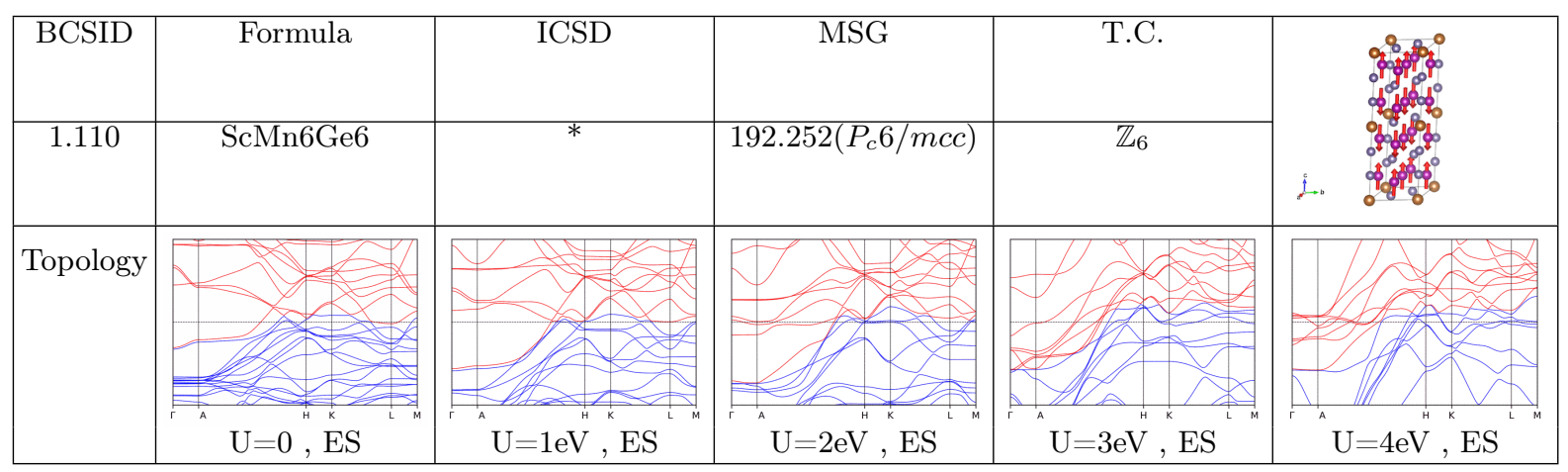

TABLE CCLXXXVIII. Topology phase diagram of ScMn6Ge6.

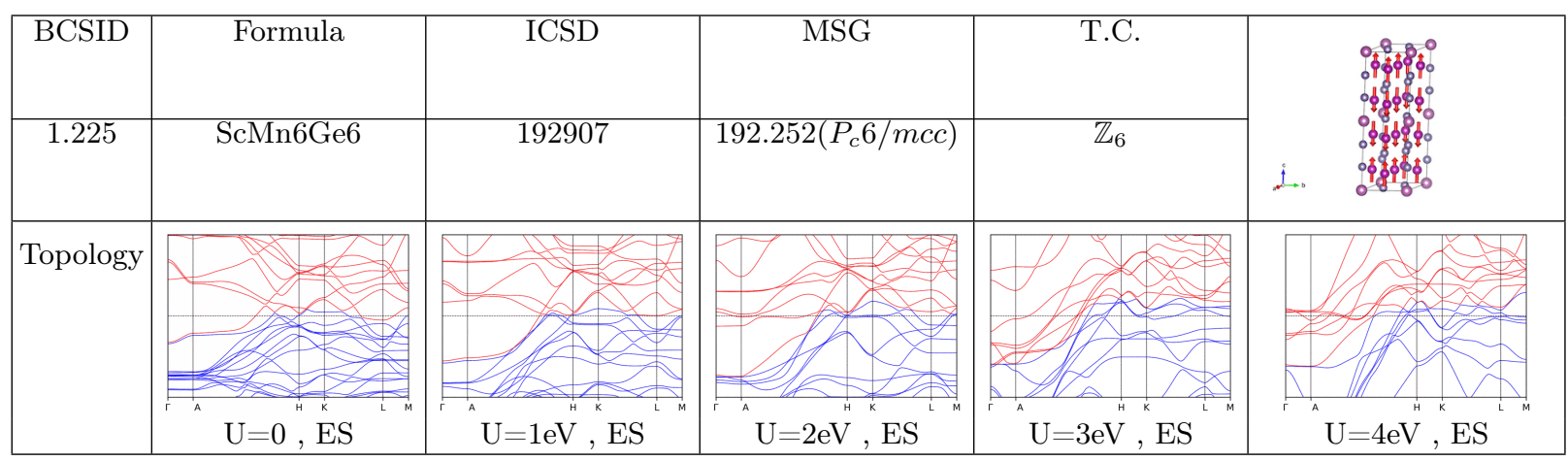

TABLE CCLXXXIX. Topology phase diagram of ScMn6Ge6.

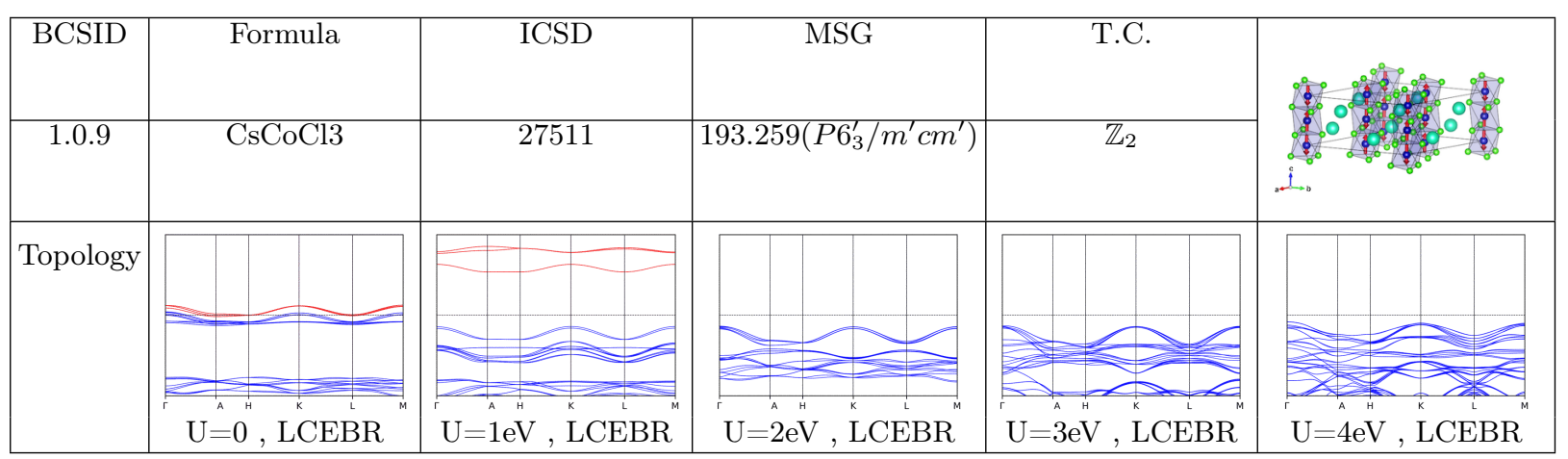

TABLE CCXC. Topology phase diagram of CsCoCl3.

\begin{tabular}{|c|c|c|c|c|c|}
\hline BCSID & Formula & ICSD & MSG & T.C. & \\
\hline 0.118 & Ba5Co5ClO13 & 99371 & $194.268\left(P 6_{3}^{\prime} / m^{\prime} m^{\prime} c\right)$ & $\mathbb{Z}_{2}$ & $\begin{array}{r}-80 \div \\
0800 \\
\div 83:\end{array}$ \\
\hline \multirow[t]{6}{*}{ Topology } & \begin{tabular}{|l|l|l|} 
& \\
\end{tabular} & \begin{tabular}{|l|l|} 
& \\
\end{tabular} & 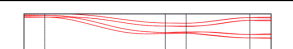 & $=1$ & \\
\hline & $\begin{array}{lll} & =1\end{array}$ & & & & \\
\hline & $\ldots<$ & 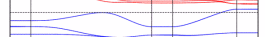 & 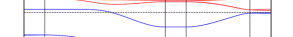 & E & \\
\hline & P & $\Longrightarrow$ & 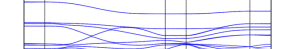 & & \\
\hline & ${ }_{A}{ }_{H K}$ & 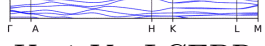 & $\mathrm{CA}_{\mathrm{H}} \mathrm{H}$ & $\mathrm{H}_{\mathrm{A}} \mathrm{HK}_{\mathrm{H}} \mathrm{L}_{\mathrm{M}}$ & $s$ \\
\hline & $\mathrm{U}=0, \mathrm{LCEBR}$ & $\mathrm{U}=1 \mathrm{eV}, \mathrm{LCEBR}$ & $\mathrm{U}=2 \mathrm{eV}, \mathrm{LCEBR}$ & $\mathrm{U}=3 \mathrm{eV}, \mathrm{LCEBR}$ & $\mathrm{U}=4 \mathrm{eV}, \mathrm{ES}$ \\
\hline
\end{tabular}

TABLE CCXCI. Topology phase diagram of Ba5Co5ClO13. 


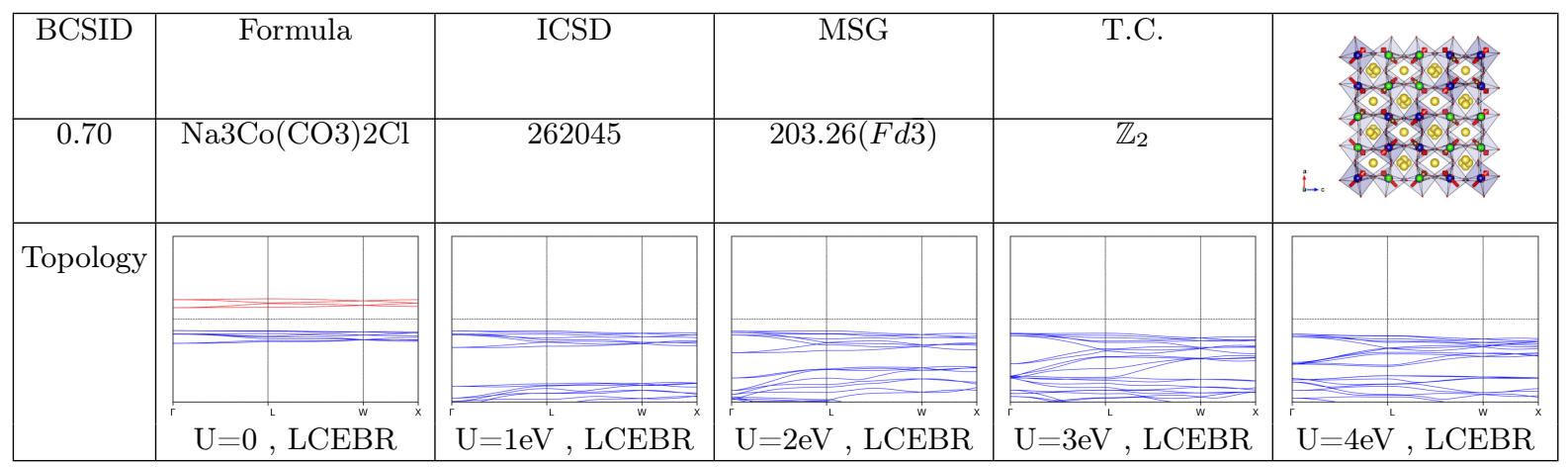

TABLE CCXCII. Topology phase diagram of $\mathrm{Na} 3 \mathrm{Co}(\mathrm{CO} 3) 2 \mathrm{Cl}$.

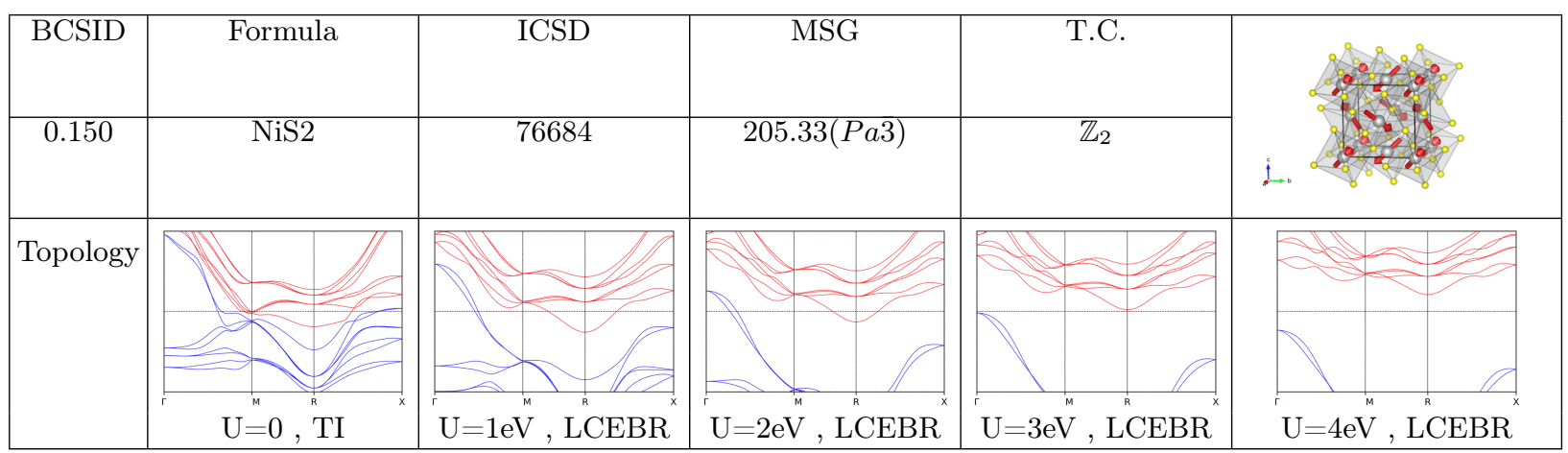

TABLE CCXCIII. Topology phase diagram of NiS2.

\begin{tabular}{|c|c|c|c|c|c|}
\hline BCSID & Formula & ICSD & MSG & T.C. & \\
\hline 0.20 & $\mathrm{MnTe} 2$ & $*$ & $205.33($ Pa3) & $\mathbb{Z}_{2}$ & \\
\hline \multirow[t]{2}{*}{ Topology } & & & & & \\
\hline & $\mathrm{U}=0,{ }^{M},{ }^{R} E B R$ & $\mathrm{I} U=1 \mathrm{eV}, \mathrm{LCEBR}^{\mathrm{R}}$ & $\mathrm{I} U=2 \mathrm{eV}^{\mu}, \mathrm{LCEBR}^{\mathrm{R}}$ & $\mathrm{U}=3 \mathrm{eV}, \mathrm{LCEBR}^{\mathrm{n}}$ & $\mathrm{I} U=4 \mathrm{eV}^{\mathrm{M}}, \mathrm{LCEBR}^{\mathrm{R}}$ \\
\hline
\end{tabular}

TABLE CCXCIV. Topology phase diagram of MnTe2.

\begin{tabular}{|c|c|c|c|c|c|}
\hline BCSID & Formula & ICSD & MSG & T.C. & \multirow[b]{2}{*}{ L. } \\
\hline 0.2 & $\mathrm{Cd} 2 \mathrm{Os} 2 \mathrm{O} 7$ & 155771 & $227.131\left(F d 3 m^{\prime}\right)$ & $\mathbb{Z}_{2}$ & \\
\hline \multirow[t]{2}{*}{ Topology } & & $E$ & 2 & & 8 \\
\hline & $\mathrm{U}=0, \mathrm{~L} \mathrm{~S}^{\mathrm{w}}$ & $\mathrm{U}=1 \mathrm{eV}, \mathrm{LCEBR}$ & $\mathrm{U}=2 \mathrm{eV}, \mathrm{LCEBR}$ & $\mathrm{U}=3 \mathrm{eV}, \mathrm{LCEBR}$ & $\mathrm{U}=4 \mathrm{eV}, \mathrm{LCEBR}$ \\
\hline
\end{tabular}

TABLE CCXCV. Topology phase diagram of Cd2Os2O7. 


\begin{tabular}{|c|c|c|c|c|c|}
\hline BCS ID & Formula & ICSD & MSG & T.C. & \multirow{2}{*}{ 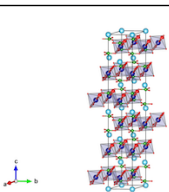 } \\
\hline 1.191 & $\mathrm{HoCr}(\mathrm{BO} 3) 2$ & 189502 & $2.7\left(P_{S} 1\right)$ & $\mathbb{Z}_{2}$ & \\
\hline \multirow[t]{2}{*}{ Topology } & & & & & \\
\hline & 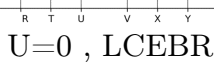 & $\mathrm{U}=2 \mathrm{eV}, \mathrm{TBD}$ & $\mathrm{U}=4 \mathrm{eV}^{\mathrm{r}}, \mathrm{LCEBR}^{\mathrm{r}}$ & $\mathrm{U}=6 \mathrm{eV}, \mathrm{TBD}$ & \\
\hline
\end{tabular}

TABLE CCXCVI. Topology phase diagram of $\mathrm{HoCr}(\mathrm{BO} 3) 2$.

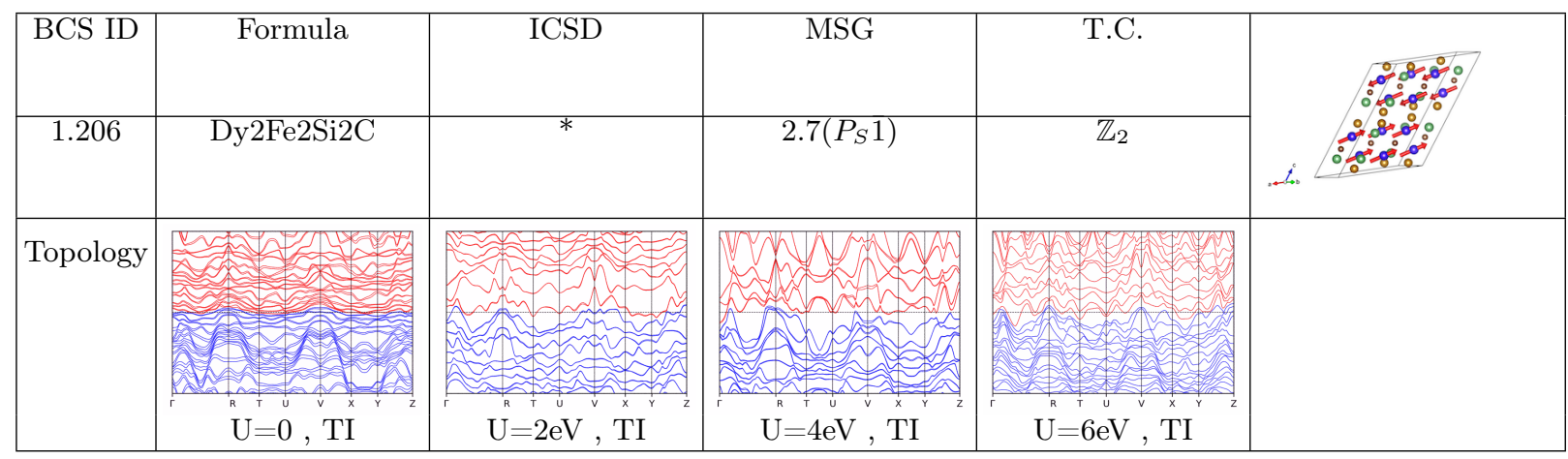

TABLE CCXCVII. Topology phase diagram of Dy2Fe2Si2C.

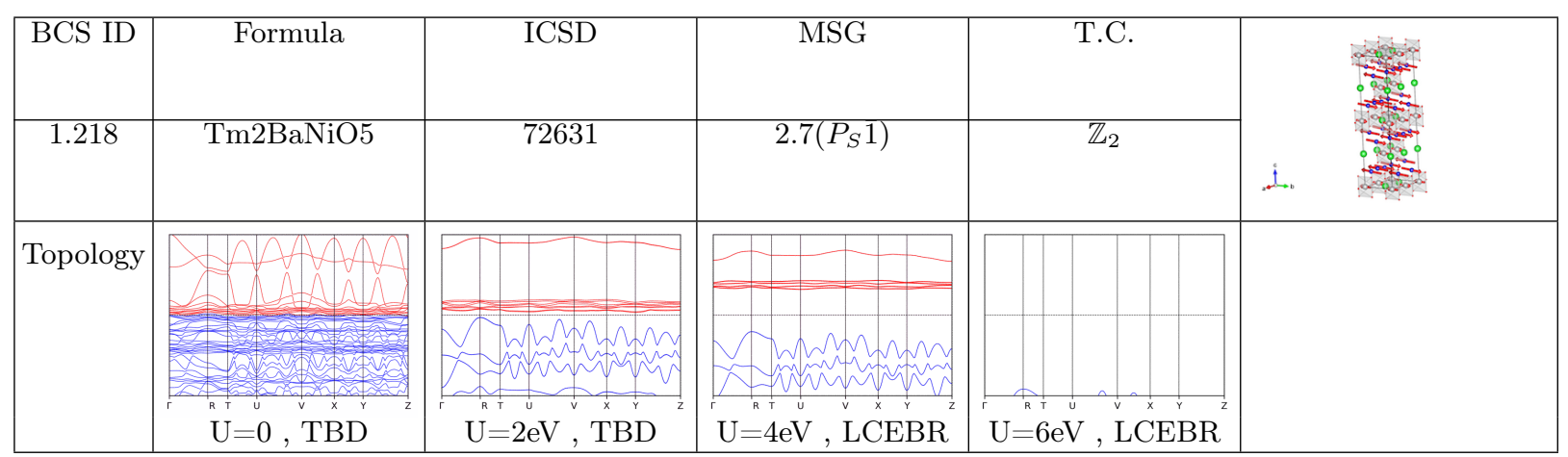

TABLE CCXCVIII. Topology phase diagram of Tm2BaNiO5.

\begin{tabular}{|c|c|c|c|c|c|}
\hline BCS ID & Formula & ICSD & MSG & T.C. & \\
\hline 1.38 & Nd2NaOsO6 & * & $2.7\left(P_{S} 1\right)$ & $\mathbb{Z}_{2}$ & \\
\hline \multirow[t]{2}{*}{ Topology } & \multirow[b]{2}{*}{$\mathrm{U}=0, \mathrm{LCEBR}$} & \multirow[b]{2}{*}{$\mathrm{U}=2 \mathrm{eV}, \mathrm{TBD}$} & 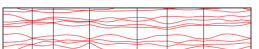 & \multirow[b]{2}{*}{$\mathrm{U}=6 \mathrm{eV}, \mathrm{TBD}$} & \\
\hline & & & $\mathrm{U}=4 \mathrm{eV}, \mathrm{LCEBR}$ & & \\
\hline
\end{tabular}

TABLE CCXCIX. Topology phase diagram of $\mathrm{Nd} 2 \mathrm{NaOsO}$. 


\begin{tabular}{|c|c|c|c|c|c|}
\hline BCS ID & Formula & ICSD & MSG & T.C. & 0 \\
\hline 0.37 & U3Al2Si3 & $T$ & $5.15\left(C 2^{\prime}\right)$ & $\mathrm{w} / \mathrm{O}$ & 8 \\
\hline Topology & s. & & & & \\
\hline & $\mathrm{U}^{\mathrm{A}}=0, \mathrm{LCEBR}$ & $\mathrm{U}={ }^{A} \mathrm{eV}, \mathrm{LCEBR}^{\mathrm{A}}$ & $\mathrm{U}=4 \mathrm{eV}^{\mathrm{A}}, \mathrm{LCEBR}^{\mathrm{A}}$ & $\mathrm{U}=6 \mathrm{eV}, \mathrm{LCEBR}$ & \\
\hline
\end{tabular}

TABLE CCC. Topology phase diagram of U3Al2Si3.

\begin{tabular}{|c|c|c|c|c|c|}
\hline BCS ID & Formula & ICSD & MSG & T.C. & \\
\hline 1.111 & $\mathrm{GdBiPt}$ & 58786 & $9.40\left(C_{c} c\right)$ & $\mathrm{w} / \mathrm{o}$ & \\
\hline \multirow[t]{2}{*}{ Topology } & 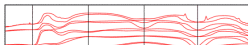 & & & & \\
\hline & $\hat{\mathrm{U}}=0, \mathrm{LCEBR}$ & $\mathrm{U}=2 \mathrm{eV}, \mathrm{LCEBR}$ & $\mathrm{U}^{\mathrm{A}}=4 \mathrm{eV}, \mathrm{LCEBR}^{\mathrm{M}}$ & $\mathrm{U}^{\mathrm{A}}=6 \mathrm{eV}, \mathrm{LCEBR}$ & \\
\hline
\end{tabular}

TABLE CCCI. Topology phase diagram of GdBiPt.

\begin{tabular}{|c|c|c|c|c|c|}
\hline BCS ID & Formula & ICSD & MSG & T.C. & \\
\hline 0.104 & ErVO3 & 185838 & $11.54\left(P 2_{1}^{\prime} / m^{\prime}\right)$ & $\mathbb{Z}_{2} \mathbb{Z}_{2} \mathbb{Z}_{4}$ & $-\frac{00}{1}=0$ \\
\hline Topology & (SA & 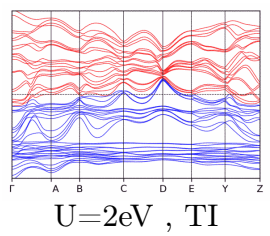 & 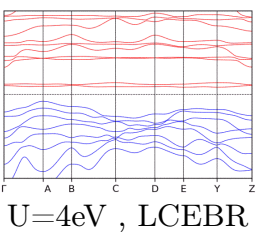 & 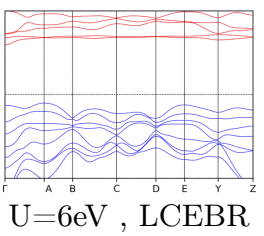 & \\
\hline
\end{tabular}

TABLE CCCII. Topology phase diagram of ErVO3.

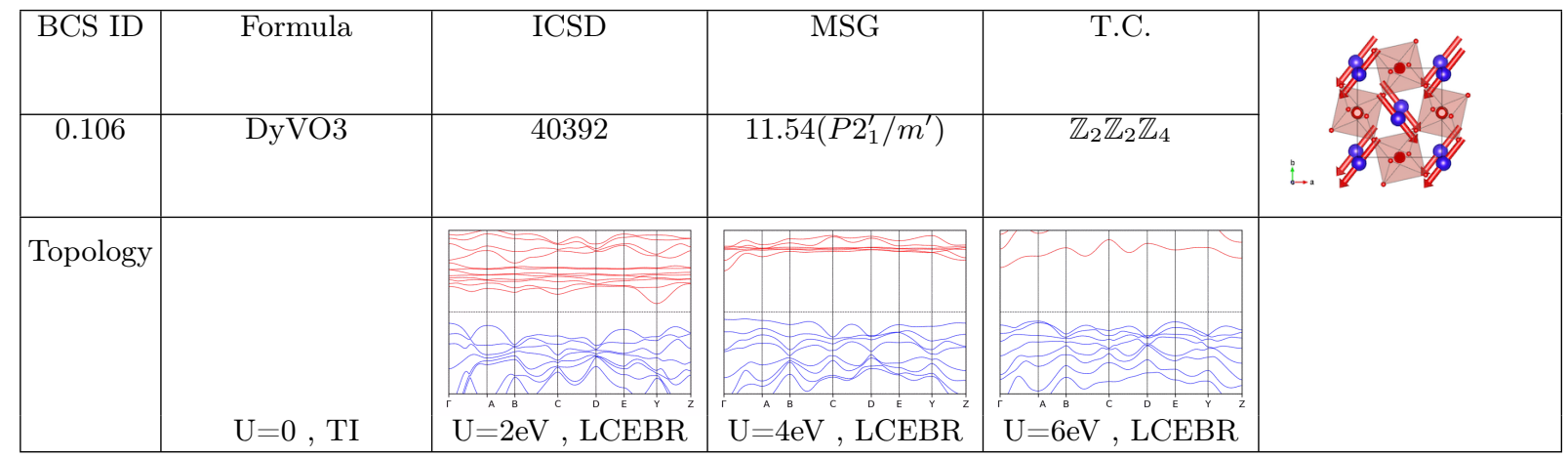

TABLE CCCIII. Topology phase diagram of DyVO3. 


\begin{tabular}{|c|c|c|c|c|c|}
\hline BCS ID & Formula & ICSD & MSG & T.C. & \\
\hline 1.171 & Tb2Fe2Si2C & & $12.63\left(C_{c} 2 / m\right)$ & $\mathbb{Z}_{2}$ & 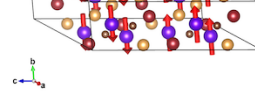 \\
\hline Topology & 4 & & & & \\
\hline & $\mathrm{U}=0, \mathrm{LCEBR}$ & $\mathrm{U}=2 \mathrm{eV}, \mathrm{TBD}$ & $\mathrm{U}=4 \mathrm{eV}, \mathrm{TBD}$ & $\mathrm{U}=6 \mathrm{eV}, \mathrm{TBD}$ & \\
\hline
\end{tabular}

TABLE CCCIV. Topology phase diagram of Tb2Fe2Si2C.

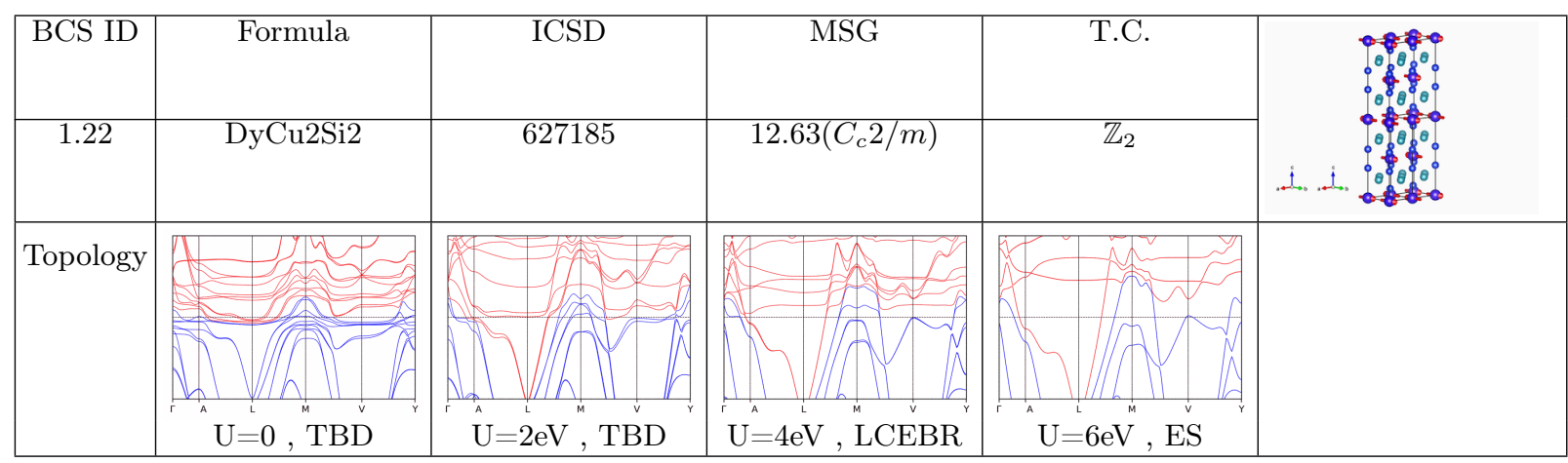

TABLE CCCV. Topology phase diagram of DyCu2Si2.

\begin{tabular}{|c|c|c|c|c|c|}
\hline BCS ID & Formula & ICSD & MSG & T.C. & \\
\hline 0.188 & CeMnAsO & 195499 & $13.67\left(P 2^{\prime} / c\right)$ & $\mathrm{w} / \mathrm{o}$ & $\dot{L}$. \\
\hline Topology & 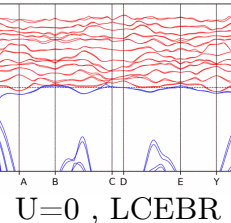 & 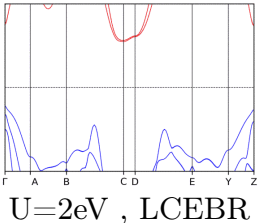 & 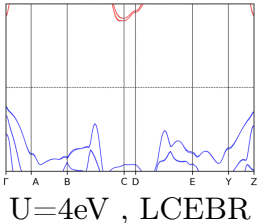 & $\mathrm{U}=6 \mathrm{eV}, \mathrm{LCEBR}$ & \\
\hline
\end{tabular}

TABLE CCCVI. Topology phase diagram of CeMnAsO.

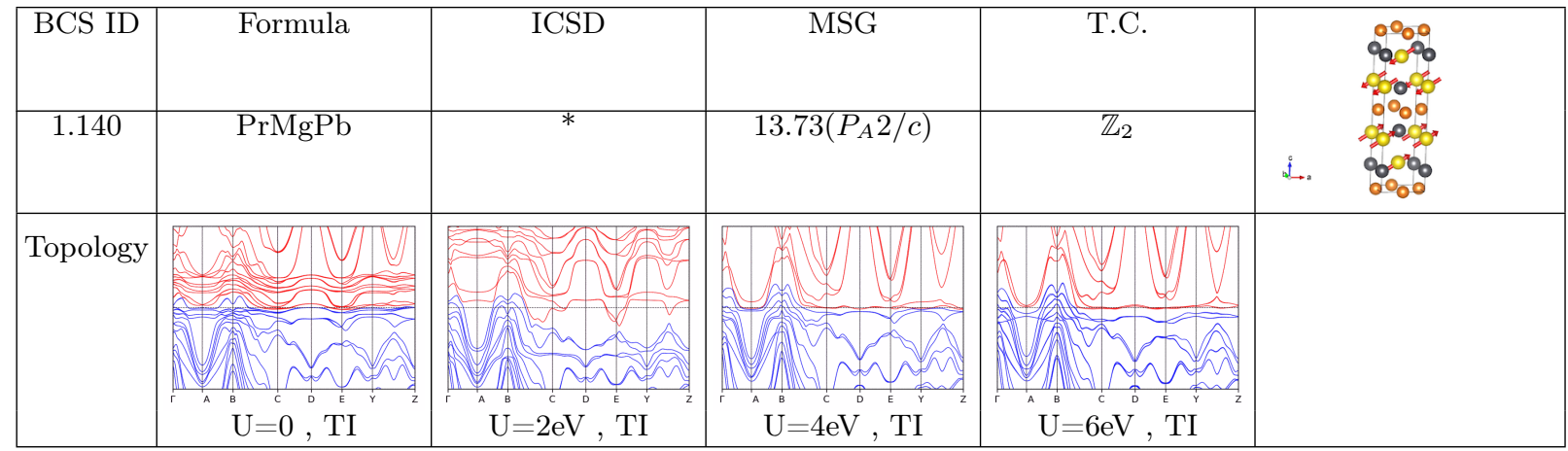

TABLE CCCVII. Topology phase diagram of PrMgPb. 


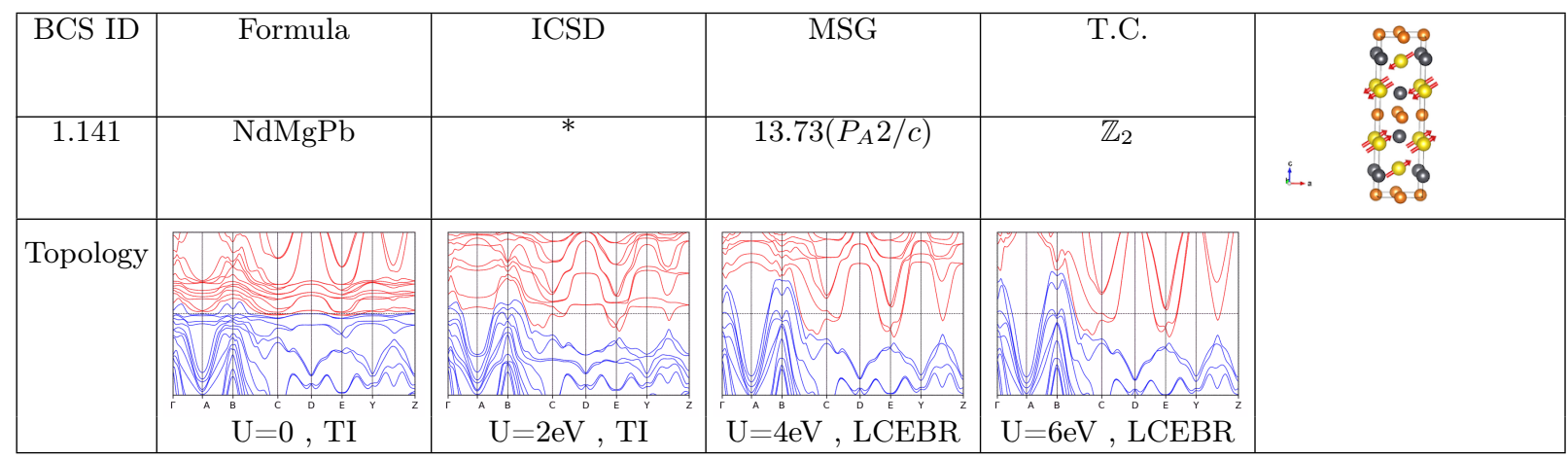

TABLE CCCVIII. Topology phase diagram of NdMgPb.

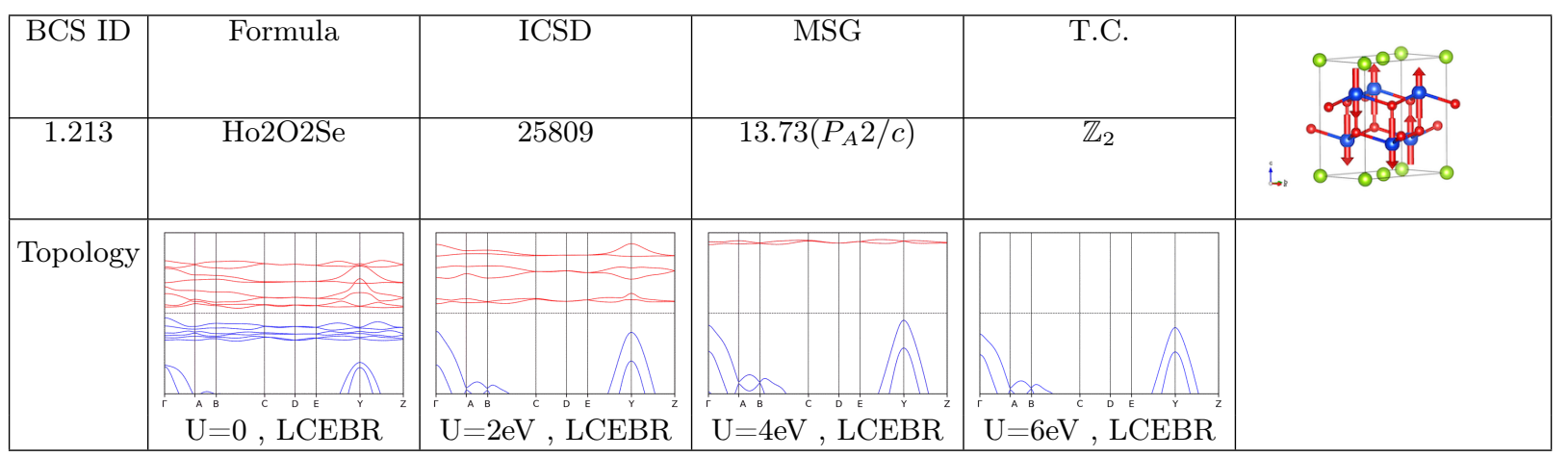

TABLE CCCIX. Topology phase diagram of Ho2O2Se.

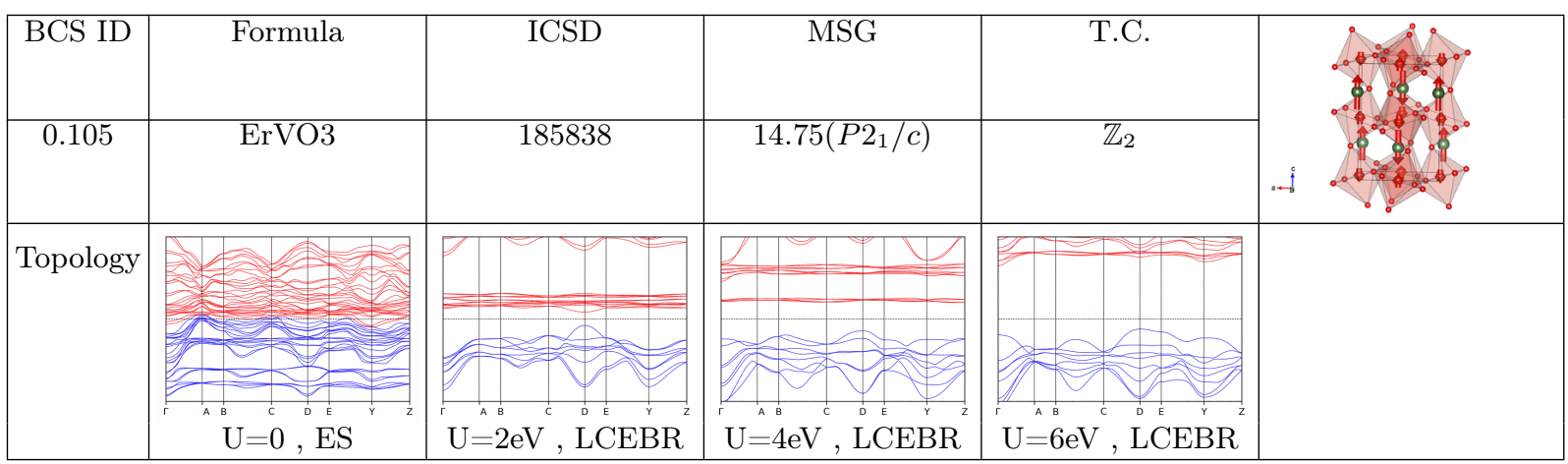

TABLE CCCX. Topology phase diagram of ErVO3.

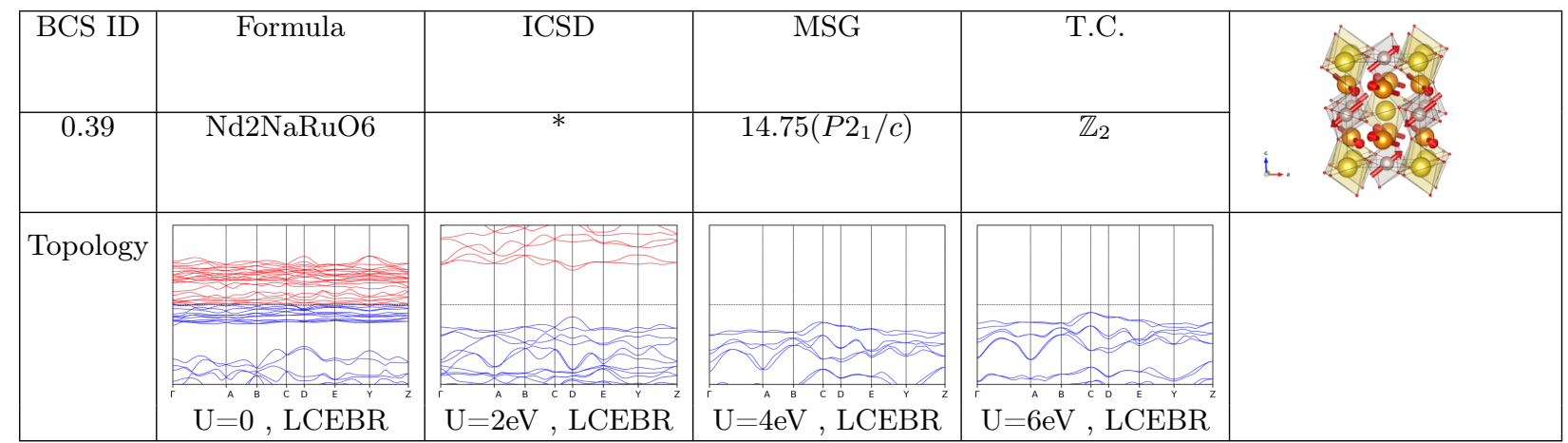

TABLE CCCXI. Topology phase diagram of Nd2NaRuO6. 


\begin{tabular}{|c|c|c|c|c|c|}
\hline BCS ID & Formula & ICSD & MSG & T.C. & \\
\hline 2.21 & $\mathrm{TbOOH}$ & 6164 & $14.78\left(P 2_{1} / c^{\prime}\right)$ & $\mathrm{w} / \mathrm{o}$ & 同 \\
\hline Topology & $\begin{array}{lll}\mathrm{N} \\
\mathrm{N}\end{array}$ & & & & \\
\hline & $\hat{\mathrm{U}}=0, \mathrm{C}, \mathrm{C} E B R$ & $\mathrm{U}^{A}=2 \mathrm{eV}^{\mathrm{c}}, \mathrm{LCEBR}^{\mathrm{E}}$ & $\mathrm{U}^{\wedge}=4 \mathrm{eV}, \mathrm{LCEBR}^{\mathrm{E}}$ & $\mathrm{U}=6 \mathrm{eV}, \mathrm{TBD}$ & \\
\hline
\end{tabular}

TABLE CCCXII. Topology phase diagram of TbOOH.

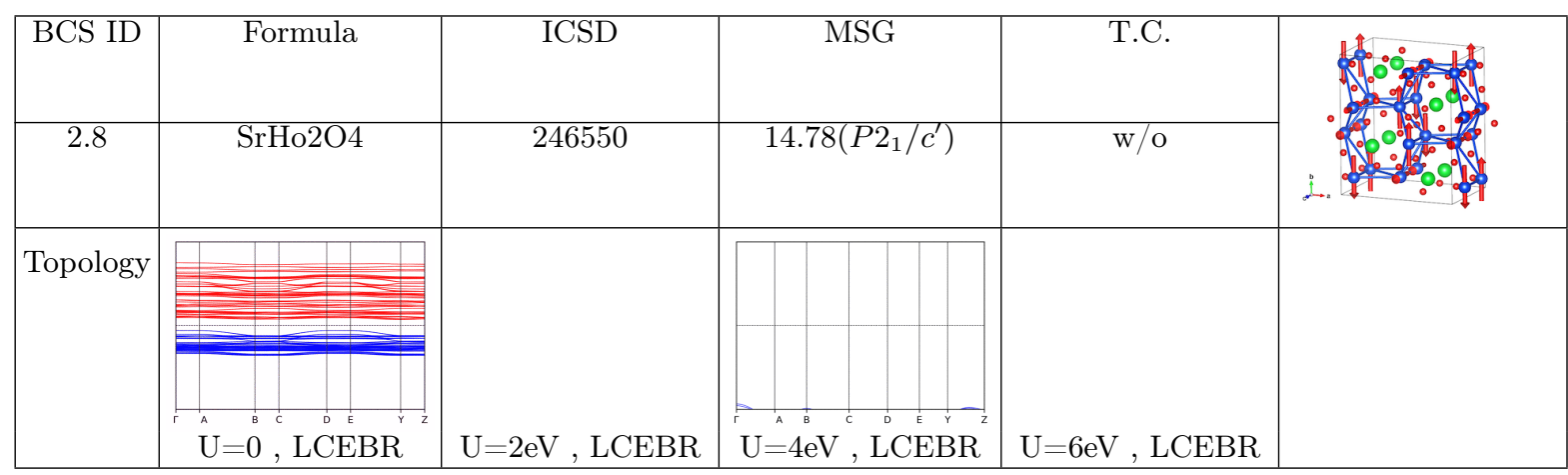

TABLE CCCXIII. Topology phase diagram of SrHo2O4.

\begin{tabular}{|c|c|c|c|c|c|}
\hline BCS ID & Formula & ICSD & MSG & T.C. & bet \\
\hline 1.95 & $\overline{\mathrm{BaNd} 2 \mathrm{O} 4}$ & 78486 & $14.80\left(P_{a} 2_{1} / c\right)$ & $\overline{\mathbb{Z}_{2}}$ & \\
\hline \multirow[t]{2}{*}{ Topology } & \multirow[b]{2}{*}{$\mathrm{U}=0, \mathrm{LCEBR}$} & \multirow[b]{2}{*}{$\mathrm{U}=2 \mathrm{eV}, \mathrm{TBD}$} & \multirow[b]{2}{*}{$\mathrm{U}=4 \mathrm{eV}, \mathrm{LCEBR}$} & & \\
\hline & & & & $\mathrm{U}=6 \mathrm{eV}, \mathrm{LCEBR}^{\mathrm{i}}$ & \\
\hline
\end{tabular}

TABLE CCCXIV. Topology phase diagram of BaNd2O4.

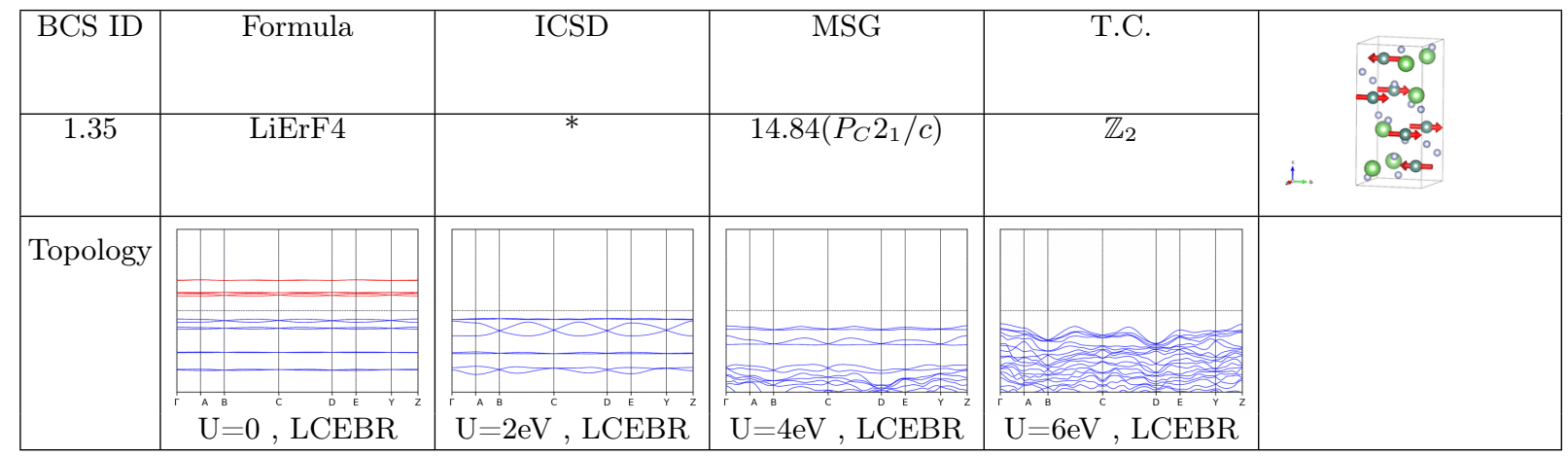

TABLE CCCXV. Topology phase diagram of LiErF4. 


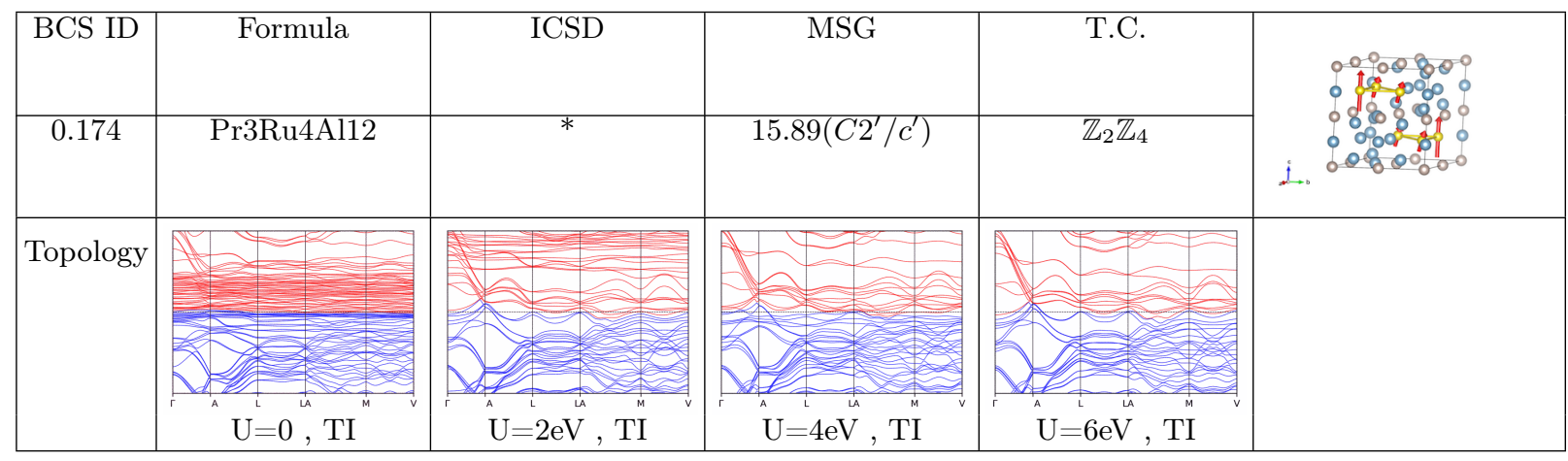

TABLE CCCXVI. Topology phase diagram of Pr3Ru4Al12.

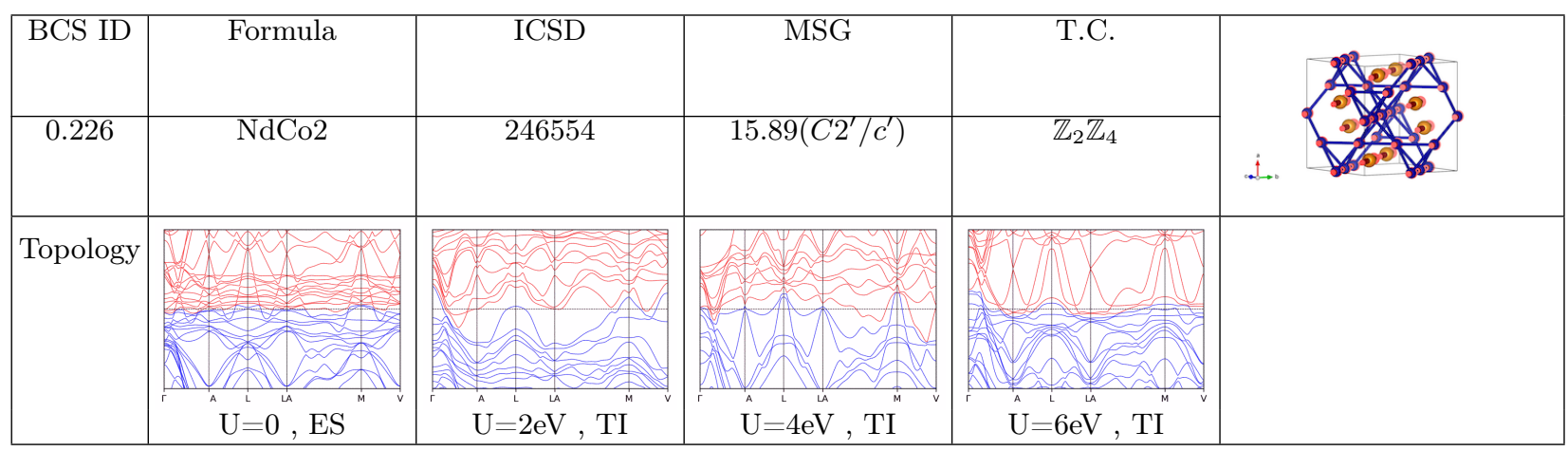

TABLE CCCXVII. Topology phase diagram of NdCo2.

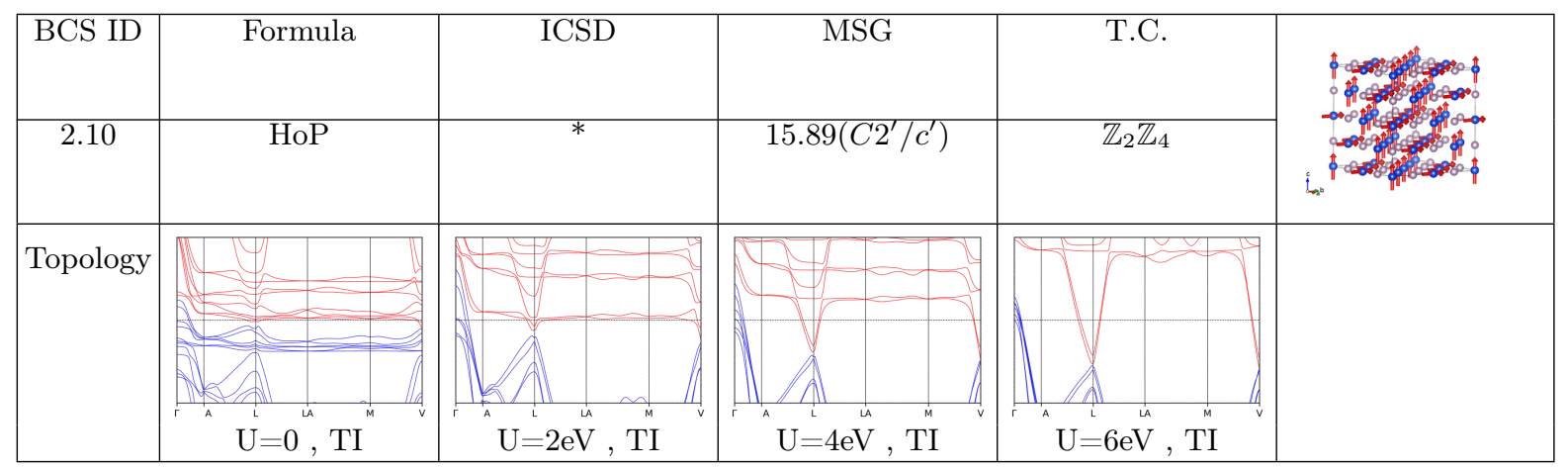

TABLE CCCXVIII. Topology phase diagram of HoP.

\begin{tabular}{|c|c|c|c|c|c|}
\hline BCS ID & Formula & ICSD & MSG & T.C. & \\
\hline 1.14 & Ho2BaNiO5 & 67930 & $15.90\left(C_{c} 2 / c\right)$ & $\mathbb{Z}_{2}$ & 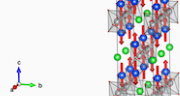 \\
\hline Topology & (N) & 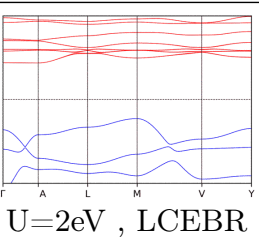 & $\mathrm{U}^{A}=4 \mathrm{eV},{ }^{\prime} \mathrm{LCEBR}$ & $\mathrm{U}^{A}=6 \mathrm{eV}, \mathrm{LCEBR}$ & \\
\hline
\end{tabular}

TABLE CCCXIX. Topology phase diagram of Ho2BaNiO5. 


\begin{tabular}{|c|c|c|c|c|c|}
\hline BCS ID & Formula & ICSD & MSG & T.C. & \\
\hline 1.15 & Er2BaNiO5 & 72630 & $15.90\left(C_{c} 2 / c\right)$ & $\mathbb{Z}_{2}$ & 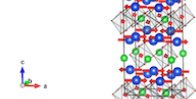 \\
\hline Topology & 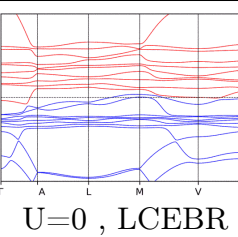 & 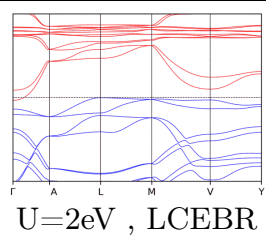 & $\mathrm{U}^{A}=4 \mathrm{eV}, \stackrel{\mathrm{L} C E B R}{ }$ & $\underbrace{}_{\mathrm{U}=6 \mathrm{eV}, \stackrel{\mathrm{LCEBR}}{ }}$ & \\
\hline
\end{tabular}

TABLE CCCXX. Topology phase diagram of Er2BaNiO5.

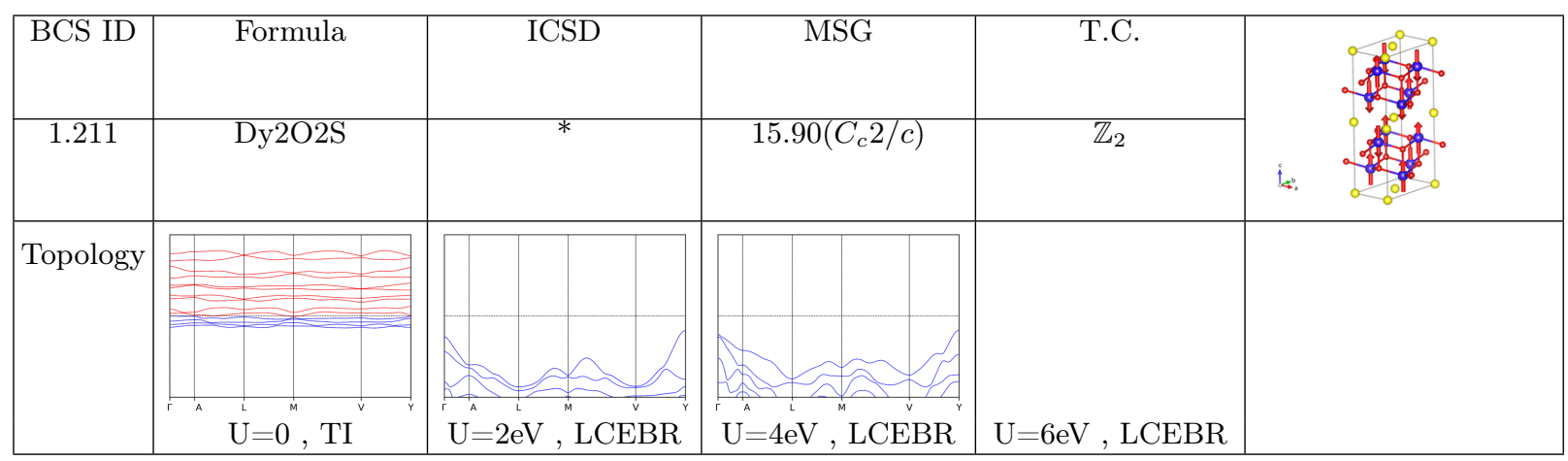

TABLE CCCXXI. Topology phase diagram of Dy2O2S.

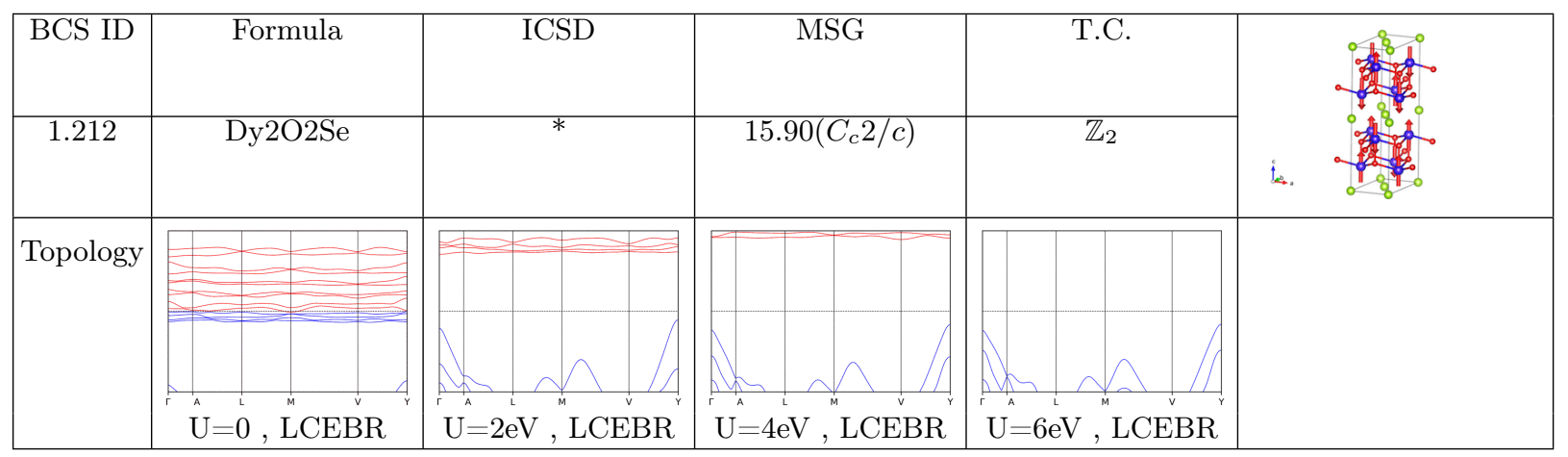

TABLE CCCXXII. Topology phase diagram of Dy2O2Se.

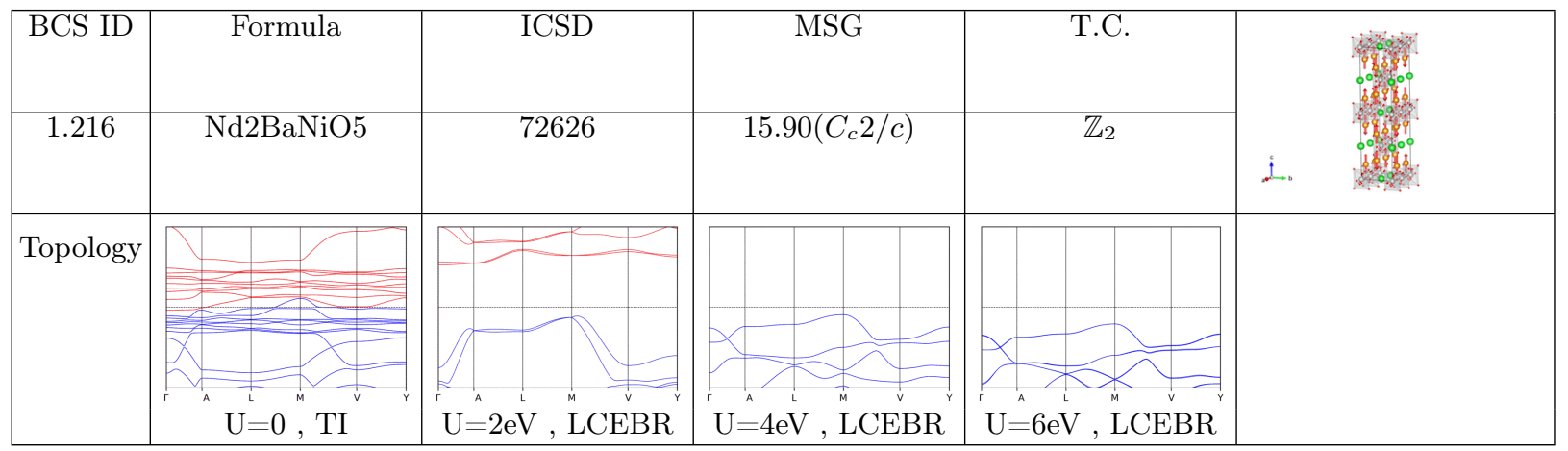

TABLE CCCXXIII. Topology phase diagram of Nd2BaNiO5. 


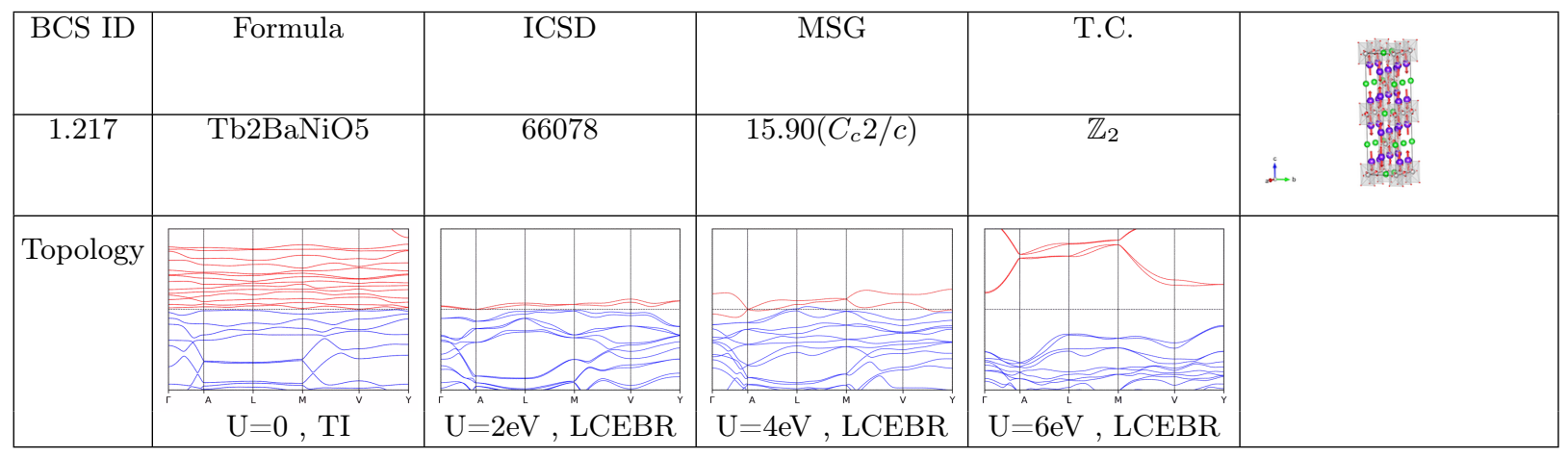

TABLE CCCXXIV. Topology phase diagram of Tb2BaNiO5.

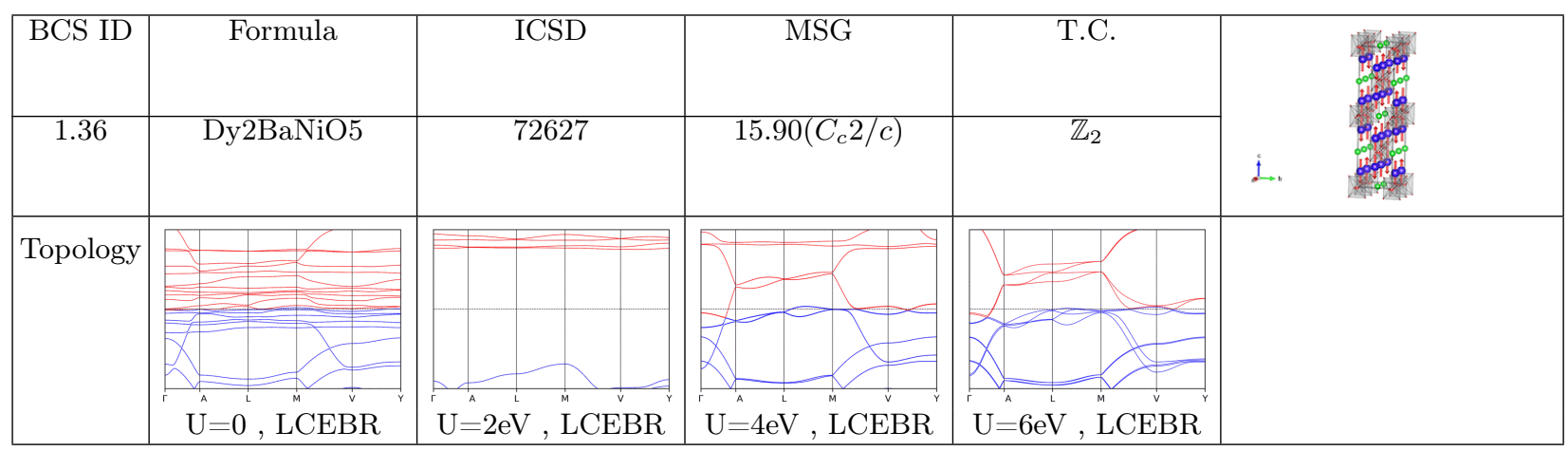

TABLE CCCXXV. Topology phase diagram of Dy2BaNiO5.

\begin{tabular}{|c|c|c|c|c|c|c|}
\hline BCS ID & Formula & ICSD & MSG & T.C. & & \\
\hline 1.53 & Er2BaNiO5 & 72630 & $15.90\left(C_{c} 2 / c\right)$ & $\mathbb{Z}_{2}$ & & 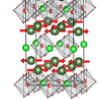 \\
\hline Topology & $\mathrm{U}_{\mathrm{A}=0, \mathrm{~L}^{\prime \prime} \mathrm{CEBR}_{\mathrm{v}}}$ & 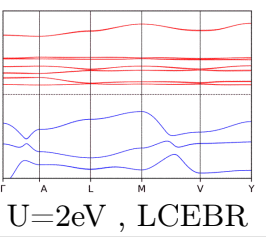 & Un=4eV, LCEBR & $\mathrm{U}=6 \mathrm{eV}, \mathrm{LCEBR}$ & & \\
\hline
\end{tabular}

TABLE CCCXXVI. Topology phase diagram of Er2BaNiO5.

\begin{tabular}{|c|c|c|c|c|c|}
\hline BCS ID & Formula & ICSD & MSG & T.C. & \\
\hline 1.20 & HoMnO3 & * & $31.129\left(P_{b} m n 2_{1}\right)$ & $\mathrm{w} / \mathrm{o}$ & $\begin{array}{l}0 \% \\
100\end{array}$ \\
\hline \multicolumn{6}{|l|}{ Topology } \\
\hline & $\mathrm{U}=0, \mathrm{LCEBR}$ & $\mathrm{U}=2 \mathrm{eV}, \mathrm{TBD}$ & $\mathrm{U}=4 \mathrm{eV}, \mathrm{TBD}$ & $\mathrm{U}=6 \mathrm{eV}, \mathrm{TBD}$ & \\
\hline
\end{tabular}

TABLE CCCXXVII. Topology phase diagram of HoMnO3. 


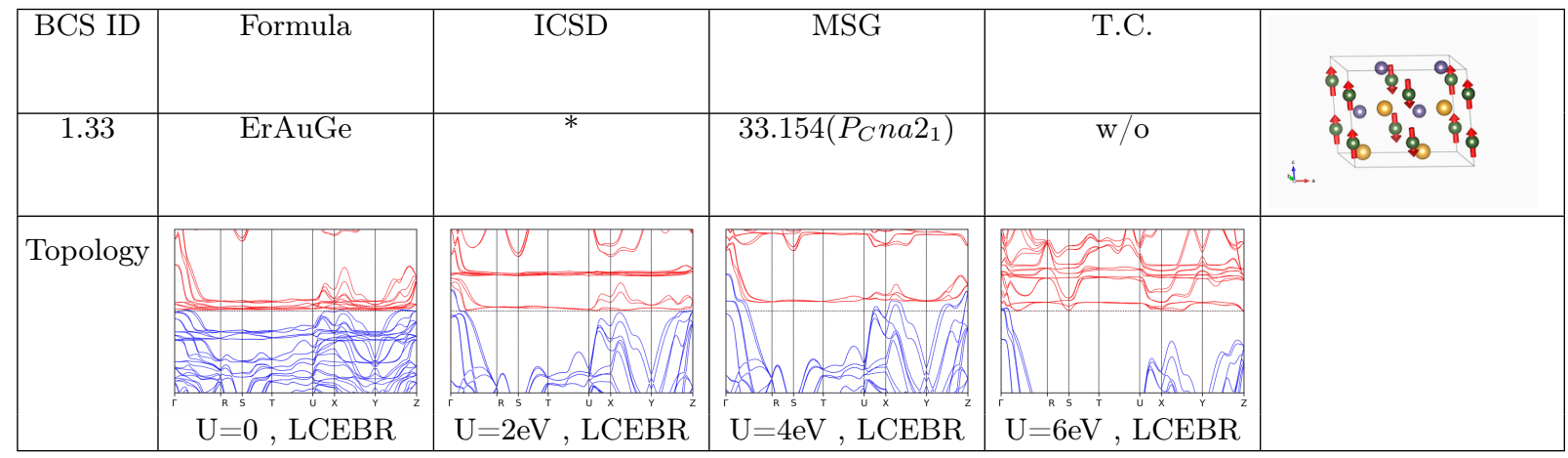

TABLE CCCXXVIII. Topology phase diagram of ErAuGe.

\begin{tabular}{|c|c|c|c|c|c|}
\hline BCS ID & Formula & ICSD & MSG & T.C. & \\
\hline 1.43 & PrNiO3 & 67718 & $36.178\left(C_{a} m c 2_{1}\right)$ & $\mathrm{w} / \mathrm{o}$ & 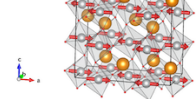 \\
\hline Topology & $\underbrace{\mathrm{K}}_{\mathrm{U}^{\mathrm{R}}=0, \mathrm{LCEBR}}$ & $\mathrm{U}^{\mathrm{r}}=\mathrm{eV}^{\mathrm{s}}, \mathrm{L}^{\mathrm{r}} \mathrm{CEBR}$ & $\frac{\mathrm{U}_{\mathrm{i}}^{\mathrm{s}}=4 \mathrm{eV}, \mathrm{ES}}{\mathrm{r}}$ & $\mathrm{U}^{\mathrm{R}} 6 \mathrm{eV}, \mathrm{LCEBR}$ & \\
\hline
\end{tabular}

TABLE CCCXXIX. Topology phase diagram of PrNiO3.

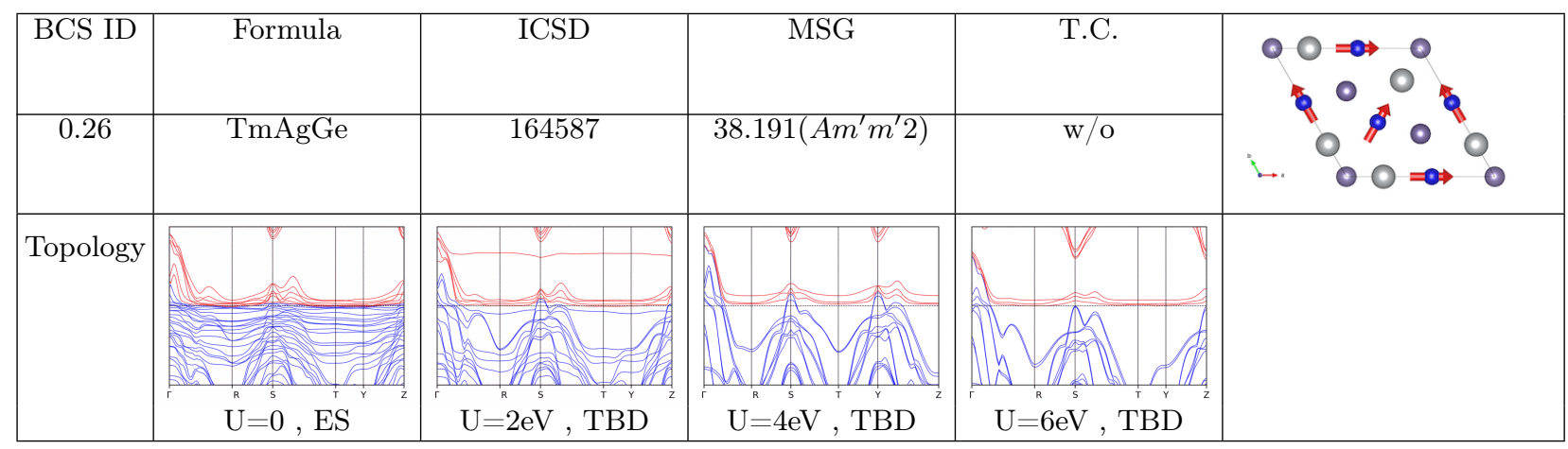

TABLE CCCXXX. Topology phase diagram of TmAgGe.

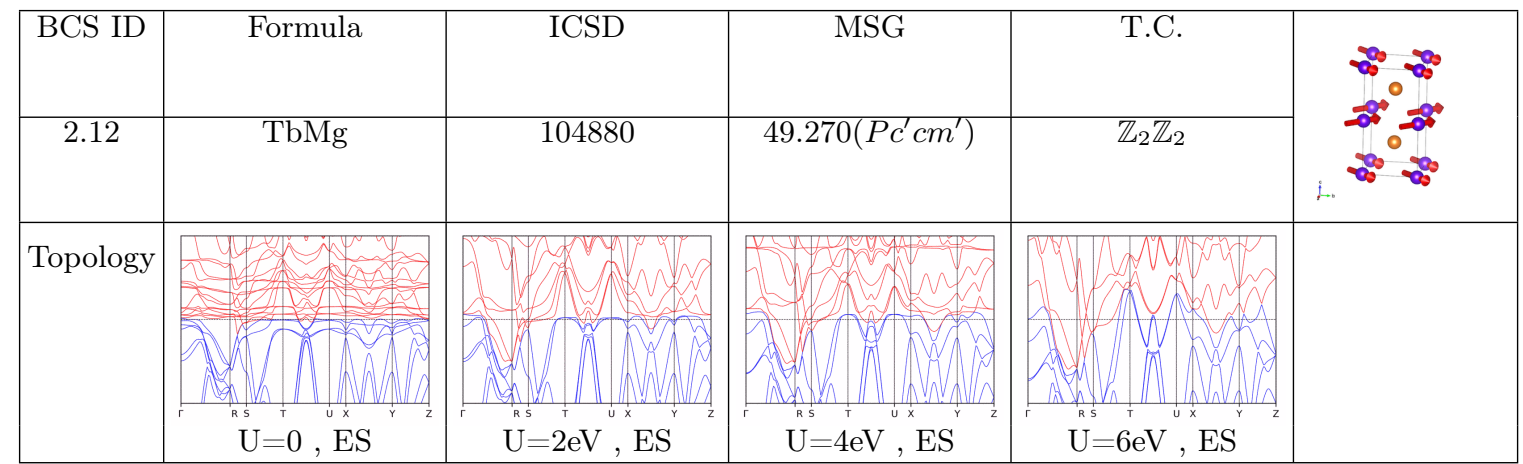

TABLE CCCXXXI. Topology phase diagram of TbMg. 


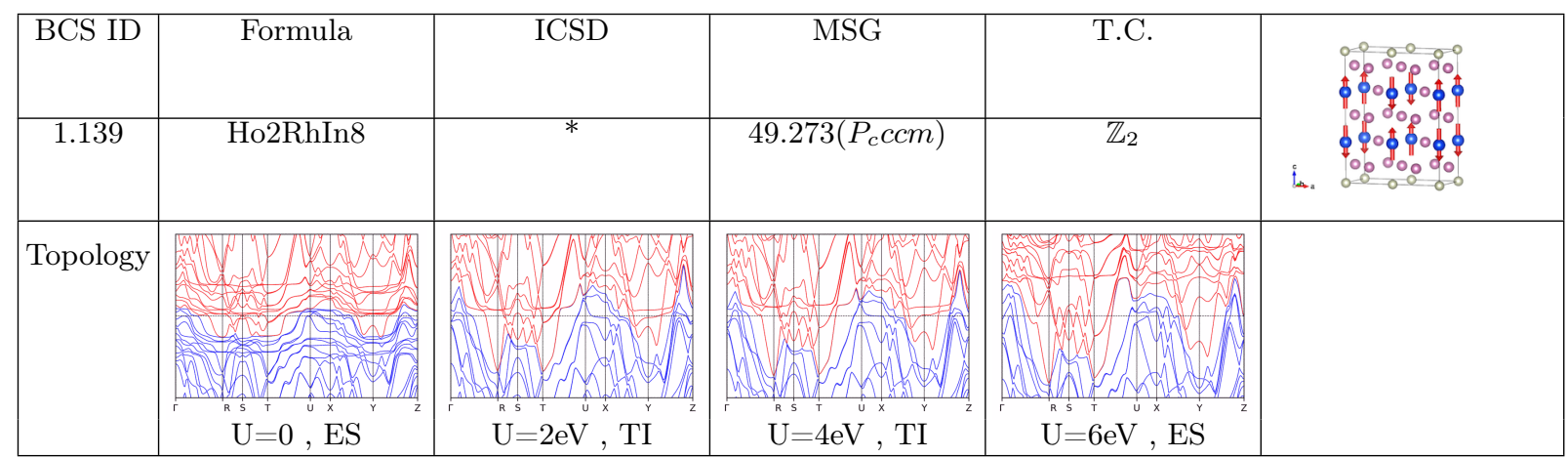

TABLE CCCXXXII. Topology phase diagram of Ho2RhIn8.

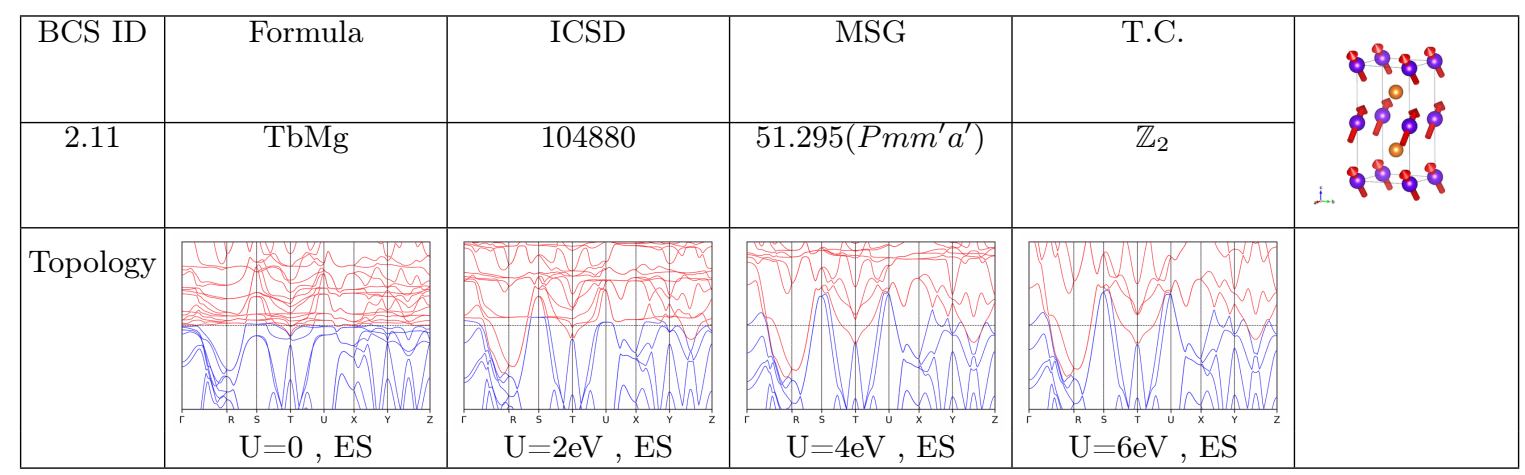

TABLE CCCXXXIII. Topology phase diagram of TbMg.

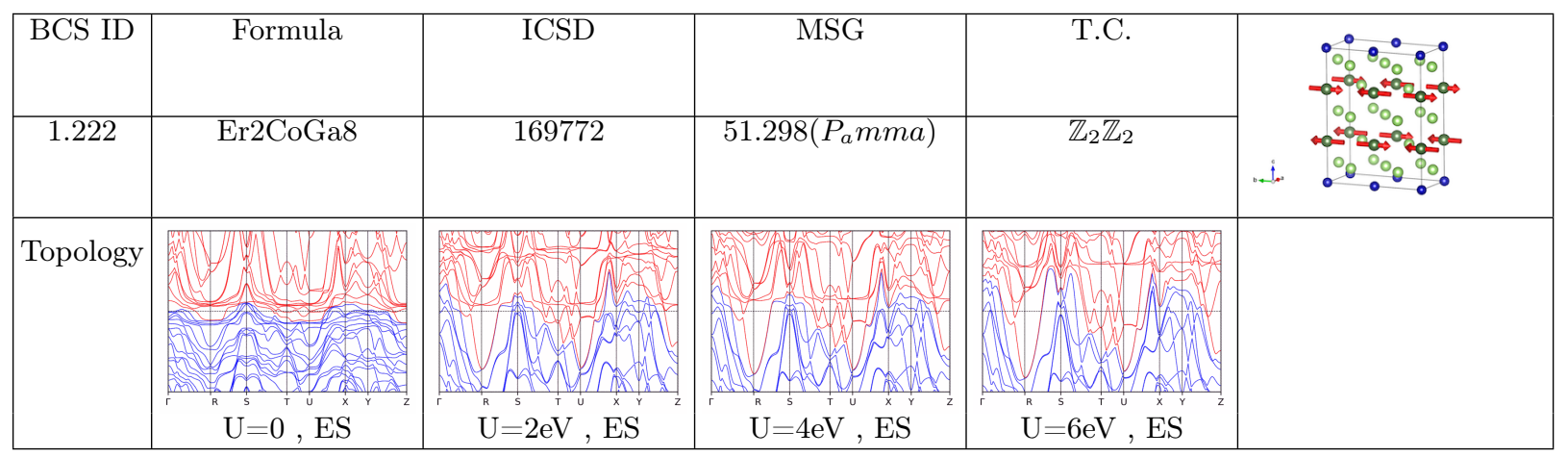

TABLE CCCXXXIV. Topology phase diagram of Er2CoGa8.

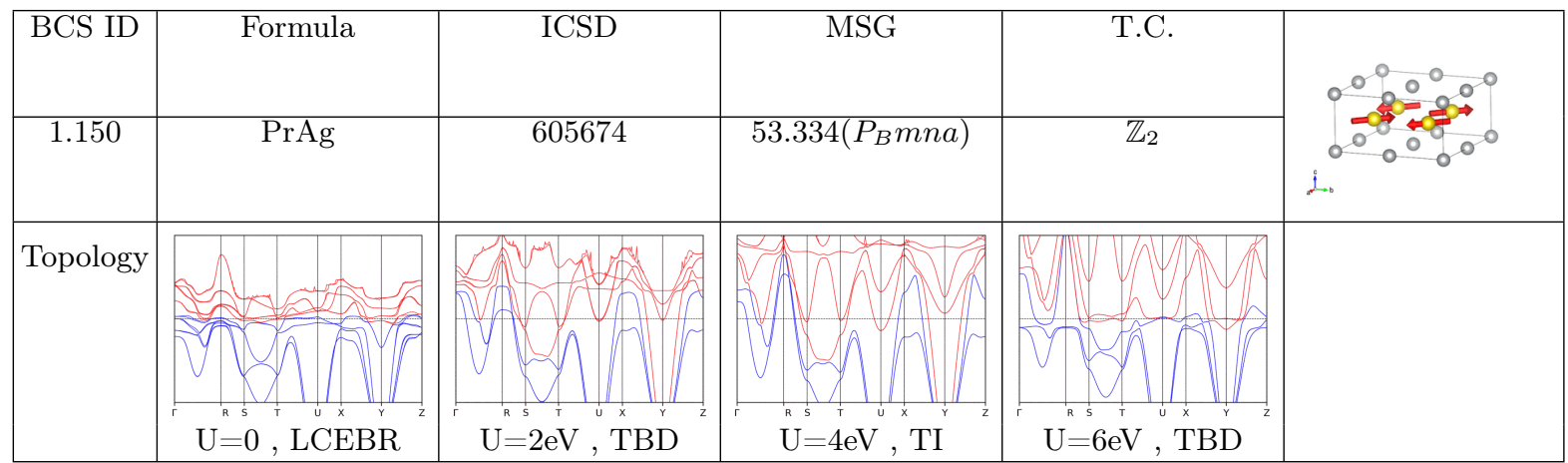

TABLE CCCXXXV. Topology phase diagram of PrAg. 


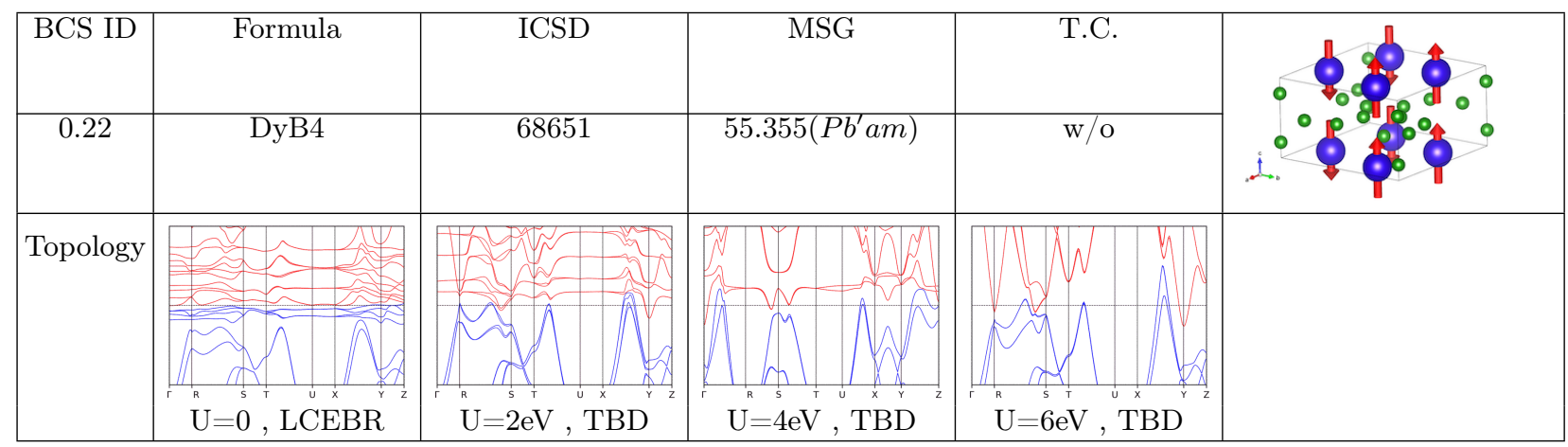

TABLE CCCXXXVI. Topology phase diagram of DyB4.

\begin{tabular}{|c|c|c|c|c|c|}
\hline BCS ID & Formula & ICSD & MSG & T.C. & \\
\hline 1.105 & $\mathrm{Gd} 2 \mathrm{CuO} 4$ & 75425 & $56.374\left({ }^{\prime} P_{A} c c n^{\prime}\right)$ & $\mathbb{Z}_{2}$ & to \\
\hline Topology & U & 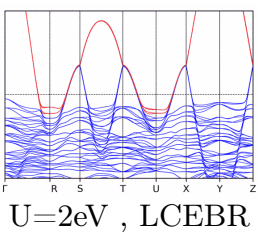 & 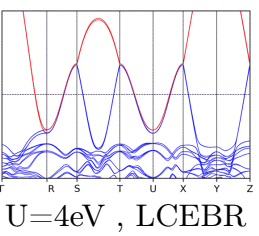 & $\mathrm{U}=6 \mathrm{eV}, \mathrm{LCEBR}$ & \\
\hline
\end{tabular}

TABLE CCCXXXVII. Topology phase diagram of Gd2CuO4.

\begin{tabular}{|c|c|c|c|c|c|}
\hline BCS ID & Formula & ICSD & MSG & T.C. & \\
\hline 1.8 & CeRu2Al10 & 59912 & $57.391\left(P_{C} b c m\right)$ & $\overline{\mathbb{Z}_{2}}$ & 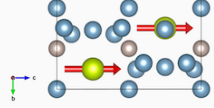 \\
\hline Topology & 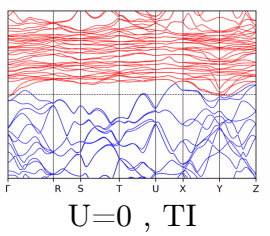 & $\sum_{\substack{\mathrm{R} \mathrm{S}^{\mathrm{T}}=2 \mathrm{eV}, \mathrm{TI} \\
\mathrm{v}}}$ & ( & 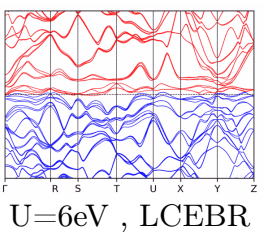 & \\
\hline
\end{tabular}

TABLE CCCXXXVIII. Topology phase diagram of CeRu2Al10.

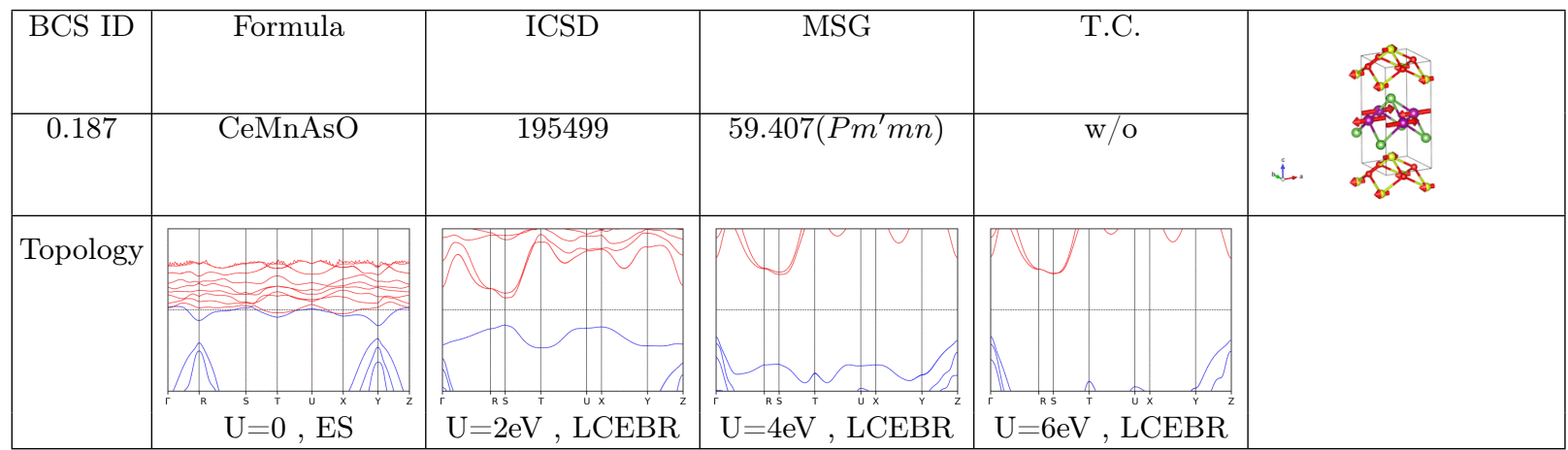

TABLE CCCXXXIX. Topology phase diagram of CeMnAsO. 


\begin{tabular}{|c|c|c|c|c|c|}
\hline BCS ID & Formula & ICSD & MSG & T.C. & \\
\hline 0.146 & EuZrO3 & & $62.444\left(P^{\prime} m^{\prime} a\right)$ & $\mathrm{w} / \mathrm{o}$ & L. Totoros \\
\hline Topology & & $\nabla$ & $\psi$ & & \\
\hline & $\mathrm{U}_{\mathrm{R}}^{\mathrm{s}} 0^{\tau}, \mathrm{L} \mathrm{LEER}^{\mathrm{x}}$ & 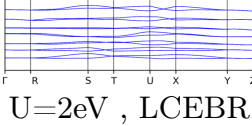 & 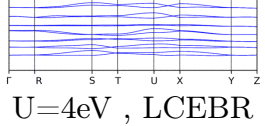 & 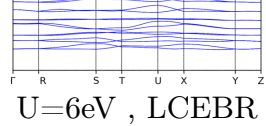 & \\
\hline
\end{tabular}

TABLE CCCXL. Topology phase diagram of EuZrO3.

\begin{tabular}{|c|c|c|c|c|c|c|}
\hline BCS ID & Formula & ICSD & MSG & T.C. & & \\
\hline 0.185 & Nd5Ge4 & 190405 & $62.447\left(P_{n} m^{\prime} a^{\prime}\right)$ & $\mathbb{Z}_{2}$ & $\infty$ & 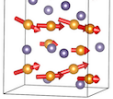 \\
\hline Topology & 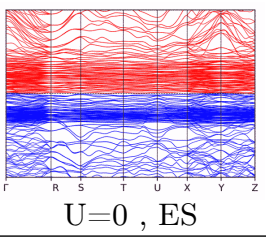 & 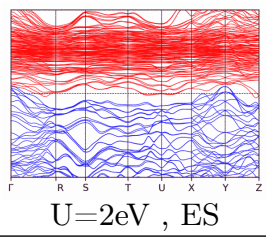 & $\mathrm{U}=4 \mathrm{eV}, \mathrm{TBD}$ & 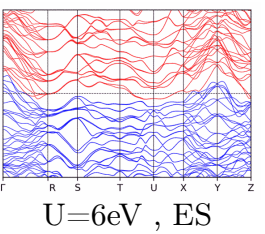 & & \\
\hline
\end{tabular}

TABLE CCCXLI. Topology phase diagram of Nd5Ge4.

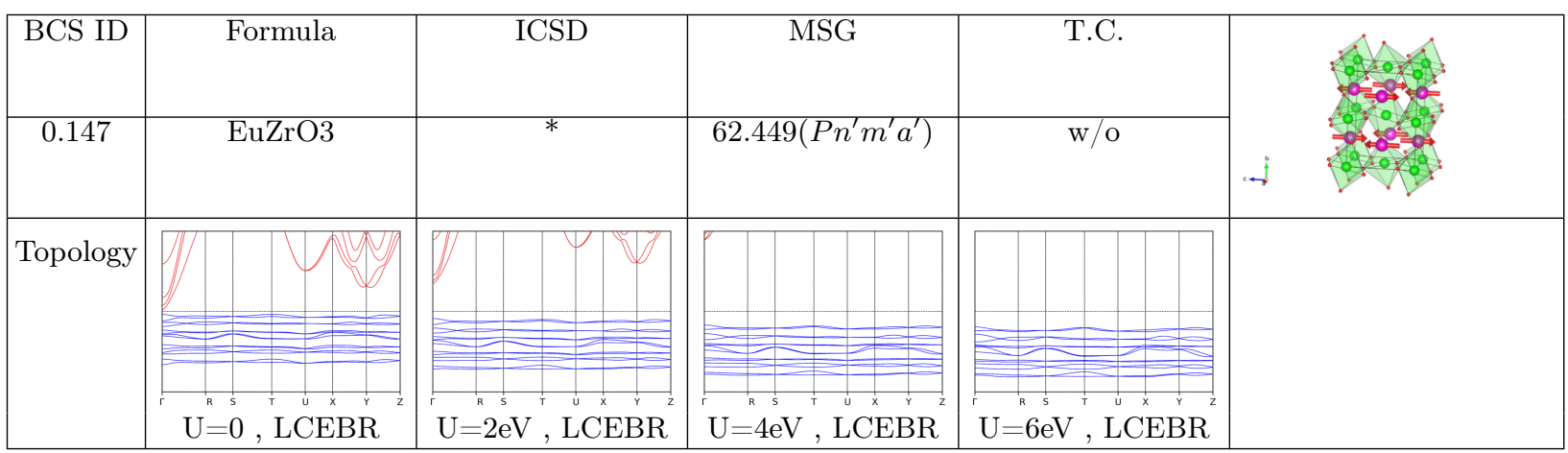

TABLE CCCXLII. Topology phase diagram of EuZrO3.

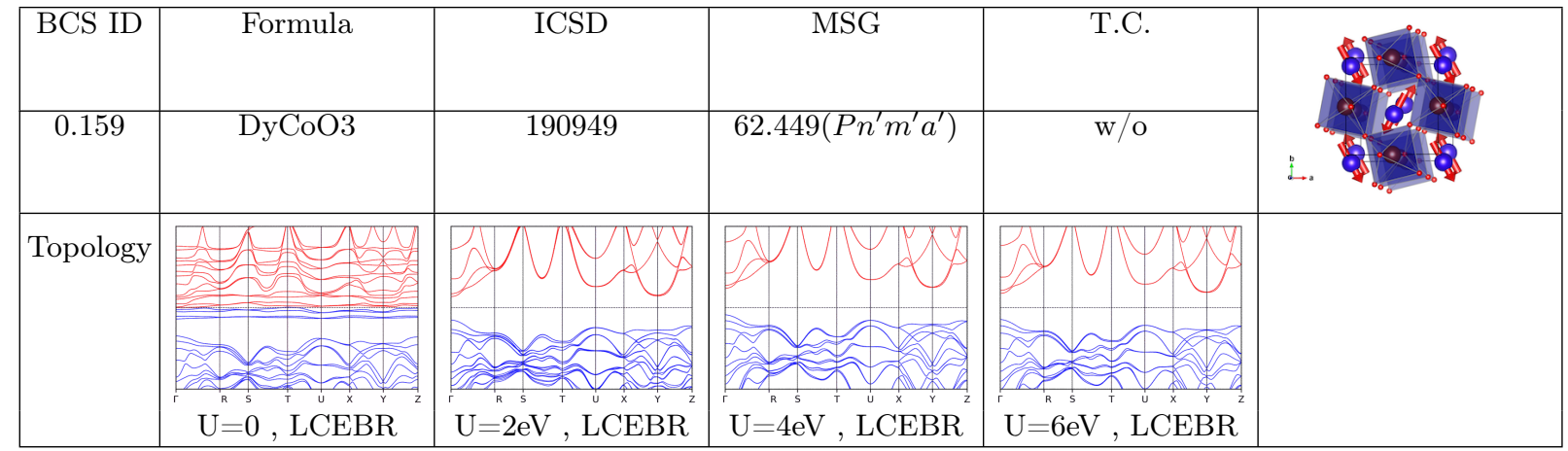

TABLE CCCXLIII. Topology phase diagram of DyCoO3. 


\begin{tabular}{|c|c|c|c|c|c|}
\hline BCS ID & Formula & ICSD & MSG & T.C. & \\
\hline 0.171 & $\overline{\mathrm{DyScO} 3}$ & 99545 & $62.449\left(P n^{\prime} m^{\prime} a^{\prime}\right)$ & $\mathrm{w} / \mathrm{o}$ & 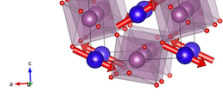 \\
\hline Topology & \begin{tabular}{|l|l|l|} 
& & \\
\end{tabular} & & & & \\
\hline & $\mathrm{U}^{\mathrm{R}}=0, \mathrm{TH}^{\mathrm{s}} \mathrm{TBD}^{\mathrm{r}}$ & $\mathrm{U}=2 \mathrm{eV}^{\mathrm{s}}, \mathrm{LCEBR}^{\mathrm{s}}$ & $\mathrm{U}=4 \mathrm{eV}, \mathrm{LCEBR}$ & $\mathrm{U}=6 \mathrm{eV}, \mathrm{LCEBR}$ & \\
\hline
\end{tabular}

TABLE CCCXLIV. Topology phase diagram of DyScO3.

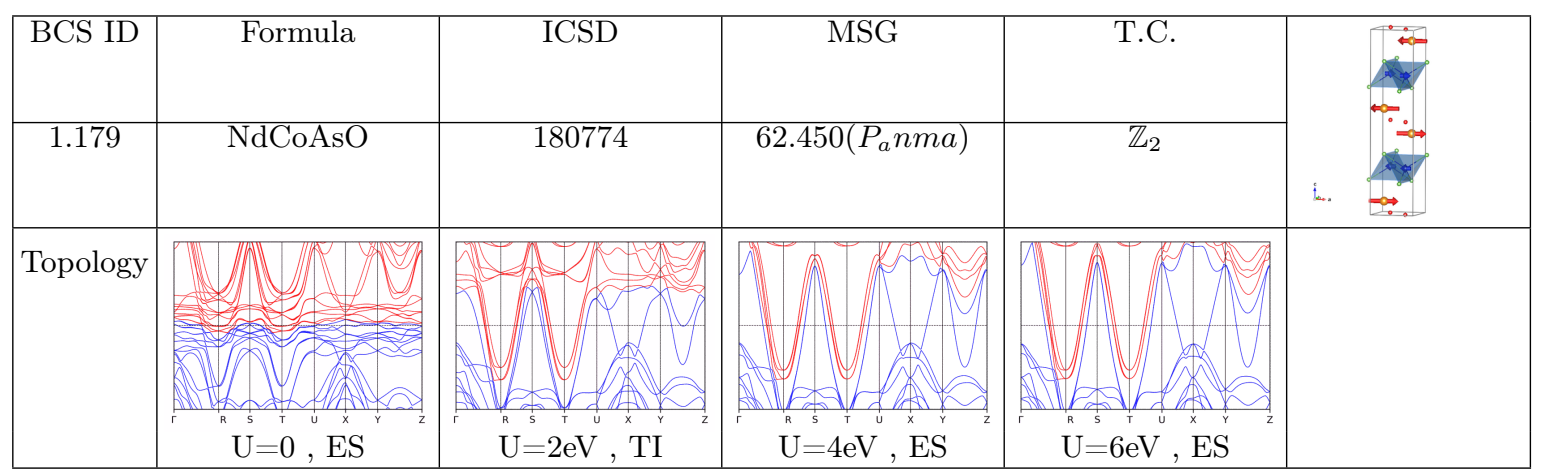

TABLE CCCXLV. Topology phase diagram of NdCoAsO.

\begin{tabular}{|c|c|c|c|c|c|}
\hline BCS ID & Formula & ICSD & MSG & T.C. & \\
\hline 0.12 & U3Ru4Al12 & 163985 & $63.461\left(\mathrm{Cmcm}^{\prime}\right)$ & $\mathrm{w} / \mathrm{o}$ & $\therefore \quad 098 \%$ \\
\hline Topology & U=0, LCEBR & $\mathrm{U}=2 \mathrm{eV}, \mathrm{TBD}$ & $\mathrm{U}^{\mathrm{R}}=4 \mathrm{eV}, \mathrm{LCEBR}$ & $\underbrace{}_{\mathrm{U}=6 \mathrm{eV},{ }^{\circ} \mathrm{LCEBR}}$ & \\
\hline
\end{tabular}

TABLE CCCXLVI. Topology phase diagram of U3Ru4Al12.

\begin{tabular}{|c|c|c|c|c|c|}
\hline BCS ID & Formula & ICSD & MSG & T.C. & \\
\hline 0.149 & Nd3Ru4Al12 & * & $63.462\left(\mathrm{Cm}^{\prime} c^{\prime} m\right)$ & $\mathbb{Z}_{2}$ & 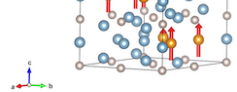 \\
\hline Topology & 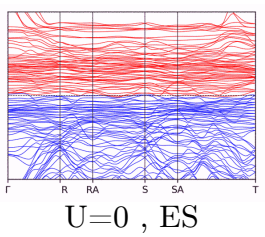 & 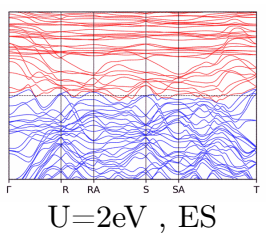 & & $\mathrm{U}=6 \mathrm{eV}, \mathrm{ES}$ & \\
\hline
\end{tabular}

TABLE CCCXLVII. Topology phase diagram of Nd3Ru4Al12. 


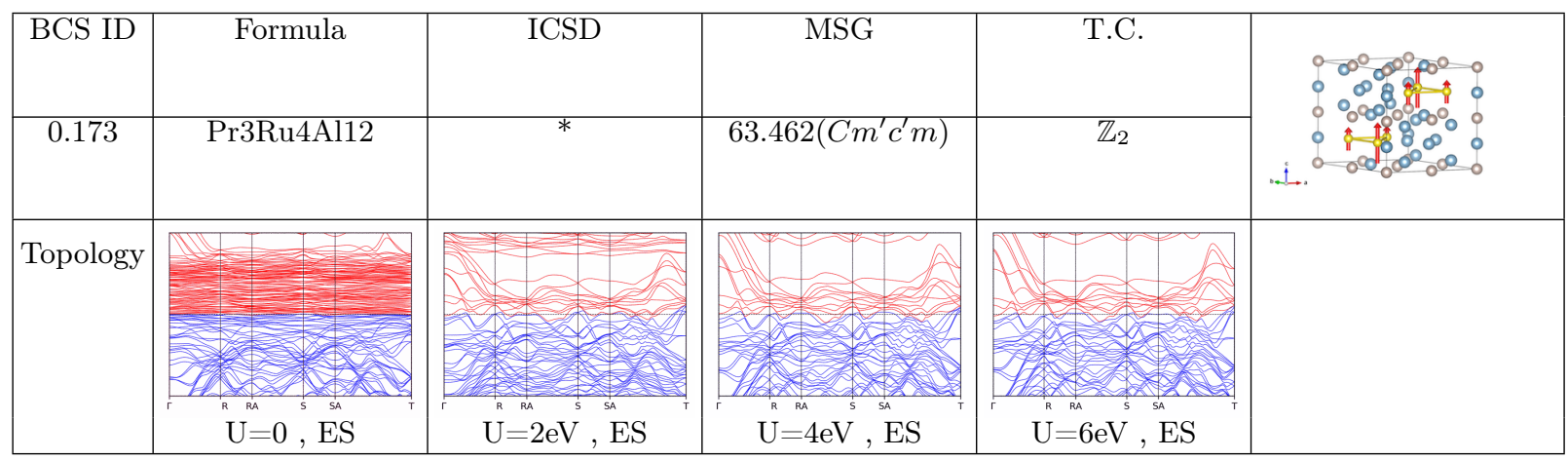

TABLE CCCXLVIII. Topology phase diagram of Pr3Ru4Al12.

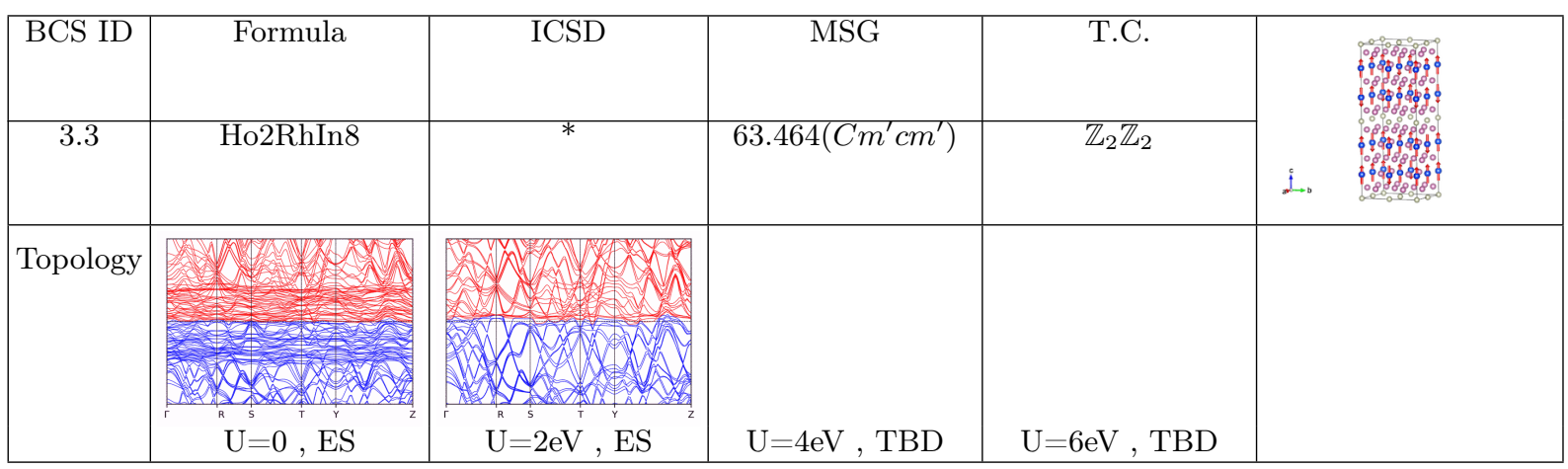

TABLE CCCXLIX. Topology phase diagram of Ho2RhIn8.

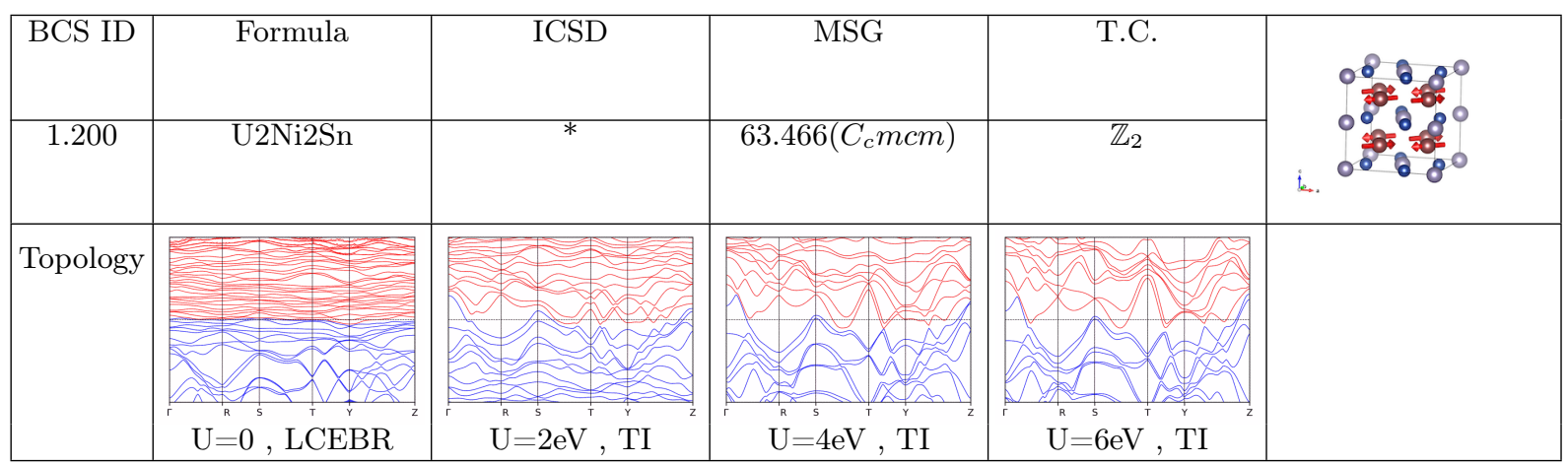

TABLE CCCL. Topology phase diagram of U2Ni2Sn.

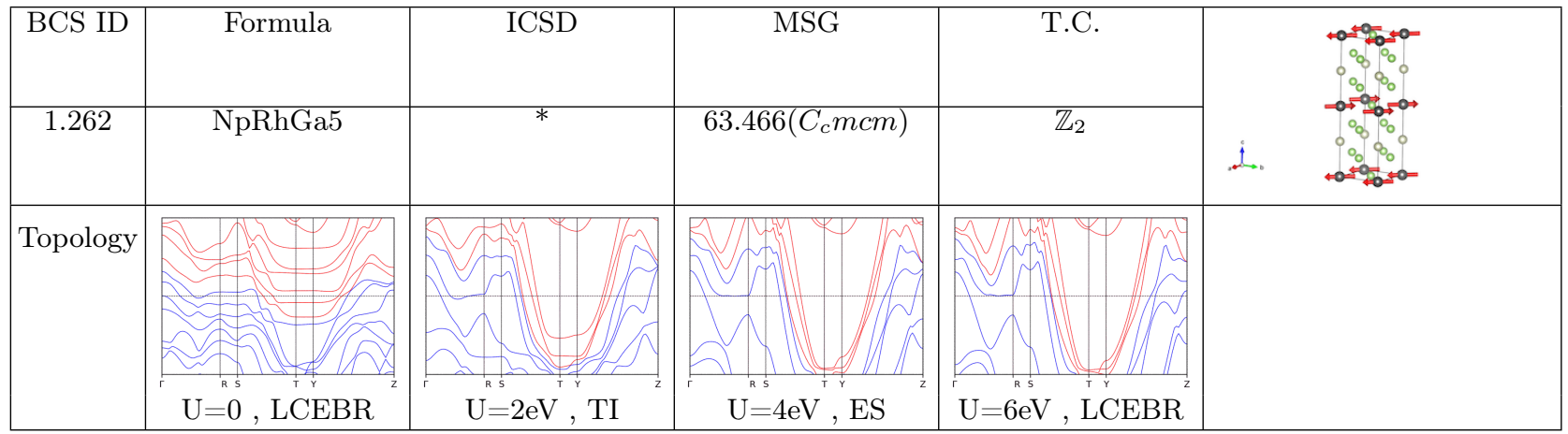

TABLE CCCLI. Topology phase diagram of NpRhGa5. 


\begin{tabular}{|c|c|c|c|c|c|}
\hline BCS ID & Formula & ICSD & MSG & T.C. & \\
\hline 1.195 & Er2Ni2In & & $63.467\left(C_{a} m c m\right)$ & $\mathbb{Z}_{2}$ & 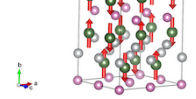 \\
\hline Topology & $\frac{\mathrm{U}_{\mathrm{U}=0, \mathrm{LCEBR}}}{{ }^{\mathrm{i}}}$ & $\begin{array}{llc}\mathrm{U}=2 \mathrm{eV}, \mathrm{LCEBR} \\
\mathrm{C}\end{array}$ & $\mathrm{U}^{\mathrm{n}}=4 \mathrm{eV}, \mathrm{TI}$ & $\mathrm{U}_{\mathrm{U}=6 \mathrm{eV}, \mathrm{LCEBR}}$ & \\
\hline
\end{tabular}

TABLE CCCLII. Topology phase diagram of Er2Ni2In.

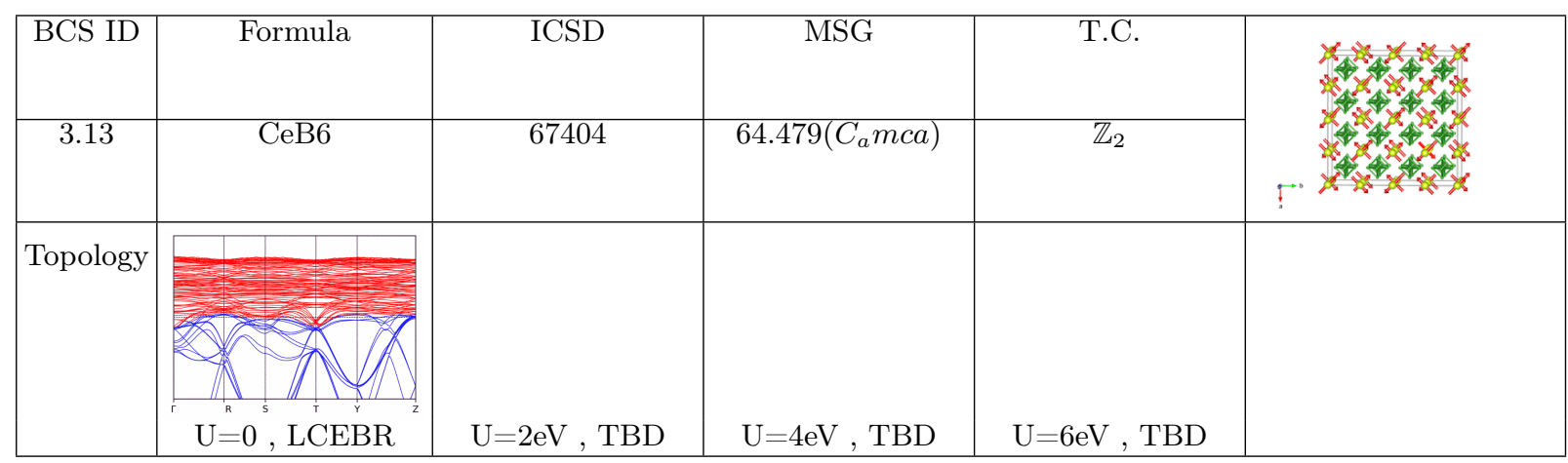

TABLE CCCLIII. Topology phase diagram of CeB6.

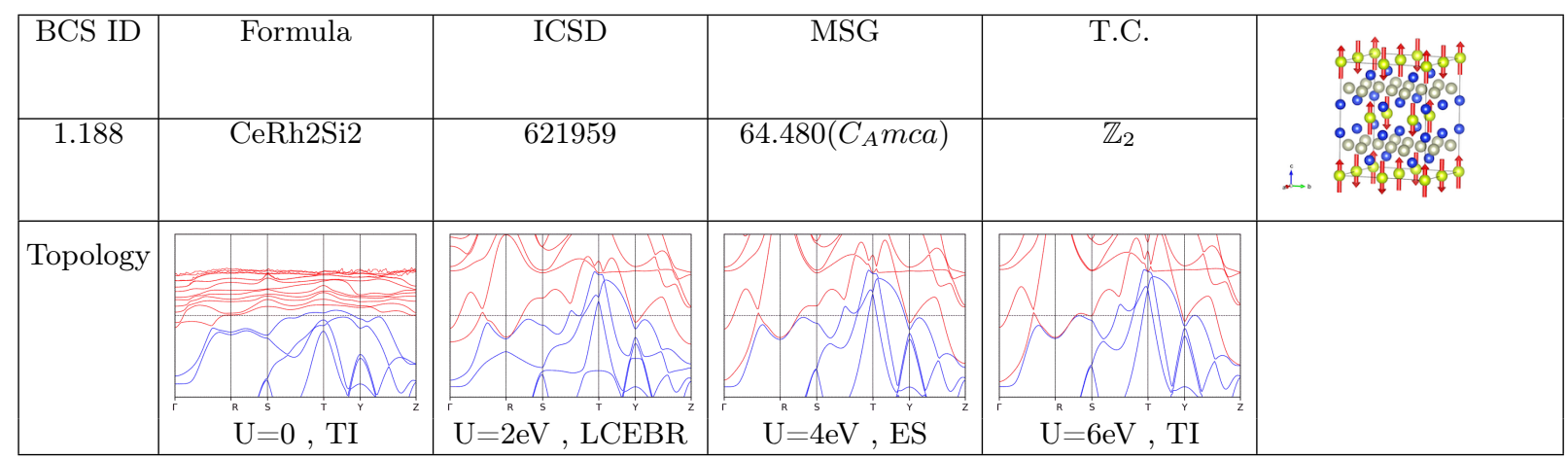

TABLE CCCLIV. Topology phase diagram of CeRh2Si2.

\begin{tabular}{|c|c|c|c|c|c|}
\hline BCS ID & Formula & ICSD & MSG & T.C. & \\
\hline 0.141 & TbGe2 & 56030 & $65.483\left(\mathrm{Cm}^{\prime} \mathrm{mm}\right)$ & $\mathrm{w} / \mathrm{o}$ & 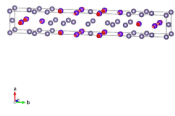 \\
\hline Topology & 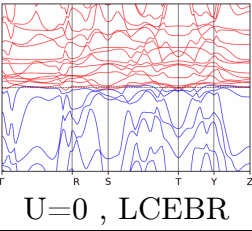 & ${ }_{\mathrm{U}=2 \mathrm{eV}, \mathrm{TBD}}$ & $\underset{\mathrm{U}=4 \mathrm{eV}, \mathrm{TBD}}{\mathrm{T}_{\mathrm{r}}}$ & $\mathrm{U}=6 \mathrm{eV}, \mathrm{LCEBR}$ & \\
\hline
\end{tabular}

TABLE CCCLV. Topology phase diagram of TbGe2. 


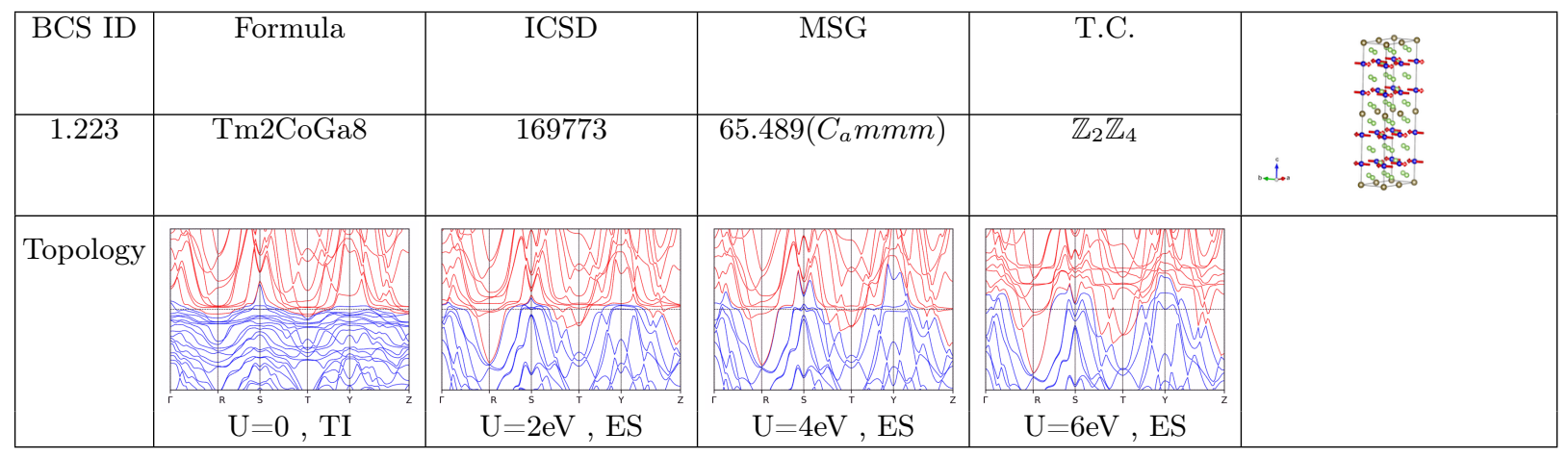

TABLE CCCLVI. Topology phase diagram of Tm2CoGa8.

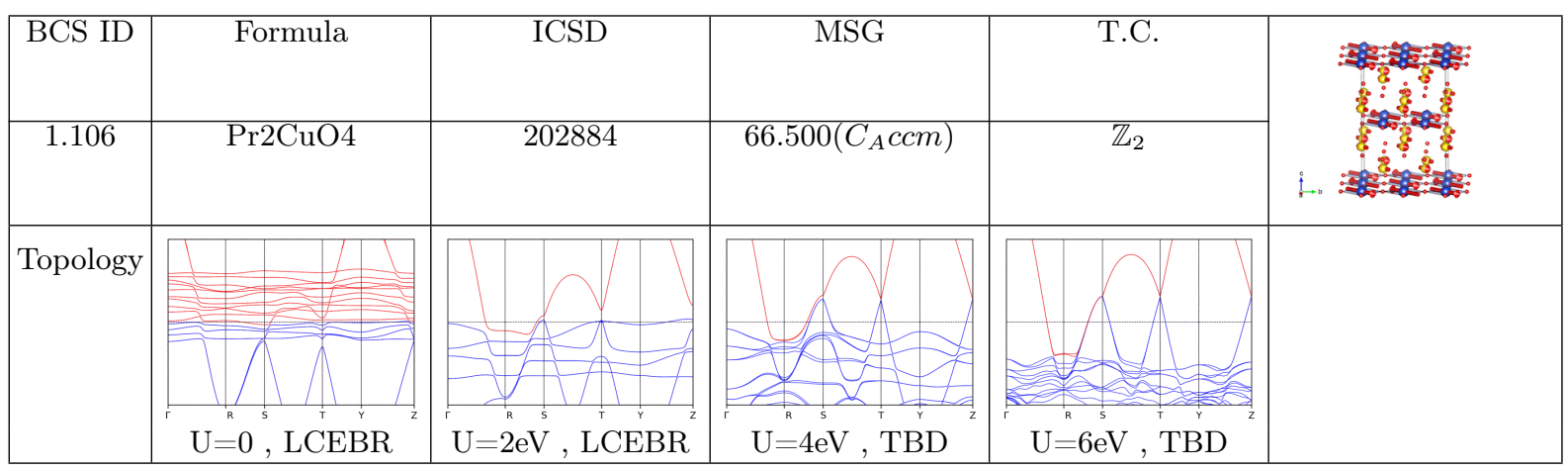

TABLE CCCLVII. Topology phase diagram of Pr2CuO4.

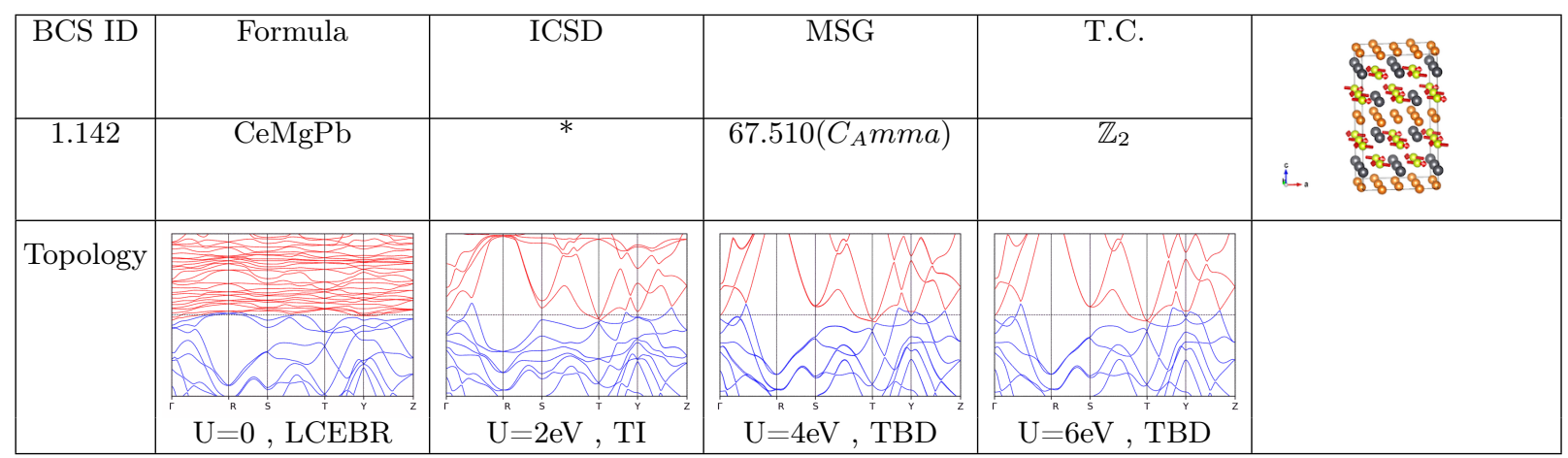

TABLE CCCLVIII. Topology phase diagram of CeMgPb.

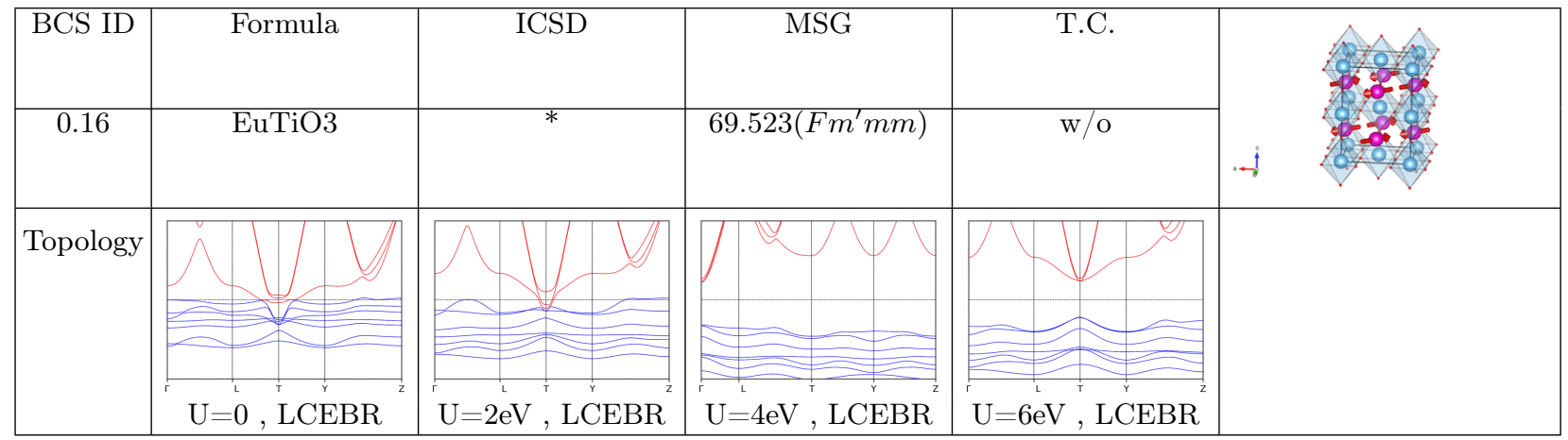

TABLE CCCLIX. Topology phase diagram of EuTiO3. 


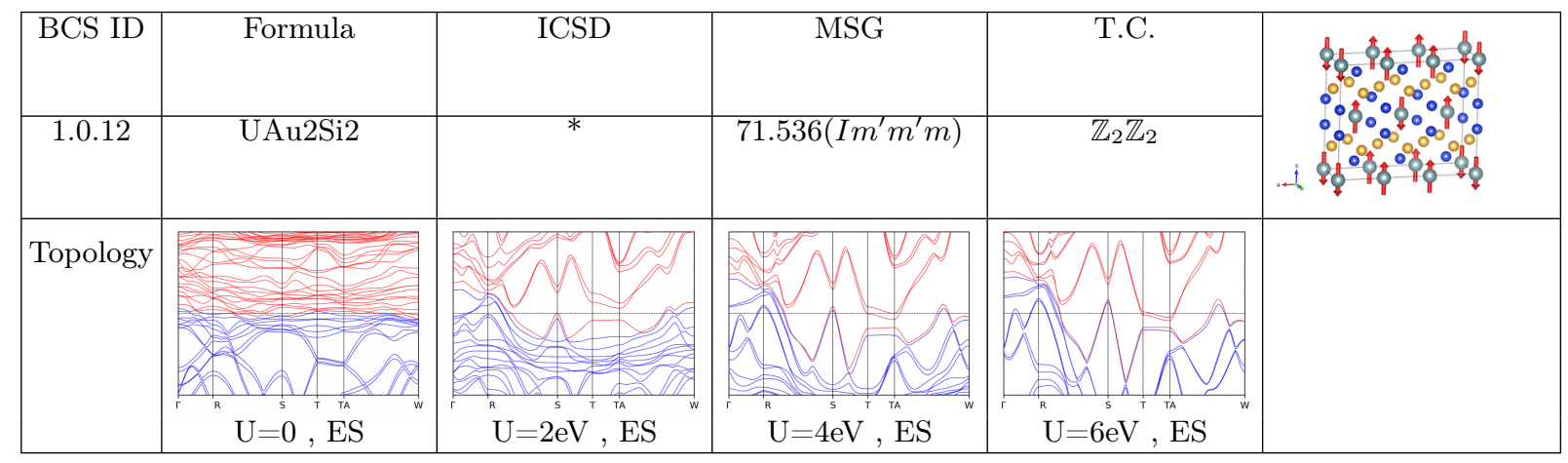

TABLE CCCLX. Topology phase diagram of UAu2Si2.

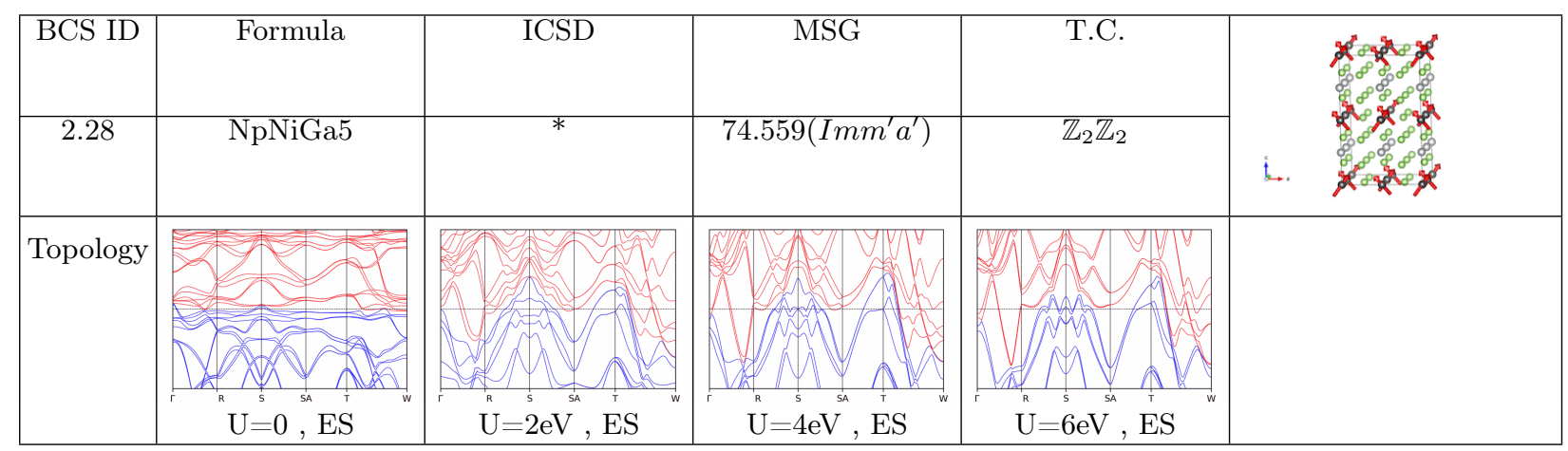

TABLE CCCLXI. Topology phase diagram of NpNiGa5.

\begin{tabular}{|c|c|c|c|c|c|}
\hline BCS ID & Formula & ICSD & MSG & T.C. & \\
\hline 1.59 & KTb3F12 & 51125 & $84.58\left(P_{I} 4_{2} / m\right)$ & $\overline{\mathbb{Z}_{4}}$ & - ito.jo \\
\hline \multirow[t]{3}{*}{ Topology } & \begin{tabular}{|l|l|} 
\\
\end{tabular} & 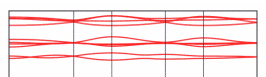 & \begin{tabular}{|l|l|l|} 
& \\
\end{tabular} & & \\
\hline & \begin{tabular}{|l|l|l|l|l|} 
\\
\end{tabular} & 目 & & & \\
\hline & 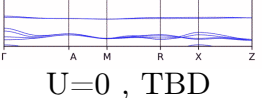 & ${\mathrm{U}=2 \mathrm{eV} \mathrm{V}^{\prime \prime}, \mathrm{LCEBR}^{\prime}}^{\mathrm{N}^{\prime}}$ & ${\mathrm{U}=4 \mathrm{eV}{ }^{\prime \prime}, \mathrm{LCEBR}^{\mathrm{n}}}^{2}$ & $\mathrm{U}=6 \mathrm{eV}, \mathrm{LCEBR}$ & \\
\hline
\end{tabular}

TABLE CCCLXII. Topology phase diagram of KTb3F12.

\begin{tabular}{|c|c|c|c|c|c|}
\hline BCS ID & Formula & ICSD & MSG & T.C. & \\
\hline 0.107 & Ho2Ge2O7 & 161912 & $92.111\left(P 4_{1} 2_{1} 2\right)$ & $\mathrm{w} / \mathrm{o}$ & \pm 0 \\
\hline Topology & \begin{tabular}{|l|l|l|l|} 
& & & \\
\end{tabular} & & & & \\
\hline & 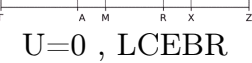 & $\mathrm{U}=2 \mathrm{eV}^{\wedge}, \mathrm{LCEBR}^{\mathrm{k}}$ & $\mathrm{U}=4 \mathrm{eV}^{\mathrm{h}}, \mathrm{LCEBR}$ & $\mathrm{U}=6 \mathrm{eV}^{\prime \prime}, \mathrm{LCEBR}^{2}$ & \\
\hline
\end{tabular}

TABLE CCCLXIII. Topology phase diagram of Ho2Ge2O7. 


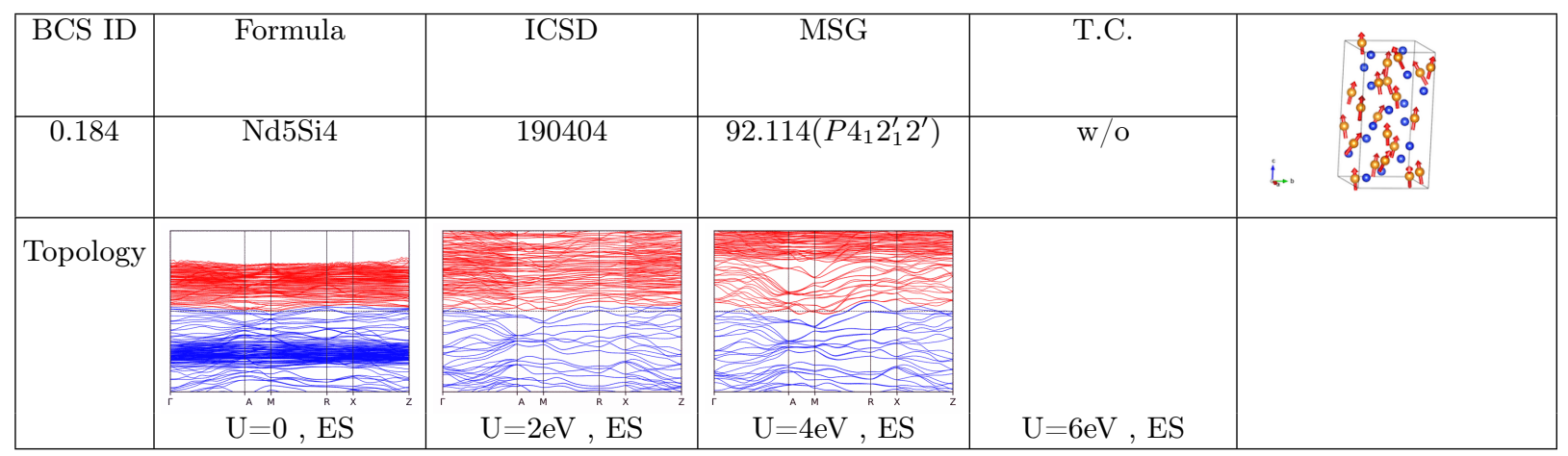

TABLE CCCLXIV. Topology phase diagram of Nd5Si4.

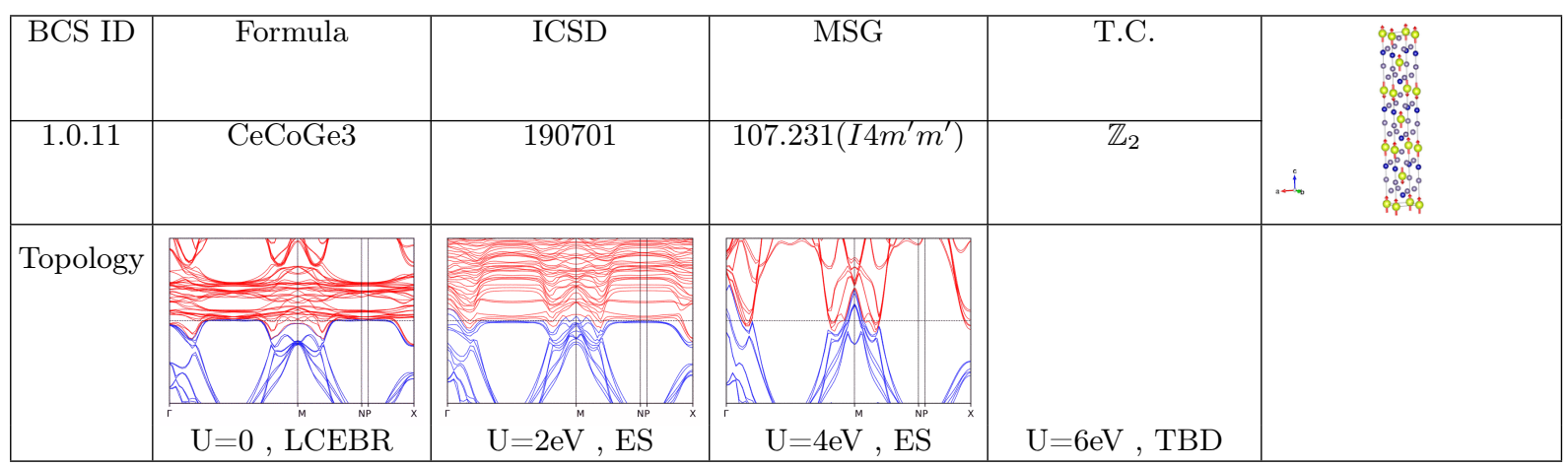

TABLE CCCLXV. Topology phase diagram of CeCoGe3.

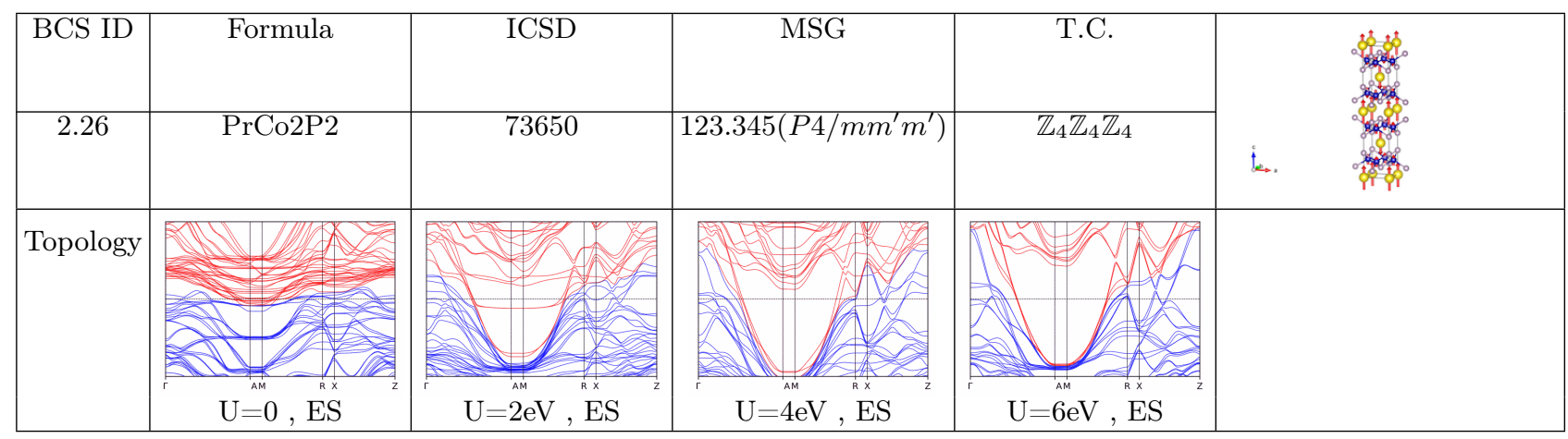

TABLE CCCLXVI. Topology phase diagram of PrCo2P2.

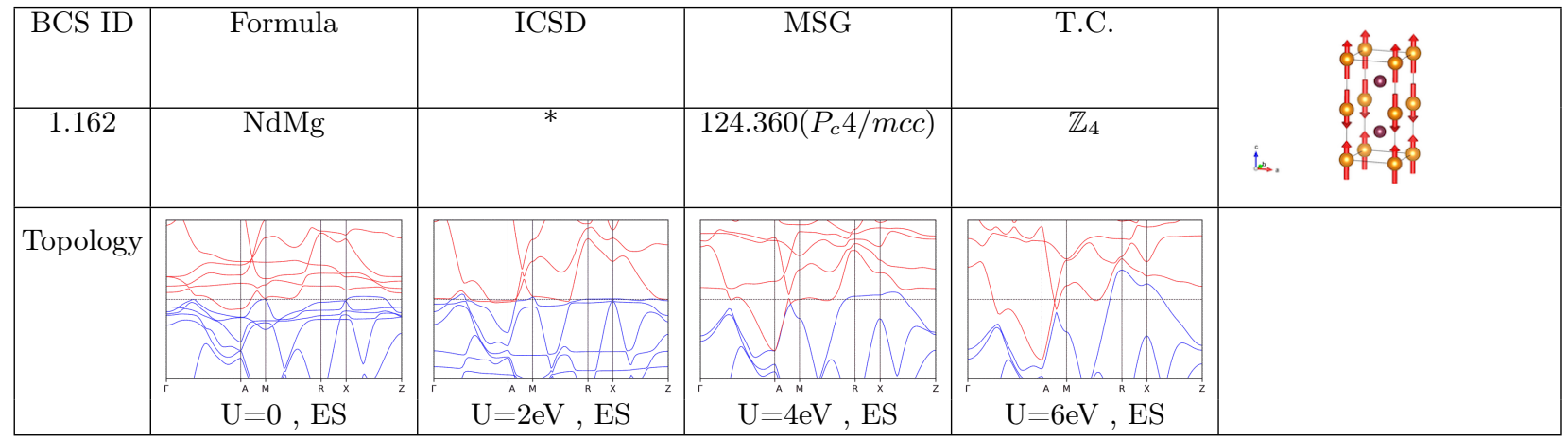

TABLE CCCLXVII. Topology phase diagram of NdMg. 


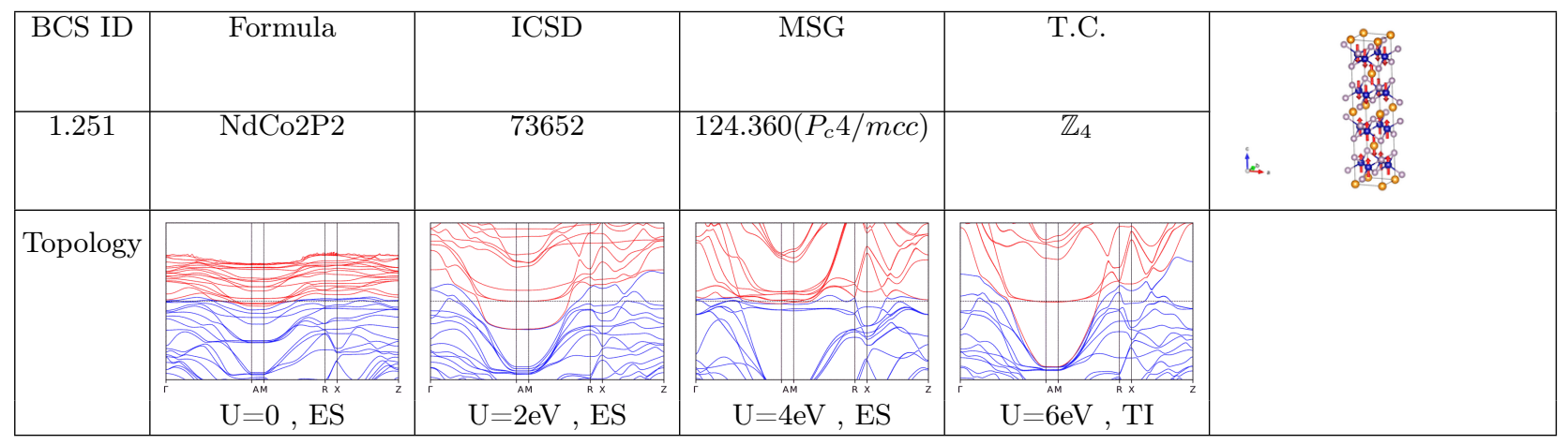

TABLE CCCLXVIII. Topology phase diagram of NdCo2P2.

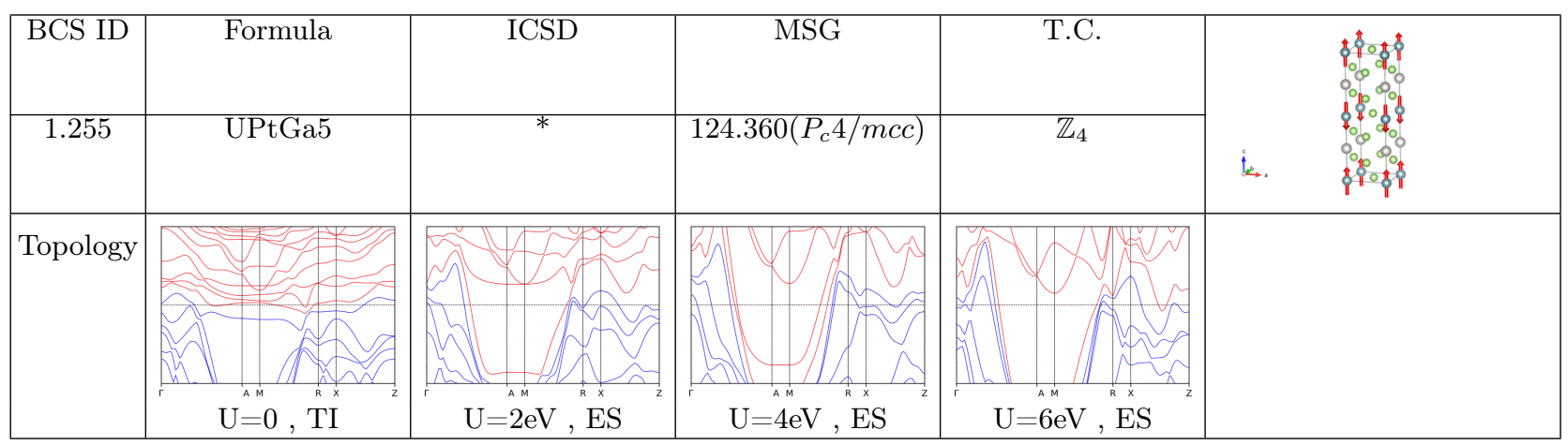

TABLE CCCLXIX. Topology phase diagram of UPtGa5.

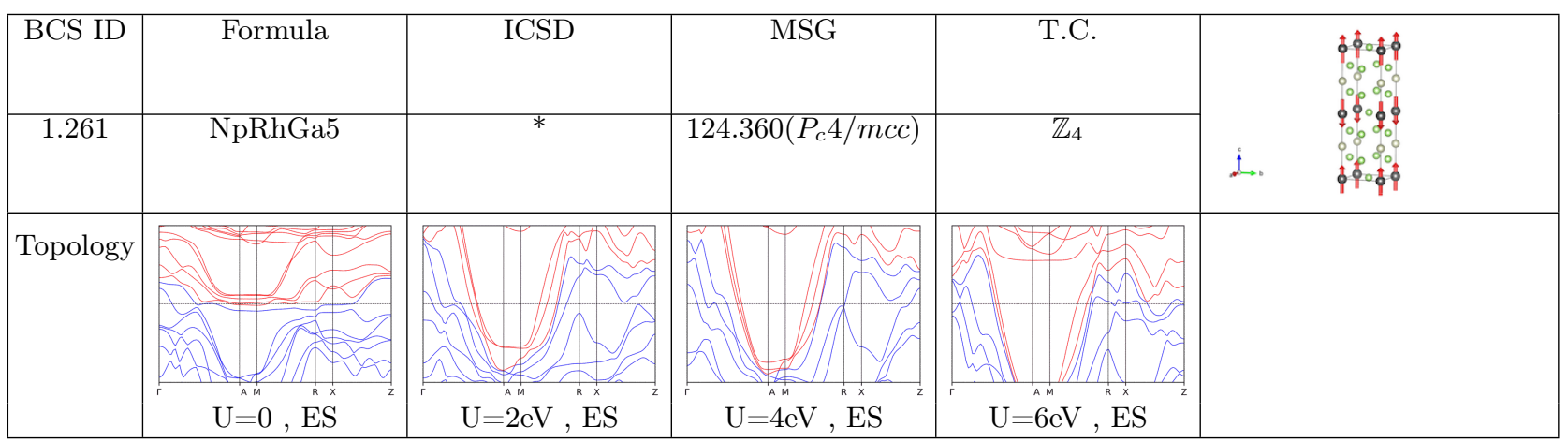

TABLE CCCLXX. Topology phase diagram of NpRhGa5.

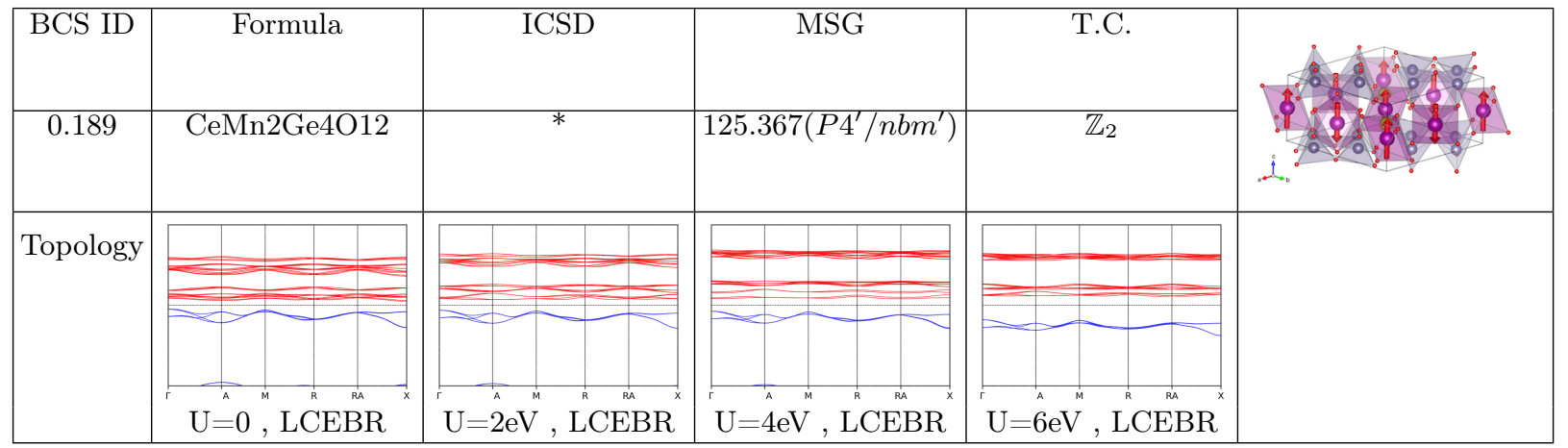

TABLE CCCLXXI. Topology phase diagram of CeMn2Ge4O12. 


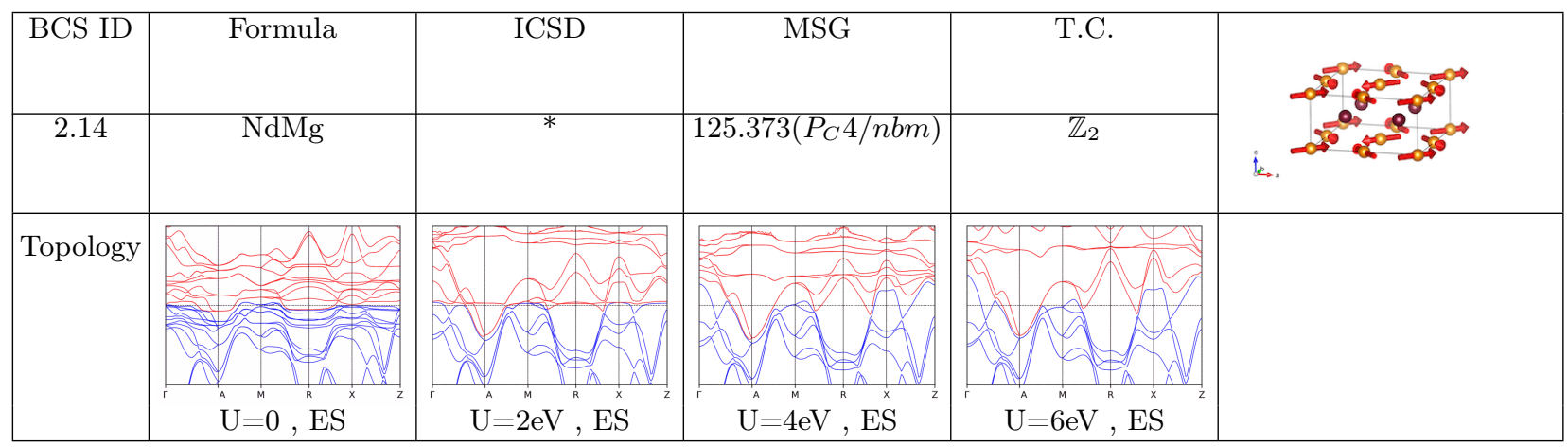

TABLE CCCLXXII. Topology phase diagram of NdMg.

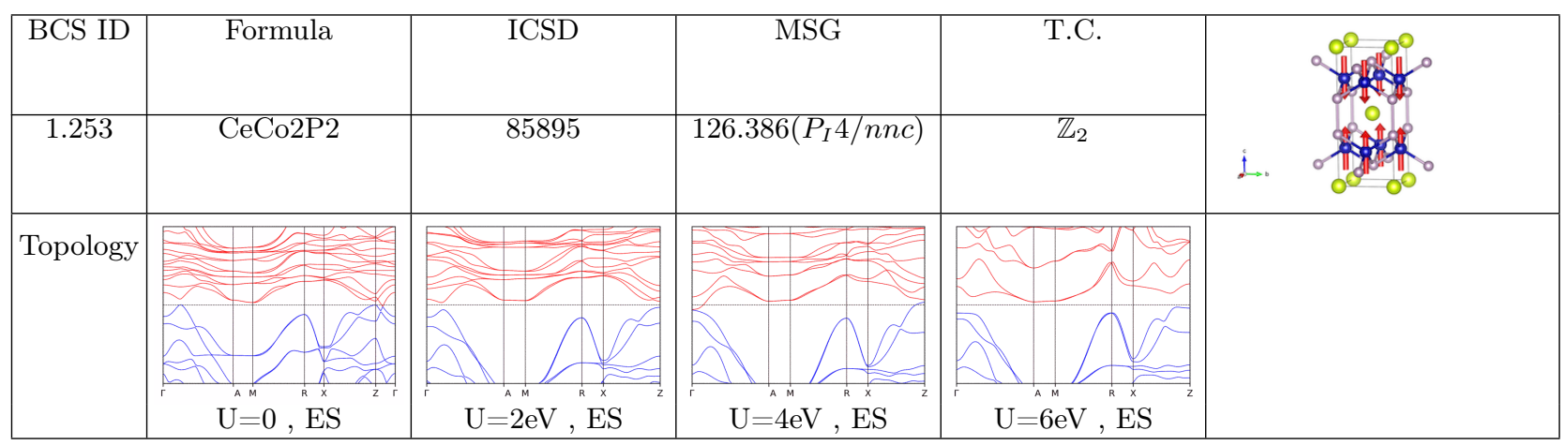

TABLE CCCLXXIII. Topology phase diagram of CeCo2P2.

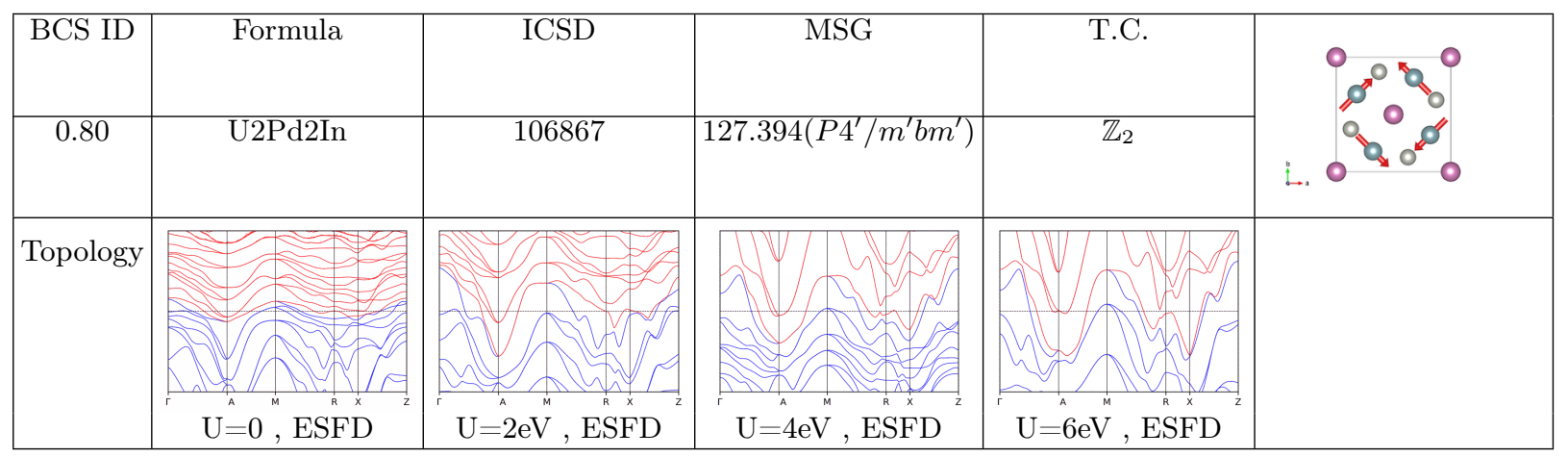

TABLE CCCLXXIV. Topology phase diagram of U2Pd2In.

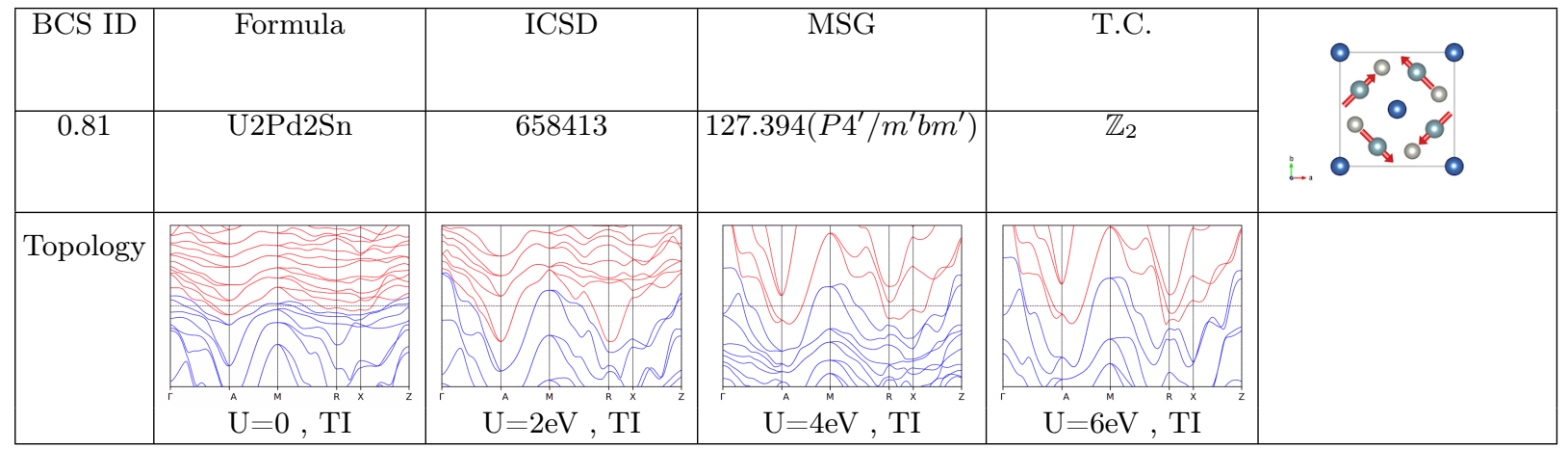

TABLE CCCLXXV. Topology phase diagram of U2Pd2Sn. 


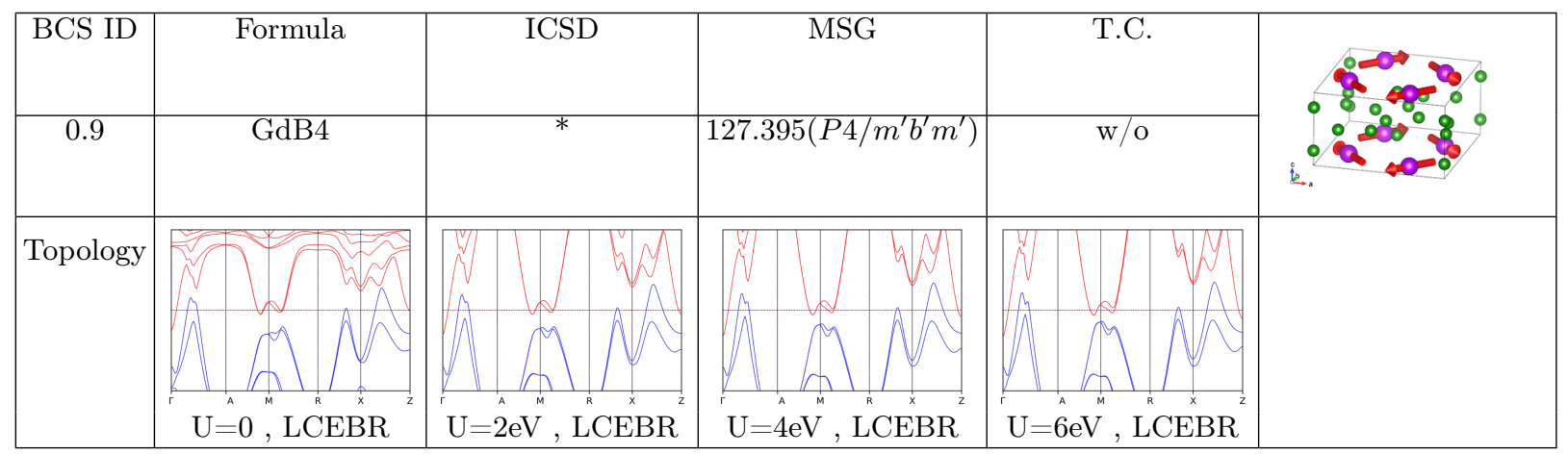

TABLE CCCLXXVI. Topology phase diagram of GdB4.

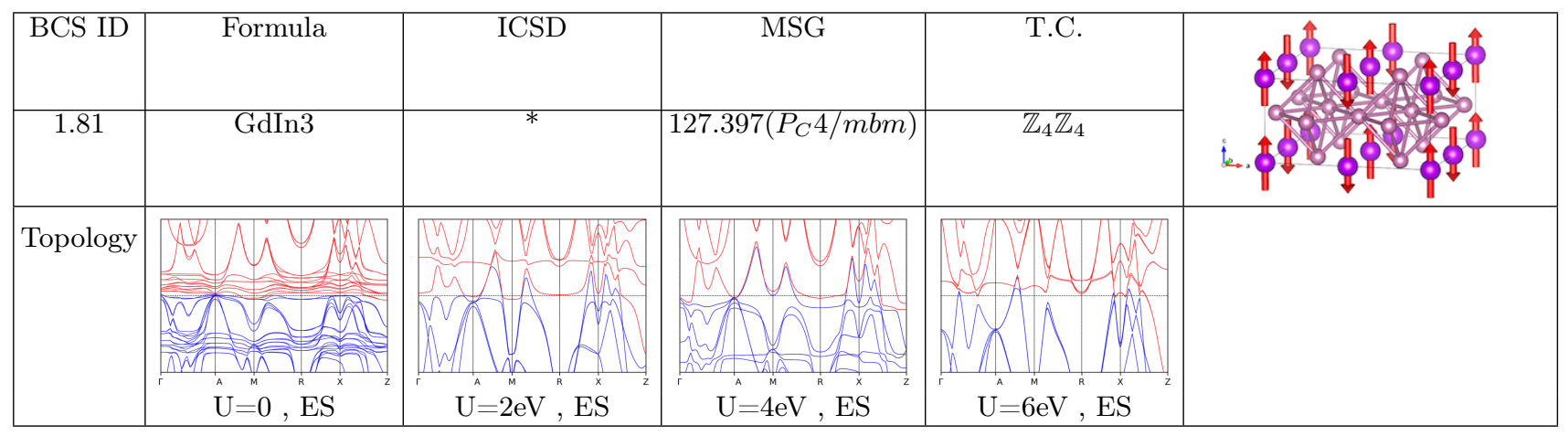

TABLE CCCLXXVII. Topology phase diagram of GdIn3.

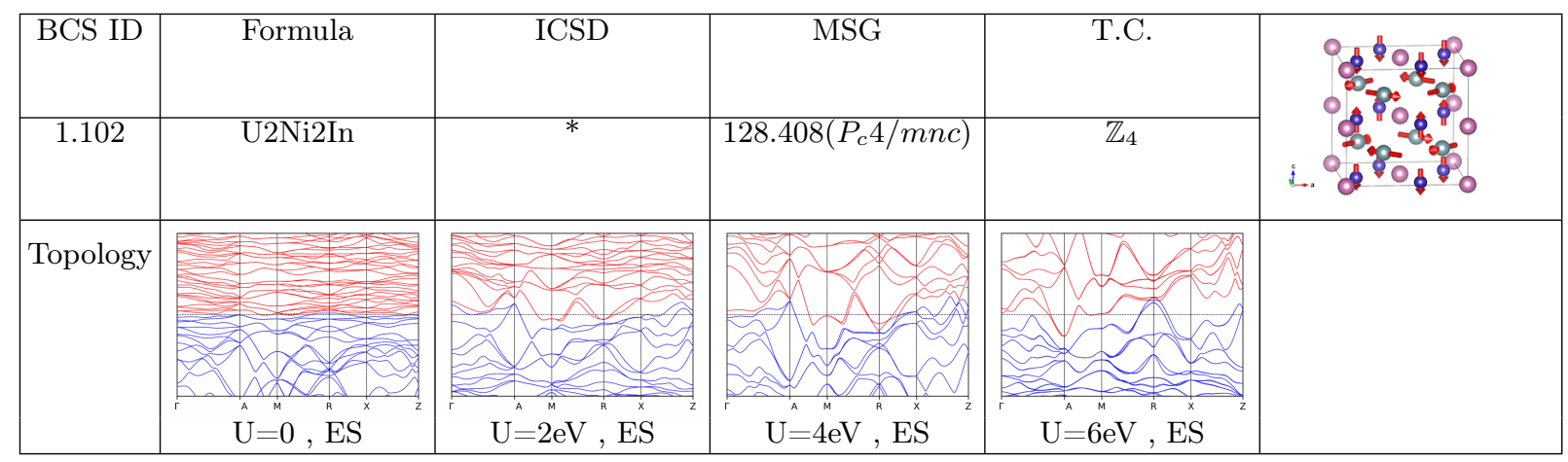

TABLE CCCLXXVIII. Topology phase diagram of U2Ni2In.

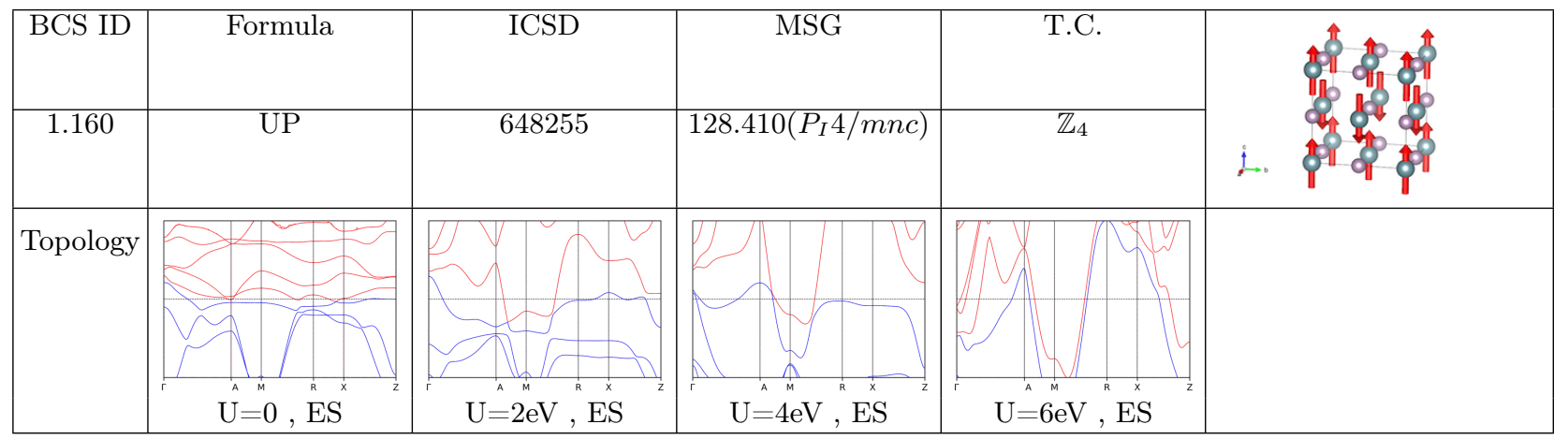

TABLE CCCLXXIX. Topology phase diagram of UP. 


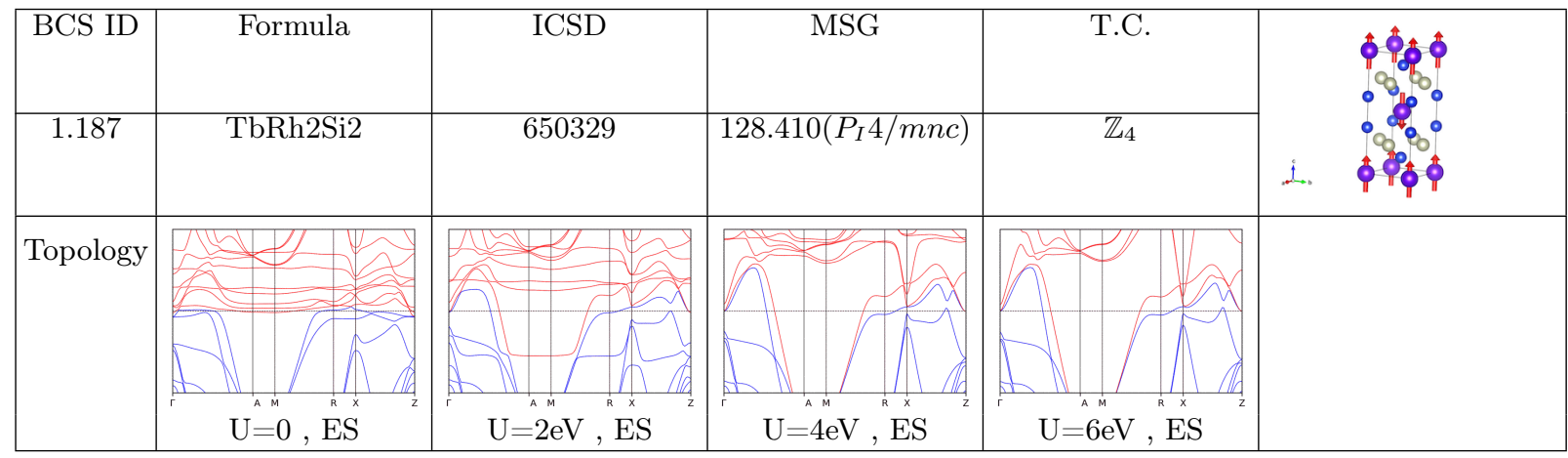

TABLE CCCLXXX. Topology phase diagram of TbRh2Si2.

\begin{tabular}{|c|c|c|c|c|c|}
\hline BCS ID & Formula & ICSD & MSG & T.C. & \\
\hline 1.208 & UAs & $*$ & $128.410\left(P_{I} 4 / m n c\right)$ & $\mathbb{Z}_{4}$ & 4. $\$ 00 \%$ \\
\hline Topology & & & & & \\
\hline & $\mathrm{U}^{A}=0,{ }^{\mathrm{A}} \mathrm{ES}$ & $\mathrm{U}=2 \mathrm{eV}^{\mathrm{m}}, \mathrm{E}^{\mathrm{x}}$ & $\mathrm{U}^{\mathrm{A}}=4^{m} \mathrm{eV}, \mathrm{ES}$ & $\mathrm{U}^{\mathrm{A}}=6^{\mathrm{m}} \mathrm{eV},{ }^{\mathrm{n}}$ & \\
\hline
\end{tabular}

TABLE CCCLXXXI. Topology phase diagram of UAs.

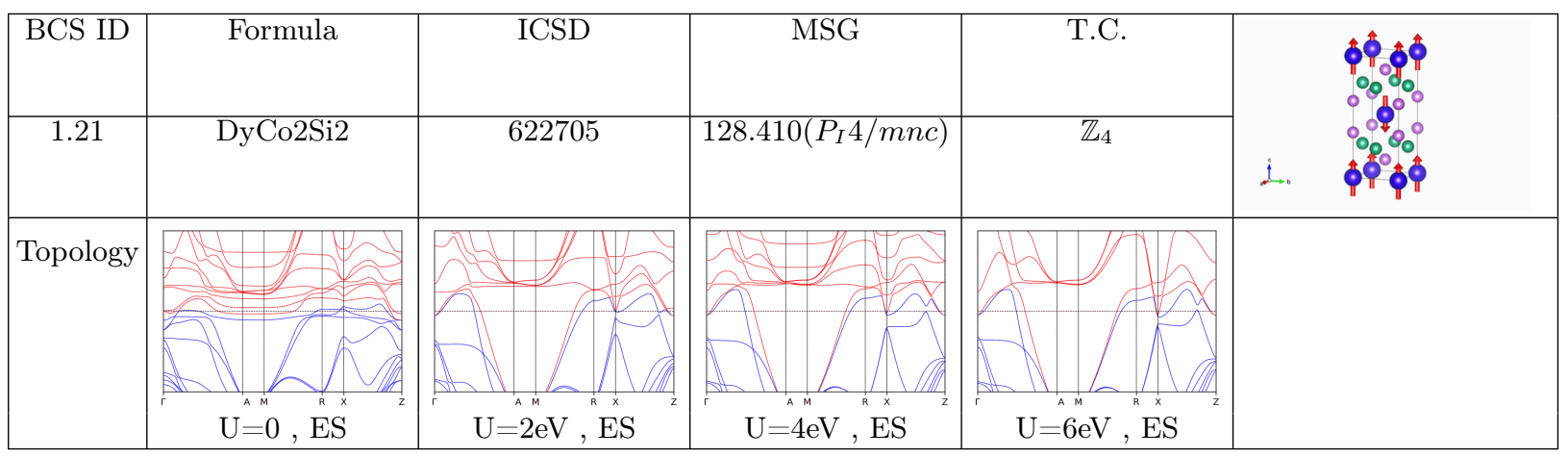

TABLE CCCLXXXII. Topology phase diagram of DyCo2Si2.

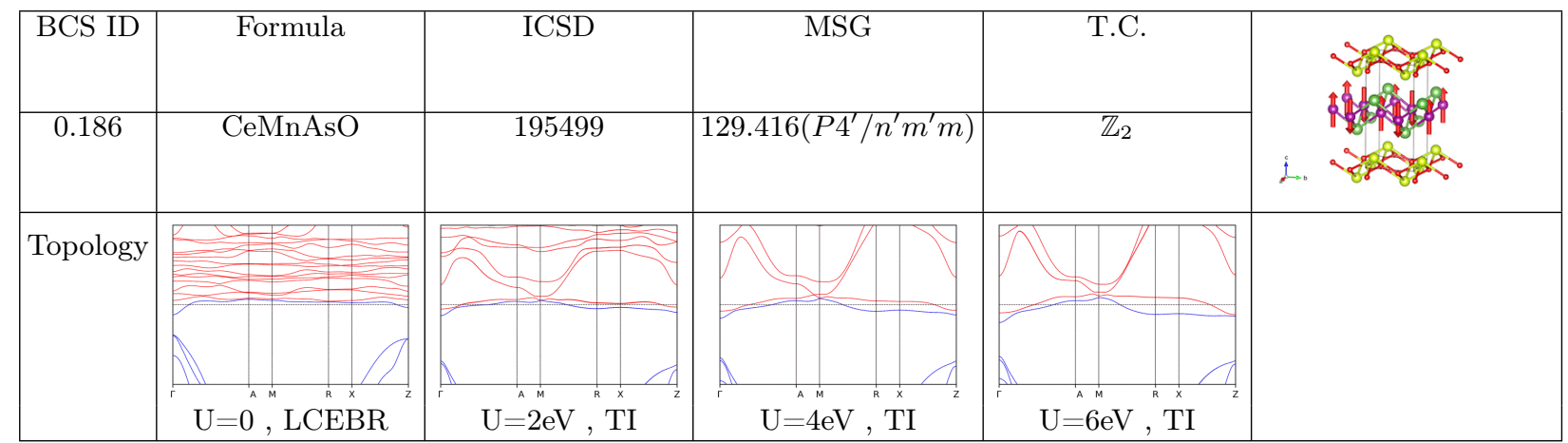

TABLE CCCLXXXIII. Topology phase diagram of CeMnAsO. 


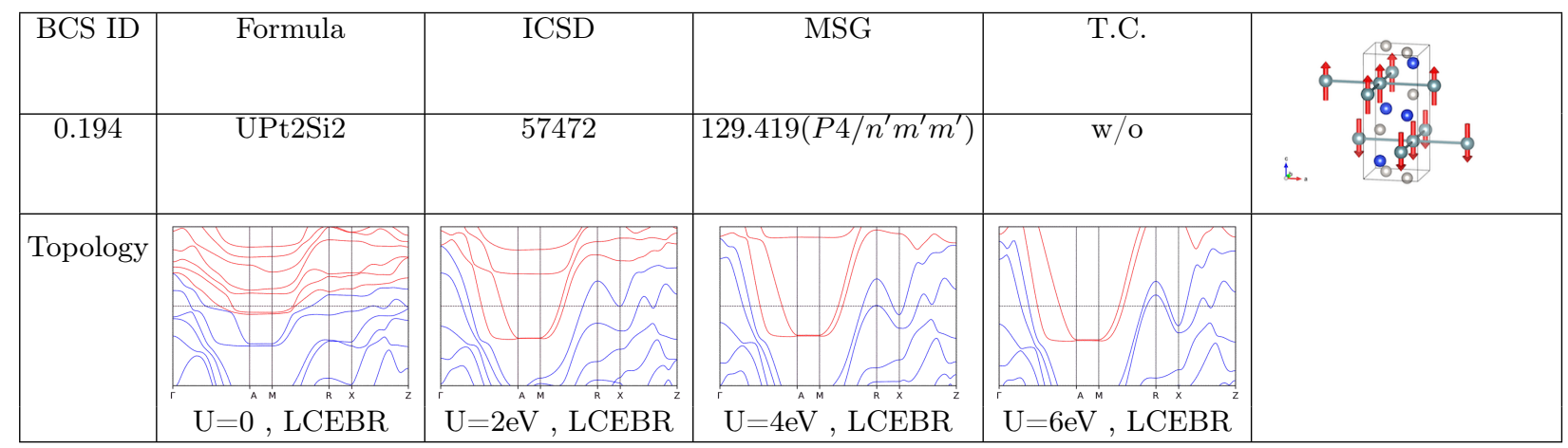

TABLE CCCLXXXIV. Topology phase diagram of UPt2Si2.

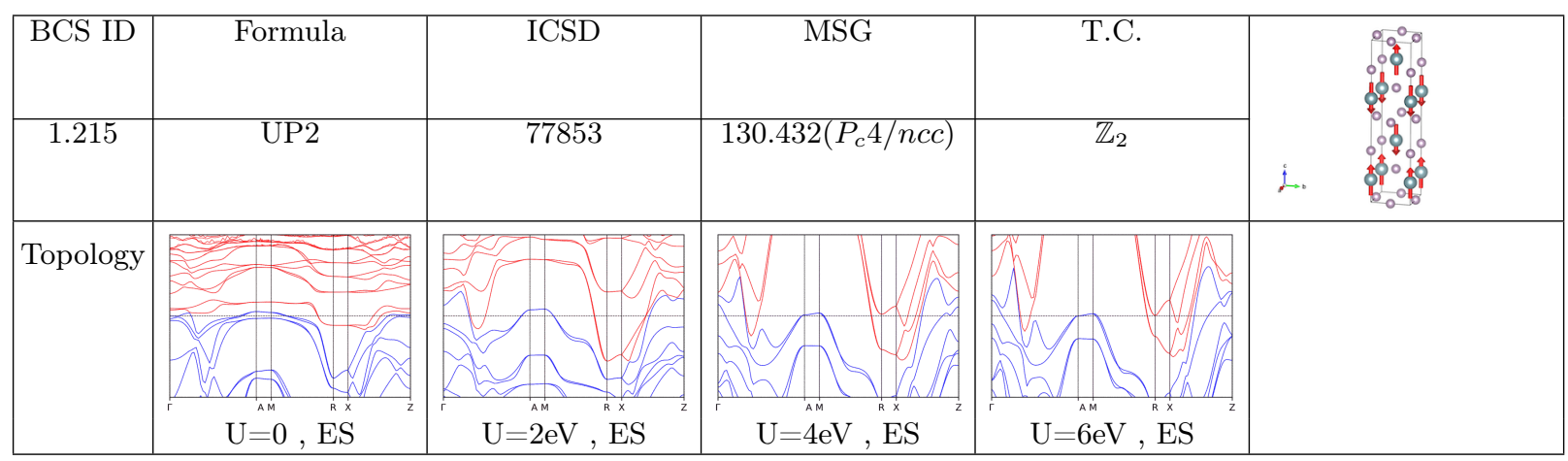

TABLE CCCLXXXV. Topology phase diagram of UP2.

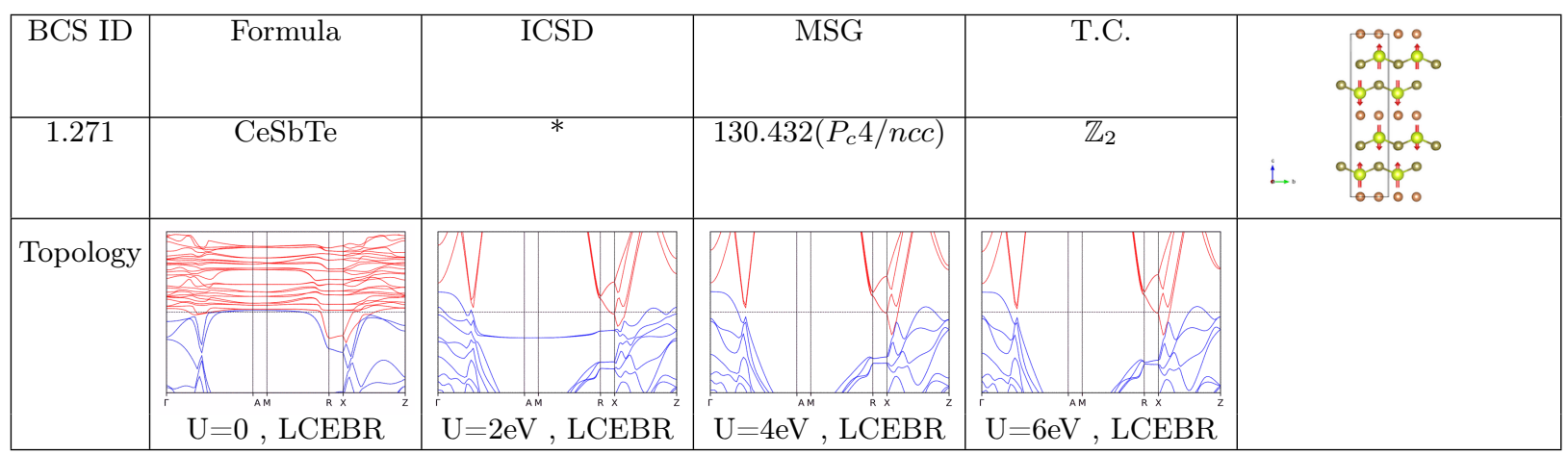

TABLE CCCLXXXVI. Topology phase diagram of CeSbTe.

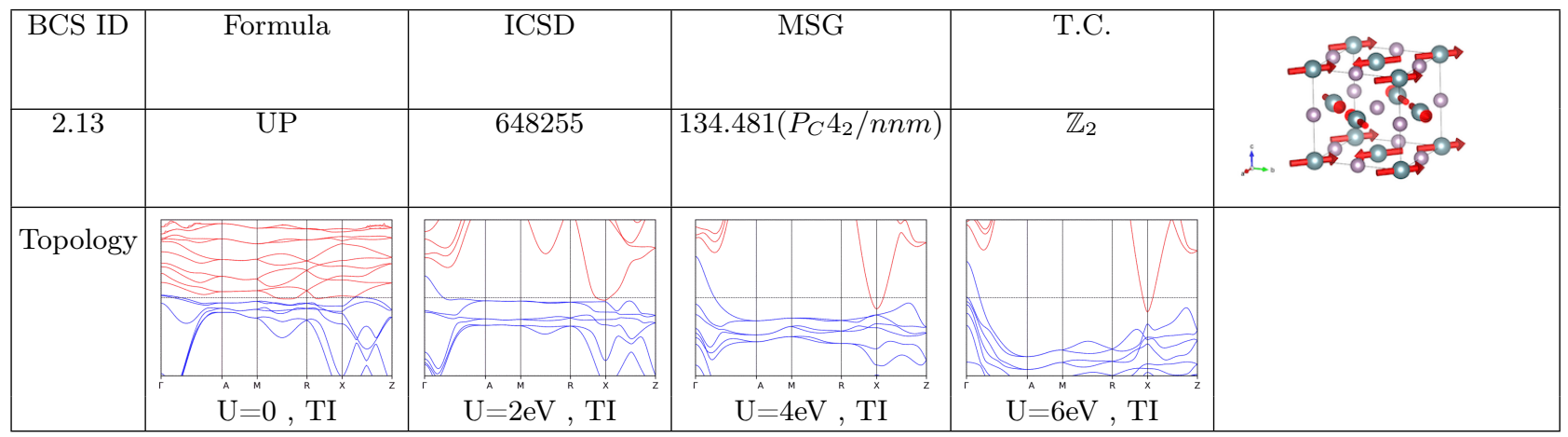

TABLE CCCLXXXVII. Topology phase diagram of UP. 


\begin{tabular}{|c|c|c|c|c|c|}
\hline BCS ID & Formula & ICSD & MSG & T.C. & \\
\hline 2.20 & $\overline{\mathrm{UAs}}$ & * & $134.481\left(P_{C} 4_{2} / n n m\right)$ & $\mathbb{Z}_{2}$ & 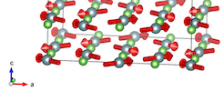 \\
\hline Topology & tich & $\frac{2}{\hat{\mathrm{U}}=2 \mathrm{eV}, \mathrm{ES}}$ & $\frac{\mathrm{U}_{\mathrm{U}=4 \mathrm{eV}, \mathrm{TI}}^{\prime \prime}}{\mathrm{N}^{\prime \prime}}$ & PI: & \\
\hline
\end{tabular}

TABLE CCCLXXXVIII. Topology phase diagram of UAs.

\begin{tabular}{|c|c|c|c|c|c|}
\hline BCS ID & Formula & ICSD & MSG & T.C. & \\
\hline 2.6 & $\mathrm{Nd} 2 \mathrm{CuO} 4$ & 202885 & $134.481\left(P_{C} 4_{2} / n n m\right)$ & $\mathbb{Z}_{2}$ & $2^{8} e^{0}$ \\
\hline Topology & 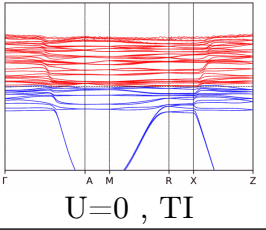 & ${\mathrm{U}=2 \mathrm{eV}^{A}, \mathrm{LCEBR}^{2}}^{C}$ & $\mathrm{U}=4 \mathrm{eV}, \mathrm{LCEBR}$ & $\mathrm{U}=6 \mathrm{eV}, \mathrm{LCEBR}$ & \\
\hline
\end{tabular}

TABLE CCCLXXXIX. Topology phase diagram of Nd2CuO4.

\begin{tabular}{|c|c|c|c|c|c|}
\hline BCS ID & Formula & ICSD & MSG & T.C. & \\
\hline 1.103 & U2Rh2Sn & 246630 & $135.492\left(P_{c} 4_{2} / m b c\right)$ & $\overline{\mathbb{Z}_{4}}$ & 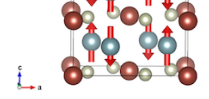 \\
\hline Topology & 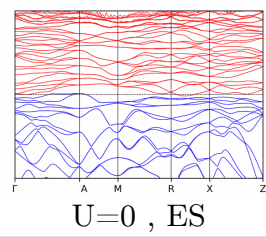 & 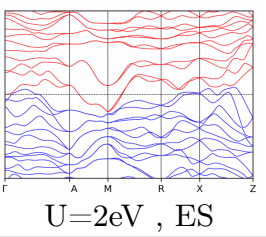 & $\underset{\mathrm{U}={ }^{\mathrm{M}} \mathrm{eV}, \mathrm{ES}}{\mathrm{B}}$ & $\underset{\mathrm{U}=\mathrm{C}^{\mathrm{M}} \mathrm{eV}, \mathrm{ES}}{\mathrm{C}}$ & \\
\hline
\end{tabular}

TABLE CCCXC. Topology phase diagram of U2Rh2Sn.

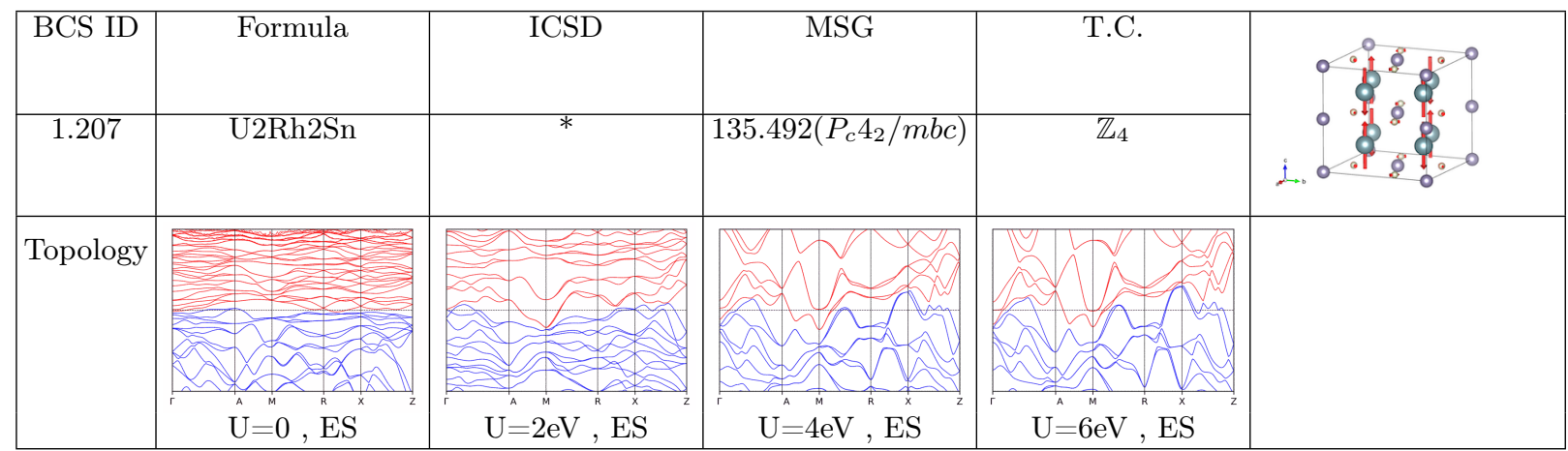

TABLE CCCXCI. Topology phase diagram of U2Rh2Sn. 


\begin{tabular}{|c|c|c|c|c|c|}
\hline BCS ID & Formula & ICSD & MSG & T.C. & \\
\hline 1.254 & UNiGa5 & & $140.550\left(I_{c} 4 / \mathrm{mcm}\right)$ & $\mathbb{Z}_{4}$ & 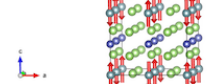 \\
\hline Topology & $\mathrm{U}=0, \mathrm{ES}$ & $\mathrm{U}=2 \mathrm{eV}^{\prime \prime}, \mathrm{T}$ & $\mathrm{U}=4 \mathrm{eV}, \mathrm{TI}^{\mathrm{m} \times}$ & ${ }_{\mathrm{U}=6 \mathrm{eV}, \mathrm{LCEBR}}$ & \\
\hline
\end{tabular}

TABLE CCCXCII. Topology phase diagram of UNiGa5.

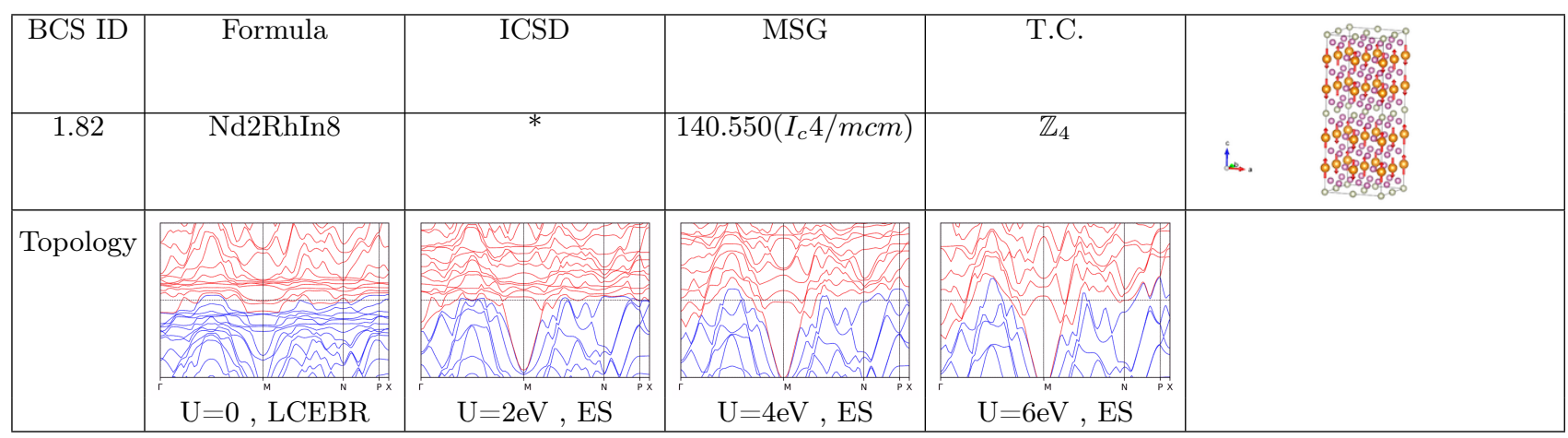

TABLE CCCXCIII. Topology phase diagram of Nd2RhIn8.

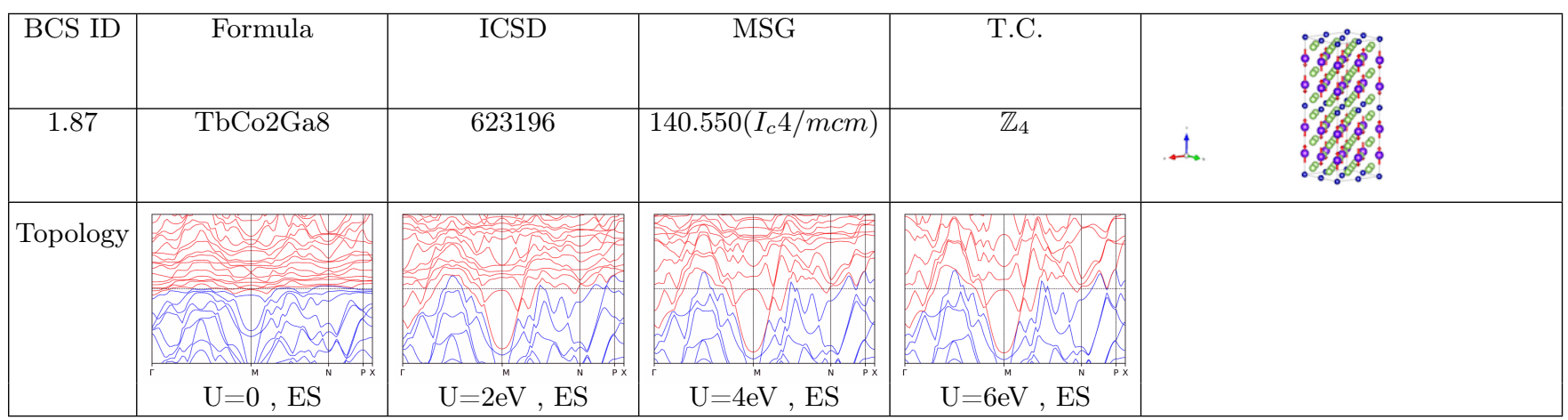

TABLE CCCXCIV. Topology phase diagram of TbCo2Ga8.

\begin{tabular}{|c|c|c|c|c|c|}
\hline BCS ID & Formula & ICSD & MSG & T.C. & \\
\hline 0.154 & Er2Ru2O7 & 97533 & $141.554\left(I 4_{1}^{\prime} / a m^{\prime} d\right)$ & $\mathbb{Z}_{2}$ & 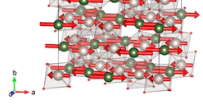 \\
\hline Topology & $\mathrm{U=0,,^{ \prime \prime } \mathrm { LCEBR }}$ & U & $\mathrm{U}_{\mathrm{U}=4 \mathrm{eV}, \mathrm{LCEBR}}$ & 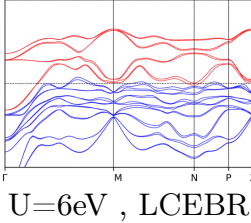 & \\
\hline
\end{tabular}

TABLE CCCXCV. Topology phase diagram of Er2Ru2O7. 


\begin{tabular}{|c|c|c|c|c|c|}
\hline BCS ID & Formula & ICSD & MSG & T.C. & \\
\hline 0.29 & Er2Ti2O7 & 24152 & $141.554\left(I 4_{1}^{\prime} / a m^{\prime} d\right)$ & $\mathbb{Z}_{2}$ & $\therefore \quad$ \\
\hline Topology & \begin{tabular}{|l|l|} 
\\
\end{tabular} & $\underbrace{\mathrm{U}=2 \mathrm{eV}, \mathrm{LCEBR}}_{\mathrm{U}}$ & $\mathrm{U}=4 \mathrm{eV}, \mathrm{LCEBR}$ & 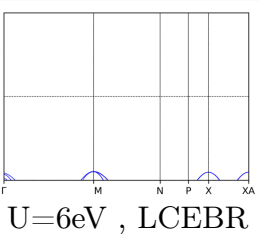 & \\
\hline
\end{tabular}

TABLE CCCXCVI. Topology phase diagram of Er2Ti2O7.

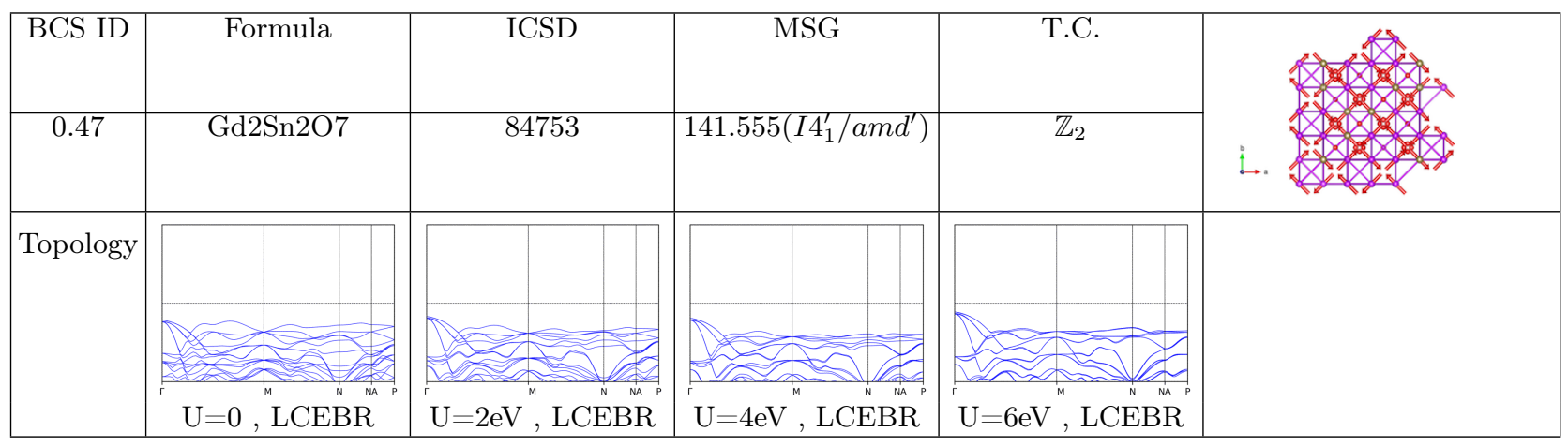

TABLE CCCXCVII. Topology phase diagram of Gd2Sn2O7.

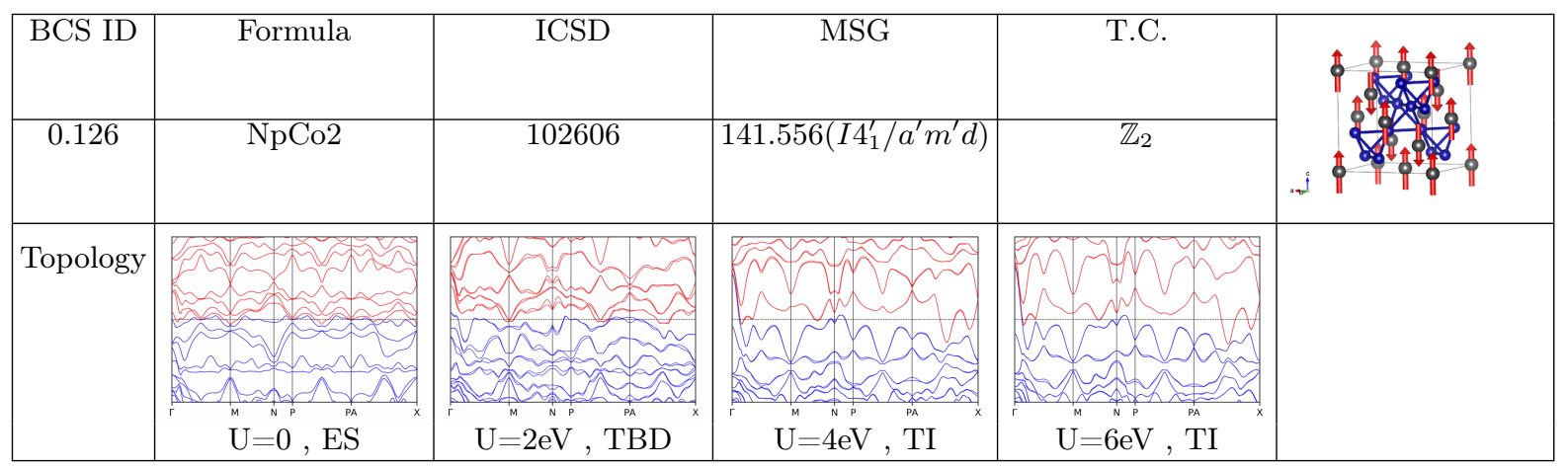

TABLE CCCXCVIII. Topology phase diagram of NpCo2.

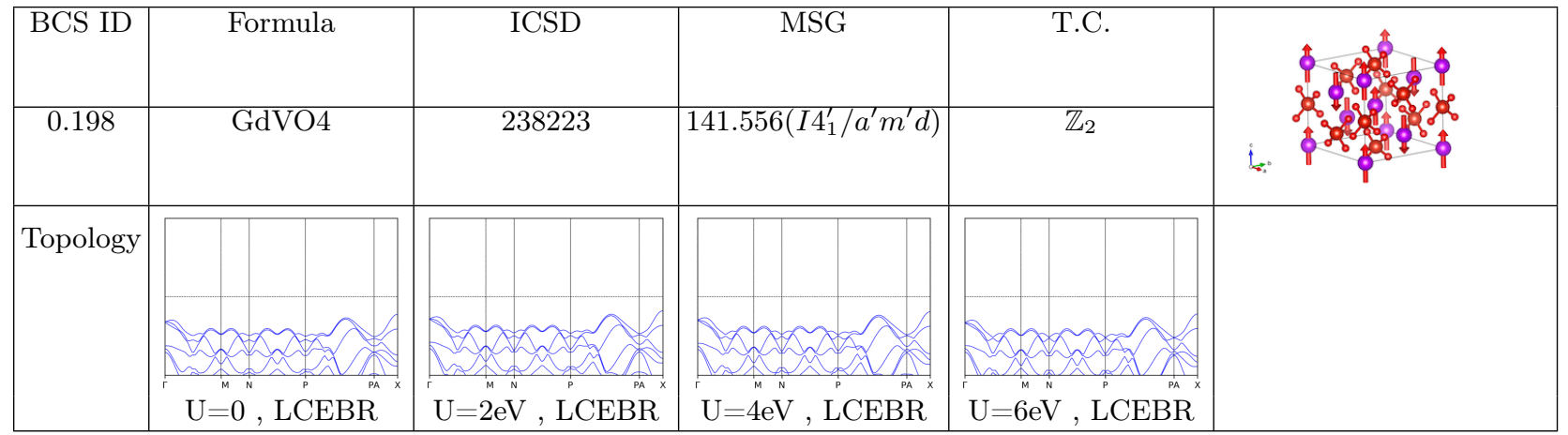

TABLE CCCXCIX. Topology phase diagram of GdVO4. 


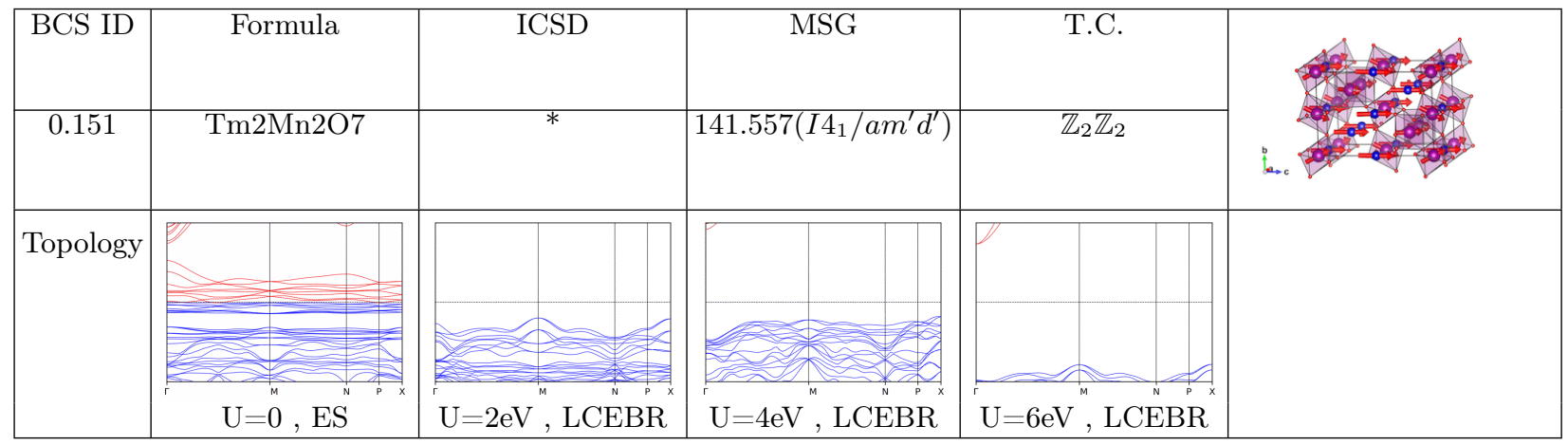

TABLE CD. Topology phase diagram of Tm2Mn2O7.

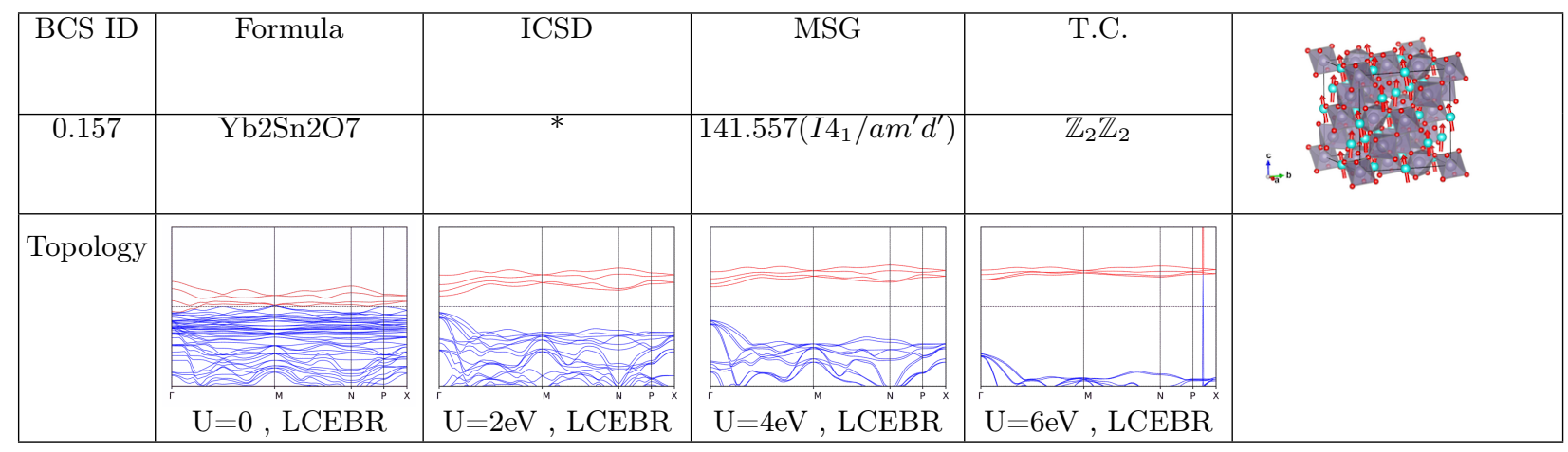

TABLE CDI. Topology phase diagram of Yb2Sn2O7.

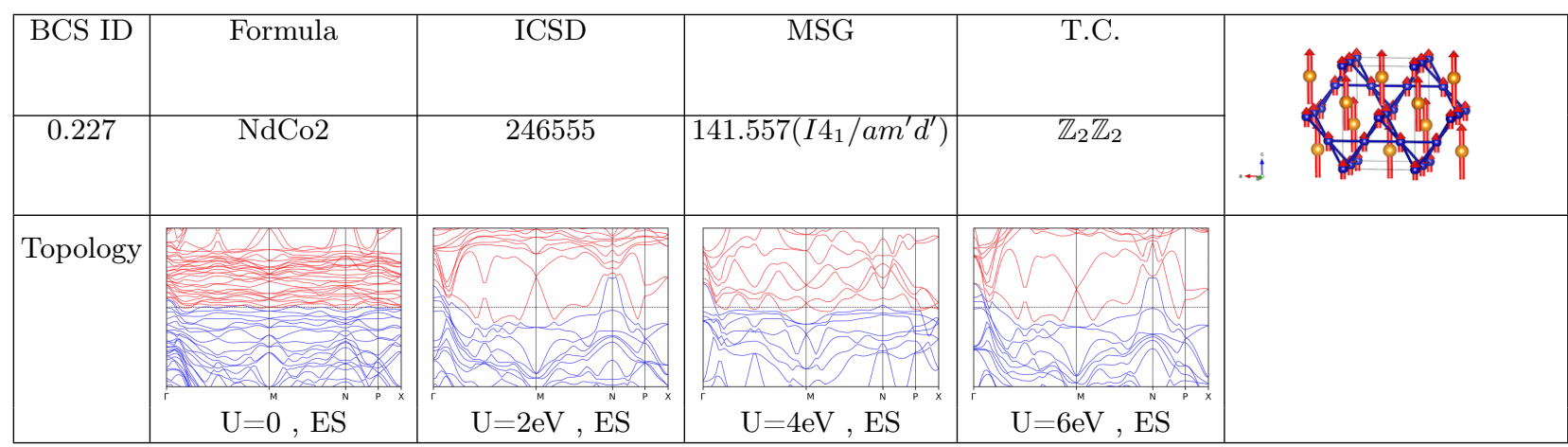

TABLE CDII. Topology phase diagram of NdCo2.

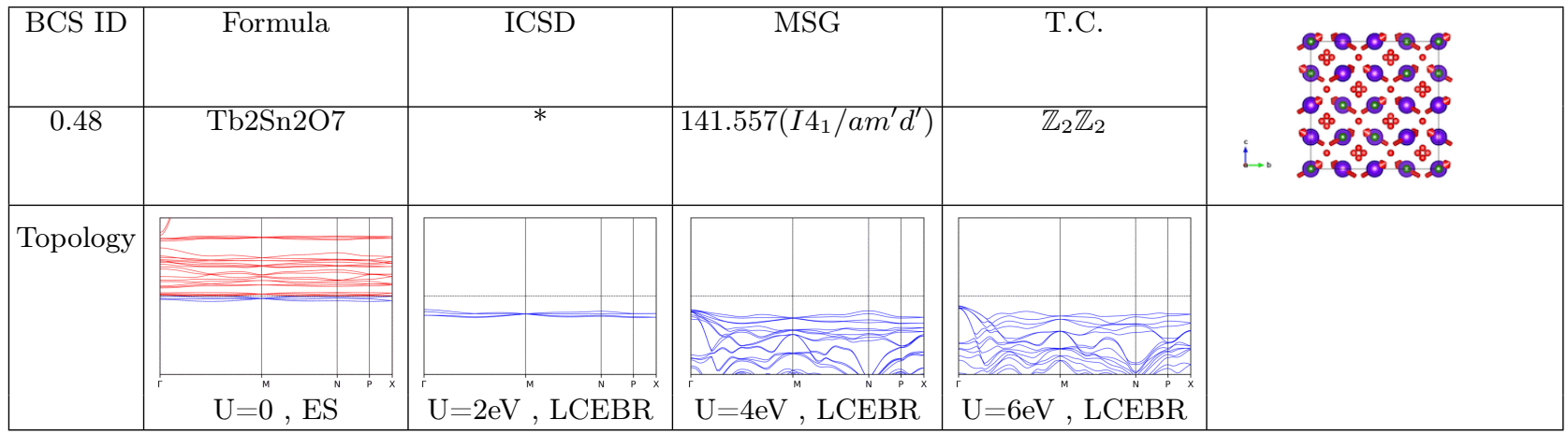

TABLE CDIII. Topology phase diagram of Tb2Sn2O7. 


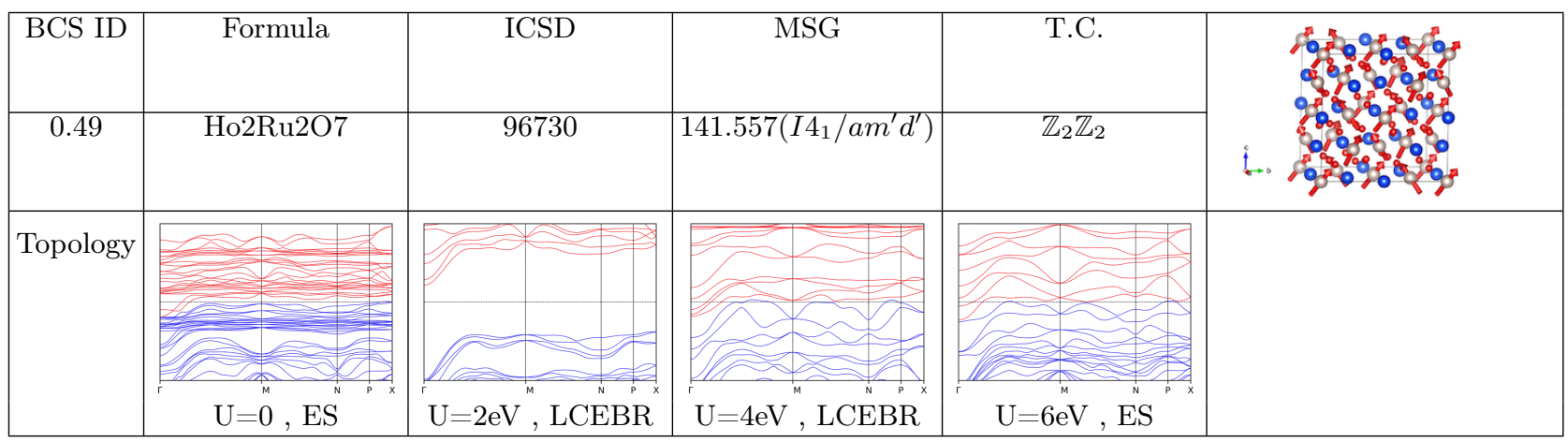

TABLE CDIV. Topology phase diagram of Ho2Ru2O7.

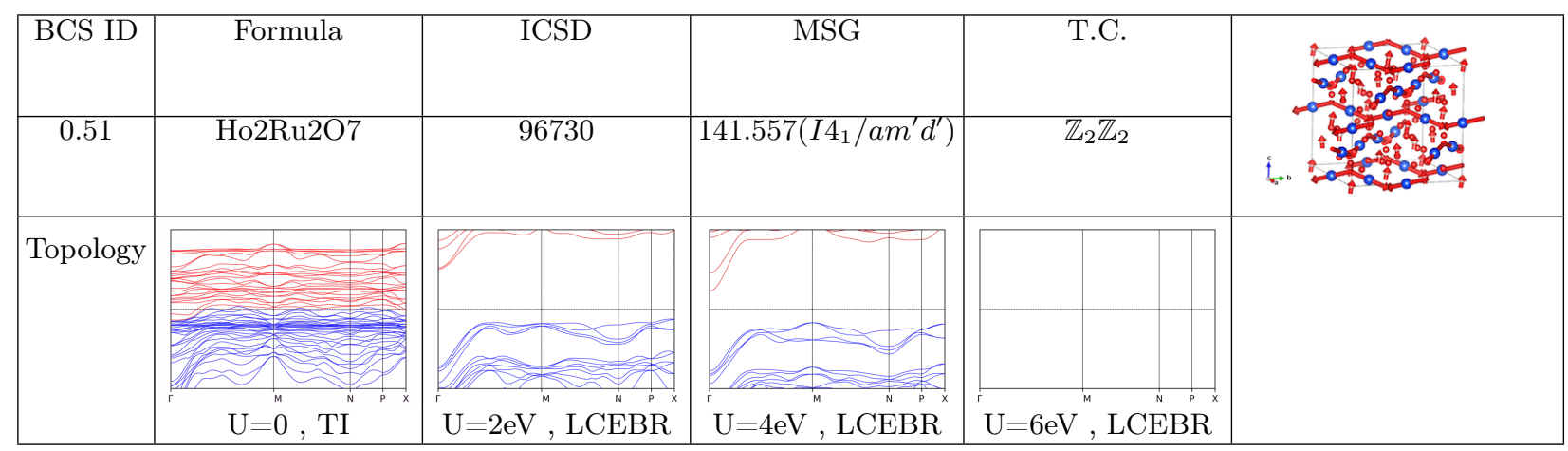

TABLE CDV. Topology phase diagram of Ho2Ru2O7.

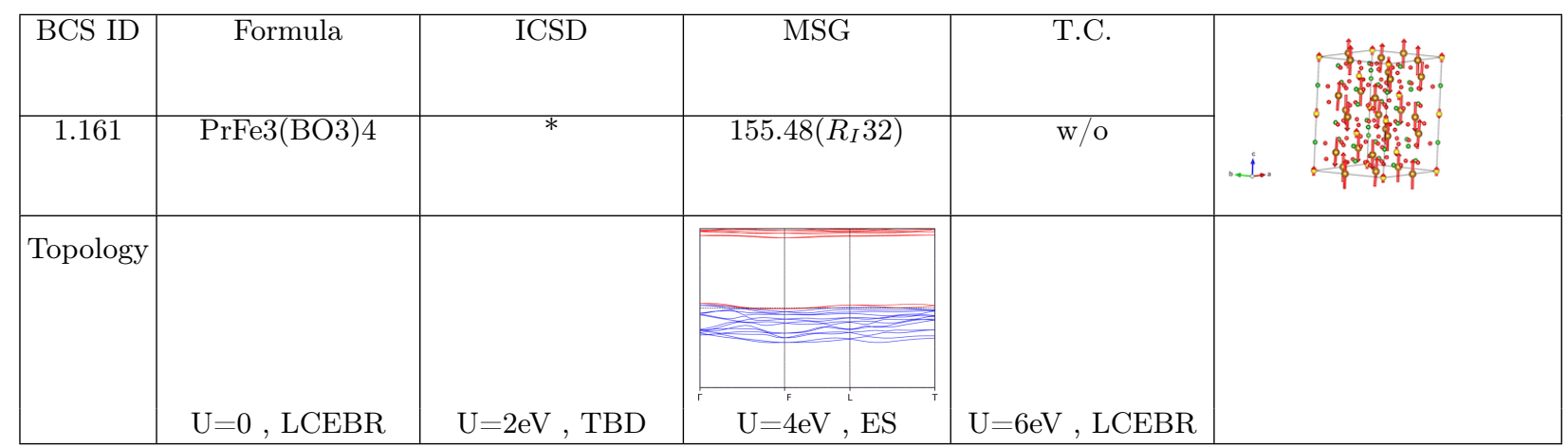

TABLE CDVI. Topology phase diagram of PrFe3(BO3)4.

\begin{tabular}{|c|c|c|c|c|c|}
\hline BCS ID & Formula & ICSD & MSG & T.C. & \\
\hline 0.169 & U3As4 & 42168 & $161.71\left(R 3 c^{\prime}\right)$ & $\mathrm{w} / \mathrm{o}$ & 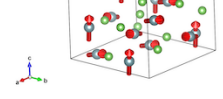 \\
\hline Topology & (m=0,ES & $\mathrm{U}=2 \mathrm{eV}, \mathrm{ES}$ & $\mathrm{U}=4 \mathrm{eV}, \mathrm{LCEBF}$ & U=6eV, LCEBR & \\
\hline
\end{tabular}

TABLE CDVII. Topology phase diagram of U3As4. 


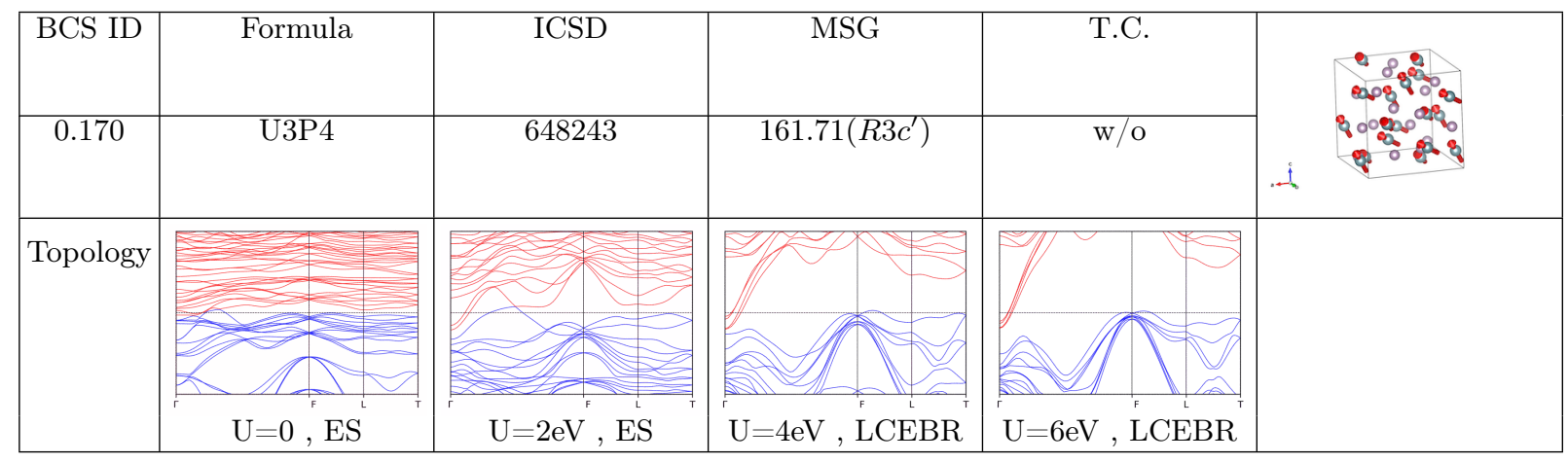

TABLE CDVIII. Topology phase diagram of U3P4.

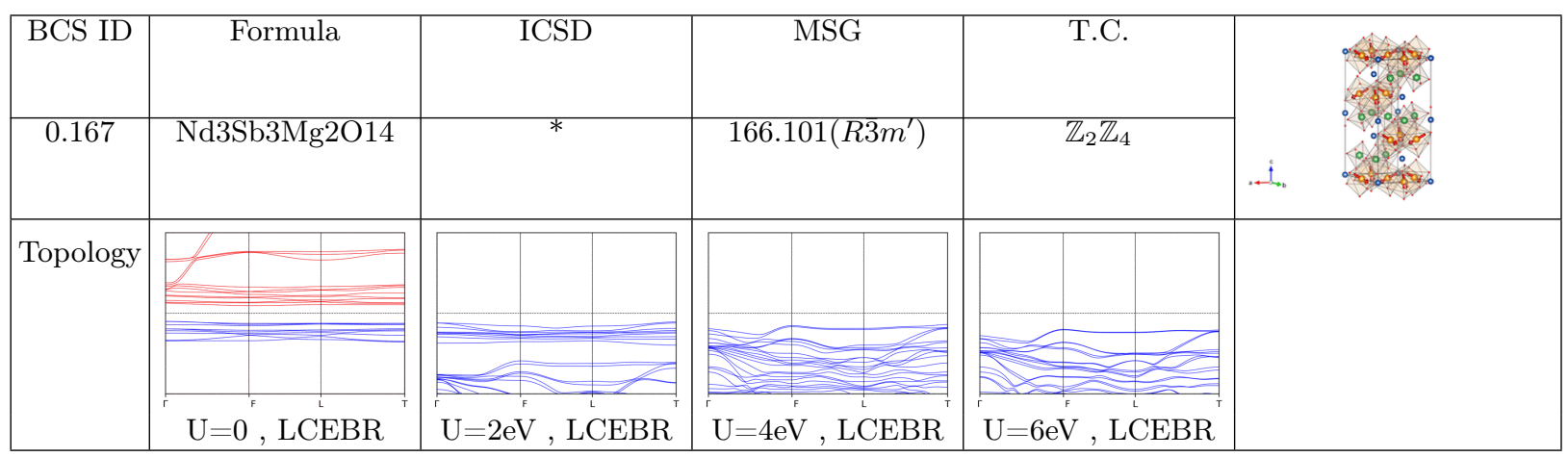

TABLE CDIX. Topology phase diagram of Nd3Sb3Mg2O14.

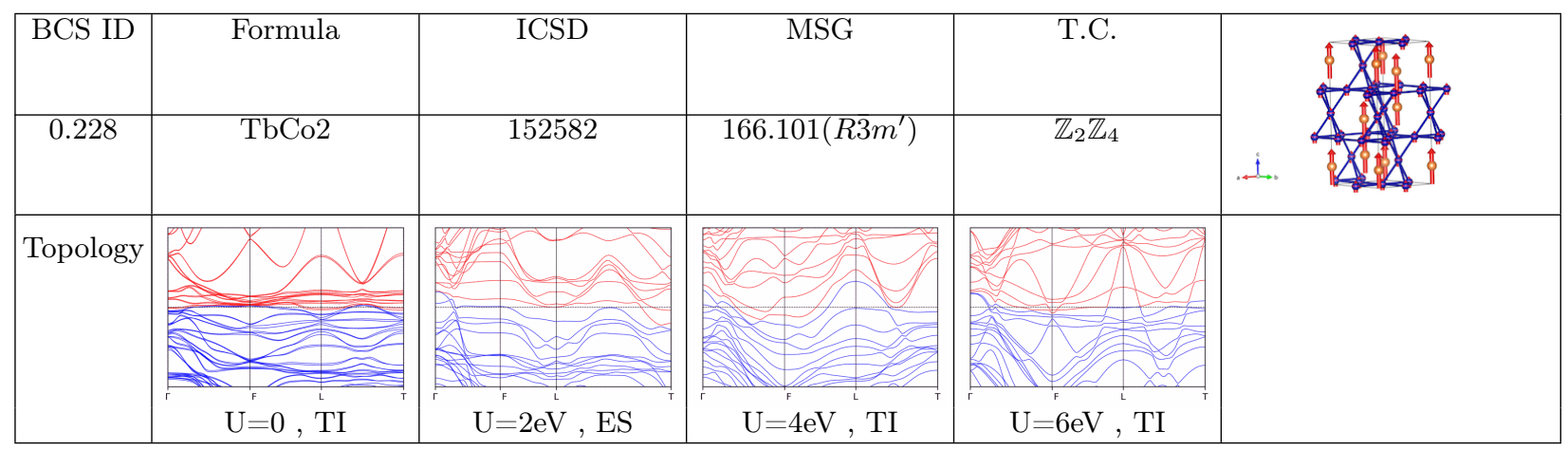

TABLE CDX. Topology phase diagram of TbCo2.

\begin{tabular}{|c|c|c|c|c|c|}
\hline BCS ID & Formula & ICSD & MSG & T.C. & \\
\hline 0.77 & Tb2Ti2O7 & 151747 & $166.101\left(R 3 m^{\prime}\right)$ & $\mathbb{Z}_{2} \mathbb{Z}_{4}$ & \\
\hline Topology & \begin{tabular}{|l|l|} 
PH & +12 \\
\end{tabular} & & & & \\
\hline & $\mathrm{U}=0, \mathrm{ES}$ & $\mathrm{U}=2 \mathrm{eV}, \mathrm{LCEBR}$ & $\mathrm{U}=4 \mathrm{eV}, \mathrm{LCEBR}$ & $\mathrm{U}=6 \mathrm{eV}, \mathrm{LCEBR}$ & \\
\hline
\end{tabular}

TABLE CDXI. Topology phase diagram of Tb2Ti2O7. 


\begin{tabular}{|c|c|c|c|c|c|}
\hline BCS ID & Formula & ICSD & MSG & T.C. & \\
\hline 1.189 & TbMg3 & 9750 & $167.108\left(R_{I} 3 c\right)$ & $\mathbb{Z}_{2}$ & os \\
\hline Topology & U=0,LCEBR & $\mathrm{U}=2 \mathrm{eV}, \mathrm{LCEBR}$ & $\mathrm{U}=4 \mathrm{eV}, \mathrm{TBD}$ & $\mathrm{U}=6 \mathrm{eV}, \mathrm{TBD}$ & \\
\hline
\end{tabular}

TABLE CDXII. Topology phase diagram of TbMg3.

\begin{tabular}{|c|c|c|c|c|c|}
\hline BCS ID & Formula & ICSD & MSG & T.C. & \\
\hline 0.32 & HoMnO3 & 92838 & $185.197\left(\mathrm{P}_{3} \mathrm{~cm}\right)$ & $\mathrm{w} / \mathrm{O}$ & i. 0.80 \\
\hline Topology & $\underbrace{\mathrm{H}^{\mathrm{i}}}_{\overrightarrow{\mathrm{U}}=0, \hat{\mathrm{LCEBR}}^{\mathrm{k}}}$ & U ${ }^{A}=2 \mathrm{eV},{ }^{\circ}{ }^{k} \mathrm{LCEBR}^{\mathrm{k}}$ & 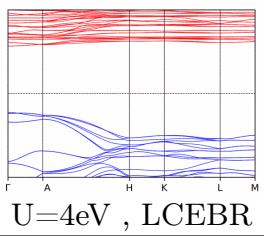 & $\mathrm{U}=6 \mathrm{eV}, \mathrm{LCEBR}$ & \\
\hline
\end{tabular}

TABLE CDXIII. Topology phase diagram of HoMnO3.

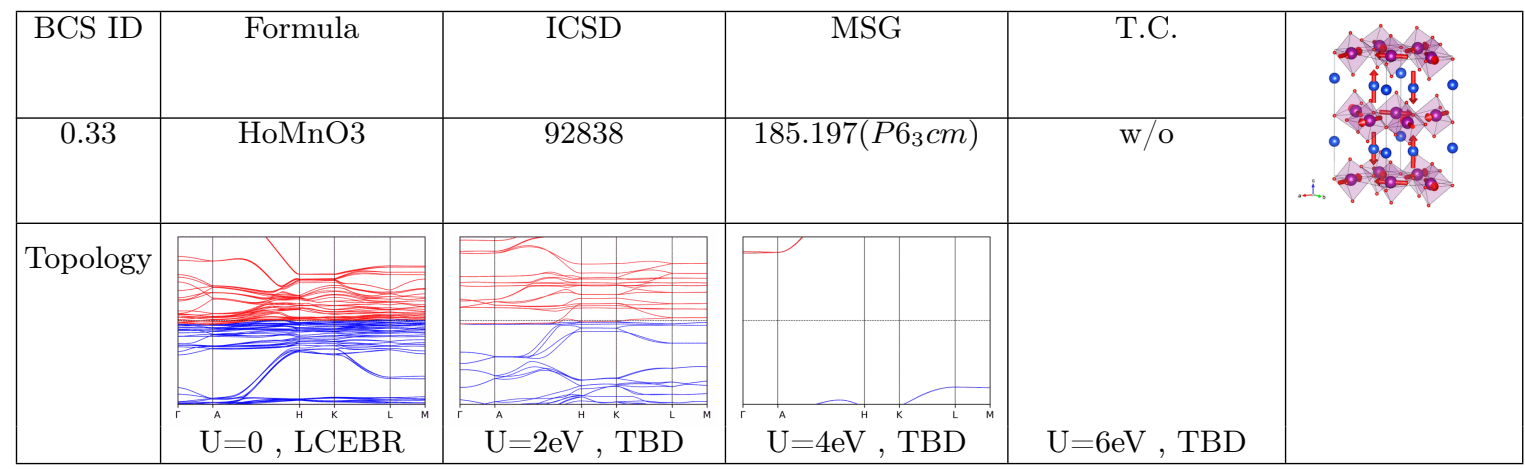

TABLE CDXIV. Topology phase diagram of HoMnO3.

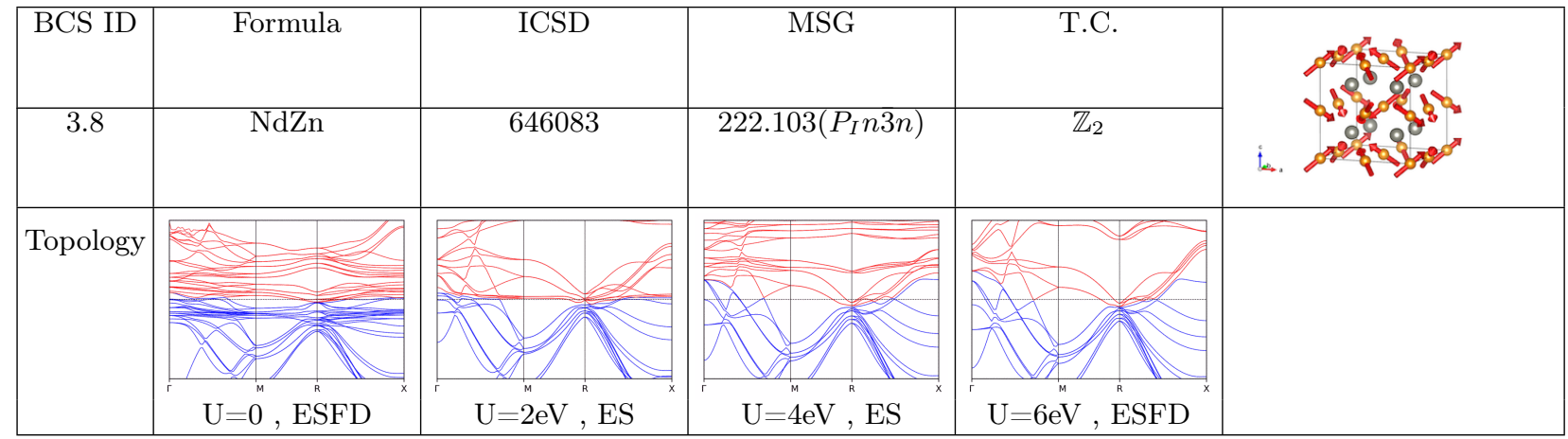

TABLE CDXV. Topology phase diagram of NdZn. 


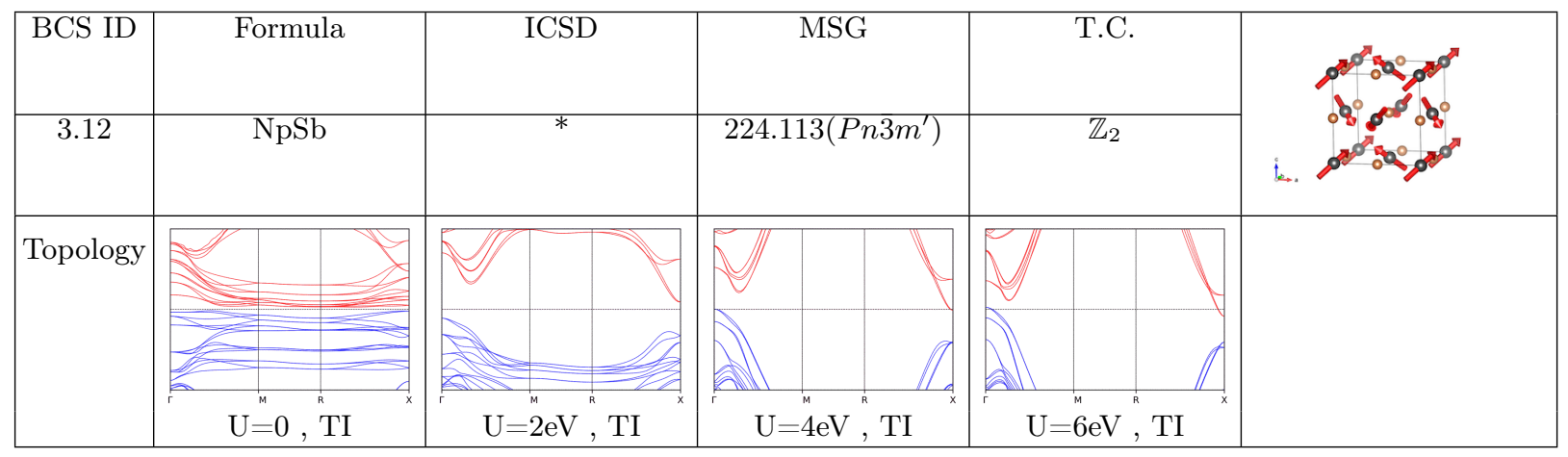

TABLE CDXVI. Topology phase diagram of NpSb.

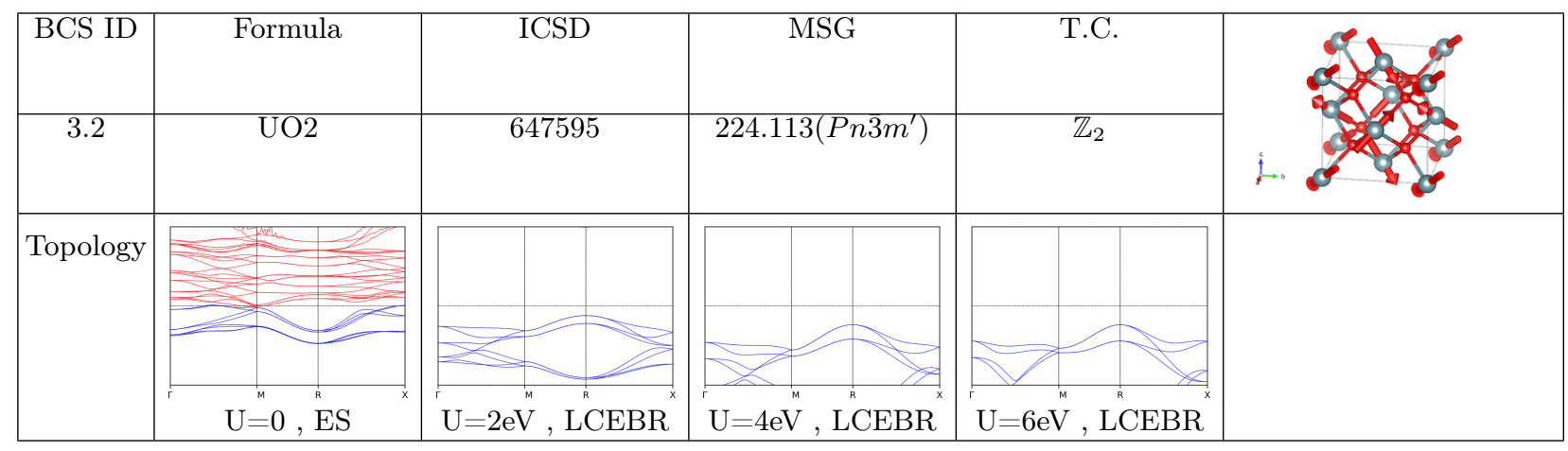

TABLE CDXVII. Topology phase diagram of UO2.

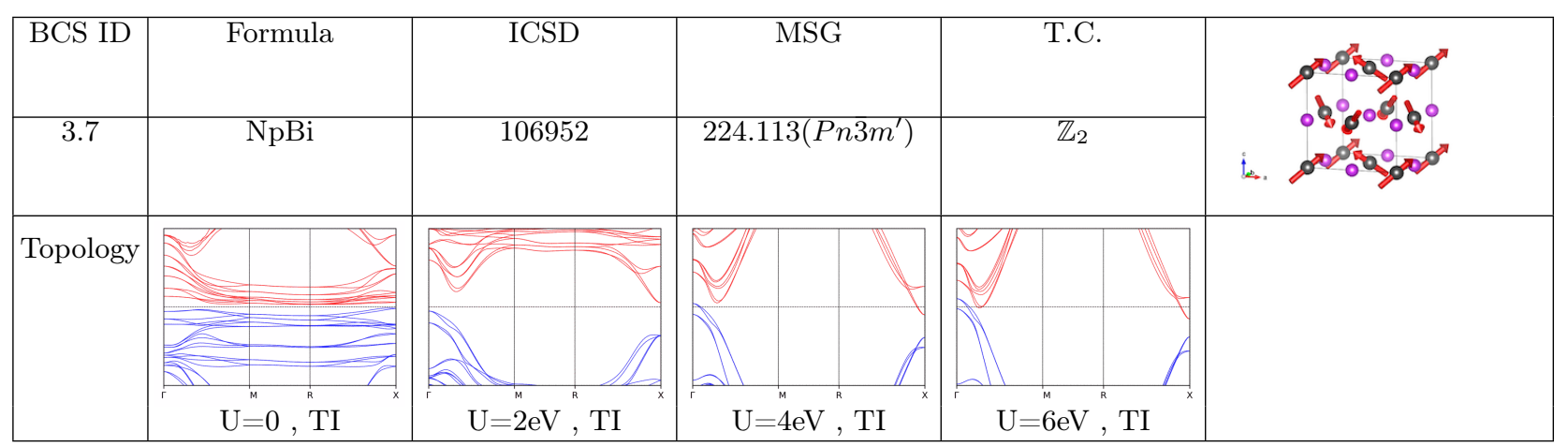

TABLE CDXVIII. Topology phase diagram of NpBi.

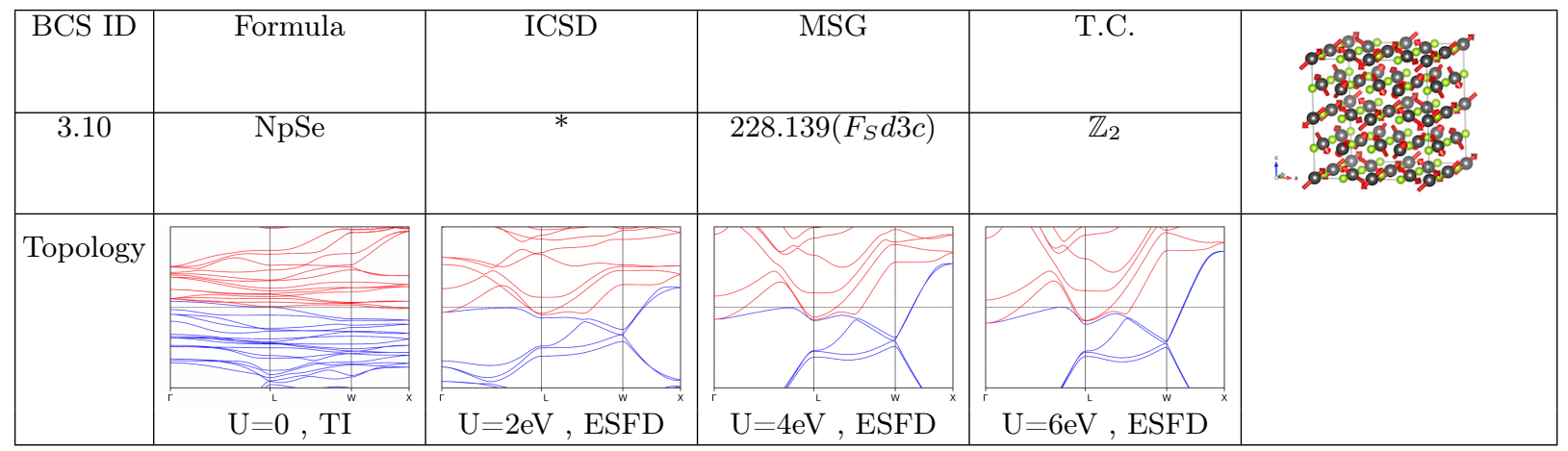

TABLE CDXIX. Topology phase diagram of NpSe. 


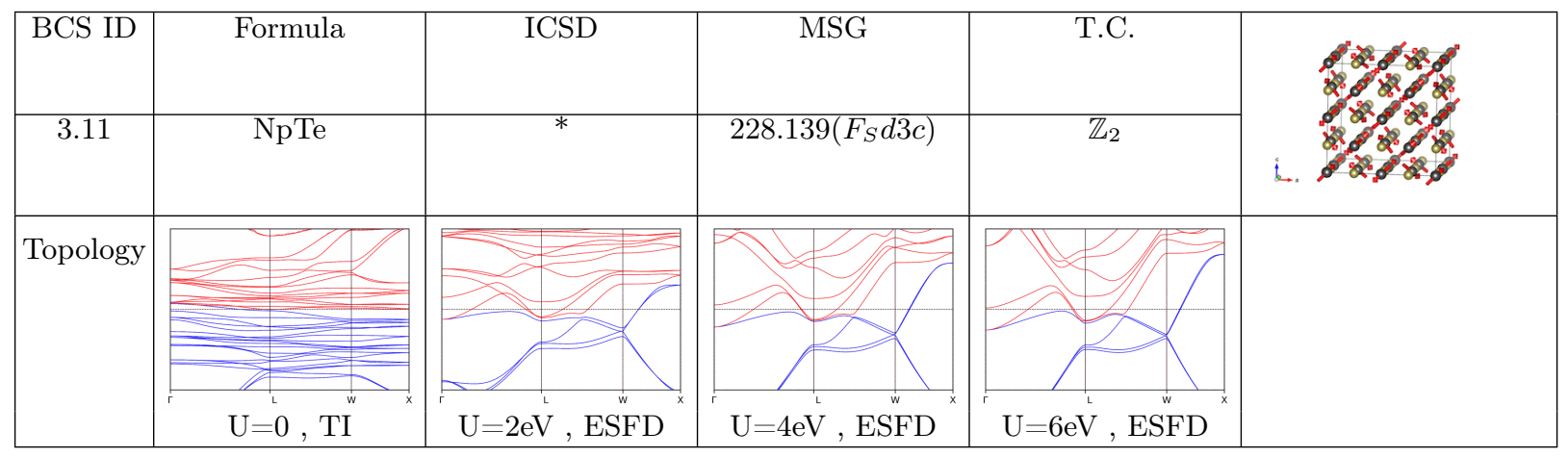

TABLE CDXX. Topology phase diagram of NpTe.

\begin{tabular}{|c|c|c|c|c|c|}
\hline BCS ID & Formula & ICSD & MSG & T.C. & \\
\hline 3.9 & $\mathrm{NpS}$ & 44382 & $228.139\left(F_{S} d 3 c\right)$ & $\mathbb{Z}_{2}$ & 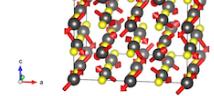 \\
\hline Topology & (N=0,ES & $\mathrm{U}=2 \mathrm{eV}, \mathrm{ESFD}$ & $\mathrm{U}=4 \mathrm{eV}, \mathrm{ESFD}$ & $\mathrm{U}=6 \mathrm{eV}, \mathrm{ESFD}$ & \\
\hline
\end{tabular}

TABLE CDXXI. Topology phase diagram of NpS.

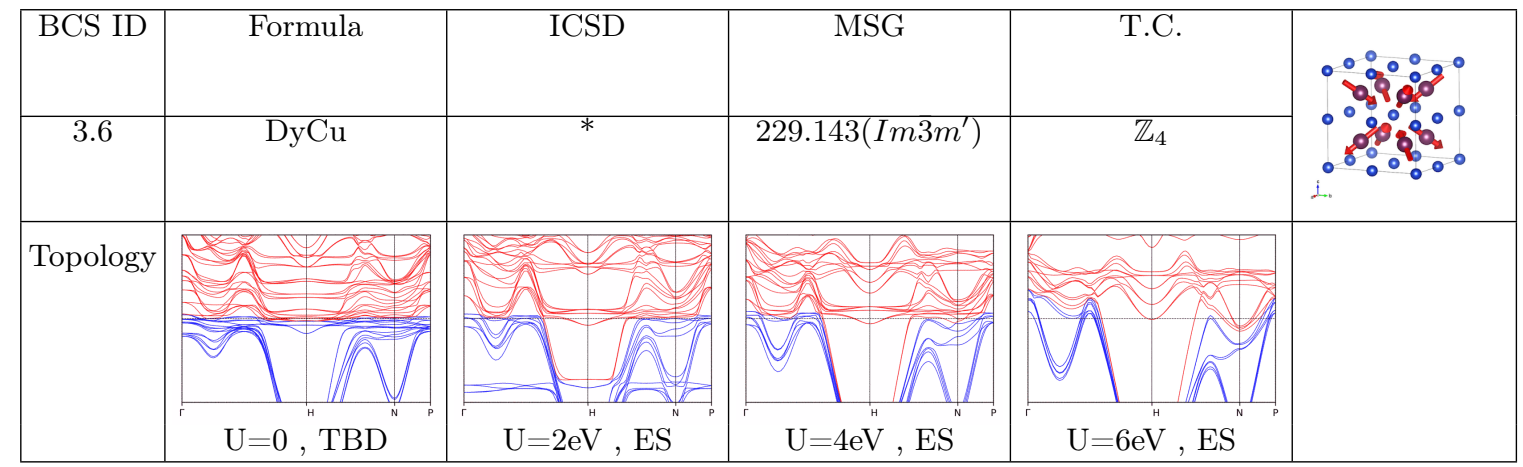

TABLE CDXXII. Topology phase diagram of DyCu. 
[1] Qi Wang, Yuanfeng Xu, Rui Lou, Zhonghao Liu, Man Li, Yaobo Huang, Dawei Shen, Hongming Weng, Shancai Wang, and Hechang Lei, "Large intrinsic anomalous hall effect in half-metallic ferromagnet $\mathrm{Co}_{3} \mathrm{Sn}_{2} \mathrm{~S}_{2}$ with magnetic weyl fermions," Nature communications 9, 1-8 (2018).

[2] Enke Liu, Yan Sun, Nitesh Kumar, Lukas Muechler, Aili Sun, Lin Jiao, Shuo-Ying Yang, Defa Liu, Aiji Liang, Qiunan Xu, et al., "Giant anomalous hall effect in a ferromagnetic kagome-lattice semimetal," Nature physics 14, 1125-1131 (2018).

[3] MM Otrokov, II Klimovskikh, H Bentmann, D Estyunin, A Zeugner, ZS Aliev, S Gaß, AUB Wolter, AV Koroleva, AM Shikin, et al., "Prediction and observation of an antiferromagnetic topological insulator," Nature 576, 416-422 (2019).

[4] Barry Bradlyn, L. Elcoro, Jennifer Cano, M. G. Vergniory, Zhijun Wang, C. Felser, M. I. Aroyo, and B. Andrei Bernevig, "Topological quantum chemistry," Nature 547, 298-305 (2017)

[5] M. G. Vergniory, L. Elcoro, Claudia Felser, Nicolas Regnault, B. Andrei Bernevig, and Zhijun Wang, "A complete catalogue of high-quality topological materials," Nature 566, 480-485 (2019).

[6] Tiantian Zhang, Yi Jiang, Zhida Song, He Huang, Yuqing He, Zhong Fang, Hongming Weng, and Chen Fang, "Catalogue of topological electronic materials," Nature 566, 475-479 (2019).

[7] Luis Elcoro, Benjamin J Wieder, Zhida Song, Yuanfeng Xu, Barry Bradlyn, and B Andrei Bernevig, "Magnetic topological quantum chemistry," arXiv preprint arXiv:2010.00598 (2020).

[8] C. L. Kane and E. J. Mele, " $Z_{2}$ topological order and the quantum spin hall effect," Phys. Rev. Lett. 95, 146802 (2005).

[9] B Andrei Bernevig, Taylor L Hughes, and Shou-Cheng Zhang, "Quantum spin hall effect and topological phase transition in HgTe quantum wells," Science 314, 1757-1761 (2006).

[10] Haijun Zhang, Chao-Xing Liu, Xiao-Liang Qi, Xi Dai, Zhong Fang, and Shou-Cheng Zhang, "Topological insulators in $\mathrm{Bi}_{2} \mathrm{Se}_{3}, \mathrm{Bi}_{2} \mathrm{Te}_{3}$ and $\mathrm{Sb}_{2} \mathrm{Te}_{3}$ with a single dirac cone on the surface," Nature physics 5, 438-442 (2009).

[11] Timothy H Hsieh, Hsin Lin, Junwei Liu, Wenhui Duan, Arun Bansil, and Liang Fu, "Topological crystalline insulators in the SnTe material class," Nature communications 3, 982 (2012).

[12] A. Burkov and Leon Balents, "Weyl Semimetal in a Topological Insulator Multilayer," Physical Review Letters 107, $127205(2011)$

[13] Xiangang Wan, Ari M Turner, Ashvin Vishwanath, and Sergey Y Savrasov, "Topological semimetal and fermi-arc surface states in the electronic structure of pyrochlore iridates," Physical Review B 83, 205101 (2011).

[14] Gang Xu, Hongming Weng, Zhijun Wang, Xi Dai, and Zhong Fang, "Chern semimetal and the quantized anomalous hall effect in $\mathrm{HgCr}_{2} \mathrm{Se}_{4}, "$ Phys. Rev. Lett. 107, 186806 (2011).

[15] Zhijun Wang, Yan Sun, Xing-Qiu Chen, Cesare Franchini, Gang Xu, Hongming Weng, Xi Dai, and Zhong Fang, "Dirac semimetal and topological phase transitions in $\mathrm{A}_{3} \mathrm{Bi}(\mathrm{A}=\mathrm{Na}, \mathrm{K}, \mathrm{Rb})$," Physical Review B 85, 195320 (2012).

[16] Bohm-Jung Yang and Naoto Nagaosa, "Classification of stable three-dimensional Dirac semimetals with nontrivial topology," Nature Communications 5, 4898 (2014).

[17] Hongming Weng, Chen Fang, Zhong Fang, B Andrei Bernevig, and Xi Dai, "Weyl semimetal phase in noncentrosymmetric transition-metal monophosphides," Physical Review X 5, 011029 (2015).

[18] Shin-Ming Huang, Su-Yang Xu, Ilya Belopolski, Chi-Cheng Lee, Guoqing Chang, BaoKai Wang, Nasser Alidoust, Guang Bian, Madhab Neupane, Chenglong Zhang, et al., "A weyl fermion semimetal with surface fermi arcs in the transition metal monopnictide TaAs class," Nature communications 6, 7373 (2015).

[19] S. M. Young, S. Zaheer, J. C. Y. Teo, C. L. Kane, E. J. Mele, and A. M. Rappe, "Dirac semimetal in three dimensions," Phys. Rev. Lett. 108, 140405 (2012)

[20] Robert-Jan Slager, Andrej Mesaros, Vladimir Juričić, and Jan Zaanen, "The space group classification of topological band-insulators," Nature Physics 9, 98-102 (2013).

[21] Chao-Xing Liu, Rui-Xing Zhang, and Brian K. VanLeeuwen, "Topological nonsymmorphic crystalline insulators," Phys. Rev. B 90, 085304 (2014)

[22] Zhijun Wang, Aris Alexandradinata, Robert J Cava, and B Andrei Bernevig, "Hourglass fermions," Nature 532, 189-194 (2016).

[23] Benjamin J Wieder, Barry Bradlyn, Zhijun Wang, Jennifer Cano, Youngkuk Kim, Hyeong-Seok D Kim, Andrew M Rappe, CL Kane, and B Andrei Bernevig, "Wallpaper fermions and the nonsymmorphic dirac insulator," Science 361, $246-251$ (2018).

[24] Hoi Chun Po, Ashvin Vishwanath, and Haruki Watanabe, "Symmetry-based indicators of band topology in the 230 space groups," Nature communications 8, 50 (2017).

[25] Jorrit Kruthoff, Jan de Boer, Jasper van Wezel, Charles L. Kane, and Robert-Jan Slager, "Topological Classification of Crystalline Insulators through Band Structure Combinatorics," Physical Review X 7, 041069 (2017).

[26] Zhida Song, Tiantian Zhang, Zhong Fang, and Chen Fang, "Quantitative mappings between symmetry and topology in solids," Nature Communications 9, 3530 (2018)

[27] Eslam Khalaf, Hoi Chun Po, Ashvin Vishwanath, and Haruki Watanabe, "Symmetry indicators and anomalous surface states of topological crystalline insulators," Phys. Rev. X 8, 031070 (2018).

[28] Feng Tang, Hoi Chun Po, Ashvin Vishwanath, and Xiangang Wan, "Comprehensive search for topological materials using symmetry indicators," Nature 566, 486-489 (2019).

[29] Haruki Watanabe, Hoi Chun Po, and Ashvin Vishwanath, "Structure and topology of band structures in the 1651 magnetic space groups," Science advances 4, eaat8685 (2018). 
[30] Max Hirschberger, Satya Kushwaha, Zhijun Wang, Quinn Gibson, Sihang Liang, Carina A Belvin, Bogdan Andrei Bernevig, Robert Joseph Cava, and Nai Phuan Ong, "The chiral anomaly and thermopower of weyl fermions in the half-heusler GdPtBi," Nature materials 15, 1161-1165 (2016).

[31] Hao Yang, Yan Sun, Yang Zhang, Wu-Jun Shi, Stuart SP Parkin, and Binghai Yan, "Topological weyl semimetals in the chiral antiferromagnetic materials $\mathrm{Mn}_{3} \mathrm{Ge}$ and $\mathrm{Mn}_{3} \mathrm{Sn}$," New Journal of Physics 19, 015008 (2017).

[32] Hang Li, Shun-Ye Gao, Shao-Feng Duan, Yuan-Feng Xu, Ke-Jia Zhu, Shang-Jie Tian, Jia-Cheng Gao, Wen-Hui Fan, Zhi-Cheng Rao, Jie-Rui Huang, Jia-Jun Li, Da-Yu Yan, Zheng-Tai Liu, Wan-Ling Liu, Yao-Bo Huang, Yu-Liang Li, Yi Liu, Guo-Bin Zhang, Peng Zhang, Takeshi Kondo, Shik Shin, He-Chang Lei, You-Guo Shi, Wen-Tao Zhang, Hong-Ming Weng, Tian Qian, and Hong Ding, "Dirac surface states in intrinsic magnetic topological insulators EuSn $\mathrm{As}_{2}$ and $\mathrm{MnBi}_{2 n} \mathrm{Te}_{3 n+1}$, , Phys. Rev. X 9, 041039 (2019).

[33] DF Liu, AJ Liang, EK Liu, QN Xu, YW Li, C Chen, D Pei, WJ Shi, SK Mo, P Dudin, et al., "Magnetic weyl semimetal phase in a kagomé crystal," Science 365, 1282-1285 (2019).

[34] Ilya Belopolski, Kaustuv Manna, Daniel S Sanchez, Guoqing Chang, Benedikt Ernst, Jiaxin Yin, Songtian S Zhang, Tyler Cochran, Nana Shumiya, Hao Zheng, et al., "Discovery of topological weyl fermion lines and drumhead surface states in a room temperature magnet," Science 365, 1278-1281 (2019).

[35] Dongqin Zhang, Minji Shi, Tongshuai Zhu, Dingyu Xing, Haijun Zhang, and Jing Wang, "Topological axion states in the magnetic insulator $\mathrm{MnBi}_{2} \mathrm{Te}_{4}$ with the quantized magnetoelectric effect," Phys. Rev. Lett. 122, 206401 (2019)

[36] J.-R. Soh, F. de Juan, M. G. Vergniory, N. B. M. Schröter, M. C. Rahn, D. Y. Yan, J. Jiang, M. Bristow, P. Reiss, J. N. Blandy, Y. F. Guo, Y. G. Shi, T. K. Kim, A. McCollam, S. H. Simon, Y. Chen, A. I. Coldea, and A. T. Boothroyd, "Ideal weyl semimetal induced by magnetic exchange," Phys. Rev. B 100, 201102 (2019).

[37] Simin Nie, Gang Xu, Fritz B Prinz, and Shou-cheng Zhang, "Topological semimetal in honeycomb lattice LnSI," Proceedings of the National Academy of Sciences 114, 10596-10600 (2017).

[38] Jinyu Zou, Zhuoran He, and Gang Xu, "The study of magnetic topological semimetals by first principles calculations," npj Computational Materials 5, 1-19 (2019).

[39] Guiyuan Hua, Simin Nie, Zhida Song, Rui Yu, Gang Xu, and Kailun Yao, "Dirac semimetal in type-iv magnetic space groups," Phys. Rev. B 98, 201116 (2018).

[40] A.V. Shubnikov, N.V. Belov, W.T. Holser, and Institut kristallografii im. A.V. Shubnikova, Colored Symmetry (Macmillan, 1964).

[41] Benjamin J. Wieder, Zhijun Wang, Jennifer Cano, Xi Dai, Leslie M. Schoop, Barry Bradlyn, and B. Andrei Bernevig, "Strong and fragile topological dirac semimetals with higher-order fermi arcs," Nature Communications 11, 627 (2020).

[42] Yuanfeng Xu, Zhida Song, Zhijun Wang, Hongming Weng, and Xi Dai, "Higher-order topology of the axion insulator $\mathrm{EuIn}_{2} \mathrm{As}_{2}, "$ Phys. Rev. Lett. 122, 256402 (2019)

[43] Benjamin J Wieder and B Andrei Bernevig, "The axion insulator as a pump of fragile topology," arXiv preprint arXiv:1810.02373 (2018).

[44] Liang Fu and C. L. Kane, "Topological insulators with inversion symmetry," Phys. Rev. B 76, 045302 (2007)

[45] Frank Wilczek, "Two applications of axion electrodynamics," Physical review letters 58, 1799 (1987).

[46] Xiao-Liang Qi, Taylor L Hughes, and Shou-Cheng Zhang, "Topological field theory of time-reversal invariant insulators," Physical Review B 78, 195424 (2008).

[47] Roger S. K. Mong, Andrew M. Essin, and Joel E. Moore, "Antiferromagnetic topological insulators," Phys. Rev. B 81, 245209 (2010)

[48] Andrew M. Essin, Joel E. Moore, and David Vanderbilt, "Magnetoelectric polarizability and axion electrodynamics in crystalline insulators," Phys. Rev. Lett. 102, 146805 (2009)

[49] Taylor L. Hughes, Emil Prodan, and B. Andrei Bernevig, "Inversion-symmetric topological insulators," Phys. Rev. B 83, $245132(2011)$

[50] Ari M. Turner, Yi Zhang, Roger S. K. Mong, and Ashvin Vishwanath, "Quantized response and topology of magnetic insulators with inversion symmetry," Phys. Rev. B 85, 165120 (2012).

[51] Eslam Khalaf, "Higher-order topological insulators and superconductors protected by inversion symmetry," Phys. Rev. B 97, $205136(2018)$

[52] Cai-Zhen Li, An-Qi Wang, Chuan Li, Wen-Zhuang Zheng, Alexander Brinkman, Da-Peng Yu, and Zhi-Min Liao, "Reducing electronic transport dimension to topological hinge states by increasing geometry size of dirac semimetal josephson junctions," Phys. Rev. Lett. 124, 156601 (2020).

[53] Noam Morali, Rajib Batabyal, Pranab Kumar Nag, Enke Liu, Qiunan Xu, Yan Sun, Binghai Yan, Claudia Felser, Nurit Avraham, and Haim Beidenkopf, "Fermi-arc diversity on surface terminations of the magnetic weyl semimetal Co $\mathrm{Cn}_{2} \mathrm{~S}_{2}$," Science 365, 1286-1291 (2019).

[54] K Kuroda, T Tomita, M-T Suzuki, C Bareille, AA Nugroho, Pallab Goswami, M Ochi, M Ikhlas, M Nakayama, S Akebi, et al., "Evidence for magnetic weyl fermions in a correlated metal," Nature materials 16, 1090 (2017).

[55] Nicodemos Varnava, Ivo Souza, and David Vanderbilt, "Axion coupling in the hybrid wannier representation," Phys. Rev. B 101, 155130 (2020).

[56] Luis Elcoro, Barry Bradlyn, Zhijun Wang, Maia G. Vergniory, Jennifer Cano, Claudia Felser, B. Andrei Bernevig, Danel Orobengoa, Gemma de la Flor, and Mois I. Aroyo, "Double crystallographic groups and their representations on the Bilbao Crystallographic Server," Journal of Applied Crystallography 50, 1457-1477 (2017).

[57] Jennifer Cano, Barry Bradlyn, Zhijun Wang, L. Elcoro, M. G. Vergniory, C. Felser, M. I. Aroyo, and B. Andrei Bernevig, "Building blocks of topological quantum chemistry: Elementary band representations," Physical Review B 97, 035139 (2018). 
[58] M. G. Vergniory, L. Elcoro, Zhijun Wang, Jennifer Cano, C. Felser, M. I. Aroyo, B. Andrei Bernevig, and Barry Bradlyn, "Graph theory data for topological quantum chemistry," Phys. Rev. E 96, 023310 (2017).

[59] Zhida Song, Sheng-Jie Huang, Yang Qi, Chen Fang, and Michael Hermele, "Topological states from topological crystals," Science Advances 5 (2019), 10.1126/sciadv.aax2007.

[60] Luis Elcoro, Zhida Song, and B. Andrei Bernevig, "Application of induction procedure and smith decomposition in calculation and topological classification of electronic band structures in the 230 space groups," Phys. Rev. B 102, 035110 (2020).

[61] Wladimir A. Benalcazar, B. Andrei Bernevig, and Taylor L. Hughes, "Quantized electric multipole insulators," Science 357, 61-66 (2017)

[62] Frank Schindler, Ashley M. Cook, Maia G. Vergniory, Zhijun Wang, Stuart S. P. Parkin, B. Andrei Bernevig, and Titus Neupert, "Higher-order topological insulators," Science Advances 4, eaat0346 (2018).

[63] Frank Schindler, Zhijun Wang, Maia G. Vergniory, Ashley M. Cook, Anil Murani, Shamashis Sengupta, Alik Yu Kasumov, Richard Deblock, Sangjun Jeon, Ilya Drozdov, Hélène Bouchiat, Sophie Guéron, Ali Yazdani, B. Andrei Bernevig, and Titus Neupert, "Higher-order topology in bismuth," Nature Physics 14, 918-924 (2018).

[64] Zhida Song, Zhong Fang, and Chen Fang, "(d-2)-Dimensional Edge States of Rotation Symmetry Protected Topological States," Physical Review Letters 119, 246402 (2017).

[65] Josias Langbehn, Yang Peng, Luka Trifunovic, Felix von Oppen, and Piet W. Brouwer, "Reflection-Symmetric Second-Order Topological Insulators and Superconductors," Physical Review Letters 119, 246401 (2017).

[66] Hoi Chun Po, Haruki Watanabe, and Ashvin Vishwanath, "Fragile Topology and Wannier Obstructions," Physical Review Letters 121, 126402 (2018)

[67] Jennifer Cano, Barry Bradlyn, Zhijun Wang, L. Elcoro, M. G. Vergniory, C. Felser, M. I. Aroyo, and B. Andrei Bernevig, "Topology of Disconnected Elementary Band Representations," Phys. Rev. Lett. 120, 266401 (2018).

[68] Barry Bradlyn, Zhijun Wang, Jennifer Cano, and B. Andrei Bernevig, "Disconnected elementary band representations, fragile topology, and Wilson loops as topological indices: An example on the triangular lattice," Physical Review B 99, 045140 (2019)

[69] Junyeong Ahn, Sungjoon Park, and Bohm-Jung Yang, "Failure of Nielsen-Ninomiya Theorem and Fragile Topology in Two-Dimensional Systems with Space-Time Inversion Symmetry: Application to Twisted Bilayer Graphene at Magic Angle," Physical Review X 9, 021013 (2019)

[70] Zhi-Da Song, Luis Elcoro, Yuan-Feng Xu, Nicolas Regnault, and B. Andrei Bernevig, "Fragile phases as affine monoids: Classification and material examples," Phys. Rev. X 10, 031001 (2020).

[71] S. V. Gallego, J. M. Perez-Mato, L. Elcoro, E. S. Tasci, R. M. Hanson, K. Momma, M. I. Aroyo, and G. Madariaga, "MAGNDATA: towards a database of magnetic structures. I. The commensurate case," Journal of Applied Crystallography 49, 1750-1776 (2016).

[72] G. Kresse and J. Furthmüller, "Efficient iterative schemes for ab initio total-energy calculations using a plane-wave basis set," Phys. Rev. B 54, 11169-11186 (1996)

[73] Fabien Tran and Peter Blaha, "Accurate band gaps of semiconductors and insulators with a semilocal exchange-correlation potential," Phys. Rev. Lett. 102, 226401 (2009).

[74] XiaoYu Deng, Lei Wang, Xi Dai, and Zhong Fang, "Local density approximation combined with gutzwiller method for correlated electron systems: Formalism and applications," Phys. Rev. B 79, 075114 (2009)

[75] Jiacheng Gao, Quansheng Wu, Clas Persson, and Zhijun Wang, "Irvsp: to obtain irreducible representations of electronic states in the vasp," arXiv preprint arXiv:2002.04032 (2020).

[76] Nathan C Frey, Matthew K Horton, Jason M Munro, Sinéad M Griffin, Kristin A Persson, and Vivek B Shenoy, "High-throughput search for magnetic and topological order in transition metal oxides," arXiv preprint arXiv:2006.01075 (2020).

[77] Qiunan Xu, Zhida Song, Simin Nie, Hongming Weng, Zhong Fang, and Xi Dai, "Two-dimensional oxide topological insulator with iron-pnictide superconductor LiFeAs structure," Phys. Rev. B 92, 205310 (2015).

[78] Dongfei Wang, Lingyuan Kong, Peng Fan, Hui Chen, Shiyu Zhu, Wenyao Liu, Lu Cao, Yujie Sun, Shixuan Du, John Schneeloch, Ruidan Zhong, Genda Gu, Liang Fu, Hong Ding, and Hong-Jun Gao, "Evidence for majorana bound states in an iron-based superconductor," Science 362, 333-335 (2018)

[79] Steve M. Young and Benjamin J. Wieder, "Filling-enforced magnetic dirac semimetals in two dimensions," Phys. Rev. Lett. 118, $186401(2017)$

[80] Hoi Chun Po, Ashvin Vishwanath, and Haruki Watanabe, "Symmetry-based indicators of band topology in the 230 space groups," Nature Communications 8, 50 (2017).

[81] J Zak, "Band representations of space groups," Physical Review B 26, 3010 (1982).

[82] J Zak, "Berry's phase for energy bands in solids," Physical review letters 62, 2747 (1989).

[83] L Michel and J Zak, "Elementary energy bands in crystals are connected," Physics Reports 341, 377-395 (2001).

[84] Chen Fang, Matthew J. Gilbert, and B. Andrei Bernevig, "Bulk topological invariants in noninteracting point group symmetric insulators," Phys. Rev. B 86, 115112 (2012)

[85] Yoonseok Hwang, Junyeong Ahn, and Bohm-Jung Yang, "Fragile topology protected by inversion symmetry: Diagnosis, bulk-boundary correspondence, and wilson loop," Phys. Rev. B 100, 205126 (2019)

[86] Juan L. Mañes, "Fragile phonon topology on the honeycomb lattice with time-reversal symmetry," Phys. Rev. B 102, 024307 (2020)

[87] A. Alexandradinata, J. Höller, Chong Wang, Hengbin Cheng, and Ling Lu, "Crystallographic splitting theorem for band representations and fragile topological photonic crystals," arXiv:1908.08541 [cond-mat, physics:physics] (2019), arXiv: 
1908.08541.

[88] J.M. Perez-Mato, S.V. Gallego, E.S. Tasci, L. Elcoro, G. de la Flor, and M.I. Aroyo, "Symmetry-Based Computational Tools for Magnetic Crystallography," Annual Review of Materials Research 45, 217-248 (2015).

[89] Guoqing Chang, Su-Yang Xu, Benjamin J. Wieder, Daniel S. Sanchez, Shin-Ming Huang, Ilya Belopolski, Tay-Rong Chang, Songtian Zhang, Arun Bansil, Hsin Lin, and M. Zahid Hasan, "Unconventional chiral fermions and large topological fermi arcs in RhSi," Phys. Rev. Lett. 119, 206401 (2017)

[90] Jennifer Cano, Barry Bradlyn, and MG Vergniory, "Multifold nodal points in magnetic materials," APL Materials 7, 101125 (2019).

[91] Guoqing Chang, Benjamin J Wieder, Frank Schindler, Daniel S Sanchez, Ilya Belopolski, Shin-Ming Huang, Bahadur Singh, Di Wu, Tay-Rong Chang, Titus Neupert, et al., "Topological quantum properties of chiral crystals," Nature materials 17, 978 (2018).

[92] Ivo Souza, Nicola Marzari, and David Vanderbilt, "Maximally localized wannier functions for entangled energy bands," Physical Review B 65, 035109 (2001).

[93] MP Lopez Sancho, JM Lopez Sancho, JM Lopez Sancho, and J Rubio, "Highly convergent schemes for the calculation of bulk and surface green functions," Journal of Physics F: Metal Physics 15, 851 (1985).

[94] QuanSheng Wu, ShengNan Zhang, Hai-Feng Song, Matthias Troyer, and Alexey A. Soluyanov, "Wanniertools : An open-source software package for novel topological materials," Computer Physics Communications 224, 405 - 416 (2018)

[95] Feng Lu, JianZhou Zhao, Hongming Weng, Zhong Fang, and Xi Dai, "Correlated topological insulators with mixed valence," Phys. Rev. Lett. 110, 096401 (2013).

[96] Hongming Weng, Jianzhou Zhao, Zhijun Wang, Zhong Fang, and Xi Dai, "Topological crystalline kondo insulator in mixed valence ytterbium borides," Phys. Rev. Lett. 112, 016403 (2014).

[97] Yuanfeng Xu, Changming Yue, Hongming Weng, and Xi Dai, "Heavy weyl fermion state in CeRu $\mathrm{Sn}_{6}$, , Phys. Rev. X 7, $011027(2017)$

[98] K. Held, "Electronic structure calculations using dynamical mean field theory," Advances in Physics 56, 829-926 (2007), https://doi.org/10.1080/00018730701619647.

[99] Nicola Lanatà, Hugo U. R. Strand, Xi Dai, and Bo Hellsing, "Efficient implementation of the gutzwiller variational method," Phys. Rev. B 85, 035133 (2012).

[100] Y. X. Yao, C. Z. Wang, and K. M. Ho, "Including many-body screening into self-consistent calculations: Tight-binding model studies with the gutzwiller approximation," Phys. Rev. B 83, 245139 (2011)

[101] Nicodemos Varnava, Ivo Souza, and David Vanderbilt, "Axion coupling in the hybrid wannier representation," arXiv preprint arXiv:1912.11887 (2019).

[102] Wladimir A. Benalcazar, B. Andrei Bernevig, and Taylor L. Hughes, "Electric multipole moments, topological multipole moment pumping, and chiral hinge states in crystalline insulators," Physical Review B 96, 245115 (2017). 